\title{
DAILY LIFE IN THE LAND OF BAMBUK: AN ARCHAEOLOGICAL STUDY OF POLITICAL ECONOMY AT DIOUBOYE, SENEGAL
}

\author{
by \\ Cameron D. Gokee \\ A dissertation submitted in partial fulfillment \\ of the requirements for the degree of \\ Doctor of Philosophy \\ (Anthropology) \\ in The University of Michigan \\ 2012
}

Doctoral Committee:

Professor Carla M. Sinopoli, Co-Chair

Professor Henry T. Wright, Co-Chair

Professor Joyce Marcus

Assistant Professor Rudolph T. Ware

Curator Ibrahima Thiaw, Th. Monod Museum of African Art 
(C) Cameron D. Gokee 2012 
Á la communauté de la Maison Rose 


\section{ACKNOWLEDGEMENTS}

The doctoral research presented in the (many) pages that follow is the end result of a journey equally academic and personal. I am profoundly aware of and thankful for conversations with friends, helpful criticisms from colleagues, and perceptive comments from local villagers that have shaped my thoughts on archaeology in West Africa. So before delving into the nuances of political economy, settlement patterns, Mande history, and pottery sherd counts that inform my interpretations of daily life at Diouboye, I would like to express my gratitude to the people and institutions that have made this journey both possible and worthwhile.

As an undergraduate student at Case Western Reserve University from 1998 to 2002, I was fortunate to take several courses in anthropological archaeology with Dr. Jim Shaffer, who would prove to be an exceptional adviser. Not only did Dr. Shaffer encourage me to attend field school, but he also inspired me to pursue graduate studies and embark upon the journey inscribed in this dissertation. I also thank Dr. Brian Redmond for introducing me to archaeological method and theory and supervising my undergraduate research at the Cleveland Museum of Natural History. Field school with the University of Arizona in 2000 was a formative experience; I thank Dr. Barbara Mills, her TAs, and all the attendees of the Debitage Ball for teaching me how to both work and play hard at archaeology.

I owe my competence as a field archaeologist to participation on several projects over the past decade. While I have learned much from my fellow diggers and screeners, I am above all thankful to the field directors who showed me how to balance research design, logistics, and leadership with a good sense of humor-Lynn Evans (Mackinac State Historic Parks), Tom Levy (UC San Diego), and Steve Post (NM Office of Archaeological Studies). I also extend my thanks to Jim Anderson (North Island College) for schooling me in the art of ground survey and to Steve Lakatos (NM Office of 
Archaeological Studies) for teaching me that nothing straightens a unit profile like two cups of coffee and a Razorback shovel.

In Senegal, Hamady Bocoum (Direction du Patrimoine Culturel) has provided encouragement and steadfast support for my fieldwork at Diouboye, where he initially sent me in 2007. I am also indebted to Augustin Holl for organizing that first mission to Diouboye and other sites along the Falémé River in 2007, for generously loaning me field equipment and a vehicle in 2008, and for introducing me to "field conditions" in Senegambian archaeology.

Fieldwork in rural West Africa poses countless logistical challenges and I owe my sanity, and quite possibly my life, to Tamsir Maïga who accompanied me to Diouboye in 2007 and again in 2008. I offer my most profound gratitude to the students from IFANUCAD who joined me in 2008 and 2009—Matar Ndiaye, Aimé Kantoussan, and Massal Diagne-for haggling with taxi drivers, advising me on local customs, and working hard each day in the field despite the difficulties of rural living. The research presented in this dissertation simply would not have been possible without their companionship.

In the village of Sansanding, the family of Yero Diallo provided us with a place to stay in 2008 and 2009 and, more importantly, their endless hospitality. I warmly thank Samba Diallo, our guide to sites along the Falémé River in 2007, who became an invaluable field assistant in subsequent seasons. I also thank the several workmen from Sansanding who got up before dawn each morning and kept things moving in the field: Hassan, Ibrahima, Samba "Didi”, Amadou, and Hamed.

The Kantoussan family offered me their exceptional hospitality, which seems to extend across most of Senegal, when I was operating on a shoestring budget in 2009. I am most grateful to Magloire Kantoussan and his family who provided me with a place to live and work in Dakar, and to Stella Kantoussan who opened her home to me in Tambacounda.

I have dedicated this dissertation to the community of the Maison Rose in Dakar. Rozy Fredericks, David Ansari, Sarah Zimmerman, Abdou Mbodj, Allison Demas, Sophie Coly, Brandon County, Toby Warner, and Sarah Hardin, among many others, have deeply enriched my understanding of Senegal through conversations, excursions, and cuisine. This wonderful and remarkably fluid group of people embodies a community 
in the best possible sense, and I thank them for making Senegal my second home during my year as Fulbright fellow in 2007-2008.

Also in Dakar, the teranga of researchers and students in the archaeology lab at IFAN made several months of analysis both productive and enjoyable. First, I thank Seydou Camara for his tireless commitment to helping students such as myself search out field reports and long-forgotten boxes of sherds amid the labyrinth of the IFAN collections. Second, I thank director Ibrahima Thiaw for offering constructive criticisms and possible directions for my research at all stages; this dissertation has benefitted enormously from his guidance as a member of my committee. I also thank Ndèye Sokhna Guèye for answering my countless questions about pottery production in Senegambia, and Abdoulaye Camara for speaking with me at length about his experiences at Diouboye and other sites along the Falémé River. Finally, I thank the students at IFAN who warmly welcomed me into their community over the past several years: Djidere Baldé, Fatoumata Camara, Mamadou Diallo, Michel Diouf, Babacar Faye, Demba Kébé, Abdoulaye Mbaye, and Amy Collé Seck, among many others.

Within and beyond the walls of IFAN, I thank François Richard for his advice on analyzing materials of European origin and for inspiring me through his own research on archaeological landscapes in Senegambia. My appreciation goes also to Marilee Wood for offering her expert opinion on the glass beads recovered from Diouboye. I thank Susan McIntosh and Anne Haour for patiently responding to emails about questions that arose in the course of pottery analysis in 2008-2009; Susan also generously sent me portions of the monograph she is publishing with Roderick McIntosh and Hamady Bocoum on the results of their work along the middle Senegal River. I am, of course, solely responsible for any lingering errors in the analysis or interpretation of materials in this dissertation.

I am especially grateful to Marie-José and Howard Opper, who wrote up an excellent report on their findings from fieldwork at Diouboye in 1990 and patiently answered my questions about the site over email-the research presented in the following pages has benefitted tremendously from their pioneering work.

The main funding for fieldwork in Senegal (2007-2010) came from a Fulbright IIE fellowship and a National Science Foundation Dissertation Improvement Grant 
(BCS-1038733). At the University of Michigan, this research received support from a Rackham International Research Award (International Institute), an African Initiative Grant (Department of Afroamerican and African Studies), an Anthropology Dissertation Grant (Department of Anthropology), and the James B. Griffin and Graduate Radiocarbon Years Before Present Funds (Museum of Anthropology). I thank the Rackham School of Graduate Studies and the College of Literature, Science, and the Arts for supporting my graduate studies at the University of Michigan through fellowships and employment opportunities over the past eight years.

At the University of Michigan, I thank first and foremost my committee members in the Department of Anthropology_Carla Sinopoli, Henry Wright, and Joyce Marcus_for their unwavering support of my research in Senegal and for their critical readings of the words and thoughts presented in the pages that follow. I also thank Butch Ware, who joined my committee from the Department of History, for discussing ways to draw upon the rich historiography of Senegambia, and for helping me stay excited about my research over the past two years. Beyond this dissertation research, my thoughts on archaeology have been greatly shaped through courses and conversations with many other faculty at the University of Michigan, particularly Kent Flannery, John O’Shea, John Speth, Bob Whallon, and Norm Yoffee. From the Department of Afroamerican and African Studies, I thank Howard Stein, Martin Murray, and students in the Winter 2012 “capstone” seminar for providing a forum to work through the some of the ideas in this dissertation.

On a more personal note, I have been everyday thankful for my fellow students in the Department of Anthropology. From day one, I have found incredible support from my cohort in the archaeology program—Khori Newlander, Amy Nicodemus, and Uthara Suvrathan. Over the past eight years, my understanding of archaeology, among other things, has grown through conversations, contests, and conference road trips with Casey Barrier, Veronique Belisle, Lacey Carpenter, Jamie Clark, Allison Davis, Paul Duffy, Matt Gallon, Ryan Hughes, Matt Kroot, Dan Pugh, Colin Quinn, and Howard Tsai, among many, many others. Moreover, the cadre of students working in Africa has formed a community of its own; my knowledge of and approach to archaeology in West Africa continues to grow through conversations over gin and tonics with Anne Compton, Andy 
Gurstelle, and Amanda Logan. I especially thank Amanda for surviving that first journey to Sansanding in 2007; I wish I could say the same for the fuel tank on the Land Rover.

Two alumni of the African archaeology community—Stephen Dueppen and Daphne Gallagher-deserve special mention as my informal mentors for most of my time at the University of Michigan. From reading proposal drafts, to advising field methods, to sending me care packages of cookies and coffee, Stephen and Daphne have offered constant support, even as they continue to challenge me to grow as a scholar. And I greatly appreciate their collaboration on the analysis of faunal and macrobotanical remains from Diouboye presented in the appendices to this dissertation.

On an even more personal note, I am deeply grateful for the love and high-caliber editorship of Alice Wright; I could not have written this dissertation without her partnership and the much-needed entertainment of our cats Little Dude and Pisgah. Finally, I thank all of my friends and family outside of academe for their unconditional support over the past decade. My father Dean and my sister Corianne have encouraged me to pursue my interests, wherever they might take me, and the spirit of my mother Barbara has been with me every step of the way. 


\section{TABLE OF CONTENTS}

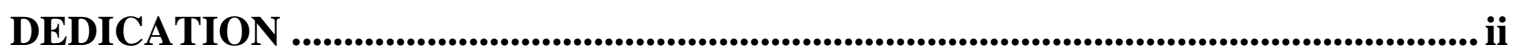

ACKNOWLEDGEMENTS ..............................................................................ii

LIST OF FIGURES

LIST OF TABLES ............................................................................................................. xxii

LIST OF APPENDICES …..............................................................................

CHAPTER 1. IN SEARCH OF BAMBUK............................................................. 1

POLITICAL ECONOMY OF VILLAGE COMMUNITIES …………........................ 6

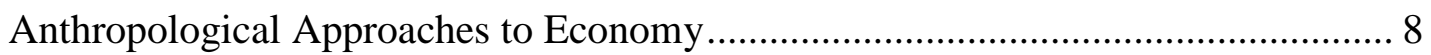

Social Institutions and Scale ......................................................................... 9

Village Communities ..................................................................................... 10

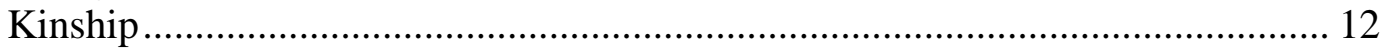

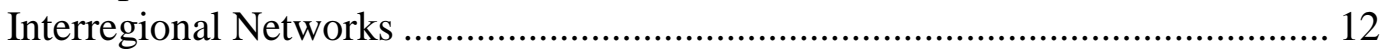

West African Historiography ................................................................................. 13

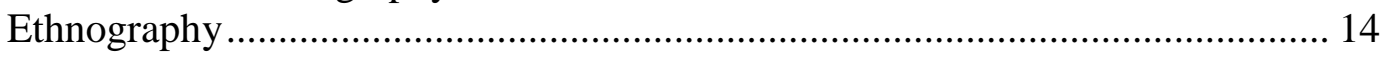

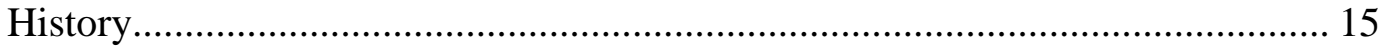

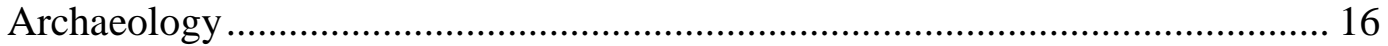

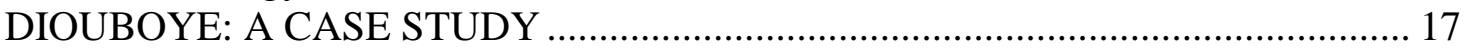

History of Archaeological Research .................................................................. 19

Central Falémé Archaeological Project (CFAP)....................................................... 20

Research Questions ................................................................................... 22

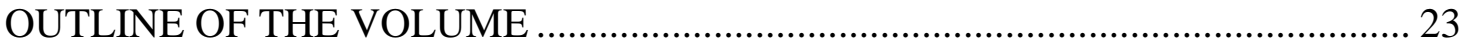

\section{CHAPTER 2. IRON AGE ECONOMICS IN WEST AFRICA:} ETHNOGRAPHIC AND HISTORICAL PERSPECTIVES............ 25

IRON AGE ECONOMICS ………………………........................................ 28

Economic Specialization: Locational and Appropriational Movements .................... 29

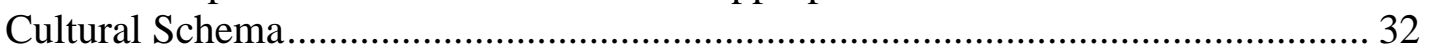

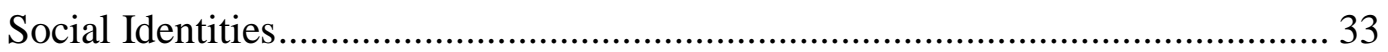

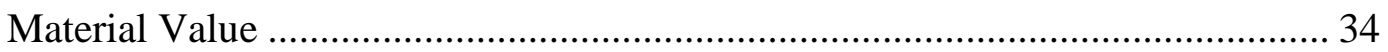

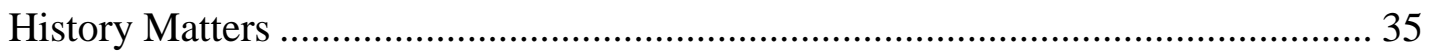


A VIEW FROM PRECOLONIAL WEST AFRICA.................................................. 35

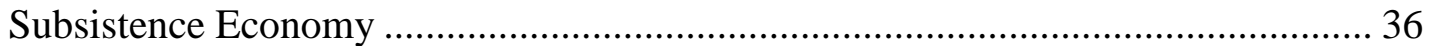

The Kin-Ordered Mode of Production................................................................... 36

Coordination and Cooperation (and Conflict) ..................................................... 38

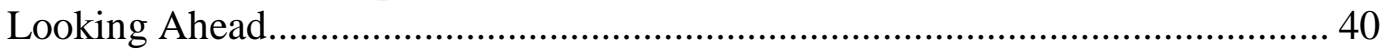

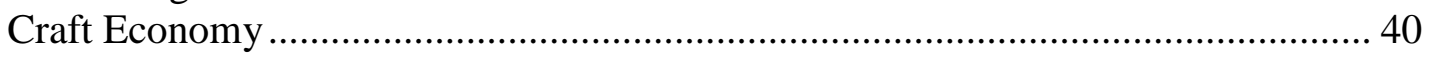

Sudanian Caste Systems …………………………….................................. 41

Crafting History ............................................................................................ 44

Looking Ahead.................................................................................................. 45

Long-Distance Exchange ............................................................................................ 45

Social Networks (Down-The-Line Exchange).................................................... 46

Gift-Giving ................................................................................................. 46

Bride Wealth and Inheritance ......................................................................... 47

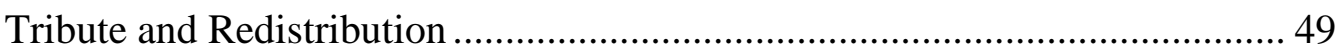

Market Exchange ………………………..................................................... 49

Merchant Communities and Trade...................................................................... 50

Looking Ahead........................................................................................... 51

ARCHAEOLOGY OF POLITICAL ECONOMY AT DIOUBOYE ........................... 51

Materiality .................................................................................................... 53

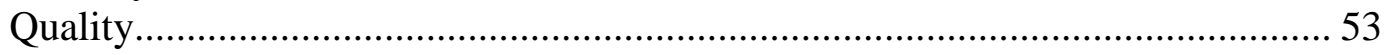

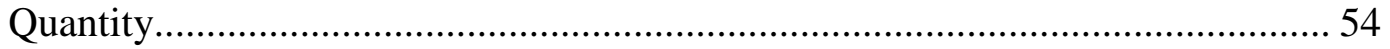

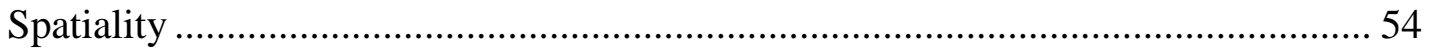

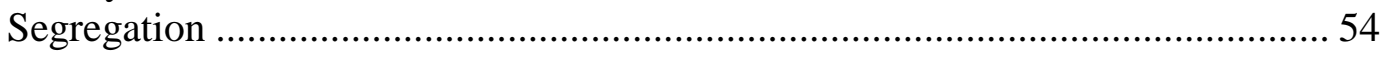

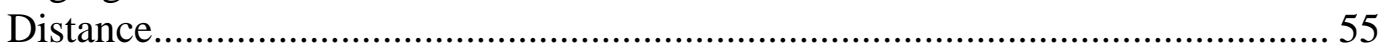

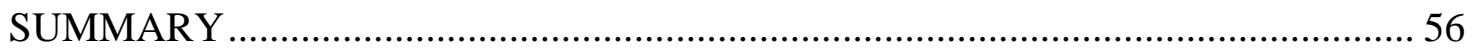

CHAPTER 3. GEOGRAPHY OF THE UPPER SENEGAL REGION................... 58

LANDSCAPE: A CONCEPTUAL FRAMEWORK............................................... 59

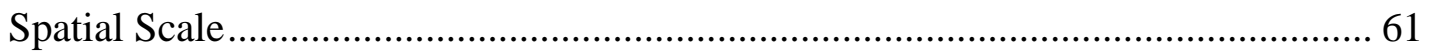

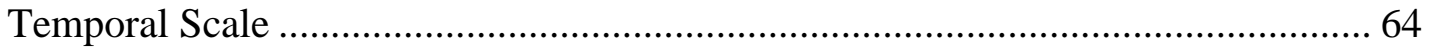

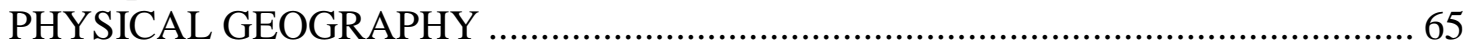

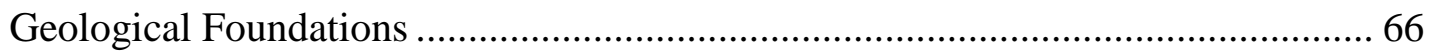

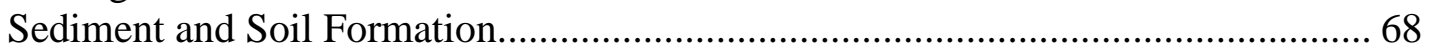

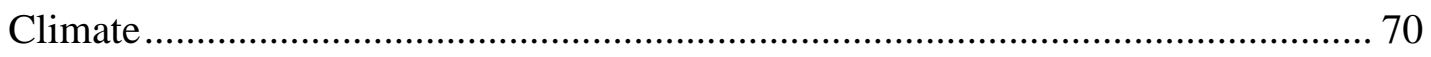

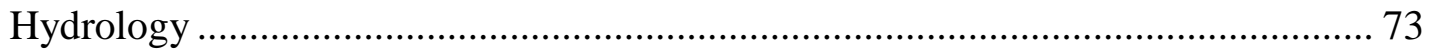

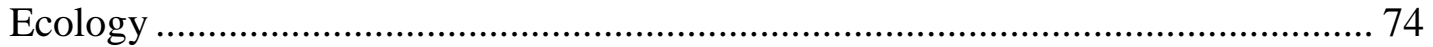

Holocene Trends in Climate and Ecology .............................................................. 79

HUMAN ECOLOGY IN THE WEST AFRICAN SAVANNA ………………............. 82

Subsistence Regimes......................................................................................... 82

Storage and Social Capital ............................................................................... 84

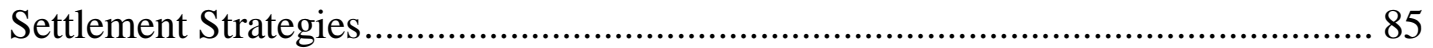

Labor Pooling and Scheduling................................................................................ 86

Ritual and Social Memory ………………........................................................... 87

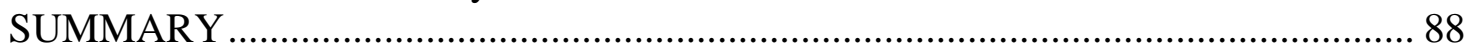


CHAPTER 4. DIOUBOYE AND THE HISTORICAL LANDSCAPE...................... 89

ARCHAEOLOGICAL LANDSCAPES IN WEST AFRICA ………………………........ 89

Mobility and Sedentism ...................................................................................... 90

Relative Mobility and the Archaeological Landscape ............................................. 92

Political Economy ……………………….............................................................. 93

Political Economy and the Archaeological Landscape......................................... 96

Ideology and Representation................................................................................... 97

MADINA-SADATOU: A LANDSCAPE IN THE LONGUE-DURÉE ........................ 98

Late Acheulean and Middle Stone Age (MSA) ........................................................ 100

Late Acheulean and MSA Components in the Madina-Sadatou Study Area ..... 101

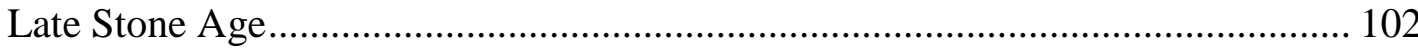

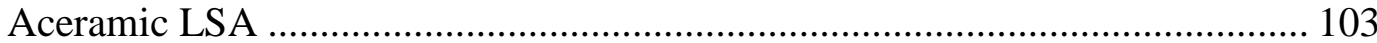

Ceramic LSA (cLSA) in the Sahara and Sahel ................................................. 104

Ceramic LSA (cLSA) in the Savanna and Equatorial Forest ............................. 107

LSA Components in the Madina-Sadatou Study Area ....................................... 111

Iron Age ....................................................................................................... 117

Early Iron Age (500 BC - AD 500): Food, Metals, and Villages ......................... 118

Late Iron Age (AD 500-1500): Pathways to Complexity.................................... 120

Iron Age Occupations in (and around) the Upper Senegal Region ..................... 124

An Historical Sketch of the Upper Senegal (AD 800-1500) ............................ 125

Archaeological Landscapes and Ceramic Spheres ......................................... 127

Iron Age Components in the Madina-Sadatou Study Area ................................. 132

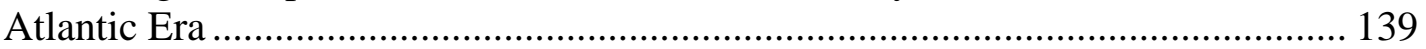

An Historical Sketch of the Upper Senegal Region (AD 1500-1905)................ 140

Archaeological Landscapes and Ceramic Ethnoarchaeology ............................. 145

Atlantic Era Components in the Madina-Sadatou Study Area ............................. 146

Tontèko Phase (AD 1500-1700) ................................................................ 151

Tomboura Phase (AD 1700-1860).............................................................. 152

Karé Phase (AD 1860-1925)...................................................................... 153

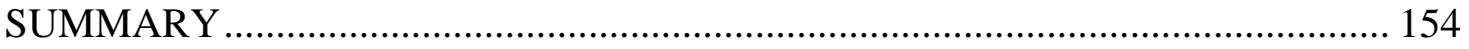

CHAPTER 5. DAILY LIFE IN DIOUBOYE: THE LOCAL LANDSCAPE....... 157

COMPARATIVE LANDSCAPE DYNAMICS IN MADINA-SADATOU............... 157

Geography and Resources.................................................................................. 157

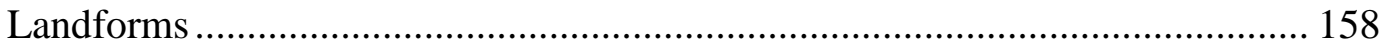

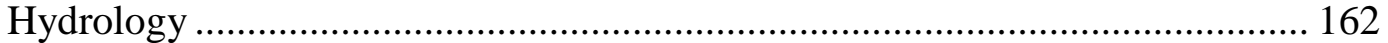

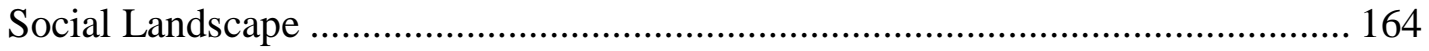

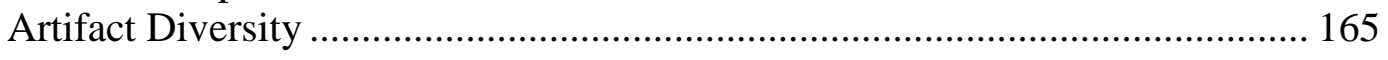

Rank-Size Distribution.................................................................................. 167

Inter-Site Proximity and Segregation................................................................ 169

SUMMARY: DIOUBOYE AND THE POLITICAL (ECONOMIC) LANDSCAPE171

CHAPTER 6. DAILY LIFE IN DIOUBOYE:

ARCHITECTURE AND ACTIVITIES............................................. 173

FROM PRESENT DATA TO PAST BEHAVIOR ................................................. 174

Site Formation: Material and Spatial Dimensions ................................................... 174 


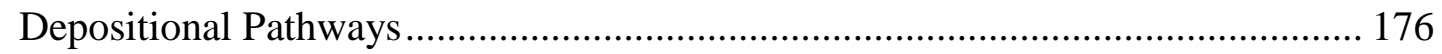

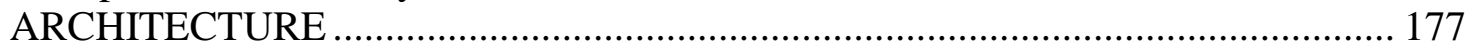

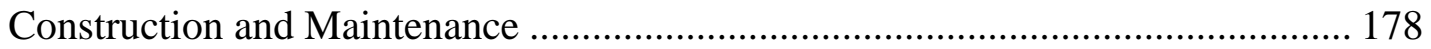

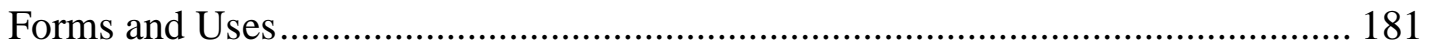

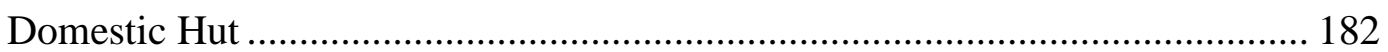

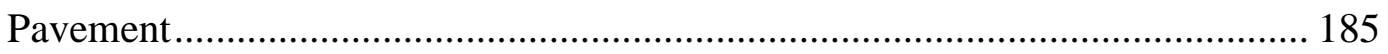

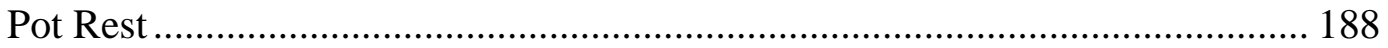

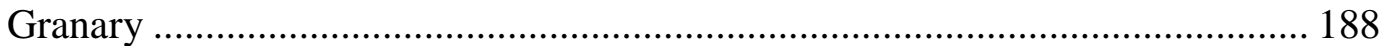

Other Unfilled Features: Possible Granaries................................................. 189

Small Filled Feature................................................................................. 189

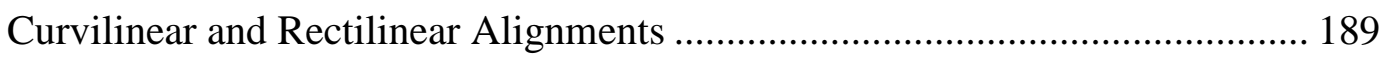

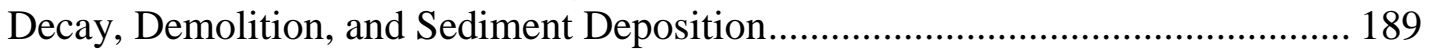

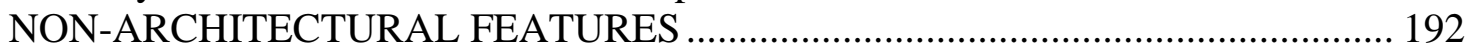

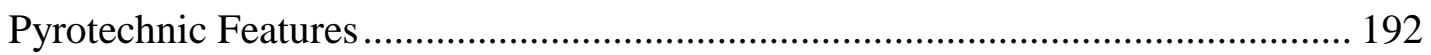

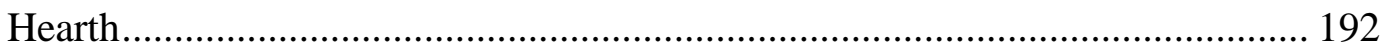

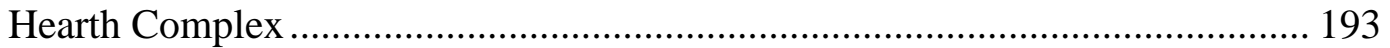

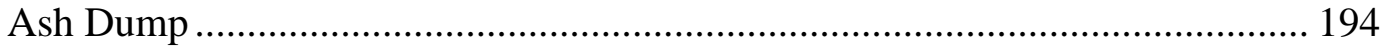

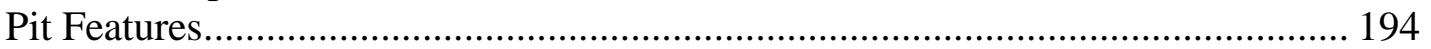

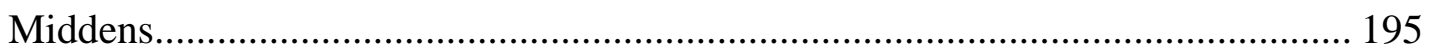

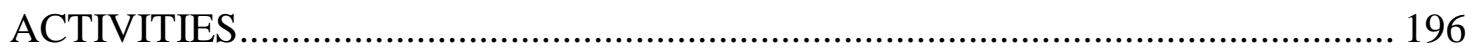

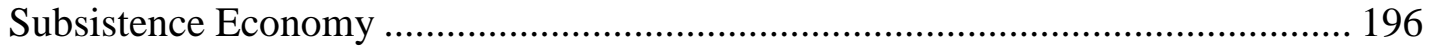

Food Production and Procurement.................................................................. 196

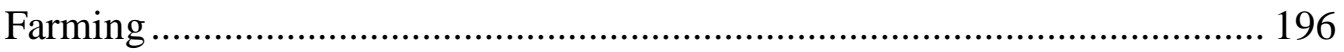

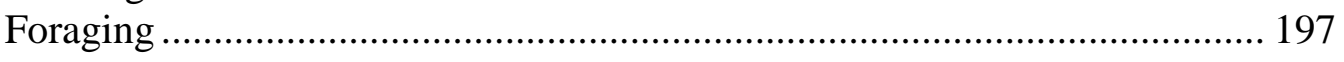

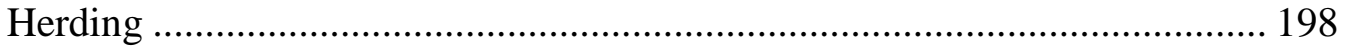

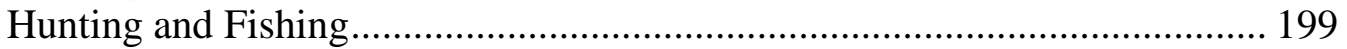

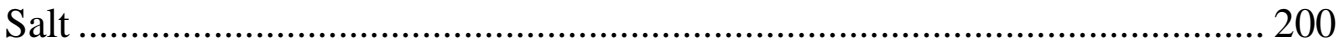

Food Preparation and Consumption............................................................ 200

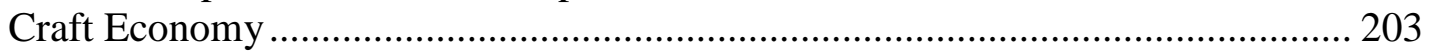

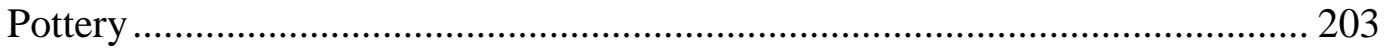

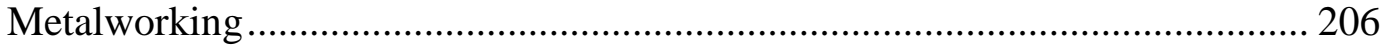

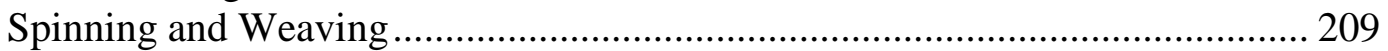

Chipped Stone Reduction and Use ............................................................... 209

Groundstone Manufacture and Use ........................................................... 211

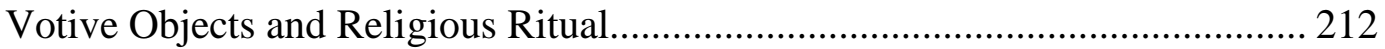

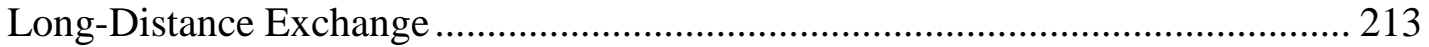

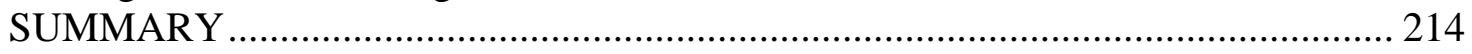

\section{CHAPTER 7. RESIDENTIAL AND NON-RESIDENTIAL AREAS:} DEPOSITION AND HISTORIES OF DAILY LIFE ...................... 215

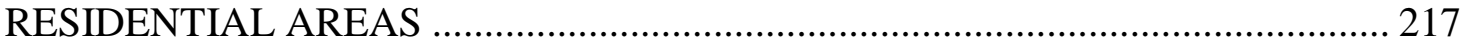

Residential Area A (Unit A1) .......................................................................... 217

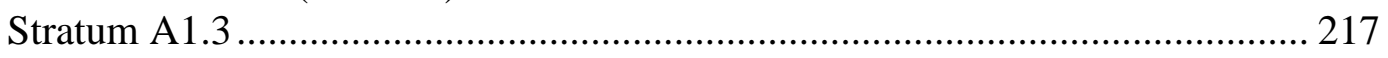

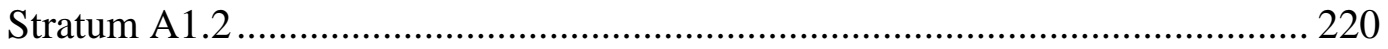

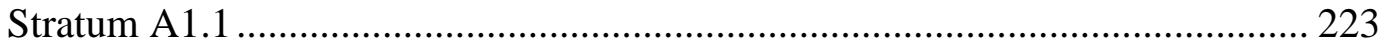

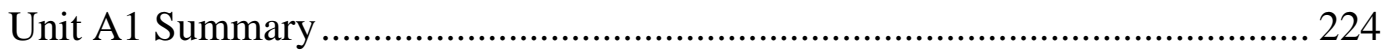


Architecture and Space ................................................................................ 224

Artifacts, Organic Remains, and Material Practice ………………………..... 225

Residential Area A (Unit A2) .................................................................. 228

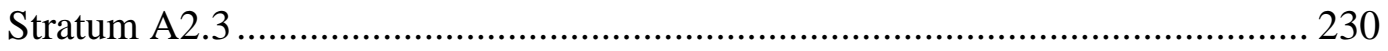

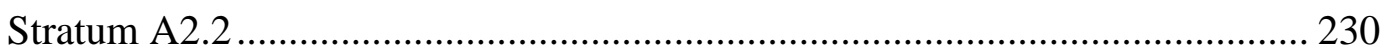

Stratum A2.1 ……............................................................................ 234

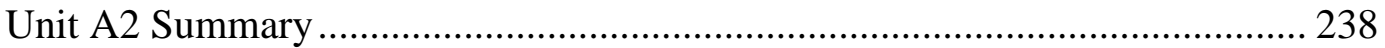

Architecture and Space ............................................................................. 238

Artifacts, Organic Remains, and Material Practice ……………………….... 238

Residential Area F (Unit F)......................................................................... 243

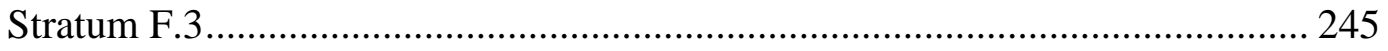

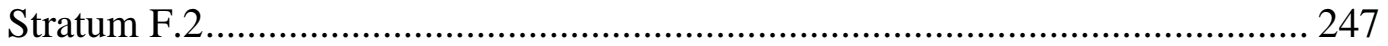

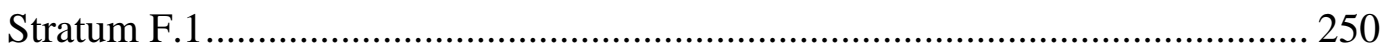

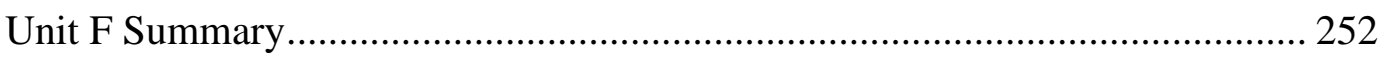

Architecture and Space ………………………………............................ 252

Artifacts, Organic Remains, and Material Practice ........................................ 252

Residential Area G (Unit G) ........................................................................ 255

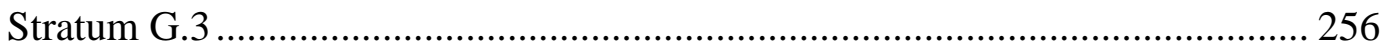

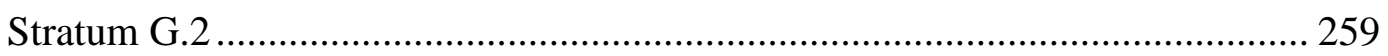

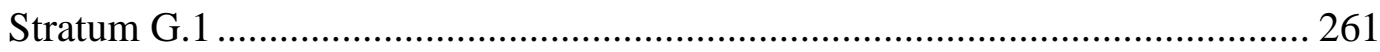

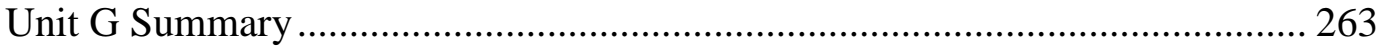

Architecture and Space ............................................................................... 263

Artifacts, Organic Remains, and Material Practice ………............................. 264

Residential Area H (Unit H) .......................................................................... 267

Stratum H.4 (Feature 28) ............................................................................ 269

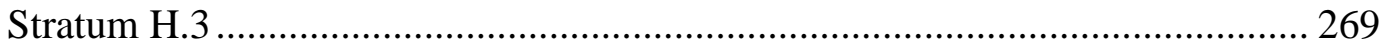

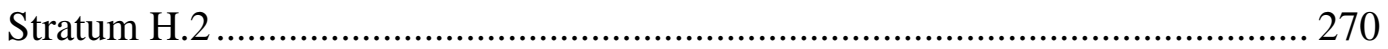

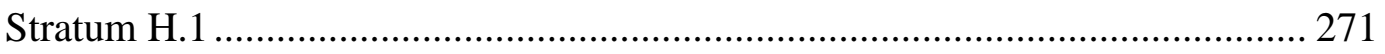

Unit H Summary ………….................................................................................. 273

Architecture and Space ............................................................................ 273

Artifacts, Organic Remains, and Material Practice ........................................ 273

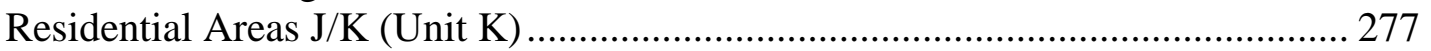

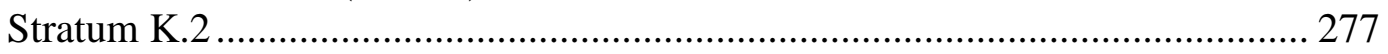

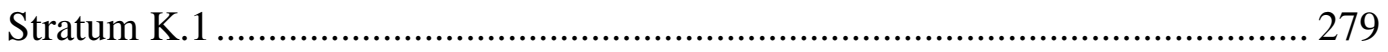

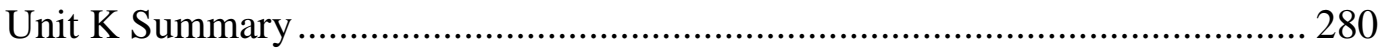

Architecture and Space …………………………………...................... 280

Artifacts, Organic Remains, and Material Practice ……………………….... 280

Residential Area N (Unit N) .......................................................................... 282

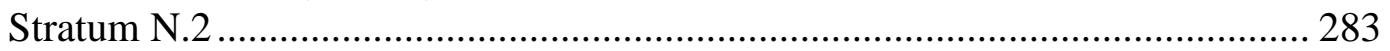

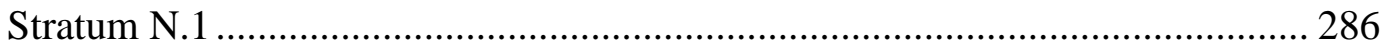

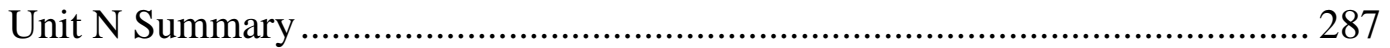

Architecture and Space …………………………………........................... 287

Artifacts, Organic Remains, and Material Practice ……………..................... 287

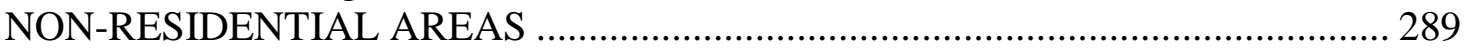

Area C Macrolithic Scatter (Unit C)................................................................ 289

Area S Cairns (Unit S) ..................................................................................... 292 
Area L Cairns (Trench L) .................................................................................... 294

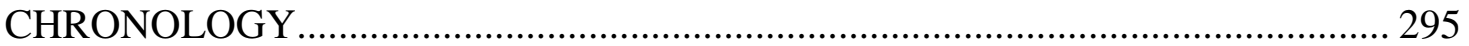

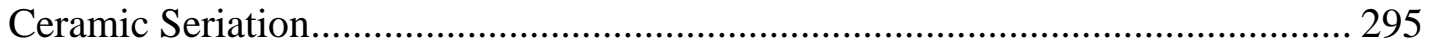

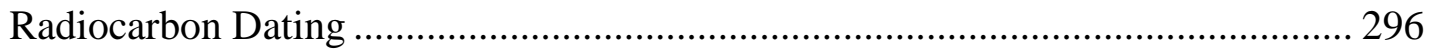

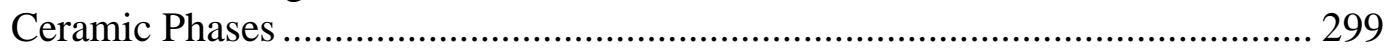

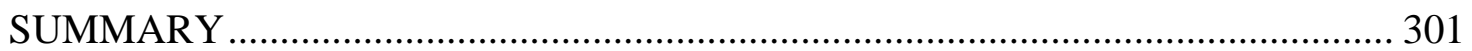

\section{CHAPTER 8. RESIDENTIAL WARDS: \\ SPATIAL ORDERING OF THE VILLAGE ECONOMY ............. 302}

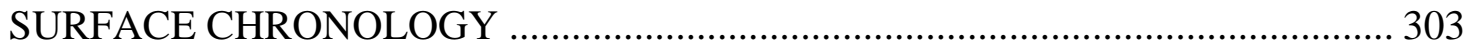

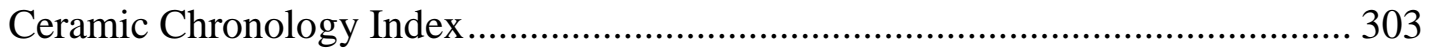

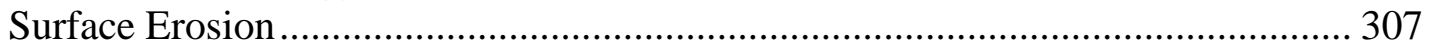

Surface Chronology of Intra-Site Areas ................................................................ 309

SPATIAL DIMENSIONS OF THE VILLAGE ECONOMY …................................ 310

Activity Areas .............................................................................................. 310

Intra-Site Areas and Wards ................................................................................. 313

Subsistence Economy: Food Preparation and Consumption ................................. 314

Craft Economy ………................................................................................... 319

Pottery Production ……………………………………............................... 319

Chipped Stone Production and Use ............................................................... 323

Groundstone Axes................................................................................ 328

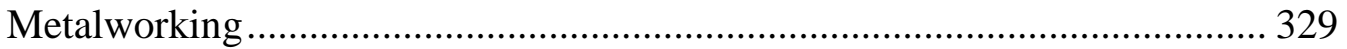

Long-Distance Exchange ............................................................................ 330

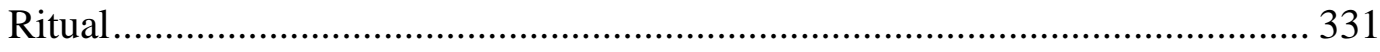

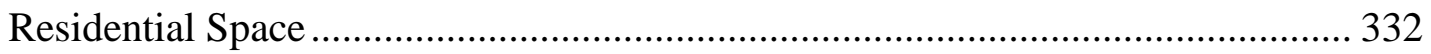

Estimating Population .................................................................................. 332

Architecture and the Production of Space ....................................................... 333

Activities and Formal Space ............................................................................ 336

Granaries: Subsistence Economy (Storage) and Social Segmentation ............. 336

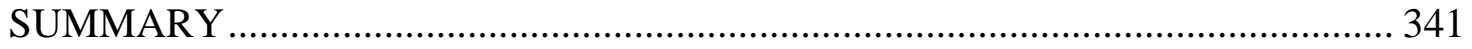

CHAPTER 9. TIME, SPACE, AND COMMUNITY IN BAMBUK ...................... 343

DIOUBOYE: HISTORY OF A VILLAGE COMMUNITY …………..................... 344

Early Diouboye A/B Phases (AD 950-1100).......................................................... 344

Space and Architecture ............................................................................. 344

Subsistence Economy ……………….......................................................... 346

Craft Production and Consumption .................................................................... 347

Long-Distance Exchange ............................................................................... 348

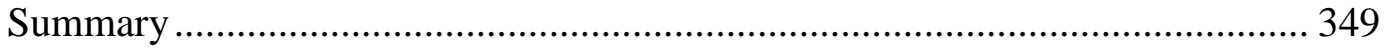

Middle Diouboye Phase (AD 1100-1250) ........................................................... 351

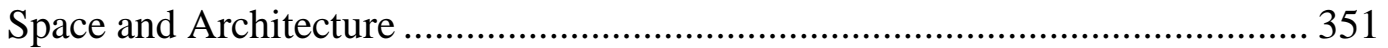

Subsistence Economy ………………………………................................. 352

Craft Production and Consumption ................................................................. 354

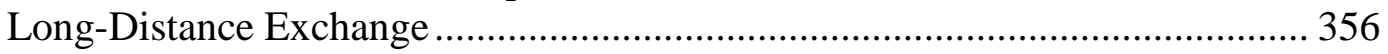

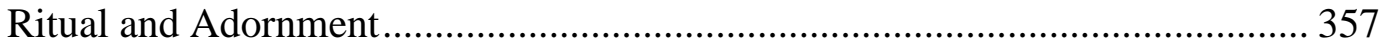

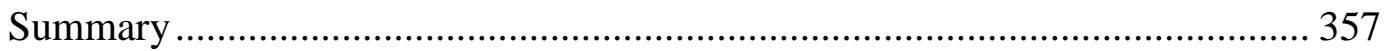




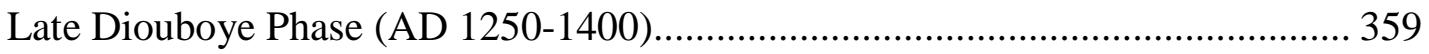

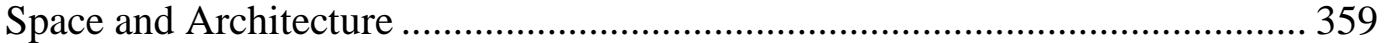

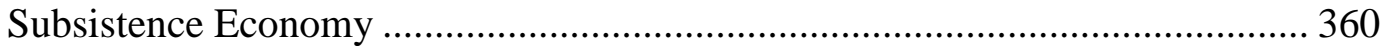

Craft Production and Consumption ............................................................. 362

Long-Distance Exchange .......................................................................... 363

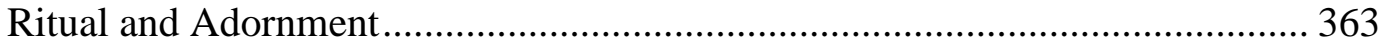

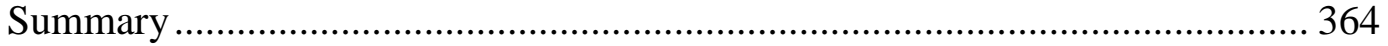

DIOUBOYE AND POLITICAL ECONOMY IN IRON AGE WEST AFRICA ...... 366

Economics of Community Life.................................................................. 366

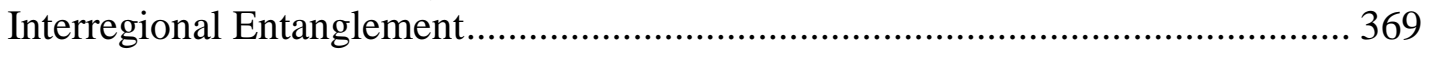

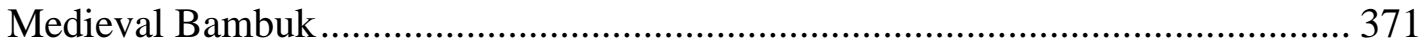

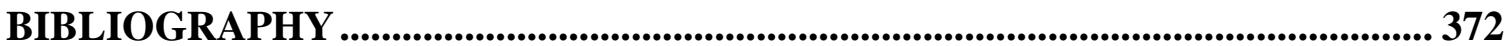




\section{LIST OF FIGURES}

Figure 1.1: $\quad$ Map of medieval West Africa.................................................................... 4

Figure 1.2: $\quad$ Map of Diouboye showing residential areas and wards ........................... 11

Figure 2.1: $\quad$ Schematic model of concepts and processes of political economy ........... 31

Figure 2.2: $\quad$ Conceptual map of links between material and spatial dimensions of the archaeological record and past political-economic processes.............. 52

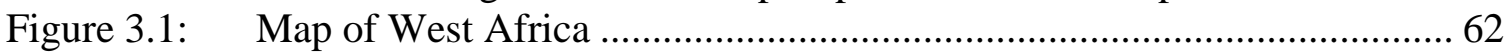

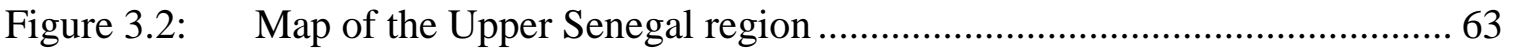

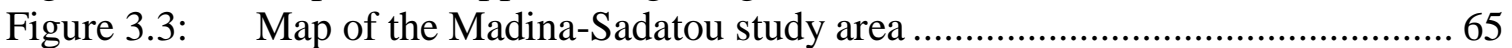

Figure 3.4: $\quad$ Map of geological units in West Africa...................................................67 67

Figure 3.5: Flood recession garden on east bank of Falémé River near Tomboura .... 70

Figure 3.6: $\quad$ Map of rainfall isohyets across West Africa............................................. 71

Figure 3.7: Interannual variability around mean precipitation across West Africa .... 72

Figure 3.8: Mean monthly distribution of precipitation at $900 \mathrm{~mm}$ isohyet in the Upper Senegal region............................................................................ 72

Figure 3.9: $\quad$ Monthly flow of the Falémé River .......................................................... 74

Figure 3.10: Map of major bioclimatic zones in West Africa......................................... 76

Figure 3.11: Holocene climate trends in the West African savanna ............................... 80

Figure 4.1: $\quad$ Map of the Madina-Sadatou study area showing sites identified through guided survey and the 2008 pedestrian survey zone .................... 99

Figure 4.2: Late Acheulean, MSA, and/or aceramic LSA chipped stone artifacts

from Madina-Sadatou ......................................................................... 102

Figure 4.3: $\quad$ LSA chipped stone artifacts ................................................................. 112

Figure 4.4: $\quad$ FAL-1042 (LSA encampment) looking east down to Falémé River....... 112

Figure 4.5: Ceramic Late Stone Age (cLSA) ceramic and groundstone artifacts ..... 113

Figure 4.6: Ceramic LSA pottery sherd types and vessel forms ................................ 114

Figure 4.7: Late Acheulean, MSA/LSA, and cLSA sites in the pedestrian survey

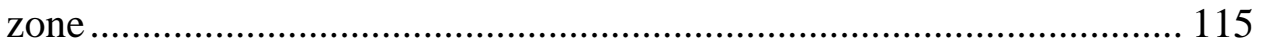

Figure 4.8: $\quad$ Map of medieval states in West Africa....................................................... 121

Figure 4.9: Iron Age (Diouboye phase) pottery from surveyed sites ......................... 133

Figure 4.10: $\quad$ Iron Age (Early/Middle Diouboye phase) sites in the pedestrian survey zone ........................................................................................... 135

Figure 4.11: Iron Age (Middle/Late Diouboye phase) sites in the pedestrian survey zone .......................................................................................... 136

Figure 4.12: Iron Age (Diouboye phase) settlement size distribution.......................... 139

Figure 4.13: Map of Senegambian polities and populations during the Atlantic Era . 141

Figure 4.14: $\quad$ Map of the lower Falémé River in the early-18 ${ }^{\text {th }}$ century AD ................ 143

Figure 4.15: Sample of beads from Karé (FAL-1038) ............................................... 147 
Figure 4.16: Atlantic Era miscellaneous artifacts....................................................... 148

Figure 4.17: Toranga (FAL-1056) showing rectilinear granary foundations and granary from present-day hamlet .......................................................... 148

Figure 4.18: Atlantic Era pottery from surveyed sites ................................................ 149

Figure 4.19: Atlantic Era settlement site size distribution ........................................... 150

Figure 4.20: Atlantic Era sites in the pedestrian survey zone ...................................... 153

Figure 5.1: Thiessen-adjusted catchment zones for Iron Age settlement sites........... 160

Figure 5.2: $\quad$ Regression of sedimentary terrace area by settlement size...................... 161

Figure 5.3: Boxplot of distances to the Falémé River for Iron Age and Atlantic

Era settlement classes ......................................................................... 164

Figure 5.4: Boxplot of jar-to-pot ratio for Iron Age and Atlantic Era settlement

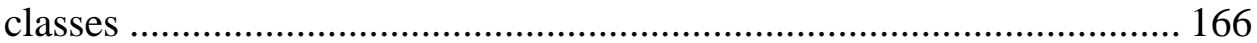

Figure 5.5: $\quad$ Rank-size plot for Iron Age settlement sites in the survey area .............. 168

Figure 5.6: $\quad$ Rank-size plot for Atlantic Era settlement sites in survey area .............. 168

Figure 5.7: Coefficient $A$ values and confidence intervals for settlement rank-

size distributions across horizons and phases ......................................... 169

Figure 6.1: $\quad$ Profile of terre pisé wall and room interior (Feature 71) .......................... 179

Figure 6.2: $\quad$ Profile of wattle-and-daub wall and room interior (Feature 106)........... 180

Figure 6.3: $\quad$ Profile of granary foundation with melt/collapse from an overlying earthen wall structure (Feature 102) ......................................................... 181

Figure 6.4: Distribution of the maximum dimensions of unfilled stone features and earthen wall foundations ................................................................. 184

Figure 6.5: Distribution of the maximum dimensions of filled stone features.......... 184

Figure 6.6: $\quad$ Wattle-and-daub hut, Sansanding, Senegal ............................................ 186

Figure 6.7: Concrete pavement and stone pot rest, Sansanding, Senegal .................. 186

Figure 6.8: $\quad$ Surface of Area $\mathrm{H}$ showing range of stone feature types......................... 187

Figure 6.9: $\quad$ Profile of hearth complexes with oxidized horizons and ash lenses

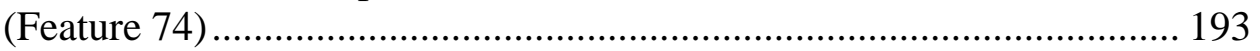

Figure 6.10: Profile of cylindrical pit (Feature 82) ………………............................ 195

Figure 6.11: Possible farming implements................................................................ 197

Figure 6.12: Contemporary herd of cattle near Sansanding, Senegal ......................... 198

Figure 6.13: Hunting and fishing equipment........................................................... 199

Figure 6.14: Vessel types from Diouboye .............................................................. 202

Figure 6.15: Grit temper showing granitic rock nodules from Area A (Unit A2); and coarse grain inclusion (5X) in pottery sherd from Diouboye .......... 204

Figure 6.16: Examples of hand wheels from Area A (Unit A2) at Diouboye; and in contemporary use at the village of Ngayène Sabakh, Senegal ........... 204

Figure 6.17: Carved chevron roulette decoration common to Diouboye pottery........ 205

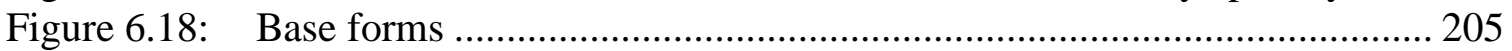

Figure 6.19: $\quad$ Possible forge in Area A (Unit A, Feature 14) ........................................ 207

Figure 6.20: Iron and copper artifacts made by diverse techniques ............................. 208

Figure 6.21: Crucible with possible copper prill from Area A (Unit A2).................... 208

Figure 6.22: Chipped stone tools........................................................................... 210

Figure 6.23: Polished hachette production stages ....................................................... 212

Figure 6.24: Miscellaneous glass, carnelian, shell, and terracotta beads from

IFAN survey at Diouboye. 213 
Figure 7.1: $\quad$ Excavation units in residential and non-residential areas ...................... 216

Figure 7.2: $\quad$ Unit A1 stratigraphic profile........................................................... 218

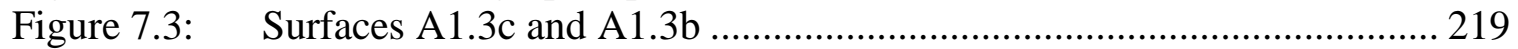

Figure 7.4: $\quad$ Surfaces A1.2d and A1.2c ................................................................. 221

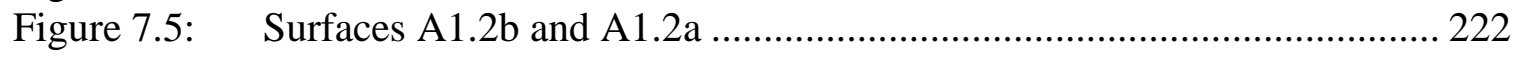

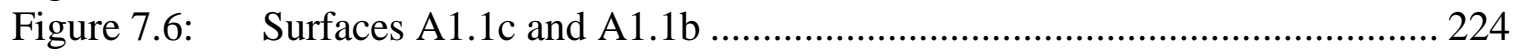

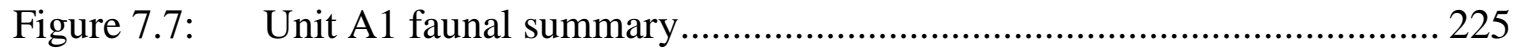

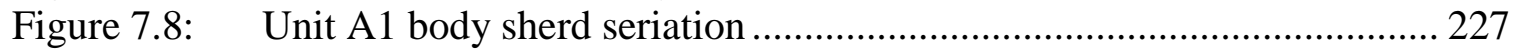

Figure 7.9: Unit A2 stratigraphic profile......................................................... 229

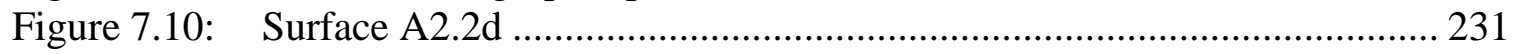

Figure 7.11: Surface A2.2b .............................................................................. 233

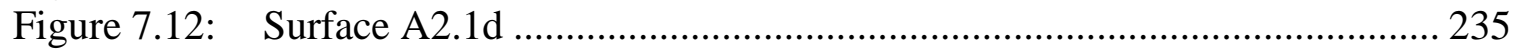

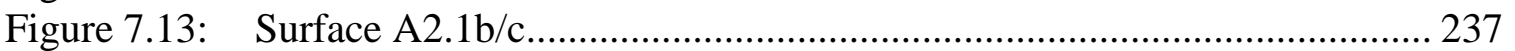

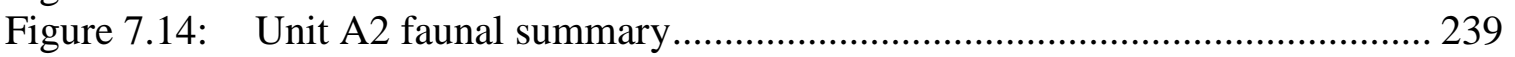

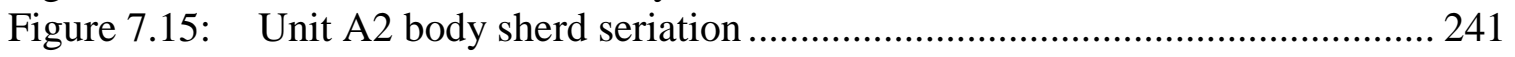

Figure 7.16: Unit F stratigraphic profile............................................................ 244

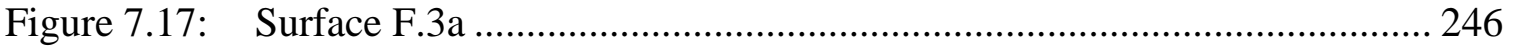

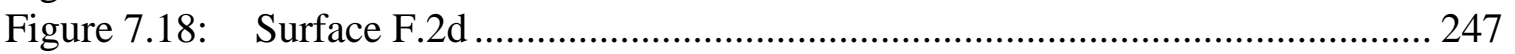

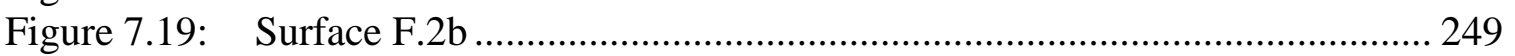

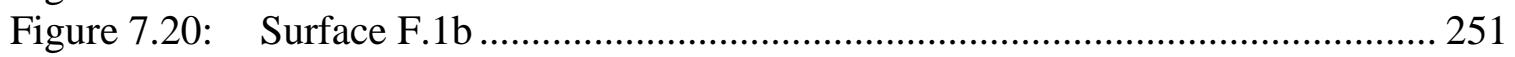

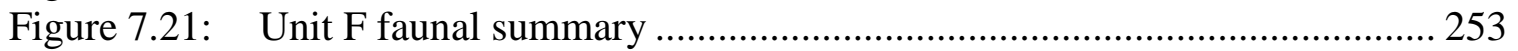

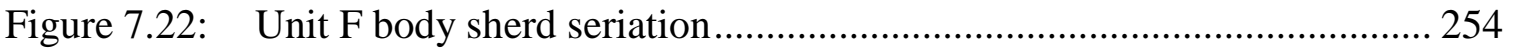

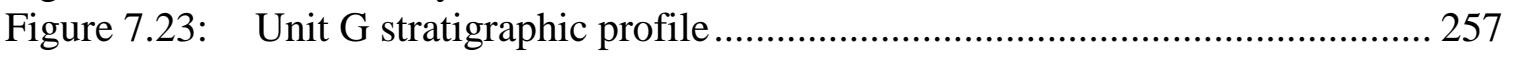

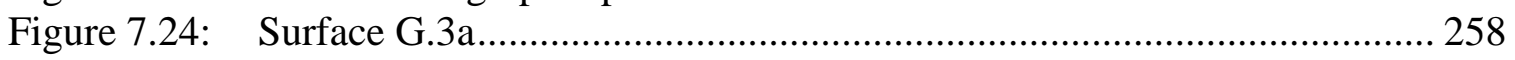

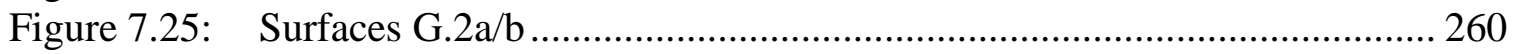

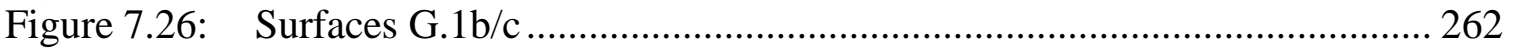

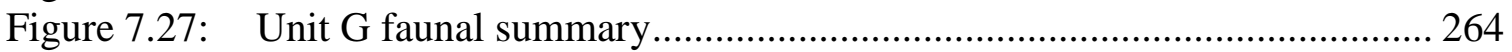

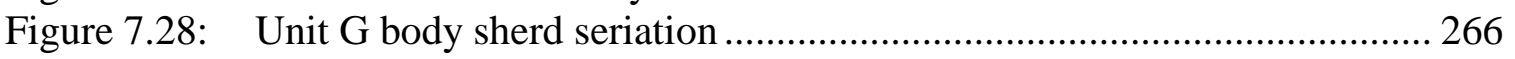

Figure 7.29: Unit H stratigraphic profile............................................................. 268

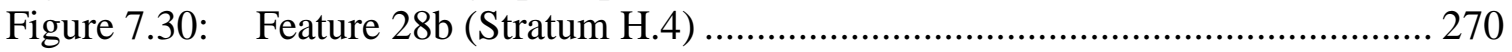

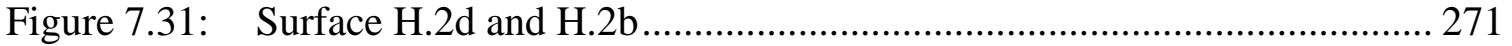

Figure 7.32: Surface H.1c...................................................................................... 272

Figure 7.33: Unit H faunal summary ................................................................ 274

Figure 7.34: Unit H body sherd seriation ............................................................. 275

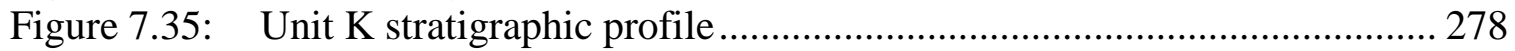

Figure 7.36: Surface K.2a and Surface K.1b.......................................................... 279

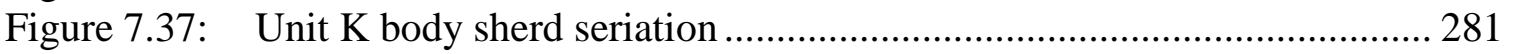

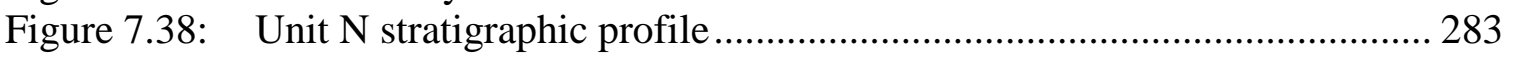

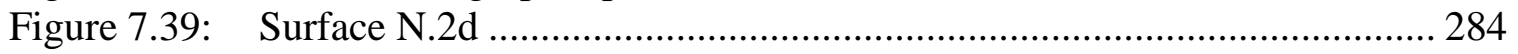

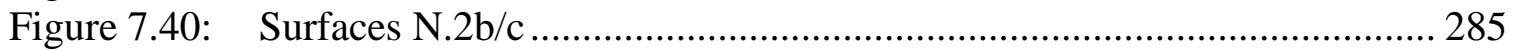

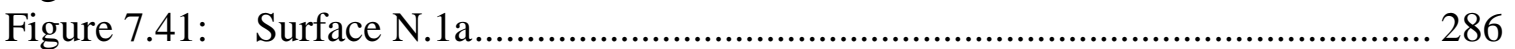

Figure 7.42: Unit N body sherd seriation .............................................................. 288

Figure 7.43: Unit C showing profile and sterile alluvium beneath Feature C-01 ...... 290

Figure 7.44: Bivariate plot of Unit C macrolithic flake thickness and width.............. 292

Figure 7.45: Unit S (Feature S-01) ...................................................................... 293

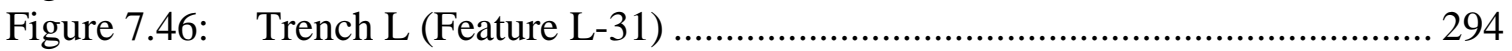


Figure 7.47: Radiocarbon dates from the Central Falémé Archaeological Project..... 298

Figure 7.48: Chronological assessment of excavated substrata in residential areas ... 300

Figure 8.1: $\quad$ Relative surface abundance of button carved body sherds .................... 305

Figure 8.2: $\quad$ Relative surface abundance cord-wrapped stick body sherds................. 305

Figure 8.3: $\quad$ Relative surface abundance of braided cord body sherds ...................... 306

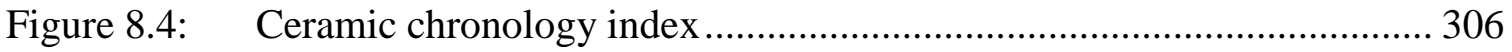

Figure 8.5: $\quad$ Surface model of ceramic chronology index ........................................ 307

Figure 8.6: $\quad$ Surface model of erosion as slope length-steepness (LS)...................... 308

Figure 8.7: $\quad$ Surface distribution of artifact sets .................................................. 313

Figure 8.8: $\quad$ Relative distribution of groundstone tool types by area ....................... 315

Figure 8.9: $\quad$ Relative abundance of vessel size by area ........................................ 316

Figure 8.10: $\quad$ Relative abundance of vessel forms by area .................................... 317

Figure 8.11: $\quad$ Relative abundance of vessel types by area...................................... 318

Figure 8.12: $\quad$ Relative abundance of body sherd paste types by area.......................... 320

Figure 8.13: Relative distribution of inflected rim types by area.............................. 320

Figure 8.14: $\quad$ Relative distribution of simple, collared, and ledged rim types by area. 321

Figure 8.15: $\quad$ Distribution of base types by ward .................................................. 321

Figure 8.16: Relative abundance of rim sherd surface treatment by area .................. 322

Figure 8.17: $\quad$ Relative abundance of chipped stone raw material by area................... 324

Figure 8.18: Relative distribution of quartz artifact types by area ............................ 326

Figure 8.19: $\quad$ Relative distribution of chert artifact types by area ............................... 326

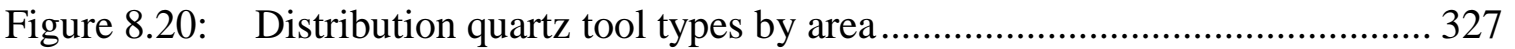

Figure 8.21: Distribution of chert tool types by area............................................. 327

Figure 8.22: Relative abundance of chert and quartz tool types by area type............. 328

Figure 8.23: Relative distribution of hematite reduction stages and debitage

counts by area .............................................................................. 329

Figure 8.24: $\quad$ Relative distribution of slag types and mass by area ............................ 330

Figure 8.25: Relative abundance of surface features by area ................................... 335

Figure 8.26: Distributions of first two nearest-neighbor distances for granaries

and possible granaries ..................................................................... 338

Figure 8.27: Relative abundance of storage features and granary groups................... 340

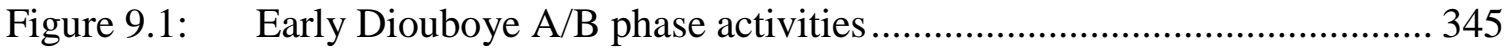

Figure 9.2: $\quad$ Relative abundance of vessel types (Early Diouboye phase) ................. 346

Figure 9.3: Relative distribution of lithic raw material (Early Diouboye phase)...... 347

Figure 9.4: $\quad$ Relative abundance of rim forms (Early Diouboye phase).................... 348

Figure 9.5: $\quad$ Relative distribution of glass, copper, and carnelian artifacts ............... 349

Figure 9.6: Relative distribution of cowrie shells ............................................... 349

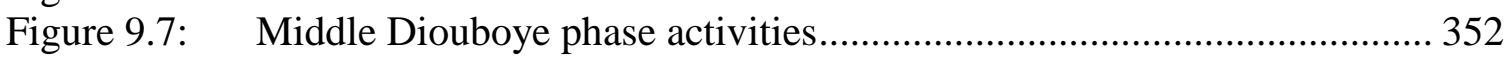

Figure 9.8: $\quad$ Relative abundance of vessel types (Middle Diouboye phase) .............. 353

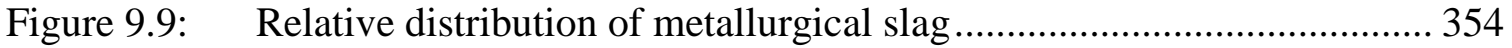

Figure 9.10: Relative distribution of iron artifacts ............................................... 355

Figure 9.11: Relative distribution of lithic raw material (Middle Diouboye phase) ... 356

Figure 9.12: Relative abundance of rim forms (Middle Diouboye phase).................. 356

Figure 9.13: Late Diouboye phase activities ........................................................... 360

Figure 9.14: $\quad$ Relative abundance of vessel types (Late Diouboye phase).................. 361

Figure 9.15: Relative abundance of rim forms (Late Diouboye phase) ...................... 362 
Figure 9.16: Relative distribution of lithic raw material (Late Diouboye phase) ....... 363

Figure A.1: $\quad$ Survey through dense savanna groundcover and more typical sparse groundcover .................................................................................. 427

Figure B.1: Digital elevation model of Diouboye showing major control points...... 458

Figure B.2: $\quad$ Modern agro-pastoral practices including livestock pen north of Area M; and land clearance for cultivation south of Areas J/K............. 459

Figure B.3: $\quad$ View north across eroded southwestern slope of Area A ...................... 460

Figure B.4: $\quad$ Surface slope model of Diouboye.................................................... 461

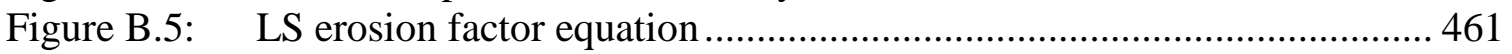

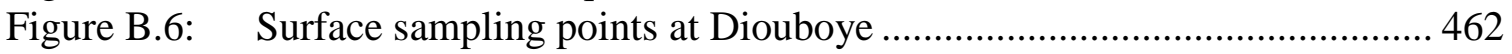

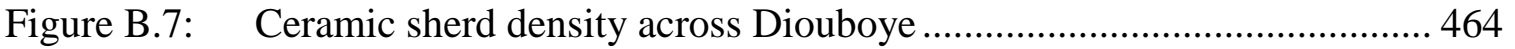

Figure B.8: $\quad$ Intra-site areas and wards defined for Diouboye .................................. 465

Figure B.9: $\quad$ Surface features (granary foundations) in Area A ................................ 467

Figure B.10: View south across Area C................................................................ 468

Figure B.11: View north across Area F ............................................................. 469

Figure B.12: View west across heavily deflated surface of Area G ......................... 469

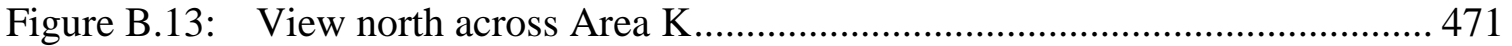

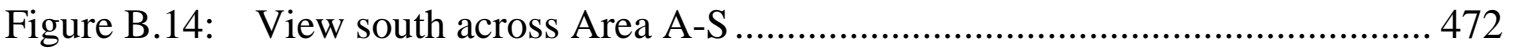

Figure C.1: Trench AZ stratigraphic profile ....................................................... 475

Figure C.2: $\quad$ Trench AZ body sherd seriation ...................................................... 475

Figure D.1: $\quad$ Paste color distributions for the Diouboye and Survey assemblages ..... 506

Figure D.2: Bivariate plots of four clay compositional groups plotted against the first two principal components and the elemental vectors plotted against the first two principal components ........................................ 510

Figure D.3: Bivariate plot of magnesium and cerium concentrations in four clay compositional groups .................................................................... 511

Figure D.4: $\quad$ Bivariate plot of calcium oxide and iron oxide concentrations in four compositional groups ............................................................... 511

Figure D.5: $\quad$ Boxplots of uranium (U) and scandium (Sc) concentrations for compositional groups and inclusion types ......................................... 512

Figure D.6: Distribution of compositional groups by horizon/phase....................... 513

Figure D.7: Relative distribution of sphericity and roundness of grain types in the petrographic sherd (thin section) sample ............................................ 516

Figure D.8: $\quad$ Ordinal size distribution of grain types in the petrographic sherd sample (thin section) ....................................................................... 517

Figure D.9: $\quad$ Ordinal size distribution for grain types in the Survey assemblage (cross-section) .................................................................................. 517

Figure D.10: Distribution of compositional groups by inclusion type........................ 518

Figure D.11: Relative distribution of inclusion density in the Survey assemblage ..... 519

Figure D.12: Ternary diagram of clay/silt matrix, grog, and grit inclusions in the petrographic sherd sample (thin section) ........................................... 519

Figure D.13: Photomicrographs (5X) of paste types and possible sub-types in the petrographic sherd sample .............................................................. 520

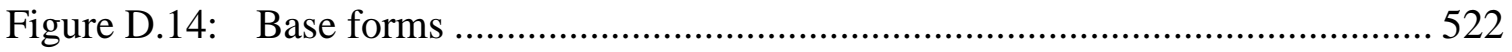

Figure D.15: Bivariate plot of base diameter by base height for circular pedestal base sherds in the Diouboye assemblage ....................................................... 523 
Figure D.16: Distribution of body sherd thickness for the Survey assemblage ........... 524

Figure D.17: Distribution of body sherd thickness for the Diouboye assemblage....... 524

Figure D.18: Distribution of pot lid diameters for the Diouboye assemblage ............. 526

Figure D.19: Formal variables measured on rim sherds ....................................... 527

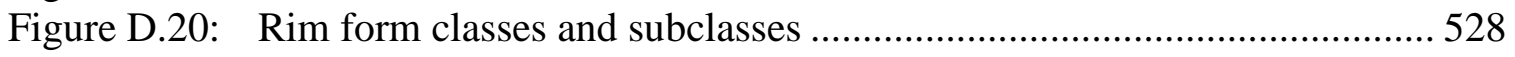

Figure D.20: Rim form classes and subclasses, continued......................................... 529

Figure D.21: Bivariate plot of rim length/thickness and rim angle for inflected rims in the Survey assemblage .................................................................. 531

Figure D.22: Bivariate plot of rim length/thickness and rim angle for inflected rims in the excavated Diouboye assemblage .............................................. 531

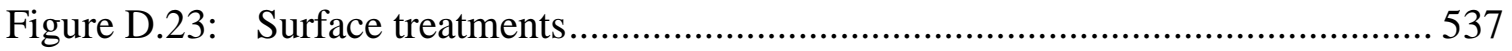

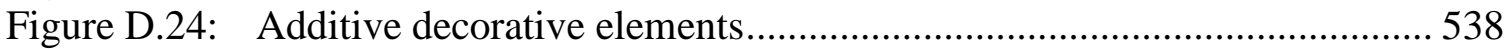

Figure D.25: Twisted, knotted, and composite cord roulettes..................................... 540

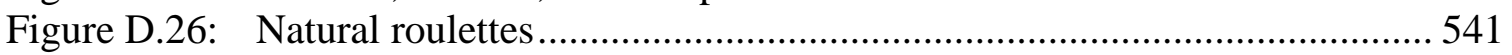

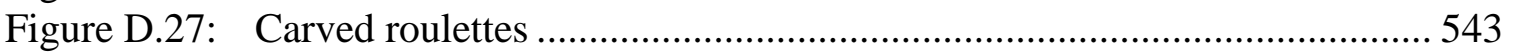

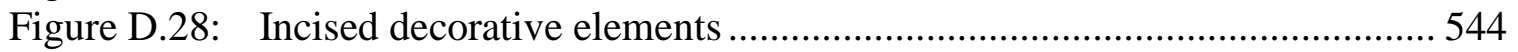

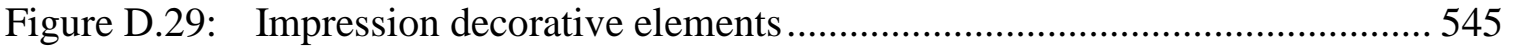

Figure D.30: Schematic oxidation patterns recorded for sherd cross-sections ............ 547

Figure D.31: Correspondence analysis plot of rim and body sherd types in the Survey assemblage.......................................................................... 554

Figure D.32: Correspondence analysis plot of body sherd types and substrata from

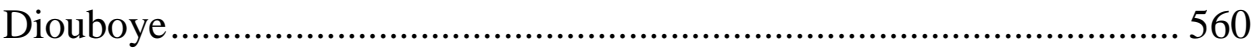

Figure D.33: Correspondence analysis plot of body sherd types and strata from Diouboye.................................................................................... 561

Figure D.34: Bivariate plot of orifice diameter and shoulder angle for rim sherds in the Survey assemblage ...................................................................... 562

Figure D.35: Bivariate plot of orifice diameter and shoulder angle for rim sherds in the Diouboye assemblage ............................................................... 563

Figure D.36: Relative abundance of rim subclass by vessel form for rim sherds in the Survey assemblage ................................................................ 564

Figure D.37: Relative abundance of rim subclass by vessel form for rim sherds in the excavated Diouboye assemblage ..... 565

Figure E.1: $\quad$ Boxplot of weights of unworked (raw) material and lightly worked (core) nodules of quartz and chert in chipped stone assemblages ......... 571

Figure E.2: $\quad$ Relative abundance of raw materials in chipped stone assemblages ...... 572

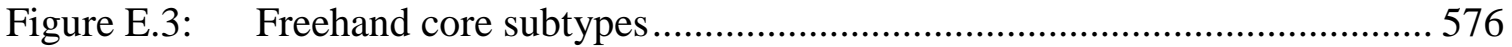

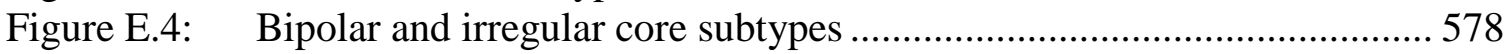

Figure E.5: $\quad$ Freehand and bipolar flake subtypes................................................ 580

Figure E.6: $\quad$ Relative abundance of chipped quartz artifact types ........................... 582

Figure E.7: $\quad$ Relative abundance of chipped chert artifact types ............................... 582

Figure E.8: Cumulative frequencies of distal cortex on detached pieces .................. 584

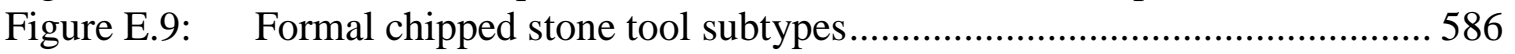

Figure E.10: Informal chipped stone tool subtypes ............................................... 588

Figure E.11: Relative abundance of informal and formal quartz tool subtypes .......... 590

Figure E.12: Relative abundance of informal and formal chert tool subtypes ............ 590

Figure E.13: Relative abundance of raw materials in groundstone assemblages ........ 595 
Figure E.14: Groundstone percussion tool types ................................................... 597

Figure E.15: Hafted groundstone tool types and subtypes ...................................... 599

Figure E.16: Bivariate plot of axe head width and length ........................................60

Figure E.17: Relative abundance of axe head subtypes.......................................... 601

Figure E.18: Distribution of hematite debitage and hachette by assemblage.............. 602

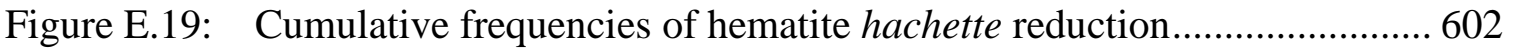

Figure E.20: Relative distribution axe wear by subtype and assemblage................... 604

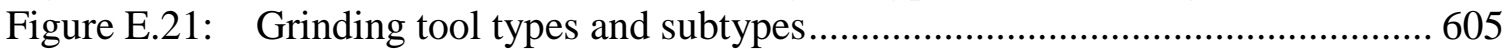

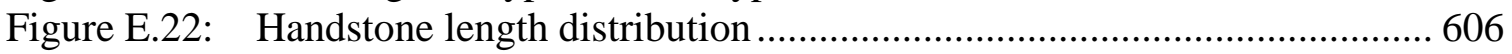

Figure E.23: Relative abundance of grinding implements by material type............... 607

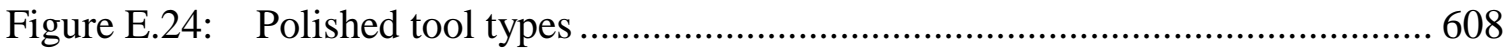

Figure F.1: Gold extraction in the Falémé River ..................................................... 614

Figure F.2: $\quad$ Pit slag specimen from the site of Golmi on the lower Falémé River .... 615

Figure F.3: Blade and projectile tool types in the Diouboye assemblage ................. 618

Figure F.4: Wire and rod types in the Diouboye Assemblage .................................. 619

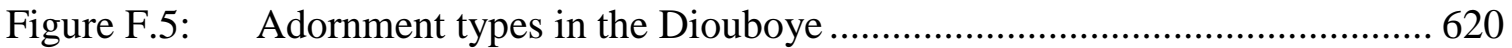

Figure F.6: Blade, projectile, and nail types in the Survey assemblage ................... 621

Figure G.1: $\quad$ Smoking pipe stem decoration and base shapes ................................. 626

Figure G.2: Miscellaneous terracotta artifact subclasses ....................................... 628

Figure G.3: Bivariate plot of length and diameter for perforated terracotta objects .. 629

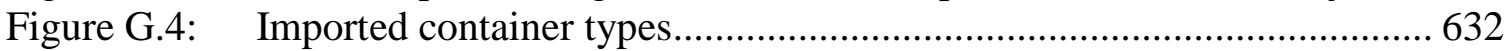

Figure G.5: $\quad$ Glass, stone, bone, and shell ornament types from Diouboye ................ 634

Figure G.6: Glass bead types from Atlantic Era sites ......................................... 636 


\section{LIST OF TABLES}

Table 4.1: $\quad$ Summary of chipped stone artifacts from MSA/LSA and cLSA sites ... 117

Table 4.2: $\quad$ Summary of surface finds and functions of Iron Age sites..................... 138

Table 4.3: $\quad$ Summary of sites seriated by temporally diagnostic surface finds......... 150

Table 5.1: $\quad$ Summary of landforms within one-kilometer site catchments ............... 159

Table 5.2: $\quad$ Summary of distance to Falémé River and nearest channel .................... 163

Table 5.3: $\quad$ Summary of artifact classes by horizon/phase and site type................... 165

Table 5.4: $\quad$ Nearest-neighbor coefficient R for surveyed sites by phase................... 170

Table 6.1: Variables recorded for the classification of architectural features.......... 183

Table 6.2: $\quad$ Summary of architectural feature attributes......................................... 183

Table 6.3: $\quad$ Summary of artifact attributes by depositional context ......................... 191

Table 6.4: $\quad$ Summary of attributes for pyrotechnic features..................................... 192

Table 6.6: $\quad$ Summary of attributes for pit features ................................................. 195

Table 6.7: $\quad$ Summary of vessel types based on form and size................................. 201

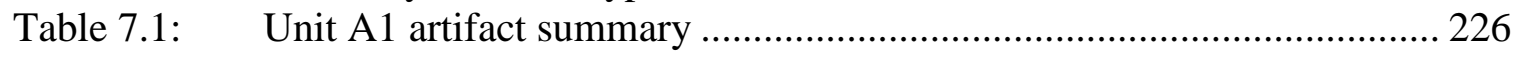

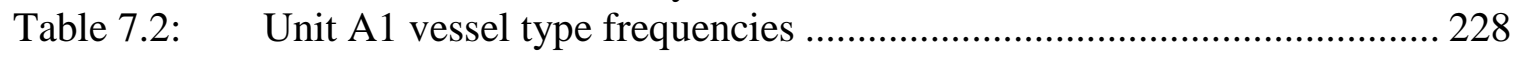

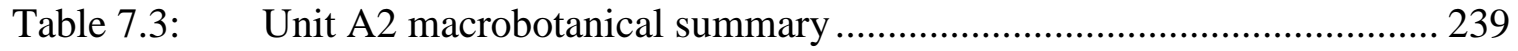

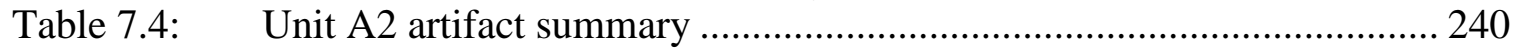

Table 7.5: $\quad$ Unit A2 vessel type frequencies ........................................................ 242

Table 7.6: $\quad$ Unit F macrobotanical summary...................................................... 253

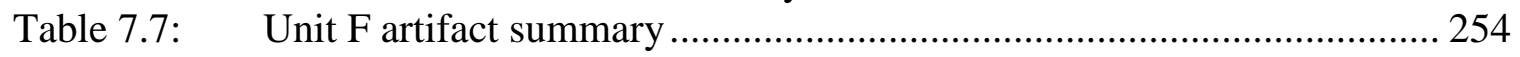

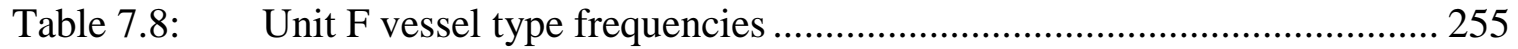

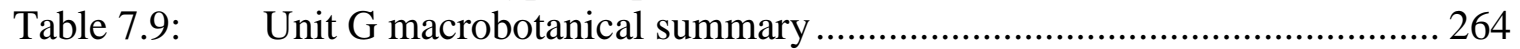

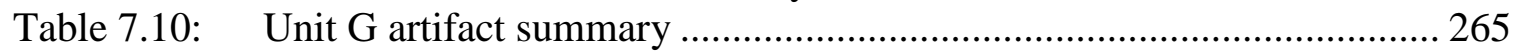

Table 7.11: $\quad$ Unit $G$ vessel type frequencies ........................................................... 266

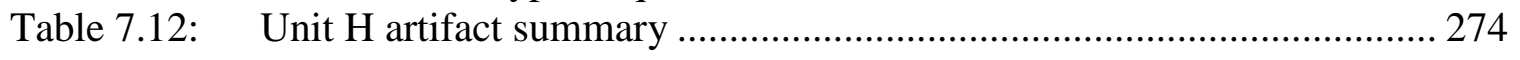

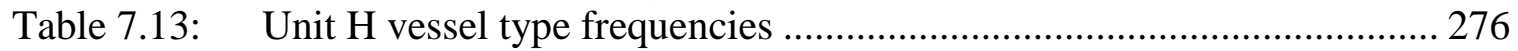

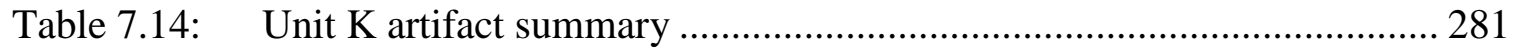

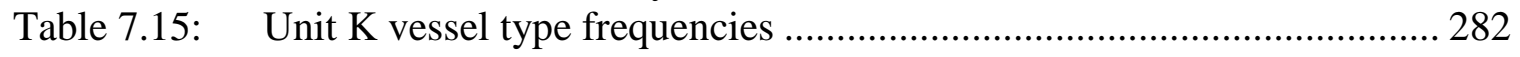

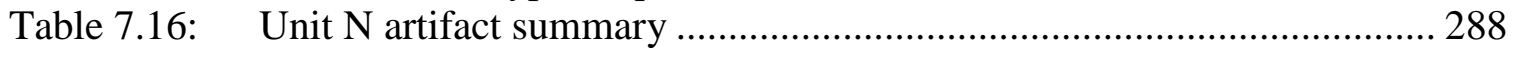

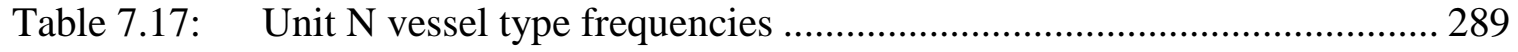

Table 7.18: $\quad$ Unit C lithic (mudstone) artifact summary ............................................. 291

Table 7.19: $\quad$ Summary of excavated strata by ceramic phase ................................... 296

Table 7.20: Radiocarbon dates from the Central Falémé Archaeological Project..... 297

Table 8.1: Chronological assessment of surface contexts....................................... 309

Table 8.2: $\quad$ Correlation matrix of surface artifact categories and erosion factors ..... 311

Table 8.3: $\quad$ Summary of relative artifact class abundance by artifact set.................. 312

Table 8.4: $\quad$ Summary of artifact abundance (systematic collection) by area ............ 314 
Table 8.5: Correlation matrix of interpolated counts of lithic artifact categories .... 324

Table 8.6: $\quad$ Summary of metal artifacts from systematic (and purposive) surface collections by CFAP ......................................................................... 330

Table 8.7: $\quad$ Summary of small finds from systematic (and purposive) collections by CFAP ................................................................................ 331

Table 8.8: $\quad$ Population estimates for residential areas.......................................... 333

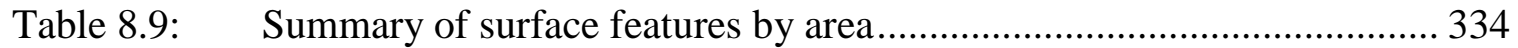

Table 8.10: $\quad$ Summary of unconstrained artifact sets by surface feature type ............ 336

Table 8.11: $\quad$ Summary of nearest neighbor distance for stone architectural features . 338

Table 8.12: Nearest neighbor statistics for all stone architectural features............... 339

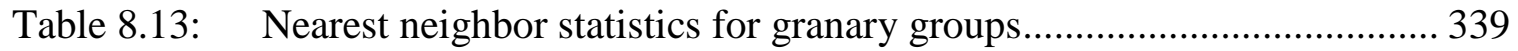

Table B.1: $\quad$ Major control points for ground survey at Diouboye ............................ 458

Table B.2: $\quad$ Summary of sampling points by area................................................ 463

Table D.1: $\quad$ Sherds sampled for petrographic and compositional analyses ............... 503

Table D.2: $\quad$ Operating parameters for the laser ablation system and ICP-MS ........... 507

Table D.3: $\quad$ Major oxide and minor element concentrations in sampled sherds ........ 508

Table D.4: $\quad$ Variables recorded for ceramic paste inclusions .................................. 515

Table D.5: Cross-tabulation of inclusion types in the Survey assemblage................ 518

Table D.6: $\quad$ Formal variables recorded for base sherds.......................................... 522

Table D.7: $\quad$ Summary of base forms by assemblage ............................................... 523

Table D.8: $\quad$ Summary of handle forms by assemblage ........................................... 525

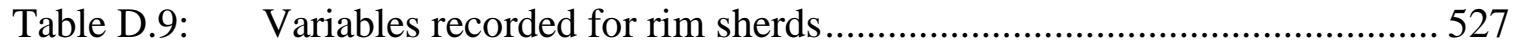

Table D.10: $\quad$ Variables recorded for surface decoration ............................................... 534

Table D.11: Summary of decorative elements by paste type for body sherds in the Survey assemblage........................................................................ 549

Table D.12: $\quad$ Summary of body sherd types in the Survey assemblage...................... 550

Table D.13: Summary of decorative elements by paste type for rim sherds in the Survey assemblage...................................................................... 551

Table D.14: $\quad$ Summary of rim form subclass by paste type for rim sherds in the

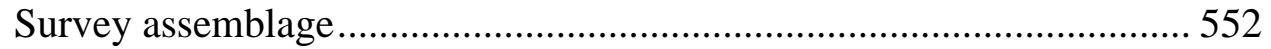

Table D.15: $\quad$ Summary of rim types in the Survey Assemblage.................................. 553

Table D.16: Summary of surface treatment, thickness, and paste type bydecorative elements for body sherds in the excavated Diouboye assemblage ......... 557

Table D.17: $\quad$ Summary of lip decoration and surface treatment by rim form subclass for rim sherds in the excavated Diouboye assemblage ......................... 558

Table D.18: $\quad$ Summary of fiber roulette decoration by rim form subclass for rim sherds in the excavated Diouboye assemblage ................................... 558

Table D.19: Summary of carved roulette decoration by rim form subclass for rim sherds in the excavated Diouboye assemblage ..................................... 559

Table D.20: Summary of impressed and incised decoration by rim form subclass for rim sherds in the excavated Diouboye assemblage ........................ 559

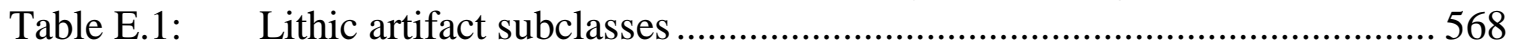

Table E.2: $\quad$ Summary of chipped stone artifacts from systematic survey ................. 568

Table E.3: $\quad$ Raw materials in the chipped stone and groundstone assemblages........ 570

Table E.4: $\quad$ Variables recorded for core specimens ................................................. 575

Table E.5: $\quad$ Variables recorded for detached pieces ................................................ 579 
Table E.6: $\quad$ Mean sizes of detached piece types .................................................. 583

Table E.7: Variables recorded for informal and formal chipped stone tools ........... 585

Table E.8: $\quad$ Summary of formal and informal quartz tool subtypes by site............... 589

Table E.9: $\quad$ Summary of formal and informal chert tool subtypes by site................. 589

Table E.10: Diversity indices for quartz informal blades and formal tool subtypes with bootstrapped confidence intervals ........................................... 591

Table E.11: Diversity indices for chert informal blades and formal tool subtypes with bootstrapped confidence intervals ................................................ 591

Table E.12: Summary of informal and formal tool subtype size (length)................. 592

Table E.13: Distribution of sites with groundstone by archaeological component.... 593

Table E.14: Variables recorded for groundstone tool form and size......................... 596

Table E.15: Variables recorded for hafted groundstone tools ................................... 598

Table E.16: Summary of formal attributes for fully-ground, semi-ground, and preform hachettes from Survey and Diouboye assemblages ................. 600

Table E.17: Variables recorded for wear on groundstone tools ................................. 603

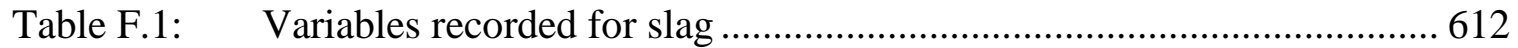

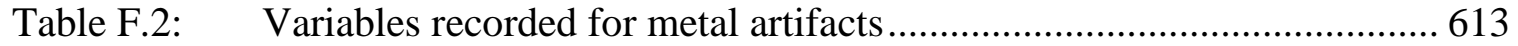

Table F.3: $\quad$ Summary of slag types in the Survey and Diouboye assemblages ......... 615

Table F.4: $\quad$ Summary of metal artifact counts for surveyed sites............................. 622

Table G.1: $\quad$ Variables recorded for terracotta artifacts............................................. 624

Table G.2: $\quad$ Variables recorded for terracotta smoking pipes .................................. 625

Table G.3: $\quad$ Summary of smoking pipe collar and base shapes by Atlantic Era ceramic component ................................................................... 627

Table G.4: $\quad$ Summary of glass bead counts by sample from Diouboye...................... 634

Table G.5: $\quad$ Summary of European glass bead counts by period of manufacture...... 636

Table H.1: $\quad$ Summary of faunal NISP by excavation unit ...................................... 652

Table I.1: $\quad$ Summary of macrobotanical remains identified in Unit A2 ................... 656

Table I.2: $\quad$ Summary of macrobotanical remains identified in Unit F..................... 656

Table I.3: $\quad$ Summary of macrobotanical remains identified in Unit G.................... 656 


\section{LIST OF APPENDICES}

APPENDIX A. THE MADINA-SADATOU STUDY AREA: SURVEY METHODS AND SITE INVENTORY ........................... 425

APPENDIX B. DIOUBOYE: SURFACE ARCHAEOLOGY .................................. 456

APPENDIX C. DIOUBOYE: EXCAVATION METHODS AND

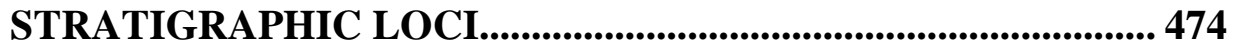

APPENDIX D. POTTERY ANALYSIS AND CLASSIFICATION ........................... 499

APPENDIX E. LITHIC ARTIFACT ANALYSIS AND CLASSIFICATION....... 566

APPENDIX F. METALLURGICAL ARTIFACTS .................................................6 610

APPENDIX G. MISCELLANEOUS ARTIFACTS ..................................................... 623

APPENDIX H. HARVESTING THE FALÉMÉ RIVER AND ITS SHORES: PRELIMINARY RESULTS FROM ANALYSES OF FAUNA FROM DIOUBOYE (by Stephen Dueppen)...................................... 640

APPENDIX I. PRELIMINARY RESULTS OF MACROBOTANICAL ANALYSIS OF SEEDS AND FRUITS FROM DIOUBOYE (by Daphne Gallagher) 


\section{CHAPTER 1 IN SEARCH OF BAMBUK}

Nestled amid the upper branches of the Senegal River, the legendary gold fields of Bambuk have captivated the imagination of traders, rulers, and scholars for more than a millennium. Although the toponym Bambuk does not appear until AD 1337 in a manuscript by the Arab geographer Ibn Fadl Allāh al-‘Umarī (Levtzion and Hopkins 2000:261), the flow of gold from this auriferous region certainly influenced the growth of interregional trade and the shifting fortunes of major polities, including medieval Ghana (Wagadu) and Mali, during the preceding centuries (Bovill 1995; Levtzion 1980; Monteil 1968). While the role of gold in financing these states may be somewhat overstated (A. Bathily 1975:39-40), it seems certain that a number of towns in the region, including Barisa and Ghiyaru on the upper Senegal River (Figure 1.1), profited from their role as intermediaries between the gold of Bambuk and trans-Saharan trade routes to the north.

The notoriety of Bambuk spread rapidly across the circum-Mediterranean world during early-second millennium $\mathrm{AD}$, thanks in part to the growing number of secondhand tales by North African merchants and travelers. Meanwhile, the pilgrimage of Mansa Musa, the ruler of Mali, to Mecca in AD 1324 offered a direct testament to the gold wealth of West Africa. According to eye witness accounts recorded by al-'Umar̄̄ (Levtzion and Hopkins 2000:270-271), the Mansa spent so much gold supporting the tens of thousands of people in his procession through Cairo that the value of this metal in Egypt declined by some ten to twenty percent for several years!

Lured by these tales of Bambuk gold, a growing number of Portuguese, French, and English merchants made their way down the Atlantic coast of Africa and into the Senegambian interior after the late-15 ${ }^{\text {th }}$ century AD (Curtin 1975a; Hair 1984). Although wealth in gold would by and large prove illusory, the discovery of other exportable commodities such as gum, cotton, and, most notoriously, slaves, would trigger the 
turbulent growth of European commercial interests in West Africa, culminating with the consolidation of colonial rule in the late- $19^{\text {th }}$ century AD.

As a result of these global economic entanglements, the historiography of

Bambuk and other major auriferous zones across West Africa has largely neglected the dynamic lifeways of local people, privileging instead the story of the mineral resource that existed beneath their feet. Where they do appear in regional histories, communities from gold-producing regions are often rendered as static projections backwards from an ethnographic present (Curtin 1973; Perinbam 1988), or as "silent” traders negotiating with merchants from complex polities (Bovill 1995:82). This latter portrayal can be traced to a passage written by the geographer Yāqūt around AD 1224:

[Arab merchants] strive onwards with much suffering until they reach the place which separates them from the owners of the gold. When they arrive there, they beat great drums which they have brought with them, and which may be heard from the horizon where these people of the Sudan live... When the merchants know that those people have heard the drum, they produce whatever they have brought of the aforementioned wares...Then they go away from that place a distance of one day's travelling. Then the Sūdān [Black people] come with the gold, put a certain amount of gold alongside each kind, and withdraw. Then the merchants come after them and each one takes the gold which he finds beside his merchandise. They leave the merchandise and depart after having beaten their drums. Nothing is known about what is beyond these people, and I think that there is no living being there on account of the scorching sun (Levtzion and Hopkins 2000:169-170).

A reinterpretation of this and related passages by historian Paulo de Moraes-Farias (1974) strongly suggests that silent trade was more myth than fact as it ignores other important elements of these trade interactions. Missing from this particular account is any reference to the indigenous merchants, called Wangara, who brokered trade between Arab-Berber caravans from North Africa and people occupying the auriferous zones (Perinbam 1973; Stewart 1979; Wilks 1982a). In fact, the conflation of the name Wangara with the physical realm of Bambuk in Arab geographies dating from the late-first millennium AD underscores the success of these middlemen in guarding access to gold and other commodities exported from lands south of the Sahara (Curtin 1973:623). Of course, this 
strategy on the part of Wangara precluded early geographers from producing more detailed accounts of communities in places such as Bambuk.

Many of the early medieval Arabic geographies do, however, make reference to the vast cultural landscape of Lamlam, which lay beyond the periphery of the political and economic networks branching across the West African savanna at this time. While the appellation Lamlam suggests that the local language was unintelligible to outside authors, Ibrahima Thiaw (1999:40) has argued that this toponym refers to a decentralized system of organization around politico-territorial units called lamanat. In either case, the people of this realm were not viewed favorably by those from more centralized societies. According to the geographer Muhammad al-Sharīf al-Idrīsī writing in AD 1154, polities and towns along the Senegal River sponsored merchants and raiders who:

...make forays into the land of Lamlam and capture its inhabitants. They bring them to their own countries, and sell them to visiting merchants. In the whole land of Lamlam there are only two small village-like towns, one called Malal [Mali?] and the other Daw...Their speech does not resemble that of the people of Maqzāra [Fulfulde/Wolof], nor that of the people of Ghāna [Soninke] (Levtzion and Hopkins 2000:108)

In the language of contemporary anthropology, this quote depicts Lamlam as a network of small-scale communities whose ethno-linguistic differences and decentralized social organization made them easy prey for their more powerful neighbors. However, in the same account al-Idrīsī goes on to describe their subsistence economy and trade:

In the Nil [sic] are species of fish, big and small, which serve as food...They catch them, salt and preserve them...Their adornments are of copper, little beads, strings of glass beads... and various kinds of false onyx manufactured from glass (Levtzion and Hopkins 2000:108)

Contrasting with the above portrayal of hapless victims, the people of Lamlam evidently valued objects such as copper ornaments and glass beads, and made frequent use of salt-all commodities obtained from their supposedly predatory neighbors to the north. 


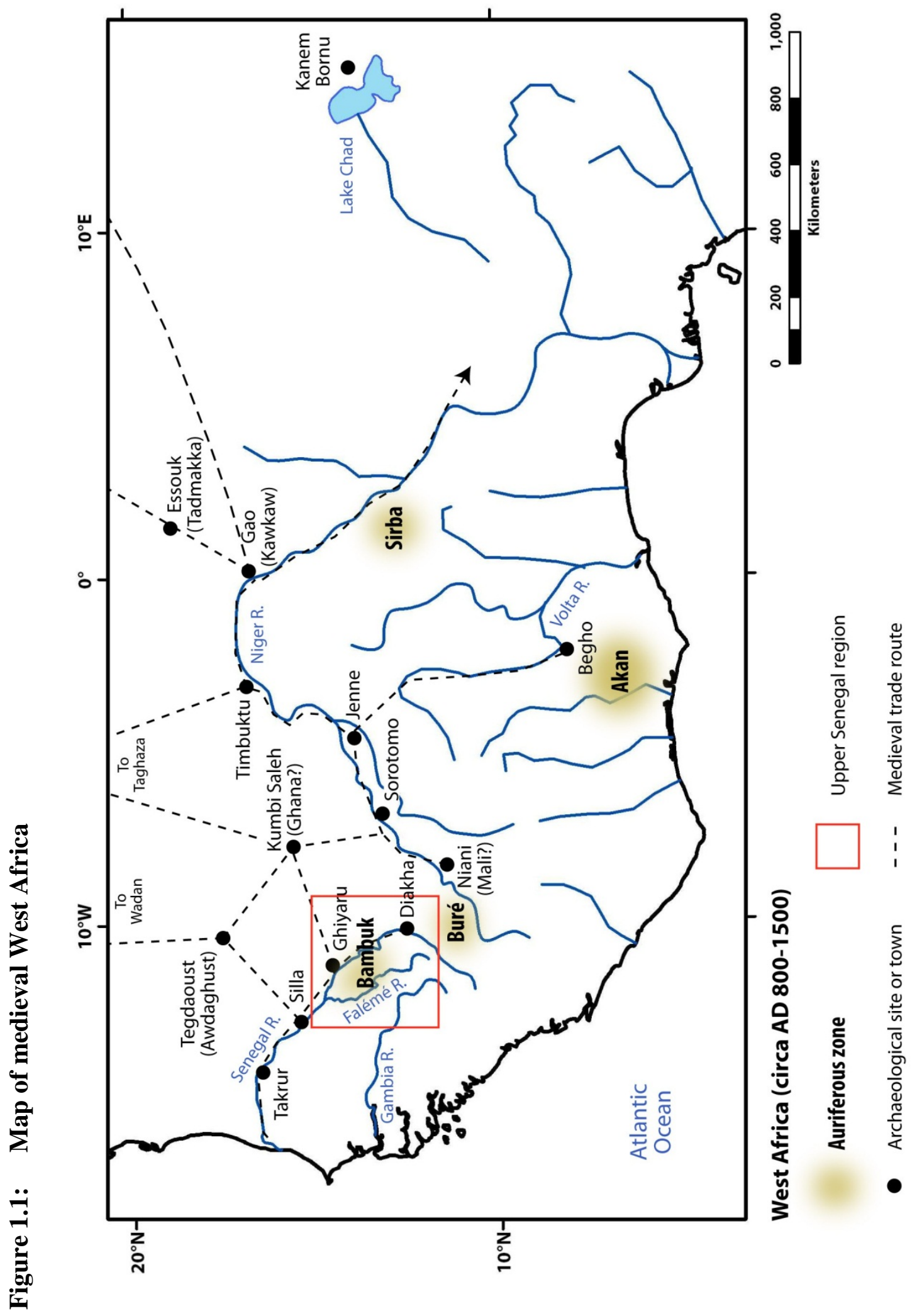


Although these brief accounts of the inhabitants of Lamlam undoubtedly gloss over diverse customs that existed across the upper Senegal and upper Niger River basins (including Bambuk), their internal contradictions still manage to suggest that political relations within and between the diverse societies of medieval West Africa have been vastly oversimplified. Rather, it appears that Bambuk and other lands beyond the veil of written history from the late-first to early-second millennium AD fostered a mosaic of communities whose relations both to each other and to interregional political economies were considerably dynamic across space and time.

The history of Bambuk comes into sharper focus after the $16^{\text {th }}$ century AD when a growing number of European travelers on the Senegal and Gambia Rivers began to describe local customs and political institutions (e.g., Boucard 1974 [1729]; Coste d'Arnobat 1789; Hair 1984; Labat 1728; M. Park 1816; Raffenel 1856). These accounts are often amalgamated to portray late precolonial societies in Bambuk as confederations of several villages with politico-ritual authority vested in local leaders (e.g., Curtin 1973:629-630; Delafosse 1912:360-361). Based on explicit or implicit assumptions about cultural conservatism in such societies, this portrait has, in many cases, been deployed to characterize earlier communities in gold-producing regions and other peripheries of transSaharan trade (e.g., Brooks 1993:33-48). While the historical characterization of Bambuk as a decentralized social landscape may very well be accurate (or so I will argue in this study), it is important to recognize that local communities actively maintained and potentially transformed multiscalar political economies and cultural traditions as they engaged with historical processes playing out across West Africa over the longue-durée.

Such local negotiations and their ramifications emerge to a greater, though not full, extent from the oral histories recounted by people occupying Bambuk and surrounding lands. Beyond the horizon of second- or third-hand memory, the safeguarding of oral traditions in many West African societies often falls to the official historians or bards (griots) of a given community (A. Bathily 1989:14-29; Conrad 1995). The narratives recounted by griots play an important role in legitimizing the authority of ruling lineages and/or immigrant groups vis-à-vis communities with ancestral ties to the land (Jansen 1996; D. R. Wright 1985), despite their malleability in particular cultural and political contexts (Austen 1999; Tonkin 1982). It follows that oral histories in West 
Africa, as with written accounts, also tend to express the perspective of centralized societies and their ruling lineages at the expense of smaller communities and lowerranking lineages.

Drawing upon the rich historiography available for the Upper Senegal region, I set out in this study to provide a deeper exploration of social life within Bambuk during the early-second millennium $\mathrm{AD}$, coincident with the expansion of interregional trade and complex polities across West Africa. Specifically, I present and interpret the results of an archaeological study of Diouboye, a village community that occupied the banks of the Falémé River around AD 1000-1400. While I continue to make use of ethnographic and historic evidence throughout this study, my archaeological excavations at Diouboye and survey of the surrounding landscape reveal that communities in gold-producing peripheries both shaped, and were shaped by, their dynamic positions within the politicaleconomic mosaic of medieval West Africa.

\section{POLITICAL ECONOMY OF VILLAGE COMMUNITIES}

In order to illuminate the social life of early village communities in Bambuk, and their relations to regional polities known from historical sources, I approach the study of Diouboye from the perspective of political economy. This framework rests upon substantial bodies of anthropological and historical theory that outline the ways in which economic processes (i.e., production, distribution/exchange, and consumption) structured political relations at different scales. Most famously deployed to study shifting power relations among societies caught up in the modern world system (Roseberry 1988; Wolf 1982), political economy has further proven useful for scholarship on interregional systems of the pre-capitalist past (Cobb 1993; Frank and Gills 1993; Peregrine and Feinman 1996). Beyond the study of relations between societies, political economy offers a framework for comparative and historical studies of relations within societies. Research in this latter vein usually falls under the rubric of historical materialism first distilled by Karl Marx (1973). Today, however, political economy subsumes a diverse set of views on the means and relations of production, as well as the ideologies that underwrite them, across contemporary, historic, and archaeological contexts (e.g., Bernbeck and McGuire 2011; Childe 1951; Feinman and Nicholas 2004; Meillassoux 1986; Polanyi 1944). By 
focusing on economic processes embedded within multiscalar social relationships, this study aims to elucidate how Diouboye emerged at the intersection of both local institutions within Bambuk and global processes unfolding across West Africa.

This is not, however, to suggest that the whole of social life at Diouboye can, or should, be viewed through the lens of political economy. Critical scholarship has argued forcefully that “culture matters" insofar as embodied gestures, beliefs, and values shape social action and vice versa (e.g., Bourdieu 1977; Sewell 1992). The rise of the modern world system, for example, did more than just reallocate material resources and property rights from indigenous communities to European states. The associated periods of historical entanglement had deep and lasting repercussions for the ways in which people on both sides of the encounter assigned value to material objects, landscapes, and human subjects (Cooper 2005; Keane 2007; Sahlins 1981; Thomas 1991). Myriad other case studies have documented how cultural habits, values, memories, and beliefs underpin diverse forms of community, from the village to the nation state, although whether these factors operated as integrative forces or contested ideologies clearly depends on historical context (Anderson 1983; Eriksen 1993). But importantly, these cultural schemas emerge and evolve through many of the same material practices that institute political-economic relations.

The centrality of material relationships within political economy further makes possible my archaeological study of Diouboye. In looking beyond vague historical references to social life in Bambuk, the material artifacts and features of the archaeological record provide a complementary, and sometimes contradictory, testament of the routine activities and singular events implicated in processes of economic production, exchange, and consumption. And, as should be clear from the foregoing discussion of historiography in West Africa, a strongly material perspective offers a way to engage with longstanding interests in the economic history of this region over the longue-durée.

Since Chapter 2 elaborates on anthropological and historical views of political economy in West Africa, I focus in the remainder of this chapter on briefly defining the key elements of this perspective as it applies to archaeological research at Diouboye. 


\section{Anthropological Approaches to Economy}

Over the past half-century or so, scholars across the social sciences have rejected, or at least tempered, the axiom that rational choice underlies formal economics; most now acknowledge that economic decisions are always embedded within social institutions, interpersonal relations, embodied routines, and cultural schema (e.g., Bourdieu 2005; Douglas and Isherwood 1979; Giddens 1984; Granovetter 1985; Greif 2006; Halperin 1994; Henrich et al. 2004; North 1990; Simon 1985). Drawing implicitly or explicitly on Marxist thought, this critique has coalesced within anthropology around Karl Polanyi’s (1957) notion of "substantive” economy as the social processes through which people produce, distribute or exchange, and consume material resources in order to satisfy their wants as both biological beings and cultural subjects.

According to this view, continual efforts to meet biological and cultural wants make manifest and reproduce structural relations, rules, and norms through sets of recurrent social practices called institutions. Defined as recursive social constructs that extend across space and endure through time (Giddens 1984:16-34), political-economic institutions include households, kinship systems, political gift-giving, markets, and ceremonial networks, and innumerable other examples. Whatever the particular case, institutions persist insofar as they coordinate interaction among social actors and material and non-material resources in more-or-less legitimate ways. Importantly for archaeology, the material and spatial dimensions of practice are precisely what lend institutions a certain amount of inertia within social and economic life (see Chapter 2).

It follows that institutional arrangements play a significant role in assigning value to material resources key to the satisfaction of cultural as well as biological wants. Without delving into a vast anthropological literature on material culture, I would simply emphasize that value depends not only on the intrinsic qualities of objects, but also on the ways in which objects move (or do not move) through economic practices embedded within social institutions (Appadurai 1986; Keane 1997; Robb 1999a; Thomas 1991; Weiner 1985). In addition to practices of exchange and consumption, value also emerges from the technologies of production that assemble material capital, labor, and practical knowledge (Dobres and Hoffman 1994; Lechtman 1977; Lemmonier 1986). During production, socioeconomic relations, embodied habits, and cultural values become 
physically imprinted in material objects - thus contributing to the relative inertia of institutions mentioned above.

The political economy, then, refers to the ways in which institutions shape access to material (and non-material) resources and, by extension, the structural relationships among individuals and social collectives that channel the exercise of power (Moe 2005; Roseberry 1988; Wolf 1982). Although historical and archaeological studies of political economy have mainly attended to the hierarchical configuration of institutions in stratified states and other centralized societies (Earle 2002; Feinman and Nicholas 2004), or their beginnings in decentralized or nonstratified societies (Price and Feinman 1995; Spencer 1993), it is quite possible to evaluate non-hierarchical institutional arrangements in much the same terms (Aldenderfer 1993; Bayman 2002; Cobb 1993; McGuire and Saitta 1996; Wiessner 2002). In this study I consider political economy as both the asymmetrical (or otherwise hierarchical) and symmetrical relations that emerge as social actors engage in economic production, distribution or exchange, and consumption. This perspective is essential to the study of how economies structure social life within and between communities of different scales. As I elaborate in the next chapter, this is particularly the case in West Africa where non-hierarchical institutional arrangements have historically been important in even the most complex societies.

\section{Social Institutions and Scale}

In this study I draw a general distinction between centralized and decentralized societies as two idealized types amid an otherwise continuous field of economic processes, political relations, and cultural logics. By centralized society, I refer to the consolidation of political authority within one or few households, kin groups, or communities; socioeconomic relations are correspondingly more asymmetrical within and/or between these social actors or groups (McGuire 1983). In contrast, a decentralized society exhibits the dispersion of political authority among households, kin groups, villages, or perhaps across a confederacy of settlements; symmetrical socioeconomic relations predominate over asymmetrical ones. As I elaborate in Chapter 2, scholars have demonstrated that even centralized states in West Africa were fairly decentralized when

compared to similar polities elsewhere in the world (S. K. McIntosh 1999a). In order to 
explore the regional social forms and dynamics that exist between these idealized types in the Upper Senegal region, I consider societies and broader "cultural spheres” from the perspective of (and relative to) a local reference point—namely, the village of Diouboye.

It is also essential to keep in mind that, whether one prefers the designation “stateless" (Fortes and Evans-Pritchard 1940), "nonstratified" (Cobb 1993), or "tribe” and "chiefdom" (Sahlins and Service 1960), the concept of decentralized society subsumes a tremendous diversity of possible political-economic relations (Feinman and Neitzel 1984). Moreover, such relations are inherently dynamic through time (Fowles 2002) and should not be reduced to "traditional" lifeways. For this reason, I will briefly unpack the concepts of village community, kinship, and interregional networks that together institute the (political) economic processes of production, exchange, and consumption in many, if not most, decentralized societies.

\section{Village Communities}

In this study I focus on one particular community within the social landscape of historical Bambuk, namely the Iron Age village of Diouboye (Figure 1.2). As defined by archaeologists Jason Yaeger and Marcello Canuto (2000:5), a community is an institution that simultaneously emerges from and coordinates both cooperative and competitive social interactions among people at a scale somewhere between the minimal kin group and society as an abstract whole. Importantly, these interactions reaffirm community identity, often in distinction to external relations, and they play out within specific places

on a regular basis. For the community of Diouboye, such places included the village itself as well as other locales across the immediate landscape.

The village - as a discrete settlement form, basis for community identity, and unit of political-economic analysis - has offered a valuable frame for archaeological inquiry around the world (e.g., Bandy 2004; Byrd 1994; Dueppen 2012; Flannery 1972b, 2002; Meskell 1998; Robb 2007). In general, village communities include several hundred to several thousand people who reside in the same settlement for most of the year. While people may come and go from a village at different tempos, the community maintains institutional coherence by coordinating regular interaction among internal social segments such households, lineages, age sets, genders, craftspeople, religious 
practitioners, ethnicities, and so forth. In turn, the institutions deriving from coresidence, continually work to shape the village as a built environment and spatial unit particularly amenable to archaeological analysis.

Whereas villages in centralized societies face oversight from non-local actors such as a chieftaincy or a state bureaucracy, those in decentralized societies exercise much greater (though never complete) economic and political independence. In the latter case, political asymmetries between villages and/or their constituent segments may ebb and flow at different tempos, but over the long-term these maintain a more-or-less symmetrical field of social relations through kinship and interregional networks.

Figure 1.2: Map of Diouboye showing residential areas and wards

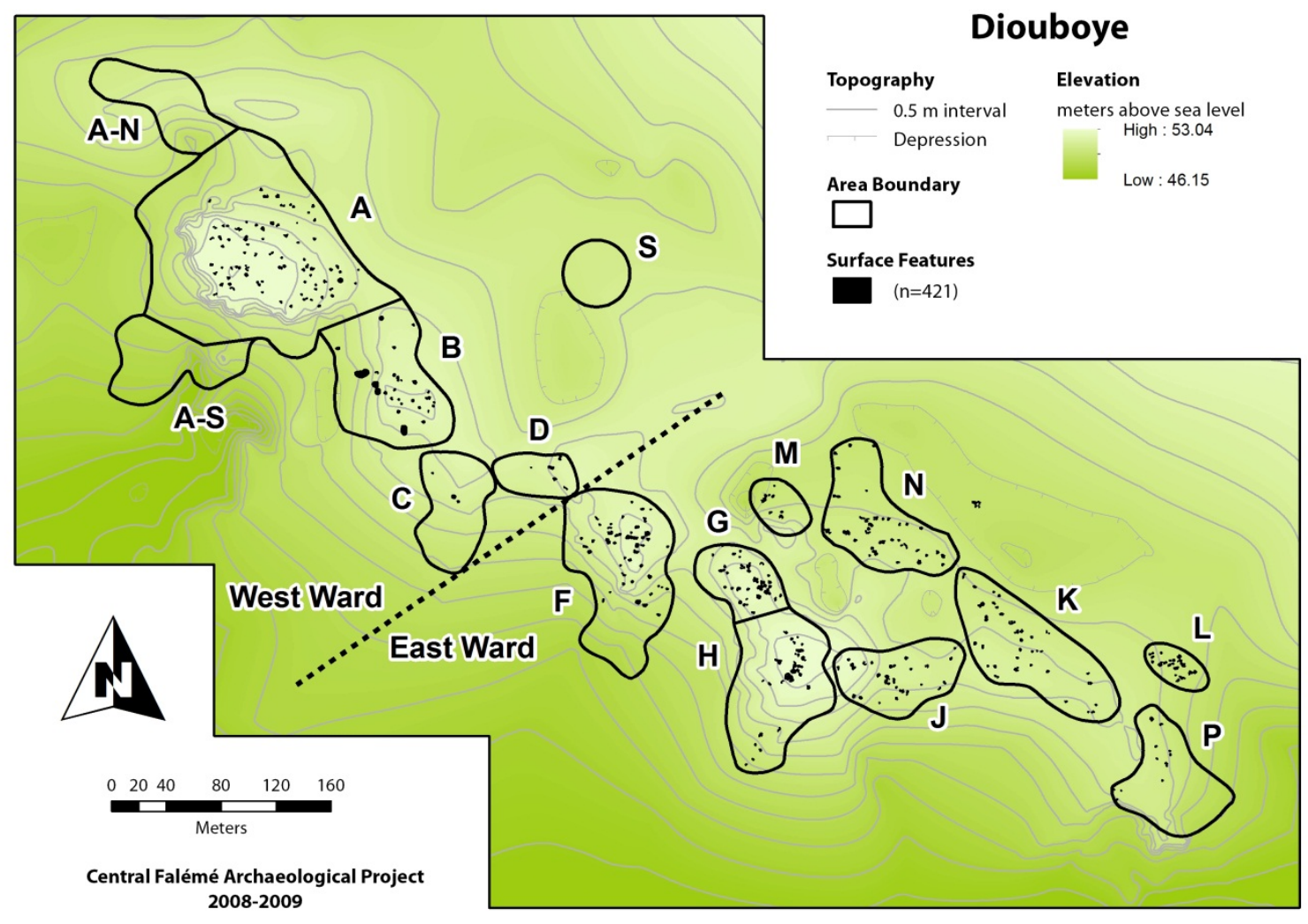




\section{Kinship}

Kinship accounts for much of the institutional matrix governing economic processes of production, exchange, and consumption in village communities (Cobb 1993; Meillassoux 1960; Siskind 1978; Terray 1972; Wolf 1982:88-100). Put simply, kinship appeals to symbolic constructs of “relatedness," including consanguinity, marriage, and filiation to harness labor through rights-in-people and to mobilize material resources through rights-in-things. In the absence of hierarchical political orders or juridical systems, kinship often provides the cultural logic for differentiating and linking people and objects and for translating rights in one to rights in the other through structured practices of exchange, marriage, and inheritance (Guyer 1995; Strathern 1985). Although power differentials based on gender and age exist within all kin groups, whether single households or entire lineages, political relations between kin groups can vary from relatively symmetrical to hierarchical even within the context of a single village.

Of particular relevance to village communities, corporate kin groups such as households or lineages mobilize human, material, and non-material resources through interactions grounded in specific places (Wolf 1982:89). This is not to discount the capacity of kinship networks to relate people near and far, but rather to highlight the role of residential spaces such as houses, compounds, and wards in coordinating kin relations for a common economic purpose. In this regard, the residential areas defined at Diouboye (Figure 1.2) provide a significant archaeological basis for tracing the dynamic relations among residential groups, presumably unified through kinship, within the associated village community.

\section{Interregional Networks}

Interregional networks comprise the myriad institutions that connect communities grounded in local places, and their internal segments, across the social landscape. For example, the idiom of kinship that structures corporate groups within a village also plays a key role in positioning such groups within supra-local networks. Greater physical and social distance, however, often translate as ancestral clan membership or ethnic identity, rather than direct descent — to the effect that economic coordination may no longer be a given. As a result, supra-local kinship networks may merge with gift-giving, marriage 
partnerships, ritual cults, and other institutional "sodalities" that mediate economic processes of production, exchange, and consumption between villages and corporate kin groups (Braun and Plog 1982; Ford 1972; Parkinson 2002; Wiessner 2002). Within decentralized societies, these sorts of economic networks underpin — or undermineasymmetries of political power among participating corporate groups and their leaders. In fact, some archaeologists distinguish between pathways for the evolution of permanent social inequality based on participation in interregional networks or on the mobilization of corporate groups (Blanton et al. 1996; Feinman 2000; Price and Feinman 1995; Spencer 1993). This perspective emphasizes the fact that interregional networks can operate as resources for local political economies, and vice versa.

Interregional economic connections that transcend the fluid boundaries between decentralized and centralized societies embed a mixture of symmetrical and hierarchical (asymmetrical) political relations. The former have received considerable attention through core-periphery models and similar perspectives focused on trade and the hierarchical ordering of social actors within complex political economies (Algaze 1993; Frank and Gills 1993; Peregrine and Feinman 1996; Wallerstein 1974). While power always inheres in relations between decentralized and centralized societies, scholars have become increasingly interested in the relative heterogeneity of power within these relations, particularly as small-scale communities negotiate their roles as producers and consumers vis-à-vis complex polities and merchant diasporas (Klein 2001; Sinopoli 2003; Stein 2002). As I elaborate in the Chapter 2, scholars working in Africa have drawn upon such insights to develop the concept of political-economic mosaic (sensu Kusimba and Kusimba 2005), which attends to the diversity of relations that inhere within societies and between communities at overlapping spatial scales.

\section{West African Historiography}

Given the long-standing exploitation of natural resources in West Africa by outsiders - from medieval North African merchants to historic colonial powers to contemporary multi-national corporations - it should come as no surprise that political economy has featured prominently in historical and anthropological studies of the subcontinent. As cogently summarized by Ann Stahl (2004a), research in this vein has 
focused mainly on understanding the place of Africa in global entanglements of the past few centuries, highlighting the richness and diversity of complex societies in Africa, and, by extension, working to overturn negative stereotypes of the African past. While these foci have proven incredibly productive, they have necessarily diverted attention from the decentralized societies interspersed both geographically and chronologically among their more centralized counterparts. At the same time, the available ethnographic, historic, and archaeological evidence suggests that village communities within societies of all scales have been vital to interregional political-economic mosaics in West Africa for at least two millennia, if not longer (Horton 1971; Stahl 2004b). The trick, then, is to mobilize these evidentiary sources for the archaeological study of social landscapes such as Bambuk, without mapping the present uncritically onto the past.

At a minimum, the use of ethnographic and historic sources in archaeological research requires that points of comparison be made explicit and that productive points of disagreement among oral, written, and material data be explored (Richard 2009). A direct-historic approach attending to source-side concerns (Stahl 1993a) remains beyond the scope of the present study, which focuses on one particular community, rather than a long-term historical sequence extending back from the ethnographic present. For this reason, it is important to briefly elaborate on the ways in which I draw these sources into the archaeological study of Diouboye in the following chapters.

\section{Ethnography}

Ethnographic accounts from West Africa describe diverse ways in which economic processes intersect with the politico-ritual structures, social identities, and cultural norms and values in small-scale rural communities (Chapter 2), as well as commonalities related to shared history and adaptation to highly seasonal environments (Chapter 3). In both these regards, I duly recognize that village communities of the colonial and post-colonial past are separated from those of the early-second millennium $\mathrm{AD}$ by several centuries of ecological change, state formation, slave-raiding and warfare, widespread conversion to Islam, and the continual reshaping of ethnic and linguistic boundaries. That said, a similar landscape and shared cultural history make contemporary 
societies of the Upper Senegal region the most appropriate analogues for their predecessors in the region.

The persistence of non-industrial subsistence and craft economies across much of rural West Africa means that ethnoarchaeology and material culture studies can also provide valuable insights on the social and logistical parameters that shaped, literally, material artifacts and architecture in the past (Roux 2007). While I draw heavily on these sorts of studies in Chapter 6 and Appendices D-G, it is important to bear in mind the effects of capitalism and other historical processes on supposedly "traditional" technological systems (Stahl and Cruz 1998).

\section{History}

As noted earlier, many historical studies of West Africa have focused on the more centralized societies and complex trade networks that dominated the medieval landscape (e.g., Bovill 1995; Levtzion 1980; Monteil 1968). For the Upper Senegal region around Diouboye (Chapter 4), I make some use of translated primary sources including medieval geographies (Levtzion and Hopkins 2000) and oral accounts recorded in the past two or three centuries (e.g, I. D. Bathily 1969; Delafosse 1912; Niane 1965; P. Smith 1965). In the absence of regional archaeological data, I make more extensive use of secondary sources summarizing the history Ghana and Mali, and smaller polities such as Gajaaga, to frame their possible relations with communities at or beyond their peripheries.

In the pursuit of political economies in small-scale communities, I also draw upon research in historical linguistics. While this field has opened considerable ground in the study of evolving political mosaics in the Great Lakes region of East Africa (Schoenbrun 1998), scholarship in West Africa has focused more heavily on the history of identities such as caste and ethnicity (A. Bathily 1975; Conrad and Frank 1995b; Tamari 1991). As I elaborate in Chapter 2, this research is valuable to the present study insofar as these identities have emerged alongside specialized subsistence, craft, and mercantile economies that transcend diverse speech communities across the West African savanna. 


\section{Archaeology}

The material remains — including artifacts, architecture, and landscapes - that emerged from and lent structural inertia to past social institutions and political economies stand to offer unique insights into the decentralized societies so often marginalized in historical narratives. This is particularly the case for the political-economic periphery of Bambuk, when historical accounts of the medieval era provide only tantalizing glimpses into the heterogeneous relations that existed between local communities, faraway political centers, and itinerant merchants.

Throughout much of the $20^{\text {th }}$ century AD, archaeological research on the Iron Age in West Africa focused on iron technologies and the rise of complex societies (Mauny 1961; Shaw et al. 1993). As a "handmaiden to history," early archaeology emphasized the ways in which complex technologies and "civilization” supposedly made their way into sub-Saharan Africa from the circum-Mediterranean world (see discussion in S. K. McIntosh and R. J. McIntosh 1988). More recent scholarship documenting the autochthonous character of these developments has, out of necessity, continued to focus on political centers located along major rivers and terrestrial trade routes-including Kumbi Saleh (Berthier 1997), Tegdaoust (Devisse 1983), Sinçu Bara (Bocoum and McIntosh 2002), Jenné-jeno (S. K. McIntosh 1995), Gao (Insoll 1996), Tadmakka (Nixon 2009), and Timbuktu (S. K. McIntosh and R. J. McIntosh 1986). The result has been to overturn preconceptions about the ultimate causality of external influences on African social developments and, in so doing, to highlight the diverse pathways to politicaleconomic complexity taken by societies across the continent (S. K. McIntosh 1999a; Stahl 2004b). At the same time, archaeologists have become aware of the need to contextualize these nodes of complexity within their broader socio-historical landscapes.

As a result, archaeologists have increasingly turned their attention to Iron Age communities situated outside major political centers. As I discuss in Chapter 4, research has focused both on villages documenting cultural continuity and change over the longuedurée (Dueppen 2008; Mayor et al. 2005; Thiaw 1999; Togola 2008), as well as on networks of settlements and other places that comprise political landscapes (Gallagher 2010; A. Lawson 2003; Richard 2007). Altogether, these studies highlight the diverse and 
dynamic fields of social organization experienced by small-scale communities, and their differing levels of integration within broader cultural spheres and political economies.

In this regard, the archaeology of West Africa from the $16^{\text {th }}$ century AD onwards has illustrated the myriad ways in which decentralized societies experienced and participated in the reorientation of interregional political-economic networks from the savanna towards the Atlantic littoral (DeCorse 2001b; Ogundiran and Falola 2007). As I elaborate in Chapter 4, research in this vein shows how the study of material and spatial practices can be set in productive tension with historical sources to understand interregional entanglements from the perspective of both global systems and local communities (e.g., Ogundiran 2009; Stahl 1999a). This perspective is fundamental to understanding how village communities within Bambuk participated in the mosaic political economy of West Africa in the centuries prior to the Atlantic Era.

\section{DIOUBOYE: A CASE STUDY}

Sprawling along an archaic levee less than one kilometer east of the Falémé River, the archaeological site of Diouboye (pronounced JU-boy) comprises a series of four or five low mounds interspersed with artifacts and architectural debris spread over nearly 12 hectares (Figure 1.2). During the course of its occupation circa AD 1000-1400, this village would have been situated at the western edge of Bambuk, and certainly within the decentralized social landscape described by medieval geographers as Lamlam. At the same time, the size and preservation of this large village - which forms a well-known local landmark — contrasts with the surrounding archaeological landscape of ephemeral settlements and activity areas. For these reasons, Diouboye invites and stands ready to address questions about the position of small-scale communities within the increasingly complex historical landscape of West Africa.

Today, Diouboye lies within the arrondissement of Kénieba in the Department of Bakel. With a population density of approximately ten people per square kilometer, this is one of the most sparsely populated administrative regions in Senegal. Excepting some transhumant Fulbe pastoralists, the contemporary population resides in dispersed settlements ranging in size from extended family hamlets to larger villages of several hundred people. These latter are usually poly-ethnic communities with people from the 
self-ascribed ethnic groups of Fulbe, Soninke, Jakhanke, Malinke, and Wolof found throughout the broader Upper Senegal region. Beyond this ethno-linguistic heterogeneity, people are invariably Muslim, many belonging to the prominent Sufi brotherhood of Tijaniyyah. Notably, this diverse social geography finds expression in historical traditions concerning Diouboye itself: Fulbe communities in the immediate vicinity attribute the site to the jihads of al-Hajj Umar Tall in the $19^{\text {th }}$-century AD, while a Malinke community at the village of Kénieba claims much earlier ancestral ties to the site.

Economically, the Upper Senegal does not achieve the per-capita agricultural and pastoral returns of the more fertile Middle Senegal and the Gambia-Siin-Saalum basins, nor does it possess the more substantial mineral wealth of the Upper Niger region to the south. The region is nearly devoid of infrastructure; the dirt tracks linking villages along the lower Falémé River quickly become inundated with mud during the summer monsoon season (and inaccessible to all but the most obdurate $4 \times 4$ vehicles), essentially depriving the populace of basic health services and required goods and services. The infrastructure that does exist—wells, telephones, schools, mosques, and so forth—has discouraged flexible land use and settlement strategies, possibly to the long-term detriment of ecological sustainability around permanent villages.

It would be a mistake, however, to project the contemporary cultural landscape of the lower Falémé onto the one occupied by the inhabitants of Diouboye. Oral and written accounts of the Upper Senegal region (A. Bathily 1989; A. F. Clark 1999; Curtin 1975a; Gomez 1992; Robinson 1985), alongside a growing number of archaeological studies (Altschul et al. 2010; Gokee 2011; Thiaw 1999, 2012), reveal that present-day regional political economies and ethno-linguistic identities were profoundly shaped during the tumultuous history of the past four or five centuries-a period that includes the structural violence of the Atlantic slave trade, the widespread conversion to Islam, large-scale population movements, and ultimately, the imposition of French colonial rule. Prior to ecological degeneration and population decline in the early- $20^{\text {th }}$ century $A D$, the Upper Senegal was a veritable breadbasket capable of producing ample, if not surplus, quantities of millet, sorghum, groundnuts, and even cotton, maize, and rice. And, as previously mentioned, the historical land of Bambuk, situated in the heart of the Upper Senegal region, was an important source of gold throughout medieval times. 
To the extent that these regional narratives note the persistence of decentralized societies along the lower Falémé River, the question becomes how and why this particular landscape perpetuated small-scale, yet certainly dynamic, communities in the face of interregional trends towards ever more complex political economies. Addressing this question entails a methodological shift to a multi-scalar archaeological study of the material and spatial practices intersecting within a village community such as Diouboye.

\section{History of Archaeological Research}

The ruins of Diouboye are well-known to people from nearby sedentary villages, as well as the pastoralists and their livestock migrating annually along the Falémé River. Although Diouboye appears on the Dalafi topographic map (1:200,000) published by the Direction des Travaux Geographiques et Cartographiques of Senegal, the earliest known archaeological observations were made by Abdoulaye Camara and colleagues from the Institut Fondamental d'Afrique Noir (IFAN) in May 1983. However, the focus of their mission on Stone Age occupations precluded any systematic investigation beyond collecting several pottery sherds to document the Iron Age component at the site (IFAN collections SEN 83.114.2).

Several years later, Camara accompanied Marie-José and Howard Opper to the site where they collected surface artifacts and excavated a small test unit over a circular stone hut foundation (Opper and Opper 1990). A handful of beads and copper ornaments recovered during this mission attested to participation by the people of Diouboye within interregional exchange networks sometime around the early-second millennium AD, while earthenware sherds, flaked stone tools, and terracotta figurines documented local craft production and ritual practices.

During a third archaeological reconnaissance in 1996, Hamady Bocoum, Edmund Dioh, and Seydou Camara drafted the first plan of Diouboye and undertook further surface collection of materials including earthenware pottery, terracotta ornaments, slag, a spindle whorl, a polished hematite hachette (celt), and flaked mudstone blades (IFAN collections SEN 96.52.2). On the basis of these finds, the site was tentatively determined to represent a transitional Late Stone Age to Iron Age occupation. 
Despite their differing objectives, these missions all highlighted in one way or another the anomalous presence of Diouboye within an archaeological landscape otherwise lacking in large and well-preserved settlement sites.

\section{Central Falémé Archaeological Project (CFAP)}

Intrigued by these prior findings, I set out for the lower Falémé River in June 2007 to explore its potential for an archaeological study of the poorly understood transition from Late Stone Age (LSA) to Iron Age occupations in West Africa (see discussion in Chapter 4). Accompanied by colleagues from the University of Michigan, IFAN, and a local guide, we visited several sites around the Jakhanke village of Tomboura and the Fulbe villages of Sansanding, Goundafa, Diombalou, and Madina Foulbé (see Appendix A); I would come to define this territory as the Madina-Sadatou study area (Chapter 3). Through cursory observations and judicious surface collections, we documented several ephemeral LSA sites with flaked quartz and chert, abandoned villages with European artifacts dating to the Atlantic Era, and at least three settlements (including Diouboye) with earthenware pottery and iron tools consistent with an Iron Age occupation (Chapter 4). The results indicated that region-oriented research could viably situate the village of Diouboye within its broader social and historical landscape.

In order to evaluate the relative chronology and stratigraphic preservation at Diouboye, which had ample surface remains (Appendix B), the 2007 mission also involved a small test excavation. As described in Appendix C, this sounding (Trench AZ) confirmed the excellent preservation of artifacts, including faunal and macrobotanical remains, and an occupation history confined to the Iron Age. Although these results precluded a study of the LSA to Iron Age transition, they demonstrated the potential to study a village situated on the periphery of Bambuk coincident with the expansion of centralized societies and trade networks across West Africa.

With this possibility in mind, I returned in January-April 2008 to initiate a study of the political and economic processes materialized within and beyond the village. The CFAP field crew included Matar Ndiaye from the Univesité Cheikh Anta Diop (UCAD), veteran field worker Tamsir Maïga, and three young men from Sansanding. Together we excavated two smaller units (Appendix C) and undertook systematic surface collection, 
mapping, and ground survey (Appendix B) at Diouboye over the course of eight weeks. We also conducted a full-coverage survey of nearly 72 square kilometers along the Falémé River to chart spatial, temporal, and social relations between Diouboye and the broader physical and historical landscape (Appendix A).

Building upon the results of this fieldwork, I directed further excavation, surface collection, and mapping at Diouboye in March-June 2009 with the assistance of Aimé Kantoussan and Massal Diagne (UCAD) and five local workmen from Sansanding. Over the course of 13 weeks we opened an additional eight excavation units of various sizes and completed the mapping of surface features across the entire site. In order to flesh out the regional interpretation of LSA and Atlantic Era sites documented during survey, we also undertook systematic surface collection and opened four small test units at the multicomponent site of FAL-1075, located approximately one kilometer southeast of Diouboye on the east bank of the Falémé River (Chapter 4).

We sorted, washed, and catalogued all of the materials recovered through excavation and surface collection at a make-shift field laboratory in Sansanding. Although we examined and discarded a substantial number of artifacts such as pottery sherds and chipped stone debris in the field, we transported a representative set of artifacts to IFAN at the end of each field season for more detailed analysis and curation; I describe the sampling procedures in Appendices A-G.

Although I personally analyzed most artifacts during the 2008 and 2009 seasons and a follow-up trip to IFAN in 2010, Aimé Kantoussan and Massal Diagne assisted with the study of pottery and chipped stone artifacts. From 2009 to 2012, I undertook spatial and statistical analyses of these and other material data at the University of Michigan using ArcGIS (ESRI 2010), JMP 9.0 (SAS Institute 2011), PAST 2.07 (Hammer et al. 2001), and other software packages where noted. Beginning in 2010, Stephen Dueppen and Daphne Gallagher began the analysis of, respectively, the faunal and macrobotanical remains from Diouboye at the University of Oregon; their preliminary findings are appended to this volume. 


\section{Research Questions}

With these anthropological perspectives on the political economy of village communities and the particular historiography of medieval West Africa as a backdrop, I initiated the Central Falémé Archaeological Project to address two interrelated questions about Diouboye and its immediate landscape:

(1) How did political-economic institutions shape, and emerge from, material and spatial practices within and beyond this village community? And how did these institutions evolve or persist through several centuries of occupation in the early-second millennium AD?

(2) How did (or did) the political economy of Diouboye, enmeshed within the decentralized social milieu of Bambuk, engage with the expansion of interregional exchange networks and shifting relations among more centralized societies across West Africa at this time?

With these questions as guideposts, I advance two general claims in the pages that follow. First, I argue that economic processes most vital to the community-subsistence production, craft manufacture, and long-distance exchange-never formed the basis for institutionalized political asymmetries among residential groups at Diouboye, although they probably underwrote differences along lines of age and gender within groups, and connections to people occupying smaller settlements in the vicinity. Rather, these economic processes were part of dynamic, yet relatively symmetrical, political relations, potentially fostered by an egalitarian ethos in the face of interregional trends towards more centralized and hierarchical institutional arrangements.

This possibility leads to my second, and more tentative, claim that the village community never fell directly under the direct control of major interregional polities. On the one hand, I suggest that Diouboye participated in the Mande cultural sphere centered on the upper Gambia and Niger Rivers (Pays Mande) to the south-a reading with implications for Mande history to which I return periodically throughout this study. On the other hand, the village also participated in long-distance networks emanating from polities such as Takrur and Gajaaga on the Senegal River to the north. Altogether, this suggests that small-scale communities of Bambuk actively negotiated their dynamic position within the complex political-economic mosaic of medieval West Africa. 


\section{OUTLINE OF THE VOLUME}

Drawing mainly upon the results of fieldwork and analyses conducted under the auspices of CFAP and summarized in the nine appendices, I pursue these claims through a study in three parts. The first part, comprising Chapter 2, elaborates the anthropological and historical perspectives, introduced above, on the political economy of village communities, particularly those occupying the West African savanna. In so doing, I outline my methodological approach to inferring past economic processes from their material and spatial dimensions inscribed in the archaeological record. I further lay out the ways in which these dimensions structured the political economy of Diouboye at two general scales — within the village itself and across the surrounding landscape.

Focusing on this latter scale, the second part of this study (Chapters 3-5) examines how physical, historical, and social aspects of the landscape shaped, and were shaped by, the political economy of Diouboye. Chapter 3 first presents a brief overview of landscape approaches in the archaeology of West Africa, then proceeds to situate Diouboye within its broader physical surroundings - including geomorphology, ecology, and long-term climate change. This discussion also explores how these parameters have shaped the economic strategies of contemporary agro-pastoral communities occupying similar physical spaces across the West African savanna.

Drawing upon these insights, Chapter 4 orients Diouboye within both the cultural landscape of West Africa and, more specifically, the occupational history of the MadinaSadatou study area. The material and spatial data collected during survey by CFAP define at least four broad horizons of occupation within Madina-Sadatou by diverse groups of foragers, herders, and farmers over the course of the Holocene epoch. When compared to archaeological and historical evidence from across the West African savanna, these data suggest that local populations in all periods maintained important social and economic ties to people and places beyond the immediate landscape. In the case of Diouboye, these connections place the community at the juncture of two political and cultural spheresone to the north encompassing the polities of Ghana, Gajaaga, and Takrur, and one to the south and east associated with the Pays Mande, the heartland of the Malian empire.

Chapter 5 employs a GIS-based analysis to compare the Iron Age landscape experienced by the residents of Diouboye to the earlier Late Stone Age and later Atlantic 
Era landscapes. By situating the aggregation of people at Diouboye within an historical context, it becomes possible to interpret the political and economic relations between this village and contemporaneous settlements and places of activity elsewhere in the vicinity.

The third part of this study (Chapters 6-8) focuses on the economic processes and political relations inhering among social actors in the village itself. Chapter 6 summarizes the activities and built environment that gave shape to life at Diouboye on a daily basis. In so doing, the chapter advances a middle-range methodology for interpreting material and spatial dimensions of the archaeological record as past socioeconomic practices of food and craft production and long-distance exchange in the following two chapters.

Chapter 7 first details the temporal sequence of material practices within each residential and non-residential area excavated at Diouboye, and concludes by anchoring the relative chronology of these sequences to associated radiometric dates. Deploying a micro-scale GIS analysis, Chapter 8 then considers synchronous surface artifacts and features to interpret the spatial dimensions of these material practices, paying particular attention to economic differentiation and integration within and between the East and West Wards of the village.

After weaving these interpretations into an historical narrative of Diouboye, Chapter 9 returns to situate this community within the anthropological study of local and interregional political economies. The archaeological study of Diouboye that I present in the following pages offers new insights onto the ways in which institutions emerged at multiple scales through the practices of food production, craft manufacture, and longdistance exchange comprising daily life in the land of Bambuk. 


\section{CHAPTER 2 \\ IRON AGE ECONOMICS IN WEST AFRICA: ETHNOGRAPHIC AND HISTORICAL PERSPECTIVES}

In a seminal contribution to the comparative study of cultural economies entitled Stone Age Economics (1972), anthropologist Marshall Sahlins identified the household as a fundamental social unit of production, distribution, and consumption in decentralized and peasant societies. Since then, archaeological studies of nascent social inequality have deployed this “domestic mode of production” to explore when and how hierarchical political relations evolve out of the surplus labor mobilized from supposedly autonomous households (see papers in Feinman and Nicholas 2004; Price and Feinman 1995)—a perspective that archaeologist Timothy Earle calls Bronze Age Economics (2002). Meanwhile, the concept of the household has provided a foil for scholarship, particularly on kinship in Africa, which emphasizes the corporate lineage in structural processes of economic production and social reproduction (see Guyer 1981). While these contrasting perspectives highlight the diversity of historical pathways to social inequality or complexity (S. K. McIntosh 1999c), they share the vital recognition that dynamic relations among social actors simultaneously depend upon and make possible the economic processes embedded within them at multiple scales.

An explicit recognition of these recursive sets of relations underlies the so-called institutional paradigm (Halperin 1994), which is critical for charting entanglements between local communities and centralized polities and mercantile networks both in and beyond West Africa. As noted in Chapter 1, studies within the frame of global political economy have helped to deconstruct the myth of "pristine” small-scale communities by showing how interregional connections shaped their institutions, identities, and cultural schemas during the onset and fluorescence of the “modern” era (e.g., Sahlins 1981; Wolf 1982). Moving beyond an emphasis on domination or penetration by the forces of capitalism (Frank and Gills 1993; Wallerstein 1974), research in this vein has also 
scrutinized the ways in which seemingly peripheral communities actively negotiated or resisted their place in emergent world systems and colonial projects (e.g., Cooper 2005; Mintz 1977; Roseberry 1989; Thornton 1992; D. R. Wright 2010). While such research concentrates mainly on events and processes of the past few centuries, it is important to recognize that cultural encounters and political-economic entanglements are not without precedent in earlier periods.

Archaeological studies have repeatedly shown that complex stratified politieswhenever and wherever they emerge-invariably sustain and depend upon relations with other societies that are organized at different scales (e.g., Sinopoli 1994, 2005; Stein 1998, 2002). A recent trend in the archaeology of Africa has been to view past political landscapes as mosaics assembling communities with diverse economies, technologies, social identities, embodied habits, religious beliefs, and cultural schema (Kusimba and Kusimba 2005:393). Within and between these communities, people may relate to one another synergistically through cooperation and coordination and/or antagonistically through conflict and competition. As these relations continuously emerge from, and define access to, material and non-material resources, they shape economic processes and form potential channels for political authority.

According to archaeologist Ann Stahl (2004b), the mosaic concept draws upon three critical insights made by ethnographers and historians working in Africa. First, these scholars have increasingly recognized that the ethno-linguistic boundaries drafted by earlier colonial administrators, missionaries, anthropologists, and other outside observers have fixed inherently dynamic social groups and subjectivities into supposedly static phenomena (Amselle 1998; Irvine 2008; Mudimbe 1988; Wilmsen 1989). This is not to suggest that ethnicities and other cultural boundaries did not exist in precolonial times, but simply to assert that the synchronic mapping of tribes, kingdoms, and similar ethnographic units may obscure the fluid patterns of kinship, religious practice, language, and cultural value that wove across them in the past.

The second insight, most cogently expressed by contributors to The African Frontier (Kopytoff 1987a), is that principles of segmentation, land tenure, and gerontocracy have led to continual cycles and shifts in political power between centralized societies and their frontiers over the longue-durée. Specifically, emigration 
from centralized "metropoles" generally resulted from factional competition and fission over various economic and social resources. In their move to the geo-cultural frontier, émigrés usually encountered autochthonous populations with customs and technology similar to their own, but whose status as "first-comers" entitled them to rights over land and, by extension, local political authority. When it was neither possible nor desirable to drive first-comers from the region, the new arrivals had to negotiate the reproduction of their old metropolitan social order through idioms of kinship, political hierarchy, and practices of reciprocity and redistribution (e.g., Jansen 1996; D. R. Wright 1985). Over time, these strategies could prove mutually reinforcing, yet remarkably fluid, in the production of political-economic mosaics among social actors and groups.

The third insight is that authority in many complex societies across precolonial Africa depended on the capacity of political leaders to integrate or "compose" material sources of power, including land, labor, and material capital, with non-material sources of power, including oratory or ritual knowledge (Guyer and Belinga 1995; S. K. McIntosh 1999a; Schoenbrun 1998). The associated forms of wealth—in things, in people, in knowledge- -blur the distinction between the means and the relations of staple or wealth finance that underwrite conventional models of political hierarchy and centralization. The result has been an enduring emphasis on authority grounded in the coordination, rather than outright control, of diverse social actors and groups, thereby contributing to complex constellations of political and economic relations.

Altogether, the concept of mosaic highlights the diversity of multi-scalar social institutions and identities that defined political economies in West Africa over the past two millennia. Against this theoretical and historical backdrop, I set out in this chapter to consider how such mosaics emerged from and gave rise to heterogeneous subsistence and craft economies and expanding long-distance exchange networks. Moving from general to specific, I first explore the concept of "Iron Age" political economies by looking at the ways in which specialization in production and exchange processes contributed to increasingly political and cultural differentiation at multiple socio-spatial scales. From there, I provide a schematic description of the political economies that have historically structured village communities in the West African savanna and that undoubtedly played a role in producing dynamic Iron Age landscapes. Finally, I introduce archaeological 
approaches to the study of political economy in and beyond such communities through material and spatial dimensions of structured practice. This discussion lays the groundwork for the subsequent chapters, which trace the emergence of Diouboye at the intersection of local economic practices, historical traditions, and global connections.

\section{IRON AGE ECONOMICS}

The punctuated appearance of complex polities and long-distance trade across West Africa over the past two millennia corresponds with the advent of marked economic heterogeneity—long considered a hallmark of "Iron Age” societies associated with nascent urban centers, stratified states, and extensive territorial integration (Childe 1951; Claessen 1984; Engels 1972 [1884]; Flannery 1972a; Friedman and Rowlands 1978; Johnson and Earle 2000:245-264; Marcus 2008; M. E. Smith 2004; H. T. Wright 1986; Yoffee 2005). Since most, if not all, early centralized societies were enmeshed within regional mosaics and interregional networks alongside decentralized ones, it bears asking: how did (or did) economic processes of production, distribution or exchange, and consumption shape communities within and between societies at these different scales?

In this study I focus on three economic spheres that became increasingly heterogeneous alongside the development of centralized societies in West Africa over the past two millennia: (1) subsistence economy; (2) craft economy; and (3) long-distance exchange. Subsistence economy subsumes the practices of production, exchange, storage, cooking, and commensalism through which people transform plants and animals (and salt) into edible foodstuffs. Craft economy, as conventionally defined by archaeologists (J. E. Clark and Parry 1990; Costin 1991), includes the manufacture of goods ranging from mundane containers and tools to prestige objects and ritual paraphernalia, whose exchange, display, and consumption sustain political and cultural life. Importantly for political economy in West Africa, some archaeologists have argued that performers of non-material arts - musicians, dancers, bards, and so forth—likewise fall within the rubric of craftspeople (Sinopoli 2003:160-171). Finally, I reserve the term long-distance exchange for those processes that move goods beyond their regions of origin. Modes of long-distance exchange can vary from circulation of professional merchants engaged in specialized trade to down-the-line mechanisms embedded in more localized acts of 
reciprocity, redistribution, and barter (Polanyi 1957; Renfrew 1975). While these aspects of the economy implicate distinctive resources and relationships, their intersections in practice remain vital to the reproduction of structural relations within and between village communities such as Diouboye. In the remainder of this section I sketch the ways in which subsistence economies, craft economies, and long-distance exchange become instituted within idealized "Iron Age" worlds—-that is, patchworks of centralized and decentralized societies intersecting at multiple scales.

\section{Economic Specialization: Locational and Appropriational Movements}

In this study I view economic heterogeneity primarily through the lens of specialization: an economic process of surplus production or exchange that is undertaken by one set of people, for consumption by other people or groups within a broader social milieu. Defined in this way, specialization extends the concept of a division of labor in society to encompass economic relationships between societies, including those organized at different scales. The material goods made and moved through specialized economies can include subsistence staples, utilitarian craft items, and prestige or "wealth" objects and commodities, while less tangible specialized services can vary from transportation and trade to the performance of religious ritual and maintenance of historical narratives. Most specialized economies depend on complex technologies that bring tools and other material capital together with the people whose practical and discursive knowledge makes production and exchange possible (Dobres 2000; Lechtman 1977; Lemmonier 1986).

The assembly of particular material and non-material resources entails what anthropologist Rhoda Halperin (1994), drawing upon a Marxist reading of works by Karl Polanyi $(1944,1957)$, has called "locational movements." Initially conceived as "changes of place" during economic processes of production, Halperin has correctly pointed out that distribution and consumption practices also involve locational movements of resources to people, or people to resources (or people as resources!). In the course of locational movements of and around resources, institutional relations emerge through "appropriational movements" or "changes of hands" within the economy. That is to say, a farmer harvesting crops (locational movement) is simultaneously reproducing the 
institutional structures of his or her household, village, or state (appropriational movement). Conversely, one sort of appropriational movement, for example state finance, can depend on locational movements of staples, wealth goods, or labor. As shown in Figure 2.1, locational and appropriational movements are not sequential steps, but rather two aspects in any given moment of economic practice.

The institutions that both emerge from and give rise to economic heterogeneity or specialization have dramatic effects on the organization of societies more generally. On the one hand, even as specialization concentrates the costs of acquiring capital and technological knowledge among only a subset of people in society, it encourages the returns on labor and other resources to increase through economies of scale (Childe 1951; Durkheim 1984 [1893]; Engels 1972 [1884]). Where specialization entails diversification of the economic base, it may even mitigate the risk to a social system and its constituent actors (R. J. McIntosh 2005:110-111; O'Shea 1981)—a benefit which no doubt plays a major role in stabilizing and legitimizing specialized economies when and where they emerge.

Yet the accrual of benefits from specialization—reduced risk and high returns on labor, resources, and knowledge-ultimately depends on the exchange of surplus goods and services among individuals or corporate groups. Institutions based on political symmetry among autonomous villages (Ford 1972; O'Shea 1981) or merchants operating outside the purview of a state (Curtin 1984; Greif 2006) often prove remarkably robust given a shared set of cultural norms and the incentive of future trade. Conversely, in more hierarchical contexts, institutions enforce contracts and structure the flow of goods from specialists to consumers, usually to the benefit of the latter and detriment of the former (Brumfiel and Earle 1987). In this sense, specialization within spheres of subsistence, craft production, and long-distance exchange may recursively structure the emergence of asymmetrical relations across a given social landscape.

Whatever the nature of the institutions that arise from economic specialization, they may all be characterized according to intersecting axes of variability within locational and appropriation movements: scale, concentration, and intensity (inspired by Costin 1991). Scale refers to the size and organization of corporate groups specializing in a given pursuit; these range from households and lineages to villages, classes, or 
ethnicities. The closely related concept of concentration alludes to the spatial distribution of these specialized economic processes and their corresponding corporate groups.

Intensity describes the relative amount (and regularity) of time, labor, and material (and non-material) resources devoted to an economic pursuit (e.g., part-time versus full-time specialization).

Figure 2.1: Schematic model of concepts and processes of political economy

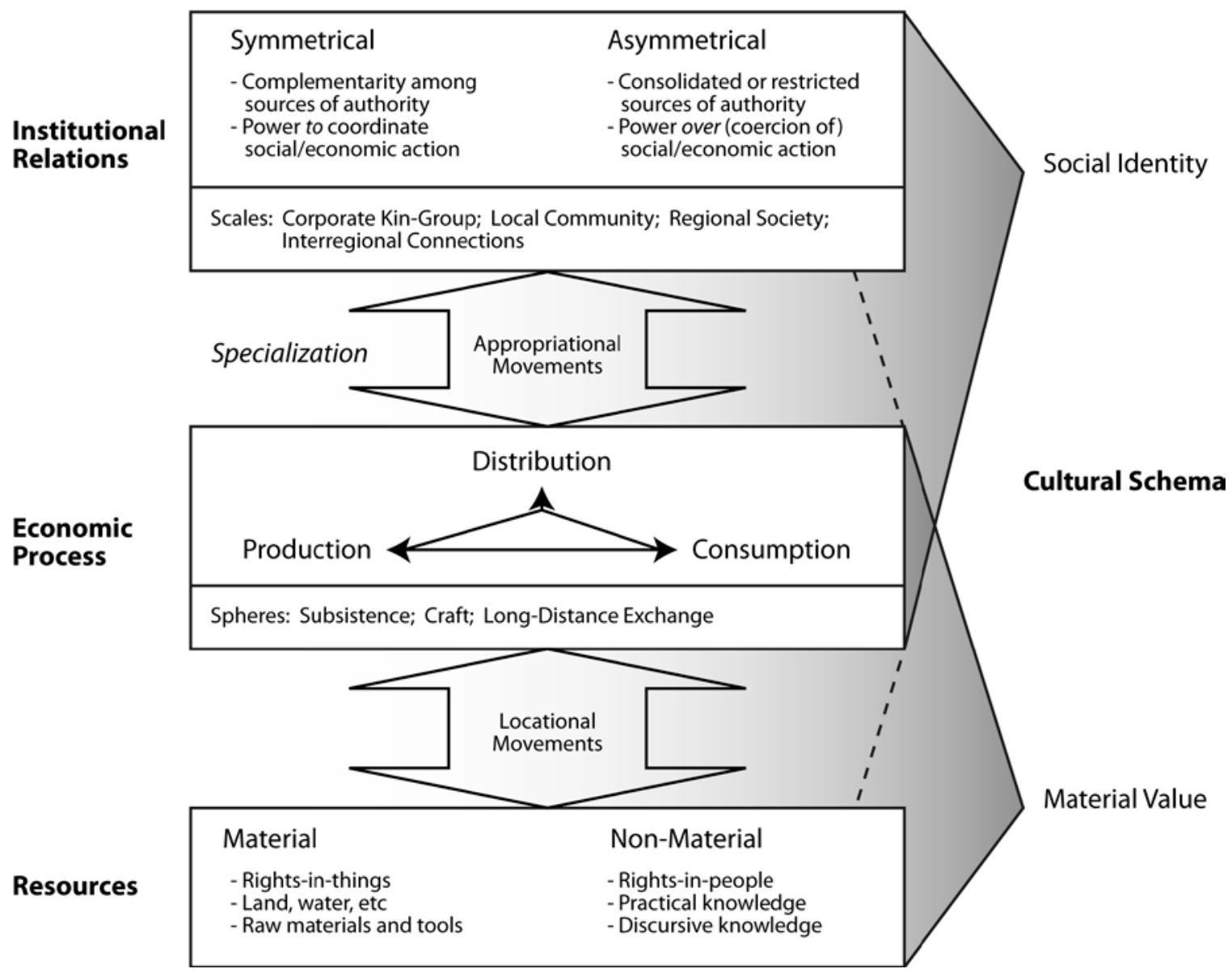

In decentralized societies, these variables of economic specialization play out largely along lines of gender and age, and at the scale of village communities or corporate kin groups. In the absence of political hierarchies governing exchange or redistribution, people tend to engage in specialized economic activities at a low to modest intensity 
scheduled around seasonal variability. At this level, specialization effectively reduces risk through diversification and social networks of material exchange, even as these networks inevitably shape access to and exercise of power between individuals and corporate groups. Centralized societies support more complex divisions of labor, greater concentrations of specialists, and more intensive production and exchange. While symmetrical relations between "specialized" societies may be predicated on economic complementarity (Stein 2002), asymmetrical relations often entail the extraction of tribute from "peripheral” regions by political “cores” (Peregrine and Feinman 1996; Wallerstein 1974). The ways in which villages, and their constituent segments, do or do not specialize in the material economy thus stand to illuminate political and cultural relations across regional mosaics and interregional networks. Of course, appropriational movements of non-material resources such as social obligations, practical knowledge, and ritual power also underwrite coordination and competition in both decentralized societies (e.g., Aldenderfer 1993; Fowles 2003). It follows that, beyond the economic underpinnings of social structure and process, institutions are never entirely reducible to the flow of material resources.

\section{Cultural Schema}

As a specific sort of non-material resource, cultural schemas play a vital role in legitimizing economic processes and political relations. By cultural schemas, I refer to the systems of semiotic interpretation that emerge from interactions among subjects and objects in the reproduction of social structures (Sewell 1992). At a fundamental level, schemas make such interactions possible by establishing the very possibility of an ontological distinction between subjects and objects (Foucault 1970; Keane 1997; Latour 1993). A discussion of material semiotics lies well beyond the scope of this study, but I would like to briefly consider two particular themes: (1) how social identities and distinctions become mapped onto economic specialization (or vice versa); and (2) how material resources accrue value within specific trajectories. These issues are particularly relevant for the study of "Iron Age" landscapes where political-economic processes often cross social and cultural boundaries. 


\section{Social Identities}

Economic practices shape social identities insofar as they call upon and reproduce different sets of practical and discursive knowledge. Practical knowledge includes the embodied gestures, habits, and savoir-faire that people acquire and reproduce, usually without remark, on a day-to-day basis. Discursive knowledge, at least from an economic perspective, includes people’s conscious understandings of material resources, including the physical landscape, and of processes for the transformation, distribution, and consumption of these resources. Economic specialization leads to, and often depends on, the heterogeneous distribution of this knowledge according to social statuses such as gender, age, kinship, caste, class, and ethnicity (Costin and Wright 1998; Dobres 2000; Hegmon 1998). These categories, in turn, shape the transmission and acquisition of knowledge over time. This is not to imply that all identities coalesce around economic processes, but rather that specializations often emerge among existing social distinctions, or become a basis for the construction of new ones.

Identities provide points of distinction and articulation between communities. On the one hand, different subsistence regimes, craft technologies and styles, and strategies of long-distance exchange and trade can all work to establish and maintain social boundaries as points of distinction. On the other hand, some identities may articulate people across these boundaries, particularly where they historically share, for example, cultural schemas about gendered economic practices, craft production as a source of ritual power, land tenure and political status, or norms of hospitality. The ways in which communities perpetuate social identities at overlapping scales can thus provide insights into how people within them perceived and legitimized underlying institutions.

Asymmetrical political relations often depend on or create identities of relative objectification. In decentralized societies, for example, people often "belong” to corporate groups such as households or lineages in two senses- "that is, they are members of the group and also part of its wealth, to be disposed of in its best interests” (Kopytoff and Miers 1977:10). Whereas rights-in-people sustain asymmetries along lines of gender or age within corporate groups insofar as collective rights are administered by men or elders, they may also generate asymmetries between corporate groups or societies that form the basis for institutions of slavery (Meillassoux 1986; Miers and Kopytoff 
1977). Social identities at these multiple scales not only shape personhood, but also the potential for people to become "commoditized" alongside material resources.

\section{Material Value}

At the same time that they shape the identities of human subjects, economies reproduce the semiotic value(s) of material objects that frame and mediate interpersonal interactions. As I mentioned in the preceding chapter, these values emerge through culturally instituted processes of production, distribution/exchange, and consumption (Appadurai 1986; Halperin 1994:85-142; Keane 1997; Robb 1999a; Thomas 1991), or, in some cases, exemption from such processes (Weiner 1985). This sort of cultural relativity holds equally true for both "symbolic" and "utilitarian" value since material resources are only recognized as such vis-à-vis cultural schema (Sewell 1992).

The inculcation of material value occurs most explicitly within markets and in public politico-ritual ceremonies (Keane 1997; Rappaport 1979), but for the most part the reproduction and transformation of cultural schema in relation to material objects is much more subtle: "[A]ny practice carried out in the material world depends on, and validates, many different cultural structures, even when, to the actors involved, most are unconscious or not salient and the obvious point of the thing is to get some functional task done or solve an annoying problem” (Robb 1999b:8). This means that material values may shift subtly as objects move across, or work to constitute, the fluid boundaries between communities.

Such materiality not only emerges from, but is fundamental to, social institutions and cultural schema involved in political economy. Practices of material production, exchange, and consumption, all situated within physical places, are precisely what allow institutions and structures to extend across (social) space and persist through time (Giddens 1984). It follows that the cultural economic processes enrolling material objects and physical places can provide key insights into political relations, including asymmetries of power, that inhere within and between communities at different scalesfrom small villages to entire landscapes. 


\section{History Matters}

Having fleshed out the roles of locational and appropriational movements and cultural schema in a general sense, it is important to emphasize that political economies exist within, or as, specific histories whose multiple tempos-from the events of daily practice, to the conjuncture of institutional processes, to the longue-durée of structural reproduction and change-profoundly shape the character of societies and their constituent communities (Baker 2003; Braudel 1980; Sahlins 1981; Sewell 1992). Decentralized societies, for example, have institutional arrangements oriented towards the diffusion of social authority over the span of generations, even as particular events may allow households, lineages, villages, and other factions to accrue political power through economic practices over the short-term. Over time, these processes can, of course, generate permanent changes in structures, as both resources and cultural schema.

Additionally, the ordering (and unpredictability) of events and processes can profoundly shape future events and processes. Structural principles and institutions do not emerge de novo in every single social interaction, but depend upon existing constellations of resources and interpersonal relations. Material resources such as land, houses, surplus staples, and wealth objects take time to accumulate, and, once accumulated, structure future social actions. Specialized economies also require the development of practical know-how, including knowledge of the landscape and materials involved in producing food and manufacturing and exchanging crafts and other commodities. Since existing social institutions constrain the possible pathways of subsequent developments, it is vital to contextualize a particular political economic mosaic within the deeper history of the accompanying landscape.

\section{A VIEW FROM PRECOLONIAL WEST AFRICA}

Moving from this abstract view of "Iron Age” political economies in general, I turn now to develop a more specific, if admittedly schematic, view of political economy in village communities across precolonial and ethnographic West Africa. While this exercise risks perpetuating the trope of "traditionalism" in rural societies, my goal is ultimately to outline the material and spatial dimensions of dynamic economic processes, political institutions, and cultural schema. In so doing, this discussion forms the backdrop 
for a closer look at historical landscapes in Bambuk and the Upper Senegal region (Chapter 4) and the interpretation of archaeological data from Diouboye (Chapters 4-9).

\section{Subsistence Economy}

People across the West African savanna have long maintained a diverse array of subsistence strategies and culinary practices. Since these feature in later discussions of human ecology (Chapter 3) and technologies of food storage, preparation, and consumption (Chapter 6), I will simply note here that complementary specializations in pastoralism, fishing, and different forms of agriculture (i.e., flood-recession, rain fed) were widespread within and between precolonial societies (Burnham 1980; Casey 1998). More to the point, two aspects of these subsistence regimes commonly figure in the village economies - the kin-ordered mode of production and institutions for coordination and cooperation among lineages and other corporate groups.

\section{The Kin-Ordered Mode of Production}

Although Marshall Sahlins (1972:74-78) anticipated many of the problems with abstracting the autonomous household from its broader cultural milieu, this concept has proven especially inadequate for the study of precolonial (and contemporary) political economies in Africa. Conventionally, the concept of the lineage has offered a better accounting of political economies predicated on both wealth-in-things and wealth-inpeople (Fortes 1953; Meillassoux 1960; Miers and Kopytoff 1977). That said, I argue that reconciling the structural perspectives of household, lineage, and community with each other, and with historical process, lies in a more flexible view of the means and relations of production through kinship networks.

This kin-ordered mode of production refers to economic processes embedded within corporate groups based on the idiom of common descent (Cobb 1993:46-49; Siskind 1978; Southall 1988; Terray 1972; Wolf 1982:88-96). Kinship does not necessarily (or only) designate biological relatedness; it also refers to social genealogies grounded in coresidence and networks of mutual obligation. Regarding the political economy, "kinship can then be understood as a way of committing social labor to the transformation of nature through appeals to filiation and marriage, and to consanguinity 
and affinity” (Wolf 1982:91). Since kinship also structures the transmission of material resources and practical and discursive knowledge across generations, it forms a key axis for reproducing relations of production, as well as exchange and consumption, over the longue-durée. In so doing, kinship provides a basis for identities such as class and ethnicity that emerge through relations within and between societies.

Kinship was fundamental to the organization of subsistence production in many, if not all, precolonial societies of West Africa-from semi-autonomous villages to centralized states (Coquery-Vidrovitch 1976; Fortes 1953; Meillassoux 1960; Southall 1988; Terray 1972). At one end of the political spectrum, decentralized societies maintained fairly unspecialized subsistence economies at the scale of the segmentary lineage and/or local community. For instance, Tallensi and LoDaaga communities of the Upper Volta region (Fortes 1940; Goody 1957; Labouret 1931) historically consisted of more-or-less autonomous extended households, each engaging in a similar suite of cultivation and light herding activities. In the Upper Senegal region, kin groups within the village-based communities of Bassari and Coniagui (Tenda) and Malinke communities likewise engaged in a similar suite of flood-recession (décrue) and rain-fed farming activities (Lestrange 1955; Leynaud and Cissé 1978; Pélissier 1966), regardless of their differing historical relations to stratified states. In these examples, the relative "wealth" of any single household or lineage was tied closely to the number of individuals that could be mobilized to work the land. Meanwhile, ritual and other forms of discursive knowledge provided avenues to social authority independent of differential access to material resources between kin groups (see also S. K. McIntosh 1999c:11-14).

At the other end of the political spectrum, kinship also structured the subsistence economies of centralized polities where authority rarely depended upon the mobilization of subsistence (staple) surplus. In her search for an “African mode of production,” historian Catherine Coquery-Vidrovitch (1976:92) states that "even in the most presumably 'despotic' states (the medieval Sudanic kingdoms, the kingdom of the Kongo, Ashanti, Dahomey), the prestige of the sovereign...never completely effaced tribopatriarchal [kin-based] authority. At most, the kingship took the form of a superimposed bureaucracy which nonetheless respected the structures of rural life.” The persistence of kin-ordered subsistence production in more centralized societies lay in an 
abundance of land that made it possible for kin groups to "vote with their feet" if wouldbe elites tried to subvert the strategies of storage or reciprocity supporting local subsistence regimes (S. K. McIntosh 1999c:18-19). Ultimately, craft economies and longdistance exchange provided a more reliable basis for asymmetrical status differences within and between societies of different scales, a point to which I return below.

In sum, the logic of kinship largely structured local subsistence economies across the heterogeneous political mosaic of precolonial West Africa. It follows that the kinordered mode of subsistence production can frame the study of village-level economies not only within decentralized societies, but also in relation to more centralized ones. Moreover, in any given historical context, the kin groups engaged in subsistence production also comprise a baseline for measuring the scale of craft and exchange practices (and their corresponding political institutions). I turn now to examine how subsistence specializations beyond the kin group structured the political landscape at different scales.

\section{Coordination and Cooperation (and Conflict)}

The types of institutions linking corporate kin groups are potentially quite diverse, but two that have emerged alongside subsistence specializations in West Africa are interethnic complementarities and territorial confederations (S. K. McIntosh 1999c:9). The example of inter-ethnic complementarity par excellence comes from the Middle Niger region of present-day Mali. Here, the broad inland floodplain of the Niger River adjoins a climate with strong seasonality and inter-annual variability yielding a dynamic patchwork of ecological niches most effectively exploited by specialists (R. J. McIntosh 2005:101-123). The technologies and knowledge of specific subsistence practices underlie ethnic identities such as Somono (deep river fishing), Bobo (shallow water fishing), Nono/Marka (rice cultivation), Bambara (millet and sorghum farming), and Peul (herding); the result is in an extremely heterogeneous political-economic mosaic (Gallais 1962; Sundstrom 1972). While these subsistence specializations and ethnicities have changed through time (see Chapter 4), their "accommodation" within the microcosm of the Middle Niger has historically depended upon three sorts of institutionalized relations: politic-ritual rights afforded first-comers such as the Bozo fishing communities over late- 
comers such as the Bambara millet farmers; fictive kinship mandating reciprocity between ethnic groups; and the perpetuation of mythical, or at least legendary, debt relations (R. J. McIntosh 2005:112-113). Even if these relations did not always prevent scheduling conflicts over access to flooded lands and pasturage, they were effective enough to ensure the continuation of specialized subsistence economies over the past several centuries without centralized political oversight.

Less complex complementarities exist elsewhere across the West African savanna between sedentary farmers and transhumant Fulfulde-speaking pastoralists known variously as Fulbe, Peul, Fula, or Fulani (C. Ba 1986; Kesteloot et al. 1993; Stenning 1957). In central Nigeria, for example, the Fulani provide sedentary farming communities with milk and meat, seasonal labor, and manure for fallowed fields in exchange for dry season grazing rights (Waters-Bayer and Bayer 1994). The prospect of future dealings is generally sufficient to ensure cooperation, but conflicts can arise during the wet season if (or when) Fulani cattle trample crops. When this happens, leaders from each group meet to negotiate a payment (nowadays cash) for the damages. In contrast to the Middle Niger, inter-ethnic complementarities depend largely on the projection of the intra-group kinbased political and judicial structures onto the field of inter-group interactions. Over the long-term, demographic shifts and climate change have led periodically to regional conflicts, particularly in Senegambia (C. Ba 1986; A. Bathily 1989:238-246; Pélissier 1966:358-361), but the resilience of farmer/herder interactions and identities suggests that these specializations have historically been beneficial to both groups.

A final example comes from the territorial confederations and historical polities of sedentary Ful6e (e.g., P. Diagne 1967; Gomez 1992; Robinson 1985). In the Middle Senegal region, territorial units called leydi oriented along the river each include fishing waters, seasonally flooded zones for rice farming, and upland areas for rain-fed farming. Accordingly, each territory conjoins three groups of subsistence specialists-fisherfolk (cuballo), pastoralists (pullo), and farmers (tooroodo)—all speaking Fulfulde and sharing common social institutions and cultural schema (Schmitz 1994). The fluidity of these social identities may in fact underlie the emergence of regional subsistence specializations since sedentary farming communities could "slough off" people into fishing and herding groups during poor agricultural years as a risk-buffering strategy ( $\mathrm{T}$. 
K. Park 1992). At the same time, these groups participated equitably in scheduling access to lands and adjudicating conflicts within the territory. A number of endogamous classes (see below) ranked some lineages within each group above others, and local leaders were selected from these on a rotating basis. As summarized by Susan McIntosh (1999b), this institutional configuration offered an alternative logic to the inter-ethnic complementarity of the Middle Niger region, yet it may have similar time depth in mediating relations among subsistence specialists.

\section{Looking Ahead}

In sum, subsistence economies across precolonial West Africa generally shared a kin-ordered mode of production organized at the scale of the household or lineage. While these corporate groups were largely unspecialized with regards to food production in their local communities, they often maintained supra-local complementarities as specialists at a regional scale. As I discuss in Chapter 4, these heterogeneous subsistence economies, and their corresponding political institutions, emerged across West Africa during the Early Iron Age, and were almost certainly in place by the occupation of Diouboye in the early-second millennium AD.

For these reasons, I contend that residential kin groups provide an appropriate unit of social (and spatial) analysis within the archaeological site of Diouboye and across its immediate landscape. Even as these groups coordinated labor and exchanged surplus foodstuffs with each other, I will argue that the subsistence economy of this village did not, in contrast to the complex mosaics associated with political centers along the Senegal and Niger Rivers, form the basis for political relations beyond the local territory.

\section{Craft Economy}

The craft economy of precolonial West Africa subsumes the production and exchange of material goods including pottery, metal tools and ornaments, carved wood, cloth, ritual objects, and even architecture, as well as performances of dance, music, and history. Crafts and craftspeople have received attention from scholars interested in art and aesthetics (e.g., Berns 1989; Frank 1998), social identities (e.g., Arnoldi et al. 1996; Conrad and Frank 1995b; Herbert 1993; McNaughton 1988), ethnoarchaeological 
principles (e.g., LaViolette 2000; Roux 2007), and, of course, the interpretation of the archaeological record comprising, in large part, the remains of crafts (e.g., Livingstone Smith 2007; Mayor 2010). Although these studies touch upon political economy in different ways, this has not been a major focus of research in West Africa where full-time specialists attached to political elites were uncommon in historical contexts (griots being a notable exception).

Craft specialization can occur at multiple and overlapping scales, but in keeping with the kin-ordered mode of production I focus on the concentration and intensity of craft production within corporate kin groups. Across the savanna, craft specialization at this scale has helped to underwrite the so-called caste system that, in one form or another, cut across ethno-linguistic boundaries and urban/rural divisions (LaViolette 2000; Tamari 1991). Notably, historians postulate the emergence of this system alongside the expansion of Mali during the Late Iron Age, coincident with the occupation of Diouboye. Therefore, the extent to which the craft economy did, or did not, map onto the kinordered mode of subsistence production within this community can help to illuminate how people within Bambuk related politically and culturally to their neighbors.

\section{Sudanian Caste Systems}

A tripartite distinction between nobles/commoners, craftspeople, and slaves was found commonly, albeit in various guises, among diverse precolonial societies of the West African (Sudanian) savanna (P. Diagne 1967; Tamari 1991). In theory, these were each endogamous orders of one or more lineages whose political status transcended the inter-ethnic or territorial complementarities described above. In practice, however, the identities of individuals and lineages (even those of slaves) were somewhat flexible across space and time (Amselle 1998; Conrad and Frank 1995b; Kopytoff and Miers 1977). According to Bonnie Wright, this system "rather than being composed of hierarchically ranked groups, is really best understood as a set of groups differentiated by innate capacity or power sources. The inequalities of the system are less matters of rank than of culturally defined realms of power, and the conjunction of all these realms constitutes the social universe” (quoted in Conrad and Frank 1995a:12). In sum, the intersection between this social order and the aforementioned groups of subsistence 
specialists generated a field of identities that emerged from and shaped the capacity for political action across the historical landscape of the West African savanna.

Of these endogamous political orders, only specialized craftspeople have been described as castes by ethnographers and historians. Although the term has come under attack on the grounds that the social mobility available to craftspeople in West Africa differed markedly from the rigid class system of India (Conrad and Frank 1995a; Dilley 2000), I would counter that this latter was more dynamic than is often assumed (Sinopoli 2003:98-100). Recognizing these limitations, I retain the term caste for thinking about craft specialization and interregional cultural institutions throughout this study.

The castes recognized within any given community, and the status of their members, varied across West Africa. Diverse Mande-speaking societies, for example, distinguished lineages of commoner/noble descent (horonw) from those of craftspeople (nyamakalaw). The latter typically included the three castes of bard (jèliw), blacksmith (numuw), and leatherworker (gárankew), with pottery production undertaken by women of blacksmith lineages (Conrad and Frank 1995b; Frank 1998; Leynaud and Cissé 1978). Beyond this generality, the political context of, commitment to, and intensity of specialized craft production differed from place to place.

Among rural villages of the Pays Mande, for example, blacksmiths have long held an ambiguous role in relation to the social hierarchies of horonw lineages (McNaughton 1988). Blacksmiths provide iron tools, ornaments, and masks, political advice, and ritual support for local leaders, even as they face public contempt by these same individuals. According to historian Patrick McNaughton (1988:10-11), this reflects the prevalence of social alliances manifest as "joking-relationships” between lineages and ethnic groups. In fact, misinterpretations of this relation by outside observers may explain the idea that nyamakalaw craftspeople were low-ranking, despite their central role in the cultural economy of local communities and the interregional political landscape.

By contrast, the Mande-speaking Bambara living in and around the modern town of Jenne in the Middle Niger region form a distinct minority and include none of the numuw blacksmiths highly regarded elsewhere across the West African savanna and in the Pays Mande (LaViolette 2000:24-25). In this urban context, Bambara have historically purchased iron goods, and even pottery, from Somono craftspeople, an 
occupational ethnic group that arose under Malian hegemony during the early- to midsecond millennium AD. Whereas craft specialists in rural areas work on a part-time basis around subsistence pursuits, their counterparts at Jenne practice more intense, full-time production to meet the demands of a larger market of consumers.

The caste system of Fulbe populations occupying the Middle Senegal region reveals important points of similarity and difference with Pays Mande. The freeborn lineages of farmers, fishermen, and pastoralists described above did not intermarry with Fulbe craftspeople (nyeenybe) who included the three main castes of bards (awlube), blacksmiths (wayilbe), and leatherworkers (sakkeebe), in addition to woodworkers (lawbe) and bard/weavers (maabuube); potters included women of blacksmith, weaver, and woodworker lineages and sometimes their own gender-specific caste group called buurnaabe (Dilley 2000, 2004). Despite their greater number, Fulbe castes offered less room for social mobility than Mande ones. According to Roy Dilley (2004:54), this is due to the fact that the powers and practices associated with craft production (but not the craft objects themselves) have been considered dangerous or impure to Muslim consumers. Rather than ally with craftspeople as a source of symbolic and economic power, members of the freeborn/noble class have maintained a careful social distance.

In contrast, Tenda-speaking communities, such as Bassari and Coniagui villages, occupying the upper Gambia River drainage do not participate in the caste systems of their Mande and Fulbe neighbors (Thiam 2010). According to ethnographer Monique Lestrange (1955:32-37), within a given village or territory some women specialize in making pottery and some men specialize as blacksmiths, and basket-weavers, but beyond this gender-based division of labor anyone can invest in learning the technical and ritual knowledge of craft production. Similarly, Diola communities of the lower Casamance-a historically decentralized landscape to the west of the Mande state of Kaabu-undertake pottery production and other crafts at the scale of extended family household (Linares de Sapir 1969). In fact, the organization of craft production by corporate kin groups or villages without an overarching ideology of status difference may have been part of longterm resistance by all of these decentralized societies to the hegemony of medieval Mali and other centralized polities over the past several centuries. Altogether, the idealized 
Mande and Fulbe caste systems present a similar cultural logic that took root in some rural communities, but not in others, over the past millennium.

\section{Crafting History}

The above ethnographic sketch should not be taken to mean that the statuses of craftspeople were static. These identities have undoubtedly evolved as commoners and craftspeople of diverse cultural backgrounds traded, migrated, and negotiated sociopolitical frontiers across West Africa, continually reshuffling the social matrices that lay at the intersection of ethnicity and caste (Conrad and Frank 1995b). Among Malinke communities of the Pays Mande, for example, jèliw bards often undertake leatherworking in competition with "pure" gárankew leatherworkers who hold to an historical identity as Soninke immigrants (Frank 1995). Competition between these groups of leatherworkers claiming different ethnic origins suggests that craftspeople were more mobile, or less likely to assimilate into host communities, than their non-caste counterparts.

More broadly, diverse attitudes towards craft specialization among centralized and decentralized societies hint at longer-term cultural processes. Using written sources and comparative linguistics, Tal Tamari $(1991,1995)$ has traced structural differences between the Wolof/Fulbe and Mande caste systems to the emergence of these identities sometime during the early to mid-second millennium $\mathrm{AD}$. While the precise historical mechanisms underlying caste formation in each of these societies remain unclear, two scenarios invite consideration. The first is that lineages associated with the economic and ritual powers of blacksmithing once held more substantial authority, but were relegated to a lower social echelon by non-specialists and would-be elites in order to maintain greater political symmetry within their societies. This trope can be found in narratives recounting the origins of Mali (Niane 1965; Tamari 2005) and the emergence of Takrur, an early state in the Middle Senegal region (A. Ba 2002:80-83). A second scenario suggests that joking relationships formed the conceptual basis for emergent caste identities between senior "noble” lineages and junior “caste” lineages as part of a broader trend towards political centralization in the early-second millennium AD (Tamari 1991:239-240). By no means mutually exclusive, both of these scenarios place the consolidation of the caste system within the heterogeneous political-economic landscape of the Late Iron Age. 
Tracing loan words for caste identities across the most widely spoken languages of the West African savanna, Tamari (1995) has also made a solid case for the subsequent expansion of the caste system under the hegemony of Mali during the $13-15^{\text {th }}$ centuries AD. Archaeological evidence in support of this interpretation, summarized in Chapter 4, includes specialized craft production workshops at urban centers within the medieval Mande political-economic sphere and the emergence of a more coherent technological tradition of pottery manufacture. What remains to be seen is when, how, and to what extent (or even if) this caste system mapped onto political relations between craftsproducing and crafts-consuming lineages in regions such as Bambuk, on the periphery of the Mande world.

\section{Looking Ahead}

Craft economies were integral to the reproduction of political status and identity within and between diverse societies of the West African savanna over the last few centuries, if not the past two millennia. Ethnographic and historical sources suggest that a caste system based on endogamy and differential access to authority emerged first within

the Mande cultural sphere centered on the upper Niger River, and perhaps simultaneously within the Wolof/Fulbe sphere in northern Senegambia, coincident with the expansion centralized polities during the early- to mid-second millennium AD.

The craft economy of Diouboye can therefore provide critical insights into the position of this community within the increasingly complex and heterogeneous politicaleconomic landscape of the Late Iron Age. In the following chapters I will argue that craft traditions at Diouboye had affinities with those of the Pays Mande and, over four centuries of occupation, production shifted increasingly to the specialized purview of one residential kin group in the village. At the same time, the available evidence gives no indication for the political marginalization of craftspeople or their reduced participation in other economic processes such as subsistence pursuits and long-distance exchange.

\section{Long-Distance Exchange}

Long-distance exchange subsumes a diverse array of pathways through which goods move across regional social boundaries and physical space (several days travel or 
more). Across precolonial West Africa, these pathways generally fell within the two dominant modes of: (1) down-the-line reciprocity or redistribution; and (2) specialized trade via merchant diasporas (e.g., Brooks 1993; Curtin 1975a; Mitchell 2005; Posnansky 1973). As material objects circulated through these exchange systems, they accrued value vis-à-vis other objects and social actors, thereby contributing to the structural inertia and, usually, legitimacy, of symmetrical and/or hierarchical institutions (e.g., Appadurai 1986; Halperin 1994:85-142; Weiner 1985; Wiessner 2002). In this regard, goods of exotic origin provide a tangible index of participation in supra-local networks, which can be an important source of authority in political economies emphasizing the capacity to coordinate diverse groups of people.

\section{Social Networks (Down-The-Line Exchange)}

Social institutions and interpersonal networks at all scales embed processes of material transaction such as gift-giving, bride wealth, inheritance, redistribution/tribute, and barter or trade. While these institutions mainly shape the circulation of localized resources such as staples, crafts, labor, and property rights, they can also work to move exotic objects in a "down-the-line" progression across social and physical space over the course of days, years, and generations. For precolonial societies in West Africa, the latter exotic items included cowries, metals, and glass beads often recovered by archaeologists, as well as less durable commodities such as salt and cloth, and perhaps people

Framed by their potential roles in long-distance exchange, some examples of these institutions from village-based societies in West Africa illustrate the reproduction of political relations at both local and interregional scales.

\section{Gift-Giving}

For most of the past century, economic anthropologists have pointed to the gift as the cultural-economic practice par excellence in many preindustrial societies (Bourdieu 1977; Malinowski 1984 [1922]; Mauss 1990 [1950]; Polanyi 1957:253; Sahlins 1972:149-183). Gifts reaffirm friendships and other social alliances among individuals, lineages, and other corporate groups, such as those described above for subsistence complementarities. In a classic ethnography on the historically decentralized Tiv 
communities of northern Nigeria, Paul and Laura Bohannon (1968) describe gifts as a means of allocating subsistence resources beyond the local kin group, particularly at the beginning of the growing season when stocks of stored yams and grains from the previous harvest may be running low in some villages, but not in others. Known as "sending hunger," people insist that these gifts need not be reciprocated, although they do affirm relations through which goods may return in subsequent years. Some gift relations are extensions of kinship, but others constitute special friendships (hur-or) that transcend lineage divisions. Additionally, the idiom of the gift can be found in different sorts of judicial processes as a means for compensating another individual or group for wrongs committed. All in all, these and many other institutions for gift-giving form a means for creating and maintaining symmetrical political relations across space and time.

Yet the bond established between giver and recipient provides a latent source of political asymmetry as debts are made and repaid at many interlocking social scales and tempos (Bourdieu 1977). This is particularly the case for hierarchical Wolof communities in Senegal, where gift-giving helps to maintain joking relations and the political order of clientship associated with the caste system (Irvine 1974). Smiths, griots, and other specialists, for example, can publically demand gifts from elites and commoners, particularly during public ceremonies. In order to avoid the potential stigma of receiving a gift, persons of equal status often make reciprocal loans in private where the danger of public recall nevertheless forms a political debt.

In sum, the exchange of gifts can contribute to the circulation of subsistence staples, crafts, and prestige items of local and non-local origin. In so doing, the allocation of these material resources among individuals, corporate groups, and communities shapes the reproduction (and potential transformation) of symmetrical and hierarchical political relations across these scales.

\section{Bride Wealth and Inheritance}

The kin-ordered mode of production established both wealth-in-people and wealth-in-knowledge as strategies for the production of authority in precolonial African societies (Guyer and Belinga 1995). By accumulating wealth in staples or other goods, corporate kin groups could transform labor and material surpluses into the social debts 
described above, as well as into vital reproductive and property rights. In this regard, institutions of bride wealth and inheritance structured the biological and socio-material reproduction of households and lineages.

Bride wealth refers to the payment of movable property by the lineage of a groom in exchange for a bride. This constitutes a primary mechanism through which corporate kin groups transform material resources into reproductive rights and further "wealth-inpeople” (Bossen 1988). As anthropologist Edmund Leach (2004 [1959]) noted for Kachin communities in southeast Asia, marriage reproduces relations between lineages that can cycle dramatically from symmetrical to asymmetrical and back again over the course of generations — or become institutionalized as permanent differences in political rank (Friedman 1979). According to Claude Meillassoux (1960), bride wealth among decentralized societies in West Africa has long been instrumental in the reproduction of relations between lineages and also within them. To the extent that elders within lineages or other corporate kin groups maintain control of property rights, including those in lineage members themselves, they stand to exercise and legitimize their authority through marriage transactions involving younger kin. Where joking partnerships between lineages or other groups depend upon the metaphor of age relations, these can become the ideological basis for political ranking. In addition to livestock, the marriage of individuals from different villages can involve the gradual movement of more exotic objects or ornaments over long distances.

Inheritance, meanwhile, refers to the legitimate transmission of rights over material resources such as land, livestock, houses, and personal effects following the death of an individual. In his landmark study of the decentralized LoDaaga societies described above, Jack Goody (1962) outlined two models of inheritance based on single and double descent systems. With the former, all property rights of the deceased pass to members of the corporate matrilineal or patrilineal group. In the latter system, however, immovable property (i.e., land, house) go to the patrilineal group, while movable property (i.e., livestock, wealth objects) go the matrilineal group. These systems are by no means static and Goody was careful to highlight points of tension underlying their structural change. Notably for archaeology, inheritance grounds corporate kin groups to spaces within villages, including rights to work surrounding lands, and fuels the 
circulation of material objects in and out of these spaces on a generational basis. This latter point is true regardless of descent system, so long as both patrilineal and matrilineal groups extend beyond the boundaries of the village.

In sum, bride wealth and inheritance contribute to the circulation of subsistence staples, crafts, and exotic items both within and between corporate groups and village communities, thereby contributing to the reproduction of political relations among them.

\section{Tribute and Redistribution}

Tribute and redistribution play an important role in the movement of staple and exotic goods across social space at multiple scales. While both of these economic processes begin with the movement of material resources towards a political center, their differences lie in the accumulation of goods by leaders on the one hand, and their reallocation among contributors on the other (Polanyi 1957:253-254). In either case, the initial pooling of resources entails political centralization at some scale.

While redistribution and tribute did occur within the subsistence economies of precolonial West African societies, particularly centralized ones, their potential to underwrite political hierarchies was usually tempered by the relative autonomy of corporate kin groups. Accordingly, these economic processes more often directed the flow of exotic objects and craft goods, particularly those charged with prestige or power (Coquery-Vidrovitch 1976; S. K. McIntosh 1999c).

\section{Market Exchange}

Before moving to discuss trade across social boundaries, it is important to note that local markets were important mechanisms for the distribution of surplus staples and craft goods such as pottery or iron tools within precolonial societies across West Africa (Bohannan and Dalton 1962). While trade and barter depend strongly on the value of objects themselves, they also require social institutions to ensure the safe conduct of traders and to establish equivalent values across classes of goods (Ford 1972). For example, the decentralized societies of the Upper Voltaic region have long maintained local earth shrines that, along with their associated ritual specialists, provided the judicial umbrella required to ensure the safe conduct of barter and trade within weekly markets 
(Fortes 1940; Goody 1957). Although the value ascribed to exotic objects made them more susceptible to inclusion in political economies, it remains possible that market exchange worked to move some of these objects across the landscape.

\section{Merchant Communities and Trade}

Given the historiography of West Africa described in Chapter 1, it should come as no surprise that the workings of long-distance trade are reasonably well-understood (e.g., Brooks 1993; Curtin 1975a; Mitchell 2005; Perinbam 1973; Posnansky 1973; Stewart 1979; Wilks 1982a, 1982b). While contact between North Africa and West Africa gradually escalated following the introduction of the domestic camel around AD 400 (Garrard 1982), it was Islam that provided both an impetus and ideology for transSaharan trade from the late-first millennium AD onward (Brett 1983; Insoll 2003; Levtzion 2000; Mitchell 2005). In fact, archaeological research at several medieval towns across the Sahel and savanna has identified merchant districts where Islamic-influenced material culture documents direct interaction with foreign traders (see Chapter 4).

Historically, the indigenous trading communities of West Africa—known as Wangara, Juula, or Jakhanke (see Chapter 1) —were invariably Muslims with ties to the medieval states of Ghana and, later, Mali. These so-called merchant diasporas comprised mobile caravans and landlord-brokers. The latter would settle beside or within local communities where intermarriage and the adoption of customs allowed them to participate in networks of exchange and trade. Caravans acquiring exportable commodities or passing through an area could look to these brokers for lodging and protection, their safety guaranteed by the status of their hosts in the local community.

Although their origins have been debated (A. Bathily 1989; S. K. McIntosh 1981; P. Smith 1965), Jakhanke communities, known both for their strong devotion to Islam and pacifism, dominated trade along waterways and overland routes in the Upper Senegal region by the time that Europeans began to explore the Atlantic coast in the $15-16^{\text {th }}$ centuries AD, (Brooks 1993). Yet with subsistence and craft economies historically little different from Malinke, Soninke, and Fulbe villages, the question remains open as to how the Jakhanke and other merchants engaged with (or settled down among) local communities in Bambuk during the early-second millennium AD. 


\section{Looking Ahead}

In sum, long-distance exchange in precolonial West Africa occurred both through direct trade administered by merchant diasporas and through down-the-line exchange enmeshed within more localized institutions of inheritance, bride wealth, reciprocity, redistribution, and barter. In the following chapters I argue that long-distance exchange practices at Diouboye were undertaken primarily, if not solely, through down-the-line mechanisms. This is not to discount the possibility for direct trade, but rather to suggest that there is no evidence for a landlord-broker living in or near the village. Furthermore, I will suggest that differential participation in exchange activities worked to reinforce the political organization of craft and subsistence economies within the community. While these political relations were not necessarily asymmetrical, they do suggest that social differences were becoming more firmly entrenched concomitant with the emergences and expansion of centralized medieval polities such as Mali.

\section{ARCHAEOLOGY OF POLITICAL ECONOMY AT DIOUBOYE}

As stated in Chapter 1, the questions guiding this study seek to situate Diouboye amid intersecting, and potentially dynamic, fields of political-economic relations organized at multiple scales - the local (the village and its environs), the regional (Upper Senegal region), and the interregional (West Africa). More specifically, I propose to examine these relations across the interconnected economic spheres of subsistence, craft, and long-distance exchange using an archaeological approach. This begins with the recognition that economic processes-including practices of production, distribution, use, and/or consumption - enroll people, material resources, and the spatial relations among them. It follows that the locational and appropriational movements relating economic processes to resources and to sociopolitical institutions generate, in part, the material and spatial dimensions of the archaeological record (Spaulding 1960). As depicted in Figure 2.2, aspects of materiality (quantity, quality) and spatiality (segregation, distance) thus allow one to infer or deduce past locational and appropriational movements and, by extension, the behavioral adaptations, technologies, political institutions, and cultural schemas in which they were embedded-in short, the political economy. 
Figure 2.2: Conceptual map of links between material and spatial dimensions of the archaeological record and past political-economic processes

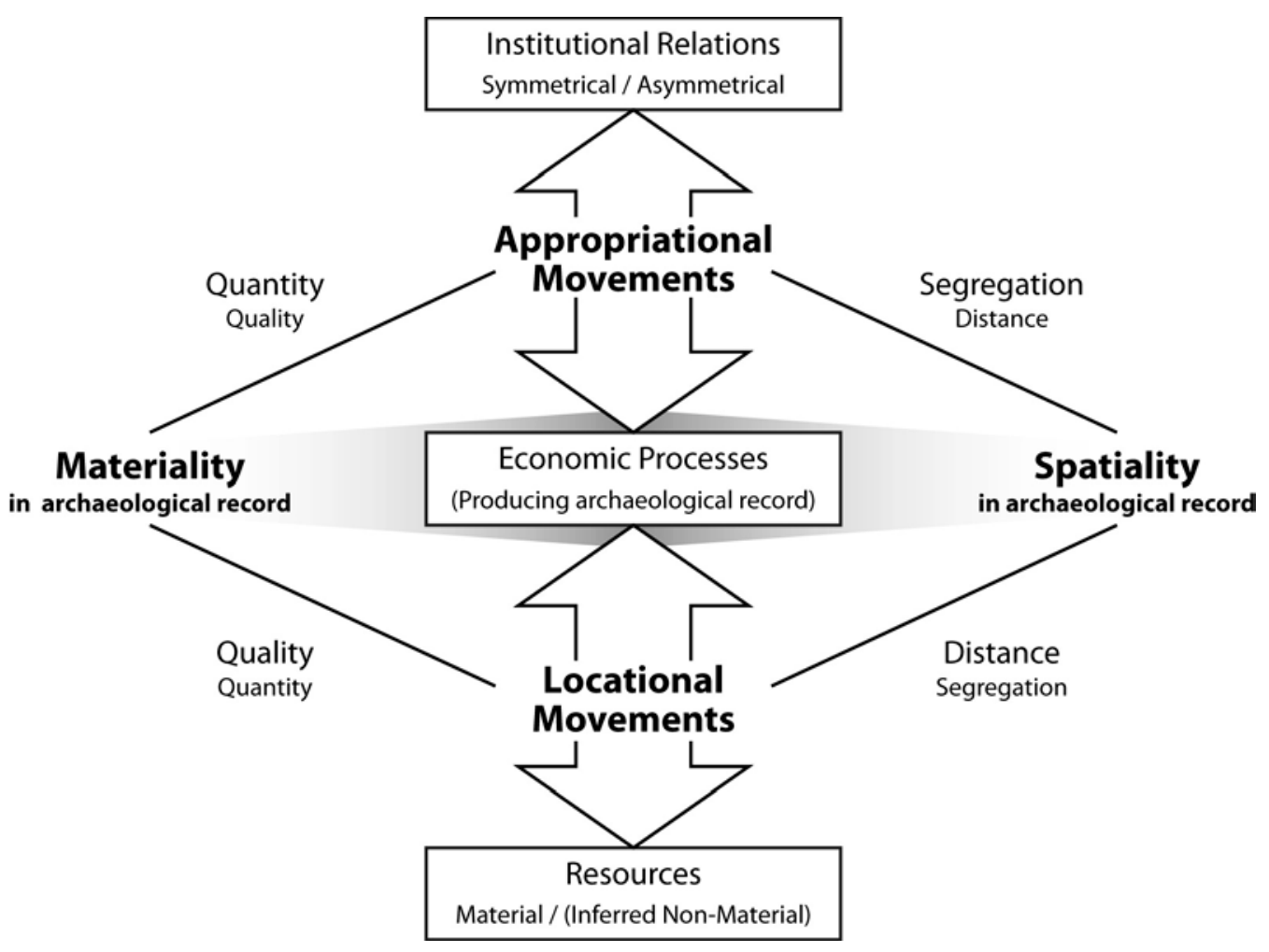

After accounting for the natural and post-depositional agencies that also shape the archaeological record (see Chapter 6), aspects of materiality and spatiality together provide a broad conceptual frame for studying the political economy of Diouboye.

Importantly, these dimensions presuppose one another; material objects occupy and relate to one another through space, in an abstract or absolute sense, while space itself emerges as a reality insofar as it enjoins material objects and embodied subjects (Lefebvre 1991). In what follows, I define materiality and spatiality as a common language for tacking back and forth between the social past and the archaeological present at multiple scalesfrom artifacts, features, and sites on up to regional landscapes and interregional entanglements. 


\section{Materiality}

Economic processes, by definition, implicate material resources through locational movements involving practices of production, distribution, and consumption. As described throughout this chapter, these resources comprise landholdings, the built environment, and rights-in-things, including those such as raw materials and tools employed in technologies of production. At one and the same time, the materiality of these processes, as appropriational movements, contributes to the reproduction and potential transformation of political institutions. In this study, I first consider such movements within subsistence, craft, and long-distance exchange economies using two material dimensions of the archaeological record—quality and quantity.

\section{Quality}

By quality, I refer not to a subjective ranking of material objects (as in, high quality versus low quality), but rather to the tangible properties of the archaeological record and the objects and features comprising it. In the appendices to this study, I expend a great deal of ink describing the qualities - material composition, size and shape, decoration, patterns of wear, and so forth — of artifacts and other components of the archaeological record from Diouboye and surrounding sites on the Falémé River.

These physical qualities play a vital role in mapping past locational movements since they shape the potential value of material resources, and provide a basis for inferring non-material ones. In the first instance, a finely-made pot is an object entailing rights of ownership at some scale, and also comprising, on account of its form and decoration, a tool for diverse subsistence, craft, and exchange practices. In the second instance, the techniques of pottery production and use inferred from physical traces of shaping and wear can reveal non-material resources of practical craft knowledge and discursive use knowledge.

As these resources become enrolled in processes of production, distribution, and consumption, they bear upon appropriational movements at multiple scales. A finelymade pot exchanged through reciprocal gift-giving, for example, might work to institute symmetrical relations between social actors, while a pot coerced as tribute might work to reproduce hierarchical relations. In both contexts, the material qualities of the pot (that is 
to say, its "style”) could signal and reproduce social identities and political boundaries among participants in the economy.

\section{Quantity}

Quantity refers to the absolute or relative numbers of a given category of artifact, feature, or other material element in the archaeological record. Whereas the appendices of this study focus on defining and interpreting the qualities of objects, the following chapters mainly concern themselves with measuring and comparing their quantity at multiple scales.

The quantitative dimension of the archaeological record plays an important role in mapping appropriational movements in political economies. This is particularly the case in decentralized societies where political statuses emerge from variability along a continuum of (rather than discrete differences in) participation in economic processes. Social actors and groups who habitually consume more than they produce across economic spheres of subsistence, craft, and long-distance exchange, for example, may occupy a higher position of authority within the overarching political institutions. Regarding locational movements, the quantity of resources drawn into economic processes also has implications for understanding the overall complexity and the number of people (as consumers and sources of labor) within a political economy.

\section{Spatiality}

The recognition that social actors and groups occupy space in predictable and culturally mediated ways (E. T. Hall 1966) provides an important inferential bridge to the social scale of past events and processes. In order to chart locational and appropriational economic movements at multiple scales, I focus on two dimensions of spatiality linking them to the archaeological record—segregation and distance.

\section{Segregation}

Segregation refers to the partitioning of space, either through the formal construction of physical barriers, or the informal repetition of material practices within a bounded place. In most archaeological studies, including the present one, segregation 
provides the basis for defining units of structured social and economic action-activity areas, houses, sites, polities, and so forth.

In this regard, segregation provides the basis for tracing appropriational movements among social groups at multiple scales. Household compounds and settlement sites, for example, emerge from structured interactions among kin groups and village communities. A comparison of the qualities and quantities of material remains produced by, distributed among, and consumed within (segregated to) these places allow one to draw inferences about the political institutions in which they were embedded. Regarding locational movements, segregation provides a basis for asserting ownership over material resources (e.g., landforms, built environments) and shaping the transmission of non-material ones.

\section{Distance}

Distance provides a measure of the physical and social space separating people, objects, and places. In practice, distance and segregation are inseparable since the latter depends largely upon proximal relations. The segregation of space into a village, for example, derives from the greater proximity among internal household compounds (and their associated kin groups), compared to their greater distance from compounds in another village (and its associated community). In this study I employ physical distance to describe relations between socio-spatial units at different scales.

The invocation of rights over material resources, and their actual locational movements into the economy, usually depends on their distance from interested social actors and groups. Similarly, distance shapes access to the non-material resources, such as practical and discursive knowledge, embedded within relations between people residing close together or far apart.

Whereas segregation helps to define the social actors involved in appropriational movements, distance provides a basis for reproducing political relations and access to authority. For instance, the equidistant spacing of villages across a landscape may underscore symmetrical participation in the production of space, while aggregation of settlement usually corresponds to the compositional and/or coercive power of political leaders vis-à-vis their role in local economies. This same logic can, of course, apply to 
distances at other scales - between huts within a compound, between compounds, and across the West African savanna.

\section{SUMMARY}

I began this chapter by summarizing critiques of the autonomous, bounded, and strongly hierarchical polity as a model for social complexity in West Africa during the Iron Age (S. K. McIntosh 1999a; Stahl 2004b). Following these critics, I suggested that the waxing and waning of centralized polities throughout the precolonial era was intimately bound up with the fortunes of smaller-scale communities at their interstices, including those occupying the auriferous realm of Bambuk in the Upper Senegal region.

With this premise as a background, I then sketched a model for villages ensconced in "Iron Age" worlds whose heterogeneous economies lay embedded within a web of symmetrical and hierarchical political relations at scales ranging from the kingroup, to the village community, to the regional and interregional polity. I focused on three economic spheres that became increasingly specialized across the social landscape of the West African savanna during the Late Iron Age-subsistence, craft, and longdistance exchange. I then outlined how economic processes of production, distribution, and consumption within each of these spheres structured, and emerged from, the locational movements of material and non-material resources and the appropriational movements of political institutions. I argued that cultural schemas play a vital role in assigning value to different resources and identities to the people who appropriate them. Ultimately, the usefulness of this model lies in its capacity to situate Diouboye within fields of cultural and political-economic relation playing out at different spatial scales and historical tempos.

I then attempted to mobilize this general model in the context of precolonial West Africa by summarizing some of the key institutions known to have structured politicaleconomic relations within and beyond village communities. These included: the kinordered mode of production and complementarities in the subsistence economy; caste systems and ritual power in the craft economy; and supra-local social networks and merchant diasporas as means of long-distance exchange. While this survey is by no means comprehensive, I have tried to pre-emptively "thicken" the description of the 
political-economic arrangements for which Diouboye provides, or conspicuously lacks, archaeological evidence.

Finally, in the last part of the chapter I defined the methodological frame that guides my interpretation of daily life and political economy at Diouboye. The following chapters will consider the material (qualitative, quantitative) and spatial (segregation, distance) dimensions of the archaeological record to map the locational and appropriational movements argued to constitute the political economy of this village at the intersection of its broader historical landscape and relations among its kin-ordered residential groups. 


\section{CHAPTER 3 GEOGRAPHY OF THE UPPER SENEGAL REGION}

Physical and social geography profoundly shape the political economy of village communities to the extent that economic processes of production, distribution, and consumption depend on the material qualities of, and spatial relations among, resources such as land, livestock, tools, and the labor of other social actors. At the same time, institutional dynamics within and beyond such communities may work to reshape their broader geographic settings. In this chapter, I begin to explore how this dialectic contributed to the political economy of Diouboye during the early- to mid-second millennium $\mathrm{AD}$ - a topic that I pursue through the following two chapters.

Drawing upon the tenets of historical geography (Baker 2003), I consider the interplay among social actors, institutions, and physical space beyond Diouboye from the viewpoint of landscape. In Africanist archaeology, this approach has generally fallen under the rubric of historical ecology with its explicit focus on past climatic variability and the ecosystems of which humans were an indelible part (Crumley 1994; R. J. McIntosh et al. 2000). As I discuss in the first part of this chapter, it is important not to conflate the concept of environment, as the physical and social milieu that people occupy, with landscape, as the relations between people and this milieu (Baker 2003:78; Knapp and Ashmore 1999:20-21). Landscapes emerge as people respond to their experiences and perceptions of the material world through social practices and technological developments that can, in turn, reshape or transform it. A landscape perspective thus directs attention to the interplay between physical geography and human actions at multiple temporal and spatial scales while bearing in mind that processes at one level are shaped by and through those operating at others.

In the second part of this chapter, I present an overview of the physical geography of the historical landscape experienced by Diouboye. Specifically, I turn to evidence from geology, pedology, hydrology, ecology, and climate studies to summarize long-term 
trends, and short-term variations, in the surrounding physical environment. Here, macrobotanical and zooarchaeological data from Diouboye also document localized ecological and climatic conditions during the early-second millennium AD.

The third part of this chapter traces long-term changes in the climate and ecology of the Upper Senegal region over the past several millennia. This is critical for assessing the fit between ethnohistoric models of human ecology and the actual conditions experienced by the inhabitants of Diouboye, as well as by earlier and later populations discussed in Chapter 4.

The last part of this chapter moves to consider how these parameters of physical geography have historically structured human ecology in the Upper Senegal region. Building upon the introduction to subsistence economies of the West African savanna in Chapter 2, I elaborate on some of the common socioeconomic strategies of food production, storage, mobility, labor organization, and religious ritual that communities have developed to ensure their survival within the strongly seasonal environment of the tropical savanna. The material and spatial dimensions of such strategies provide a basis for interpreting locational and appropriational movements within the subsistence economy of Diouboye.

\section{LANDSCAPE: A CONCEPTUAL FRAMEWORK}

Despite a gradual shift in terminology from "settlement" to "landscape” archaeology over the past three decades, archaeologists have long recognized that evidence for human occupation and other activities at a regional scale, however defined, can yield important insights into the structure and history of past societies (e.g., R. M. Adams 1981; Fish and Kowalewski 1990; Hodder and Orton 1976; G. A. Johnson 1977; Knapp and Ashmore 1999; J. R. Parsons 1971; Rossignol and Wandsnider 1992; Willey 1951; Wright and Johnson 1975). To briefly summarize an extensive literature, settlement archaeology emerged within the paradigm of processualism to evaluate the fit between models of past social systems and the spatial relations among sites and their

physical surroundings. With an implicit focus on the economic underpinnings of such systems, these studies have ranged widely from Adams’ (1981) appraisal of evolving urban settlement in Mesopotamia, to Steponaitis’s (1981) exposé of early tributary 
economies in Formative Mesoamerica, to Binford's (1980) model of collector and forager mobility strategies. Importantly, settlement approaches have developed hand in hand with methods for survey and systematic sampling that today form the bread and butter of regional studies in archaeology (Ammerman 1981; Nance 1983; Plog et al. 1978; Read 1986). Even as scholars address methodological issues and explore new theoretical interests, the tenets of settlement archaeology remain foundational to the discipline.

Over the past three decades, archaeologists have paid increasing attention to the ways in which social actors within "systems" articulated with a more broadly conceived landscape. On the one hand, this trend stems from a methodological interest in identifying past activities and significant natural places between or beyond the traditional “site” (Dunnell and Dancey 1983; Rossignol and Wandsnider 1992). And, on the other hand, it mirrors an increasing awareness across the disciplines of cultural anthropology, history, and geography that landscapes are more than simply passive backdrops for social processes: they emerge from a continuous dialectic between humans and their physical surroundings (e.g., Baker 2003; Bender 1993). Landscape discourse provides a venue in which archaeology, with its potential to reflect upon the longue durée, can weigh in on interdisciplinary discussions of social and environmental change (e.g., Balée 1998; Bollig and Bubenzer 2009; Crumley 1994). Presently, landscape archaeology forms a broad conceptual umbrella for evolutionary and historical ecology (Butzer 1982; R. J. McIntosh et al. 2000), studies of materiality of power, religion, and ideology (Bradley 1998; Norman and Kelly 2004; A. T. Smith 2003), phenomenology (Ingold 1993; Tilley 1994), and research on the production of social identity and memory (Knapp and Ashmore 1999; Yoffee 2007), to name but a few current directions. Needless to say, it is necessary to summarize some of the main tenets of my perspective in this study.

In the broadest sense, landscape is a genre of space — an abstract concept referring to the social (re)production of (spatial) relations among material objects, human subjects and the cultural schema through which the latter ascribe meaning to the former (E. T. Hall 1966; Lefebvre 1991; Soja 1989). While these three aspects-the material, the social, and the representational - may be said to comprise the totality of space, it is the act of production, as discussed by Henri Lefebvre (1991), that gives space a certain "abstract universality” for the study of society. Production also animates space through 
time, moving it into the realm of historical (and archaeological) study. And, as space grounds the dialectic between structure and practice in the material world (Giddens 1984; Soja 1989), it provides a critical interface between units and scales of analysis (i.e., regions, sites, features) and the economic processes, sociopolitical institutions, and cultural schema that I seek to understand and explain in the present study.

The distinction between landscape and other categories of space is largely an analytical one. In this and subsequent chapters, landscape shall refer to the production of space through and between places - those relatively discrete physical localities, whether natural or built, that become loci of socioeconomic action and cultural representation (Ashmore 2002; Rossignol and Wandsnider 1992). The archaeology of landscape then rests on the potential to identify such places and their interrelations (material, spatial, and temporal), a task constrained by the extent to which people modified them (Binford 1982), and by the vagaries of post-depositional processes (Schiffer 1987). Because the study of landscape also depends on analytical scale (Crumley 1979), it is imperative to define a study region appropriate to the research agenda.

\section{Spatial Scale}

The spatial scale(s) employed in a study of an historical landscape would ideally conform to those recognized by past communities and polities, but these often prove difficult to identify where social boundaries and territories were regularly contested, or where they are historically unknown. As a result, archaeologists must often define analytical units around geographical features of some presumed cultural significance. In this study I view the historical landscape of West Africa from the vantage of one particular time and place, namely, the village of Diouboye during the early to mid-second millennium AD. Following from my interest in the political economy of this community, I consider the spatial/material dimensions of the surrounding landscape at three nested scales - the local landscape of the central Falémé River basin, the regional landscape of the Upper Senegal River basin, and the interregional landscape of the West African subcontinent. Although I have already made mention of these in the preceding chapters, it will be useful to offer more concrete descriptions before proceeding to a discussion of the archaeological literature in Chapter 4. 
West Africa (Figure 3.1) conventionally subsumes the vast expanse of Africa stretching 3200 kilometers from the Cap Vert peninsula of Senegal in the west to the Lake Chad basin in the east, and 1500 kilometers from the Gulf of Guinea in the south to the Sahara Desert in the north. Not surprisingly, the physical terrain and ecology of this subcontinent are incredibly heterogeneous, as are the 300 million or more people who occupy it today. While acknowledging this diversity, societies across West Africa do share many historical developments and experiences - from the origins of food production several thousand years ago to the yoke of colonial exploitation in the earlytwentieth century AD (Chapter 4). This vast landscape also defines the maximal extent of interregional networks considered to have shaped the political economy of Diouboye.

Figure 3.1: $\quad$ Map of West Africa

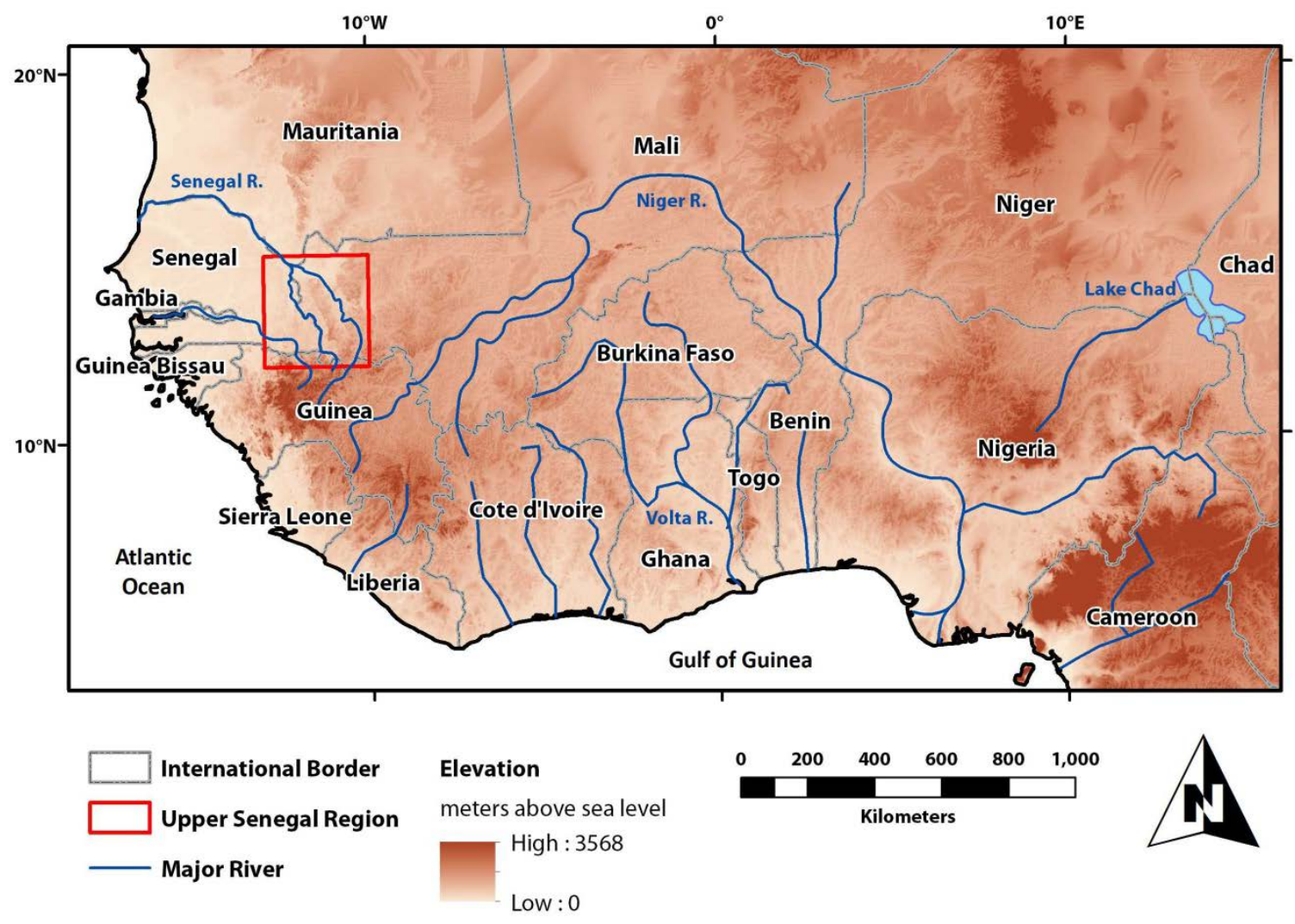


Figure 3.2: Map of the Upper Senegal region

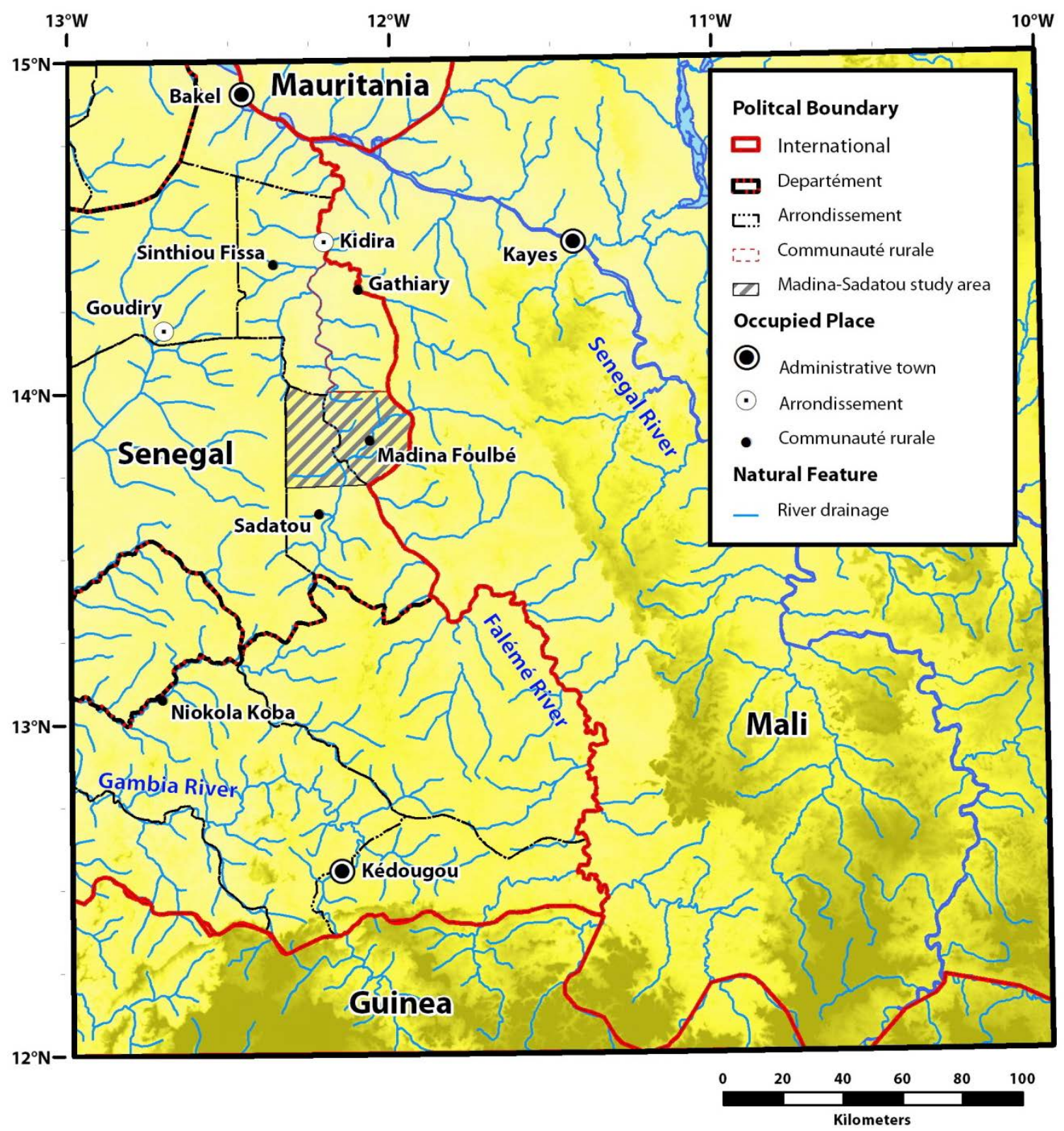

The Upper Senegal region comprises the area drained by the Senegal River between its broad middle valley floodplain to the northwest and its headwaters in the Futa Jallon highlands to the south (Figure 3.2). The Gambia and Falémé Rivers, the latter being the largest tributary to the Senegal, also traverse this region from their origins in the Futa Jallon. Although the topography and ecology of the Upper Senegal are quite 
diverse, its position as a crossroads between the Sahel and the savanna forest has long given the region a degree of physical and social coherence apart from surrounding areas, such as the Middle Senegal region and the Pays Mande of the upper Niger River. Given this loose geographic integrity, this study works from the premise that the political economy of this region played a role in mediating between the community of Diouboye and vast interregional networks.

At a scale commensurate with the landscape experienced on a more regular basis by people residing at Diouboye, I defined the Madina-Sadatou study area (Figure 3.3) according to a combination of natural topography and two modern administrative units. These latter include the entire communauté rurale of Madina Foulbé and the northern half of Sadatou. Straddling the Falémé River, this study area extends to the international border with Mali in the east and south (midway to the Tomboura Escarpment) and the Bundu Hills in the west, beyond which lays the watershed of the Gambia River; the northern boundary of these administrative units occurs at $14^{\circ} \mathrm{N}$. Although MadinaSadatou corresponds loosely to the "kingdom” of Combredougou depicted on an early map of the Upper Senegal region (see Figure 4.14), the coherence of this territory cannot be assumed for earlier times. In any event, this central segment of the Falémé River basin presents a modicum of geographical integrity and narrows archaeological analysis to a manageable scale of places within a one-day round-trip walk from Diouboye.

\section{Temporal Scale}

According to historians of French annales school (Baker 2003:18-24), most notably Ferdinand Braudel (1980), the temporal scales of the landscape can range from the single event (événement), to the cycling of social processes on the order or years and decades (conjuncture), to the long-term transformation of institutional configurations and cultural schema (longue durée). While the annales school envisioned physical geography as structuring the longue durée, more recent scholarship emphasizes the emergence of history out of the interplay between material and social (and representational) landscapes at multiple tempos (e.g., Crumley 1994; Ingold 1993; R. J. McIntosh et al. 2000). Interannual and decadal variability in critical resources, for instance, may require flexibility in technological systems, while short-term catastrophes can bring about transformations of 
social institutions. Meanwhile, the day-to-day exploitation of the natural environment has undeniable consequences over the long-term. Although this study focuses on the several centuries bracketed by the initial settlement and then abandonment of Diouboye, I will contextualize this period within the longue durée of the surrounding landscape in Chapter 4. A comparison of earlier and later occupations within the same regional or local landscape can point to ways in which the physical environment conditioned economic processes and political relations at different scales.

Figure 3.3: Map of the Madina-Sadatou study area

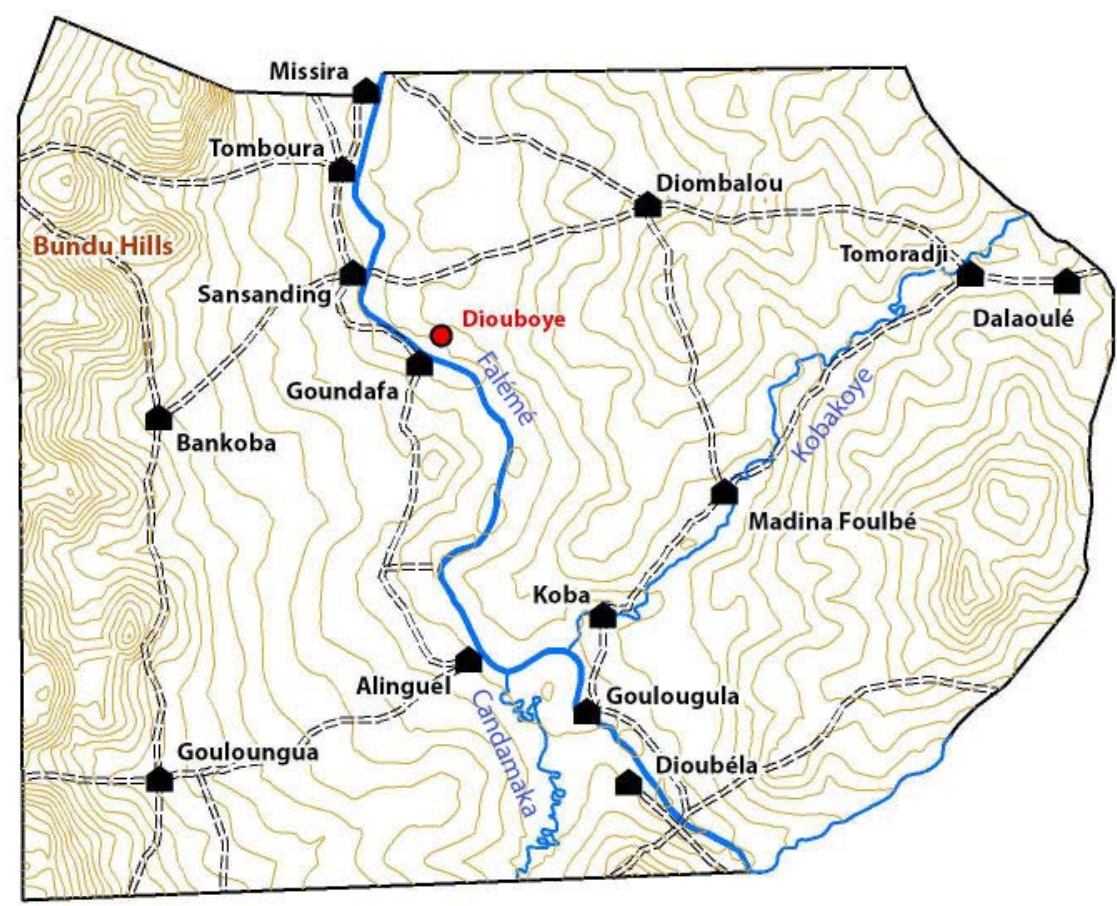

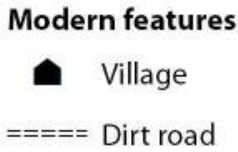

Elevation

5 meter contour

Drainage

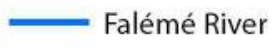

Major tributary
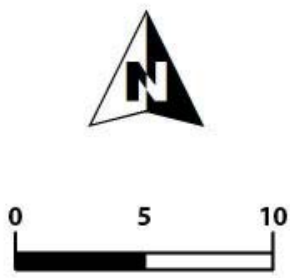

Kilometers

\section{PHYSICAL GEOGRAPHY}

This section draws upon research in the natural sciences to characterize the contemporary physical setting of the Upper Senegal region and, where possible, the Madina-Sadatou study area. Specifically, I outline the spatial and temporal distribution and variability in material resources including minerals, soils, rainfall and hydrology, and the diverse ecosystems these sustain. 


\section{Geological Foundations}

The three principal geological entities forming the West African subcontinentearly Precambrian cratons (ca. >1500 Mya), late Precambrian mobile belts (ca. 1100-500 Mya), and diverse sedimentary basins (ca. <1000 Mya) —all converge to contribute to the overall geophysical diversity of the Upper Senegal region (Bassot 1966; Michel 1973; Wright et al. 1985). The rocks and minerals manifest in these geological units directly shape the spatial distribution of raw materials for chipped stone and groundstone tools, iron and gold working, pottery manufacture, and even architecture. Natural and cultural processes of erosion on these parent rocks create the soils and topography affecting ecology and water drainage_-critical parameters in both foraging and agro-pastoral subsistence economies.

The Precambrian craton extending across most of southern West Africa (Figure 3.4) comprises an extremely stable quartzo-feldspathic basement with volcanic intrusions of granite and quartzite (Wright et al. 1985). Across the western portion of this craton, a Birimian greenschist "supracrustal” component hosts the dispersed deposits of diamonds, gold, bauxite, and chromite largely responsible for the mineral wealth of the region (Wright et al. 1985:45-49). These geological facies typically yield low quantities of iron ore, but a large deposit of magnetite in the upper Falémé River basin proves a notable exception. In the Madina-Sadatou study area, exposed Birimian schists and metamorphosed granites form a series of exposed inselbergs and terraces to the east of the river channel where they severely limit the amount of cultivable alluvium. They may yield rocks historically exploited for pottery temper and groundstone tools such as gabbro, amphibolite, and hematite (see Appendices D-E).

The Mauritanide belt of sedimentary, metamorphic, and volcanic rocks lies to the north and west of this major craton (Wright et al. 1985:51-55). While the composition of this belt varies along its longitudinal axis, the Falémé and Bakel groups of the Bundu Hills mainly comprise deformed conglomerates, sandstones, quartzites, and schists with few exploitable minerals (Bassot 1966:140-170, 204-206). Nevertheless, these geological groups, particularly the pinkish-gray "Bundu” sandstones found near the Madina-Sadatou study region, are the probable source of quartz and blue chert exploited by Late to Middle 
Stone Age toolmakers (Camara and Duboscq 1990:298; Michel 1973:70), either directly from exposed veins or, more likely, as cobbles transported into the river bed by run-off.

Figure 3.4: Map of geological units in West Africa (after Wright et al. 1985:7)

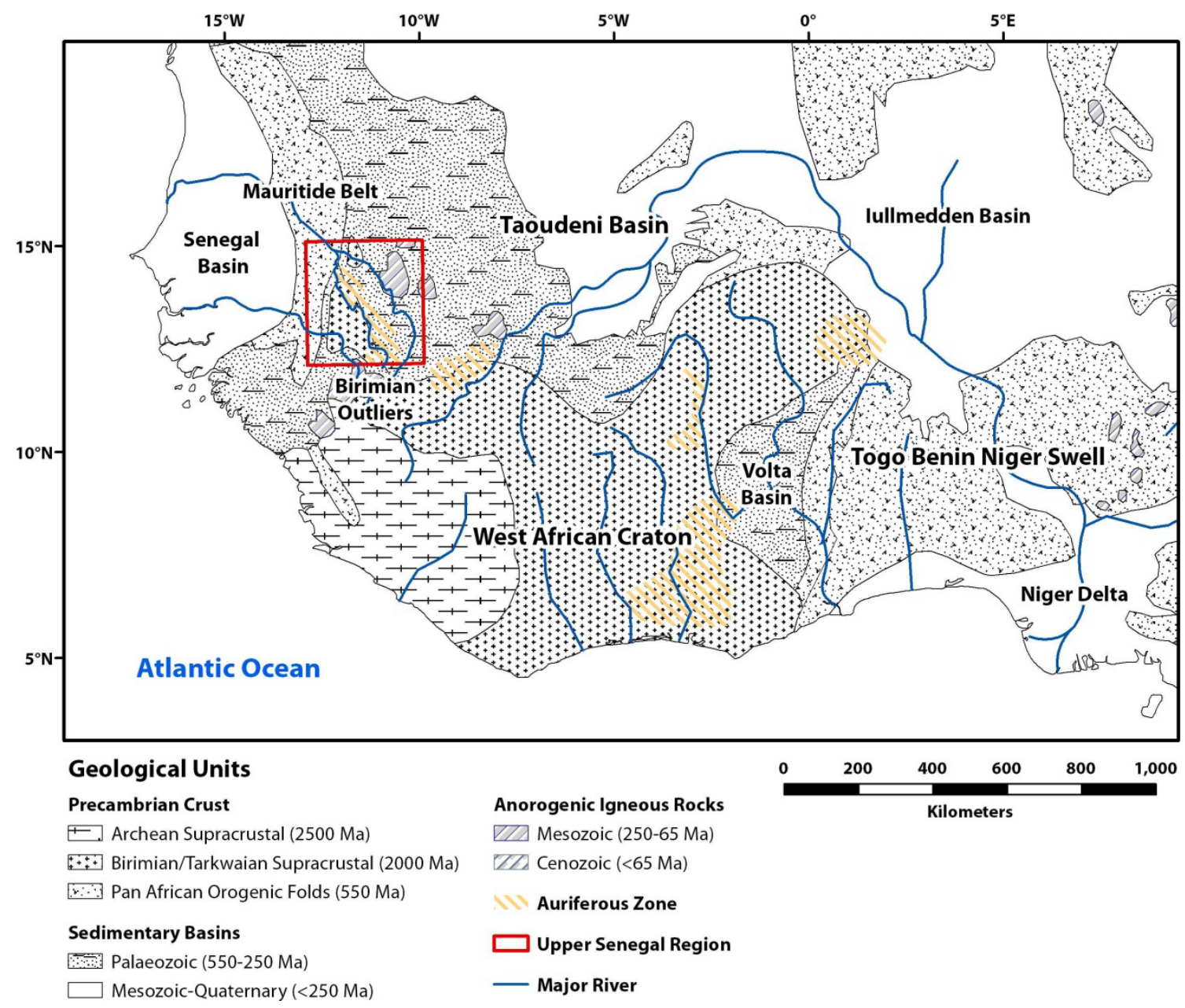

Nestled amid remnant Birimian facies and folds of the Falémé/Bakel groups, the Madina-Sadatou study area contrasts with the surrounding sedimentary basins of the Upper Senegal region (Wright et al. 1985:82-84, 111-112). The Manding Plateau and the Taoudeni Basin to the east contain sandstone, shale, dolomites, chert/flint, but few other exploitable minerals. Stretching west to the Atlantic Ocean, the beds of limestone, shale, 
and marl comprising the Senegal Basin yield neither commoditized minerals, nor ample sources of workable quartz and chert.

\section{Sediment and Soil Formation}

Across subtropical West Africa, several mechanical and chemical processes work to decompose these primary geological units into sediments and soils for growing crops, building houses, and even smelting iron (Young 1976). In the Upper Senegal region, the primary agents of sediment formation include climate (temperatures and annual cycles of precipitation) and vegetation (Michel 1973). Seasonal rainfall dissolves some of the minerals present in parent rocks while running water erodes, transports, and deposits sedimentary particles, gravels, and rocks. The root systems of plants break down these parent materials even as they discourage erosion. These and other in situ chemical and biotic activities transform sediments into soil (Rapp and Hill 1998:30; Young 1976:6484). In addition to their properties for cultivating crops, the properties of different soils and sediments made them amenable for economic activities across Madina-Sadatou ranging from hut construction to pottery production.

Laterite, a durable soil horizon found throughout Africa, forms when seasonal rainfall leaches iron from upper soil horizons to precipitate in lower ones as insoluble iron oxides (Young 1976:154-167). Once formed, laterite can be easily fractured, often appearing as gravel-sized particles in other soil horizons, or as quarried blocks in architectural features and on archaeological sites. Because laterite consists mainly of ferric oxides (50-90\%), it may also provide a source of ore for iron smelting (Sassoon 1963). On the other hand, laterite less than 50 centimeters below the ground surface can pose a serious problem for agriculture by prohibiting root penetration or lowering moisture retention in the overlying soils. For this reason, people in subtropical West Africa generally use laterite-rich soils for wet-season pasturage.

Along the lower Falémé River, alternating wet and dry climatic episodes have led to the formation of younger soils through down-cutting of exposed parent materials and alluvial deposition in heterogeneously sorted horizons some eight to ten meters thick (Michel 1973:282-285, 514-516). In the western part of the Madina-Sadatou study area, poorly drained vertisols and slightly alkaline soils become quite sticky and even support 
standing pools of water during the wet season from June to October. Although such soil conditions are not amenable to rain-fed agriculture in West Africa, they are ideal for many of the savanna grasses that sustain livestock herds (Kowal and Kassam 1978; Young 1976:182-187). The montmorillonite clay present in vertisols (>35\%) also makes them a good source of raw material for hand-built pottery (Rice 1987:61). In the eastern part of the study area, the weathering of Birimian schists generates leached ferruginous soils with modest levels of moisture retention. Found throughout subtropical West Africa, such soils are excellent for the cultivation of maize, tobacco, and cotton (Kowal and Kassam 1978; Young 1976:134-138). However, in drier climes or where water retention is low, these soils will only support crops with a relatively short growing season such as millet and sorghum.

Since the mid-Holocene epoch, the lower Falémé River has become entrenched within these soil horizons to occupy a channel some 300-700 meters wide. Within this channel, alluvial deposition continues to produce beds of sorted sand, gravel, and large cobbles, as well as small levees of finer sediments (Michel 1973:421-427, 629-630). Active and fossil alluvial deposits provide indirect access to the mineral resources mentioned above, including worn cobbles for chipped and groundstone tool production, iron ores, and placer deposits of gold. Where levees occur today in Madina-Sadatou, they may retain sufficient moisture for the cultivation of small gardens, but they do not permit the large scale flood-recession agriculture found on the true floodplains of the larger Senegal and Niger Rivers.

Anthropogenic processes of soil loss and degradation have undoubtedly shaped the physical landscape of the Madina-Sadatou study area. Although the long-term effects of land clearance, cultivation, and livestock grazing are difficult to estimate without more detailed geomorphological studies, these have all contributed to soil erosion and degradation along river margins in the Upper Senegal region for at least the past several centuries, if not millennia (A. F. Clark 1995; Hayward and Oguntoyinbo 1987:161-162; Kowal and Kassam 1978:166-173). Importantly for this study, the removal of groundcover and subsequent erosion have enhanced the visibility of many archaeological sites, even as they have obliterated countless others (see Appendix A). 
Figure 3.5: Flood recession garden on east bank of Falémé River near Tomboura

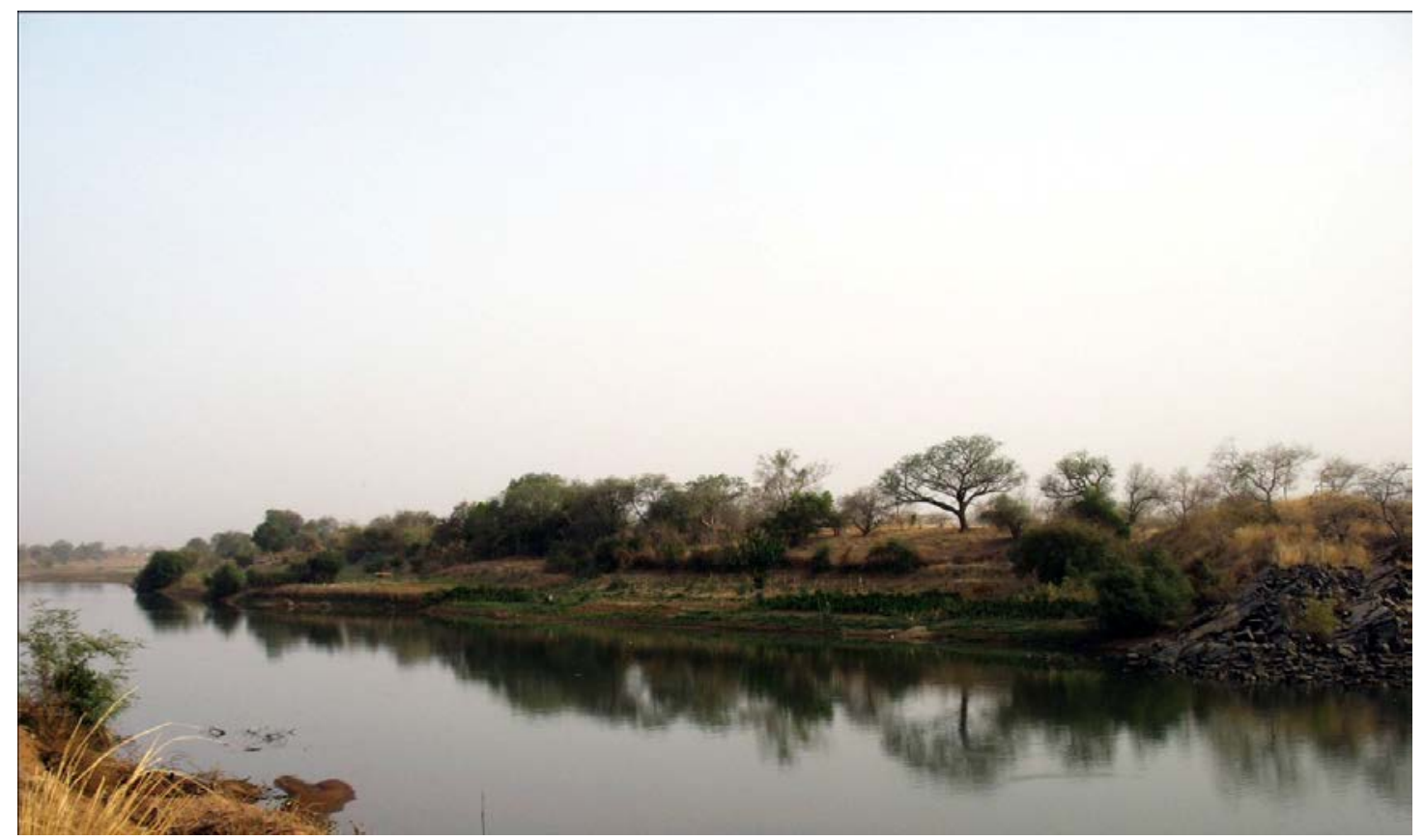

\section{Climate}

Precipitation in West Africa is strongly seasonal and commonly increases as one moves south from the Sahara Desert (Figure 3.6). This pattern arises from the annual movement of the Inter-Tropical Convergence Zone (ITCZ) where humid monsoonal air currents from the Gulf of Guinea cool as they encounter northeasterly trade winds, leading to heavy rainstorms (Gill 1991:27-37; Hayward and Oguntoyinbo 1987; Nicholson and Grist 2001). Owing to sea surface and landmass temperatures and the position and intensity of the African Easterly Jet, the ITCZ migrates each year from its southern position $\left(4^{\circ} \mathrm{N}\right)$ in January to its northern one (around $18^{\circ} \mathrm{N}$ ) in July. As the ITCZ moves south during the dry season, a northeasterly trade wind called the Harmattan returns to sweep dusty air down from the Sahara. While this system largely explains strong seasonality across the subcontinent, some longitudinal variation occurs where coastal systems, such as the Canary Current along the Senegambian littoral, ameliorate temperature differences between land and sea to affect a daily pattern of east/west wind changes. 
Figure 3.6: Map of rainfall isohyets across West Africa (from FAO/UNEP 1984; Rogers and Randolph 1986)

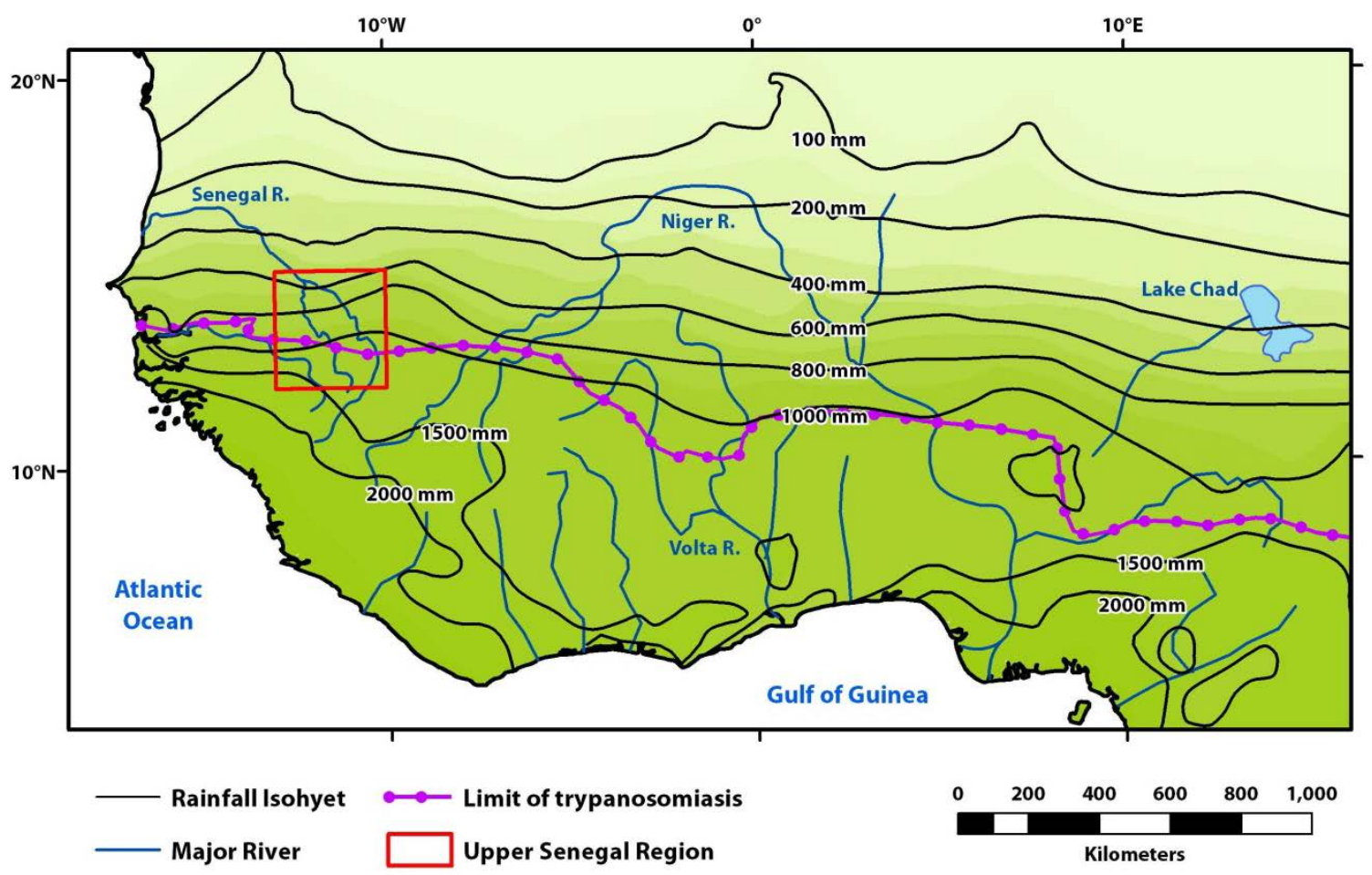

Alongside precipitation, temperature is the second major determinant of climate patterns across West Africa (Hayward and Oguntoyinbo 1987). In the Upper Senegal region, the winter solstice and the dusty air of the Harmattan reduce solar radiation during the dry season, contributing to "low" atmospheric temperatures in January when the daily average is only $33^{\circ} \mathrm{C}$. Temperatures increase steadily throughout the dry season to reach a maximum of $40^{\circ} \mathrm{C}$ in April and May before declining with the advent of the wet season to a mere $31^{\circ} \mathrm{C}$ in August. This wet season “cooling” results from the high potential evapotranspiration that simultaneously reduces the soil moisture required for early crop growth. Daily temperature variation ranges from $10^{\circ} \mathrm{C}$ during the wet season to $16^{\circ} \mathrm{C}$ during the dry season. For this reason, people usually design domestic architecture to both dissipate heat during the day and to provide nighttime insulation during the dry season when temperatures can dip below $15^{\circ} \mathrm{C}$ (Hayward and Oguntoyinbo 1987:200201; Prussin 1974:185-186). 
Figure 3.7: Interannual variability around mean precipitation across West Africa (after L’Hôte et al. 2002)

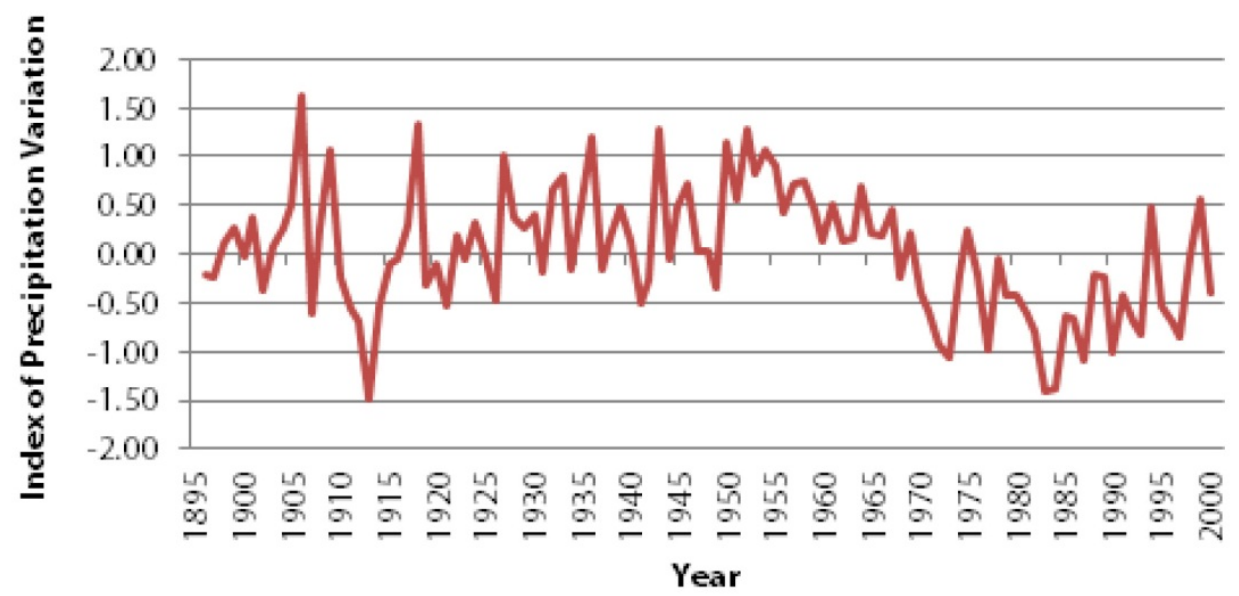

Figure 3.8: Mean monthly distribution of precipitation at $900 \mathrm{~mm}$ isohyet in the Upper Senegal region (after Pélissier and Bâ 1980)

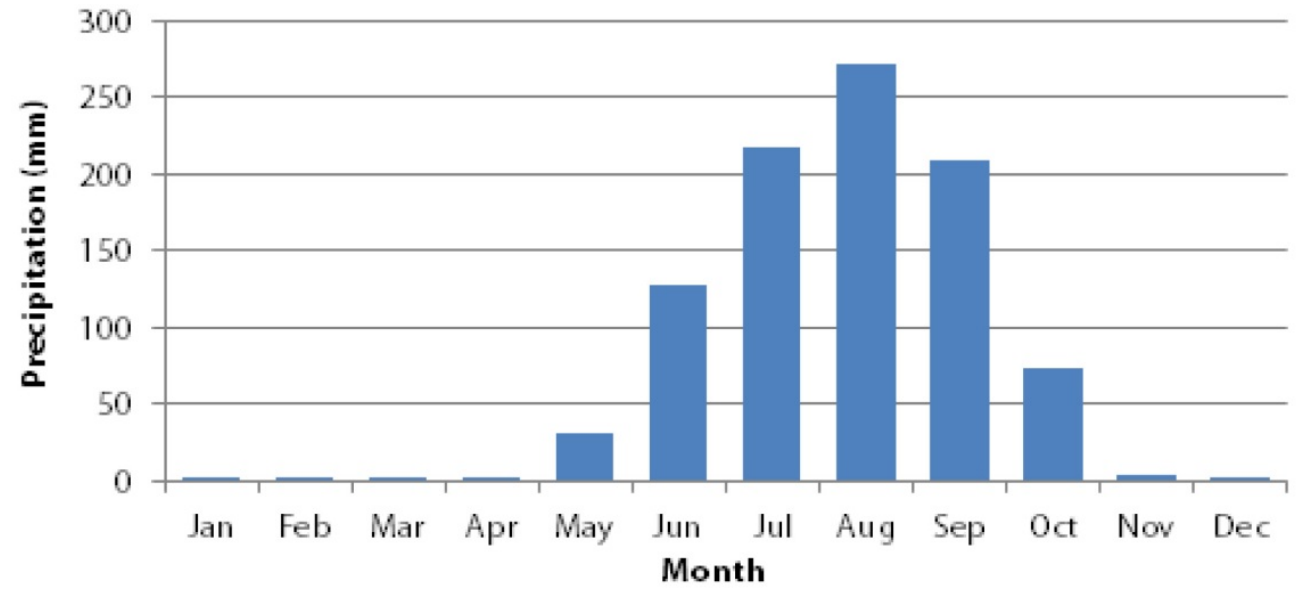

Beyond the regularity of this climate regime and its latitudinal isohyets, rainfall can vary markedly over time and space-with severe consequences for local ecological systems and subsistence economies. In the Madina-Sadatou study area where annual precipitation today averages some 800-900 millimeters, the duration of the wet season varies by as much as $30-40 \%$ on a year-to-year basis around the long-term mean (Nicholson 1989:49-50); this makes the start and duration of the effective growing season 
unpredictable. The daily and weekly timing of rainfall, particularly at the onset of the wet season, can greatly affect yields at small spatial scales, even from village to villagemuch rain can flood or wash out sprouting crops, too little rain can stunt them (Kowal and Kassam 1978:69-79). Moreover, these average annual patterns are the product of multi-year, even decades-long, cycles of rainfall above and below the mean. In her studies of climate trends across subtropical West Africa over the past five centuries, Sharon Nicholson $(1980,1989,1994)$ has identified at least six anomalous modes of significantly wetter or drier conditions lasting one or more decades and marked by abrupt, unpredictable shifts between them. While the magnitude of these shifts in earlier times is unknown, it is probably safe to assume that past sedentary communities developed strategies for mitigating the risks posed to the subsistence economy by radical and somewhat stochastic variation in rainfall at a multitude of temporal scales within and beyond individual lifespans.

\section{Hydrology}

By collecting locally variable rainfall and run-off over large areas, the major rivers of West Africa provide a relatively predictable source of water for irrigating crops, watering livestock, attracting fish and game, and otherwise supporting human economies and social interaction (Brooks 1993; Grove 1985; Shahin 2002). The Niger and the Senegal Rivers even boast interior deltas amenable to flood recession agriculture and the settlement of large populations (S. K. McIntosh 1999b). In Senegambia the gentle westward descent of the Senegal Basin contributes to the low velocity discharge of the Senegal and Gambia Rivers and their saline "reverse" estuaries. Since all of these rivers originate in the equatorial highlands of present-day Guinea, their patterns of seasonal and interannual variability are strongly correlated (Grove 1985; Hayward and Oguntoyinbo 1987:153-163; Shahin 2002). Most rivers reach their lowest levels, or even stop flowing, by the end of the dry season in April, and rapidly rise in June with the return of the summer monsoons. On an interannual basis, radical changes in precipitation levels can produce exponentially more dramatic differences in river levels. On the Niger River, for example, a 15\% variation away from mean annual rainfall within the river catchment results in a 33\% change in annual discharge (Grove 1985:10)! 
The Falémé River, which forms a dominant feature of the Madina-Sadatou study area, flows north some 625 kilometers from its headwaters in the Futa Jallon highlands to its confluence with the Senegal River (Shahin 2002:377-390). Squeezed between the Bundu Hills in the west and the Tomboura Escarpment in the east, the main channel emerges at the top of a strongly hierarchical network of steep tributaries flowing east or west and draining a basin of some 28,900 square kilometers; in the Madina-Sadatou study area these include the seasonal Kobakoye and Candamaka tributaries (Figure 3.3). During the dry season, the relative impermeability of the metamorphic basement keeps the water table near the surface of the river bed. Where standing pools of water do not persist, people can excavate shallow wells to provide water for themselves and their livestock. As discussed in Chapter 4, the perennial availability of water within and along the river channel, in contrast to the difficulty of digging wells through laterite duricrust, may partially explain the preferential location of past villages along the Falémé and its tributaries.

\section{Figure 3.9: Monthly flow of the Falémé River (www.sage.wisc.edu/riverdata)}

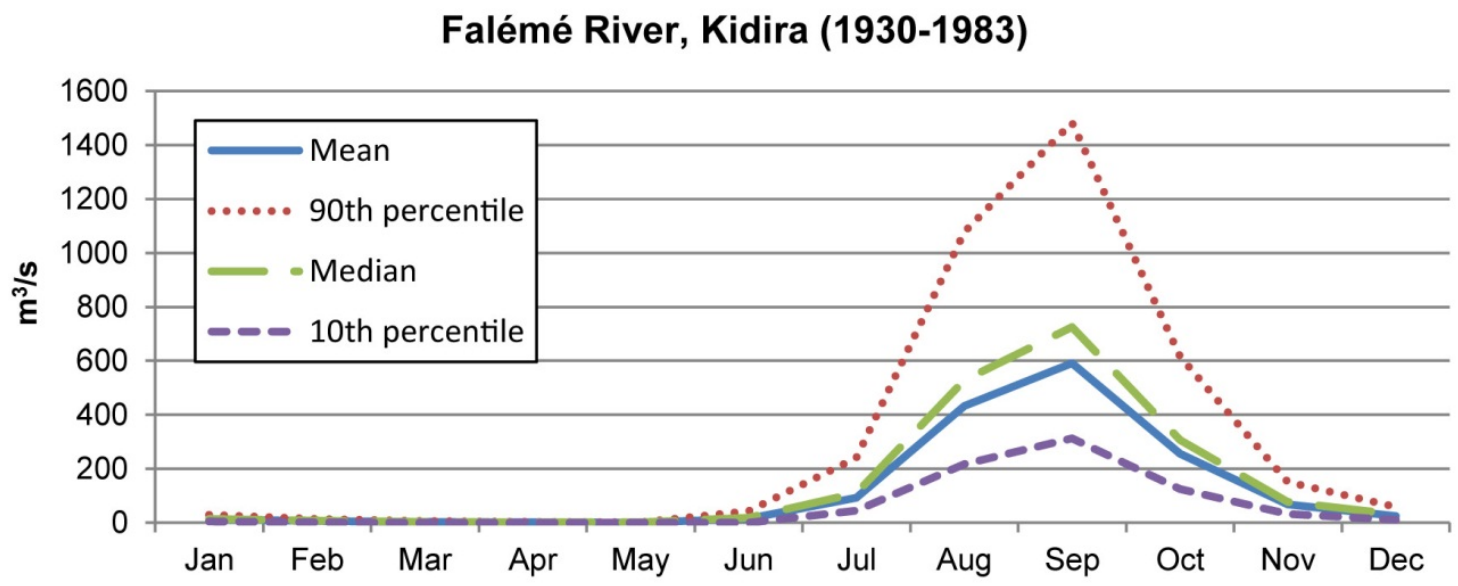

\section{Ecology}

South of the Sahara Desert, patterns of rainfall give rise to four conventional bioclimatic zones: the Sahel steppe grassland, the semi-arid Sudanian savanna, the humid 
Guinean savanna woodland, and the tropical rainforest (G. W. Lawson 1986). While the clinal distribution of precipitation produces diffuse and dynamic boundaries between these zones, they do describe broad differences in the plant and animal resources available for human exploitation (Hayward and Oguntoyinbo 1987:164-178; Koechlin 1997; Kowal and Kassam 1978). Importantly for the present study, they provide a frame for reconstructing past climate and ecological conditions in the Upper Senegal region.

The Sahel grassland receives 100-500 millimeters of annual precipitation (with high spatial and temporal variability) during a short wet season lasting anywhere from 30-95 days (Gillet 1986; Koechlin 1997). Adaptations by the vegetation, mainly low grasses and thorn scrub, include a long dormant period, reduced evaporation with spiny branches, thorns, and hairy leaves, and conservation of moisture and nutrients in underground storage organs. The few animal species endemic to this environment, such as gerbils and several reptiles, survive the long dry season with decreased daytime activity and low water requirements, while larger species such as gazelle, hartebeest, and lions have historically occupied gallery forests along perennial water sources.

Where annual rainfall in the southern Sahel exceeds 350 millimeters, people today cultivate drought-resistant crops such as pearl millet. Even here, the insufficient duration (55 days) and intensity of precipitation leads to utter crop failure more often than one year in ten (Kowal and Kassam 1978:110). The seasonal vegetation provides pasturage for camels, cattle, sheep, and goats (A. B. Smith 1992) although seasonal cycles require people to develop strategies for moving livestock among sources of food and water.

The Sudanian savanna receives 500-900 millimeters of annual precipitation during a wet season lasting three to five months (Kowal and Kassam 1978; Sanford and Isichei 1986). Although this zone boasts more tree cover than the Sahel, including economically important species such as baobab and palms, the major plants are grasses, sedges, and scrub adapted to the lengthy dry season. During the wet season, larger browsing fauna (and their predators) migrate north into the savanna to exploit rich vegetation; predominant species include duiker, oribi, kob, giant eland, and buffalo. 
Figure 3.10: Map of major bioclimatic zones in West Africa (WWF 2004)

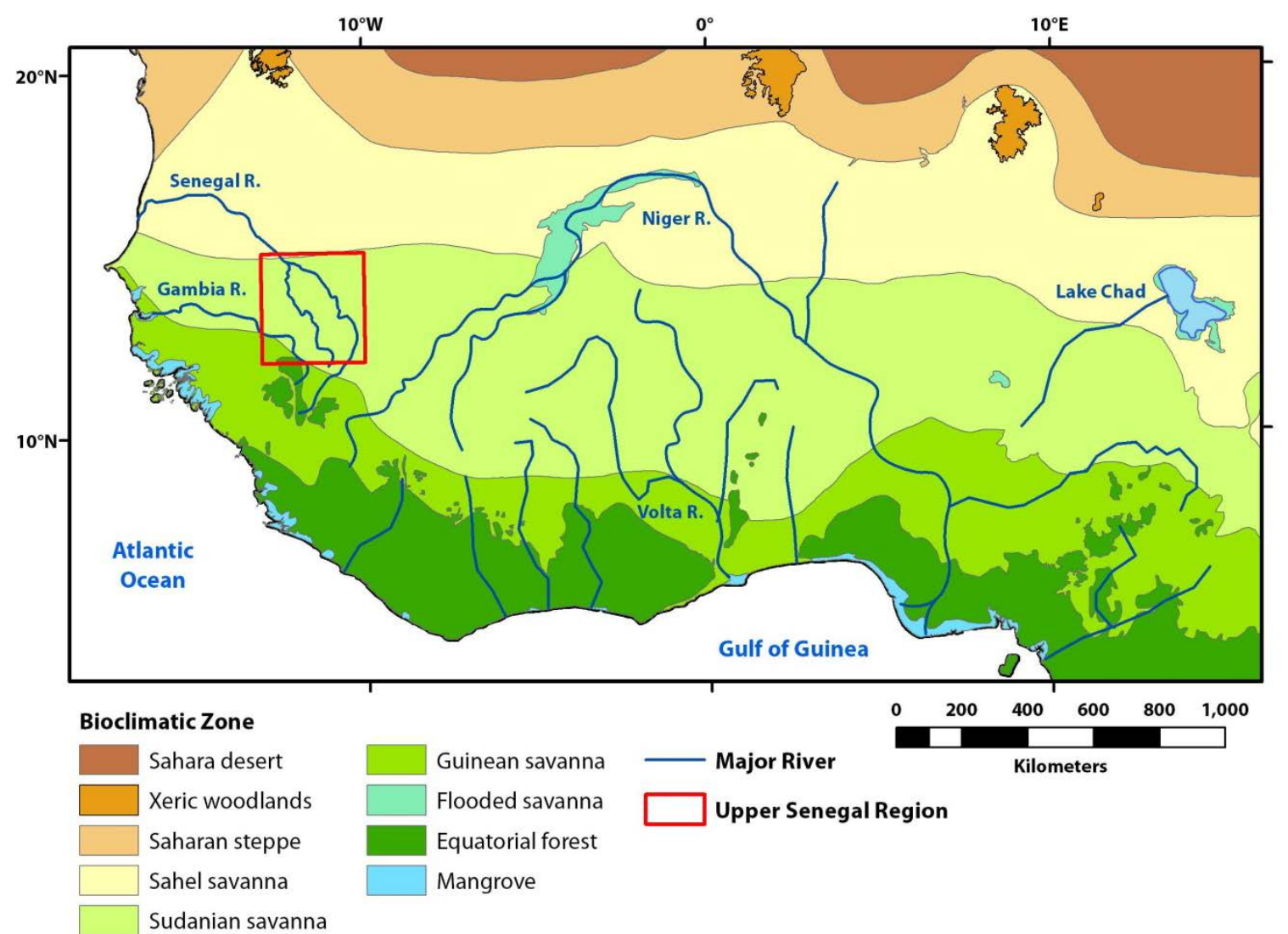

With a more predictable 95-140 days of rainfall, the Sudanian savanna zone is amenable to the cultivation of crops such as pearl millet, sorghum, groundnuts, and cotton using short fallow, shifting, and permanent cultivation strategies depending on local soil and hydrology. As with the Sahel, this zone provides ample pasturage for herd animals as well as horses, whose numbers are kept in check by the distribution of the tsetse fly (see below). This ecological system is highly susceptible to anthropogenic factors such as overgrazing, cultivation and loss erosion, firewood consumption, and uncontrolled burning.

The Madina-Sadatou study area lies today in the Sudanian savanna, but in times past (including the occupation of Diouboye) it would have fallen within Guinean savanna woodland, which receives 900-1500 millimeters of precipitation per year during a 140200 day wet season (Kowal and Kassam 1978; Sanford and Isichei 1986). In addition to 
baobab, stands of deciduous trees with broad leaves, including African locust bean, shea, and oil palm, form a mosaic interspersed with patches of tall tussocky grasses and scrub. In addition to the wild fauna present in Sudanian savanna, the Guinean savanna harbors species adapted to wetter or more closed environments, such as forest elephants, warthog, roan antelope, and monkeys. Alongside the savanna crops mentioned above, people today cultivate rice, maize, manioc, and yams with rotational fallowing of established field systems. Domestic livestock typically include sheep, goats, and dwarf cattle resistant to sleeping sickness as well as domesticated guinea fowl and chicken.

The equatorial rainforest receives more than 1500 millimeters of annual precipitation throughout the year although a month long “dry” season occurs along the Guinea coast in July as the ITCZ reaches its northernmost extent (Swaine and Hall 1986). Given this rainfall regime, large deciduous trees form a closed canopy that discourages grasses. By clearing land and engaging in periodic bush fallow, people grow most of the aforementioned crops, although rice, millet, and yams are staples. Additionally, the many forest plants such as kola and melegueta pepper have long provided products for both local consumption and long-distance exchange.

Within these broad bioclimatic zones, many smaller-scale habitats exist according to local soil conditions, relief, and hydrology. The Falémé River, for example, nurtures a narrow gallery forest hosting modest populations of savanna-forest animal species and providing a rapidly replenished source of wood for human habitations and fires. The river itself also comprises an important habitat. During the wet season, its deep and highly oxygenated waters foster high levels of aquatic vegetation, species of large-bodied fish such as Nile perch, and large dangerous game such as crocodiles and hippopotami. By the late dry season, the river condenses into shallow pools potentially harboring tilapia, catfish, and other species that do well in deoxygenated waters. As noted above, these pools, and the barrages built by humans, also provide a perennial source of water for game and livestock.

The savannas and forests of West Africa host a number of diseases and parasites detrimental to humans and their livestock and crops-including malaria, river blindness, sleeping sickness (trypanosomiasis), and insect pests. Malarial parasites (Plasmodium spp.) transmitted by mosquitoes have been infecting human hosts in Africa since the 
Pleistocene epoch, but cultivation practices over the past two or three thousand years have increased transmission rates and corresponding human resistance in the form of sickle cell hemoglobin (Webb 2005). While malaria is endemic to all of the sub-Saharan bioclimatic zones, local factors that either increase mosquito populations (e.g., cultivation practices, proximity to standing water) or decrease partial immunities (e.g., inconstant exposure) can exacerbate the disease and mortality rates in sedentary populations.

The trypanosome parasites responsible for sleeping sickness are transmitted by the tsetse fly (Glossina spp.) whose northernmost distribution corresponds roughly to the 1000 millimeter rainfall isohyet across West Africa (see Figure 3.7), but can fluctuate dramatically from year to year on the basis of rainfall and available moisture along river channels (Rogers and Randolph 1986). Although this disease sometimes affects humans, it is lethal to many domesticated animals. In regions with tsetse, people have responded by breeding dwarf species of "trypanotolerant” cattle and goats (Blench 1993). In general, exposure to these insect-borne diseases can be dramatically reduced simply by settling some distance from open water.

Although the majority of present-day crop destruction in West Africa derives from the introduction of non-native pests, some of the most damaging ones, including grasshoppers, cereal stemborers, and the African bollworm have long been endemic (Abate et al. 2000; Hayward and Oguntoyinbo 1987:177-178). Compounding the variability of crop yields based on the vagaries of rainfall, the ubiquitous presence of grasshoppers can cause trivial losses or complete decimation (which occurs roughly one year in five across the Sahel). In other bioclimatic zones where people cultivate maize, sorghum, and pearl millet, stemborers can bring about average yield losses of 15-40\%. Because insect cycles are somewhat chaotic, contemporary farmers across West Africa employ a number of strategies for mitigating the damage from observable pests. These include inter-cropping different species (which also enhances soil fertility and reduces erosion), early planting to avoid known hatchings, using botanical extracts or animal urine as natural pesticides, and performing religious rituals. While the time depth of these practices is uncertain, it stands to reason that subsistence economies in the savanna have long depended on people's capacity to deal with a number of unpredictable climatic and ecological factors. 


\section{Holocene Trends in Climate and Ecology}

By integrating variables such as air and ocean currents, precipitation, and land and sea surface temperatures with anthropogenic processes such as deforestation, models of contemporary West African climate and ecological systems provide a starting point for reconstructing the dynamic conditions of the past several millennia (Brooks 1989; R. J. McIntosh 2005:73-79; Nicholson 1979). Although it is not yet possible to identify local variability across the Upper Senegal region, it is probably safe to assume that past bioclimatic zones encompassed a similar amount of spatial and temporal heterogeneity as present ones (R. J. McIntosh 2005:73-89; Nicholson 1979). These, in turn, can be reconstructed from disparate, if admittedly sparse, lines of data including pollen sequences (Lézine 1989; Maley 1981; Médus 1984; Salzmann and Waller 1998), sediment cores from hydrological systems (Kröpelin et al. 2008; Lézine and Casanova 1989; Michel 1973; Shanahan et al. 2006; Talbot et al. 1984), paleobotanical and paleofaunal assemblages (Ballouche and Neumann 1995), and historic documents (Becker 1985; A. F. Clark 1995). For better or worse, these data show a heavy bias towards coastal regions, Lake Bosumptwi in Ghana, and a few extant and fossil lake basins in the Sahel. Climate historians also caution that environmental data, such as pollen, have complex depositional histories that reflect anthropogenic processes as much as environmental ones (Lézine 1989:329; Salzmann and Waller 1998:67-69).

Nevertheless, scholars have integrated these data into a number of broadly comparable historical climate models (e.g., Brooks 1989; 1993; Brunk and Gronenborn 2004; Kröpelin et al. 2008; Kuper and Kröpelin 2006; Mayor et al. 2005; R. J. McIntosh 1993, 2005; Munson 1981; Nicholson 1978b, 1979; Vernet 2002). Without going into a detailed review of the data, I briefly extrapolate these to the Upper Senegal region.

Beginning around 10,000 BC, the early-Holocene epoch in West Africa witnessed a gradual return to increasingly humid conditions following the extreme aridity of the terminal Pleistocene. Based upon evidence from pollen cores and lake and river sediments across East and West Africa, including cores from the Senegal and Gambia Rivers, precipitation levels reached a maximum around 8000-6500 BC. Well-documented sediment sequences from Lake Bosumtwi and Lake Chad record deep-lake conditions for this period instigated by a humid maximum (Brunk and Gronenborn 2004; Shanahan et 
al. 2006), while sediment and pollen sequences from several Saharan fossil lakes point to the florescence of a "green" Sahara (Kuper and Kröpelin 2006; Sereno et al. 2008).

Punctuated by humid phases, the climate became steadily more arid from 6000 to 2500 BC based upon evidence of declining lake levels and tree cover in the present-day Saharan and Sahel zones (Kröpelin et al. 2008; Sereno et al. 2008). Although this desiccation continued from 2500 to $300 \mathrm{BC}$, the later onset of arid conditions circa 17001200 BC at Lake Bosumptwi (Shanahan et al. 2006) compared to 2800-2300 BC in the present-day Sahara (Kröpelin et al. 2008) underscores the slow southward progression of this process. The Senegal River slowly took shape throughout this era, first as a gulf extending inland several hundred kilometers, and later as an elongated estuary, permitting the initial occupation of what is today the Middle Senegal Valley (Bocoum 2000:163169; Michel 1973). This period culminated in a stable, moderately arid phase documented across subtropical West Africa from approximately 300 BC to AD 300.

Figure 3.11: Holocene climate trends in the West African savanna (redrawn from Mayor et al. 2005:50; R. J. McIntosh 2005:80)

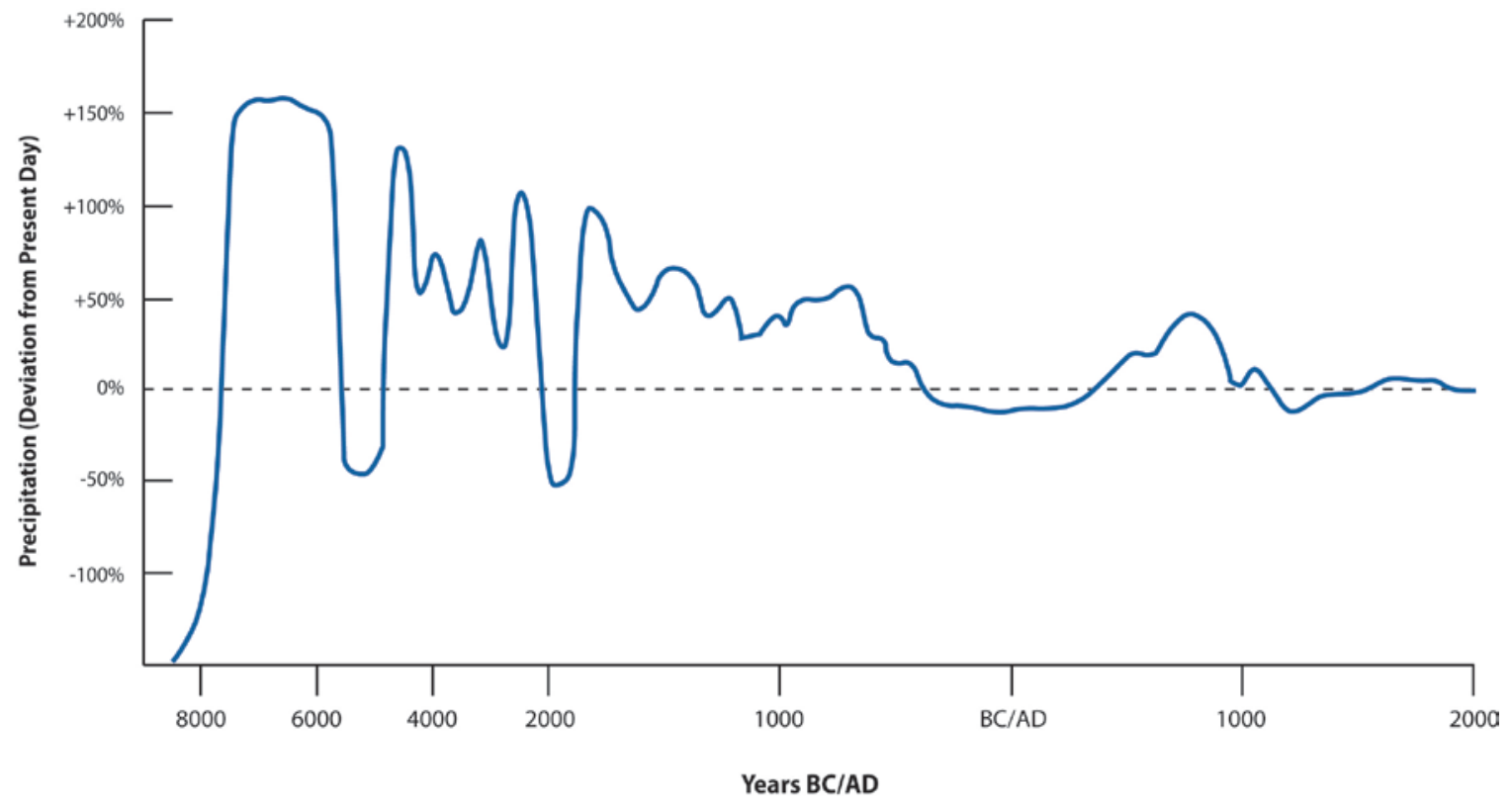


Marking the onset of the contemporary climate system in West Africa, the period AD 300-700 witnessed an increase in annual precipitation to levels perhaps 25-50\% greater than today (R. J. McIntosh 2005:84). These conditions reached a humid "stable optimum” with significantly less temporal and spatial variability during the period AD 700-1100. Archaeological data from Senegambia and the Middle Niger region also point to more humid conditions at this time (Bocoum 2000; R. J. McIntosh 2005), while historical accounts by medieval Arab chroniclers suggest that the boundaries of the Sahel and Sudanian savanna lay further north than they do today (Nicholson 1979). Assuming a sustained 20\% increase in annual rainfall, the Upper Senegal region would have fallen comfortably within the domain of the Guinean woodland savanna (see Brooks 1993). Notably, this period corresponds to the settlement of Diouboye.

From this time onward, historic evidence tends to outweigh ecological data in the reconstruction of climate trends, resulting in more nuanced (and more contested) interpretations (McCann 1999). The period AD 1100-1500 saw an overall decline in rainfall, and corresponding increase in the amplitude of variability, marking a “catastrophic discontinuity” from early stable conditions (R. J. McIntosh 2005:84) and a southward shift in the bioclimatic zones, perhaps by several hundred kilometers. This interpretation rests upon environmental data, historical accounts of drought and population movement, and the southward penetration of the horse trade into presumably tsetse free areas (Brooks 1989:35-36). Sharon Nicholson (1979), however, argues that this drier period was much shorter, falling between AD 1300 and 1450. This latter interpretation finds tentative support from the faunal data at Diouboye where people largely exploited riverine resources for several centuries (Appendix $\mathrm{H}$ ). Whatever the case, the climate of the Madina-Sadatou study area appears to have become increasingly arid by the time people abandoned the village in the $14^{\text {th }}$ century AD.

Relatively humid conditions returned to West Africa in the early- $16^{\text {th }}$ century AD although scholars disagree about the duration. Historian George Brooks (1989:37) sees the return of dry conditions by AD 1630, while Sharon Nicholson (1978a, 1979) has suggested that despite three severe droughts, humid conditions persisted into the $18^{\text {th }}$ century AD. Fine-grained data leave no question that West Africa experienced slightly 
more humid conditions in the mid $-19^{\text {th }}$ to early- $20^{\text {th }}$ centuries $\mathrm{AD}$, before the onset of an ongoing drought in the 1960s (L’Hôte et al. 2002; Nicholson 1978a).

Alongside the more durable geological elements of the landscape, these dynamic climate and ecological conditions have shaped the material resources available to communities living along the Falémé River. Rock sources, soil conditions, seasonal and perennial water channels, and the range of biota on the landscape are consistent from year to year, even as they shift over the course of generations, centuries, and millennia. Meanwhile, spatial and temporal variability in annual rainfall, and by extension the distribution of plant yields, create the risk of unpredictable short-falls in food procurement and production (Halstead and O'Shea 1989; R. J. McIntosh et al. 2000). Since these material resources underwrite economic processes-particularly those within the subsistence economy-their spatial and temporal organization provides an entry point into the political economies of the past communities relying upon them.

\section{HUMAN ECOLOGY IN THE WEST AFRICAN SAVANNA}

Building upon the discussion of subsistence economy begun in Chapter 2, this section briefly develops some of the key technological and social strategies employed by communities occupying the dynamic landscape of the Upper Senegal, and the West African (that is, Sudanian/Guinean) savanna more broadly. Focusing on those societies with food-producing economies, I consider production regimes, physical and social storage, relative mobility, labor organization, and social memory. Because such strategies exist within institutional arrangements and cultural schema, their failure to mitigate unforeseen catastrophes (or adapt to major climatic transgressions) can create ruptures and the possibility of historical transformation within these structures. Of course, social institutions do not merely function to alleviate ecological risk (see Halperin 1994:55-84); in many cases they contribute directly to insecurity in access to food and other resources.

\section{Subsistence Regimes}

In outlining the ecology of West Africa, I made mention of the main subsistence resources available to people occupying the Upper Senegal region both today and in the past. These include a host of wild plants and animals, as well as several major 
domesticates, all of which have become enmeshed within subsistence economies via long-term dialogue between ecology, political economy, and cultural tastes.

Over the past millennium, people have developed a diverse resource portfolio whose agricultural, pastoral, and wild products help to buffer against the vagaries of climate and pests and to obtain important vitamins and minerals (Gill 1991:76-79). Farmers, for example, may employ fallowing and intercropping of millet, sorghum, and cowpea to reduce the danger of crop pests, preserve soil quality over time, and enhance labor returns. Where high spatial and temporal variability in rainfall, and thus crop yields, exist around single villages, farmers often choose to cultivate non-adjacent fields, which they then sow heavily (Graef and Haigis 2001). People also plant gardens close to their homes where they can guard against pests and where the deposition of human waste and other organic refuse provides excellent fertilizer.

Overall, this system forms a graduated series of catchment "rings" radiating outward from individual homesteads, villages, or other settlements (Burnham 1980:159160; Gallagher 2010:88-90; Pélissier 1966). Farmers generally situate their gardens and permanent field systems demanding greater labor inputs nearer to their place of residence, ideally within a one-kilometer walk (Stone 1996:131-138). For rotational or shifting cultivation beyond this distance, people may even build semi-permanent field huts, or move their residences each season, to supervise crops susceptible to foraging animals and other pests (Lestrange 1955:18-21). Interspersed among these field systems, and lying beyond them, are non-domesticated trees and even wild bush lands from which people collect firewood, gather leaves, bark, and other products, and hunt game.

Livestock, including cattle, sheep, and goats, complement agricultural production in the savanna by providing secondary products such as milk as well as primary ones such as meat and leather (Burnham 1980; Casey 1998; A. B. Smith 1992). During the dry season, livestock may be corralled in fields where they consume crop stubble and enrich the soil with their droppings. With the arrival of the rainy season, people shepherd herds to distant pasturage so as not to interfere with staple crop yields or important wild grasses. In fact, the capacity to move livestock across the landscape, as a sort of "wealthon-the-hoof," makes them a valuable counterpart to staple crops, which must suffer the vagaries of local rainfall. 
Given the value placed on livestock for social transactions, people seldom slaughter them and instead obtain protein from wild game and fish. While hunters' associations and cultural proscriptions may encourage (or prohibit) the killing of specific species such as lions, hyenas, or warthog (Cissé 1964; Lestrange 1955:26-27), many others are, quite literally, fair game. In addition to the targeted hunting of large-bodied animals such as buffalo and elephant, people may procure smaller game opportunistically in their gardens or through collective hunting drives organized at the community level (Dueppen 2012:25). For people residing near major river systems such as the Falémé, fish and other aquatic resources often contribute substantially to local diets.

As discussed in Chapter 2, the labor, practical knowledge, and mobility entailed by different production activities generally leads to specialization at some scale-whether individuals within a household, lineage groups within a village or territory, or even ethnic groups across a regional landscape. It follows that diversified subsistence regimes in the West African savanna depend upon, and reproduce, institutional relations for scheduling access to land and other resources, organizing labor, and exchanging foodstuffs between producers and consumers.

\section{Storage and Social Capital}

In the West African savanna, sedentary communities have developed a number of processing and storage techniques to prolong staples depending on their perishability (Gill 1991:105-106). Non-perishable foods such as grains, oils, and nuts can, in theory, store well for a number of years if protected from moisture in structures ranging from sturdy earthen wall structures to ephemeral baskets (Prussin 1972). The humidity of the rainy season, however, introduces bacteria and mold that easily damage food stores, reducing the effective storage duration of even non-perishables to less than one year (Burnham 1980:154; Casey 1998:54). Semi-perishable foods such as tubers can be stored for up to a year, but are usually consumed prior to the subsequent growing season. Perishable foods such as dairy products, vegetables, soft fruits, fish, and meat can be processed for short-term storage (i.e., smoking, sun-drying, salt-curing), but require consumption within several days or weeks. As noted in Chapter 2, communities in West 
Africa may make decisions about the storage of different foodstuffs at the scale of the individual, household, or corporate kin group.

The seasonality of savanna ecology also encourages people to "store" the labor invested in food production as informal social debt-a common strategy for mitigating subsistence risk in many small-scale societies (Halstead and O'Shea 1989; O'Shea 1981). In West Africa, as discussed in Chapter 2, this delayed-return reciprocity often lies embedded within kinship relations extending beyond the local community. Rather than move foodstuffs, people in households experiencing a poor harvest can generally call upon relatives living in other villages for extended periods of time to conserve food stores in their own compounds (Casey 1998). In the Middle Senegal region, farming households deal with short-term subsistence crises by "shunting” members into other economic pursuits such as fishing or herding (S. K. McIntosh 1999b:158; T. K. Park 1992). Overall, the appropriational movement of social storage within the subsistence economy depends upon locational movements of people to resources or resources to people-thus shaping the logic of mobility and settlement.

\section{Settlement Strategies}

In conjunction with local geography and corresponding subsistence regimes, people residing in the West Africa savanna have adopted settlement strategies ranging from dispersed single-family homesteads to aggregated urban complexes. Dispersed and aggregated settlement patterns also occur at a finer scale between homestead-based and village-based communities. These different settlement strategies arise, in part, as a response to the fallowing and labor requirements of local agricultural practices-more extensive field systems encouraging homesteads, more intensive ones appearing around villages_-as well as demographic pressure and political organization (Burnham 1980; Horton 1971; Shipton 1984). As settled communities persist and expand, exhausting local soils (and other resources), they may undergo processes of fission in which frontierspeople depart to settle new lands.

Pastoralism, of course, requires a certain degree of mobility, whether seasonal transhumance between a sedentary village and wet season pastures, or a more fully nomadic existence throughout the year (Burnham 1980; A. B. Smith 1992). While young 
men in some communities are responsible for the livestock of their respective corporate kin groups (Lestrange 1955:27), herd management throughout much of West Africa is undertaken by, or contracted out to, ethnic Fulbe herders within a system of complementary exchange (see Chapter 2).

Given the importance of residential mobility for understanding past landscapes in West Africa, I explore this topic in greater detail in Chapter 4.

\section{Labor Pooling and Scheduling}

Since labor is often the greatest constraint on the potential output of savanna subsistence regimes, the coordination and scheduling of social actors and resources is a prime concern for political economies at all scales (Burnham 1980; Casey 1998). Many farming communities, for example, experience an enormous labor bottleneck during peak times of planting, harvesting, and processing. In order to deal with this extreme seasonality, the Kofyar, an agricultural society in Nigeria, organize locational movements within the subsistence economy at three scales (Stone 1996; Stone et al. 1990). First, a flexible gender-based division of labor within household compounds (homesteads) allows men, women, and children to work together at the time-sensitive tasks of field clearing, planting, and weeding throughout the rainy season. Second, neighboring homesteads cooperate to exchange labor along age and gender lines in the maintenance of different field systems. Finally, larger work parties of 30-100 people undertake the labor-intensive task of "ridging" fields to aerate and drain the soil around recently sprouted millet and sorghum. This labor pooling occurs on the basis of delayed-reciprocity, but field owners always reciprocate more directly with fresh millet beer to encourage camaraderie. While the specifics differ according to local ecology and cultural history, the Kofyar illustrate the interplay between the labor bottlenecks arising from savanna agriculture and the political economies of sedentary communities across West Africa.

In contrast to the intense labor requirements of the rainy season, farming communities experience a surplus of labor during the dry season (Gill 1991). With their granaries (hopefully) full, people may continue to procure wild plant products, game, and fish as important supplements to their diet; they also find more time to engage in craft production for local use or regional markets. This is the ideal time to construct earthen 
wall structures or repair damage meted about by the previous rains. Travel also becomes manageable and people make time to renew supra-local social and political relations and partake in long-distance exchange. In the modern global economy, people in rural areas frequently migrate to urban centers in search of seasonal wage-labor.

Competing with the labor demands of agricultural production, the returns on fishing are often greatest during the rainy season as piscine reproduction and growth cycles upward with seasonal water levels (Sundstrom 1972). Pastoralists, on the other hand, experience their heaviest labor demands during the dry season when herds of livestock, particularly cattle, must be moved frequently to areas of available pasturage (A. B. Smith 1992).

A diversified subsistence economy creates the potential for conflicts over land, water, and other resources by people engaged in foraging, farming, herding, and fishing. As previously discussed in Chapter 2, increasing specialization thus entails institutional relations for scheduling access to these resources and mediating conflicts when they arise to ensure mutual benefits within the regional economy (Burnham 1980; Casey 1998; R. J. McIntosh 2005; S. K. McIntosh 1999b). These institutions usually involve kinship or joking partnerships that depend upon, and reproduce, the identities and political statuses of specialists at multiple scales; that is, they find legitimacy through cultural schema.

\section{Ritual and Social Memory}

Social strategies for mitigating risk in the seasonal West African savanna depend not only upon political relations within and between social actors, communities, and physical geography, but also the perpetuation of these relations across space and time. In this regard, ritual provides a means through which people reproduce the cultural schema enabling economic coordination and cooperation (Chwe 2001; Rappaport 1979). In rural communities, for example, ritualized events that involve feasting, such as harvest festivals and weddings, tend to occur at the end of the rainy season when surplus foodstuffs can be stored in the body and as social debt (Casey 1998:55). And, to the extent that ceremonies demand livestock, they help to perpetuate exchange relations between sedentary farmers and mobile pastoralists. At the same time that secret associations steeped in ritual teach individuals with the practical and discursive 
knowledge required to take down large game (Cissé 1964), they usually provide an avenue for achieving status and for regulating political affairs across kinship divisions (S. K. McIntosh 1999c:12-14). All this is not to say that religious ritual only works to perpetuate political economic institutions, but it often plays an important role.

At an inter-generational scale, the social memory encoded in ritual can also help communities weather longer-term cycles in ecological degradation or amelioration, perhaps punctuated by once-in-a-lifetime catastrophes (R. J. McIntosh 2000; Minc 1986). When social mechanisms for exploiting diverse resources, storage, and sharing are insufficient to ensure community survival, oral traditions and beliefs can provide a template for the success of migration or the activation of long-distance social relations. These examples are by no means exhaustive, but they point to the ways in which the representational dimension of the landscape shapes the historical production of subsistence economies at multiple scales.

\section{SUMMARY}

This chapter began by introducing the landscape perspective that frames my study of political economic relations extending beyond Diouboye to other communities in the Upper Senegal region and beyond. I proceeded to summarize those spatial and material dimensions of the physical landscape-geology and local relief, soils, rainfall and hydrological networks, and ecology—-that have historically structured economic (mainly subsistence) processes and institutions in the West African savanna. While recognizing that these elements, particularly soil conditions and ecology, are also affected by humans, I then discussed some of the key parameters on locational and appropriational movements set by the structure of savanna landscapes. More specifically, I looked at some common socioeconomic strategies employed by agrarian or agro-pastoral communities to reduce the subsistence risk resulting from the annual unpredictability of precipitation, livestock diseases, and pests. Against the background of the Holocene climate history, these strategies provide a means of interpreting some of the regional archaeological evidence presented in the following chapter. 


\section{CHAPTER 4 DIOUBOYE AND THE HISTORICAL LANDSCAPE}

Village communities exist within political-economic and cultural networks that extend across space and endure and evolve through time. In the present chapter I move to situate Diouboye within such networks by attending to the material, spatial, and historical relations inhering among places, and people, across the surrounding landscape. Drawing upon the brief introduction of landscape presented in Chapter 3, I first consider how archaeologists have deployed this perspective in West Africa, thereby highlighting themes relevant to the study of Madina-Sadatou as an historical landscape. In the second part of this chapter, I present trends in the past political economies and cultural dynamics of this study area, focusing primarily on the longue durée of the Holocene epoch. Specifically, I consider how data from my archaeological survey along the Falémé River articulates with a schematic outline of archaeological horizons across West Africa, as well as with selected oral and textual sources documenting the landscape history of the Upper Senegal region. This exercise, along with the preceding discussion of physical geography and human ecology, sets the scene for the comparative analysis of Diouboye and the political economy of its local landscape in Chapter 5.

\section{ARCHAEOLOGICAL LANDSCAPES IN WEST AFRICA}

Prior to the 1980s, surface prospection in West Africa served mainly to locate sites for excavation and to map out broad zones of shared archaeological "culture" (Bower 1986; De Barros 1990). Since the introduction of systematic survey methods nearly three decades ago (Connah 1984; S. K. McIntosh and R. J. McIntosh 1980), landscape-oriented research in West Africa has come to focus on three interrelated methodological and theoretical issues: (1) mobility and sedentism; (2) political economy (social complexity); and (3) ideology and representation. In this section, I discuss each of these in turn, paying attention to their relative value for interpreting the archaeological 
landscape encompassed within the Madina-Sadatou study area. By archaeological landscape, I refer to the spatial and material dimensions among places (i.e., sites) involved in past political economies, cultural traditions, and historical transformations.

\section{Mobility and Sedentism}

A strict dichotomy between sedentary and mobile societies fits neither with the archaeological record of West Africa nor with ethnographic observations of residential mobility in agro-pastoral communities (see Chapter 3). To be sure, mounded tell sites do attest to long-term settlements in many regions; they are even prevalent in the Middle Niger and Méma regions (Haaland 1980; S. K. McIntosh 1995; S. K. McIntosh and R. J. McIntosh 1980; Togola 2008) and in the Lake Chad Basin (Breunig 2004; Connah 1981; Holl 2002) where annual flood regimes make artificial mounds the "least worst" locale for long-term sedentary occupation. Meanwhile, rock shelters and ephemeral encampments occupied by highly mobile foragers and pastoralists do occur in the Sahara and Sahel (Holl 1989; MacDonald 1997), as well as in the savanna forest (Casey 2003; Davies 1980). Nevertheless, a growing commitment to regional archaeology has revealed that many, if not most, agro-pastoral societies in West Africa over the past three millennia generated expansive networks of shallow occupation sites occupied for one to several generations, perhaps by single communities (Gallagher 2010; A. Lawson 2003; Richard 2007; Thiaw 1999). Ultimately, this research demonstrates that strategies of mobility/sedentism vary continuously along spatial and temporal axes; and they do so according to complex constellations of ecological, economic, political, and cultural factors.

As discussed in Chapter 3, mobility is a fundamental strategy for coping with subsistence risk in foraging and agro-pastoral economies, particularly in the savanna where unpredictable climate variation and pests can lead to periodic catastrophes. This strategy can be quite multifaceted since "mobility is a property of individuals, who may move in many different ways: alone or in groups, frequently or infrequently, over long or short distance” (R. L. Kelly 1992:44). Foragers and pastoralists, for example, organize their inter-annual moves among wild resources and social encounters along a continuum between residential and logistical mobility (Binford 1980; Wiessner 1982a). The former 
entails movement of the entire social group between short-term camps, while the latter involves small task groups coordinating their activities from a longer-term camp.

Sedentism extends logistical mobility into the year-round occupation of a settlement by at least some segment of a community, often leading to intensified food production and investment in permanent storage facilities and domestic architecture to mitigate the potential risks associated with loss of mobility (R. L. Kelly 1992:49-54). Of course, logistical forays and inter-annual moves remain critical within and between sedentary communities as means to acquire non-local resources, manage field systems and livestock, and maintain supra-local social networks (Graham 1994; Stone 1996).

Over time, climate change and/or declining yields from available land and other resources can lead to the abandonment of settlements as communities relocate to unoccupied land or, as is more often the case, disperse to join existing settlements or pioneer new ones (Prussin 1969; Stone 1996). The tempo and intensity of these processes depends, in part, on the subsistence regime (Gallagher 2010) — whether pastoral, fully sedentary agriculture, or shifting agriculture.

This sort of ecological perspective on mobility has proven most useful in archaeological studies where regional patterns of habitation and other activities can be explained by geographic and climatic variables, given some basic assumptions about strategies of risk management (e.g., Butzer 1982; R. J. McIntosh 2005). In West Africa, this perspective also finds support from historical traditions of diverse agrarian communities that explain the abandonment and settlement of villages in terms of ecology and climate change (Goody 1967; Pélissier 1966; Stone 1996).

Just as often, however, ethnographic and historical accounts of mobility and migration emphasize individual and external agencies, social structures, and traditions (Kopytoff 1987b). Non-ecological factors affecting relative mobility at different scales can include access to labor (Stone et al. 1990), land tenure systems and territorial claims (T. K. Park 1992; Shipton 1994), household and community fission (Goody 1967; Turner 1957), sustained political violence or slave raiding (Hawthorne 2003:155-159), and systems of trade and exchange (Brooks 1993; Curtin 1975a). In West Africa these may be further bound up with ideologies and religious practices that sustain residence and migration patterns, or help to underwrite social boundaries between, for example, 
transhumant pastoralists and sedentary farmers (Levy and Holl 2002; R. J. McIntosh 2005). Documentation of relative mobility, with implications for locational and appropriational movements, can thus yield insights into past political and cultural economies - to the extent that it can be teased from the material and spatial dimensions of archaeological landscapes.

\section{Relative Mobility and the Archaeological Landscape}

At the local level of discrete places on the landscape, patterns of relative mobility can affect the forms, diversity, and scale of material practices that generate artifacts and architecture (Binford 1982; R. L. Kelly 1992; Rafferty 1985). All else being equal, infrequent visits and/or very specialized activities at a given locale tend to produce a relatively homogeneous material assemblage suited to specific tasks, while longer-term or sedentary occupations generate a greater diversity of artifacts suited to a broader suite of tasks. The physical setting and seasonal exploitation of plant and animal resources can also influence temporal patterns of site use and mobility within a community (Berelov 2006). Most strikingly, relative sedentism affects the scale and spatial structure of material investment in a place. Sedentary communities often build more durable houses and storage facilities than do relatively mobile ones, and they tend to dispose of their accumulated rubbish out of the way of domestic traffic (Hayden and Cannon 1983). In fact, this spatial segregation usually extends to other economic practices structured on the basis of gender roles, relative specialization, and inter-household relations (Graham 1994; Kent 1982). Alongside refuse disposal, architectural construction and decay further contribute to stratigraphic build-up and the deposition of datable artifacts useful for inferring the span of settlement occupation-a crucial variable for establishing the relative contemporaneity of places across a past landscape.

A comparison of material assemblages and spatial distance between places at a regional scale can also elucidate past strategies of sedentism and mobility. In many foraging and pastoral societies, for example, residential mobility produces a large number of generalized encampments resulting from frequent moves of an entire band, while logistical mobility produces fewer camps or settlements, surrounded by more numerous locations for the procurement of specific resources (Binford 1980; Holl 1989; R. L. Kelly 
1992). In sedentary societies, on the other hand, the short-distance displacement of individual households can actually contribute to the formation of mounded sites over time (Holl 1987), while the pioneering of homesteads and/or the displacement of entire villages can generate networks of relatively ephemeral occupation sites in close proximity to one another (A. Lawson 2003:18). Longer-distance migrations can generate temporal and material discontinuities in regional occupation, often in association with the closure or abandonment of heavy or immobile site furniture (Graham 1994). At the same time, it is important to recall that archaeological landscapes emerge not only from relative mobility at multiple scales, but also from the political economies and cultural schema in which such movements are enmeshed.

\section{Political Economy}

Regional or landscape approaches in archaeology offer an incredible vantage from which to view the evolutionary and historical pathways of past political economies (e.g., Covey 2006; Duffy 2010; Liu 1996; Plog 1986; A. T. Smith 2003; Stanish 2003; Wright and Johnson 1975). This is certainly the case in West Africa where archaeologists have employed regional data to examine the emergence of rank (Holl 1993, 2002), politicaleconomic mosaics (R. J. McIntosh and S. K. McIntosh 2003; R. J. McIntosh et al. in press; S. K. McIntosh 1999b), the management of trade routes (A. Lawson 2003), the production of monumental landscapes (S. K. McIntosh and R. J. McIntosh 1993b), and trajectories of interregional entanglement and cultural contact over the longue durée (Richard 2007; Thiaw 1999, 2012). Often couched in the rubric of "social complexity," these studies offer a counterpoint to commonly held assumptions about the relation between social institutions and settlement patterns in geographic models predicated upon principles of market economy, vertical integration, and impermeable social boundaries (Crumley 1979). Drawing upon these insights, I briefly consider the interplay between political relations, as appropriational movements within the economy, and the production of landscapes across West Africa, particularly those oriented along rivers such as the Falémé.

As discussed in Chapter 2, institutions comprise symmetrical and/or hierarchical (asymmetrical) political relations among social actors and groups. Physical aspects of 
landscape become enrolled in the reproduction of these relations because they structure when, where, and how people gain access to various resources. In addition to material resources such as arable land, livestock pastures, raw materials for crafts, and other culturally valued objects, these include non-material (social) resources such as corporate labor, marriage partners, political alliances, and places of religious significance. The movement of these resources into subsistence, craft, and exchange economies depends not only on locational movements, as exemplified by the concept of mobility above, but also the appropriational movements that shape, and are shaped by, symmetrical and hierarchical relations at multiple scales across the landscape.

Symmetrical relations among communities, polities, ethnic groups and other social actors on the regional stage work to segregate space (and the resources therein) in equivalent or complementary ways. In a cogent synopsis of dominant spatial models for regional analysis, archaeologist Carole Crumley (1979:144) defined this process as heterarchical organizational structure "in which each element possesses the potential of being unranked (relative to other elements) or ranked in a number of different ways.” Put another way, the symmetrical ordering of elements across the landscape-places or catchments in the parlance of this study — emerges either from their homogeneous composition or the necessity for symbiotic interaction among the people occupying them.

Some of the ethnographic examples mentioned in Chapters 2-3 illustrate the heterarchical organization of political-economic landscapes. The small-scale and shifting farming communities among the Kofyar, (Stone 1996), Tiv (Bohannan and Bohannan 1968), and LoDaaga (Goody 1967), for example, occupy households and homesteads, each with comparable access to cultivable land and other material resources. In spite of their potential for political and economic autonomy, these social units maintain supralocal relations based on balanced reciprocity of labor and foodstuffs. These interactions not only perpetuate social life and culture; they may also function to mitigate subsistence risk in the savanna.

Across the more complex social mosaic of the Middle Niger region, diverse ethnic groups with complementary subsistence and craft specializations occupy neighboring villages or urban wards within towns, creating socio-spatial boundaries that some scholars read explicitly as heterarchical organization (R. J. McIntosh 1998). 
Although material evidence for specialized economic practices, such as fishing and blacksmithing, reveals heterogeneity among past settlements within this landscape (M. E. Clark 2003), the absence of clear evidence for hierarchical political differences (i.e., monumental architecture, differential access to prestige goods) suggests that relations among specialists were predicated upon political symmetry, rather than hierarchy (R. J. McIntosh and S. K. McIntosh 2003; S. K. McIntosh and R. J. McIntosh 1993a). These examples are by no means comprehensive, but they do highlight some of the ways in which the economic practices sustaining political symmetries work to segregate space in complementary ways.

On the other hand, hierarchical relations among social actors both emerge from and reproduce control over material and non-material resources (and cultural schema) across the landscape. In cases where highly valued resources are spatially restricted or regionally heterogeneous, communities may leverage political authority through control over local resources (Hirth 1978), suffer exploitation by non-local polities (Peregrine and Feinman 1996; Wallerstein 1974), or negotiate their position somewhere in-between (Stein 1998, 2002). At the same time, centralized or hierarchical polities exert and legitimize their authority, in part, by segregating space, and distancing (or aggregating) groups within it, independent of the natural distribution of material resources (Richard 2007; A. T. Smith 2003). Contrasting with heterarchy, the hierarchical ordering of places and catchments across the landscape thus arises from their heterogeneous composition and/or the potential for some people to coerce economic action from others.

In an ethnological comparison of historically ranked societies in East Africa, Donna Taylor (1975) found that political-economic hierarchy beyond a single settled community produced complementary differences in settlement size as high status groups consumed more material resources and invested in more social capital than low status ones. Such processes led to an uneven pattern of spatial segregation with resources flowing towards political elites. Similarly, ports of trade along the West African littoral, and much earlier in the Sahel, have historically accrued authority over their hinterlands vis-à-vis control of long-distance exchange. Again, these examples are meant to be illustrative, not definitive, and one can expect that both heterarchical and hierarchical 
dimensions will coexist at multiple scales within any given landscape-particularly for the complex political-economic mosaics of precolonial West Africa.

\section{Political Economy and the Archaeological Landscape}

Alongside patterns of residential mobility described above, symmetrical and hierarchical dimensions of political economy structure the intensity and diversity of activities among settlements and other places on the landscape.

Spatial dimensions of an archaeological landscape comprise the segregation of space within and around these places, as well as their distances to surrounding geographic features (catchment zone). Where settlements and other sites are positioned to exploit different catchments, it may be fair to speak of differentiation in the resource base, particularly if artifact assemblages point to locally specialized economic activities. After controlling for proximity to landforms such as rock outcrops, cultivable soils, and rivers involved in the locational movement of resources, the distances between settlements say something about the institutional relations structuring practices of production, distribution/exchange, and consumption within and among them. This assumes, of course, some correspondence between spatial distance and social affinity (see discussion in Crumley 1979). Settlement aggregation, which segregates occupied and non-occupied space, further increases the possibility of regular interactions among people within the associated community. Given these assumptions, efforts to infer the relative hierarchy or heterarchy of past political landscapes must consider the material dimensions of the archaeological record within and between sites.

The intensity and diversity of activities performed at any given place emerge from its position(s) within the landscape, as discussed for relative mobility. For permanent settlements, qualitative and quantitative aspects of architectural and artifactual assemblages provide a rough index of rank and/or relative autonomy within regional networks. This is because independent or high ranking communities tend towards selfsufficiency within, or control over, many economic, political, and cultural institutions.

Scalar relations among sites further inform the interpretation of their relative positions within the political economy. In theory, larger settlements shape interactions among more people on a regular basis than smaller ones; this translates into greater 
"wealth-in-people" and "wealth-in-knowledge" available to the local community and its political leaders. Where settlements could not have been supported by staple production within their catchments, one can make inferences about the mobilization of staples or labor from neighboring communities (Steponaitis 1981; H. T. Wright 2000). While many studies have assumed a relation between site size and sociopolitical hierarchy (G. A. Johnson 1977), it has become increasingly clear, particularly in Africa, that settlement aggregation can emerge via symmetrical integration and, conversely, political ranking does not always generate settlement hierarchies (Duffy 2010; R. J. McIntosh 2005; Richard 2007; Taylor 1975). This is not to discount the use of settlement size as a proxy for the scale of face-to-face interactions within a community, but only to suggest that one must consider complementary lines of material and spatial evidence for appropriational, as opposed to locational, movements within past economies.

\section{Ideology and Representation}

Despite the relative novelty of landscape approaches in West Africa, scholars have long recognized the importance of ideology or cultural schema in structuring the use of space and legitimizing the ritual and economic production of political authority within it. The megalithic cemeteries of Senegambia, for example, physically anchored social identity and politico-ritual authority to the landscape through the committal of the dead and the construction of ritual spaces (Holl et al. 2007; Jouenne 1930). Monumental earthworks produced by the state-level societies of Benin and Hueda provide further statements of political and ritual forms of power (Darling 1984; Norman and Kelly 2004). From the perspective of village-level societies, archaeologist Scott MacEachern (2002) has examined how cosmology and the material landscape articulated in the production of social authority at the scale of dispersed households (rather than nucleated villages) in the Mandara highlands of Cameroon. For better or worse, Madina-Sadatou has neither the ethnographic nor historic evidence that informs most of these studies, nor undisputable archaeological manifestations of ritual landscapes, such as the shrines found elsewhere in West Africa (Insoll et al. 2009). As a result, I generally refrain from interpreting the representational aspect of past landscapes in this study. It is nonetheless important to bear in mind that shifting material and spatial dimensions of regional political economies 
shaped, and were shaped by, cultural schema referencing the physical (and social) landscape (Conrad 2008).

\section{MADINA-SADATOU: A LANDSCAPE IN THE LONGUE-DURÉE}

In this section I position Diouboye within the historical landscape of MadinaSadatou by considering archaeological and historical evidence from this study area within the even broader contexts of the Upper Senegal region and the West African savanna. I already made mention of historical sources in Chapter 1, so I will simply reiterate that oral histories and traditions provide rich narratives, but their potential to shift across space and time according to the orator, audience, and social context often requires that one triangulate among several accounts to approximate some nominally “objective” past (Vansina 1985). As discussed below, historians concerned foremost with political economy in the Upper Senegal have applied this methodology to integrate oral histories collected during fieldwork over the past several decades with earlier renditions collected by European travelers and administrators beginning in the $16^{\text {th }}$ century $\mathrm{AD}$ (e.g., A. Bathily 1989; Curtin 1975a; Gomez 1992). Alongside local accounts and outsider observations, geographies written by Arab scholars after the ninth century AD provide a schematic understanding of politics and trade in sub-Saharan Africa in which goldproducing lands such as Bambuk figure prominently (Levtzion and Hopkins 2000). Meanwhile, West African scholars began to inscribe their own histories using the Arabic orthography that had accompanied the spread of Islam into the region (de Moraes-Farias 2003). Since I rely heavily upon secondary syntheses of the historical processes that have played out in the Upper Senegal region, I acknowledge that further attention to the biases and tensions among oral and textual sources and archaeological evidence could produce a richer understanding of the past landscape (Richard 2007, 2009; Stahl 2001).

As I mentioned in Chapter 1, archaeology in the Upper Senegal region has only just begun to contribute substantively to understandings of the historical landscape. Despite several important reconnaissance missions, areas with systematic survey and excavation remain few and far between, requiring the inference of broader trends through integration with supplementary historical evidence. Fortunately, a systematic investigation along the lowermost stretch of the Falémé River by Ibrahima Thiaw (1999) 
Figure 4.1: Map of the Madina-Sadatou study area showing sites identified through guided survey and the 2008 pedestrian survey zone
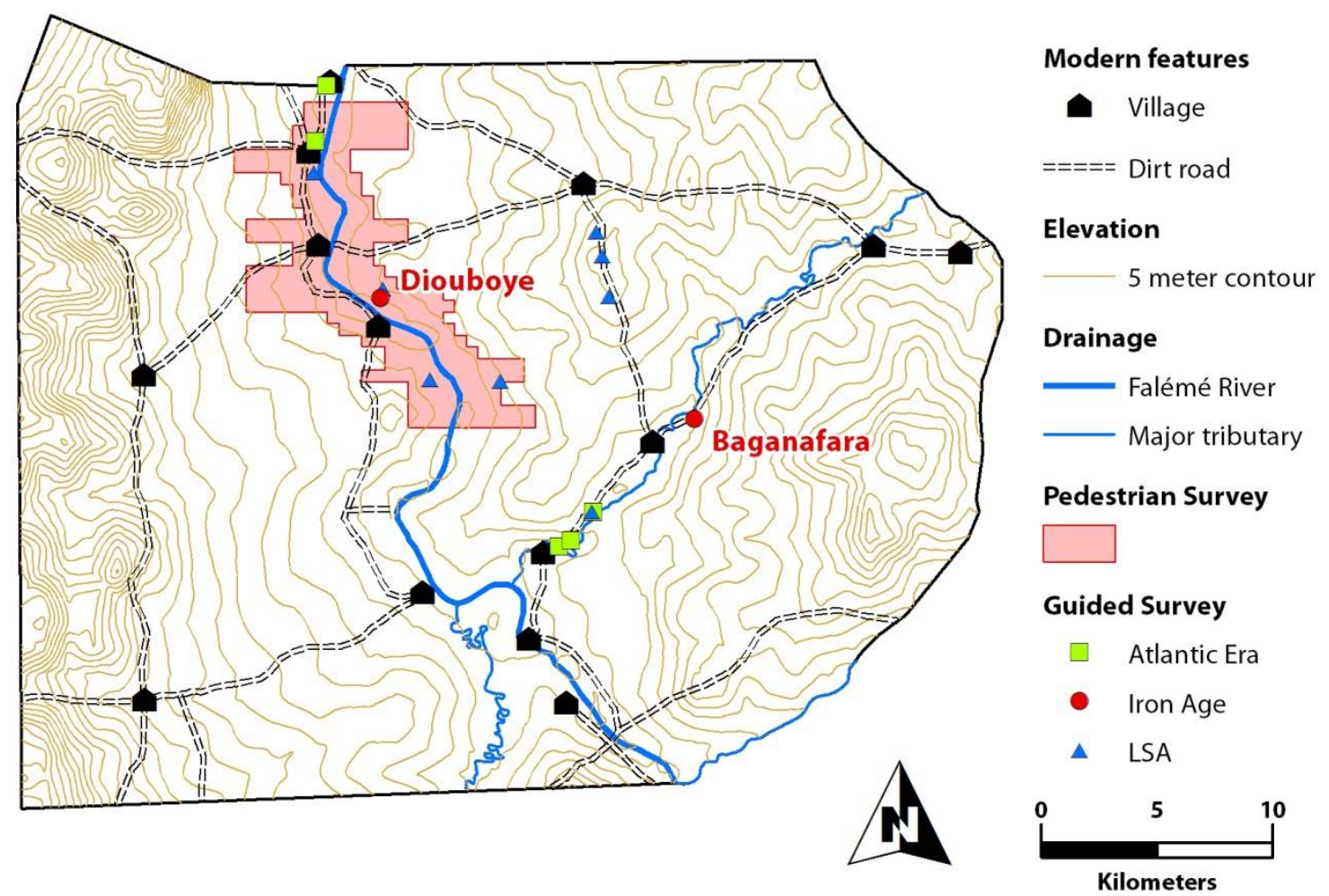

provides an invaluable chronological and material baseline for the present research in and around Diouboye. Ongoing fieldwork near Sabodala in southeastern Senegal (Altschul et al. 2010) will undoubtedly help to interpret and refine the archaeological sequence that I have documented in the Madina-Sadatou study area.

In order to situate Diouboye within its historical landscape, I conducted a fullcoverage survey of 71.75 square kilometers centered on this site and oriented north-south along a 14-kilometer stretch of the Falémé River (Appendix A). This survey documented 146 sites of longer-term occupation or repetitive activity alongside 65 isolated occurrences resulting from singular events. Guided, vehicular reconnaissance identified nine other sites that do not figure prominently in the following analyses and discussion (Figure 4.1). Systematic and/or diagnostic surface collections at most of the sites encountered on survey yielded artifacts sensitive to chronological and functional interpretations. These include earthenware pottery (Appendix D), chipped stone and 
groundstone artifacts (Appendix E), metallurgical artifacts (Appendix F), and diverse terracotta and glass objects (Appendix G). Despite the palimpsest of surface materials on many sites, it was possible to define three broad archaeological horizons similar to those employed elsewhere in West Africa-(Middle-Late) Stone Age, Iron Age, and Atlantic Era - each of which has been subdivided into local phases on the basis of differences in material culture. This archaeological sequence provides an analytical point of entry into the landscape of Madina-Sadatou over the longue durée and facilitates engagement with historical and archaeological evidence for the broader social trajectories and institutions in which Diouboye was enmeshed.

\section{Late Acheulean and Middle Stone Age (MSA)}

During the early- $20^{\text {th }}$ century AD, a series of archaeological missions identified Mode 1 and Mode 2 chipped stone tool industries ${ }^{1}$ at localities across the Upper Senegal region, including a number of sites along the lower Falémé River (see Ravisé 1975). It was not until the 1980s, however, that Abdoulaye Camara and Bertrand Duboscq began more systematic research to enhance understanding of early human occupation and technological development in the region. By correlating datable alluvial episodes with surface and subsurface observations from sites along the Falémé River, including Djita and Sansanding, these scholars proposed a techno-chronological sequence spanning much of the late Pleistocene (Camara and Duboscq 1984, 1987, 1990). In sum, the uppermost alluvial terraces that formed during OIS 9 (334,000-301,000 BP) generally yield rare Mode 1 tools and debitage made from local quartzite and quartz; these are often heavily weathered and sometimes encrusted with laterite. Alluvial deposits from OIS 7 (242,000186,000 BP) and OIS 5 (127,000-71,000 BP) appear to envelop a Late Acheulean (and perhaps Sangoan) techno-complex with Mode 1 chopping tools, Mode 2 bifaces, and infrequent Mode 3 scrapers with light retouch — these all reduced from local chert, finegrained sandstone or mudstone, and quartzite. Eroded terraces presumably dating to the terminal Pleistocene OIS 2 (27-10,000 BP) are associated with a Middle Stone Age (MSA) techno-complex defined by relatively smaller Mode 1 and Mode 2 tools and elevated frequencies of Mode 3 and Mode 4 tools made from chert/flint, quartzite, and

\footnotetext{
${ }^{1}$ Refer to Appendix D for a summary of the terminology used to describe chipped stone tool assemblages.
} 
quartz. Notably, the latter tool categories include leaf-shaped bifacial points, lanceolates, and retouched blades similar to those from Mousterian/Aterian assemblages in the Sahara (Hugot 1967). Despite the uncertainty of a chronology based on alluvial deposits, this framework mirrors better known sequences elsewhere across Africa (J. D. Clark 1982). Accordingly, the Upper Senegal region may have been largely abandoned during the extremely arid conditions of the terminal Pleistocene described briefly in Chapter 3.

On pace with the evolutionary transition from archaic to anatomically modern Homo sapiens documented by fossils from East Africa, the growing diversity of Late Acheulean and MSA lithic assemblages in West Africa provides some evidence for the gradual emergence of "modern” capacities for abstract thinking and planning depth, economic adaptability, technological innovation, and symbolic behavior (Barham and Mitchell 2008; McBrearty and Brooks 2000). Although the Senegambian data are currently too sparse to weigh in on these issues (A. Diop 2000), they do fit with a model of mobile foragers whose reliance on resources near permanent water allowed global climatic cycles to drive regional abandonment, technological innovation, and reoccupation with increasingly specialized resource procurement strategies (Holl 1989). According to this model, the subsequent reoccupation of the Upper Senegal region during the climatic amelioration of the early- to mid-Holocene (see below) simply represents the latest episode in a much longer demographic cycle.

\section{Late Acheulean and MSA Components in the Madina-Sadatou Study Area}

With their excavations at Sansandé, Camara and Duboscq (1984) demonstrated the potential contributions of the Madina-Sadatou study area to Late Acheulean and MSA archaeology in West Africa. Since these components were not a focus of my research, the Late Acheulean assemblages encountered on six sites (Figure 4.6), were merely subject to purposive sampling of obvious Mode 1-2 tools. These tools, along with their associated debitage, were generally made from sandstone, quartz, and chert, the latter with heavy patination (Figure 4.2). The distribution of sites with Late Acheulean artifacts near the Falémé River channel may be more a reflection of site preservation and exposure, than any preferential use of the landscape. 
Figure 4.2: $\quad$ Late Acheulean (a-c), MSA (c-d), and/or aceramic LSA (e-g) chipped stone artifacts from Madina-Sadatou: (a) Mode 2 hand axe; (b-c) Mode 3 flake tools; (d) Mode 3 sidescraper; (e-f) Projectile points; and (g) Quartz sidescraper

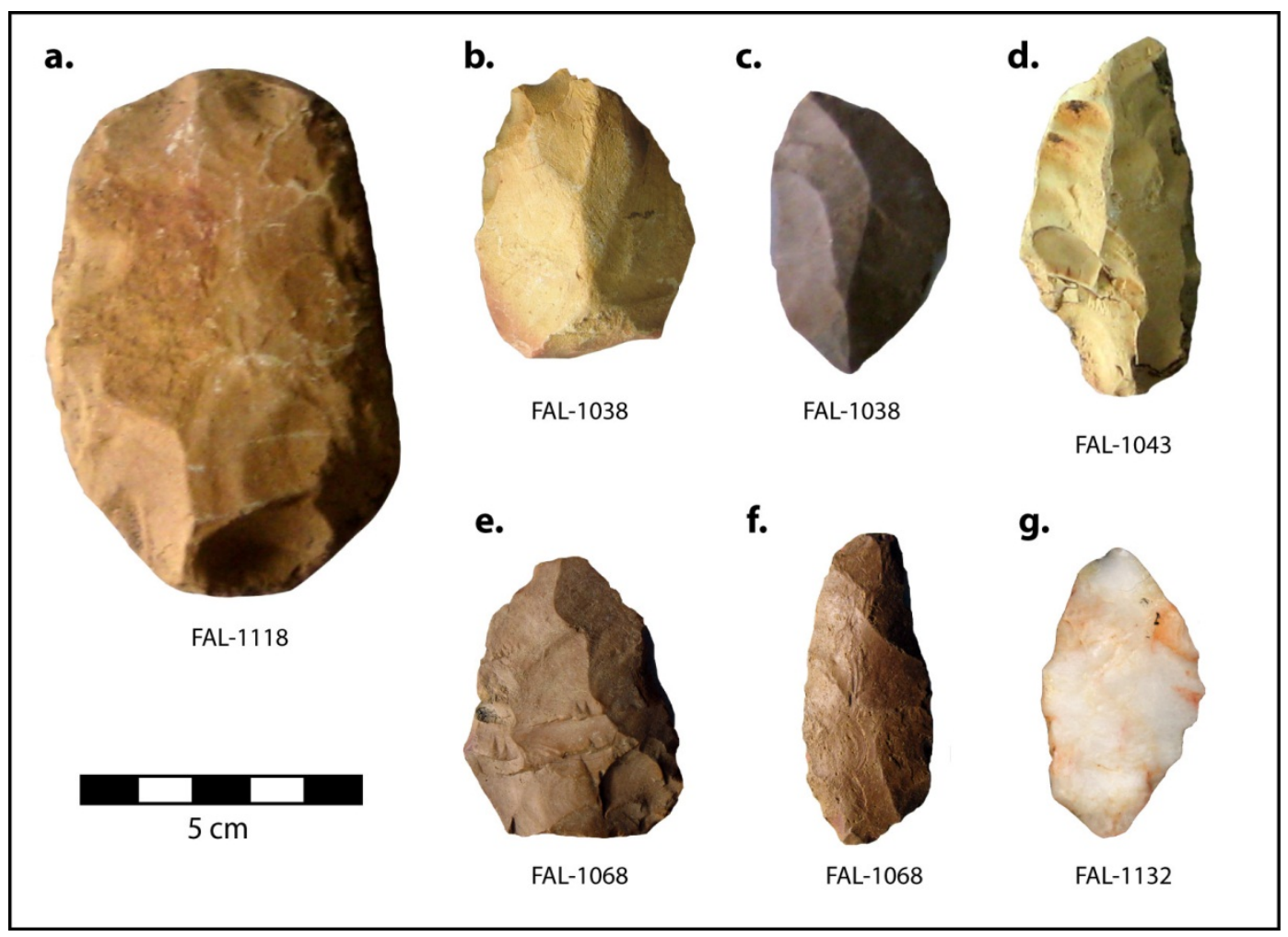

Mode 3 tools, including side scrapers and leaf-shaped projectile points made from mudstone, quartz, and chert/flint (Figure 4.2), define an MSA component in the study area. Pending a detailed chipped stone analysis, I could not, however confidently distinguish these from the aceramic LSA materials present at many surveyed sites (see below). Altogether, survey documented 48 sites and 27 isolated occurrences with an MSA and/or aceramic LSA component classified by the presence of Mode 3 and Mode 5 chipped stone artifacts and the absence of pottery.

\section{Late Stone Age}

Across the Upper Senegal region, oral traditions of later Soninke immigrants recount an initial occupation by people occupying caves and riverside encampments where they subsisted on wild plants, game, and fish-their "primitive" culture now lost in 
an era of relative "obscurity” (A. Bathily 1989:64). This trope, common to many local histories, may attempt to subvert the territorial rights of autochthonous populations by implying a sort of predestination for the late-comers who would ultimately control historical knowledge (Kopytoff 1987b; D. R. Wright 1985). For this reason, oral traditions should not be read as empirical signposts for the transition from mobile/foraging to sedentary/food-producing societies. They do have some veracity in that, at some point in the past, people did rely upon hunting, fishing, and gathering- $\mathrm{a}$ lifeway attested along the lower Falémé River by ephemeral sites littered with pottery and chipped and groundstone tools.

Elsewhere across West Africa and the Sahara Desert, archaeological research has done much to reveal the complex evolutionary pathways of subsistence economies, and their integrated settlement strategies, that have arisen since the dawn of the Holocene circa $8000 \mathrm{BC}(10,000 \mathrm{BP})$. Here I follow the convention of subsuming this period within the Late Stone Age (LSA) ${ }^{2}$ —an archaeological horizon defined by the sustained use of Mode 5 "microlithic" stone tool industries that began sporadically across much of Africa between 50,000 and 20,000 BP (Ambrose 2002; Cornelissen 2002; Deacon 1984; Holl 1989; Kusimba 2003; McBrearty and Brooks 2000). The LSA forms an especially uneasy rubric in West Africa where people adopted Mode 5 industries unevenly across space and time, and where Mode 3 “macrolithic” industries remained in use through the first millennium AD (MacDonald and Allsworth-Jones 1994). While some scholars have abandoned this terminology in favor of a purely calendric chronology (Barham and Mitchell 2008), I find in this study that the LSA provides a useful frame for relating data from the Madina-Sadatou study area to regional (and admittedly quite diverse) sequences of technological and socio-economic change.

\section{Aceramic LSA}

The innovation or adoption of microlithic technology in West Africa during the terminal Pleistocene remains poorly understood given a current paucity of welldocumented and datable sites (Casey 2003; Hugot 1967). Because the extreme aridity of

\footnotetext{
${ }^{2}$ See S. K. McIntosh and R. J. McIntosh (1983) and Stahl (1999) for critical discussions of space-time nomenclature in West Africa
} 
this period caused the Sahara Desert to expand dramatically (Kuper and Kröpelin 2006), it is no surprise that the earliest aceramic LSA ${ }^{3}$ assemblages in West Africa occur at sites such as Shum Laka (Cornelissen 2003; Lavachery 2001), Abidjan-Bingerville (Chenorkian 1983), and Iwo Eleru (Shaw and Daniels 1984), all situated in southerly and upland regions where savanna and forest mosaics harbored more predictable resources for mobile foragers (Cornelissen 2002). Microlithic segments in these assemblages, made predominantly from local quartz, may have been mounted in compound tools, presumably for exploiting a broad spectrum of plant and animal resources from diverse and/or seasonal habitats (Casey 2000), although microwear analyses show that not all microliths were hafted (see Barham and Mitchell 2008:288; Kusimba 2003:180-183). Rather than a unidirectional progression towards complex hunting equipment, Barham and Mitchell (2008) argue that Mode 5 tools formed part of strategies for reducing subsistence risk based on greater residential mobility (Binford 1980), conservation of lithic raw material (Bousman 2005), and regional exchange networks (Ambrose 2002; Wiessner 1982b). Until further data bring this picture of aceramic LSA lifeways into focus, it should suffice to say that microlithic technology, and associated forms of socioeconomic organization, must have set the stage for the changes that would unfold across West Africa during the early- to mid-Holocene.

\section{Ceramic LSA (cLSA) in the Sahara and Sahel}

During the early-Holocene epoch (circa 8000-6000 BC), the West African climate became increasingly humid, transforming the Sahara into an opulent savanna interspersed with lakes and seasonal wetlands (Kuper and Kröpelin 2006; Roset 1987). As people from ecological refuges in Central and North Africa gradually dispersed across the region, they developed a more diverse toolkit with Mode 5 microlithic technology, groundstone implements, carved bone harpoons, and a distinctive dotted and wavy-line decorated pottery (Haaland 1992; Sutton 1974)—-the hallmarks of the ceramic LSA ${ }^{4}$ (cLSA) in the Sahara (J. D. Clark 1967). This material assemblage has been found at well-preserved and datable sites along fossil lake and river margins and along seasonal

\footnotetext{
${ }^{3}$ Paléolithique terminal and épipaléolithique in the French nomenclature

${ }^{4}$ Néolithique in the French nomenclature; also “Aqualithic civilization” (Sutton 1974)
} 
watercourses in upland massifs (Holl 1989, 2004; Roset 1987). In both cases, it seems that semi-permanent occupation near reliable water sources led to intensive exploitation, if not low-level management, of wild game, fish, and dense stands of edible grasses (Garcea 2006), the latter prepared with the aid of pottery and grinding stones. Isotopic and craniometric analyses of 17 early-Holocene burials from the lakeside cemetery of Gobero in Niger point to limited mobility, and, interestingly enough, demographic continuity with late Pleistocene populations from North Africa (Sereno et al. 2008). In the eastern Sahara, sites from Nabta Playa with permanent huts, bell-shaped storage pits, and deep wells suggest that a reduction in mobility corresponded with delayed-return reciprocity and social concepts of ownership (Wendorf and Schild 1998).

A growing amount of evidence suggests that people in the eastern Sahara also domesticated cattle around this time. For example, the recovery of cattle bones from Nabta Playa and Bir Kiseiba in western Egypt, well outside the ecological range of wild cattle, may derive from incipient herd management (Wendorf and Schild 1998:100-101). This interpretation finds support from linguistic, morphological, and genetic evidence for an African, as opposed to Asian, origin of some present-day cattle breeds (Blench and MacDonald 2000). Overall, these data are consistent with models for the intensification of herd management in response to stochastic rainfall patterns in the Sahara, particularly with a relapse to modest aridity circa 6000-5000 BC (Garcea 2006; Marshall and Hildebrand 2002). Under these conditions, herd animals such as wild cattle and Barbary sheep would have provided a fairly predictable food supply (storage-on-the-hoof) that moved between patches of adequate rainfall and consumed a broader spectrum of plants than human foragers (generalization-by-proxy).

Whatever the origin of domesticated cattle, pastoral subsistence regimes steadily spread with the mid-Holocene return of more humid conditions from 5000 to 2500 BC. Unambiguous domestic cattle remains appear at sites spanning the western Sahara and Sahel from the Lake Chad basin and central Saharan massifs to the Atlantic littoral, and as far south as the Niger River and the presumed barrier of the tsetse zone (Gautier 1987; Holl 1998; A. B. Smith 1992). Faunal remains and other artifacts from many of these sites, along with isotopic analyses of human remains from Karkarichinkat and Gobero (Finucane et al. 2008; Sereno et al. 2008), indicate that pastoralism was only one part of 
complex herder-fisher-forager subsistence strategies that ranged from semi-sedentary to highly mobile depending on seasonality and scheduling constraints.

A subsistence economy incorporating cattle coincided with transformations in social and cultural institutions across the Sahara and Sahel. The emergence of ritual cattle slaughter and monumental burial, perhaps in response to stochastic climate change (Di Lernia 2006), shows that livestock were more than sources of primary and secondary products - they had cultural value, and may have begun to function as a medium of giftgiving, bride wealth, and other forms of exchange across social boundaries. Meanwhile, the widespread distribution of Saharan pottery styles—including simple vessels decorated with incisions, comb, and cord-wrapped tool impressions (Close 1995)—may attest to social networks capable of disseminating information about ecological and disease conditions over large territories, and mediating access to resources in times of subsistence crisis (A. B. Smith 1992:26-30). Coinciding with pastoral principles of ownership, cemeteries and rock art paintings found across the Sahara during the mid-Holocene symbolize a greater attachment to place, and perhaps the maintenance of territorial boundaries (Holl 1998). This certainly seems to be the case at the Gobero cemetery where craniometric data show that people were physiologically distinct from their predecessors in the region (Sereno et al. 2008), further pointing to large-scale demographic movement across the Sahara throughout the cLSA.

Beginning around $3000 \mathrm{BC}$, cattle may have also begun to underwrite political asymmetries across these social networks. The stone tumuli previously associated with cattle burials became increasingly used as human tombs, perhaps representing a shift from collective ritual and memory to an emphasis on individual status (Di Lernia 2006:60-61). More provocatively, Kevin MacDonald (1998) has argued that these tumuli, along with trade in prestige objects such as polished stone hachettes and arm rings, correspond to the emergence of rank from institutions for corporate ownership and the accumulation of cattle wealth by nascent elites. Meanwhile, nucleated households and settlement hierarchies hint at increasingly political differentiation in the Dhar Tichitt region of Mauritania from 1800 to 400 BC (Holl 1985a, 1993; Munson 1980).

Excavations in the Dhar Tichitt region have yielded pottery sherds with pearl millet impressions documenting the cultivation of this crop after 1800 BC (Amblard and 
Pernès 1989), presumably around the scheduling constraints of pastoralism (Holl 1985b). This pattern fits with the earliest archaeobotanical evidence for domesticated millet at several LSA sites in the Tilemsi Valley of northern Mali dating to the third millennium BC (Manning et al. 2011). Since the earliest evidence for domestic plants actually corresponds to a period of climatic amelioration, environmental pressures do not seem to provide a sufficient explanation for domestication in West Africa (Vernet 2002). The above data, along with excavated sequences in northern Burkina Faso and the Lake Chad basin, suggest that a gradual reduction in herder-forager mobility simply intensified exploitation of wild grains (Breunig and Neumann 2002; MacDonald 1996; MacDonald et al. 2009; Marshall and Hildebrand 2002). Whatever the case, the domestication of pearl millet during the late cLSA did not mark a "Neolithic revolution" in the southern Sahara and Sahel. Rather it formed one part of a diverse subsistence economy that also included pastoralism, hunting, fishing, and harvesting wild plants. This subsistence regime did, however, set the stage for the development of more specialized agro-pastoral economies in West Africa alongside the widespread shift to sedentism and adoption of iron technology after 500 BC.

\section{Ceramic LSA (cLSA) in the Savanna and Equatorial Forest}

The LSA occupation of the West African savanna and forest zones, generally termed the Guinean cLSA, differs from Saharan sequence discussed so far (J. D. Clark 1967). With climatic amelioration during the early- to mid-Holocene, populations employing an aceramic LSA chipped stone tool industry, often with quartz microliths, continued to occupy southern sites such as Rim (Andah 1979) and Iwo Eleru (Shaw and Daniels 1984); they also expanded northward into the upper Volta and upper Niger River basins (Eyo 1972; Frank et al. 2001; MacDonald 1997; York 1978). Groundstone tools and infrequent pottery sherds decorated with mat, comb, punctate, and cord-wrapped stick impressions only showed up after 6000 BC at sites such as Shum Laka (Lavachery 2001) and the K6 rockshelter in Ghana (Stahl 1985), suggesting the southward diffusion of these technologies from the Sahara. After 3500 BC, cLSA occupations became more widespread from Senegal to Cameroon (Atherton 1972; Chenorkian 1983; Gabel 1976; Ravisé 1970; Shaw 1944), though this may reflect uneven fieldwork and/or post- 
depositional disturbances, rather than demographic expansion. Beyond the prevalence of quartz microliths, all of these LSA assemblages demonstrate modest diversity across space and time, with periodic shifts toward macrolithic Mode 3 tools possibly related to the exploitation of resources requiring more intensive processing (Lavachery 2001; MacDonald and Allsworth-Jones 1994). Altogether, the sparse data suggest that both ecological and socio-demographic factors contributed to broad regional differences across the cLSA landscape of West Africa.

Since tsetse flies stalled the mid-Holocene penetration of domestic animals into the southern savanna and equatorial forest, local subsistence regimes differed from those to the north. For example, the remains of oil-producing plants (i.e., oil palm, canarium) at Shum Laka (Lavachery 2001), Bosumpra Cave (A. B. Smith 1975), and Kintampo tradition sites in present-day Ghana (D'Andrea et al. 2006) may point to the intensification of foraging practices, if not low-level food production (Stahl 1993b). Domesticates from the Sahara and Sahel made their way into the savanna forest after 2000 BC when Kintampo tradition sites provide unambiguous evidence for the cultivation of domestic pearl millet and cow pea (D'Andrea and Casey 2002; D'Andrea et al. 2007) and possible access to domestic livestock (Stahl 1985; Watson 2005). The timing and mechanisms for the domestication of yams-now integral to the savannaforest subsistence economy — are currently unknown, though phytolith analyses now place banana arboriculture in Central Africa as early as the first millennium BC (Lejju et al. 2006). The appearance of domesticated plants and animals did not, however, stimulate an economic revolution during the cLSA; most assemblages show a continuing reliance on wild species.

This subtle economic shift entailed, at least in the Kintampo tradition, a reduction in residential mobility with emphasis on longer-term occupation of rock shelters and the construction of wattle-and-daub architecture (Casey 2000; Stahl 1985, 1986; Watson 2005). Although the transition to sedentary villages with agro-pastoral economies in this region remains poorly understood (Stahl 1994:79-81), it appears to have occurred throughout the first millennium $\mathrm{BC}$ alongside the adoption of iron technology. 


\section{LSA Occupations in (and around) the Upper Senegal Region}

Although they have not figured prominently in discussions of Holocene socioeconomic evolution, a number Senegambian LSA sites have been identified by archaeologists (Corbeil et al. 1948; Guitat 1970, 1972; Laforgue 1925; Lame 2000; Ravisé 1975). Dating to the period after 3500 BC, sites in the vicinity of Cap-Manuel and Khant on the Atlantic littoral have yielded pottery, groundstone axes, chipped stone tools (including microliths) and carved bone and wood harpoons that document the exploitation of coastal resources (Ravisé 1970). In the “dunal” zone east of the Cap-Vert peninsula, a number of sites have yielded geometric microliths and ovoid or hemispherical pottery vessels (sometimes with everted rims) tempered with quartz and decorated over the entire surface with wavy-line comb and impressions from cordwrapped elements; these sites suggest that elevated sea levels pushed coastal foragers inland around 2500-2000 BC (Lame 2000). The paucity of LSA sites elsewhere in western Senegambia and along the Senegal River almost certainly results from limited fieldwork and/or the eradication of LSA assemblages by geological processes.

Early scholarship on cLSA occupations in Senegambia viewed pottery and microlithic tools from the Saharan cLSA and polished stone tools and macrolithic tools from the Guinean cLSA as evidence for participation in both of these two techno-cultural spheres (Corbeil et al. 1948; Laforgue 1923). While this observation may hold true at a broad scale, future research will need to disentangle the demographic histories and social networks of cLSA populations from their shared technological adaptations to dynamic ecological conditions.

In this regard, the Falémé and upper Senegal River basins remain a relatively untapped, yet potentially quite rich, source of data on LSA occupations in West Africa. Surface collections from early vehicular surveys identified a rather distinctive LSA tradition with groundstone and polished hematite axes, quartz- or grit-tempered pottery, and chipped stone tool industries of quartz and chert, supposedly with low frequencies of microliths (Laforgue 1924; Ravisé 1975; Zeltner 1916). In his much more systematic survey, Ibrahima Thiaw (1999:247-248) observed an LSA component particularly rich in netweights at numerous shallow sites $(n=86)$ on the west bank of the lower Falémé River. These sites were generally long and narrow, oriented parallel to the river bed on levees 
around one hundred meters to the west, suggesting repeated occupation by residentially mobile fisher-foragers. Three thermoluminescence dates obtained by Anne Ravisé (cited in Camara and Duboscq 1987:514) from a site near Senoudebou confirm that LSA populations occupied the Falémé River valley to at least the early-first millennium BC. Although they may have subsequently abandoned the region during an arid transgression from $300 \mathrm{BC}$ to $\mathrm{AD} 300$, it remains possible that such populations persisted alongside the arrival of settled villagers during the Late Iron Age.

Hundreds of kilometers to the east, excavated sequences at the rock shelters of Korounkorokalé in the Upper Niger region (MacDonald 1997; Szumowski 1956) and Fanfannyégèné I in the Upper Senegal region (Huysecom 1990) provide a very rough point of comparison for LSA materials found on the lower Falémé. Stratified deposits at Korounkorokalé reveal a transition from aceramic to ceramic LSA occupations around 4000 BC. At the same time, continuity in the microlithic chipped stone industry, made predominantly from quartz, and limited faunal data highlight a long-term emphasis on riparian resources with gradual intensification in plant exploitation attested by the introduction of groundstone technology. Although the stratified sequence from Fanfannyégèné I dates much later (1000-800 BC), it, too, documents a trend towards greater densities of pottery and groundstone tools over time. Neither site yielded evidence for domestic plants or animals. Pottery sherds from LSA occupations at both sites are mainly thin, friable, and decorated with cord-wrapped cord roulette and impression.

Continuity in microlithic chipped stone industries led Kevin MacDonald (1997) to argue for the adoption of ceramic technology by indigenous foraging populations who gradually intensified food production and shifted from a strategy of residential to logistical foraging throughout the mid- to late-Holocene. While I hesitate to directly extrapolate this model to the lower Falémé River basin, it does provide a hypothetical construct that fits present data for the comparatively late adoption of intensified foraging and/or food production and a gradual shift from residential to logistical mobility, with lithic production extending into the first millennium $\mathrm{AD}$ concomitant with the widespread adoption of iron metallurgy. 


\section{LSA Components in the Madina-Sadatou Study Area}

Late Stone Age artifacts are a nearly ubiquitous element on the Madina-Sadatou landscape. Along the eroded slopes leading back from the main Falémé River channel, it was difficult to walk thirty meters on survey without encountering at least one quartz flake. Discrete LSA sites with concentrated artifacts from repetitive or long-term use are also quite prevalent. Systematic survey documented 67 sites and nine isolated occurrences with a cLSA component including Mode 5 microlithic artifacts, groundstone tools, polished hematite hachettes, and distinct pottery with quartz/feldspar grit temper and decorative elements of shorn grass and/or composite cord-wrapped roulettes and impressions (Figures 4.3-4.6). Pending a detailed analysis of the chipped stone assemblage, it has not been possible to distinguish aceramic LSA assemblages potentially conflated with cLSA ones, or with Iron Age components, likewise featuring chipped stone artifacts. For this reason, aceramic LSA components are likely underrepresented in the surveyed sample. The common co-occurrence of LSA assemblages with later, more expansive Atlantic Era components at many sites also made it difficult to define the areal extent and depth of the former. 
Figure 4.3: LSA chipped stone artifacts: (a) bladelets; (b) microlith trapezoids; (c) microlith trapezoid/scraper; (d) microlith triangles; (e) microlith segments; (f) geometric point; (g) bifacial point; (h) discoid core

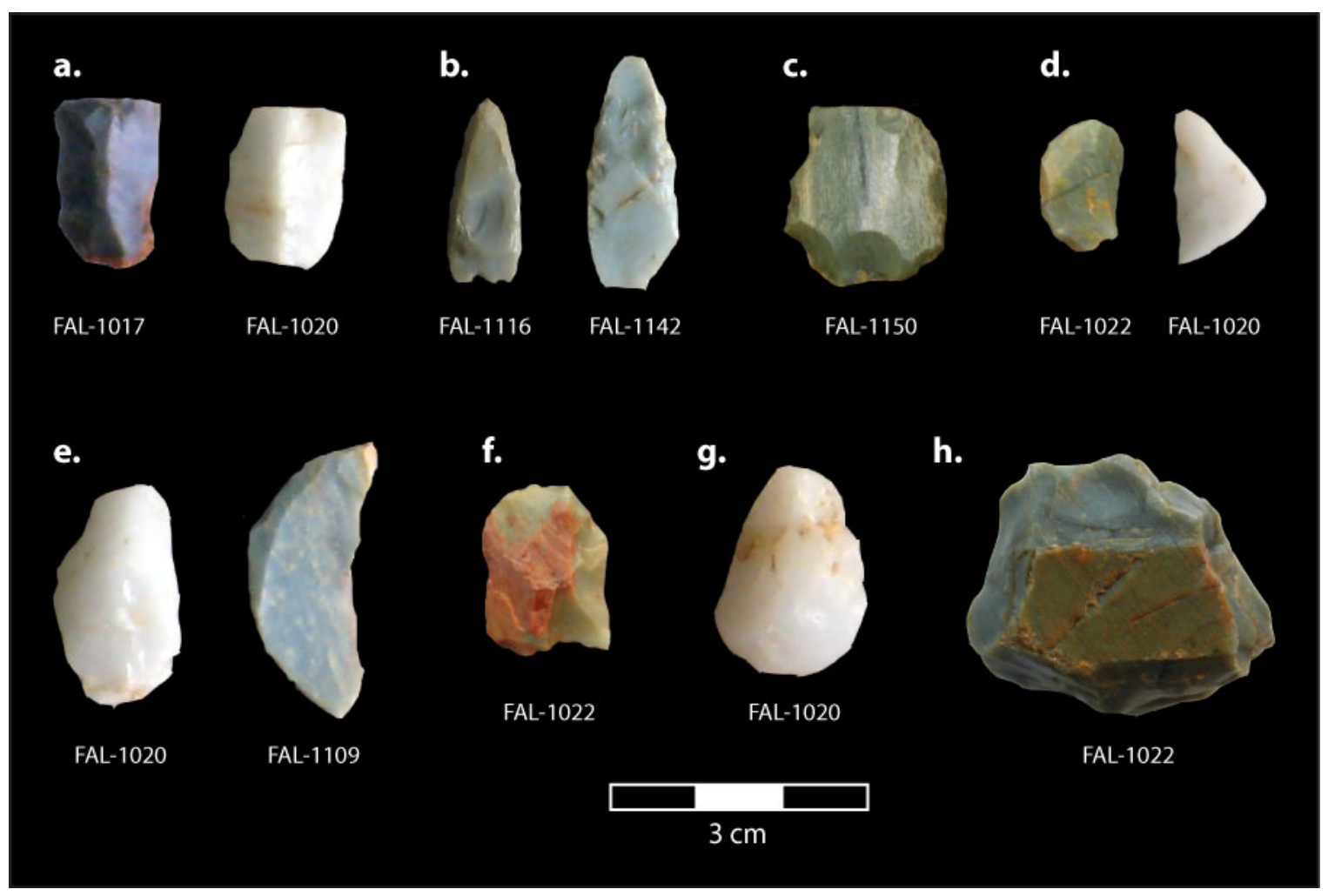

Figure 4.4: FAL-1042 (LSA encampment) looking east down to Falémé River

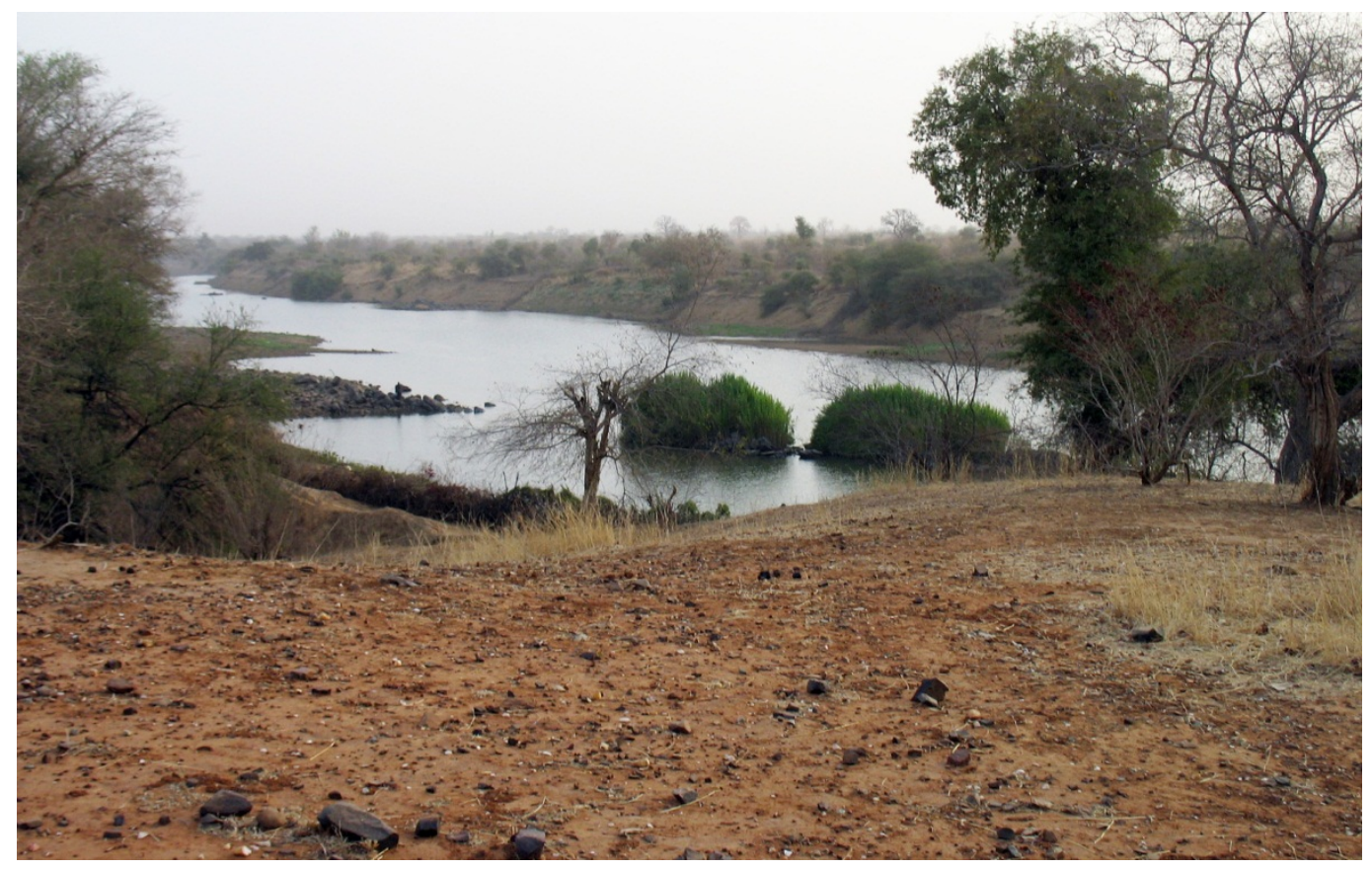


Figure 4.5: Ceramic Late Stone Age (cLSA) ceramic and groundstone artifacts: (a-b) cord-wrapped cord impressed sherds; (b-d) shorn grass roulette sherds; (e) handstone fragment; (f) groundstone axe; (g-h) elliptical, semi-polished hachettes; (i) large hematite large axe blade fragment

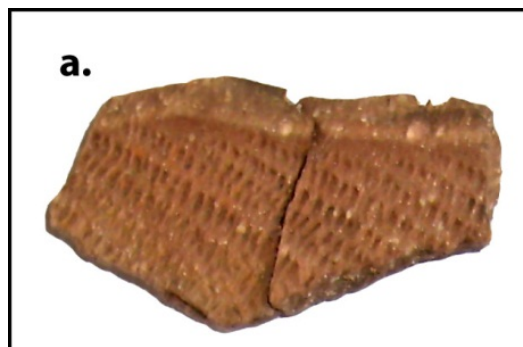

FAL-1036

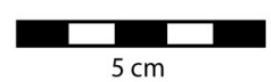

e.

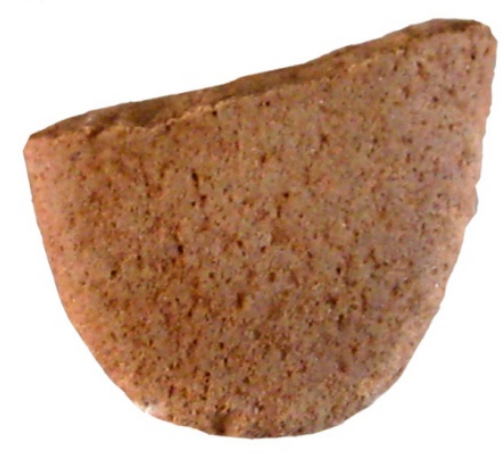

FAL-1041

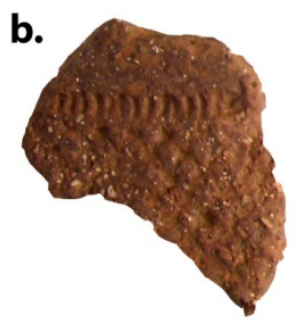

FAL-1084

f.

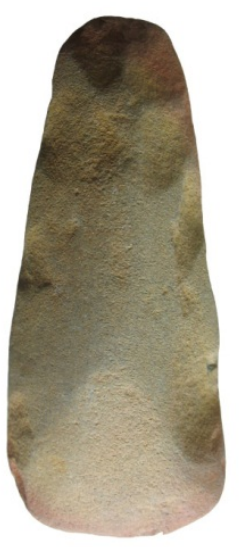

FAL-1038

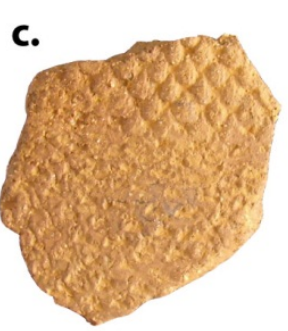

FAL-1062

g.

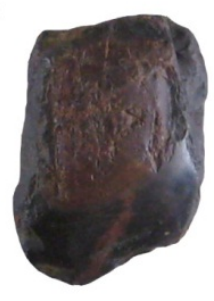

FAL-1020 d.

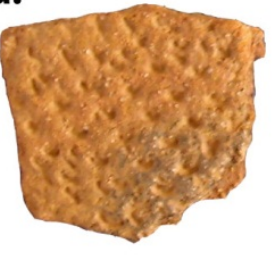

FAL-1121

h.

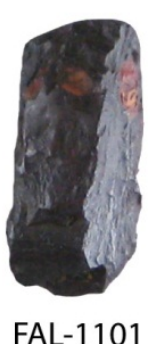

i.

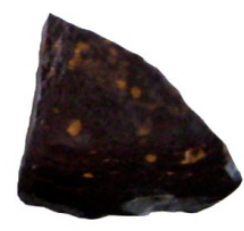

FAL-1131 
Figure 4.6: Ceramic LSA pottery sherd types and vessel forms

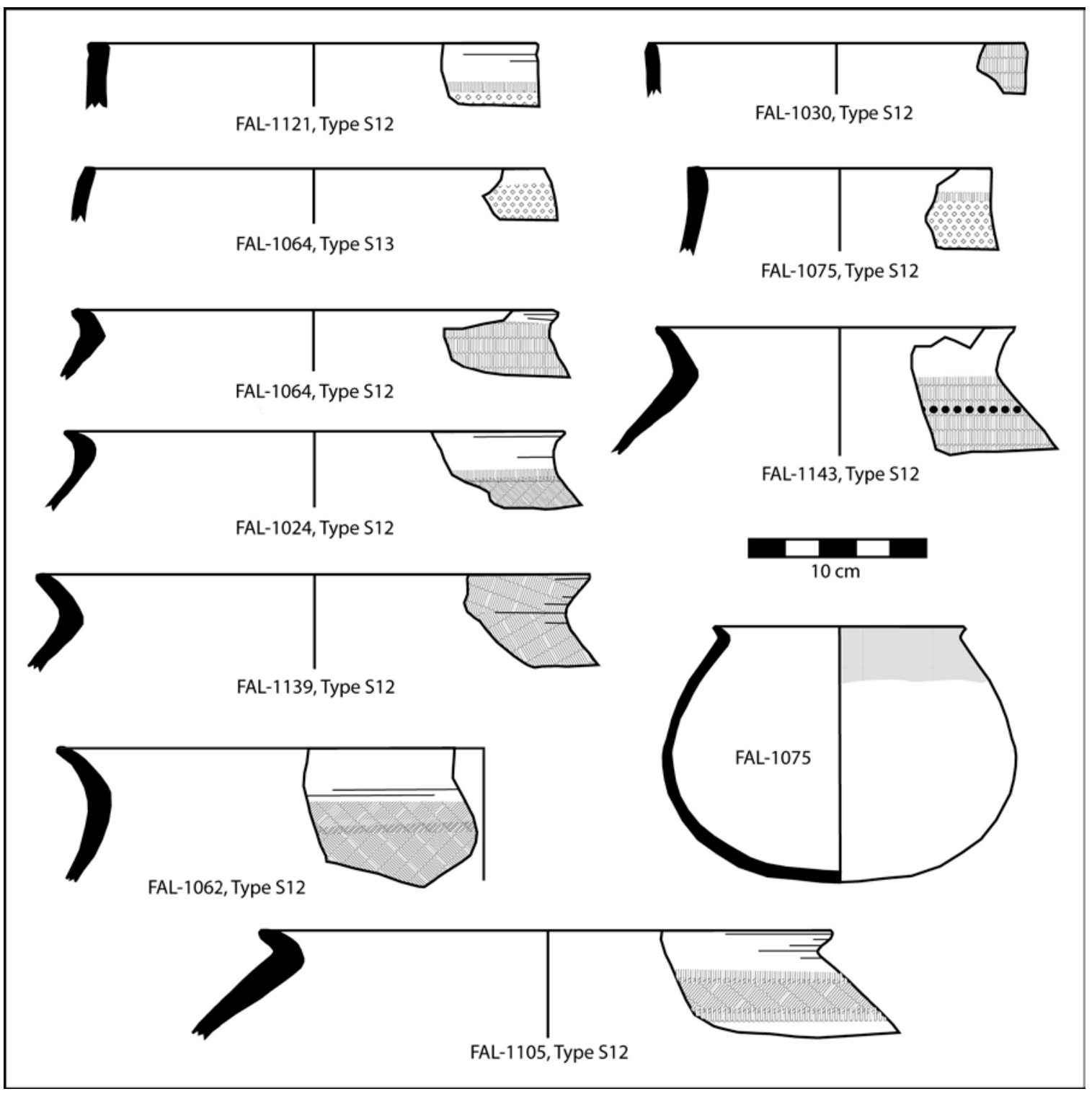


Figure 4.7: Late Acheulean, MSA/LSA, and cLSA sites in the pedestrian survey zone

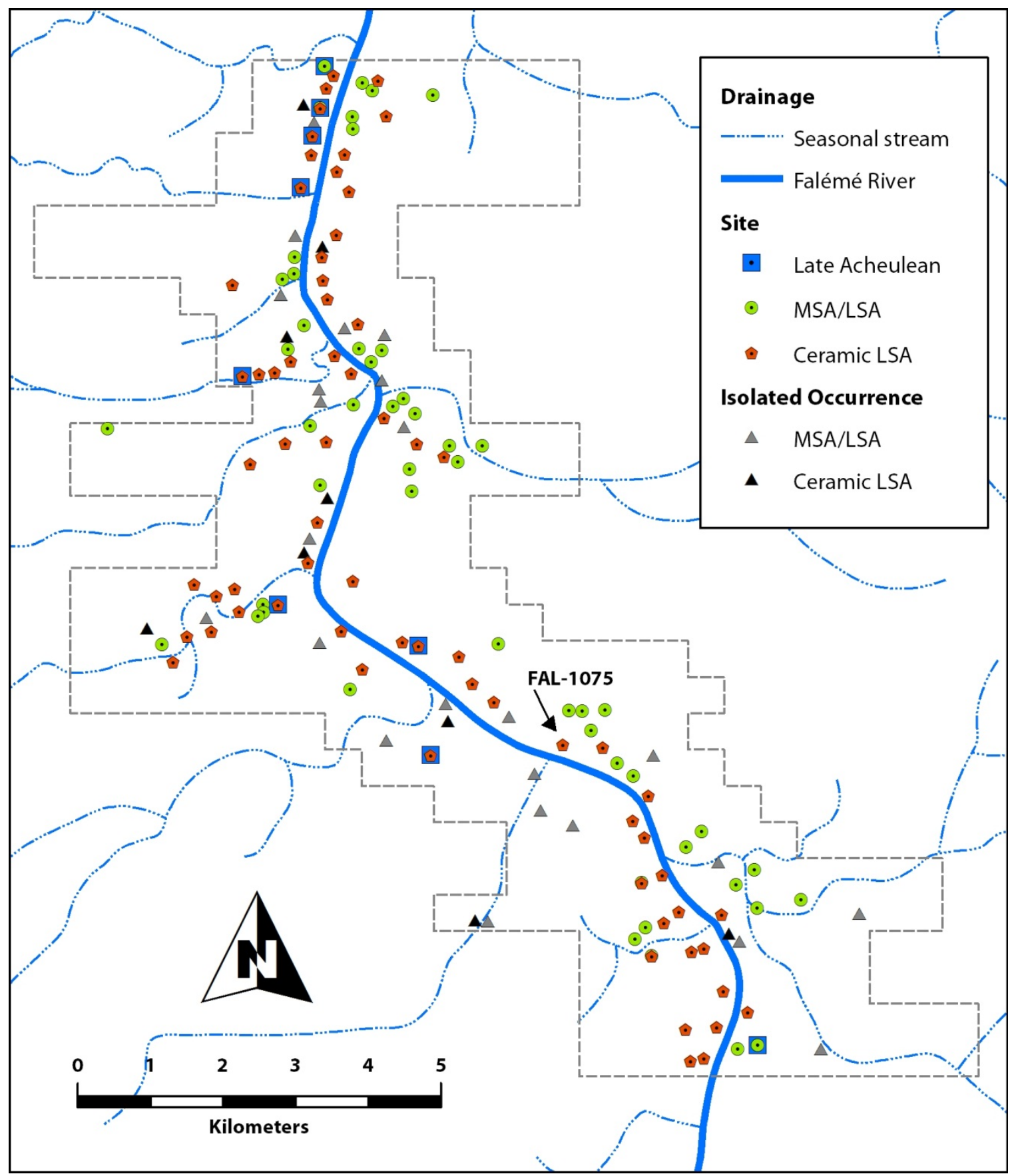


In order to augment this surface study of LSA occupations in Madina-Sadatou, one member of the 2009 CFAP team, Massal Diagne (2010), initiated excavations at FAL-1075. This multi-component site lies 650 meters to the southeast of Diouboye, directly above a steep embankment leading down to the Falémé River. Erosion has revealed materials associated with two somewhat discrete surface components-cLSA in the northern part of the site (FAL-1075-01) and Atlantic Era (see below) in the southern part where some cLSA materials are also present (FAL-1075-02). Concurring with surface observations made during pedestrian survey (see also Thiaw 1999:247), two test units (1x2 $\mathrm{m}$ and 1x6 $\mathrm{m}$ ) revealed an extremely deflated cLSA component confined to the 20 centimeters of weathered soil lying above a compact alluvium. In his study of the chipped stone artifacts from this site, Diagne (2010) identified a tool industry with microlithic elements produced from cobbles of local quartz and blue-green chert. The assemblage also included cLSA pottery (see above) and polished hematite tools, but no terracotta netweights; this was surprising given the proximity of the river and the ubiquity of these artifacts at LSA sites along the lowermost Falémé River. Overall, the stratigraphic superficiality of FAL-1075 gives the impression of a place occupied briefly as part of an economic strategy dependent upon residential mobility.

Aceramic and ceramic LSA components need not imply a strict chronological relation. Some of the former may simply represent logistical tasks that did not result in the deposition of pottery or groundstone artifacts. In this regard, a comparison of aggregate data from systematic samples of lithic artifacts (Table 4.1)—including the relative frequencies and mean sizes of cores, reduced shatter and flakes, and formal tools-reveals similar sequences of reduction across both components, suggesting that they are not functionally distinct. This pattern could support the contention that MSA/LSA component sites did not derive from logistical tasks involving specialized toolkits or specific reduction stages, but rather from an earlier regional occupation. A slight reduction in core and flake size likewise points to more conservative use of both quartz and chert at cLSA sites, perhaps as a reduction in mobility resulted in less frequent moves to ideal raw material sources. This could also be consistent with an increase in the relative abundance, and increasing size range, of formal tools for performing more complex of specific logistical tasks. 
LSA occupations were not a major focus of my research, so I submit the foregoing observations and interpretations as grounds for further investigation. For the moment, however, they suggest that the Falémé River provided an important element within the landscape that would be exploited by later Iron Age populations in the region.

Table 4.1: $\quad$ Summary of chipped stone artifacts from MSA/LSA and cLSA sites

\begin{tabular}{|c|c|c|c|c|c|c|c|c|c|}
\hline \multirow{2}{*}{$\begin{array}{l}\text { Component } \\
\text { Raw Material } \\
\end{array}$} & \multirow{2}{*}{$\begin{array}{c}\text { Total } \\
\text { N }\end{array}$} & \multicolumn{2}{|c|}{ Core } & \multicolumn{2}{|c|}{ Shatter } & \multicolumn{2}{|c|}{ Flake } & \multicolumn{2}{|c|}{ Formal Tool } \\
\hline & & $\%$ & $\begin{array}{l}\text { Weight } \\
\text { Avg. (g) }\end{array}$ & $\%$ & $\begin{array}{l}\text { Weight } \\
\text { Avg. (g) }\end{array}$ & $\%$ & $\begin{array}{l}\text { Weight } \\
\text { Avg. (g) }\end{array}$ & $\%$ & $\begin{array}{l}\text { Weight } \\
\text { Avg. (g) }\end{array}$ \\
\hline MSA/LSA & 437 & 2.3 & 19.05 & 41.4 & 2.37 & 54.9 & 2.31 & 1.4 & 6.32 \\
\hline Quartz & 13,885 & 1.4 & 22.18 & 45.8 & 2.31 & 51.7 & 2.25 & 1.0 & 6.67 \\
\hline Chert & 5257 & 4.8 & 16.64 & 29.5 & 2.80 & 63.4 & 2.54 & 2.3 & 6.71 \\
\hline Mudstone & 295 & 0.0 & - & 39.8 & 8.93 & 55.1 & 12.03 & 5.1 & 17.5 \\
\hline Ceramic LSA & 15,454 & 2.6 & 19.58 & 43.8 & 3.09 & 50.7 & 2.34 & 2.9 & 11.70 \\
\hline Quartz & 10,574 & 1.6 & 18.35 & 47.7 & 2.82 & 49.3 & 2.06 & 1.4 & 8.47 \\
\hline Chert & 4518 & 4.9 & 15.56 & 34.6 & 3.18 & 55.3 & 2.37 & 5.2 & 4.09 \\
\hline Mudstone & 362 & 4.5 & 87.00 & 46.4 & 10.23 & 32.4 & 14.07 & 16.7 & 49.2 \\
\hline
\end{tabular}

\section{Iron Age}

As I have already discussed, the West African Iron Age was associated with the emergence and maturation of complex agro-pastoral economies, increasingly specialized craft production, and interregional exchange networks. This archaeological horizon began with widespread adoption of iron technology in the first millennium BC and drew to a close with the rise of the Atlantic world system in the $16^{\text {th }}$ century AD. That said, the nomenclature is somewhat awkward, not least because the transition from stone to iron technologies took place at drastically different tempos across West Africa (Holl 2000; MacDonald 1997). Using European contact to mark the end of this horizon also carries the dangerous implication of a supposed rupture between antiquity and modernity, a device that has too often averted the historical gaze from critical points of cultural continuity and transformation in non-European societies. It is essential to keep these 
critiques of the Iron Age rubric in mind, even as I retain this nomenclature ${ }^{5}$ to situate the village community of Diouboye within trajectories of sociopolitical change across West Africa circa 500 BC to AD 1500.

The distinction between an Early and Late Iron Age is subjective; I place this division around AD 500 to highlight both the economic changes documented in the archaeological record (see below) and the contribution of written and oral sources to the interpretation of "medieval” West African political economies. Chapter 1 noted the contribution of Arabic geographies and inscriptions to the historiography of the Late Iron Age, as well as the oral traditions and epics that bring histories of cultural interaction, economic transformation, and political conflict into sharper focus. Keeping in mind that these historical sources were produced by individuals in specific social contexts, they continue to provide an important frame for archaeological understandings of the period.

In what follows, I employ archaeological and historical evidence to sketch out some broad trends across the West African savanna regarding the adoption of metallurgy and agro-pastoral subsistence regimes during the Early Iron Age, and the increasing complexity of regional (and local) political economies during the Late Iron Age. This background provides a context for exploring the historical landscape of the Upper Senegal region, and the Madina-Sadatou study area more specifically, during the occupation of Diouboye.

\section{Early Iron Age (500 BC - AD 500): Food, Metals, and Villages}

The transition from mobile forager to sedentary agro-pastoral economies remains one of the least understood processes in West African prehistory (Kahlheber and Neumann 2007; Neumann 2003). While people variably integrated pearl millet and cowpea into their subsistence economies throughout the second to first millennia BC, other domesticates that would become critical to full-time agriculture—sorghum, rice, okra, and Bambara groundnut-only appear sporadically prior to AD 500 at a few sites in the Sahel and savanna including Daima (Connah 1981), Elkido (Magnavita 2002), and

\footnotetext{
${ }^{5}$ Alternative designations such as "prehistory" and "proto-history" (préhistoire and protohistoire in the French nomenclature) emphasize the nature of evidentiary sources, rather than trends in social processes.
} 
Jenné-jeno (S. K. McIntosh 1995), and after AD 700 at the site of Arondo (see below). On the other hand, sites in the Middle Senegal (R. J. McIntosh et al. in press) and Upper Volta regions (Dueppen 2008; Gallagher 2010) cultivated only pearl millet and fonio throughout the first millennium AD. Sampling issues, both in terms of sites excavated and macrobotanical preservation, may be at play, but the emerging portrait is one in which a relatively heterogeneous suite of subsistence regimes co-existed across the savanna prior to the second millennium AD.

The diversification and intensification of cultivated plants in some regions may have arisen during a period of sustained aridity circa 300 BC to AD 300 as population densities increased in the well-watered Lake Chad basin and Inland Niger Delta (Kahlheber and Neumann 2007). Even as people continued to exploit a wide array of wild plant resources throughout the Iron Age (Neumann 2003:82-83), a growing diversity of crops and domestic animals must have entailed increasingly specialized subsistence economies and political institutions for mitigating risk within sedentary communities, and for coordinating access to resources such as water, arable land, and pasture (R. J. McIntosh 1993). These institutions likely involved some of the kinship and territorial rights, social statuses, and ritual powers that would underwrite complex politicaleconomic mosaics during the Late Iron Age (see below).

The heterogeneous diffusion of copper and iron metallurgy across West Africa had ramifications for evolving agro-pastoral systems and political economies during this period (Holl 2000). The ultimate origins of metal technology in sub-Saharan Africawhether adoption from abroad or independent innovation-remain subject to a debate that lies well beyond the scope of this thesis (see discussions in Alpern 2005; Holl 2009; Killick 2004; Woodhouse 1998). The earliest uncontested evidence for metal production appears in the Agadez region of the southern Sahara, where kilns and slag from copper manufacture date to the early-first millennium BC (Killick et al. 1988). Evidence for copper production appears on sites dating from 800 to 200 BC, concurrent with the development of iron metallurgy. At the site of Walaldé in the Middle Senegal Valley, for example, artifacts and smelting debris document the working of copper likely obtained from mines at Akjoujt to the north (Deme and McIntosh 2006). Alongside copper ornaments, more than two dozen iron tools and fragments from this site are 
contemporaneous with well-accepted evidence for early iron production in the lower Niger region (Okafor 1993) and the Mandara highlands (MacEachern 1996). Throughout the subsequent Early Iron Age, iron and copper technologies spread to nearly every corner of West Africa where they were shaped by local political and cultural contexts (Holl 2000; Woodhouse 1998). As part of a more specialized craft economy, metal production, distribution, and display would have provided media for economic, political, and ritual power (De Barros 2000; Herbert 1984, 1993)—material and non-material resources that would ultimately underwrite complex political economies.

Technologies for food and metal production developed alongside a regional trend towards increasing sedentism (S. K. McIntosh and R. J. McIntosh 1983). Prior to 500 BC, sedentary village sites such as those of Dhar Tichitt and Djiganyai in southeastern Mauritania (Holl 1993; MacDonald et al. 2009) and Daboya in Ghana (Shinnie and Kense 1989) were far outnumbered by contemporaneous sites attesting to more mobile lifeways. The Early Iron Age, however, witnessed the settlement of numerous sites in the Lake Chad basin (Connah 1981; Holl 2002; Magnavita 2004), the Middle Niger region (S. K. McIntosh 1995), the Middle Senegal region (Bocoum and McIntosh 2002; R. J. McIntosh et al. in press) and Voltaic region (Dueppen 2008; Effah-Gyamfi 1985); many of these would ultimately evolve into village confederations and urban configurations.

\section{Late Iron Age (AD 500-1500): Pathways to Complexity}

By AD 500 the climate of West Africa had become amenable to the expansion of agro-pastoral subsistence regimes across the savanna and Sahel as ecological variability began to resemble that of the present day (see Chapter 3). More humid conditions around the turn of the millennium, however, would have pushed the boundaries for rain-fed cultivation and tsetse fly (as a barrier to domestic livestock) further north into the Sahel.

With a growing emphasis on staple crops and sedentism, this period witnessed the evolution of urban centers and stratified states associated with an increasing complex mosaic of political economies. As mentioned in Chapter 1, early studies based on Arabic geographies and limited archaeological evidence proposed that trans-Saharan trade provided an external "stimulus" for these interregional processes (e.g., Levtzion 1980; Mauny 1961). However, it should be clear from the above discussion that Late Iron Age 
institutions for the production of staple and craft goods, coordination of land and labor, and long-distance exchange all had antecedents in the period prior to sustained contact with North Africa. Additionally, archaeological research on and around Jenné-jeno in the Inland Niger Delta (S. K. McIntosh 1995) shows that dense populations, possibly supported by staple imports and specialized craft production, existed just prior to, or concomitant with, early Arab accounts in the ninth century AD. By the early-second millennium AD, urban centers also emerged farther south in the Pays Mande (Filipowiak 1979), in the Voltaic region (Anquandah 1993; Effah-Gyamfi 1985), and in the equatorial forests around the lower Niger River (Andah 1982; Okpoko 1987).

Figure 4.8: Map of medieval states in West Africa

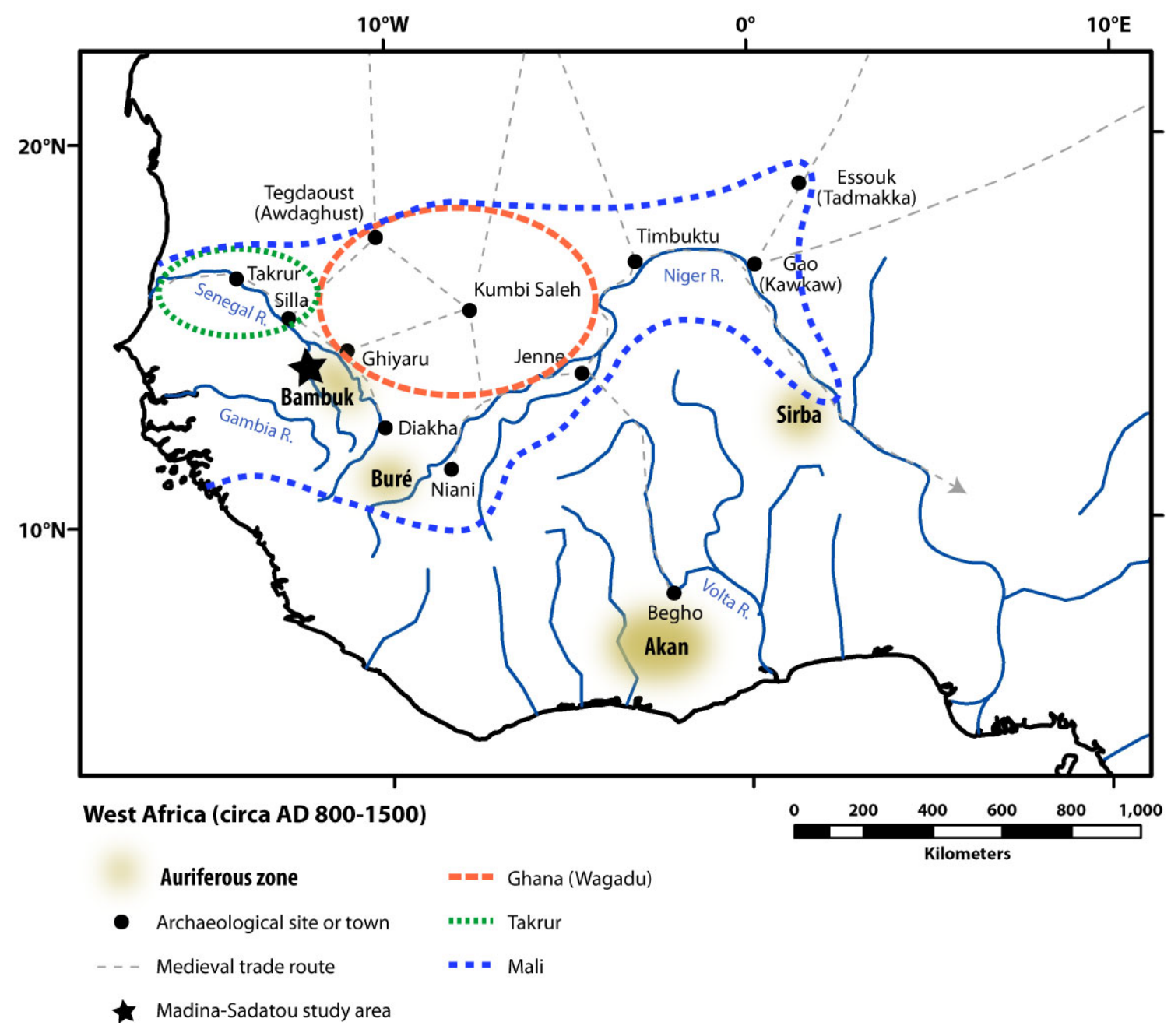


Historical sources have documented the emergence, interaction, and dissolution of a number of major and minor polities in the West African savanna during this period. Major polities most directly implicated in the political economy of the Upper Senegal region include Ghana, Takrur, and Mali, the detailed (and debated) histories of which lie beyond the scope of this study. To briefly summarize, the Soninke polity of Ghana emerged sometime during the mid-first millennium $\mathrm{AD}$ in the northern savanna between the lucrative trade routes of the Senegal and Niger Rivers, the upper reaches of which provided access to the gold sources of Bambuk and Buré (A. Bathily 1975; Bovill 1995; Levtzion 1980); the capital of this state may have been located at the archaeological site of Kumbi Saleh (Berthier 1997). In a cogent summary of the available archaeological and historical evidence, Susan McIntosh (2008) surmises that Ghana rose to regional prominence by negotiating alliances with trans-Saharan traders, tying the ideology of sacred kingship to gold production, and supporting the merchant diasporas (Wangara or Jakhanke) who extracted gold from places such as Bambuk, which were outside the immediate control of the state. Having dismantled the myth of Almoravid conquest (Conrad and Fisher 1983; Masonen and Fisher 1996), historians now link the decline of Ghana to a broader reorganization of trans-Saharan trade and regional political relations during the $11-12^{\text {th }}$ centuries AD.

By the end of the first millennium AD, the polity of Takrur came to dominate the Middle Senegal region, perhaps in competition with the town/state of Silla (A. Ba 2002; Fall 1982). Scholars have variously attributed the rise of the ruling Jaa-Ogo dynasty to control over iron production, the introduction of Islam, and/or long-distance trade in salt, iron, and textiles (A. Ba 2002; Bocoum 2000; Delafosse 1912). Focused more on the control or coordination of localized subsistence and craft production, the political economy of Takrur did not depend on access to the gold of Bambuk, presumably making it less susceptible to perturbations in trans-Saharan trade. By most accounts, however, Takrur expanded its regional hegemony during the early-second millennium AD until finally coming under vassalage to Mali.

According to the well-known epic, Sunjata Keita founded this Mande state sometime around AD 1235 by subduing his rivals, including the blacksmith king Soumaoro Kanté of Sosso (Niane 1965). Under the reign of Sunjata and descendent rulers 
(mansa), Mali rapidly expanded outwards from the Pays Mande to control much of the Niger and Senegal River basins, as well as the trading êntrepots of the Sahel. However, this vast territory almost certainly comprised a dynamic political-economic mosaic, and the local experience of Malian hegemony for many rural subsistence and craft economies remains to be understood. What seems certain, however, is that Mali maintained indirect, or perhaps direct, control over long-distance exchange, including access to the auriferous regions of Buré and probably Bambuk (Delafosse 1912; Niane 1989). The regional power of Mali began to fade in the $15^{\text {th }}$ century AD as first non-Mande polities including Djolof (Bomba 1977) and Gajaaga (A. Bathily 1989), and later Mande vassals including Kaabu (Niane 1989) and states along the Gambia River (Quinn 1972), asserted their autonomy.

Trans-Saharan trade, though not the ultimate cause of political centralization in West Africa, was nonetheless critical for acquiring wealth and constituting social status and identity throughout the Late Iron Age (S. K. McIntosh 2008; Stahl 2004b). Historical sources describe how towns such as Awdaghust, Tadmekka, and Gao served as êntrepots between North African merchants bearing salt, copper, glass, cloth, and horses from the Mediterranean world and West African merchants (i.e., Wangara, Juula, and Jakhanke) bearing gold, slaves, exotic spices, nuts, and skins from the southern savanna and forest (Levtzion 1980). Archaeological fieldwork at many of these sites has generated material evidence for trade in at least the more durable of these commodities, as well as for Islamic influences from North Africa on architecture, pottery, and religious practice (Devisse 1983; Insoll 1997; Nixon 2009). From the late-first millennium AD, trade relations became a conduit for the introduction of Islam into many West African societies, first as a means to sustain trade (Brett 1983), and later as a political and spiritual force in its own right (Levtzion 2000). As discussed in Chapters 1-2, trade networks extending southward from the Sahel implicated a series of merchant diasporas whose shared identities facilitated long-distance trade between agents embedded in local communities (Brooks 1993; Curtin 1975a). Exotic objects such as glass and carnelian beads and copper reveal the extent of past trade networks across the political mosaic that stretched from the western Senegambia to the lower Niger River (Posnansky 1973).

Interspersed within and beyond Late Iron Age political centers and trade networks, most people continued to live in pastoral encampments or modest settlements 
organized around principles of kinship and coresidence. Following my discussion in Chapter 1, I found it useful to speak of diverse, though often interlocking, pathways of social complexity within this milieu (following S. K. McIntosh 1999c; Stahl 2004b). A growing body of archaeological research on decentralized societies and village-based communities demonstrates the diversity of political economies to be found in these settings (e.g., Gallagher 2010; A. Lawson 2003; Richard 2007; Stahl 2001; Thiaw 1999), as well as their potential long-term dynamics as communities cycled between egalitarian and hierarchical social structures (Dueppen 2012). It is within such a milieu that people settled and occupied the village of Diouboye.

Although the Iron Age drew to a close with the advent of an Atlantic world system after AD 1500, this was not an historical rupture. In fact, significant demographic and political changes had already begun to take place across West Africa throughout the $15^{\text {th }}$ century $\mathrm{AD}$ alongside the emergence of a more unpredictable climatic regime (Brooks 1989). As noted above, this period witnessed the decline of Mali and the growth of smaller regional polities. The $14-15^{\text {th }}$ centuries also encompassed a regional trend toward political instability wrought by Fulbe migrations from the Middle Niger region across Senegambia and the establishment of the states in northern Guinea (Futa Jallon) and along the middle Senegal River (Futa Toro) (Delafosse 1912). Not surprisingly, then, settlement dynamics shifted dramatically as people abandoned many towns and villages, including Diouboye. In most cases, however, the interplay between landscape, history, and political economy at these regional and local scales remains to be explored.

\section{Iron Age Occupations in (and around) the Upper Senegal Region}

Over the past century, the Iron Age occupation of Senegambia has figured prominently in the fieldwork undertaken by an eclectic group of amateur colonial officials, expatriate academics, and Senegalese scholars (Richard 2009). Whether framed by an early curiosity with mortuary monuments or more recent concerns with diverse social trajectories in West Africa, fieldwork has focused on four archaeological culture areas defined according to their predominant site types (Bocoum 2000; Descamps 1979; Martin and Becker 1984). These include: the dense array of shell mounds dotting the Atlantic littoral (Descamps and Thilmans 1979; Linares de Sapir 1971); the megalithic 
cemeteries of the central Gambia and Siin-Saalum River basins (Gallay et al. 1982; Holl et al. 2007; Thilmans et al. 1980); the earthen tumuli of northwestern Senegal (Joire and Duchemin 1955; S. K. McIntosh and R. J. McIntosh 1993b); and the network of settlement and iron smelting sites found across the Middle Senegal region (Bocoum and McIntosh 2002; R. J. McIntosh et al. in press; Thilmans and Ravisé 1980).

The archaeology of Iron Age occupations along the Falémé River, by contrast, only began to develop in the 1990s (Thiaw 1999). Given this dearth of research, I first present first a brief history of the Upper Senegal to AD 1500 sketching out regional political economies and their entanglement within the broader historical processes outlined above. I then integrate this narrative with archaeological data available from within and immediately surrounding this region.

\section{An Historical Sketch of the Upper Senegal (AD 800-1500)}

According to oral traditions (I. D. Bathily 1969), the upper Senegal River, including the tributary basins of the lower Falémé and Xoolimbine, came under the political dominion of Ghana in the late-first millennium AD. At that time, the region became known as Gajaaga (derived from the Soninke word for "west”; also known as Galam). As throughout much of precolonial West Africa, this appellation probably referred less to a physical territory than to a social dimension of the landscape, namely

the hegemony of Soninke elites over diverse communities speaking Mande, Fulfulde, and possibly Tenda languages. To the west of Gajaaga lay the polities of Takrur, Silla, and Djolof. To the south lay the Pays Mande, the heartland of medieval Mali centered on the upper Niger River. At the interstices of these politico-cultural spheres lay the goldproducing lands of Buré and Bambuk; this latter was sparsely inhabited by small communities seeking refuge from the predations of more centralized polities (see Chapter 1). Although the historiography of the Upper Senegal presents a vague consensus on how such toponyms mapped onto the regional landscape (Figure 4.7), it is essential, particularly in this archaeological study, to recognize their potential fluidity over the longue durée.

In his masterful history of Gajaaga prior to the slave trade, Abdoulaye Bathily (1989) suggests that the initial occupants of the Upper Senegal may have been Mande- 
speaking foragers or small-scale farmers (see above). In the mid-first millennium AD Soninke populations began to immigrate into the region with the growth and decline of Ghana and/or the influx of a merchant diaspora from the town of Dia on the Niger River (A. Bathily 1989:73-78; S. K. McIntosh 1981; P. Smith 1965). While later Soninke griots have recounted this process as an amicable encounter, they also portray the emerging dynasty of political elites as powerful warriors easily capable of subjugating an indigenous populace. Whatever the case, this Soninke dynasty, known as the Sempera Bacili, ultimately established some form of rule throughout Gajaaga by the early-second millennium AD, coincident with the apogee of imperial Ghana. Indeed, the similarities in political structure between Ghana and historical Gajaaga suggest that the latter emerged as a vassal state to control the lucrative trade routes along the upper Senegal River.

With the decline of Ghana in the $11-12^{\text {th }}$ centuries AD, the Sempera Bacili maintained control of Gajaaga and even managed to expand their political dominion over the Upper Senegal region — a process driven, in part, through political fission and internecine strife within the elite dynasty (A. Bathily 1989:152-163). I propose that this political landscape formed what Igor Kopytoff (1987b) calls an "internal African frontier" in which the reproduction of complex social structures hinges upon migration and encounter with other populations (see Chapter 2). In the case of Gajaaga, this involved the expansion of a hierarchical political system with an elite gerontocracy of Soninke nobles (tunka) and warriors (mangu) ruling over a more diverse populace of commoners (jambuuruni). Historical sources suggest that craft specialists (nyaxamalo) such as blacksmiths, potters, and griots also held a distinct status, perhaps as endogamous castes (A. Bathily 1989; Tamari 1991). Slaves held the lowest rank in Gajaaga and, although some achieved status as warriors, many were sold northwards into the transSaharan trade. Muslim clerics (marabouts) played both a religious and judicial role for the Sempera Bacili dynasty. At the same time, the perpetuation and success of these Late Iron Age institutions in Gajaaga, and particularly the legitimacy of the ruling class, may have depended on their vassalage to imperial powers such as Ghana and Mali.

At or beyond the shifting frontiers of Soninke and Mande hegemony in the Upper Senegal region lay the geo-cultural landscapes of Bambuk and Lamlam introduced in Chapter 1. Although the relation between these local landscapes and regional polities 
remains murky, the village of Diouboye, situated on the banks of the Falémé River, would have been well-positioned to monitor commerce between Bambuk and Mande states to the south and Ghana, Gajaaga, and Takrur to the north. At the same time, proximity to the river might have made this community vulnerable to raiders potentially preying upon the decentralized societies of Lamlam. All said, material and spatial dimensions of the archaeological landscape of Madina-Sadatou stand to inform on the ways in which local political economies articulated with regional ones at this time.

\section{Archaeological Landscapes and Ceramic Spheres}

Although archaeological research in the Upper Senegal has been limited, the available data from several Iron Age contexts do provide a point of comparison for the events and processes known from historical sources. First and foremost, Ibrahima Thiaw’s (1999) survey along 50 kilometers of the lowermost Falémé River provides an invaluable spatial and material baseline for interpreting other Iron Age landscapes in the vicinity-particularly that of the Madina-Sadatou study area just 30-40 kilometers upriver. Phase IVB (circa AD 950-1500) sites identified by Thiaw included only three village sites (2-5 ha) and five small-scale iron-smelting sites tentatively assigned to this horizon. These sites clustered in the northern half of the study area, perhaps indicating an ecological and/or social deterrent to settlement further south along the Falémé River.

Thiaw (1999) also undertook excavation at one of these village sites called Arondo, a low mound (5 ha) situated on the Senegal River some 500 meters northwest of its confluence with the Falémé. Three units revealed 3.40 meters of stratified deposits built up through mud-brick walls, hearths, and borrow pits and the accumulation of refuse from subsistence activities and craft production. Faunal remains attested to consumption of domesticated cattle and sheep/goat (and possibly chicken) supplemented by a greater emphasis on wild bovid species, hippopotamus, crocodile, turtle, catfish, and Nile perch (Watson 1999); these latter were acquired with the aid of terracotta netweights. Macrobotanical remains recorded the cultivation of millet and sorghum (after AD 700) alongside the exploitation of tree crops including baobab and jujube (Gallagher 1999). Slag and iron artifacts from metallurgical production and consumption appeared in the 
earliest levels at Arondo, while ten copper alloy items only documented the import of this metal from the savanna after AD 700.

Alongside these exotic objects, the pottery sequence from Arondo hints at the dynamic integration of this village community into the increasingly complex political economies of the Middle Niger and Middle Senegal regions. Based on the proximity of Arondo to the middle Senegal Valley, Thiaw (1999:174-177) adapted the four-phase ceramic chronology developed for Iron Age settlements in this region (Bocoum and McIntosh 2002; R. J. McIntosh et al. in press), while noting some interesting differences. During Phase II (AD 400-600), the earliest occupants of Arondo produced long-neck and everted-rim jars decorated with red slip and knotted cord roulettes similar to those found at contemporaneous sites along the middle Senegal River, in addition to thin-walled and T-rim vessels known from the Pays Mande and Middle Niger regions. These early assemblages also included small numbers of sherds with carved roulette designs similar to those from Diouboye and other sites to the south (see below). Phase III/IVA (AD 600950) pottery assemblages included more collared and everted rims with red slip, channeling, and/or twisted cord roulette impressions most similar to those from the Middle Senegal region at this time.

Over the past several decades, survey and excavation in the Middle Senegal region has identified a network of iron-smelting emplacements and settlements ranging from small villages to proto-urban configurations (Martin and Becker 1984). Salvage operations and controlled excavation at some of these sites, including Podor, Siwré, Cuballel, and Sinçu Bara indicate that the region was occupied by iron-producing people who supplemented their agro-pastoral subsistence economy with fishing, hunting and gathering throughout the first millennium AD (Bocoum 2000; Bocoum and McIntosh 2002; Chavane 1985; R. J. McIntosh et al. in press; Thilmans and Ravisé 1980). At these sites, similar grog-tempered pottery assemblages included simple, everted, and shortcollared rim forms decorated with incisions and punctates; body decorations included folded, twisted, and braided cord roulette. During this period, settlement patterns gradually shifted from small hamlets bordering the river, to a network of modest villages ( $<3 \mathrm{ha}$ ) and logistical sites, these latter possibly representing encampments by fisherfolk or pastoralists (R. J. McIntosh et al. in press). Although copper had been traded into the 
region during the first millennium BC (Deme and McIntosh 2006), the absence of this and other imported goods throughout the early- to mid-first millennium AD suggests that local communities were not yet participating in trans-Saharan trade networks.

Coincident with the historical emergence of Takrur and Silla during ninth to tenth centuries AD, the Middle Senegal archaeological landscape underwent a number of changes (Bocoum and McIntosh 2002; Chavane 1985; S. K. McIntosh 1999b). Sinçu Bara expanded to reach a maximum size of 67 hectares, signaling the emergence of a multi-tiered settlement hierarchy. Pottery assemblages from this period (Phase IV) included vertical short- and long-collared rims with cordons and collars and sherds decorated with twisted cord roulette, but the appearance of distinctive, heavily burnished wares with high relief surfaces at Sinçu Bara other sites in the eastern end of the middle Senegal Valley could mark a trend towards regionalization and/or interaction with Soninke towns represented by sites such as Tegdaoust and Kumbi Saleh (Chavane 1985:127; Thilmans and Ravisé 1980:126-135). Glass beads and copper objects also made their reappearance at this time; these latter included bells and harness elements resembling those from towns in the Sahel and associated in burials with possible warriorelites (Garenne-Marot 1995). While these prestige items suggest that trade was important to the emergence and display of political hierarchy, Susan McIntosh (1999b) has suggested that the political economy of Takrur depended upon coordinated interactions among economic specialists and other social actors described in Chapter 2-including farmers, herders, fishers, and slaves (T. K. Park 1992; Schmitz 1994). Whatever the case, the Middle Senegal region persisted as a sphere of material interaction throughout the second millennium $\mathrm{AD}$ as people continued to produce burnished pottery with high relief channels and cordons.

To the south of Bambuk in the Pays Mande, archaeological research on Iron Age occupations remains limited to a 1965-1968 field campaign in and around Niani (Filipowiak 1968, 1979), a putative capital of medieval Mali (Conrad 1994; Hunwick 1973). A series of trenches and horizontal exposures of earthen and stone architecture and refuse from subsistence practices, craft production, and perhaps ritual have, however, generated a wealth of data interpreted to fit Mande historical narratives. During an early phase of occupation (AD 500-900), the settlement began as a cluster of villages with 
wattle-and-daub huts less than one kilometer north of the Sankarani River, a major tributary to the Niger. Pottery assemblages included spherical and ovoid vessels with simple and lightly everted or short-collared rims decorated with slip and/or incisions; some vessels also had distinctive ring or footed bases. Modes of surface decoration included twisted cord, folded strip, and carved button and chevron roulette (Filipowiak 1979:Figures 38, 43, 50, 52). Corresponding to the regional dominance of Mali circa AD 1200-1700, Niani grew to encompass an area of nearly ten square kilometers, including a number of areas that Wladyslaw Filipowiak (1979) interprets as wards (quartiers) variously occupied by ruling elites, Muslim traders, and commoners. This overall increase in economic specialization and political hierarchy has been attributed to the emergence of trade networks evidenced by imported copper and glass objects, and later by terracotta smoking pipes, as well as northerly cultivars such as millet, lentils, and turnips. Architectural styles evolved to include circular huts with laterite block foundations and pisé wall construction. Evidence for local craft specialization includes iron tools and slag, spindle whorls, and earthenware pottery-these latter apparently consisting of variations on earlier forms and decorative motifs.

Downriver from Niani, ongoing work at the Iron Age site of Sorotomo (MacDonald et al. 2011) has turned up pottery vessels, including narrow-mouthed jars, with everted and thickened rims, red slip, and folded strip, braided cord, and fish vertebrae roulettes. Meanwhile, at the rockshelter of Korounkorokalé, noted above for its LSA sequence, have yielded metallurgical slag and a suite of pottery with thickened rims and decoration from roulettes of fish vertebrae, folded strips, and carved wood (MacDonald 1997:184). The presence of one footed base could further anchor this site within a putative ceramic sphere centered on the Pays Mande and Middle Niger region (Bedaux 1980; Bedaux and Raimbault 1993), as discussed below.

To the southwest of Bambuk in the Gambia River basin, Iron Age societies buried their deceased in megalithic stone (laterite) circles and tumuli, often with iron spears and copper jewelry attesting to specialized craft production and participation in long-distance exchange networks (Holl and Bocoum 2006; Thilmans et al. 1980). The density of copper sumptuary goods, including the accoutrement of horse-riding warriors, in some of the later tumuli points to the expansion of hierarchical political economies southward from 
the Middle Senegal region during the mid-second millennium AD. Although pottery assemblages from cemeteries and shallow settlement sites have been ordered into a chronological sequence (Gallay 2010), their remarkable diversity suggests that the central Gambia formed a zone of interaction among people from diverse cultural traditions, including Mande communities tracing their origins to the period of Malian expansion (Niane 1989; Quinn 1972; D. R. Wright 1985). In this regard, it is interesting to note that some of the formal and decorative elements associated with Late Iron Age occupations in the Pays Mande-including carved chevron and button roulettes and short-collared rims on spherical vessels - appear at some settlement sites, but not at many others (Gallay 2010; A. Lawson 2003; S. K. McIntosh and R. J. McIntosh 1993b).

Building upon a synthesis of spatio-temporal distributions in West African pottery decorations by Alexandre Livingstone Smith (2007), ceramic data from the above sites and regions permit the definition of three broad ceramic spheres supported by other classes of material culture. Given the paucity of well-dated ceramic sequences, these undoubtedly gloss a tremendous, and as of yet unknown, amount of variability in material practice across space and time. That said, these offer a useful heuristic for situating Diouboye and the Iron Age landscape of Madina-Sadatou within broader networks of socioeconomic and cultural interaction.

A Middle Senegal ceramic sphere, corresponding to Phase III/IV pottery assemblages at Sinçu Bara (Bocoum and McIntosh 2002), Cubalel (R. J. McIntosh et al. in press), and Arondo (Thiaw 1999), includes globular vessel forms mainly tempered with grog and decorated with folded strip, twisted cord, and braided cord roulettes. From the late-first millennium $\mathrm{AD}$ onwards, some vessels boast high relief channels, cordons, geometric imprints, and a deep reddish-purple slip. Cordons and channels also appear as decorative elements on everted and long- and short-collared rims, which often have a red slip. Perforated terracotta discs are often found in association with these ceramics at sites along the Senegal River (Thilmans and Ravisé 1980).

A Niger-Gambia ceramic sphere, corresponding to assemblages from Niani (Filipowiak 1979) and some settlements and cemetery sites in the Gambia River drainage (Gallay 2010; A. Lawson 2003), includes globular vessel forms tempered with grog, sand, and laterite; many vessels also have ring or footed bases. Folded strip, twisted cord, 
and carved wooden roulettes are predominant decoration techniques, while red slip and comb or horizontal incisions often adorn vessel shoulders and lips. Simple, vertical, and everted rim forms are common, although ledged and carinated rims are not unknown. Iron tools from Niani include small blades and tanged projectile points, while the spears recovered from megalithic burials are considerably larger and more variable.

A Middle Niger ceramic sphere has been well-documented in the Inland Niger Delta (S. K. McIntosh 1995; S. K. McIntosh and R. J. McIntosh 1980), the Bandiagara plateau (Bedaux and Lange 1983; Mayor et al. 2005), and in the Méma region (Togola 2008) southeast of Kumbi Saleh (Berthier 1997). Despite variability across these assemblages, Iron Age pottery throughout this region shares a few general features. Vessels tend to be globular or ovoid, often with everted, flared, carinated, or thickened rims; long-necked jars are also a distinctive vessel type. The pedestal and footed vessel bases found at Niani also characterize this ceramic sphere (Bedaux 1980). Body decorations include twisted cord, braided cord, and folded strip roulette, with some complex variations on the latter. Vessel shoulders and rims often display a red slip and incisions or comb impressions.

\section{Iron Age Components in the Madina-Sadatou Study Area}

The seriated sequence of material culture from Diouboye provides an analytical and chronological anchor for mapping the Iron Age landscape of the Madina-Sadatou study area. Artifacts and surface features similar to those from Diouboye were found at another 32 sites and 10 isolated occurrences in the pedestrian survey zone. Pottery assemblages from this component included sherds with grog and grit temper and decorations of twisted and braided cord, folded strip, and cord-wrapped stick roulette, as well as carved button and chevron roulettes. Vessel rims exhibited simple, everted, and collared forms, and often displayed a red slip and/or incisions and punctate decoration. Iron artifacts and stone features that I have interpreted as granary bases and other domestic architecture (see Chapter 6) helped to define an Iron Age surface component. 
Figure 4.9: Iron Age (Diouboye phase) pottery from surveyed sites

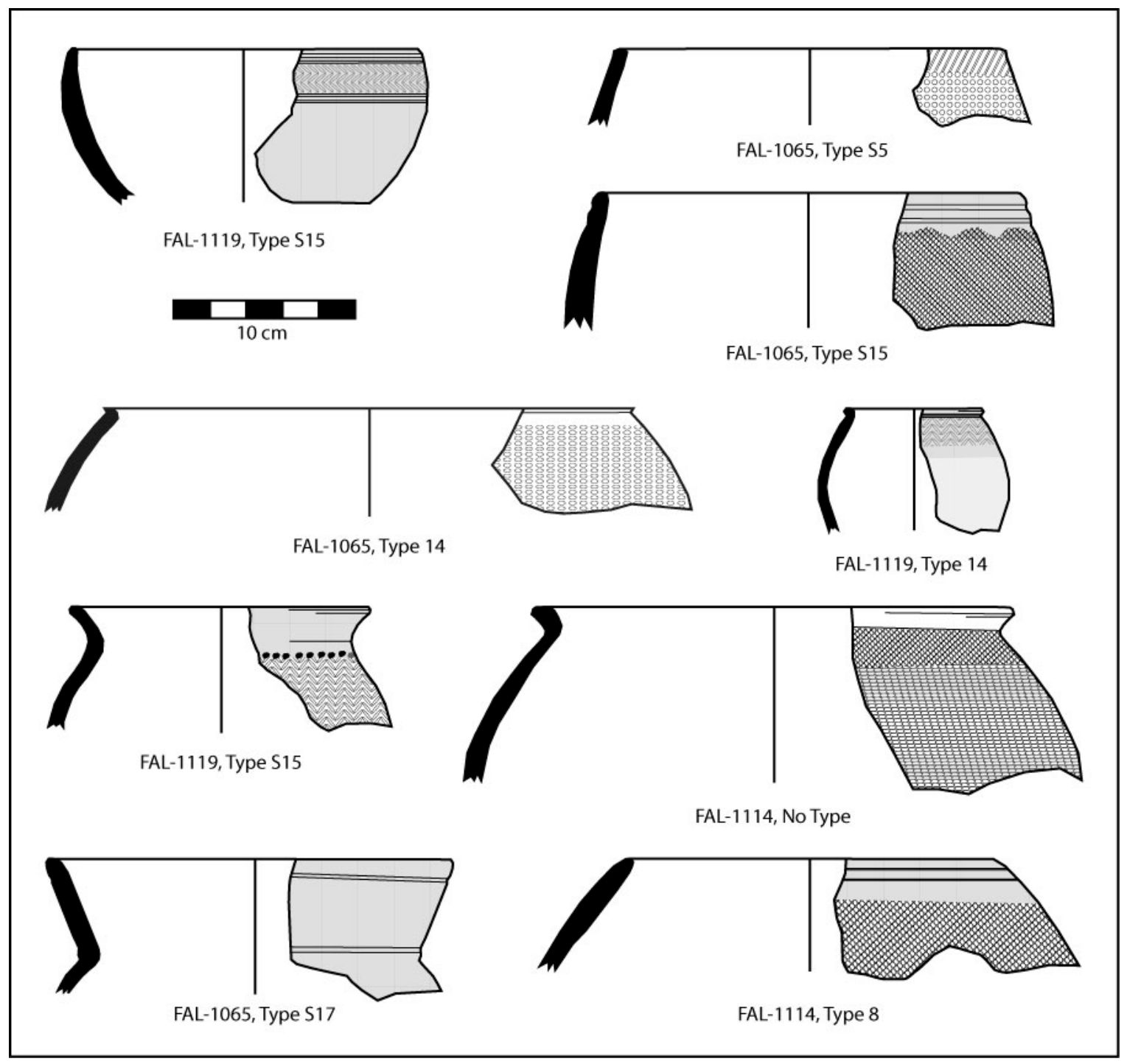

Based on the presence/absence of temporally sensitive sherd types (see Chapter 8), it was possible to assign occupations at many sites to one or both of the Diouboye Early/Middle (AD 950-1200) or Diouboye Middle/Late (AD 1200-1400) phases. The shallowness of most settlement sites suggests that an absence of Early Diouboye phase (button-carved roulette) sherds at the latter sites is not simply a sampling bias.

Several sites, however, deserve special mention. Despite their fine grit inclusions characteristic of cLSA pottery, the sherd assemblages from FAL-1006 and FAL-1016 in the northern end of the pedestrian survey zone were classified as an Iron Age component 
based on the presence of red slip and twisted cord, and cord-wrapped stick roulette decoration. The few sherds from sites FAL-1133 and FAL-1134 in the southern end of the survey zone had a very coarse grit paste similar to those from the later Tontèko phase, but were assigned to the Iron Age on the basis of their carved roulette decoration. Surface finds from Baganafara (FAL-1148), a site documented during guided survey, also resembled those from Diouboye, strongly suggesting that the two villages were contemporaneous. While the classifications do not greatly affect the interpretations of the following discussion and analysis, the Iron Age landscape of the Upper Senegal region, and even the Madina-Sadatou study area, certainly exhibited more economic and cultural diversity than depicted in the available data.

The relationship between cLSA and Iron Age sites, and their associated communities, in the study area remains unknown. A sharp discontinuity in technological traditions, subsistence practices, and settlement patterns is consistent with regional abandonment during the arid phase of $300 \mathrm{BC}$ to $\mathrm{AD} 300$. However, the current paucity of interregional data precludes a rigorous assessment of this proposal.

I do suggest that the Iron Age witnessed a resettlement of the region by people sharing material practices, and perhaps cultural structures, with communities to the south. In light of the foregoing discussion, the pottery assemblages from Diouboye and contemporary sites align more closely with the Niger-Gambia ceramic sphere to the south, than with the Middle Senegal sphere to the north. At a superficial level, Diouboye phase pottery traditions exhibited carved roulette decoration that never appeared farther to the north, except in low frequencies at Arondo. Conversely, the heavy cordons and channeling characteristic of the Middle Senegal ceramic sphere never appear on Diouboye phase ceramics. At a more fundamental level, formal vessel elements including circular pedestal bases and vertical and everted rims, rather than the long-collared ones found in the Middle Senegal region, may point to the transmission of technical knowledge within communities of craft specialists that extended north from the Pays Mande. This interpretation is consistent with later Soninke accounts of encountering Malinke communities upon their immigration into the Upper Senegal region. 
Figure 4.10: Iron Age (Early/Middle Diouboye phase) sites in the pedestrian survey zone

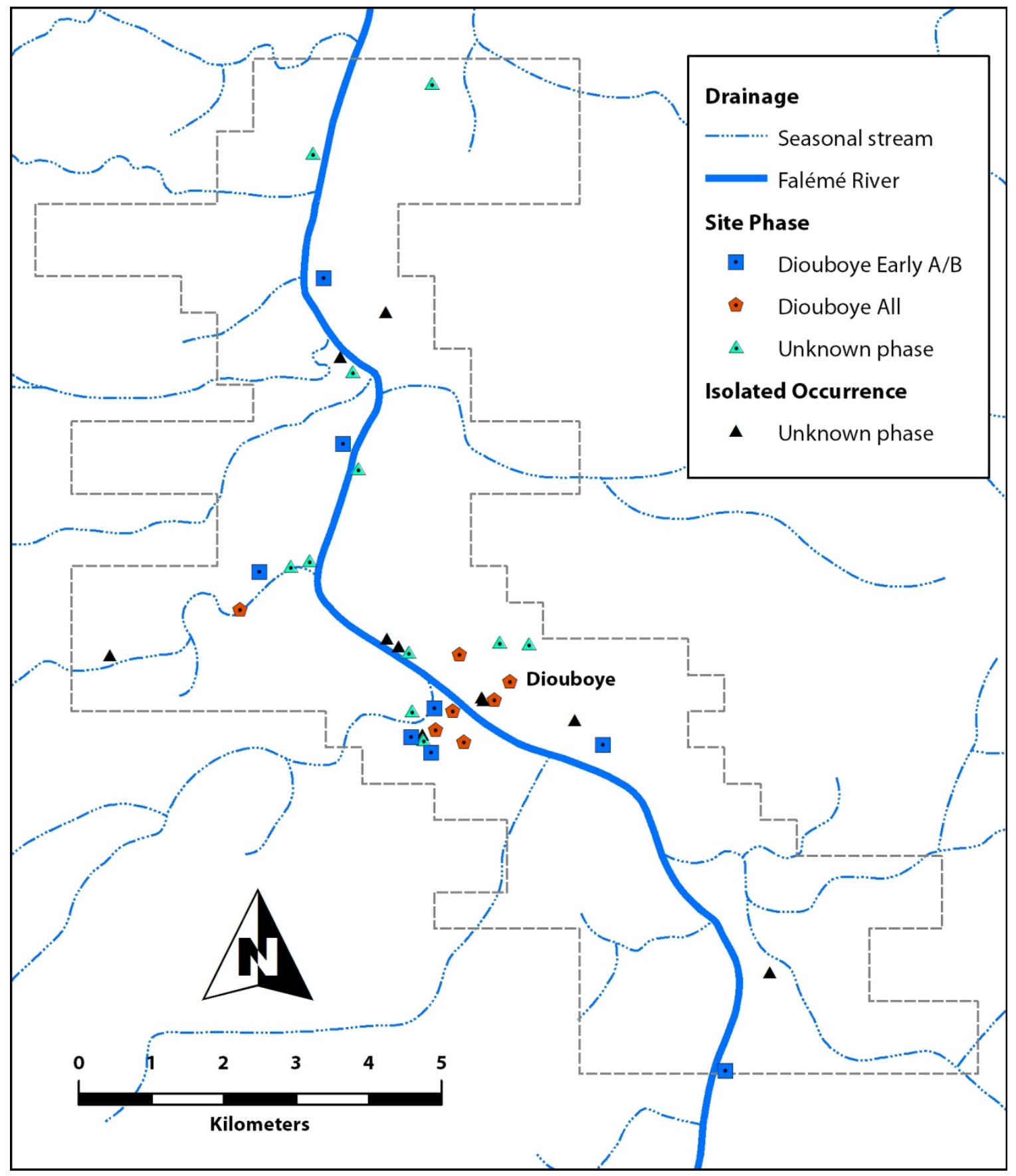


Figure 4.11: Iron Age (Middle/Late Diouboye phase) sites in the pedestrian survey zone

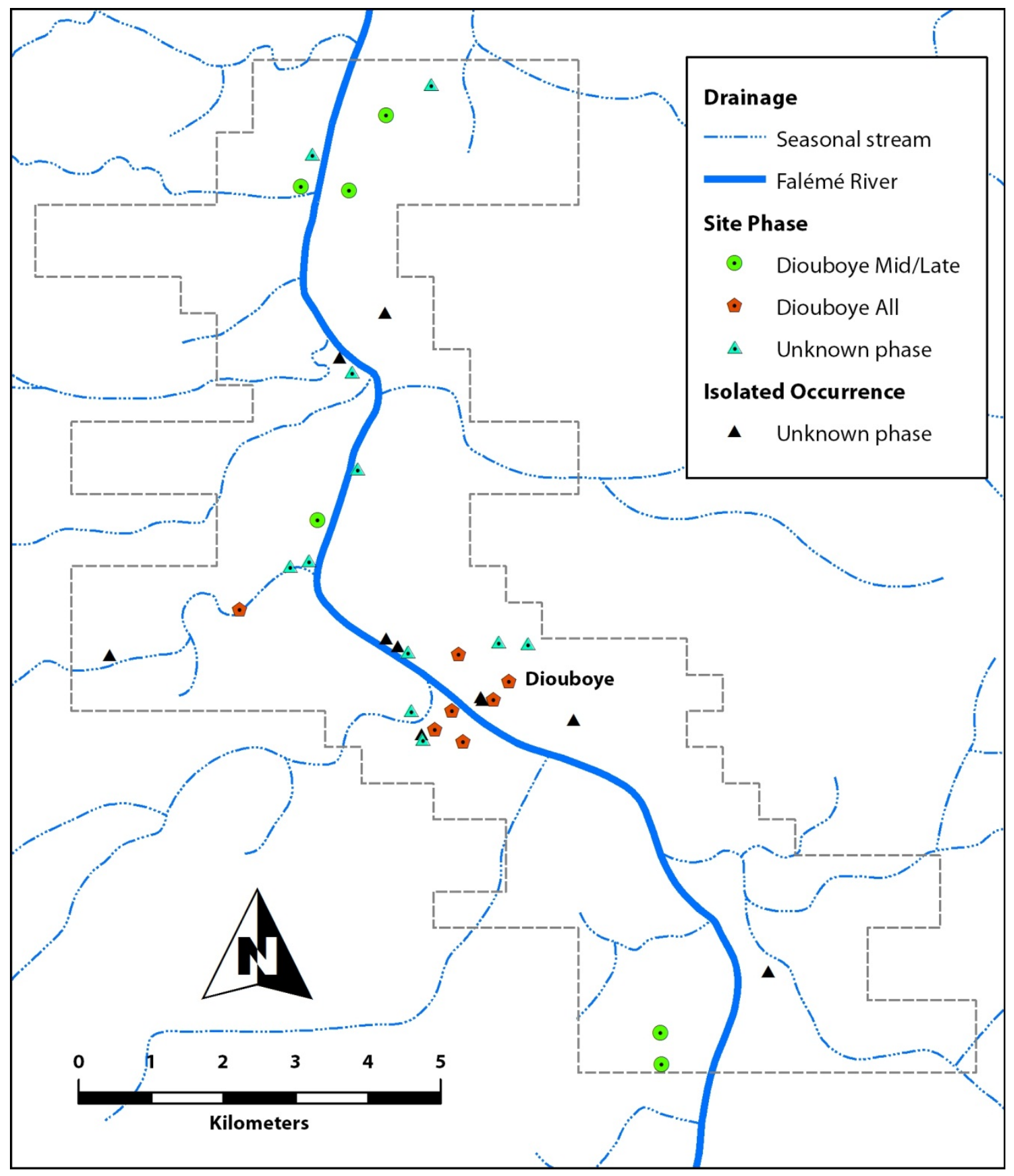


The Iron Age landscape charted in the course of survey reveals a substantial clustering of activity around Diouboye (Figures 4.10-4.11). Ten isolated occurrences consisted of small (46-328 $\mathrm{m}^{2}$ ) scatters of pottery sherds and occasional chipped stone debris, with one locale (FAL-IO-022) comprising a low-density array of sherds strewn over more than one half hectare. Sites of more intensive or long-term activity were substantially larger ( $468 \mathrm{~m}^{2}$ to $12 \mathrm{ha}$ ) and yielded a greater diversity of surface remains. Material and spatial variables (i.e., site size and artifact diversity) together formed the basis for the following classification of places within the Iron Age landscape as settlements and other logistical sites.

Settlement sites $(\mathrm{n}=21)$ had evidence for domestic architecture in the form of stone granary bases or similar features, coupled with modest to high densities of artifacts. These included groundstone tool fragments, chipped stone debris, iron tools and ornaments, and pottery suited to a variety of subsistence tasks including water storage and cooking (Table 4.2). Sometimes these sites occurred as low mounds consistent with long-term occupation and refuse disposal. As shown in Figure 4.12, these settlements fell into three size classes that I have termed homesteads ( $<1.5$ ha), hamlets ( 1.5 to $4.0 \mathrm{ha}$ ), and villages ( $>4.0$ ha). Notably, I excluded Tomboura East 1 (FAL-1126) from sizebased analyses since a large Atlantic Era component at this site obscured the boundaries of the underlying Iron Age one.

Logistical sites ( $\mathrm{n}=11$ ) of repeated but indeterminate activity had modest densities of artifacts attributable to an Iron Age component, but lacked concrete evidence for permanent architecture. Because these sites resembled homesteads in both areal extent and artifact diversity (Table 4.2), it is possible that they derive from an even more ephemeral form of occupation such as seasonal field huts or pastoral encampments (R. J. McIntosh et al. in press). One site had possible pit furnaces of unknown date. Several of these logistical sites (as well as isolated occurrences) appeared more than two kilometers from the nearest settlement, suggesting they arose from specialized tasks such as hunting, collecting, mining, or perhaps even clandestine ritual.

While material and spatial relations among these sites highlight the central place of Diouboye within the local political economy, I postpone discussion of this pattern until the comparative analysis in the following chapter. For the moment, I simply call attention 
to the low density of Iron Age settlements along the lower Falémé River at a time when populations occupying the Sahel and the Niger, Senegal, and Gambia River basins became increasingly concentrated in towns. To be sure, the rock-laden soils of the Madina-Sadatou study area and persistence of tsetse into the early-second millennium AD were probably not conducive to supporting large agro-pastoral populations. But the villages that do exist, including Diouboye, Baganafara, and those Phase IVB sites identified by Thiaw (1999), appear to represent communities set apart from one another by distances of ten to twenty kilometers. If this settlement pattern holds true for future data, it would suggest that village communities maintained relatively symmetrical political relations with one another at a regional scale. I would further hypothesize that subtle differences in the resources available from local ecology, geomorphology, and proximity to trade routes or polities such as Takrur, Gajaaga, and Mali provided a basis for economic complementarity and cultural interaction among these communities.

Table 4.2: $\quad$ Summary of surface finds and functions of Iron Age sites

\begin{tabular}{|c|c|c|c|c|c|c|c|c|c|c|c|}
\hline \multicolumn{2}{|c|}{ Locale Type } & \multirow{3}{*}{$\begin{array}{c}\text { Area } \\
\text { ha } \\
\mu \\
\end{array}$} & \multirow{3}{*}{$\begin{array}{c}\text { Stone } \\
\text { Feature } \\
\text { present } \\
\end{array}$} & \multicolumn{4}{|c|}{ Lithic Tools } & \multicolumn{4}{|c|}{ Metallurgy } \\
\hline & Total & & & \multicolumn{2}{|c|}{ Chipped } & \multicolumn{2}{|c|}{ Ground } & \multicolumn{2}{|c|}{ Iron } & \multicolumn{2}{|c|}{ Slag } \\
\hline & $n$ & & & $n$ & $\%$ & $n$ & $\%$ & $n$ & $\%$ & $n$ & $\%$ \\
\hline Settlement & 22 & 2.0 & Yes & 16 & $73 \%$ & 16 & $73 \%$ & 6 & $27 \%$ & 7 & $32 \%$ \\
\hline Diouboye & 1 & 11.9 & Yes & 1 & $100 \%$ & 1 & $100 \%$ & 1 & $100 \%$ & 1 & $100 \%$ \\
\hline Baganafara & 1 & 8.8 & Yes & 1 & $100 \%$ & 1 & $100 \%$ & 1 & $100 \%$ & 1 & $0 \%$ \\
\hline Goundafa & 1 & 5.3 & Yes & 1 & $100 \%$ & 1 & $100 \%$ & 1 & $100 \%$ & 1 & $100 \%$ \\
\hline Hamlet & 5 & 2.3 & Yes & 3 & $100 \%$ & 5 & $100 \%$ & 1 & $20 \%$ & 2 & $40 \%$ \\
\hline Homestead & 14 & 0.4 & Yes & 10 & $71 \%$ & 10 & $71 \%$ & 2 & $14 \%$ & 2 & $14 \%$ \\
\hline Non-Settlement & 21 & 0.2 & No & 10 & $48 \%$ & 7 & $33 \%$ & 0 & $0 \%$ & 1 & $5 \%$ \\
\hline Logistical & 11 & 0.3 & No & 7 & $78 \%$ & 7 & $78 \%$ & 0 & $0 \%$ & 1 & $9 \%$ \\
\hline Isolated Occ. & 10 & 0.07 & No & 3 & $30 \%$ & 0 & $0 \%$ & 0 & $0 \%$ & 0 & $0 \%$ \\
\hline Total & 43 & 1.1 & - & 26 & $60 \%$ & 23 & $53 \%$ & 6 & $14 \%$ & 8 & $19 \%$ \\
\hline
\end{tabular}


Figure 4.12: Iron Age (Diouboye phase) settlement size distribution

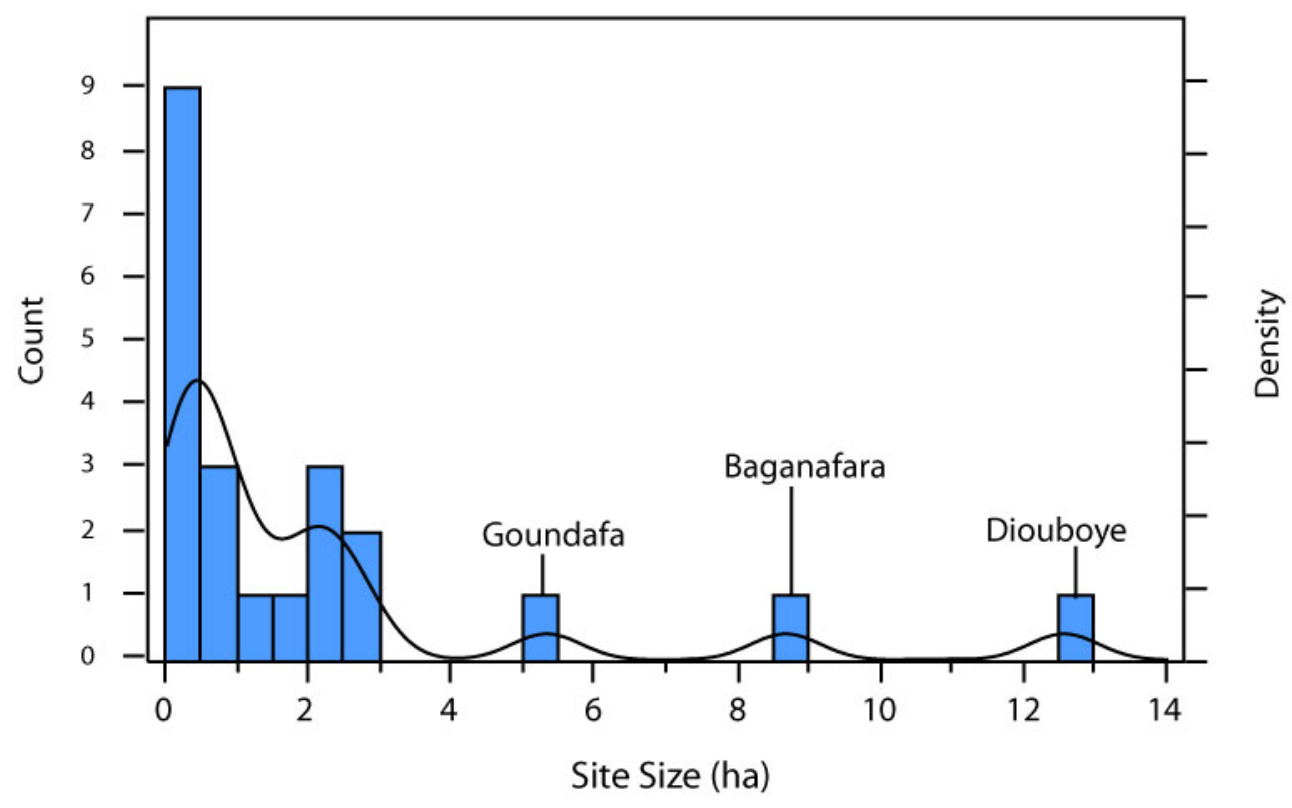

\section{Atlantic Era}

The Atlantic Era marks a tumultuous reorientation of political economies across West Africa that began with European contact in the late- $15^{\text {th }}$ century AD and ended with the colonial "scramble for Africa" at the end of the $19^{\text {th }}$ century AD. This period witnessed the waning hegemony of savanna states and the rising affluence of coastal polities facing outwards onto the evolving Atlantic world system (Thornton 1992). Drawing upon a wealth of oral and textual sources, historians have scrutinized and debated the major events and cultural economic processes of this era-including the Atlantic slave trade (Barry 1998; Curtin 1975a; Diouf 2003), the spread of Islam as a political and spiritual force (Drame 2009; Gomez 1992; Hanson 1996; Robinson 1985), and the construction of ethno-linguistic identities (Amselle 1998; Irvine 2008). Much of this recent research has generally worked to position African communities (or at least some communities) as active participants in the production of history, even as the Atlantic commoditization of gold, guns, and slaves shaped indigenous political economies and politics of identity across states and "stateless" societies alike. 
Over the past three decades, archaeologists in West Africa have begun to interpret the entangled histories of the Atlantic Era through diverse strands of material data (e.g., DeCorse 2001b; Ogundiran and Falola 2007). Reflecting global trends in historical archaeology, this research has considered landscapes and the built environment (K. G. Kelly and Norman 2007; Monroe 2010; Monroe and Ogundiran 2012; Richard 2007), the exchange and consumption of exotic goods (Ogundiran 2002; Richard 2010; Stahl 2004b), the reorganization of craft economies and subsistence regimes (Gijanto 2011a, 2011b; Ogundiran 2009), and mortuary and religious practices (Norman 2009, 2011; Stahl 2008). Beyond their potential to situate the Atlantic conjuncture within the longuedurée of African history, these archaeological data offer a perspective on people too often “silenced” in historical narratives — peasants, women, craftspeople, and slaves (Thiaw 2011). In much the same vein, the potential dissonance within and between classes of historical and material evidence can help to tease out central tensions in the production of historical knowledge (Reid and Lane 2004; Schmidt 2006; Stahl 2001). At the same time, collaboration between ethnoarchaeology and archaeology has begun to address the production of contemporary social identities that accompanied these processes (Guèye 1998; Mayor 2010; Sall 2005; Thiaw 2010).

While archaeology along the Falémé River has already begun to contribute to Atlantic Era historiography in the Upper Senegal (Gokee 2011; Thiaw 1999, 2012), I focus here on the ways in which settlement, political economy, and ideology shaped the archaeological landscape circa AD 1500-1905. This exercise provides a baseline for interpreting material and spatial dimensions of the preceding Iron Age landscape in the next chapter.

\section{An Historical Sketch of the Upper Senegal Region (AD 1500-1905)}

When Portuguese explorers sailing down the African coast began to travel up the Senegal River in the late- $15^{\text {th }}$ century, they quickly recognized an important avenue for trade with inland regions, including the legendary gold fields of Bambuk (Curtin 1973; Hair 1984). In the following centuries, ever more frequent French and British mercantile expeditions along the Senegal and Gambia Rivers charted and wrote about the physical and social landscape of the Upper Senegal region, including the lower Falémé (Boucard 
1974 [1729]; e.g., Coste d'Arnobat 1789; Durand 1802; Jobson 1999 [1623]; Labat 1728; M. Park 1816; Raffenel 1856). At the same time, African scholars writing in Arabic participated in and reflected upon the unfolding processes of trade, political transformation, militarism, and religious conversion (Hunwick 2003), although such accounts are fairly sparse for the Upper Senegal (Gomez 1992:10). Oral traditions recorded during this and later periods provide further chronological resolution (e.g., I. D. Bathily 1969; N'Diaye et al. 1971) My objective in the following pages is not to critically assess these written and oral sources, a task best left to historians (A. Bathily 1989; A. F. Clark 1999; Gomez 1992), but rather to continue the schematic regional narrative left off previously at the end of the Iron Age.

Figure 4.13: Map of Senegambian polities and populations during the Atlantic Era

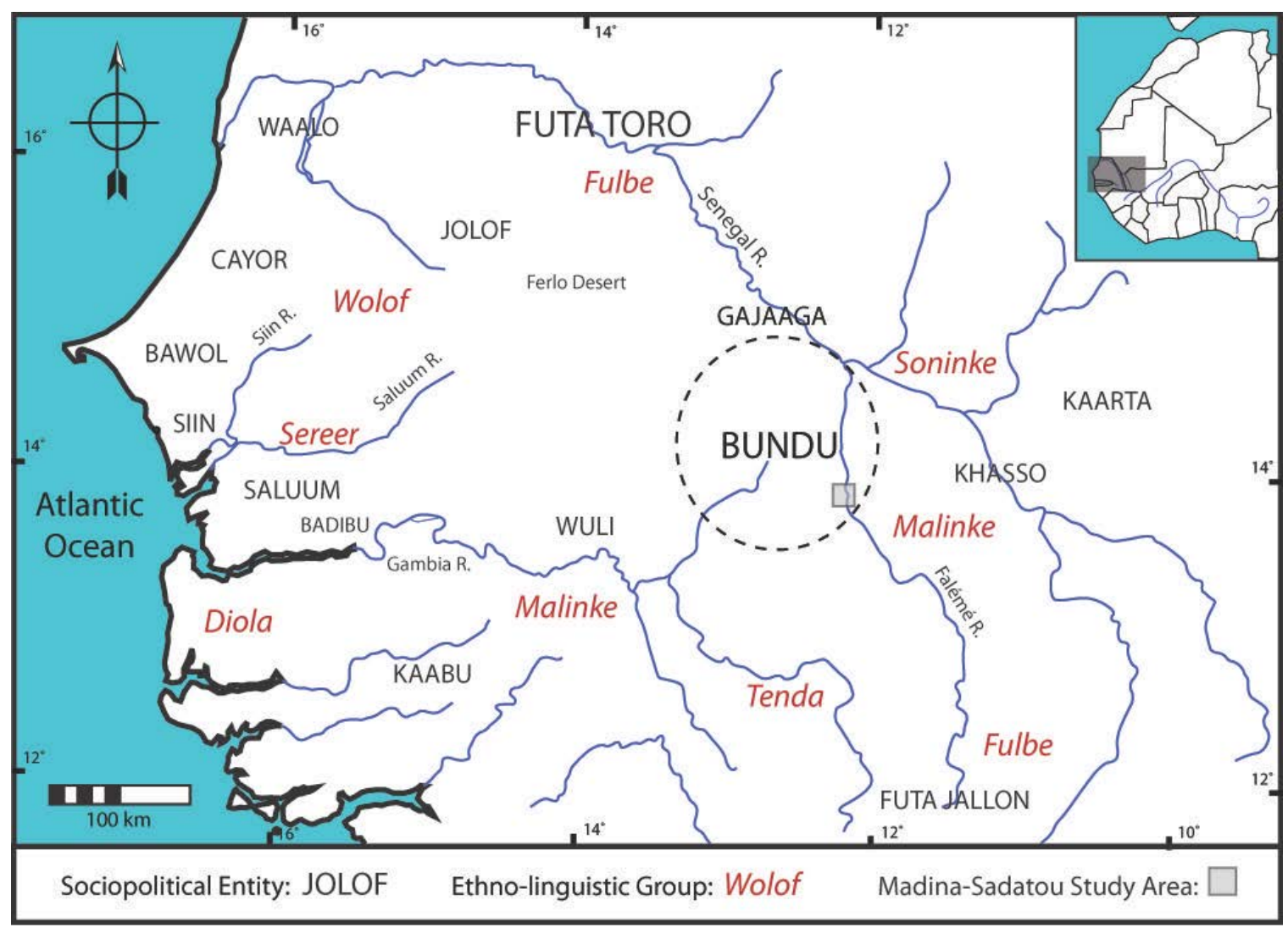


Throughout the $16^{\text {th }}$ and $17^{\text {th }}$ centuries $\mathrm{AD}$, internal and external stresses on Soninke-ruled states in the Upper Senegal, particularly Gajaaga, worked to reshape the political landscape. By the onset of this period, the Songhai Empire, centered at Gao on the Niger Bend, had superseded Mali as the major interregional power (Hunwick 2003), though its influence in Gajaaga appears to have been limited (A. Bathily 1989:237-238).

The disruption of trans-Saharan trade by conflicts between Songhai and Morocco, however, may have pushed merchants into trade networks oriented towards the Atlantic, resulting in an influx of iron, salt, alcohol, tobacco, beads, and firearms - traded for gold, gum, slaves, and other commodities (Curtin 1975a). Meanwhile, relations between sedentary and pastoral populations became increasingly tense, perhaps on account of ecological degradation across the Sahel and savanna (A. Bathily 1989:238-246; Webb 1995). With access to horses, and now guns, Fulbe leaders in the Middle Senegal region seized upon fomenting militarism to establish the polities of Futa Toro and Khasso, the latter wrested from Soninke control over the uppermost stretch of the Senegal River.

By the early-18 ${ }^{\text {th }}$ century AD, immigrant Fulbe led by the Muslim cleric Malik Sy carved out the southern part of Gajaaga to form Bundu, a theocratic polity (almamate) near the confluence of the Senegal and Falémé Rivers (Curtin 1975b; Gomez 1985; N'Diaye et al. 1971). According to historian Michael Gomez (1992), the formation and growth of Bundu over the next two centuries lay in the ability of Fulbe immigrants to forge a political dynasty among communities of diverse ethno-linguistic heritage and religious practice, in contrast with the militarism of other Fulbe states in Senegambia. Unlike the Sempera Bacili of Gajaaga, the dynastic rulers of Bundu were theocrats (almaami) whose authority found legitimacy through Islamic ideology. In this regard, local Jakhanke clerics greatly shaped the pragmatism of the state and tolerance towards non-Muslims within it. Nevertheless, Bundu did employ jihad to justify military campaigns against the people of Bambuk, Wuli, and Tenda to the south (Gomez 1992:2631). Bundu engulfed the territory of Combredougou comprising the Madina-Sadatou study area (Figure 4.14) sometime during the mid- $18^{\text {th }}$ and early-19 ${ }^{\text {th }}$ centuries AD. Historical accounts of this expansion describe the people encountered in Madina-Sadatou as Malinke living in small villages caught within an ongoing conflict between Bundu and Farabana, a political center in Bambuk, for control over Niger-Gambia trade. 
Figure 4.14: Map of the lower Falémé River in the early-18 ${ }^{\text {th }}$ century AD (redrawn from Labat 1728)

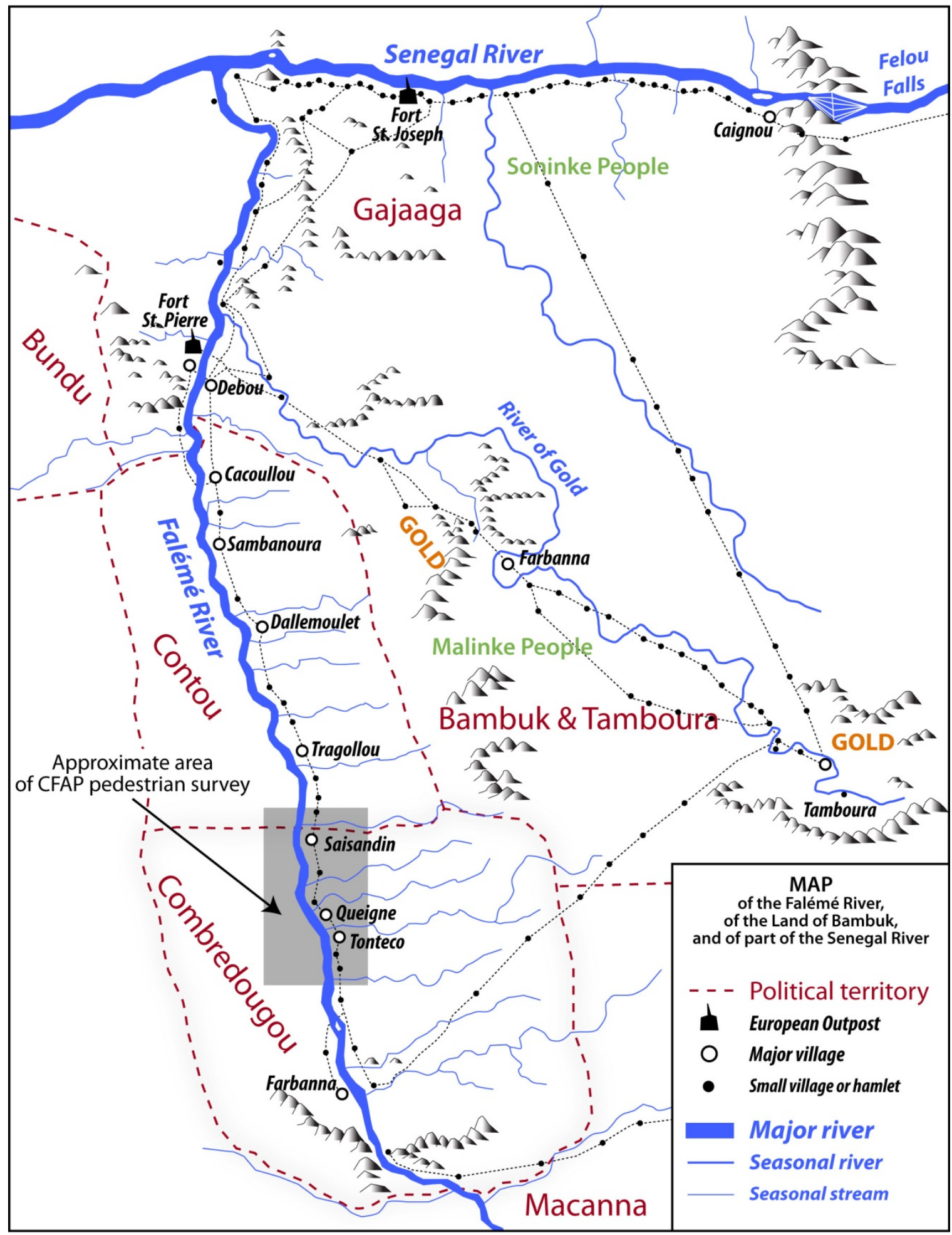


This conjuncture underscores the importance of Atlantic trade to the political economy of Bundu and other states across the Upper Senegal during this era. By the late$17^{\text {th }}$ century AD, French and British merchants had overcome the difficulties of seasonal navigation and began to build a series of outposts along the upper reaches of the Senegal and Gambia Rivers. In Bundu and Gajaaga, political elites acquired firearms and other valued commodities such as liquor not only through their control of the trade in (nonMuslim) slaves acquired from the south and east, but also through the production of surplus foodstuffs needed to sustain enslaved people prior to their shipment downriver to Atlantic ports (A. Bathily 1986; Gomez 1992). Of course, a political economy grounded in violence created stresses within and between these polities, thus fueling internecine conflict and regional fragmentation during the first half of the $19^{\text {th }}$ century AD, even as renewed French and British trading interests created opportunities for external alliancebuilding. European travelers to Bundu noted a growing number of fortified villages (tata) in a trend mirrored across Senegambia during this tumultuous period.

These divergent interests came to a head in the mid- $19^{\text {th }}$ century $\mathrm{AD}$ as a growing French influence shifted from the economic sphere to the political one (A. F. Clark 1996; Gomez 1992). The response in Futa Toro was the rise of militant Islam under al-Hajj Umar Tall, who from 1855 to 1857 fought the French and their allies for control of the eastern Senegambia (Robinson 1985). Many Fulbe factions within Bundu supported this rebellion with firearms traded from British holdings in the Gambia. Nonetheless, Umar Tall ultimately lost the conflict, precipitating his call for jihad against states along the Niger River. Passing through Bundu in 1858, Tall confiscated foodstuffs to support his military and encouraged several thousand people to join his campaign. ${ }^{6}$ Political stability returned to Bundu under the almaami Bokar Saada who, from 1860 to 1885, signed treaties with the French and (over)taxed the local populace to wage war against adjacent polities. The more stable period leading up to the formalization of French colonial rule in 1905 witnessed the return of many people who had left Bundu in the two or three decades prior (A. F. Clark 1994).

\footnotetext{
${ }^{6}$ During an initial visit to Diouboye, our guide from Sansanding, a Ful6e village, recounted how Umar Tall had burned the village to the ground after the community refused to offer food and shelter to his army.
} 


\section{Archaeological Landscapes and Ceramic Ethnoarchaeology}

As with the preceding Iron Age, the archaeological evidence for the mundane material practices that underlay the economic transformations and political intrigue of the Atlantic Era comes almost entirely from excavation and survey along the lower Falémé River by Ibrahima Thiaw (1999, 2012). Some 43 historic period sites—including several fortified tata and European êntrepots situated amid numerous ephemeral "plage” sitescluster in the northern and southern ends of the survey zone, possibly demarcating an uninhabited frontier between Gajaaga and Bundu circa 1700 to 1900 (Thiaw 1999:250). This temporal designation derives from the presence of independently dated tobacco pipes, metal nails, and glass beads (DeCorse et al. 2003), as well as "subactuelle” pottery similar to that found in historic contexts elsewhere across Senegambia (see below). These assemblages (Phase V) include short, everted vessel rims decorated with slip, twisted cord roulette, cordons and other appliqués, and fingertip and stick impressions; they usually exhibit some form of organic temper mixed with sand or grog. Another 15 settlement sites yielded Phase IVC pottery assemblages including long-neck jars decorated with twisted cord roulette, slip, and incisions or light channels bearing a strong resemblance to Soninke pottery in Mali (Gallay 1970) and loosely attributable to the period AD 1300-1600 (Thiaw 1999:177-178). The presence of slag at many of these sites may indicate the persistence of a local, albeit low intensity, iron industry.

In order to elucidate African and European interactions during this period, Thiaw opened four test units at the ruins of Tata Almamy and Fort Senoudebou (1999:114-122). This latter had been built and occupied by the French from 1845 to 1862, after which it was conceded to almaami Bokar Saada and his entourage (Gomez 1992:148). Largely confirming historical accounts, excavations turned up cattle and sheep remains, tobacco pipes, musket balls and gunflints from both French and British sources, machine cut and early wire nails, glass beads, and wine and champagne bottle fragments. These latter are not surprising in light of the initial French occupation and the lifestyle of Bokar Saada, who "esteemed" wine and generally "lived beyond the boundaries of shari'a" (Gomez 1992:151). The recovery of Phase V pottery from excavated contexts corroborates a ceramic chronology in which this tradition immediately predates ones documented ethnographically. 
In this regard, a comparison of ethnographic pottery production and consumption in the Middle and Upper Senegal regions illuminates social processes both present and past. Ethnoarchaeological surveys by N. Sokhna Guèye $(1998,2002)$ and Agnès Gelbert (1999, 2001, 2003) have defined two fairly discrete pottery traditions, each made by women from an endogamous class of craftspeople (Chapter 2). In Futa Toro (Middle Senegal region), Fulbe potters temper their clay with grog and animal dung, apply coils above a hollowed lump of clay, squeeze the lightly everted rims into shape, and decorate their jars with red slip, incisions, and appliqués. This pottery tradition bears a strong resemblance to the "subactuelle" pottery recovered from excavation and surface collections at historic sites (ca. AD 1600-1900) occupied by sedentary and pastoral Fulbe. According to Guèye (2002:30), this could point to the emergence of specialized production and exchange and restricted knowledge transmission within the endogamous networks of craftspeople known today.

In Gajaaga and Bundu, diverse Soninke, Fulbe, and Malinke potters all temper their clay with grog and straw, apply coils above a molded base, vertically scrape the everted rims into shape, and decorate their jars with incisions, rocker stamping, and a dark, organic coating produced from baobab leaves (Gelbert 1999, 2001, 2003); this tradition bears greater resemblance to the Phase V or "subactuelle" pottery assemblages from the lower Falémé. By considering social parameters on the transmission of techniques such as temper and rim formation, Gelbert (1999:221-222) has concluded that this tradition developed in part through the actual immigration of Fulbe potters from Futa Toro into Gajaaga and Bundu. If this is the case, then the transition from Phase IVC to Phase V pottery observed by Thiaw (1999) may signal the demographic shift wrought by the expansion of Bundu along the Falémé River (Gokee 2011). At the same time, these ethnoarchaeological studies caution against the interpretation of ethnic identity from crafts whose underlying technologies intersect with political economies and cultural schema in complex ways.

\section{Atlantic Era Components in the Madina-Sadatou Study Area}

Surface assemblages assigned to the Atlantic Era had artifacts known to post-date European contact such as terracotta pipes, glass beads and bottles, iron nails, and metal 
coins (Figures 4.15-4.16) —all in association with pottery sherds documenting traditions of manufacture antecedent to the ethnographic ones described above (Figure 4.18). All told, this component appeared on 51 sites and 26 isolated occurrences in the pedestrian survey zone, and an additional four locales documented during guided survey. Almost two-thirds of these locales could be further assigned to one of three tentative phases of Atlantic Era occupation defined by temporally diagnostic surface finds (Table 4.3), test excavations at FAL-1075, and cross-checking against historical sources. These include an early Tontèko phase (AD 1500-1700), a late Karé phase (AD 1860-1925), and an intervening, more ambiguously dated, Tomboura phase (AD 1700-1860).

\section{Figure 4.15: Sample of beads from Karé (FAL-1038)}

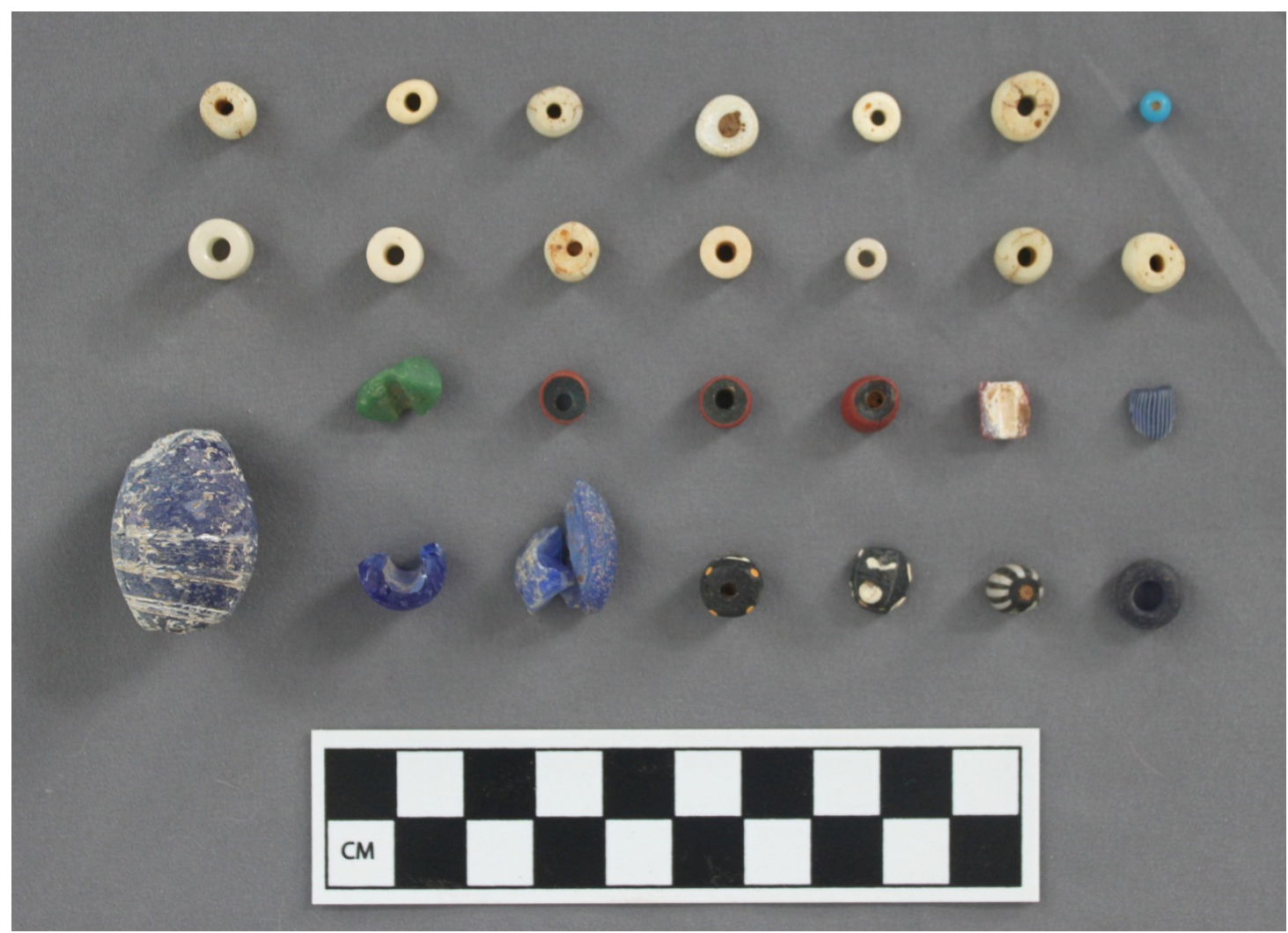


Figure 4.16: Atlantic Era miscellaneous artifacts: (a) smoking pipe stems; (b) spindle whorls; (c) silver coin; (d) gunflint; (e) iron projectile point; (f) hand-forged and machine-cut iron nails

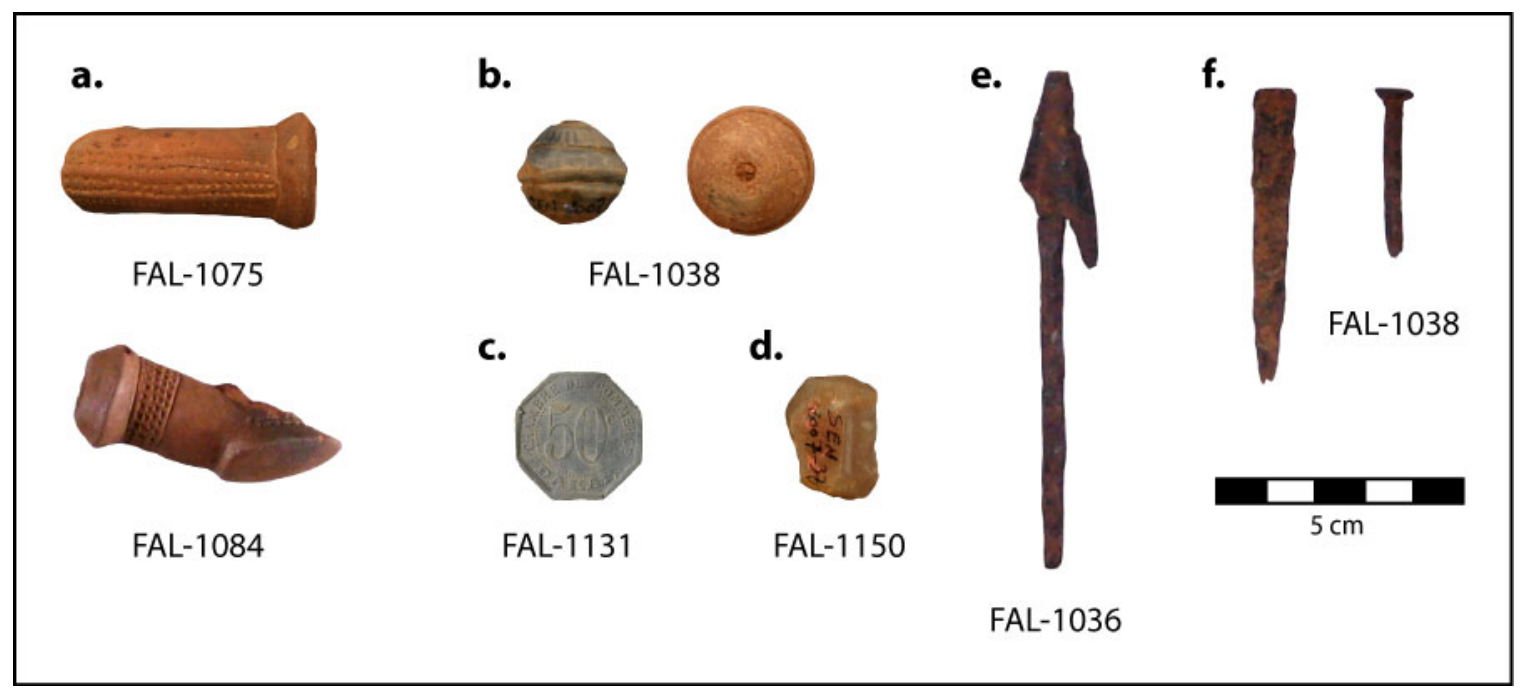

Figure 4.17: Toranga (FAL-1056) showing rectilinear granary foundations and granary (inset) from present-day hamlet

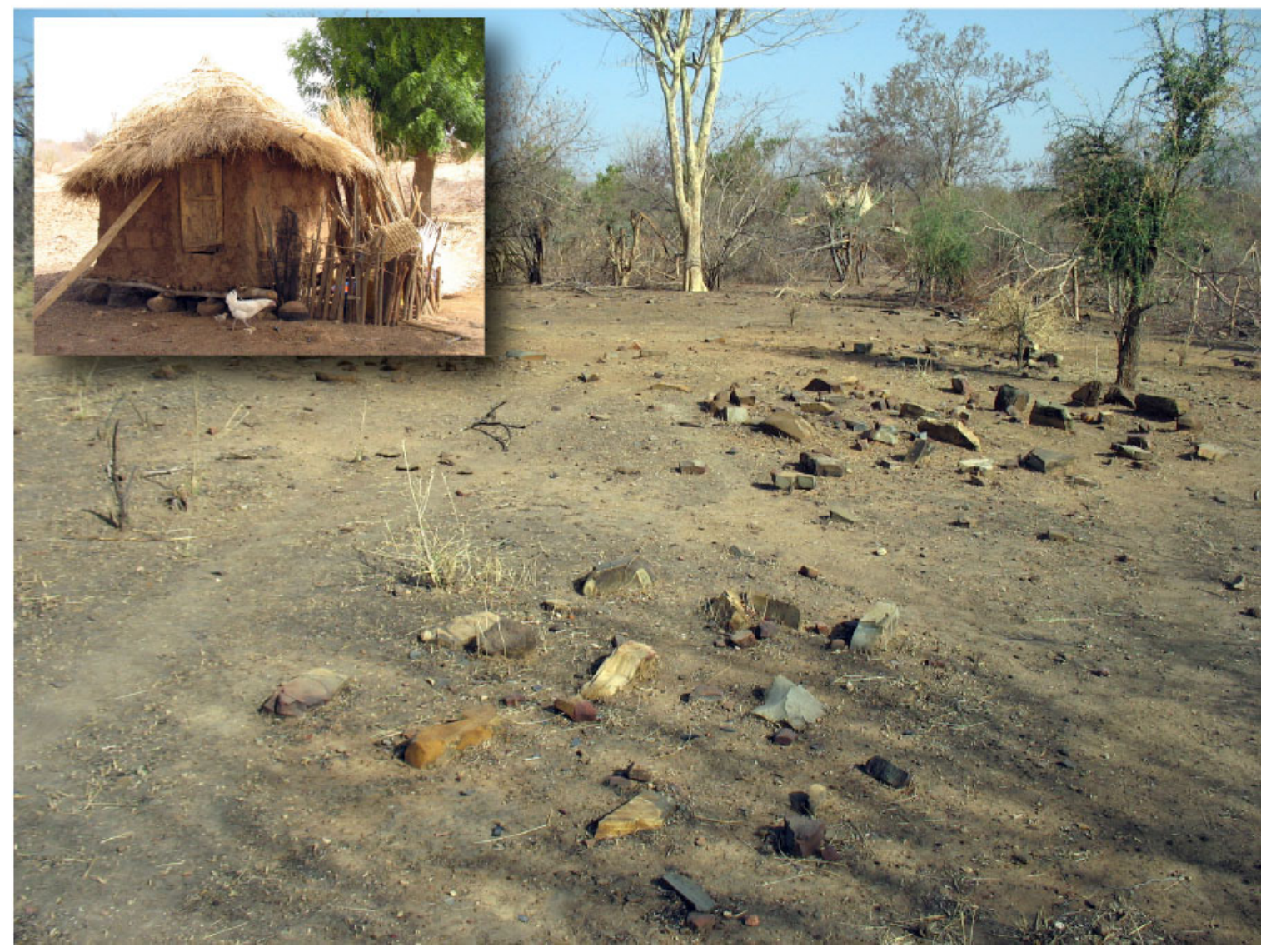


Figure 4.18: Atlantic Era pottery from surveyed sites

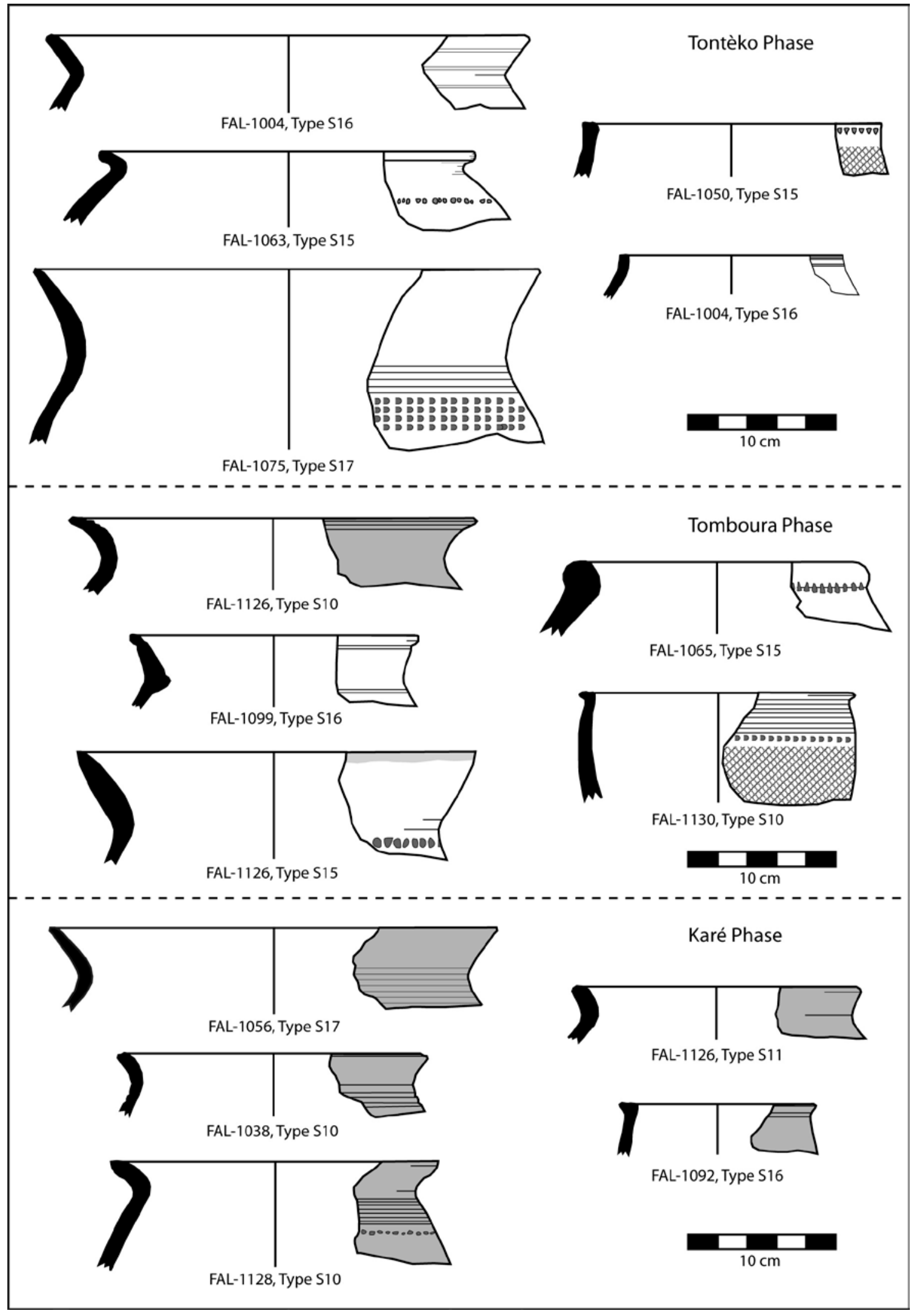


Table 4.3: Summary of sites seriated by temporally diagnostic surface finds

\begin{tabular}{|c|c|c|c|c|c|c|c|c|}
\hline \multirow[t]{2}{*}{ Site } & \multirow{2}{*}{$\begin{array}{c}\text { Ceramic } \\
\text { Phase }\end{array}$} & \multirow[t]{2}{*}{ Pipe } & \multicolumn{3}{|c|}{ Bead } & \multicolumn{2}{|c|}{ Nail } & \multirow[t]{2}{*}{ Coin } \\
\hline & & & 18th c. & 17-19th c. & Late 19th c. & Forged & Cut & \\
\hline F.1075 & Tontèko & 8 & & & & & & \\
\hline Tontèko & Tontèko & 2 & & & & & & \\
\hline F.1004 & Tomboura & 4 & 3 & & & 4 & & \\
\hline $\begin{array}{c}\text { Tomboura } \\
\text { East } 1\end{array}$ & Tomboura & & 1 & & & 2 & & \\
\hline $\begin{array}{l}\text { Tomboura } \\
\text { North }\end{array}$ & $\begin{array}{l}\text { Tomboura/ } \\
\text { Karé }\end{array}$ & 2 & & 64 & 4 & & 1 & "1926" \\
\hline Karé & Karé & & & 41 & 23 & 3 & 4 & "1924" \\
\hline Toranga & Karé & 1 & & & 1 & & 1 & \\
\hline
\end{tabular}

Figure 4.19: Atlantic Era settlement site size distribution

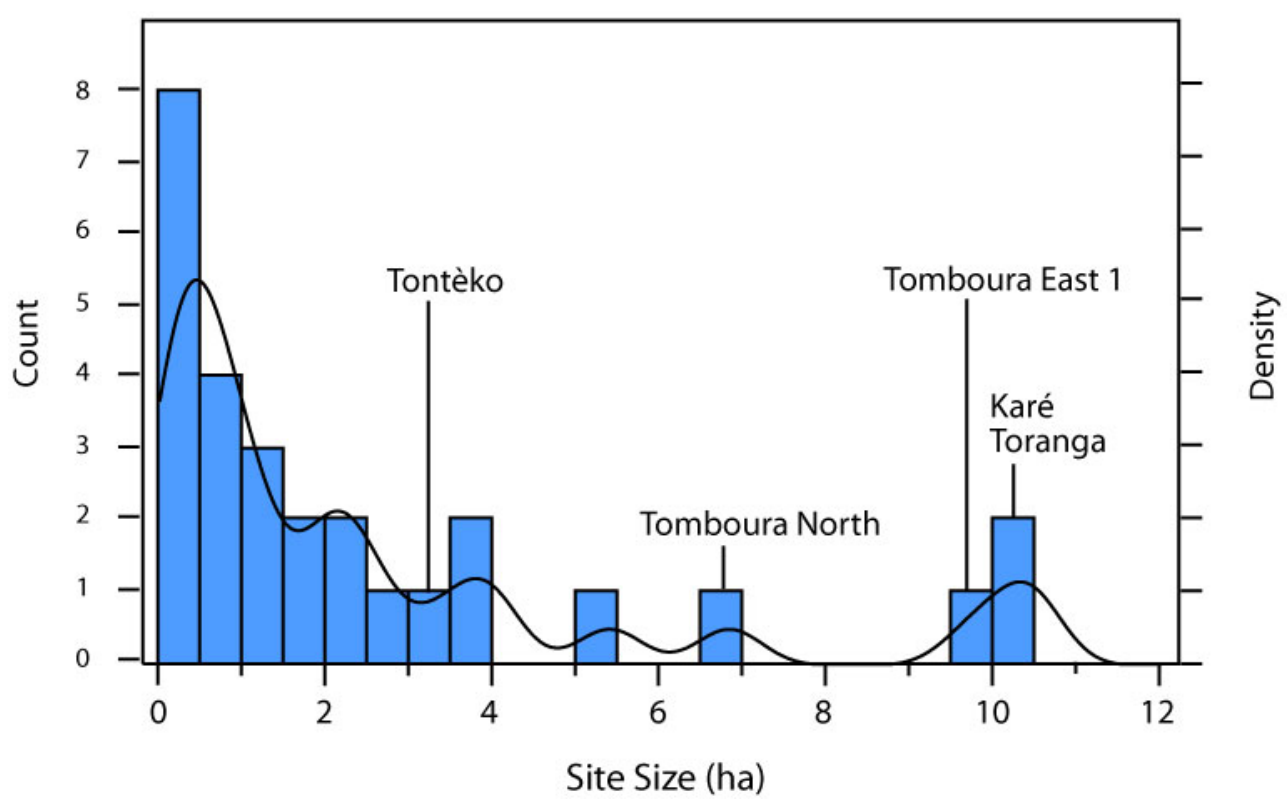

The Atlantic Era occupation produced logistical “plage” sites from short-term occupation or repetitive tasks in addition to relatively sedentary settlements. These latter had rectilinear stone granary foundations (similar to those in present-day villages), modest densities of pottery, and diverse metal and glass artifacts. Figure 4.19 shows the distribution of three size classes defined for settlements: homesteads ( $<1.5$ ha), hamlets 
(1.5-4.0 ha), and villages (> 4.0 ha). Logistical sites had modest densities of artifacts attributable to the Atlantic Era, but lacked evidence for permanent architecture. One site with tentative pit furnaces may also date to this period.

\section{Tontèko Phase (AD 1500-1700)}

Tontèko phase assemblages included terracotta smoking pipes, iron tools, and sherds with coarse grit inclusions and decorations of twisted cord roulette, incision, channeling, and comb impression (Appendix D). These sherds strongly resemble descriptions of Phase IVC pottery from the lowermost Falémé, possibly made by historic Soninke communities. Tontèko phase settlements contained limited frequencies of groundstone artifacts and remnants of domestic structures, including stone granary foundations and patches of burnt plaster and daub. Altogether, pedestrian survey identified this component at two isolated occurrences, six logistical sites, and six settlement sites ranging in size from small homesteads to the large hamlet ( $3 \mathrm{ha}$ ) of Tontèko itself (Figure 4.19). These sites formed three clusters spaced four to five kilometers apart along the river, a pattern consistent with small communities with generalized economies.

In order to complement these surface data and anchor the Tontèko phase more securely within a regional chronology, I opened two test units (1x1 m) in the southern half of FAL-1075, a Tontèko phase hamlet (described above for its cLSA component). One unit (S-1) exposed the collapsed remnants of a nearly complete pottery vessel visible eroding from the western edge of the site. This unit attained sterile soil at a depth of only 25 centimeters below surface, coincident with the deflated surface of cLSA occupation observed in the northern half of the site. A second unit (S-2) excavated next to a rectilinear stone granary foundation revealed a homogeneous stratum of compact silty clay loam with no signs of post-depositional disturbance and low frequencies of pottery sherds, faunal remains, and charcoal to a depth of 50 centimeters below surface. A charred wood fragment from Level 3 of this unit (40-50 cm below surface) yielded a

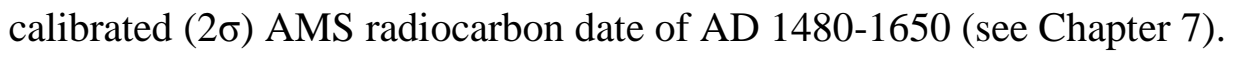

The shallowness of archaeological deposits at FAL-1075 mandates a cautious interpretation of this date, but it does coincide nicely with relative dating of the Tontèko 
phase based on the appearance of terracotta pipes in West Africa after the $16^{\text {th }}$ century AD (S. K. McIntosh et al. 2003; Philips 1983; Shaw 1960), and the absence of European goods that accompanied the expansion of Atlantic trade into the region during the $18^{\text {th }}$ century AD. Although FAL-1075 may not be the earliest Tontèko phase site in the Madina-Sadatou study area, this absolute date suggests a hiatus in local occupation following the abandonment of Diouboye. According to this scenario, which will require future investigation, the Tontèko phase settlements could represent a reoccupation of the Madina-Sadatou study area by Soninke, or more perhaps Jakhanke, communities as people steadily emigrated out of Gajaaga to take advantage of a slightly wetter climate, and better cultivation conditions, along the Falémé River.

\section{Tomboura Phase (AD 1700-1860)}

The Tomboura phase fills an ill-defined chronological space bracketed by the preceding Tontèko phase and subsequent Karé phase. And I would emphasize that the boundaries between these phases may owe as much or more to past spatial and social distinctions than to temporal change in material culture. Furthermore, the occupation of any given settlement could have straddled these analytical phases. That said, the Tomboura phase component included pottery sherds with channeled decoration, organic surface coating, and grit and grog inclusions. This component also included small numbers of hand-forged and early machine-cut iron nails and imported glass beads dated to the $18^{\text {th }}$ century AD. Pedestrian survey identified a Tomboura phase material component on three logistical sites and 11 settlements with deposits of collapsed daub and rectilinear granary foundations indicative of sedentary occupations; these settlements ranged from small homesteads to the village of Tomboura East 1 (9.7 ha). Tomboura phase sites cluster in the northern and southern ends of the survey zone, suggesting that two or three village-based communities occupied the survey area, a proposal that finds support from historic maps of the area (Figure 4.14). 
Figure 4.20: Atlantic Era sites in the pedestrian survey zone

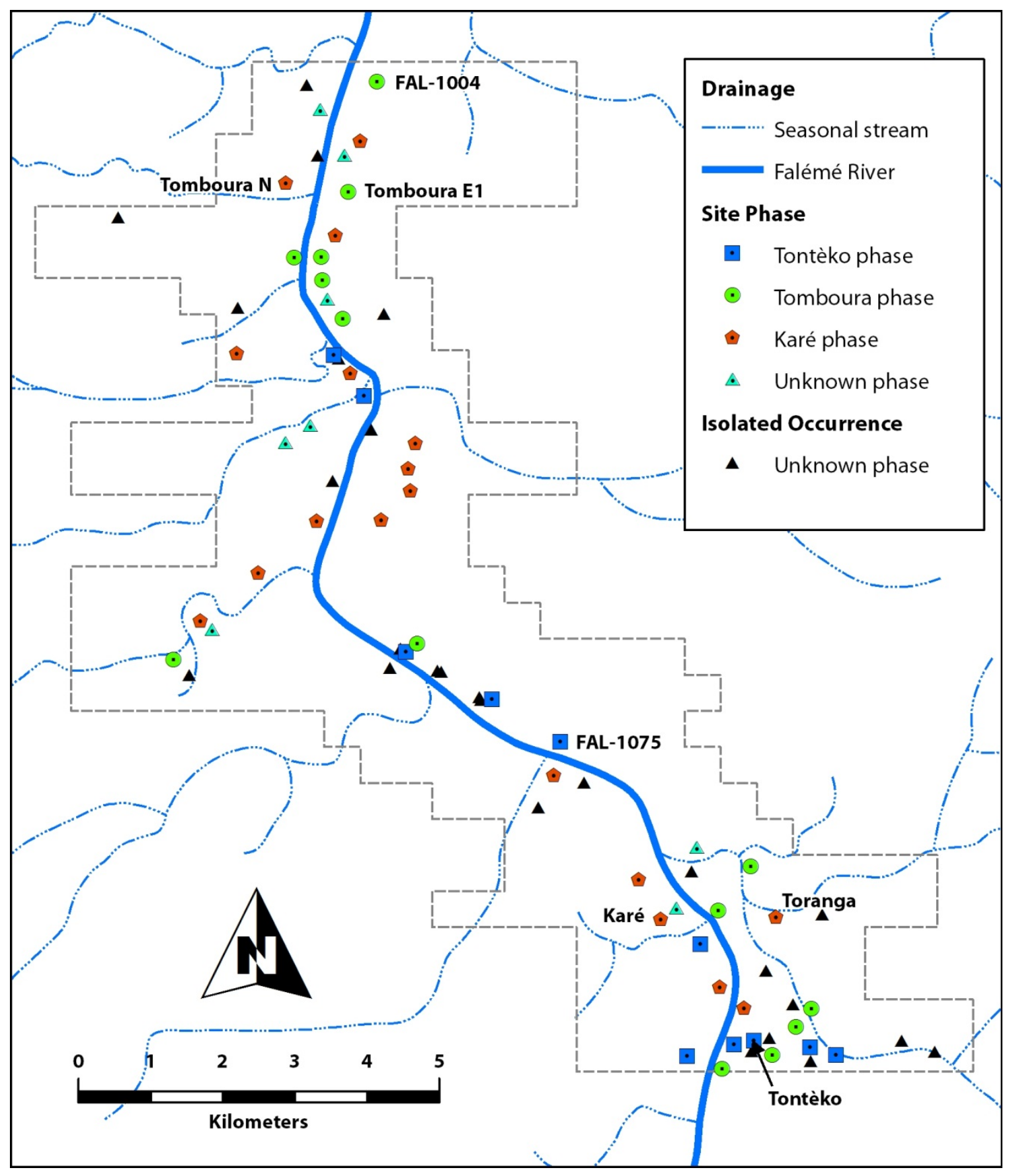

Karé Phase (AD 1860-1925)

The Karé phase component has a terminus post quem in the mid- $19^{\text {th }}$ century AD on the basis of imported bottles, Bohemian glass beads, machine cut and early wire nails, 
and even two colonial-era coins. The proposed transitional date of 1860 corresponds to the turbulent episode of conflict and depopulation that accompanied al-Hajj Umar Tall's passage through the area. Given a fuzzy boundary with the preceding phase, I lump together sites with a Karé or Tomboura phase component in this study; this also permits comparison with Phase V sites (1700-1900) to the north.

Local people identified several sites with Karé phase materials as villages occupied by their grandparents in the early- $20^{\text {th }}$ century AD (see also Opper and Opper 1990:30). It is therefore no surprise that our pedestrian survey identified a Karé phase component at numerous locales, including four isolated occurrences, seven logistical sites, and 11 settlements ranging from large homesteads to the village of Karé (10.4 ha). An additional three Karé phase settlements, including a fortified tata, were identified along the Kobakoye drainage during vehicular survey (Figure 4.1). Overall, these sites are slightly more dispersed than those in earlier periods, perhaps as larger, more stable, villages required frequent or sustained logistical trips for farming, hunting, herding, or gathering.

The material dimensions of Atlantic Era sites and spatial interrelations across the landscape of Madina-Sadatou highlight demographic change and perhaps a shift in the political economies of local village communities. Following the abandonment of Diouboye, the Tontèko phase occupation marks the resettlement of the study area by people whose craft economy links them to ethnographic and historic Soninke or Jakhanke communities. Alongside the expansion of Phase IVC settlement further to the north (Thiaw 1999), these sites could signal a long-term migration southward along the Falémé, coincident with the growth of Gajaaga. The material and spatial continuity of the Tomboura and Karé phases suggests that the expansion of the theocratic Bundu state did not entail a dramatic social rupture, although population densities in the Madina-Sadatou study area may have ebbed and flowed at scales beyond the resolution of the available archaeological data.

\section{SUMMARY}

The archaeological landscape of the Madina-Sadatou study area has emerged from a discontinuous sequence of occupation beginning in the Pleistocene epoch several 
hundred thousand years ago, and continuing until the present day. In order to situate the village community of Diouboye within this vast historical trajectory, this chapter has ordered data from pedestrian survey into a chronological sequence focusing on the past several millennia (mid- to late-Holocene). In this regard, I have made extensive use of well-dated archaeological evidence from neighboring regions and secondary historical sources. Although I have taken these historical narratives largely at face value, the rich material record of Madina-Sadatou suggests that finer resolution data, particularly for the Atlantic Era, could offer insights onto the production of frontiers by the regional polities of Gajaaga and Bundu and European mercantile interests—a task that Ibrahima Thiaw (2012) has already undertaken for the region around Senoudebou.

At a minimum, the chronology developed in this chapter suggests that the settlers of Diouboye arrived with a full-blown sedentary craft economy, and presumably subsistence regime; they were not the immediate descendants of the mobile foraging (and herding?) communities of the cLSA occupation. Although there appears to have been a hiatus in occupation following Diouboye's abandonment, affinities in material culture with Tontèko phase hamlets could suggest the ebb and flow of people belonging to the same general social milieu. Pots, of course, are not people, and this scenario certainly merits more rigorous investigation through future work at both Diouboye and Tontèko phase sites in the study area.

The data from pedestrian survey provide a point of departure for understanding how the Iron Age landscape of Diouboye fit into the political-economic mosaic of the Upper Senegal region and beyond. Affinities in material culture, including iron and pottery forms, suggest that the settlers of this village maintained ties to a sphere of interaction centered on the Pays Mande of the upper Niger River and along the Gambia Rivers to the south. The absence of distinctive channeled and cordoned pottery from the Middle Senegal region does not necessarily indicate lack of some interaction with peoples to the north, as a discussion of exchange items will reveal in Chapters 6-7. Rather, I would suggest that material dimensions of the survey data show that Diouboye participated in different sorts of economic and cultural relations with other communities at multiple scales. At the same time, local settlement dynamics reveal that Diouboye supported a political economy somewhat different than that of later villages enmeshed 
within the political networks of Gajaaga and Bundu. Interpreting the material and spatial dimensions of the Iron Age landscape can therefore benefit from comparison to the Atlantic Era one, and to the structure of resources such as land and water- the task of the next chapter. 


\section{CHAPTER 5 \\ DAILY LIFE IN DIOUBOYE: THE LOCAL LANDSCAPE}

In this chapter, I look more closely at the landscape experienced and exploited on a day-to-day basis by the community of Diouboye. My interpretation hinges upon a comparison with subsequent Atlantic Era settlements and, to a lesser extent, earlier LSA encampments in the same space. Referring to the regional geography presented in Chapter 3, I first consider the extent to which the spatial structure of physical landforms and hydrology, as potential economic resources, shaped settlement and other practices of place-making across these three horizons. I then explore the material (diversity, site size) and spatial dimensions (segregation, proximity) of the Iron Age and Atlantic Era landscapes presented in Chapter 4 to assess how resources-both material and nonmaterial-were moved and appropriated into the economy of Diouboye and nearby communities. Thus contextualized, I argue that the village of Diouboye, on the whole, maintained a privileged position in local institutional networks by mobilizing subsistence labor, specializing in craft production, and possibly controlling access to long-distance exchange. In comparison to Atlantic Era occupations in the region, I further suggest that Diouboye existed within a fairly decentralized landscape largely outside the control of contemporaneous polities such as Ghana, Gajaaga, Takrur, or Mali.

\section{COMPARATIVE LANDSCAPE DYNAMICS IN MADINA-SADATOU Geography and Resources}

As discussed in Chapter 3, the physical landscape of the Upper Senegal region has long supported a diverse suite of resources for hunting, herding, fishing, and farming, as well as for crafts such as wood and leatherworking, weaving, metalworking, and, of course, potting. The Senegal and Falémé Rivers and their tributaries further provide avenues for transhumance and trade across ecological zones ranging from the Sahel in the north to the equatorial forest in the south. I turn now to examine how the spatial 
distribution of the landforms and water affected settlement and economic tasks undertaken by people living in the Madina-Sadatou study area over the past millennium. I unfortunately lack data on the distribution of raw materials for chipped stone, groundstone, pottery, and perhaps metal production that likewise influenced locational movements within the craft economy, although one has the impression that the Falémé River bed provides a predictable source for most rocks and minerals.

Since the pedestrian survey zone encompassed fairly uniform terrain and vegetation, I assume that distance is proportional to the cost of travel between any two sites, or from a site across its catchment. Even as the Falémé River swells to impede foot travel during the rainy season, it provides a potential avenue for wooden pirogues-a technology cited by medieval geographers for transporting salt up and down the Senegal River (Al-Idrisi in Levtzion and Hopkins 2000:106-107). In my conversations with them, farmers from Goundafa claimed to have no trouble crossing the river daily to tend their fields directly adjacent to Diouboye during the wet season.

\section{Landforms}

The resource potential of the catchment surrounding a settlement is a function of its ecology and geomorphology. Because micro-ecology within the pedestrian survey zone along the Falémé River depends largely upon distance from the main channel, I consider this factor below alongside hydrology. Geomorphology, however, exhibited some spatial heterogeneity not wholly attributable to distance from the river. Using aerial imagery publically available on Google Earth (accessed 2008) with an ostensible resolution of 15 meters adequate for the following coarse-grained summary, I defined three landforms types in a GIS database: (1) river channel and embankment; (2) sediment terrace; and (3) laterite or bedrock terrace. The classification and distribution of these landforms were subject to "ground-truthing" with GPS in each transect during systematic survey in 2008. Since differential erosion and groundcover may have biased the visibility of archaeological sites across these landforms, the following comparisons across horizons make use of relative site frequencies and catchment patterns. 
Table 5.1: Summary of landforms within one-kilometer site catchments

\begin{tabular}{|c|c|c|c|c|c|c|c|c|c|c|}
\hline \multirow{2}{*}{\multicolumn{2}{|c|}{$\begin{array}{c}\text { Horizon } \\
\text { Type/Phase } \\
\text { n } \\
\end{array}$}} & \multicolumn{3}{|c|}{$\begin{array}{l}\text { Sediment Terrace } \\
\text { Area }\left(\mathrm{km}^{2}\right)\end{array}$} & \multicolumn{3}{|c|}{$\begin{array}{c}\text { Channel/Embankment } \\
\text { Area }\left(\mathrm{km}^{2}\right)\end{array}$} & \multicolumn{3}{|c|}{$\begin{array}{l}\text { Laterite/Bedrock } \\
\text { Area }\left(\mathrm{km}^{2}\right)\end{array}$} \\
\hline & & $\mu$ & $\sigma$ & $\%$ & $\mu$ & $\sigma$ & $\%$ & $\mu$ & $\sigma$ & $\%$ \\
\hline Random points & 214 & 2.44 & 0.73 & 77.6 & 0.12 & 0.13 & 3.6 & 0.59 & 0.77 & 18.8 \\
\hline Stone Age & 148 & 2.61 & 0.38 & $83.1 \%$ & 0.21 & 0.11 & $6.7 \%$ & 0.32 & 0.37 & $10.2 \%$ \\
\hline MSA/LSA Sites & 51 & 2.52 & 0.43 & $80.3 \%$ & 0.20 & 0.09 & $6.2 \%$ & 0.42 & 0.42 & $13.5 \%$ \\
\hline MSA/LSA IO & 24 & 2.58 & 0.43 & $82.3 \%$ & 0.21 & 0.11 & $6.8 \%$ & 0.34 & 0.44 & $11.0 \%$ \\
\hline cLSA Sites & 64 & 2.67 & 0.31 & $84.9 \%$ & 0.22 & 0.11 & $7.1 \%$ & 0.25 & 0.29 & $8.1 \%$ \\
\hline cLSA IO & 9 & 2.77 & 0.26 & $88.3 \%$ & 0.22 & 0.13 & $7.0 \%$ & 0.15 & 0.32 & $4.7 \%$ \\
\hline Iron Age & 42 & 2.52 & 0.48 & $80.0 \%$ & 0.24 & 0.09 & $7.6 \%$ & 0.39 & 0.51 & $12.4 \%$ \\
\hline All Sites & 32 & 2.55 & 0.52 & $81.2 \%$ & 0.23 & 0.08 & $7.3 \%$ & 0.36 & 0.54 & $11.5 \%$ \\
\hline Early/Mid Dbye & 16 & 2.53 & 0.49 & $80.6 \%$ & 0.24 & 0.07 & $7.8 \%$ & 0.37 & 0.49 & $11.6 \%$ \\
\hline Mid/Late Dbye & 11 & 2.62 & 0.42 & $83.4 \%$ & 0.24 & 0.07 & $7.6 \%$ & 0.28 & 0.41 & $9.0 \%$ \\
\hline $\mathrm{IO}$ & 10 & 2.42 & 0.35 & $77.1 \%$ & 0.25 & 0.10 & $8.1 \%$ & 0.46 & 0.39 & $14.8 \%$ \\
\hline Atlantic Era & 77 & 2.59 & 0.46 & $82.7 \%$ & 0.21 & 0.11 & $6.7 \%$ & 0.33 & 0.50 & $10.5 \%$ \\
\hline All Sites & 51 & 2.66 & 0.25 & $84.7 \%$ & 0.22 & 0.11 & $6.9 \%$ & 0.27 & 0.27 & $8.5 \%$ \\
\hline Tontèko & 11 & 2.46 & 0.29 & $78.2 \%$ & 0.24 & 0.12 & $7.7 \%$ & 0.44 & 0.36 & $14.1 \%$ \\
\hline Tomboura/Karé & 32 & 2.71 & 0.20 & $86.2 \%$ & 0.21 & 0.10 & $6.6 \%$ & 0.23 & 0.22 & $7.3 \%$ \\
\hline IO & 22 & 2.57 & 0.70 & $79.1 \%$ & 0.21 & 0.13 & $6.4 \%$ & 0.47 & 0.76 & $14.5 \%$ \\
\hline
\end{tabular}

Table 5.1 summarizes the relative proportions for these three landforms within one-kilometer catchments of each site and isolated occurrence documented on survey; half-kilometer and two-kilometer catchments yielded similar results. Not surprisingly, sites were preferentially located on sediment terraces and near the river channel. A twotailed Kolmogorov-Smirnov (K-S) test of the difference between cumulative distributions of two non-parametric samples (see Harrower 2010), found site catchments for all horizons to contain significantly less laterite/bedrock $(Z=1.89, \rho=0.002)$ and significantly more sediment terrace $(Z=1.64, \rho=0.009)$ and river channel $(Z=3.72, \rho<0.001)$ than those of the random sample of 214 points spaced at least 50 meters across the survey zone.

Interestingly, settlement size only relates weakly to amounts of sedimentary (cultivable) terrace for communities engaged in food production during the second millennium AD. Using catchments adjusted by Thiessen polygons to approximate boundaries between settlement sites for each horizon (Figure 5.1), one finds a similar 
Figure 5.1: Thiessen-adjusted catchment zones for Iron Age settlement sites

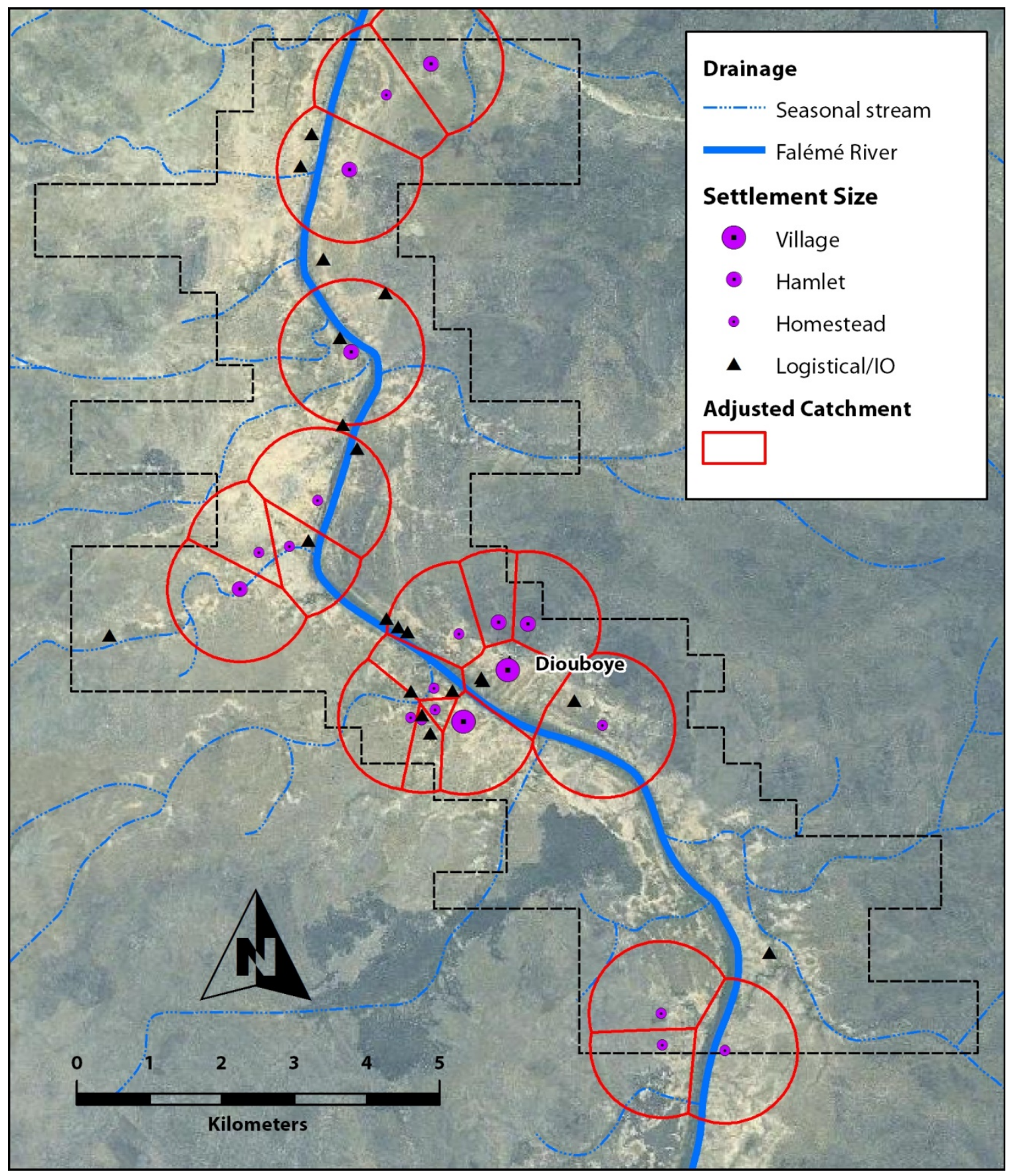


positive correlation between site size, as a proxy for population, and access to cultivable landforms in both the Iron Age and Atlantic Era (Figure 5.2). With a conservative estimate of 25 people per hectare of site area, and 0.5 hectares of land per person required for millet cultivation without fallow (Gallagher 2010:96), one can model the minimal amount of cultivable land required to support a sedentary population at each settlement. All sites above and to the left of this threshold in Figure 5.2, including most homesteads and hamlets, could have sustained themselves by cultivating land within the one kilometer from the settlement, if not closer.

\section{Figure 5.2: $\quad$ Regression of sedimentary terrace area by settlement size}

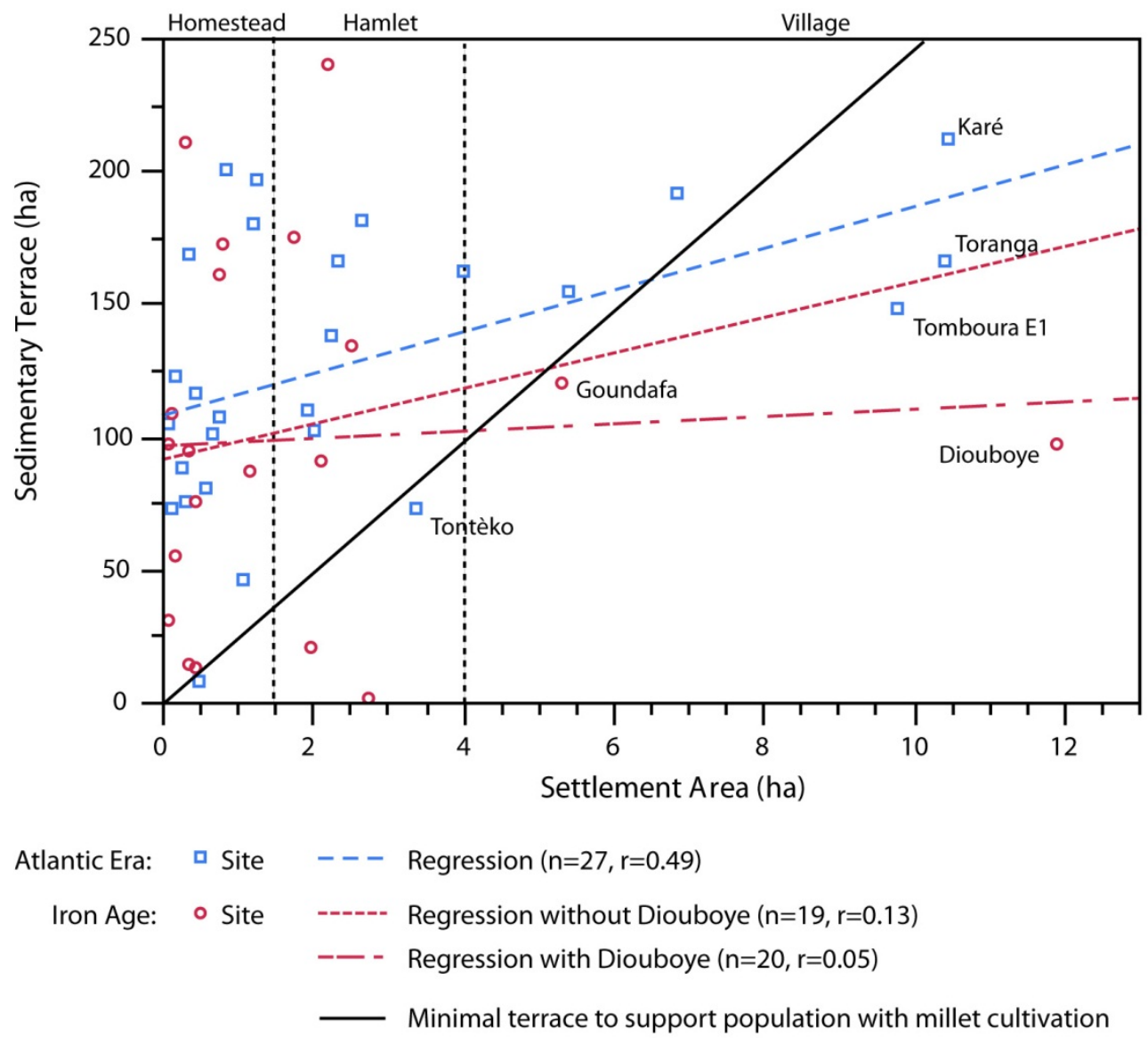


Bounded to the east by a ridge of eroded laterite and sandstone, Diouboye has relatively limited cultivable area for its size compared to other sedentary sites in the study area. This suggests that maximizing cultivable land was not a prime factor in village settlement and growth, meaning that the community at its peak must have relied upon the production and/or acquisition of food beyond millet cultivation in its catchment. Several scenarios are equally feasible: a significant reliance on pastoral or wild foodstuffs; the clearance of fields more than one kilometer from the village; seasonal movement out to homesteads or logistical sites (possible field houses); or the mobilization of staples from outlying settlements through kinship or other corporate relations. None of these scenarios are mutually exclusive. Whatever the case, people living at Diouboye must have engaged in relatively greater locational movement of subsistence resources than their compatriots in nearby settlements, including that of Goundafa across the river.

\section{Hydrology}

The foregoing consideration of catchment productivity as a function of a single landform, namely sediment terraces, does not consider the potential importance of seasonal hydrology. A number of seasonal channels transect the Madina-Sadatou region, but the Falémé River is the most prominent hydraulic feature within the north-south corridor of the pedestrian survey area. As discussed in Chapter 3, the river today flows nine months per year, but some low-lying basins provide a perennial source of water for wild game, fish, herds, and people. In the absence of higher resolution data on groundcover, distance from the river also provides a proxy measure for relative access to resources from riparian bush and savanna-forest habitats. Again not surprisingly, Table 5.2 shows that sites in the pedestrian survey zone generally lie within one half to one kilometers of the Falémé River (Rank 1) or its seasonal tributaries (Ranks 2-3), a significantly shorter distance than found for the random sample of points $(Z=3.77$, $\rho<0.001)$. Although erosion may enhance site visibility near the riverbed, this potential bias is partially offset by the greater visibility of sites on exposed bedrock and laterite terraces located some distance away. 
Table 5.2: $\quad$ Summary of distance to Falémé River and nearest channel

\begin{tabular}{|c|c|c|c|c|c|c|c|c|}
\hline \multicolumn{2}{|c|}{ Horizon } & \multirow{2}{*}{\multicolumn{2}{|c|}{$\begin{array}{c}\text { Falémé River } \\
\text { Distance (m) }\end{array}$}} & \multicolumn{5}{|c|}{ Nearest Channel } \\
\hline & Phase & & & \multicolumn{2}{|c|}{ Distance (m) } & \multicolumn{3}{|c|}{ Rank (\% of row) } \\
\hline & $\mathrm{n}$ & $\mu$ & $\sigma$ & $\mu$ & $\sigma$ & 1 & 2 & 3 \\
\hline Random Points & 214 & 1175 & 829 & 439 & 377 & $33.0 \%$ & $6.1 \%$ & $60.9 \%$ \\
\hline Stone Age & 148 & 572 & 562 & 231 & 178 & $54.7 \%$ & $10.1 \%$ & $35.2 \%$ \\
\hline MSA/LSA Sites & 51 & 617 & 561 & 259 & 221 & $45.1 \%$ & $7.8 \%$ & $47.1 \%$ \\
\hline MSA/LSA IO & 24 & 647 & 594 & 241 & 187 & $50.0 \%$ & $4.2 \%$ & $45.8 \%$ \\
\hline cLSA Sites & 64 & 483 & 474 & 202 & 135 & $62.5 \%$ & $14.1 \%$ & $23.4 \%$ \\
\hline cLSA IO & 9 & 755 & 957 & 260 & 137 & $66.7 \%$ & $11.1 \%$ & $22.2 \%$ \\
\hline Iron Age & 42 & 474 & 499 & 270 & 260 & $61.9 \%$ & $11.9 \%$ & $26.2 \%$ \\
\hline All Sites & 32 & 429 & 315 & 272 & 274 & $59.4 \%$ & $12.5 \%$ & $28.2 \%$ \\
\hline Early/Mid Dbye & 16 & 417 & 279 & 228 & 166 & $56.3 \%$ & $12.5 \%$ & $31.3 \%$ \\
\hline Mid/Late Dbye & 11 & 362 & 265 & 237 & 195 & $72.7 \%$ & $9.1 \%$ & $18.2 \%$ \\
\hline $\mathrm{IO}$ & 10 & 620 & 872 & 262 & 223 & $70.0 \%$ & $10.0 \%$ & $20.0 \%$ \\
\hline Atlantic Era & 77 & 585 & 624 & 218 & 179 & $57.1 \%$ & $5.2 \%$ & $37.7 \%$ \\
\hline All Sites & 51 & 495 & 462 & 211 & 175 & $58.8 \%$ & $7.8 \%$ & $33.3 \%$ \\
\hline Tontèko & 11 & 409 & 484 & 155 & 115 & $63.6 \%$ & $0.0 \%$ & $36.4 \%$ \\
\hline Tomboura/Karé & 32 & 514 & 461 & 242 & 204 & $59.4 \%$ & $9.4 \%$ & $31.3 \%$ \\
\hline $\mathrm{IO}$ & 22 & 762 & 840 & 232 & 189 & $53.8 \%$ & $0.0 \%$ & $46.2 \%$ \\
\hline
\end{tabular}

As shown in Figure 5.3, the distribution of settlements relative to the Falémé River was similar for both periods, with a bimodal distribution of settlements falling within 500 meters of the Falémé, or at some distance of 500-1000 meters, generally along one of the larger seasonal tributaries. Although the relationship between settlement size class (i.e., homestead, hamlet, village) and distance to the Falémé River did not differ significantly between Tontèko and Tomboura/Karé phase components $(\mathrm{Z}=0.63$, $\rho=0.822$ ), there was a significant shift between the Iron Age and Atlantic Era occupations $(\mathrm{Z}=1.37, \rho=0.048)$. The reasons underlying the move of Atlantic Era villages back from the main river channel are unknown, but could include a move to increase cultivable land near the river, changes in the landscape from erosion, or to distance communities from mosquitos (malaria), dangerous animals, and/or foreign aggressors. 
Figure 5.3: Boxplot of distances to the Falémé River for Iron Age and Atlantic Era settlement classes

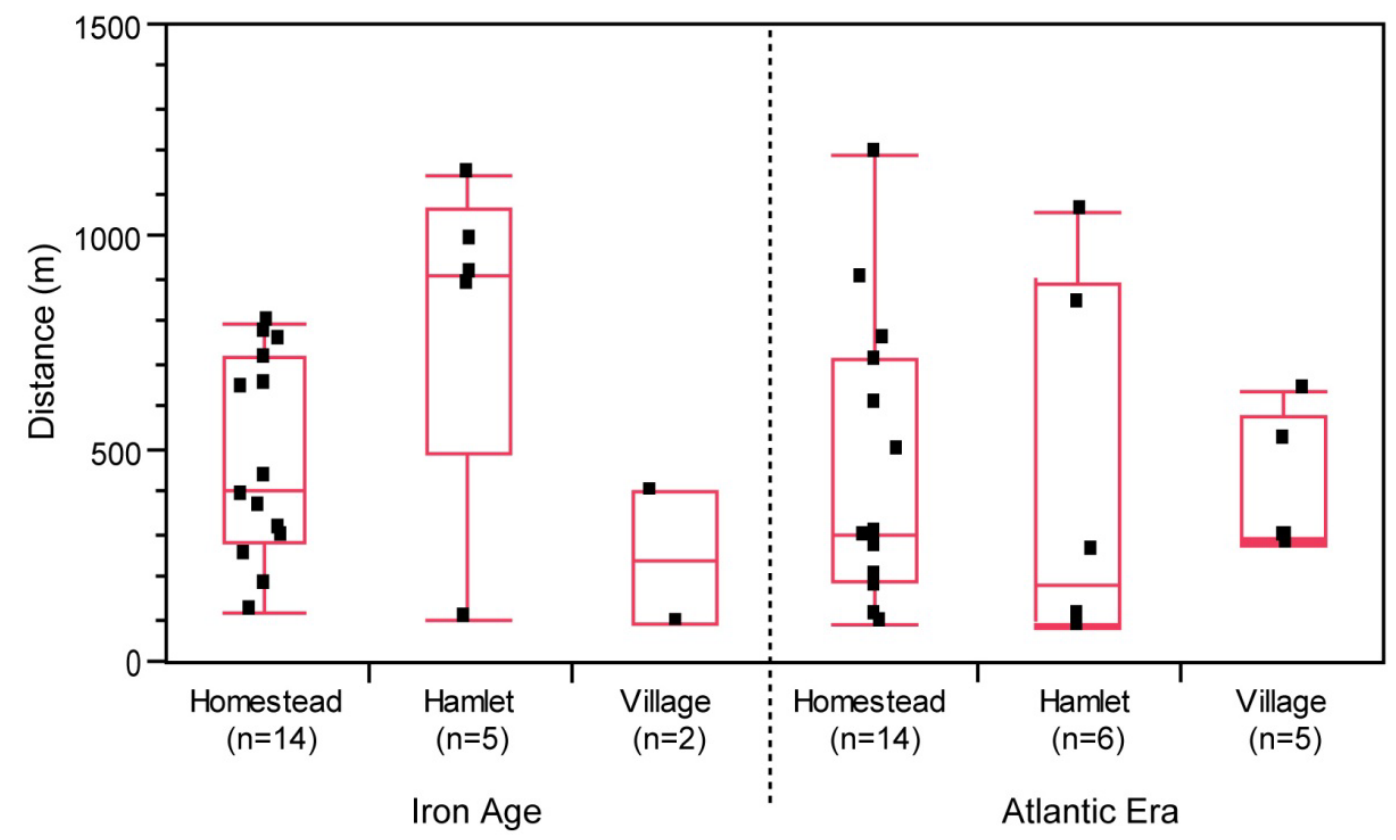

\section{Social Landscape}

With this summary of material resources afforded by local geomorphology, I turn now to map the contours of institutions shaping economic practices across the Iron Age landscape by considering architectural and artifactual aspects of sites, and their spatial interrelations, in the study area. First, I examine the diversity of these artifact assemblages, particularly pottery, in order to evaluate the range of subsistence, craft, and long-distance exchange activities undertaken at different locales. I then use distributions of settlement size, which presumably approximate the scale of social groups occupying them, to think about the distribution of non-material (social) resources across the landscape, and their potential appropriation into local economies. Finally, I consider how spatial ordering of these economic processes underwrote symmetrical and asymmetrical political relations among the people residing at neighboring settlements. 


\section{Artifact Diversity}

Documenting the diversity of material artifacts at a site or other locale provides a well-established method for drawing inferences about the diversity or complexity of economic activities, provided that one can adequately control for sample size and other biases (Baxter 2001; Cochrane 2003; Conkey 1989; Kintigh 1984; Plog and Hegmon 1993; Sullivan and Tolonen 1998). The diagnostic sampling of sites surveyed in the Madina-Sadatou study area, while sufficient for identifying temporally sensitive artifacts, did not generate the samples required for quantitative measures of assemblage diversity. While recognizing that large sites with greater numbers of rare items often have correspondingly more diverse assemblages, a summary of non-pottery artifacts involved in some subsistence, craft, and exchange practices hints at subtle differences in the organization of local economies between the Iron Age and Atlantic Era (Table 5.3).

During the Iron Age, evidence for some activities involving metal tools and supra-local

Table 5.3: Summary of artifact classes by horizon/phase and site type

\begin{tabular}{|c|c|c|c|c|c|c|c|c|c|}
\hline \multirow{2}{*}{\multicolumn{2}{|c|}{$\begin{array}{l}\text { Horizon } \\
\qquad \text { Settlement Type }\end{array}$}} & \multicolumn{4}{|c|}{ Metal } & \multicolumn{3}{|c|}{ Terracotta } & \multirow{3}{*}{$\begin{array}{c}\text { Glass } \\
\text { Bead } \\
n\end{array}$} \\
\hline & & \multirow{2}{*}{$\begin{array}{c}\text { Slag } \\
n\end{array}$} & \multirow{2}{*}{$\begin{array}{c}\text { Nail } \\
n\end{array}$} & \multirow{2}{*}{$\begin{array}{c}\text { Tool } \\
n\end{array}$} & \multirow{2}{*}{$\begin{array}{c}\text { Projectile } \\
n\end{array}$} & \multirow{2}{*}{$\begin{array}{l}\text { Net - } \\
\text { weight } \\
n\end{array}$} & \multirow{2}{*}{$\begin{array}{c}\text { Spindle } \\
n\end{array}$} & \multirow{2}{*}{$\begin{array}{c}\text { Pipe } \\
n \\
\end{array}$} & \\
\hline & $n$ & & & & & & & & \\
\hline Iron Age & 28 & 6 & - & 1 & 2 & 3 & - & - & 2 \\
\hline Unknown & 9 & 1 & - & - & - & - & - & - & - \\
\hline Homestead & 14 & 2 & - & - & 1 & 1 & - & - & - \\
\hline Hamlet & 5 & 2 & - & - & - & - & - & - & - \\
\hline Village & 2 & 1 & - & 1 & 1 & 2 & - & - & 2 \\
\hline Tontèko Phase & 12 & 3 & 1 & 1 & 2 & 1 & - & 2 & - \\
\hline Unknown & 7 & 1 & - & - & - & - & - & - & - \\
\hline Homestead & 2 & - & - & - & 1 & - & - & - & - \\
\hline Hamlet & 3 & 2 & 1 & 1 & 1 & 1 & - & 2 & - \\
\hline $\begin{array}{l}\text { Tomboura/ } \\
\text { Karé Phase }\end{array}$ & 32 & 7 & 8 & 5 & 3 & 3 & 4 & 4 & 6 \\
\hline Unknown & 9 & - & - & - & 1 & - & - & - & - \\
\hline Homestead & 12 & 3 & 1 & 1 & 1 & 1 & 1 & 2 & 1 \\
\hline Hamlet & 6 & 2 & 2 & - & - & 1 & 1 & 1 & 1 \\
\hline Village & 5 & 2 & 5 & 4 & 1 & 1 & 2 & 1 & 4 \\
\hline
\end{tabular}


exchange occurred mainly at Diouboye, while netweights turned up at this and the other major village (Goundafa) in the survey zone. On the other hand, the presence of slag at outlying sites suggests that metalworking was more widespread. Although this pattern further applies to the limited data from the Tontèko phase, the Tomboura/Karé phases mark a shift with evidence for more diverse economic activities occurring more frequently in homesteads and hamlets. All in all, the available data point tentatively to greater specialization within the craft, exchange, and perhaps subsistence economies of Diouboye than those of later villages in the region - an interpretation that merits further study with finer-grained surface and subsurface collections from surveyed sites.

The systematic sampling of pottery assemblages on surveyed sites provides a basis for more robust comparison of economic practices, or at least some of those practices within the subsistence economy. Over the past two or three millennia, people occupying the study area made and used three general pottery forms-bowls, pots, and jars-each useful for different sorts of daily tasks (see Appendix D). Since bowls for serving were rare on surveyed sites, I only consider pots, probably associated with food preparation and serving, and jars more likely used for transportation, storage, and perhaps brewing. Despite several outlier assemblages with a jar-to-pot ratio greater than $4: 1$, the relative frequencies of these vessel forms were consistent across settlement sites both

\section{Figure 5.4: Boxplot of jar-to-pot ratio for Iron Age and Atlantic Era settlement classes}

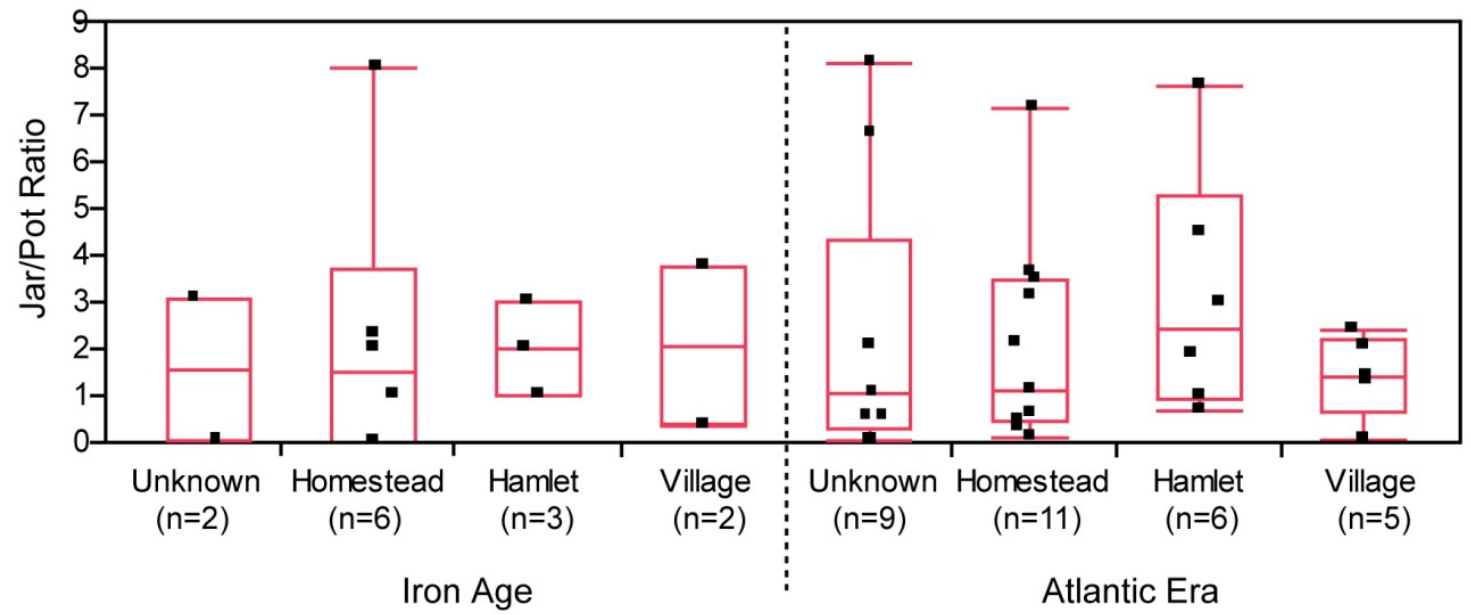


within and between archaeological horizons (Figure 5.4). Tangentially, the similarity between assemblages from homestead and logistical sites may support the interpretation of the latter as small ephemeral settlements, rather than locales for more specialized tasks.

\section{Rank-Size Distribution}

Rank-size distributions provide a visual measure of the extent to which a population (assumed to be proportional with settlement size) is concentrated within, or distributed across settlements in a study area (Drennan and Peterson 2004; Falconer and Savage 1995; G. A. Johnson 1977; Paynter 1982). Many studies, particularly of urban landscapes, rely upon deviation from a log-normal distribution to determine the shape of a regional rank-size plot as primate (one primate center), convex (several central places), or some combination of thereof-each presumably associated with a past socioeconomic system of logistical and appropriational movements (Savage 1997).

Figures 5.5-5.6 show that Iron Age and Atlantic Era settlements each exhibit a lightly convex rank-size distribution although the latter arcs above the log-normal distribution. Given the arbitrariness of log-normality for interpreting settlement patterns, particularly in rural villagescapes, I found it more useful to compare the rank-size distributions for these horizons directly to one another. Adopting a method developed by Robert Drennan and Christian Peterson (2004), I employed the RSBOOT program to calculate the A coefficient of area above/below a log-normal baseline for each rank-size distribution and bootstrap (1000 resamples) the confidence intervals (Figure 5.7).

In the final analysis, site size rankings did not differ significantly between phases within each horizon (nor from log-normality where $A=0$ ), although this coefficient may have been confounded by the inability to measure the phase-by-phase evolution of multicomponent sites from surface finds. A significant difference $(\rho<0.20)$ between the Iron Age and Atlantic Era occupations, however, may suggest that the latter comprised a relatively convex settlement system associated with low levels of political-economic integration among villages (G. A. Johnson 1977:498) or may result from the sampling of a "peripheral" area (Paynter 1982:148-149). Both of these scenarios dovetail nicely with the documented historical expansion of Gajaaga and Bundu in the region. The Iron Age 
Figure 5.5: Rank-size plot for Iron Age settlement sites in the survey area

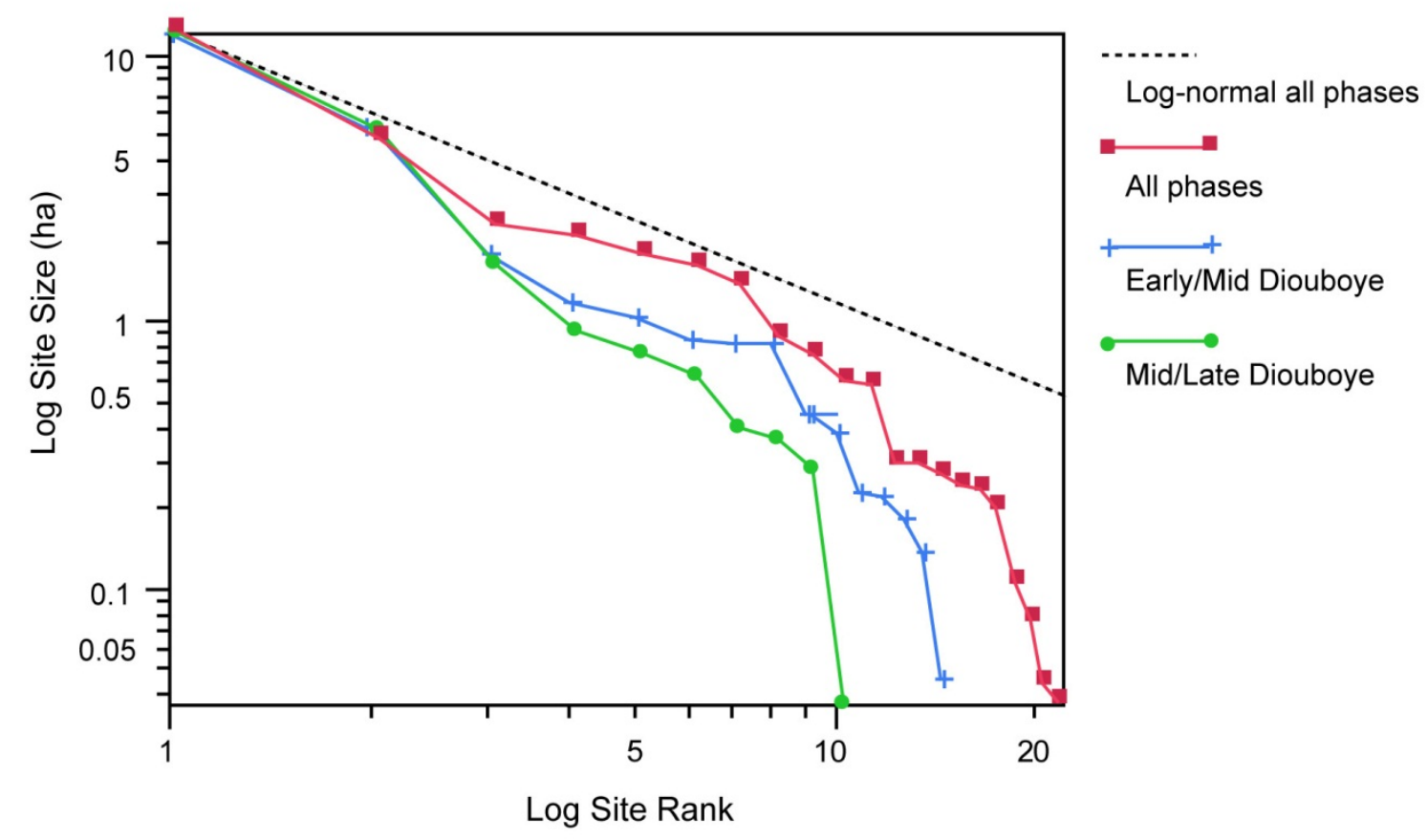

Figure 5.6: Rank-size plot for Atlantic Era settlement sites in survey area

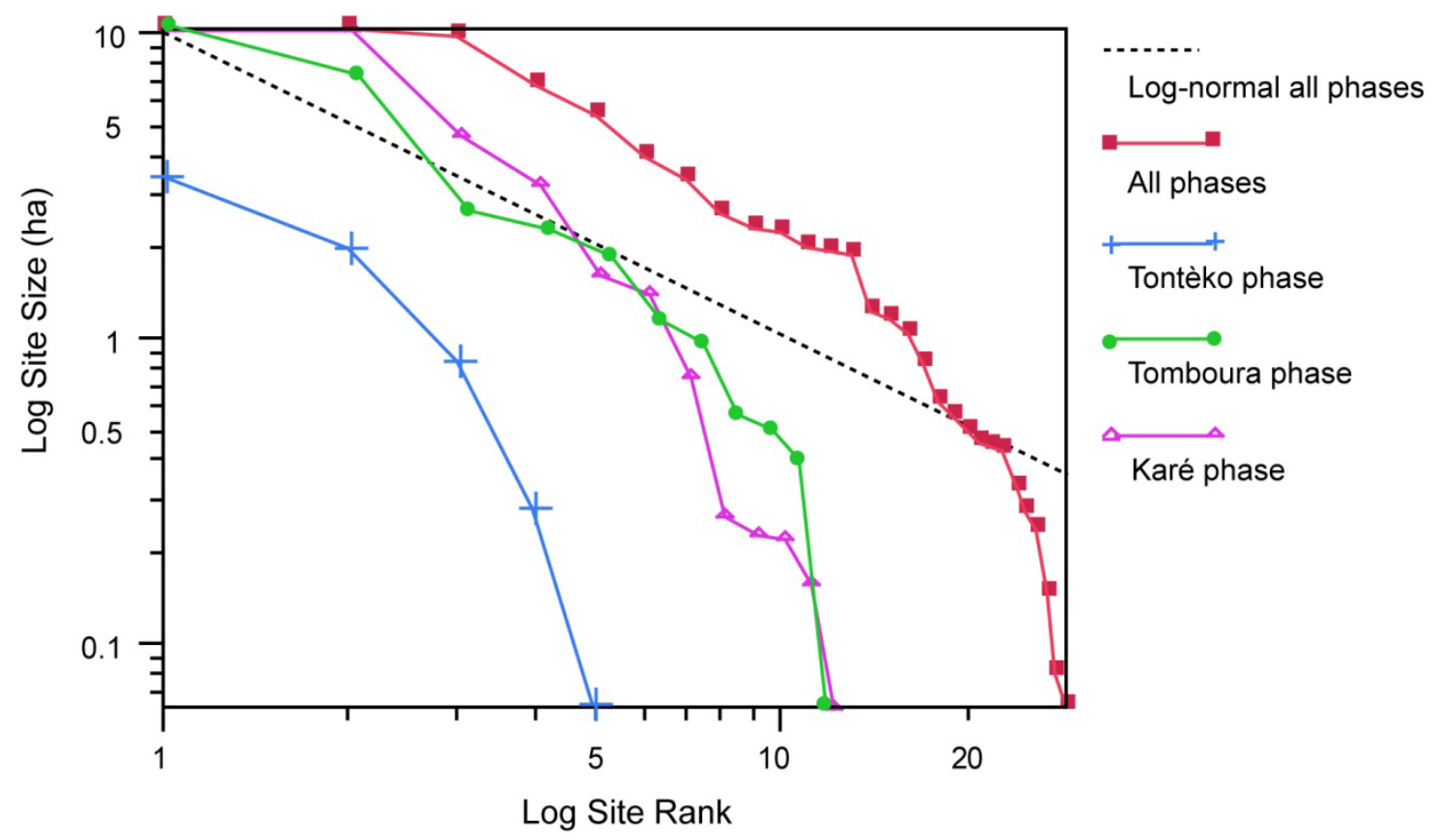


occupation, on the other hand, exhibits a relatively primo-convex distribution when compared to both its own log-normal baseline and to the Atlantic Era. This pattern could emerge from the juxtaposition of two divergent settlement systems-one integrated around a primate center (Diouboye), and the other involving a more decentralized confederation of central places (Falconer and Savage 1995:41). In this latter regard, the wide spacing among Iron Age villages (such as Baganafara) in the Madina-Sadatou study area, and among the Phase IVB sites identified to the north (Thiaw 1999), could represent a political landscape of symmetrical relations among small, economically independent communities. An assessment of this scenario will clearly depend upon further mapping of the archaeological landscape within and beyond the study area.

Figure 5.7: Coefficient $A$ values and confidence intervals (99\%, 90\%, and $80 \%)$ for settlement rank-size distributions across horizons and phases

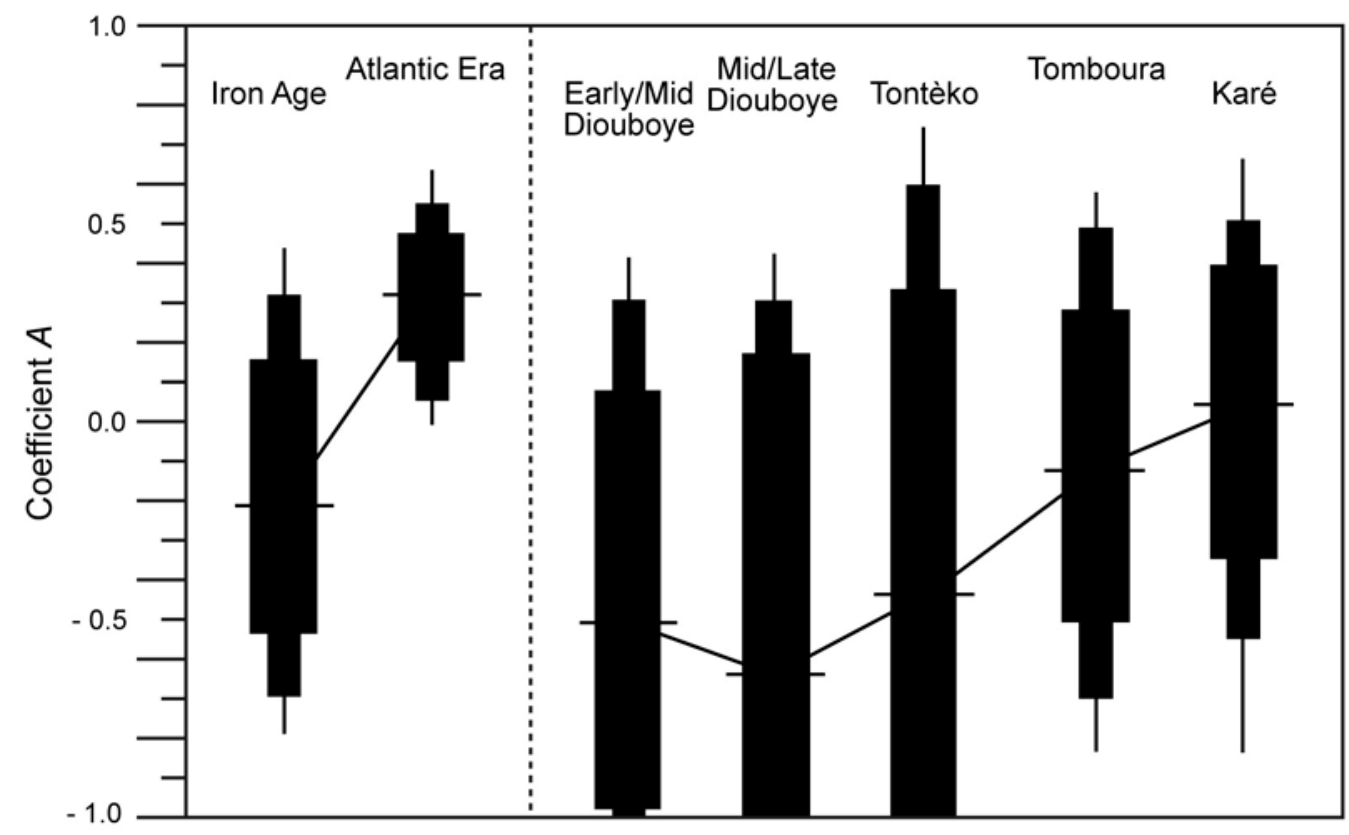

\section{Inter-Site Proximity and Segregation}

Even a cursory glance at phase-by-phase occupations in Chapter 4 reveals that sites generally cluster around larger villages, perhaps signaling the presence of co- 
residential communities demarcated by socio-spatial boundaries. The question, then, is to what extent these spatial relations of proximity among places, and segregation between them, relate to locational and appropriational movements of resources through the local political economy.

In this regard, Nearest Neighbor analysis provides an effective, albeit coarsegrained, measure of clustering or dispersal based on the distance from each site to its nearest neighbor. Since these distances formed a non-normal distribution in most periods, I employed median values to calculate $\mathrm{R}$, a coefficient for the observed deviation in intersite distances from a random distribution (Washburn 1974). A summary of the results in Table 5.4 shows strong and statistically significant clustering $(\mathrm{R}<1)$ across the survey zone $\left(71.75 \mathrm{~km}^{2}\right)$ for all periods prior to the Karé phase. While an emphasis on settlement along the Falémé River partly explains this clustering, the data also suggest a subtle reduction in settlement clustering between the Iron Age and the Atlantic Era. These patterns support the trend seen in the rank-size distribution with the importance of a "primate" village in the Iron Age giving way to a more dispersed pattern of central place villages surrounded by smaller-scale settlements and logistical sites or encampments during the Atlantic Era.

Table 5.4: $\quad$ Nearest-neighbor coefficient $R$ for surveyed sites by phase

\begin{tabular}{|l|c|c|c|c|c|c|}
\hline \multicolumn{1}{c|}{$\begin{array}{c}\text { Horizon } \\
\text { Phase }\end{array}$} & $\mathrm{N}$ & $\begin{array}{c}\text { Median } \mathbf{D}_{\text {obs }} \\
\text { (meters) }\end{array}$ & $\begin{array}{c}\text { Median } \mathbf{D}_{\text {exp }} \\
\text { (meters) }\end{array}$ & \multicolumn{3}{c|}{ R } \\
$\left(\mathrm{D}_{\text {obs }} / \mathrm{D}_{\text {Exp }}\right)$ & $\sigma$ & $\mathrm{Z}$ \\
\hline \hline Stone Age & & & & & & \\
\hline MSA/LSA & 51 & 283 & 557 & 0.509 & 0.043 & -6.31 \\
\hline cLSA & 67 & 345 & 486 & 0.709 & 0.033 & -4.28 \\
\hline Iron Age & & & & & & \\
\hline All Sites & 32 & 429 & 704 & 0.610 & 0.069 & -3.97 \\
\hline Early/Mid Dbye Phase & 16 & 499 & 995 & 0.502 & 0.138 & -3.58 \\
\hline Mid/Late Dbye Phase & 11 & 672 & 1200 & 0.560 & 0.201 & -2.62 \\
\hline Atlantic Era & & & & & & \\
\hline All Sites & 52 & 373 & 552 & 0.675 & 0.043 & -4.21 \\
\hline Tontèko & 11 & 704 & 1200 & 0.587 & 0.201 & -2.46 \\
\hline Tomboura & 15 & 718 & 1028 & 0.698 & 0.148 & -2.10 \\
\hline Karé & 14 & 1165 & 1064 & 1.095 & 0.158 & 0.64 \\
\hline
\end{tabular}




\section{SUMMARY: DIOUBOYE AND THE POLITICAL (ECONOMIC) LANDSCAPE}

The question of how Diouboye participated in broader spheres of political, economic, and cultural interaction beyond the boundaries of the village forms one of the central themes in this dissertation. Referring back to the model of small-scale political economies presented in Chapter 2, I briefly summarize the interpretations developed in this and the previous two chapters concerning the ways in which the community as a whole engaged with people and places across local, regional, increasingly global landscapes. Given the modest scale of region-oriented research in eastern Senegal, and western Mali for that matter, most of these interpretations remain provisory pending further fieldwork in and around the Madina-Sadatou study area.

First of all, the village of Diouboye benefitted from a diverse suite of material resources found throughout the Upper Senegal region and in close proximity along the lower Falémé River basin. As a perennial source of water in a highly seasonal savanna landscape, the river itself could have sustained wild animals, including fish and many riparian species, as well as herds of livestock and, of course, people. Although the climate became increasingly arid throughout the early-second millennium $\mathrm{AD}$, conditions would have nevertheless supported savanna-forest trees and cultivation of domestic crops such as pearl millet. Furthermore, the unique geology of the region provided local people with a constant source of rocks for chipped stone tools, grinding stones, and perhaps even polished axes. People also could have exploited alluvial gold deposits eroding down from the Tomboura escarpment. Given these parameters, it should come as no surprise that, as illustrated in this chapter, Iron Age communities settled close to the river-whatever other dangers it may have posed.

Second, at an interregional and historical scale, it is noteworthy that Diouboye lay at the interstices of at least three political economic or cultural spheres identified from historical and archaeological evidence for the Late Iron Age. To the north and east, merchant diasporas associated with the waning power of Ghana, and expansion of Gajaaga, may have fostered the commoditization of resources found throughout the Upper Senegal, including gold, cotton, and slaves. To the north and west lay the increasingly centralized agro-pastoral societies of the Middle Senegal region, including Takrur, Silla, and later the Djolof state. And to the south lay the decentralized village 
societies of the upper Gambia River basin and, beyond these, the Pays Mande - the heartland of the Malian state. As discussed in Chapter 4, the people of Diouboye shared a number of material practices for pottery and metal production, settlement organization, and subsistence with communities to the south, although subsequent chapters will reveal important exchange connections with networks to the north. Although the results of a rank-size analysis presented in this chapter must remain tentative pending the collection of more regional data, the primo-convex distribution of settlements suggests that Diouboye maintained symmetrical political relations with other large villages throughout the Falémé River basin as a whole, even the community sat atop potentially asymmetrical relations across the immediate landscape.

Third, a comparison of material assemblages and site catchments provides a clue as to how local subsistence and craft economies shaped, and were shaped by, political relations between Diouboye and surrounding settlements. Although the village had access to the rich ecology of the Falémé River and its halo of riparian bush, it seems likely that residents depended, in part, upon subsistence production beyond the capacity of adjacent cultivable lands. Whether staple resources flowed through corporate kin networks or came directly from the seasonal trips away from the village, or even a concerted emphasis on hunting and fishing, the political institutions within the community evidently had sufficient inertia to prevent fission into smaller settlement groups. The limited surface data may also point to a greater diversity of socioeconomic practices at Diouboye (further attested with excavation data in Chapter 7) in comparison to outlying settlements and ephemeral logistical sites. Whether or not the community specialized in these activities, the size of the Diouboye suggests a larger population whose labor and practical and discursive knowledge undoubtedly enriched the base of non-material resources available for appropriation within the local political economy. The question of how these non-material and material resources actually became enrolled into economic processes and political relations within the community now requires a closer look at material and spatial dimensions of the archaeological record of Diouboye proper-the topic of the next three chapters. 


\section{CHAPTER 6 \\ DAILY LIFE IN DIOUBOYE: ARCHITECTURE AND ACTIVITIES}

Having explored the historical landscape extending outwards across space and time from Diouboye, I turn now to focus on the ways in which people within this village experienced, reproduced, and transformed economic processes-including the underlying parameters and broader structural contexts—on a regular, if not daily, basis. In this chapter I will outline those activities attested in the archaeological record of Diouboye (and note those conspicuously absent) before moving on to present the specific evidence for where and when people engaged in such activities.

Activities (or behaviors) provide a bridge between their material and spatial effects in the archaeological record and the political economies and histories that they help to constitute as social practices. The epistemological limits of this bridge, often predicated on analogical argument, have fueled a decades-long discussion of middlerange reasoning in archaeology (e.g., Ascher 1961; Binford 1967, 1985; Fogelin 2007b; Gould 1985; Raab and Goodyear 1984; Wylie 1982, 2002), and I take the position here that no single interpretive methodology will be appropriate to all theoretical positions or empirical case studies (following Dobres and Robb 2005; Tschauner 1996). Beyond logical coherence, robust middle-range interpretation should maintain transparencyeither between archaeological data and past activities, or between activities and their social significance (Yellen 1977:1-12). This requires a discussion of similarities and differences among ethnographic analogues, experimental findings, and the available archaeological evidence, as well as relevant assumptions about the underlying causality of the observations subject to comparison.

In the present chapter I am concerned primarily with "first-order" middle-range interpretations comparing the material correlates of behaviors documented in experimental studies and ethnographic and historic accounts, mainly from sub-Saharan Africa, with actual lines of archaeological evidence from Diouboye. After controlling for 
the effects of natural and post-depositional processes, these correlates provide a way to link archaeological data from specific contexts (Chapters 7-8) to a suite of possible economic practices of the sort described in Chapter 2. For the purposes of analysis and discussion, it is useful to draw a distinction between activities that produce the built environment and those that occur within and beyond this formal space. This distinction is not, however, meant as an absolute since most economic processes enroll the physical resources of the built environment into material practices of production, exchange, and consumption, and, more importantly, spatial practices of distancing and segregation.

\section{FROM PRESENT DATA TO PAST BEHAVIOR}

Since geological and biological processes of sediment deposition, erosion, and disturbance always work alongside economic acts of production, exchange, use, and consumption to generate material and spatial dimensions of the archaeological record (see Chapter 2), any interpretation of past practices needs to account for the depositional and post-depositional processes that intercede between them and the observed data. Here archaeologist Michael Schiffer (1987) has proposed that the formal, spatial, frequency, and relational dimensions of archaeological assemblages can provide grounds for inferring the additive processes that brought about their formation. Beyond contextualizing these assemblages, the sediments of stratified sites themselves comprise “artifacts” of anthropogenic and natural processes to be interpreted from their formal composition (Rapp and Hill 1998). In this section I briefly repackage these insights into a middle-range framework for interpreting past economic practices at Diouboye on the basis on material and spatial dimensions of the archaeological record.

\section{Site Formation: Material and Spatial Dimensions}

The material (qualitative and quantitative) and spatial (distance and segregation) dimensions of an archaeological dataset emerge in the course of production, use, and consumption activities, as well as processes of discard and redeposition. The qualitative (or formal) dimension describes the physical properties of artifacts, features, and sediments in the archaeological record. While some properties, such as raw materials, shape, decoration, and patterns of wear, result from choices and activities within a past 
technological system, other properties, such as size, breakage, and degree of erosion and corrosion, can likewise point to and contextualize processes of (re)deposition. The species-specific forms of faunal elements, for example, document the taxa procured and consumed at a site, but taphonomic variables such as cut marks, breakage, carbonization, and gnaw wear can reveal patterns of food preparation, discard, and redeposition by dogs or burrowing insects (Schiffer 1987:188-189). For this reason, the analyses of artifacts from Diouboye and surrounding sites included variables related to socio-technological activities, as well as those related to depositional and post-depositional processes (see Appendices D-F).

The quantitative (or frequency) dimension of an archaeological dataset indexes the intensity or duration of production and use/consumption activities, including rates of attrition accrued in the course of discard and redeposition. A high quantity of sherds, for example, can result from intensive use and breakage at a given locale, secondary disposal in a dump, or simply repeated trampling. Fortunately, several studies have shown that relative sherd abundance, when considered along with qualitative properties of erosion (size) and function, provides a robust measure of past pottery use and consumption (Mayor 1992; Mills 1989). Throughout this study I therefore compare absolute and relative frequencies within (and between) artifact classes to interpret their trajectories through past behavioral and archaeological contexts.

Distance and segregation present a slightly different, but parallel, take on the spatial and relational dimensions of archaeological datasets described by Schiffer (1987). Distance is a relative measure of physical space among artifacts and built features, while segregation corresponds to their relative partitioning via patterned interactions. These dimensions encapsulate the ways in which people occupied and reproduced social space, but they are strongly subject to alteration by natural agents ranging from wind and water to roots, insects, and domestic animals. This is not to say that spatial relations are too spurious to be useful, but simply that their interpretation must take account of depositional and post-depositional processes in addition to the past economic practices of anthropological interest.

The properties of sediments around archaeological remains can provide critical information about natural and cultural processes of deposition (Rapp and Hill 1998). 
First, the formal composition of a deposit-including its clastic, chemical, and organic constituents-provides a signature of the geological processes that produced it. In the absence of geoarchaeological analysis at Diouboye, I relied mainly on in-field assessments of particle texture (i.e., sand, loam, silt, clay) to determine the agencies responsible for soil deposition. For example, aeolian and hydrological soil transport both tend to sort sediment particles according to size, while anthropogenic processes such as earthen wall collapse often result in a more homogeneous distribution of inclusions (see below). Color also provided a crude proxy for the chemical and organic composition of a deposit, including relative degree of oxidation and richness of decomposed biotic matter.

Second, the boundaries between stratified archaeological sediments can testify to continuity and change in depositional systems. Whereas gradual transitions usually derive from continuous deposition over the long-term, comformable boundaries of abrupt transition point to punctuated changes and uncomformable boundaries truncating bedding planes point to processes of erosion. Altogether these properties form the basis for interpreting depositional contexts of archaeological strata at Diouboye (Appendix C) for comparison with other material and spatial dimensions of the data as described above.

\section{Depositional Pathways}

Economic practices generate the archaeological record through several overlapping or interlocking pathways. While ethnographic studies have observed diverse pathways under a range of socioeconomic conditions (e.g., Hayden and Cannon 1983; Kent 1982; Kroll and Price 1991; Murray 1980; Wilson 1994), in this study I adopt a relatively simple distinction among primary, secondary, and de facto refuse (following Schiffer 1987:47-98). Primary refuse includes those objects deposited at their location of

production or last use. In sedentary villages such as Diouboye, where people tend to clean domestic activity areas on a regular basis, this usually comprises small, residual materials swept into depressions or trapped by architectural features. By contrast, areas for segregated and/or intense activities (say, for craft production) may be less carefully maintained and thus accumulate larger debris or expended tools. Secondary refuse refers to those materials deposited away from their initial use location, usually in areas designated specifically for this purpose such as pits, middens, and gardens (see below); 
these deposits generally exhibit a greater quantity and diversity of artifacts than their surrounding deposits (Wilson 1994). Secondary disposal, as I employ it here, subsumes all subsequent redeposition as general fill or earthen wall melt. Finally, de facto refuse includes those usable objects left in place following the abandonment of an area. These pathways of refuse disposal, when considered as an integral component of past economic processes, help to contextualize the activities of production, exchange, and use or consumption that preceded them.

\section{ARCHITECTURE}

Even as the built environment works to frame human action, it usually comprises an important resource for the reproduction and transformation of economic and cultural structures (E. T. Hall 1966; Lawrence and Low 1990; Lefebvre 1991; Rapoport 1990). In fact, physical structures such as houses often become iconic of the social structures-the family, kin group, class, and so forth-reproduced within them (Bourdieu 1973; DonleyReid 1990; Fleisher and LaViolette 2008; Keane 1995). Accordingly, I draw a broad distinction in this study between the formal space given shape by durable architecture and the informal space of interactions among people and material culture that occur within and beyond the built environment.

Despite this attention to durability, formal space is neither permanent nor is the social experience of it unchanging. Across the West African savanna, conventional earthen wall technology employs widely available materials, such as dried mud, wood, and occasionally stone, in order to reduce labor investment and allow for some modicum of flexibility in residential patterns and mobility (Denyer 1978; Prussin 1974). Because earthen walls are vulnerable to natural elements in this environment, annual acts of remodeling and construction contribute to the continual reproduction and reshaping of social relations at different scales within a community (Agorsah 1986). As a result, it is possible to consider vernacular architecture in West African settings as the result of a process, or an integrated set of production, use, and maintenance activities (McGuire and Schiffer 1983), which differ from other activities insofar as they produce formal space.

A number of architectural and ethnoarchaeological studies have documented the construction, use, decay, maintenance, and abandonment/destruction of earthen wall 
architecture in West Africa, particularly as it contributes to the formation of archaeological sites (Agorsah 1985, 1986; Denyer 1978; Holl 1987; R. J. McIntosh 1974, 1977; Ogundele 2005; Prussin 1969, 1974). While the function and social significance of built features in the archaeological record is often difficult to interpret (David 1971), these studies do provide a framework for considering how architecture at Diouboye articulated with other activities — a critical step towards better understanding how formal space emerged from and shaped economic practices and social institutions within the community. In this section I focus specifically on architectural structures that enclose or demarcate space. The non-architectural pyrotechnic and pit features likewise implicated in the production of space at Diouboye are discussed in the following section.

\section{Construction and Maintenance}

Following Roderick McIntosh’s (1974) exposé of earthen wall architecture in central Ghana, it was possible to identify at least two different construction techniques at Diouboye - terre pisé and wattle-and-daub. Both techniques began with the excavation of soil with sufficient loam and clay content, presumably from a "borrow pit" near, if not directly adjacent to, the site of construction. Because the composition of soil obtained from each pit differed slightly, no two walls had an identical composition although they usually included modest densities of laterite gravel and reddish-yellow clay from natural sediments at the bottom of borrow pits (see also R. J. McIntosh 1977). Where such pits cut through occupation deposits, the resulting walls must have incorporated artifacts from previous phases, leading to long-term "upward" movement of archaeological materials at the site (Holl 1987:131; R. J. McIntosh 1974:159). In some cases this made earthen wall structures difficult to distinguish relative to the surrounding archaeological matrix beyond a subtle change in sediment density or firmness.

Terre pisé (also called puddled mud or tauf) construction at Diouboye involved layering courses of mud to form curved walls some 16-26 centimeters thick. Consistent with the inclusion of natural alluvium, the resulting wall stubs were a yellowish-red silt to clay loam, often with greater quantities of laterite gravel (2-5\%) than surrounding sediments. For the most part, people did not excavate a wall trench or prepare a formal floor prior to the construction of a circular terre pisé room; the initial occupation floors 
were hardened through pounding or simply by repeated foot traffic and sweeping. Over the course of occupation, however, most rooms underwent one or two resurfacing episodes, perhaps alongside more general wall and thatch roof repair.

Based on ethnographic observations from elsewhere in West Africa (David 1971:115-119; R. J. McIntosh 1974:159), such terre pisé structures would have required new plaster every couple of years over the course of a 15-20 year lifespan, although walls with good upkeep could have survived several decades. Changes in temperature and humidity undoubtedly weakened terre pisé walls at Diouboye over the long-term, but the primary cause of decay would have been undercutting by heavy seasonal rainfall, which would have formed a talus of coarse material and associated "melt" of finer sediment fanning out from the base of the wall (Carter and Pagliero 1966). People today mitigate erosion of the exterior wall base by reinforcing this area with large stones (Agorsah 1985:106; R. J. McIntosh 1974:163) and the presence of broad stone circles in surface contexts (see below) suggests that the occupants of Diouboye adopted a similar strategy.

\section{Figure 6.1: $\quad$ Profile of terre pisé wall and room interior (Feature 71)}

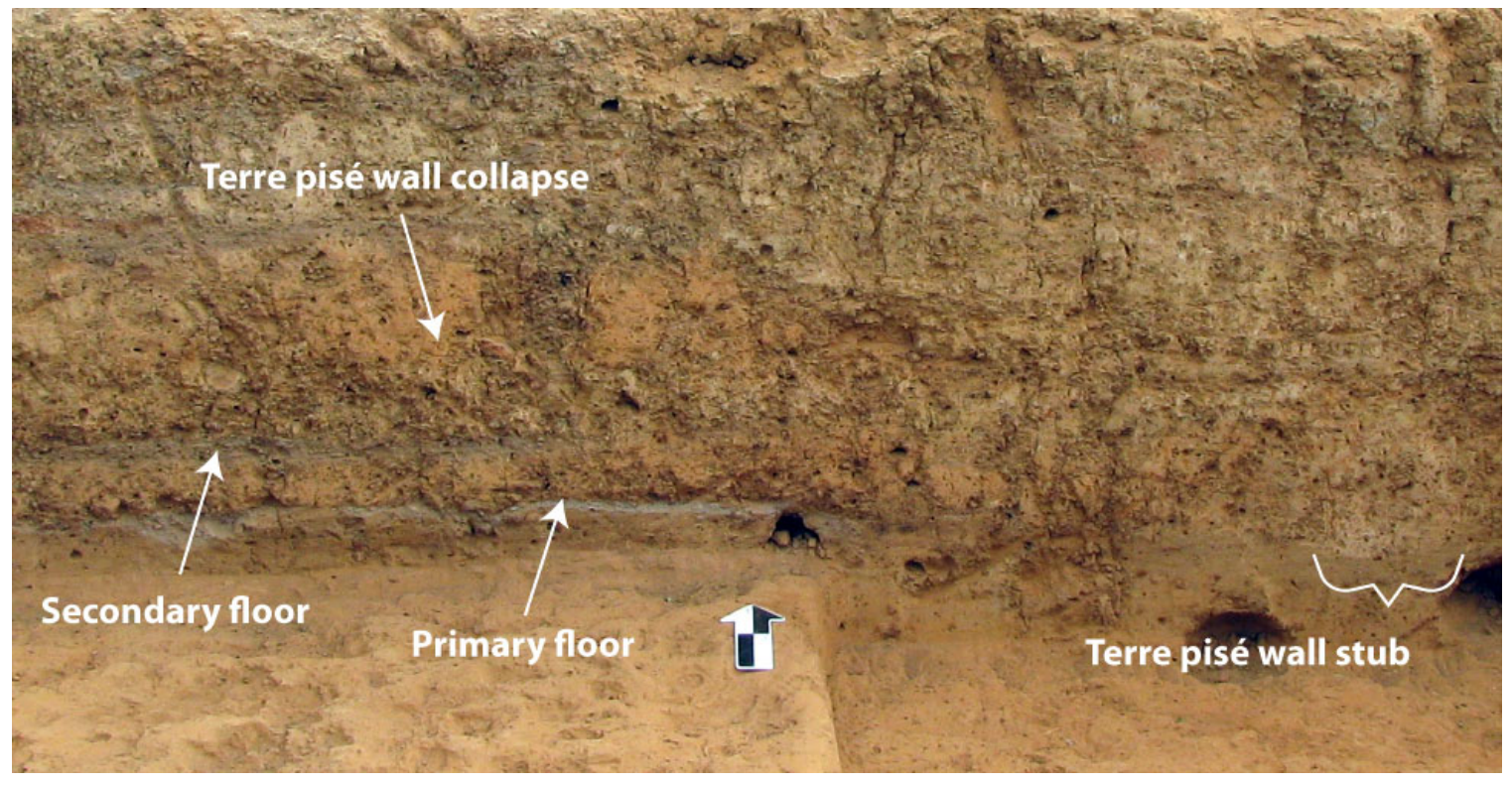


Less frequent than terre pisé at Diouboye, wattle-and-daub construction involved applying mud to the interior and exterior surfaces of a wooden frame grounded in a shallow trench; this technique produced walls with high clay content and only seven to ten centimeters in thickness. As in terre pisé structures, floors in wattle-and-daub rooms were resurfaced at least once over the course of their brief use life, which McIntosh (1974:163) has estimated at only seven years. Even with continual upkeep and resistance to erosion, wattle-and-daub walls were susceptible to termites, inevitably hastening their demise. In fact, termite activity at Diouboye, both past and present, helps to explain the dearth of evidence for wattle-and-daub architecture and wooden furniture such as beds, benches, and fences since the small cavities created by insects in archaeological deposits made it difficult to identify unambiguous post molds.

\section{Figure 6.2: $\quad$ Profile of wattle-and-daub wall and room interior (Feature 106)}

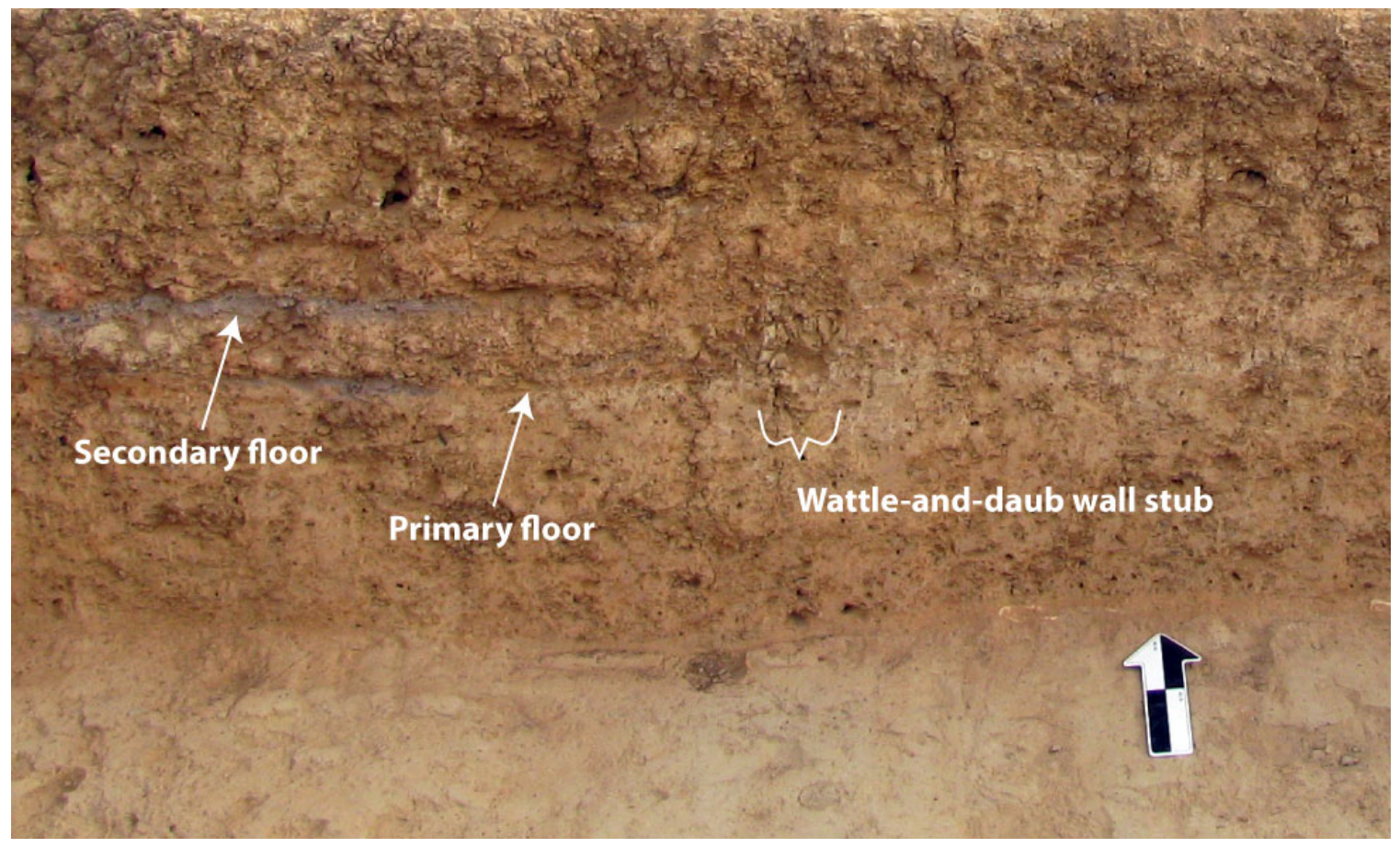


In addition to reinforcing earthen walls, the inhabitants of Diouboye also employed angular rocks, readily available from the weathered sandstone ridge east of the site, to build a variety of other architectural features. The forms of these installations varied from rectilinear to circular although reuse of rocks and processes of erosion usually worked to alter their initial arrangement. Some stone features identified during excavation revealed the remnants of an overlying earthen wall, suggesting that many of these foundations supported granaries or other structures.

Figure 6.3: Profile of granary foundation with melt/collapse from an overlying earthen wall structure (Feature 102)

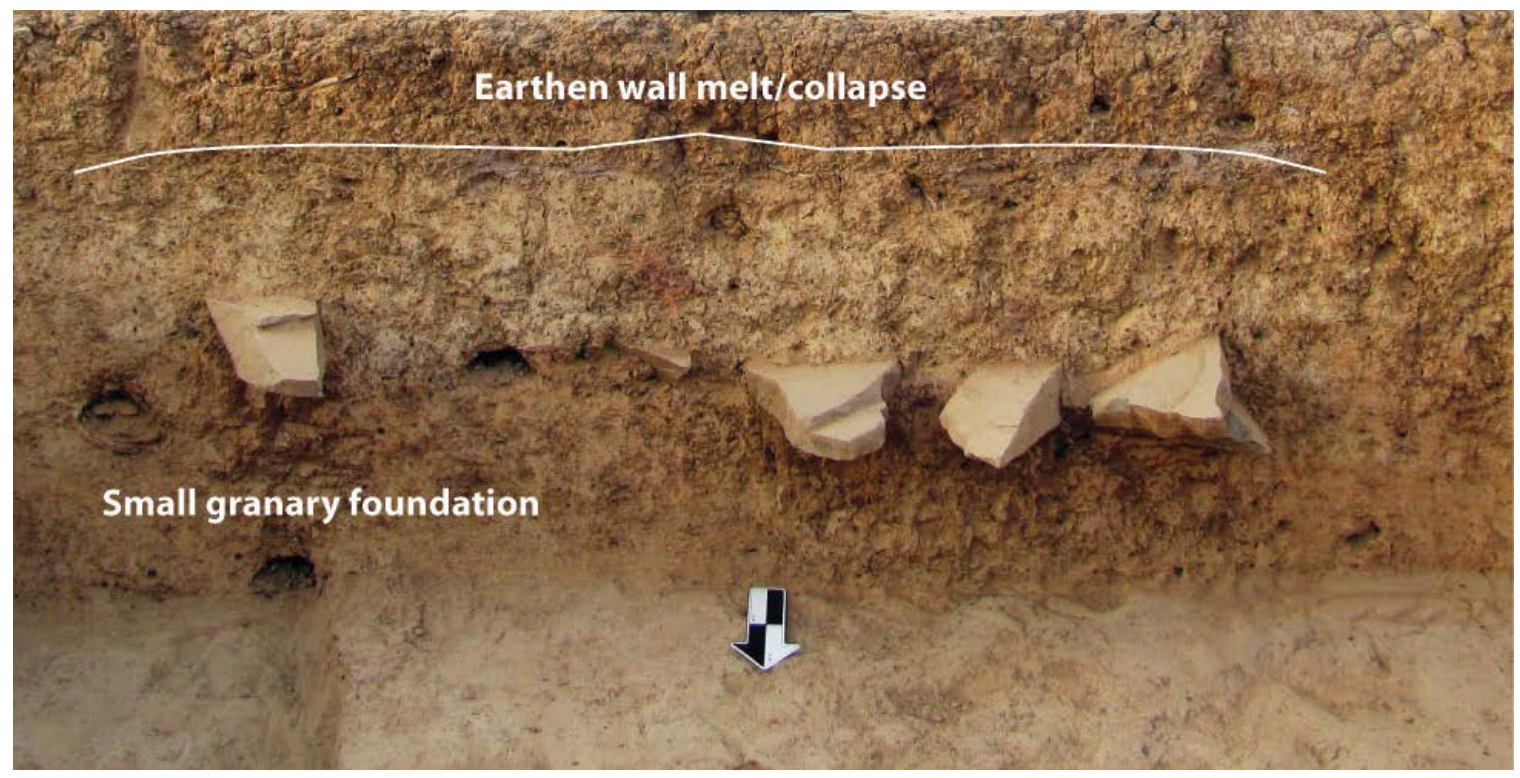

\section{Forms and Uses}

The materials and technologies available to the people of Diouboye must have placed certain constraints on their architecture, but the actual forms built within these constraints resulted from a number of decisions about both utilitarian requirements and local aesthetics or symbolism (Denyer 1978; Lawrence and Low 1990; McGuire and Schiffer 1983; Prussin 1969; Steadman 1996). Even as most architectural features were put to a variety of uses throughout their lifecycles, such changes would have been guided 
by their initial forms. For instance, a sleeping hut of general form and size (5-15 $\left.\mathrm{m}^{2}\right)$ could be easily repurposed into a kitchen, storage room, or livestock pen. On the other hand, features designed for a more specific purpose, including pot rests or platforms, shrines, and granaries, may have been less adaptable. Whatever their use, people could have accentuated the formal properties of these ritual and vernacular structures depending on their semiotic values, as in the rounding of granaries to resemble the bellies of fertile women (Fiedermutz-Laun 2005).

Recognizing the complex and dynamic relations between form and use, in this section I outline the range of economic practices associated with the architectural features observed at Diouboye. Using attributes known to have some significance in ethnographic settings (Table 6.1), I first identify discrete feature types that presumably played different roles within the built environment of the village. I then use evidence from subsurface archaeological contexts to interpret the activities that took place in and around these feature types, including their own construction, maintenance, and decay. Given the evidential bias towards the final activities accompanying each feature, these types should be considered provisional and open to refinement through more detailed analyses or the accumulation of more contextual data.

\section{Domestic Hut}

Domestic huts at Diouboye were circular earthen wall structures with a modal exterior diameter of 1.90-3.86 meters (Figure 6.4). In excavation contexts these appeared as terre pisé or wattle-and-daub wall stubs enclosing compact interior floors, often with evidence for small hearths and episodes of resurfacing. Domestic huts appeared in surface contexts as open rings of sandstone rocks presumably laid down as a foundation or to retard erosion. Notably, many of the curvilinear stone alignments observed on the site surface (see below) may have been partial reinforcements or door foundations, such as those observed in subsurface Features 66 and 115 (see Chapter 7). 
Table 6.1: $\quad$ Variables recorded for the classification of architectural features

\begin{tabular}{|l|l|}
\hline \multicolumn{1}{|c|}{ Variable } & \multicolumn{1}{c|}{ Description (Attribute) } \\
\hline \hline Form & Overall shape of the feature (circular; rectangular; curvilinear; linear; irregular) \\
\hline Completeness & $\begin{array}{l}\text { Preservation of the feature (complete; incomplete, but size estimable; incomplete, } \\
\text { but size inestimable; unknown) }\end{array}$ \\
\hline Stone Quantity & Quantity of stones clearly associated with the feature \\
\hline Stones Interior & Presence of stones inside the feature (filled; partially filled; unfilled; unknown) \\
\hline Earthen Wall Type & Description of wall material, if present (terra pisé; wattle-and-daub; unknown) \\
\hline Earthen Wall Width & Maximum width of earthen wall stub, if present \\
\hline Ash Present & Presence of moderate to high ash density (>2\%) in the surrounding soil matrix \\
\hline Maximum \\
Dimension
\end{tabular}

Table 6.2: $\quad$ Summary of architectural feature attributes

\begin{tabular}{|c|c|c|c|c|c|c|c|c|c|c|c|}
\hline \multirow[t]{3}{*}{ Type } & \multicolumn{2}{|c|}{ Context } & \multicolumn{4}{|c|}{ Interior Stones } & \multirow{3}{*}{$\begin{array}{c}\text { Max } \\
\text { Dimension } \\
\text { Meters } \\
\text { range }\end{array}$} & \multirow{2}{*}{\multicolumn{2}{|c|}{$\begin{array}{c}\text { Earth } \\
\text { Wall } \\
\text { Present }\end{array}$}} & \multirow{2}{*}{\multicolumn{2}{|c|}{$\begin{array}{c}\text { Ash } \\
\text { Present }\end{array}$}} \\
\hline & & Total & \multicolumn{2}{|c|}{ Present } & \multicolumn{2}{|c|}{ Absent } & & & & & \\
\hline & & $n$ & $n$ & $\%$ & $n$ & $\%$ & & $n$ & $\%$ & $n$ & $\%$ \\
\hline \multirow{2}{*}{$\begin{array}{l}\text { Stone } \\
\text { foundation }\end{array}$} & Surface & 337 & 145 & $43 \%$ & 123 & $36 \%$ & $0.50-3.86$ & - & - & - & - \\
\hline & Excavation & 20 & 11 & $55 \%$ & 6 & $30 \%$ & $0.50-1.90$ & 7 & $35 \%$ & 6 & $30 \%$ \\
\hline \multirow{2}{*}{$\begin{array}{l}\text { Stone } \\
\text { alignment }\end{array}$} & Surface & 59 & - & - & - & - & - & - & - & - & - \\
\hline & Excavation & 3 & - & - & - & - & - & 2 & $67 \%$ & 0 & $0 \%$ \\
\hline \multirow{2}{*}{$\begin{array}{l}\text { Earthen } \\
\text { wall stub }\end{array}$} & Surface & 1 & - & - & - & - & - & 1 & $100 \%$ & - & - \\
\hline & Excavation & 13 & 0 & $0 \%$ & 13 & $100 \%$ & $1.90-3.30$ & 13 & $100 \%$ & 6 & $46 \%$ \\
\hline
\end{tabular}


Figure 6.4: Distribution of the maximum dimensions of unfilled stone features and earthen wall foundations

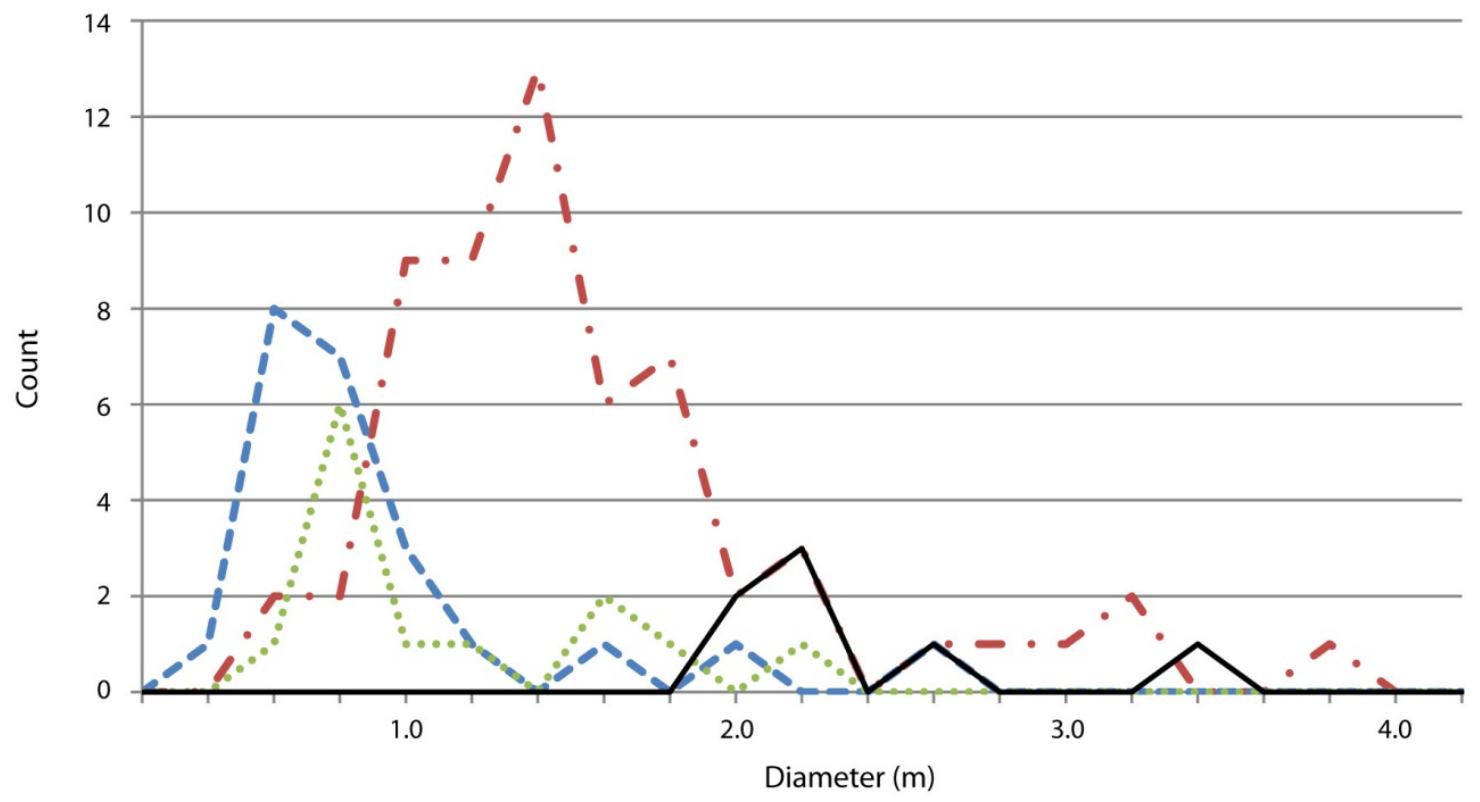

Feature Form: $-\ldots$ Circular $\quad-$ Rectangular $\ldots$... Irregular — Earthen Wall

Figure 6.5: Distribution of the maximum dimensions of filled stone features

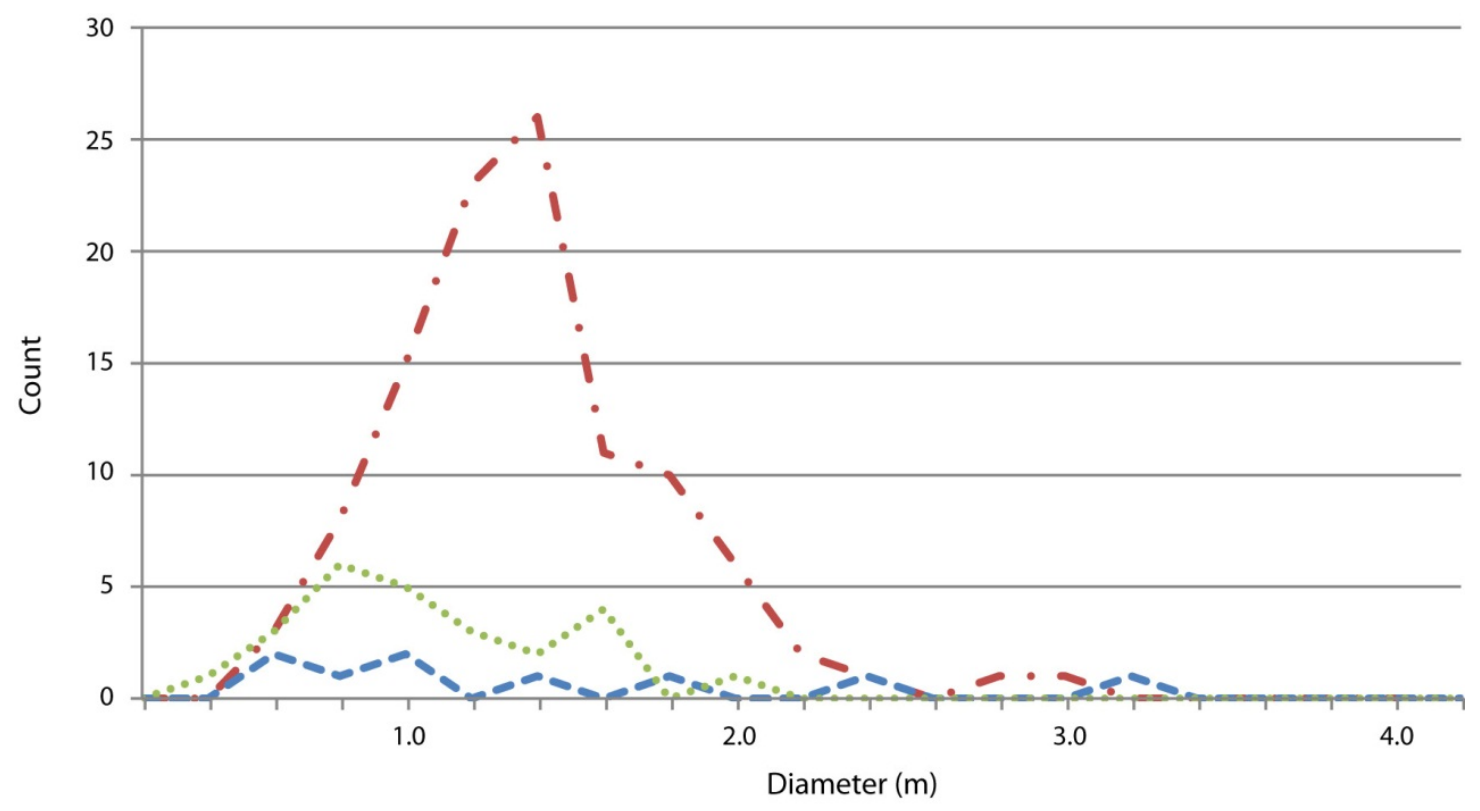

Feature Form: $-\cdots$ Circular $\ldots-$ Rectangular $\ldots .$. Irregular 
The term "domestic" denotes a relative flexibility in the use of these huts over time, but many probably began as sleeping quarters and/or storage facilities for one or a few individuals. Their interior area (3.80-10.52 $\left.\mathrm{m}^{2}\right)$ was consistent with the size of sleeping rooms in contemporary West African villages (David 1971:126; R. J. McIntosh 1977:189; Ogundele 2005:32, 45) although it certainly falls on the smaller end of this spectrum (Lestrange 1955:38). Small room sizes could have been a function of labor investment in thatch roof repair, the size of social units, or architectural tradition.

Evidence within domestic huts pointed to sleeping and storage functions, rather than food preparation or rubbish disposal. First, the floors in most rooms displayed the tell-tale oxidized sediment of an informal hearth (see below). Unlike the thick hearth complexes produced through daily food preparation in open courtyards, these small features were more consistent with the low-level heat required to warm a room during the cool nights of the dry season. Second, the overlying room fill often contained modest densities of ceramic and faunal remains on par with those found in general deposits, rather than the high densities characteristic of refuse deposits (see Table 6.3 below). Room fill was, however, distinguished by the presence of larger sherds protected from trampling, presumably, by subsequent wall collapse. Although direct evidence was lacking, domestic huts at Diouboye may have further served to welcome guests, store or prepare food, brew beer, produce craft goods, or conduct rituals.

\section{Pavement}

Pavements were filled stone features, regardless of form, with a maximum linear dimension greater than 2.05 meters (Figure 6.5). Despite their close association with earthen wall melt and/or collapse in excavated contexts (Features 96, 123, and 130), the absence of wall stubs suggested that pavements were platforms for food preparation and other activities conducted in open courtyards during the rainy season. This interpretation is consistent with pavement construction in local villages today (Figure 6.7), as well as with the high densities of faunal remains and sherds from cooking vessels associated with these features in subsurface contexts (see Table 6.3 below). Notably, some of the larger features interpreted as granary foundations could have served as platforms (David 1971:120). 
Figure 6.6: Wattle-and-daub hut, Sansanding, Senegal

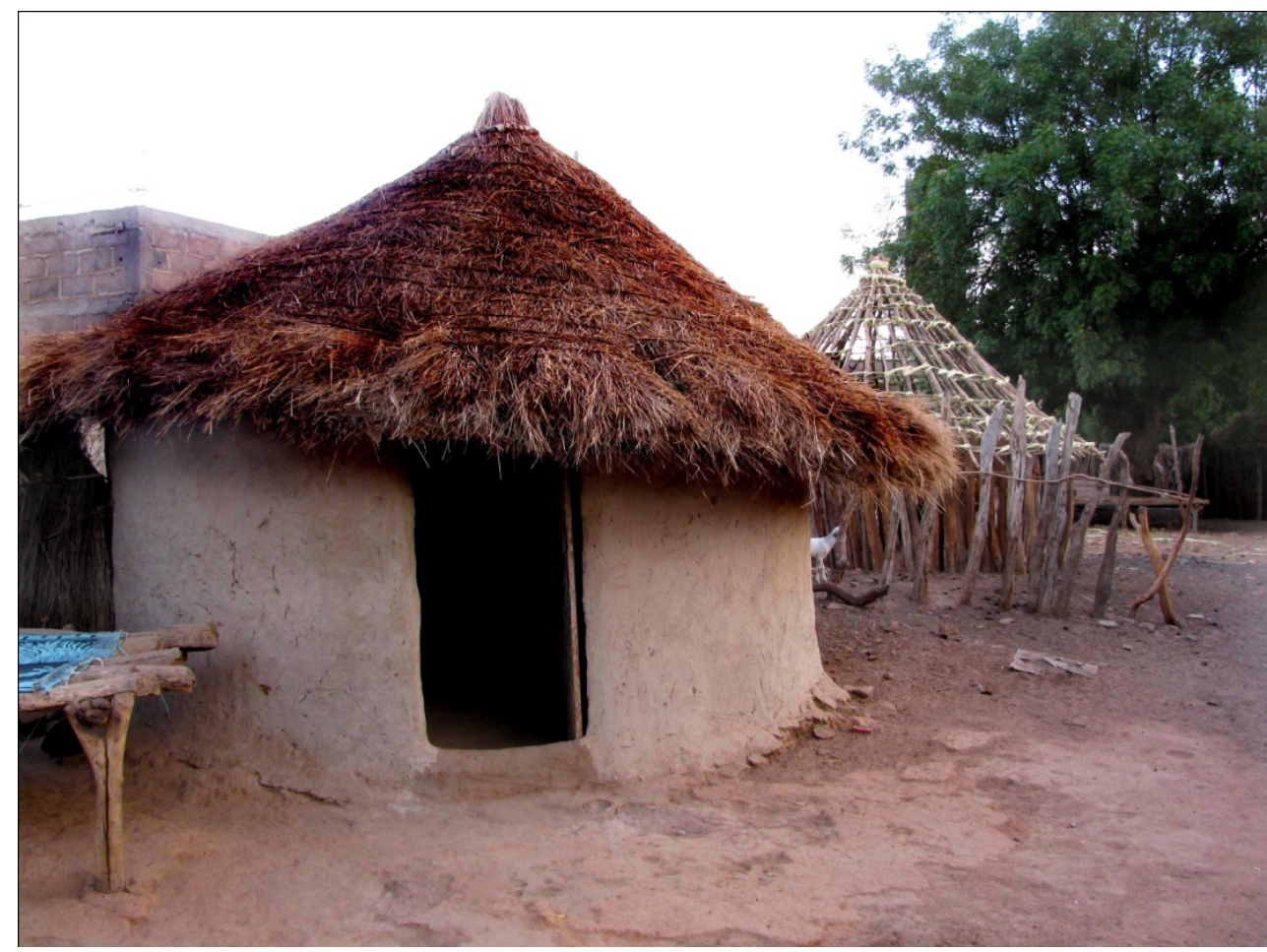

Figure 6.7: Concrete pavement and stone pot rest, Sansanding, Senegal

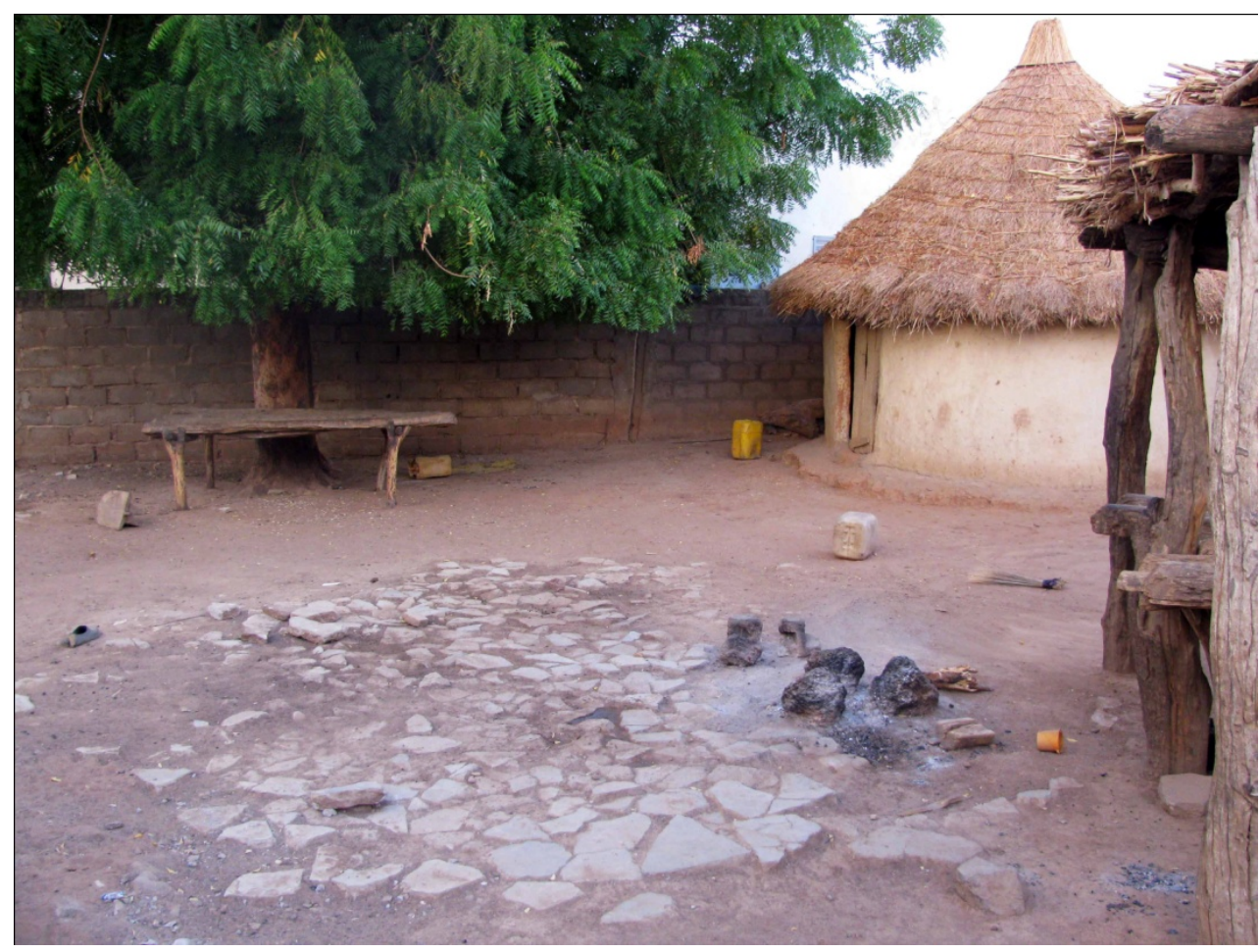


Figure 6.8: Surface of Area $\mathrm{H}$ showing range of stone feature types

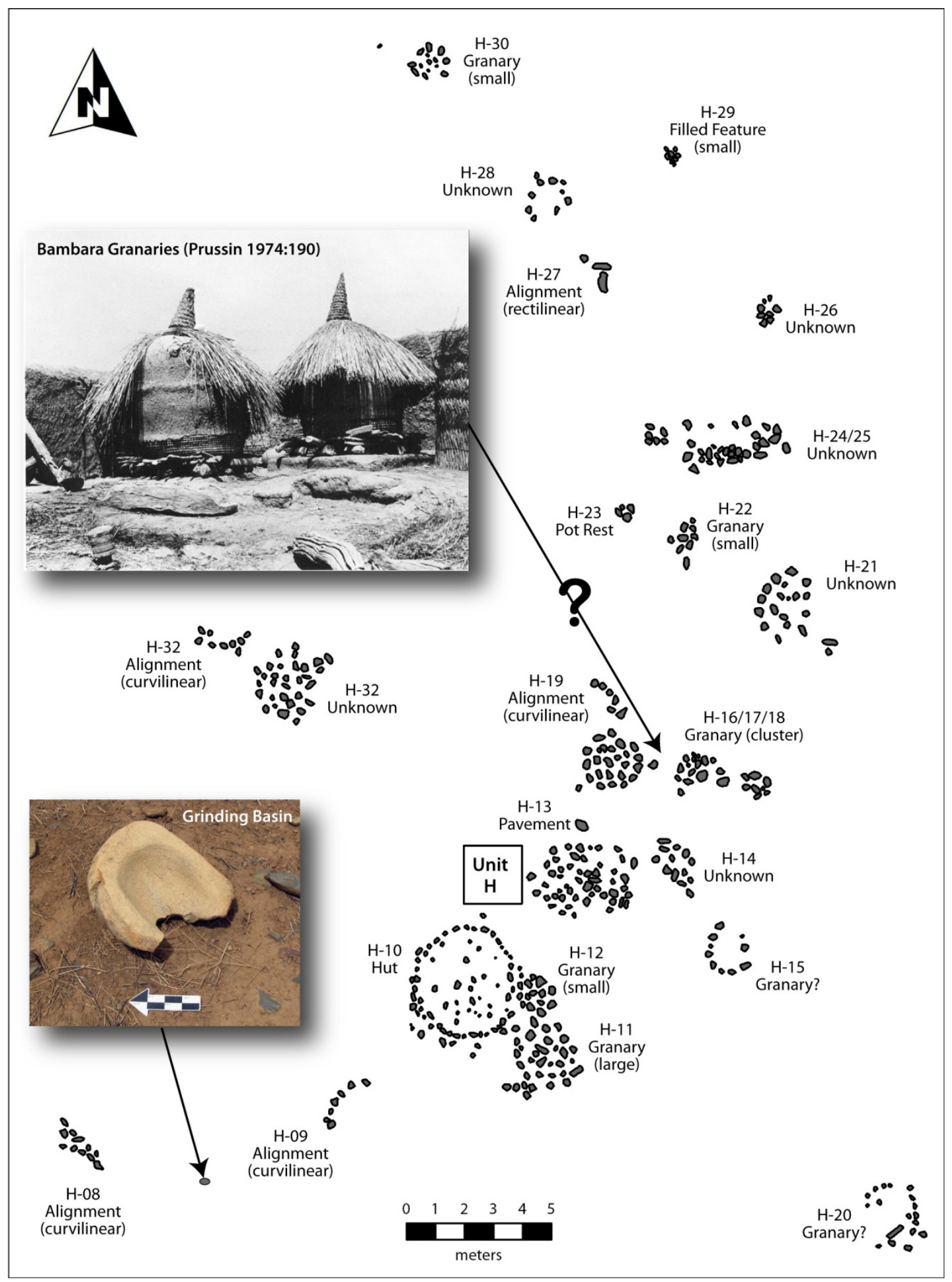




\section{Pot Rest}

Pot rests were arrangements of three to seven stones measuring less than 0.80 meters in diameter and presumably designed to support a globular pottery vessel, although they could also have supported baskets, grinding stones, or other implements. People today use such pot rests for cooking (Figure 6.7), but the lack of fire-cracking on sandstone rocks at Diouboye, and their complete absence in or near hearths, suggests that they elevated water storage vessels to aid with cooling through evaporation (David 1971:120). The association of a reconstructable storage vessel with a pot rest (A-28) in one surface context and the absence of earthen wall collapse around such features in excavated contexts together lend support to this interpretation.

\section{Granary}

Medium-sized filled features, regardless of form, were classified as either small (0.81-1.60 m) or large (1.61-2.38 m) granaries on the basis of subtle bimodality in their size distribution. This reading comes first of all from their resemblance to contemporary granaries in the West African savanna. Commonly, these are elevated above ground with either stones or wooden posts, depending on local availability, as a precaution against seasonal flooding, termites, and other pests (Ogundele 2007; Prussin 1972). The size and strong tendency towards circular form in the granary foundations at Diouboye were also consistent with the architectural mechanics required for storing grain. In an ethnographic comparison of earthen wall granaries across northern Ghana, Labelle Prussin has pointed out that "[s]ince cereal grains in bulk generally behave according to hydraulic laws, the function of a granary closely approximates that of a water container” (1972:145). It follows that people usually build granaries of durable mud brick or terre pisé, this latter technique obtaining maximum compressive strength in circular structures.

An overlying deposit of compact clay-enriched earthen wall melt or collapse found in six of nine excavated contexts indicated that these foundations did indeed support some form of earthen wall superstructure. At the same time, it remains possible that the overlying granaries were actually large baskets, as found today in some Bambara villages (Prussin 1974:190). The question that remains to be resolved is how, or if, the 
storage requirements of different grains, the size of social units, and/or stylistic traditions factored into the bimodal size distribution of these features.

\section{Other Unfilled Features: Possible Granaries}

Unfilled features larger than pot rests and smaller than domestic huts (0.81-2.40 m) were probably granaries, although they could have also been grinding stands or even pot rests for large brewing vessels. The largest such features could even have been small kitchens, ritual huts, or specialized storage areas for jars containing water, beer, or other comestibles. Importantly, this indeterminacy does not sway the interpretations of villagescale political economy developed in later chapters.

\section{Small Filled Feature}

Filled stone features in the smallest size modality (0.56-0.78 m) could have been grinding stone supports, elements of dry season kitchens, shrines, or associated with roasting pits. Since these features occurred in small numbers at Diouboye, this indeterminacy does not greatly impact the results of analyses in subsequent chapters.

\section{Curvilinear and Rectilinear Alignments}

Linear alignments with lengths of 1.20-4.60 meters corresponded to intact segments of earthen wall foundations and/or partially dismantled features. Since it was not possible to distinguish between these two possibilities for any given feature, I treated them as functionally indeterminate during analyses.

\section{Decay, Demolition, and Sediment Deposition}

At any given moment, the huts and other structures within each residential area of Diouboye coexisted at different phases in their lifecycles of construction, maintenance, and decay or collapse (see David 1971; R. J. McIntosh 1974; Ogundele 2005). An adequate understanding of these processes is important because they not only preserved (or eradicated) evidence for the history of each architectural feature, but they were also the primary agents of sediment deposition at the site (see also B. A. Hall 1994; Holl 
1987). Additionally, the intentional filling of hardened sediment from collapsed walls or borrow pits may have helped to improve drainage across open courtyard spaces.

While some structures, such as those converted into refuse dumps, may have decomposed gradually, most earthen walls showed evidence for at least partial demolition. When terre pisé walls were pulled down or collapsed, they often protected a foundational wall stub (Figure 6.1) as well as de facto refuse documenting final activities in the structure. Although wattle-and-daub wall stubs were rare (but see Figure 6.2), sediment discoloration and the ubiquity of medium to coarse daub fragments suggests that they were widespread at Diouboye; people may have simply dismantled them to recycle the wooden poles. Where wall stubs were not preserved, these deposits of wall collapse provided important clues to sequences of construction, maintenance, and demolition in each residential area.

In some cases, the pounding and/or repeated use of floor surfaces produced extremely firm lenses of fine sediment identified as possible hut foundations. Most excavated house floors yielded ash lenses, but in no case did they or their associated wall fragments exhibit the extreme oxidation expected from the conflagration of an entire structure, which experimental evidence indicates can reach temperatures of $1000^{\circ} \mathrm{C}$ over a period of 24-48 hours (Friede and Steel 1980). It stands to reason that most, if not all, of the structures excavated at Diouboye were pulled down in the course of spatial remodeling, rather than burned to the ground by raiders.

The number of complete stone features identified in excavation contexts shows that people did not always reuse the sandstone rocks from a dismantled architectural feature or foundation. In some cases, the labor costs involved in digging them out from beneath a collapsed structure may have outweighed the costs of importing fresh material from the nearby sandstone escarpment. In other cases, the superposition of nearly identical features in a stratigraphic sequence indicates that people sometimes employed this sort of architectural "footprint" to support later structures. Overall, the sheer density of rock features in some occupation horizons, which was comparable to the density of those abandoned on the site surface, shows that remodeling was not an insurmountable obstacle to the interpretation or residential space based, in part, on the presence or absence of such features (Chapters 7-8). 
In order to distinguish the depositional processes contributing to each deposit, I compared variables of soil color, texture, and the type and density of non-artifact inclusions to the patterns expected from R. McIntosh's (1977) study of earthen wall architecture in Ghana. Remarkably, the summary statistics for ceramic and faunal artifacts from these intuited deposition types matched up well with the experimental expectations (Table 6.3). Earthen wall stubs, melt, and collapse generally had fewer and smaller sherds, resulting from the inclusion of occupation debris, than surrounding general fill and midden deposits. The tendency towards greater densities of larger sherds in room and pit fill likely coincided with the protection of artifacts from trampling and other forms of attrition. The high densities of faunal remains associated with stone features, particularly granaries could indicate that open space between the rocks naturally collected household sweepings and debris (Ogundele 2005:43). The modest size and density of artifacts in general fill deposits was consistent with diverse processes of earthen wall erosion and collapse, intentional filling, and the discard of domestic refuse.

Table 6.3: Summary of artifact attributes by depositional context

\begin{tabular}{|c|c|c|c|c|c|c|c|c|c|}
\hline Depositional Cor & $\begin{array}{l}\text { ext } \\
\text { Total }\end{array}$ & \multicolumn{2}{|c|}{$\begin{array}{c}\text { Sherd Weight } \\
\text { Grams }\end{array}$} & \multicolumn{2}{|c|}{$\begin{array}{c}\text { Sherd Ratio } \\
\qquad \mathrm{N} / \mathrm{m}^{3}\end{array}$} & \multicolumn{2}{|c|}{$\begin{array}{c}\text { Sherd Density } \\
\mathrm{kg} / \mathrm{m}^{3}\end{array}$} & \multicolumn{2}{|c|}{$\begin{array}{c}\text { Fauna Density } \\
\mathrm{g} / \mathrm{m}^{3}\end{array}$} \\
\hline & $n$ & $\mu$ & $\sigma$ & $\mu$ & $\sigma$ & $\mu$ & $\sigma$ & $\mu$ & $\sigma$ \\
\hline $\begin{array}{l}\text { Stone foundation, } \\
\text { curvilinear }\end{array}$ & 12 & 13.01 & 7.12 & 676 & 1196 & 7.77 & 11.67 & 1594 & 1224 \\
\hline $\begin{array}{l}\text { Stone foundation, } \\
\text { irregular }\end{array}$ & 5 & 20.28 & 16.29 & 674 & 440 & 15.88 & 9.35 & 5037 & 5730 \\
\hline $\begin{array}{l}\text { Stone foundation, } \\
\text { rectangular }\end{array}$ & 1 & 5.00 & - & 23 & - & 0.11 & - & 0 & - \\
\hline Pyrotechnic feature & 9 & 12.82 & 5.64 & 267 & 287 & 3.67 & 5.28 & 405 & 362 \\
\hline Earth wall stub & 6 & 6.99 & 1.85 & 158 & 215 & 1.17 & 1.65 & 245 & 187 \\
\hline Earth wall collapse & 14 & 10.92 & 2.55 & 200 & 186 & 1.99 & 1.45 & 221 & 180 \\
\hline Earth wall melt & 5 & 9.04 & 7.14 & 146 & 101 & 1.65 & 2.03 & 235 & 212 \\
\hline Fill, general & 55 & 9.83 & 4.20 & 471 & 371 & 4.04 & 2.76 & 639 & 544 \\
\hline Fill, pit/midden & 22 & 12.83 & 5.01 & 1080 & 788 & 13.40 & 10.45 & 2586 & 2212 \\
\hline Fill, room & 9 & 18.56 & 7.76 & 671 & 813 & 11.43 & 15.49 & 559 & 536 \\
\hline
\end{tabular}




\section{NON-ARCHITECTURAL FEATURES}

Whereas architecture strongly shaped formal space within Diouboye, many other elements of the built environment—including pyrotechnic features, open courtyards, refuse pits, and middens-likely emerged incidentally through the economic practices and daily routines of informal space. The life histories of these features, as with architecture, can attest to the structured array of activities resulting in their production, use, and abandonment. In this section I continue to employ ethnographic observations as a backdrop against which to interpret the archaeological evidence for such activities at Diouboye.

\section{Pyrotechnic Features}

Cooking, roasting, metalworking, ritual immolation, and other activities involving fire at Diouboye rarely took place around a heavily built feature such as a pit. In order to understand how fire structured space and associated subsistence and craft practices across Diouboye, I have tentatively classified the resulting pyrotechnic features on the basis of diameter, depth, shape, and associated ash, charcoal, and artifacts.

\section{Table 6.4: Summary of attributes for pyrotechnic features}

\begin{tabular}{|c|c|c|c|c|c|c|c|}
\hline \multicolumn{2}{|l|}{ Feature Type } & \multirow{2}{*}{$\begin{array}{c}\text { Diameter } \\
\text { Meters } \\
\text { range }\end{array}$} & \multirow{2}{*}{$\begin{array}{l}\text { Depth } \\
\text { Meters } \\
\text { range }\end{array}$} & \multirow{2}{*}{$\begin{array}{c}\text { Color } \\
\text { Munsell } \\
\text { hue }\end{array}$} & \multicolumn{3}{|c|}{ Ash Density (Estimated) } \\
\hline & $\begin{array}{c}\text { Cotal } \\
n\end{array}$ & & & & $\begin{array}{c}<2 \% \\
n\end{array}$ & $\begin{array}{c}2-10 \% \\
n\end{array}$ & $\begin{array}{c}>10 \% \\
n\end{array}$ \\
\hline Hearth & 16 & $0.42-0.90$ & $0.01-0.07$ & 7.5YR - 5R & 2 & 10 & 4 \\
\hline Hearth complex & 8 & $0.90-2.30$ & $0.02-0.13$ & 5YR - 7.5YR & 0 & 5 & 3 \\
\hline Forge (?) & 1 & 1.58 & 0.05 & $5 Y R$ & 0 & 0 & 1 \\
\hline Roasting pit (?) & 1 & 1.30 & 0.21 & 7.5YR & 0 & 1 & 0 \\
\hline
\end{tabular}

\section{Hearth}

Hearths were shallow horizons of reddish oxidized soil resulting from small fires with a sustained temperature over $500^{\circ} \mathrm{C}$ (Rapp and Hill 1998:37). At Diouboye, these were less than 90 centimeters in diameter and often accompanied by a lens of fine 
grayish-white ash from the wood, dung, or other material burned as fuel. Hearths often, but not always, occurred within domestic huts where they must have provided a place for preparing food and/or a source of warmth during the cool evenings of the dry season.

\section{Hearth Complex}

Hearth complexes included three or more layers of oxidized sediment interspersed with thin lenses of gray ash resulting from the long-term use of a single space for firerelated activities (Figure 6.9). Since the placement of a fire might shift from day to day, these complexes had diffuse spatial boundaries and measured more than one meter in diameter. A number of subsistence and craft activities could have contributed to the evolution of hearth complexes, but their modest numbers of pottery sherds and faunal remains (Table 6.3) suggest that food preparation was a predominant activity. Moreover, hearth complexes never directly adjoined other architectural features, suggesting that they anchored the relatively open space of courtyards and gained some degree of permanence within the built environment as structures rose and fell around them.

Figure 6.9: Profile of hearth complexes with oxidized horizons and ash lenses (Feature 74)

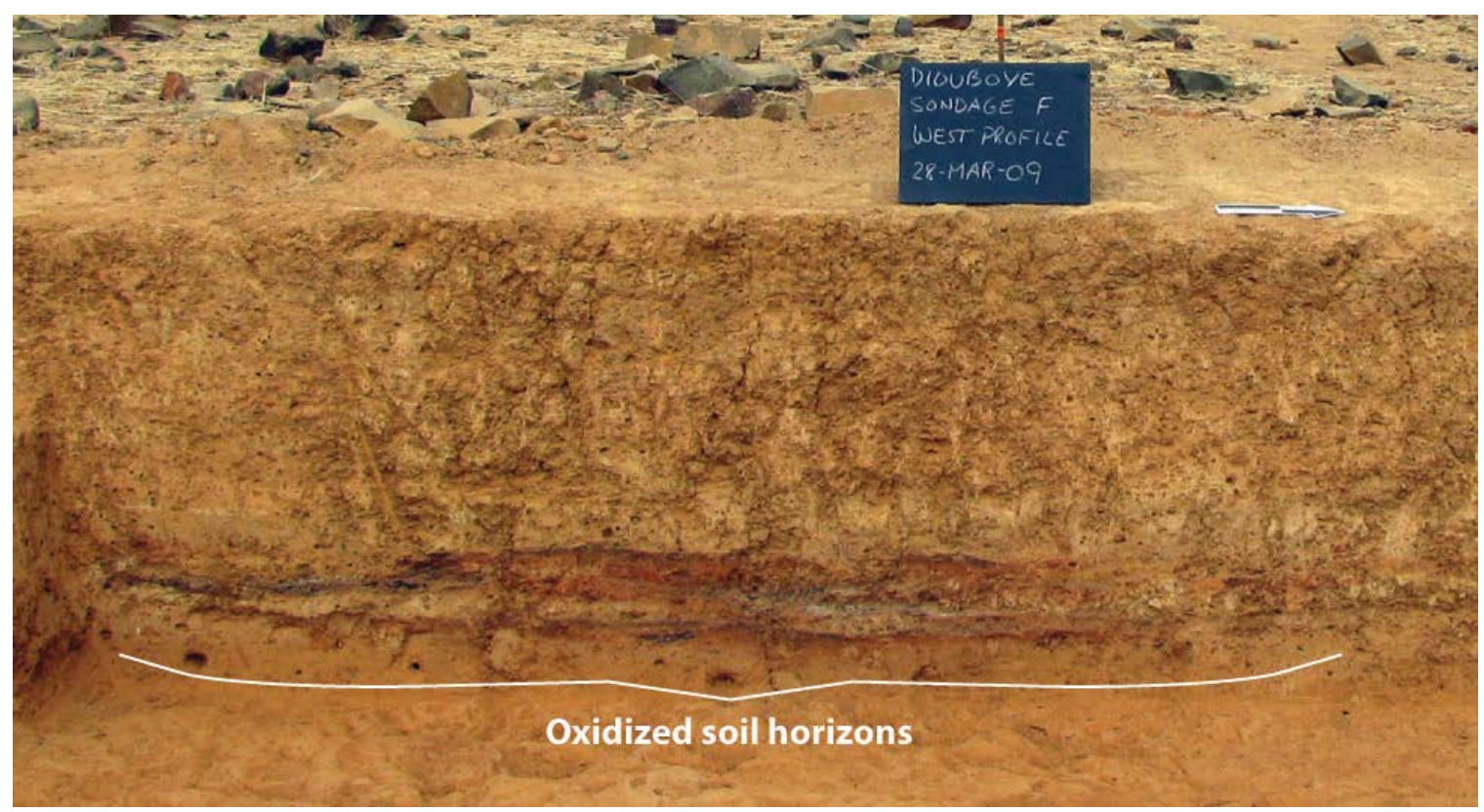


Two irregular pyrotechnic features merit mention. As described in the following chapter, one hearth complex (Feature 14) associated with extremely high quantities of ash and elevated frequencies of glassy and pit slag (see Appendix F) in the surrounding deposits was probably an informal forge for metalworking. Another hearth (Feature 65) set into a circular depression with oxidized walls and an adjacent stack of stones may have been a roasting pit.

\section{Ash Dump}

Ash dumps were shallow lenses of ash and cinders not found in association with oxidized soil. The majority of these deposits occurred in proximity to hearths, hinting at episodes of fire management or hearth cleaning, but some may have derived from the toppling of burned wooden structures.

\section{Pit Features}

The residents of Diouboye sometimes dug pits through previous anthropogenic deposits. Based on their size and overall form, I have classified these as amorphous, cylindrical, and shallow pits (Table 6.6). Although the impetus for shallow pits remains unclear, the larger amorphous and cylindrical pits could have been sources of sediment for earthen walls (see above). Whatever their origins, all pits were ultimately filled with ceramic sherds, animal remains, ash, and other secondary refuse from subsistence and craft activities (Table 6.3). Cylindrical pits (Figure 6.10) likely accumulated refuse from nearby activities within the same residential compound, while the large amorphous pits contained a greater diversity of ceramics and other artifacts, perhaps from their use as communal middens by several neighboring compounds (see Hayden and Cannon 1983:140-146; Wilson 1994). 
Table 6.6: Summary of attributes for pit features

\begin{tabular}{|c|c|c|c|c|c|c|c|}
\hline Pit Form & $\begin{array}{r}\text { tal } \\
n\end{array}$ & $\begin{array}{c}\text { Diameter } \\
\text { Meters } \\
\text { range }\end{array}$ & $\begin{array}{l}\text { Depth } \\
\text { Meters } \\
\text { range }\end{array}$ & $\begin{array}{c}\text { Volume } \\
\mathrm{m}^{3} \\
\text { range }\end{array}$ & $\begin{array}{c}\text { Ceramic } \\
\text { Density } \\
\mathrm{kg} / \mathrm{m}^{3} \\
\text { range }\end{array}$ & $\begin{array}{c}\text { Fauna } \\
\text { Density } \\
\text { g/m³ } \\
\text { range }\end{array}$ & $\begin{array}{c}\text { Ash } \\
\text { Estimated } \\
\text { range }\end{array}$ \\
\hline Amorphous & 2 & $>2.00$ & $1.20-1.70$ & $>4.2-13.6$ & $6.17-36.38$ & $1402-4809$ & $<2 \%$ \\
\hline Cylindrical & 4 & $0.72-1.40$ & $0.70-0.90$ & $0.29-1.39$ & $7.23-14.05$ & $216-1969$ & $2-10 \%$ \\
\hline Shallow & 3 & $0.65-1.00$ & $0.17-0.32$ & $0.07-0.13$ & 2.96 & 1792 & $2-10 \%$ \\
\hline
\end{tabular}

\section{Figure 6.10: Profile of cylindrical pit (Feature 82)}

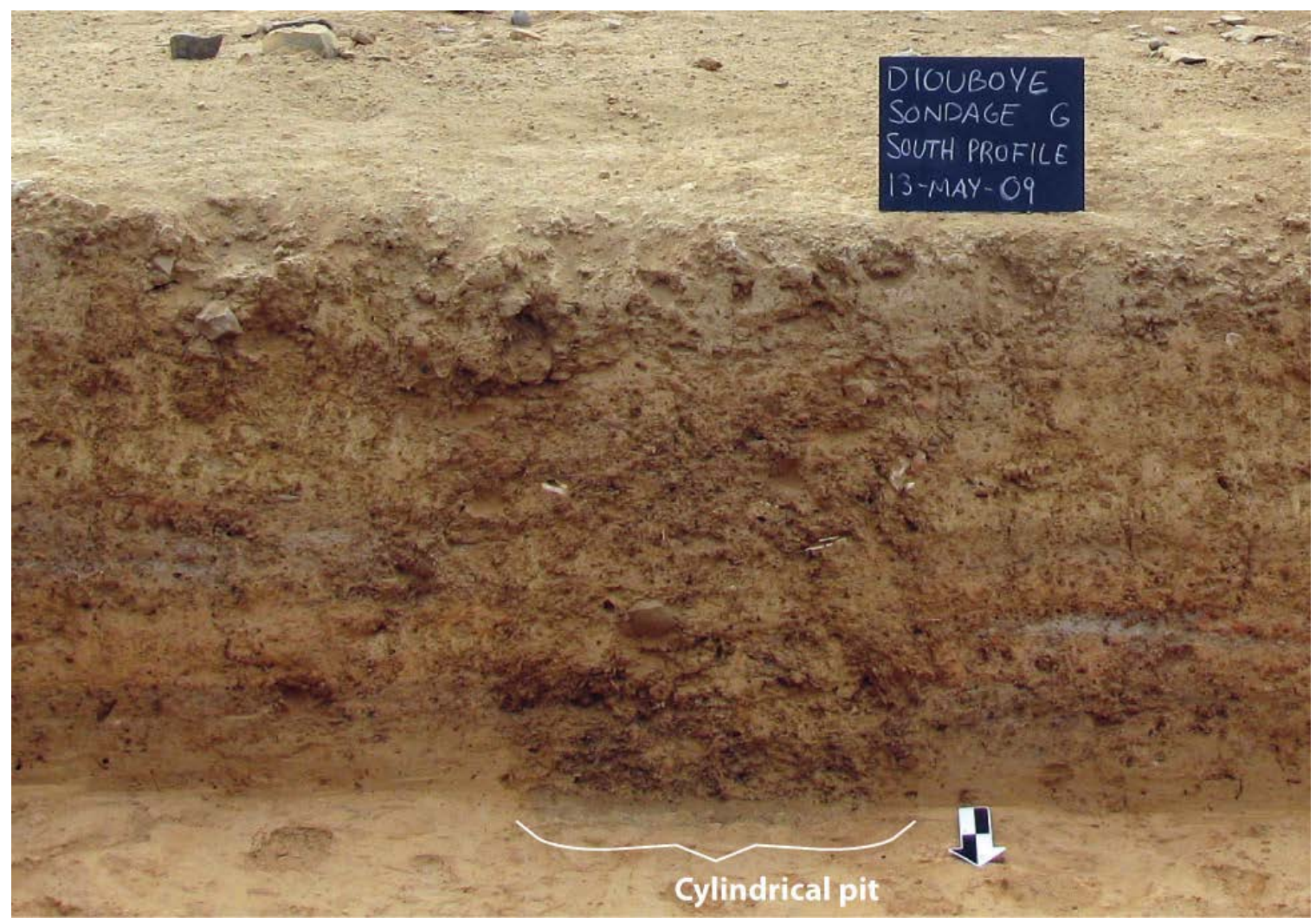

\section{Middens}

The occupants of Diouboye disposed of articles from subsistence and craft activities (and sometimes long-distance exchange) in secondary refuse dumps (middens). Insofar as refuse disposal took place in, and perpetuated the location of, low activity and 
low traffic areas, this process helped to segregate space within and between residential and non-residential areas of the village. In fact, sheet middens, identified as strong brown sediments with exceptionally high densities of charcoal, sherds, and faunal remains (see Table 6.3) formed directly above most amorphous and cylindrical refuse-filled pits. This suggests that once people designated a space for refuse disposal, they continued to use it that way for quite some time. And yet, the presence of domestic huts and hearth complexes directly above midden deposits at Diouboye shows that such spaces were not forever contaminated, but could be reappropriated as necessary.

\section{ACTIVITIES}

In this section I briefly summarize the sorts of economic practices that took place in, around, and beyond the abovementioned architectural features on a daily basis. Although I focus on the spheres of subsistence, craft, and long-distance exchange central to this study, I also make mention of limited evidence for ritual and bodily adornment. In so doing, I note that evidence for economic practices has been shaped by pathways of discard, including primary refuse (defining potential activity areas) or secondary refuse disposal in pits or middens, the particularities of which are discussed in Chapters 7-8.

\section{Subsistence Economy}

During the early-second millennium AD, the climate and geography of the lower Falémé River basin was amenable to subsistence strategies grounded in hunting, gathering, farming, and possibly herding (see Chapter 3). Not surprisingly, then, multiple lines of evidence confirmed that residential groups across Diouboye practiced a diverse array of subsistence pursuits.

\section{Food Production and Procurement}

\section{Farming}

At a minimum, the residents of Diouboye cultivated pearl millet, presumably on the alluvium found within a one-kilometer catchment of the village. As detailed in the analysis by Daphne Gallagher (see Appendix I), carbonized caryopses from this domesticate cropped up in several excavated contexts across the site. The small sample of 
identified grains precludes a full assessment of their contribution to overall diet, but the ratio of granaries to earthen wall stubs in excavated units (5:3) appears to match or exceed the relative amount of space dedicated to grain storage in ethnographic West African villages heavily engaged in farming (e.g., David 1971; Lestrange 1955; Prussin 1969, 1972). While recognizing the differential preservation of stone versus earthen architecture, this pattern suggests that millet was a staple, if not the staple, foodstuff within Diouboye.

Clearing land and harvesting and threshing grain required tools such as axes, hoes, and blades. Although they could have served several purposes, a number of such tools made from both iron and stone (Figure 6.11) further support the contention that the people of Diouboye engaged in some level of food production.

Figure 6.11: Possible farming implements: (a) small iron hoe; (b) mudstone blade; and (c) large groundstone axe

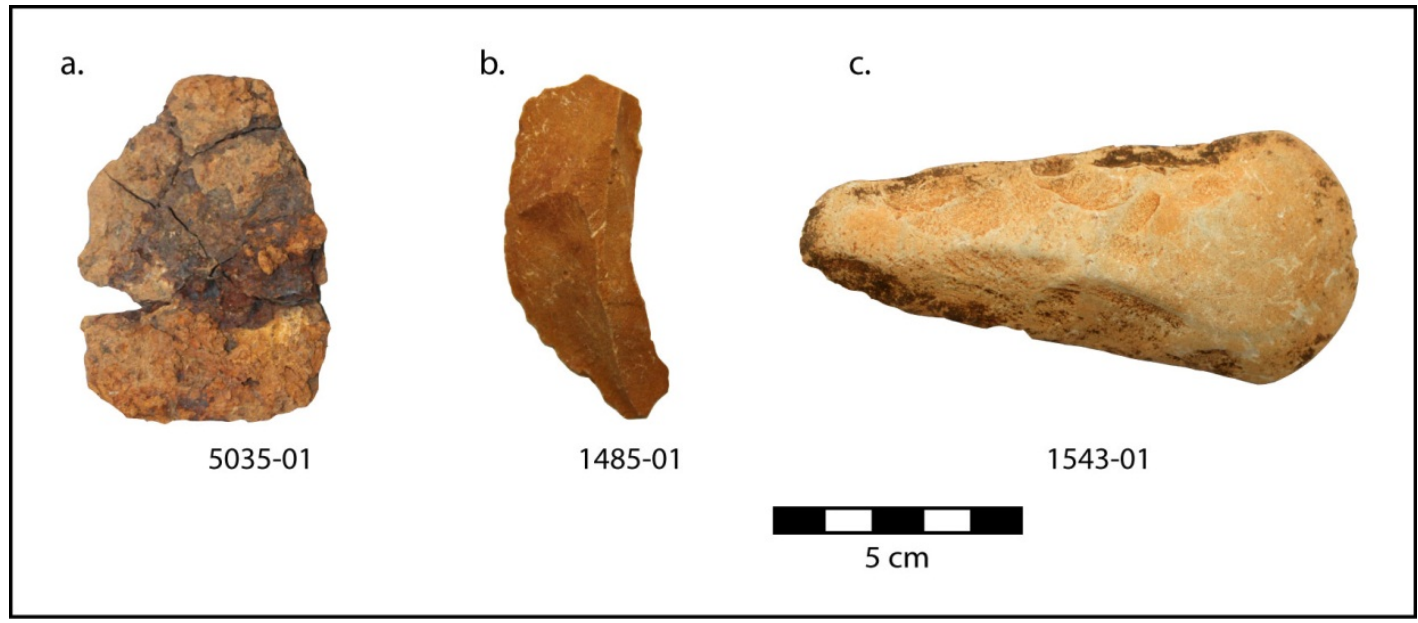

\section{Foraging}

The occupants of Diouboye collected and consumed the fruits and seeds of wild trees such as baobab, jujube, and desert date (Gallagher, Appendix I), all of which would have been available in cultivated fields surrounding the village and the savanna forest beyond. Although no direct evidence remains, people almost certainly made use of the 
leaves and bark of these plants (and many others) for sauces, beverages, textiles, and so forth. They also exploited wood and grasses to build huts and furniture and to make the fires attested by the charcoal and ash interspersed throughout excavated deposits. In this regard, groundstone axes and iron blades may provide indirect evidence for felling trees and clearing fields.

\section{Herding}

While perennial access to water and vast stretches of land ideal for grazing could have supported substantial herds around Diouboye, the small proportions of positively identified cattle (5\%) and sheep/goat (4.5\%) bones in the fauna sample analyzed by Stephen Dueppen suggest that these animals played a minor role in the overall diet (Appendix H). Depending on the specific breeds, however, seasonal transhumance may have been required to mitigate the effects of sleeping sickness. Whether the villagers were raising their own herds, or bartering with specialized pastoralists, remains an open question. In either case, the relative emphasis on cattle could signal the value of these animals within the local political economy as currency in marriage transactions, politicoritual feasts, and establishing social debts.

\section{Figure 6.12: Contemporary herd of cattle near Sansanding, Senegal}

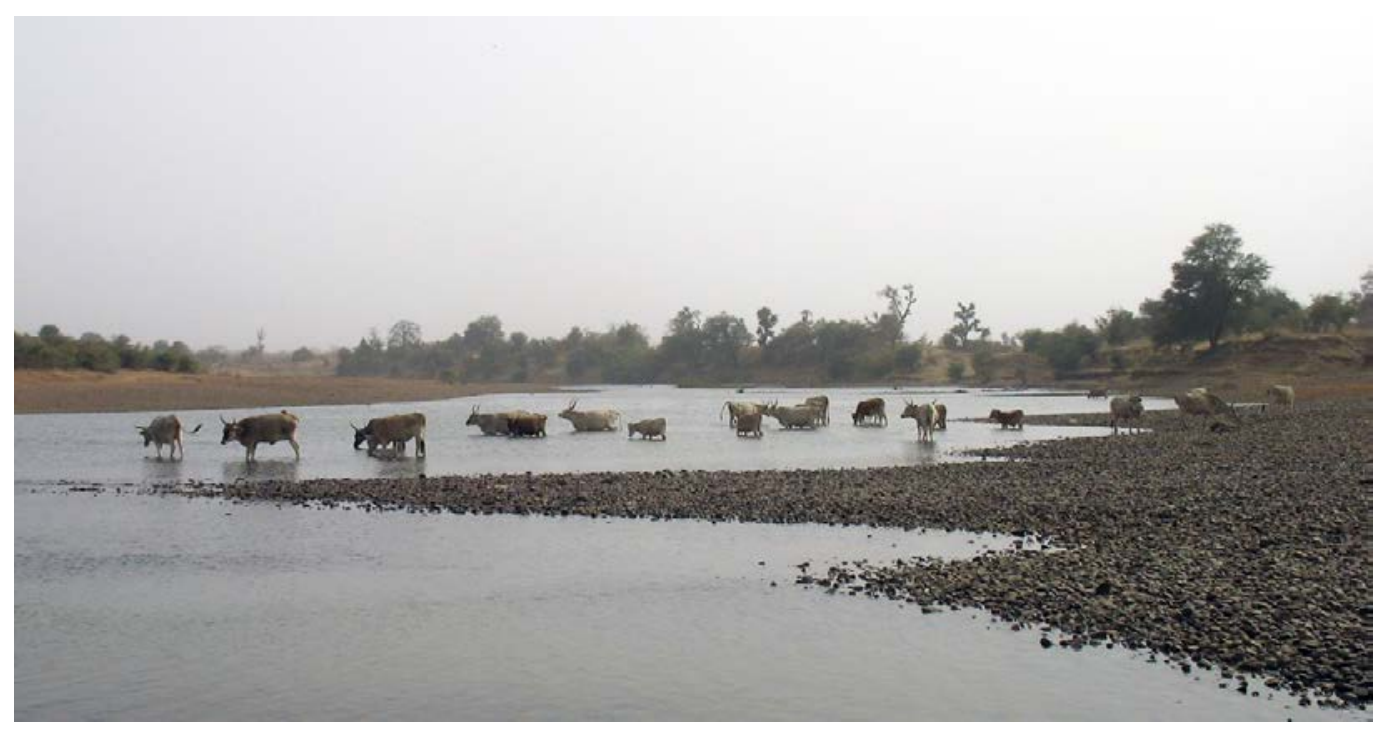




\section{Hunting and Fishing}

Interestingly, the people of Diouboye focused on the exploitation of large-bodied game and fish (Dueppen, Appendix H). From the savanna forest and riparian thickets around the village, they hunted animals ranging in size from wild guinea fowl and hares, to small bush duiker, to African buffalo and elephants. They also took down hippopotami and crocodiles in the Falémé River itself. These game may point to relatively targeted exploitation, rather than the opportunistic garden hunting and collective hunting documented at contemporaneous sites in West Africa (Dueppen 2008:242). In addition to unambiguous iron points and knives, hunters may have employed chipped quartz and chert tools for taking down game or for processing meat and hides (Figure 6.13).

Figure 6.13: Hunting and fishing equipment: (a) mudstone bifacial point; (b) large iron projectile point; (c) quartz bifacial point base; (d) small iron projectile point; (e) possible iron harpoon fragment; (f) cigar-shaped netweight; and (g) globular netweights

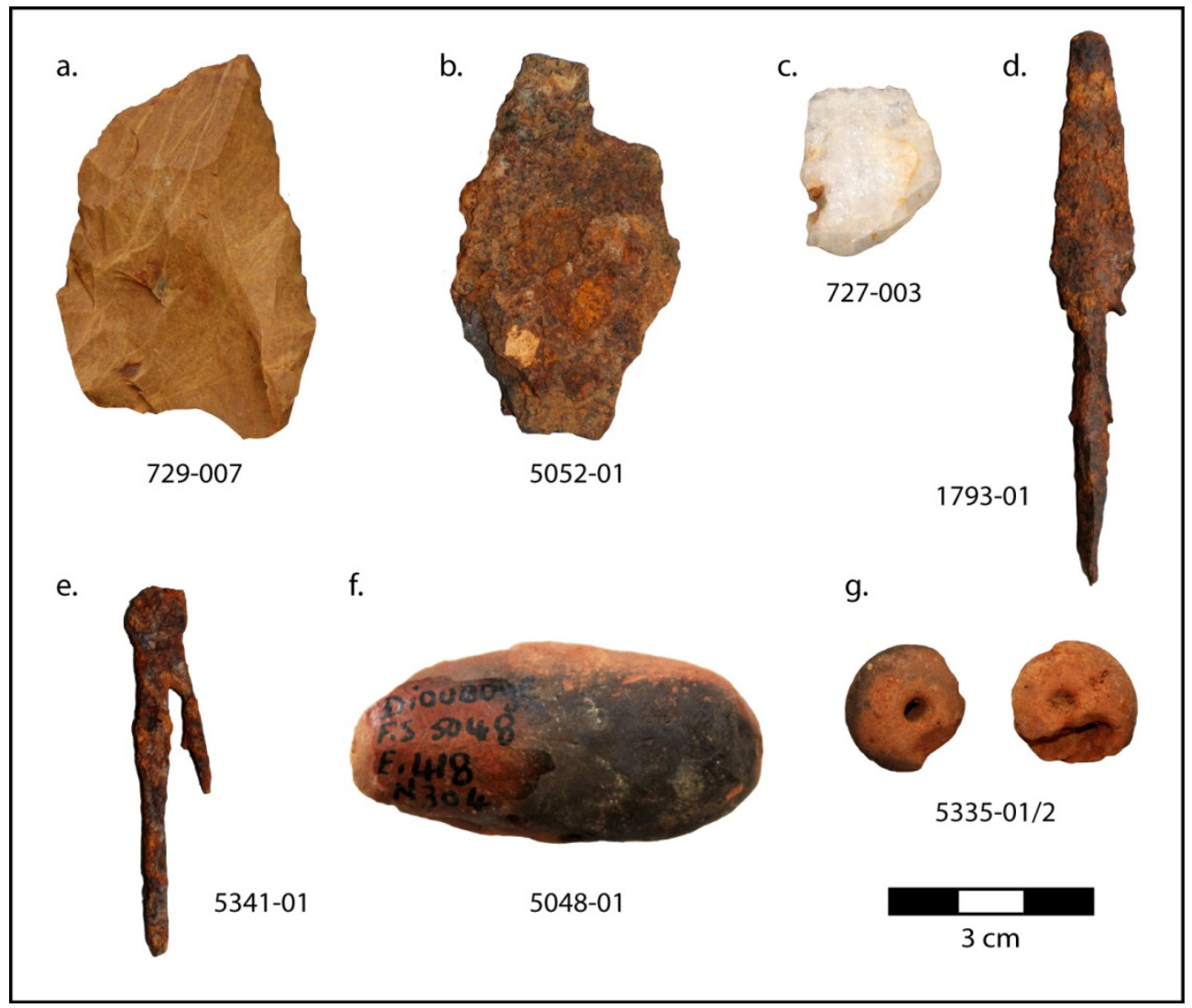


An emphasis on large-bodied fish species, particularly Nile perch and catfish, shows that people mainly fished the deeper central channels of the Falémé River, possibly with the aid of hooks, harpoons, and weighted nets (Van Neer 2008:128), all found at Diouboye (Figure 6.13). People may have salt-dried or smoked fish for longerterm storage (Holl 2002:244-247), but the absence of unambiguous smoking huts or fishdrying platforms, combined with the perennial availability of fish, lends no support to this hypothesis.

Salt

Speaking of salt, this essential dietary supplement may have required specialized extraction, as it did historically in villages where salty earth and brackish water were not readily available. In the Lake Chad basin of northern Cameroon, for example, people relied upon a complex procedure of boiling cattle dung or salty plant leaves to obtain salt-a process associated with distinctive ceramic pottery forms (Holl 2002:243-244). Alternatively, the residents of Diouboye may have obtained salt through exchange networks oriented along the Senegal River to the Atlantic Ocean or toward the Teghaza salt mines of the Sahara Desert (Alexander 1993). This latter scenario remains plausible given the presence of other objects obtained via long-distance exchange (see below).

\section{Food Preparation and Consumption}

After harvesting, threshing, and storing millet and other edible plants, people prepared them into specific dishes. In order to obtain meal for steaming couscous, boiling porridge and sauces, and perhaps even baking unleavened bread, people across the village employed stone slabs for grinding and stone mortars for pounding and crushing (see Figure 6.8 above). The presence of flat and round handstones, along with the quartz hammerstones needed to rejuvenate grinding slabs (see Appendix E), suggest that the residents of Diouboye maintained a suite of plant processing techniques. Today in northern Ghana, for example, handstones not only serve different functions based on their size, but each woman within a household compound may guard her own stone or stones (Casey 2000:53-58). Additionally, women could have processed grains in wooden mortars, as they do today in rural West African villages. 
Pending a taphonomic analysis of the faunal remains themselves, wild and domesticated animals were probably butchered and processed for their hides in or near the village. Iron knives and chipped stone flakes and informal scrapers could have been used for scraping hides and working leather (Frank 1998; Weedman 2006), while chert flakes employed as burins may have aided with this latter task.

Over the centuries, the residents of Diouboye stored, transported, prepared, and consumed foodstuffs using thousands of ceramic vessels whose fragments comprised the bulk of artifacts recovered from the site. Rim sherds, for example, documented at least six vessel types whose qualitative aspects of form presumably corresponded in some way with their principal uses (see Appendix D for detailed discussion). As summarized in Table 6.7, people likely used jars for storage and transport since their restricted orifices would prevent spillage and/or control access to their contents, although these same features could have made the useful for cooking.

People most likely employed pots with less restricted orifices for preparing different sorts of dishes. Small pots, for example, would have been ideal for heating small quantities of sauce, while larger pots had the capacity for boiling larger quantities of millet and other bulky starches. Alternative uses for these latter included washing and soaking, brewing beer, and perhaps boiling water in conjunction with couscous steamers evidenced by infrequent sherds with circular perforations.

Table 6.7: Summary of vessel types based on form and size (see Appendix D)

\begin{tabular}{|c|c|c|c|}
\hline \multirow{2}{*}{$\begin{array}{l}\text { Vessel Orifice } \\
\text { Size }\end{array}$} & \multicolumn{3}{|c|}{ Vessel Form } \\
\hline & Bowl & Pot & Jar \\
\hline Small & $\begin{array}{l}\text { - Orifice } \leq 15 \mathrm{~cm} \\
\text { - Serving food } \\
\text { - Washing }\end{array}$ & $\begin{array}{l}\text { - Orifice } \leq 18 \mathrm{~cm} \\
\text { - Cooking sauces and/or } \\
\text { cereals }\end{array}$ & $\begin{array}{l}\text { - Orifice } \leq 15 \mathrm{~cm} \\
\text { - Transporting water }\end{array}$ \\
\hline Large & $\begin{array}{l}\text { - } \text { Orifice > } 15 \mathrm{~cm} \\
\text { - Serving food } \\
\text { - Washing } \\
\text { - Tanning } \\
\text { - Steaming grain } \\
\text { (couscous steamer?) }\end{array}$ & $\begin{array}{l}\text { - Orifice > } 18 \mathrm{~cm} \\
\text { - Cooking cereals } \\
\text { - Washing/soaking } \\
\text { - Storing water } \\
\text { - Brewing beer } \\
\text { - Steaming grain }\end{array}$ & $\begin{array}{l}\text { - Orifice > } 15 \mathrm{~cm} \\
\text { - Cooking } \\
\text { - Storing water } \\
\text { - Brewing beer }\end{array}$ \\
\hline
\end{tabular}


Figure 6.14: Vessel types from Diouboye: (a) small pot; (b) small jar; (c) small and large bowls; (d) large pot; (e) large jar A; (f) large jar B (globular)

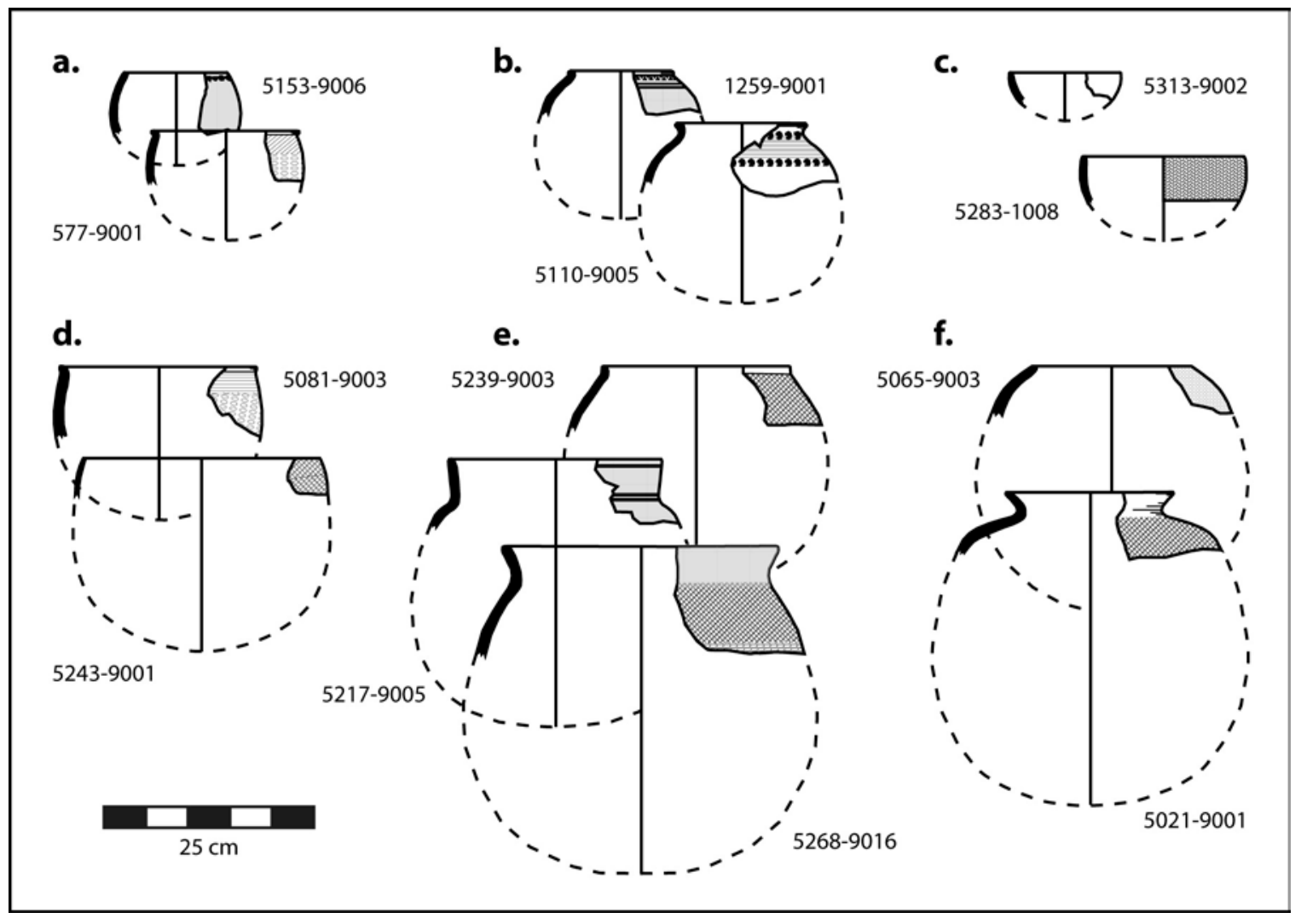

While some of the large unrestricted rims may have belonged to couscous steamers, the majority probably derived from bowls used for serving food, washing, or covering other vessels (lids). Since the preponderance of jars and pots discussed in subsequent chapters indicates that people were consuming couscous and boiled grain dishes, the near absence of small bowls suggests that consumption of daily meals took place around a communal dish or pot, although unpreserved wooden calabashes cannot be ruled out as personal bowls. Alternatively, Susan McIntosh (1995:160) has hypothesized that low numbers of serving vessels in some Iron Age contexts from the Middle Niger region correspond to a focus on baking unleavened bread in shallow depressions. This cooking practice could help to explain the presence of small informal hearths identified in domestic areas at Diouboye-a thought-provoking interpretation that merits further study. 


\section{Craft Economy}

The subsistence economy of Diouboye depended in large part on locally-made crafts. While the production of some craft goods, including metal objects, pots, and possibly groundstone tools, may have become increasingly specialized throughout the history of the community, other craft activities such as woodworking, leatherworking, and weaving were probably more generalized. Deferring a discussion of specialization until the following chapters, here I summarize the evidence for craft production and use practices not already mentioned above in the context of the subsistence economy.

\section{Pottery}

The potters supplying Diouboye with cooking and storage wares employed a fairly discrete range of techniques across five general stages of production-clay procurement, paste preparation, vessel shaping, decoration, and firing (see Appendix D). Despite gradual changes in many techniques, these formed a coherent technological tradition over the several centuries of village occupation.

If contemporary clay sources are any indication of past reality (Gelbert 1999), then potters could have obtained workable clay from the Falémé riverbed, seasonal channels, or even termite mounds. These sources and other raw materials, including grit temper and firing fuel (grass or dung), were all available within one kilometer from the village, minimizing the need for intensive transport. A compositional analysis described in Appendix D found that the potters of Diouboye employed two different types of clay, in addition to a third possible clay type not found at sampled sites from earlier or later archaeological horizons.

Once carried back to the village, potters augmented their clay with either crushed sherds (grog) or grit from a pinkish-white granitic rock composed of feldspar, quartz, and mica (Figure 6.15). The use of grit with all three clay types and the recovery of hunks of this rock at Diouboye suggest that potters intentionally controlled for this inclusion in their clay recipes.

Potters shaped vessels by hand, presumably with the aid of a wooden or ceramic hand wheel, such as the one tentatively identified in Area A (Figure 6.16). By rotating this dish, potters could have steadily built up coils on a rounded base made from a mold 
or hollowed lump. Depending on the overall form of the vessel, they would either fold the rim into an everted or flared lip or add an additional coil of clay to produce a gently flared or beaded collar.

Figure 6.15: Grit temper showing (a) granitic rock nodules from Area A (Unit A2); and (b) coarse grain inclusion $(5 X)$ in pottery sherd from Diouboye

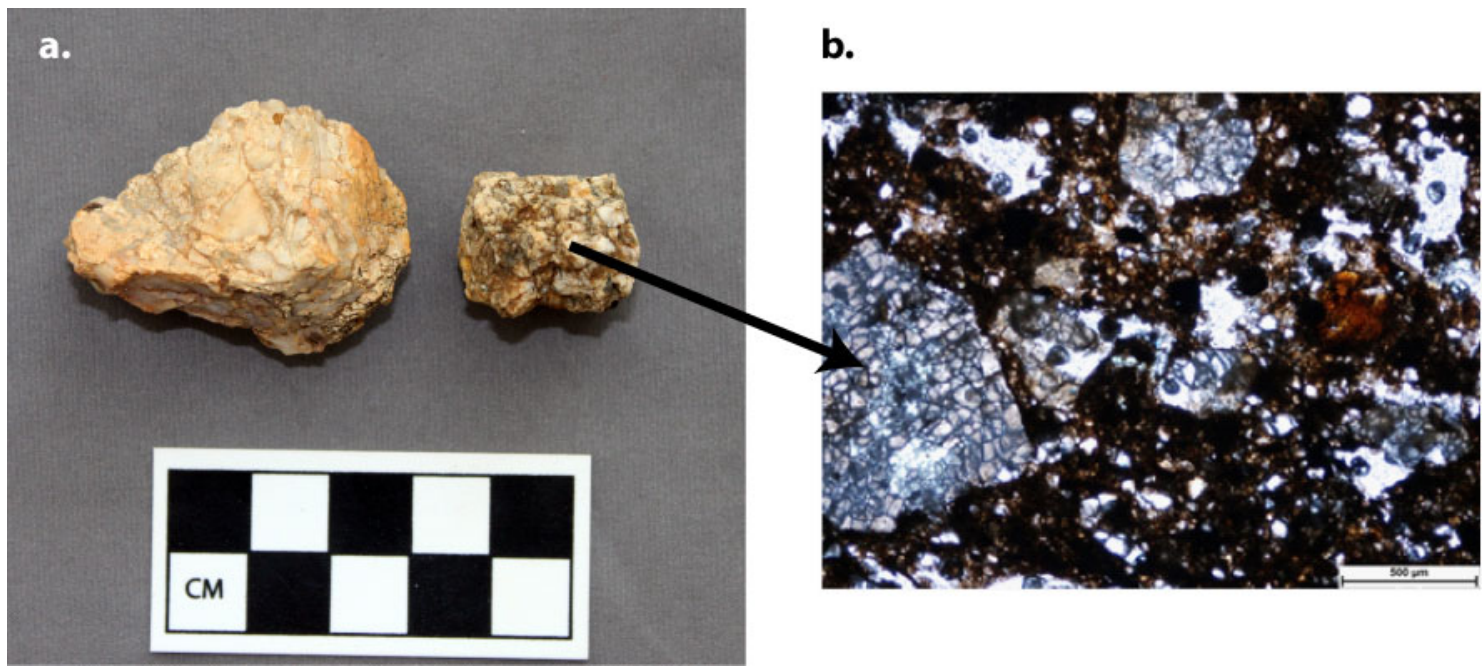

Figure 6.16: Examples of hand wheels from (a) Area A (Unit A2) at Diouboye; and (b) in contemporary use at the village of Ngayène Sabakh, Senegal

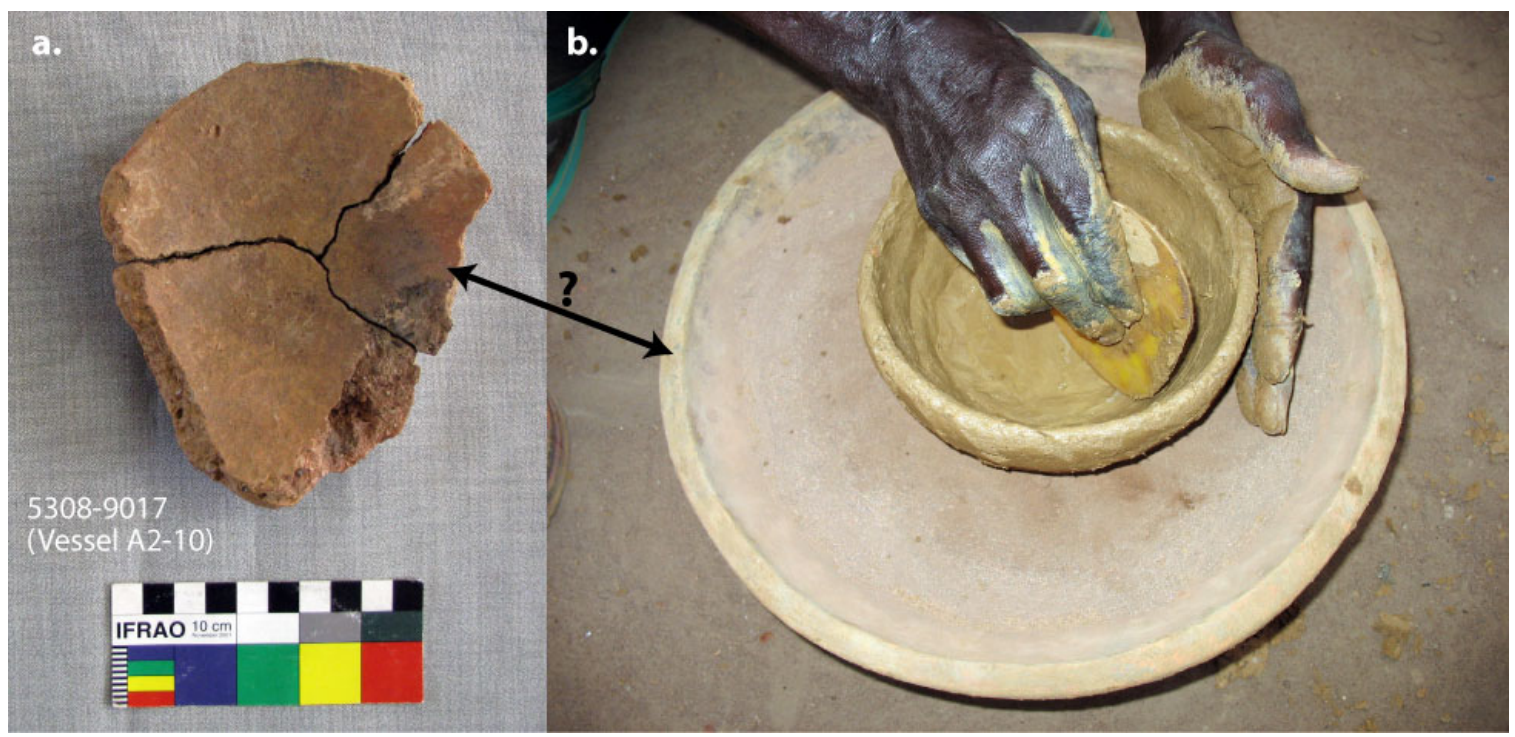


Figure 6.17: Carved chevron roulette decoration common to Diouboye pottery

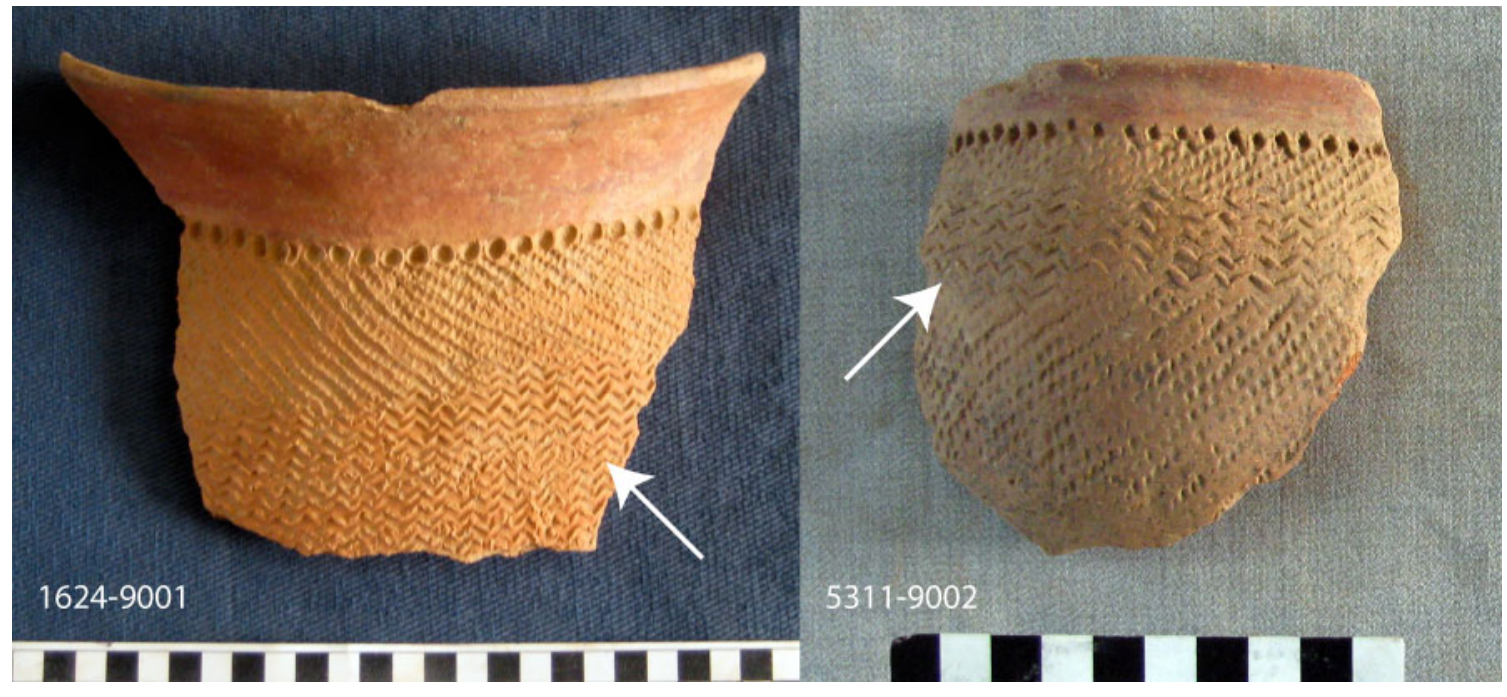

Figure 6.18: Base forms: (a) footed and (b) conical pedestal

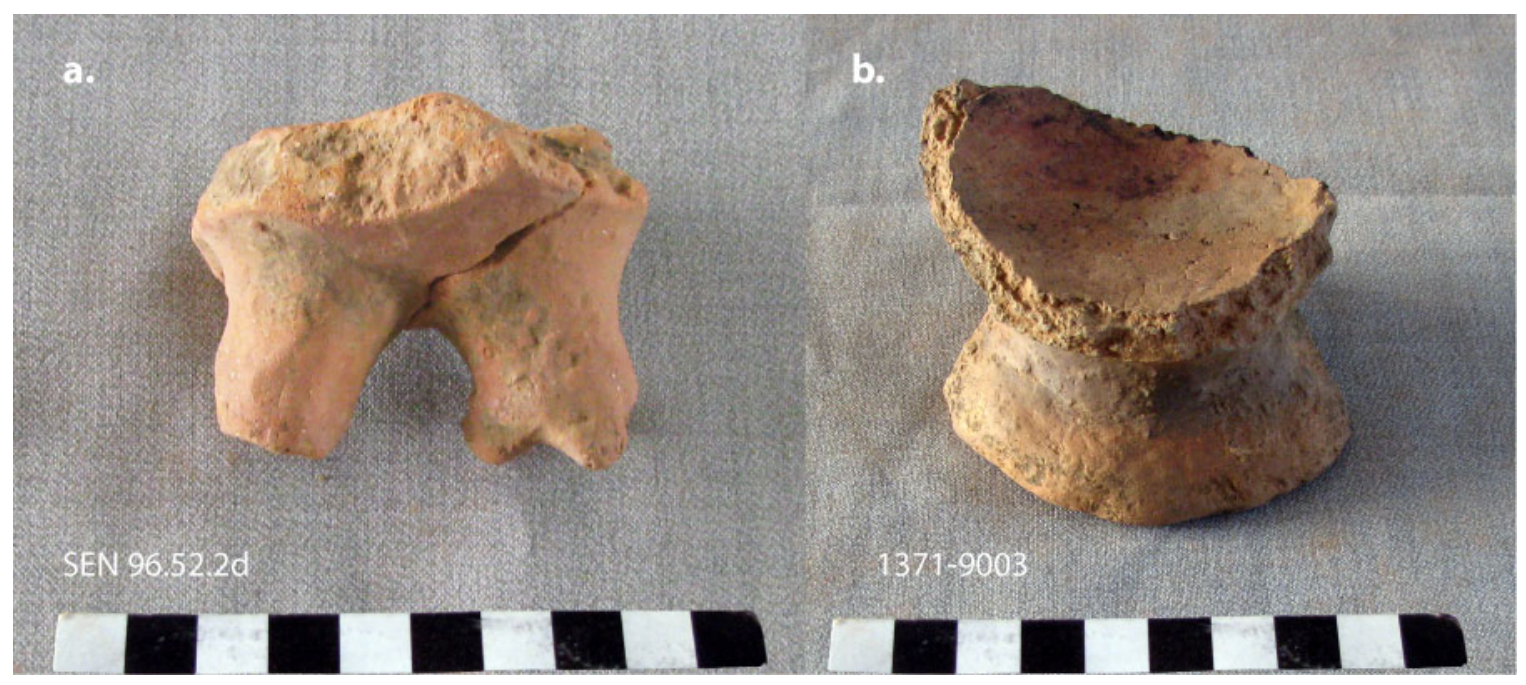

As elsewhere across the West African savanna, potters made extensive use of twisted cord, knotted cord, composite cord-wrapped stick, and carved roulettes to decorate the globular bodies of their wares. Carved roulettes, although they declined throughout the site occupation, were particularly interesting since they link the pottery tradition of Diouboye to that of the Niger-Gambia ceramic spheres described in Chapter 4 
(see also Livingstone Smith 2007). Potters decorated the upper body and rims of their wares with red slip and incised, comb, and stamped decorations. In fact, the application of carved chevron roulette to the shoulder of most jars and larger pots seemed to define a distinctive ceramic “type” for the site (Figure 6.17).

Based on the heterogeneous levels of oxidation and reduction visible in sherd cross-sections, potters probably fired their wares in large bonfires under conditions of moderate air flow.

\section{Metalworking}

The opening of the earth to obtain ores and their transformation into tools and ornaments were technologically complex tasks and, by extension, potential sources of economic and ritual power in precolonial West Africa. For these reasons, metalworking seems to have been a specialized element of the craft economy within Diouboye, a topic that I address in the following chapters.

People obtained workable metals and finished objects through a variety of social or technological pathways. The distinct geology of the Falémé River basin gave the inhabitants of Diouboye relatively unimpeded access to iron ores and alluvial gold (Chapter 3). Since unambiguous furnaces and smelting slags were not observed during systematic survey in the Madina-Sadatou study area (Chapter 4), the small quantities of slag from Diouboye and surrounding sites probably derived from processing raw bloom or forging iron into finished objects. According to this scenario, smiths at Diouboye would have obtained iron through exchange with outlying communities. Based on ethnographic and historic accounts, it is more likely that the villagers did exploit local gold deposits by digging, sorting, and panning in the Falémé River bed (see Appendix F), but these practices have left no archaeological evidence for inferring their material or spatial dimensions.

Although it is possible that people occupying the Upper Senegal region smelted copper from degraded Birimian greenstone deposits, long-distance exchange was a more likely source for this precious red metal. Copper artifacts traceable to the Akjoujt mines of Mauritania were traded along the Senegal River by at least the mid-first millennium BC (Deme and McIntosh 2006) and even appeared in late-first millennium AD contexts 
at the mound complex of Arondo at the Falémé-Senegal confluence (Thiaw 1999:219222). Pending a compositional analysis, the presence of copper at Diouboye by the earlysecond millennium $\mathrm{AD}$ would be consistent with the expansion of networks for copper exchange across Senegambia by this time.

The techniques used by blacksmiths at Diouboye to produce finished objects must have depended on both the metal at hand and, in the case of iron, on the quality of the bloom. Iron smithing, for example, involved the hammering of metal heated in a forge at temperatures of $450-800^{\circ} \mathrm{C}$ to form the diverse tools and ornaments used and worn by members of the community (Figures 6.11, 6.13, 6.20). Although some forges in the Upper Senegal region were historically quite complex (Appia 1965:347-349), many others consisted simply of a bellows, bed of charcoals, water basin, and set of hammers and other tools (McNaughton 1988:28-29). This layout accords well with an abnormal pyrotechnic feature associated with significantly elevated amounts of slag (Figure 6.19) and interpreted as a forge for low intensity blacksmithing.

Assuming that the few copper objects characterize the suite of known techniques, then smiths must have fashioned small decorative objects (Figure 6.20) from drawn

Figure 6.19: Possible forge in Area A (Unit A, Feature 14)

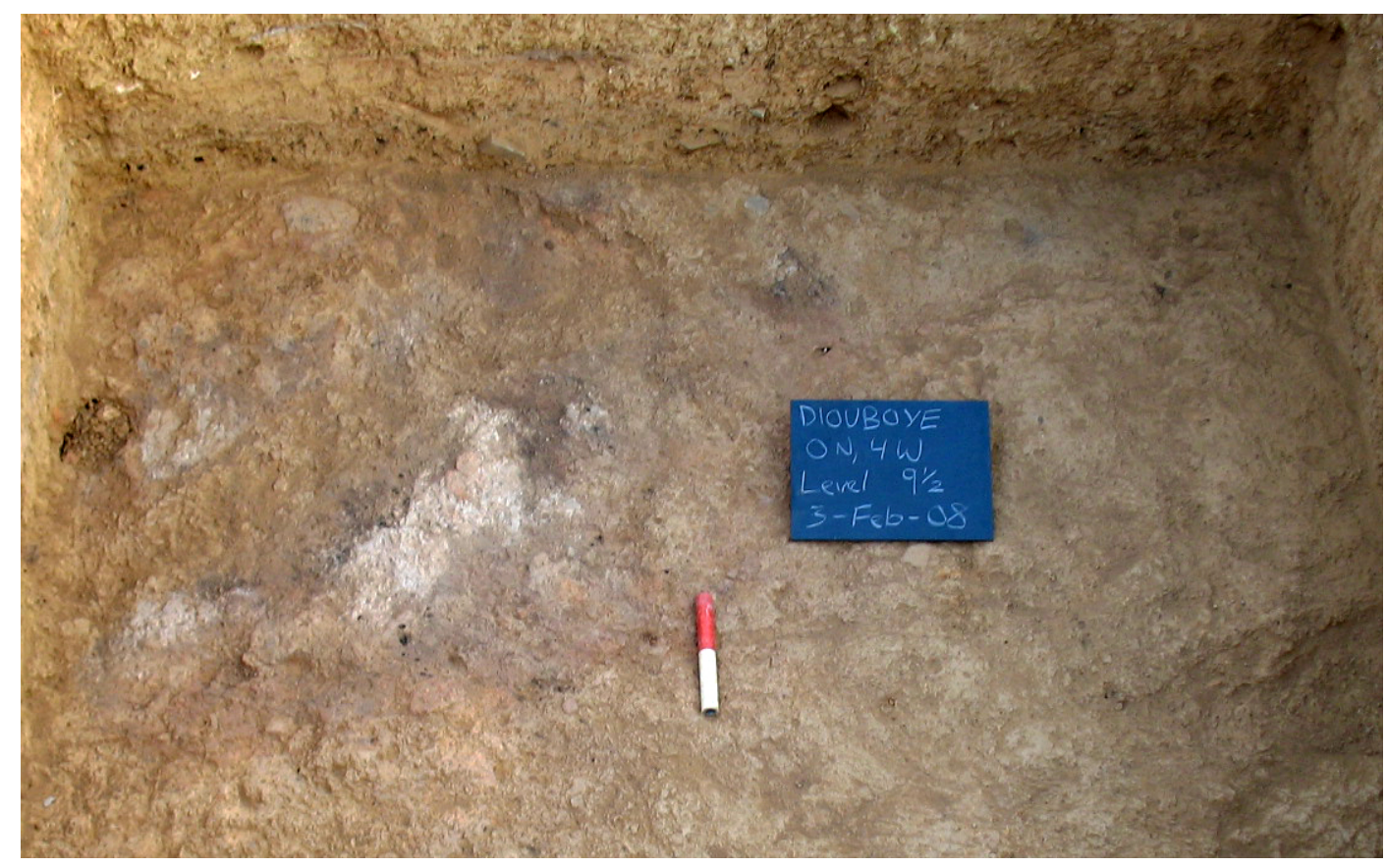


plates and wires or perhaps cast them from molten copper produced in small conical crucibles (Figure 6.21) similar to those found at other Late Iron Age sites in the region (Filipowiak 1979). The use of this latter technology is particularly interesting since it also could have been used to cast gold.

Figure 6.20: Iron and copper artifacts made by techniques of (a) hammering (and welding?); (b-c) hammering (and drawing?); and (d) casting?

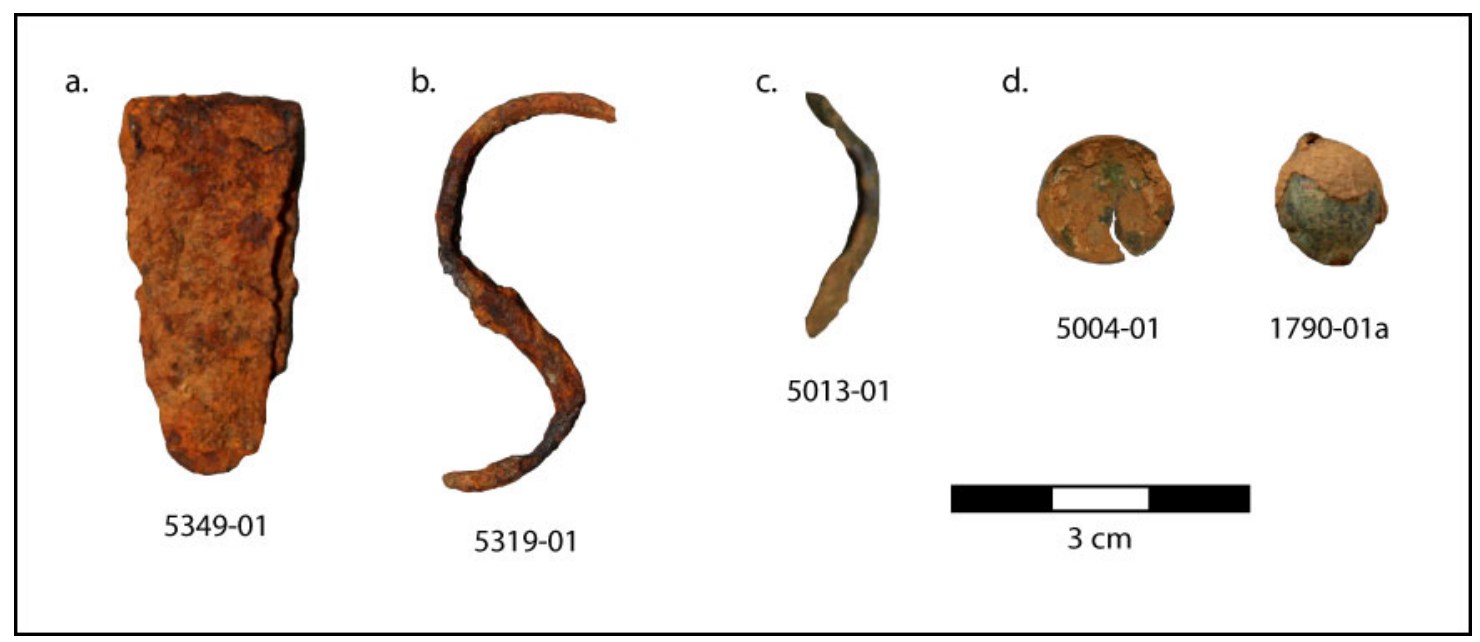

Figure 6.21: Crucible with possible copper prill from Area A (Unit A2)

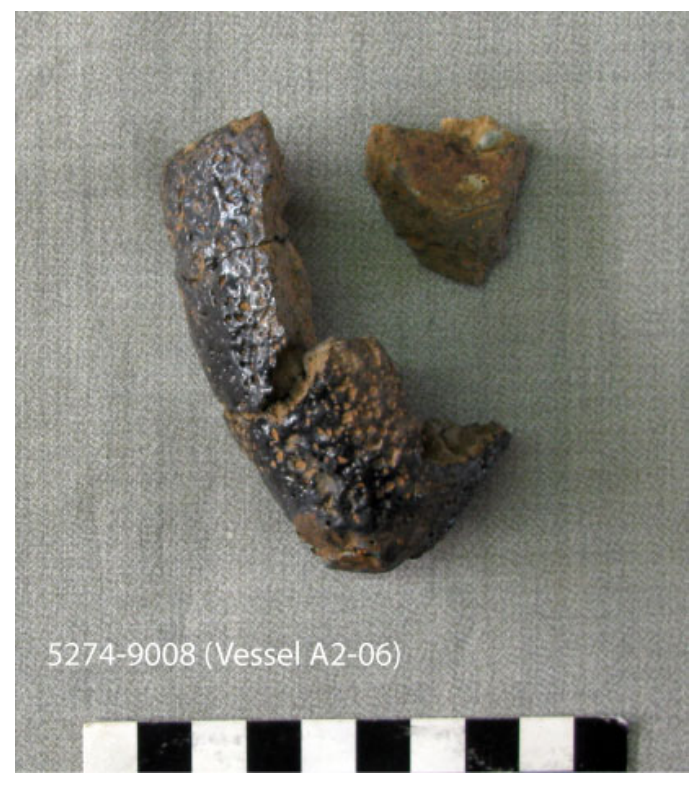




\section{Spinning and Weaving}

With the aid of ceramic spindle whorls (Appendix G), the residents of Diouboye spun yarn from fibers that may have included wool, palm (Raphia sp.), or wild grasses. Although the site yielded no direct evidence for weaving, people may have employed horizontal strip looms similar to those described by al-Bakri for the town of Silla on the Senegal River and attested by a small sample of textile fragments recovered from other archaeological sites dating from the late-first millennium AD onwards (Kriger 2006:7080). Indirectly, the diversity and complexity of twisted and braided cord roulettes applied to pottery belie a strong knowledge of the techniques for manipulating fibers.

\section{Chipped Stone Reduction and Use}

In addition to iron tools, the residents of Diouboye made and used chipped stone tools for a variety of purposes within the subsistence and perhaps craft economy. This claim remains tentative in the absence of well-dated activity areas from excavated contexts, but the greater exploitation of quartz, use of bipolar reduction, presence of larger informal tools, and dearth of microliths all distinguished the chipped stone assemblage of Diouboye from those of three nearby Late Stone Age sites (Appendix E). While I expect this claim to be provocative given the view that sedentary villagers in West Africa had largely abandoned chipped stone technologies by the second millennium AD, I would simply note that such artifacts are regularly found in Late Iron Age contexts (e.g., Gallagher 2010; MacDonald 1997), and even remain in use today (Weedman 2006). In part, this reflects the incredible spatial and social variation in the adoption of, and access to, iron technology in Africa over the past three millennia (Holl 2000), but it also attests to a willingness to efficiently exploit available geological resources.

The inhabitants of Diouboye had relatively direct access to workable cobbles of quartz, chert, siliceous mudstone, and perhaps even hematite, from the bed of the Falémé River or exposed sedimentary strata within a two-kilometer catchment (see Chapter 3). The predominance of quartz (65\%) in comparison to chert (28\%) across the site suggests that people were not concerned with searching for fine-grained materials or perhaps that they preferred quartz for specific tasks such as scraping. Quartz flakes may have also derived from the hammerstones used to regenerate groundstone basins (see below). 
People reduced cobbles of quartz and chert using bipolar reduction and, possibly, freehand reduction, evidently with little regard for the type of material being worked. Put simply, this technique involves placing a nodule of raw material (sometimes wrapped in cloth) on an anvil, then smashing it from above. Although bipolar reduction produces lots of angular shatter, it also quickly generates quite a number of flakes with a variety of sharp or steep edges. The presence of flakes, formal tools, and the occasional freehand core further suggests that people did employ freehand reduction, primarily to shape bipolar pieces or retouch tools. At Diouboye, both of these techniques could have been learned and practiced by people throughout the village in the course of day-to-day economic activities.

Figure 6.22: Chipped stone tools: (a-b) utilized blades; (c) utilized flake with sharp margins; (d) wedged blade with steep margin; (e) scraper; (f) wedged blade with steep margin and light retouch; (g) keeled core with utilized margins; (h) utilized flake with moderate margins

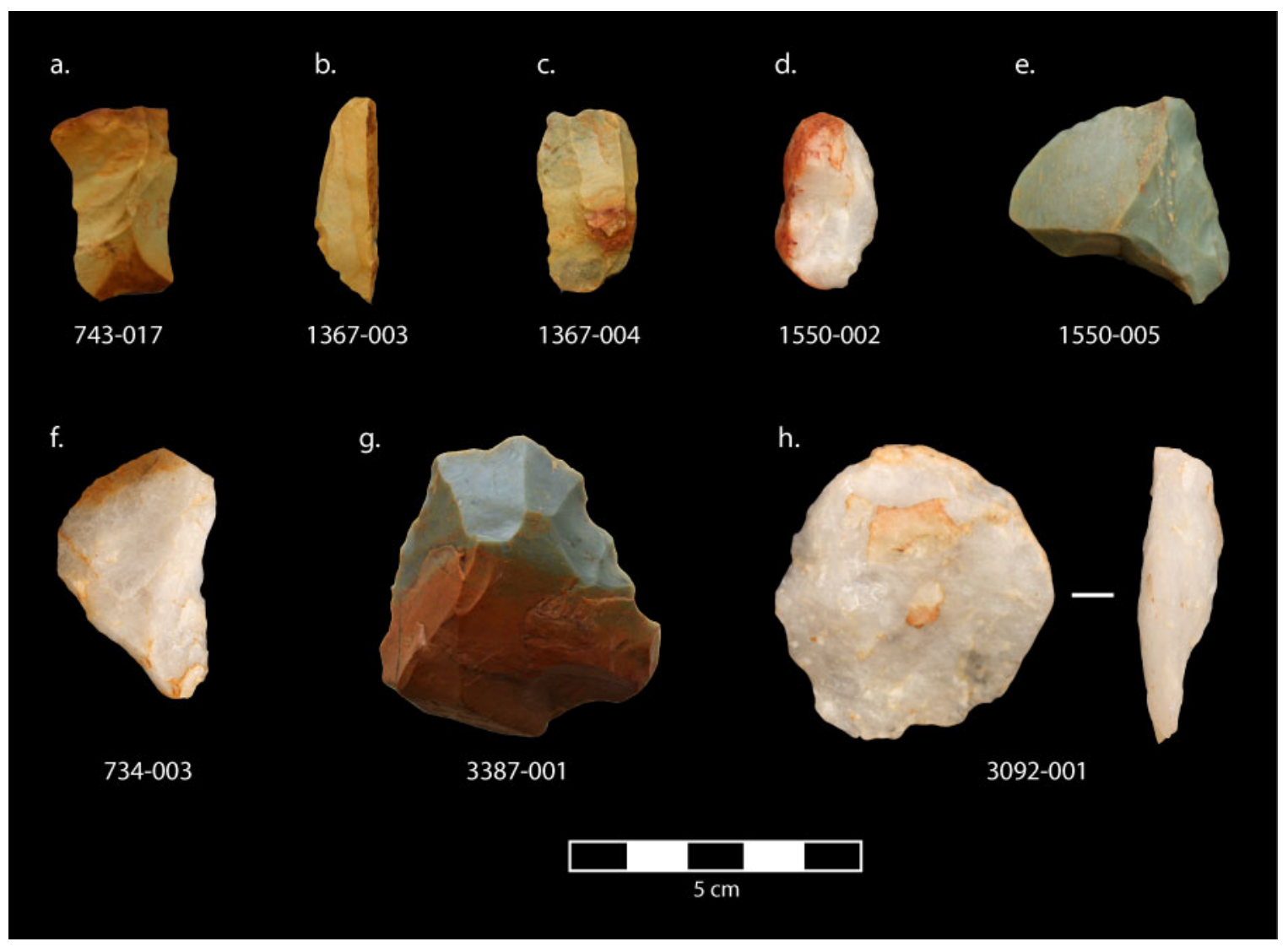


In fact, the majority of tools for both quartz and chert were utilized flakes with light edge wear; this means that many of the flakes utilized briefly went undetected during analysis. Some of the formal tools (and, indeed, all of the lithic artifacts) recovered from the site may represent redeposition from LSA contexts, but it is quite possible that people further reduced flakes to produce the occasional scraper, burin, projectile point, blade, or even microlith (Figure 6.22). While the uses of these informal and formal tools at Diouboye are not known, the chipped stone assemblage does show that people used stone tools for a wide array of tasks in their daily lives; more valuable iron may have been reserved for specific activities.

Macrolithic scatters of long mudstone blades, flakes, and other reduction debris provided the best evidence for in situ tool manufacture at Diouboye, although their surface distribution made dating tenuous. As shown in Figure 6.11 above, the blades generated in this fashion could have been used for harvesting or threshing grain.

\section{Groundstone Manufacture and Use}

In addition to the use of groundstone tools such as grinding slabs, handstones, and quartz hammerstones for crushing and pounding foodstuffs, the people of Diouboye also made use of groundstone axes and polished hematite hachettes and other tools (Appendix E). What's more, the recovery of hematite debitage and tool preforms at the site (Figure 6.23), outnumbering those found in LSA sites, may indicate that such tools were actually manufactured by the Iron Age populace. Given the strong association of groundstone axes with LSA contexts across West Africa, this provocative hypothesis will demand scrutiny with further data.

While larger groundstone axes probably served for clearing land (see above) as part of the overall subsistence economy, patterns of chipping and polish on the smaller hachettes hints at their use in subsistence and craft activities such as shaping the horns of livestock, working leather or wood, or practicing religious rituals. 
Figure 6.23: Polished hachette production stages: (a) blank; (b) preform; (c) semiground; (d) fully-ground; and (e) a quartz hachette (Unit F)

a.

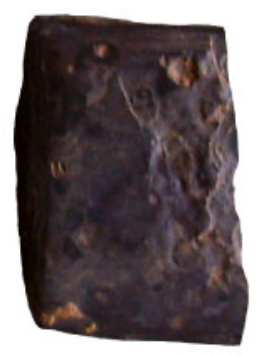

$1462-01$ b.

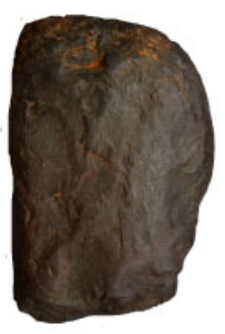

3368-01
C.

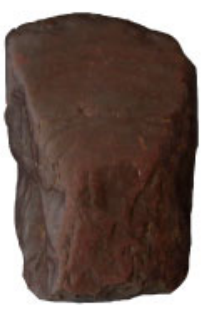

3015-01 d.

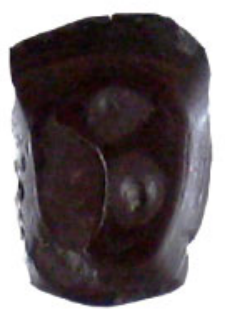

$1138-01$ e.

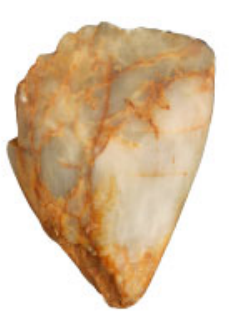

5109-01

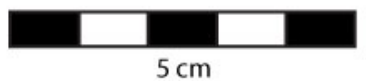

\section{Votive Objects and Religious Ritual}

Religious rituals are often difficult to distinguish in the archaeological record, in part because nearly all activities can become "ritualized" when their repetitive performance creates a sense of formalism, traditionalism, and invariance (Fogelin 2007a:58). And, in many cases, religious ritual is simply one facet of a subsistence or craft activity, such as hunting specific animals or manufacturing iron (Cissé 1964; Herbert 1993; McNaughton 1988). For this reason, many of the abovementioned economic activities may have had a religious dimension lying, for the moment, beyond the grasp of archaeological analysis.

Votive objects provided the most conspicuous evidence for ritual. In addition to the hematite hachettes described above, people made, used, and discarded distinctive terracotta items including rare zoo- and anthropomorphic figurines as well as "bracelets" (Appendix G). In the absence of contextual evidence, such figurines could be interpreted as either ritual objects or children's toys — as have similar objects found at Iron Age sites across West Africa (e.g., Holl 2002; S. K. McIntosh 1995). 


\section{Long-Distance Exchange}

Many subsistence products and crafts undoubtedly circulated in and around Diouboye through down-the-line exchange embedded in kinship relations, gift-giving, marriage contracts, politico-ritual feasts, and so forth, but people also participated in exchange for objects with value stemming, in part, from their non-local origins. In addition to copper and possibly salt, imported objects included cowries from the Indian Ocean, glass beads traded from North Africa, and carnelian and facetted quartz beads from towns across the Sahel and savanna such as Gao (Appendix G). The origins of these materials hint at participation within two interaction spheres-one centered on the Middle to Upper Senegal River, and another emanating from the Pays Mande along the Niger River-but it is important to recall that these commodities did not necessarily move in one direction across the interregional landscape. While it remains possible that itinerant merchant groups such as the Wangara or Jakhanke traded directly with the occupants of Diouboye, both the diversity and low frequency of non-local objects points to their circulation through the same sorts of inter-community exchanges characterizing the distribution of local goods.

Figure 6.24: Miscellaneous glass, carnelian, shell, and terracotta beads from IFAN survey at Diouboye (Opper and Opper 1990)

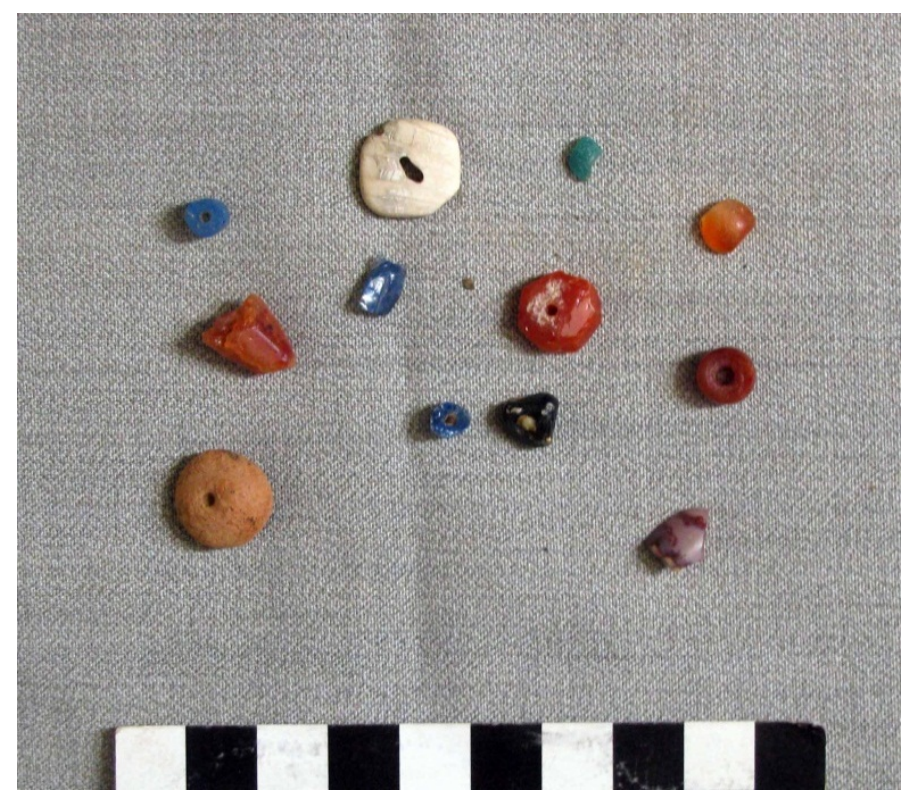


The goods exchanged against these non-local items are a matter of speculation. Early accounts by Arab geographers indicate that, by the late-first millennium AD, the Upper Senegal region was producing and exporting surpluses of grain, cotton, and possibly gold and slaves (A. Bathily 1989). Given the modest size and geographic position of Diouboye, it would be difficult to argue that the community produced a considerable surplus of grain or cotton, so it seems more likely people exported gold, groundstone, and other local commodities.

\section{SUMMARY}

This chapter had two goals for shifting this archaeological study from the regional landscape to the more localized political economy of Diouboye attested by surface and subsurface data from the site. First, I outlined the middle-range methodology used to sort out the material and spatial dimensions of past economic processes from those generated in the course of natural, and later cultural, post-depositional processes at the site. Second, I briefly summarized the architecture and subsistence, craft, and exchange activities undertaken by residents of the village (drawing upon data presented in much greater detail in Appendices D-I). This sketch of what the residents of Diouboye were doing on a day-to-day basis forms a background for a closer look at the actual material and spatial dimensions of these activities and, by extension, locational and appropriational movements comprising the political economy of this Iron Age village - the subject of the next two chapters. 


\section{CHAPTER 7 \\ RESIDENTIAL AND NON-RESIDENTIAL AREAS: DEPOSITION AND HISTORIES OF DAILY LIFE}

As the people of Diouboye engaged in diverse material practices at specific times and places, they reproduced the subsistence and craft economies, kinship relations, politico-ritual institutions, exchange networks, and myriad other social institutions intersecting to comprise the village community. The palimpsest of the archaeological record, however, makes it difficult, if not impossible, to tease apart practices as singular events. For this reason, I employ the normative architectural episodes and activities discussed in the previous chapter to organize the material evidence from excavations at Diouboye into discrete temporal and spatial units. Mirroring the definition of social institutions from Chapter 1, these recurrent sets of practices likewise stand to reveal the economic processes of production, exchange, and consumption enrolled in sociopolitical relations across the village.

In this chapter I reconstruct the social history of Diouboye from six residential areas associated with day-to-day subsistence and craft activities at the scale of the household compound (or compounds). In addition, I present evidence for the more specialized activities and post-depositional disturbances responsible for non-residential areas of the site (Appendix B). As detailed in Appendix C, my excavation strategy (20082009) for reconstructing the cultural economy and settlement history of the village involved opening 79 square meters distributed across three larger units (16-24 $\left.\mathrm{m}^{2}\right)$ and four smaller units (4-6 $\left.\mathrm{m}^{2}\right)$ in residential areas, and three test units (1-4 $\left.\mathrm{m}^{2}\right)$ in nonresidential areas (Figure 7.1). These units provided an adequate sample for intra-site spatial comparison across areas, while the excavation of two units in Area A (excluding the 2007 exploratory Trench AZ) further documented spatial variation within one residential area. 
While superimposed depositional contexts provided a relative measure of elapsed time, it is important to recall that each one of these analytic units encapsulated a unique duration - from an episode of hut construction to the discard of refuse over a generation. For the purposes of developing a site-level chronology at the end of this chapter, I present the sequence from each excavation unit according to strata encompassing similar depositional loci and substrata. This necessarily involves a discussion of the horizontal relations among architecture and other loci within each unit, but I defer a more detailed discussion of spatial organization within Diouboye to the following chapter. The analysis and classification of artifacts described throughout this chapter can be found in Appendices D-I.

Figure 7.1: Excavation units in residential and non-residential areas

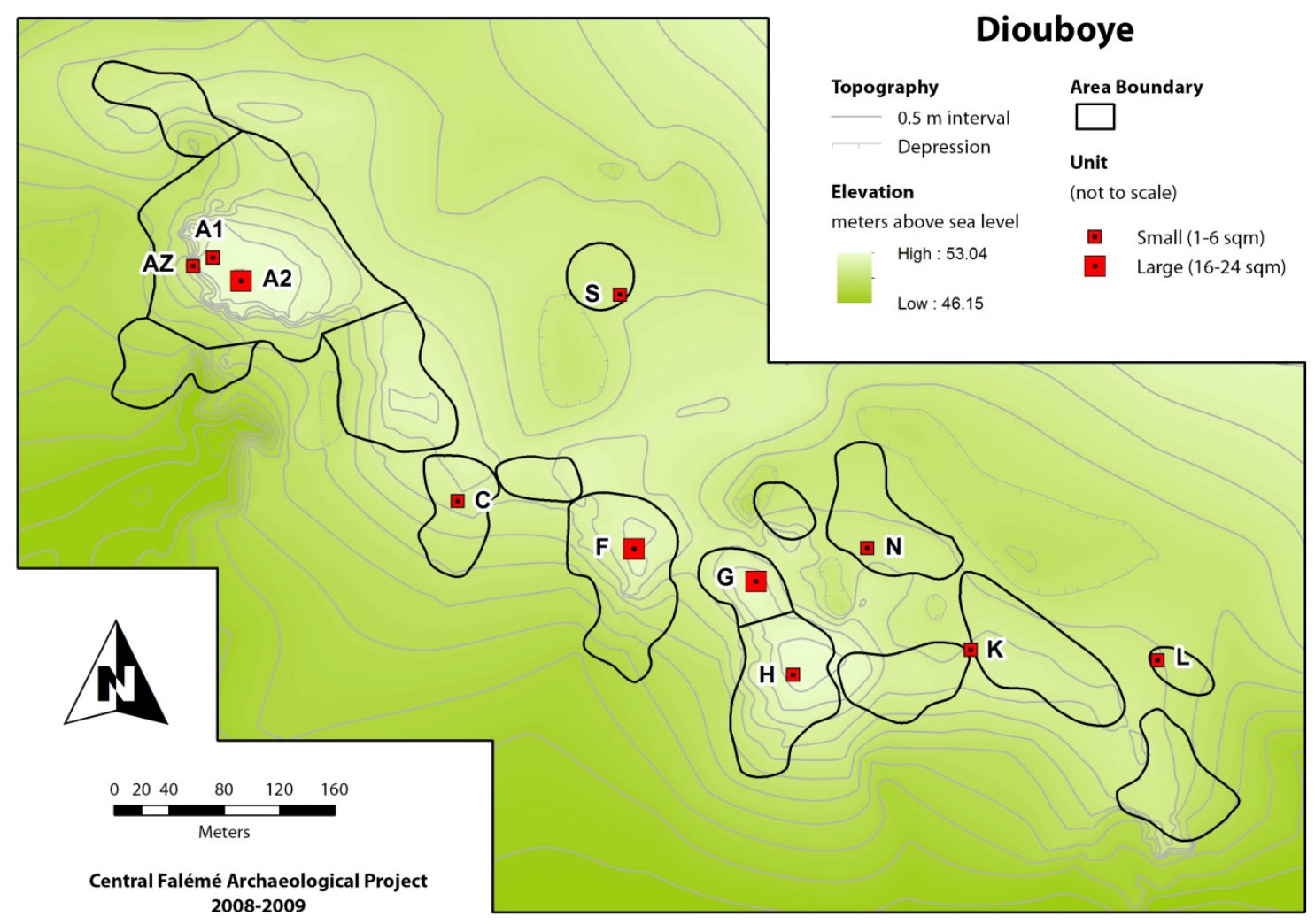




\section{RESIDENTIAL AREAS}

\section{Residential Area A (Unit A1)}

As the first unit opened during the 2008 field season, I placed Unit A1 (2x2 m) near the apex of Area A, the highest mound at Diouboye, with the intention of obtaining the deepest possible stratified sequence. Sloping gently down to the north and east, the surface of the unit was a loose, reddish-yellow sandy clay loam littered with laterite gravel, a few scattered sandstone rocks, and numerous pottery sherds, including several large fragments from a partially reconstructable vessel.

While the earlier exploratory Trench AZ (Appendix C) had established a stratified sequence to a depth of 0.90 meters, Unit A1 ultimately revealed 1.26 meters of archaeological deposits above the natural alluvium that underlies the site. As shown in Figure 7.2, the three strata observed in this unit subsumed 12 successive substrata of architecture and depositional activities spanning all three of the occupation phases defined for Diouboye in the final part of this chapter.

\section{Stratum A1.3}

Lying above an extremely firm, yellowish-red alluvium with no evidence of human activity, the lowermost cultural deposit encountered in Unit A, Substratum A1.3e (1.14-1.26 m), was a firm horizon of reddish-yellow clay loam representing the accumulation of general fill during the initial occupation of this residential area. Evidence for human activity included modest frequencies of charcoal, ceramic sherds, and faunal remains, in addition to two episodes of ash disposal (Feature 16 and Feature 17).

Directly atop this fill was an array of four to five possible post molds (Feature 12). Although their similarity to termite burrows made it challenging to identify post molds at Diouboye, the curvilinear arrangement, similarity in mold depth (1.14-1.19 m), and charcoal-enriched fill all lend support to the interpretation of Feature 12 as the perimeter of a wooden structure, such as a wattle-and-daub hut, on Surface A1.3d. ${ }^{7}$ This interpretation gains support from the overlying Substratum A1.3d (1.01-1.17 m) where greater concentrations of charcoal and daub were present in the southern half of the unit.

\footnotetext{
${ }^{7}$ Surfaces are named according to the overlying substratum.
} 


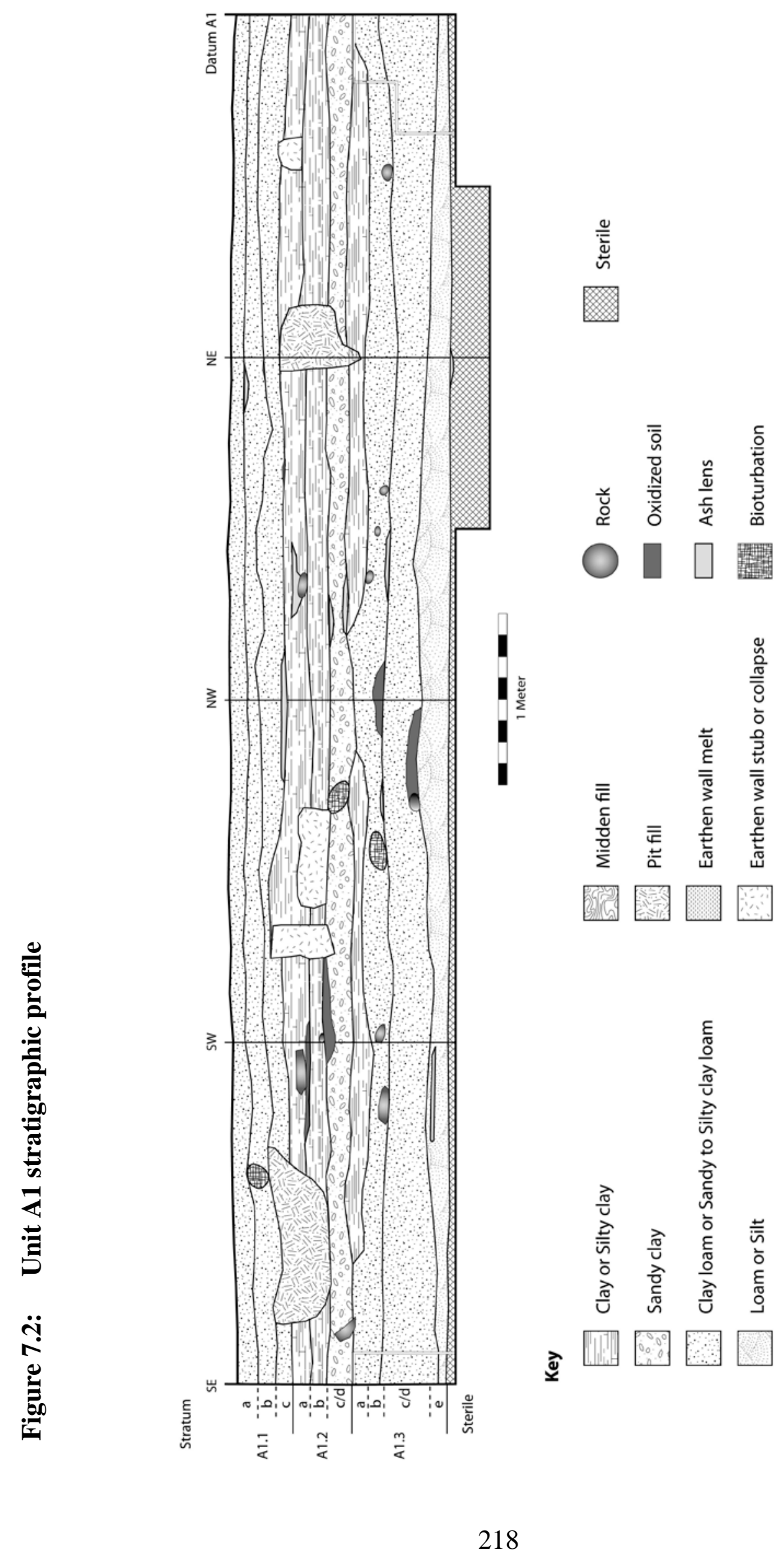


Very modest frequencies of sherds and faunal remains in this substratum pointed to a general accumulation of soil and domestic debris. Two carnelian beads in this deposit attested to participation in regional exchange networks, or perhaps even directly traded with itinerant merchants.

Subsequently, multiple episodes of pyrotechnic activity in the northwestern corner of the unit produced Feature 14 on Surface A1.3c (Figure 7.3). This elongated, shallow trench (5-6 cm in depth) was marked by substantial oxidation of the underlying sediment and lined with a thick lens of white ash and charcoal (see Figure 6.19) —attributes consistent with bellowing and heating coals to the temperatures needed to forge iron. The interpretation of Feature 14 as a small forge finds support with the presence of a single bent iron rod fragment and the (relatively) high density of glassy and pit slags ( $\mathrm{n}=19$, $48.9 \mathrm{~g})$ both in the surrounding sediment and the overlying Substratum A1.3c (0.91-1.04 m). This latter deposit otherwise comprised a general fill with modest levels of daub hinting at its formation, in part, through the collapse of a nearby earthen wall structure.

Figure 7.3: $\quad$ Surfaces A1.3c (left) and A1.3b (right)

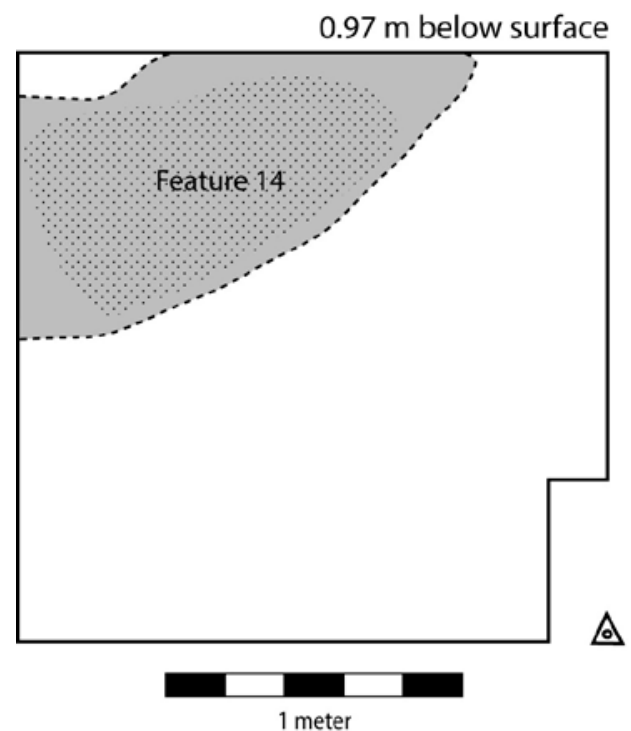

Earthen wall stub or collapse

is Midden fill

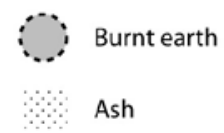

Key

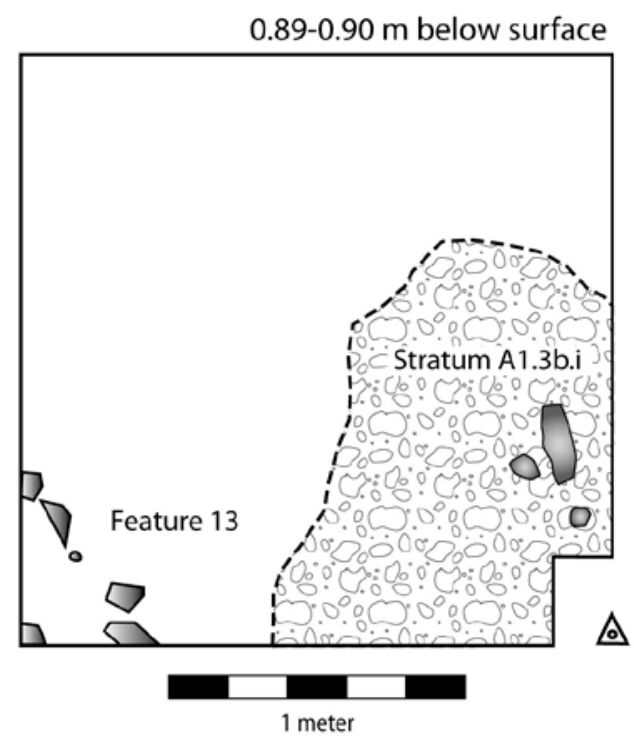
Sherd
監 Bioturbation
Sandstone
Intrusive feature 
Feature 13, an alignment of small sandstone blocks, appeared in the southwestern corner of Unit A1, marking the otherwise poorly defined Surface A1.3b (Figure 7.3). The purpose of this architectural feature is unknown, but it may have been part of a rectilinear alignment. Whatever the case, it evidently fell into disuse with a shift towards refuse disposal. Substratum A1.3b.i (0.81-0.92 m) was a moderately firm deposit of sandy clay loam with high densities of daub, charcoal, and discarded artifacts, including one groundstone fragment and one quartz bead, that together pointed to a sheet midden and/or episode of earthen wall collapse. Lying atop Feature 13 and the remainder of Surface A1.3b, Substratum A1.3b.ii (0.80-0.91 m) was a firm deposit of silty clay with artifacts, including the fragmented remains of an iron hoe blade and a hematite blade fragment, all suggesting quotidian activities and natural sediment deposition within a compound.

Medium daub and fine charcoal inclusions suggested that Substratum A1.3a (0.71-0.82 m) formed partly through earthen wall maintenance, as well as household activities and refuse disposal. In fact, a lens of coarse sand and gravel in the eastern half of the unit could have resulted from an episode of construction or surface leveling. Feature D was a poorly preserved hearth of burnt sediment and an ash lens that marked the bottom of this substratum where no clear occupation floor was otherwise discernible.

\section{Stratum A1.2}

Feature 11, a hearth complex lying atop the well-defined Surface A1.2d (Figure 7.4), marked the transition to an increasingly formal use of space in this corner of Area A. This feature comprised a thick horizon (3-4 cm) of at least three superimposed layers of burnt sediment and ash. However, the near absence of artifacts beyond a few animal bones and an expended handstone fragment suggested that the area was swept regularly, possibly due to its location within a domestic courtyard. Above this probable occupation floor, the high densities of medium daub and charcoal and haphazard sandstone rocks in Substratum A1.2d (0.58-0.72 m) indicated deposition through a combination of domestic activities and earthen wall demolition. This substratum also yielded two dark green, drawn glass beads (Type Gb-A).

Following the deposition of the Substratum A1.2d, a combination of architectural and domestic production activities established Surface A1.2c (Figure 7.4) as a compact 
Figure 7.4: Surfaces A1.2d (left) and A1.2c (right)
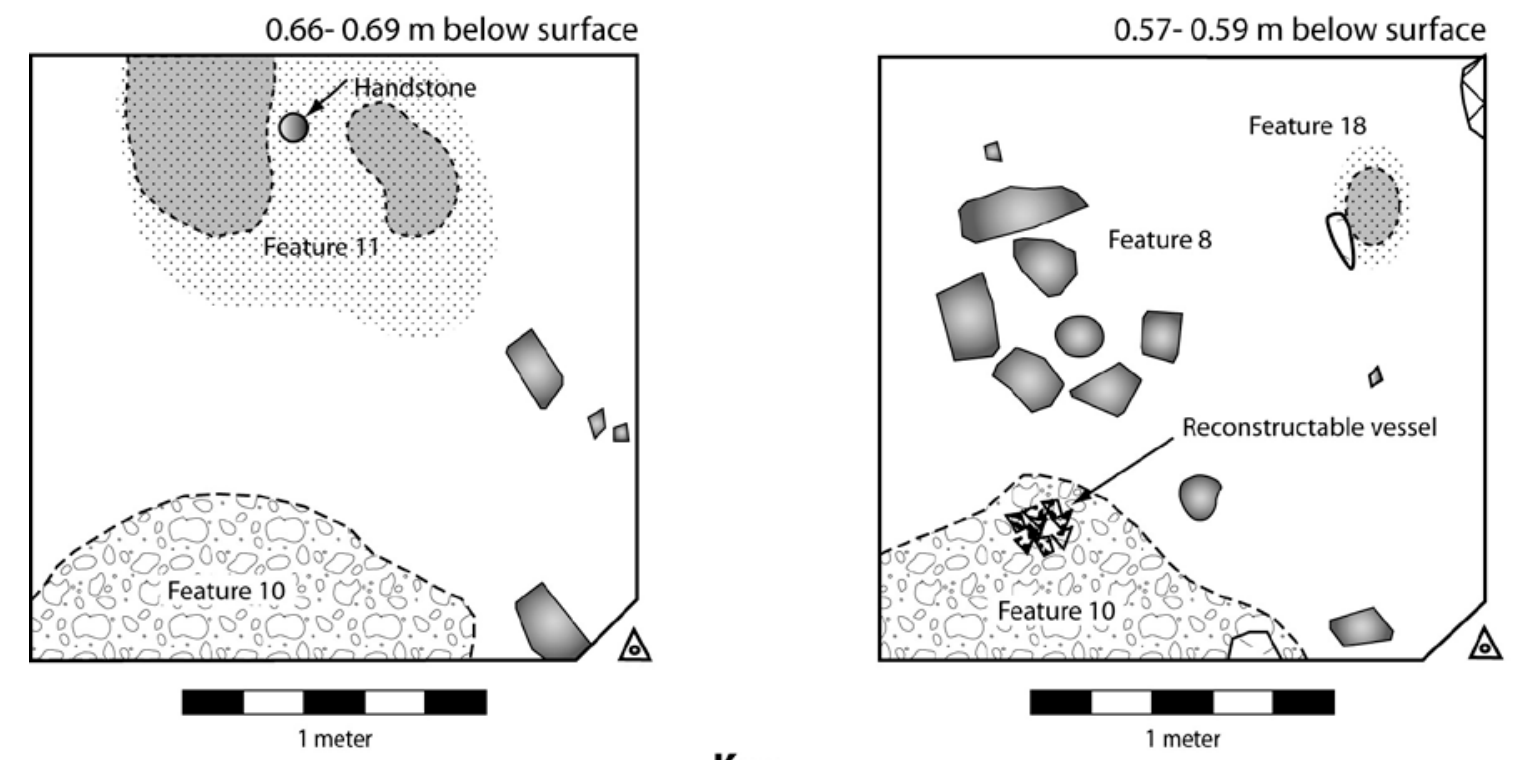

Key
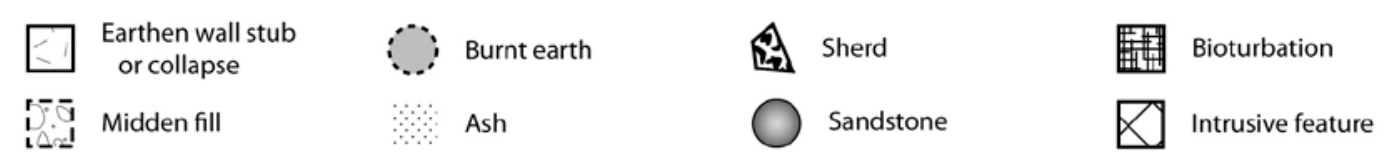

and clearly defined occupation floor. Feature 8, a nearly complete circular installation of seven sandstone rocks measuring some 0.80 meters in diameter, comprised a small filled feature of unknown function, possibly for supporting a small granary. Feature 18, an informal hearth, hints at nearby food preparation. The overlying Substratum A1.2c (0.49$0.65 \mathrm{~m}$ ) was firm silty clay whose sparse artifacts, including an iron rod fragment, charcoal, and daub, suggested gradual accumulation through domestic activities such as food production accompanied by regular sweeping of this space.

Intruding through the southern part of Stratum A1.2, Feature $10(0.53-0.81 \mathrm{~m})$ was a shallow and amorphous depression filled with a friable to firm silty clay loam with coarse charcoal mottles, faunal elements, a small terracotta bead, and large pottery sherds, including those from a reconstructable small pot. This feature comprised either the northern margin of a larger depression or simply a sheet midden contemporaneous with the occupations represented by Substrata A1.2b-c. 
Substratum A1.2b (0.40-0.51 m) was a compact horizon of silty clay with several sandstone fragments, but very low densities of charcoal and other artifacts, including one round bone bead, altogether consistent with a period of earthen wall maintenance and/or demolition. This substratum witnessed the use of two informal hearths (Feature E and Feature 9) and the final use of Feature 8, which continued to be visible at this level (Figure 7.5). Feature L, an earthen wall stub or collapsed block of terre pisé just visible in the west profile, also corresponded to this stratigraphic episode.

Whatever its origins, Feature L appeared on the well-defined Surface A1.2a (Figure 7.5) where it laid directly southwest of Feature 7, an extremely firm clay loam derived from episodic wall melt or collapse from an unidentified earthen wall structure. Crossing the southern half of the unit, Feature 6 comprised a rectilinear alignment of sandstone rocks measuring 22 centimeters in width. Since the associated soil did not differ from one side of the feature to the other, it likely served to support or reinforce

Figure 7.5: $\quad$ Surfaces A1.2b (left) and A1.2a (right)

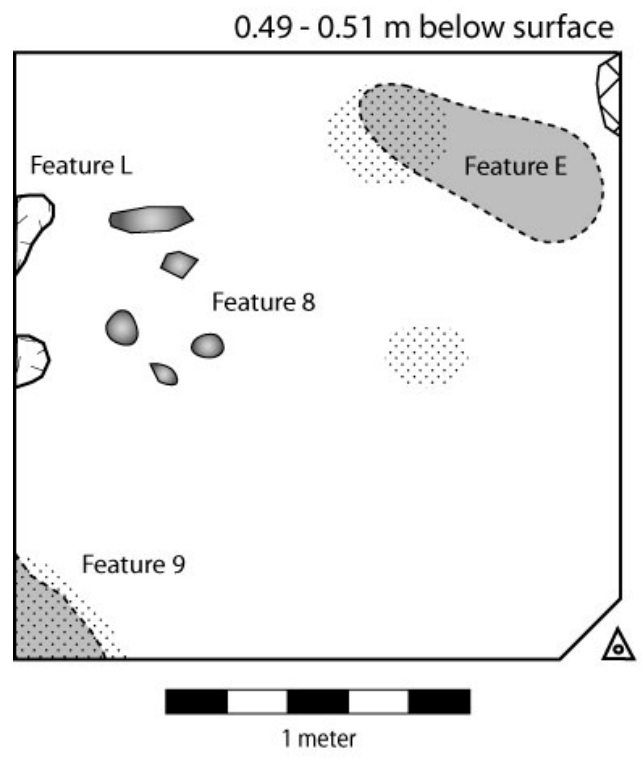

Key
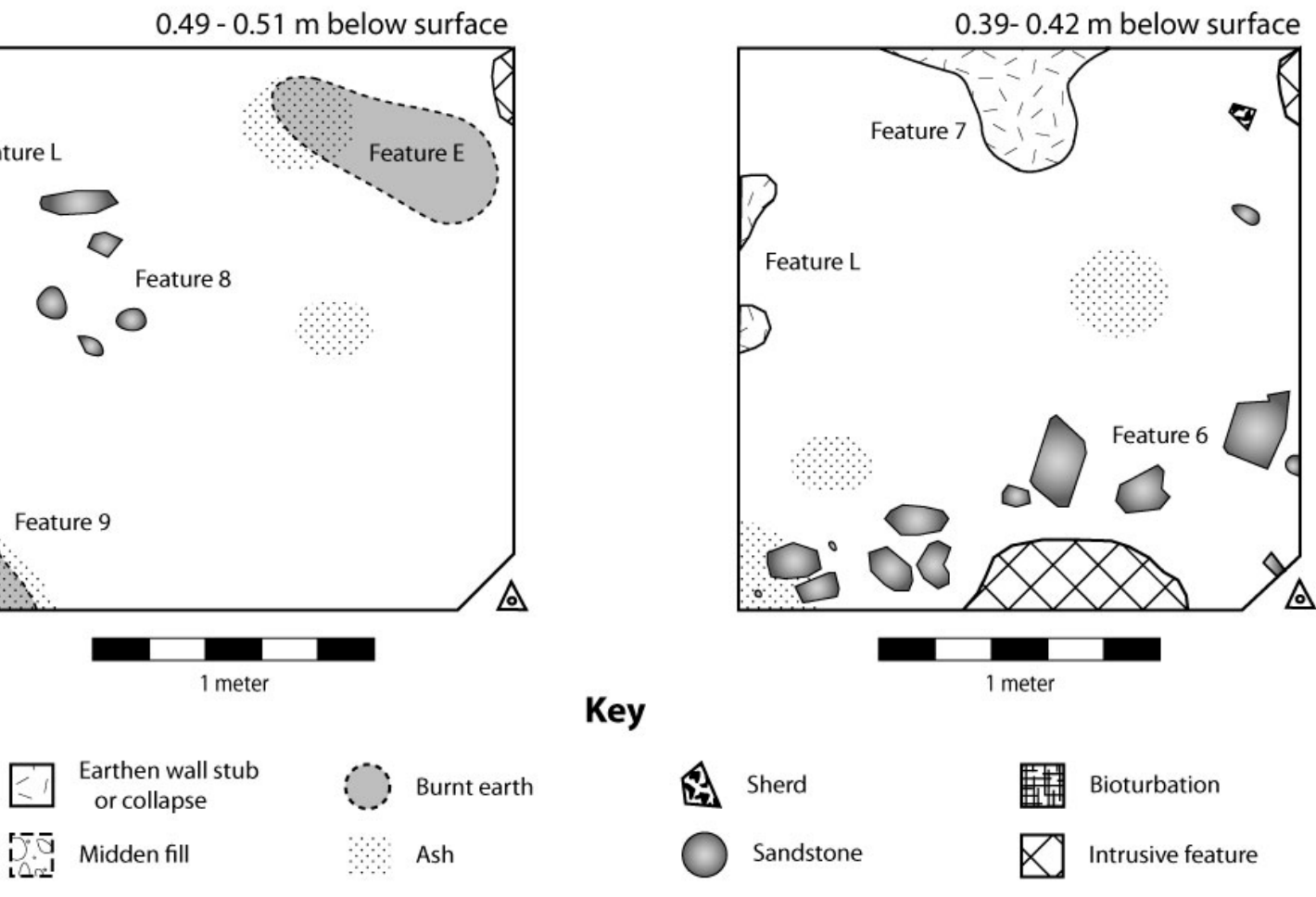
an otherwise unidentified structure. Substratum A1.2a (0.29-0.42 m) was a compact deposit of silty clay with modest frequencies of charcoal and artifacts, including two compound glass beads (Type Gb-D), consistent with general accumulation through food production activities and earthen wall collapse. A fragment of copper wire recovered from Feature 7 yielded the earliest evidence for access to copper in Unit A1.

\section{Stratum A1.1}

Located at the transition to the uppermost stratum, Feature $\mathrm{N}$ was the edge of a pit of unknown dimensions. Since this feature was not excavated as a discrete unit, its specific contents are not known, but the clear boundary, shallow depth, and density of large faunal elements visible in profile all suggest that this pit was dug down from the compact living floor of Surface A1.1c to dispose of waste from a discrete butchery episode. The well-defined Surface A1.1c (Figure 7.6) supported a shallow hearth (Feature 5), possibly within the confines of a structure demarcated by the earthen wall stubs/collapse of Feature 7 and Feature L. The overlying Substratum A1.1c (0.21-0.30 m) was a general fill with light charcoal and daub inclusions and modest artifact frequencies, including one piece of slag (5.9 g).

Intruding as far down as Substratum A1.2b, Feature 4 was a shallow pit filled with firm, reddish-brown silty clay loam and high densities of very coarse daub and charcoal; this matrix was consistent with the collapse or discard from a pyrotechnic feature such as a roasting pit. Indeed, Surface A1.1b supported two modest hearths (Feature 2 and Feature 3) as well as Substratum A1.1b.ii (0.19-0.25 m) whose elevated charcoal and artifact frequencies pointed to the intensive discard of refuse from food production. Substratum A1.1b.i (0.10-0.21 m) contained modest levels of artifacts, including a copper ornament, likely resulting from quotidian domestic activities.

Finally, the uppermost Substratum A1.1a (0.00-0.13 m) was weathered topsoil easily distinguished from the underlying, more compact substrata by the presence of several ash lenses and patches of burnt soil classed together as Feature 1, a very poorly preserved hearth complex. Even taking into account probable deflation, artifact densities in this substratum were not very high. Four pieces of slag ( 4.9 g) derived from metal working in the vicinity (see Unit A2 below), just prior to abandonment of the area. 
Figure 7.6: Surfaces A1.1c (left) and A1.1b (right)

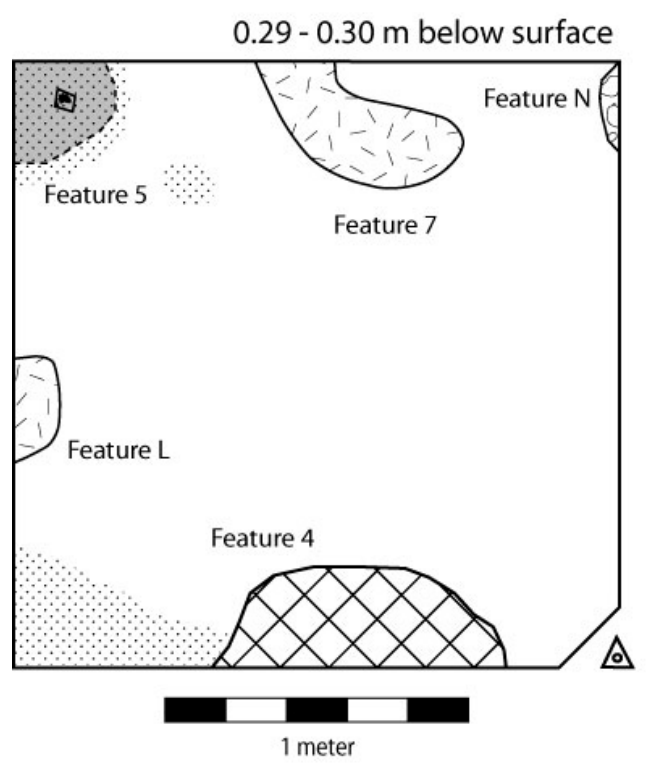

Earthen wall stub or collapse

$\overline{\bar{D}} \overline{\mathrm{a}} \mathrm{i}$ Midden fill

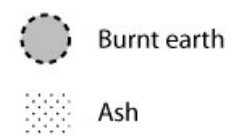

Key

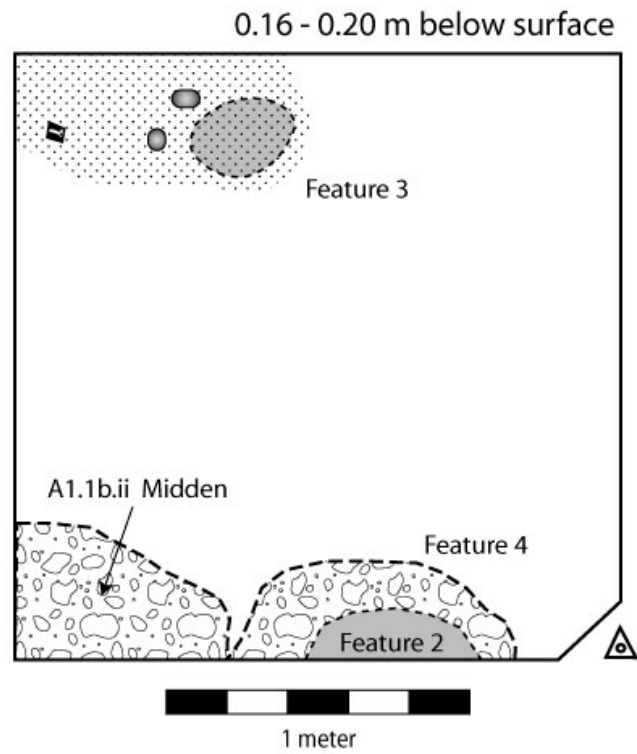

1. Sherd Sandstone Bioturbation

\section{Unit A1 Summary}

Architecture and Space

Stratum A1.3 represented the gradual shift from informal accumulation of residential occupation debris to a more formal use of space within Area A. Architectural elements, including a sandstone alignment (Feature 13), modest daub frequencies, and a possible post mold alignment (Feature 12), documented the construction and maintenance of wattle-and-daub and/or pisé wall structures. A possible blacksmith forge (Feature 14) and associated slag showed that some individuals occupying this residential area possessed the knowledge and skill to work iron, and possibly copper, although the relative formality of this “workshop” remains unclear. Stratum A1.2 witnessed an increasing formalization of space with cycles of earthen wall construction, maintenance, and demolition alongside the production of semi-formal areas of refuse disposal and small hearths. Stratum A1.1 marked a return to the less formal use of space as 
architectural features give way to pits and hearths presumably associated with food preparation, consumption, and refuse disposal.

\section{Artifacts, Organic Remains, and Material Practice}

The faunal sample, analyzed by Stephen Dueppen (Appendix H), from Unit A1 included 70 identifiable specimens (Figure 7.7). These remains reveal fairly limited consumption of sheep/goat although some of the unidentified terrestrial mammals, which comprise the bulk of the sample, may have been domesticates. Overall, this dataset demonstrates that the occupants of Area A obtained most of their meat by exploiting both savanna forest and riparian habitats along the Falémé River for diverse terrestrial and aquatic game. As noted by Dueppen in Appendix H, an emphasis on large-bodied prey, including some megafauna (hippopotamus), may be more consistent with hunting expeditions, than with communal or garden-hunting strategies that yield higher proportions of small game.

\section{Figure 7.7: Unit A1 faunal summary (from Appendix H)}

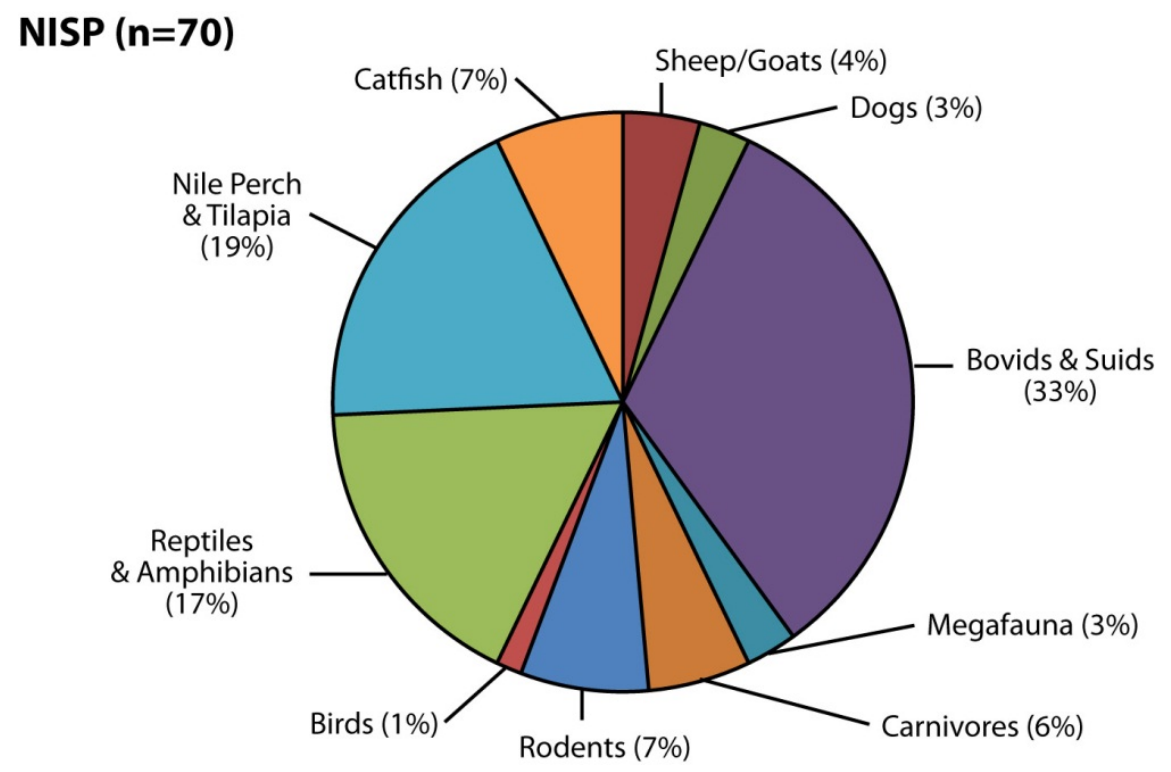


Table 7.1: $\quad$ Unit A1 artifact summary

\begin{tabular}{|c|c|c|c|c|c|c|c|}
\hline Sub & $\mathrm{m}^{3}$ & $\begin{array}{c}\text { Ceramic } \\
n\end{array}$ & $\begin{array}{c}\text { Lithic } \\
\mathrm{n}\end{array}$ & $\begin{array}{c}\text { Slag } \\
\mathrm{n}\end{array}$ & Groundstone & Tools & Ornaments \\
\hline A1.1a & 0.31 & 531 & 14 & 4 & \multirow{3}{*}{$\begin{array}{l}1 \text { x Hematite } \\
\text { axe preform } \\
1 \text { x Handstone }\end{array}$} & \multirow{3}{*}{-} & \multirow{3}{*}{$\begin{array}{l}1 \text { x Copper } \\
\text { ornament }\end{array}$} \\
\hline A1.1b & 0.40 & 162 & 2 & 0 & & & \\
\hline A1.1c & 0.31 & 221 & 0 & 1 & & & \\
\hline A1.1 & 1.02 & 914 & 16 & 5 & 2 & - & 1 \\
\hline $\mathrm{A} 1.2 \mathrm{a}$ & 0.40 & 211 & 1 & 0 & \multirow[b]{4}{*}{$1 \mathrm{x}$ Handstone } & \multirow{4}{*}{$\begin{array}{l}1 \times \text { Copper wire } \\
1 \times \text { Iron rod } \\
\text { fragment }\end{array}$} & \multirow{4}{*}{$\begin{array}{c}2 \times \text { Glass bead } \\
\text { (Gb-A) } \\
2 \times \text { Glass bead } \\
\text { (Gb-D) } \\
1 \times \text { Round bone } \\
\text { bead } \\
1 \times \text { Terracotta } \\
\text { bead } \\
1 \times \text { Terracotta } \\
\text { figurine frag }\end{array}$} \\
\hline $\mathrm{A} 1.2 \mathrm{~b}$ & 0.59 & 624 & 0 & 0 & & & \\
\hline $\mathrm{A} 1.2 \mathrm{c}$ & 0.41 & 219 & 0 & 0 & & & \\
\hline $\mathrm{A} 1.2 \mathrm{~d}$ & 0.41 & 248 & 0 & 0 & & & \\
\hline A1.2 & 1.81 & 1302 & 1 & 0 & 1 & 2 & 7 \\
\hline A1.3a & 0.31 & 345 & 2 & 0 & \multirow{5}{*}{$\begin{array}{l}1 \times \text { Hematite } \\
\text { blade } \\
1 \times \text { Handstone }\end{array}$} & \multirow{5}{*}{$\begin{array}{l}1 \times \text { Iron hoe } \\
\text { blade } \\
1 \times \text { Iron rod } \\
\text { fragment }\end{array}$} & \multirow{5}{*}{$\begin{array}{c}1 \times \text { Quartz bead } \\
\text { (Sb-1) } \\
2 \times \text { Carnelian } \\
\text { bead (Cb-1) }\end{array}$} \\
\hline A1.3b & 0.41 & 854 & 2 & 0 & & & \\
\hline A1.3c & 0.49 & 273 & 2 & 9 & & & \\
\hline A1.3d & 0.53 & 180 & 4 & 10 & & & \\
\hline A1.3e & 0.40 & 48 & 0 & 0 & & & \\
\hline A1.3 & 2.14 & 1700 & 6 & 19 & 2 & 2 & 3 \\
\hline Total & 4.97 & 3916 & 23 & 24 & 5 & 4 & 11 \\
\hline
\end{tabular}

The 54 flotation samples collected from Unit A1 currently await analysis. While these may document plant use and environmental conditions in and around Area A, the poor preservation and meager macrobotanical data recovered from Unit A2 (below) suggest that the interpretive potential of these data could ultimately prove limited.

An iron hoe in Stratum A1.3 showed that people employed iron implements for agro-pastoral tasks, while the presence of handstones in all three strata suggests that they processed foodstuffs in or around this space throughout the occupation of Area A.

The earthenware pottery assemblage from Unit A1 demonstrated a clear seriation of decorative attributes (Figure 7.8) useful for fitting the associated stratigraphic units into a relative chronology. Table 7.2 reveals a general trend in the use of ceramic vessels (see Appendix D for a discussion of the ceramic typologies employed throughout this 
chapter). Bowls and large pots were present in Strata A1.3 and A1.2 where their association with hearths and refuse deposits supports the interpretation of general food preparation and consumption activities in the vicinity. Notably, the proportions of both large and small jars increased with a shift to more informal space in Stratum A1.1.

Beyond the presence of two iron artifacts, slag, and a possible forge in Stratum A1.3, evidence for craft specialization was minimal in Unit A1. The lowermost and uppermost strata yielded angular shatter and flakes of chert $(n=8)$, quartz $(n=9)$, and hematite ( $n=3)$, while quartz shatter and flakes primarily accounted for lithic artifacts in the uppermost stratum. A single flint flake in Stratum A1.2 could indicate a decline in chipped stone reduction and use away from this space associated with food preparation activities. Whatever the case, a copper wire (possible hook) and an iron rod fragment from this latter stratum show that people did make use of tools other than pottery.

\section{Figure 7.8: Unit A1 body sherd seriation}

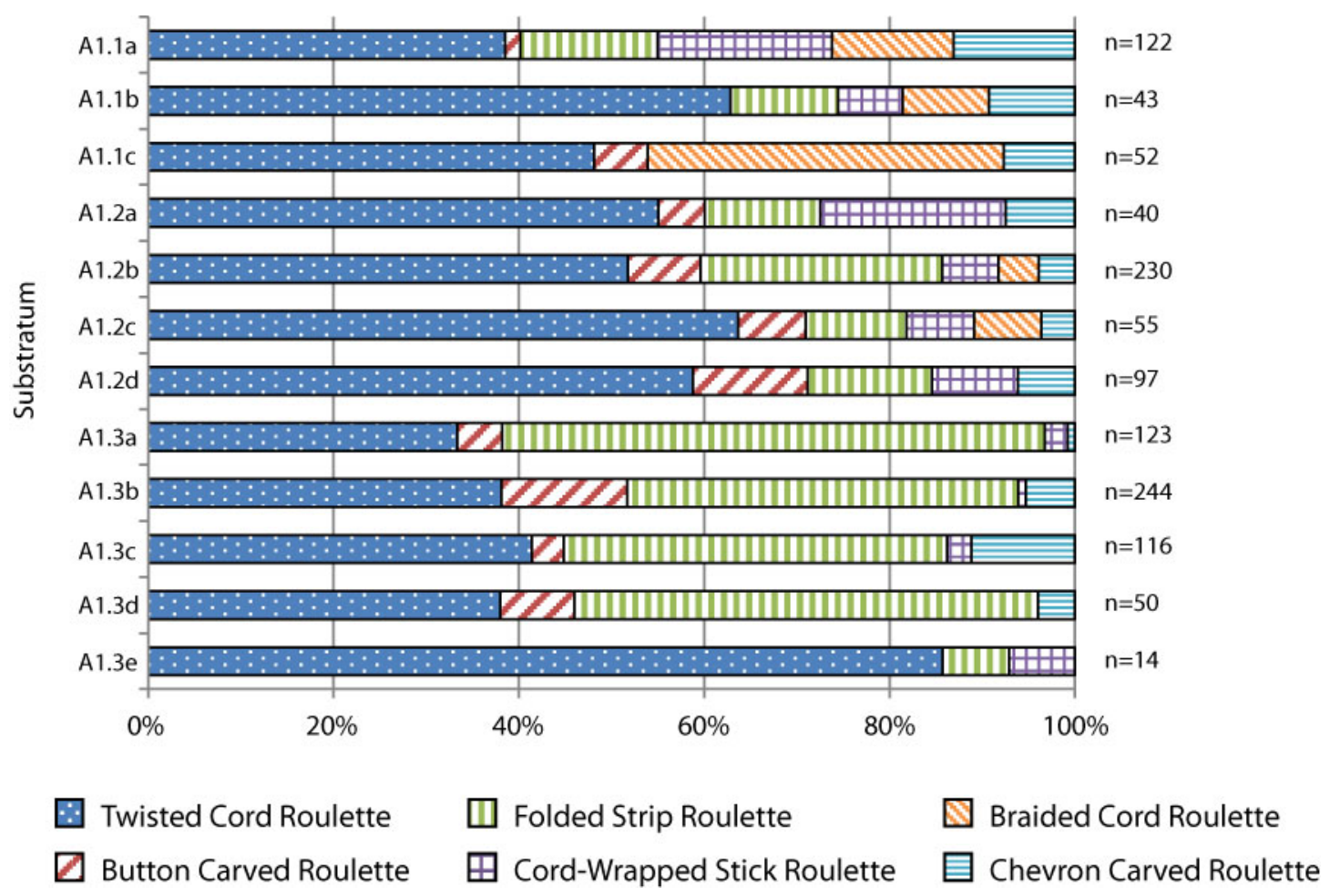


Table 7.2: $\quad$ Unit A1 vessel type frequencies

\begin{tabular}{|c|c|c|c|c|c|c|c|c|c|c|c|c|c|}
\hline \multirow{3}{*}{\multicolumn{2}{|c|}{$\begin{array}{r}\text { Substratum } \\
\text { Total } \\
n\end{array}$}} & \multirow{2}{*}{\multicolumn{2}{|c|}{$\begin{array}{c}\text { Bowl } \\
\text { All }\end{array}$}} & \multicolumn{4}{|c|}{ Pot } & \multicolumn{6}{|c|}{ Jar } \\
\hline & & & & \multicolumn{2}{|c|}{ Small } & \multicolumn{2}{|c|}{ Large } & \multicolumn{2}{|c|}{ Small } & \multicolumn{2}{|c|}{ Large A } & \multicolumn{2}{|c|}{ Large B } \\
\hline & & $n$ & $\%$ & $n$ & $\%$ & $n$ & $\%$ & $n$ & $\%$ & $n$ & $\%$ & $n$ & $\%$ \\
\hline A1.1a & 27 & 0 & $0.0 \%$ & 2 & $7.4 \%$ & 0 & $0.0 \%$ & 6 & $22.2 \%$ & 2 & $7.4 \%$ & 2 & $7.4 \%$ \\
\hline A1.1b & 14 & 0 & $0.0 \%$ & 2 & $14.3 \%$ & 0 & $0.0 \%$ & 1 & $7.1 \%$ & 2 & $14.3 \%$ & 3 & $21.4 \%$ \\
\hline A1.1c & 10 & 0 & $0.0 \%$ & 0 & $0.0 \%$ & 0 & $0.0 \%$ & 4 & $40.0 \%$ & 0 & $0.0 \%$ & 0 & $0.0 \%$ \\
\hline A1.1 & 51 & 0 & $0.0 \%$ & 4 & $7.8 \%$ & 0 & $0.0 \%$ & 11 & $21.6 \%$ & 4 & $7.8 \%$ & 5 & $9.8 \%$ \\
\hline $\mathrm{A} 1.2 \mathrm{a}$ & 11 & 0 & $0.0 \%$ & 1 & $9.1 \%$ & 0 & $0.0 \%$ & 2 & $18.2 \%$ & 0 & $0.0 \%$ & 2 & $18.2 \%$ \\
\hline $\mathrm{A} 1.2 \mathrm{~b}$ & 34 & 1 & $2.9 \%$ & 3 & $8.8 \%$ & 8 & $23.5 \%$ & 3 & $8.8 \%$ & 2 & $5.9 \%$ & 0 & $0.0 \%$ \\
\hline $\mathrm{A} 1.2 \mathrm{c}$ & 11 & 0 & $0.0 \%$ & 0 & $0.0 \%$ & 0 & $0.0 \%$ & 0 & $0.0 \%$ & 1 & $9.1 \%$ & 2 & $18.2 \%$ \\
\hline $\mathrm{A} 1.2 \mathrm{~d}$ & 12 & 0 & $0.0 \%$ & 1 & $8.3 \%$ & 1 & $8.3 \%$ & 0 & $0.0 \%$ & 0 & $0.0 \%$ & 1 & $8.3 \%$ \\
\hline A1.2 & 68 & 1 & $1.5 \%$ & 5 & $7.4 \%$ & 9 & $13.2 \%$ & 5 & $7.4 \%$ & 3 & $4.4 \%$ & 5 & $7.4 \%$ \\
\hline A1.3a & 14 & 2 & $14.3 \%$ & 1 & $7.1 \%$ & 3 & $21.4 \%$ & 1 & $7.1 \%$ & 0 & $0.0 \%$ & 1 & $7.1 \%$ \\
\hline A1.3b & 56 & 4 & $7.1 \%$ & 9 & $16.1 \%$ & 7 & $12.5 \%$ & 3 & $5.4 \%$ & 3 & $5.4 \%$ & 4 & $7.1 \%$ \\
\hline A1.3c & 23 & 1 & $4.3 \%$ & 7 & $30.4 \%$ & 5 & $21.7 \%$ & 1 & $4.3 \%$ & 0 & $0.0 \%$ & 0 & $0.0 \%$ \\
\hline A1.3d & 12 & 0 & $0.0 \%$ & 5 & $41.7 \%$ & 1 & $8.3 \%$ & 2 & $16.7 \%$ & 0 & $0.0 \%$ & 0 & $0.0 \%$ \\
\hline A1.3e & 2 & 0 & $0.0 \%$ & 1 & $50.0 \%$ & 0 & $0.0 \%$ & 1 & $50.0 \%$ & 0 & $0.0 \%$ & 0 & $0.0 \%$ \\
\hline A1.3 & 107 & 7 & $6.5 \%$ & 23 & $21.5 \%$ & 16 & $15.0 \%$ & 8 & $7.5 \%$ & 3 & $2.8 \%$ & 5 & $4.7 \%$ \\
\hline Total & 226 & 16 & $3.5 \%$ & 64 & $14.2 \%$ & 50 & $11.1 \%$ & 48 & $10.6 \%$ & 20 & $4.4 \%$ & 30 & $6.6 \%$ \\
\hline
\end{tabular}

Carnelian, quartz, and glass beads from the earliest two strata, and a copper ornament from Stratum A1.1, confirmed that the residents of Area A took part in exchange oriented towards both northern (trans-Saharan) and eastern (Niger River) trade circuits. In addition, these materials indicate that people residing in this area engaged early on in interregional trade or long-distance exchange networks. These objects also provide a glimpse into the role of personal adornment within the village community.

\section{Residential Area A (Unit A2)}

Situated in the south-central stretch of the intact Area A mound, Unit A2 began as a horizontal exposure $(4 \times 4 \mathrm{~m})$ of the open space between several circular sandstone installations to the north, east, and south. Laterite gravel and crushed sherds, including several partially reconstructable vessels, scattered across the surface of the unit attested to minor deflation of a possible refuse disposal area. It was therefore hoped that excavation 


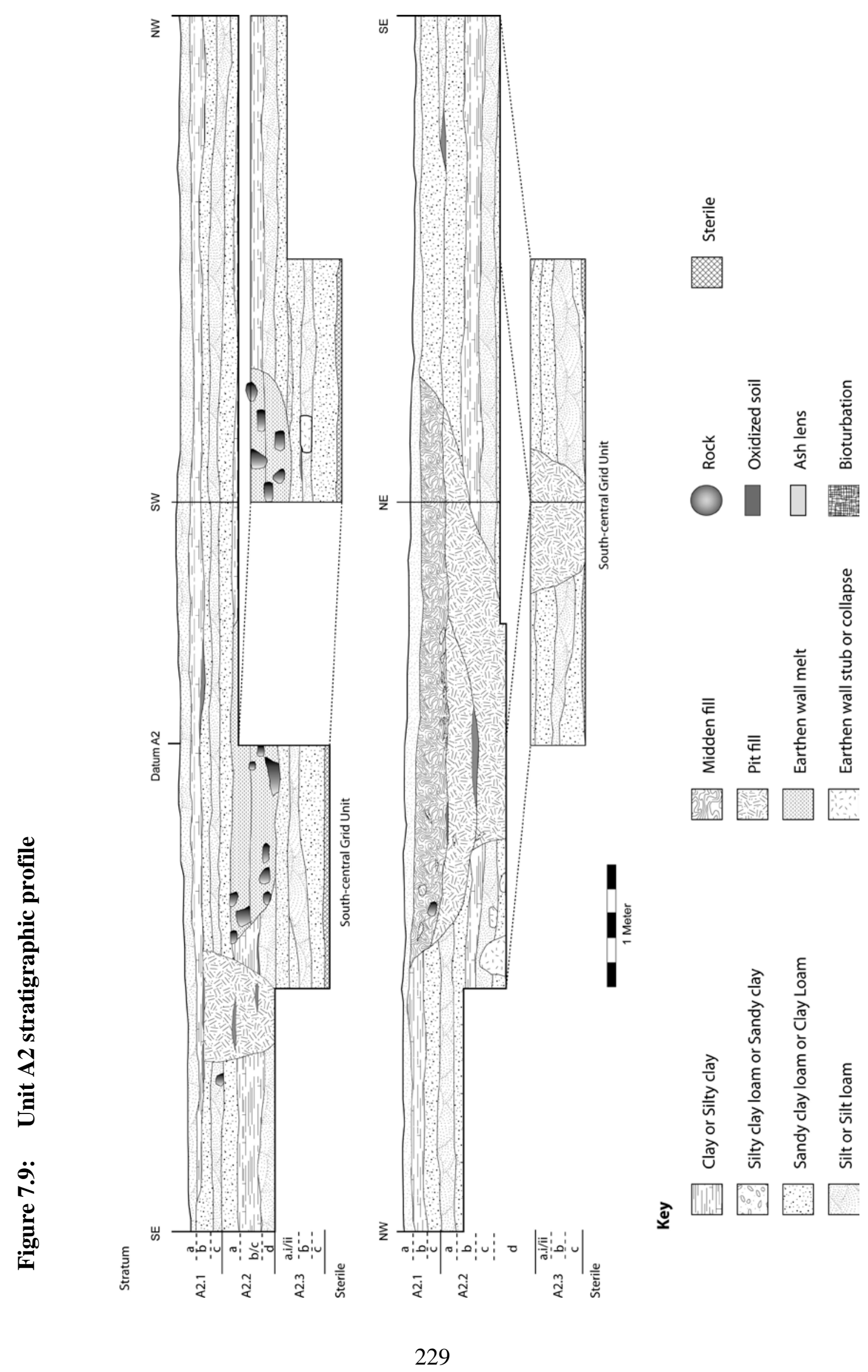


of this unit would unearth midden deposits and architectural features suitable for comparison with those observed in Unit A1 and elsewhere across Diouboye.

Unit A2 was quite successful in this endeavor, exposing archaeological deposits to a depth of 1.21 meters below surface. Three strata, subsuming 11 substrata, encapsulated several pit features, midden deposits, and at least eight domestic structures (Figure 7.8). In order to completely expose an earthen wall hut foundation in the uppermost stratum (Feature 115), I extended Unit A2 by two meters in the west to a depth of 0.53 meters below surface. As a result, it was only possible to reach sterile soil in the south-central grid square (2x2 m) and in one pit feature.

\section{Stratum A2.3}

The natural alluvium beneath Unit A2, visible at a depth of 1.21 meters in the south-central grid unit and in Feature 122 (below), was an extremely firm, reddish-yellow silty clay loam with no evidence of human activity. The first cultural activity in this area produced Substratum A2.3c (0.93-1.21 m) whose pottery sherds and faunal remains documented a general suite of domestic activities including food storage, preparation, and consumption. Dipping slightly into this initial occupation horizon, Substratum A2.3b (0.81-1.05 m) subsequently developed as a sheet midden with high densities of daub, charcoal, and other artifacts, including a single cowrie shell. Yielding high densities of daub, the amorphous Substratum A2.3a.ii (0.81-0.94 m) marked an episode of earthen wall melt and maintenance followed by an episode of collapse with the more widespread Substratum A2.3a.i (0.73-0.85 m).

\section{Stratum A2.2}

The construction of Feature 130, a large granary foundation with an estimated diameter of 1.90 meters, defined Surface A2.2d (Figure 7.10) and a transition towards the more formal production of space within a household compound. The area beneath this granary was prepared with a lens of laterite gravel, while an associated matrix of extremely firm silty clay with fine daub inclusions appeared to represent the erosion and/or demolition of an overlying earthen wall structure. Several meters to the north on the same occupation surface, Feature 132 was a poorly preserved terre pisé wall stub 
interrupted by an intrusive pit. Above this surface, Substratum A2.2d (0.65-0.77 m) was an extremely firm silty clay loam with low artifact densities consistent with a relatively rapid episode of earthen wall collapse, or perhaps routine sweeping of this space as part of a household courtyard.

Figure 7.10: Surface A2.2d

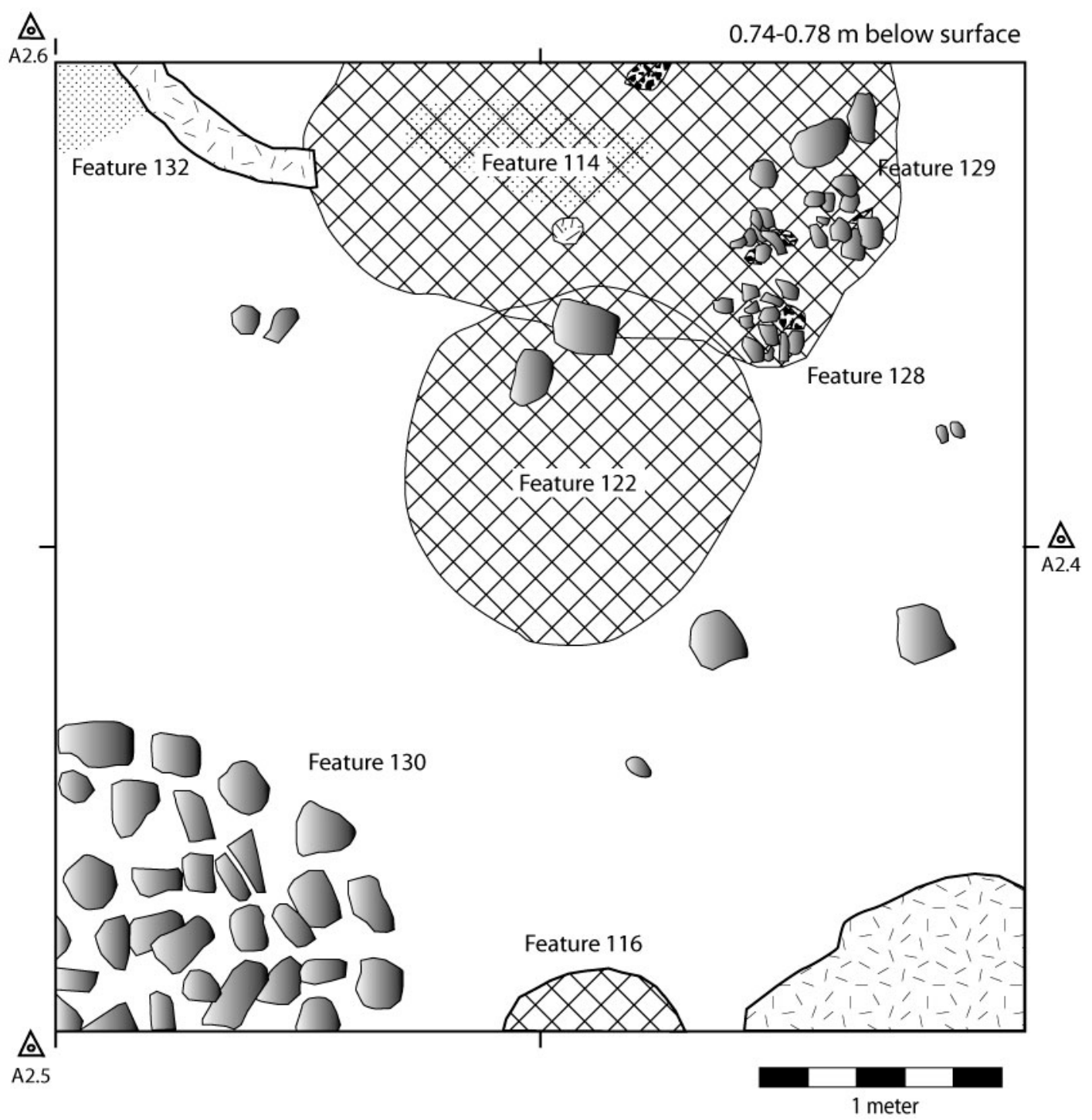

Key

Earthen wall stub
or collapse

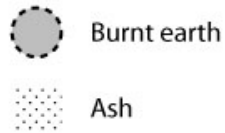

Ash

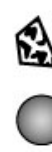

0

Sherd

Sandstone

Bioturbation
$\square$ Intrusive feature 
The use of Features 130 and 132 evidently continued alongside the deposition of Substratum A2.2c.i (0.50-0.72 m), a general fill formed, in part, through gradual earthen wall melt. A possible deposit of wall melt or collapse surrounding the Feature 132 wall stub contained three discoidal terracotta whorls, two round carnelian beads, and one wellpreserved small iron projectile point. Substratum A2.2c.ii was a firm deposit of earthen wall melt or collapse associated with the demolition of Feature 130. Several angular sandstone rocks in Substratum A2.2c.i could have derived from the removal of other foundations prior to the remodeling of this space.

Surface A2.2b (Figure 7.11) was a compact occupation surface that, despite the intrusion of several pit features, supported at least four stone foundations. Feature 123 was a large granary foundation constructed, intriguingly enough, on the footprint of Feature 130, yet stones from the latter were not recycled to construct the former. The associated soil matrix was a compact earthen wall melt containing small pottery sherds and faunal remains possibly trapped beneath the structure.

To the east, the absence of earthen wall melt around Feature 125 and Feature 126 lent support to their classification as pot rests. However, subtle differences in their diameters ( $0.78 \mathrm{~m}$ and $0.65 \mathrm{~m}$, respectively) and number of stones, alongside a layer of crushed sandstone (3-5 cm thick) beneath the former, could indicate functional differences between the two features. The soil matrix around Feature 127, a small, filled feature composed of ten rocks (diameter $0.80 \mathrm{~m}$ ), was indistinguishable from the overlying Substratum A2.2b. Finally, the extremely firm, silty clay of Substratum A2.2b (0.44-0.57 $\mathrm{m}$ ) accumulated as fill around these features, although low artifact counts indicated periodic sweeping of the space within Unit A2. In contrast to the preceding horizon, Substratum A2.2b yielded no evidence for craft production or other specialized activities in the immediate vicinity.

Substratum A2.2a (0.35-0.53 m) was a distinct layer of moderately firm, sandy clay loam with elevated frequencies of daub, charcoal, faunal remains, ceramics, and other artifacts including one terracotta bead, a terracotta bracelet fragment, one globular terracotta netweight, a piece of slag, an iron s-shaped rod fragment, and a quartz hammerstone. Based on heightened concentrations of charcoal visible in profile, this deposit was either a sheet midden or demolished architectural features on the underlying 
Surface A2.2a. Whatever the case, this substratum marked a clear transition between the domestic architecture and episodes of fill in Stratum A2.2 and the increasingly middenlike deposits of Stratum A2.1.

Figure 7.11: Surface A2.2b

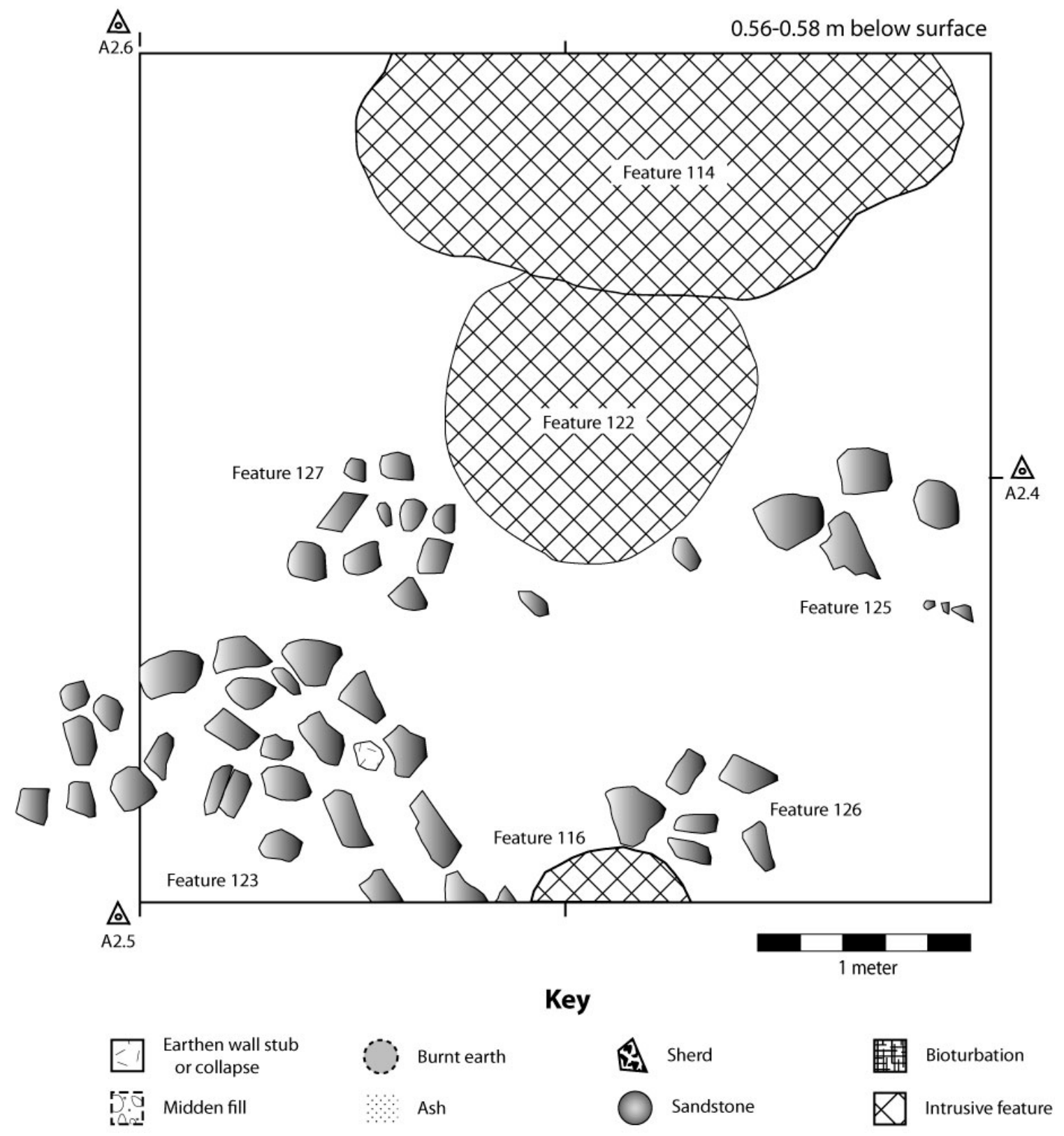




\section{Stratum A2.1}

Substratum A2.1d (0.25-0.39 m) represented a continuation of the sheet midden begun in Substratum A2.2a albeit with a decrease in charcoal and substantial increase in the density of ceramic sherds and faunal remains, including one handstone fragment, a terracotta bead, a possible figurine fragment, and two iron knife blades—one large and one small. At some point early in the formation of this midden, Feature 122, a cylindrical pit measuring at least 1.40 meters in diameter, was excavated to a depth of 0.85 meters below the transitional Surface A2.1d (Figure 7.12). Attesting to subsistence and craft production activities in the vicinity, the pit fill was dense with pottery sherds and faunal remains, as well as an iron harpoon, a spherical copper ornament, and one terracotta bead, and one globular terracotta netweight. A scatter of sandstone rocks on Surface A2.1d could represent the remnants of architectural foundations whose stones were repurposed.

Marking a shift from midden to architectural space, Feature 115 was a circular, terre pisé wall foundation measuring 2.25 meters in diameter and set with an arc of nine small rocks demarcating a possible entryway in the northeast. Concurrent with the use of this modest hut, Substratum A2.1c (0.13-0.32 m) comprised a general occupation fill with high sherd frequencies balanced by low densities of charcoal and daub; other artifacts included a terracotta bead and a circular polishing stone. Given the richness of the surrounding matrix, it seems unlikely that Feature 115 was simply a sleeping hut. Rather, it may have formed part of a space for food storage and preparation, craft production, and/or ritual activity. These activities find support from the recovery of a small iron knife blade and sherds from a reconstructable pot on the interior floor of this structure.

Several small ash dumps, including Feature 119 and Feature 120, helped to distinguish the poorly defined occupational floor of Surface A2.1b (Figure 7.12). At this level, people also excavated a cylindrical pit, Feature 116, presumably to obtain soil for earthen wall construction. Whatever its purpose, the pit was filled with moderate levels of refuse from domestic activities including sherds, animal bones, and ash. Substratum A2.1b (0.03-0.27 $\mathrm{m})$ accumulated on this surface through general household activities such as food preparation and consumption. A deposit of earthen wall collapse within Feature 115, and thick ash lens possibly associated with burning in the structure, indicate 


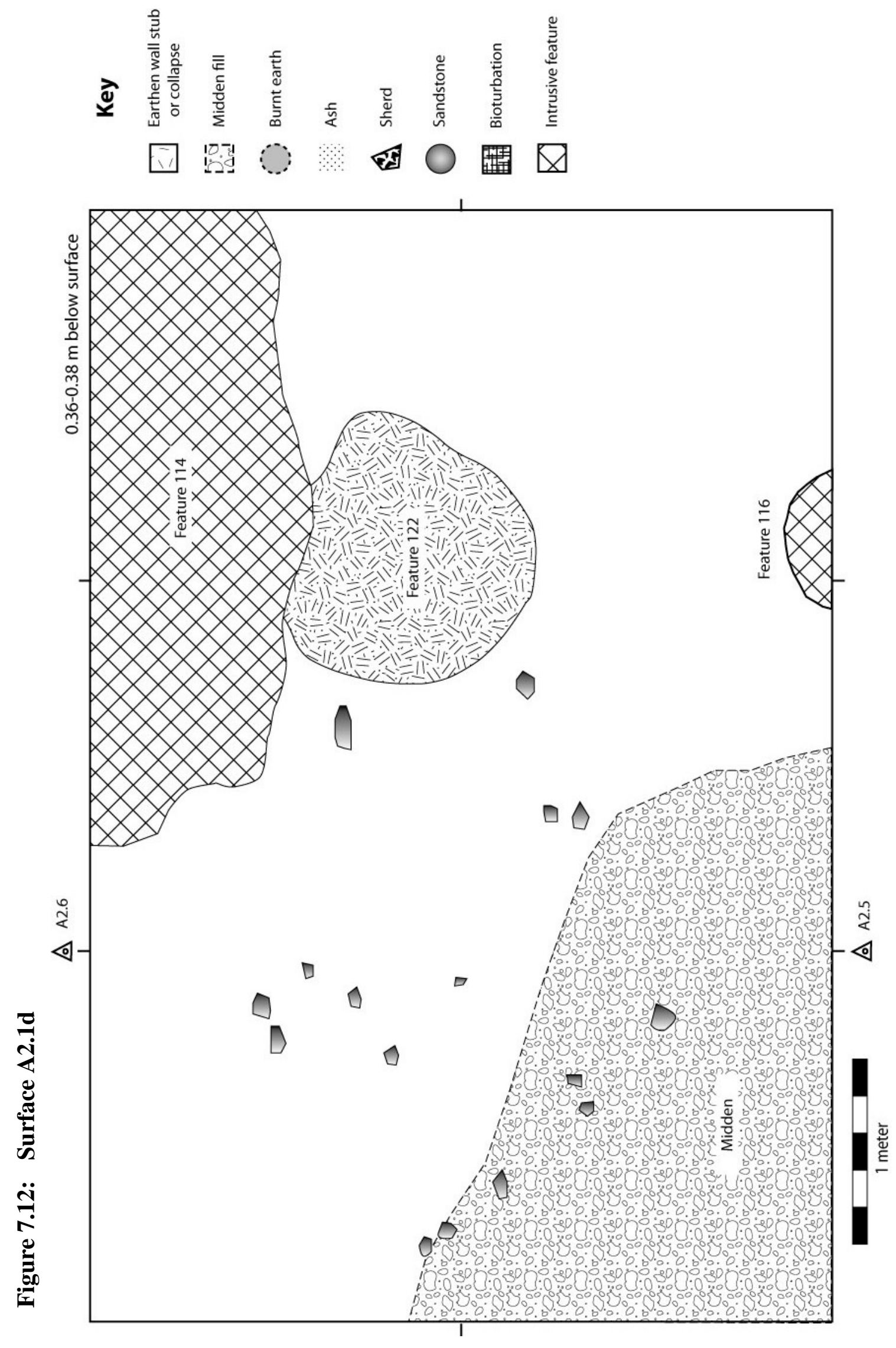


that people continued to use the hut alongside the deposition of Substratum A2.1b, but it ultimately collapsed toward the end of this stratigraphic episode.

Immediately following, or perhaps contemporaneous with, the accumulation of Stratum A2.1b, the occupants of Area A excavated a broad and amorphous borrow pit (Feature 114). Although the bottom of this pit was not reached during excavation, its contours were visible in the north profile and in the cross-section of Feature 122 (see above), pointing to a maximum depth of 1.20 meters below the surface. The depression was subsequently filled with at least two major episodes of refuse disposal and capped with a more diffuse sheet midden. Feature 114c, the lowermost deposit of fill, contained substantial frequencies of refuse including ceramic sherds, large faunal remains, three discoidal terracotta whorls, a grinding slab fragment, and a large iron knife blade folded in half. Along the bottom of Feature 114 at this level, two small dumps of small sandstone rocks (Feature 128, Feature 129) hinted at discrete episodes of disposal, possibly related to roasting or other food preparation techniques. Feature $114 \mathrm{~b}$, the intermediate deposit of fill yielded fewer artifacts (including one cowrie), but marked an increase in the number of ash lenses, suggesting the preferential disposal of waste from hearths. Feature 114a comprised the uppermost deposit of fill, including the spread of a sheet midden across the northern half of Unit A2. As with the lowermost level, this deposit contained substantial quantities of large animal bones and pottery sherds, including fragments from at least eight partially reconstructable vessels and items of personal adornment, including one globular terracotta netweight and one molded glass bead (Type Gb-F). A number of small finds recovered throughout the fill attested to the disposal of refuse from craft production activities - including a rhyolite hammerstone, a heavily worn plate interpreted as a potter's hand wheel (Figure 6.16), several large fragments of the granitic rock used to temper some earthenware pottery (Figure 6.15), and a vitrified conical crucible (Figure 6.21). The deposition of these objects in the Feature 114a midden strongly suggests that people undertook pottery production and blacksmithing in the vicinity. 


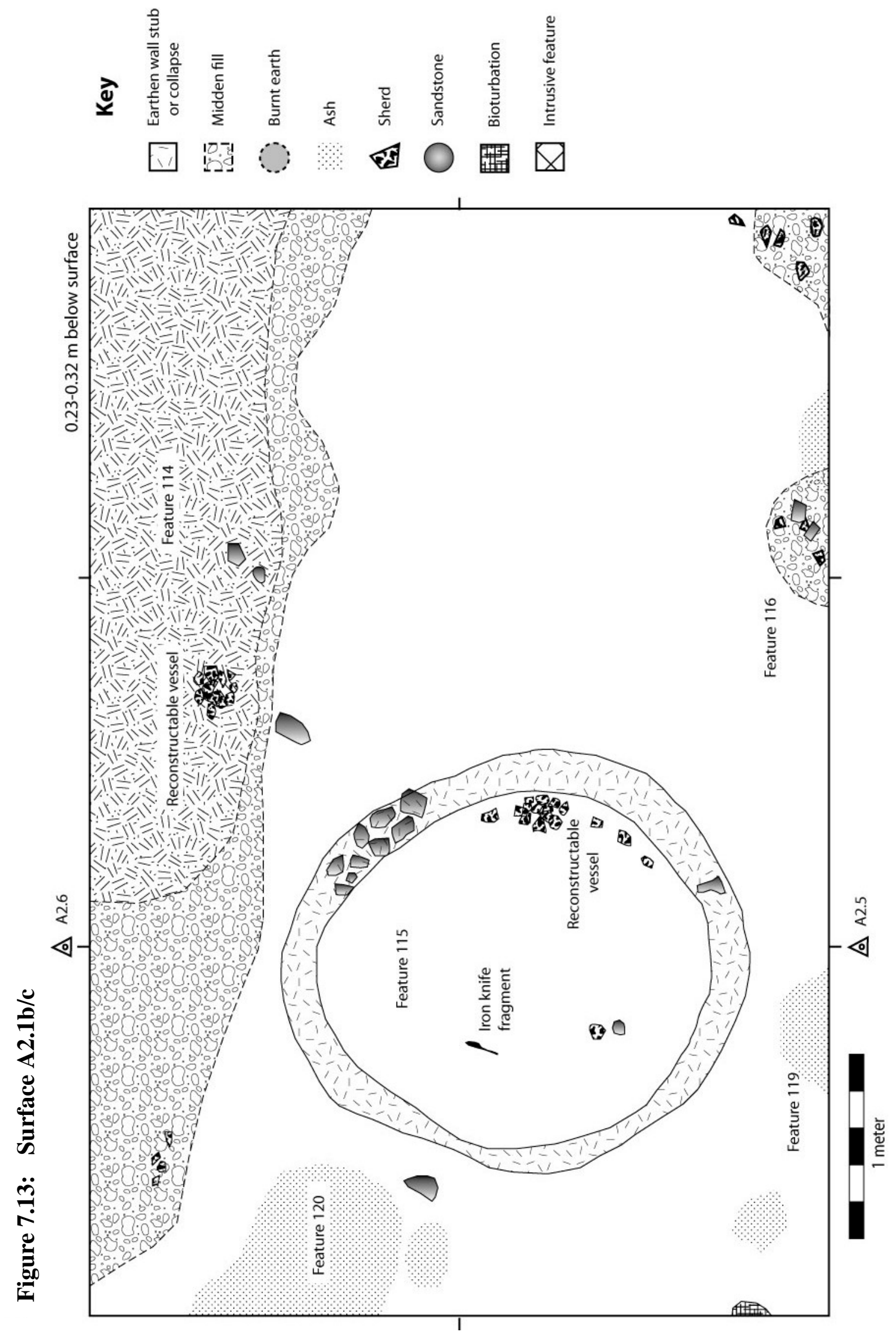


In the southern half of Unit A2, Substratum A2.1a (0.03-0.14) developed as the weathered matrix of underlying soil horizons. In the northern half of the unit, this deposit reflected deflation of the Feature 114a midden, yielding high quantities of sherds, faunal remains, and several objects of personal adornment including one quartz bead, and terracotta bead and bracelet fragments. An amphibolite hammerstone and quartz ( $\mathrm{n}=36$ ) and chert $(\mathrm{n}=16)$ debitage documented lithic reduction, while a modest amount of general slag $(n=8,41.7 \mathrm{~g})$ posited metallurgical activities in the area.

\section{Unit A2 Summary}

Architecture and Space

Stratum A2.3 derived from the relatively informal use of space involving a wide array of household activities such as food preparation, storage, and consumption, and refuse disposal. Although daub in Substratum A2.3a hinted at the presence of a nearby earthen wall structure, it remains unknown whether this comprised part of a household compound. Stratum A2.2 witnessed a shift to the more formal use of domestic space with at least two major cycles of constructing, maintaining, and demolishing stone foundations and earthen wall architecture. The sheet midden of Substratum A2.2a began the increasing use of this space for rubbish disposal. This trend reversed briefly in Stratum A2.1c where Feature 115 marked a return to the formalized production of domestic space. As this structure fell into disuse, the occupants of Area A dug the broad pit of Feature 114, only to later fill it with refuse from food and craft activities, these latter including metal working, pottery manufacture, and possibly even spinning and weaving.

\section{Artifacts, Organic Remains, and Material Practice}

The analyzed faunal sample from Unit A2 included 218 identifiable specimens from both general fill and pit contexts (Figure 7.14). Although the consumption of cattle and sheep/goat may have been limited, some of the unidentified terrestrial mammals, which comprise an even greater proportion of the sample, may have been domesticates. This dataset highlights the exploitation of all nearby habitats by the occupants of Area A, but with an emphasis on large-bodied prey, including some megafauna (hippopotamus). 
An iron harpoon and two terracotta netweights (Table 7.4) were undoubtedly part of a much more complex tool kit for exploiting these diverse resources.

The macrobotanical remains identified in 25 flotation samples by Daphne Gallagher (Appendix I) documented the consumption of domesticated pearl millet along with the exploitation of numerous wild species, particularly baobab fruit (Table 7.3). Diverse weedy species originated in the surrounding savanna environment.

Figure 7.14: Unit A2 faunal summary (from Appendix H)

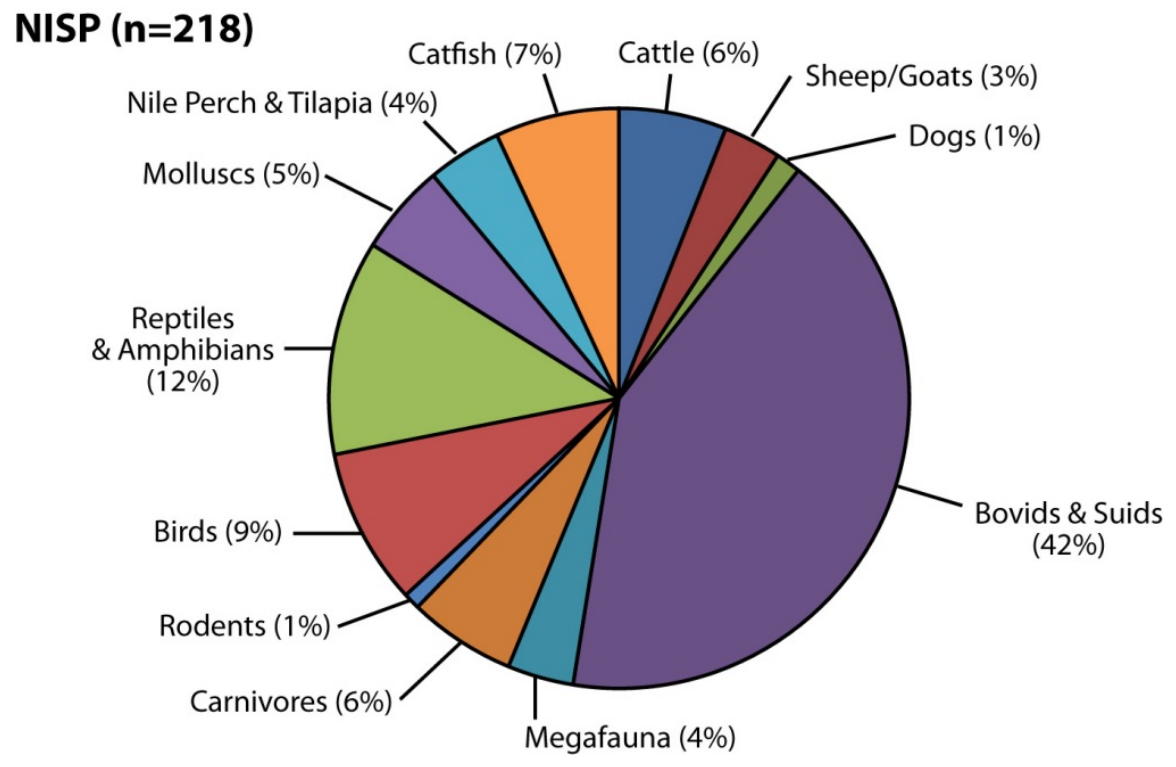

Table 7.3: Unit A2 macrobotanical summary (from Appendix I)

\begin{tabular}{|l|c|c|c|c|c|c|c|}
\hline Stratum & Pearl & \multicolumn{3}{|c|}{ Tree Fruits } & \multicolumn{3}{c|}{ Weedy Herbs } \\
& Millet & \multicolumn{2}{|c|}{ Baobab } & Jujube & $\begin{array}{c}\text { Desert } \\
\text { Date }\end{array}$ & Legume & Grass \\
Other \\
\hline \hline A2.1 & & $\mathrm{x}$ & $?$ & $\mathrm{x}$ & $\mathrm{x}$ & & \\
\hline A2.2 & & $\mathrm{x}$ & & & & & \\
\hline A2.3 & $\mathrm{x}$ & $\mathrm{x}$ & $?$ & & $\mathrm{x}$ & $\mathrm{x}$ & $\mathrm{x}$ \\
\hline
\end{tabular}


Table 7.4: Unit A2 artifact summary

\begin{tabular}{|c|c|c|c|c|c|c|c|}
\hline Subs & $\frac{\operatorname{tum}}{\mathrm{m}^{3}}$ & $\begin{array}{c}\text { Ceramic } \\
\mathrm{n}\end{array}$ & $\begin{array}{c}\text { Lithic } \\
\mathrm{n}\end{array}$ & $\begin{array}{c}\text { Slag } \\
n\end{array}$ & Groundstone & Tools & Ornaments \\
\hline A2.1a & 0.81 & 2082 & 55 & 8 & \multirow[b]{4}{*}{$\begin{array}{l}1 \mathrm{x} \text { Hammerstone } \\
1 \mathrm{x} \text { Handstone }\end{array}$} & \multirow[b]{4}{*}{$\begin{array}{l}1 \mathrm{x} \text { Iron rod } \\
\text { fragment }\end{array}$} & \multirow{4}{*}{$\begin{array}{c}1 \times \text { Copper } \\
\text { ornament } \\
1 \text { x Quartz bead } \\
\text { (Sb-1) } \\
2 \times \text { Terracotta } \\
\text { bead } \\
2 \times \text { Terracotta } \\
\text { figurine frag } \\
1 \times \text { Terracotta } \\
\text { bracelet frag }\end{array}$} \\
\hline $\mathrm{A} 2.1 \mathrm{~b}$ & 2.51 & 2561 & 1 & 0 & & & \\
\hline A2.1c & 1.55 & 1886 & 0 & 0 & & & \\
\hline A1.1d & 1.37 & 4006 & 3 & 0 & & & \\
\hline Ft. 114 & 3.65 & 7031 & 2 & 0 & $\begin{array}{l}1 \times \text { Hammerstone } \\
1 \times \text { Grinding slab }\end{array}$ & $\begin{array}{l}1 \times \text { Large iron } \\
\text { knife } \\
3 \times \text { Spindle } \\
\text { whorl } \\
1 \times \text { Netweight }\end{array}$ & $\begin{array}{c}1 \times \text { Glass bead } \\
(\text { Gb-F) } \\
1 \times \text { Cowrie }\end{array}$ \\
\hline Ft. 122 & 1.34 & 1265 & 1 & 0 & & $\begin{array}{c}1 \times \text { Iron } \\
\text { harpoon } \\
1 \times \text { Netweight }\end{array}$ & $\begin{array}{l}1 \times \text { Terracotta } \\
\text { bead }\end{array}$ \\
\hline A2.1 & 11.23 & 18,831 & 62 & 8 & 4 & 8 & 10 \\
\hline A2.2a & 1.93 & 3065 & 0 & 1 & \multirow{4}{*}{$\begin{array}{l}1 \text { x Quartz } \\
\text { hammerstone }\end{array}$} & \multirow{4}{*}{$\begin{array}{c}1 \times \text { Small iron } \\
\text { point } \\
1 \times \text { Iron rod } \\
\text { fragment } \\
3 \times \text { Spindle } \\
\text { whorl } \\
1 \times \text { Netweight }\end{array}$} & \multirow{4}{*}{$\begin{array}{c}2 \times \text { Carnelian } \\
\text { bead }(\text { Cb-1) } \\
1 \times \text { Terracotta } \\
\text { bead } \\
1 \times \text { Terracotta } \\
\text { bracelet frag }\end{array}$} \\
\hline $\mathrm{A} 2.2 \mathrm{~b}$ & 0.90 & 404 & 0 & 0 & & & \\
\hline $\mathrm{A} 2.2 \mathrm{c}$ & 1.43 & 489 & 0 & 0 & & & \\
\hline $\mathrm{A} 2.2 \mathrm{~d}$ & 0.65 & 174 & 0 & 0 & & & \\
\hline A2.2 & 4.91 & 4132 & 0 & 1 & 1 & 6 & 4 \\
\hline A2.3a & 0.57 & 144 & 0 & 0 & \multirow{3}{*}{ - } & \multirow{3}{*}{ - } & \multirow{3}{*}{$1 \times$ Cowrie } \\
\hline A2.3b & 0.47 & 519 & 0 & 0 & & & \\
\hline A2.3c & 0.73 & 247 & 0 & 0 & & & \\
\hline$A 2.3$ & 1.77 & 910 & 0 & 0 & - & - & 1 \\
\hline Total & 17.91 & 23,873 & 62 & 9 & 5 & 14 & 15 \\
\hline
\end{tabular}

Iron and groundstone implements present in the upper two strata indicated that the occupants of Area A processed these foodstuffs in the vicinity, although the relatively low frequencies of these tools relative to other artifacts suggest that these activities were not particularly intensive.

The earthenware pottery assemblage in Unit A2 demonstrated a clear seriation of decorative attributes (Figure 7.15) useful for fitting the associated stratigraphic units into a relative chronology. As shown in Table 7.5, the rim sherd assemblage revealed a trend 
in usage similar to that observed in Unit A1. Although bowls were rare throughout the unit, the relative frequency of large pots generally declined over time, while jars increased correspondingly. Notably, the large globular jars (Large B type) that predominated in Unit A1 were virtually absent in this assemblage, despite the much larger sample size. Regardless of the spatial dynamics within Unit A2, the assemblages from all three strata could have been associated with general household tasks for preparing and consuming food and transporting and storing water.

Among the high frequencies of artifacts in Unit A1, particularly in the refusefilled pits of Feature 114 and Feature 122, several rare artifacts (Table 7.4) provided evidence for craft specialization beyond the actual earthenware pot sherds and iron tools themselves. For instance, several pieces of slag and a conical crucible in the uppermost stratum demonstrated that people residing at Area A not only practiced technologies for working iron, a metal potentially smelted locally, but also for working copper, a metal obtained through supra-local exchange (see Appendix F).

\section{Figure 7.15: Unit A2 body sherd seriation}

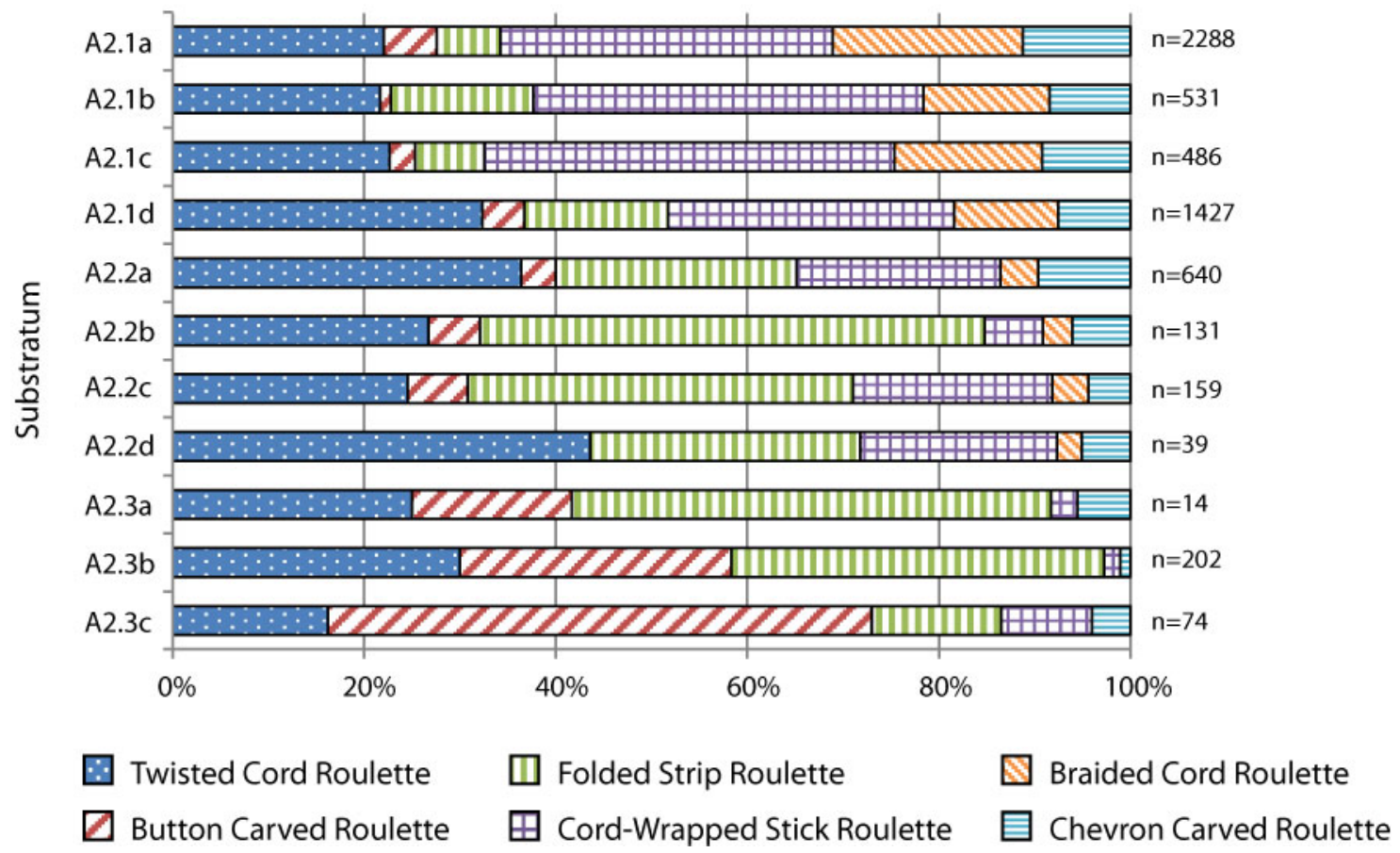


Table 7.5: Unit A2 vessel type frequencies

\begin{tabular}{|c|c|c|c|c|c|c|c|c|c|c|c|c|c|}
\hline \multirow{3}{*}{\multicolumn{2}{|c|}{$\begin{array}{c}\text { Substratum } \\
\text { Total } \\
n \\
\end{array}$}} & \multirow{2}{*}{\multicolumn{2}{|c|}{$\begin{array}{c}\text { Bowl } \\
\text { All }\end{array}$}} & \multicolumn{4}{|c|}{ Pot } & \multicolumn{6}{|c|}{ Jar } \\
\hline & & & & \multicolumn{2}{|c|}{ Small } & \multicolumn{2}{|c|}{ Large } & \multicolumn{2}{|c|}{ Small } & \multicolumn{2}{|c|}{ Large A } & \multicolumn{2}{|c|}{ Large B } \\
\hline & & $n$ & $\%$ & $n$ & $\%$ & $n$ & $\%$ & $n$ & $\%$ & $n$ & $\%$ & $n$ & $\%$ \\
\hline A2.1a & 108 & 5 & $4.6 \%$ & 3 & $2.8 \%$ & 3 & $2.8 \%$ & 6 & $5.6 \%$ & 3 & $2.8 \%$ & 1 & $0.9 \%$ \\
\hline F. 114 & 394 & 9 & $2.3 \%$ & 63 & $16.0 \%$ & 30 & $7.6 \%$ & 22 & $5.6 \%$ & 21 & $5.3 \%$ & 2 & $0.5 \%$ \\
\hline A2.1b & 158 & 1 & $0.6 \%$ & 16 & $10.1 \%$ & 9 & $5.7 \%$ & 10 & $6.3 \%$ & 8 & $5.1 \%$ & 1 & $0.6 \%$ \\
\hline A2.1c & 139 & 2 & $1.4 \%$ & 15 & $10.8 \%$ & 6 & $4.3 \%$ & 12 & $8.6 \%$ & 3 & $2.2 \%$ & 1 & $0.7 \%$ \\
\hline A2.1d & 196 & 3 & $1.5 \%$ & 22 & $11.2 \%$ & 7 & $3.6 \%$ & 13 & $6.6 \%$ & 7 & $3.6 \%$ & 0 & $0.0 \%$ \\
\hline F. 122 & 73 & 2 & $2.7 \%$ & 10 & $13.7 \%$ & 8 & $11.0 \%$ & 5 & $6.8 \%$ & 4 & $5.5 \%$ & 0 & $0.0 \%$ \\
\hline A2.1 & 1068 & 22 & $2.1 \%$ & 129 & $12.1 \%$ & 63 & $5.9 \%$ & 68 & $6.4 \%$ & 46 & $4.3 \%$ & 5 & $0.5 \%$ \\
\hline $\mathrm{A} 2.2 \mathrm{a}$ & 186 & 4 & $2.2 \%$ & 23 & $12.4 \%$ & 8 & $4.3 \%$ & 8 & $4.3 \%$ & 3 & $1.6 \%$ & 2 & $1.1 \%$ \\
\hline A2.2b & 18 & 0 & $0.0 \%$ & 3 & $16.7 \%$ & 0 & $0.0 \%$ & 2 & $11.1 \%$ & 1 & $5.6 \%$ & 0 & $0.0 \%$ \\
\hline A2.2c & 30 & 1 & $3.3 \%$ & 4 & $13.3 \%$ & 0 & $0.0 \%$ & 4 & $13.3 \%$ & 1 & $3.3 \%$ & 0 & $0.0 \%$ \\
\hline $\mathrm{A} 2.2 \mathrm{~d}$ & 18 & 0 & $0.0 \%$ & 5 & $27.8 \%$ & 2 & $11.1 \%$ & 0 & $0.0 \%$ & 1 & $5.6 \%$ & 0 & $0.0 \%$ \\
\hline A2.2 & 252 & 5 & $2.0 \%$ & 35 & $13.9 \%$ & 10 & $4.0 \%$ & 14 & $5.6 \%$ & 6 & $2.4 \%$ & 2 & $0.8 \%$ \\
\hline A2.3a & 3 & 0 & $0.0 \%$ & 0 & $0.0 \%$ & 0 & $0.0 \%$ & 0 & $0.0 \%$ & 1 & $33.3 \%$ & 0 & $0.0 \%$ \\
\hline A2.3b & 26 & 0 & $0.0 \%$ & 4 & $13.8 \%$ & 5 & $17.2 \%$ & 0 & $0.0 \%$ & 0 & $0.0 \%$ & 0 & $0.0 \%$ \\
\hline A2.3c & 16 & 0 & $0.0 \%$ & 2 & $12.5 \%$ & 5 & $31.3 \%$ & 2 & $12.5 \%$ & 0 & $0.0 \%$ & 0 & $0.0 \%$ \\
\hline A2.3 & 45 & 0 & $0.0 \%$ & 6 & $13.3 \%$ & 10 & $22.2 \%$ & 2 & $4.4 \%$ & 0 & $0.0 \%$ & 0 & $0.0 \%$ \\
\hline Total & 1365 & 27 & $2.0 \%$ & 170 & $12.5 \%$ & 83 & $6.1 \%$ & 84 & $6.2 \%$ & 52 & $3.8 \%$ & 7 & $0.5 \%$ \\
\hline
\end{tabular}

Coincident with these materials, the uppermost substratum yielded angular shatter and flakes of chert/flint ( $\mathrm{n}=16)$ and quartz $(\mathrm{n}=35)$ along with two quartz and one chert core. The only tool in this chipped stone assemblage was a chert backed bladelet. Despite the small size of this sample, it remains the largest recovered from an excavated context at Diouboye. This could suggest that lithic tool use and/or manufacture took place in the vicinity, and that sharp debris was collected for deposition in this midden away from the courtyards of household compounds. This interpretation is consistent with the near absence of chipped stone debris in earlier substrata, particularly those of Strata A.2, where architectural foundations demarcated zones of (bare) foot traffic between granaries and other features used on a daily basis.

Several terracotta beads, bracelets, and figurine fragments found primarily, but not exclusively, in pit fill and midden deposits reflect modes of personal adornment and 
perhaps ritual activities. Six terracotta spindle whorls from Feature 114 and Stratum A2.2 further highlighted the diversity of craft production activities undertaken at Area A, although they offer no conclusive evidence for inter-household specializations for spinning and weaving textiles.

Finally, a diverse assemblage of ornaments-including cowries and carnelian, quartz, and glass beads—shows that the residents of Area A took part in exchange networks from the early era of occupation until abandonment of the site. The presence of a copper ornament in Stratum A2.3 confirmed participation in northern (trans-Saharan) trade circuits by the later period of occupation at Diouboye. As personal adornments, these exotic objects would, of course, have served as indexes of access to these networks by individuals and residential groups within the village community.

\section{Residential Area F (Unit F)}

In light of heavy erosion along the western margin of Area F, I positioned Unit F $(4 \times 4 \mathrm{~m})$ near the apex of intact deposits several meters to the southeast of this embankment. Although the unit surface was fairly level, modest amounts of laterite gravel, angular sandstone fragments, and weathered sherds on this light brown sandy clay were consistent with long-term (and on-going) processes of sheet erosion and deflation.

I situated Unit $\mathrm{F}$ among several intact surface features with the intention of sampling the semi-formal space within a household compound and establishing the occupational history in the center of Diouboye. Although the unit achieved a maximum depth of only 0.79 meters, it nevertheless gave positive returns on both accounts by documenting ten stratigraphic episodes (Figure 7.16) replete with architectural features, hearth complexes, and diverse artifacts including sherds from at least two of the occupation phases defined for Diouboye in the final part of this chapter. 

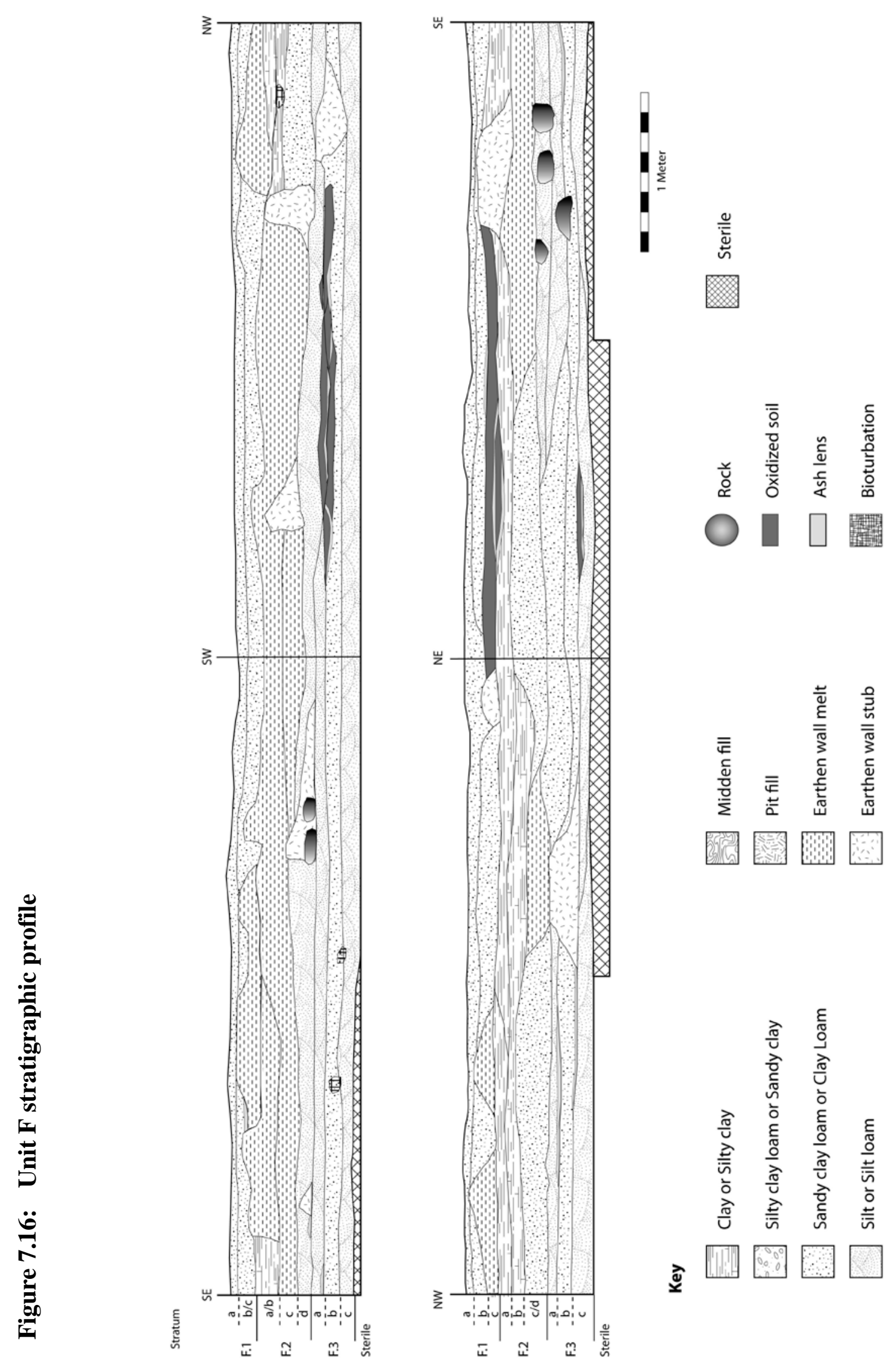

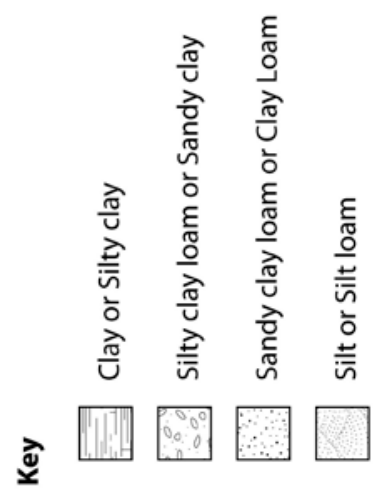




\section{Stratum F.3}

The occupation of Area F began on an extremely firm, yellowish-red silty loam alluvium encountered in Unit $\mathrm{F}$ at a minimum depth of 0.72 meters. The modest artifact and charcoal frequencies in Substratum F.3c (0.58-0.79 m), a moderately compact sandy loam transitioning to the sterile soil below, highlighted the low intensity of domestic activities during this initial episode.

Surface F.3b, however, flagged the start of a dynamic occupational sequence involving discrete domestic architecture and activity areas. Situated in the center of the unit, Feature 72 was a poorly preserved wattle-and-daub stub or trench measuring 2.10 meters in diameter; a patch of burnt sediment at the southeastern edge of the structure may have been an informal hearth, suggesting that the small hut had some specialized function. Feature 71, on the other hand, was a well-preserved terre pisé wall stub with an estimated diameter of 3.30 meters, corresponding to a domestic hut. The original floor within this latter structure displayed modest oxidation and an ash lens indicative of a small, informal hearth (sealed in place by a later repaving episode). Visible at the western edge of Unit F, Feature 74 was a substantial horizon of burnt sediment marking the repeated use of a hearth. Over time, natural processes of soil accumulation worked alongside domestic activities of food preparation, consumption, and earthen wall repair in this area to produce Substratum F.3b (0.58-0.67 m), an extremely firm deposit of general fill with fine to coarse daub inclusions.

While maintaining the same spatial layout, Features 71 and 72 continued onto Surface F.3a (Figure 7.17), coincident with several new features in the eastern half of the unit. Feature 75 was a small ash dump just south of Feature 73, a possible granary foundation with at least two sandstone rocks and a soil matrix resembling earthen wall melt with high densities of ceramic sherds and faunal remains. Feature 68 was an extremely firm sediment with daub inclusions forming either a wall melt or collapse from Feature 71, or perhaps the interior surface of a separate, unpreserved hut. Feature 69 involved a second resurfacing and final episode of fill (demolition?) within Feature 71. The overlying Substratum F.3a (0.44-0.62 m), a finely sorted sediment with low artifact frequencies, represented the general accumulation of sediment and debris from household 
activities. Developing above Feature 74, Feature 70 was a hearth complex arising from repetitive food preparation activities along the western edge of Unit F.

\section{Figure 7.17: Surface F.3a}

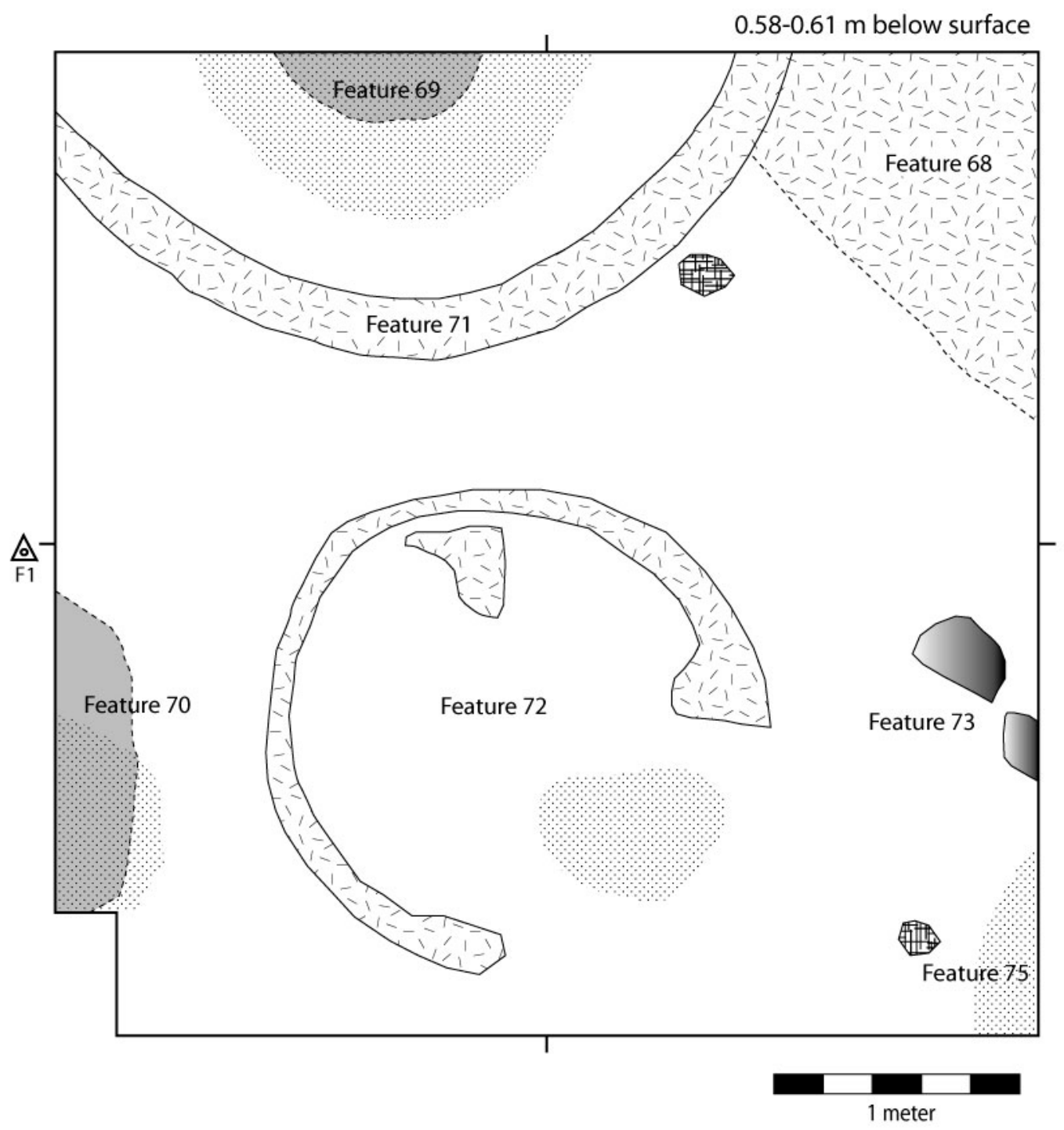

Key

Earthen wall stub or collapse

Midden fill

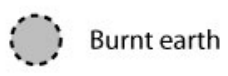

Ash
Sherd

Sandstone
Bioturbation

$\triangle$ Intrusive feature 
Figure 7.18: Surface F.2d

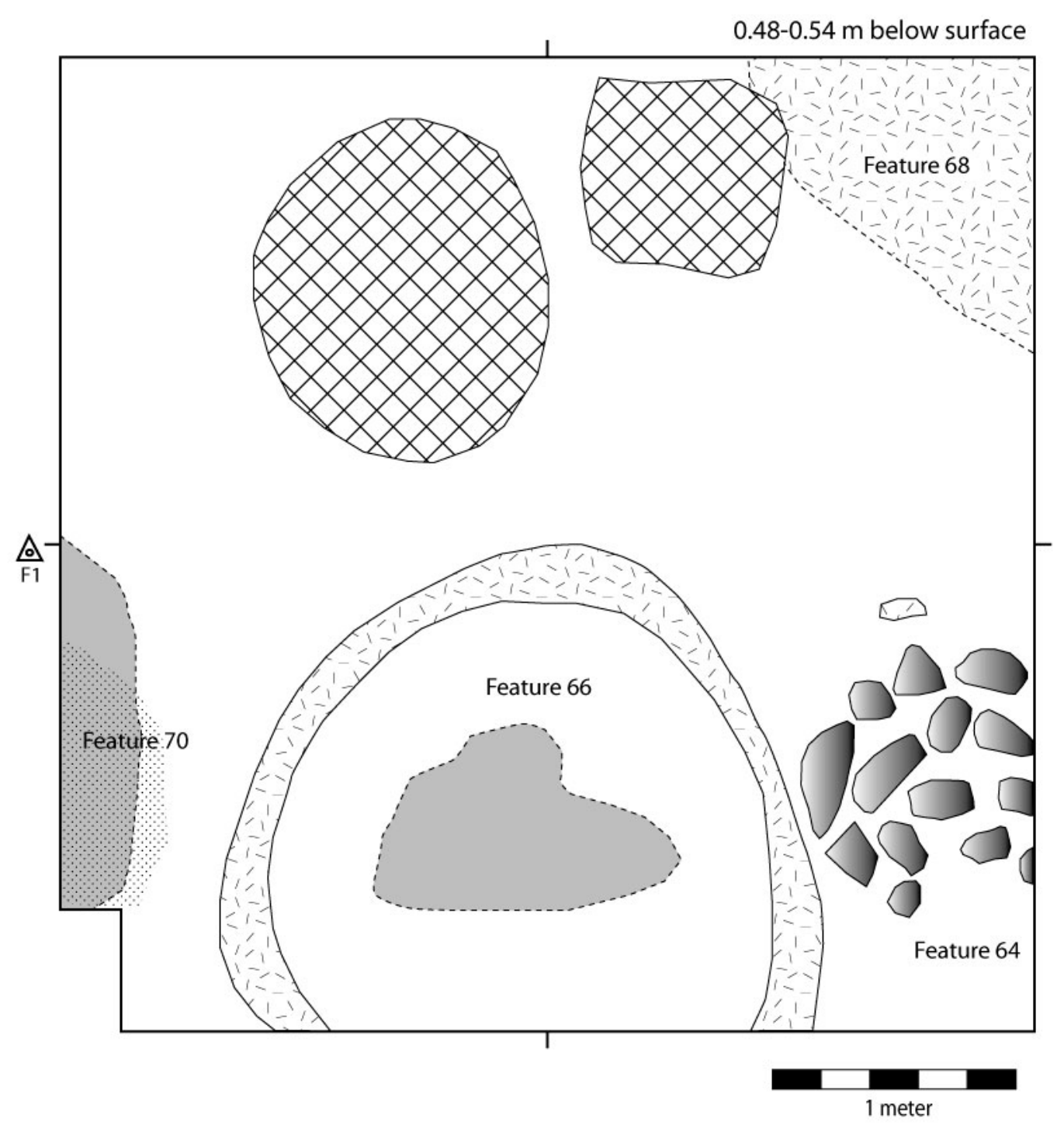

Key

Earthen wall stub or collapse

D.ं.

Midden fill
Burnt earth

Ash
14. Sherd

Sandstone
Bioturbation

Intrusive feature

\section{Stratum F.2}

Although the hearth complex of Feature 70 continued to define a semi-formal space in the southwestern corner of Unit F, Surface F.2d (Figure 7.18) marked a slight reorientation of the surrounding architectural features. Built directly above Feature 73, 
for example, Feature 64 was a small granary foundation composed of at least 13 sandstone rocks and measuring 1.15 meters in diameter. The associated soil had higher densities of daub and faunal remains than the surrounding matrix, consistent with debris trapped beneath an eroding earthen wall superstructure. Immediately to the west, Feature 66 comprised the poorly preserved terre pisé wall stub of a domestic hut measuring 2.50 meters in diameter; two underlying sandstone slabs in the southwest profile demarcated a possible doorway. The interior floor of Feature 66 was compact with some ash and light oxidation highlighting the location of an informal hearth in the middle of this domestic hut. Despite the intrusion of Features 62 and 65, the northern half of the unit preserved a relatively open space. Substratum F.2d (0.38-0.49 m) accumulated above this occupation surface as earthen wall melt combined with the soil and debris from generalized household activities attested by the ceramic and faunal assemblages.

Confined to the southeastern grid unit, Substratum F.2c.ii (0.23-0.40 m) was a thick deposit of earthen wall melt and collapse around the base of Feature 66. Although artifact frequencies were minimal, this sediment did contain six pieces of lithic debitage and a nearly complete polished quartz hachette. Concentrated in the northern and western parts of the unit, Substratum F.2c.i (0.23-0.48 m) was a reddish-brown, sandy clay loam with medium daub inclusions and laterite gravel resembling the natural alluvium beneath the site. For this reason, the deposit was classified as melt/collapse from earthen wall structures on Surface F.2c, although it could also have been an intentional fill to level this space prior to subsequent construction activity.

Signaling a reorientation of the space within Unit F, Feature 61, a possible terre pisé wall stub, was laid down during, or immediately following, the deposition of Substratum F.2c.i on Surface F.2b (Figure 7.19). Feature 65 was a shallow pit lined with light brown clay; the presence of subsequent oxidation, two sandstone rocks, and a fill rich with charcoal and faunal remains all suggest that this pyrotechnic feature was some sort of roasting pit. Feature 62 comprised a square stack of twelve sandstone rocks set into a shallow depression. Classified as a small filled feature, the proximity of Feature 62 to Feature 65 hints at a related function although rocks from the former displayed no firecracking. Feature 60 was an irregular scatter of five sandstone rocks, possibly the remains of a dismantled installation. With few artifacts, but a modest density of fine charcoal, 
Substratum F.2b (0.24-0.32 m) consisted of a general fill (or possible earthen wall melt) concentration in the northern half of Unit F around and above Features 62 and 65.

Figure 7.19: Surface F.2b

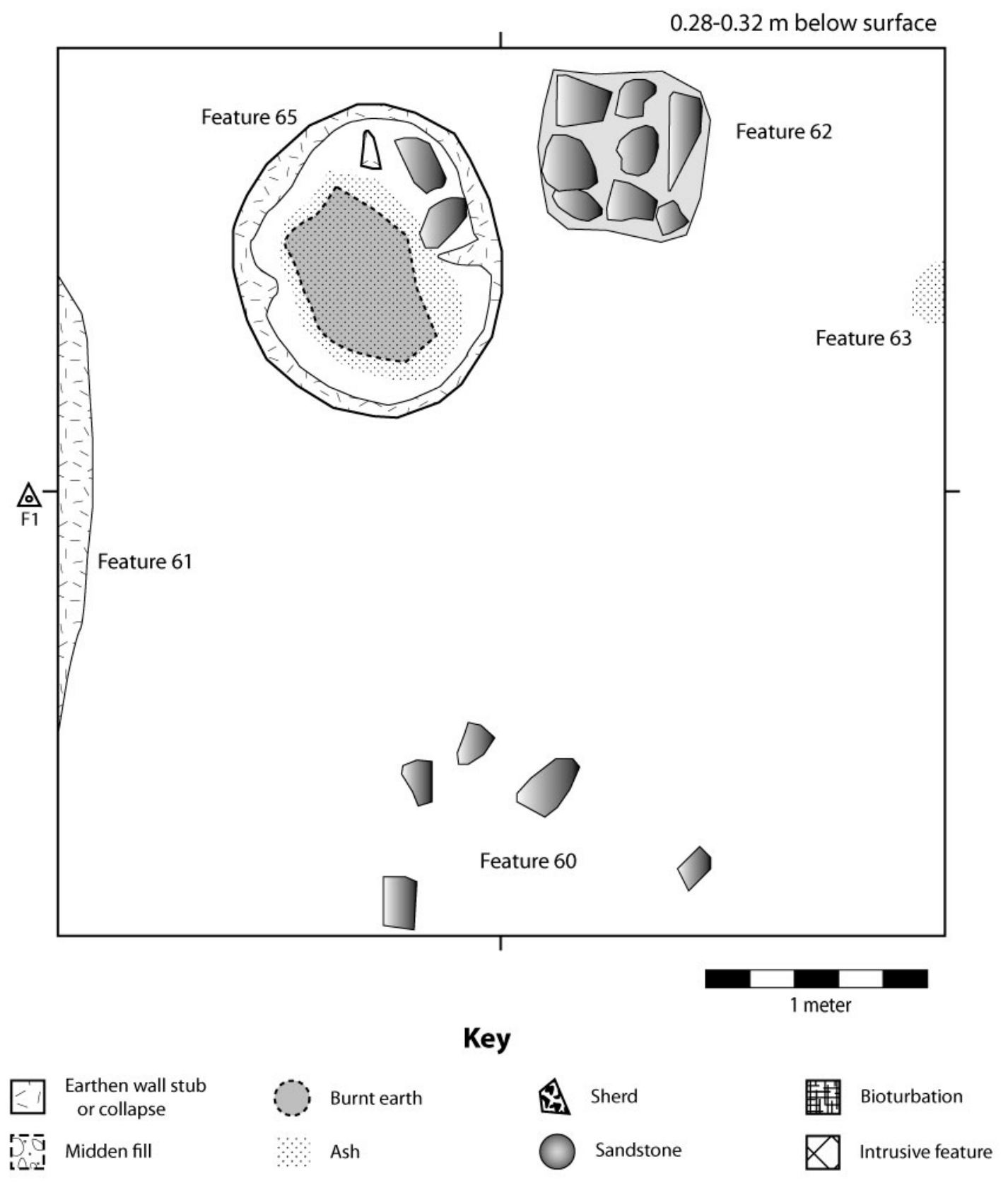


Substratum F.02a.ii (0.17-0.31 m) was a firm silty clay horizon with coarse daub inclusions and laterite pea gravel derived from earthen wall collapse and leveling prior to subsequent activity in this space. Exhibiting less daub, but more charcoal, Substratum F.2a.i (0.13-0.28 m) was an extremely firm silty clay loam deposit consistent with episodic deposition through earthen wall melt and collapse. Together these deposits set the stage for a further reshaping of this residential space in Stratum F.1.

\section{Stratum F.1}

The poorly preserved Surface F.1b/c (Figure 7.20) marked the final episode of residential activity in Unit F. Here Feature 57 comprised a round surface of extremely compact silty clay loam with light oxidation and ash highlighting either the presence of an ephemeral hearth or, more likely, the interior floor of a small hut. The matrix yielded few artifacts, but it did include the remnants of a large, and highly fragmented, iron projectile point or knife blade. Feature 58/59 was an amorphous hearth complex with several layers of heavily burnt soil and ash. Measuring at least 1.23 meters in diameter, Feature 54 was a semi-circular installation of seven sandstone rocks classified as a possible granary foundation. Found as an amorphous deposit across this surface, Substratum F.1c (0.10-0.18 m) appeared to result from the gradual decay or collapse of earthen walled structures, including perhaps Feature 57.

Positioned directly above Feature 58/59, Feature 52/53 represented the continued use of this hearth complex with three additional layers of burnt sediment and ash in the eastern half of Unit F. A major episode of earthen wall collapse and soil deposition worked to produce Substratum F.1b (0.04-0.19 m), a brownish-yellow silty clay loam with medium daub inclusions unevenly blanketing Surface F.1b/c. Substratum F.1a (0.000.10 ) was friable sandy loam topsoil generated from the subsequent weathering of this underlying matrix. Together these strata contained modest frequencies of artifacts, including several pieces of lithic debitage, slag (2.7 g) and groundstone fragments, and a solitary glass bead, although their proximity to the surface leaves open the possibility for contamination from elsewhere across Area F. In this regard, an exponentially greater number of sherds in this stratum, compared to all lower ones, seemed to be due to the deflation of this intra-site area via processes of wind and sheet erosion. 
Figure 7.20: Surface F.1b

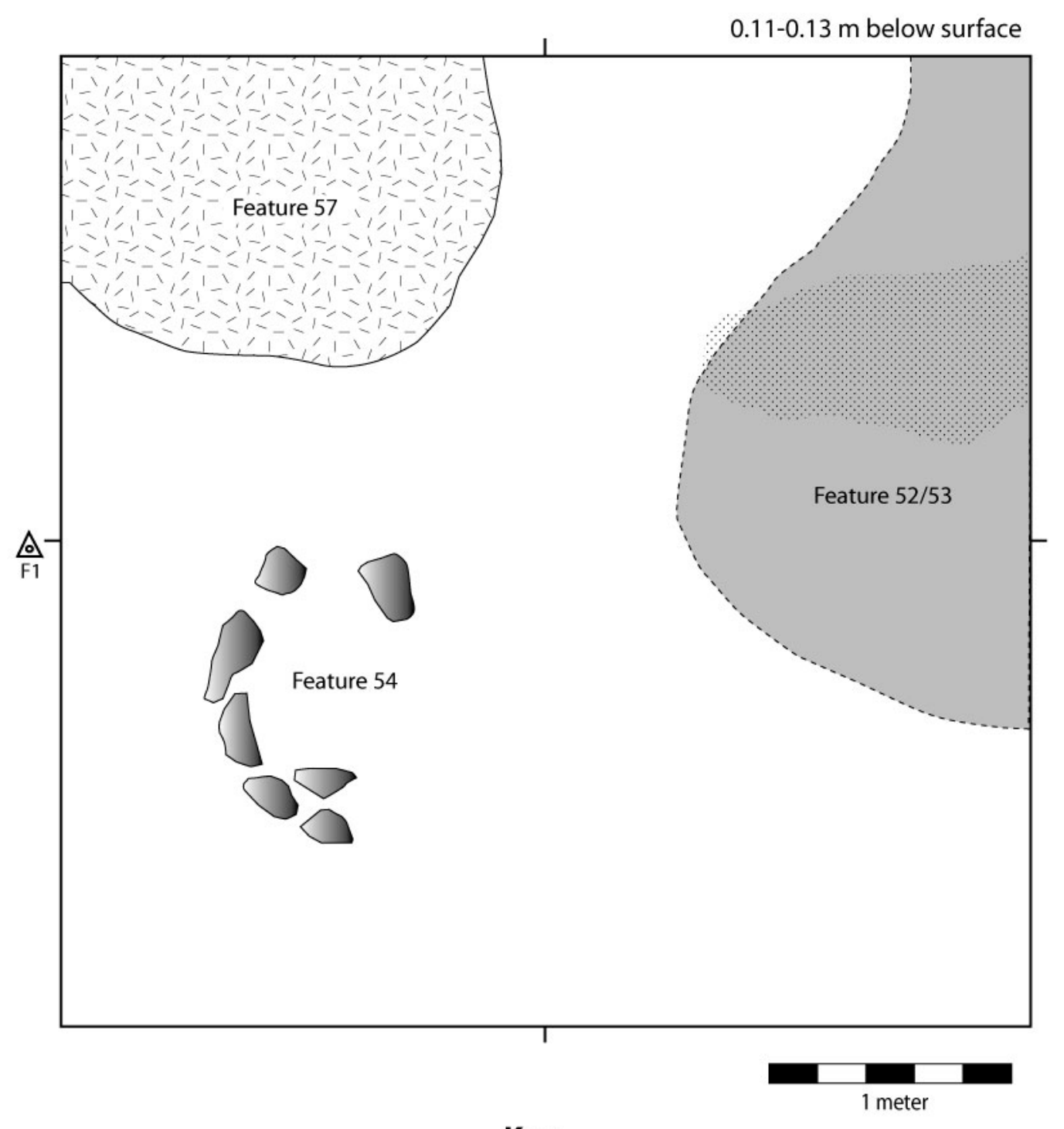

Key

Earthen wall stub
or collapse

S. Midden fill

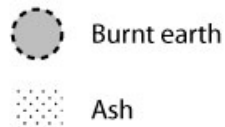

Ash
1. Sherd

Sandstone
Bioturbation

Intrusive feature 


\section{Unit F Summary}

Architecture and Space

Stratum F.3 encompassed a rapid formalization of the space encompassed by Unit F with the construction and maintenance of at least two earthen wall huts (Features 71 and 72), a possible granary foundation (Feature 73), and a well-defined hearth area (Feature 70) directly above a compact alluvium that underlies the entire site. Low artifact densities suggest that this space was swept regularly, an interpretation that would also account for the comparatively high densities of small sherds and faunal remains trapped within Feature 72. Stratum F.2 witnessed continuity in the use and reuse of space within this residential compound as the occupants of Area F built and remodeled a domestic hut (Feature 66), a granary (Feature 64), and a possible roasting pit (Features 62 and 65). Again, low artifact densities point to regular cleaning, with most of this stratum developing through earthen wall collapse and leveling. Stratum F.1 involved a final reorientation of this residential space to include one possible earthen wall hut (Feature 57), a circular stone foundation of unknown function (Feature 54), and a semi-formal hearth complex presumably associated with food preparation.

\section{Artifacts, Organic Remains, and Material Practice}

The relatively clean residential contexts within Unit F yielded a modest sample of only 27 identifiable faunal specimens (Figure 7.21). Domesticates, including cattle and possible dog, were not abundant in comparison to the substantial proportion of diverse terrestrial mammals. The small sample size notwithstanding, the only aquatic resource found in Unit F was a single freshwater bivalve shell, possibly suggesting a greater focus on hunting terrestrial species such as small bovids and antelopes.

The macrobotanical remains from 23 flotation samples (Table 7.6) included some grass caryopses of a size consistent with domesticated millet. Wild species such as baobab fruit and jujube documented the use of plants from nearby savanna habitats. 
Figure 7.21: Unit F faunal summary (from Appendix H)

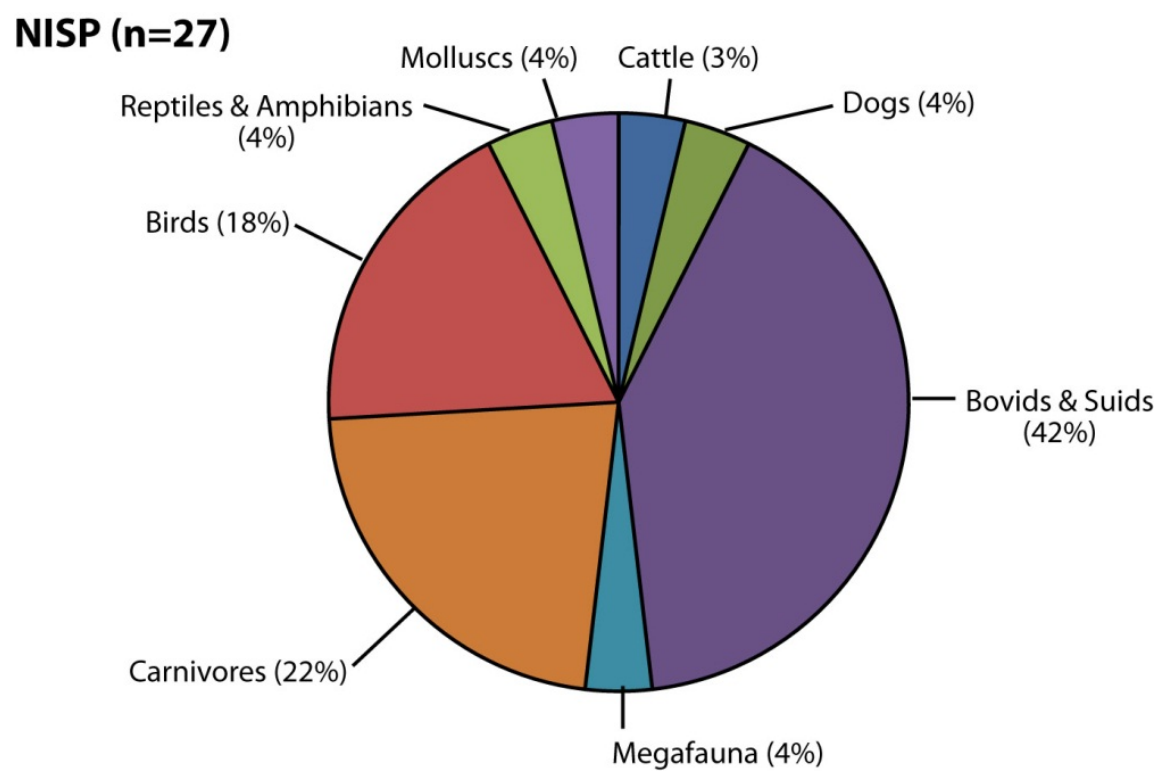

Table 7.6: Unit F macrobotanical summary (from Appendix I)

\begin{tabular}{|l|c|c|c|c|c|c|c|}
\hline Stratum & Pearl & \multicolumn{3}{|c|}{ Tree Fruits } & \multicolumn{3}{c|}{ Weedy Herbs } \\
& Millet & \multicolumn{2}{|c|}{ Baobab Jujube $\begin{array}{c}\text { Desert } \\
\text { Date }\end{array}$} & Legume & Grass & Other \\
\hline \hline F.1 & & $\mathrm{x}$ & & & & $\mathrm{x}$ & \\
\hline F.2 & $?$ & $\mathrm{x}$ & & & $\mathrm{x}$ & $\mathrm{x}$ & \\
\hline F.3 & $?$ & $\mathrm{x}$ & $\mathrm{x}$ & & & & \\
\hline
\end{tabular}

Given low sherd frequencies in Unit F, decorative attributes demonstrated a rough but clear seriation of the earthenware pottery assemblage (Figure 7.22) amenable for fitting the associated stratigraphic units into a relative chronology. Likewise, the small sample of rim sherds assigned to vessel types revealed tentative trends in pottery use. In general, bowls and pots were present in Stratum F.3, while jars slowly increased in frequency in Strata F.2 and F.1. Overall, the predominance of small jars and pots wellsuited for cooking throughout the sequence was consistent with the continued presence of hearth complexes demarcating semi-formal space for, among other things, preparing and consuming meals. 
Table 7.7: $\quad$ Unit $F$ artifact summary

\begin{tabular}{|c|c|c|c|c|c|c|c|}
\hline Str & $\mathrm{m}^{3}$ & $\begin{array}{c}\text { Ceramic } \\
\mathrm{n}\end{array}$ & $\begin{array}{c}\text { Lithic } \\
\mathrm{n}\end{array}$ & $\begin{array}{c}\text { Slag } \\
\mathrm{n}\end{array}$ & Groundstone & Tools & Ornaments \\
\hline F.1a & 1.20 & 2422 & 6 & 1 & \multirow{3}{*}{$\begin{array}{l}1 \text { x Hammerstone } \\
1 \text { x Grinding slab } \\
\text { fragment }\end{array}$} & \multirow{3}{*}{$\begin{array}{l}1 \text { x Large iron } \\
\text { blade }\end{array}$} & \multirow{3}{*}{$\begin{array}{l}1 \text { x Glass bead } \\
\text { type Gb-B }\end{array}$} \\
\hline F.1b & 1.06 & 161 & 3 & 0 & & & \\
\hline F.1c & 0.82 & 125 & 0 & 0 & & & \\
\hline F.1 & 3.08 & 2708 & 9 & 1 & 2 & 1 & 1 \\
\hline F.2a & 0.90 & 35 & 0 & 0 & \multirow{4}{*}{$\begin{array}{l}1 \text { x Quartz } \\
\text { hachette }\end{array}$} & \multirow{4}{*}{$\begin{array}{l}1 \text { x Unknown } \\
\text { iron frag }\end{array}$} & \multirow{4}{*}{$\begin{array}{l}1 \times \text { Iron } \\
\text { bracelet frag }\end{array}$} \\
\hline F.2b & 0.67 & 18 & 0 & 0 & & & \\
\hline F.2c & 1.66 & 128 & 6 & 0 & & & \\
\hline F.2d & 1.08 & 65 & 0 & 0 & & & \\
\hline F.2 & 4.31 & 246 & 6 & 0 & 1 & 1 & 1 \\
\hline F.3a & 2.01 & 341 & 2 & 0 & \multirow{3}{*}{ - } & \multirow{3}{*}{ - } & \multirow{3}{*}{ - } \\
\hline F.3b & 0.86 & 83 & 0 & 0 & & & \\
\hline F.3c & 1.99 & 106 & 4 & 0 & & & \\
\hline$F .3$ & 4.86 & 530 & 6 & 0 & - & - & - \\
\hline Total & 12.25 & 3484 & 21 & 1 & 3 & 2 & 2 \\
\hline
\end{tabular}

Figure 7.22: Unit $F$ body sherd seriation

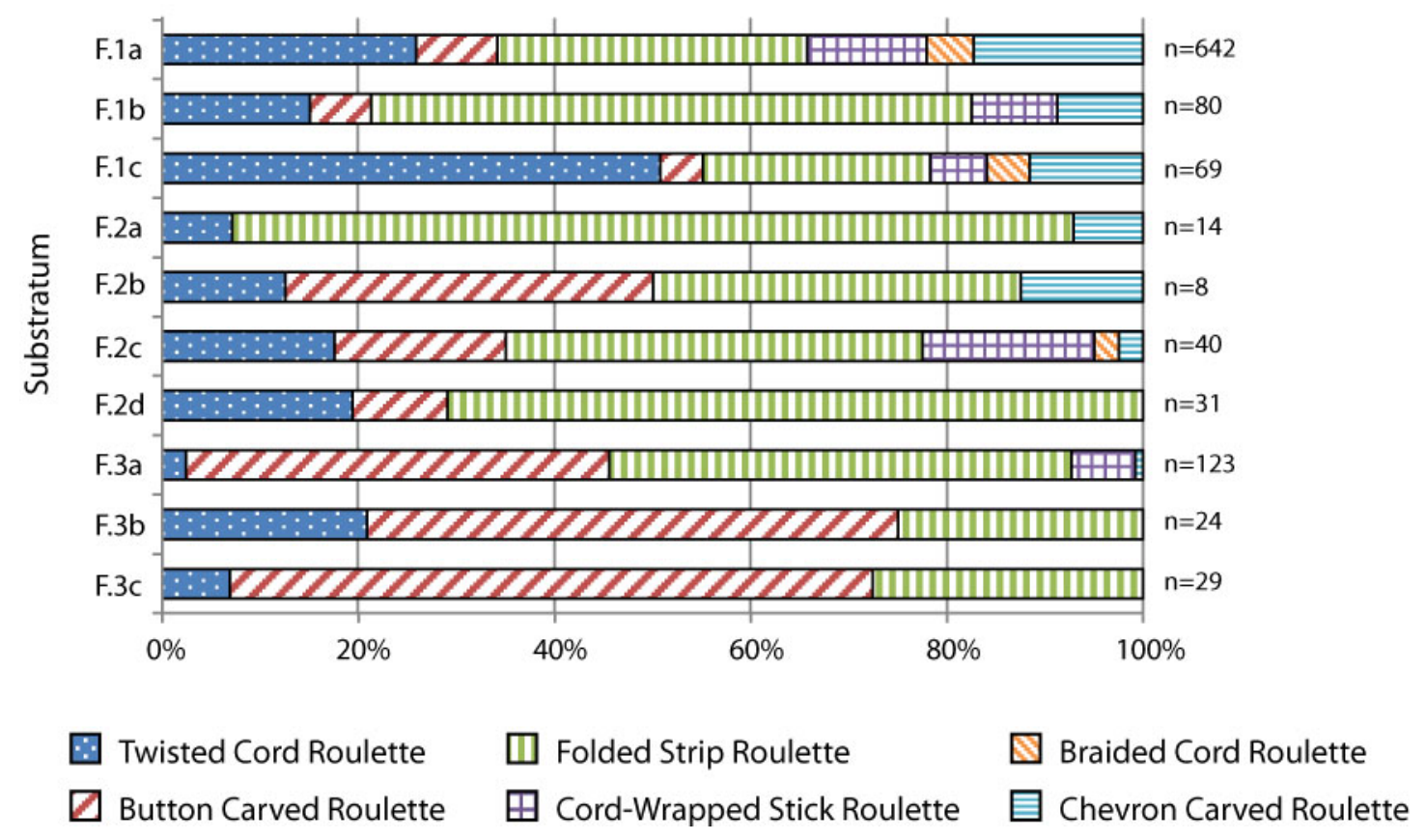


Table 7.8: $\quad$ Unit $F$ vessel type frequencies

\begin{tabular}{|c|c|c|c|c|c|c|c|c|c|c|c|c|c|}
\hline \multirow{3}{*}{\multicolumn{2}{|c|}{$\begin{array}{r}\text { Substratum } \\
\text { Total } \\
n\end{array}$}} & \multirow{2}{*}{\multicolumn{2}{|c|}{$\begin{array}{c}\text { Bowl } \\
\text { All }\end{array}$}} & \multicolumn{4}{|c|}{ Pot } & \multicolumn{6}{|c|}{ Jar } \\
\hline & & & & \multicolumn{2}{|c|}{ Small } & \multicolumn{2}{|c|}{ Large } & \multicolumn{2}{|c|}{ Small } & \multicolumn{2}{|c|}{ Large A } & \multicolumn{2}{|c|}{ Large B } \\
\hline & & $n$ & $\%$ & $n$ & $\%$ & $n$ & $\%$ & $n$ & $\%$ & $n$ & $\%$ & $n$ & $\%$ \\
\hline F.1a & 77 & 1 & $1.3 \%$ & 5 & $6.5 \%$ & 11 & $14.3 \%$ & 8 & $10.4 \%$ & 6 & $7.8 \%$ & 0 & $0.0 \%$ \\
\hline F.1b & 7 & 0 & $0.0 \%$ & 1 & $14.3 \%$ & 2 & $28.6 \%$ & 1 & $14.3 \%$ & 1 & $14.3 \%$ & 0 & $0.0 \%$ \\
\hline F.1c & 8 & 0 & $0.0 \%$ & 2 & $25.0 \%$ & 1 & $12.5 \%$ & 0 & $0.0 \%$ & 3 & $37.5 \%$ & 0 & $0.0 \%$ \\
\hline F.1 & 92 & 1 & $1.1 \%$ & 8 & $8.7 \%$ & 14 & $15.2 \%$ & 9 & $9.8 \%$ & 10 & $10.9 \%$ & 0 & $0.0 \%$ \\
\hline F.2a & 2 & 0 & $0.0 \%$ & 0 & $0.0 \%$ & 0 & $0.0 \%$ & 0 & $0.0 \%$ & 0 & $0.0 \%$ & 1 & $50.0 \%$ \\
\hline F.2b & 0 & 0 & $0.0 \%$ & 1 & $7.7 \%$ & 2 & $15.4 \%$ & 2 & $15.4 \%$ & 1 & 7.7\% & 0 & $0.0 \%$ \\
\hline F.2c & 13 & 0 & $0.0 \%$ & 0 & $0.0 \%$ & 0 & $0.0 \%$ & 0 & $0.0 \%$ & 0 & $0.0 \%$ & 0 & $0.0 \%$ \\
\hline F.2d & 4 & 0 & $0.0 \%$ & 1 & $5.3 \%$ & 2 & $10.5 \%$ & 2 & $10.5 \%$ & 1 & $5.3 \%$ & 1 & $5.3 \%$ \\
\hline F.2 & 19 & 1 & $4.2 \%$ & 4 & $16.7 \%$ & 8 & $33.3 \%$ & 1 & $4.2 \%$ & 0 & $0.0 \%$ & 0 & $0.0 \%$ \\
\hline F.3a & 24 & 1 & $12.5 \%$ & 1 & $12.5 \%$ & 0 & $0.0 \%$ & 3 & $37.5 \%$ & 1 & $12.5 \%$ & 0 & $0.0 \%$ \\
\hline F.3b & 8 & 0 & $0.0 \%$ & 1 & $9.1 \%$ & 1 & $9.1 \%$ & 2 & $18.2 \%$ & 1 & $9.1 \%$ & 0 & $0.0 \%$ \\
\hline F.3c & 11 & 2 & $4.7 \%$ & 6 & $14.0 \%$ & 9 & $20.9 \%$ & 6 & $14.0 \%$ & 2 & $4.7 \%$ & 0 & $0.0 \%$ \\
\hline F.3 & 43 & 3 & $1.9 \%$ & 15 & $9.7 \%$ & 25 & $16.2 \%$ & 17 & $11.0 \%$ & 13 & $8.4 \%$ & 1 & $0.6 \%$ \\
\hline Total & 154 & 1 & $1.3 \%$ & 5 & $6.5 \%$ & 11 & $14.3 \%$ & 8 & $10.4 \%$ & 6 & $7.8 \%$ & $\mathbf{0}$ & $0.0 \%$ \\
\hline
\end{tabular}

Although the occupants of Area F made use of iron tools (and perhaps jewelry), Unit F yielded no evidence for more specialized craft production activities beyond a solitary piece of slag. This was not too surprising given both the small artifact sample and an emphasis on food-related activities within this space. Chipped stone debitage of quartz $(\mathrm{n}=11)$, chert/flint $(\mathrm{n}=9)$, and hematite $(\mathrm{n}=1)$ were consistent with redeposition or low intensity lithic tool use and/or repair, while a hammerstone and grinding slab fragment spoke to possible grain-processing activities only in the final phase of residence.

A single glass bead and a small quartz hachette provided some evidence for social practices beyond the quotidian tasks of the residential compound.

\section{Residential Area G (Unit G)}

Beginning as a trench ( $2 \times 4 \mathrm{~m})$ near the apex of the Area $\mathrm{G}$ mound, two additional grid squares later expanded Unit $\mathrm{G}(4 \times 4 \mathrm{~m})$ to expose a possible wattle-and-daub structure (Feature 86) in Stratum G.3. Sloping gently down toward the east, the surface of Unit $\mathrm{G}$ was a loose, brown silt loam with high densities of heavily crushed ceramic 
sherds and laterite gravel from considerable deflation. A number of sandstone surface features nevertheless implied that the space immediately around Unit G formed part of a household compound during the terminal phase of occupation in Area G.

The purpose of Unit $\mathrm{G}$ was to delineate the history of architecture, activities, and depositional episodes leading to the formation of this small, yet discrete, mound. As illustrated in Figure 7.23, Unit G unveiled a sequence of stratified deposits extending to a depth of 0.83 meters below surface and documenting diverse earthen wall and sandstone architectural features, cylindrical borrow pits, hearths, and artifacts attesting to food preparation and consumption, craft production, and supra-local exchange. Seriation of pottery from the three observed strata, divisible into eight substrata, showed that occupation spanned all three phases defined for Diouboye in the final part of this chapter.

\section{Stratum G.3}

Appearing at a minimum depth of 0.81 meters below surface, the alluvium underlying Area $\mathrm{G}$ was extremely firm, reddish-yellow silty clay bearing no evidence of human activity beyond a few intrusive sherds. Substratum G.3c (0.69-0.83 m) had a similar soil matrix, but incorporated mottled patches of daub and charcoal alongside substantial quantities of ceramic sherds, faunal remains, three pieces of chipped stone debitage, and a single cowrie shell. These materials suggested that Area $\mathrm{G}$ began as a residential area where people undertook a suite of household tasks, and engaged in supralocal exchange.

Refuse disposal became the predominant use of the space within Unit $\mathrm{G}$ during the period bracketed by Substratum G.3b. Feature 98 comprised a sheet midden enriched with charcoal, sherds, and faunal remains in the northern half of the unit, while to the south Feature 99 defined a smaller midden deposit with similar artifact densities but more ash lenses. 

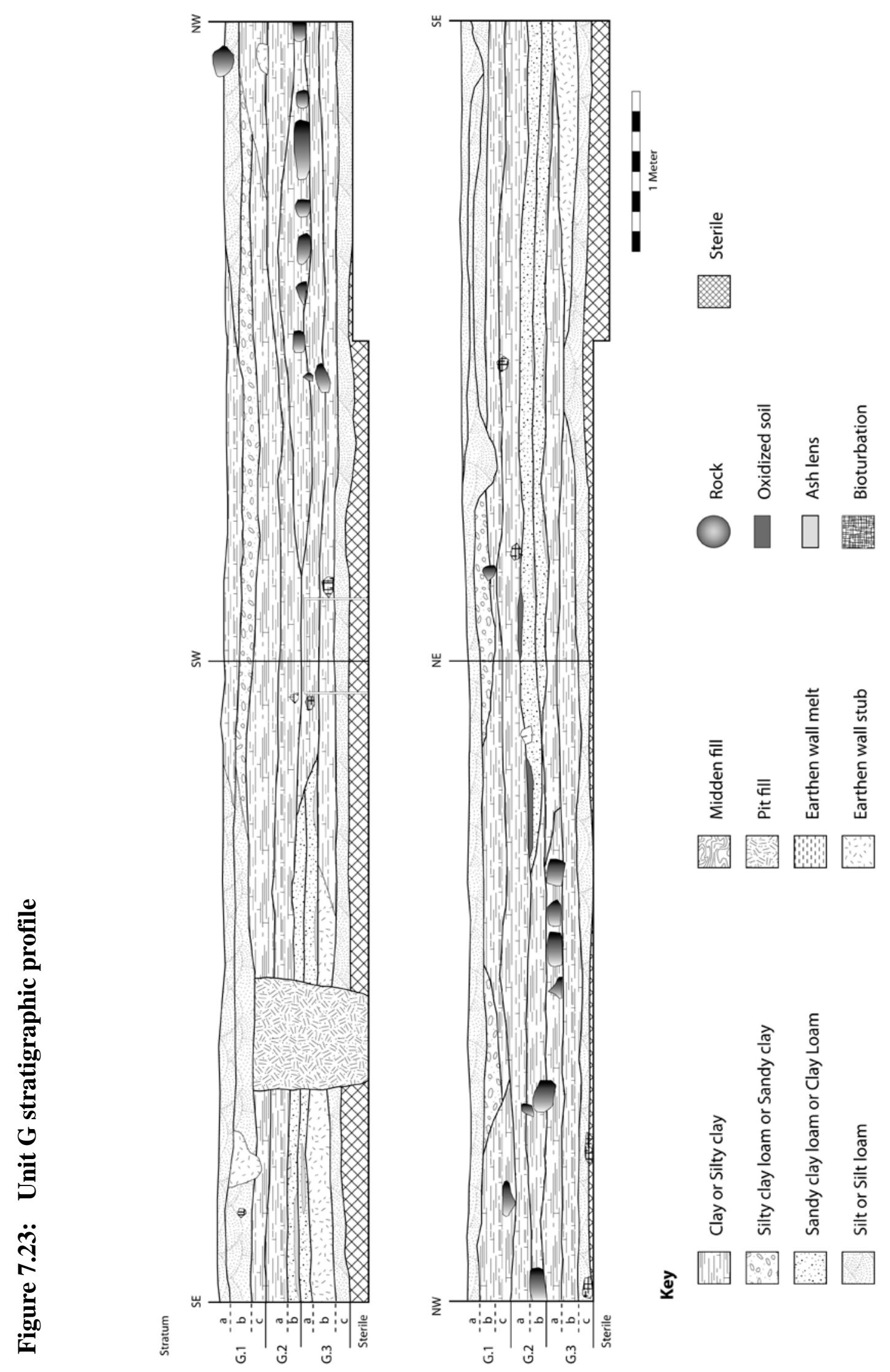
Figure 7.24: Surface G.3a
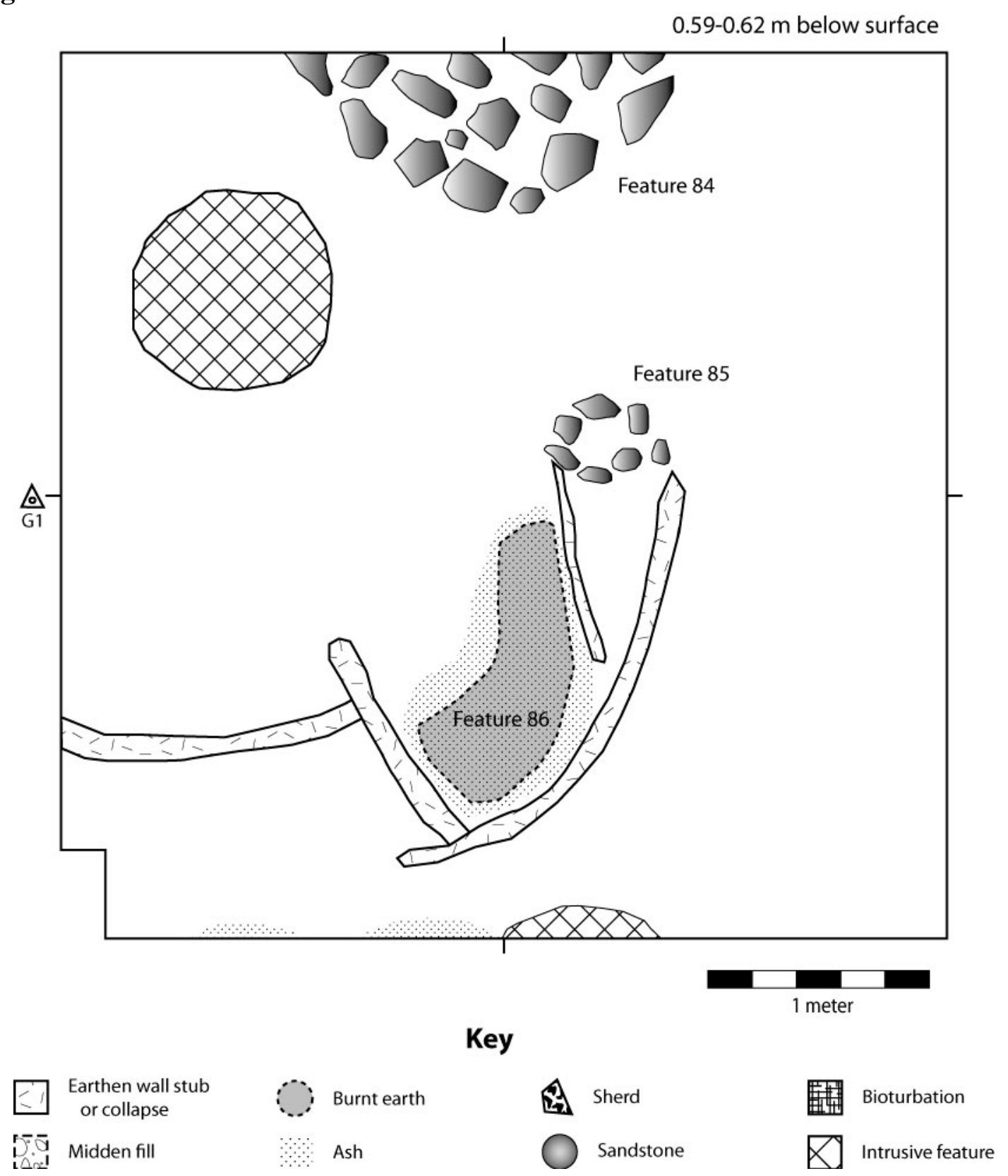

Above these deposits, Substratum G.3b.ii (0.59-0.72 m) constituted a general fill from household activity coupled with the natural accumulation of fine clay. Marked by greater frequencies of laterite gravels and daub inclusions in the northern half of the unit, Substratum G.3b.i (0.60-0.74 m) appeared to represent an episode of intentional leveling or perhaps earthen wall collapse from an otherwise undocumented structure. 
Several architectural elements on Surface G.3a (Figure 7.24) defined a more formalized use of the space within Unit G. Although the curvilinear alignment of Feature 86 first resembled some sort of bioturbation, the actual sediment was friable yellowishbrown clay nearly devoid of other inclusions. Given a clear interior surface with light oxidation and ash deposits from the in situ burning of a small hearth, this feature was classified as a series of superimposed wattle-and-daub wall stubs/trenches. Immediately post-dating these possible earthen walls, Feature 85 formed a small pot rest of seven sandstone blocks measuring 0.57 meters in diameter. Extending out from the north profile, Feature 84 was a large granary foundation with an estimated diameter of 1.701.90 meters. It was unclear, however, whether the high densities of artifacts (including one cowrie) within and below this feature derived from contemporaneous activities, or from the underlying midden (Feature 98).

Concurrent with the use of these features, Substratum G.3a.iii (0.47-0.64 m), a reddish-yellow silty clay, accumulated in the center of Unit $\mathrm{G}$ as a general fill with intermittent episodes of wall melt. Along the east profile, Substratum G.3a.ii (0.47-0.61 $\mathrm{m}$ ) emerged through the disposal of refuse including ash and charcoal, ceramic sherds, faunal remains (including one cowrie), a sharpening stone, and a small iron knife fragment. Substratum G.3a.i (0.48-0.68 m) appeared in the northwestern part of the unit as a firm deposit with high densities of laterite pea gravel and medium to coarse daub inclusions signaling the collapse of earthen wall structures from Surface G.3a.

\section{Stratum G.2}

Surface G.2b (Figure 7.25) marked a reorientation of this residential space, particularly with features concentrated in the northwestern part of the unit. Feature 96 was a small, filled circular installation, measuring 0.70-0.80 meters in diameter, but with no evidence of an overlying earthen wall structure. Although slightly clipped by an intrusive cylindrical pit, Feature 93 formed a platform (possible granary foundation) of sandstone rocks built immediately to the west of Feature 84. The associated matrix differed slightly from the surrounding fill with elevated frequencies of charcoal, ash, and faunal remains, including one cowrie and large sherds from a reconstructable longnecked jar. Representing a continuation of Substratum G.3a.ii in the eastern half of the 
unit, Substratum G.2b.ii (0.37-0.51 m) was a layer of artifact-rich fill. Elsewhere across the unit, Substratum G.2b.i (0.42-0.56 m) accrued as an extremely firm horizon of silty clay with modest artifact frequencies, presumably the result of generalized household tasks for food preparation and consumption and episodic wall melt induced by rain.

Figure 7.25: Surfaces G.2a/b

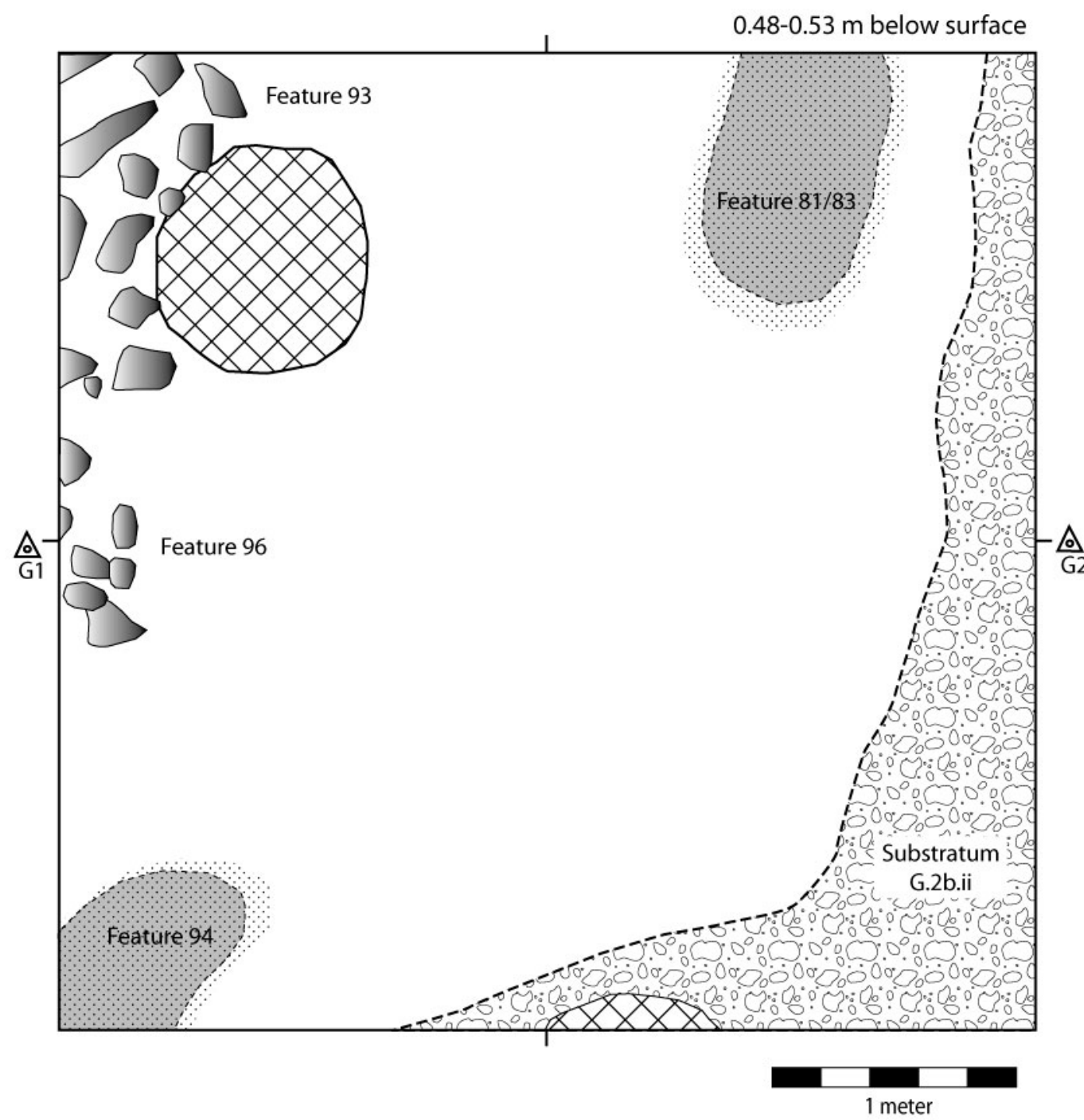

Key

Earthen wall stub or collapse

i.

Midden fill
Burnt earth

Ash
E. Sherd

Sandstone
Bioturbation

$\triangle$ Intrusive feature 
Surface G.2a (Figure 7.25) witnessed the continued use of Feature 93 alongside two semi-formal hearth areas. Feature 81/83 was a thick $(4-5 \mathrm{~cm})$ hearth complex of heavily burnt sediment and ash, providing evidence for repeated pyrotechnic activity, presumably cooking, in the northeast grid unit. The oxidized sediment of Feature 94 also defined a possible hearth, although it lacked an associated ash lens. Substratum G.2a (0.29-0.48 $\mathrm{m}$ ) covered this surface as a firm deposit of brownish-yellow silty clay with very modest artifact frequencies, pointing to accumulation through earthen wall collapse and general domestic activity within this semi-formal household courtyard.

\section{Stratum G.1}

Material practices consistent with residential occupation persisted onto the wellpreserved Surface G.1c (Figure 7.26). Although an intrusive pit precluded a diameter estimate, Feature 89 was a curvilinear terre pisé wall stub of very compact clay loam with a lightly oxidized interior surface — possibly marking the foundation of a domestic hut. Directly to the east, Feature 92 formed a discrete patch of reddish, burnt sediment corresponding to a small, informal hearth, while two sandstone rocks in the northern profile (Feature 91) were suggestive of an adjacent foundation. Domestic activities and natural soil accumulation on this surface led to the formation of Substratum G.1c.ii (0.20$0.40 \mathrm{~m}$ ), an extremely firm silty clay with modest artifact frequencies. In the northwestern part of the unit, Substratum G.1c.i (0.23-0.39 m) consisted of a moderately firm earthen wall melt, and possible collapse, around and above Feature 89.

Surface G.1b (Figure 7.26) yielded no evidence for architectural features beyond Feature 87, a probable pot rest of six angular sandstone rocks in the center of Unit G. Otherwise it would seem that the occupants of Diouboye used this space to obtain sediment for earthen wall construction and to dispose of domestic refuse. Visible in the southern profile by virtue of friable fill and dense inclusions of charcoal, ash, and artifacts, Feature 82 was a cylindrical pit excavated to a depth of at least 0.90 meters (see Figure 6.10). In the northwest grid square, Feature 90 comprised a cylindrical pit (0.94 m diameter) dug to a depth of 0.82 meters below Surface G.1b. Whatever the initial purpose of this pit, the fill was a heterogeneous deposit with high densities of charcoal, ceramic sherds, faunal elements, and sandstone rocks—some possibly deriving from Features 93 
which would have been encountered during its excavation. In addition, Feature 90 contained a polishing stone, a handstone and moderately worn grinding basin, and several terracotta objects including a figurine fragment, a discoidal spindle whorl, and a globular netweight.

Figure 7.26: Surfaces G.1b/c

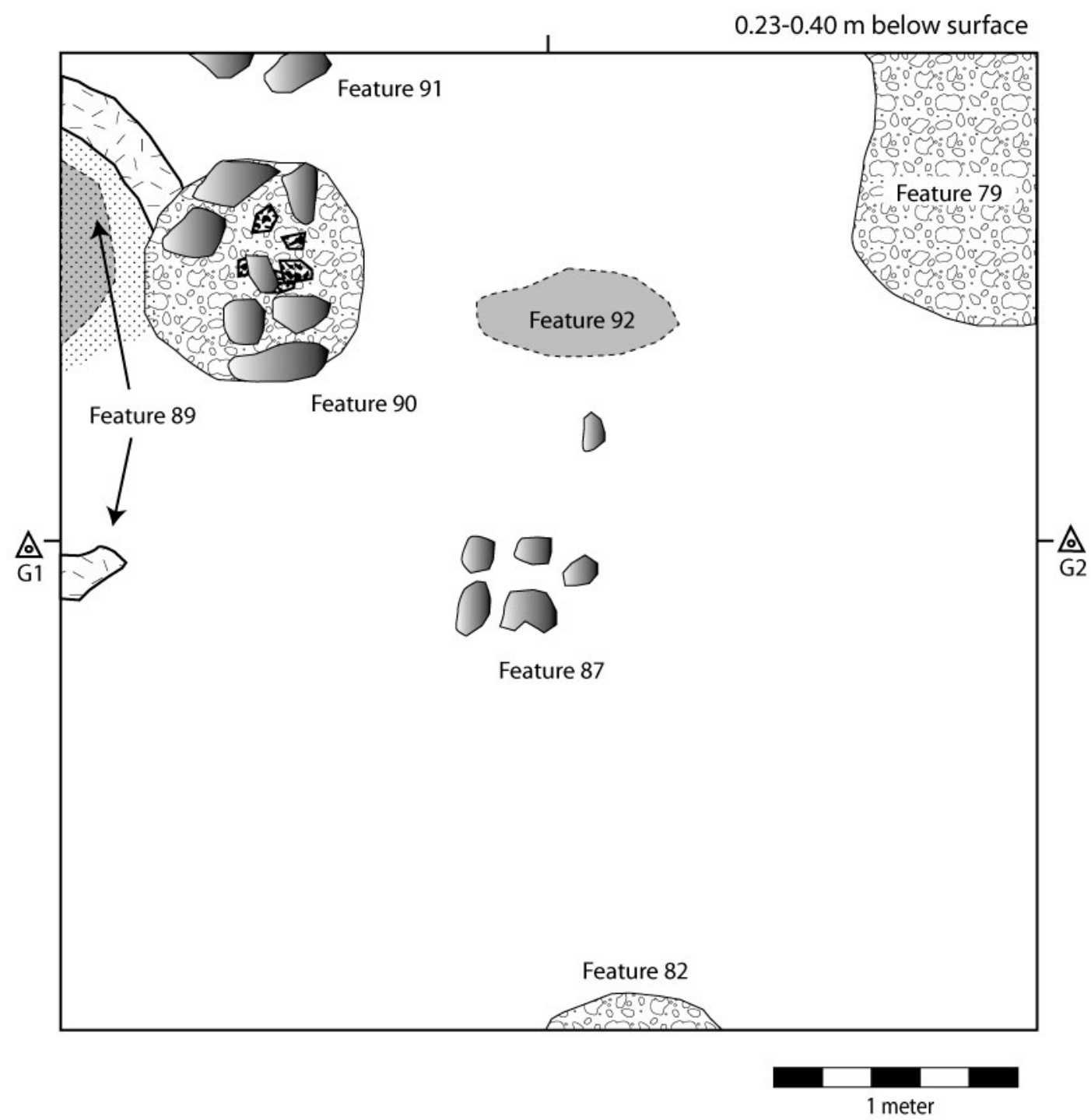

Key

Earthen wall stub or collapse

i.
Burnt earth

Ash
E. Sherd

Sandstone
Bioturbation

$\triangle$ Intrusive feature 
Meanwhile Feature 79 developed as a friable horizon of sandy clay loam with elevated artifact densities and inclusions of burnt daub consistent with the controlled discard of household refuse and/or debris from earthen wall maintenance or collapse. In the western half of the unit, Substratum G.1b.ii $(0.10-0.27 \mathrm{~m})$ was a moderately firm, silty clay loam with modest cultural detritus, including one cowrie, one carnelian bead, and two spherical terracotta spindle whorls. Substratum G.1b.i (0.10-0.24 m), on the other hand, was a pale brown loamy sand with elevated densities of artifacts (including an ivory bead and bracelet fragment), but only limited amounts of daub and charcoal. This latter was a unique form of deposit at Diouboye interpreted as an intentional leveling episode within this courtyard space, perhaps to create a water permeable surface and reduce mud during the rainy season.

Three deposits forming the last occupational episode in Unit G differed according to the character of the underlying matrix from which they had weathered. At the same time, Substrata G.1a.i-iii (0.000-0.18 m) all contained similar amounts of organic detritus, ash, and artifacts, including several pieces of lithic debitage and an iron hook.

\section{Unit G Summary}

\section{Architecture and Space}

In sum, Stratum G.3 documented the gradual establishment of a household compound within a space that had previously accumulated some debris from nearby activities. Feature 86 indicates that some early structures were made of wattle-and-daub, while the installation of at least two stone foundations (Features 84 and 85) quickly moved to formalize this residential area. Stratum G.2 simply represented an evolution of this spatial plan to include two stone foundations (Features 93 and 96), two hearths (Features 81/83 and 94), and a possible refuse area to the east. Finally, Stratum G.1 marked a shift to semi-formal space in Unit G with the presence of an earthen wall structure (Feature 89) and two stone installations (Features 87 and 91) counterbalanced by the excavation of two cylindrical borrow pits (Features 82 and 90) and the subsequent disposal of refuse in these and a sheet midden. 
Artifacts, Organic Remains, and Material Practice

Diverse contexts, including general fill and pit fill, within Unit G yielded a sample of 72 identifiable faunal specimens (Figure 7.27). These remains pointed to the limited consumption of cattle and sheep/goat although some of the terrestrial mammals, which comprise the more than half of the sample, may have been domesticates. Overall, this dataset suggests that the occupants of Area G obtained most of their meat by hunting medium-sized game in the savanna surrounding the village. They also harvested mollusks and fish from the Falémé River, but no larger aquatic species were present in the analyzed assemblage.

Figure 7.27: Unit G faunal summary (from Appendix $\mathbf{H}$ )

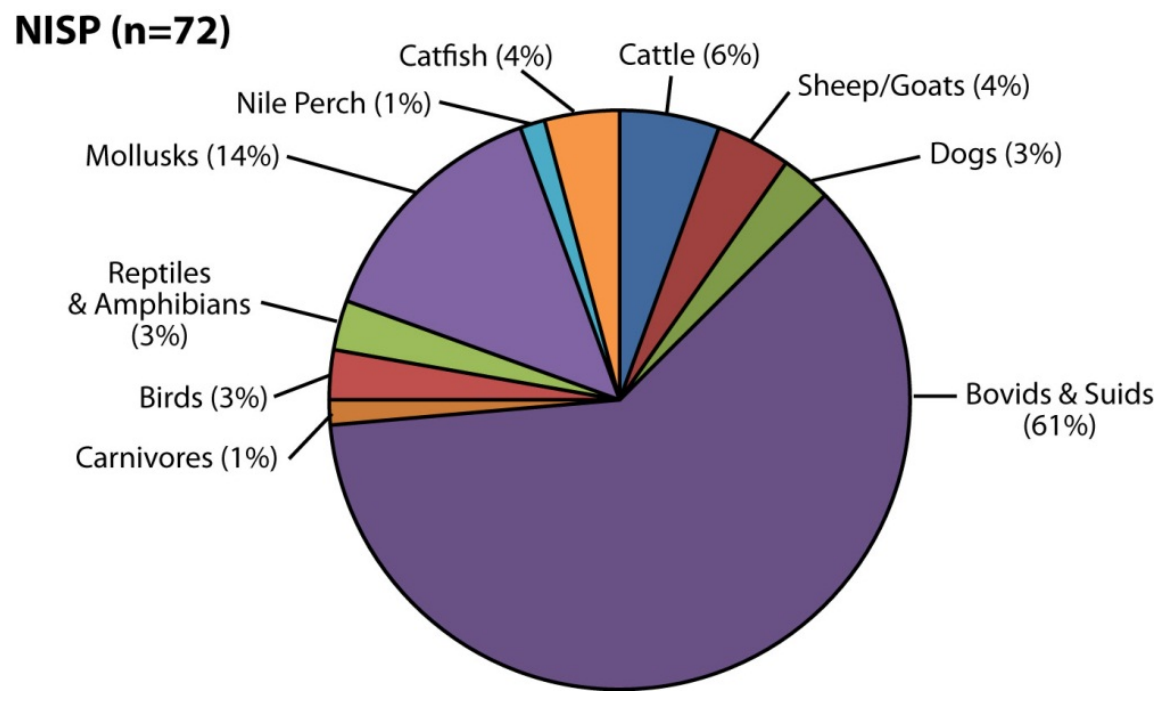

Table 7.9: Unit G macrobotanical summary (from Appendix I)

\begin{tabular}{|c|c|c|c|c|c|c|c|}
\hline \multirow[t]{2}{*}{ Stratum } & \multirow{2}{*}{$\begin{array}{l}\text { Pearl } \\
\text { Millet }\end{array}$} & \multicolumn{3}{|c|}{ Tree Fruits } & \multicolumn{3}{|c|}{ Weedy Herbs } \\
\hline & & Baobab & Jujube & $\begin{array}{c}\text { Desert } \\
\text { Date } \\
\end{array}$ & Legume & Grass & Other \\
\hline G.1 & & $\mathrm{x}$ & & & & & $\mathrm{x}$ \\
\hline G.2 & & $\mathrm{x}$ & & & & $\mathrm{x}$ & \\
\hline G.3 & & $\mathrm{x}$ & & & & $\mathrm{x}$ & \\
\hline
\end{tabular}


The macrobotanical remains from 17 flotation samples (Table 7.9) did not yield any clear domesticates although this likely arose from issues of preservation and sampling rather than an absence of grains in the diet of people occupying Area G. Baobab shells and wild sedges/grasses document the surrounding savanna habitat.

The several groundstone and chipped stone artifacts found in the Unit G sequence may have been used for food preparation - the former for processing grains, the latter from making or retouching informal stone tools. In this regard, it is noteworthy that the chipped stone assemblage included more chert/flint $(n=10)$ than quartz $(n=8)$ and hematite $(n=1)$; small freehand flakes $(n=10)$ were also more numerous than angular shatter $(n=6)$ and cores $(n=2)$. The near absence of these artifacts around hearths in Stratum G.2, and their greater presence in Feature 90 and other midden contexts suggested that refuse from knapping was subject to preferential disposal away from heavily trafficked spaces within the compound.

Table 7.10: Unit G artifact summary

\begin{tabular}{|c|c|c|c|c|c|c|c|}
\hline & $\mathrm{m}^{3}$ & $\begin{array}{c}\text { Ceramic } \\
\mathrm{n}\end{array}$ & $\begin{array}{c}\text { Lithic } \\
n\end{array}$ & $\begin{array}{c}\text { Slag } \\
\mathrm{n}\end{array}$ & Groundstone & Tools & Ornaments \\
\hline G.1a & 2.15 & 4790 & 5 & 0 & \multirow{3}{*}{$\begin{array}{l}1 \text { x Rhyolite } \\
\text { hammerstone }\end{array}$} & \multirow{3}{*}{$\begin{array}{l}1 \times \text { Iron hook } \\
2 \times \text { Bent iron } \\
\text { rod } \\
2 \times \text { Spindle } \\
\text { whorl }\end{array}$} & \multirow{3}{*}{$\begin{array}{c}1 \text { x Carnelian } \\
\text { bead (Cb-2) } \\
1 \text { x Ivory } \\
\text { bracelet } \\
1 \text { x Bone bead }\end{array}$} \\
\hline G.1b & 1.47 & 1041 & 1 & 0 & & & \\
\hline G.1c & 1.83 & 502 & 0 & 0 & & & \\
\hline Ft. 90 & 0.74 & 537 & 7 & 0 & $\begin{array}{l}1 \text { x Handstone } \\
1 \text { x Grinding slab } \\
1 \text { x Polishing } \\
\text { stone } \\
\end{array}$ & $\begin{array}{l}1 \times \text { Spindle } \\
\text { whorl } \\
1 \times \text { Netweight }\end{array}$ & $\begin{array}{l}1 \times \text { Terracotta } \\
\text { figurine frag }\end{array}$ \\
\hline G.1 & 6.19 & 6870 & 13 & 0 & 4 & 7 & 4 \\
\hline G.2a & 1.28 & 316 & 1 & 0 & \multirow{2}{*}{ - } & \multirow{2}{*}{ - } & \multirow{2}{*}{$\begin{array}{l}1 \times \text { Cowrie } \\
1 \times \text { Terracotta } \\
\text { bracelet frag }\end{array}$} \\
\hline G.2b & 1.36 & 388 & 0 & 0 & & & \\
\hline G.2 & 2.64 & 704 & 1 & 0 & - & - & 2 \\
\hline G.3a & 1.52 & 605 & 2 & 0 & \multirow{3}{*}{$\begin{array}{l}1 \times \text { Sharpening } \\
\text { stone }\end{array}$} & \multirow{3}{*}{$\begin{array}{l}1 \text { x Small iron } \\
\text { knife } \\
1 \text { x Copper } \\
\text { wire }\end{array}$} & \multirow{3}{*}{3 x Cowrie } \\
\hline G.3b & 1.20 & 276 & 3 & 0 & & & \\
\hline G.3c & 1.39 & 447 & 3 & 0 & & & \\
\hline G.3 & 4.11 & 1328 & 8 & 0 & 1 & 2 & 3 \\
\hline Total & 12.94 & 8902 & 22 & $\mathbf{0}$ & 5 & 9 & 9 \\
\hline
\end{tabular}


Figure 7.28: Unit G body sherd seriation

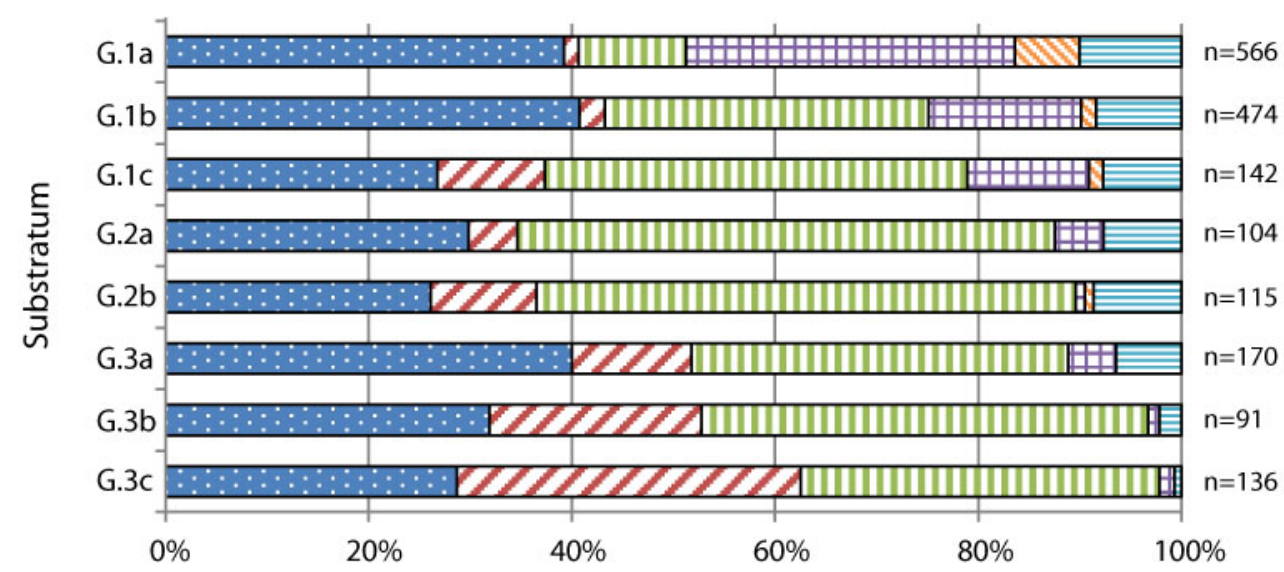

Table 7.11: Unit $G$ vessel type frequencies

\begin{tabular}{|c|c|c|c|c|c|c|c|c|c|c|c|c|c|}
\hline \multirow{3}{*}{\multicolumn{2}{|c|}{$\begin{array}{r}\text { Substratum } \\
\text { Total } \\
n\end{array}$}} & \multirow{2}{*}{\multicolumn{2}{|c|}{$\begin{array}{c}\text { Bowl } \\
\text { All }\end{array}$}} & \multicolumn{4}{|c|}{ Pot } & \multicolumn{6}{|c|}{ Jar } \\
\hline & & & & \multicolumn{2}{|c|}{ Small } & \multicolumn{2}{|c|}{ Large } & \multicolumn{2}{|c|}{ Small } & \multicolumn{2}{|c|}{ Large A } & \multicolumn{2}{|c|}{ Large B } \\
\hline & & $n$ & $\%$ & $n$ & $\%$ & $n$ & $\%$ & $n$ & $\%$ & $n$ & $\%$ & $n$ & $\%$ \\
\hline G.1a & 192 & 1 & $0.5 \%$ & 15 & $7.8 \%$ & 6 & $3.1 \%$ & 10 & $5.2 \%$ & 2 & $1.0 \%$ & 2 & $1.0 \%$ \\
\hline G.1b & 86 & 3 & $3.5 \%$ & 11 & $12.8 \%$ & 7 & $8.1 \%$ & 7 & $8.1 \%$ & 3 & $3.5 \%$ & 0 & $0.0 \%$ \\
\hline G.1c & 30 & 1 & $3.3 \%$ & 2 & $6.7 \%$ & 2 & $6.7 \%$ & 2 & $6.7 \%$ & 1 & $3.3 \%$ & 1 & $3.3 \%$ \\
\hline G.1 & 308 & 5 & $1.6 \%$ & 28 & $9.1 \%$ & 15 & $4.9 \%$ & 19 & $6.2 \%$ & 6 & $1.9 \%$ & 3 & $1.0 \%$ \\
\hline G.2a & 25 & 0 & $0.0 \%$ & 4 & $16.0 \%$ & 2 & $8.0 \%$ & 5 & $20.0 \%$ & 2 & $8.0 \%$ & 0 & $0.0 \%$ \\
\hline G.2b & 23 & 2 & $8.7 \%$ & 2 & $8.7 \%$ & 0 & $0.0 \%$ & 2 & $8.7 \%$ & 1 & $4.3 \%$ & 1 & $4.3 \%$ \\
\hline G.2 & 48 & 2 & $4.2 \%$ & 6 & $12.5 \%$ & 2 & $4.2 \%$ & 7 & $14.6 \%$ & 3 & $6.3 \%$ & 1 & $2.1 \%$ \\
\hline G.3a & 42 & 0 & $0.0 \%$ & 6 & $14.3 \%$ & 3 & $7.1 \%$ & 3 & $7.1 \%$ & 3 & $7.1 \%$ & 1 & $2.4 \%$ \\
\hline G.3b & 17 & 0 & $0.0 \%$ & 4 & $23.5 \%$ & 3 & $17.6 \%$ & 3 & $17.6 \%$ & 1 & $5.9 \%$ & 0 & $0.0 \%$ \\
\hline G.3c & 17 & 0 & $0.0 \%$ & 5 & $29.4 \%$ & 3 & $17.6 \%$ & 0 & $0.0 \%$ & 0 & $0.0 \%$ & 0 & $0.0 \%$ \\
\hline G.3 & 76 & 0 & $0.0 \%$ & 15 & $22.4 \%$ & 9 & $13.4 \%$ & 6 & $9.0 \%$ & 4 & $6.0 \%$ & 1 & $1.5 \%$ \\
\hline Total & 432 & 7 & $1.6 \%$ & 49 & $11.3 \%$ & 26 & $6.0 \%$ & 32 & $7.4 \%$ & 13 & $3.0 \%$ & 5 & $1.2 \%$ \\
\hline
\end{tabular}


The occurrence of iron implements throughout the sequence showed that people in Area $\mathrm{G}$ had access to metal objects, even as the absence of slag suggested that smithing activities took place elsewhere. On the other hand, three terracotta spindle whorls from Stratum G.1 indicated that the residents of Area G were directly engaged in spinning and perhaps weaving.

The earthenware pottery assemblage in Unit G demonstrated a clear seriation of decorative attributes (Figure 7.28) useful for fitting the associated stratigraphic units into a relative chronology. As illustrated in Table 7.11, the proportions of pottery rim types remained relatively constant throughout the sequence where large pots and jars, though outnumbered by their smaller counterparts, highlighted diverse cooking, storage, transportation, and other activities in the vicinity. Large bowls were entirely absent from the ceramic assemblage, while the low numbers of small bowls in the upper two strata pointed to a shift in consumption practices from the earliest occupation of Area G.

Practices of personal adornment and exchange were remarkably well-represented in the artifact assemblage from Unit G (Table 7.10). Three cowrie shells and a piece of copper wire from the lowermost stratum highlighted participation in supra-local exchange networks right from the earliest occupation in Area G. The consumption of these and similar items, including terracotta, bone, and carnelian ornaments, continued throughout the occupation. In this regard, it is noteworthy that people apparently discarded (or lost) these objects in domestic contexts; they were not confined to a discrete ritual space within the village.

\section{Residential Area H (Unit H)}

I positioned Unit $\mathrm{H}(2 \times 2 \mathrm{~m})$, the second unit opened during the 2008 field season, near two well-preserved granary foundations marking the interior of a household compound on the apex of Area H. The unit surface, sloping slightly down to the south, was a friable silty clay loam littered with laterite gravel, a few small sandstone fragments, and heavily crushed sherds indicative of deflation and trampling by livestock.

I excavated this unit to sample the stratigraphic sequence from the southernmost mound at Diouboye to obtain temporal and material data comparable with those from Unit A1. Surprisingly, Unit H reached a maximum depth of 2.95 meters below ground 


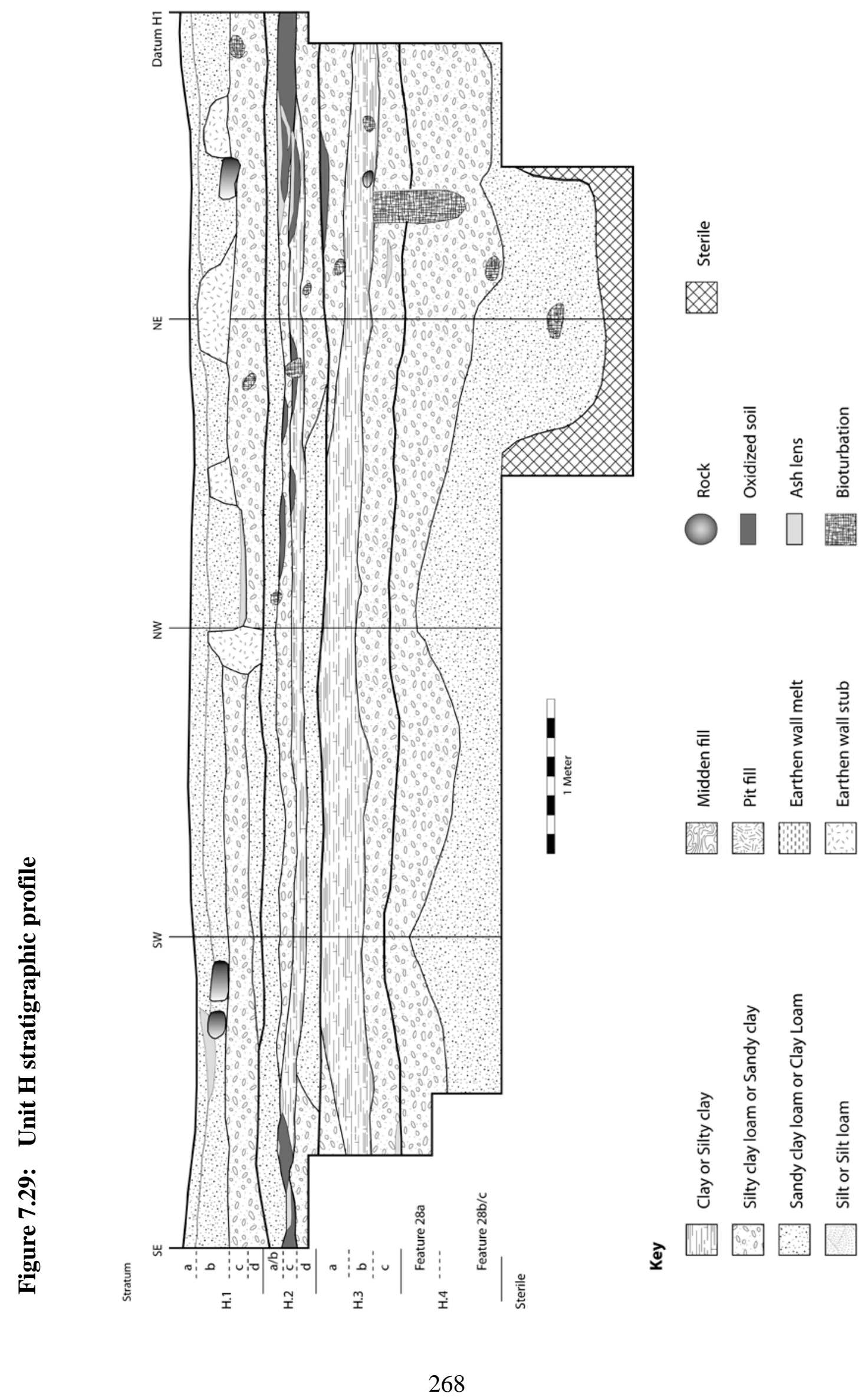


surface due to the large borrow pit (Feature 28), the fill of which constituted the lowermost stratum. Above this deposit, two additional strata recorded successive episodes of architecture and residential activities. Subsuming 12 substrata altogether (Figure 7.29), the archaeological sequence in Unit $\mathrm{H}$ spanned all three of the occupation phases defined for Diouboye.

\section{Stratum H.4 (Feature 28)}

The archaic levee underlying Diouboye appeared as a yellowish-red silty clay void of cultural material and visible in profile at a depth of 2.15 meters below surface. The uneven topography of this deposit evidently resulted from the excavation of a broad pit, the shape of which could not be fully ascertained within the confines of Unit $\mathrm{H}$. The fill within this depression (Feature 28), which reached a depth of 2.95 meters below surface in the northeast corner of the unit, amounted to the entirety of Substratum H.4, a sandy to silty clay loam filled with ash, charcoal, and substantial quantities of sherds and faunal remains. The lowermost layer, Feature 28c (1.93-2.95 m), was a friable soil matrix with medium to coarse charcoal mottles and diffuse boundaries with the surrounding alluvium; this deposit could have resulted from bioturbation of the rich fill from overlying layers. Feature 28b (1.72-2.00 m) encompassed a dense cluster of sherds from at least five nearly complete pottery vessels (Figure 7.26). Feature 28a (1.32-2.04 m), an upper layer of relatively firm fill, exhibited light bands of laterite gravel and contained less charcoal than the lower layers.

\section{Stratum H.3}

With discrete ash dump episodes on its upper surface, Substratum H.3c (1.20-1.45 m) was an extremely firm horizon of silty clay loam marking the transition from pit fill below to the semi-formal use of space above. Although the subsequent Substratum H.3b (1.03-1.21 m) and Substratum H.3a (0.88-1.05 m) lacked architectural elements, their light brown color, firm texture, and modest inclusions of charcoal, daub, sherds, and faunal remains (and even the fragments of a small iron projectile point) nevertheless pointed to deposition through residential activities such as preparing and consuming food and building and maintaining earthen wall structures in the immediate vicinity. 
Figure 7.30: Feature 28b (Stratum H.4)
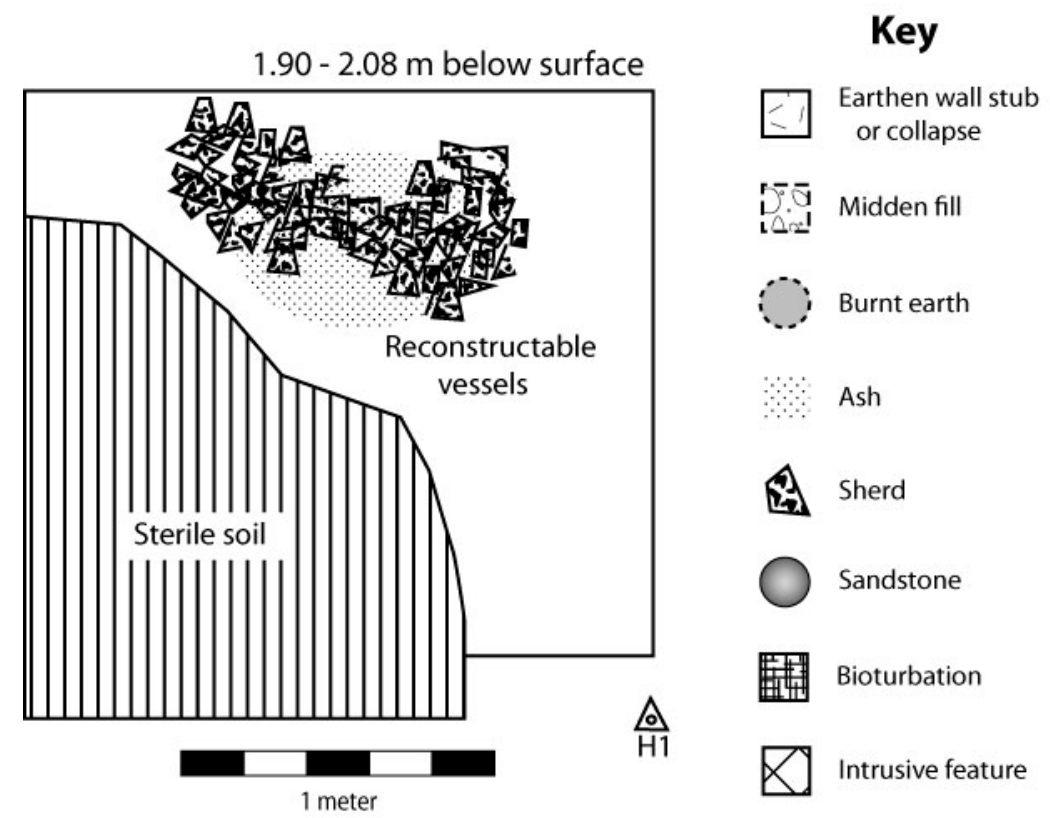

\section{Stratum H.2}

Surface H.2d (Figure 7.30), the earliest clear occupation surface in Unit $\mathrm{H}$, marked a sharp transition to a semi-formal space apparently situated within the confines of a household compound. In the eastern half of the unit, Feature J outlined a broad, informal hearth area of heavily oxidized sediment. Above this feature, Substratum H.2d.i (0.74-0.81 m) was a deposit of extremely firm light brown silty clay with modest sherd densities resembling general fill, but with oxidation resulting from repeated cooking activity. Elsewhere across the western half of the unit, Substratum H.2d.ii (0.81-0.95 m) lacked evidence for oxidation, but modest frequencies of sherds, faunal remains, two handstones, and daub suggest that this deposit accumulated in the course of general household activities and nearby erosion or collapse of earthen wall structures.

The next three occupation surfaces maintained this same spatial plan. Feature $\mathrm{H}$, an informal hearth complex with two to three layers of oxidized sediment interlaced with lenses of ash, covered the eastern half of Surface H.2c. Meanwhile Substratum H.2c (0.70-0.79 m) developed as a firm layer of silty clay with debris from domestic activities and two fragments of a large iron knife. Feature $G$ formed yet another informal hearth 
Figure 7.31: Surface H.2d (left) and H.2b (right)

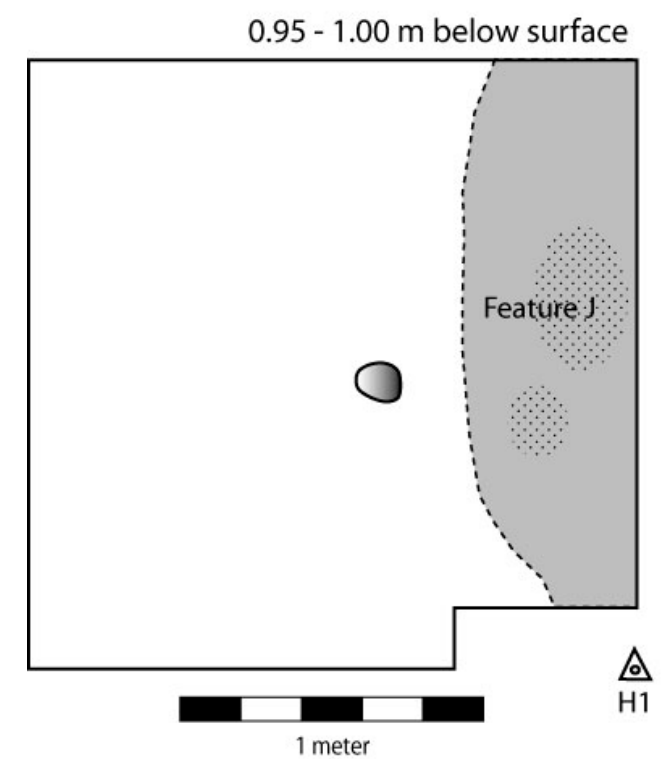

Earthen wall stub or collapse Midden fill
Burnt earth Ash

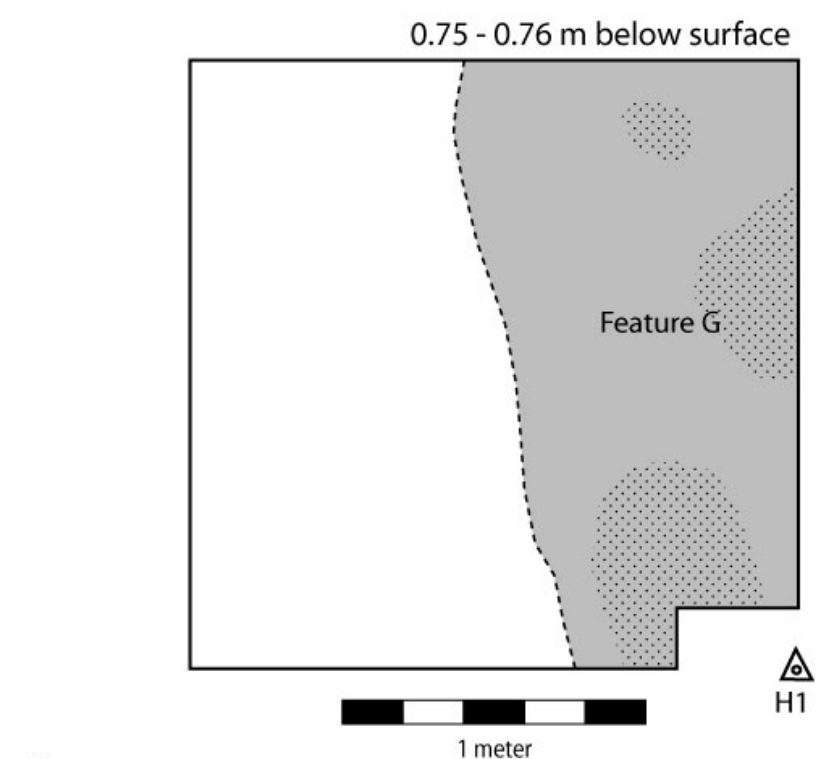

Key

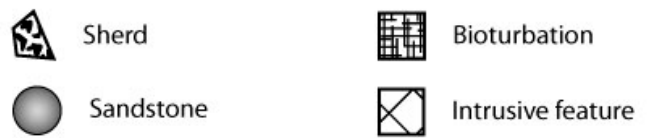

complex on the well-defined Surface H.2b (Figure 7.31), itself capped with Substratum H.2b (0.63-0.76 m), a deposit of sandy clay loam with ceramic sherds, faunal remains, and the corroded remnants of a large iron projectile point. Finally, the Feature F hearth complex on Surface H.2a represented the last phase of this layout; Substratum H.2a (0.49-0.67 m) was a sandy clay loam with modest frequencies of daub and charcoal and other artifacts, documenting food preparation and consumption activities alongside earthen wall repair or demolition in preparation for subsequent structures.

\section{Stratum H.1}

Substratum H.1d (0.42-0.56 m) was a friable clay loam with modest daub and artifact densities (including a corroded iron point or knife blade) representing a continuation of the underlying Substratum H.2a possibly associated with the construction of Feature 22, a circular terre pisé wall stub, in the northwestern corner of the unit. The interior of this structure did not exhibit any replastering episodes; the single floor surface 
was covered with a thin lens of ash prior to the subsequent deposition of fill and earthen wall melt/collapse containing a large cigar-shaped netweight.

The surrounding area, Surface H.1c, was an extremely firm occupation floor with no evidence for the semi-formal hearths that had characterized Stratum H.2. Situated just above this surface, Feature 21 was a curvilinear alignment of three unevenly spaced sandstone rocks, possibly placed as wall supports for an unidentified structure, or simply displaced following the demolition of a stone foundation. The former scenario fits well with the Substratum H.1c (0.32-0.42 m) matrix-an extremely firm deposit of sandy clay with coarse daub, charcoal, sherds, and faunal remains indicating a mixture of earthen wall melt and accumulated debris from household activities.

The overlying Substratum H.1b (0.13-0.35 m) and Substratum H.1a (0.00-0.17 m) together formed a friable to moderately firm horizon of silty clay loam that likewise appeared to derive from earthen wall collapse and/or weathering of the underlying matrix. The modest frequencies of cultural materials from these substrata, including the surface context, suggested that deflation was not too extensive in this part of Area H. At the same time, the recovery of a single ceramic prosser-molded bead (Type 103) dating to at least the $19^{\text {th }}$ century $\mathrm{AD}$ revealed some disturbance of the uppermost substrata.

Figure 7.32: Surface H.1c

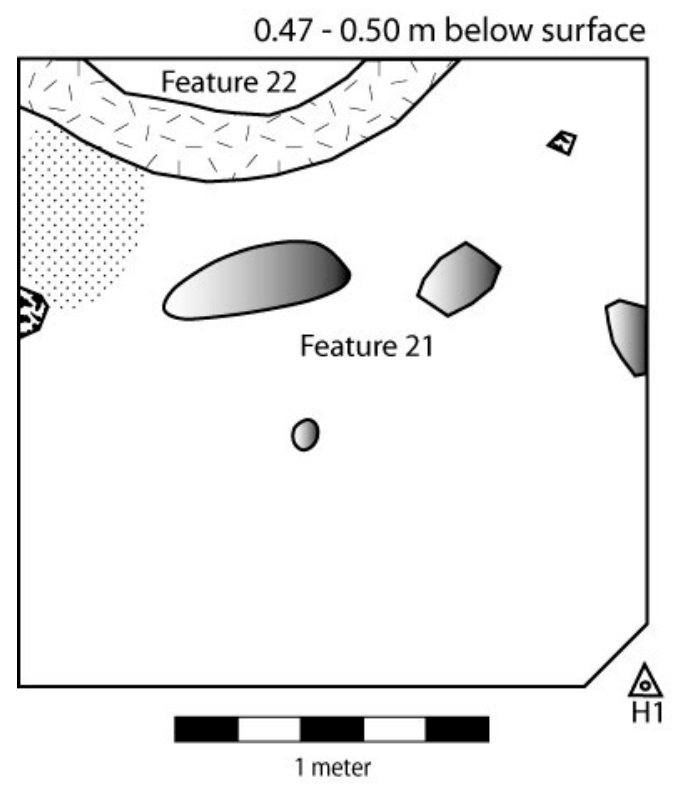

\section{Key}

Earthen wall stub or collapse

Midden fill

Burnt earth

Ash

Sherd

Sandstone

卧 Bioturbation

$\square$ Intrusive feature 


\section{Unit H Summary}

Architecture and Space

The earliest two strata in Unit $\mathrm{H}$ depicted the use of this space as an amorphous borrow pit and/or natural embankment that served as a dump for refuse including charcoal and ash, broken pottery, and faunal remains. During the period encapsulated by Stratum H.2, Area H evolved into a more formal residential space with a sequence of informal hearths layered one atop the other in the eastern half of Unit H. This spatial arrangement came to an end with the construction of a small earthen wall hut (Feature 22) and the emplacement of several angular sandstone cobbles (Feature 21) in Stratum H.1. Based on their depth, these features most likely predated the surface features in Area $\mathrm{H}$, which date to the final phase of occupation in this part of the site.

\section{Artifacts, Organic Remains, and Material Practice}

Diverse contexts, including general fill, features, and pits, yielded a sample of 42 identifiable faunal specimens (Figure 7.33). Cattle and sheep/goat remains were relatively more abundant in Unit $\mathrm{H}$ than in other excavated contexts, but a preponderance of terrestrial mammals, particularly medium-sized game from the savanna, was consistent with meat consumption patterns across the village. Assorted aquatic resources rounded out the remainder of the assemblage.

The 19 flotation samples collected from Unit H currently await analysis although several specimens of unambiguous pearl millet have been identified (Daphne Gallagher, personal communication). Given the two early dates initially obtained for the stratified sequence in this area, two of these millet grains were submitted for direct AMS radiocarbon dating, as described in the next section. 
Figure 7.33: Unit $\mathbf{H}$ faunal summary (from Appendix $H$ )

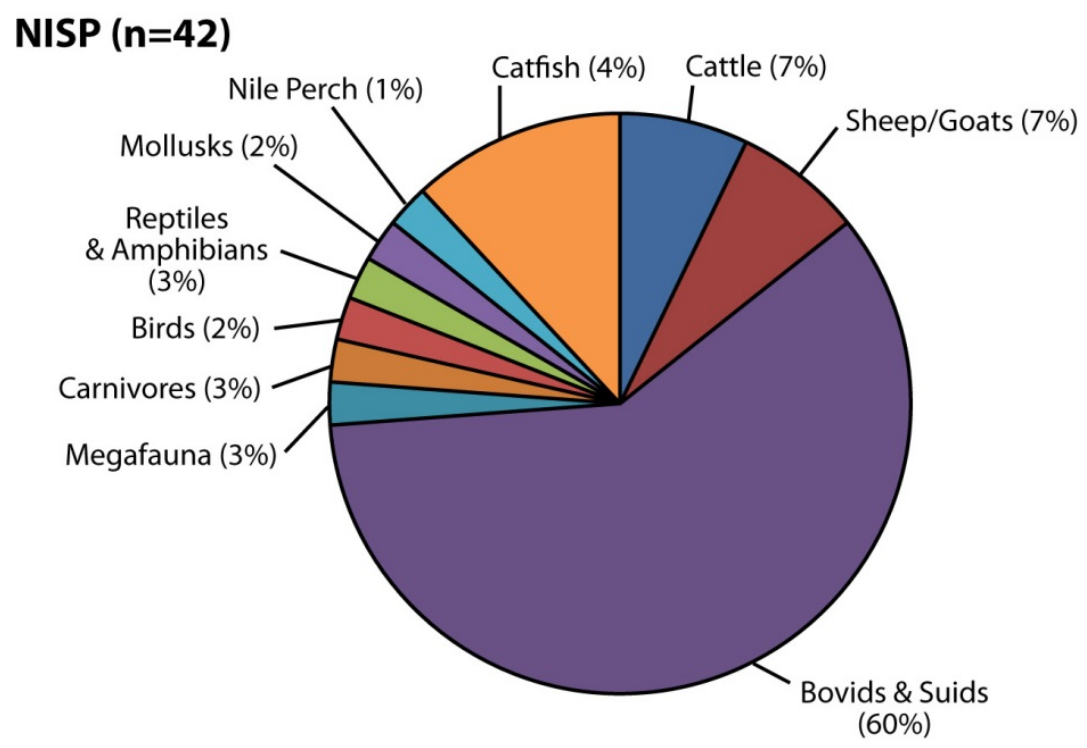

Table 7.12: $\quad$ Unit $H$ artifact summary

\begin{tabular}{|c|c|c|c|c|c|c|c|}
\hline Stratun & $\mathrm{m}^{3}$ & $\begin{array}{c}\text { Ceramic } \\
\mathrm{n}\end{array}$ & $\begin{array}{c}\text { Lithic } \\
n\end{array}$ & $\begin{array}{c}\text { Slag } \\
\mathrm{n}\end{array}$ & Groundstone & Tools & Ornaments \\
\hline H.1a & 0.28 & 180 & 5 & 0 & \multirow{4}{*}{ - } & \multirow{4}{*}{$\begin{array}{l}1 \times \text { Iron frag } \\
1 \times \text { Knife/point } \\
\text { blade frag } \\
1 \times \text { Netweight }\end{array}$} & \multirow{4}{*}{ - } \\
\hline H.1b & 0.82 & 263 & 2 & 0 & & & \\
\hline H.1c & 0.28 & 174 & 2 & 0 & & & \\
\hline H.1d & 0.44 & 129 & 2 & 0 & & & \\
\hline H.1 & 1.82 & 746 & 11 & 0 & - & 3 & - \\
\hline H.2a & 0.49 & 262 & 3 & 0 & \multirow{4}{*}{2 x Handstone } & \multirow{4}{*}{$\begin{array}{l}1 \times \text { Large iron } \\
\text { point } \\
1 \times \text { Large iron } \\
\text { knife }\end{array}$} & \multirow{4}{*}{$\begin{array}{l}2 \times \text { Terracotta } \\
\text { figurine frag }\end{array}$} \\
\hline H.2b & 0.27 & 49 & 3 & 0 & & & \\
\hline H.2c & 0.26 & 121 & 3 & 0 & & & \\
\hline H.2d & 0.51 & 233 & 1 & 0 & & & \\
\hline H.2 & 1.53 & 665 & 10 & 0 & 2 & 2 & 2 \\
\hline H.За & 0.45 & 200 & 7 & 1 & \multirow{3}{*}{ - } & \multirow{3}{*}{$\begin{array}{c}1 \times \text { Small iron } \\
\text { point frag }\end{array}$} & \multirow{3}{*}{ - } \\
\hline H.3b & 0.66 & 239 & 1 & 0 & & & \\
\hline H.3c & 0.69 & 318 & 5 & 0 & & & \\
\hline H.3 & 1.80 & 757 & 13 & 1 & - & 1 & - \\
\hline H.4 & 3.03 & 1934 & 9 & 0 & - & - & $\begin{array}{c}1 \times \text { Terracotta } \\
\text { figurine frag } \\
\end{array}$ \\
\hline Total & 7.56 & 4102 & 43 & 1 & 2 & 6 & 3 \\
\hline
\end{tabular}


Stone and iron artifacts potentially associated with subsistence and other household tasks were found throughout the Unit H sequence. The former included two sandstone handstones and pieces of chert $(n=10)$, quartz $(n=30)$, and hematite $(n=4)$ debitage, which attained their highest frequencies in the pit fill and midden deposits of Strata H.3 and H.4, and in wall collapse of Stratum H.1. The latter included the remnants of at least five separate iron implements. Sampling issues aside, the presence of these artifacts throughout the sequence was at least consistent with the long-term production of a semi-formal space within Unit $\mathrm{H}$ for daily practices of food preparation and consumption practices. A solitary piece of slag notwithstanding, this unit did not yield any evidence for craft production activities in the immediate vicinity.

Figure 7.34: Unit $\mathbf{H}$ body sherd seriation

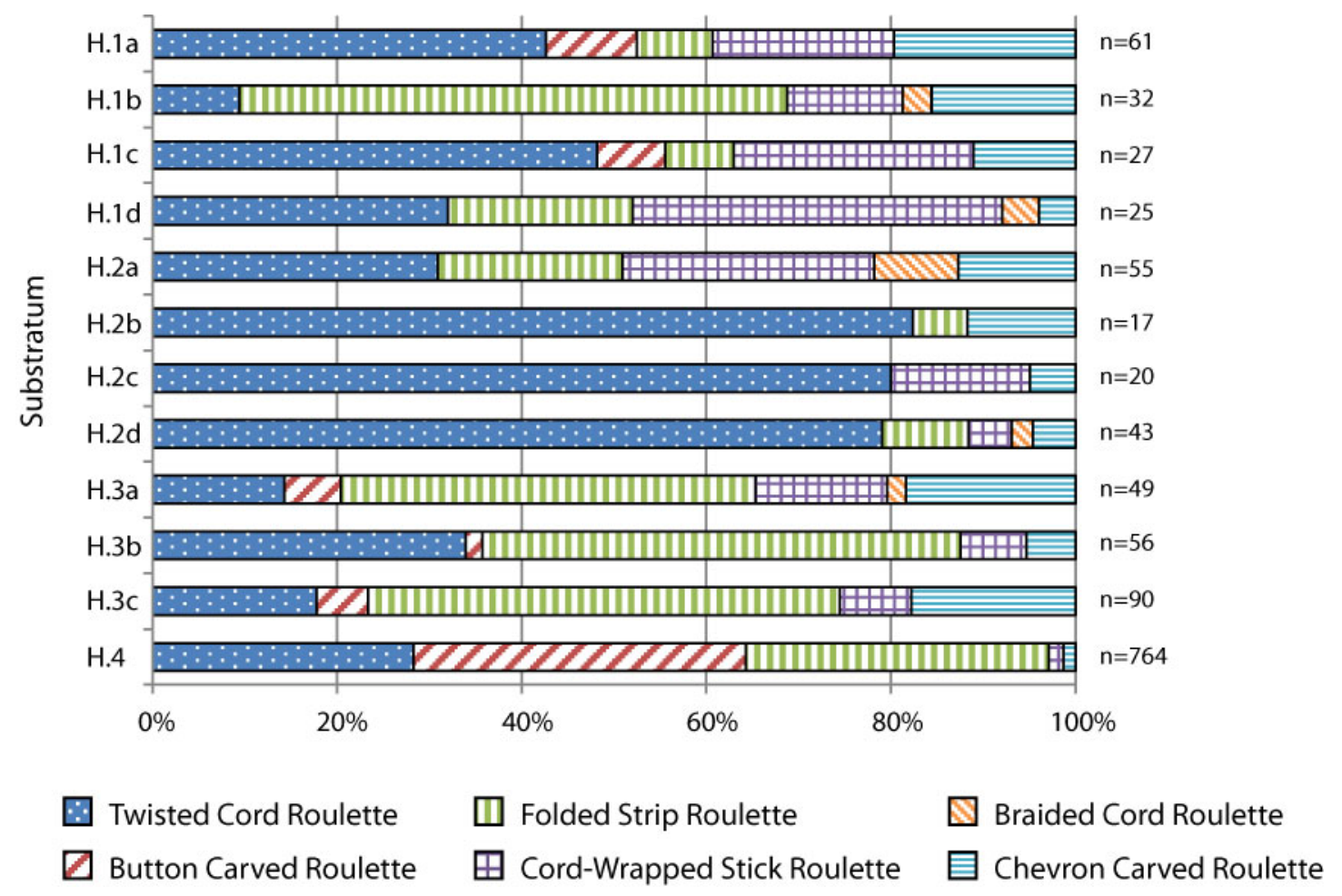


Table 7.13: Unit $H$ vessel type frequencies

\begin{tabular}{|c|c|c|c|c|c|c|c|c|c|c|c|c|c|}
\hline \multirow{3}{*}{\multicolumn{2}{|c|}{$\begin{array}{r}\text { Substratum } \\
\text { Total } \\
n\end{array}$}} & \multirow{2}{*}{\multicolumn{2}{|c|}{$\begin{array}{c}\text { Bowl } \\
\text { All }\end{array}$}} & \multicolumn{4}{|c|}{ Pot } & \multicolumn{6}{|c|}{ Jar } \\
\hline & & & & \multicolumn{2}{|c|}{ Small } & \multicolumn{2}{|c|}{ Large } & \multicolumn{2}{|c|}{ Small } & \multicolumn{2}{|c|}{ Large A } & \multicolumn{2}{|c|}{ Large B } \\
\hline & & $n$ & $\%$ & $n$ & $\%$ & $n$ & $\%$ & $n$ & $\%$ & $n$ & $\%$ & $n$ & $\%$ \\
\hline H.1a & 9 & 0 & $0.0 \%$ & 1 & $11.1 \%$ & 3 & $33.3 \%$ & 1 & $11.1 \%$ & 0 & $0.0 \%$ & 0 & $0.0 \%$ \\
\hline H.1b & 12 & 0 & $0.0 \%$ & 0 & $0.0 \%$ & 0 & $0.0 \%$ & 3 & $25.0 \%$ & 1 & $8.3 \%$ & 1 & $8.3 \%$ \\
\hline H.1c & 8 & 0 & $0.0 \%$ & 3 & $37.5 \%$ & 0 & $0.0 \%$ & 1 & $12.5 \%$ & 1 & $12.5 \%$ & 0 & $0.0 \%$ \\
\hline H.1d & 9 & 2 & $22.2 \%$ & 0 & $0.0 \%$ & 1 & $11.1 \%$ & 1 & $11.1 \%$ & 0 & $0.0 \%$ & 0 & $0.0 \%$ \\
\hline H.1 & 38 & 2 & $5.3 \%$ & 4 & $10.5 \%$ & 4 & $10.5 \%$ & 6 & $15.8 \%$ & 2 & $5.3 \%$ & 1 & $2.6 \%$ \\
\hline H.2a & 12 & 1 & $8.3 \%$ & 2 & $16.7 \%$ & 2 & $16.7 \%$ & 2 & $16.7 \%$ & 1 & $8.3 \%$ & 1 & $8.3 \%$ \\
\hline H.2b & 3 & 0 & $0.0 \%$ & 1 & $33.3 \%$ & 1 & $33.3 \%$ & 1 & $33.3 \%$ & 0 & $0.0 \%$ & 0 & $0.0 \%$ \\
\hline H.2c & 3 & 0 & $0.0 \%$ & 0 & $0.0 \%$ & 0 & $0.0 \%$ & 2 & $66.7 \%$ & 0 & $0.0 \%$ & 0 & $0.0 \%$ \\
\hline H.2d & 17 & 1 & $5.9 \%$ & 2 & $11.8 \%$ & 7 & $41.2 \%$ & 3 & $17.6 \%$ & 0 & $0.0 \%$ & 0 & $0.0 \%$ \\
\hline H.2 & 35 & 2 & $5.7 \%$ & 5 & $14.3 \%$ & 10 & $28.6 \%$ & 8 & $22.9 \%$ & 1 & $2.9 \%$ & 1 & $2.9 \%$ \\
\hline H.За & 9 & 0 & $0.0 \%$ & 0 & $0.0 \%$ & 1 & $11.1 \%$ & 4 & $44.4 \%$ & 0 & $0.0 \%$ & 0 & $0.0 \%$ \\
\hline H.3b & 19 & 0 & $0.0 \%$ & 4 & $21.1 \%$ & 2 & $10.5 \%$ & 7 & $36.8 \%$ & 1 & $5.3 \%$ & 1 & $5.3 \%$ \\
\hline H.3c & 31 & 0 & $0.0 \%$ & 8 & $25.8 \%$ & 7 & $22.6 \%$ & 2 & $6.5 \%$ & 2 & $6.5 \%$ & 0 & $0.0 \%$ \\
\hline H.3 & 59 & 0 & $0.0 \%$ & 12 & $20.3 \%$ & 10 & $16.9 \%$ & 13 & $22.0 \%$ & 3 & $5.1 \%$ & 1 & $1.7 \%$ \\
\hline H.4 & 81 & 4 & $4.9 \%$ & 10 & $12.3 \%$ & 18 & $22.2 \%$ & 4 & $4.9 \%$ & 6 & $7.4 \%$ & 8 & $9.9 \%$ \\
\hline Total & 213 & 8 & $3.8 \%$ & 31 & $14.6 \%$ & 42 & $19.7 \%$ & 31 & $14.6 \%$ & 12 & $5.6 \%$ & 11 & $5.2 \%$ \\
\hline
\end{tabular}

The earthenware pottery assemblage in Unit $\mathrm{H}$ demonstrated a relatively clear seriation of decorative attributes (Figure 7.34) useful for fitting the associated stratigraphic units into a relative chronology; the high number of sherds decorated with folded strip roulette in Substratum H.1b could reflect the redeposition of materials from lower strata incorporated into the earthen wall matrix of Feature 22 or other structures.

The small sample of ceramic rim sherds (Table 7.13) merited a cautious interpretation of vessel use. The predominance of smaller pots and jars throughout the sequence coincided nicely with the use of space within Unit $\mathrm{H}$ for cooking meals, transporting water, and similar activities in the vicinity. On the other hand, the assemblage of large jars in Stratum H.4, several of which were nearly complete, could have derived from the same domestic tool kit for storage and/or large-scale food preparation activities such as beer brewing. 


\section{Residential Areas J/K (Unit K)}

A cluster analysis of surface features after the 2009 field season distinguished Area $\mathrm{K}$ and Area $\mathrm{J}$ in the eastern extent of Diouboye. Although the surface here exhibited very low artifact densities, the presence of several sandstone rocks and installations indicated that Areas $\mathrm{J} / \mathrm{K}$ had hosted household compounds. I opened Unit K (2x2 m) in the space between these two areas, just five meters to the west of a large granary foundation on the surface of Area K.

Beyond further sampling past spatial and material practices in the eastern part of Diouboye, the objective of my excavation in Unit $\mathrm{K}$ was to understand the depositional processes leading to the formation of this off-mound area. Confirming the hypothesis of a relatively brief occupation, Unit K revealed stratified archaeological deposits to a depth of only 0.55 meters below the ground surface (Figure 7.35). The two strata, and five substrata, defined for this sequence documented domestic activities associated with pottery sherds from the earliest two occupation phases at Diouboye.

\section{Stratum K.2}

The archaic levee underlying Diouboye appeared in Unit $\mathrm{K}$ at a minimum depth of 0.46 meters where it formed an extremely firm alluvium of reddish-yellow silty clay. Above this, Substratum K.2c (0.35-0.55 m) was a firm, yellowish-red horizon of sandy clay loam with low densities of pottery sherds, faunal remains, and charcoal flecks accounting for a gradual escalation of cultural activity in this area. In the southern half of the unit, Substratum K.2b.ii (0.30-0.43 m) accumulated as a general fill deposit, while Substratum K.2b.i (0.26-0.38 m) to the north represented a midden-like deposit enriched with pottery sherds, faunal remains, and charcoal and ash from nearby food preparation and other domestic activities.

Beyond marking a stratigraphic boundary with the overlying Substratum K.2a (0.11-0.33 m), a firm sandy clay loam, Surface K.2a (Figure 7.36) was only recognizable for Feature 77, a thick deposit of earthen wall melt in the northwest corner of the unit. Feature 78, a shallow pit also appeared on this surface. 


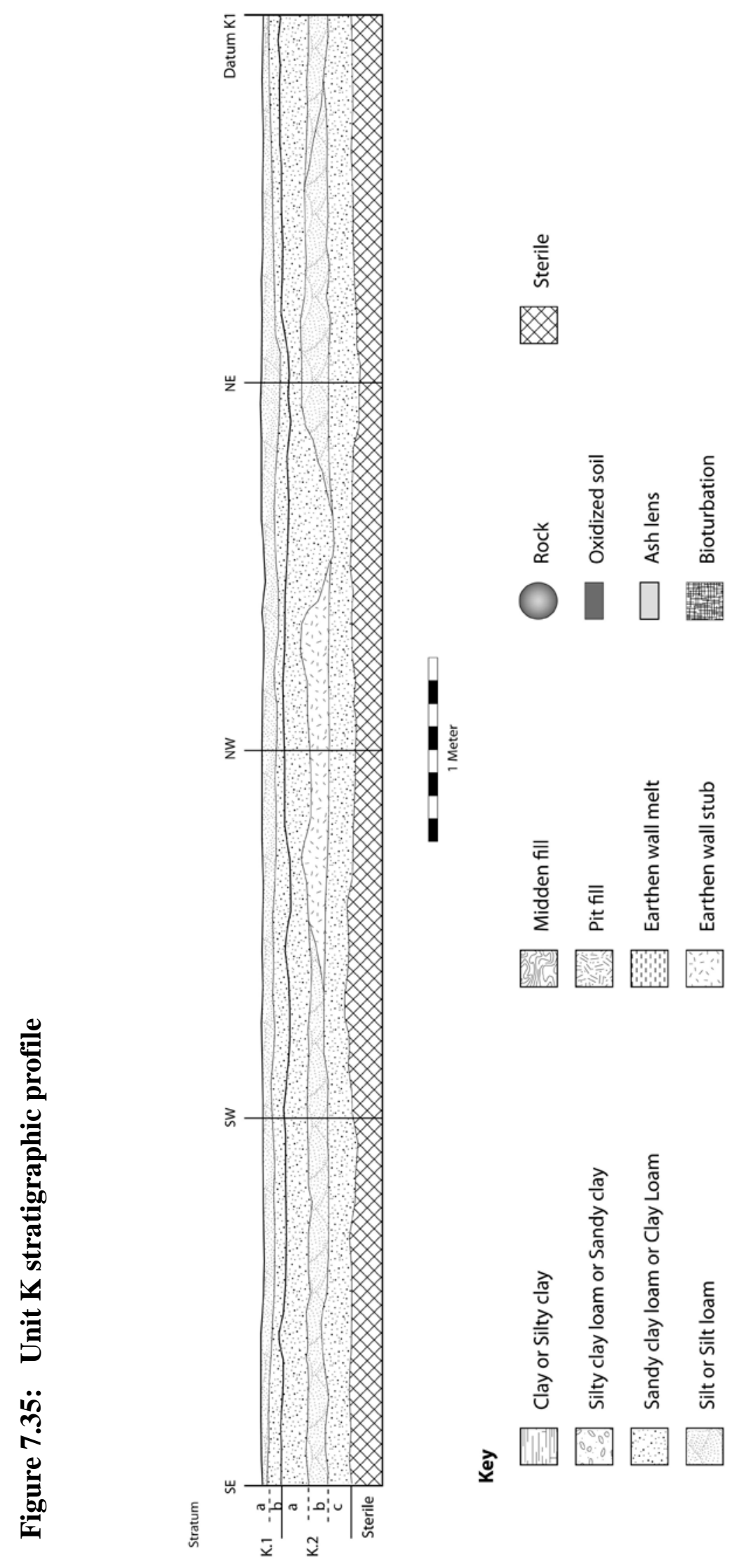


Figure 7.36: Surface K.2a (left) and Surface K.1b (right)

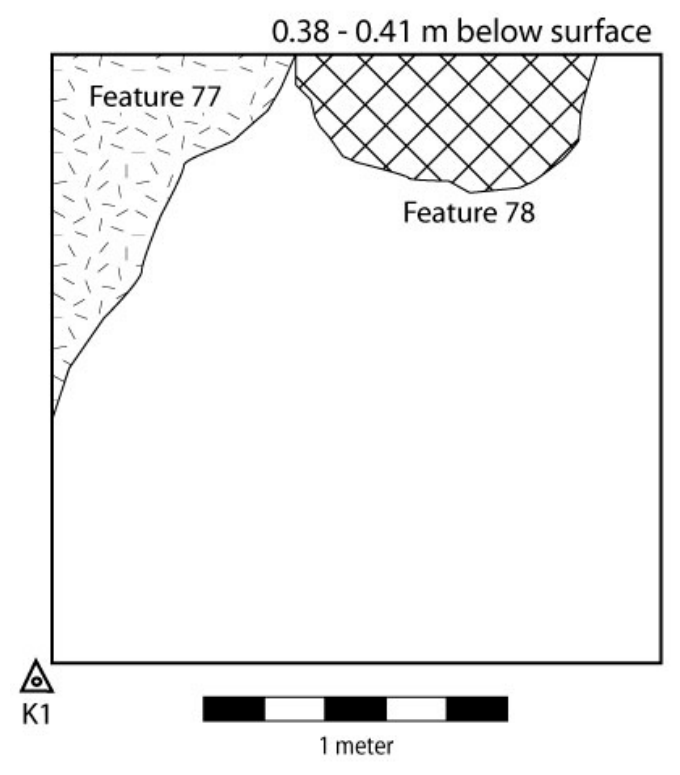

Key

Earthen wall stub or collapse S.ju Midden fill

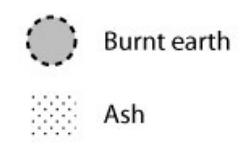

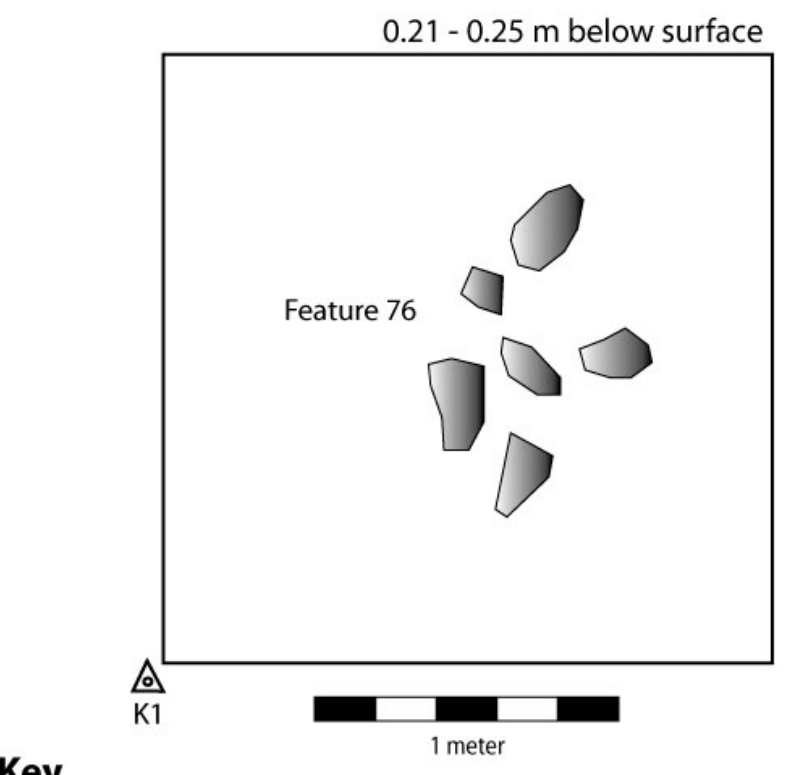

Sherd

Sandstone
Bioturbation

Intrusive feature

\section{Stratum K.1}

Marking the presence of an otherwise ill-defined Surface K.1b, Feature 76 was a cluster of six sandstone rocks with a diameter of 1.05 meters representing either a disaggregated pot rest or partially dismantled granary foundation. Whatever the case, the sediment associated with this feature did not differ from the surrounding matrix. The overlying Substratum K.1b (0.04-0.17 m) was a firm horizon of sandy clay loam with very modest frequencies of pottery and faunal remains that, combined with the presence of daub, hinted at partial formation through earthen wall melt and/or collapse. Substratum K.1a (0.00-0.11 m) was friable topsoil weathered from these underlying deposits, but with low artifact densities pointing to only limited deflation. 


\section{Unit K Summary}

Architecture and Space

Unit K provided a small window onto spatial and material practices in Areas $\mathrm{J} / \mathrm{K}$, revealing continuity in a relatively informal space lying outside heavily trafficked zones within household compounds or specialized activity areas. While Stratum K.2 contained some cultural debris, the absence of clear occupation surfaces and architecture pointed to redeposition from activities conducted elsewhere. The possible wall melt of Feature 77 and the subsequent placement of several sandstone rocks (Feature 76) at the transition to Stratum K.1 demonstrated a subtle shift towards increasing formality just prior to the abandonment of this area.

\section{Artifacts, Organic Remains, and Material Practice}

Given the relative paucity of artifacts in this unit, the faunal assemblage yielded only three identifiable specimens including one element from each of the classes of very small duiker, small bovid, and very large bovid. These were consistent with the abundance of terrestrial mammals observed in other excavated units.

The eight flotation samples collected from Unit K currently await analysis although the shallow depth of this unit suggests that disturbance from roots and insects may have biased the results. Nevertheless, a carbonized baobab shell submitted for direct AMS radiocarbon dating returned an age consistent with occupation of the site during the early-second millennium AD (see following section).

With the exception of six pieces of quartz debitage from the uppermost substratum, the small artifact samples from Unit K yielded no evidence for more specialized subsistence or craft production activities. In the rim sherd assemblage (Table 7.15) the frequencies of large pots and jars were greater than those of their smaller counterparts throughout the sequence, but the small sample size means this pattern may not adequately represent activities at Areas $\mathrm{J} / \mathrm{K}$ as a whole.

Fortunately, the pottery assemblage demonstrated a clear seriation (Figure 7.37) sufficient for fitting the associated strata into the site-level ceramic chronology developed at the end of this chapter. 
Table 7.14: Unit K artifact summary

\begin{tabular}{|c|c|c|c|c|c|c|c|}
\hline & $\mathrm{m}^{3}$ & $\begin{array}{c}\text { Ceramic } \\
\mathrm{n}\end{array}$ & $\begin{array}{c}\text { Lithic } \\
\mathrm{n}\end{array}$ & $\begin{array}{c}\text { Slag } \\
\mathrm{n}\end{array}$ & Groundstone & Tools & Ornaments \\
\hline K.1a & 0.30 & 124 & 6 & 0 & \multirow{2}{*}{ - } & \multirow{2}{*}{ - } & \multirow{2}{*}{ - } \\
\hline K.1b & 0.24 & 39 & 0 & 0 & & & \\
\hline K.1 & 0.54 & 163 & 6 & 0 & - & - & - \\
\hline K.2a & 0.68 & 112 & 0 & 0 & \multirow{3}{*}{-} & \multirow{3}{*}{ - } & \multirow{3}{*}{ - } \\
\hline $\mathrm{K} .2 \mathrm{~b}$ & 0.59 & 150 & 0 & 0 & & & \\
\hline K.2c & 0.30 & 118 & 0 & 0 & & & \\
\hline K.2 & 1.57 & 380 & 0 & 0 & - & - & - \\
\hline Total & 2.11 & 543 & 6 & $\mathbf{0}$ & - & - & - \\
\hline
\end{tabular}

Figure 7.37: Unit K body sherd seriation

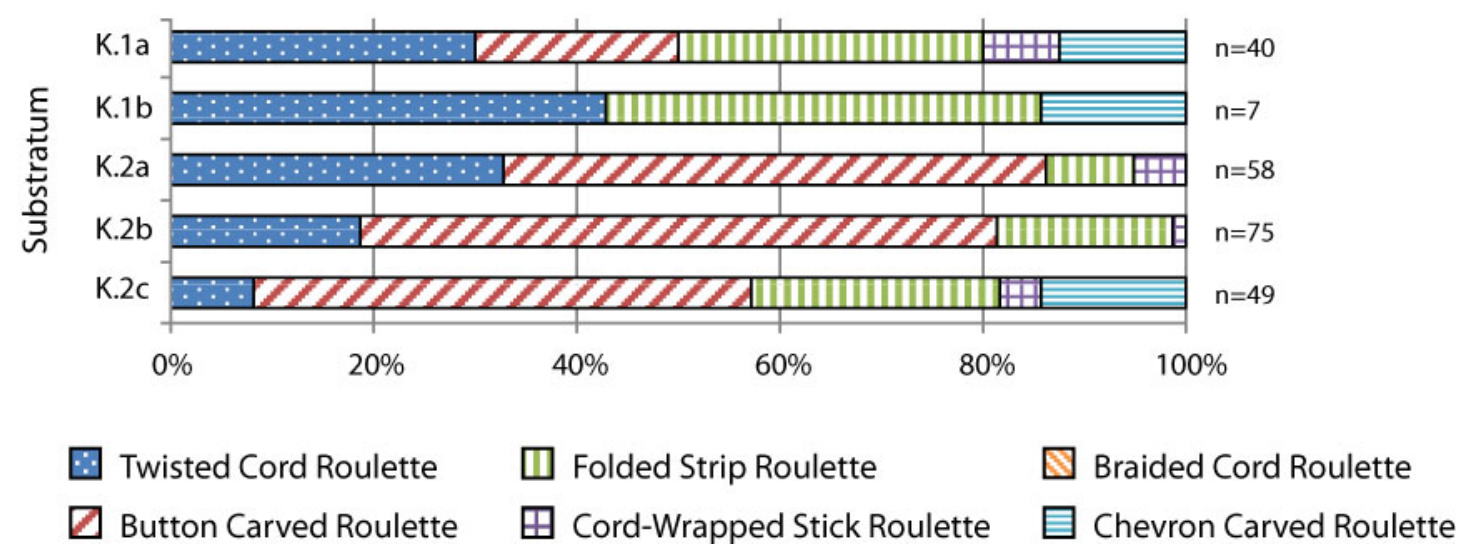


Table 7.15: Unit $\mathrm{K}$ vessel type frequencies

\begin{tabular}{|c|c|c|c|c|c|c|c|c|c|c|c|c|c|}
\hline \multirow{3}{*}{\multicolumn{2}{|c|}{$\begin{array}{r}\text { Substratum } \\
\text { Total } \\
n\end{array}$}} & \multirow{2}{*}{\multicolumn{2}{|c|}{$\begin{array}{c}\text { Bowl } \\
\text { All }\end{array}$}} & \multicolumn{4}{|c|}{ Pot } & \multicolumn{6}{|c|}{ Jar } \\
\hline & & & & \multicolumn{2}{|c|}{ Small } & \multicolumn{2}{|c|}{ Large } & \multicolumn{2}{|c|}{ Small } & \multicolumn{2}{|c|}{ Large A } & \multicolumn{2}{|c|}{ Large B } \\
\hline & & $n$ & $\%$ & $n$ & $\%$ & $n$ & $\%$ & $n$ & $\%$ & $n$ & $\%$ & $n$ & $\%$ \\
\hline K.1a & 5 & 0 & $0.0 \%$ & 1 & $20.0 \%$ & 2 & $40.0 \%$ & 0 & $0.0 \%$ & 0 & $0.0 \%$ & 0 & $0.0 \%$ \\
\hline K.1b & 1 & 0 & $0.0 \%$ & 0 & $0.0 \%$ & 0 & $0.0 \%$ & 0 & $0.0 \%$ & 0 & $0.0 \%$ & 0 & $0.0 \%$ \\
\hline K.1 & 6 & 0 & $0.0 \%$ & 1 & $16.7 \%$ & 2 & $33.3 \%$ & 0 & $0.0 \%$ & 0 & $0.0 \%$ & 0 & $0.0 \%$ \\
\hline K.2a & 13 & 0 & $0.0 \%$ & 1 & $7.7 \%$ & 4 & $30.8 \%$ & 0 & $0.0 \%$ & 3 & $23.1 \%$ & 0 & $0.0 \%$ \\
\hline $\mathrm{K} .2 \mathrm{~b}$ & 5 & 0 & $0.0 \%$ & 0 & $0.0 \%$ & 0 & $0.0 \%$ & 1 & $20.0 \%$ & 0 & $0.0 \%$ & 0 & $0.0 \%$ \\
\hline K.2c & 10 & 1 & $10.0 \%$ & 2 & $20.0 \%$ & 3 & $30.0 \%$ & 1 & $10.0 \%$ & 1 & $10.0 \%$ & 1 & $10.0 \%$ \\
\hline K.2 & 28 & 1 & $3.6 \%$ & 3 & $10.7 \%$ & 7 & $25.0 \%$ & 2 & $7.1 \%$ & 4 & $14.3 \%$ & 1 & $3.6 \%$ \\
\hline Total & 34 & 1 & $2.9 \%$ & 4 & $11.8 \%$ & 9 & $26.5 \%$ & 2 & $5.9 \%$ & 4 & $11.8 \%$ & 1 & $2.9 \%$ \\
\hline
\end{tabular}

\section{Residential Area N (Unit N)}

Based on surface artifacts and features, Area N was unmistakably the result of residential occupation. As a relatively level expanse sloping gently down to the north, it was important to understand the timing and duration of settlement activity at an offmound area of the site that with less evidence for disturbance than Areas $\mathrm{J} / \mathrm{K}$.

In order to address the issue of chronology, I opened Unit N (2x2 m) at the highest point of Area $\mathrm{N}$ along its southern margin. Not only was this location far from the possible disturbance by a modern trail, but low frequencies of laterite gravel and pottery sherds attested to minimal deflation. Extending to a depth of 0.76 meters below surface, Unit $\mathrm{N}$ exposed seven substrata organized into two discrete strata yielding evidence of hearths, stone foundations, and earthen wall structures constituting part of a household compound (Figure 7.38). In fact, I expanded Unit $\mathrm{N}$ by one meter to the west to further expose a moderately well-preserved wattle-and-daub wall and trench (Feature 106). According to the associated ceramic sequence, these deposits accounted for the first two of the three occupation phases defined for Diouboye. 


\section{Figure 7.38: Unit $\mathbf{N}$ stratigraphic profile}
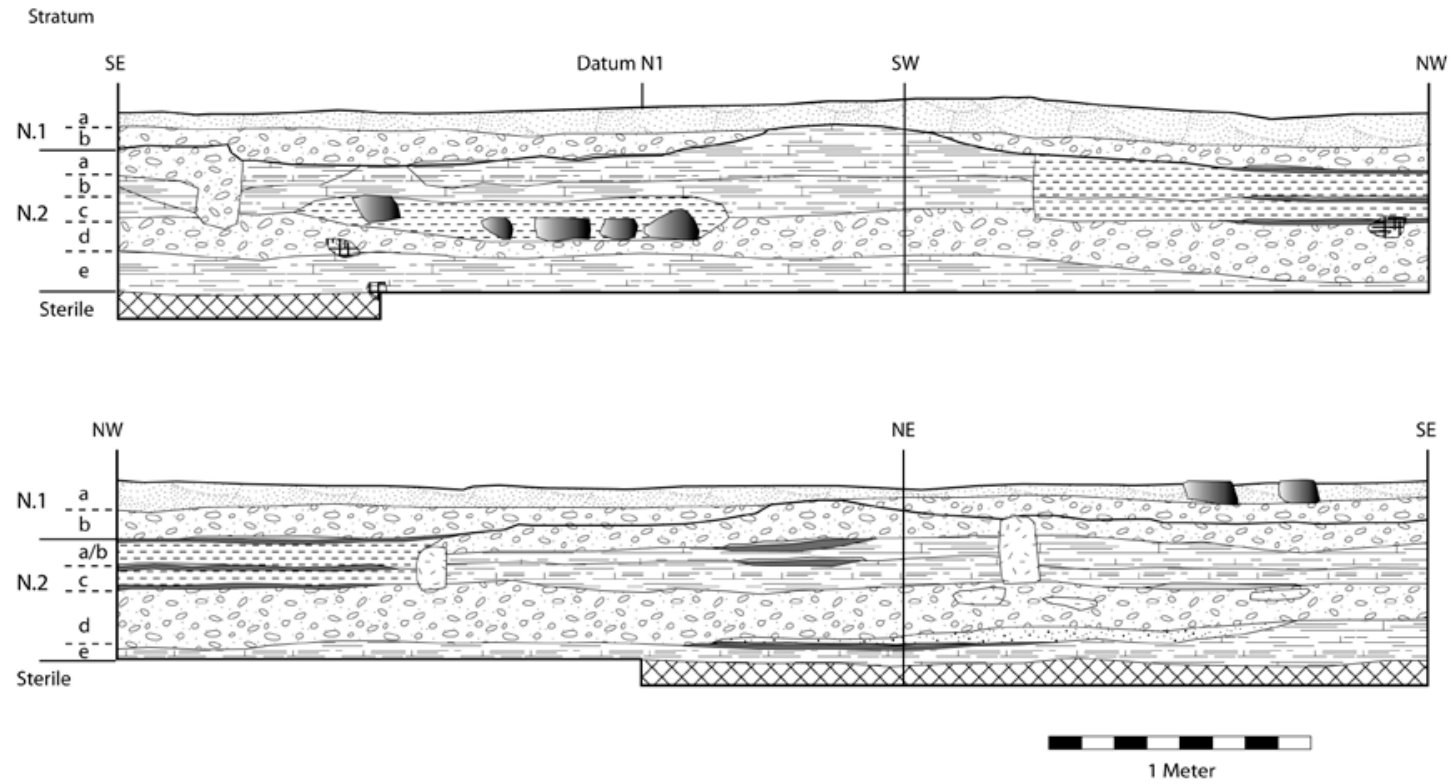

Key

\begin{tabular}{|c|c|c|}
\hline Clay or Silty clay & Midden fill & Rock \\
\hline Silty clay loam or Sandy clay & Pit fill & Oxidized soil \\
\hline Sandy clay loam or Clay Loam & Earthen wall melt & Ash lens \\
\hline Silt or Silt loam & Earthen wall stub & Bioturbation \\
\hline
\end{tabular}

\section{Stratum N.2}

The archaic levee underlying Diouboye initially appeared in Unit $\mathrm{N}$ as an extremely firm, very pale brown clay alluvium some 0.73 meters below surface. The initial activities represented by Substratum N.2e (0.62-0.76 m), a reddish-yellow silty clay horizon, produced low frequencies of pottery sherds, faunal remains, and daub consistent with general domestic tasks. Surface N.2d (Figure 7.39), an occupation floor defined by horizontal ceramic sherds, marked a rapid shift towards formalization of this space with Feature 113, an informal hearth complex in the northeastern corner of the unit. Although no further features were present on this surface, the accumulation of Substratum N.2d (0.43-0.69 m), a silty clay loam with inclusions of laterite gravel and 
Figure 7.39: Surface N.2d

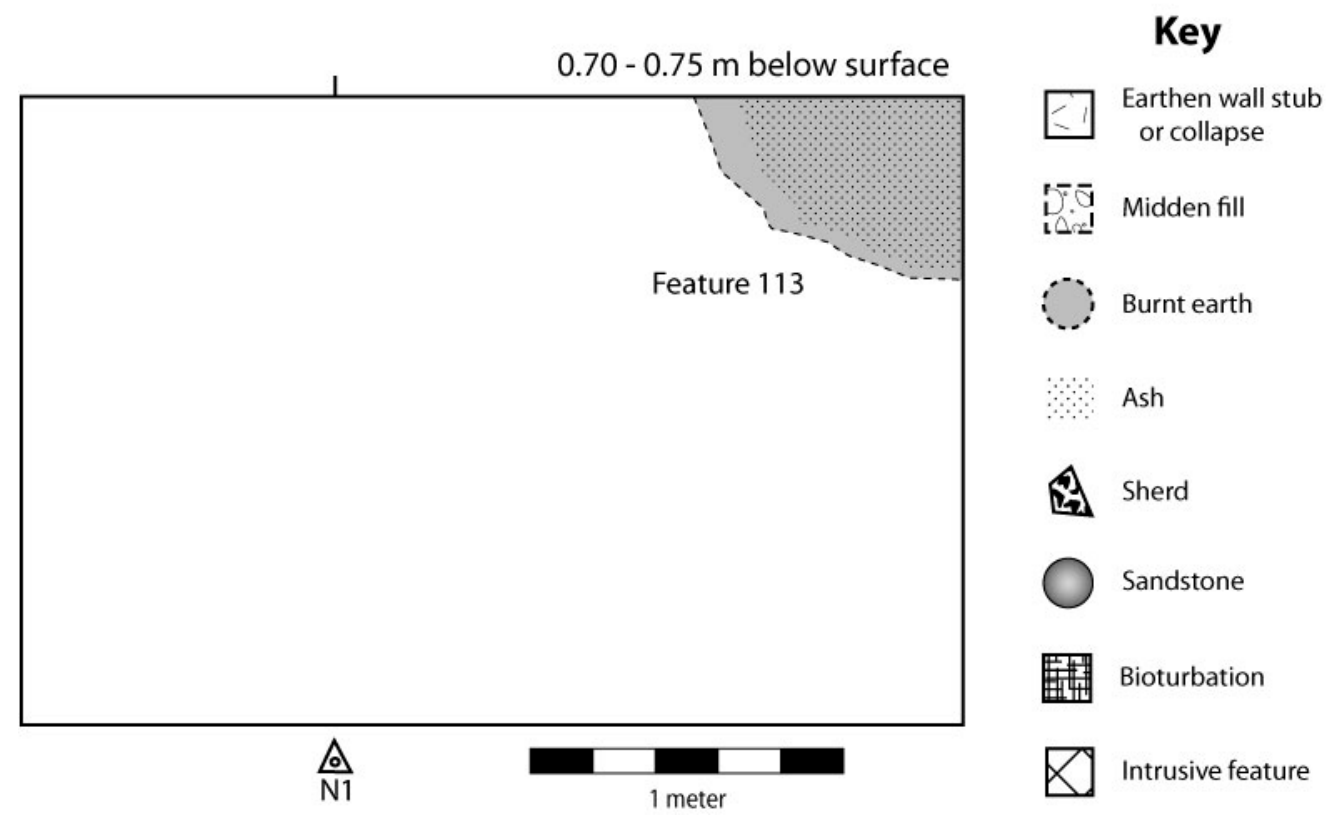

medium daub, was probably due, in part, to episodes of earthen wall construction, melt, and demolition. Marking the emergence of household space structured by architecture, Feature 112 appeared in the upper half of this substratum as a poorly preserved earthen wall stub framing an interior floor with light oxidation and ash.

Surface N.2c (Figure 7.40) established the spatial layout that would endure throughout the remainder of Stratum N.2. The southern profile of the unit cut into Feature 102, a small granary foundation with an estimated diameter of 1.20 meters, while the western half of the unit exposed Feature 106, a segment of a domestic hut with wattleand-daub walls and a compact interior floor with evidence for a small hearth (see Figure 6.2). Using the visible arc of wall, the exterior diameter of this structure was estimated at 2.26 meters. Substratum N.2c (0.32-0.47 m) was an extremely firm deposit of silty clay that formed gradually outside these features and with cultural debris including moderate frequencies of faunal remains, ceramic sherds, and daub inclusions, presumably from episodic wall repair and/or collapse. At least once during the period encapsulated by this substratum, Feature 106 received a new interior surface visible as a compact layer of fill solidified with light oxidation, presumably resulting from a small hearth. 
Figure 7.40: Surfaces N.2b/c
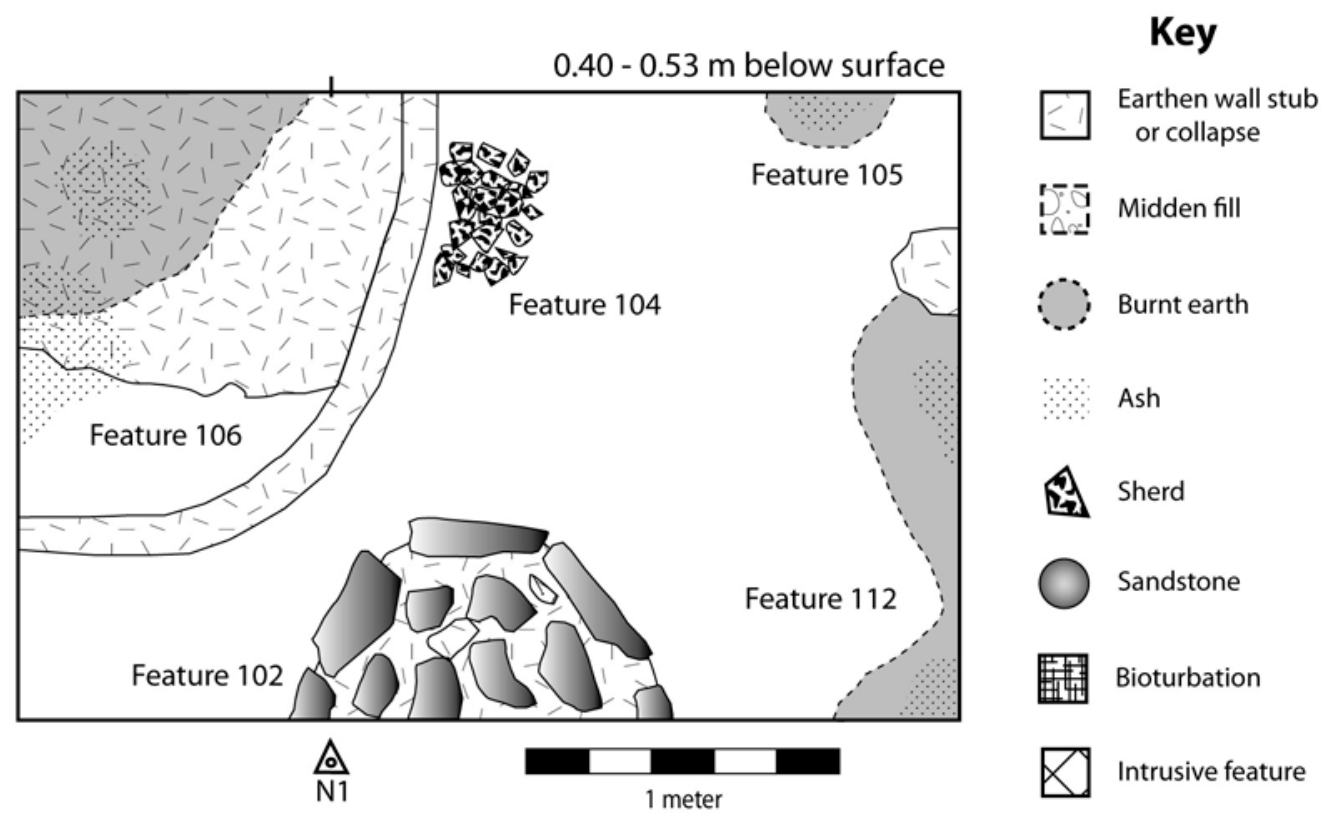

Sometime during the occupation represented by Surface N.2b (Figure 7.40), the earthen wall superstructure of Feature 102 collapsed to form a substantial deposit of overlying wall melt/collapse (see Figure 6.3). At the same time, the oxidized sediment and ash lens of Feature 105 marked an informal hearth in the northeastern corner of the unit, while Feature 104 comprised a small jar evidently broken and left in situ. The overlying Substratum N.2b (0.28-0.38 m), an extremely firm deposit of silty clay dense with daub inclusions indicated that this space experienced the rapid demolition and leveling of earthen wall structures, with the effect of preserving Features 102 and 104. Remarkably, the use of Feature 106 appeared to continue into the subsequent substrata with another resurfacing episode coincident with the establishment of an exterior hearth (Feature 103). Finally, Substratum N.2a (0.27-0.33 m), a sandy to silty clay with modest daub, charcoal, pottery sherds, and faunal remains appeared to constitute the product of general domestic activities including meal preparation and consumption and earthen wall repair. Both this matrix and the fill from Feature 106 contained a single cowrie shell. 


\section{Stratum N.1}

Stratum N.1 involved a restructuring of space through the establishment of several features dating to the terminal phase of occupation in Area N. Feature 109, a horizon of burnt sediment and ash occupying the spatial footprint of Feature 106, appeared to represent the demolition of this structure. Substratum N.1b.ii (0.15-0.29 m) and Substratum N.1b.i (0.11-0.22 m) corresponded to general fill with limited pottery sherds, faunal remains, and charcoal deriving form household activities in the vicinity.

Despite its shallow depth, Surface N.1a (Figure 7.41) defined a clear occupation floor. Associated features included a small hearth (Feature 101) and a partially dismantled circular stone foundation (Feature 100), tentatively classified as a small granary, with a diameter of 0.92 meters. The overlying Substratum N.1a (0.00-0.19 m) was a loose to friable silt topsoil with very modest artifact frequencies suggesting that post-depositional processes of deflation were relatively limited in Area N.

Figure 7.41: Surface N.1a

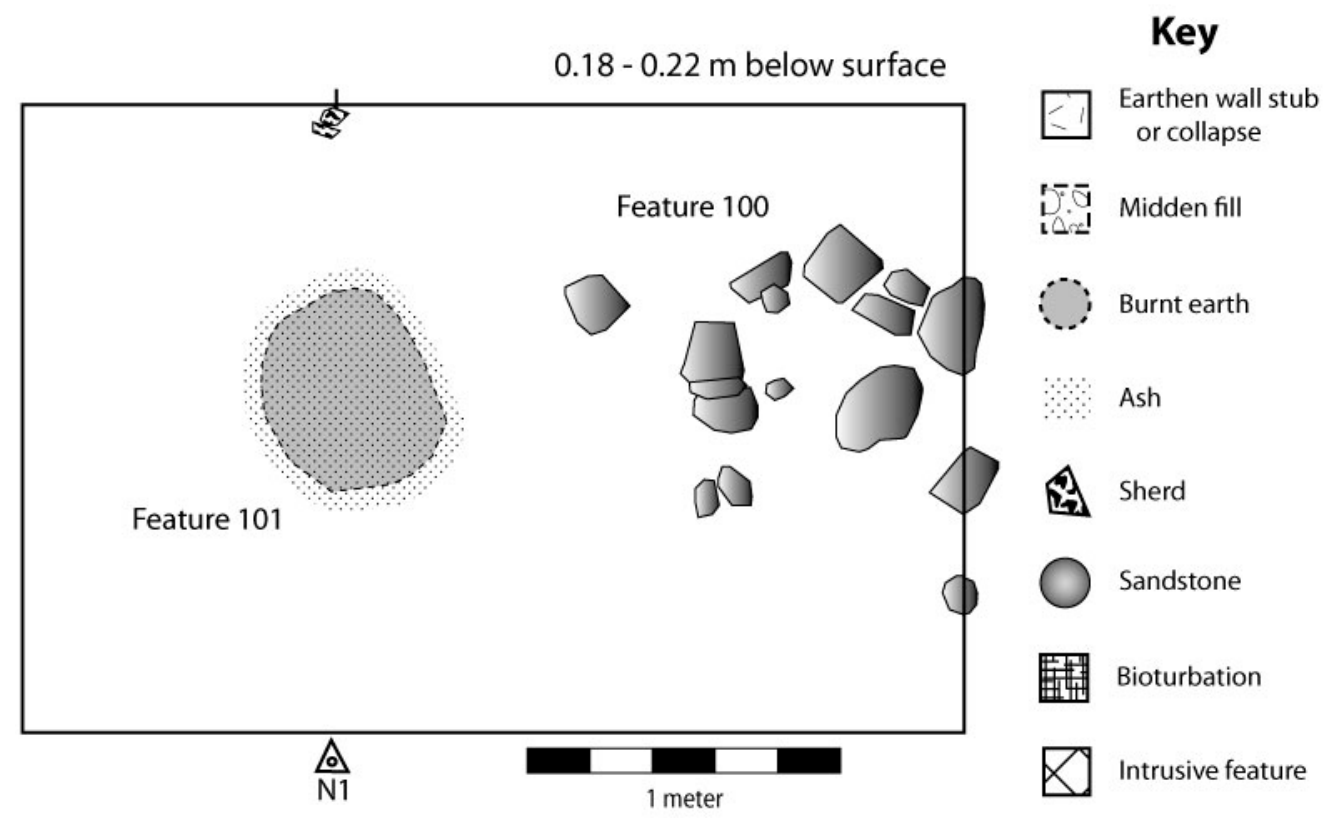




\section{Unit N Summary}

Architecture and Space

The stratigraphic sequence uncovered in Unit $\mathrm{N}$ confirmed that the non-mounded surface of Area $\mathrm{N}$ represented a shorter duration of residence in comparison with mounded areas at the site. Stratum N.2, which accounted for most of the occupation, represented the establishment of a household compound with a wattle-and-daub hut (Feature 106), a series of hearths (Features 105, 112, and 113), and even a medium-sized granary (Feature 102). Several episodes of earthen wall and floor rejuvenation alongside the accumulation of sediment and cultural debris emphasized how people maintained the relative formality of this space. Although the demolition of these structures and subsequent construction of a possible granary foundation (Feature 100) did mark a transition in Stratum N.1, the space within Unit N continued to function as part of a household compound evidenced by nearby surface features.

\section{Artifacts, Organic Remains, and Material Practice}

The reuse and cleaning of domestic space within Unit $\mathrm{N}$ resulted in a small sample of artifacts. The faunal assemblage yielded only five identifiable specimens including one element of cattle, one of sheep/goat, and three from the medium antelope class. These data were consistent with the modest consumption of domesticates and relative to wild terrestrial mammals observed in other excavated units.

The ten flotation samples collected from general fill and features in this unit currently await analysis; the extent of disturbance from roots and insects is unknown.

Decorative attributes in the pottery assemblage from Unit $\mathrm{N}$ seriated nicely across the depositional sequence (Figure 7.42), a fact which would prove useful for orienting stratigraphic contexts within a site-level chronology. Despite their low frequencies in each substratum, the distributions of ceramic rim sherds differed substantially between the upper and lower strata (Table 7.17). Bowls were absent from Stratum N.2 where smaller jars and pots were found alongside several hearths indicating that people were preparing and consuming meals in the vicinity of Unit N. Stratum N.1, on the other hand, witnessed a substantial increase in the number of larger pots and globular jars (type Large B), perhaps representing a shift toward storage and/or transportation activities in the area. 
Table 7.16: Unit $\mathbf{N}$ artifact summary

\begin{tabular}{|c|c|c|c|c|c|c|c|}
\hline & & $\begin{array}{c}\text { Ceramic } \\
\mathrm{n}\end{array}$ & $\begin{array}{c}\text { Lithic } \\
n\end{array}$ & $\begin{array}{c}\text { Slag } \\
\mathrm{n}\end{array}$ & Groundstone & Tools & Ornaments \\
\hline N.1a & 0.79 & 342 & 0 & 0 & \multirow{2}{*}{$\begin{array}{l}1 \text { x Unidentified } \\
\text { fragment }\end{array}$} & \multirow{2}{*}{ - } & \multirow{2}{*}{$\begin{array}{l}1 \times \text { Unidentified } \\
\text { terracotta frag }\end{array}$} \\
\hline N.1b & 0.72 & 205 & 4 & 0 & & & \\
\hline$N .1$ & 1.51 & 547 & 4 & 0 & 1 & - & 1 \\
\hline N.2a & 0.31 & 83 & 0 & 0 & \multirow{5}{*}{ - } & \multirow{5}{*}{ - } & \multirow{5}{*}{2 x Cowrie } \\
\hline N.2b & 0.28 & 70 & 0 & 0 & & & \\
\hline N.2c & 0.35 & 103 & 0 & 0 & & & \\
\hline $\mathrm{N} .2 \mathrm{~d}$ & 1.11 & 119 & 0 & 0 & & & \\
\hline N.2e & 0.42 & 74 & 2 & 0 & & & \\
\hline$N .2$ & 2.47 & 449 & 2 & 0 & - & - & 2 \\
\hline Total & 3.98 & 996 & 6 & $\mathbf{0}$ & 1 & - & 3 \\
\hline
\end{tabular}

Figure 7.42: Unit $\mathbf{N}$ body sherd seriation

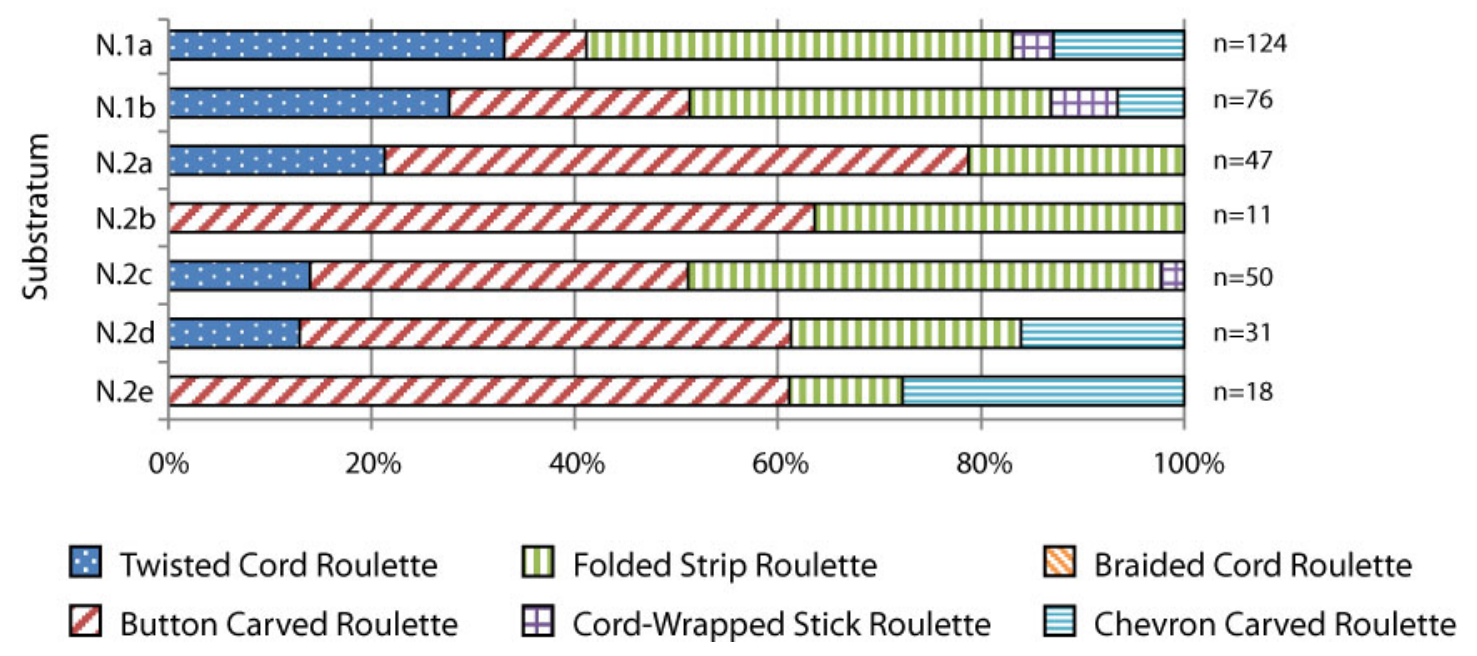

Beyond an informal chert scraper from Substratum N.2e, the artifact assemblage from Unit $\mathrm{N}$ yielded no tools from craft production. The remainder of the chipped stone sample included relatively small quartz $(n=1)$ and chert $(n=4)$ debitage.

The two cowries recovered from Substratum N.2c demonstrated some access to, and consumption of, non-local items by the residents of Area $\mathrm{N}$ right from the first phase of occupation. 
Table 7.17: Unit $\mathbf{N}$ vessel type frequencies

\begin{tabular}{|c|c|c|c|c|c|c|c|c|c|c|c|c|c|}
\hline \multirow{3}{*}{\multicolumn{2}{|c|}{$\begin{array}{r}\text { Substratum } \\
\text { Total } \\
n\end{array}$}} & \multirow{2}{*}{\multicolumn{2}{|c|}{$\begin{array}{c}\text { Bowl } \\
\text { All }\end{array}$}} & \multicolumn{4}{|c|}{ Pot } & \multicolumn{6}{|c|}{ Jar } \\
\hline & & & & \multicolumn{2}{|c|}{ Small } & \multicolumn{2}{|c|}{ Large } & \multicolumn{2}{|c|}{ Small } & \multicolumn{2}{|c|}{ Large A } & \multicolumn{2}{|c|}{ Large B } \\
\hline & & $n$ & $\%$ & $n$ & $\%$ & $n$ & $\%$ & $n$ & $\%$ & $n$ & $\%$ & $n$ & $\%$ \\
\hline N.1a & 19 & 1 & $5.3 \%$ & 0 & $0.0 \%$ & 8 & $42.1 \%$ & 0 & $0.0 \%$ & 1 & $5.3 \%$ & 1 & $5.3 \%$ \\
\hline N.1b & 15 & 1 & $6.7 \%$ & 2 & $13.3 \%$ & 3 & $20.0 \%$ & 1 & $6.7 \%$ & 4 & $26.7 \%$ & 3 & $20.0 \%$ \\
\hline N.1 & 34 & 2 & $5.9 \%$ & 2 & $5.9 \%$ & 11 & $32.4 \%$ & 1 & $2.9 \%$ & 5 & $14.7 \%$ & 4 & $11.8 \%$ \\
\hline N.2a & 4 & 0 & $0.0 \%$ & 0 & $0.0 \%$ & 0 & $0.0 \%$ & 0 & $0.0 \%$ & 2 & $50.0 \%$ & 0 & $0.0 \%$ \\
\hline N.2b & 5 & 0 & $0.0 \%$ & 1 & $20.0 \%$ & 1 & $20.0 \%$ & 1 & $20.0 \%$ & 2 & $40.0 \%$ & 0 & $0.0 \%$ \\
\hline N.2c & 4 & 0 & $0.0 \%$ & 1 & $25.0 \%$ & 0 & $0.0 \%$ & 0 & $0.0 \%$ & 1 & $25.0 \%$ & 0 & $0.0 \%$ \\
\hline $\mathrm{N} .2 \mathrm{~d}$ & 8 & 0 & $0.0 \%$ & 0 & $0.0 \%$ & 1 & $12.5 \%$ & 2 & $25.0 \%$ & 3 & $37.5 \%$ & 0 & $0.0 \%$ \\
\hline N.2e & 3 & 0 & $0.0 \%$ & 1 & $33.3 \%$ & 0 & $0.0 \%$ & 0 & $0.0 \%$ & 0 & $0.0 \%$ & 0 & $0.0 \%$ \\
\hline$N .2$ & 24 & 0 & $0.0 \%$ & 3 & $12.5 \%$ & 2 & $8.3 \%$ & 3 & $12.5 \%$ & 8 & $33.3 \%$ & 0 & $0.0 \%$ \\
\hline Total & 58 & 2 & $3.4 \%$ & 5 & $8.6 \%$ & 13 & $22.4 \%$ & 4 & $6.9 \%$ & 13 & $22.4 \%$ & 4 & $6.9 \%$ \\
\hline
\end{tabular}

\section{NON-RESIDENTIAL AREAS}

\section{Area C Macrolithic Scatter (Unit C)}

Based on high densities of surface pottery and sandstone cobbles, along with one intact granary foundation, I have classified Area $\mathrm{C}$ as a heavily deflated residential area (Appendix B). At the same time, the presence of slag, groundstone fragments, and chipped stone artifacts, including one macrolithic scatter of concentrated mudstone debitage, suggested that people also undertook more specialized subsistence and/or craft activities within this space.

In order to investigate this possibility, I opened Unit C (1x1 m) as a small probe in the southwestern quadrant of a macrolithic scatter (Feature C-01) measuring some 2.80 meters in diameter. As shown in Figure 7.43, excavation of three levels (Surface, 0-5 cm; $5-11 \mathrm{~cm}$ ) revealed a relatively thin layer of mudstone debitage lying directly above a firm yellowish red alluvium with no visible inclusions. Silty clay interspersed among the debitage derived from post-depositional wind and water action.

This unit, located some 0.54 meters downslope from the highest point on Area C, nevertheless confirmed that stratified deposits do not exceed a depth of 0.20-0.30 meters across this area. This shallow depth represents, in part, deflation from long-term sheet erosion, but it is also suggests that depositional practices were less intensive than those at 
mounded areas of the site-either because such practices occurred over a shorter period of time at Area $\mathrm{C}$, or because they did not involve as many architectural elements. The former scenario currently seems more likely given the surface frequencies of angular sandstone cobbles, consistent with at least one phase of residential occupation.

Figure 7.43: Unit $\mathrm{C}$ showing profile and sterile alluvium beneath Feature C-01

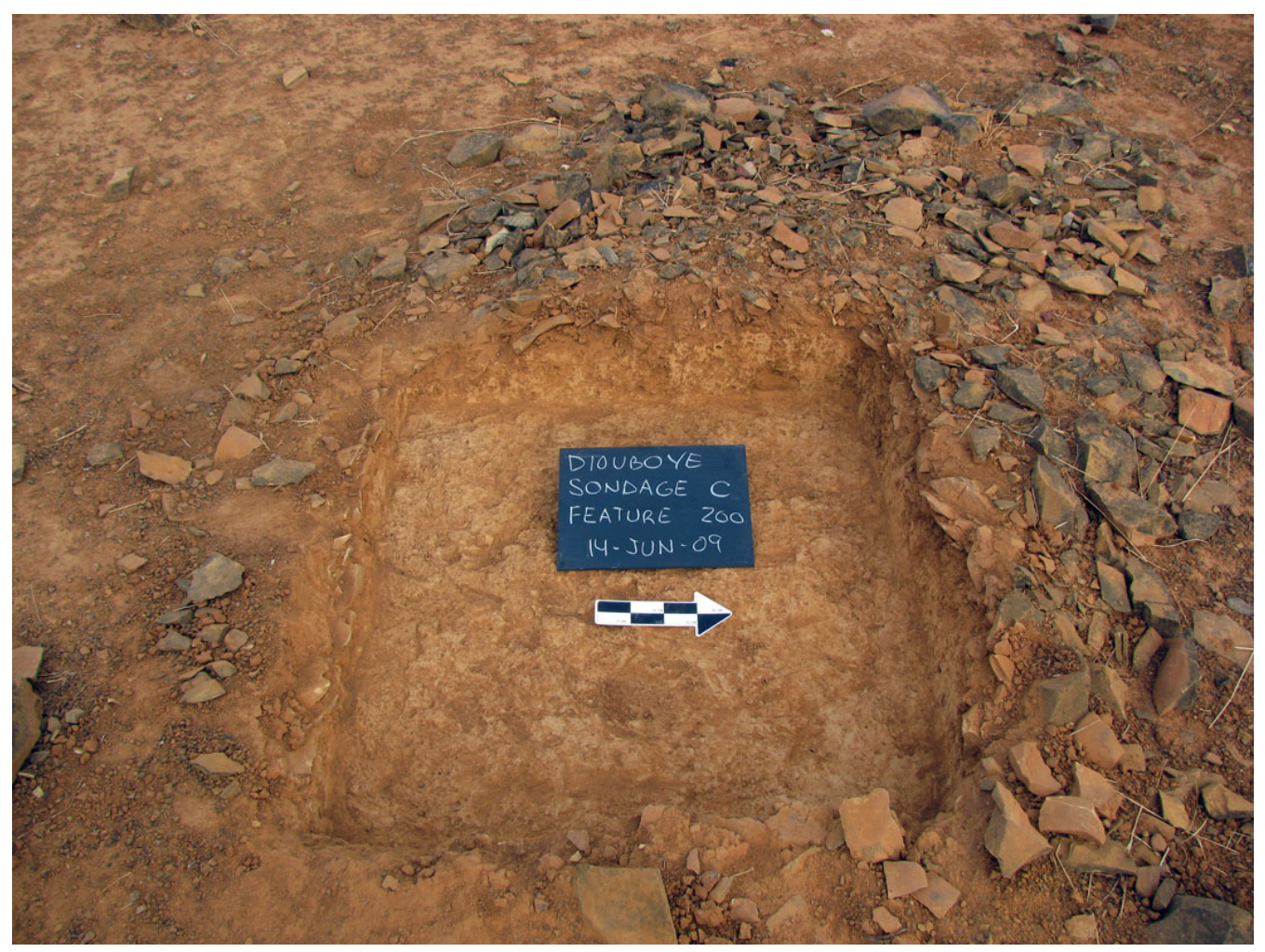

Table 7.18 summarizes the sample of mudstone debitage recovered from Unit C, which was dominated by freehand flakes and angular shatter-these latter including both chips with no dorsal scars and chunks with dorsal scars (see Appendix E). The clear boundaries of this deposit and greater frequencies of chunks in the upper layer of debris were consistent with an in situ and relatively discrete reduction episode(s). 
Table 7.18: $\quad$ Unit $C$ lithic (mudstone) artifact summary

\begin{tabular}{|c|c|c|c|c|c|c|c|c|c|c|c|c|}
\hline \multirow{3}{*}{$\begin{array}{c}\text { Level } \\
\text { Depth } \\
\mathrm{cm} \\
\end{array}$} & \multirow{2}{*}{\multicolumn{2}{|c|}{ Total }} & \multirow{2}{*}{\multicolumn{2}{|c|}{$\begin{array}{c}\text { Core } \\
\text { Freehand }\end{array}$}} & \multicolumn{4}{|c|}{ Angular Shatter } & \multicolumn{4}{|c|}{ Flake } \\
\hline & & & & & \multicolumn{2}{|c|}{ Chip } & \multicolumn{2}{|c|}{ Chunk } & \multicolumn{2}{|c|}{ Freehand } & \multicolumn{2}{|c|}{ Unknown } \\
\hline & $n$ & $\mathrm{~kg}$ & $n$ & $\%$ & $n$ & $\%$ & $n$ & $\%$ & $n$ & $\%$ & $n$ & $\%$ \\
\hline 0 & 488 & 4.5 & 0 & $0.0 \%$ & 266 & $54.5 \%$ & 162 & $33.2 \%$ & 26 & $5.3 \%$ & 34 & $7.0 \%$ \\
\hline $0-5$ & 2867 & 13.3 & 0 & $0.0 \%$ & 1984 & $69.2 \%$ & 310 & $10.8 \%$ & 412 & $14.4 \%$ & 161 & $5.6 \%$ \\
\hline 5-11 & 442 & 2.3 & 1 & $0.2 \%$ & 370 & $83.7 \%$ & 47 & $10.6 \%$ & 7 & $1.6 \%$ & 17 & $3.8 \%$ \\
\hline Total & 3797 & 20.0 & 1 & $0.0 \%$ & 2620 & $69.0 \%$ & 519 & $13.7 \%$ & 445 & $11.7 \%$ & 212 & $5.6 \%$ \\
\hline
\end{tabular}

Given the size and relative homogeneity of this assemblage, I recorded metric and formal variables for approximately 50\% of the freehand flakes in each excavated level $(n=211)$. As shown in Figure 7.44, the dimensions of length and width were remarkably consistent across the distribution of larger and smaller specimens. The observed platform types were predominantly flat (64\%) although cortical (21.3\%) and abraded/crushed (14.7\%) platforms were also present. Along with one polyhedral freehand core, the number of flat striking platforms suggested that this assemblage derived from a prepared core, freehand reduction strategy for producing elongated mudstone flakes and shatter. Although no formal tools were present in the assemblage from Unit C, some 20 flakes with both sharp and steep utilized margins were recovered during surface collection across Diouboye.

Based on the presence of similar macrolithic industries at several late-Holocene sites across West Africa, MacDonald and Allsworth-Jones (1994) have suggested that informal prepared core tools were a response to coarse-grained raw materials since they otherwise appear on Late Stone Age to early Iron Age sites across environments ranging from the semi-arid Sahel to the tropical forest. This explanation seems insufficient to account for macrolithic tool production at Diouboye, given the predominance of quartz and chert raw materials at sites throughout the study area. Rather, I hypothesize that people chose mudstone to produce blade-like flakes larger in size than would be possible with the small nodules of local chert and quartz. In lieu of complex tools using microliths, larger mudstone flakes would have been appropriate for cutting or processing softer materials in tasks such as reaping grain or preparing meals. 
Figure 7.44: Bivariate plot of Unit C macrolithic flake thickness and width showing tenth-quantile density estimate contours and confidence interval ellipses $(90 \%)$ for large and small flakes

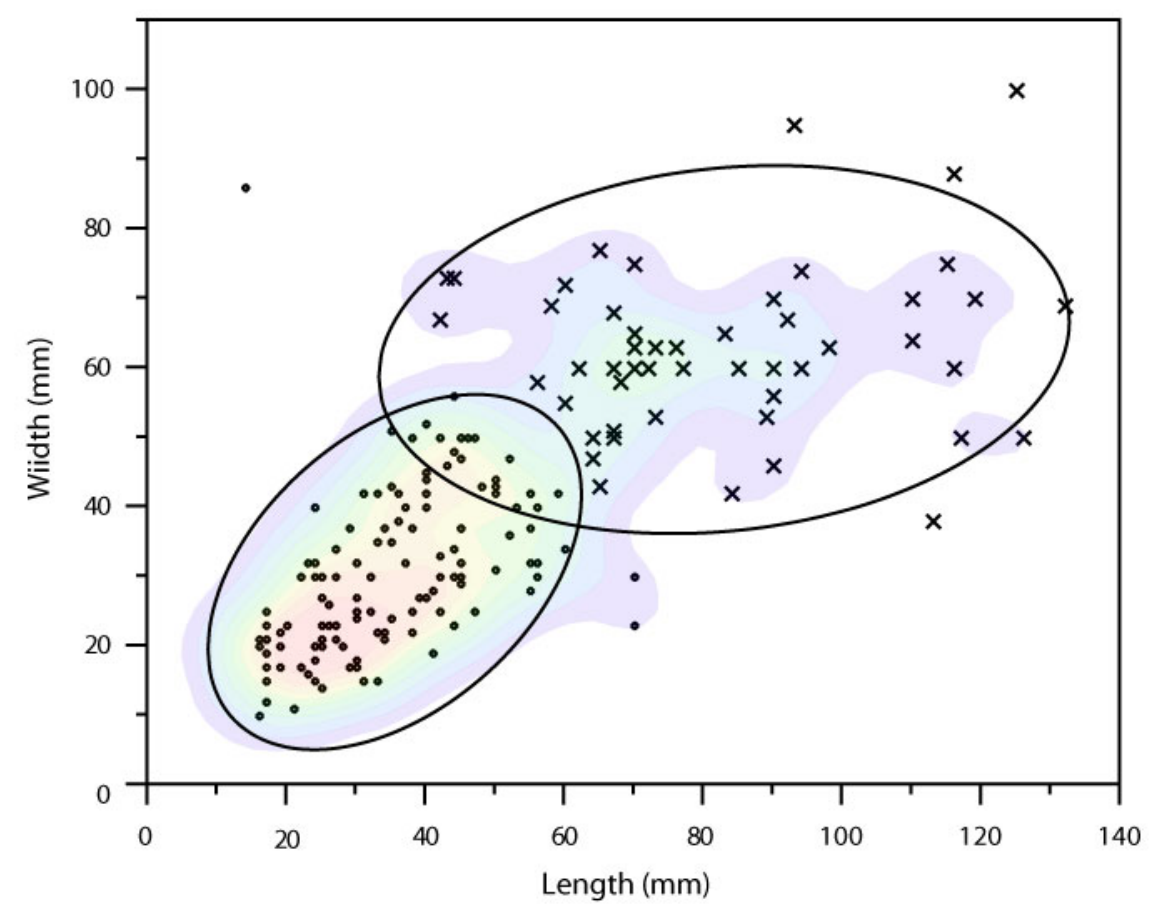

Regardless of the ultimate use of macrolithic mudstone flakes, Unit C has demonstrated that people produced them during the occupation of Diouboye in relatively discrete episodes on the outskirts of residential spaces in Area C and Area B.

\section{Area S Cairns (Unit S)}

The surface of Area S, situated approximately 100 meters east of Area A, was covered with numerous sandstone rocks similar to those derived from architectural features elsewhere across the site. However, the near absence of surface artifacts and unambiguous architectural foundations (there were numerous irregular alignments) led me to investigate the formation processes responsible for these stones. For this reason, I centered Unit S (2x2 m) on Feature S-01, an irregular concentration of 18 angular rocks measuring 1.45 meters in diameter and resembling a disaggregated granary foundation (Figure 7.45). 
Figure 7.45: Unit S (Feature S-01)
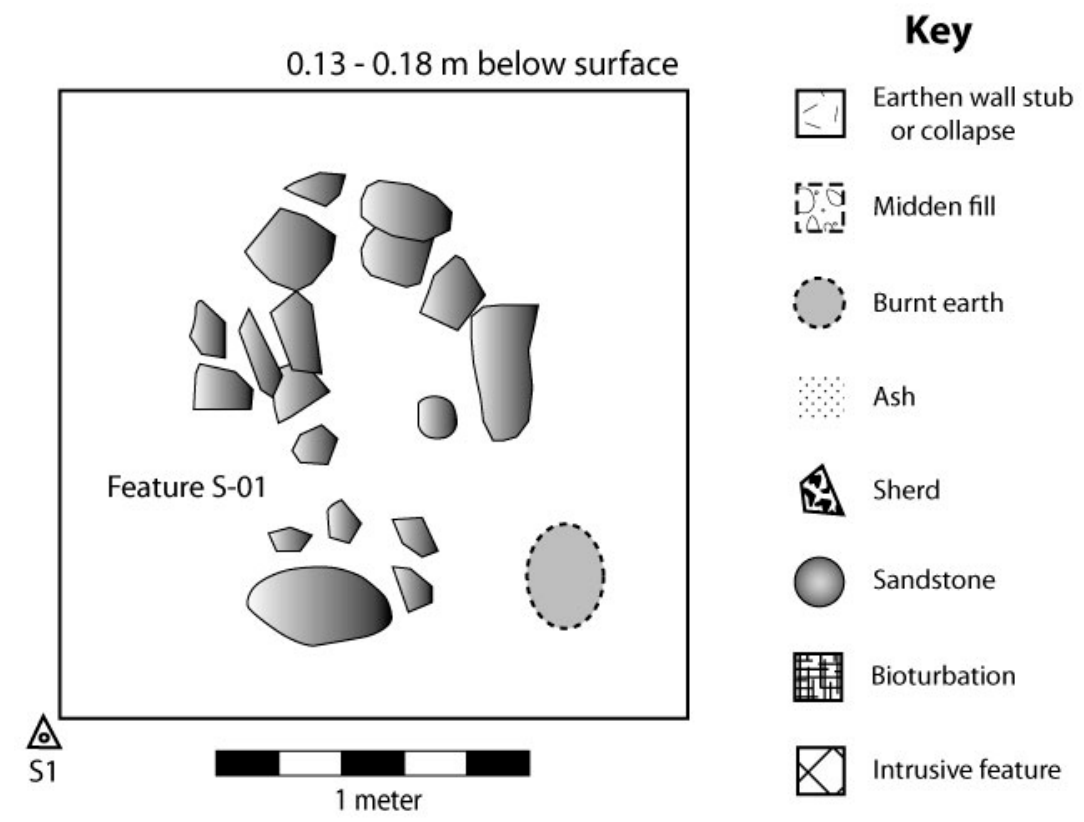

Excavation of Unit S exposed one relatively homogeneous stratum lying above a firm brownish-yellow deposit of silty clay loam encountered at a depth of 0.25 meters below surface. Directly above this sterile alluvium, Substratum S.1b (0.15-0.25 m) comprised a friable horizon of sandy clay loam with some lightly oxidized sediment and a few sherds and faunal elements attesting to nearby cultural activities. Feature S-01, described above, sat above this deposit surrounded by Substratum S.1a (0.00-0.15 m), a loose brown sandy clay loam of weathered topsoil. Of the eight rim sherds recovered from these deposits, it was possible to identify pieces of four large pots, one small pot, one small jar, and one large jar.

The modest amount of daub, charcoal, and artifacts observed in the soil matrix around and below Feature S-01 do confirm that Area S was the site of some architectural activity contemporaneous with the occupation of Diouboye. However, the shallow depth of archaeological deposits and incoherent spatial distribution of sandstone rocks indicate that such activity was short-lived, with the current irregular stone alignments representing post-depositional disturbance from field clearance and plowing by farmers past and/or present. 


\section{Area L Cairns (Trench L)}

Although 24 sandstone cairns dotted the surface of Area L, the corresponding dearth of loose sandstone fragments and other artifacts indicated that these features derived from depositional practices quite different from those responsible for the residential areas of Diouboye. Suspecting that these cairns formed a cemetery associated with the village, I excavated an exploratory Trench L (1x2 m) across Feature L-31 to test for the presence of a burial pit.

Feature L-31 was a rectilinear arrangement of 16 sandstone rocks oriented roughly northeast/southwest and measuring 1.76 meters in length (Figure 7.46). As an exploratory trench, I directed workmen to screen only those deposits with visible charcoal or sherds (and to stop excavation upon any encounter with bone). However, excavation revealed neither cultural deposits, nor bones of any kind to a depth of 1.15 meters below ground. The sediment throughout was a firm to extremely firm, yellowishred silty clay alluvium with absolutely no inclusions, although bioturbation and decaying roots appeared in the trench profile.

Figure 7.46: Trench L (Feature L-31)
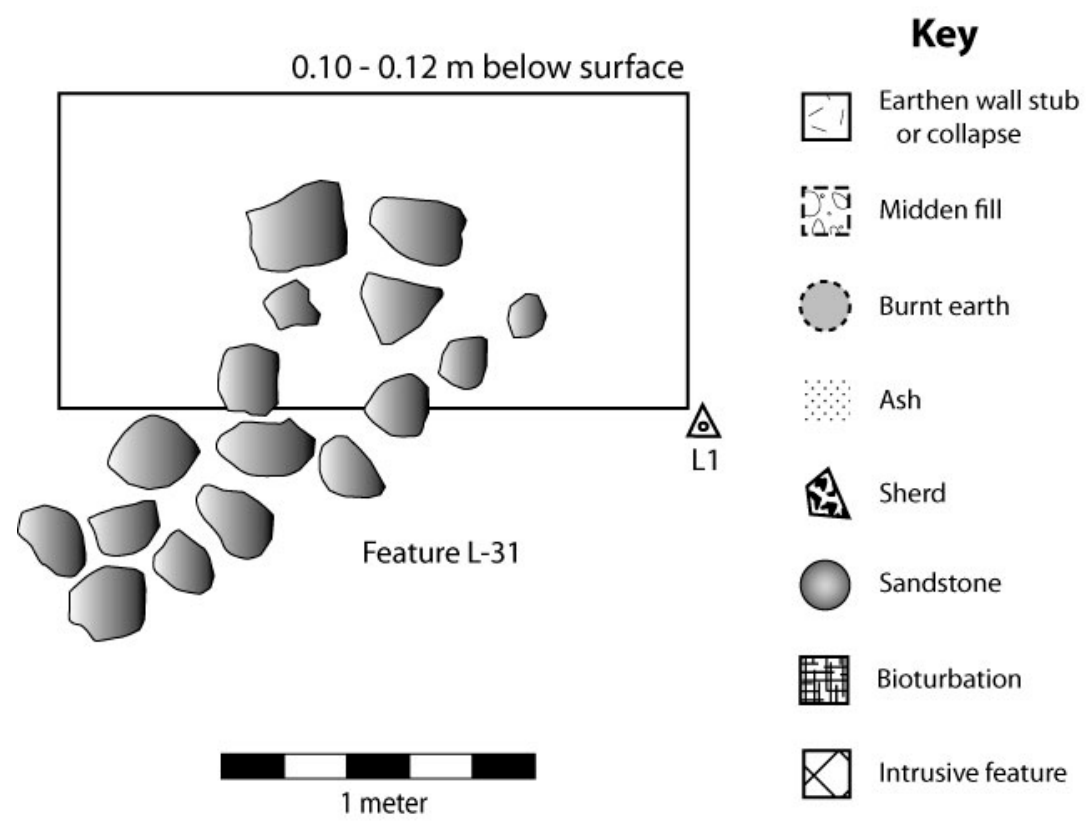
Based on these results, I have interpreted the irregular alignments and cairns of Area $\mathrm{L}$ as the dismantling and redeposition of surface features by farmers in order to open up more space for cultivation in the eastern extent of the site. And for this reason, I have generally excluded Area L from spatial analyses throughout this study.

\section{CHRONOLOGY}

While the above stratigraphic sequences establish the relative chronology within each excavated unit, it is desirable to compare the timing of events between these sequences, and to anchor them within the broader historical processes playing out across West Africa during the Late Iron Age. In this section I summarize the ceramic phases for Diouboye derived from the seriation of temporally sensitive body sherd types (see Appendix D). After applying this relative chronology to the strata detailed in the preceding sections, I present radiocarbon dates obtained on associated charcoal to bracket the resulting ceramic/occupation phases within estimated calendar dates. These phases frame the intra-site comparison of material and spatial practices in the next two chapters.

\section{Ceramic Seriation}

In order to construct a ceramic chronology for Diouboye, I isolated those sherd attributes most sensitive to temporal change in the stratified sequences. As I discuss in Appendix D, body sherd types defined by their decoration techniques—including braided cord roulette, button carved roulette, chevron carved roulette, cord-wrapped stick roulette, folded strip roulette, and twisted cord roulette—clearly seriated by stratigraphic level. Using correspondence analysis (CA), a multidimensional scaling procedure (Baxter 2003:204-206; Duff 1996; Usman 2003), in conjunction with K-means cluster analysis, it was possible to identify those strata with similar type frequencies. Table 7.19 summarizes the resulting ceramic chronology.

Despite the success of this seriation, it is worth repeating my core assumption that observed trends in decorative sherd types primarily reflect stylistic "drift" occurring at a similar pace across Diouboye. Since this assumption rarely holds true in practice where numerous social mechanisms also shape patterns of pottery decoration and household consumption, absolute dating methods provide a check on site-level ceramic chronology. 
Table 7.19: Summary of excavated strata by ceramic phase

\begin{tabular}{|c|c|c|c|c|c|c|c|c|}
\hline \multirow[t]{2}{*}{ Phase } & \multirow[t]{2}{*}{ Stratum } & \multicolumn{7}{|c|}{ Body Sherd Type (Decoration) } \\
\hline & & $\mathrm{n}$ & $\begin{array}{l}\text { Twisted } \\
\text { Cord }\end{array}$ & $\begin{array}{l}\text { Button } \\
\text { Carved }\end{array}$ & $\begin{array}{l}\text { Folded } \\
\text { Strip }\end{array}$ & $\begin{array}{l}\text { Cord- } \\
\text { Wrapped } \\
\text { Stick }\end{array}$ & $\begin{array}{l}\text { Braided } \\
\text { Cord }\end{array}$ & $\begin{array}{c}\text { Chevron } \\
\text { Carved }\end{array}$ \\
\hline \multirow{5}{*}{$\begin{array}{l}\text { Late } \\
\text { Diouboye }\end{array}$} & A2.1 & 4732 & $25.10 \%$ & $4.40 \%$ & $10.10 \%$ & $34.70 \%$ & $15.90 \%$ & $9.60 \%$ \\
\hline & A1.1 & 217 & $45.60 \%$ & $2.30 \%$ & $10.60 \%$ & $12.00 \%$ & $18.40 \%$ & $11.10 \%$ \\
\hline & G.1 & 1182 & $38.30 \%$ & $3.00 \%$ & $22.80 \%$ & $22.90 \%$ & $3.80 \%$ & $9.10 \%$ \\
\hline & H.1 & 145 & $34.50 \%$ & $5.50 \%$ & $21.40 \%$ & $22.80 \%$ & $1.40 \%$ & $14.50 \%$ \\
\hline & Late & 6276 & $28.51 \%$ & $4.09 \%$ & $12.77 \%$ & $31.42 \%$ & $13.37 \%$ & $9.67 \%$ \\
\hline \multirow{11}{*}{$\begin{array}{l}\text { Middle } \\
\text { Diouboye }\end{array}$} & H.2* & 135 & $60.00 \%$ & $0.00 \%$ & $11.90 \%$ & $14.80 \%$ & $4.40 \%$ & $8.90 \%$ \\
\hline & A2.2 & 969 & $33.40 \%$ & $4.10 \%$ & $31.50 \%$ & $19.10 \%$ & $3.70 \%$ & $8.20 \%$ \\
\hline & A1.2 & 422 & $55.20 \%$ & $8.50 \%$ & $19.90 \%$ & $8.30 \%$ & $3.30 \%$ & $4.70 \%$ \\
\hline & H.3 & 195 & $21.50 \%$ & $4.60 \%$ & $49.70 \%$ & $9.20 \%$ & $0.50 \%$ & $14.40 \%$ \\
\hline & F.1 & 791 & $26.90 \%$ & $7.70 \%$ & $33.90 \%$ & $11.30 \%$ & $4.30 \%$ & $15.90 \%$ \\
\hline & K.1 & 47 & $31.90 \%$ & $17.00 \%$ & $31.90 \%$ & $6.40 \%$ & $0.00 \%$ & $12.80 \%$ \\
\hline & N.1 & 200 & $31.00 \%$ & $14.00 \%$ & $39.50 \%$ & $5.00 \%$ & $0.00 \%$ & $10.50 \%$ \\
\hline & A1.3 & 547 & $38.90 \%$ & $8.60 \%$ & $45.50 \%$ & $1.60 \%$ & $0.00 \%$ & $5.30 \%$ \\
\hline & G.2 & 219 & $27.90 \%$ & $7.80 \%$ & $53.00 \%$ & $2.70 \%$ & $0.50 \%$ & $8.20 \%$ \\
\hline & F.2 & 93 & $16.10 \%$ & $14.00 \%$ & $58.10 \%$ & $7.50 \%$ & $1.10 \%$ & $3.20 \%$ \\
\hline & Middle & 3618 & $34.77 \%$ & $7.15 \%$ & $35.47 \%$ & $10.56 \%$ & $2.57 \%$ & $9.46 \%$ \\
\hline \multirow{4}{*}{$\begin{array}{l}\text { Early } \\
\text { Diouboye } \\
\text { B }\end{array}$} & G.3 & 392 & $34.40 \%$ & $21.20 \%$ & $38.00 \%$ & $2.80 \%$ & $0.00 \%$ & $3.60 \%$ \\
\hline & A2.3 & 290 & $25.90 \%$ & $34.10 \%$ & $33.80 \%$ & $3.80 \%$ & $0.00 \%$ & $2.40 \%$ \\
\hline & H.4 & 764 & $14.10 \%$ & $67.70 \%$ & $16.80 \%$ & $0.80 \%$ & $0.00 \%$ & $0.70 \%$ \\
\hline & Early B & 1451 & $22.00 \%$ & $48.30 \%$ & $26.00 \%$ & $1.90 \%$ & $0.00 \%$ & $1.80 \%$ \\
\hline \multirow{6}{*}{$\begin{array}{l}\text { Early } \\
\text { Diouboye } \\
\text { A }\end{array}$} & C.1 & 5 & $40.00 \%$ & $40.00 \%$ & $0.00 \%$ & $20.00 \%$ & $0.00 \%$ & $0.00 \%$ \\
\hline & F.3 & 174 & $5.70 \%$ & $48.30 \%$ & $40.80 \%$ & $4.60 \%$ & $0.00 \%$ & $0.60 \%$ \\
\hline & N.2 & 157 & $12.70 \%$ & $52.90 \%$ & $27.40 \%$ & $0.60 \%$ & $0.00 \%$ & $6.40 \%$ \\
\hline & S.1 & 41 & $0.00 \%$ & $51.20 \%$ & $39.00 \%$ & $7.30 \%$ & $0.00 \%$ & $2.40 \%$ \\
\hline & K.2 & 182 & $20.30 \%$ & $56.00 \%$ & $16.50 \%$ & $3.30 \%$ & $0.00 \%$ & $3.80 \%$ \\
\hline & Early A & 559 & $12.30 \%$ & $52.20 \%$ & $28.70 \%$ & $3.40 \%$ & $0.00 \%$ & $3.40 \%$ \\
\hline
\end{tabular}

*Substratum reassigned from Late to Middle Diouboye phase based on radiocarbon dates (see below)

\section{Radiocarbon Dating}

Table 7.20 and Figure 7.47 summarize the calibrated radiocarbon dates obtained for charcoal samples from Diouboye, in addition to one from FAL-1075. For reasons of logistics and cost, I submitted three samples to Beta Analytic and eleven samples to the NSF-Arizona AMS Laboratory. The resulting dates place the occupation of Diouboye 
comfortably within the late-first to early-second millennium $\mathrm{AD}$, significantly earlier than the $15-17^{\text {th }}$ century AD date associated with Atlantic Era (Tontèko Phase) pottery at the site of FAL-1075 (see Chapter 4).

While dated materials generally upheld the ceramic chronology of their associated strata (Figure 7.47), three samples were clear outliers. Beta-249335 and Beta-24934, from the upper and lower levels of Unit $\mathrm{H}$, yielded dates that were not only statistically indistinguishable from one another, but also significantly earlier than all others at the site. In fact, two additional dates on carbonized pearl millet seeds (AA-94748, AA-94749) from the intervening contexts of Stratum H.2 fell firmly in line with the relative ceramic chronology of the site. This suggests that the previous late-first millennium AD dates from Area H derived from “old wood” for construction or fuel, rather than bioturbation. This interpretation finds support from the depositional integrity of the excavated substrata, at least within Strata H.2 and H.3, suggesting that the upward migration of archaeological materials was minimal or absent in Unit $\mathrm{H}$.

Table 7.20: Radiocarbon dates from the Central Falémé Archaeological Project

\begin{tabular}{|c|c|c|c|c|c|c|}
\hline \multirow[t]{2}{*}{ Lab/Sample } & \multirow[t]{2}{*}{ Site } & \multirow[t]{2}{*}{ Unit } & \multirow[t]{2}{*}{ Substratum } & \multirow[t]{2}{*}{ Locus Matrix } & \multicolumn{2}{|c|}{ Date (calAD) } \\
\hline & & & & & $\begin{array}{c}\text { Lower CI } \\
(2 \sigma) \\
\end{array}$ & $\begin{array}{c}\text { Upper CI } \\
(2 \sigma) \\
\end{array}$ \\
\hline Beta-249335 & Diouboye & $\mathrm{H}$ & H.1c & Fill, room & 660 & 880 \\
\hline Beta-249334 & Diouboye & $\mathrm{H}$ & H.3c & Fill, midden & 670 & 880 \\
\hline AA-91145 & Diouboye & A2 & A2.1a & Fill, pit & 1256 & 1390 \\
\hline AA-91144 & Diouboye & $\mathrm{A} 2$ & A2.1d & Fill, midden & 1161 & 1276 \\
\hline AA-91146 & Diouboye & A2 & A2.3b & Fill, midden & 1020 & 1164 \\
\hline AA-91147 & Diouboye & $\mathrm{F}$ & F.2a & Earthen wall melt & 1039 & 1215 \\
\hline AA-91148 & Diouboye & $\mathrm{F}$ & F.3b & Fill, general & 1030 & 1208 \\
\hline AA-91150 & Diouboye & G & G.3a & Stone foundation & 990 & 1155 \\
\hline AA-95748 & Diouboye & $\mathrm{H}$ & H.2b & Hearth & 1042 & 1254 \\
\hline AA-95749 & Diouboye & $\mathrm{H}$ & H.2d & Hearth & 1058 & 1280 \\
\hline AA-91149 & Diouboye & $\mathrm{K}$ & K.2a & Earthen wall collapse & 1275 & 1394 \\
\hline AA-95750 & Diouboye & $\mathrm{K}$ & K.2c & Fill, general & 1028 & 1224 \\
\hline AA-91143 & Diouboye & $\mathrm{N}$ & N.2b & Earthen wall collapse & 985 & 1155 \\
\hline Beta-290993 & FAL-1075 & 1 & 1.03 & Fill, general & 1480 & 1650 \\
\hline
\end{tabular}


Figure 7.47: Radiocarbon dates from the Central Falémé Archaeological Project (calibrated using OxCal 4.1)

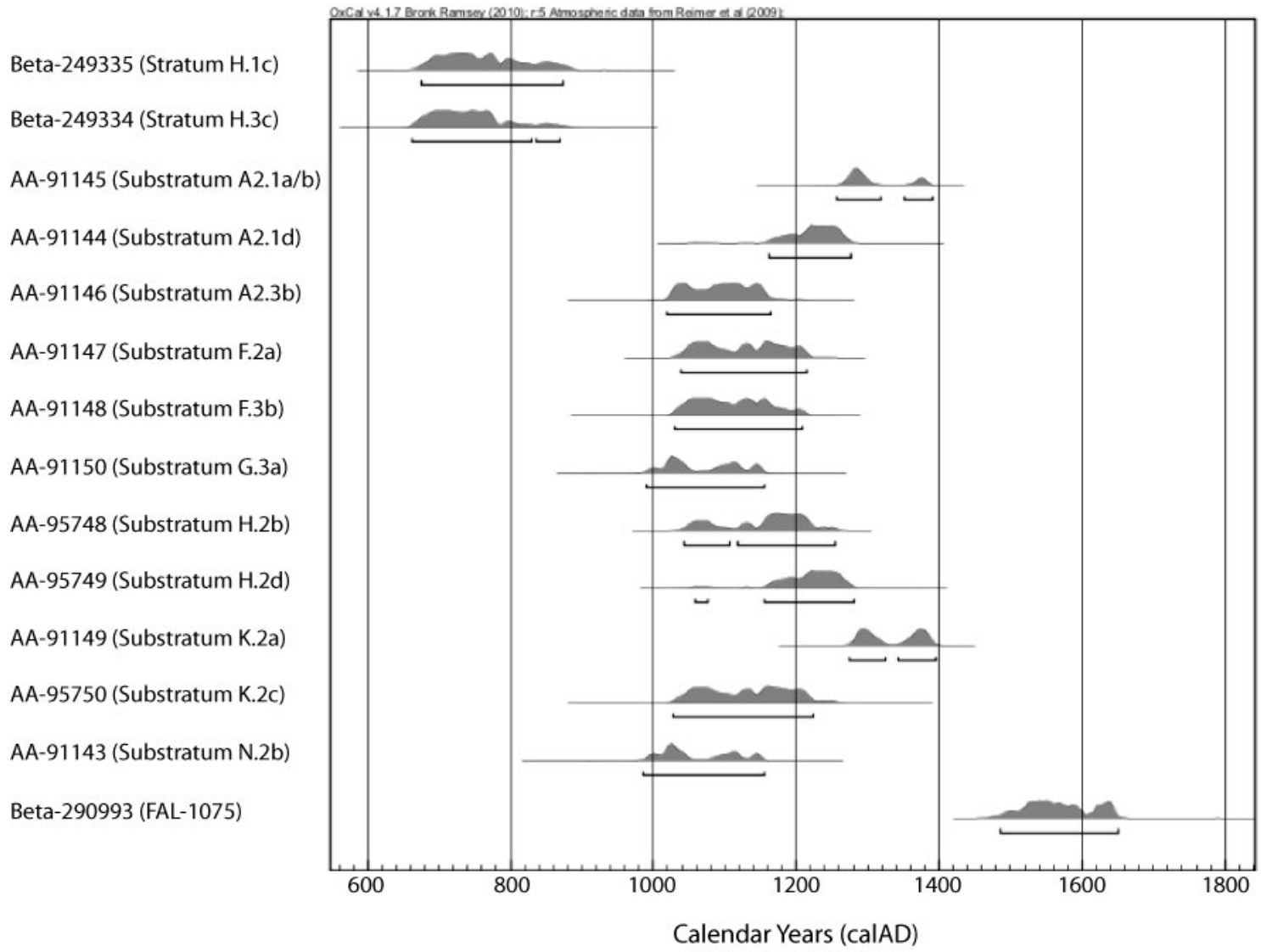

Conversely, a third outlier sample (AA-91149) in Unit K yielded a date older than expected for the associated Early Diouboye phase ceramics. While Areas J/K were at some point a residential area, the absence of occupation surfaces, hearths, stone installations, or other unambiguous evidence for primary deposition in Unit $\mathrm{K}$ made it difficult to identify bioturbation or plowing that could have pushed younger charcoal into older deposits, particularly for the shallow depth of this sample (30-33 cm below surface). In contrast to this charcoal of questionable origin, a carbonized baobab shell (AA-95750) from the lowermost Substratum K.2c provided a more secure date for its associated cultural deposits (baobab fruits would have been harvested annually). The date for this sample further coincides with the dating of Early to Middle Diouboye Phase ceramics elsewhere across the site. 


\section{Ceramic Phases}

After accounting for outliers from Area $\mathrm{H}$ and Area $\mathrm{K}$, the remaining ten dates were consistent with site stratigraphy (Figure 7.48); that is, earlier dates came from lower deposits within units A2 and F. They also indicate that seriated distributions of ceramics in each unit do, in fact, represent temporal change, rather than some other social variable. Still, the following phases should be considered tentative first estimates pending further radiometric analysis of samples from Diouboye.

Early Diouboye A/B Phase (AD 950-1100): Five radiocarbon samples associated with this ceramic phase yielded dates ranging from the late-tenth century $\mathrm{AD}$ to the beginning of the $13^{\text {th }}$ century AD. Since these all came from the middle to upper substrata within their respective strata, I estimate the fuzzy boundary between the early and middle phase of site occupation at AD 1100. The initial settlement of Diouboye may have begun earlier than suggested by the confidence intervals for these dates, perhaps during the early- to mid- $10^{\text {th }}$ century AD.

Middle Diouboye Phase (AD 1100-1250): The single radiocarbon sample clearly associated with this ceramic phase in Substratum F.2a dated from the mid- $11^{\text {th }}$ to early $13^{\text {th }}$ century AD. Based on dates falling in this range, Substrata H.2d and H.2b were also reassigned to this phase, despite their ostensibly later ceramic assemblages. While I have attributed substrata with Middle Diouboye phase ceramic assemblages to the latter part of the range bracketed by these three dates, it is important to recognize that their confidence intervals overlap substantially with those for dates from the Early Diouboye phase, making the two phases statistically indistinguishable from the standpoint of radiocarbon dating.

Late Diouboye Phase (AD 1250-1400): Two samples from Substrata A2.1a/b and A2.1d furnished dates associated with this phase. Collected from directly above Middle Diouboye phase deposits in Unit A2, the earlier sample had a date of AD 1161-1270. This provided a basis for setting the lower phase boundary at AD 1250. A second sample from relatively deep fill in Feature 122, but representing a deposition from Stratum A2.1, yielded a substantially later date of AD 1256-1390. Along with the outlying date from Substratum K.2a, this suggests that human activity of one form or another continued at Diouboye through the mid to late $14^{\text {th }}$ century AD. 
Interestingly enough, these dates further suggest that Diouboye was abandoned just prior to the advent of an Atlantic Era occupation of the region represented by a $16^{\text {th }}$ century AD date from the Tontèko phase component at site FAL-1075 (Beta-290993).

Figure 7.48: Chronological assessment of excavated substrata in residential areas

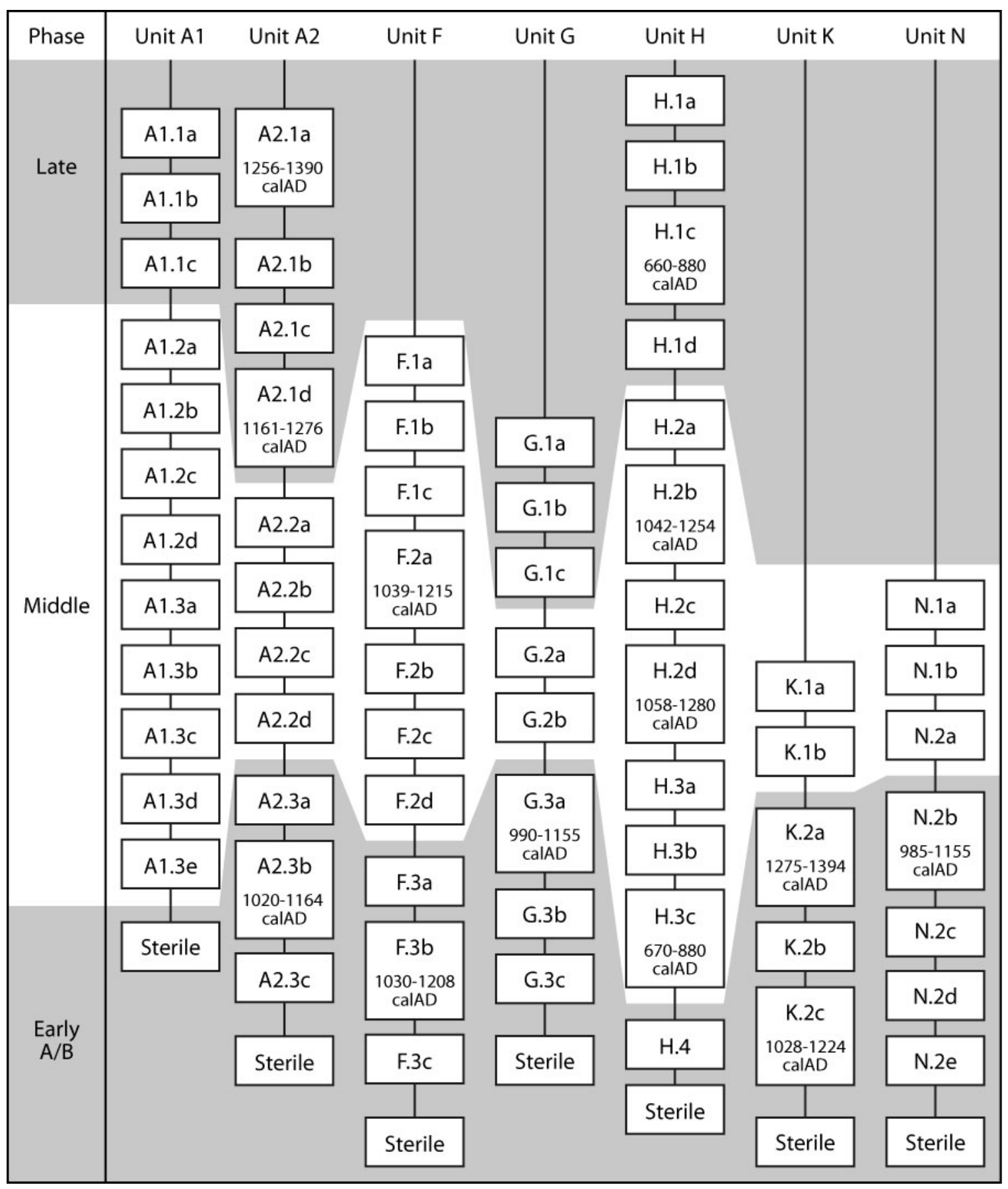




\section{SUMMARY}

The excavation of six residential and three non-residential areas at Diouboye documented sequences of subsistence and craft production activities alongside the construction of domestic architecture such as huts, granaries, and hearths. Altogether, these data indicate that Diouboye was settled on a previously unoccupied levee of compact alluvium parallel to the Falémé River sometime around the beginning of the second millennium AD. Seriation of the ceramic assemblages from each unit, combined with 13 radiocarbon dates, orient the ensuing occupations into three general occupation phases spanning three or four centuries until site abandonment prior to AD 1400. These data and their interpretation as sequences of past material practice form the basis for a comparison of economic processes and political institutions across the village in the next two chapters. 


\section{CHAPTER 8 \\ RESIDENTIAL WARDS: SPATIAL ORDERING OF THE VILLAGE ECONOMY}

Drawing upon the changes and continuities observed in specific stratigraphic sequences, I turn in this chapter to evaluate the spatial and material dimensions of economic activities and political institutions by looking at surface archaeological data from across Diouboye. As detailed in Appendix B, I documented surface architecture and artifacts through a program of systematic collection and feature recording, as well as ground survey to model local topography and erosional processes. In this way I defined the 16 areas of residential and/or non-residential activity already introduced in the preceding chapter. Additionally, I defined two broader spatial units — an East Ward and a West Ward - that form the basis for further comparative analysis in the present chapter.

In the sections that follow, I first situate the surface remains from Diouboye within the chronological grid established in Chapter 7. This task involves working around some of the biases introduced through on-going processes of erosion to identify surface materials from more-or-less coeval activities within each area. I then examine the organization of subsistence, craft, and long-distance exchange practices attested by relations among surface artifacts within and between areas. Finally, I consider how such economic activities, and possibly social relations, related to the formalization of space around architectural elements such as granaries.

Despite the biases of post-depositional processes, the material and spatial dimensions of these data underscore clear differences in the use of space. Residential areas were associated with quotidian tasks such as preparing and consuming meals, making craft goods, and engaging in exchange and politics, while non-residential areas were used for specialized tasks or refuse disposal. The spatial coherence of residential areas further suggests that they each represent one or more household compounds occupied by members of a lineage or similar corporate kin group. The evidence I present 
in this chapter suggests that some activities, such as grain storage, grinding, metalworking, and perhaps chipped stone tool use, differed across residential areas, particularly between the two wards, even as many other subsistence, craft, and longdistance exchange activities did not. Alongside the results of excavation, these subtle distinctions gleaned from surface data contribute to interpretations of the village political economy explored in the next chapter.

\section{SURFACE CHRONOLOGY}

A comparison of architecture and activities across the surface of Diouboye depends first and foremost on understanding how these data fit into the overall sequence of occupation. Here, the ceramic chronology developed in the previous chapter provides a basic framework for inferring the temporal dimensions of surface contexts-including phase(s) of occupation and relative degree of stratigraphic deflation. In this latter regard, models of surface erosion based on local topography, artifact densities, and qualitative infield assessments can help to evaluate some of the natural transformation processes that have generated the surface pottery assemblages.

\section{Ceramic Chronology Index}

Since the ceramic chronology for Diouboye consists of three phases defined by seriating body sherds, it may not directly apply to surface assemblages where processes other than stylistic change, such as long-term deflation, can significantly alter the relative frequencies of artifacts. By way of a work-around, I focused on the three body sherd types — button carved (CrB1/2), cord-wrapped stick (CwS1/2), and braided cord $(\mathrm{BrS} / \mathrm{D})$ - whose relative frequencies changed most dramatically in the stratified sequences from excavated units (see Table 7.19). When considered for all analyzed sherds, not just the seriated types, button carved sherds accounted for $32.5 \%$ of all those from Early Diouboye A/B deposits, but largely fell out of fashion during the Middle Diouboye phase, and only accounted for 2.3\% of sherds from the Late Diouboye phase. Sherds decorated with cord-wrapped stick roulette, on the other hand, comprised a mere $1.5 \%$ of the Early Diouboye A/B assemblage, and steadily increased to $18.1 \%$ of the Late Diouboye assemblage. Braided cord sherds were completely absent from Early Diouboye 
A/B strata and accounted for only $1.7 \%$ of sherds from Middle Diouboye strata, yet became more definitive of Late Diouboye strata where they formed $7.7 \%$ of the total body sherd assemblage. Although the proportions of twisted twine, folded strip, and chevron roulette sherds also contributed to the seriation of stratified deposits, their subtler temporal trends made them a poor choice for assessing surface chronology.

In order to date surface materials using these temporally sensitive button carved, cord-wrapped stick, and braided cord sherd types, I first calculated their relative abundance as a proportion of analyzed sherds at each sampling point (see Appendix B), excluding points with fewer than three sherds. I then employed natural-neighbor interpolation (2.6 m resolution) to model the proportions of these types across the entire site surface. While this method is less powerful than geostatistical techniques such as kriging (Conolly and Lake 2006:90-100), the natural-neighbor interpolation of any given point uses the relative overlap of its own Thiessen polygon with those of neighboring points to provide a model of local variability that exactly fits the initial data points, yet exhibits considerable smoothness.

Figure 8.1 illustrates the interpolated proportions of carved button sherds across the surface of Diouboye. This type occurred in high concentrations, though at densities slightly less than those found among strata assigned to the Early Diouboye A/B phase, along the eroded margins of most residential areas, and across the crowns of Area B, Area C, and Area A-S. The few sampling points with surface artifacts in Areas $\mathrm{J} / \mathrm{K}$ also yielded modest proportions of button roulette sherds.

Figures 8.2-8.3 illustrate the interpolated proportions of cord-wrapped stick and braided cord sherds found more frequently in strata dating from the Middle to Late Diouboye phases. By comparison with button carved sherds, both of these types appeared in greater proportions across the upslope portions of residential areas, particularly the mounded ones, as well as Area B, Area C, and Area A-S. They were, however, absent from the admittedly small sherd samples in Areas $\mathrm{J} / \mathrm{K}$. 
Figure 8.1: Relative surface abundance of button carved body sherds

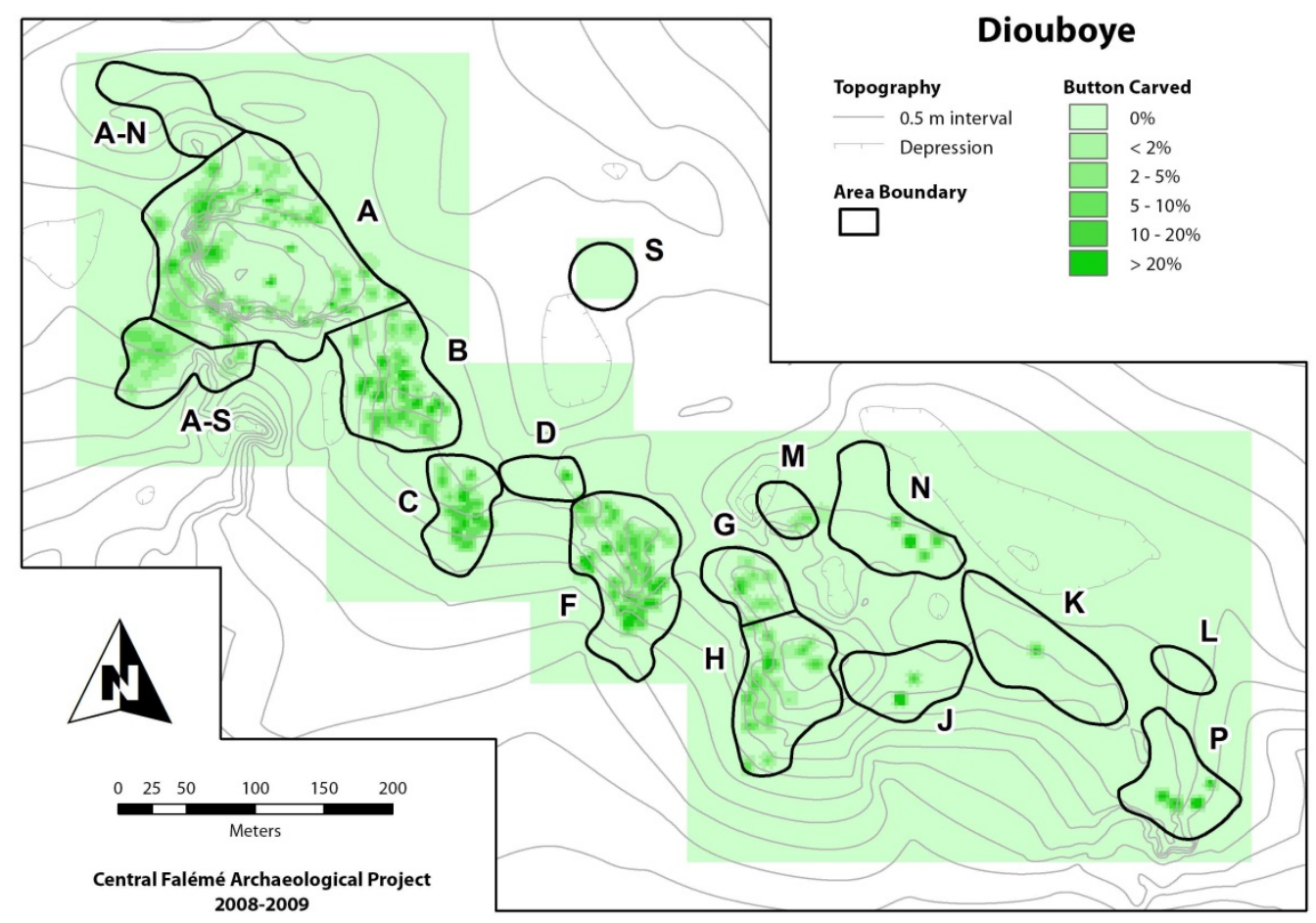

Figure 8.2: $\quad$ Relative surface abundance cord-wrapped stick body sherds

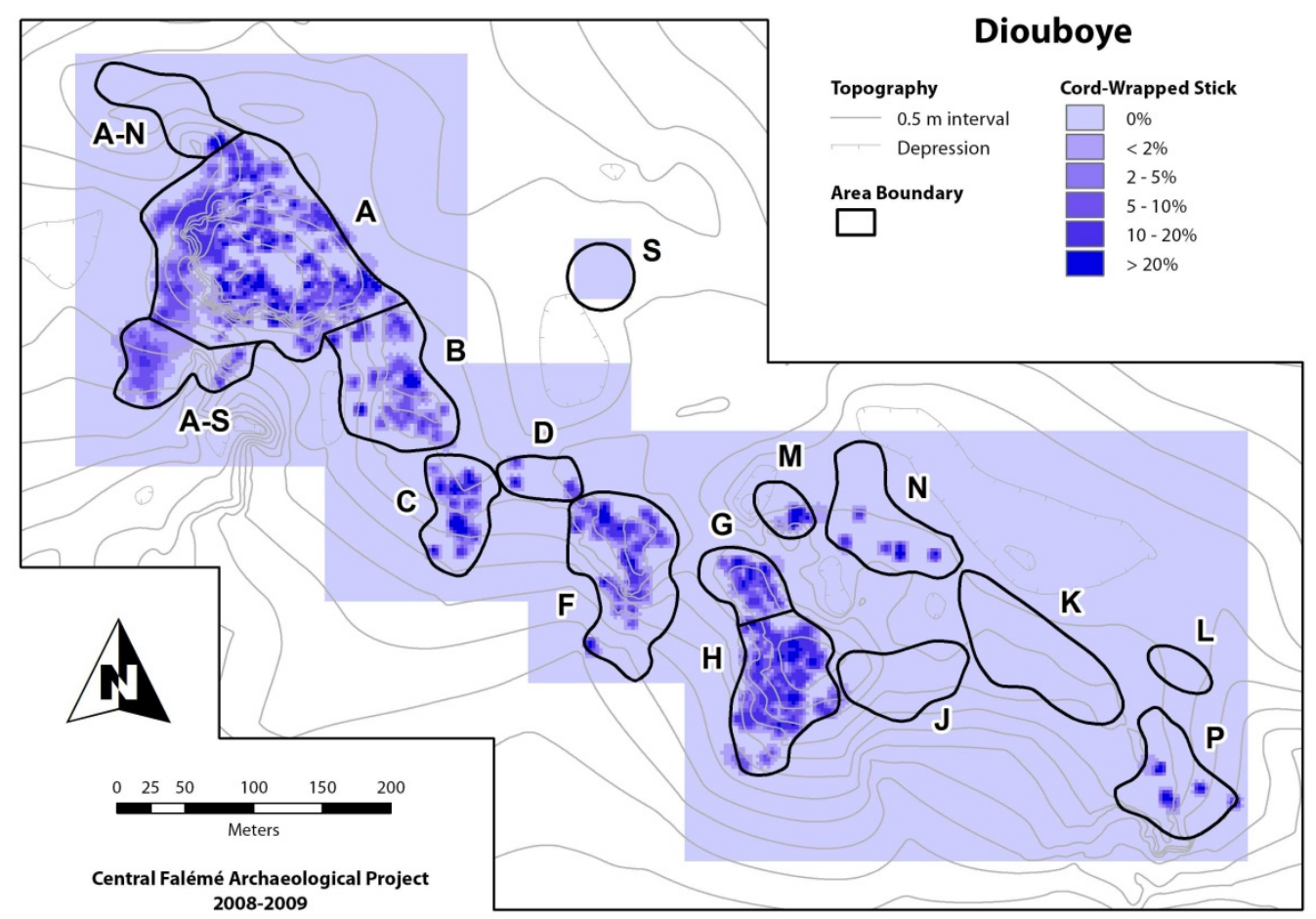


Figure 8.3: $\quad$ Relative surface abundance of braided cord body sherds

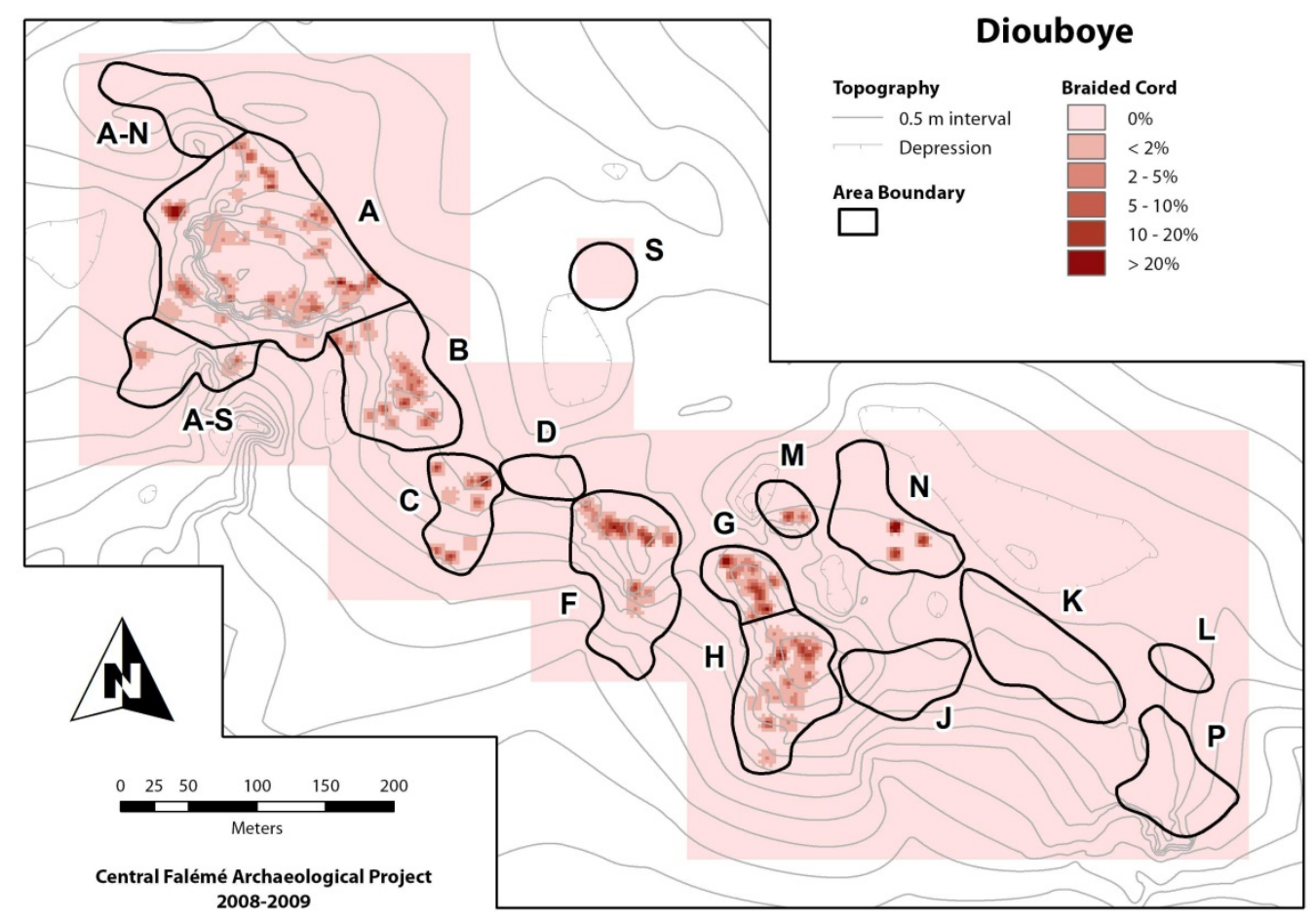

To view a composite representation of these patterns I developed a ceramic chronology index of the number of carved button (CrB1/2) sherds relative to cordwrapped stick (CwS) sherds across the surface of the site. Because these proportions did not have the same uniform distributions, I transformed them into square roots and divided by their sum to give a maximum value of 1.0 for surface points with only cord-wrapped stick sherds, and a minimum value of-1.0 for points with only carved button sherds (Figure 8.4). Figure 8.5 displays the distribution of this index across the site surface. The eroded and deflated edges of Area A-S, Area F, and Area G/H notwithstanding, the observed pattern largely confirms those noted above for the individual sherd types.

Figure 8.4: Ceramic chronology index

$$
\text { Ceramic Chronology Index }=\frac{\sqrt{\text { proportion CwS }}-\sqrt{\text { proportion CrB1/2 }}}{\sqrt{\text { proportion } \mathrm{CwS}}+\sqrt{\text { proportion CrB1/2 }}}
$$


Figure 8.5: Surface model of ceramic chronology index

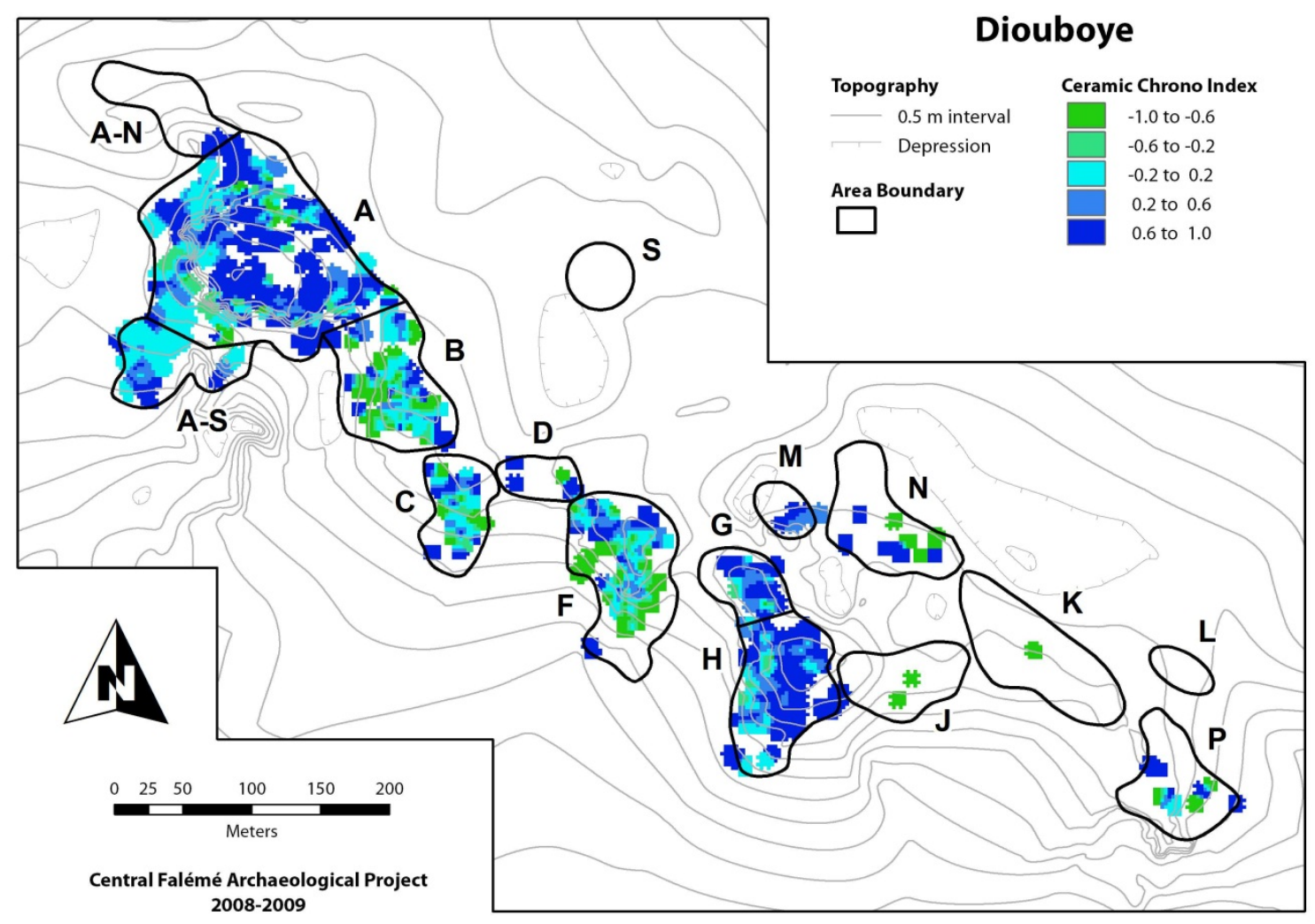

The relative concentrations of sherds decorated with cord-wrapped stick were consistent with a Late Diouboye phase assemblage atop Area A, Area F, Area G, Area H, Area M, and Area P, but were more consistent with earlier or multi-phase assemblages across Area B, Area C, Area D, and Area A-S.

\section{Surface Erosion}

To help distinguish between earlier and multi-phase assemblages, I had to account for the effects of hydraulic redeposition, which I characterized along a continuum from sheet to rill erosion (Rapp and Hill 1998:67-77). Sheet erosion occurs across areas with limited slope where accumulated precipitation with low energy flow of water transports finer sediment particles such as sand and loam, but not coarser ones such as gravel and artifacts. Rill erosion occurs on relatively steep surfaces where run-off coalesces into 
discrete channels with high energy flow and a greater capacity to transport sediments and artifacts downslope.

The LS erosion factor, a composite measure of slope steepness and hydraulic accumulation described in Appendix B, provides a means of evaluating the relative influences of these processes on surface assemblages. Figure 8.6 shows the distribution of LS values across the surface of Diouboye where lower values $(<1.0)$ demarcate areas of sheet erosion and higher values (>3.0) correspond to rill erosion. A visual comparison with Figure 8.5 above reveals that elevated densities of carved button sherds around the margins of Area A, Area B, Area F, Area H, and Area P coincided with zones of rill erosion, suggesting that they represent the exposure and downslope movement of artifacts from Early to Middle Diouboye phase deposits. By contrast, the palimpsests of sherds from multiple phases in Area $\mathrm{C}$ and Area A-S coincided with zones of sheet erosion and more likely represent the gradual deflation of local archaeological deposits.

Figure 8.6: $\quad$ Surface model of erosion as slope length-steepness (LS)

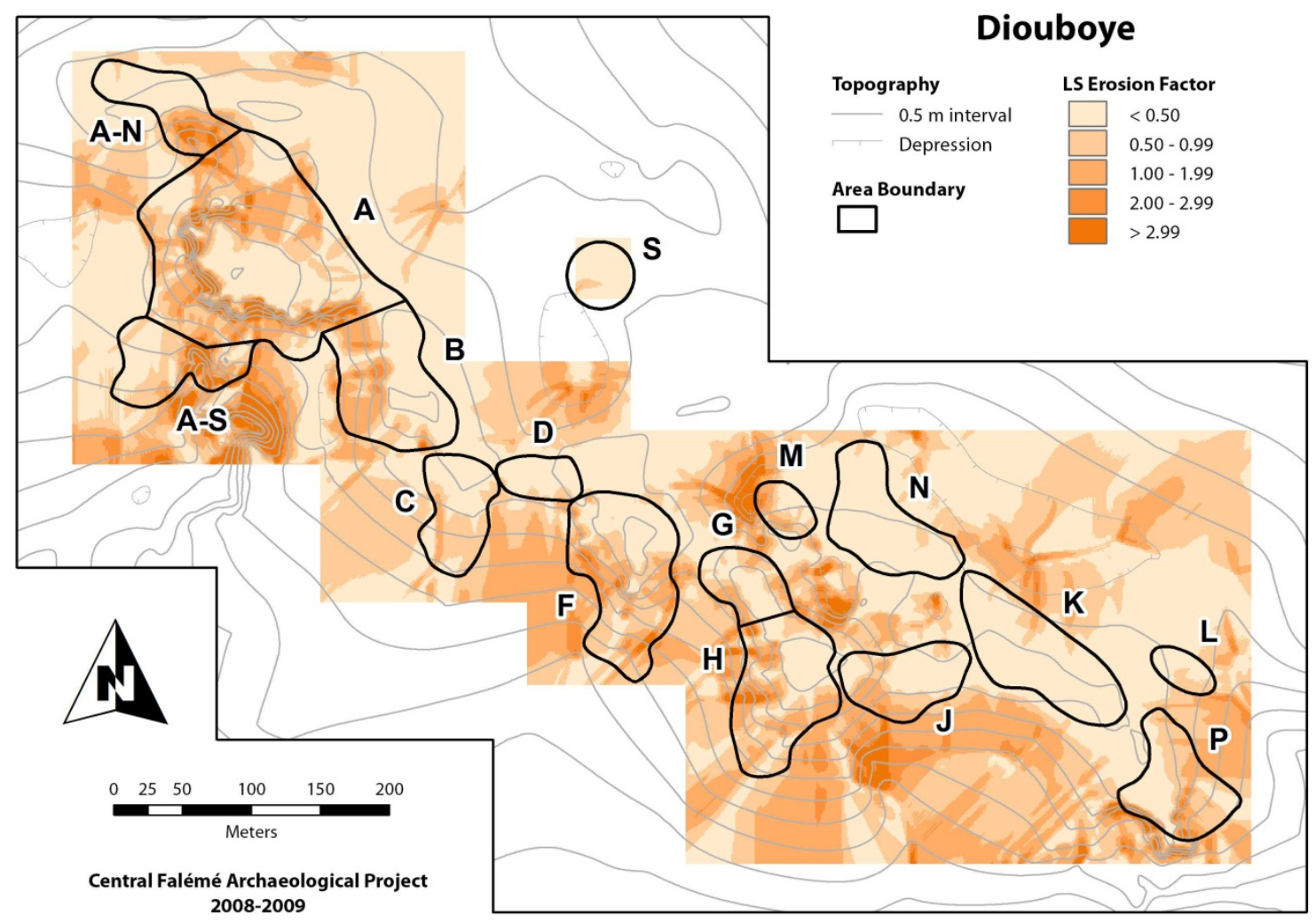


All in all, the surface erosion model indicates that concentrations of artifacts and architectural features situated immediately around the crown of most residential areas have experienced only modest deflation, and may be employed, with due caution, in the spatial analyses described below.

\section{Surface Chronology of Intra-Site Areas}

On the basis of the above ceramic chronology index, counterbalanced by an assessment of rill and sheet erosion and the stratigraphy of excavated sequences, I have interpreted the chronology of surface remains from each area, or at least the relatively intact core of each area, as listed in Table 8.1. Although more detailed modeling of erosional and/or depositional processes could go a long way towards refining this chronology, at present it offers a clear frame for situating surface remains within the three phases of occupation at Diouboye.

Table 8.1: Chronological assessment of surface contexts

\begin{tabular}{|l|c|c|c|c|c|}
\hline \multicolumn{1}{|c|}{ Area } & Type & Stratified Phases & Surface Phase & Rill Erosion & Sheet Erosion \\
\hline \hline Area A-N & Non-Res & - & - & Modest & Light \\
\hline Area A & Residential & Early B to Late & Late & Heavy & Light \\
\hline Area A-S & Non-Res & - & Middle & Modest & Modest \\
\hline Area S & Disturbed & Early A? & - & Light & Light \\
\hline Area B & Residential & - & Middle/Late & Heavy & Modest \\
\hline Area C & Residential? & Early A/B? & Middle/Late & Light & Heavy \\
\hline Area D & Residential? & - & Middle & Light & Modest \\
\hline Area F & Residential & Early A to Middle & Late & Heavy & Modest \\
\hline Area G & Residential & Early B to Late & Late & Heavy & Modest \\
\hline Area H & Residential & Early B to Late & Late & Modest & Light \\
\hline Area J/K & Residential & Early A to Middle & Middle & Modest & Light \\
\hline Area L & Disturbed & None & - & Light & Light \\
\hline Area M/N & Residential & Early A to Middle & Middle/Late & Modest & Light \\
\hline Area P & Residential? & - & Middle/Late? & Heavy & Light \\
\hline
\end{tabular}




\section{SPATIAL DIMENSIONS OF THE VILLAGE ECONOMY}

As summarized in Chapter 6, the diverse artifacts from Diouboye provide a means for evaluating the relative intensity and concentration of economic activities undertaken amidst and beyond the formal space defined by domestic huts, granaries, pot rests, and other architectural features. In this section I examine the material and spatial relations among surface data in order to develop a temporally coarse-grained, yet panoramic, view of subsistence, craft production, and long-distance exchange practices at multiple scales across the village.

\section{Activity Areas}

Beyond the issue of surface chronology, systematic surface collection across Diouboye aimed to identify relatively segregated spaces of food preparation and/or consumption, craft production, or refuse disposal—activity areas in archaeological parlance (e.g., Kent 1987). Of course, segregation varies according to the type of activity under consideration. Courtyards or domestic huts, for instance, usually accumulate artifacts used or made in a number of activities, while workshops more often accumulate debris from a restricted set of activities (Kent 1990). Further complicating the archaeological recognition of activity areas, multi-purpose tools such as stone blades or metal knives are ultimately discarded in only one of their use locales. Depositional pathways (e.g., primary versus secondary disposal) and post-depositional processes (e.g., erosion, decay) also work to aggregate or decompose "depositional sets" of artifacts from otherwise segregated activities (Carr 1984; Gregg et al. 1991; Schiffer 1987). Moreover, one cannot assume that the depositional sets representing similar activities, let alone different ones, will be of the same size, shape, or even relative artifact composition.

To work around these issues, I adopted an "unconstrained" approach (following Whallon 1984) in which one interpolates the relative densities of pertinent artifact types across the site surface, then employs multivariate analyses to identify points of similar artifact composition. A clustering analysis on these points can then parse out activity areas of variable size and shape. Because this approach cannot distinguish palimpsests created by overlapping activities or post-depositional processes (see Carr 1984, 1987), I

found it useful to consider spatial coherence in the density of individual artifact types and 
to measure the relative influence of erosion on a point-by-point basis using the surface erosion model described above.

Using data from systematic surface collection points, I first interpolated (naturalneighbor method, $2.6 \mathrm{~m}$ resolution) the counts of those artifact types presumably associated with subsistence and craft technologies at Diouboye-including functional rim sherd types, chipped stone debris, groundstone, and slag. Given their low frequencies, I excluded metal tools and ornaments, glass beads, figurine fragments, and other small finds from spatial analysis at this scale. After summing the artifact counts in each interpolated cell, I calculated the absolute and relative frequencies of each artifact type across a resampled (5.0 m resolution) grid of 2106 cells consistent with the resolution of the initial sampling strategy.

Multivariate analyses revealed relatively weak relationships among major artifact classes across the 2106 cells (Table 8.2). Chipped stone artifacts, for example, exhibited a very modest correlation with groundstone $\left(\mathrm{r}^{2}=0.338\right)$ and slag $\left(\mathrm{r}^{2}=0.367\right)$ at any given location. Slag demonstrated almost no correlation with rims from large and small pottery vessels, which were positively correlated with each other $\left(r^{2}=0.603\right)$. More specific artifact subclasses and types did not offer stronger correlations although, as discussed below, they did prove valuable for distinguishing activities between residential and nonresidential areas.

Table 8.2: $\quad$ Correlation matrix of surface artifact categories and erosion factors

\begin{tabular}{|l||rcccc|rrr|}
\hline & $\begin{array}{c}\text { Vessel } \\
\text { Large }\end{array}$ & $\begin{array}{c}\text { Vessel } \\
\text { Small }\end{array}$ & $\begin{array}{c}\text { Chipped } \\
\text { Stone }\end{array}$ & $\begin{array}{c}\text { Ground } \\
\text {-stone }\end{array}$ & Slag & $\begin{array}{c}\text { LS } \\
\text { Value }\end{array}$ & $\begin{array}{c}\text { Ceramic } \\
\text { Density }\end{array}$ & $\begin{array}{c}\text { Stone } \\
\text { Density }\end{array}$ \\
\hline \hline Vessel Large & 1.000 & 0.603 & 0.155 & 0.104 & 0.097 & 0.000 & 0.441 & 0.239 \\
\hline Vessel Small & 0.603 & 1.000 & 0.115 & 0.143 & 0.085 & -0.026 & 0.436 & 0.179 \\
\hline Chipped Stone & 0.155 & 0.115 & 1.000 & 0.338 & 0.367 & -0.047 & 0.194 & 0.035 \\
\hline Groundstone & 0.104 & 0.143 & 0.338 & 1.000 & 0.155 & 0.014 & 0.145 & 0.121 \\
\hline Slag & 0.097 & 0.085 & 0.367 & 0.155 & 1.000 & -0.049 & 0.305 & 0.038 \\
\hline LS Erosion & 0.000 & -0.026 & -0.047 & 0.014 & -0.049 & 1.000 & 0.034 & 0.171 \\
\hline Ceramic Density & 0.441 & 0.436 & 0.194 & 0.145 & 0.305 & 0.034 & 1.000 & 0.437 \\
\hline Stone Density & 0.239 & 0.179 & 0.035 & 0.121 & 0.038 & 0.171 & 0.437 & 1.000 \\
\hline
\end{tabular}


Table 8.2 further shows that interpolated artifact counts either had a weakly positive or weakly negative correlation with indices of erosion, including LS values and the densities of surface ceramics and sandstone cobbles. In fact, the exclusion of 631 grid cells with an LS value greater than 1.0 did not significantly impact the resulting correlations among artifacts. On the whole, post-depositional erosion has not significantly affected relations among surface materials, at least not at the scale of the entire site.

In order to evaluate whether the positive (albeit weak) correlations among surface artifacts were depositional sets resulting from the spatial segmentation of craft and/or subsistence activities, I employed cluster analysis (Ward's method) to define seven sets of grid cells with similar proportions of large and small vessel rims (see classification in Appendix D), chipped stone, groundstone, and slag. As summarized in Table 8.3, grid cells with relatively high percentages of rim sherds were assigned to Artifact Sets 1, 2, and 3, while those with greater proportions of chipped stone, slag, and groundstone were assigned, respectively, to Artifact Sets 4/5, 6, and 7.

Table 8.3: Summary of relative artifact class abundance by artifact set

\begin{tabular}{|c|c|c|c|c|c|c|c|c|c|c|c|}
\hline \multirow{3}{*}{\multicolumn{2}{|c|}{$\begin{array}{c}\text { Artifact Set } \\
\text { Total } \\
n \\
\end{array}$}} & \multicolumn{10}{|c|}{ Artifact Class } \\
\hline & & \multicolumn{2}{|c|}{ Large Vessel } & \multicolumn{2}{|c|}{ Small Vessel } & \multicolumn{2}{|c|}{ Chipped Stone } & \multicolumn{2}{|c|}{ Groundstone } & \multicolumn{2}{|c|}{ Slag } \\
\hline & & $\mu$ & $\sigma$ & $\mu$ & $\sigma$ & $\mu$ & $\sigma$ & $\mu$ & $\sigma$ & $\mu$ & $\sigma$ \\
\hline 1 & 233 & 0.654 & 0.226 & 0.079 & 0.104 & 0.085 & 0.149 & 0.002 & 0.010 & 0.005 & 0.023 \\
\hline 2 & 70 & 0.043 & 0.081 & 0.914 & 0.106 & 0.015 & 0.037 & 0.003 & 0.019 & 0.000 & 0.000 \\
\hline 3 & 408 & 0.181 & 0.143 & 0.346 & 0.111 & 0.188 & 0.168 & 0.013 & 0.032 & 0.005 & 0.020 \\
\hline 4 & 663 & 0.081 & 0.105 & 0.066 & 0.073 & 0.509 & 0.276 & 0.025 & 0.051 & 0.025 & 0.049 \\
\hline 5 & 437 & 0.007 & 0.017 & 0.003 & 0.011 & 0.966 & 0.055 & 0.009 & 0.023 & 0.000 & 0.003 \\
\hline 6 & 138 & 0.113 & 0.186 & 0.106 & 0.145 & 0.275 & 0.279 & 0.053 & 0.093 & 0.353 & 0.202 \\
\hline 7 & 157 & 0.111 & 0.166 & 0.110 & 0.148 & 0.182 & 0.247 & 0.466 & 0.312 & 0.004 & 0.019 \\
\hline
\end{tabular}

When projected onto the site-level grid (Figure 8.7), these artifact sets revealed two general patterns. First, "unconstrained” spatial clusters of variable shape and size did occur within the surface artifact assemblage. Even after dismissing clusters with fewer than four adjacent grid cells (less than $100 \mathrm{~m}^{2}$ ), and those affected by rill erosion, it was 
Figure 8.7: Surface distribution of artifact sets

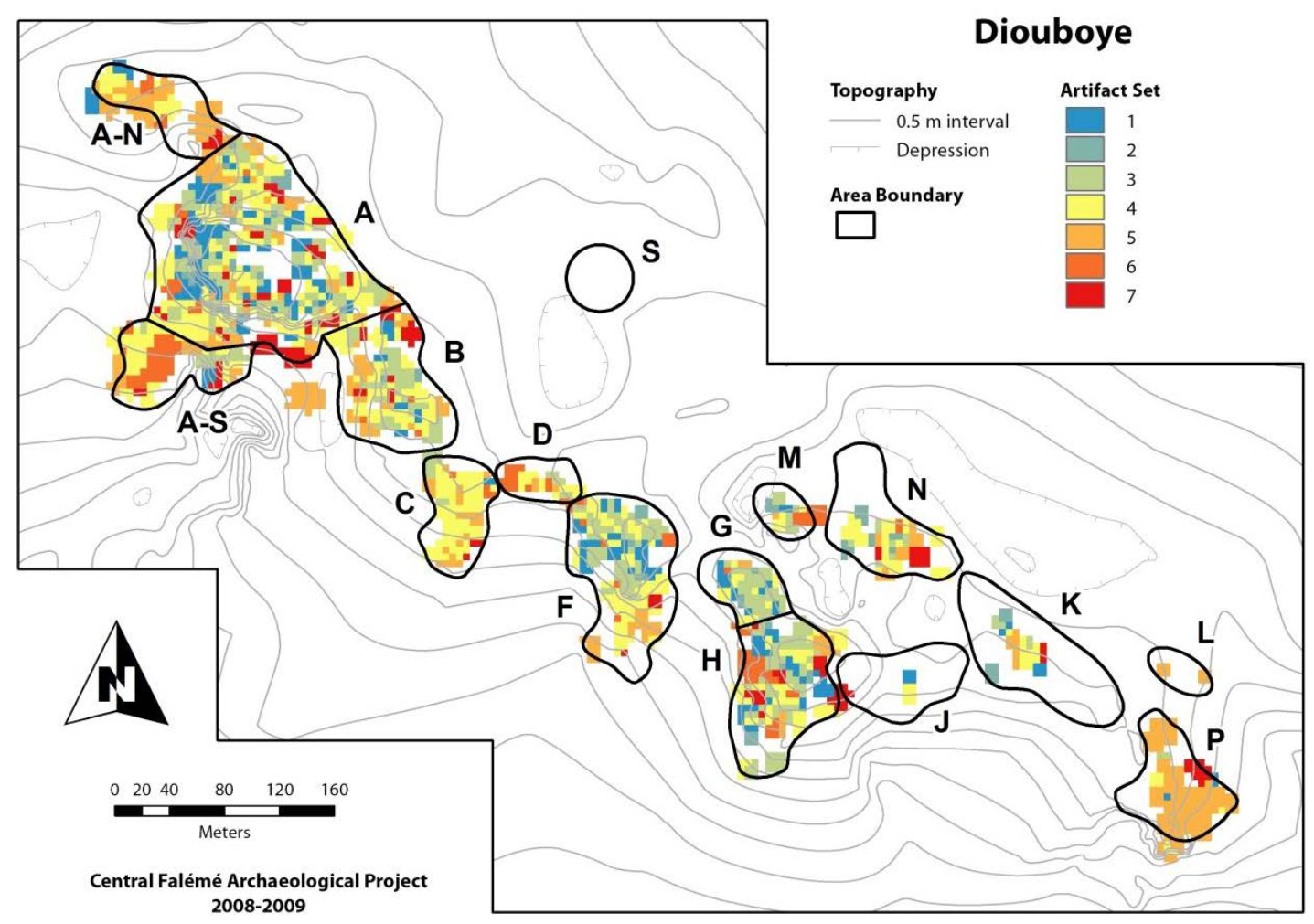

possible to define activity areas with greater concentrations of rim sherds related to food storage and culinary activities, in contrast to those with higher proportions of chipped stone, groundstone, and slag presumably associated with more specialized subsistence and craft activities. Second, these activity areas were small and fairly diverse in residential areas, particularly Area A, perhaps resulting from the segmentation of quotidian household activities and refuse disposal. While these patterns unfortunately exhaust the limited interpretive potential of small-scale activity areas at Diouboye, they do call attention to differences among intra-site areas-Artifact Sets 1-4 predominating in residential areas and Artifact Sets 5-7 occurring more commonly in non-residential ones.

\section{Intra-Site Areas and Wards}

The actual frequencies of the artifacts recovered from surface contexts clearly underline differences between areas and wards (Table 8.4). As predicted by the unconstrained clustering of artifact sets, household activities and depositional practices 
within Area A, Area F, and Area G generated substantial quantities of pottery, even as they demonstrated considerable diversity in the ratio of rims from large and small vessels. Chipped stone, groundstone, and slag occurred more frequently in non-residential areas, even though these materials exhibited considerable heterogeneity across the site. Area A$\mathrm{N}$ and Area $\mathrm{P}$, for example, had substantially greater quantities of chipped stone debris, while Area D had the highest concentrations of slag. Keeping in mind the issues of surface chronology and post-depositional bias, I turn now to investigate what these differences reveal about the structuring of socioeconomic practices between nonresidential and residential areas, these latter each interpreted as the space occupied by one or more household compounds comprising some form of corporate kin group.

Table 8.4: $\quad$ Summary of artifact abundance (systematic collection) by area

\begin{tabular}{|l|c|cc|cc|cc|cc|cc|}
\hline \multicolumn{2}{|c|}{$\begin{array}{r}\text { Area/Ward } \\
\text { Artifact Total }\end{array}$} & \multicolumn{9}{c|}{ Large Vessel } & \multicolumn{2}{c|}{ Small Vessel } & \multicolumn{2}{c|}{ Chipped Stone } & \multicolumn{2}{c|}{ Groundstone } & \multicolumn{2}{c|}{ Slag } \\
& $n$ & $n$ & $\%$ & $n$ & $\%$ & $n$ & $\%$ & $n$ & $\%$ & $n$ & $\%$ \\
\hline \hline Area A-N & 107 & 3 & $2.8 \%$ & 0 & $0.0 \%$ & 93 & $86.9 \%$ & 2 & $1.9 \%$ & 6 & $5.6 \%$ \\
\hline Area A & 5135 & 171 & $3.3 \%$ & 94 & $1.8 \%$ & 580 & $11.3 \%$ & 53 & $1.0 \%$ & 17 & $0.3 \%$ \\
\hline Area A-S & 1561 & 19 & $1.2 \%$ & 16 & $1.0 \%$ & 333 & $21.3 \%$ & 12 & $0.8 \%$ & 60 & $3.8 \%$ \\
\hline Area B & 1881 & 45 & $2.4 \%$ & 41 & $2.2 \%$ & 276 & $14.7 \%$ & 26 & $1.4 \%$ & 10 & $0.5 \%$ \\
\hline Area C & 1383 & 41 & $3.0 \%$ & 25 & $1.8 \%$ & 553 & $40.0 \%$ & 32 & $2.3 \%$ & 17 & $1.2 \%$ \\
\hline Area D & 199 & 4 & $2.0 \%$ & 4 & $2.0 \%$ & 43 & $21.6 \%$ & 3 & $1.5 \%$ & 12 & $6.0 \%$ \\
\hline West Ward & $\mathbf{1 0 , 4 4 8}$ & $\mathbf{2 8 3}$ & $\mathbf{2 . 7 \%}$ & $\mathbf{1 8 0}$ & $\mathbf{1 . 7 \%}$ & $\mathbf{2 0 6 0}$ & $\mathbf{1 9 . 7 \%}$ & $\mathbf{1 2 8}$ & $\mathbf{1 . 2 \%}$ & $\mathbf{1 2 2}$ & $\mathbf{1 . 2 \%}$ \\
\hline Area F & 2542 & 158 & $6.2 \%$ & 73 & $2.9 \%$ & 480 & $18.9 \%$ & 10 & $0.4 \%$ & 23 & $0.9 \%$ \\
\hline Area G & 841 & 57 & $6.8 \%$ & 32 & $3.8 \%$ & 30 & $3.6 \%$ & 2 & $0.2 \%$ & 5 & $0.6 \%$ \\
\hline Area H & 2501 & 62 & $2.5 \%$ & 47 & $1.9 \%$ & 94 & $3.8 \%$ & 13 & $0.5 \%$ & 23 & $0.9 \%$ \\
\hline Area J/K & 118 & 6 & $5.1 \%$ & 1 & $0.8 \%$ & 27 & $22.9 \%$ & 2 & $1.7 \%$ & 1 & $0.8 \%$ \\
\hline Area M/N & 368 & 10 & $2.7 \%$ & 9 & $2.4 \%$ & 51 & $13.9 \%$ & 3 & $0.8 \%$ & 4 & $1.1 \%$ \\
\hline Area P/L & 804 & 4 & $0.5 \%$ & 4 & $0.5 \%$ & 631 & $78.5 \%$ & 5 & $0.6 \%$ & 0 & $0.0 \%$ \\
\hline East Ward & $\mathbf{7 1 7 4}$ & $\mathbf{2 9 7}$ & $\mathbf{4 . 1 \%}$ & $\mathbf{1 6 6}$ & $\mathbf{2 . 3 \%}$ & $\mathbf{1 3 1 3}$ & $\mathbf{1 8 . 3 \%}$ & $\mathbf{3 5}$ & $\mathbf{0 . 5 \%}$ & $\mathbf{5 6}$ & $\mathbf{0 . 8 \%}$ \\
\hline
\end{tabular}

\section{Subsistence Economy: Food Preparation and Consumption}

Organic plant and animal remains were mainly absent from surface contexts, but more durable artifacts associated with the underlying subsistence technologies were well 
in abundance. Due to the complex relations inhering between artifact form and function, many of these tools, including pots, stone or metal knives, projectile points, and grinding slabs and handstones, probably served a variety of practical uses, only some of which pertained directly to subsistence. For this reason, I defer discussion of chipped stone and iron tools to focus here on the groundstone and ceramic technologies more reliably associated with food storage, preparation, and consumption practices.

Groundstone artifacts could have been used to process any number of organic or inorganic materials, but the predominance of flat or lightly concave grinding slabs $(n=12)$, rather than basin-shaped mortars $(n=1)$, alongside a number of one-handed and two-handed handstones $(n=28)$, strongly suggests that these implements served primarily for processing grain (see Chapter 6). Grinding slabs, handstones, and hammerstones also occurred in similar ratios in each residential area with a sample size greater than two (Figure 8.8), consistent with their use as part of a groundstone toolkit. This lends support to the hypothesis that people employed hammerstones to rejuvenate grinding slabs in addition to other possible uses for crushing minerals, mashing foodstuffs, or reducing chipped stone cores.

Figure 8.8: Relative distribution of groundstone tool types by area

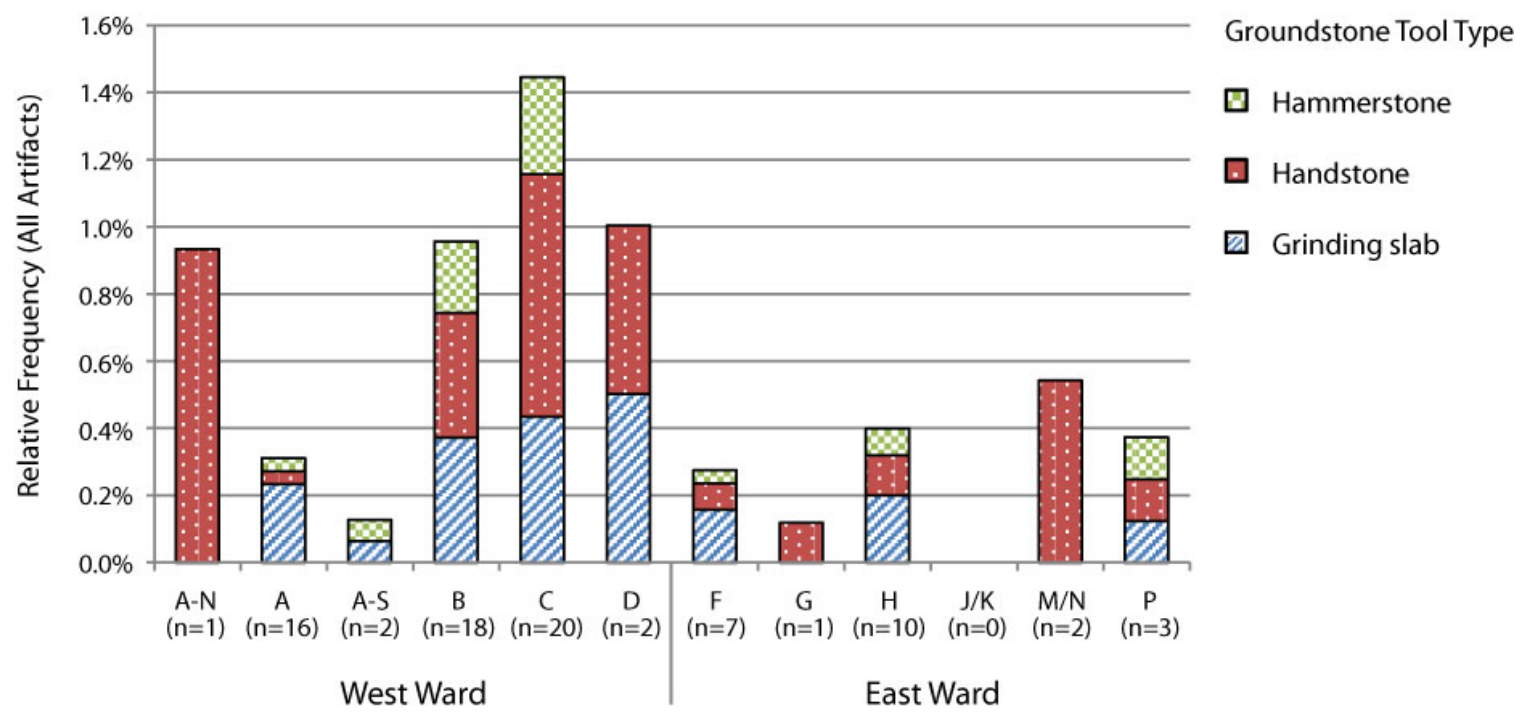


Figure 8.8 shows that groundstone toolkits comprised a greater proportion of the surface artifacts in Area B and Areas C/D, despite the absence of discrete groundstone activity areas within these spaces (Figure 8.6). While grinding activities were not strongly segregated within the village, they were evidently more intense in the West Ward during the Middle to Late Diouboye phases. Explanations for this intensity include the greater production and processing of staples, a preference for cuisine prepared with more coarsely ground grain (David 1998:25-28; Gallagher 2010:161), the presence of a communal space for intra-household grinding activities, or a greater proportion of married women with their own groundstone implements (Casey 2000:53; David 1998:23). Given these possibilities, it is not yet evident if, or at what scale, grinding activities were specialized within the community.

Turning to storage vessels and cookware, Figure 8.7 above may have revealed a predominance of sherds from both large and small vessels (artifact sets 1-3) at residential areas, but a closer look at the rim sherd assemblage (Figure 8.9) found larger vessels to account for a greater proportion of the sherds than their smaller counterparts - the main exceptions being those areas with extremely small samples.

Figure 8.9: $\quad$ Relative abundance of vessel size by area

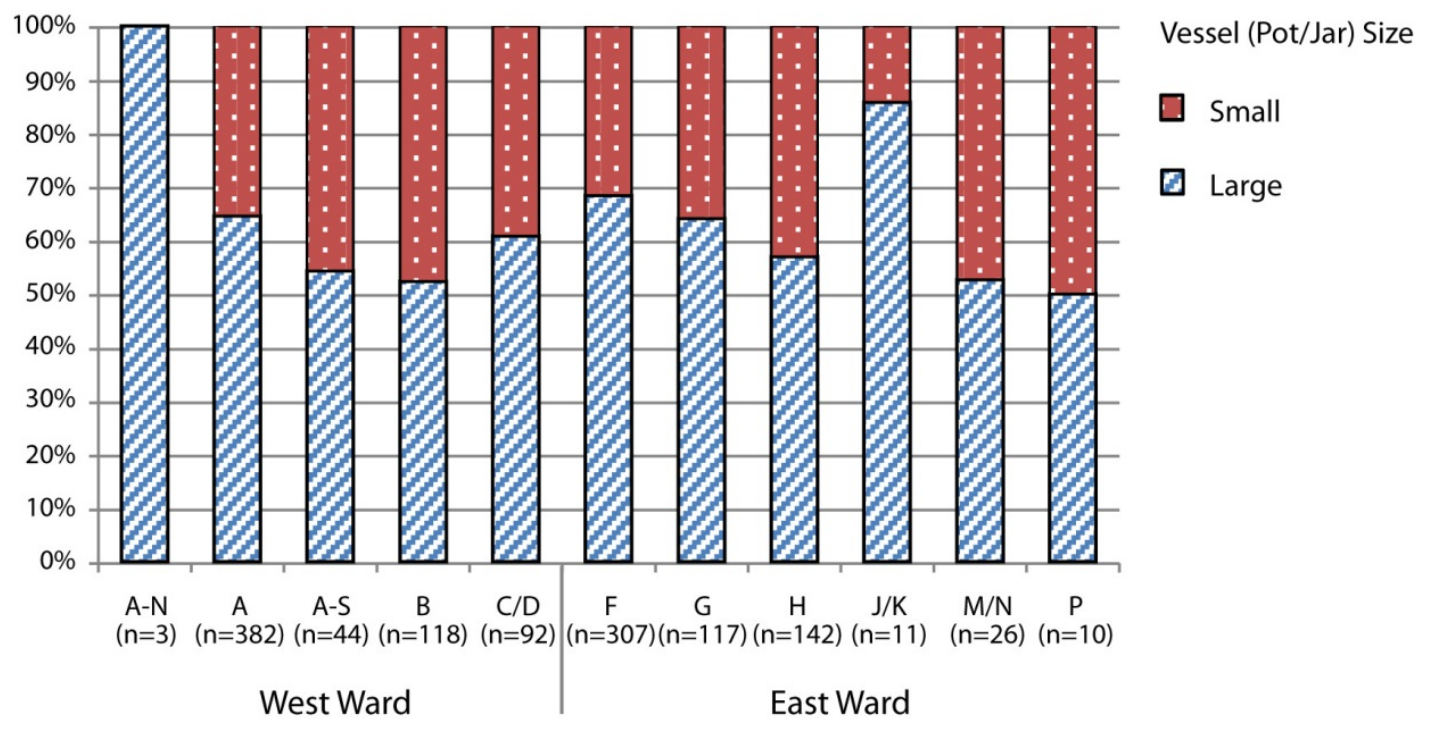


Figure 8.10: Relative abundance of vessel forms by area

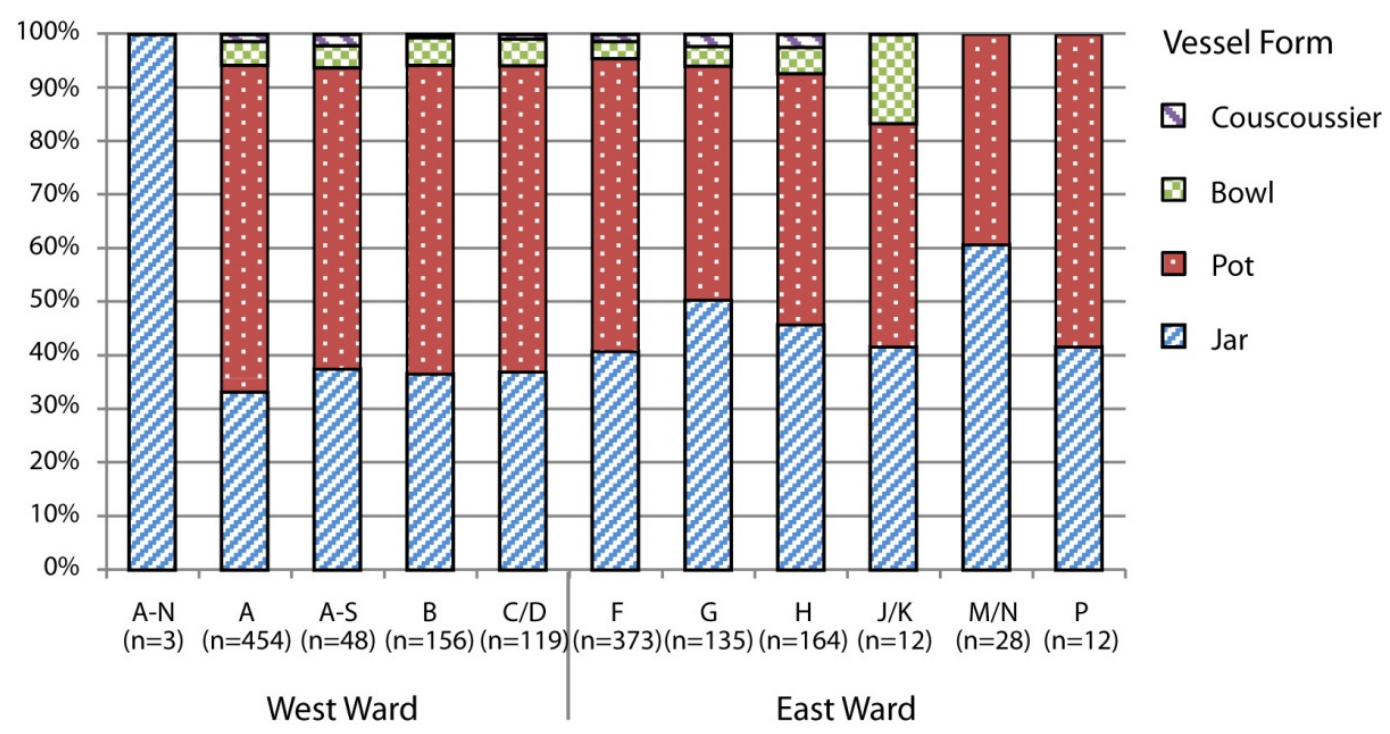

Bowl, pot, and jar rims demonstrated subtle variability across residential areas, particularly those from different wards (Figure 8.10). Bowl rims and couscoussier fragments, potentially derived from the same sorts of vessels, turned up in similarly low numbers across the site. Meanwhile, jar $(\mathrm{n}=322)$ and pot $(\mathrm{n}=363)$ rims occurred in similar frequencies in the East Ward, but pot $(n=462)$ substantially outnumbered jar $(n=263)$ rims in the West Ward.

Figure 8.11 displays both these trends together as the relative abundance of five functional vessel types defined according to form and size. While stratigraphic deflation (De Barros 1982) and differential breakage rates (Mayor 1992) may have affected the counts of these types, their proportions should nevertheless reflect the suite of economic practices involving ceramic containers in each area (Mills 1989). On the basis of ethnographic analogy linking vessel form to function, I assume that people used bowls primarily for serving, pots and small jars for cooking and carrying water, and large jars for storage (see Chapter 6). Accordingly, the residential pottery assemblages from Diouboye fit nicely with a general trend among ethnographic households in West Africa to have two or three times as many serving and water carrying vessels as storage vessels (e.g., David and Hennig 1972; Gallay 1970). 
Figure 8.11: Relative abundance of vessel types by area

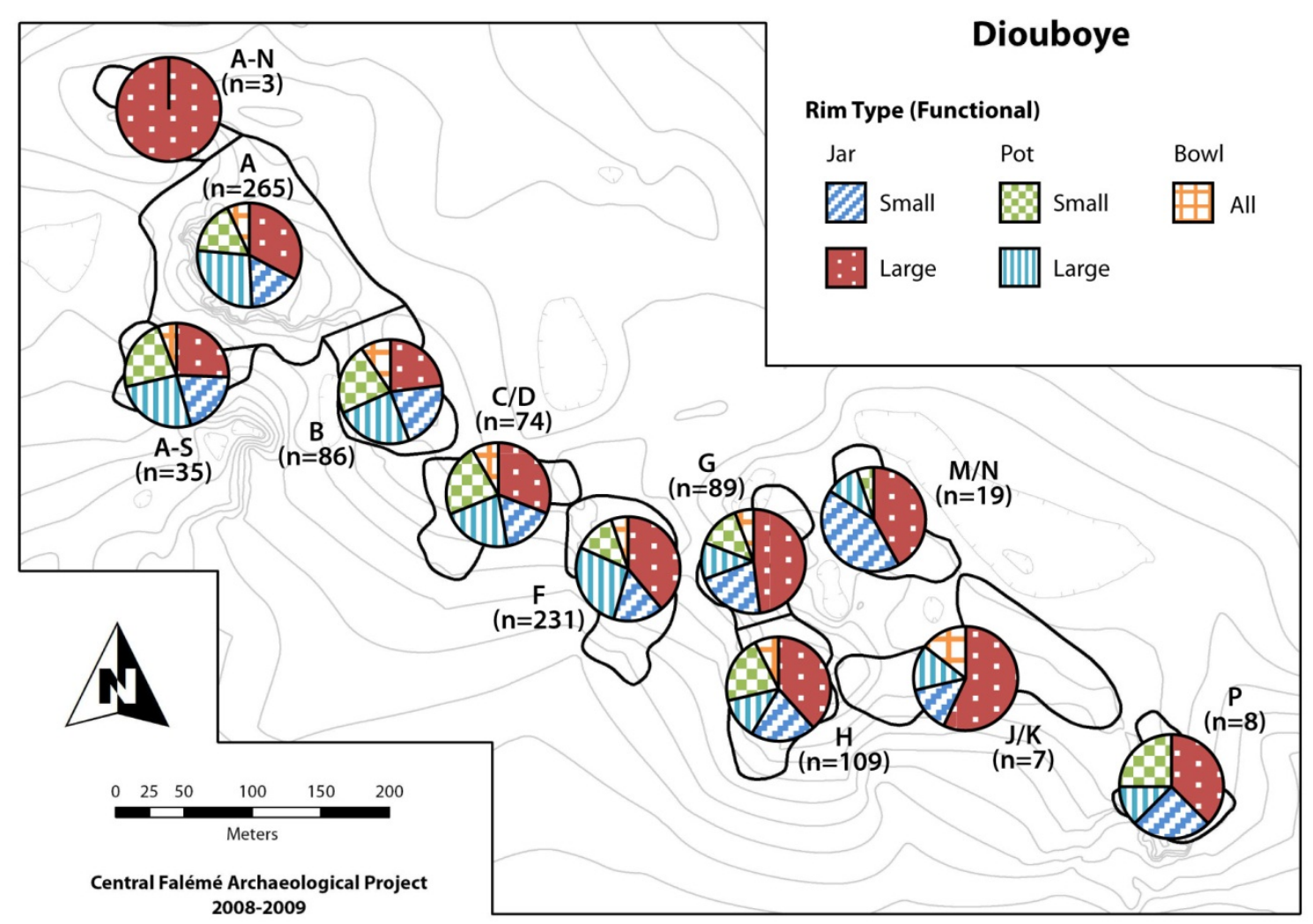

The similar proportions of functional vessel types across residential and nonresidential areas within each ward suggests that practices involving food storage, transportation, cooking, and serving were not strongly segregated into discrete activity areas within the village during the Middle to Late occupation phases. At the same time, a comparison of surface assemblages reveals a significant difference in the associations of these pottery types with each of the two wards ( $\left.n=926, \chi^{2}=14.35, \rho=0.006\right)$. While I hesitate to interpret these patterns as "specialized" subsistence activities, they do underscore possible differences in culinary regimes (Ashley 2010) or perhaps the internal social organization of food preparation and consumption according to social segments within each ward—a possibility further explored below and in the next chapter. 


\section{Craft Economy}

Beyond subsistence practices, earthenware pottery and other durable objects from the surface of Diouboye document the technologies of their own production. Drawing upon my analysis of these ceramic (Appendix D), lithic (Appendix E), and metallurgical (Appendix F) artifacts, I turn now to examine the socio-spatial organization of craft technologies across residential and non-residential areas of the village.

\section{Pottery Production}

The raw materials, tools, and gestures deployed in pottery manufacture-from clay procurement and processing, to vessel formation and decoration, to firing-have generated a number of quantitative and qualitative differences visible in the rim and body sherds from Diouboye. The usual caveats regarding deflation and other post-depositional disturbance notwithstanding, techniques of pottery production differed across the site in ways most parsimoniously explained by differential patterns of consumption. At a minimum this confirms that local potters participated in a single technical tradition, even if they did not belong to the same household or corporate kin group.

Surface materials manifested two predominant paste recipes with grog and, to much lesser extent, grit inclusions (Figure 8.12). Although grit-tempered sherds were significantly associated with the East Ward $\left(n=11,048, \chi^{2}=9.79, \rho=0.002\right)$, this trend appears to derive from the temporal shift towards grog temper observed in stratified sequences. In fact, the higher proportions of grit-tempered wares in Areas J/K and Area $\mathrm{N}$ were consistent with Middle Diouboye phase assemblages from excavated strata.

Rim forms, as a proxy measure of shaping techniques, occurred in similar proportions in the pottery assemblages from each area, even after controlling for different vessel forms (i.e., bowl, pot, jar, and unknown). Figure 8.13 shows that, with the exception of non-residential Area A-N and Area P, people used and discarded vessels with similar proportions of vertical collar (EvC), everted angular (ErA), everted round (ErR), flared angular (EfA), and flared round (EfR) rims. As with paste types, the low numbers of everted round rims in the Areas $\mathrm{J} / \mathrm{K}$, Area $\mathrm{M}$, and Area $\mathrm{N}$ may be related to the abandonment of these areas by the Late Diouboye phase. 
Figure 8.12: Relative abundance of body sherd paste types by area

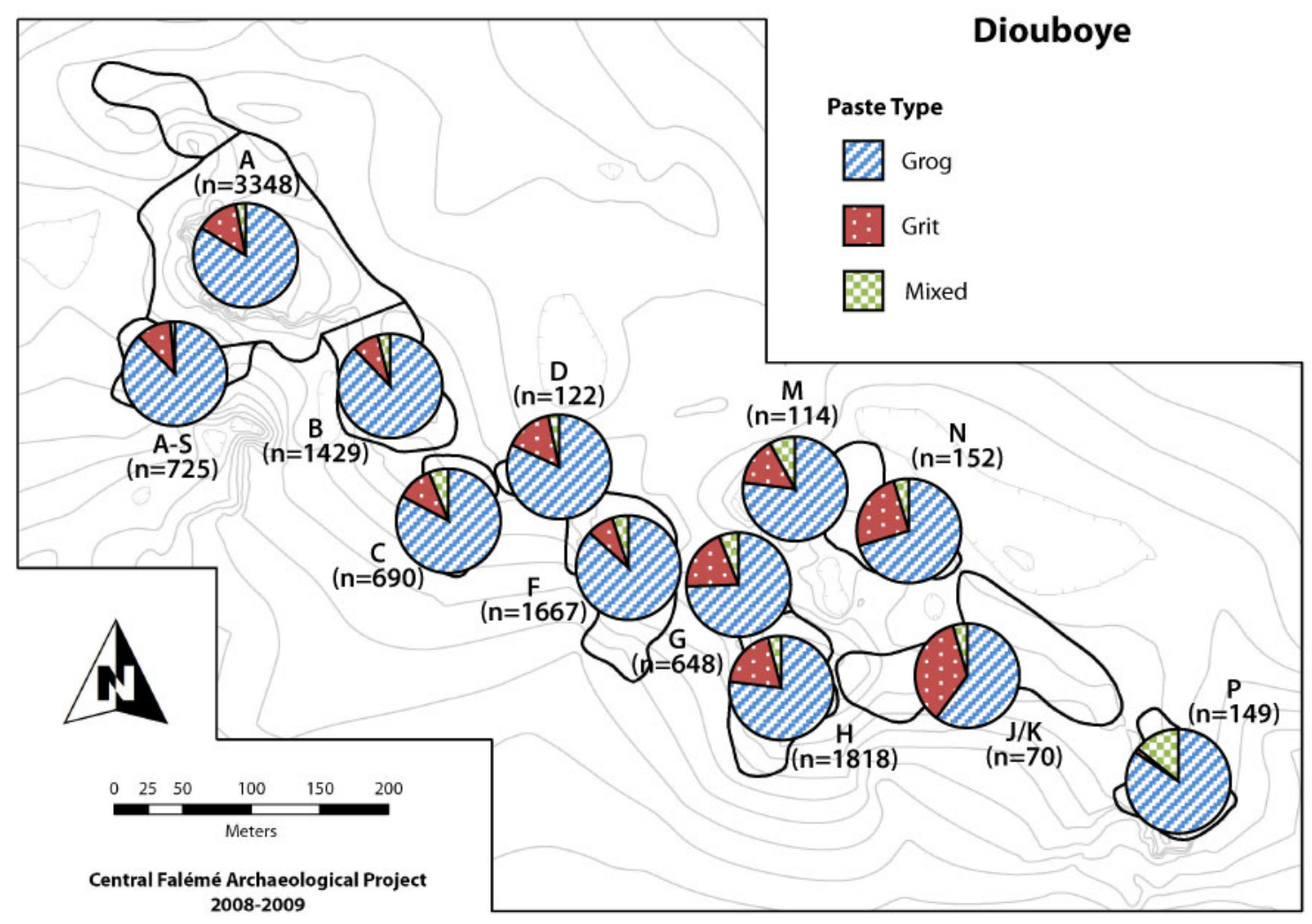

Figure 8.13: Relative distribution of inflected rim types by area

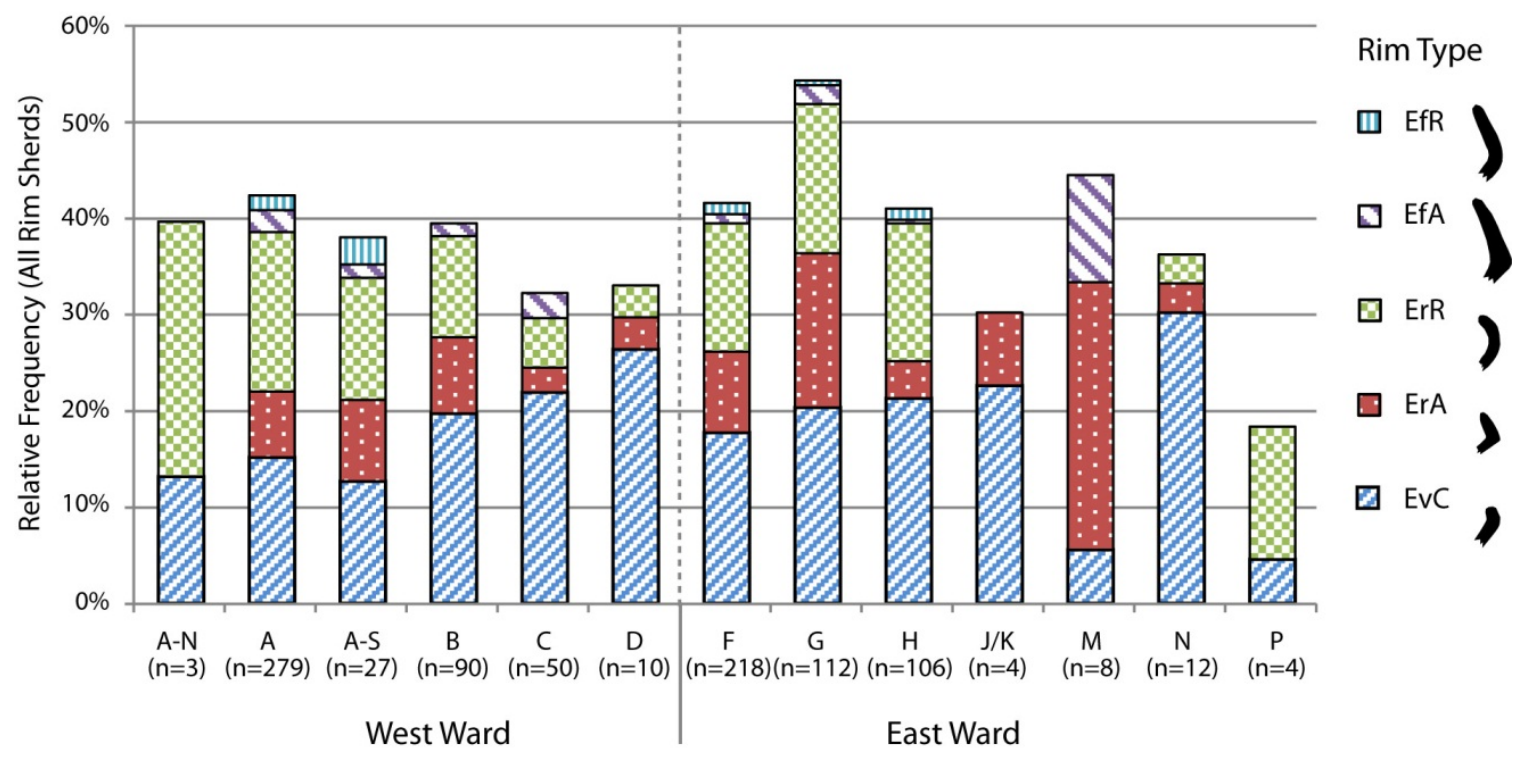


Figure 8.14: Relative distribution of simple, collared, and ledged rim types by area

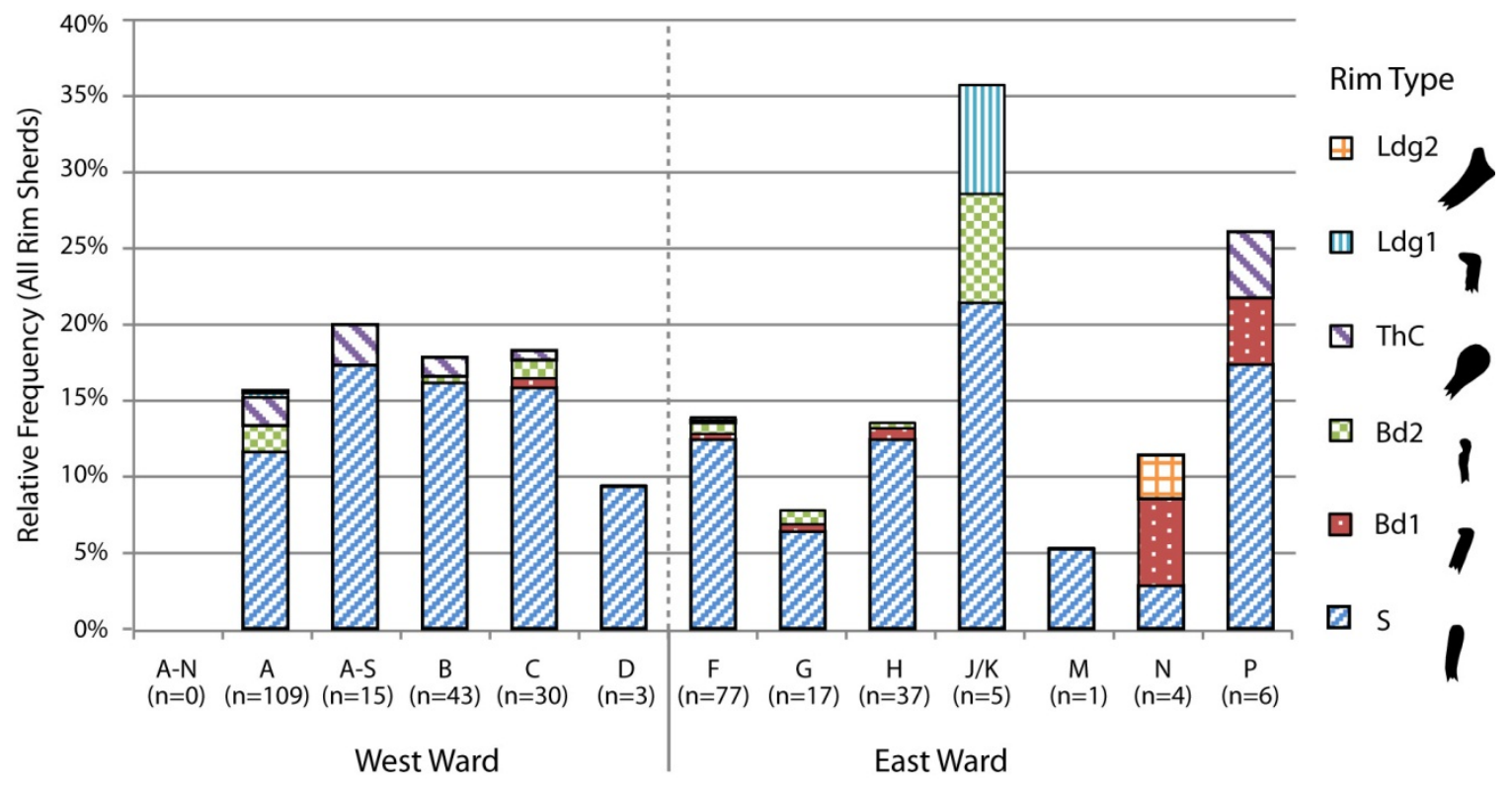

Figure 8.15: Distribution of base types by ward

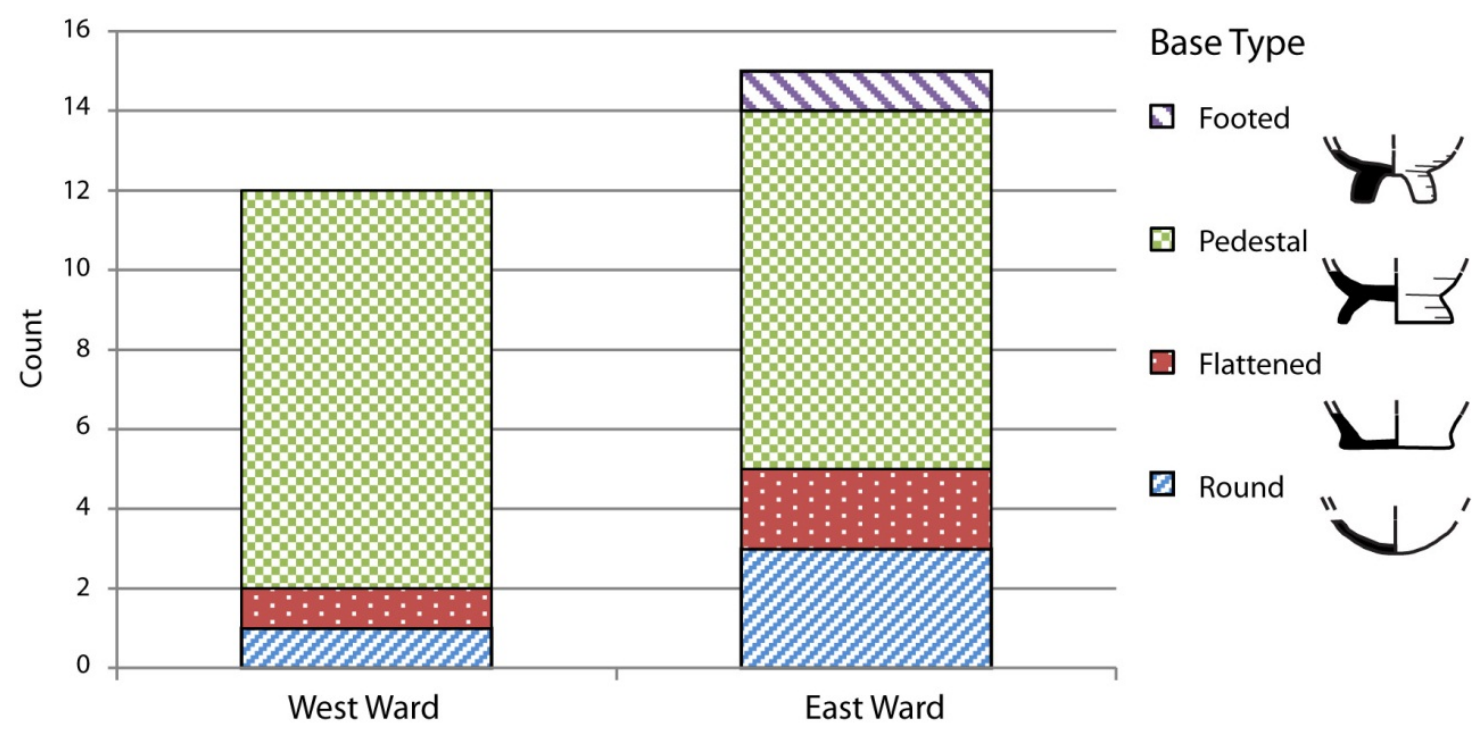

Figure 8.14 shows slightly more diversity among ledged and collared rims, with thickened collar (ThC) rims found mainly in the West Ward, and everted bead (Bd1) rims found more often in the East Ward. Despite the small sample sizes of these latter 
subclasses, this pattern may be significant insofar as the thickened collar rim form is more commonly found in the Niger River basin (e.g., MacDonald 1997:184; Togola 2008:58), than in the Middle Senegal region. The small samples of base sherds further highlight technical parallels in pottery across both wards (Figure 8.15).

While rouletted pottery motifs changed significantly over time in each excavated sequence at Diouboye, this was not the case for surface treatment. For this reason, the relative abundance of these techniques can be compared across surface assemblages representing different occupational phases. In this regard, Figure 8.16 shows that residential areas generally had similar proportions of rim sherds treated with slip (1020\%) and polish (3-10\%). The absence of slipped rims at Areas J/K may reflect the small sample size, while the exceptionally high incidence of red slip at Area G may in fact correspond to a greater use of decorated pottery in this area, a trend borne out by comparison of excavated pottery assemblages in the following chapter.

\section{Figure 8.16: Relative abundance of rim sherd surface treatment by area}

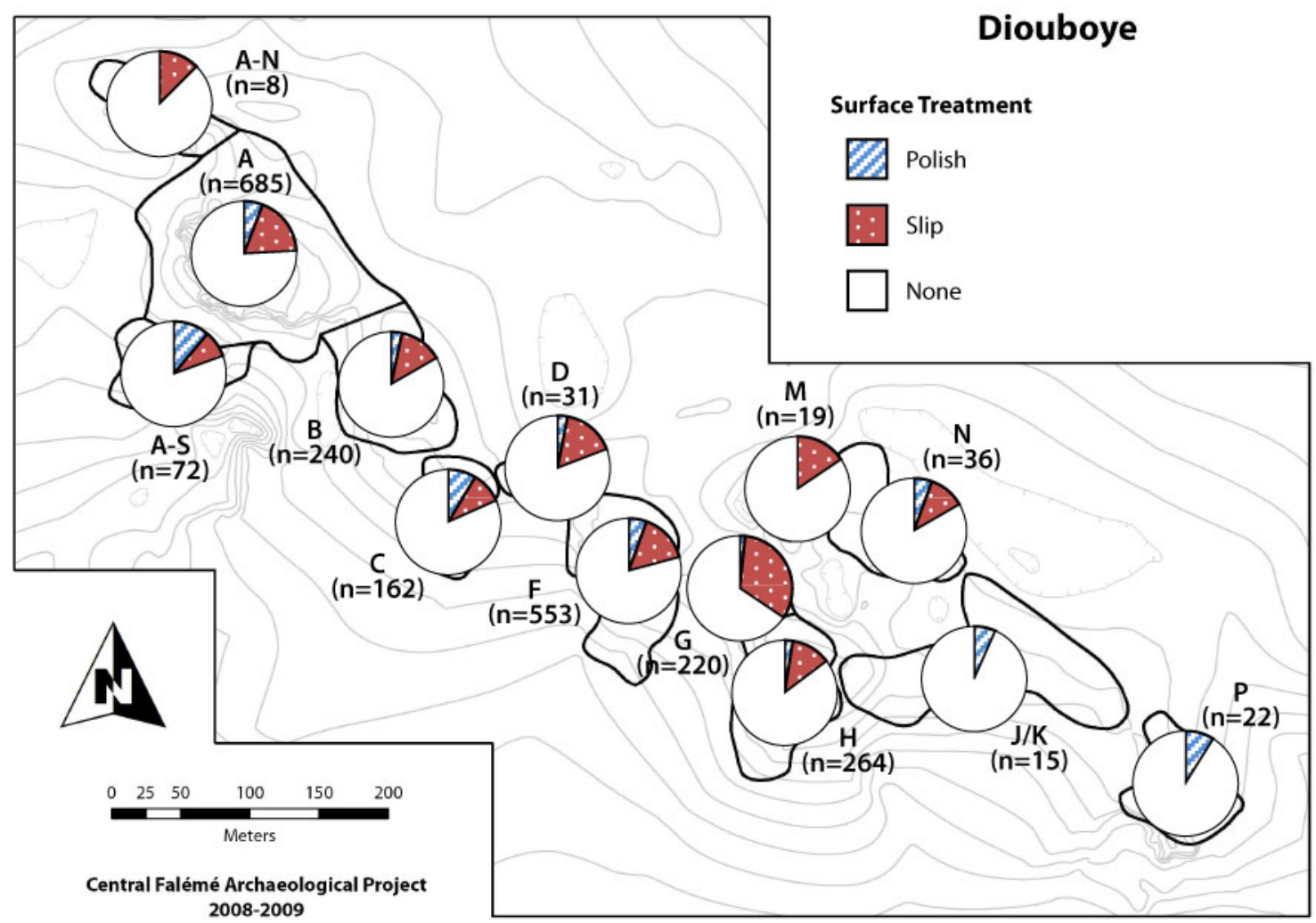


All in all, the potters furnishing Diouboye selected techniques from a repertoire of paste recipes, base and rim styles, and decorative elements grounded in the dynamic logistical and social contingencies of a shared tradition during the Middle to Late phases of occupation. Even if people of each residential area ward produced their own potterya hypothesis that could explain subtle differences in rim formation techniques-the practical knowledge (i.e., gestures, tools, and techniques) associated with pottery production was nevertheless shared among craftspeople across the village. That is to say, it appears that people did not deploy pottery production to signal social boundaries within the community.

\section{Chipped Stone Production and Use}

Some chipped stone artifacts collected from the surface of Diouboye undoubtedly derived from redeposition in the course of earthen wall construction and decay. Nonetheless, significant differences between this assemblage and those from nearby LSA sites were consistent with the economies of sedentary village and mobile hunter-gathererherder communities, respectively (see Appendix E). Accordingly, these artifacts provide some insights onto the socio-spatial organization of chipped stone reduction and use at Diouboye.

As illustrated above in Figure 8.7, grid cells with elevated concentrations of chipped stone artifacts (Artifact Sets 4-5) occurred across Diouboye, but were more frequent in non-residential areas. A closer look at the interpolated counts of lithic artifact categories shows modest positive correlations among debitage, including angular shatter, bipolar flakes, and freehand flakes (Table 8.5). The weaker positive correlations between these detached pieces and raw material nodules, cores, and formal tools undoubtedly results from the low frequencies of these artifacts. The weak, if not negative, correlation between hammerstones and debitage further supports the contention that the former served primarily to rejuvenate other groundstone implements, not to knap chipped stone. Overall, these data suggest that practices of primary reduction were not spatially segregated from those of secondary reduction and/or tool use. 
Table 8.5: Correlation matrix of interpolated counts of lithic artifact categories

\begin{tabular}{|c|c|c|c|c|c|c|c|}
\hline & $\begin{array}{c}\text { Raw } \\
\text { Material }\end{array}$ & Core & Shatter & $\begin{array}{l}\text { Bipolar } \\
\text { Flake }\end{array}$ & $\begin{array}{l}\text { Freehand } \\
\text { Flake }\end{array}$ & $\begin{array}{c}\text { Formal } \\
\text { Tool }\end{array}$ & $\begin{array}{c}\text { Hammer } \\
\text {-stone }\end{array}$ \\
\hline Raw Material & 1.000 & 0.193 & 0.374 & 0.382 & 0.314 & 0.187 & -0.007 \\
\hline Core & 0.193 & 1.000 & 0.370 & 0.362 & 0.427 & 0.269 & 0.102 \\
\hline Shatter & 0.374 & 0.370 & 1.000 & 0.699 & 0.747 & 0.445 & 0.064 \\
\hline Bipolar Flake & 0.382 & 0.362 & 0.699 & 1.000 & 0.656 & 0.358 & 0.012 \\
\hline Freehand Flake & 0.314 & 0.427 & 0.747 & 0.656 & 1.000 & 0.445 & 0.058 \\
\hline Formal Tool & 0.187 & 0.269 & 0.445 & 0.358 & 0.445 & 1.000 & -0.005 \\
\hline Hammerstone & -0.007 & 0.102 & 0.064 & 0.012 & 0.058 & -0.005 & 1.000 \\
\hline
\end{tabular}

Figure 8.17: Relative abundance of chipped stone raw material by area

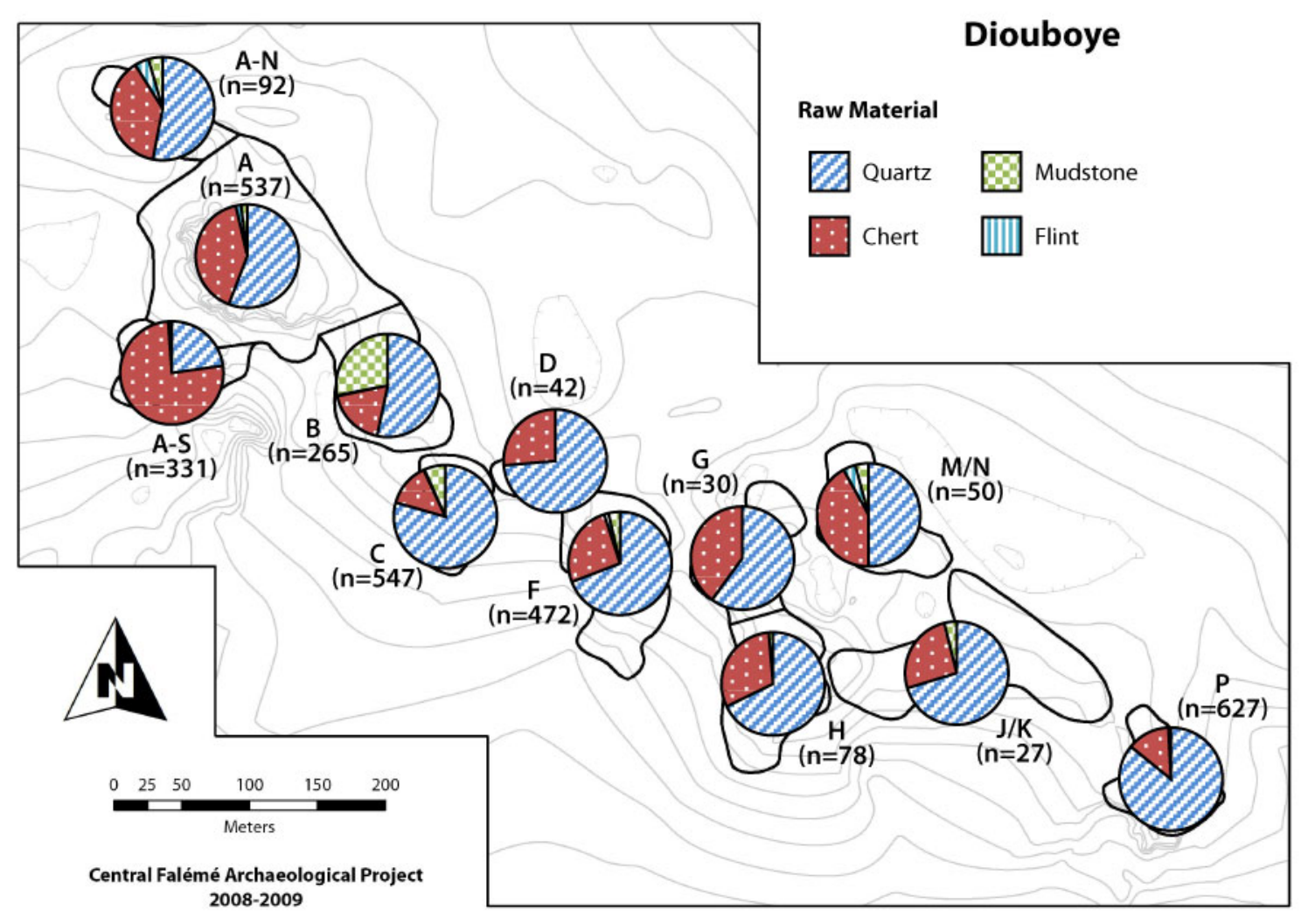


Spatial relations among chipped stone artifacts become more salient when one considers their distribution across areas and wards. The relative frequencies of raw materials, for example, varied markedly across the site, with the highest proportions of chert occurring in the Area A-S (Figure 8.17). Meanwhile, the reduction of mudstone was more-or-less restricted to Area B and Area C, where seven macrolithic scatters appeared outside the more formalized space defined by architectural foundations and other domestic debris.

Despite this variability in raw material, the relative abundance of lithic artifact types was relatively constant across the site, a pattern that holds for the samples of quartz (Figure 8.18), chert/flint (Figure 8.19), and mudstone artifacts (see Unit C in Chapter 7). At both residential and non-residential areas, people reduced quartz to exhaustion to obtain sharp bipolar and freehand flakes, but rarely undertook secondary reduction to make formal tools with this material. For chert, on the other hand, the greater numbers of freehand flakes point to less expedient reduction culminating, in some cases, with formal tools such as blades and scrapers. While chipped stone reduction may have been more intense in non-residential areas (Area A-N, Area A-S, Area P, and perhaps also Areas C/D) where chipped stone artifacts accounted for a greater proportion of all artifacts, the actual technical knowledge and skills associated with stone tools were not strongly segregated within the village community.

Differences in the intensity of lithic reduction between residential and nonresidential areas do, however, raise the question: what were the villagers of Diouboye using stone tools for? Given the low frequencies of formal tools, Figure 8.20 and Figure 8.21 display the combined counts of both formal and informal tool types recovered from each area. Assuming that form had some bearing on overall histories of use (Appendix E), these data support the contention that people employed chipped stone tools for a similar range of cutting, scraping, perforating, and perhaps fire-starting tasks across residential areas, despite the availability of iron tools. 
Figure 8.18: Relative distribution of quartz artifact types by area

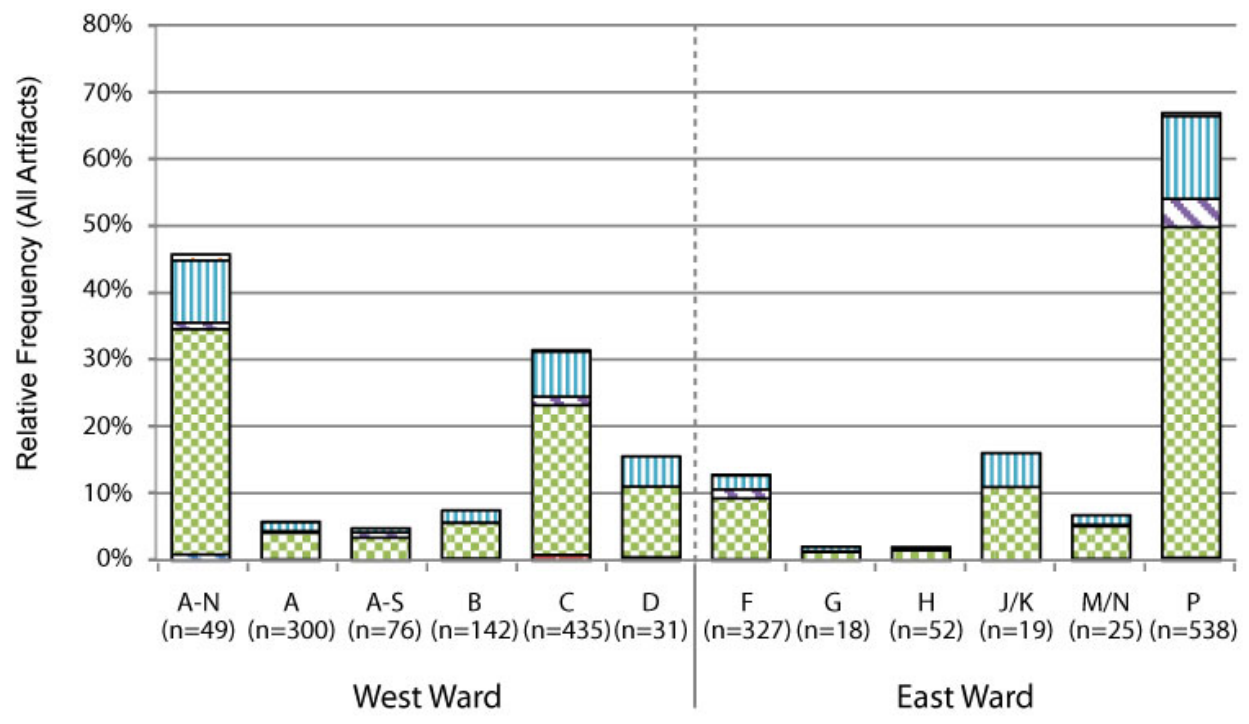

Artifact Type

$\square$ Tool

प Freehand flake

Bipolar flake

$\square$ Angular shatter

Core

므 Raw material

Figure 8.19: Relative distribution of chert artifact types by area

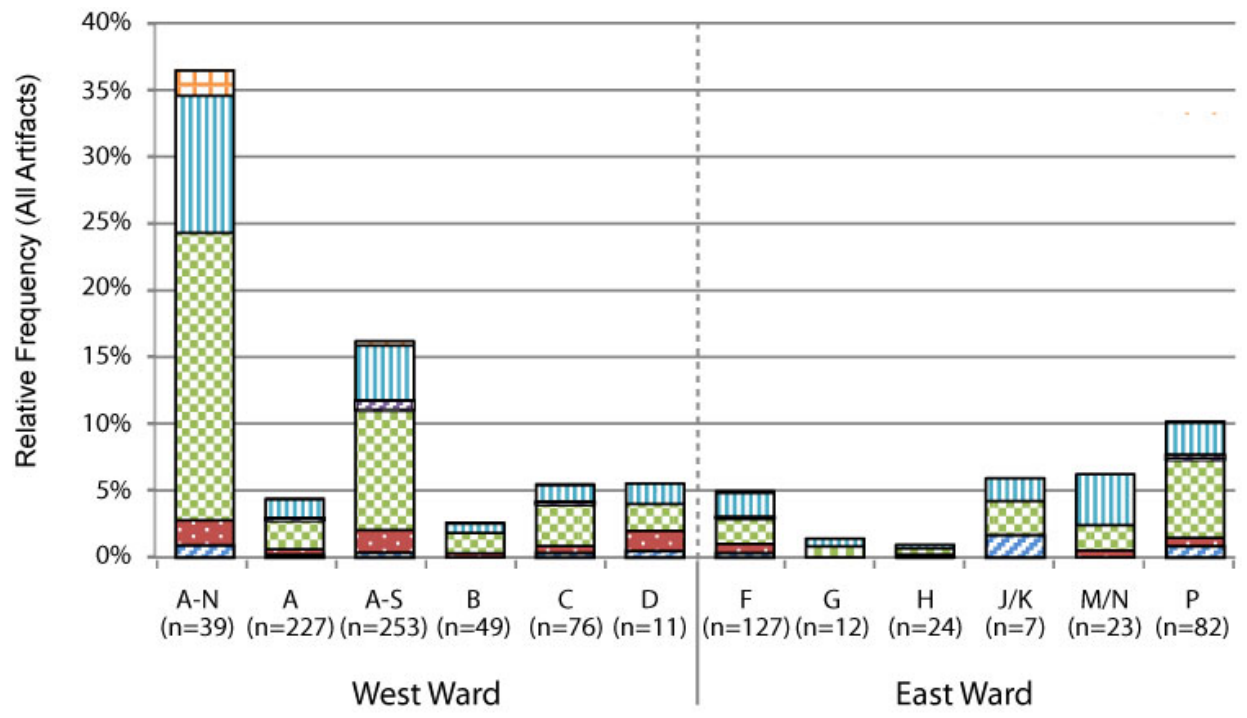

Artifact Type

$\square$ Tool

प Freehand flake

$\square$ Bipolar flake

$\square$ Angular shatter

C Core

Raw material

West Ward

East Ward 
Figure 8.20: Distribution quartz tool types by area

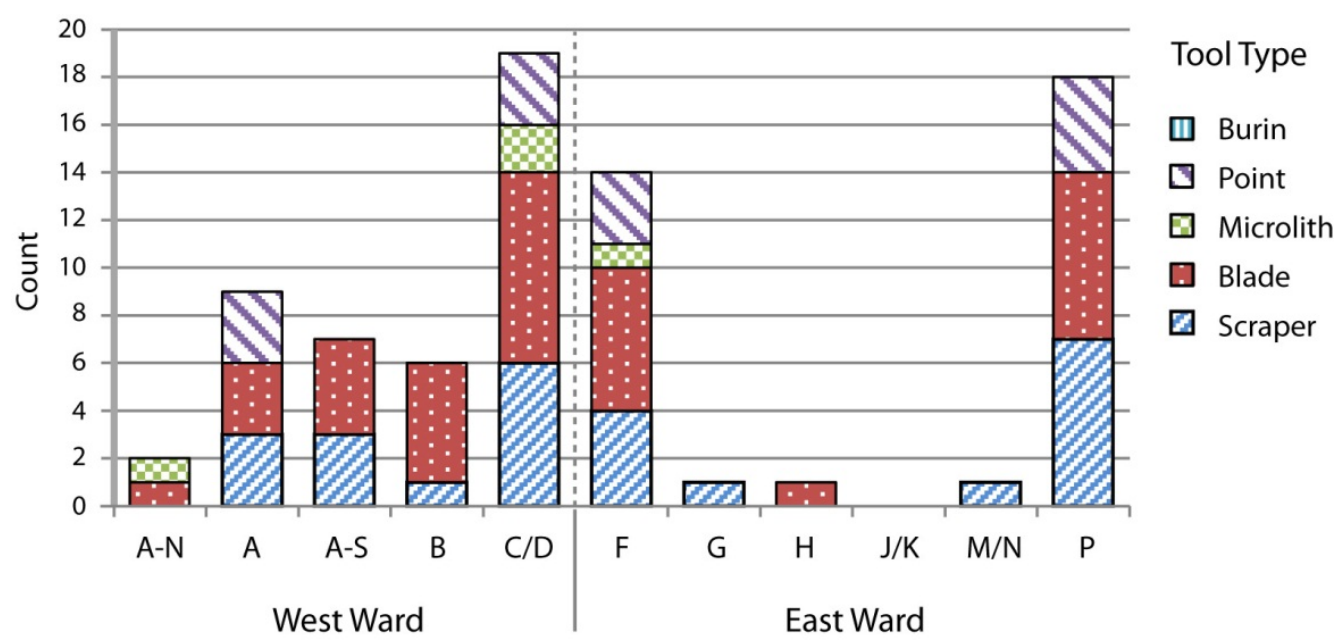

Figure 8.21: Distribution of chert tool types by area

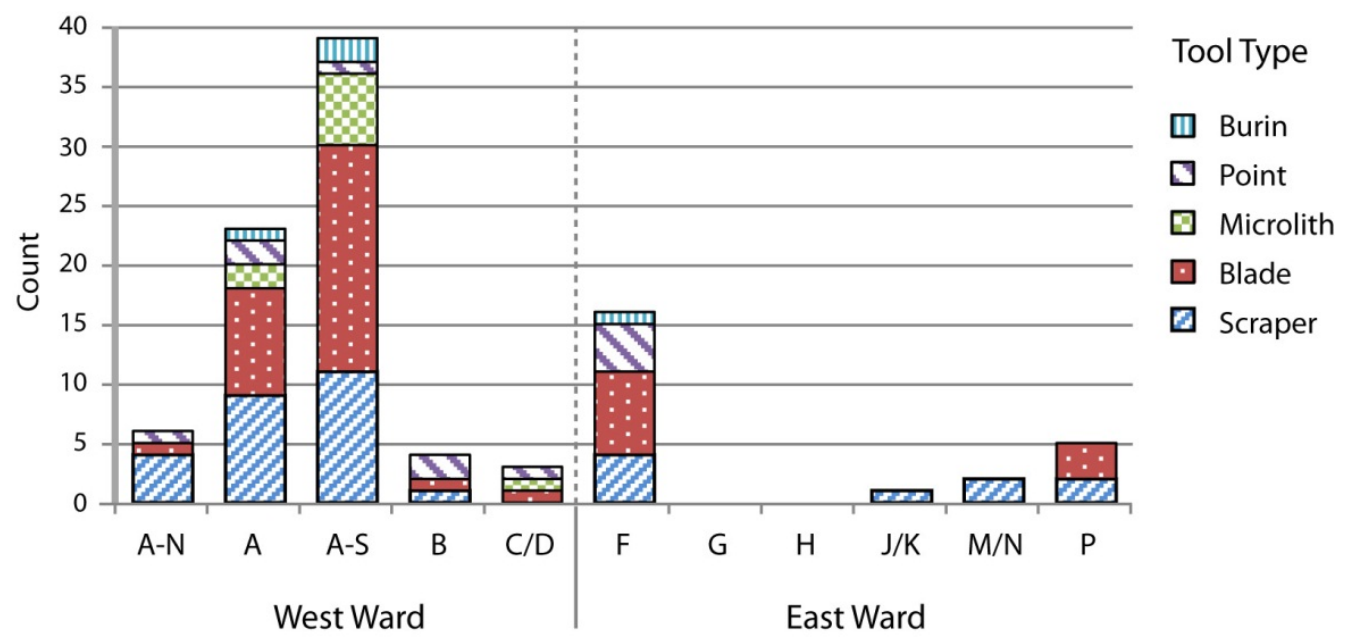

Figure 8.22 shows that, regardless of any differences in the exploitation of quartz and chert, the proportions of tool types did not differ with respect to residential and nonresidential areas $\left(n=135, \chi^{2}=1.57, \rho=0.831\right)$. The exception was Area A-S, whose tool proportions did differ significantly from those of other non-residential areas $(n=77$, $\left.\chi^{2}=9.16, \rho=0.047\right)$. The greater number of blades and lower number of projectile points in this latter area could point to the segregation of craft activities such as hide-processing or metal working (see below). 
Figure 8.22: Relative abundance of chert and quartz tool types by area type

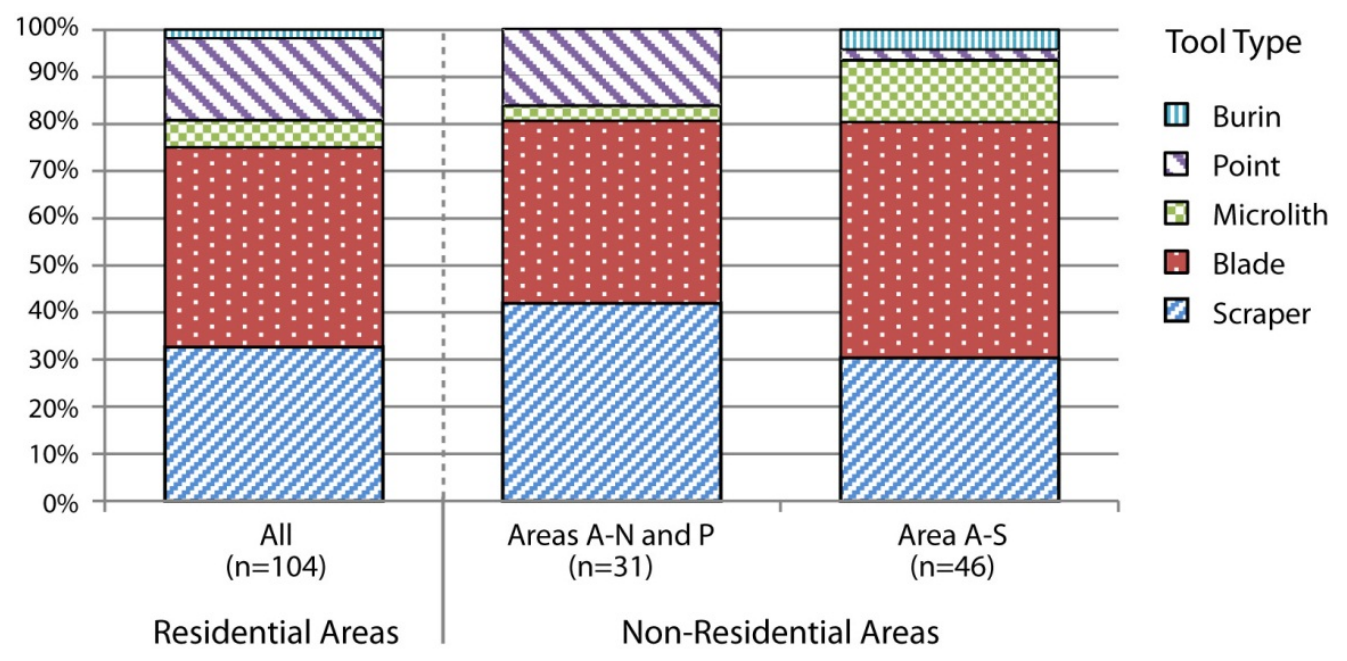

\section{Groundstone Axes}

Although I have already touched upon groundstone tools related to food processing, analyses of the spatial distributions of hematite debitage and axe heads, including both large specimens and hachettes, can inform on their production and uses. Exhibiting various stages of reduction and use (see Appendix E), Figure 8.23 indicates that axe heads, as with other groundstone implements, occurred in greater relative frequencies in the West Ward. While the small sample sizes merit a cautious interpretation, the modest positive association of blanks/preforms with the West Ward ( $\left.n=38, \chi^{2}=3.71, \rho=0.076\right)$ suggests that production or reworking of these objects may have been spatially restricted, or at least more intense, in this part of the village. The axes themselves, however, were used and/or displayed in the East Ward, where the presence of hematite flakes documented their percussion or other techniques of wear. If people employed these for shaping horns or some other ritual purpose, then specialized production of or access to these artifacts (via exchange) by the residents of the West Ward could have constituted an important source of political or ritual power in the community. 
Figure 8.23: Relative distribution of hematite reduction stages and debitage counts by area

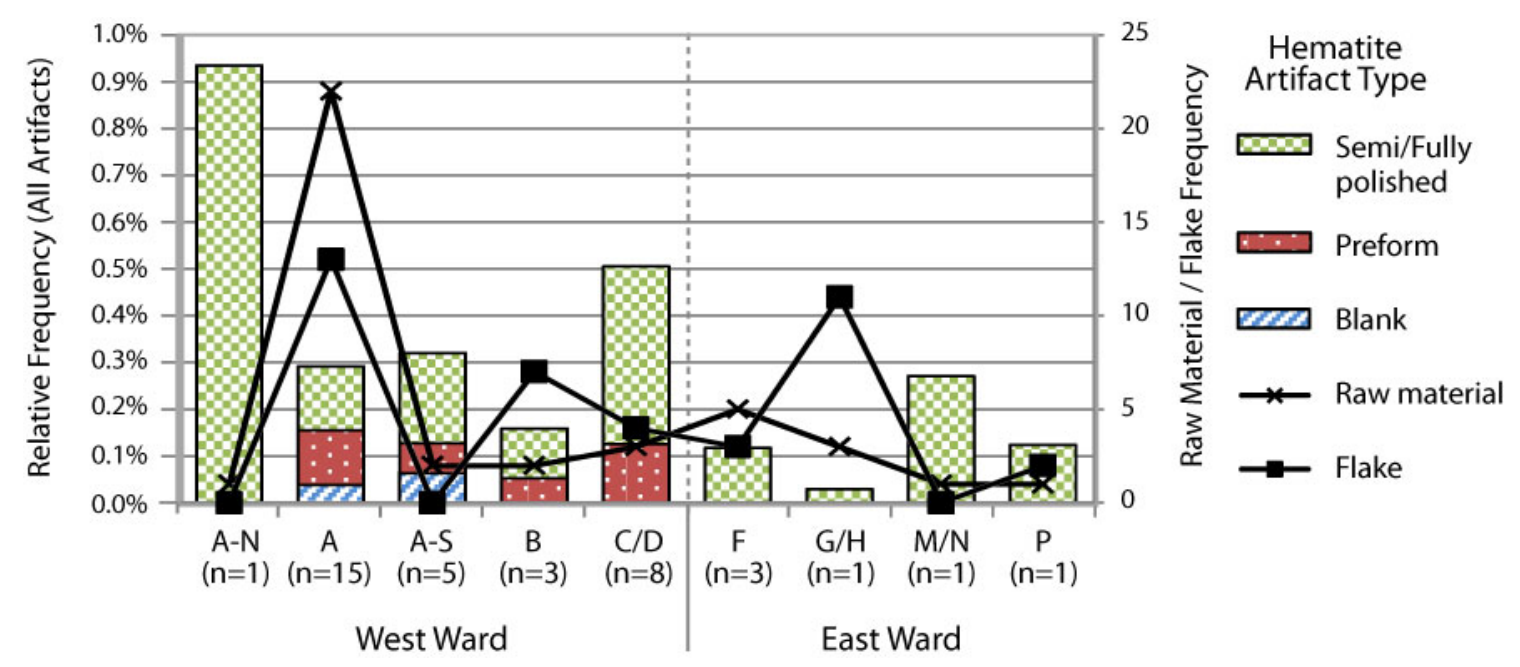

\section{Metalworking}

Pending the detailed compositional analyses needed to fully assess metalworking practices at the Diouboye, the macroscopic analysis of iron and copper artifacts and metallurgical artifacts (see Appendix F) from surface contexts allow for a preliminary interpretation of metal technologies in the village community. For example, one can see in Figure 8.7 above that depositional sets with elevated amounts of slag (Artifact Set 6) occurred in Area A-N, Area A-S, Area D, and along the eroded western slope of Area H. The absence of furnace or tuyère pipe fragments and the relatively low volume of slag, compared with Iron Age smelting sites found elsewhere across Senegambia, strongly suggest that these slags derived from smithing, rather than smelting, activities. While the surface data indicate that metal working was not completely segregated within one ward, smithing was nevertheless more intense in the West Ward where slag densities were both greater, and relatively restricted to non-residential areas (Figure 8.24). These patterns, in turn, hint at greater specialization on the part of people residing at Area A and Area B (and perhaps Area F) during the Middle to Late phases of occupation. While metal artifacts recovered from surface contexts were far too few to draw firm conclusions about patterns of exchange and consumption (Table 8.6), these data at least confirm that people across Diouboye had equitable access to iron and copper metals for use and display. 
Figure 8.24: Relative distribution of slag types and mass by area

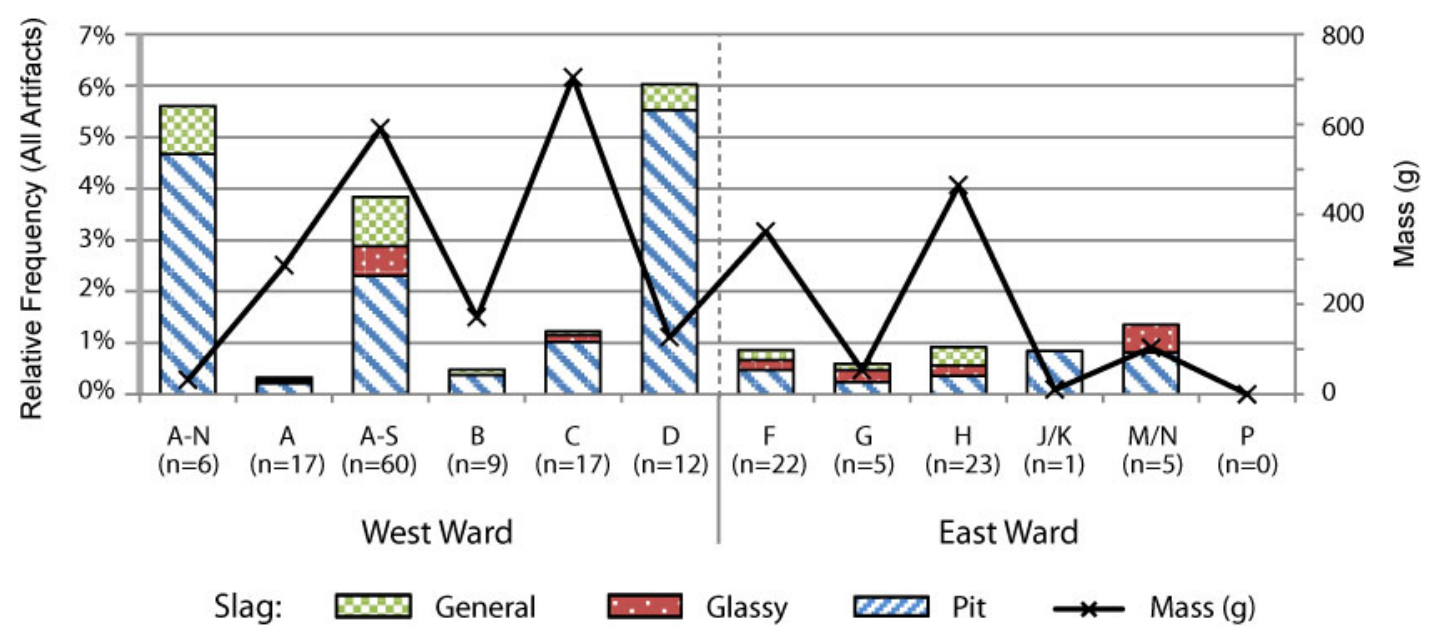

Table 8.6: Summary of metal artifacts from systematic (and purposive) surface collections by CFAP

\begin{tabular}{|c|c|c|c|c|c|}
\hline \multirow[t]{2}{*}{ Area/Ward } & \multicolumn{4}{|c|}{ Iron } & \multirow{2}{*}{$\begin{array}{c}\text { Copper } \\
\text { Ornament }\end{array}$} \\
\hline & Wire/Rod & Blade & Bracelet & Unknown & \\
\hline Area A & $2(3)$ & & & & \\
\hline Area A-S & & 2 & & & \\
\hline Area B & 1 & 1 & & & \\
\hline West Ward & 6 & 2 & - & - & - \\
\hline Area $\mathrm{F}$ & 1 & 1 & 1 & 1 & \\
\hline Area $\mathrm{H}$ & & 1 & & 1 & (2) \\
\hline Area $\mathrm{N}$ & & 1 & & & \\
\hline Area P & & & & & 1 \\
\hline East Ward & 1 & 3 & 1 & 2 & 3 \\
\hline Total & 7 & 5 & 1 & 2 & 3 \\
\hline
\end{tabular}

\section{Long-Distance Exchange}

Beads made of non-local carnelian, glass, and copper provided evidence for longdistance exchange practices by the community of Diouboye (see Appendix G). As shown in Table 8.7, the small numbers of such exotic objects indicate that people across the site maintained exchange through down-the-line mechanisms of gift-giving, bride wealth and inheritance, inter-village exchange, and so forth. The seeming association of glass beads 
Table 8.7: Summary of small finds from systematic (and purposive) collections by CFAP

\begin{tabular}{|c|c|c|c|c|c|c|c|c|}
\hline \multirow[t]{2}{*}{ Area/Ward } & \multicolumn{4}{|c|}{ Beads } & \multicolumn{4}{|c|}{ Terracotta Object } \\
\hline & Carnelian & Copper & Glass & Ivory & Bead & Figurine & Netweight & Unk \\
\hline Area A & (1) & & (2) & $2(2)$ & & & (1) & \\
\hline Area A-S & & & 1 & & & & & 1 \\
\hline Area B & & & 2 & & & 2 & & \\
\hline Area C & & & 1 & & & 1 & & \\
\hline Area D & & & & 1 & & & & \\
\hline West Ward & 1 & - & 6 & 5 & - & 3 & 1 & 1 \\
\hline Area F & & & & & & & 1 & \\
\hline Area $G$ & 1 & & & & & 1 & & \\
\hline Area $\mathrm{H}$ & & (2) & & & (1) & 1 & 1 & \\
\hline Area P & & 1 & & & & & & \\
\hline East Ward & 1 & 3 & - & - & 1 & 2 & 2 & 1 \\
\hline Total & 2 & 3 & 6 & 5 & 1 & 5 & 3 & 2 \\
\hline
\end{tabular}

with the West Ward, and copper ornaments with the East Ward, most likely resulted from small sample sizes since previous collections by Marie-José and Howard Opper (1990) had recovered one copper ornament from Area A, and ten glass and carnelian beads along the modern dirt track that cuts across Area P. Altogether, these data give the impression of equal participation in exchange networks by corporate kin groups across the village.

\section{Ritual}

Unambiguous evidence for religious ritual was limited in the surface assemblages from Diouboye. As already mentioned, polished hematite axes may have held some religious value although this hypothesis remains untested. Additionally, ivory and terracotta ornaments (Table 8.7) could have been items of personal adornment or components of ritual regalia. Beyond the five figurine fragments recovered by CFAP, previous surface collections had recovered two terracotta bracelets, one phallic figurine, and one zoomorphic figurine in the vicinity of Area A (Opper and Opper 1990). Whatever their actual uses, the distribution of all these potential ritual objects suggests that their associated activities took place primarily in residential areas. That is, they were not spatially segregated or socially restricted within the village. 


\section{Residential Space}

Beyond the activity areas defined by recurrent artifact associations, several hundred sandstone rock foundations document the relatively formalized production of space within each residential area. Here I first employ these features to model demography across Diouboye. I then consider dimensions of proximity/distance and segregation among the foundations of permanent structures, particularly granaries, to explore the socio-spatial organization of the subsistence economy at multiple scales within and between residential areas.

\section{Estimating Population}

The social structuring of the economy depends in large part on the number of people who live together and interact on a regular basis. A general estimate of the population within a settled community_and its constituent households or corporate kin groups - can therefore help to map the potential mobilization and reproduction of wealthin-people as both labor and knowledge. Although comparative ethnography has demonstrated a positive, yet highly variable, correlation between population and overall area or number of huts or rooms within a settlement (Hassan 1978:55-59; Naroll 1962; Wiessner 1974), archaeological interpretations must account for post-depositional effects on these variables.

In a classic ethnoarchaeological study of Bé, a Fulani village in Cameroon, Nicholas David (1971) provides a clear analog for estimating population dynamics at Diouboye. Not only do the two communities share similar agro-pastoral subsistence (and presumably mobility) regimes, they both have household compounds of circular huts arranged around semi-formal courtyards with hearths, middens, and pot rests. All of these features are important because the adult population per compound in Bé correlated better with the number of huts ( $\mathrm{r}=0.863)$, than with compound surface area ( $\mathrm{r}=0.674)$ and granary groups provided a crude measure for the number of household heads in the village. In order to estimate the maximum adult (post-pubescent) population of each residential area at Diouboye, I calculated the space covered by household compounds as a five-meter buffer around extant surface features. These areal measures of minimum "residential space” further comprised the grounds for the spatial analyses of features 


\section{Table 8.8: $\quad$ Population estimates for residential areas}

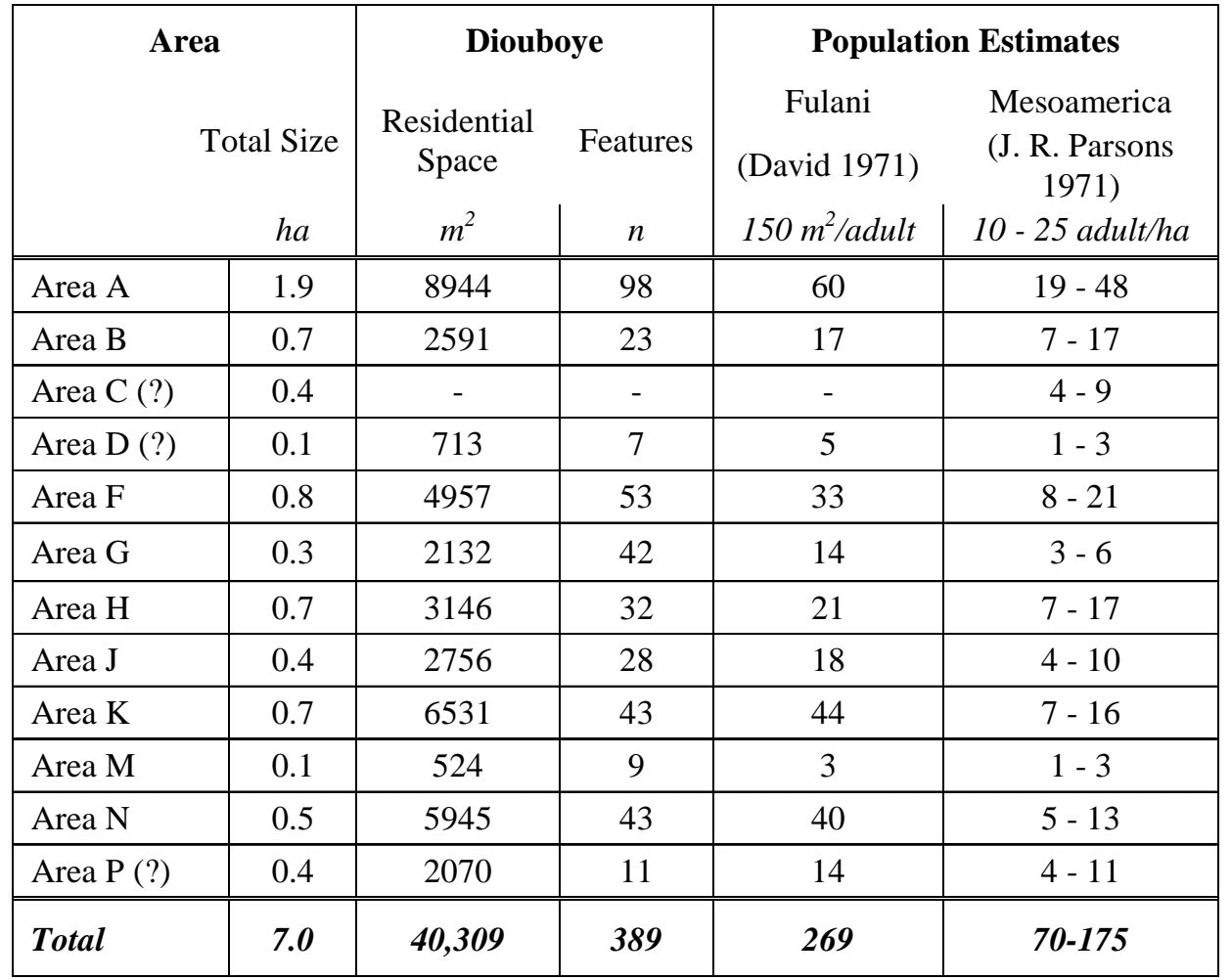

discussed below. Table 8.8 outlines the estimated population of Diouboye using density values derived from the study of Bé, in addition to several other well-accepted formulas for converting site area into population.

The estimated village population during the Middle Diouboye phase, when all areas were occupied, lies somewhere around 100-250 adults, with perhaps 40-60 children and infants based on the demography of rural villages in northern Guinea (Lestrange 1955:4-5). These estimates are inevitably crude, but they provide at least some impression of the number of people that would have co-habited and interacted within each residential area and across the village community on a day-to-day basis.

\section{Architecture and the Production of Space}

While artifact sets document the spatial organization of subsistence and craft practices in a general sense, the durable elements of the built environment, including domestic huts, granaries, pavements, pot rests, and grinding stations, would have further 
Table 8.9: $\quad$ Summary of surface features by area

\begin{tabular}{|c|c|c|c|c|c|c|c|c|c|c|c|}
\hline \multirow{3}{*}{\multicolumn{2}{|c|}{$\begin{array}{l}\text { Area/Ward } \\
\text { Feature Total }\end{array}$}} & \multicolumn{10}{|c|}{ Feature Type } \\
\hline & & \multirow{2}{*}{\multicolumn{2}{|c|}{ Hut }} & \multirow{2}{*}{\multicolumn{2}{|c|}{ Pavement }} & \multicolumn{6}{|c|}{ Granary } \\
\hline & & & & & & \multicolumn{2}{|c|}{ Small } & \multicolumn{2}{|c|}{ Large } & \multicolumn{2}{|c|}{ Possible } \\
\hline & $n$ & $n$ & $\%$ & $n$ & $\%$ & $n$ & $\%$ & $n$ & $\%$ & $n$ & $\%$ \\
\hline Area A & 75 & 2 & $2.7 \%$ & 0 & $0.0 \%$ & 25 & $33.3 \%$ & 3 & $4.0 \%$ & 9 & $12.0 \%$ \\
\hline Area B & 22 & 0 & $0.0 \%$ & 0 & $0.0 \%$ & 9 & $40.9 \%$ & 2 & $9.1 \%$ & 4 & $18.1 \%$ \\
\hline Area D & 7 & 0 & $0.0 \%$ & 0 & $0.0 \%$ & 2 & $28.6 \%$ & 1 & $14.3 \%$ & 1 & $14.3 \%$ \\
\hline West Ward & 104 & 2 & $1.9 \%$ & 0 & $0.0 \%$ & 36 & $34.6 \%$ & 6 & $5.8 \%$ & 14 & $13.5 \%$ \\
\hline Area F & 42 & 0 & $0.0 \%$ & 3 & $7.1 \%$ & 12 & $28.6 \%$ & 5 & $11.9 \%$ & 5 & $11.9 \%$ \\
\hline Area G & 35 & 0 & $0.0 \%$ & 0 & $0.0 \%$ & 8 & $22.9 \%$ & 6 & $17.1 \%$ & 4 & $11.5 \%$ \\
\hline Area $\mathrm{H}$ & 29 & 1 & $3.4 \%$ & 1 & $3.4 \%$ & 6 & $20.7 \%$ & 3 & $10.3 \%$ & 4 & $13.8 \%$ \\
\hline Area J & 26 & 0 & $0.0 \%$ & 0 & $0.0 \%$ & 4 & $15.4 \%$ & 2 & $8.3 \%$ & 3 & $12.5 \%$ \\
\hline Area $\mathrm{K}$ & 41 & 0 & $0.0 \%$ & 0 & $0.0 \%$ & 13 & $31.7 \%$ & 4 & $9.3 \%$ & 8 & $18.7 \%$ \\
\hline Area M & 5 & 0 & $0.0 \%$ & 0 & $0.0 \%$ & 2 & $40.0 \%$ & 0 & $0.0 \%$ & 0 & $0.0 \%$ \\
\hline Area N & 43 & 1 & $2.3 \%$ & 0 & $0.0 \%$ & 5 & $11.6 \%$ & 4 & $9.3 \%$ & 15 & $34.9 \%$ \\
\hline Area P & 11 & 0 & $0.0 \%$ & 0 & $0.0 \%$ & 2 & $18.2 \%$ & 0 & $0.0 \%$ & 1 & $9.1 \%$ \\
\hline East Ward & 232 & 2 & $0.9 \%$ & 4 & $1.7 \%$ & 52 & $22.4 \%$ & 24 & $10.3 \%$ & 28 & $12.1 \%$ \\
\hline Total & 336 & 4 & $1.2 \%$ & 4 & $1.2 \%$ & 88 & $26.2 \%$ & 30 & $8.9 \%$ & 34 & $10.1 \%$ \\
\hline
\end{tabular}

Table 8.9: $\quad$ Summary of surface features by area, continued

\begin{tabular}{|c|c|c|c|c|c|c|c|c|c|c|c|}
\hline \multirow{3}{*}{\multicolumn{2}{|c|}{$\begin{array}{l}\text { Area/Ward } \\
\text { Feature Total }\end{array}$}} & \multicolumn{10}{|c|}{ Feature Type } \\
\hline & & \multirow{2}{*}{\multicolumn{2}{|c|}{$\begin{array}{l}\text { Filled } \\
\text { Small }\end{array}$}} & \multirow{2}{*}{\multicolumn{2}{|c|}{ Pot Rest }} & \multicolumn{4}{|c|}{ Alignment } & \multicolumn{2}{|c|}{ Unknown } \\
\hline & & & & & & \multicolumn{2}{|c|}{ Curvilinear } & \multicolumn{2}{|c|}{ Rectilinear } & \multirow[b]{2}{*}{$n$} & \multirow[b]{2}{*}{$\%$} \\
\hline & $n$ & $n$ & $\%$ & $n$ & $\%$ & $n$ & $\%$ & $n$ & $\%$ & & \\
\hline Area A & 75 & 2 & $2.7 \%$ & 7 & $9.3 \%$ & 6 & $8.0 \%$ & 8 & $10.7 \%$ & 11 & $14.7 \%$ \\
\hline Area B & 22 & 0 & $0.0 \%$ & 0 & $0.0 \%$ & 0 & $0.0 \%$ & 1 & $4.5 \%$ & 6 & $27.3 \%$ \\
\hline Area D & 7 & 0 & $0.0 \%$ & 1 & $14.3 \%$ & 0 & $0.0 \%$ & 2 & $28.6 \%$ & 0 & $0.0 \%$ \\
\hline West Ward & 104 & 2 & $1.9 \%$ & 8 & $7.7 \%$ & 6 & $5.8 \%$ & 11 & $10.6 \%$ & 17 & $16.3 \%$ \\
\hline Area F & 42 & 1 & $2.4 \%$ & 2 & $4.8 \%$ & 4 & $9.5 \%$ & 4 & $9.5 \%$ & 6 & $14.3 \%$ \\
\hline Area G & 35 & 1 & $2.9 \%$ & 2 & $5.7 \%$ & 1 & $2.9 \%$ & 4 & $11.4 \%$ & 9 & $25.7 \%$ \\
\hline Area $\mathrm{H}$ & 29 & 1 & $3.4 \%$ & 1 & $3.4 \%$ & 3 & $10.3 \%$ & 3 & $10.3 \%$ & 6 & $20.7 \%$ \\
\hline Area J & 26 & 1 & $4.2 \%$ & 1 & $4.2 \%$ & 2 & $8.3 \%$ & 1 & $4.2 \%$ & 12 & $46.2 \%$ \\
\hline Area $\mathrm{K}$ & 41 & 0 & $0.0 \%$ & 2 & $4.7 \%$ & 0 & $0.0 \%$ & 4 & $9.3 \%$ & 10 & $24.4 \%$ \\
\hline Area M & 5 & 1 & $20.0 \%$ & 1 & $20.0 \%$ & 0 & $0.0 \%$ & 0 & $0.0 \%$ & 1 & $20.0 \%$ \\
\hline Area $\mathrm{N}$ & 43 & 0 & $0.0 \%$ & 1 & $2.3 \%$ & 3 & $7.0 \%$ & 2 & $4.7 \%$ & 12 & $27.9 \%$ \\
\hline Area P & 11 & 0 & $0.0 \%$ & 1 & $9.1 \%$ & 1 & $9.1 \%$ & 2 & $18.2 \%$ & 4 & $36.4 \%$ \\
\hline East Ward & 232 & 5 & $2.2 \%$ & 11 & $4.7 \%$ & 14 & $6.0 \%$ & 20 & $8.6 \%$ & 60 & $25.9 \%$ \\
\hline Total & 336 & 7 & $2.1 \%$ & 19 & $5.7 \%$ & 20 & $6.0 \%$ & 31 & $9.2 \%$ & 77 & $22.9 \%$ \\
\hline
\end{tabular}


structured these economic processes across Diouboye. As detailed in Appendix B, I documented 421 surface features across the site, 390 of which were classified as types of stone architectural foundations on the basis of formal dimensions of size, shape, and number of constituent stones (see Chapter 6). An additional 54 features have been excluded from this analysis due to their association with strong surface erosion or slope (LS value $>2.0$ ) factors that puts their relative chronology in question. Although the other architectural features (Table 8.9) were unlikely to all have been contemporaneous, an average lifespan of two or three decades for earthen wall structures (David 1971; Prussin 1974), combined with the possible reuse of stone foundations, suggests that the majority of these features were in use simultaneously at each area.

Figures 8.25 shows that, despite the heterogeneity within each ward and modest numbers of unidentifiable features, residential areas shared similar proportions of surface feature types, including a predominance of those interpreted as granary foundations.

Figure 8.25: Relative abundance of surface features by area

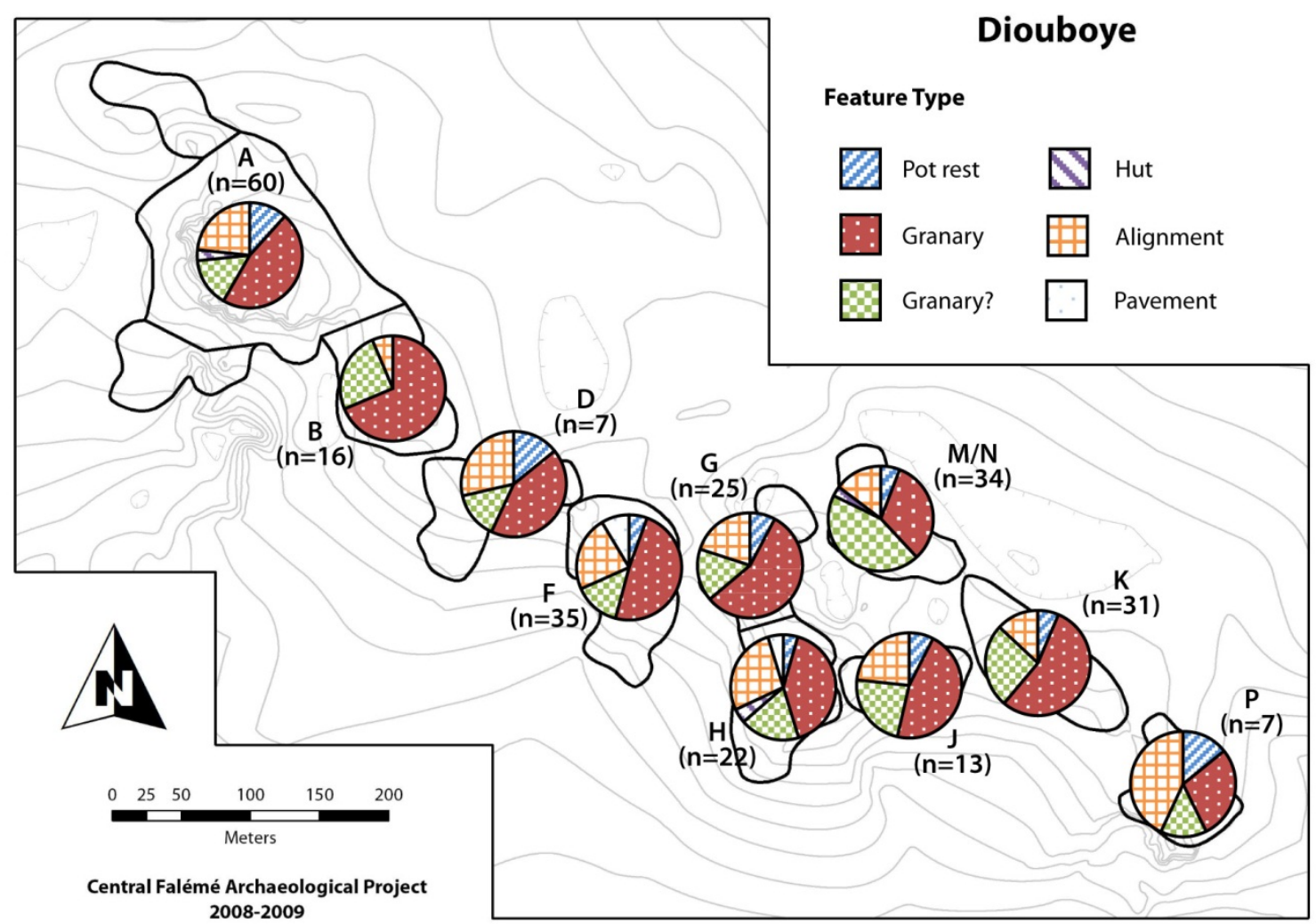


Table 8.10: Summary of unconstrained artifact sets by surface feature type

\begin{tabular}{|c|c|c|c|c|c|c|c|c|c|c|c|}
\hline \multirow[t]{3}{*}{ Feature Type } & \multicolumn{11}{|c|}{ Artifact Set } \\
\hline & \multirow{2}{*}{$\begin{array}{c}\text { Total } \\
n\end{array}$} & \multicolumn{2}{|r|}{1} & \multicolumn{2}{|c|}{$2 / 3$} & \multicolumn{2}{|c|}{$4 / 5$} & \multicolumn{2}{|c|}{7} & \multicolumn{2}{|c|}{ None } \\
\hline & & $n$ & $\%$ & $n$ & $\%$ & $n$ & $\%$ & $n$ & $\%$ & $n$ & $\%$ \\
\hline Hut & 4 & 0 & $0.0 \%$ & 2 & $50.0 \%$ & 2 & $50.0 \%$ & 0 & $0.0 \%$ & 0 & $0.0 \%$ \\
\hline Pavement & 4 & 2 & $50.0 \%$ & 1 & $25.0 \%$ & 1 & $25.0 \%$ & 0 & $0.0 \%$ & 0 & $0.0 \%$ \\
\hline Pot Rest & 12 & 3 & $25.0 \%$ & 4 & $33.3 \%$ & 3 & $25.0 \%$ & 0 & $0.0 \%$ & 2 & $16.7 \%$ \\
\hline Granary & 99 & 18 & $18.2 \%$ & 36 & $36.4 \%$ & 29 & $29.3 \%$ & 6 & $6.1 \%$ & 10 & $10.1 \%$ \\
\hline Unfilled & 35 & 7 & $20.0 \%$ & 9 & $25.7 \%$ & 11 & $31.4 \%$ & 2 & $5.7 \%$ & 6 & $17.1 \%$ \\
\hline $\begin{array}{l}\text { Filled Feature, } \\
\text { Small }\end{array}$ & 6 & 0 & $0.0 \%$ & 4 & $66.7 \%$ & 2 & $33.3 \%$ & 0 & $0.0 \%$ & 0 & $0.0 \%$ \\
\hline Alignment & 43 & 10 & $23.3 \%$ & 18 & $41.9 \%$ & 11 & $25.6 \%$ & 2 & $4.7 \%$ & 2 & $4.7 \%$ \\
\hline Unknown & 57 & 7 & $12.3 \%$ & 18 & $31.6 \%$ & 24 & $42.1 \%$ & 2 & $3.5 \%$ & 6 & $10.5 \%$ \\
\hline Total & 260 & 47 & $18.1 \%$ & 92 & $35.4 \%$ & 83 & $31.9 \%$ & 12 & $4.6 \%$ & 26 & $10.0 \%$ \\
\hline
\end{tabular}

Activities and Formal Space

Unfortunately, the surface data from Diouboye did not express a coherent relationship between architectural features and subsistence and craft economies, at least at the scale of activity areas. Table 8.10 summarizes the spatial associations between feature types and the artifact sets representing similar concentrations of large or small vessels, chipped stone, and groundstone (see Table 8.3 above); no architectural features occurred in association with slag (Artifact Set 6). Nor did the relative frequencies of types within these major artifact classes (e.g., small jars, large pots, cores, hammerstones, etc.) correlate in any meaningful way with surface feature types.

While it is quite probable that the residents of Diouboye did, in fact, structure daily practices around their built environment, the resolution of these surface data is simply too coarse to identify such relationships beyond the broad distinctions between residential areas described above.

\section{Granaries: Subsistence Economy (Storage) and Social Segmentation}

Although cluster analysis of surface features was able to parse out residential areas at Diouboye (Appendix B), it was less appropriate for identifying meaningful spatial relations between individual household compounds. This is because many rural 
communities in West Africa arrange their compounds around an open courtyard, with the result that any given domestic structure may actually lie closer to those of a different compound than to others within the same compound (David 1971:120). The ethnographic data do, however, indicate that huts and granaries can define socio-spatial segments (and perhaps even wealth differences) within rural village communities. Given the paucity of hut foundations identified on the surface of Diouboye, I focus here on granaries.

Granaries provide an ideal archaeological unit for spatial analysis since they correspond to the scale at which food is prepared, shared, and consumed on a regular basis - whether by nucleated households, individual wives and their children, or some other normative social unit. Among the Fulani of Bé, for example, granaries similar to those identified at Diouboye fall under the control of male household heads responsible for sorghum cultivation, although some female ex-slaves also own granaries (David 1971:121-122). Labelle Prussin's (1972) ethnographic survey of granary architecture in six West African societies further reveals patterns of ownership ranging from an individual woman and her children to the supra-compound patriclan! Deferring for the moment what sorts of social units controlled granaries (and their contents) at Diouboye, let it suffice to say that spatial dimensions of proximity/distance and segregation among these features offer a window into political-economic organization within and between residential areas.

In order to visualize spatial relations among granary foundations and other architectural elements on the surface of Diouboye, I calculated the three nearest neighbors (measured from center points) first among all features, and then among granaries and possible granaries. While the mean and standard deviation of nearest neighbor distance (Table 8.11) revealed no strong pattern by feature type, the distribution of first and second nearest neighbor distances for granaries and possible granaries (Figure 8.26) illustrates a tendency towards spacing at distances of less than five meters. In the course of surface mapping, this pattern was readily apparent with the clustering of granary foundations and similar features in groups of two or three (see Figure B.9). Based on this trend, I have grouped all granary and possible granary foundations with central points less than 2.5 meters apart into "granary groups" for the purposes of subsequent spatial analysis. 
Table 8.11: Summary of nearest neighbor distance for stone architectural features

\begin{tabular}{|l|c|cc|}
\hline \multicolumn{1}{|c|}{ Feature Type } & Total & Nearest Neighbor Distance (m) \\
& $\mathrm{n}$ & $\mu$ & $\sigma$ \\
\hline \hline Hut Foundation & 4 & 3.47 & 2.03 \\
\hline Pavement & 4 & 3.78 & 1.25 \\
\hline Pot Rest & 16 & 4.07 & 4.06 \\
\hline Granary, small & 86 & 5.25 & 5.60 \\
\hline Granary, large & 30 & 3.23 & 1.68 \\
\hline Granary, possible & 53 & 4.40 & 2.51 \\
\hline Filled Feature, small & 5 & 3.53 & 3.42 \\
\hline Alignment, curvilinear & 20 & 4.07 & 2.54 \\
\hline Alignment, rectilinear & 31 & 4.51 & 1.80 \\
\hline Unknown & 76 & 5.13 & 3.92 \\
\hline
\end{tabular}

Figure 8.26: Distributions of first two nearest-neighbor distances for granaries and possible granaries

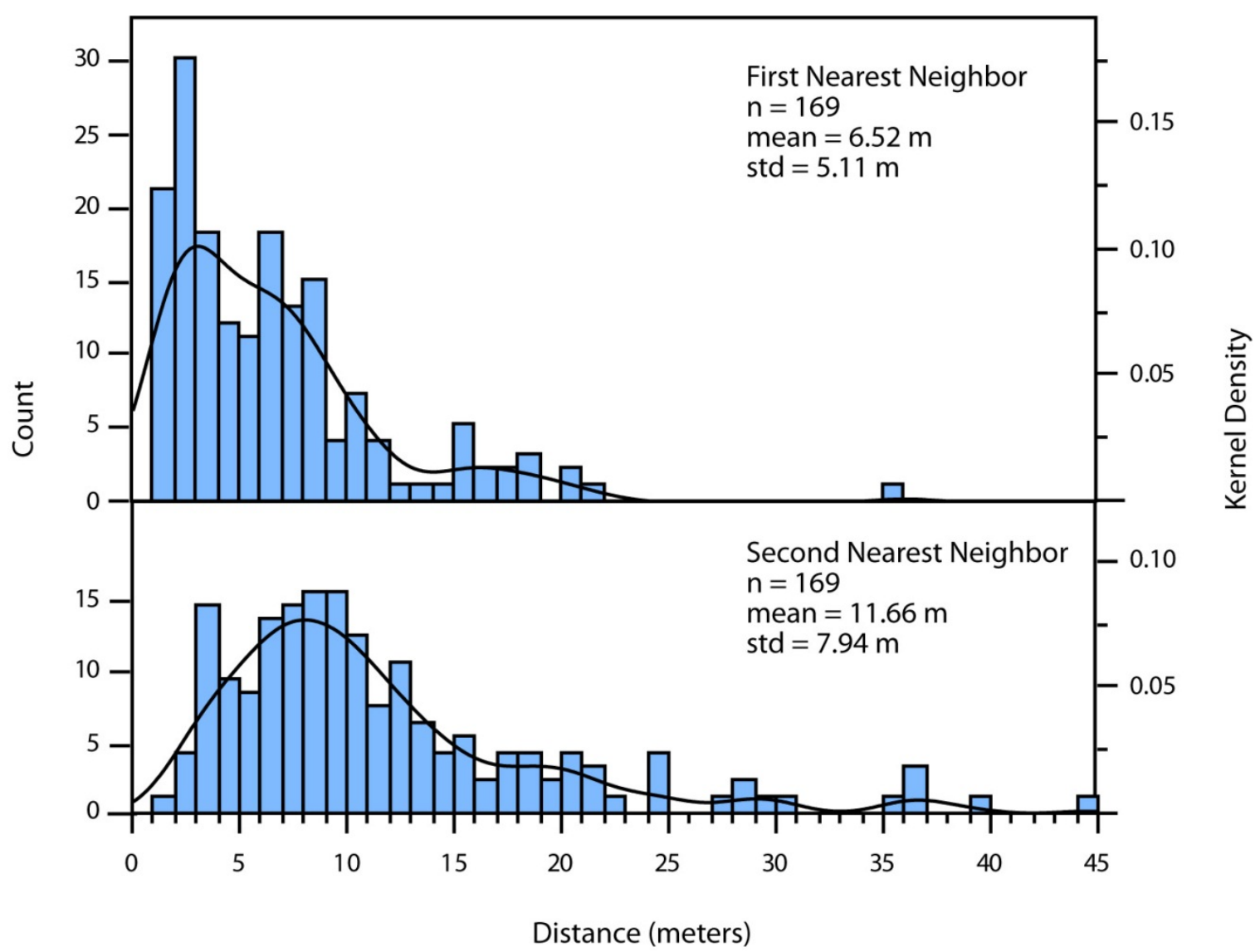


A nearest neighbor analysis based on median distance (following Washburn 1974) provided a measure of clustering or dispersion among architectural surface features within each residential area. The statistic $\mathrm{R}$, which is the observed median distance between feature center points divided by the expected distance, equals 0.00 for maximally clustered points, 1.00 for randomly dispersed points, and 2.29 for points maximally dispersed in a hexagonal lattice. Table 8.12 summarizes the $\mathrm{R}$ statistics

Table 8.12: Nearest neighbor statistics for all stone architectural features

\begin{tabular}{|l|c|c|c|c|c|c|}
\hline \multicolumn{1}{|c|}{ Area } & Size $\left.\mathbf{( m}^{\mathbf{2}}\right)$ & $\mathbf{N}$ & $\mathbf{\rho} \mathbf{( N / S i z e )}$ & $\mathbf{r}^{\text {Median }}(\mathbf{m})$ & $\mathbf{r}^{\text {Expected }}(\mathbf{m})$ & $\mathbf{R}\left(\mathbf{r}^{\mathbf{M}} / \mathbf{r}^{\mathbf{E}}\right)$ \\
\hline \hline Area A & 8944 & 98 & 0.011 & 3.59 & 2.96 & 1.21 \\
\hline Area B & 2591 & 23 & 0.009 & 5.22 & 3.29 & 1.59 \\
\hline Area D & 713 & 7 & 0.010 & 2.28 & 3.12 & 0.73 \\
\hline Area F & 4957 & 53 & 0.011 & 3.91 & 2.99 & 1.31 \\
\hline Area G & 2132 & 42 & 0.020 & 2.79 & 2.21 & 1.27 \\
\hline Area H & 3146 & 32 & 0.010 & 3.26 & 3.07 & 1.06 \\
\hline Area J & 2756 & 30 & 0.011 & 4.74 & 2.97 & 1.60 \\
\hline Area K & 6531 & 41 & 0.006 & 3.33 & 3.91 & 0.85 \\
\hline Area M & 524 & 9 & 0.017 & 2.93 & 2.36 & 1.24 \\
\hline Area N & 5945 & 43 & 0.007 & 3.93 & 3.64 & 1.08 \\
\hline Area P & 2070 & 11 & 0.005 & 7.62 & 4.25 & 1.79 \\
\hline \hline Total & $\mathbf{4 0 , 3 0 9}$ & $\mathbf{3 8 9}$ & $\mathbf{0 . 0 1 0}$ & $\mathbf{3 . 6 4}$ & $\mathbf{3 . 1 5}$ & $\mathbf{1 . 1 6}$ \\
\hline
\end{tabular}

Table 8.13: Nearest neighbor statistics for granary groups

\begin{tabular}{|l|c|c|c|c|c|c|}
\hline \multicolumn{1}{|c|}{ Area } & Size $\left.\mathbf{( m}^{\mathbf{2}}\right)$ & $\mathbf{N}$ & $\mathbf{\rho} \mathbf{( N / S i z e )}$ & $\mathbf{r}^{\text {Median }} \mathbf{( m )}$ & $\mathbf{r}^{\text {Expected }} \mathbf{( m )}$ & $\mathbf{R}\left(\mathbf{r}^{\mathbf{M}} / \mathbf{r}^{\mathbf{E}}\right)$ \\
\hline \hline Area A & 8944 & 41 & 0.005 & 6.86 & 4.57 & 1.50 \\
\hline Area B & 2591 & 13 & 0.005 & 7.55 & 4.37 & 1.73 \\
\hline Areas D/F & 5670 & 21 & 0.004 & 8.59 & 5.09 & 1.69 \\
\hline Area G & 2132 & 15 & 0.007 & 7.99 & 3.69 & 2.17 \\
\hline Area H & 3146 & 9 & 0.003 & 6.93 & 5.79 & 1.20 \\
\hline Area J & 2756 & 12 & 0.004 & 7.46 & 4.69 & 1.59 \\
\hline Area K/P & 8601 & 24 & 0.003 & 7.97 & 5.86 & 1.36 \\
\hline Area M/N & 6469 & 21 & 0.003 & 10.67 & 5.43 & 1.96 \\
\hline \hline Total & $\mathbf{4 0 , 3 0 9}$ & $\mathbf{1 5 6}$ & $\mathbf{0 . 0 0 4}$ & $\mathbf{7 . 7 3}$ & $\mathbf{4 . 9 8}$ & $\mathbf{1 . 5 5}$ \\
\hline
\end{tabular}


calculated for architectural features (excluding cairns and macrolithic scatters) from the surface of Diouboye. With the exception of Area D and Area K, these values express a modest trend towards dispersion among architectural features.

Granary groups, with a mean R statistic across areas of 1.65 (median $=1.55$ ), were significantly more dispersed in the sample of residential areas than all features together ( $t=-2.72, \rho=0.015)$. This pattern is consistent with restricted access to storage facilities by small groups of people engaged in daily food preparation and consumptionperhaps nuclear households or individual women and their children in an extended or polygamous household.

To the extent that granary groups demarcated socio-spatial units within each residential area, they provide a basis for comparing the structure of political and economic relations across the village during the Middle to Late phases of occupation. A size comparison of granary and possible granary foundations, for example, shows that Area A had relatively more medium-sized storage features than all other residential areas (Figure 8.27). At the same time, granary groups in the West Ward represent a greater proportion of all identifiable features than those areas in the East Ward, with the notable exception of Areas K/P. Assuming that granary/unfilled feature diameters correlate with

Figure 8.27: Relative abundance of storage features and granary groups

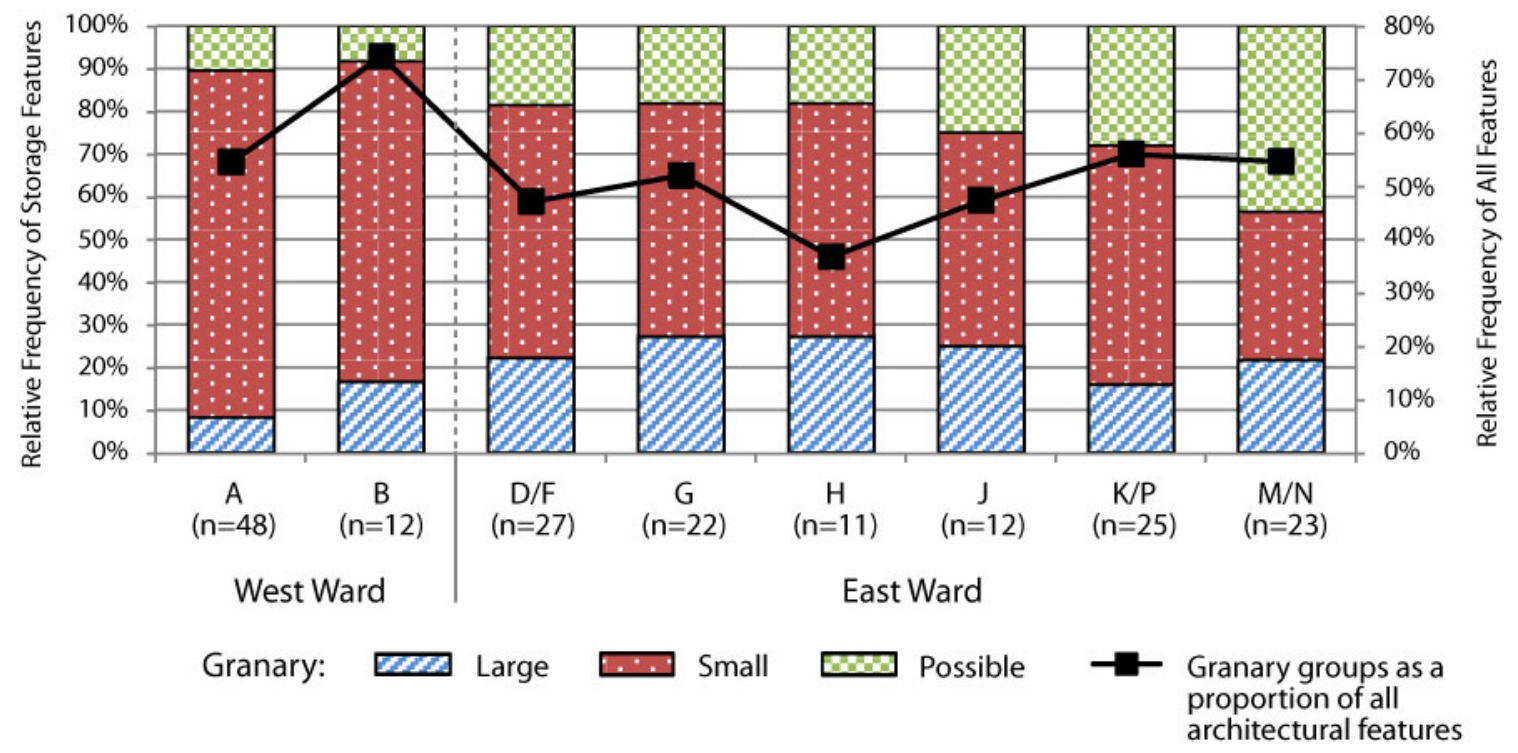


the size of overlying earthen wall structures, this pattern of smaller granaries, alongside relatively more numerous granary groups, could indicate that the corporate kin groups occupying Area A and the West Ward experienced slightly greater segmentation around more minimal social units than compounds in the East Ward. The similar degree of storage space per residential area, however, suggests that household compounds each maintained relatively equitable quantities of staples per capita.

\section{SUMMARY}

The surface archaeology of Diouboye revealed subtle differences in the material and spatial production of socioeconomic structures within the village, at least during the Middle to Late Diouboye phases. Depositional sets defined by correlated artifacts point to the segregation of some craft (and perhaps subsistence) production tasks, but the resulting activity areas generally emphasized more salient spatial differences among nonresidential and residential areas and between wards.

After excluding those areas resulting mainly from post-depositional disturbance (i.e., Area L, Area S), it was possible to distinguish residential areas with stone architectural features from non-residential areas with few surface features. Whereas earthenware pottery predominated in the artifact assemblages from the former, the latter were more heterogeneous, often with chipped stone, groundstone, and metallurgical slag highlighting a greater intensity of (specialized) craft and/or subsistence production activities. This pattern confirms that, at a minimum, the occupants of Diouboye made some distinction between more formalized domestic space, and the more informal space where non-domestic tasks and/or refuse disposal took place.

Subsistence activities, including grain processing, storage, and meal preparation and consumption, were fairly generalized among residential groups. Groundstone implements possibly related to grain processing were present in similar proportions across residential areas, albeit in substantially greater quantities in the West Ward, particularly in Areas B-D. Functional vessel types suggest that a similar suite of cooking, storage, and serving practices occurred within each residential area, although subtle, yet significant, differences between wards may point to some as of yet unknown economic, political, or cultural distinction between the groups occupying each half of the site. 
The surface data for craft activities - namely metal working, chert tool use, and groundstone axe use/manufacture-were both more diverse and more intense in Area AN, Area A-S, and Areas C/D compared to other areas. Situated in the West Ward, these areas highlight the somewhat segregated, if not specialized, organization of certain economic practices. Notably, emphasis on metal production and grinding in Areas C/D may have helped to produce spatial, and presumably social, distance between the East and West Wards within the village. At the same time, evidence for the production and use of chipped stone, groundstone, and iron tools across the site shows that the knowledge, tools, and techniques underpinning these technologies were relatively unrestricted.

Similarly, the small number of exotic goods and other ornaments found on the surface of Diouboye suggest that ritual and/or long-distance exchange through social networks did not form a basis for political distinction between residential areas and their associated corporate kin groups.

The ordering of distances among surface granary features suggests that groups in the West Ward were slightly more segmented around minimal social units undertaking food storage than their counterparts in the East Ward, particularly if one excludes the surface remains from Areas J/K dating to the Middle Diouboye phase. This pattern may indicate that, by the Late Diouboye phase, corporate kin groups in the West Ward held slightly greater access to social capital, measured as the number of minimal social units per residential areas, but otherwise people appeared to organize domestic space in a similar fashion across the village.

Altogether, the organization of space and the relative intensity of activities for grinding grain, making/using groundstone axes, chipped stone tool use, and metal smithing reveal a trend toward economic specialization within the West Ward of the village during the Middle to Late Diouboye phases. This trend is, however, counterbalanced by similar storage and culinary technologies, long-distance exchange practices, and the presence of low-intensity craft and ritual activities across all residential areas. These suggest that material (and non-material) resources underwrote more symmetrical, rather than hierarchical, political differences between households and kin groups across the community. 


\section{CHAPTER 9 \\ TIME, SPACE, AND COMMUNITY IN BAMBUK}

The evidence presented in Chapters 6-8 demonstrates that the political economy of Diouboye emerged mainly at the intersection of relations among local kin groups, and to a lesser extent, through participation in interregional networks emanating from political centers and trading êntrepots across the Iron Age landscape of West Africa. In the present chapter I pull together these lines of evidence to narrate change and continuity in the economic spheres of subsistence, craft, and long-distance exchange across three broad phases of village occupation circa AD 1000-1400. Drawing upon my theoretical, historical, and methodological discussion of village economies in precolonial West Africa (Chapter 2), I focus on the material and spatial dimensions observed across these aspects of the political economy to consider the institutions that structured life within the community on a day-to-day basis.

In the second part of this chapter, I situate this narrative within the historiography of West Africa and writing the anthropological study of small-scale communities and decentralized societies introduced in Chapter 1. Since Diouboye lay on the outskirts of the auriferous belt of Bambuk, this village offers some insights into social life beyond the “periphery” of written history promulgated by centralized polities and merchant diasporas during the medieval era. In particular, the archaeology of Diouboye suggests that smallscale communities were active participants in the production of interregional, and increasingly complex, political economies. At the same time, neither these modes of interaction nor emerging economic differences at a local scale appeared to underwrite strongly hierarchical political relations within the community. The resulting portrait reveals a nucleated village in which kinship and sodalities helped to maintain an egalitarian ethos in the face of social fission and fusion, increasingly specialized crafts production, shifting participation in interregional exchange networks, and possibly 
dynamic engagements with the more centralized societies to the south (Mali) and north (Ghana/Gajaaga, Tekrur) throughout the early-second millennium AD.

As is the case for most, if not all, archaeological projects, this study raises far more questions than it proposes to answer. I therefore conclude this study with some musings about ongoing and future research in the Upper Senegal region that will, I hope, expand and revise some of the preliminary conclusions advanced in these pages.

\section{DIOUBOYE: HISTORY OF A VILLAGE COMMUNITY}

The surface archaeology examined in Chapter 8 portrays Diouboye as a village of several coresidential (corporate kin) groups engaged in similar subsistence and exchange practices, though somewhat specialized with regards to some craft activities. By integrating these findings with the subsurface data presented in Chapter 7, I now consider how these patterns emerged over the course of three phases of occupation spanning the early-second millennium AD.

\section{Early Diouboye A/B Phases (AD 950-1100) \\ Space and Architecture}

Marked by the appearance of Early Diouboye A phase pottery assemblages, the initial settlement of Diouboye took place in Area F and Area N, and possibly Areas J/K and Area S, sometime during the final century of the first millennium AD. These residential areas formed a tight cluster on an archaic levee oriented parallel with the Falémé River to the west (Figure 9.1). This layout was consistent with subtle differences between the residential areas in access to cultivable land, water, and the other natural resources within the village catchment-perhaps related to the timing of arrival by their associated corporate kin groups.

Early Diouboye B phase pottery assemblages document the rapid expansion of settlement across Area G, Area H, and at least part of Area A. Although surface remains need to be interpreted with caution, they suggest that residential activity also began in Area B and Area M. Whatever the case, the early population of Diouboye was apparently concentrated in the East Ward where four or five residential areas surrounded a central 
depression. Yielding no cultural remains, this latter area most likely served as a plaza for conducting community affairs within the village.

Excavations revealed a rapid formalization of space in Area F, Area G, and Area $\mathrm{N}$, and possibly in the vicinity of Area S, where people built terre pisé domestic huts, wattle-and-daub structures, and granaries and pot rests on circular sandstone foundations throughout the Early Diouboye phase. Between these features, multiple hearth complexes emerged within semi-formal spaces for cooking and consumption. The midden-like fill from the lowest stratum in Area $\mathrm{H}$ may have derived from nearby domestic activities, or more likely, through rubbish disposal from Area G to the northwest.

While excavations did not reveal permanent architecture in Area A and Areas $\mathrm{J} / \mathrm{K}$, it remains possible that residential occupation was responsible for the deposition of cultural debris such as pottery sherds, ash, and charcoal in their lowermost strata.

\section{Figure 9.1: Early Diouboye A/B phase activities}

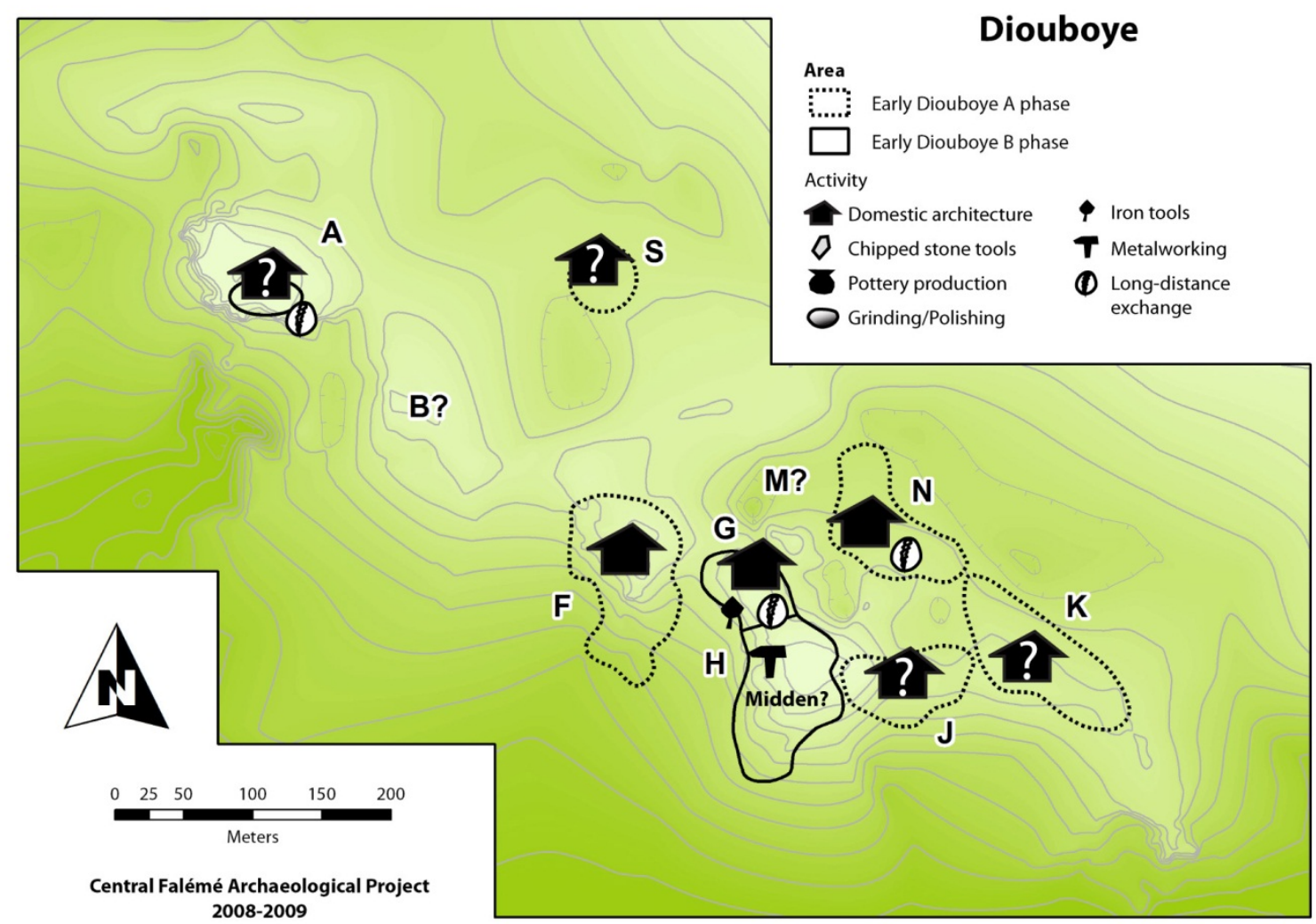




\section{Subsistence Economy}

The relatively humid conditions prevailing in the Upper Senegal during the Early Diouboye phase would have been amenable to the cultivation of pearl millet (tentatively identified in Unit G) and other savanna crops on the swath of alluvium separating the village from the Falémé River (Chapter 3). The presence of large and small granary foundations uncovered in Early Diouboye phase strata of Unit G, Unit F, and Unit N indicates that coresidential groups were each responsible for the storage of staples throughout the year.

Livestock, however, would have been difficult to sustain in this environment if the humid climate was accompanied by greater exposure to tsetse fly. Whether the occupants of Diouboye adopted smaller trypanotolerant breeds or engaged in exchange with transhumant pastoralists remains to be seen, but in either case they undoubtedly obtained most of their meat from wild resources (Appendix H). The Falémé River in particular yielded abundant, if perhaps seasonal, fish and reptiles; these were presumably exploited with the aid of harpoons and nets. Alongside water-dwelling mammals such as hippopotami, people also hunted a diverse array of terrestrial game, including carnivores, in the surrounding savanna forest. The successful and intensive exploitation of wild flora and fauna from the earliest occupation of the village suggests a people well-acquainted with this sort of riparian savanna niche.

Figure 9.2: Relative abundance of vessel types (Early Diouboye phase)

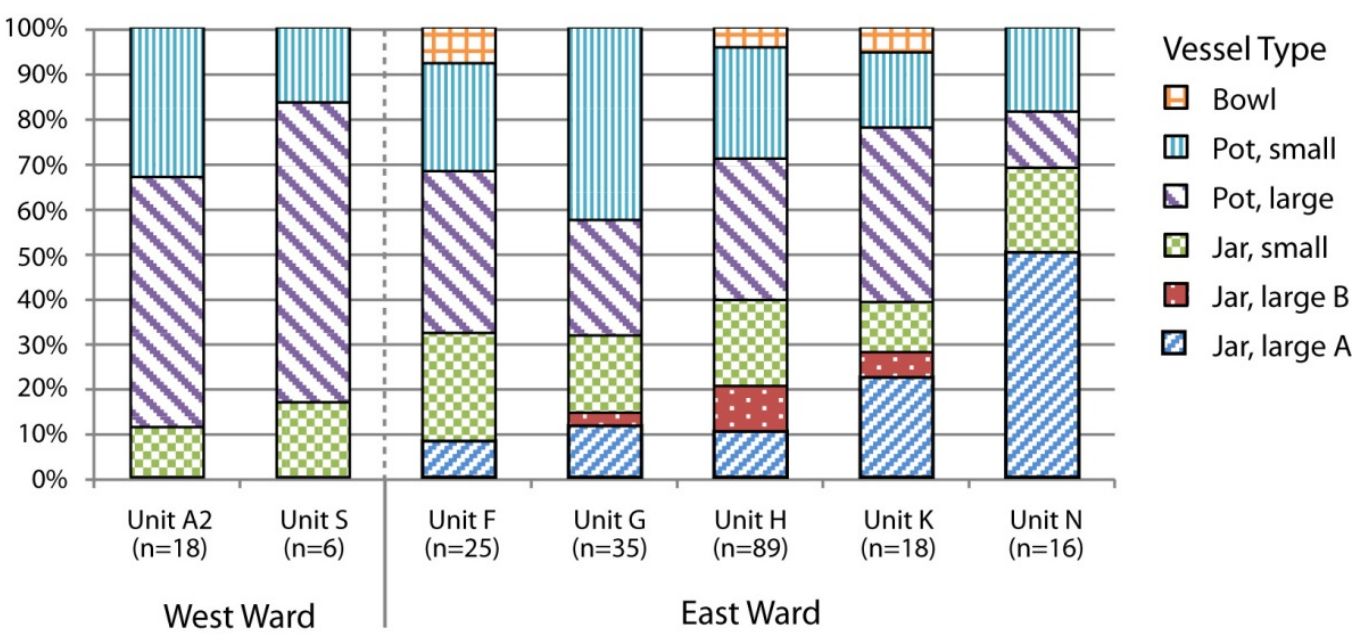


Regarding technologies of food and water transportation, cooking, and consumption, the assemblage of rim sherds from Early Diouboye phase contexts shows some consistent differences between the West and East wards (Figure 9.2), possibly contributing to patterns in the surface data. The low proportions of jars in Area A and Area S suggest that transportation and storage activities did not figure strongly in day-today happenings in these locations. When compared to the architectural evidence for residential occupation in the East Ward, this could indicate that the West Ward only hosted seasonal dwellings such as field houses or initiation huts. In either case, these data lend support to the interpretation that residential activities involving transportation and storage were concentrated in the East Ward where households each undertook a similar suite of culinary practices.

\section{Craft Production and Consumption}

Direct evidence for craft production was virtually absent in Early Diouboye phase contexts. Assuming that small numbers of lithic debitage did not derive from redeposition (Figure 9.3), then it would seem that villagers used informal stone tools in residential settings on a limited basis.

\section{Figure 9.3: Relative distribution of lithic raw material (Early Diouboye phase)}

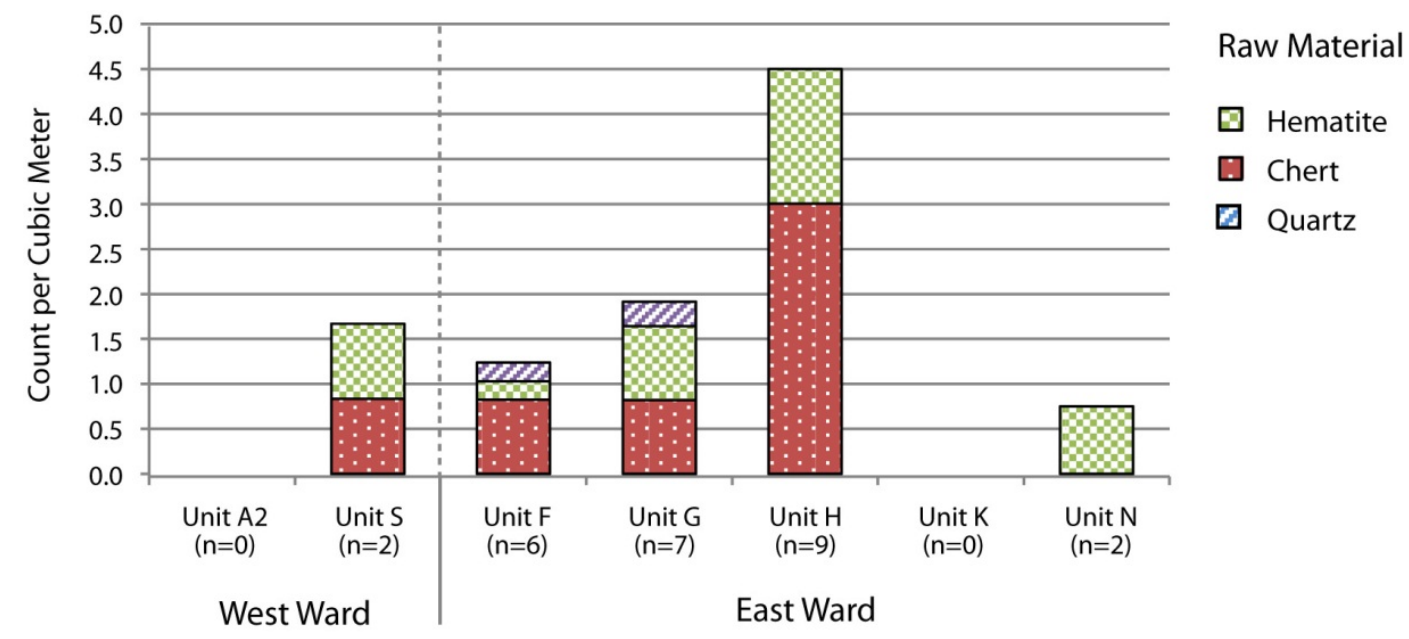


For pottery, the homogeneous distributions of paste recipes, decorative elements, and rim forms (Figure 9.4) across the site were consistent with local production within a single technological tradition, the social and spatial organization of which remains unknown. Two long-collared sherds from Area F and Area G provide a tentative stylistic link to potting traditions of the Middle Senegal region (see Chapter 4).

A small iron knife fragment from Area $G$ documented access to finished iron tools although the absence of slag in Early Diouboye phase contexts precluded any interpretation of smelting or smithing activities. It remains possible that the earliest occupants of Diouboye exchanged for iron produced further to the south or north.

\section{Figure 9.4: Relative abundance of rim forms (Early Diouboye phase)}

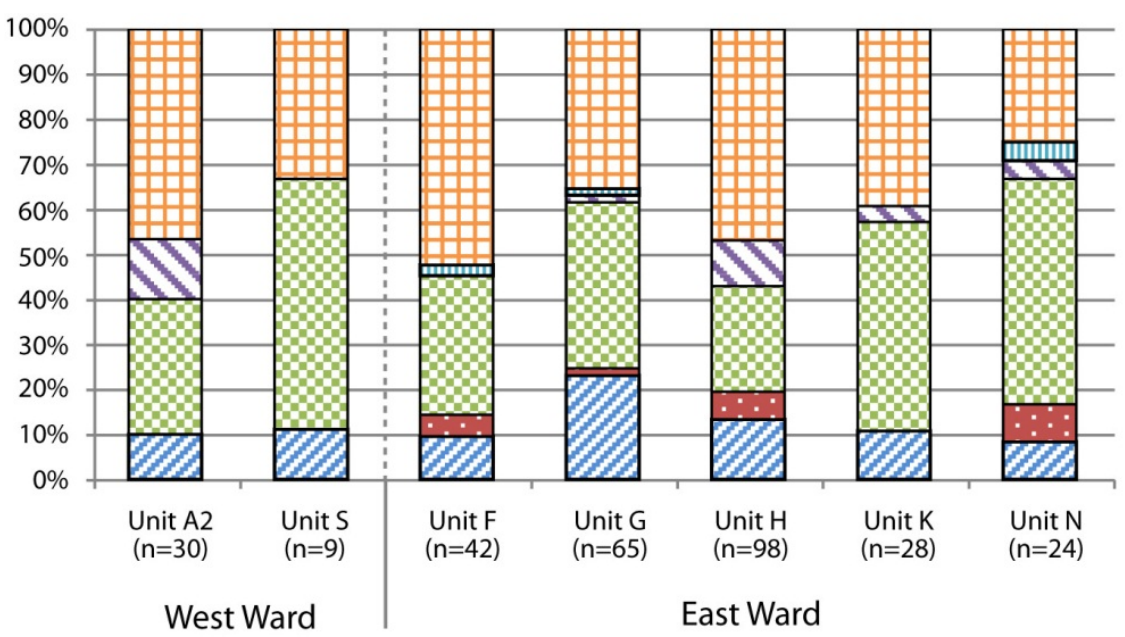

Rim Form

四 Simple (S)

四 Ledged (Ldg1/2)

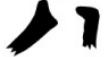

0 Collared $(\mathrm{Bd} 1 / 2, \mathrm{ThC})$

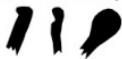

$\square$ Vertical collar (EvC)

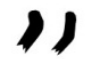

$\square$ Flared (EfA/R)

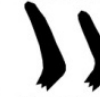

Everted (ErA/R)

1)

\section{Long-Distance Exchange}

Five cowries and a single piece of copper wire document participation by Early phase inhabitants in interregional networks (Figures 9.5-9.6). Based on the low numbers of these objects, the circulation of these goods most likely occurred as gift-giving, bride wealth, and perhaps political or ritual exchange between neighboring villages and kin groups. The absence of glass and carnelian beads (prevalent in later phases) could suggest that trade circuits for such materials were not yet open to the community of Diouboye. 
Figure 9.5: Relative distribution of glass, copper, and carnelian artifacts

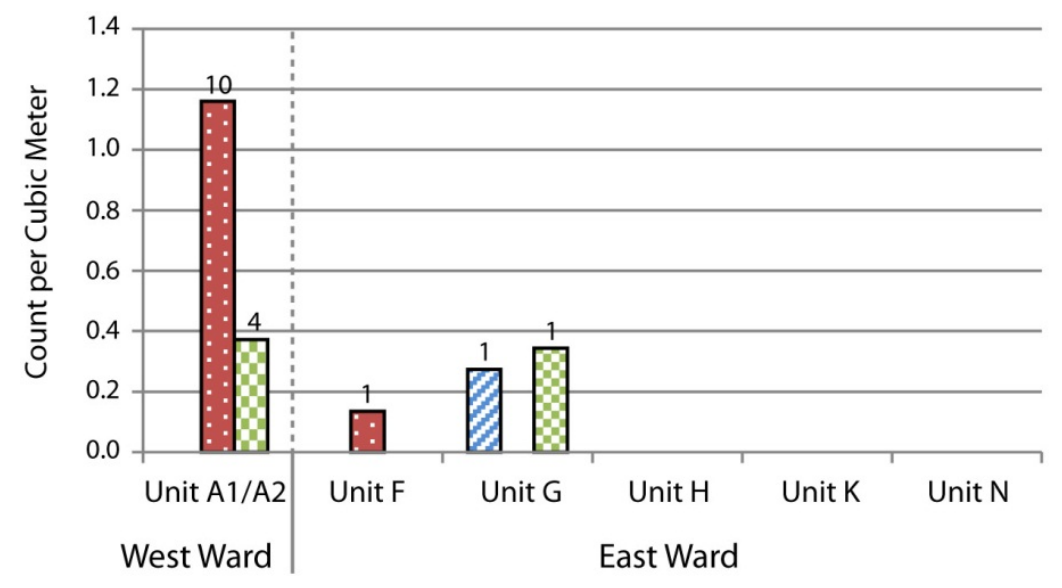

Phase

Late Diouboye

$\square$ Middle Diouboye

G Early Diouboye A/B

Figure 9.6: Relative distribution of cowrie shells

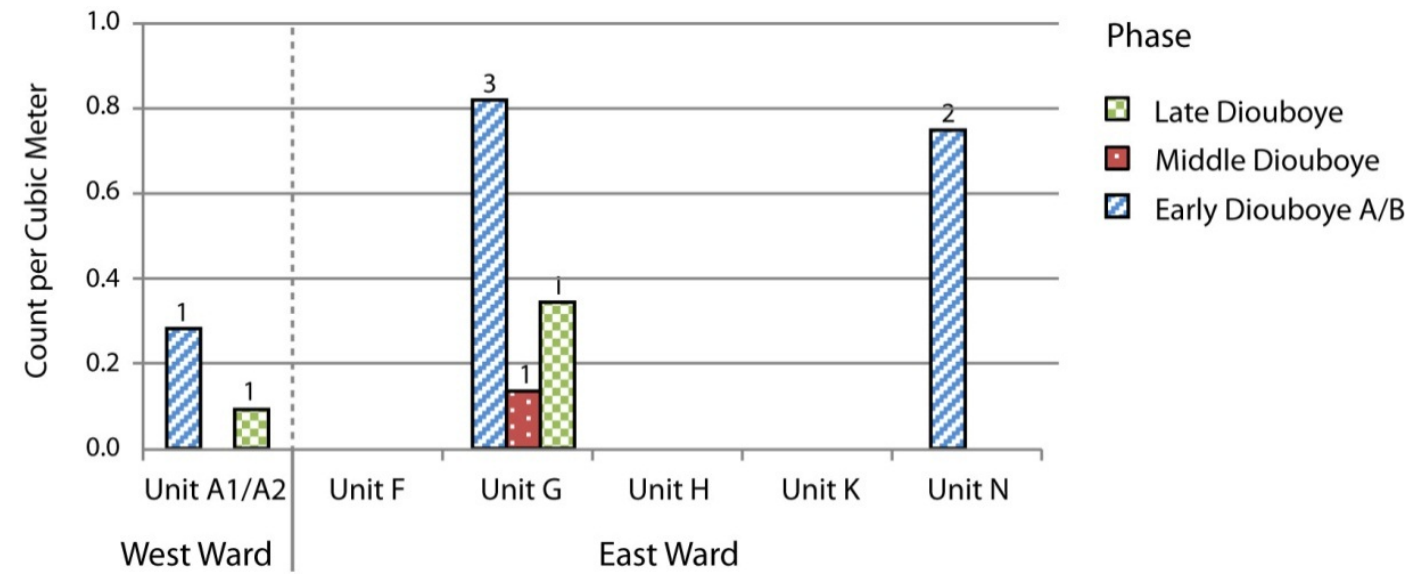

\section{Summary}

So who were the settlers of Diouboye? They arrived in the central stretch of the Falémé River valley with an architectural repertoire that included earthen wall structures of both wattle-and-daub and terre pisé, and a capacity for innovation with local materials such as sandstone rocks. Together, this constitutes evidence for an established tradition of sedentary village life. Knowledge of hunting and foraging in the rich riparian and savanna habitats along the Falémé River, along with the probable cultivation of millet, point to familiarity with similar landscapes. Given the limited emphasis on livestock and 
pottery technologies most closely resembling those of a tentative Niger-Gambia ceramic sphere, I propose that the village founders originated within, or at least participated in, the Mande cultural world centered to the south, rather than the Soninke or Fulfulde world situated in the Middle Senegal region and Sahel to the north. Indeed, the initial layout of the village with several residential areas surrounding an open plaza falls squarely within the "traditional" form of Malinke villages in the Upper Niger region (Leynaud and Cissé 1978:81-84), a point that I return to below.

The initial population of Diouboye may have included as many as 60-80 adults. The simultaneous settlement of three or four residential areas in the Early Diouboye A phase probably represents a process of immigration and fusion, rather than more protracted demographic growth through household fission. In this regard, the greater population density and evidence for domestic architecture in the East Ward may have ultimately bestowed first-comer status on the groups who resided there. Since this status formed an important and widespread source of politico-ritual power in precolonial West African societies (Chapters 1-2), I submit that residents of the East Ward held sway over community decisions about subsequent settlement and land use in and around Diouboye.

Regarding the political economy of the early village itself, the generalized suite of subsistence and long-distance exchange activities and lack of evidence for specialized craft production suggest that material resources did not underwrite hierarchical relations between residential groups. Non-material resources ensconced in political idioms of kinship, ritual power, and first-comer status may have worked to ensure more-or-less symmetrical participation in economic processes.

The regional survey data do, however, suggest that Diouboye quickly emerged as an economic and political center on the local landscape. While the precise evolution of occupation at other settlements cannot be gleaned from surface materials, they do show that Diouboye began as one of several hamlets evenly spaced along the Falémé River, and quickly established itself as a "primate" village on the local landscape by the end of the Early Diouboye phase (Chapters 4-5). Whatever the size of Goundafa at this time, the proximity of this settlement directly across the river suggests that its inhabitants formed a distinct social segment within a broader local community centered on Diouboye. 
Beyond their broad participation in a Mande cultural sphere evidenced by shared craft technologies, it remains unknown how the early occupants of Diouboye interacted politically with more centralized polities such as Takrur and Ghana/Gajaaga in the north and hypothesized Mande polities to the south and east. Access to a few non-local goods, cowries in particular, minimally suggests that regional social networks were more important than interregional trade for connecting the village to polities elsewhere across the savanna.

\section{Middle Diouboye Phase (AD 1100-1250) Space and Architecture}

The village grew to its maximum extent during the Middle Diouboye phase with the establishment of household compounds at Area A, Areas J/K, and possibly Area $\mathrm{H}$, in addition to continued residence at Area F, Area G, and Area N. Surface evidence also confirms residential occupation at Area B and Area $M$, as well as the relegation of some non-residential activities to Area A-S and possibly Areas C/D. The chronological status of Area $\mathrm{P}$ is less certain, but residential activity probably extended into this area as well.

As shown in Figure 9.7, this period witnessed the evolution of the village plan into discrete East and West Wards. The East Ward continued to form a ring of residential areas surrounding an unoccupied plaza. The implications of this arrangement for access to land, particularly by the occupants of Areas $\mathrm{M} / \mathrm{N}$, is not clear, but the linear alignment of residential areas would have been consistent with equitable access to strips of land oriented perpendicular between the village and the Falémé River to the west.

The formal production of domestic space continued in Area F, Area G, Areas $J / K$, and Area $\mathrm{N}$ where people built and maintained huts with terre pisé walls, granary foundations, and semi-formal hearth areas. Dozens of sandstone features from the Middle Diouboye phase surface contexts of Areas J/K and Area M provided further evidence for residential occupation. While Unit $\mathrm{H}$ did not yield any architecture, several superimposed hearths associated with cooking practices illustrated a transition from midden to domestic space in Area $\mathrm{H}$.

In Area A the expansion of activities from Unit A2 to Unit A1 revealed a growing formalization of space through the construction of granary foundations and terre pisé 
structures. Based on surface chronologies, people also established household compounds in Area B, and possibly Area C. This latter, however, may have persisted with Area D as an informal space for activities of grinding and/or polishing, metal working, and reducing mudstone. Around Area A, people also segregated these same craft production and possibly subsistence activities at Area A-S, and possibly Area A-N.

\section{Figure 9.7: Middle Diouboye phase activities}

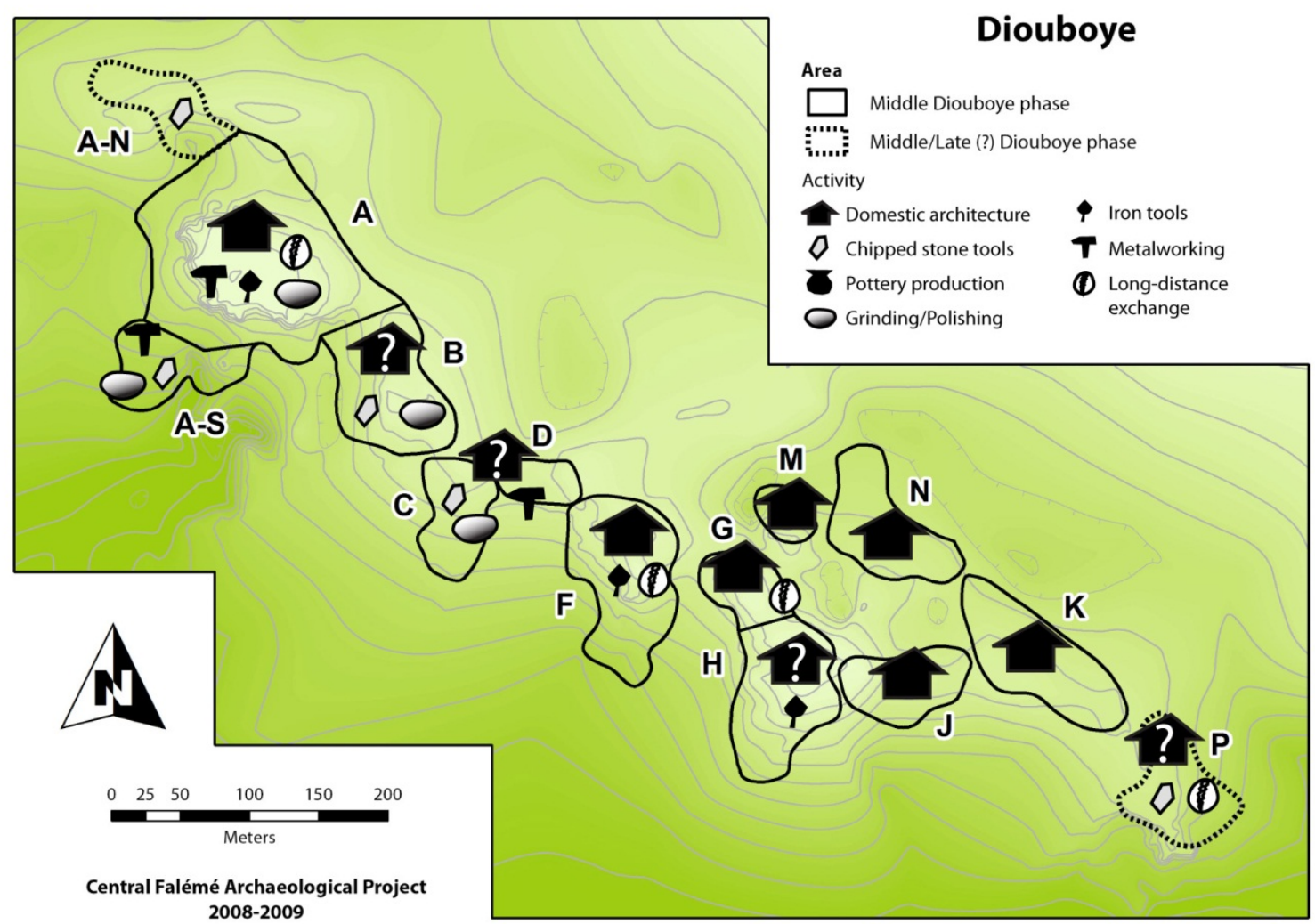

\section{Subsistence Economy}

Although the climate of the Upper Senegal region became less humid during the Middle Diouboye phase, conditions remained favorable for the cultivation of savanna crops such as pearl millet (tentatively identified in Unit F). As in the preceding phase, large and small granary foundations in subsurface contexts attested to independent 
control of storage in each residential area. The similar proportions of granaries from Middle Diouboye phase surface contexts in Area J, Areas K/P, and Areas M/N (see Figure 8.27) suggest that corporate groups residing in each area stored similar levels of surplus for consumption throughout the year.

The small samples of faunal remains (NISP) across the excavated units do not allow for comparing changes in fauna exploitation through time (Appendix $\mathrm{H}$ ). As in the preceding phase, livestock may have been seasonally or annually available in the region, depending upon access to trypanotolerant breeds. The villagers of Diouboye continued to obtain the majority of their meat from terrestrial and riparian game, as well as largebodied Nile perch and catfish from the Falémé River.

Pottery assemblages suggest that people from both wards were engaging in a similar suite of food transportation, storage, and cooking and commensal practices by the Middle Diouboye phase. Jars both large and small became frequent in Area A, while pottery assembalges from residential areas in the East Ward became more diverse (Figure 9.8). Beyond the great number of large vessels in Area $\mathrm{N}$ possibly resulting from an emphasis on storage, the preponderance of smaller jars and pots in other areas is consistent with the preparation and consumption of meals-not surprising given the recovery of most sherds from contexts associated with semi-formal hearth complexes.

Figure 9.8: Relative abundance of vessel types (Middle Diouboye phase)

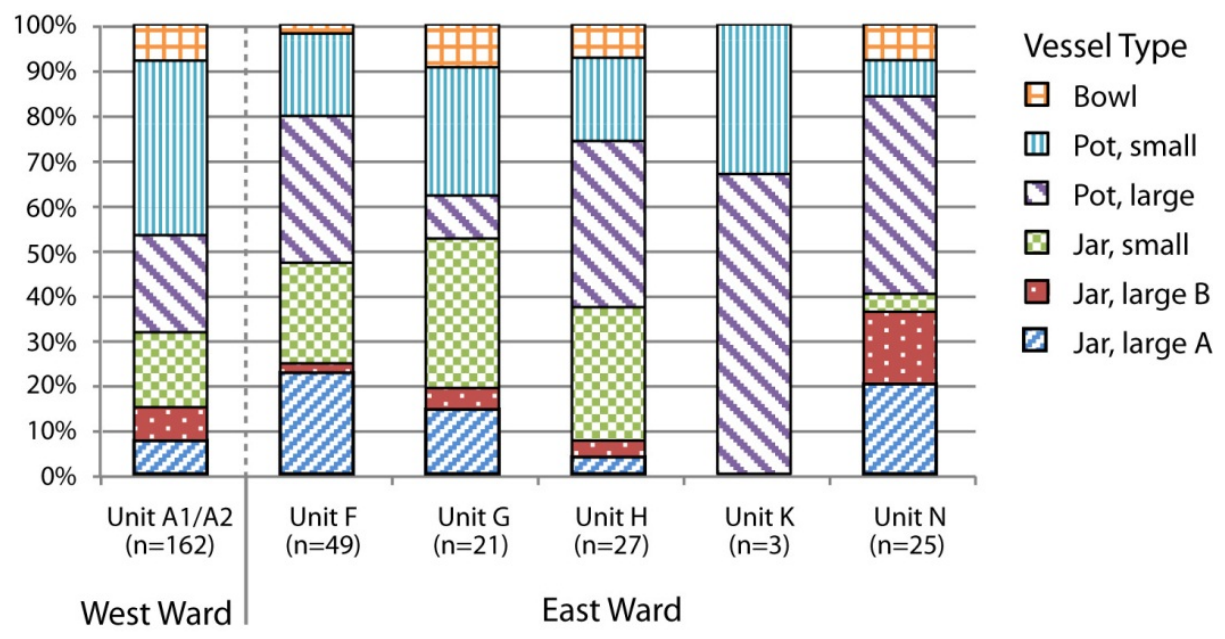


Contrasting with their absence in the earlier contexts, groundstone and iron tools became more common in strata dating to the Middle Diouboye phase. Unit F yielded a hammerstone and grinding slab fragment, while handstones turned up in Unit A1 and Unit A2. Iron tools possibly associated with hunting and farming included hoe blades and small point fragments from Area A, Area F, and Area H. Despite the small sample sizes, these artifacts underscore the use of certain crafts for generalized participation in subsistence production at the household compounds comprising each residential area.

\section{Craft Production and Consumption}

Iron tools and pottery sherds stand testament to the use and discard of locally made crafts, but additional lines of evidence indicate that craft production became more widespread and spatially segregated during the Middle Diouboye phase. A possible blacksmithing workshop with slag and chipped stone was set in close proximity to, or perhaps within, household compounds in Area A, even as the presence of these artifacts in surface contexts at Area A-S and Areas C/D suggest that metal working increasingly took place outside, or on the outskirts of, residential areas in the West Ward.

Figure 9.9: $\quad$ Relative distribution of metallurgical slag

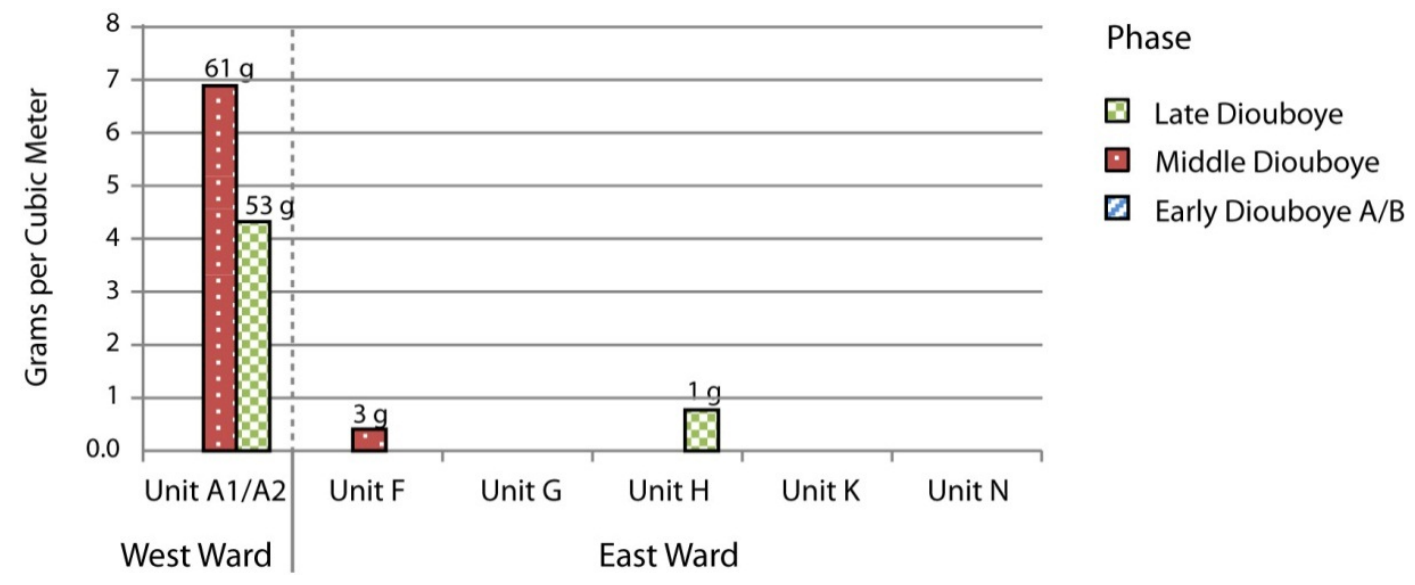


Figure 9.10: Relative distribution of iron artifacts

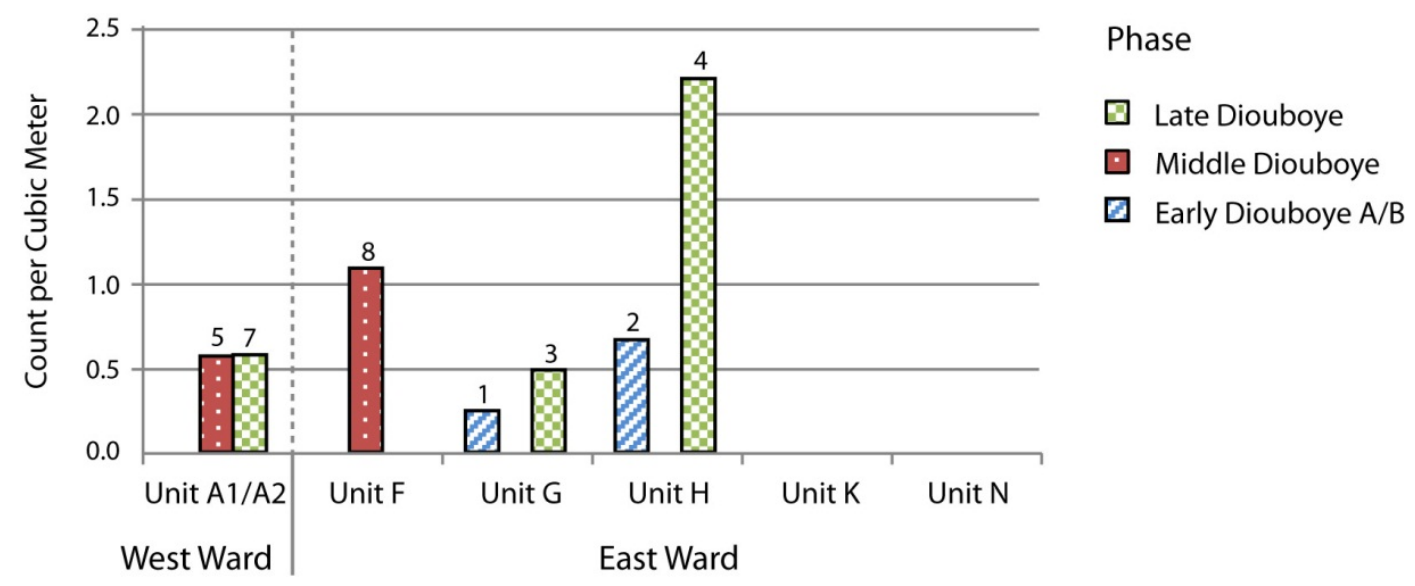

Quartz and chert debitage recovered in excavated contexts (Figure 9.11) show that activities involving informal stone tools, such as cutting, scraping, groundstone rejuvenation, or perhaps fire-starting, took place at all residential areas. The tendency for chipped stone artifacts to occur in higher densities in midden deposits, such as Stratum H.3, hints at the careful cleaning of these sharp materials from heavily trafficked residential spaces. Meanwhile, surface data point to the spatial segregation of more intensive lithic reduction activities involving quartz and mudstone within non-residential Area A-S, and possibly residential spaces including Area B, Area C, as well as Area P. The homogeneous distributions of paste recipes, decorative elements, and rim forms (Figure 9.12) across the site continued to be consistent with the local production of pottery within a single technological tradition, the social and spatial organization of which remains unknown. Given the evidence for increasingly specialized metal working in the West Ward, the residents of Area A may have likewise been responsible for pottery manufacture by this time, but testing this hypothesis requires further excavation data.

The recovery of three terracotta spindle whorls in Unit A2 suggests that spinning took place within residential contexts, although the scale of this and weaving tasks remains as of yet unknown. 
Figure 9.11: Relative distribution of lithic raw material (Middle Diouboye phase)

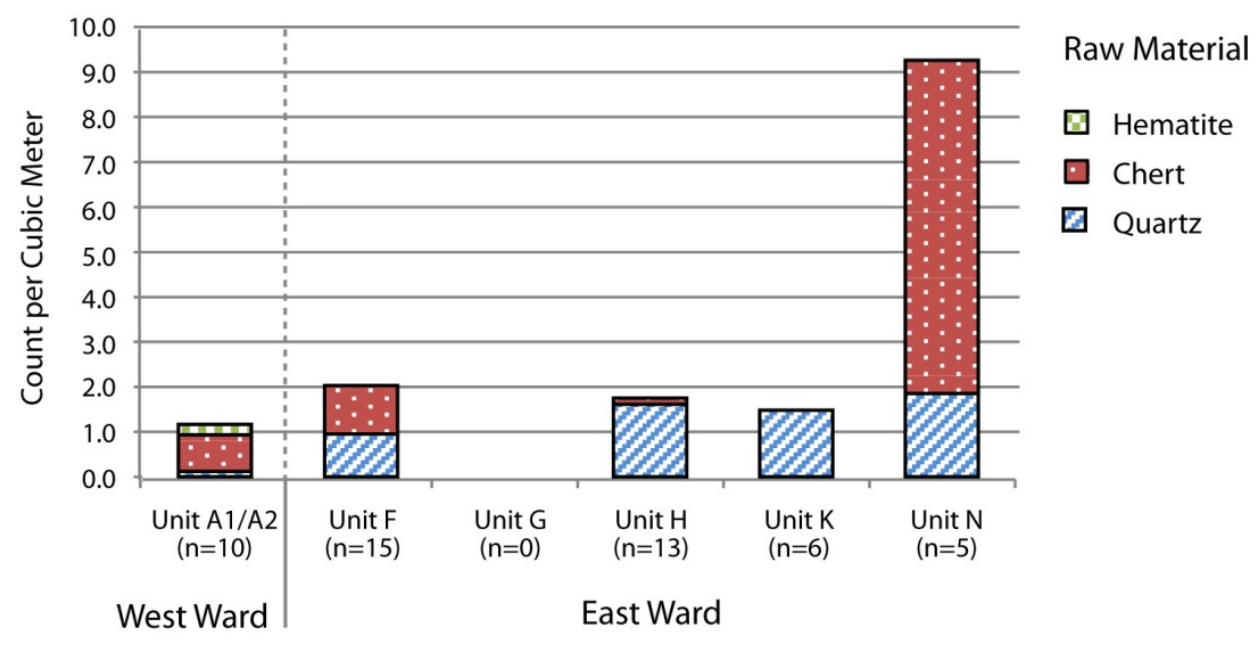

Figure 9.12: Relative abundance of rim forms (Middle Diouboye phase)

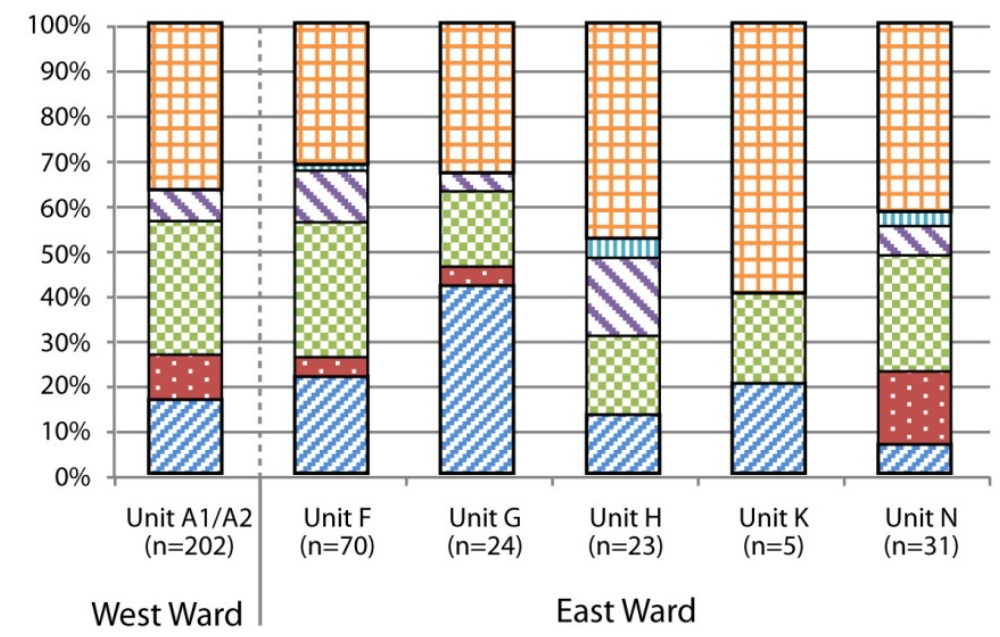

Rim Form

巴 Simple (S)

(⿴) Ledged (Ldg1/2)

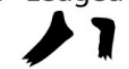

$\square$ Collared (Bd1/2,ThC)

11

$\square$ Vertical collar (EvC)

1)

$\square$ Flared (EfA/R)

)

Q Everted (ErA/R)

1)

\section{Long-Distance Exchange}

Imported artifacts were rare, but exchange for cowries, which had predominated in the Early Diouboye phase, appears to have declined at the expense of glass, carnelian, and possibly quartz beads (Figures 9.5-9.6). These objects were found primarily in the larger artifact samples of Area A, which also yielded a segment of copper wire. 
In spite of their low frequencies, it is possible to draw two inferences from these artifacts. First, interregional exchange networks may have gradually shifted to emphasize the circulation of objects with a northern, rather than eastern, origin. This trend could signal a reorientation of trade coincident with the decline of Ghana and the rise of Mali and/or the changing position of Diouboye within these networks. Second, it is notable that the residents of the West Ward continued to participate in supra-local exchange, even as they increasingly specialized in metalworking and other activities involving chipped stone and groundstone tools. In contrast to the later Sudanian caste system, craftspeople negotiated the production of their own political status through exchange practices both within the community and across social networks. In fact, active participation in exchange may have been necessary for smiths at Diouboye to import copper and iron smelted outside the region.

\section{Ritual and Adornment}

In addition to imported beads, several terracotta beads $(n=3)$, terracotta bracelet fragments $(n=2)$, and a possible iron bracelet fragment show that people from residential areas across the village made some use of local materials for adornment. In addition, a polished quartz hachette from Area F could suggest that such objects found use, possibly for ritual, within residential spaces. Along with hematite axes across Diouboye, this hachette could have been made or reworked within Area A-S, or possibly Areas C/D, where surface finds of blanks and debitage were consistent with localized production. If so, then the specialized production of symbolically charged groundstone and metal objects could have given residents of the West Ward an important source of authority within the community.

\section{Summary}

The village nearly doubled in size during the Middle Diouboye phase to achieve its maximum spatial extent and a population of perhaps 200-300 people. Along with surface remains, excavations confirmed that household compound(s) within each residential area hosted a general suite of economic practices. Minimally, these included the storage, cooking, and consumption of foodstuffs, the use of informal chipped stone 
tools, the (ritualized) use of polished hematite hachettes, and possibly spinning with discoidal terracotta spindle whorls. The growth of the village also corresponded to greater segregation of activities between wards, and between residential and non-residential areas, the latter associated with a greater intensity of metal working, hematite hachette production, and perhaps certain wood- or hide-working tasks associated with chipped stone and groundstone tools. The exchange and display of non-local objects may have become increasingly differentiated as people residing in the East Ward continued to traffic mainly in cowries, while the West Ward established greater access to glass, facetted carnelian/quartz, and copper beads.

Even with a trend towards segregation and differential intensity across economic spheres of craft production and exchange, these practices did not underwrite strongly hierarchical political relations between residential groups within the community. While the production of, or access to, some crafts and exchange objects may have been important sources of political power, particularly for the residents of the West Ward, these were not channeled into the material trappings of ranked status in subsistence practices or exchange for exotic items through social networks extending beyond the village. In this regard, a growing emphasis on craft production in the West Ward may have been a strategy to balance social authority within a community first settled by the occupants of the East Ward.

At a slightly broader scale, survey data show that Diouboye continued to be a primary economic and political center on the local landscape. While it was not possible to distinguish sites dating to the Middle Diouboye phase from those of the Late Diouboye phase, the regional settlement distribution suggests that the growth of the village was accompanied by the abandonment of several smaller homesteads (Chapters 4-5). Across the river, Goundafa may have also reached its maximum extent at this time. Whether or not people from smaller hamlets immigrated to these larger villages, this pattern of aggregation is consistent with a more defensive posture on the part of the local populace. As discussed for Diouboye in Chapter 5, the community would have required the mobilization of subsistence resources beyond the capacity of cultivable land in the immediate catchment, as well as sociopolitical structures for integrating more coresidential groups with different kinship allegiances. 
Diouboye maintained ties to the Mande cultural world through shared craft technologies, particularly pottery. An increase in glass and copper imports, however, could point to greater participation in exchange networks oriented towards the Middle to Upper Senegal regions and the trans-Saharan trade êntrepots beyond. This pattern is particularly interesting since it corresponds to the decline of the Ghana Empire, and its control over the gold of Bambuk, through the $11-12^{\text {th }}$ centuries AD. Notably, the people of Diouboye did not suffer the political fortunes of Ghana and other centralized polities in their day-to-day subsistence and craft economies, and access to interregional exchange networks appears only to have expanded. As I elaborate below, these trends point to the capacity of people within Diouboye to negotiate their position on the periphery of the multiple and overlapping political-economic spheres that ebbed and flowed across the savanna during the early- to mid-second millennium AD.

\section{Late Diouboye Phase (AD 1250-1400)}

\section{Space and Architecture}

According to the surface chronology, the Late Diouboye Phase witnessed the abandonment of Areas $\mathrm{M} / \mathrm{N}$, Areas $\mathrm{J} / \mathrm{K}$, and probably Area P. Sandstone fragments from disaggregated architectural foundations in Areas C/D suggest that residential activities replaced, or continued alongside, earlier chipped stone and metal working activities. The final plan of the village thus comprised a linear alignment of residential areas oriented parallel to the expanse of cultivable alluvium on the west, even as the wards continued to form a salient spatial division. If the ceramic chronology is any clue, then this division endured through the steady depopulation of the village culminating in the abandonment of the last two occupied areas-Area A in the West Ward and Area H in the East Wardduring the $14^{\text {th }}$ century AD.

As in earlier phases, excavations found that people in each residential area built and maintained a similar suite of architectural features, including terre pisé wall structures and granary foundations. Cylindrical borrow pits also became a feature of domestic space in Area A and Area G, although their absence in previous phases may simply represent a sampling bias. 
The evolution of domestic space at the scale of the residential area remains unknown, but it appears that Area A and Area B experienced greater segmentation around granaries than did areas in the East Ward by this final phase of occupation. This pattern could suggest that the West Ward not only had more residents and presumably “wealth-in-people,” but that extended households or other kin groups had proportionally more social units organized around ownership of granaries.

Figure 9.13: Late Diouboye phase activities

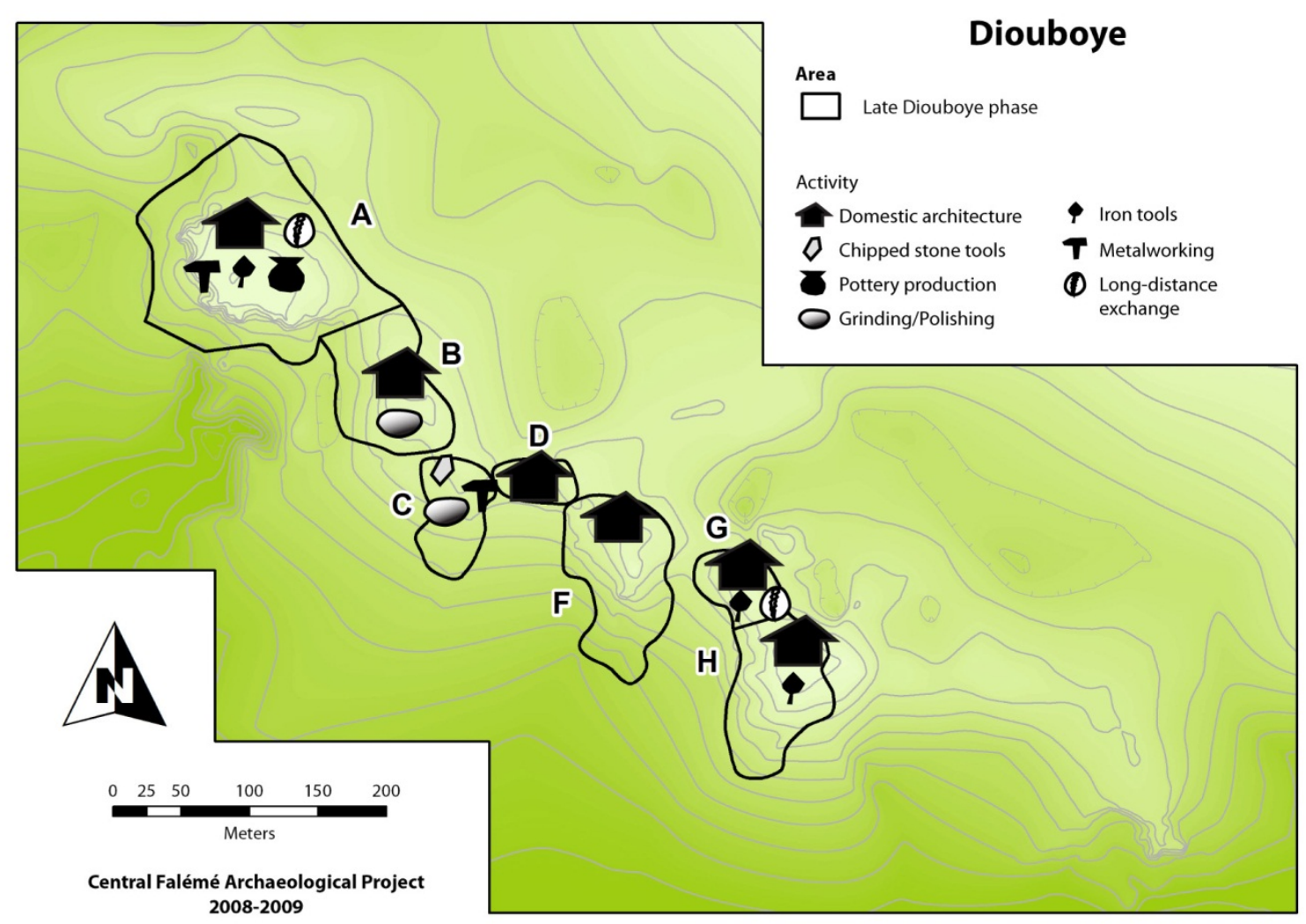

\section{Subsistence Economy}

By the end of the Late Diouboye phase circa AD 1400, the climate of the Upper Senegal region began to resemble current conditions, which remain amenable to the cultivation of pearl millet (firmly identified in Unit A2). The aforementioned granary foundations from surface contexts attest to similar storage capacities in each residential 
area, if not slightly more segmented social fields of ownership within the West Ward. As in the preceding phases, livestock may have been seasonally available in the region, but the villagers of Diouboye continued to obtain the majority of their meat from terrestrial and riparian games, as well as large-bodied Nile perch and catfish from the Falémé River.

In comparison with preceding phases, the use of jars in Area A increased to match the proportions found elsewhere across the site, while the slightly lower number of large vessels in Area $\mathrm{H}$ highlights a possible reduction in storage and transportation activities (Figure 9.14)—perhaps not surprising given the sequential use of space within Unit $\mathrm{H}$ for semi-formal cooking hearths.

Excavated handstones and other groundstone implements from Area A, Area G, and Area $\mathrm{H}$ indicate that food processing took place within domestic space, though several of these artifacts derived from midden or pit fill contexts of secondary deposition. These three residential areas also yielded tools for procuring and processing foodstuffs, including iron blades, a projectile point, a hook, a harpoon, and a large terracotta netweight. Alongside the similar diversity of wild and domesticated resources consumed in each area, these artifacts support an interpretation of equitable participation in a general subsistence economy by people occupying each residential area.

Figure 9.14: Relative abundance of vessel types (Late Diouboye phase)

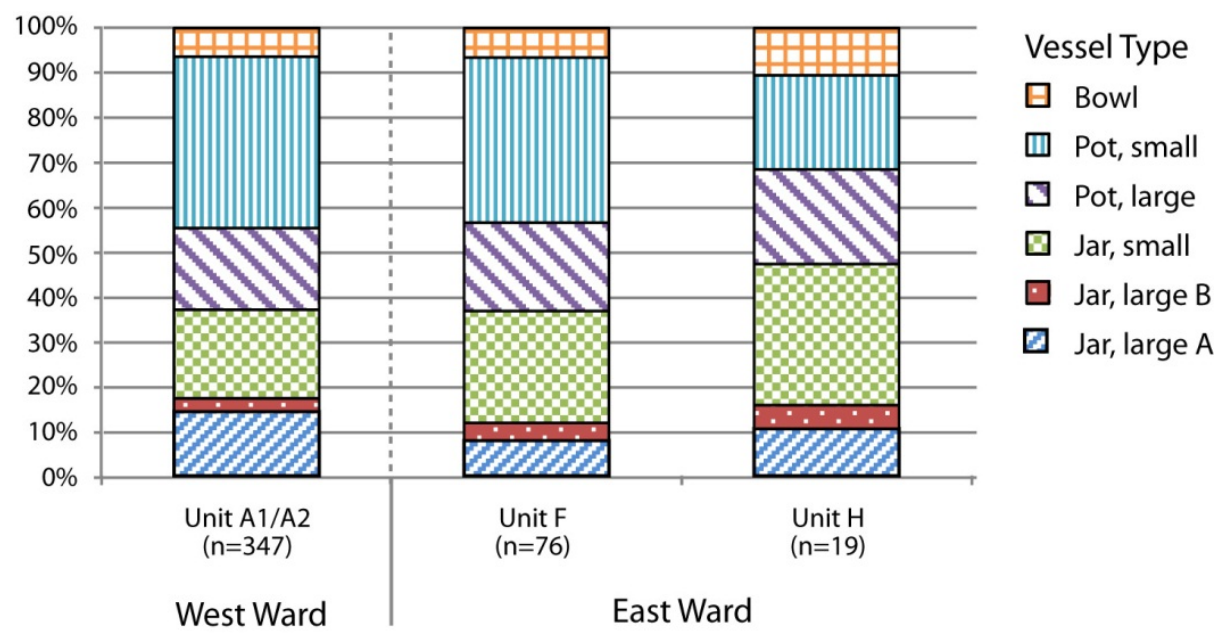




\section{Craft Production and Consumption}

The distribution of artifacts related to groundstone axe manufacture, metal working, and pottery production strongly suggest that specialized craft activities became less segregated between residential and non-residential space, even as they continued to be concentrated in the West Ward. The presence of a hematite hachette preform in Unit A1, for example, coincided with the greater proportion of blanks and preforms in the surface assemblages from Area A, Area B, and Areas C/D. Even as Area A-S fell into disuse and the installation of architectural features in Area D marked a transition to more formalized domestic space, the higher incidences of slag and the recovery of a crucible from Late Diouboye phase contexts in Area A indicate that people were working iron and copper metals in or near household compounds. While the homogeneous distribution of rim forms (Figure 9.15), paste recipes, and decorative motifs highlight the persistence of a single pottery tradition, the evidence of a hand wheel and several hunks of granitic "temper" from Unit A2 point to the co-occurrence of potting and metal working practices in this part of the village.

\section{Figure 9.15: Relative abundance of rim forms (Late Diouboye phase)}

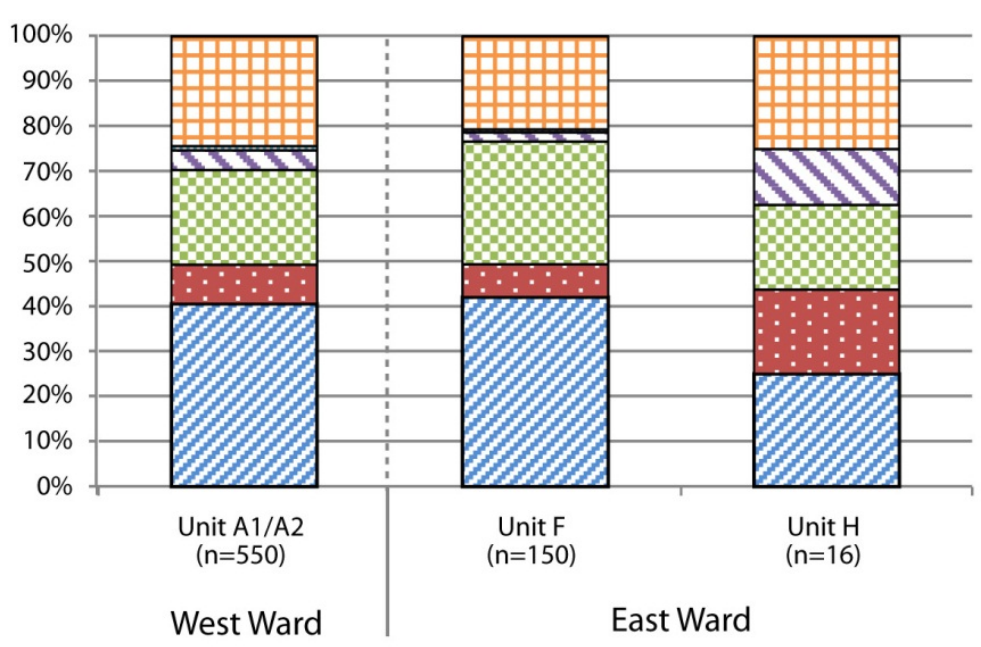

Rim Form

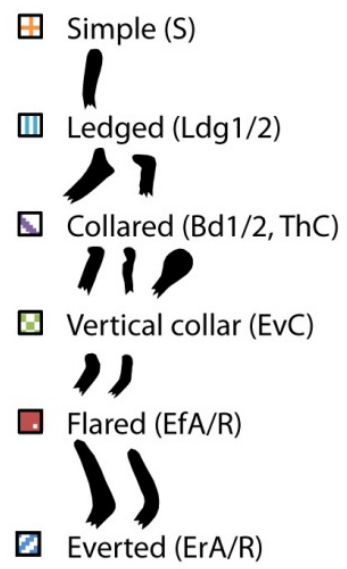

1) 
Figure 9.16: Relative distribution of lithic raw material (Late Diouboye phase)

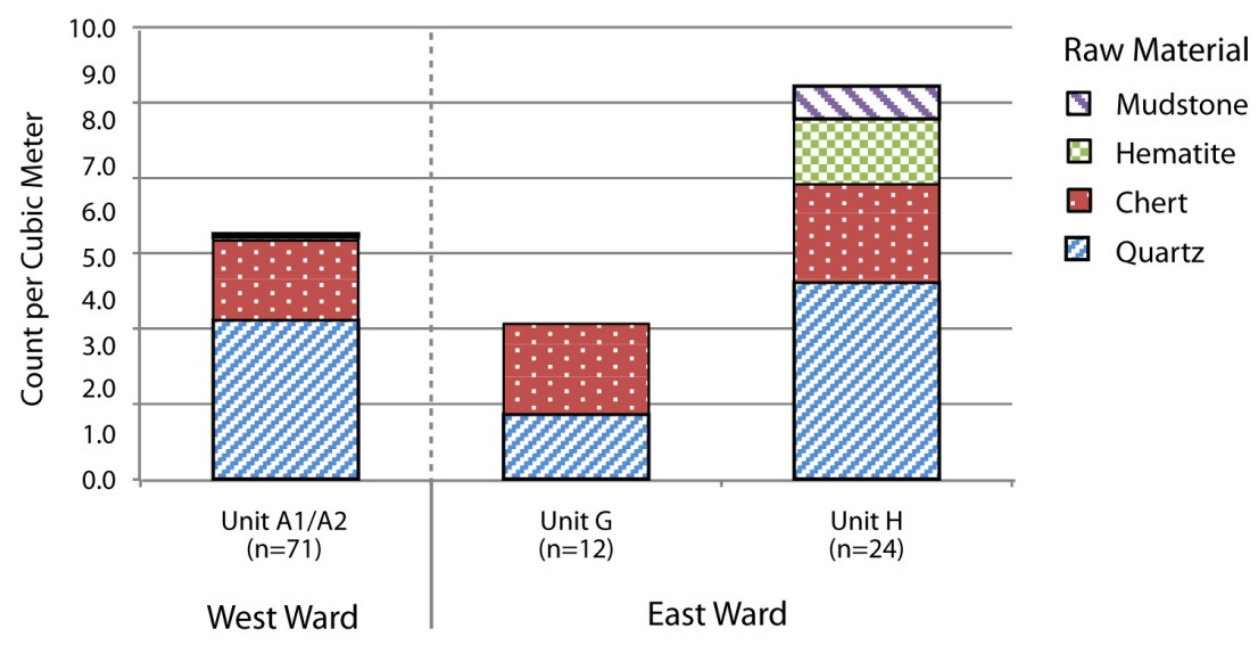

As in the preceding phase, several terracotta spindle whorls from Area A and Area G confirm that spinning (and possibly weaving) activities likewise took place within domestic space across the village. Similarly, chipped stone debris turned up in surface and subsurface contexts across the residential areas occupied during this phase although it occurred in the somewhat greater densities alongside the abovementioned evidence for metal and pottery production in Area A (Figure 9.16).

\section{Long-Distance Exchange}

A carnelian bead from Area G and two copper ornaments and one cowrie from Area A generally confirmed the pattern observed in surface assemblages for the exchange and display of non-local objects in residential contexts throughout the village. While small sample sizes make it difficult to assess the relative intensity of long-distance exchange between residential areas, these finds, along with those from surface contexts, demonstrate that the growing segregation of craft production in Area A did not preclude participation in supra-local political and economic networks.

\section{Ritual and Adornment}

Alongside the surface finds of terracotta figurines and bracelets discussed in Chapter 8 , the recovery of similar items, including several terracotta and bone beads, 
from excavated contexts shows that the residents of Diouboye continued to fashion ornaments and/or ritual paraphernalia out of local materials. The deposition of these materials in all residential areas shows that associated practices of display were not confined to one particular ward.

\section{Summary}

As the spatial plan of Diouboye returned to a linear form parallel with the Falémé River, the population steadily declined. The clustering of surface features indicates that the village continued to comprise at least two distinct wards, perhaps even until the ultimate abandonment of Area A and Area H. The causes of this exodus likely included some combination of ecological and political factors. The savanna climate had become steadily more arid, and perhaps volatile, during the period AD 1000-1400 (see Chapter 3), while three centuries of cultivation could have led to the degradation of land, woods, and other resources around Diouboye. Given the heavy focus on hunting and fishing within the riparian corridor of the Falémé River, it may have been necessary to migrate in search of perennial access to deep-water fish and ample wild game.

As I discussed in Chapter 4, the $14-15^{\text {th }}$ centuries AD coincided with transformations in the interregional political landscape as states such as Takrur, Djolof, Gajaaga, Kaabu and other vassals to Mali began to assert their autonomy across Senegambia. Meanwhile, large-scale migrations by Fulbe populations into Senegambia from the Middle Niger region may have further destabilized existing settlement strategies and political economies across the Upper Senegal region. It remains unclear how, or if, power shifts among centralized societies impacted the decentralized societies at their interstices. With the expansion of arid conditions (and southward retreat of tsetse fly and sleeping sickness), Bambuk would have been, whether for better or worse, more accessible to traders and/or slave raiders on horseback (Brooks 1993; Webb 1993). In this latter regard, the abandonment of Diouboye and possibly its environs, followed by a gradual resettlement during the $15-16^{\text {th }}$ centuries $\mathrm{AD}$ (Chapter 4 ) could signal a process of political conflict or even violence, rather than the negotiation of a cultural frontier.

Prior to abandonment, residential groups across the village continued to engage in similar subsistence and craft activities including the storage, cooking, and consumption 
of foodstuffs, the use of informal chipped stone tools, and spinning. They also participated in similar practices for the exchange and display of imported objects and the use of terracotta figurines, bracelets, and other items potentially associated with ritual.

Whether or not the craft activities attested by surface finds in Areas C/D date to this phase, the evidence does point toward the greater intensity of metal working, pottery production, and possibly hematite axe production in Area A and possibly Area B. These economic activities also became less segregated within the ward, occurring in or near the space demarcated by domestic architecture. The differential ordering of economic practices and social relations across residential areas also finds tentative support in the greater segmentation of space around surface granary groups in the West Ward.

All in all, these data highlight growing specialization in the craft economy, and continued generalization in subsistence and exchange practices, between residential areas. This could suggest that political power remained balanced among residential groups with first-comer status providing a non-material source of power for occupants of the East Ward, and the practical (and ritual) knowledge of blacksmithing and pottery manufacture providing a material source of power for occupants of the West Ward.

Settlement patterns among sites dating to the Late Diouboye phase suggest that the village continued to form a primary economic and political center within the local landscape. As I noted above, the region experienced a major depopulation between the abandonment of Diouboye and the subsequent foundation of several small hamlets and homesteads during the Tontèko phase (AD 1500-1700). Since the material practices of these later settlements, including their pottery and settlement structure, differ qualitatively from those of earlier Iron Age populations, it stands to reason that their residents had a distinct cultural background, possibly tying them to the Soninke polity of Gajaaga in the north.

In the absence of a more complete regional dataset, it remains difficult to assess the relations between Diouboye and more centralized polities beyond Bambuk, but people across the village certainly continued to engage in interregional exchange and maintain continuity in the rhythms of day-to-day economic and social life through the final phase of occupation, despite the shifting political landscape noted above. 


\section{DIOUBOYE AND POLITICAL ECONOMY IN IRON AGE WEST AFRICA}

The above synopsis of the economic processes and sociopolitical institutions that simultaneously shaped and emerged from daily life at Diouboye has already moved to address the two questions that I posed at the outset of this study. Put simply, I asked how local and supra-local relations enmeshed within subsistence, craft, and exchange economies intersected within this village community throughout its history. Thus framed, the foregoing archaeological study of political economy has, above all, worked to further illuminate variability in the small-scale communities that predominated across past social landscapes both in and beyond West Africa. Additionally, Diouboye provides unique insight into the experience of people living near the much-sought-after gold of Bambuk during the early-second millennium AD. In this final section I respond to these questions by summarizing interpretations developed throughout the preceding pages and by highlighting hypotheses and suppositions to be addressed through future fieldwork.

\section{Economics of Community Life}

An anthropological perspective of political economy has proven useful for the study of daily life in Diouboye, despite an absence of the strongly hierarchical relations conventionally considered from this perspective. Drawing upon a Marxist reading of economic anthropologist Karl Polanyi (Halperin 1994), I defined political economy as the symmetrical (power to) and asymmetrical (power over) relations that emerge (in very different ways) from processes of production, distribution, and consumption in social groups at all scales_-from nuclear households to interregional empires. In focusing on a single village community, I have worked to understand how these economic processesas locational movements of resources to people (and vice versa) and appropriational movements sustaining institutions_-played out at these multiple scales. To this end, I traced the spheres of subsistence, craft, and long-distance exchange, known to have become increasingly specialized across the mosaic Iron Age landscapes of West Africa, through their material and spatial dimensions in the archaeological record of Diouboye and the surrounding Madina-Sadatou study area.

Based on the available evidence, the subsistence economy varied remarkably little between residential groups at Diouboye and remained fairly consistent over the course of 
several centuries. Substantial quantities of faunal remains from the site, though highly fragmented, document an emphasis on hunting and fishing in the surrounding riparian savanna where people also made use of wild plants such as baobab and jujube. Closer to the village, people cultivated pearl millet for storage in earthen-walled or basket granaries built atop circular stone foundations. Equitable distributions of groundstone and iron tools for food processing, and pots for storage, cooking, and serving, also suggest that residential groups undertook similar food practices. The larger size of residential areas in the West Ward, and greater segmentation of space within Area A, could hint at greater "wealth-in-people" defined around the minimal social units (e.g., nuclear families, women with children) living in this area. In this regard, it is notable that the village probably depended in part upon subsistence activities beyond the cultivation of its immediate catchment. This appropriation of space, whether through seasonal labor or intensive exploitation of riparian resources, probably reinforced the political clout of Diouboye vis-à-vis other settlements and residential groups on the local landscape.

Food was undoubtedly part and parcel of social relations within the village, but there is no indication that the subsistence economy underwrote political statuses or cultural identities. Whatever differences may have existed in production activities (i.e., fishing, hunting, farming) or their intensity (i.e., annual surplus stored in granaries) were presumably evened out among residential groups through symmetrical relations of reciprocity on a daily, seasonal, or annual basis. The low number of faunal specimens confirmed as cattle and sheep/goat, and their equal proportions in the assemblage, could, however, suggest that subsistence regimes underwrote social boundaries at a regional scale between the villagers of Diouboye and transhumant pastoralists supplying the community with occasional livestock - today a well-established pattern in the West African savanna, and a hypothesis that will merit further consideration as more faunal samples undergo analysis.

Another intriguing possibility is that an emphasis on the exploitation of largebodied game supported the presence of a hunters' association within the community. Briefly, these associations allow individual men to share and master the ritual knowledge required to handle the dangerous power released upon killing wild game (Cissé 1964). Although historian Jan Jansen (2008) has questioned the link between contemporary 
hunters' associations and those recounted in the historical production of "traditional" Mande identity, he does acknowledge that such sodalities have long been integral to at least the ideology of collective action in rural societies. This latter interpretation has been aptly summarized by Youssouf Cissé (1964), who describes how the politico-ritual structure of Mande hunters' associations can perpetuate social relations among men from different kinship groups and offer a pathway to leadership for younger men within the community. A hunters' association could therefore have provided one means through which subsistence practices sustained symmetrical political relations within and between residential areas across Diouboye. Of course, this interpretation remains provisory pending further analysis of the faunal remains aimed at understanding which species were targeted (and avoided) and how different body parts were distributed and consumed throughout the community.

The craft economy exhibited greater variability across space and time within the village. While the organization of the craft economy, namely pottery making, metal working, stone tool use, and perhaps polished axe manufacture, remains unknown for the initial phase of occupation, these tasks all became increasingly segregated and/or intense within the West Ward during the Middle to Late Diouboye phases. Low-level craft specialization may have formed an important resource or social distinction for balancing local political authority as the community grew in size from the initial first-comers (and with authority grounded in local land rights) to include diverse late-comers. At the same time, the village may have maintained a monopoly over craft production across the local landscape since outlying hamlets and villages provided less conspicuous evidence for metalworking.

If, as I have argued, the community of Diouboye maintained political and cultural ties to the Mande world, then this trend towards craft specialization may represent the historical expansion of the Mande caste system into Bambuk. On the one hand, the relegation to craftspeople (nyamakalaw) to their own ward on the outskirts of the village beyond the central plaza — similar to the pattern observed at Diouboye—is consistent with the spatial organization of Malinke villages today in the Pays Mande (Leynaud and Cissé 1978:248-265). On the other hand, there are no substantial differences in the quantity or quality of the available material data to suggest that people living in the West 
Ward held a lower status within the community. The implication is that, despite the legendary origins of nyamakalaw status recounted in regional epics (e.g., Conrad 1995; Niane 1965), these identities have historically emerged through the negotiation of political authority at local scales-just as they continue to do today.

Non-local objects, including cowries, carnelian, quartz, and glass beads, copper items, and perhaps even iron, show that people across the community took part in longdistance exchange networks. The small sample sizes make any comparison speculative, but people occupying the West Ward of Diouboye may have participated more intensively in trade for copper and glass items, compared to residential groups in the East Ward preferring cowries. At a minimum, the trend towards craft specialization in the West Ward did not entail exclusion from access to exotic goods (contrary to expectations of presumably low status castes). If anything, residential groups engaged in metalworking may have played a larger role in exchange, particularly as they worked to acquire nonlocal copper and possibly iron for their craft. More provocatively, the capacity of local smiths to cast copper and gold could have further positioned them as key players in community connections to regional and interregional political economies.

\section{Interregional Entanglement}

In the archaeology of West Africa, political economy has proven to be a useful frame for examining global entanglements, particularly those fueled by Atlantic trade after the $16^{\text {th }}$ century AD. Prior to this period, interregional connections and the regional hegemony of West African states remain more difficult to ascertain from material data, given their limited interest in directly controlling subsistence and craft economies. Rather, state finance from Ghana to Mali depended in part upon trade in commodities headed northward across the Sahara Desert, while later the polities of Gajaaga and Bundu benefitted from trade oriented westward towards the Atlantic Ocean. Maintaining the flow of commodities from "peripheries” such as Bambuk meant leaving local structures of subsistence and craft production largely in place. That said, the three economic spheres considered in this study do provide some insights into the dynamics of regional political and cultural institutions, particularly when compared to the evidence available from contemporaneous sites. 
An emphasis on hunting in the subsistence economy of Diouboye, for example, suggests cultural connections with communities to the south in the savanna forest, rather than those to the north who were more heavily engaged in pastoralism by this time. As hypothesized above, this may signal the development of symmetrical economic relations with transhumant pastoralists migrating seasonally along the Falémé River. To speculate further, an emphasis on fishing could have benefitted from a steady supply of salt for preservation, a practice cited by the medieval geographer al-Idrisi (Levtzion and Hopkins 2000:108); salt, of course, was obtained from northern trade networks extending to the Atlantic littoral and across the Sahara Desert.

Craft practices at Diouboye had affinities with technological traditions of the Mande world of the upper Niger and Gambia River basins. Iron tools, pottery forms and decoration techniques, and circular hut and granary features with foundational stones, all demonstrate greater similarity with material culture and architecture to the south, than to the Middle Senegal region to the north. Terracotta spindle whorls could suggest that people cultivated cotton on a limited basis, this fiber having diffused across West Africa during the late-first millennium AD. As craft production became semi-specialized, possibly within the village and certainly across the local landscape, it did not, however, entail a political or cultural status marked by artifact style or differential access to staple or wealth goods.

As I mentioned, this pattern adds historical nuance to the development of the Sudanian caste systems that supposedly emerged during the early-second millennium AD in the core areas of political centralization by Mande and Wolof/Fulbe societies. If this interpretation holds up against the results of future fieldwork, it could suggest that rural communities within these political economic networks were late to adopt the caste system, or more likely, that Diouboye lay outside the hegemony of these major regional polities. As for ethnographically decentralized Tenda or Diola societies in Senegambia, people could have elected to learn the practical and ritual knowledge of a given trade. The craft economy, then, could have functioned to maintain a balance of social authority within the village community in the face of regional trends towards political hierarchy.

The abovementioned non-local objects provide the most obvious evidence for socioeconomic relations extending outward from the community of Diouboye and 
beyond the immediate landscape of the Madina-Sadatou study area. While the myriad origins of these objects - glass beads from North Africa, copper and carnelian from the Sahara Desert, cowries from the Indian Ocean-hint at participation in diverse trade networks, their low frequencies suggest either low-intensity direct trade or down-the-line exchange through institutions for gift-giving, bride wealth, inheritance, and so forth. Although direct trade with merchant diasporas such as the Jakhanke cannot be ruled out, fieldwork in Madina-Sadatou found no clear evidence for a permanent colony of traders. As with the craft economy, evidence for long-distance exchange suggests that residential groups within Diouboye circa AD 1000-1400 were able to negotiate their position within interregional networks witnessing the decline of Ghana in the Sahel and the rise of Gajaaga and Takrur on the Senegal River to the north and the Mali on the Niger River to the south.

\section{Medieval Bambuk}

The case study of Diouboye offers a point of departure for charting the dynamic landscape of Bambuk and other lands beyond the reach of medieval history. On the one hand, my reconstruction of daily life in this village supports an interpretation in which Bambuk and other gold-producing areas supported mosaics of small-scale communities living largely outside the direct political purview of major West African states. On the

other hand, I have challenged views of such communities as uniform "stateless" societies or as hapless bystanders in the economic, political, and cultural processes that played out across West Africa over the past two millennia. As archaeological research continues to illuminate the diversity of precolonial societies in eastern Senegal, it may yet become possible to understand Diouboye as one nexus of many daily lives in the land of Bambuk. 


\section{BIBLIOGRAPHY}

Abate, T., A. van Huis, and J. K. O. Ampofo

2000 Pest Management Strategies in Traditional Agriculture: An African Perspective. Annual Review of Entomology 45:631-659.

Adams, J. L.

2002 Ground Stone Analysis: A Technological Approach. Salt Lake City: University of Utah Press.

Adams, M. J.

1973 Structural Aspects of a Village Art. American Anthropologist 75(1):265-279.

Adams, R. M.

1981 Heartland of Cities: Surveys of Ancient Settlement and Land Use on the Central Floodplain of the Euphrates. Chicago: University of Chicago Press.

Adams, W. Y., and E. W. Adams

1991 Archaeological Typology and Practical Reality: A Dialectical Approach to Artifact Classification and Sorting. Cambridge: Cambridge University Press.

Agorsah, E. K.

1985 Archaeological Implications of Traditional House Construction among the Nchumuru of Northern Ghana. Current Anthropology 26(1):103-115.

1986 House Forms in Northern Volta Basin, Ghana: (Evolution, Internal Spatial Organisation and the Social Relationships Depicted). West African Journal of Archaeology 16:25-51.

Aldenderfer, $\mathrm{M}$.

1993 Ritual, Hierarchy, and Change in Foraging Societies. Journal of Anthropological Archaeology 12:1-40.

Alexander, J.

1993 The Salt Industries of West Africa: A Preliminary Study. In The Archaeology of Africa: Foods, Metals and Towns. T. Shaw, P. Sinclair, B. Andah, and A. Okpoko, eds. Pp. 652-657. London: Routledge.

Algaze, G.

1993 The Uruk World System: The Dynamics of Expansion of Early Mesopotamian Civilization. Chicago: University of Chicago Press.

Alpern, S.

2005 Did They or Didn't They Invent It? Iron in Sub-Saharan Africa. History in Africa 32(1):41-94.

Altschul, J. H., I. Thiaw, G. Wait, and M. Heilen

2010 A Slave Who Would Be King: Oral Tradition and Archaeology of the Recent Past in a Portion of the Upper Senegal River Basin. In 10th Biennual Meeting of the Society of Africanist Archaeologists. Dakar. 
Amblard, S., and J. Pernès

1989 The Identification of Cultivated Pearl Millet (Pennisetum) Amongst Plant Impressions on Pottery from Oued Chebbi (Dhar Oualata, Mauritania).

Ambrose, S. H.

2002 Small Things Remembered: Origins of Early Microlithic Industries in SubSaharan Africa. In Thinking Small: Global Perspectives on Microlithization. R. G. Elston and S. L. Kuhn, eds. Pp. 9-29. Archaeological Papers of the American Anthropological Association, Vol. 12. Washington, D.C.: American Anthropological Association.

Ammerman, A. J.

1981 Surveys and Archaeological Research. Annual Review of Anthropology 10:63-88. Amselle, J.-L.

1998 Mestizo Logics: Anthropology of Identity in Africa and Elsewhere. C. Royal, transl. Stanford: Stanford University Press.

Andah, B. W.

1979 The Later Stone Age and Neolithic of Upper Volta Viewed in a West African Context. West African Journal of Archaeology 9:87-117.

1982 Urban "Origins" in the Guinea Forest with Special Reference to Benin. West African Journal of Archaeology 12:63-71.

Anderson, B.

1983 Imagined Communities: Reflections on the Origins and Spread of Nationalism. London: Verso.

Andrefsky, W., Jr.

1994 Raw-Material Availability and the Organization of Technology. American Antiquity 59(1):21-34.

2005 Lithics: Macroscopic Approaches to Analysis. Cambridge: Cambridge University Press.

2009 The Analysis of Stone Tool Procurement, Production, and Maintenance. Journal of Archaeological Research 17(1):65-103.

Anquandah, J.

1993 Urbanization and State Formation in Ghana During the Iron Age. In The Archaeology of Africa: Food, Metals and Towns. T. Shaw, P. Sinclair, B. Andah, and A. Okpoko, eds. Pp. 642-651. New York: Routledge.

Appadurai, A., ed.

1986 The Social Life of Things. Cambridge: Cambridge University Press.

Appia, B.

1965 Les Forgerons Du Fouta-Djallon. Journal de la Société des Africanistes 35(2):317-352.

Arnoldi, M. J., C. M. Geary, and K. L. Hardin, eds.

1996 African Material Culture. Bloomington: Indiana University Press.

Arthur, J. W.

2006 Living with Pottery: Ethnoarchaeology among the Gamo of Southwest Ethiopia. Salt Lake City: University of Utah Press.

Arthur, K. W.

2010 Feminine Knowledge and Skill Reconsidered: Women and Flaked Stone Tools. American Anthropologist 112(2):228-243. 
Ascher, R.

1961 Analogy in Archaeological Interpretation. Southwestern Journal of Anthropology 17:317-325.

Ashley, C. Z.

2010 Towards a Socialised Archaeology of Ceramics in Great Lakes Africa. African Archaeological Review 27(2):135-163.

Ashmore, W.

2002 Decisions and Dispositions: Socializing Spatial Archaeology. American Anthropologist 104(4):1172-1183.

Atherton, J. H.

1972 Excavations at Kamabai and Yagala Rockshelters, Sierra Leone. West African Journal of Archaeology 2(1):39-74.

Austen, R. A., ed.

1999 In Search of Sunjata: The Mande Oral Epic as History, Literature, and Performance. Bloomington: Indiana University Press.

Ba, A.

2002 Le Takrur, Des Origines a La Conquête Par Le Mali (Vie - Xiiie Siècles). Nouakchott: Nouvelle.

Ba, C.

1986 Les Peuls Du Sénégal: Étude Géographique. Dakar: Les Nouvelles Éditions Africaines.

Bachmann, H.-G.

1993 The Identification of Slags from Archaeological Sites. Pp. 563-592. Firenze:

Ali'insegna del Giglio.

Baker, A. R. H.

2003 Geography and History: Bridging the Divide. Cambridge: Cambridge University Press.

Balée, W., ed.

1998 Advances in Historical Ecology. New York: Columbia University Press.

Ballouche, A., and K. Neumann

1995 A New Contribution to the Holocene Vegetation History of the West African

Sahel: Pollen from Oursi, Burkina Faso and Charcoal from Three Sites in Nigeria. Vegetation History and Archaeobotany 4(1):31-39.

Bandy, M. S.

2004 Fissioning, Scalar Stress, and Social Evolution in Early Village Societies.

American Anthropologist 106(2):322-333.

Barham, L.

1987 The Bipolar Technique in Southern Africa: A Replication Experiment. The South African Archaeological Bulletin 42(145):45-50.

Barham, L., and P. Mitchell

2008 The First Africans: African Archaeology from the Earliest Tool Makers to Most Recent Foragers. Cambridge: Cambridge University Press.

Barry, B.

1998 Senegambia and the Atlantic Slave Trade. Cambridge: Cambridge University Press. 
Bassot, J. P.

1966 Étude Géologique Du Sénégal Oriental Et De Ses Confins Guinéo-Maliens. Paris: Éditions BRGM.

Bathily, A.

1975 A Discussion of the Traditions of Wagadu with Some Reference to Ancient Ghana. Bulletin de l'Institut Fondamental d'Afrique Noire, Série B 37(1):2-94.

1986 La Trait Atlantique Des Esclaves Et Ses Effets Économiques Et Sociaux En Afrique: La Cas Du Galam, Royaume De L’hinterland Sénégambien Au DixHuitième Siècle. Journal of African History 27:269-293.

1989 Les Portes De L’or: Le Royaume De Galam (Sénégal) De L’ère Musulmane Au Temps De Nègriers (Viii-Xviiie Siècle). Paris: Éditions Harmattan.

Bathily, I. D.

1969 Notices Socio-Historiques Sur L'ancien Royaume Soninké Du Gadiaga. Bulletin de l'Institut Fondamental d'Afrique Noire, Série B 31(1):31-105.

Baxter, M. J.

1989 Multivariate Analysis of Data on Glass Compositions: A Methodological Note. Archaeometry 31(1):45-53.

2001 Methodological Issues in the Study of Assemblage Diversity. American Antiquity 66(4):715-725.

2003 Statistics in Archaeology. London: Arnold.

Bayman, J. M.

2002 Hohokam Craft Economies and the Materialization of Power. Journal of Archaeological Method and Theory 9(1):69-95.

Bayman, J. M., and M. G. Sanchez

1998 The Surface Archaeology of Classic Period Hohokam Community Organization. In Surface Archaeology. A. P. Sullivan, ed. Pp. 75-88. Albuquerque: University of New Mexico Press.

Becker, C.

1985 Notes Sur Les Conditions Ecologiques En Sénégambie Aux 17e Et 18e Siècles. African Economic History 14:167-216.

Bedaux, R. M. A.

1972 Tellem, Reconnaissance Archéologique D'une Culture De L'ouest Africain Au Moyen Age: Recherches Architectoniques. Journal de la Société des Africanistes 42(2):103-185.

1980 The Geographic Distribution of Footed Bowls in the Upper and Middle Niger Region. In West African Culture Dynamics: Archaeological and Historical Perspectives. B. K. Swartz, Jr. and R. E. Dumett, eds. Pp. 247-258. The Hague: Mouton.

Bedaux, R. M. A., and A. G. Lange

1983 Tellem, Reconnaissance Archéologique D'une Culture De L'ouest Africain Au Moyen-Age: La Poterie. Journal de la Société des Africanistes 53(1/2):5-59.

Bedaux, R. M. A., and M. Raimbault

1993 Les Grandes Provinces De La Céramiques Au Mali. In Vallées Du Niger. Pp. 273293. Paris: Éditions de la Réunion des Musées Nationaux.

Bender, B., ed.

1993 Landscape: Politics and Perspectives. New York: Berg. 
Berelov, I.

2006 Signs of Sedentism and Mobility in an Agro-Pastoral Community During the Levantine Middle Bronze Age: Interpreting Site Function and Occupation Strategy at Zahrat Adh-Dhra' 1 in Jordan. Journal of Anthropological Archaeology 25:117-143.

Bernbeck, R., and R. H. McGuire

2011 A Conceptual History of Ideology and Its Place in Archaeology. In Ideologies in Archaeology. R. Bernbeck and R. H. McGuire, eds. Pp. 15-59. Tucson: University of Arizona Press.

Berns, M. C.

1989 Ceramic Arts in Africa. African Arts 22(2):32-37.

Berthier, S.

1997 Recherches Archéologiques Sur La Capitale De L'empire De Ghana: Étude D'un Secteur D'habitat À Koumbi Saleh, Mauritanie, Campagnes Ii-Iii-Iv-V (19751976)-(1980-1981). Oxford: Archaeopress.

Binford, L. R.

1965 Archaeological Systematics and the Study of Culture Process. American Antiquity 31(2):203-210.

1967 Smudge Pits and Hide Smoking: The Use of Analogy in Archaeological Reasoning. American Antiquity 32(1):1-12.

1980 Willow Smoke and Dogs' Tails: Hunter-Gatherer Settlement Systems and Archaeological Site Formation. American Antiquity 45:4-20.

1982 The Archaeology of Place. Journal of Anthropological Archaeology 1:5-31.

1985 "Brand X" Versus the Recommended Product. American Antiquity 50(3):580-590.

Binford, L. R., and G. I. Quimby

1963 Indian Sites and Chipped Stone Materials in the Northern Lake Michigan Area. Fieldiana Anthropology 36(12):277-307.

Bishop, R. L., R. L. Rands, and G. R. Holley

1982 Ceramic Compositional Analysis in Archaeological Perspective. Advances in Archaeological Method and Theory 5:275-330.

Blanton, R. E., G. M. Feinman, S. A. Kowalewski, and P. N. Peregrine

1996 A Dual-Processual Theory for the Evolution of Mesoamerican Civilization. Current Anthropology 37(1):1-14.

Blench, R.

1993 Ethnographic and Linguistic Evidence for the Prehistory of African Ruminant Livestock, Horses and Ponies. In The Archaeology of Africa: Foods, Metals and Towns. T. Shaw, P. Sinclair, B. Andah, and A. Okpoko, eds. Pp. 71-103. London: Routledge.

Blench, R., and K. MacDonald, eds.

2000 The Origins and Development of African Livestock: Archaeology, Genetics, Linguistics, and Ethnography. London: University College of London Press.

Bocoum, $\mathrm{H}$.

2000 L'age Du Fer Au Sénégal. Dakar: CRI.

Bocoum, H., A. Camara, A. Diop, B. Diop, M. Lame, and M. Thiam

2002 Eléments D'archéologie Ouest-Africaine: V. Sénégal. Paris: Éditions Sépia. 
Bocoum, H., and S. K. McIntosh

2002 Excavations at Sinçu Bara, Middle Senegal Valley (Senegal). Dakar: Institut Fondamental d'Afrique Noire.

Bohannan, P., and L. Bohannan

1968 Tiv Economy. Evanston: Northwestern University Press.

Bohannan, P., and G. Dalton

1962 Markets in Africa. Evanston: Northwestern University Press.

Bollig, M., and O. Bubenzer, eds.

2009 African Landscapes: Interdisciplinary Perspectives. New York: Springer.

Bomba, V.

1977 Traditions About Ndiadiane Ndiaye, First Buurba Djolof: Early Djolof, the

Southern Almoravids, and Neighboring Peoples. Bullentin de l'Institut

Fondamental d'Afrique Noire, Série B 39(1):1-35.

Bossen, L.

1988 Toward a Theory of Marriage: The Economic Anthropology of Marriage

Transactions. Ethnology 27(2):127-144.

Boucard, C.

1974 [1729] Relation De Bambouc. Bulletin de l'Institut Fondamental d'Afrique Noire, Série B 36(2):227-245.

Bourdieu, $\mathrm{P}$.

1973 The Berber House. In Rules and Meaning: The Anthropology of Everyday Knowledge. M. Douglas, ed. Pp. 98-110. Harmondsworth: Penguin Books.

1977 Outline of a Theory of Practice. Cambridge: Cambridge University Press.

2005 The Social Structures of the Economy. C. Turner, transl. Cambridge: Polity Press. Bousman, C. B.

2005 Coping with Risk: Later Stone Age Technological Strategies at Blydefontein Rock Shelter, South Africa. Journal of Anthropological Archaeology 24:193-226.

Bovill, E. W.

1995 The Golden Trade of the Moors. Princeton: M. Weiner Publishers.

Bower, J.

1986 A Survey of Surveys: Aspects of Surface Archaeology in Sub-Saharan Africa. African Archaeological Review 4:21-40.

Bowser, B. J.

2000 From Pottery to Politics: An Ethnoarchaeological Study of Political Factionalism, Ethnicity, and Domestic Pottery Style in the Ecuadorian Amazon. Journal of Archaeological Method and Theory 7(3):219-248.

Bradley, R.

1998 The Significance of Monuments. London: Routledge.

Braudel, F.

1980 On History. S. Matthews, transl. Chicago: University of Chicago Press.

Braun, D. P.

1983 Pots as Tools. In Archaeological Hammers and Theories. J. A. Moore and A. S. Keene, eds. Pp. 107-134. New York: Academic Press.

Braun, D. P., and S. Plog

1982 Evolution of "Tribal" Social Networks: Theory and North American Evidence. American Antiquity 47:504-525. 
Brett, M.

1983 Islam and Trade in the Bilād Al-Sūdān, Tenth-Eleventh Century A.D. Journal of African History 24(4):431-440.

Breunig, $\mathrm{P}$.

2004 Environmental Instability and Cultural Change in the Later Prehistory of the Chad Basin. In Living with the Lake: Perspectives on History, Culture and Economies of Lake Chad. M. Krings and E. Platte, eds. Pp. 52-72. Cologne: Rudiger Kuoppe Verlag.

Breunig, P., and K. Neumann

2002 From Hunters and Gatherers to Food Producers: New Archaeological and Archaeobotanical Evidence from the West African Sahel. In Droughts, Food and Culture: Ecological Change and Food Security in Africa's Later Prehistory. F. A. Hassan, ed. Pp. 123-156. New York: Kluwer Academic / Plenum Publishers.

Brooks, G. E.

1989 Ecological Perspectives on Mande Population Movements, Commercial Networks, and Settlement Patterns from the Atlantic Wet Phase (Ca. 5500-2500 B.C.) to the Present. History in Africa 16:23-40.

1993 Landlords and Strangers: Ecology, Society, and Trade in Western Africa, 10001630. Boulder: Westview Press.

Brown, J.

1990 Horn-Shaping Ground-Stone Axe-Hammers. Azania 25:57-67.

Brumfiel, E. M., and T. K. Earle

1987 Specialization, Exchange, and Complex Societies: An Introduction. In Specialization, Exchange, and Complex Socieites. E. M. Brumfel and T. K. Earle, eds. Pp. 1-9. Cambridge: Cambridge University Press.

Brunk, K., and D. Gronenborn

2004 Floods, Droughts, and Migrations: The Effects of Late Holocene Lake Level Oscillations and Climate Fluctuations on the Settlement and Political History in the Chad Basin. In Living with the Lake: Perspectives on History, Culture and Economies of Lake Chad. M. Krings and E. Platte, eds. Cologne: Rudiger Kuoppe Verlag.

Burnham, P.

1980 Changing Agricultural and Pastoral Ecologies in the West African Savanna Region. In Human Ecology in Savanna Environments. D. R. Harris, ed. Pp. 147170. New York: Academic Press.

Butzer, K. W.

1982 Archaeology as Human Ecology: Method and Theory for a Contextual Approach Cambridge: Cambridge University Press.

Byrd, B. F.

1994 Public and Private, Domestic and Corporate: The Emergence of the Southwest Asian Village. American Antiquity 59(4):639-666.

Camara, A.

2000 Archéologie Du Sénégal: Le Paléolithique Ancien. In L’archéologie En Afrique De L'ouest: Sahara Et Sahel. R. Vernet, ed. Pp. 239-256. Paris: Éditions Sépia. 
Camara, A., and B. Duboscq

1984 Le Gisement Préhistorique De Sansandé, Basse Vallée De La Falémé, Sénégal: Approche Typologique Et Stratigraphique. L'Anthropologie 88(3):377-402.

1987 Contexte Chronostratigraphique Des Outillages Du Paléolithique Évolué Dans L'est Du Sénégal. L'Anthropologie 91(2):511-520.

1990 La Fouille D'un Site Acheuléen À Djita (Basse Vallée De La Falémé, Sénégal). L'Anthropologie 94(2):293-304.

Carr, C.

1984 The Nature of Organization of Intrasite Archaeological Records and Spatial Analytic Approaches to Their Investigation. Advances in Archaeological Method and Theory 7:103-222.

1987 Dissecting Intrasite Artifact Palimpsests Using Fourier Methods. In Method and Theory for Activity Area Research: An Ethnoarchaeological Approach. S. Kent, ed. Pp. 236-291. New York: Columbia University Press.

1995 A Unified Middle-Range Theory of Artifact Design. In Style, Society, and Person: Archaeological and Ethnological Perspectives. C. Carr and J. Neitzel, eds. Pp. 171-253. New York: Plenum Press.

Carter, T. H., and R. Pagliero

1966 Notes on Mud-Brick Preservation. Sumer 22:65-76.

Casey, J.

1998 The Ecology of Food Production in West Africa. In Transformations in Africa: Essays on Africa's Later Past. G. Connah, ed. Pp. 46-70. New York: Leicester University Press.

2000 The Kintampo Complex. Volume BAR International Series. Oxford: Archaeopress.

2003 The Archaeology of West Africa from the Pleistocene to the Mid-Holocene. In Under the Canopy: The Archaeology of Tropical Rainforests. J. Mercader, ed. Pp. 35-63. New Brunswick, NJ: Rutgers University Press.

Chastanet, $\mathrm{M}$.

1984 Cultures Et Outils Agricoles En Pays Soninke (Gajaaga Et Gidimaxa). Cahiers OSTROM, Séries Sciences Humaines 20(3/4):453-459.

Chavane, B. A.

1985 Villages De L'ancien Tekrour. Paris: Karthala.

Chenorkian, R.

1983 Ivory Coast Prehistory: Recent Developments. African Archaeological Review $1(1): 127-142$.

Childe, V. G.

1951 Social Evolution. London: C. A. Watts.

Childs, S. T.

1991 Style, Technology, and Iron Smelting Furnaces in Bantu-Speaking Africa. Journal of Anthropological Archaeology 10:332-359.

Childs, S. T., and D. Killick

1993 Indigenous African Metallurgy: Nature and Culture. Annual Review of Anthropology 22:317-337. 
Chwe, M. S.-Y.

2001 Rational Ritual: Culture, Coordination, and Common Knowledge. Princeton: Princeton University Press.

Cissé, Y.

1964 Notes Sur Les Sociétés De Chasseurs Malinké. Journal de la Société des Africanistes 34(2):175-226.

Claessen, H. J. M.

1984 The Internal Dynamics of the Early State. Current Anthropology 25(4):365-379.

Clark, A. F.

1994 Internal Migrations and Population Movements in the Upper Senegal Valley (West Africa), 1890-1920. Canadian Journal of African Studies 28(3):399-420.

1995 Environmental Decline and Ecological Response in the Upper Senegal Valley, West Africa, from the Late Nineteenth Century to World War I. Journal of African History 36(2):197-218.

1996 The Fulbe of Bundu (Senegambia): From Theocracy to Secularization. International Journal of African Historical Studies 29(1):1-23.

1999 From Frontier to Backwater: Economy and Society in the Upper Senegal Valley (West Africa), 1850-1920. Lanham, MD: University Press of America.

Clark, J. D.

1967 The Problem of Neolithic Culture in Subsaharan Africa. In Background to Evolution in Africa. W. W. Bishop and J. D. Clark, eds. Pp. 601-627. Chicago: University of Chicago Press.

1982 The Cultures of the Middle Paleolithic/Middle Stone Age. In The Cambridge History of Africa: From the Earliest Times to C. 500 Bc. J. D. Clark, ed. Pp. 248340, Vol. 5. Cambridge: Cambridge University Press.

Clark, J. E., and W. J. Parry

1990 Craft Specialization and Cultural Complexity. Research in Economic Anthropology 12:289-346.

Clark, M. E.

2003 Archaeological Investigations at Jenné-Jeno Settlement Complex, Inland Niger Delta, Mali, West Africa. Unpublished PhD Dissertation, Southern Methodist University Press.

Close, A. E.

1978 The Identification of Style in Lithic Artifacts. World Archaeology 10(2):223-237.

1995 Few and Far Between: Early Ceramics in North Africa. In The Emergence of Pottery: Technology and Innovation in Ancient Societies. W. K. Barnett and J. W. Hoopes, eds. Pp. 23-37. Washington, D.C.: Smithsonian Institution Press.

Cobb, C. R.

1993 Archaeological Approaches to the Political Economy of Nonstratified Societies. Archaeological Method and Theory 5:43-100.

Cochrane, G. W. G.

2003 Artefact Attribute Richness and Sample Size Adequacy. Journal of Archaeological Science 30:837-848. 
Conkey, M. W.

1989 The Use of Diversity in Stylistic Analysis. In Diversity in Archaeology. R. D. Leonard and G. T. Jones, eds. Pp. 118-129. Cambridge: Cambridge University Press.

2006 Style, Design, and Function. In Handbook of Material Culture. C. Tilley, W. Keane, S. Kuchler, M. J. Rowlands, and P. Spier, eds. Pp. 355-372. London: Sage Publications.

Connah, G.

1981 Three Thousand Years in Africa. Cambridge: Cambridge University Press.

1984 An Archaeological Exploration in Southern Borno. African Archaeological Review 2:153-171.

Conolly, J., and M. Lake

2006 Geographical Information Systems in Archaeology. Cambridge: Cambridge University Press.

Conrad, D. C.

1994 A Town Called Dakajalan: The Sunjata Tradition and the Question of Ancient Mali's Capital. Journal of African History 35(3):355-377.

1995 Blind Man Meets Prophet: Oral Tradition, Islam, and Funé Identity. In Status and Identity in West Africa: Nyamakalaw of Mande. D. C. Conrad and B. E. Frank, eds. Pp. 86-132. Bloomington: Indiana University Press.

2008 From the "Banan" Tree of Kouroussa: Mapping the Landscape in Mande Traditional History. Canadian Journal of African Studies 42(2/3):384-408.

Conrad, D. C., and H. J. Fisher

1983 The Conquest That Never Was: Ghana and the Almoravids, 1076. Ii. The Local Oral Sources. History in Africa 10(1):53-78.

Conrad, D. C., and B. E. Frank

1995a Nyamakalaya: Contradiction and Ambiguity in Mande Society. In Status and Identity in West Africa: Nyamakalaw of Mande. D. C. Conrad and B. E. Frank, eds. Pp. 1-23. Bloomington: Indiana University Press.

1995b Status and Identity in West Africa: Nyamakalaw of Mande. Bloomington: Indiana University Press.

Cooper, F.

2005 Colonialism in Question: Theory, Knowledge, History. Berkeley: University of California Press.

Coquery-Vidrovitch, C.

1976 The Political Economy of African Peasantry and Modes of Production. In The Political Economy of Contemporary Africa. P. C. W. Gutkind and I. Wallerstein, eds. Beverly Hills: Sage Publications.

Corbeil, R., R. Mauny, and J. Charbonnier

1948 Préhistoire Et Protohistoire De La Presqu'ile Du Cap-Vert Et De L’extrême Ouest Sénégalais. Bulletin de l'Institut Français d'Afrique Noire 10:378-460.

Cornelissen, E.

2002 Human Responses to Changing Environments in Central Africa between 40,000 and 12,000 B.P. Journal of World Prehistory 16(3):197-235. 
Cornelissen, E.

2003 On Microlithic Quartz Industries at the End of the Pleistocene in Central Africa: The Evidence from Shum Laka (Nw Cameroon). African Archaeological Review 20(1):1-24.

Coste d'Arnobat, C.-P.

1789 Voyage Au Pays De Bambouc. Paris: Defer de Maisonneuve.

Costin, C. L.

1991 Craft Specialization: Issues in Defining, Documenting, and Explaining the Organization of Production. Archaeological Method and Theory 3:1-56.

Costin, C. L., and R. P. Wright, eds.

1998 Craft and Social Identity. Volume 8. Washington, D.C.: American Anthropological Association.

Cotterell, B., and J. Kamminga

1987 The Formation of Flakes. American Antiquity 52(4):675-708.

Covey, R. A.

2006 How the Incas Built Their Heartland: State Formation and the Innovation of Imperial Strategies in the Sacred Valley, Peru. Ann Arbor: University of Michigan Press.

Cowgill, G. L., J. H. Altschul, and R. S. Sload

1984 Spatial Analysis of Teotihuacan: A Mesoamerican Metropolis. In Intrasite Spatial Analysis in Archaeology. H. Hietala, ed. Pp. 154-195. Cambridge: Cambridge University Press.

Crumley, C. L.

1979 Three Locational Models: An Epistemological Assessment for Anthropology and Archaeology. Advances in Archaeological Method and Theory 2:141-173.

1994 Historical Ecology: Cultural Knowledge and Changing Landscapes. Santa Fe: School of American Research Press.

Curtin, P. D.

1973 The Lure of Bambuk Gold. Journal of African History 14(4):623-631.

1975a Economic Change in Precolonial Africa: Senegambia in the Era of the Slave Trade. Madison: University of Wisconsin Press.

1975b The Uses of Oral Tradition in Senegambia: Maalik Sii and the Foundation of Bundu. Cahiers d'Études Africaines 15(58):189-202.

1984 Cross-Cultural Trade in World History. Cambridge: Cambridge University Press.

D'Andrea, A. C., and J. Casey

2002 Pearl Millet and Kintampo Subsistence. African Archaeological Review 19(3):147-173.

D'Andrea, A. C., S. Kahlheber, A. L. Logan, and D. J. Watson

2007 Early Domesticated Cowpea (Vigna Unguiculata) from Central Ghana. Antiquity 81:686-698.

D'Andrea, A. C., A. L. Logan, and D. J. Watson

2006 Oil Palm and Prehistoric Subsistence in Tropical West Africa. Journal of African Archaeology 4(2):195-222.

Daget, J., and Z. Ligers

1962 Une Ancienne Industrie Malienne: Les Pipes En Terre. Bulletin de l'Institut Francais d'Afrique Noire, Série B 24(1):12-53. 
Darling, P. J.

1984 Archaeology and History in Southern Nigeria: The Ancient Linear Earthworks of Benin and Ishan. Volume 215. Oxford: Archaeopress.

David, N.

1971 The Fulani Compound and the Archaeologist. World Archaeology 3(2):111-131.

1998 The Ethnoarchaeology and Field Archaeology of Grinding at Sukur, Adamawa State, Nigeria. African Archaeological Review 15(1):13-63.

David, N., R. Heimann, D. Killick, and M. Wayman

1989 Between Bloomery and Blast Furnace: Mafa Iron-Smelting Technology in North Cameroon. African Archaeological Review 7:183-208.

David, N., and H. Hennig

1972 The Ethnography of Pottery: A Fulani Case Seen in Archaeological Perspective. Volume Module 21. Reading, MA: Addison-Wesley.

David, N., J. Sterner, and K. Gavua

1988 Why Pots Are Decorated. Current Anthropology 29(3):365-389.

Davies, O.

1980 The Ntereso Culture in Ghana. In West African Culture Dynamics: Archaeological and Historical Perspectives. B. K. Swartz and R. E. Dumett, eds. Pp. 205-225. The Hague: Mouton Publishers.

Davison, C. C., R. D. Giauque, and J. D. Clark

1971 Two Chemical Groups of Dichroic Glass Beads from West Africa. Man 6(4):645659.

De Barros, $P$.

1982 The Effects of Variable Site Occupation Span on the Results of Frequency Seriation. American Antiquity 47(2):291-315.

1990 Changing Paradigms, Goals and Methods in the Archaeology of Francophone West Africa. In A History of African Archaeology. P. Robertshaw, ed. Pp. 155172. London: James Currey.

2000 Iron Metallurgy: Sociocultural Context. In Ancient African Metallurgy. J. O. Vogel, ed. Pp. 147-198. Walnut Creek, CA: AltaMira Press.

2001 The Effect of the Slave Trade on the Bassar Ironworking Society, Togo. In West Africa During the Atlantic Slave Trade. C. R. DeCorse, ed. Pp. 59-80. New York: Leicester University Press.

de Moraes-Farias, P. F.

1974 Silent Trade: Myth and Historical Evidence. History in Africa 1(1):9-24.

2003 Arabic Medieval Inscriptions from the Republic of Mali: Epigraphy, Chronicles, and Songhay-Tuāreg History. Volume 4. Oxford: Oxford University Press.

Deacon, J.

1984 The Later Stone Age of Southernmost Africa. Oxford: Archaeopress.

DeCorse, C. R.

1989 Beads as Chronological Indicators in West African Archaeology: A Reexamination. Beads 1:41-53.

2001a An Archaeology of Elmina: Africans and Europeans on the Gold Coast, 14001900. Washington, D.C.: Smithsonian Institution Press. 
DeCorse, C. R.

2001b West Africa During the Atlantic Slave Trade: Archaeological Perspectives. New York: Leicester University Press.

DeCorse, C. R., F. G. Richard, and I. Thiaw

2003 Toward a Systematic Bead Description System: A View from the Lower Falemme, Senegal. Journal of African Archaeology 1(1):77-110.

Delafosse, $M$.

1912 Haut-Sénégal-Niger. 3 vols. Paris: Emile Larose.

DeMarrais, E., L. J. Castillo, and T. Earle

1996 Ideology, Materialization, and Power Strategies. Current Anthropology 37(1):1531.

Deme, A., and S. K. McIntosh

2006 Excavations at Walaldé: New Light on the Settlement of the Middle Senegal Valley by Iron-Using Peoples. Journal of African Archaeology 4(2):317-347.

Denyer, $\mathrm{S}$.

1978 African Traditional Architecture: An Historical and Geographical Perspective. New York: Africana Publishing Company.

Descamps, C.

1979 Sites Protohistoriques De La Sénegambie. Annales de la Faculté des Lettres et Sciences Humaines de Dakar 9:303-313.

Descamps, C., and G. Thilmans

1979 Les Tumulus Coquilliers Des Iles Du Saloum (Sénégal). Bulletin de l'ASEQUA 54/55:81-91.

Devisse, J., ed.

1983 Tegdaoust Iii: Recherches Sur Aoudaghost, Campagnes 1960-1965, Enquêtes Générales. Paris: Éditions Recherches sur les Civilisations.

Di Lernia, S.

2006 Building Monuments, Creating Identity: Cattle Cult as a Social Response to Rapid Environmental Changes in the Holocene Sahara. Quaternary International 151:50-62.

Diagne, $\mathrm{M}$.

2010 Contribution À La Connaissance Du Néolithique De La Falémé: Étude Archéologique Du Matériel De Diouboye (Fal-1062 Et Fal-1075). Dakar: Memoire de Maitrise, Université Cheikh Anta Diop.

Diagne, $\mathrm{P}$.

1967 Pouvoir Politique Traditionnel En Afrique Occidentale: Essais Sur Les Institutions Politiques Précoloniales. Paris: Présence Africaine.

Dietler, M., and I. Herbich

1989 Tich Matek: The Technology of Luo Pottery Production and the Definition of Ceramic Style. World Archaeology 21(1):148-164.

1998 Habitus, Techniques, Style: An Integrated Approach to the Social Understanding of Material Culture and Boundaries. In The Archaeology of Social Boundaries. M. T. Stark, ed. Pp. 232-263. Washington, D.C.: Smithsonian Institution Press. 
Diez-Martín, F. et al.

2009a The Middle to Later Stone Age Transition in East Africa: New Data from Mumba Rockshelter Bed V (Tanzania) and Their Implications for the Origin of Modern Human Behavior. Journal of African Archaeology 7(2):147-173.

Diez-Martín, F., P. Sánchez, M. Domínguez-Rodrigo, A. Mabulla, and R. Barba

2009b Were Olduvai Hominins Making Butchering Tools or Battering Tools? Analysis of a Recently Excavated Lithic Assemblage from Bk (Bed Ii, Olduvai Gorge, Tanzania). Journal of Anthropological Archaeology 28:274-289.

Diez-Martín, F., P. Sánchez, M. Domínguez-Rodrigo, and M. E. Prendergast

2011 An Experimental Study of Bipolar and Freehand Knapping of Naibor Soit Quartz from Olduvai Gorge (Tanzania). American Antiquity 76(4):690-708.

Dilley, R. M.

2000 The Question of Caste in West Africa, with Special Reference to Tukulor Craftsmen. Anthropos 95:149-165.

2004 Islamic and Caste Knowledge Practices among Haalpulaar'en in Senegal: Between Mosque and Termite Mound. Edinburgh: Edinburgh University Press.

Diop, A.

2000 Archéologie Du Sénégal: Les Industries Paléolithiques Post-Acheuléennes. In L’archéologie En Afrique De L'ouest: Sahara Et Sahel. R. Vernet, ed. Pp. 257268. Paris: Éditions Sépia.

Diop, B.

1995 Survivances Traditionnelles De L'activité Potière Dans L'espace Sénégambien: La Société Artisanale. Revue Sénégalaise d'Histoire 1:25-45.

2000 Recherches Ethnoarchéologiques Sur La Céramique Au Sénégal, Production Artisanale Et Consommation Domestique. In Arts Du Feu Et Productions Artisanales: Xxe Rencontres Internationales D'archéologie Et D'histoire D'antibes. P. Benoit, P. Fluzin, P. Pétrequin, and J. Thiriot, eds. Pp. 261-285. Antibes: Éditions APDCA.

Diouf, S. A.

2003 Fighting the Slave Trade: West African Strategies. Athens, OH: Ohio University Press.

Dobres, M.-A.

2000 Technology and Social Agency: Outlining a Practice Framework for Archaeology. Malden, MA: Blackwell.

Dobres, M.-A., and C. R. Hoffman

1994 Social Agency and the Dynamics of Prehistoric Technology. Journal of Archaeological Method and Theory 1(3):211-258.

Dobres, M.-A., and J. E. Robb

2005 "Doing" Agency: Introductory Remarks on Methodology. Journal of Archaeological Method and Theory 12(3):159-166.

Dodd, W. A., Jr.

1979 The Wear and Use of Battered Tools at Armijo Rockshelter. In Lithic Use-Wear Analysis. B. Hayden, ed. Pp. 231-242. New York: Academic Press.

Donley-Reid, L. W.

1990 A Structuring Structure: The Swahili House. In Domestic Architecture and the Use of Space. S. Kent, ed. Pp. 114-126. Cambridge: Cambridge University Press. 
Douglas, M., and B. Isherwood

1979 The World of Goods: Towards an Anthropology of Consumption. New York: Basic Books.

Drame, A.

2009 Migration, Marriage, and Ethnicity: The Early Development of Islam in Precolonial Middle Casamance. In New Perspectives on Islam in Senegal: Conversion, Migration, Wealth, Power, and Femininity. M. Diouf and M. Leichtman, eds. Pp. 169-185. New York: Palgrave Macmillan.

Drennan, R. D., and C. E. Peterson

2004 Comparing Archaeological Settlement Systems with Rank-Size Graphs: A Measure of Shape and Statistical Confidence. Journal of Archaeological Science 31:533-549.

Dueppen, S.

2008 Reinventing Equality: The Archaeology of Kirikongo, Burkina Faso. Unpublished $\mathrm{PhD}$ Dissertation, University of Michigan.

2012 From Kin to Great House: Inequality and Communalism at Iron Age Kirikongo, Burkina Faso. American Antiquity 77(1):3-39.

Duff, A. I.

1996 Ceramic Micro-Seriation: Types or Attributes? American Antiquity 61(1):89-101. Duffy, P. R.

2010 Complexity and Autonomy in Bronze Age Europe: Assessing Cultural Developments in Eastern Hungary. Unpublished PhD Dissertation, University of Michigan.

Dunnell, R. C.

1978 Style and Function: A Fundamental Dichotomy. American Antiquity 43(2):192202.

Dunnell, R. C., and W. S. Dancey

1983 The Siteless Survey: A Regional Scale Data Collection Strategy. Advances in Archaeological Method and Theory 6:267-287.

Durand, J.-B. L.

1802 Voyages Au Sénégal. Paris: Henri Agasse.

Durkheim, E.

1984 [1893] The Division of Labour in Society. W. D. Halls, transl. New York: Free Press.

Earle, $\mathrm{T}$.

2002 Bronze Age Economics: The Beginnings of Political Economies. Boulder: Westview Press.

Edmonds, M. R.

1995 Stone Tools and Society: Working with Stone in Neolithic and Bronze Age Britain. London: Routledge.

Effah-Gyamfi, K.

1985 Bono Manso: An Archaeological Investigation into Early Akan Urbanism. Volume 2. Calgary: University of Calgary Press.

Engels, F.

1972 [1884] The Origin of the Family, Private Property and the State. New York: International Publishers. 
Erdogan, E. H., G. Erpul, and İ. Bayramin

2007 Use of Usle/Gis Methodology for Predicting Soil Loss in a Semiarid Agricultural Watershed. Environmental Monitoring and Assessment 131:153-161.

Eriksen, T. H.

1993 Ethnicity and Nationalism: Anthropological Perspectives. London: Pluto Press. Eyo, E.

1972 Rop Rock Shelter Excavations, 1964. West African Journal of Archaeology 2:1316.

Falconer, S. E., and S. H. Savage

1995 Heartlands and Hinterlands: Alternative Trajectories of Early Urbanization in Mesopotamia and the Southern Levant. American Antiquity 60(1):37-58.

Fall, Y.

1982 Silla: Problématique D’un Site De La Vallée Du Fleuve Du Sénégal. Archives Suisses d'Anthropologie Générale 42(2):199-216.

FAO/UNEP

1984 Final Report: Unep/Fao World and Africa Gis Data Base: FAO/UNEP

Desertification and Mapping Project.

Feinman, G. M.

2000 Corporate/Network: New Perspectives on Models of Political Action and the Puebloan Southwest. In Social Theory in Archaeology. M. B. Schiffer, ed. Pp. 3151. Salt Lake City: University of Utah Press.

Feinman, G. M., and J. Neitzel

1984 Too Many Types: An Overview of Sedentary Prestate Societies in the Americas. Advances in Archaeological Method and Theory 7:39-102.

Feinman, G. M., and L. M. Nicholas, eds.

2004 Archaeological Perspectives on Political Economies. Salt Lake City: University of Utah Press.

Fenn, T.

2006 Copper Metallurgy and Trans-Saharan Commerce: Report on Summer 2006 Field Research in the Western Agadez Region, Central Niger. Nyame Akuma 66(2):2534.

Fiedermutz-Laun, A.

2005 The House, the Hearth and the Granary: Symbols of Fertility among the West African Kasena. The Medieval History Journal 8(1):247-265.

Filipowiak, W.

1968 Contribution Aux Recherches Sur La Capitale Du Royaume De Mali À L'époque Du Haut Moyen Age (Afrique Occidentale). Archaeologia Polona 10:217-232.

1979 Études Archéologiques Sur La Capitale Médiévale Du Mali. Szczecin: Muzeum Narodowe w Szczecinie.

Finucane, B., K. Manning, and M. Toure

2008 Late Stone Age Subsistence in the Tilemsi Valley, Mali: Staple Isotope Analysis of Human and Animal Remains from the Site of Karkarichinkat Nord (Kn05) and Karkarichinkat Sud (Ks05). Journal of Anthropological Archaeology 27(1):82-92.

Fish, S. K., and S. A. Kowalewski, eds.

1990 The Archaeology of Regions: A Case for Full-Coverage Survey Washington, D.C.: Smithsonian Institution Press. 
Flannery, K. V.

1972a The Cultural Evolution of Civilizations. Annual Review of Ecology and Systematics 3:399-425.

1972b The Origins of the Village as a Settlement Type in Mesoamerica and the near East: A Comparative Study. In Man, Settlement and Urbanism. P. J. Ucko, R. Tringham, and G. W. Dimbleby, eds. Pp. 23-53. Chicago: Duckworth.

2002 The Origins of the Village Revisited: From Nuclear to Extended Households. American Antiquity 67(3):417-433.

Fleisher, J. B., and A. LaViolette

1999 Elusive Wattle-and-Daub: Finding the Hidden Majority in the Archaeology of the Swahili. Azania 34:87-108.

2008 The Changing Power of Swahili Houses, Fourteenth to Nineteenth Centuries, A.D. In The Durable House: House Society Models in Archaeology. R. A. Beck, ed. Pp. 175-197. Occasional Paper No. 35, Center for Archaeological Investigations. Carbondale: Southern Illinois University Press.

Fogelin, L.

2007a The Archaeology of Religious Ritual. Annual Review of Anthropology 36:55-71. 2007b Inference to the Best Explanation: A Common and Effective Form of Archaeological Reasoning. American Antiquity 72(4):603-625.

Ford, R. I.

1972 Barter, Gift, or Violence: An Analysis of Tewa Intertribal Exchange. In Social Exchange and Interaction. E. N. Wilmsen, ed, Vol. Anthropological Papers, No. 46. Ann Arbor: Museum of Anthropology, University of Michigan.

Fortes, $\mathrm{M}$.

1940 The Political System of the Tallensi of the Northern Territories of the Gold Coast. In African Political Systems. M. Fortes and E. E. Evans-Pritchard, eds. Pp. 239271. New York: Oxford University Press.

1953 The Structure of Unilineal Descent Groups. American Anthropologist 55(1):1741.

Fortes, M., and E. E. Evans-Pritchard

1940 African Political Systems. New York: Oxford University Press.

Foucault, M.

1970 The Order of Things: An Archaeology of the Human Sciences. New York: Random House.

Fowles, S. M.

2002 From Social Type to Social Process: Placing 'Tribe' in a Historical Framework. In The Archaeology of Tribal Societies. W. A. Parkinson, ed. Pp. 13-33. Ann Arbor: International Monographs in Prehistory.

2003 The Making of Made People: The Prehistoric Evolution of Hierocracy among the Northern Tiwa of New Mexico. Unpublished PhD Dissertation, University of Michigan.

Frank, A. G., and B. K. Gills, eds.

1993 The World System: Five Hundred Years or Five Thousand? London: Routledge. 
Frank, B. E.

1995 Soninke Gárankew and Bamana-Malinke Jeliw: Mande Leatherworkers, Identity, and Diaspora. In Status and Identity in West Africa: Nyamakalaw of Mande. D. C. Conrad and B. E. Frank, eds. Pp. 133-150. Bloomington: Indiana University Press.

1998 Mande Potters and Leatherworkers: Art and Heritage in West Africa. Washington, D.C.: Smithsonian Institution Press.

Frank, T., P. Breunig, P. Muller-Haude, K. Neumann, W. Van Neer, R. Vogelsang, and H.-P. Wotzka

2001 The Chaine De Gobnangou, Se Burkina Faso: Archaeological, Archaeobotanical, Archaeozoological and Geomorphological Studies. Sonderdruck aus Beitrage Zur Allgemeinen Und Vergleichenden Archaologie 21:127-190.

Friede, H. M., and R. H. Steel

1980 Experimental Burning of Traditional Nguni Huts. African Studies 39(2):175-181. Friedman, J.

1979 System, Structure, and Contradiction: The Evolution of Asiatic Social Formations. Walnut Creek, CA: AltaMira Press.

Friedman, J., and M. J. Rowlands

1978 Notes Towards an Epigenetic Model of the Evolution of 'Civilisation'. In The Evolution of Social Systems. J. Friedman and M. J. Rowlands, eds. Pp. 201-276. Pittsburgh: University of Pittsburgh Press.

Gabel, C.

1976 Microlithic Occurrences in the Republic of Liberia. West African Journal of Archaeology 6:21-35.

Gallagher, D. E.

1999 Analysis of Seeds and Fruits from the Sites of Arondo and Ft. Senedebu, Senegal. In Archaeological Investigations of Long-Term Culture Change in the Lower Falemme (Upper Senegal Region) A.D. 500-1900. I. Thiaw, ed. Pp. 385-431: Unpublished PhD Dissertation, Rice University.

2010 Farming Beyond the Escarpment: Society, Environment, and Mobility in Precolonial Southeastern Burkina Faso. Unpublished PhD Dissertation, Gallais, J. University of Michigan.

1962 Signification Du Groupe Ethnique Au Mali. L'Homme 2(2):106-129. Gallay, A.

1970 La Poterie En Pays Sarakolé (Mali, Afrique Occidentale). Journal de la Société des Africanistes 40(1):7-84.

1992 Traditions Céramique Et Ethnies Dans Le Delta Interieur Du Niger (Mali): Approche Ethnoarchéologique. Bulletin du Centre Genevois d'Anthropologie 3:23-46.

2010 Seriation Chronologique De La Céramique Mégalithique Sénégambienne (Sénégal, Gambie), 700 Cal Bc - 1700 Cal Ad. Journal of African Archaeology 8(1):99-129. 
Gallay, A., and E. Huysecom

1994 L'étude Ethnoarchéologique De La Céramique Du Delta Intérieur Du Niger: Un

Bilan De Cinq Missions De Terrain. Bulletin du Centre Genevois d'Anthropologie 4:78-86.

Gallay, A., G. Pignat, and P. Curdy

1982 Mbolop Tobé (Santhiou Kohel, Sénégal): Contribution a La Connaissance Du Mégalithisme Sénégambien. Archives Suisses d'Anthropologie Générale, Genève 46(2):217-259.

Garcea, E. A. A.

2006 Semi-Permanent Foragers in Semi-Arid Environments of North Africa. World Archaeology 38(2):197-219.

Garenne-Marot, L.

1995 Le Commerce Médiéval Du Cuivre: La Situation Dans La Moyenne Vallée Du Sénégal D'après Les Données Archéologiques Et Historiques. Journal des Africanistes 65(2):43-56.

Garrard, T. F.

1982 Myth and Metrology: The Early Trans-Saharan Gold Trade. Journal of African History 23:443-461.

Gautier, A.

1987 Prehistoric Men and Cattle in North Africa: A Dearth of Data and a Surfeit of Models. In Prehistory of Arid North Africa: Essays in Honor of Fred Wendorf. A. Gelbert, A.

E. Close, ed. Pp. 163-187. Dallas: Southern Methodist University Press.

1999 Technological and Stylistic Borrowings between Ceramic Traditions: A Case Study from Northeastern Senegal. In Ethno-Analogy and the Reconstruction of Prehistoric Artefact Use and Production. L. R. Owen and M. Porr, eds. Pp. 207224. Tübingen: Mo Vince Verlag.

2001 Ethnoarchaeological Study of Ceramic Borrowings: A New Methodological Approach Applied in the Middle and Upper Valleys of the Senegal River. In Ethno-Archaeology and Its Transfers. S. Beyries and P. Pétrequin, eds. Pp. 81-94. Bar International Series. Oxford: Archaeopress.

2003 Traditions Céramiques Et Emprunts Techniques Dans La Vallée Du Fleuve Sénégal. Paris: Éditions de la Maison des Sciences de l'Homme.

2005 Reconnaissance Des Techniques Et Des Méthodes De Façonnage Par L'analyse Des Macrotraces : Étude Ethnoarchéologique Dans La Vallée Du Sénégal. In Pottery Manufacturing Processes: Reconstitution and Interpretation. A. Livingstone Smith, D. Bosquet, and R. Martineau, eds. Pp. 67-78, Vol. BAR International Series 1349. Oxford: Archaeopress.

Giddens, A.

1984 The Constitution of Society: Outline of the Theory of Structuration. Berkeley: University of California Press.

Gijanto, L.

2007 Contact and Exchange During the Era of the Atlantic Trade: Preliminary Findings from Archaeological Investigations in the Gambia. Nyame Akuma 68:12-20. 
Gijanto, L.

2011a Exchange, Interaction, and Change in Local Ceramic Production in the Niumi Commercial Center on the Gambia River. Journal of Social Archaeology 11(1):21-48.

2011b Socio-Economic Interaction and Ceramic Aesthetic: Understanding West African Ceramic Production and Use in Context. Azania 46(3):250-268.

Gill, G. J.

1991 Seasonality and Agriculture in the Developing World. Cambridge: Cambridge University Press.

Gillet, $\mathrm{H}$.

1986 Desert and Sahel. In Plant Ecology in West Africa: Systems and Processes. G. W. Lawson, ed. Pp. 151-182. New York: John Wiley \& Sons.

Gokee, C. D.

2010 Crafts, Cowries, and Carved Roulettes: A Preliminary Report on Archaeological Research at Diouboye, Senegal. Nyame Akuma 74(2):2-11.

2011 Practical Knowledge and Politics of Encounter Along the Lower Falémé River, Senegambia (C. Ad 1500-1925). Azania 46(3):269-293.

Gomez, M. A.

1985 The Problem with Malik Sy and the Foundation of Bundu. Cahiers d'Études Africaines 25(100):537-553.

1992 Pragatism in the Age of Jihad: The Precolonial State of Bundu. Cambridge: Cambridge University Press.

Gondonneau, A., and M. F. Guerra

2002 The Circulation of Precious Metals in the Arab Empire: The Case of the near and the Middle East. Archaeometry 44(4):573-599.

Goody, J.

1957 Fields of Social Control among the Lodagaba. The Journal of the Royal Anthropological Institute of Great Britain and Ireland 87(1):75-104.

1962 Death, Property and the Ancestors. Stanford: Stanford University Press.

1967 The Social Organisation of the Lowiili. London: Oxford University Press.

Goodyear, A. C.

1993 Tool Kit Entropy and Bipolar Reduction: A Study of Interassemblage Lithic Variability among Paelo-Indian Sites in the Northeastern United States. North American Archaeologist 14(1):1-23.

Gosden, C.

2005 What Do Objects Want? Journal of Archaeological Method and Theory 12(3):193-211.

Gosselain, O. P.

1998 Social and Technical Identity in a Clay Crystal Ball. In The Archaeology of Social Boundaries. M. T. Stark, ed. Pp. 78-106. Washington, D.C.: Smithsonian Institution Press.

1999 In Pots We Trust. Journal of Material Culture 4(2):205-230.

2000 Materializing Identities: An African Perspective. Journal of Archaeological Method and Theory 7(3):187-217. 
Gosselain, O. P.

2008 Mother Bella Was Not a Bella: Inherited and Transformed Traditions in Southwestern Niger. In Cultural Transmission and Material Culture: Breaking Down Boundaries. M. T. Stark, B. J. Bowser, and L. Horne, eds. Pp. 150-177. Tucson: University of Arizona Press.

2010 Exploring the Dynamics of African Pottery Cultures. In The Archaeology of Regional Technologies: Case Studies from the Palaeolithic to the Age of the Vikings. R. Barndon, A. Engevik, and I. Øye, eds. Pp. 193-224. Lewiston, NY: Edwin Mellen Press.

2011 Pourquoi Le Décorer? Quelques Observations Sur Le Décor Céramique En Afrique. Azania 46(1):3-19.

Gosselain, O. P., and A. Livingstone Smith

2005 The Source: Clay Selection and Processing Practices in Sub-Saharan Africa. In Pottery Manufacturing Processes: Reconstitution and Interpretation. A. Livingstone Smith, D. Bosquet, and R. Martineau, eds. Pp. 33-47, Vol. BAR International Series 1349. Oxford: Archaeopress.

Goucher, C. L.

1981 Iron Is Iron 'Til It Is Rust: Trade and Ecology in the Decline of West African Iron-Smelting. Journal of African History 22(2):179-189.

Gould, R. A.

1985 The Empiricist Strikes Back: A Reply to Binford. American Antiquity 50(3):638644.

Graef, F., and J. Haigis

2001 Spatial and Temporal Rainfall Variability in the Sahel and Its Effects on Farmers' Management Strategies. Journal of Arid Environments 48:221-231.

Graham, M.

1994 Mobile Farmers: An Ethnoarchaeological Approach to Settlement Organization among the Rarámuri of Northwestern Mexico. Volume 3. Ann Arbor: International Monographs in Prehistory.

Granovetter, M.

1985 Economic Action and Social Structure: The Problem of Embeddedness. The American Journal of Sociology 91(3):481-510.

Gratuze, B.

1999 Obsidian Characterization by Laser Ablation Icp-Ms and Its Application to Prehistoric Trade in the Mediterranean and the near East: Sources and Distribution of Obsidian within the Aegean and Anatolia. Journal of Archaeological Science 26:869-881.

Greenfield, H. J., and D. E. Miller

2004 Spatial Patterning of Early Iron Age Metal Production at Ndondondwane, South Africa: The Question of Cultural Continuity between the Early and Late Iron Ages. Journal of Archaeological Science 31(11):1511-1532.

Gregg, S. A., K. W. Kintigh, and R. Whallon

1991 Linking Ethnoarchaeological Interpretation and Archaeological Data: The Sensitivity of Spatial Analytical Methods to Postdepositional Disturbance. In The Interpretation of Archaeological Spatial Patterning. E. M. Kroll and T. D. Price, eds. Pp. 149-196. New York: Plenum Press. 
Greif, A.

2006 Institutions and the Path to the Modern Economy: Lessons from Medieval Trade. Cambridge: Cambridge University Press.

Greiser, S. T., and P. D. Sheets

1979 Raw Material as a Functional Variable in Use-Wear Studies. In Lithic Use-Wear Analysis. B. Hayden, ed. Pp. 289-296. New York: Academic Press.

Grove, A. T., ed.

1985 The Niger and Its Neighbors: Environmental History and Hydrobiology, Human Use and Health Hazards of the Major West African Rivers. Rotterdam: A. A. Balkema.

Guèye, N. S.

1998 Poteries Et Peuplements De La Moyenne Vallée Du Fleuve Sénégal Du Xvi ${ }^{e} \mathrm{Au}$ $X x^{e}$ Siècle: Approches Ethnoarchéologique, Archéologique, Et Ethnohistorique. Unpublished PhD Thesis, Université de Paris X - Nanterre.

2002 Ethnoarchéologie, Ethnohistoire Et Interprétation De La Distribution Des Poteries De La Moyenne Vallée Du Fleuve Sénégal Du Xve Au Xxe Siècle. Nyame Akuma 57(June):21-32.

2011 Dis-Moi Quel Pot Tu as Et Je Te Dirai Qui Tu Es ! Matérialiser Les Identités Sociales Dans Les Décors Céramiques De La Moyenne Vallée Du Fleuve Sénégal (Nord Du Sénégal). Azania 46(1):20-35.

Guitat, R.

1970 Carte Et Répertoire Des Site Néolithiques Du Sénégal. Bulletin de l'Institut Fondamental d'Afrique Noire, Série B 32(4):1125-1134.

1972 Carte Et Répertoire Des Site Néolithiques Du Mali Et De La Haute-Volta. Bulletin de l'Institut Fondamental d'Afrique Noire, Série B 34(4):896-925.

Guyer, J. I.

1981 Household and Community in African Studies. African Studies Review 24(2/3):87-137.

1995 Wealth in People, Wealth in Things--Introduction. Journal of African History 36(1):83-90.

Guyer, J. I., and S. M. E. Belinga

1995 Wealth in People as Wealth in Knowledge: Accumulation and Composition in Equatorial Africa. Journal of African History 36:91-120.

Haaland, R.

1980 Man's Role in the Changing Habitat of Mema During the Old Kingdom of Ghana. Norweigan Archaeological Review 13(1):31-46.

1992 Fish, Pots, and Grain: Early and Mid-Holocene Adaptations in the Central Sudan. African Archaeological Review 10:43-64.

Hair, P. E. H.

1984 The Falls of Félou: A Bibliographical Exploration. History in Africa 11:113-130.

Hall, B. A.

1994 Formation Processes of Large Earthen Residential Mounds in Mixtequilla, Veracruz, Mexico. Latin American Antiquity 5(1):31-50.

Hall, E. T.

1966 The Hidden Dimension. Garden City, NY: Doubleday. 
Halperin, R. H.

1994 Cultural Economies Past and Present. Austin: University of Texas Press.

Halstead, P., and J. O'Shea

1989 Introduction: Cultural Responses to Risk and Uncertainty. In Bad Year

Economics. P. Halstead and J. O'Shea, eds. Pp. 1-8. Cambridge: Cambridge University Press.

Hammer, O., D. A. T. Harper, and P. D. Ryan

2001 Past: Paleontological Statistics Software Package for Eduction and Data Analysis: Palaeontologia Electronica.

Hanson, J. H.

1996 Migration, Jihad, and Muslim Authority in West Africa: The Futanke Colonies in Karta. Bloomington: Indiana University Press.

Haour, A.

2011 Putting Pots and People in the Sahelian Empires. Azania 46(1):36-48.

Haour, A. et al., eds.

2010 African Pottery Roulettes Past and Present: Techniques, Identification and Distribution. Oxford: Oxbow Books.

Harrower, M. J.

2010 Geographic Information Systems (Gis) Hydrological Modeling in Archaeology: An Example from the Origins of Irrigation in Southwest Arabia (Yemen). Journal of Archaeological Science 37(7):1447-1452.

Hassan, F. A.

1978 Demographic Archaeology. Advances in Archaeological Method and Theory 1:49-103.

Hawthorne, W.

2003 Strategies of the Decentralized: Defending Communities from Slave Raiders in Coastal Guinea-Bissau, 1450-1815. In Fighting the Slave Trade: West African Strategies. S. A. Diouf, ed. Pp. 152-169. Athens, OH: Ohio University Press.

Hayden, B., ed.

1979 Lithic Use-Wear Analysis. New York: Academic Press.

Hayden, B., and A. Cannon

1983 Where the Garbage Goes: Refuse Disposal in the Maya Highlands. Journal of Anthropological Archaeology 2(2):117-163.

Hayward, D. F., and J. S. Oguntoyinbo

1987 The Climatology of West Africa. Totowa, NJ: Barnes and Noble.

Hegmon, $\mathrm{M}$.

1998 Technology, Style, and Social Practices: Archaeological Approaches. In The Archaeology of Social Boundaries. M. T. Stark, ed. Pp. 264-279. Washington, D.C.: Smithsonian Institution Press.

2000 Advances in Ceramic Ethnoarchaeology. Journal of Archaeological Method and Theory 7(3):129-137.

Hegmon, M., and S. Kulow

2005 Painting as Agency, Style as Structure: Innovations in Mimbres Pottery Designs from Southwest New Mexico. Journal of Archaeological Method and Theory 12(4):313-334. 
Henrich, J., R. Boyd, S. Bowles, C. Camerer, E. Fehr, and H. Gintis, eds.

2004 Foundations of Human Sociality: Economic Experiments and Ethnographic

Evidence from Fifteen Small-Scale Societies. Oxford: Oxford University Press.

Henrickson, E. F., and M. M. A. McDonald

1983 Ceramic Form and Function: An Ethnographic Search and an Archaeological Application. American Anthropologist 85(3):630-643.

Herbert, E. W.

1984 Red Gold of Africa: Copper in Precolonial History and Culture. Madison: University of Wisconsin Press.

1993 Iron, Gender, and Power: Rituals of Transformation in African Societies. Bloomington: Indiana University Press.

1998 Mining as Microcosm in Precolonial Sub-Saharan Africa. In Social Approaches to the Industrial Past: The Archaeology and Anthropology of Mining. A. B. Knapp, V. C. Pigott, and E. W. Herbert, eds. Pp. 138-154. New York: Routledge.

Hirth, K. G.

1978 Interregional Trade and the Formation of Prehistoric Gateway Communities. American Antiquity 43(1):35-45.

Hodder, I.

1982 Symbols in Action: Ethnoarchaeological Studies of Material Culture. Cambridge: Cambridge University Press.

Hodder, I., and C. Orton

1976 Spatial Analysis in Archaeology. Cambridge: Cambridge University Press.

Holl, A. F. C.

1985a Background to the Ghana Empire: Archaeological Investigations on the Transition to Statehood in the Dhar Tichitt Region (Mauritania). Journal of Anthropological Archaeology 4:73-115.

1985b Subsistence Patterns of the Dhar Tichitt Neolithic, Mauritania. African Archaeological Review 3:151-162.

1987 Mound Formation Processes and Societal Transformations: A Case Study from the Perichadian Plain. Journal of Anthropological Archaeology 6:122-158.

1989 Social Issues in Saharan Prehistory. Journal of Anthropological Archaeology 8:313-354.

1993 Late Neolithic Cultural Landscape in Southeastern Mauritania: An Essay in Spatiometrics. In Spatial Boundaries and Social Dynamics: Case Studies from Food-Producing Societies. A. F. C. Holl and T. E. Levy, eds. Pp. 95-133. Ann Arbor: International Monographs in Prehistory.

1998 Livestock Husbandry, Pastoralisms, and Territoriality: The West African Record. Journal of Anthropological Archaeology 17:143-165.

2000 Metals and Precolonial African Society. In Ancient African Metallurgy. J. O. Vogel, ed. Pp. 1-81. Walnut Creek, CA: AltaMira Press.

2002 The Land of Houlouf: Genesis of a Chadic Polity, 1900 B.C.-A.D. 1800. Volume 35. Ann Arbor: Museum of Anthropology.

2004 Holocene Saharans. New York: Continuum.

2006 West African Early Towns: Archaeology of Households in Urban Landscapes. Ann Arbor: Museum of Anthropology, University of Michigan. 
Holl, A. F. C.

2009 Early West African Metallurgies: New Data and Old Orthodoxy. Journal of World Prehistory 22:415-438.

Holl, A. F. C., and H. Bocoum

2006 Variabilité Des Pratiques Funéraires Dans Le Mégalithisme Sénégambian: Le Cas De Sine Ngayène. In Senegalia: Études Sur Le Patrimoine Ouest-Africain. C. Descamps and A. Camara, eds. Pp. 224-234. Paris: Éditions Sépia.

Holl, A. F. C., H. Bocoum, S. Dueppen, and D. Gallagher

2007 Switching Mortuary Codes and Ritual Programs: The Double-Monolith-Circle from Sine-Ngayene, Senegal. Journal of African Archaeology 5(1):127-148.

Holl, A. F. C., and L. Koté

2000 Settlement Patterns, Food Production, and Craft Specialization in the Mouhoun Bend (Nw Burkina Faso): Preliminary Results of the Mobap 1997-1999 Field Seasons. West African Journal of Archaeology 28:69-101.

Horton, R.

1971 Stateless Societies in the History of West Africa. In History of West Africa. J. F. A. Ajayi and M. Crowder, eds. Pp. 78-119. London: Longman.

Hugot, H. J.

1967 Le Paléolithique Terminale Dans L'afrique De L'ouest. In Background to Evolution in Africa. W. W. Bishop and J. D. Clark, eds. Pp. 529-555. Chicago: University of Chicago Press.

Hume, I. N.

1970 A Guide to Artifacts of Colonial America. New York: Knopf.

Hunwick, J. O.

1973 The Mid-Fourteenth Century Capital of Mali. Journal of African History 14(2):195-206.

2003 Timbuktu and the Songhay Empire: Al-Sa'dīs Ta'rīkh Al-Sūdān Down to 1613, and Other Contemporary Documents. Leiden: Koninklijke Brill NV.

Hurley, W. M.

1979 Prehistoric Cordage: Identification of Impressions on Pottery. Volume 3. Washington, D.C.: Taraxacum.

Huyescom, E.

1994 Identification Technique Des Céramiques Africaines. In Terre Cuite Et Societé: La Céramique, Document Technique, Economique, Culturel: Xive Rencontres Internationales D'archéologie Et D'histoire D'antibes. P. Benoit, P. Fluzin, P. Pétrequin, and J. Thiriot, eds. Pp. 269-301. Antibes: Éditions APDCA.

Huysecom, E.

1990 Fanfannyégèné I: Un Abri-Sous-Roche À Occupation Néolithique Au Mali: La Fouille, Le Matériel Archéologique, L'art Rupestre. Stuttgart: Steiner Verlag Wiesbaden.

Ingold, T.

1993 The Temporality of the Landscape. World Archaeology 25(2):152-174.

Inizan, M.-L., M. Reduron-Ballinger, H. Roche, and J. Tixier

1995 Technologie De La Pierre Taillée. Volume 4. Meudon: Cercle de Recherches et d'Etudes Préhistoriques. 
Insoll, T.

1996 Islam, Archaeology, and History: Gao Region (Mali) Ca. Ad 900-1250. Oxford: Tempus Reparatum.

1997 Iron Age Gao: An Archaeological Contribution. Journal of African History 38:130.

2003 The Archaeology of Islam in Sub-Saharan Africa. Cambridge: Cambridge University Press.

Insoll, T., B. Kankpeyeng, and R. MacLean

2009 The Archaeology of Shrines among the Tallensi of Northern Ghana: Materiality and Interpretive Relevance. In Shrines in Africa: History, Politics, and Society. A. C. Dawson, ed. Pp. 41-70. Calgary: University of Calgary Press.

Insoll, T., D. A. Polya, K. Bhan, D. Irving, and K. Jarvis

2004 Towards an Understanding of the Carnelian Bead Trade from Western India to Sub-Saharan Africa: The Application of Uv-La-Icp-Ms to Carnelian from Gujarat, India and West Africa. Journal of Archaeological Science 31:1161-1173.

Insoll, T., and T. Shaw

1997 Gao and Igbo-Ukwu: Beads, Interregional Trade, and Beyond. African Archaeological Review 1:9-23.

Irvine, J. T.

1974 Caste and Communication in a Wolof Village. Unpublished PhD Dissertation, University of Pennsylvania.

2008 Subjected Words: African Linguistics and the Colonial Encounter. Language \& Communication 28:323-343.

Jansen, J.

1996 The Representation of Status in Mande: Did the Mali Empire Still Exist in the Nineteenth Century? History in Africa 23:87-109.

2008 From Guild to Rotary: Hunters' Associations and Mali's Search for a Civil Society International Review of Social History 53(S):249-270.

Jeske, R. J.

1992 Energetic Efficiency and Lithic Technology: An Upper Mississippian Example. American Antiquity 57(3):467-481.

Jobson, R.

1999 [1623] The Discovery of River Gambra. London: Haklyut Society.

Johnson, A. W., and T. K. Earle

2000 The Evolution of Human Societies: From Foraging Group to Agrarian State. Stanford: Stanford University Press.

Johnson, G. A.

1977 Aspects of Regional Analysis in Archaeology. Annual Review of Anthropology $6: 479-508$

Johnson, $\mathrm{M}$.

1970 The Cowrie Currencies of West Africa. Part I. Journal of African History 11(1):17-49.

Joire, J., and G. Duchemin

1955 Découvertes Archéologiques Dans La Région De Rao (Bas-Sénégal). Bulletin de l'Institut Français d'Afrique Noire, Série B 17(3):249-333. 
Jouenne, P.

1930 Les Monuments Mégalithiques Du Sénégal: Les Roches Gravées Et Leur Interprétation Cultuelle. Bulletin du Comité d'Etudes Historiques et Scientifiques de l'Afrique Occidentale Française 13(3):309-399.

Kahlheber, S., and K. Neumann

2007 The Development of Plant Cultivation in Semi-Arid West Africa. In Rethinking Agriculture: Archaeological and Ethnoarchaeological Perspectives. T. Denham, J. Iriarte, and L. Vrydaghs, eds. Pp. 320-346. Walnut Creek, CA: Left Coast Press.

Keane, W.

1995 The Spoken House: Text, Act, and Object in Eastern Indonesia. American Ethnologist 22(1):102-124.

1997 Signs of Recognition: Powers and Hazards of Representation in an Indonesian Society. Berkeley: University of California Press.

2005 Signs Are Not the Garb of Meaning: On the Social Analysis of Material Things. In Materiality. D. Miller, ed. Pp. 182-205. Durham: Duke University Press.

2007 Christian Moderns: Freedom and Fetish in the Mission Encounter. Berkeley: University of California Press.

Kelly, K. G., and N. L. Norman

2007 Historical Archaeology of Landscape in Atlantic Africa. In Envisioning Landscape: Situations and Standpoints in Archaeology and Heritage. D. Hicks, L. McAtackney, and G. Fairclough, eds. Pp. 172-193. Walnut Creek, CA: Left Coast Press.

Kelly, R. L.

1988 The Three Sides of a Biface. American Antiquity 53(4):717-734.

1992 Mobility/Sedentism: Concepts, Archaeological Measures, and Effects. Annual Review of Anthropology 21:43-66.

Kense, F. J.

1983 Traditional African Iron Working. Volume 1. Calgary: University of Calgary Press.

Kent, S.

1982 Analyzing Activity Areas: An Ethnoarchaeological Study of the Use of Space. Albuquerque: University of New Mexico Press.

1987 Method and Theory for Activity Area Research: An Ethnoarchaeological Approach. New York: Columbia University Press.

1990 Domestic Architecture and the Use of Space: An Interdisciplinary Cross-Cultural Study. Cambridge: Cambridge University Press.

Kesteloot, L., C. Barbey, and S. M. Ndongo

1993 Les Peul. In Vallées Du Niger. Pp. 173-189. Paris: Éditions de la Réunion des Musées Nationaux.

Killick, D.

2004 Review Essay: What Do We Know About African Iron-Working? Journal of African Archaeology 2(1):97-112. 
Killick, D., N. J. van der Merwe, R. B. Gordon, and D. Grébénart

1988 Reassessment of the Evidence for Early Metallurgy in Niger, West Africa. Journal of Archaeological Science 15:367-394.

Killion, T. W., J. A. Sabloff, G. Tourtellot, and N. P. Dunning

1989 Intensive Surface Collection of Residential Clusters at Terminal Classic Sayil, Yucatan, Mexico. Journal of Field Archaeology 16(3):273-294.

Kintigh, K. W.

1984 Measuring Archaeological Diversity by Comparison with Simulated Assemblages. American Antiquity 49(1):44-54.

Klein, M. A.

2001 The Slave Trade and Decentralized Societies. Journal of African History 42(1):49-65.

Knapp, A. B., and W. Ashmore

1999 Archaeological Landscapes: Constructed, Conceptualized, Ideational. In Archaeologies of Landscape: Contemporary Perspectives. W. Ashmore and A. B. Knapp, eds. Pp. 1-30. Malden, MA: Blackwell.

Koechlin, J.

1997 Ecological Conditions and Degradation Factors in the Sahel. In Societies and Nature in the Sahel. C. Raynaut, ed. Pp. 12-36. London: Routledge.

Kopytoff, I., ed.

1987a The African Frontier: The Reproduction of Traditional African Societies. Bloomington: Indiana University Press.

1987b The Internal African Frontier: The Making of African Political Culture. In The African Frontier: The Reproduction of Traditional African Societies. I. Kopytoff, ed. Pp. 3-84. Bloomington: Indiana University Press.

Kopytoff, I., and S. Miers

1977 African 'Slavery' as an Institution of Marginality. In Slavery in Africa: Historical and Anthropological Perspectives. M. Suzanne and I. Kopytoff, eds. Pp. 3-81. Madison: University of Wisconsin Press.

Kowal, J. M., and A. H. Kassam

1978 Agricultural Ecology of Savanna: A Study of West Africa. Oxford: Clarendon Press.

Kriger, C. E.

2005 Mapping the History of Cotton Textile Production in Precolonial West Africa. African Economic History 33:87-116.

2006 Cloth in West African History. Walnut Creek, CA: Altamira Press.

Kroll, E. M., and T. D. Price, eds.

1991 The Interpretation of Archaeological Spatial Patterning. New York: Plenum Press.

Kröpelin, S. et al.

2008 Climate-Driven Ecosystem Succession in the Sahara: The Past 6000 Years. Science 320(9 May 2008):765-768.

Kuijt, I., W. C. Prentiss, and D. L. Pokotylo

1995 Bipolar Reduction: An Experimental Study of Debitage Variability. Lithic Technology 20(2):116-127. 
Kuper, R., and S. Kröpelin

2006 Climate-Controlled Holocene Occupation in the Sahara: Motor of Africa's Evolution. Science 313(11 August 2006):803-807.

Kusimba, C. M., and S. B. Kusimba

2005 Mosaics and Interactions: East Africa, 2000 B.P. To the Present. In African Archaeology. A. B. Stahl, ed. Pp. 392-419. Malden, MA: Blackwell.

Kusimba, S. B.

2003 African Foragers: Environment, Technology, Interactions. Walnut Creek, CA: AltaMira Press.

L’Hôte, Y., G. Mahé, B. Somé, and J. P. Triboulet

2002 Analysis of a Sahelian Annual Rainfall Index from 1896 to 2000; the Drought Continues. Hydrological Sciences 47(4):563-572.

Labat, J.-B.

1728 Nouvelle Relation De L'afrique Occidentale. Volume 4. Paris: Guillaume Cavelier.

Labouret, $\mathrm{H}$.

1931 Les Tribus Du Rameau Lobi. Paris: Institut d'Ethnologie.

Laforgue, P.

1923 Essai Sur L'influence De L'industrie Saharienne En Afrique Occidentale Au Cours De La Période Néolithique. Bulletin de la Société Préhistorique Française 20(5):161-66.

1924 L'outillage Néolithique En Hematite De La Falémé (Sénégal). Bulletin de la Société Préhistorique Française 21(11):263-264.

1925 État Actuel De Nos Connaissances Sur La Préhistoire En Afrique Occidentale Française. Bulletin du Comité d'Études Historiques et Scientifiques de l'Afrique Occidentale Française 8(1):105-171.

Lame, $\mathrm{M}$.

2000 Archéologie Du Sénégal: Le Néolithique. In L’archéologie En Afrique De L'ouest: Sahara Et Sahel. R. Vernet, ed. Pp. 269-275. Paris: Éditions Sépia.

Latour, B.

1993 We Have Never Been Modern. C. Porter, transl. Cambridge, MA: Harvard University Press.

Lavachery, P.

2001 The Holocene Archaeological Sequence of Shum Laka Rock Shelter (Grassfields, Western Cameroon). African Archaeological Review 18(4):213-247.

LaViolette, A.

2000 Ethno-Archaeology in Jenné, Mali: Craft and Status among Smiths, Potters and Masons. Volume 838. Oxford: Archaeopress.

Lawrence, D. L., and S. M. Low

1990 The Built Environment and Spatial Form. Annual Review of Anthropology 19:453-505.

Lawson, A.

2003 Megaliths and Mande States: Sociopolitical Change in the Gambia Valley over the Past Two Millennia. Unpublished PhD Dissertation, University of Michigan. 
Lawson, G. W., ed.

1986 Plant Ecology in West Africa: Systems and Processes. New York: John Wiley \& Sons.

Leach, E. R.

2004 [1959] Political Systems of Highland Burma. Volume 44. New York: Berg.

Lechtman, $\mathrm{H}$.

1977 Style in Technology--Some Early Thoughts. In Material Culture: Styles,

Organization, and Dynamics of Technology. H. Lechtman and R. S. Merrill, eds. Pp. 3-20. St. Paul: West Publishing Co.

1996 Cloth and Metal: The Culture of Technology. In Andean Art at Dumbarton Oaks.

E. H. Boone, ed. Pp. 33-43, Vol. 1. Washington, D.C.: Dumbarton Oaks.

Lefebvre, $\mathrm{H}$.

1991 The Production of Space. D. Nicholson-Smith, transl. Malden, MA: Blackwell.

Lejju, B. J., P. Robertshaw, and D. Taylor

2006 Africa's Earliest Bananas? Journal of Archaeological Science 33:102-113.

Lemmonier, P.

1986 The Study of Material Culture Today: Toward an Anthropology of Technical

Systems. Journal of Anthropological Archaeology 5:147-186.

Leroi-Gourhan, A.

1943 Evolution Et Techniques: L'homme Et La Matière. Paris: Albin Michel.

1966 La Préhistoire. Paris: Presses Universitaires de France.

Lestrange, $\mathrm{M}$.

1955 Les Coniagui Et Les Bassari (Guinée Française). Paris: Presses Universitaires de France.

Levtzion, N.

1980 Ancient Ghana and Mali. New York: Methuen \& Co.

2000 Islam in the Bilad Al-Sudan to 1800. In The History of Islam in Africa. N.

Levtzion and R. L. Pouwels, eds. Pp. 63-92. Athens, OH: Ohio University Press.

Levtzion, N., and J. F. P. Hopkins

2000 Corpus of Early Arabic Sources for West African History. Cambridge: Cambridge University Press.

Levy, T. E., and A. F. C. Holl

2002 Migrations, Ethnogenesis, and Settlement Dynamics: Israelites in Iron Age Canaan and Shuwa-Arabs in the Chad Basin. Journal of Anthropological Archaeology 21:83-118.

Lewarch, D. E., and M. J. O'Brien

1981 The Expanding Role of Surface Assemblages in Archaeological Research. Advances in Archaeological Method and Theory 4(297-342).

Leynaud, E., and Y. Cissé

1978 Paysans Malinke Du Haut Niger: Tradition Et Développement Rural En Afrique Soudanaise. Bamako: Imprimerie Popularie du Mali.

Lézine, A.-M.

1989 Late Quaternary Vegetation and Climate of the Sahel. Quaternary Research 32:317-334 
Lézine, A.-M., and J. Casanova

1989 Pollen and Hydrological Evidence for the Interpretation of Past Climates in

Tropical West Africa During the Holocene. Quaternary Science Reviews 8:45-55.

Linares de Sapir, O.

1969 Diola Pottery of the Fogny and the Kasa. Expedition 11(3):2-11.

1971 Shell Middens of the Lower Casamance and Problems of Diola Prehistory. West African Journal of Archaeology 1:23-54.

Liu, L.

1996 Settlement Patterns, Chiefdom Variability, and the Development of Early States in North China. Journal of Anthropological Archaeology 15:237-288.

Livingstone Smith, A.

2000 Processing Clay for Pottery in Northern Cameroon: Social and Technical Requirements. Archaeometry 42(1):21-42.

2007 Histoire Du Décor À La Roulette En Afrique Subsaharienne. Journal of African Archaeology 5(2):189-216.

Livingstone Smith, A., and A. Viseyrias

2010 Shaping Kabambian Pottery: Identification and Definition of Technical Features. The Open Anthropology Journal 3:124-141.

MacDonald, K. C.

1996 The Windé Koroji Complex: Evidence for the Peopling of the Eastern Inland Niger Delta (2100-500 Bc). Préhistoire Anthropologie Méditerranéennes 5:147165.

1997 Korounkorokalé Revisited: The Pays Mande and the West African Microlithic Technocomplex. African Archaeological Review 14(3):161-200.

1998 Before the Empire of Ghana: Pastoralism and the Origins of Cultural Complexity in the Sahel. In Transformations in Africa: Essays on Africa's Later Past. G. Connah, ed. Pp. 71-103. New York: Leicester University Press.

2011 Betwixt Tichitt and the Ind: The Pottery of the Faïta Facies, Tichitt Tradition. Azania 46(1):49-69.

MacDonald, K. C., and P. Allsworth-Jones

1994 A Reconsideration of the West African Macrolithic Conundrum: New Factory Sites and an Associated Settlement in the Vallée Du Serpent, Mali. African Archaeological Review 12:73-104.

MacDonald, K. C., S. Camara, S. Canós Donnay, N. Gestrich, and D. Keita

2011 Sorotomo: A Forgotten Malian Capital? Archaeology International 13/14.

MacDonald, K. C., R. Vernet, M. Martinón-Torres, and D. Q. Fuller

2009 Dhar Néma: From Early Agriculture to Metallurgy in Southeastern Mauritania. Azania 44(1):3-48.

MacEachern, S.

1996 Iron Age Beginnings North of the Mandara Mountains, Cameroon and Nigeria. In Aspects of African Archaeology (Papers from the 10th Congress of the Panafrican Association for Prehistory and Related Studies). G. Pwiti and R. Soper, eds. Pp. 489-495. Harare: University of Zimbabwe Publications.

2002 Beyond the Belly of the House: Space and Power around the Mandara Mountains. Journal of Social Archaeology 2(2):197-219. 
Magnavita, C.

2002 Recent Archaeological Finds of Domesticated Sorghum Bicolor in the Lake Chad Region. Nyame Akuma 57(June):14-20.

2004 Towards the Emergence of Sociopolitical Complexity in the Lake Chad Region (1800 Bc - 1600 Ad). In Living with the Lake: Perspectives on History, Culture and Economies of Lake Chad. M. Krings and E. Platte, eds. Pp. 73-100. Cologne:

Maley, J. Rudiger Kuoppe Verlag.

1981 Études Palynologiques Dans Le Bassin Du Tchad Et Paléoclimatologie De L'afrique Nord-Tropicale De 30000 Ans a L'époque Actuelle. In Palaeoecology of Africa and the Surrounding Islands. J. A. Coetzee and E. M. V. Z. Bakker, eds. Pp. 45-52, Vol. 13. Rotterdam: A. A. Balkema.

Malinowski, B.

1984 [1922] Argonauts of the Western Pacific. Long Grove, IL: Waveland Press. Manning, K., R. Pelling, T. Higham, J.-L. Schwenniger, and D. Q. Fuller

2011 4500-Year Old Domesticated Pearl Millet (Pennisetum Glaucum) from the Tilemsi Valley, Mali: New Insights into an Alternative Cereal Domestication Pathway. Journal of Archaeological Science 38:312-322.

Marcus, J.

2003 Monumentality in Archaic States. In Theory and Practice in Mediterranean Archaeology: Old World and New World Perspectives. J. K. Papadopoulous and R. M. Leventhal, eds. Pp. 115-134. Los Angeles: Cotsen Institute of Archaeology.

2008 The Archaeological Evidence for Social Evolution. Annual Review of Anthropology 37:251-266.

Marshall, F., and E. Hildebrand

2002 Cattle before Crops: The Beginnings of Food Production in Africa. Journal of World Prehistory 16(2):99-143.

Martin, V., and C. Becker

1984 Inventaire Des Sites Protohistoriques De La Sénégambie. Kaolack: CNRS.

Marx, K.

1973 Grundrisse. New York: New Left Review.

Masonen, P., and H. J. Fisher

1996 Not Quite Venus from the Waves: The Almoravid Conquest of Ghana in the Modern Historiography of West Africa. History in Africa 23:197-232.

Mauny, R.

1961 Tableau Géographique De L'ouest Africain Au Moyen Age. Dakar: Institut Français d'Afrique Noire.

1963 Contribution À La Préhistoire Et À La Protohistoire De La Région De Kédougou (Sénégal Oriental). Cahiers du Centre de Recherches Anthropologiques 11(5):113-122.

Mauss, M.

1990 [1950] The Gift. W. D. Halls, transl. New York: W. W. Norton.

Mayor, A.

1992 La Durée De Vie Des Céramiques Africaines: Un Essai De Compréhension Des Mécanismes. Bulletin du Centre Genevois d'Anthropologie 3:47-70. 
Mayor, A.

2010 Ceramic Traditions and Ethnicity in the Niger Bend, West Africa. Ethnoarchaeology 2(1):5-48.

Mayor, A., E. Huysecom, A. Gallay, M. Rasse, and A. Ballouche

2005 Population Dynamics and Paleoclimate over the Past 3000 Years in the Dogon Country, Mali. Journal of Anthropological Archaeology 24:25-61.

McBrearty, S., and A. S. Brooks

2000 The Revolution That Wasn't: A New Interpretation of the Origin of Modern Human Behavior. Journal of Human Evolution 39(5):453-563.

McCann, J. C.

1999 Climate and Causation in African History. International Journal of African Historical Studies 32(2/3):261-279.

McGuire, R. H.

1983 Breaking Down Cultural Complexity: Inequality and Heterogeneity. Advances in Archaeological Method and Theory 6:91-142.

McGuire, R. H., and D. J. Saitta

1996 Although They Have Petty Captains, They Obey Them Badly: The Dialectics of Prehispanic Western Pueblo Social Organization. American Antiquity 61(2):197216.

McGuire, R. H., and M. B. Schiffer

1983 A Theory of Architectural Design. Journal of Anthropological Archaeology 2:277-303.

McIntosh, R. J.

1974 Archaeology and Mud Wall Decay in a West African Village. World Archaeology 6(2):154-171.

1977 The Excavation of Mud Structures: An Experiment from West Africa. World Archaeology 9(2):185-199.

1989 Middle Niger Terracottas before the Symplegades Gateway. African Arts 22(2):74-83.

1993 The Pulse Model: Genesis and Accommodation of Specialization in the Middle Niger. Journal of African History 34(2):181-220.

1998 The Peoples of the Middle Niger. Malden, MA: Blackwell.

2000 Social Memory in Mande. In The Way the Wind Blows: Climate, History, and Human Action. R. J. McIntosh, J. A. Tainter, and S. K. McIntosh, eds. Pp. 141180. New York: Columbia University Press.

2005 Ancient Middle Niger: Urbanism and the Self-Organizing Landscape. Cambridge: Cambridge University Press.

McIntosh, R. J., and S. K. McIntosh

2003 Early Urban Configurations on the Middle Niger: Clustered Cities and Landscapes of Power. In The Social Construction of Ancient Cities. M. Smith, ed. Pp. 103-120. Washington, D.C.: Smithsonian Institution Press.

McIntosh, R. J., S. K. McIntosh, and H. Bocoum, eds.

in press Seeking the Origins of Takrur: Archaeological Excavations and Survey in the Middle Senegal Valley. New Haven: Yale University Press. 
McIntosh, R. J., J. A. Tainter, and S. K. McIntosh, eds.

2000 The Way the Wind Blows: Climate, History, and Human Action. New York: Columbia University Press.

McIntosh, S. K.

1981 A Reconsideration of Wangara/Palolus, Island of Gold. Journal of African History 22:145-158.

1995 Excavations at Jenné-Jeno, Hambarketolo, and Kaniana (Inland Niger Delta, Mali), the 1981 Season. Berkeley: University of California Press.

1999a Beyond Chiefdoms: Pathways to Complexity in Africa. Cambridge: Cambridge University Press.

1999b Floodplains and the Development of Complex Society: Comparative Perspectives from the West African Semi-Arid Tropics. Archeological Papers of the American Anthropological Association 9(1):151-165.

1999c Pathways to Complexity: An African Perspective. In Beyond Chiefdoms: Pathways to Complexity in Africa. S. K. McIntosh, ed. Pp. 1-30. Cambridge: Cambridge University Press.

2008 Reconceptualizing Early Ghana. Canadian Journal of African Studies 42(2/3):347-373.

McIntosh, S. K., and H. Bocoum

2000 New Perspectives on Sinçu Bara, a First Millennium Site in the Senegal Valley. African Archaeological Review 17(1):1-43.

McIntosh, S. K., D. Gallagher, and R. J. McIntosh

2003 Tobacco Pipes from Excavations at the Museum Site, Jenne, Mali. Journal of African Archaeology 1(2):171-199.

McIntosh, S. K., and R. J. McIntosh

1980 Prehistoric Investigations at Jenne, Mali. Volume BAR International Series. Oxford: Archaeopress.

1983 Current Directions in West African Prehistory. Annual Review of Anthropology 12(215-258).

1986 Archaeological Reconnaissance in the Region of Timbuktu, Mali. National Geographic Research 2(3):302-319.

1988 From Stone to Metal: New Perspectives on the Later Prehistory of West Africa. Journal of World Prehistory 2(1):89-133.

1993a Cities without Citadels: Understanding Urban Origins Along the Middle Niger. In The Archaeology of Africa: Foods, Metals and Towns. T. Shaw, P. Sinclair, B. Andah, and A. Okpoko, eds. Pp. 622-641. London: Routledge.

1993b Field Survey in the Tumulus Zone of Senegal. African Archaeological Review 11:73-107.

McNaughton, P. R.

1988 The Mande Blacksmiths: Knowledge, Power, and Art in West Africa. Bloomington: Indiana University Press. 
Médus, J.

1984 Analyse Pollinique Des Sédiments Holocènes Du Lac Tanma, Sénégal. In

Palaeoecology of Africa and the Surrounding Islands. J. A. Coetzee and E. M. V.

Z. Bakker, eds. Pp. 255-264, Vol. 16. Rotterdam: A. A. Balkema.

Meillassoux, C.

1960 Essai D'interprétation Du Phénomène Économique Dans Les Sociétés

Traditionnelles D'auto-Subsistance. Cahiers d'Études Africaines 1(4):38-67.

1986 The Anthropology of Slavery: The Womb of Iron and Gold. A. Dasnois, transl.

Chicago: University of Chicago Press.

Meskell, L.

1998 An Archaeology of Social Relations in an Egyptian Village. Journal of Archaeological Method and Theory 5(3):209-243.

Michel, P.

1973 Les Bassins Des Fleuves Sénégal Et De Gambie: Etude Géomorphologique. Paris: Editions de l'ORSTOM.

Miers, S., and I. Kopytoff

1977 Slavery in Africa: Historical and Anthropological Perspectives. Madison:

University of Wisconsin Press.

Miller, D. E., and D. Killick

2004 Slag Identification at Southern African Archaeological Sites. Journal of African Archaeology 2(1):23-47.

Miller, D. E., and N. J. Van Der Merwe

1994 Early Metal Working in Sub-Saharan Africa: A Review of Recent Research. Journal of African History 35(1):1-36.

Mills, B. J.

1989 Integrating Functional Analyses of Vessels and Sherds through Models of Ceramic Assemblage Formation. World Archaeology 21(1):133-147.

Minc, L. D.

1986 Scarcity and Survival: The Role of Oral Tradition in Mediating Subsistence Crises. Journal of Anthropological Archaeology 5:39-113.

Mintz, S. W.

1977 The So-Called World System: Local Initiative and Local Response. Dialectical Anthropology 2:253-270.

Mitchell, P.

2005 African Connections: Archaeological Perspectives on Africa and the Wider World Walnut Creek, CA: AltaMira Press.

Moe, T. M.

2005 Power and Political Institutions. Perspectives on Politics 3(2):215-233.

Monroe, J. C.

2010 Power by Design: Architecture and Politics in Precolonial Dahomey. Journal of Social Archaeology 10(3):367-397.

Monroe, J. C., and A. Ogundiran, eds.

2012 Power and Landscape in Atlantic West Africa: Archaeological Perspectives. Cambridge: Cambridge University Press. 
Monteil, V.

1968 Les Empires Du Mali: Étude D’histoire Et De Sociologie Soudanaises. Paris: G.P. Maisonneuve et Larose.

Mudimbe, V. Y.

1988 The Invention of Africa: Gnosis, Philosophy, and the Order of Knowledge. Bloomington: Indiana University Press.

Munsell

2000 Munsell Soil Color Charts. New Windsor, NY: Munsell Color.

Munson, P. J.

1980 Archaeology and the Prehistoric Origins of the Ghana Empire. Journal of African History 21(4).

1981 A Late Holocene (C. 4500-2300 Bp) Climatic Chronology for the Southwestern Sahara. In Palaeoecology of Africa and the Surrounding Islands. J. A. Coetzee and E. M. V. Z. Bakker, eds. Pp. 53-60, Vol. 13. Rotterdam: A. A. Balkema.

Murray, P.

1980 Discard Location: The Ethnographic Data. American Antiquity 45(3):490-502.

N'Diaye, S. O., A. N. Skinner, and P. D. Curtin

1971 The Story of Malik Sy. Cahiers d'Études Africaines 11(43):467-487.

Nance, J. D.

1983 Regional Sampling in Archaeological Survey: The Statistical Perspective. Advances in Archaeological Method and Theory 6:289-356.

Naroll, R.

1962 Floor Area and Settlement Population. American Antiquity 27(4):587-589.

Neff, $\mathrm{H}$.

1994 Rq-Mode Prinical Components Analysis of Ceramic Compositional Data. Archaeometry 31(1):115-130.

2003 Analysis of Mesoamerican Plumbate Pottery Surfaces by Laser AblationInductively Coupled Plasma-Mass Spectrometry (La-Icp-Ms). Journal of Archaeological Science 30(1):21-35.

Neff, H., R. L. Bishop, and E. V. Sayre

1989 More Observations on the Problem of Tempering in Compositional Studies of Archaeological Ceramics. Journal of Archaeological Science 16(1):57-69.

Neumann, K.

2003 The Late Emergence of Agriculture in Sub-Saharan Africa: Archaeobotanical Evidence and Ecological Considerations. In Food, Fuel and Fields: Progress in African Archaeobotany. K. Neumann, A. Butler, and S. Kahlheber, eds. Pp. 71Niane, D. T.

92. Cologne: Heinrich Barth Institute.

1965 Sundiata: An Epic of Old Mali. G. D. Pickett, transl. Essex: Longman.

1989 Histoire Des Mandingues De L'ouest: Le Royaume Du Gabou. Paris: Éditions Karthala.

Nicholson, S. E.

1978a Climate Variations in the Sahel and Other African Regions During the Past Five Centuries. Journal of Arid Environments 1:3-24. 
Nicholson, S. E.

1978b Comparison of Historical and Recent African Rainfall Anomalies with Late Pleistocene and Early Holocene. Paleoecology of Africa and the Surrounding Islands 10(99-123).

1979 The Methodology of Historical Climate Reconstruction and Its Application to Africa. Journal of African History 20(1):31-49.

1980 The Nature of Rainfall Fluctuations in Subtropical West Africa. Monthly Weather Review 108(April):473-487.

1989 Long-Term Changes in African Rainfall. Weather 44(2):47-56.

1994 Recent Rainfall Fluctuations in Africa and Their Relationship to Past Conditions over the Continent. The Holocene 4(2):121-131.

Nicholson, S. E., and J. P. Grist

2001 A Conceptual Model for Understanding Rainfall Variability in the West African Sahel on Interannual and Interdecadal Timescales. International Journal of Climatology 21:1733-1757.

Nixon, S.

2009 Excavating Essouk-Tadmakka (Mali): New Archaeological Investigations of Early Islamic Trans-Saharan Trade. Azania 44(2):217-255.

Norman, N. L.

2009 Powerful Pots, Humbling Holes, and Regional Ritual Processes: Towards an Archaeology of Huedan Vodun, Ca. 1650-1727. African Archaeological Review 26(3):187-218.

2011 Collective and Interpretive Openings: Perforated Gbe Ritual Ceramics at Local, Regional, and Diasporic Spheres of Interpretation. Azania 46(3):250-268.

Norman, N. L., and K. G. Kelly

2004 Landscape Politics: The Serpent Ditch and the Rainbow in West Africa. American Anthropologist 106(1):98-110.

North, D. C.

1990 Institutions, Institutional Change and Economic Performance. Cambridge: Cambridge University Press.

O'Shea, J.

1981 Coping with Scarcity: Exchange and Social Storage. In Economic Archaeology: Towards an Integration of Ecological and Social Approaches. Pp. 167-183, Vol. 96. Oxford: Archaeopress.

Odell, G. H.

2001 Stone Tool Research at the End of the Millennium: Classification, Function, and Behavior. Journal of Archaeological Research 9(1):45-100.

Ogundele, S. O.

2005 Ethnoarchaeology of Domestic Space and Spatial Behavior among the Tiv and Ungwai of Central Nigeria. African Archaeological Review 22(1):25-54.

2007 Indigenous Storage Structures among the Ungwai of Central Nigeria. Anthropologist 9(3):215-220.

Ogundiran, A.

2001 Ceramic Spheres and Regional Networks in the Yoruba-Edo Region, Nigeria, 13th-19th Centuries A.C. Journal of Field Archaeology 28(1/2):27-43. 
Ogundiran, A.

2002 Of Small Things Remembered: Beads, Cowries, and Cultural Translations of the Atlantic Experience in Yorubaland. International Journal of African Historical Studies 35(2/3):427-457.

2009 Material Life and Domestic Economy in a Frontier of the Oyo Empire During the Mid-Atlantic Age. International Journal of African Historical Studies 42(3):351385.

Ogundiran, A., and T. Falola, eds.

2007 Archaeology of Atlantic Africa and the African Diaspora. Bloomington: Indiana University Press.

Okafor, E. E.

1993 New Evidence on Early Iron-Smelting from Southeastern Nigeria. In The Archaeology of Africa: Food, Metals and Towns. T. Shaw, P. Sinclair, B. Andah, and A. Okpoko, eds. Pp. 432-448. New York: Routledge.

Okpoko, A. I.

1987 The Early Urban Centres and States of West Africa. West African Journal of Archaeology 17:243-264.

Opper, M.-J., and H. Opper

1990 Beads from the Faleme River Valley: An Archaeological and Ethnographic Report of a Research Mission to Eastern Senegal. Alexandria, VA.

Ozanne, P.

1969 The Diffusion of Smoking in West Africa. Odu 11:29-42.

Park, M.

1816 Travels in the Interior Districts of Africa Performed in the Years 1795, 1796 and 1797. London: John Murray.

Park, T. K.

1992 Early Trends toward Class Stratification: Chaos, Common Property, and Flood Recession Agriculture. American Anthropologist 94(1):90-117.

Parkinson, W. A.

2002 Introduction: Archaeology and Tribal Societies. In The Archaeology of Tribal Societies. W. A. Parkinson, ed. Pp. 1-12, Vol. 15. Ann Arbor: International Monographs in Prehistory.

Parmentier, R. J.

1997 Semiotic Approaches to Meaning in Material Culture. Semiotica 116(1):42-63.

Parry, W. J., and R. L. Kelly

1987 Expedient Core Technology and Sedentism. In The Organization of Core Technology. J. K. Johnson and C. A. Morrow, eds. Pp. 285-304. Boulder, CO: Westview Press.

Parsons, I.

2011 Foraging, Food Production, and Laminar Components in the Northern Cape, South Africa, and Beyond. Journal of African Archaeology 9(1):5-14.

Parsons, J. R.

1971 Prehistoric Settlement Patterns in the Texcoco Region, Mexico. Ann Arbor: University of Michigan Museum of Anthropology. 
Paynter, R.

1982 Models of Spatial Inequality: Settlement Patterns in Historical Archaeology. New York: Academic Press.

Pélissier, $\mathrm{P}$.

1966 Les Paysans Du Sénégal: Les Civilisations Agraires Du Cayor À La Casamance. Saint-Yrieix: Fabrègue.

Pélissier, P., and C. Bâ

1980 Atlas Du Sénégal. Paris: Éditions Juene Afrique.

Peregrine, P. N., and G. M. Feinman, eds.

1996 Pre-Columbian World Systems. Madison: Prehistory Press.

Perinbam, B. M.

1973 Social Relations in the Tran-Saharan and Western Sudanese Trade: An Overview. Comparative Studies in Society and History 15(4):416-436.

1988 The Political Organization of Traditional Gold Mining: The Western Loby, C. 1850 to C. 1910. Journal of African History 29(3):437-462.

Peterson, C. E., and R. D. Drennan

2005 Communities, Settlements, Sites, and Surveys: Regional-Scale Analysis of Prehistorica Human Interaction. American Antiquity 70(1):5-30.

Pfaffenberger, B.

1992 Social Anthropology of Technology. Annual Review of Anthropology 21:491-516. Philips, J. E.

1983 African Smoking and Pipes. Journal of African History 24(3):303-319.

Plog, S., ed.

1986 Spatial Organization and Exchange: Archaeological Survey of Northern Black

Mesa. Carbondale: Southern Illinois University Press.

Plog, S., and M. Hegmon

1993 The Sample Size-Richness Relation: The Relevance of Research Questions, Sampling Strategies, and Behavioral Variation. American Antiquity 58(3):489496.

Plog, S., F. Plog, and W. Wait

1978 Decision Making in Modern Surveys. Advances in Archaeological Method and Theory 1:383-421.

Polanyi, K.

1944 The Great Transformation. New York: Farrar \& Rinehart, Inc.

1957 The Economy as Instituted Process. In Trade and Market in the Early Empires. K. Polanyi, C. M. Arensberg, and H. W. Pearson, eds. Pp. 243-269. New York: Free Press.

Posnansky, M.

1973 Aspects of Early West African Trade. World Archaeology 5(2):149-162.

Price, T. D., and G. M. Feinman, eds.

1995 Foundations of Social Inequality. New York: Plenum Press.

Prussin, L.

1969 Architecture in Northern Ghana: A Study of Forms and Functions. Berkeley: University of California Press.

1972 West African Mud Granaries. Paideuma 18:144-169. 
Prussin, L.

1974 An Introduction to Indigenous African Architecture. The Journal of the Society of Architectural Historians 33(3):182-205.

Pugh, J. C.

1975 Surveying for Field Scientists. London: Methuen.

Quinn, C. A.

1972 Mandingo Kingdoms of the Senegambia. Evanston: Northwestern University Press.

Raab, L. M., and A. C. Goodyear

1984 Middle-Range Theory in Archaeology: A Critical Review of Origins and Applications. American Antiquity 49(2):255-268.

Raffenel, A.

1856 Nouveau Voyage Dans Le Pays Des Nègres. 2 vols. Paris: Napoléon Chaix et Co.

Rafferty, J. E.

1985 The Archaeological Record on Sedentariness: Recognition, Development, and Implication. Advances in Archaeological Method and Theory 8:113-156.

Rapoport, A.

1990 Systems of Activities and Systems of Settings. In Domestic Architecture and the Use of Space. S. Kent, ed. Pp. 9-20. Cambridge: Cambridge University Press.

Rapp, G., Jr., and C. L. Hill

1998 Geoarchaeology: The Earth-Science Approach to Archaeological Interpretation. New Haven: Yale University Press.

Rappaport, R. A.

1979 Ecology, Meaning, and Religion. Berkeley: North Atlantic Books.

Ravisé, A.

1970 Industrie Néolithique En Os De Saint-Louis. Notes Africaines 128:97-102.

1975 Recensement De Sites Paléolithiques Et Néolithiques Du Sénégal. Bulletin de l'Institut Fondamental d'Afrique Noire, Série B 37(1):234-245.

Read, D., W.

1975 Regional Sampling. In Sampling in Archaeology. J. W. Mueller, ed. Pp. 45-60. Tucson: University of Arizona Press.

1986 Sampling Procedures for Regional Surveys: A Problem of Representativeness and Effectiveness. Journal of Field Archaeology 13:477-491.

Redman, C. L.

1978 Multivariate Artifact Analysis: A Basis for Multidimensional Interpretations. In Social Archaeology: Beyond Subsistence and Dating. C. L. Redman, W. T. Langhorne, Jr., M. J. Berman, N. M. Versaggi, E. V. Curtin, and J. C. Wanser, eds. Pp. 159-192. New York: Academic Press.

1987 Surface Collection, Sampling, and Research Design: A Retrospective. American Antiquity 52(2):249-265.

Redman, C. L., and P. J. Watson

1970 Systematic, Intensive Surface Collection. American Antiquity 35(3):279-291.

Reed, S., N. Bailey, and O. Onokplse

2000 Soil Science for Archaeologists, Vol. 1: Florida Agricultural and Mechanical University and Southeast Archeological Center, National Park Service. 
Reid, A. M., and P. J. Lane

2004 African Historical Archaeologies: An Introductory Consideration of Scope and Potential. In African Historical Archaeologies. A. M. Reid and P. J. Lane, eds. Pp. 1-32. New York: Kluwer Academic / Plenum Publishers.

Reid, A. M., and R. MacLean

1995 Symbolism and the Social Contexts of Iron Production in Karagwe. World Archaeology 27(1):144-161.

Renfrew, C.

1975 Trade as Action at a Distance: Questions of Integration and Communication. In Ancient Civilization and Trade. J. A. Sabloff and C. C. Lamberg-Karlovsky, eds. Pp. 3-59. School of American Research. Albuquerque: University of New Mexico Press.

Rice, P. M.

1987 Pottery Analysis: A Sourcebook. Chicago: University of Chicago Press.

Richard, F. G.

2007 From Cosaan to Colony: Exploring Archaeological Landscape Formations and Socio-Political Complexity in the Siin (Senegal), Ad 500-1900. Unpublished PhD Dissertation, Syracuse University.

2009 Historical and Dialectical Perspectives on the Archaeology of Complexity in the Siin-Saalum (Senegal): Back to the Future? African Archaeological Review 26:75-135.

2010 Recharting Atlantic Encounters: Object Trajectories and Histories of Value in the Siin (Senegal) and Senegambia. Archaeological Dialogues 17(1):1-27.

Robb, J. E., ed.

1999a Material Symbols: Culture and Economy in Prehistory. Carbondale: Southern Illinois University Press.

1999b Secret Agents: Culture, Economy, and Social Reproduction. In Material Symbols: Culture and Economy in Prehistory. J. E. Robb, ed. Pp. 3-15. Carbondale: Southern Illinois University Press.

2007 The Early Mediterranean Village: Agency, Material Culture, and Social Change in Neolithic Italy. Cambridge: Cambridge University Press.

Robertshaw, P., N. Benco, M. Wood, L. Dussubieux, E. Melchiorre, and A. Ettahiri

2010 Chemical Analysis of Glass Beads from Medieval Al-Basra (Morocco). Archaeometry 52(3):355-379.

Robinson, D.

1985 The Holy War of Umar Tal: The Western Sudan in the Mid-Nineteenth Century. Oxford: Clarendon Press.

Rogers, D. J., and S. E. Randolph

1986 Distribution and Abundance of Tsetse Flies (Glossina Spp.). Journal of Animal Ecology 55(3):1007-1025.

Roseberry, W.

1988 Political Economy. Annual Review of Anthropology 17:161-185.

1989 Anthropologies and Histories: Essays in Culture, History, and Political Economy. New Brunswick: Rutgers University Press. 
Roset, J.-P.

1987 Paleoclimatic and Cultural Conditions of Neolithic Development in the Early Holocene of Northern Niger (Aïr and Ténéré). In Prehistory of North Africa:

Essays in Honor of Fred Wendorf. A. E. Close, ed. Pp. 211-234. Dallas: Southern Methodist University Press.

Rossignol, J., and L. Wandsnider, eds.

1992 Space, Time, and Archaeological Landscapes. New York: Plenum Press.

Roux, V.

2007 Ethnoarchaeology: A Non Historical Science of Reference Necessary for Interpreting the Past. Journal of Archaeological Method and Theory 14(2):153178.

Rowan, Y. M., and J. R. Ebeling, eds.

2008 New Approaches to Old Stones: Recent Studies of Ground Stone Artifacts. London: Equinox.

Rowlands, M. J., and J.-P. Warnier

1993 The Magical Production of Iron in the Cameroon Grassfields. In The Archaeology of Africa: Foods, Metals and Towns. T. Shaw, P. Sinclair, B. Andah, and A. Okpoko, eds. Pp. 512-550. London: Routledge.

Sackett, J. R.

1977 The Meaning of Style in Archaeology: A General Model. American Antiquity 42(3):369-380.

1982 Approaches to Style in Lithic Archaeology. Journal of Anthropological Archaeology 1(1):59-112.

1985 Style and Ethnicity in the Kalahari: A Reply to Wiessner. American Antiquity 50(1):154-159.

Sahlins, M. D.

1972 Stone Age Economics. New York: Aldine de Gruyter.

1981 Historical Metaphors and Mythical Realities: Structure in the Early History of the Sandwich Islands Kingdom. Ann Arbor: University of Michigan Press.

Sahlins, M. D., and E. R. Service

1960 Evolution and Culture. Ann Arbor: University of Michigan Press.

Sall, M.

2005 Traditions Céramiques, Identités Et Peuplement En Sénégambie: Ethnographie Compareé Et Essai De Reconstitution Historique. Volume BAR International Series 1407. Oxford: Archaeopress.

Salzmann, U., and M. Waller

1998 The Holocene Vegetational History of the Nigerian Sahel Based on Multiple Pollen Profiles. Review of Palaeobotany and Palynology 100:39-72.

Sanford, W. W., and A. O. Isichei

1986 Savanna. In Plant Ecology in West Africa: Systems and Processes. G. W. Lawson, ed. Pp. 95-149. New York: John Wiley \& Sons.

Sassoon, $\mathrm{H}$.

1963 Early Sources of Iron in Africa. South African Archaeological Bulletin 18(72):176-180. 
Savage, S. H.

1997 Assessing Departures from Log-Normality in the Rank-Size Rule. Journal of Archaeological Science 24:233-244.

Schiffer, M. B.

1987 Formation Processes of the Archaeological Record. Albuquerque: University of New Mexico Press.

Schiffer, M. B., and J. M. Skibo

1997 The Explanation of Artifact Variability. American Antiquity 62(1):27-50.

Schiffer, M. B., A. P. Sullivan, and T. C. Klinger

1978 The Design of Archaeological Surveys. World Archaeology 10(1):1-28.

Schlanger, S. H.

1991 On Manos, Metates, and the History of Site Occupations. American Antiquity 56(3):460-474.

Schmidt, P. R., ed.

1996 The Culture and Technology of African Iron Production. Gainesville: University of Florida Press.

2006 Historical Archaeology in Africa: Representation, Social Memory, and Oral Traditions. Walnut Creek, CA: AltaMira Press.

Schmitz, J.

1994 Cités Noires: Les Républiques Villageoises Du Fuuta Tooro (Vallée Du Fleuve Sénégal). Cahiers d'Études Africaines 34(133-135):231-263.

Schoenbrun, D. L.

1998 A Green Place, a Good Place: Agrarian Change, Gender, and Social Identity in the Great Lakes Region to the 15th Century. Portsmouth: Heinemann.

Sereno, P. C. et al.

2008 Lakeside Cemeteries in the Sahara: 5000 Years of Holocene Population and Environmental Change. PLoS ONE 3(8):1-22.

Sewell, W. H., Jr.

1992 A Theory of Structure: Duality, Agency, and Transformation. American Journal of Sociology 98(1):1-29.

Shafer, H. J., and T. R. Hester

1991 Lithic Craft Specialization and Product Distribution at the Maya Site of Colha, Belize. World Archaeology 23(1):79-97.

Shahin, M.

2002 Hydrology and Water Resources of Africa. Dordrecht: Kluwer Academic.

Shanahan, T. M. et al.

2006 Paleoclimatic Variations in West Africa from a Record of Late Pleistocene and Holocene Lake Level Stands of Lake Bosumtwi, Ghana. Palaeogeography, Palaeoclimatology, Palaeoecology 242:287-302.

Shaw, T.

1944 Report on Investigations Carried out in the Cave Known as "Bosumpra" at Abetifi, Kwahu, Gold Coast Colony. Proceedings of the Prehistoric Society 10(1):1-67.

1960 Early Smoking Pipes: In Africa, Europe, and America. Journal of the Royal Anthropological Institute 90(2):272-305. 
Shaw, T.

1970 Igbo-Ukwu: An Account of Archaeological Discoveries in Eastern Nigeria. London: Faber and Faber.

Shaw, T., and S. G. H. Daniels

1984 Excavations at Iwo Eleru, Ondo State, Nigeria. West African Journal of Archaeology 14:1-269.

Shaw, T., P. Sinclair, B. Andah, and A. Okpoko

1993 The Archaeology of Africa: Food, Metals and Towns. New York: Routledge.

Shennan, S.

1997 Quantifying Archaeology. Edinburgh: Edinburgh University Press.

Shinnie, P. L., and F. J. Kense

1989 The Archaeology of Gonja, Ghana: Excavations at Daboya. Calgary: University of Calgary Press.

Shipton, P.

1984 Strips and Patches: A Demographic Dimension in Some Africa Land-Holding and Political Systems. Man 19(4):613-634.

1994 Land and Culture in Tropical Africa: Soils, Symbols, and the Metaphysics of the Mundane. Annual Review of Anthropology 23:347-377.

Shott, M. J.

1986 Technological Organization and Settlement Mobility: An Ethnographic Examination. Journal of Anthropological Research 42(1):15-51.

1989 Bipolar Industries: Ethnographic Evidence and Archaeological Implications. North American Archaeologist 10(1):1-24.

Sillitoe, P., and K. Hardy

2003 Living Lithics: Ethnoarchaeology in Highland Papua New Guinea. Antiquity 77:555-566.

Simon, H. A.

1985 Human Nature in Politics: The Dialogue of Psychology with Political Science. The American Political Science Review 79:293-304.

Sinopoli, C. M.

1991 Approaches to Archaeological Ceramics. New York: Plenum Press.

1994 The Archaeology of Empires. Annual Review of Anthropology 23:159-180.

2003 The Political Economy of Craft Production: Crafting Empire in South India, C. 1350-1650. Cambridge: Cambridge University Press.

2005 Polity and Economy in Fourteenth- through Seventeenth-Century South India. In A Catalyst for Ideas: Anthropological Archaeology and the Legacy of Douglas W. Schwartz. V. Scarborough and R. M. Leventhal, eds. Pp. 229-256. Santa Fe: School of American Research Press.

Siskind, J.

1978 Kinship and Mode of Production. American Anthropologist 80(4):860-872.

Smith, A., and N. David

1995 The Production of Space and the House of Xidi Sukur. Current Anthropology 36(3):441-457.

Smith, A. B.

1975 Radiocarbon Dates from Bosumpra Cave, Abetifi, Ghana. Proceedings of the Prehistoric Society 41:179-182. 
Smith, A. B.

1992 Pastoralism in Africa: Origins and Development Ecology. London: Hurst and Company.

Smith, A. T.

2003 The Political Landscape: Constellations of Authority in Early Complex Polities. Berkeley: University of California Press.

Smith, M. E.

2004 The Archaeology of Ancient State Economies. Annual Review of Anthropology 33:73-102.

Smith, P.

1965 Les Diakhanké: Histoire D'une Dispersion. Bulletins et Mémoires de la Société d'Anthropologie de Paris 8(4):231-262.

Soja, E. W.

1989 Postmodern Geographies: The Reassertion of Space in Critical Social Theory. New York: Verso.

Soper, R.

1985 Roulette Decoration on African Pottery: Technical Considerations, Dating and Distributions. African Archaeological Review 3:29-51.

Southall, A.

1988 On Mode of Production Theory: The Foraging Mode of Production and the Kinship Mode of Production. Dialectical Anthropology 12(2):165-192.

Spaulding, A. C.

1960 The Dimensions of Archaeology. In Essays in the Science of Culture. G. E. Dole and R. Carneiro, eds. Pp. 437-456. New York: Crowell.

Speakman, R. J., and H. Neff

2005 The Application of Laser Ablation-Icp-Ms to the Study of Archaeological Materials: An Introduction. In Laser Ablation-Icp-Ms in Archaeological Research. R. J. Speakman and H. Neff, eds. Pp. 1-14. Albuquerque: University of New Mexico Press.

Spencer, C. S.

1993 Human Agency, Biased Transmission, and the Cultural Evolution of Chiefly Authority. Journal of Anthropological Archaeology 12(1):41-74.

Stahl, A. B.

1985 Reinvestigation of Kintampo 6 Rock Shelter, Ghana: Implications for the Nature of Culture Change. African Archaeological Review 3:117-150.

1986 Early Food Production in West Africa: Rethinking the Role of the Kintampo Culture. Current Anthropology 27(5):532-536.

1993a Concepts of Time and Approaches to Analogical Reasoning in Historical Perspective. American Antiquity 58(2):235-260.

1993b Intensification in the West African Late Stone Age: A View from Central Ghana. In The Archaeology of Africa: Food, Metals and Towns. T. Shaw, P. Sinclair, B. Andah, and A. Okpoko, eds. Pp. 261-273. New York: Routledge.

1994 Innovation, Diffusion, and Culture Contact: The Holocene Archaeology of Ghana. Journal of World Prehistory 8(1):51-112.

1999a The Archaeology of Global Encounters Viewed from Banda, Ghana. African Archaeological Review 16(1):5-81. 
Stahl, A. B.

1999b Perceiving Variability in Time and Space: The Evolutionary Mapping of African Societies. In Beyond Chiefdoms: Pathways to Complexity in Africa. S. K. McIntosh, ed. Pp. 39-55. Cambridge: Cambridge University Press.

2001 Making History in Banda: Anthropological Visions of Africa's Past. Cambridge: Cambridge University Press.

2002 Colonial Entanglements and the Practices of Taste: An Alternative to Logocentric Approaches. American Anthropologist 104(3):827-845.

2004a Comparative Insights into the Ancient Political Economies of West Africa. In Archaeological Perspectives on Political Economies. G. M. Feinman and L. M. Nicholas, eds. Pp. 253-270. Salt Lake City: University of Utah Press.

2004b Political Economic Mosaics: Archaeology of the Last Two Millennia in Tropical Sub-Saharan Africa. Annual Review of Anthropology 33:145-172.

2008 Dogs, Pythons, Pots, and Beads: The Dynamics of Shrines and Sacrificial Practices in Banda, Ghana, 1400-1900 Ce. In Memory Work: Archaeologies of Material Practices. B. J. Mills and W. H. Walker, eds. Pp. 159-186. Santa Fe: School for Advanced Research Press.

Stahl, A. B., and M. d. D. Cruz

1998 Men and Women in a Market Economy: Gender and Craft Production in West Central Ghana, C. 1775-1995. In Gender in African Prehistory. S. Kent, ed. Pp. 205-226. Walnut Creek, CA: AltaMira Press.

Stahl, A. B., M. d. D. Cruz, H. Neff, M. D. Glascock, R. J. Speakman, B. Giles, and L. Smith

2008 Ceramic Production, Consumption and Exchange in the Banda Area, Ghana: Insights from Compositional Analyses. Journal of Anthropological Archaeology 27(3):363-381.

Stanish, C.

2003 Ancient Titicaca. Berkeley: University of California Press.

Stark, M. T.

2003 Current Issues in Ceramic Ethnoarchaeology. Journal of Archaeological Research 11(3):193-242.

Stark, M. T., R. L. Bishop, and E. Miksa

2000 Ceramic Technology and Social Boundaries: Cultural Practices in Kalinga Clay Selection and Use. Journal of Archaeological Method and Theory 7(4):295-331.

Steadman, S. R.

1996 Recent Research in the Archaeology of Architecture: Beyond the Foundations. Journal of Archaeological Research 4(1):51-93.

Stein, G. J.

1998 Heterogeneity, Power, and Political Economy: Some Current Research Issues in the Archaeology of Old World Complex Societies. Journal of Archaeological Research 6(1):1-44.

2002 From Passive Periphery to Active Agents: Emerging Perspectives in the Archaeology of Interregional Interaction. American Anthropologist 104(3):903916. 
Stenning, D. J.

1957 Transhumance, Migratory Drift, Migration: Patterns of Pastoral Fulani Nomadism. The Journal of the Royal Anthropological Institute of Great Britain and Ireland 87(1):57-73.

Steponaitis, V.

1981 Settlement Hierarchies and Political Complexity in Nonmarket Societies: The Formative Period of the Valley of Mexico. American Anthropologist 83:321-363. Stewart, M. H.

1979 The Role of the Manding in the Hinterland Trade of the Western Sudan: A Linguistic and Cultural Analysis. Bulletin de l'Institut Fondamental d'Afrique Noire, Série B 41(2):281-302.

Stoltman, J. B.

1989 A Quantitative Approach to the Petrographic Analysis of Ceramic Thin Sections. American Antiquity 54(1):147-160.

1991 Ceramic Petrography as a Technique for Documenting Cultural Interaction: An Example from the Upper Mississippi Valley. American Antiquity 56(1):103-120.

Stone, G. D.

1996 Settlement Ecology: The Social and Spatial Organization of Kofyar Agriculture. Tucson: University of Arizona Press.

Stone, G. D., R. M. Netting, and M. P. Stone

1990 Seasonality, Labor Scheduling, and Agricultural Intensification in the Nigerian Savanna. American Anthropologist 92:7-23.

Strathern, M.

1985 Kinship and Economy: Constitutive Orders of a Provisional Kind. American Ethnologist 12(2):191-209.

Sullivan, A. P., and A. S. Tolonen

1998 Evaluating Assemblage Diversity Measures with Surface Archaeological Data. In Surface Archaeology. A. P. Sullivan, ed. Pp. 143-155. Albuquerque: University of New Mexico Press.

Sundstrom, L.

1972 Ecology and Symbiosis: Niger Water Folk. Uppsala: Studia Ethnographica.

Sutton, J. E. G.

1974 The Aquatic Civilization of Middle Africa. Journal of African History 15:527546.

Swaine, M. D., and J. B. Hall

1986 Forest Structure and Dynamics. In Plant Ecology in West Africa: Systems and Processes. G. W. Lawson, ed. Pp. 47-93. New York: John Wiley \& Sons.

Szumowski, G.

1956 Fouilles De L'abri Sous Roche De Kourounkorokalé (Soudan Français). Bulletin de l'Institut Français d'Afrique Noire, Série B 18(3-4):462-508.

Talbot, M. R., D. A. Livingstone, P. G. Palmer, J. Maley, J. M. Melack, G. Delibrias, and S. Gulliksen

1984 Preliminary Results from Sediment Cores from Lake Bosumtwi, Ghana. In Palaeoecology of Africa and the Surrounding Islands. J. A. Coetzee and E. M. V. Z. Bakker, eds. Pp. 173-192. Rotterdam: A. A. Balkema. 
Tamari, T.

1991 The Development of Caste Systems in West Africa. Journal of African History 32(2):221-250.

1995 Linguistic Evidence for the History of West African "Castes". In Status and Identity in West Africa: Nyamakalaw of Mande. D. C. Conrad and B. E. Frank, eds. Pp. 61-85. Bloomington: Indiana University Press.

2005 Kinship and Caste in Africa: History, Diffusion and Evolution. In The Character of Kingship. D. Quigley, ed. Pp. 141-169. New York: Berg.

Taylor, D.

1975 Some Locational Aspects of Middle-Range Hierarchical Societies. PhD Dissertation, The City University of New York.

Teltser, P. A.

1993 An Analytic Strategy for Studying Assemblage-Scale Ceramic Variation: A Case Study from Southeast Missouri. American Antiquity 58(3):530-543.

Terray, E.

1972 Marxism and "Primitive" Societies. New York: Monthly Review Press.

Thiam, M.

2010 Poterie Et Identité: Les Bassari Et Bedik Du Sénégal Oriental. In Espaces, Culture Matérielle Et Identités En Sénégambie. I. Thiaw, ed. Pp. 68-84. Dakar: CODESRIA.

Thiaw, I.

1999 Archaeological Investigations of Long-Term Culture Change in the Lower Falemme (Upper Senegal Region) A.D. 500-1900. Unpublished PhD Dissertation, Rice University.

2010 Espaces, Culture Matérielle Et Identités En Sénégambie. Dakar: CODESRIA.

2011 Slaves without Shackles: An Archaeology of Everyday Life on Gorée Island, Senegal. Proceedings of the British Academy 168:147-165.

2012 Atlantic Impacts on Inland Senegambia: French Penetration and African Initiatives in Eighteenth and Nineteenth-Century Gajaaga and Bundu (Upper Senegal River). In Power and Landscape in Atlantic West Africa: Archaeological Perspectives. J. C. Monroe and A. Ogundiran, eds. Cambridge: Cambridge University Press.

Thilmans, G., C. Descamps, and B. Khayat

1980 Protohistoire Du Sénégal, Tome I: Les Sites Mégalithiques. Dakar: Institut Fondamental d'Afrique Noire.

Thilmans, G., and A. Ravisé

1980 Protohistoire Du Sénégal, Tome Ii: Sintiou-Bara Et Les Sites Du Fleuve. Dakar: Institut Fondamental d'Afrique Noire.

Thomas, N.

1991 Entangled Objects: Exchange, Material Culture, and Colonialism in the Pacific. Cambridge, MA: Harvard University Press.

Thomassey, P., and R. Mauny

1956 Campagne De Fouilles De 1950 À Koumbi Saleh (Ghana?). Bulletin de l'Institut Français d'Afrique Noire, Série B 18(1-2):117-140. 
Thornton, J.

1992 Africa and Africans in the Making of the Atlantic World, 1400-1680. Cambridge:

Tilley, C.

Cambridge University Press.

1994 A Phenomenology of Landscape: Places, Paths and Monuments. New York: Berg.

Togola, T.

2008 Archaeological Investigations of Iron Age Sites in the Mema Region, Mali (West Africa). Oxford: Archaeopress.

Tonkin, E.

1982 The Boundaries of History in Oral Performance. History in Africa 9:273-284.

Torrence, R., ed.

1989 Time, Energy, and Stone Tools. Cambridge: Cambridge University Press.

Tschauner, $\mathrm{H}$.

1996 Middle-Range Theory, Behavioral Archaeology, and Postempiricist Philosophy of Science in Archaeology. Journal of Archaeological Method and Theory 3(1):130.

Turner, V. W.

1957 Schism and Continuity in an African Society. Manchester: Rhodes-Livingstone Institute.

Usman, A. A.

2003 Ceramic Seriation, Sites Chronology, and Old Oyo Factor in Northcentral Yorubaland, Nigeria. African Archaeological Review 20(3):149-169.

Usman, A. A., R. J. Speakman, and M. D. Glascock

2005 An Initial Assessment of Prehistoric Ceramic Production and Exchange in Northern Yoruba, North Central Nigeria: Results of Ceramic Compositional Analysis. African Archaeological Review 22(3):141-168.

Van der Leeuw, S. E.

1993 Giving the Potter a Choice: Conceptual Aspects of Pottery Techniques. In Technological Choices: Transformation in Material Cultures since the Neolithic. P. Lemmonier, ed. Pp. 238-288. London: Routledge.

Van Doosselaere, B.

2005 Perception Stylistique E Technologie Céramique: Reconstitution Et Interprétation Des Techniques De Façonnage Des Poteries Archéologiques De Koumbi Saleh (Mauritanie, Ixème - Xvème Siècles). In Pottery Manufacturing Processes: Reconstitution and Interpretation. A. Livingstone Smith, D. Bosquet, and R. Martineau, eds. Pp. 179-199, Vol. BAR International Series 1349. Oxford: Archaeopress.

Van Neer, W.

2008 Fishing in the Senegal River During the Iron Age: The Evidence from the Habitation Mounds of Cubalel and Siouré. In Animals and People:

Archaeozoological Papers in Honor of Ina Plug S. Badenhorst, P. Mitchell, and J. C. Driver, eds. Pp. 117-130. Bar International Series, Vol. BAR International Series 1849. Oxford: Archaeopress.

Vansina, J.

1985 Oral Tradition as History. Madison: University of Wisconsin Press. 
Vernet, R.

2002 Climate During the Late Holocene in the Sahara and Sahel: Evolution and Consequences on Human Settlement. In Droughts, Food and Culture: Ecological Change and Food Security in Africa's Later Prehistory. F. A. Hassan, ed. Pp. 4763. New York: Kluwer Academic / Plenum Publishers.

Vogel, J. O., ed.

2000 Ancient African Metallurgy. Walnut Creek, CA: Altamira Press.

Wallerstein, I.

1974 The Modern World-System. New York: Academic Press.

Washburn, D. K.

1974 Nearest Neighbor Analysis of Pueblo I-Iii Settlement Patterns Along the Rio Puerco of the East, New Mexico. American Antiquity 39(2):315-335.

Waters-Bayer, A., and W. Bayer

1994 Coming to Terms: Interactions between Immigrant Fulani Cattle-Keepers and Indigenous Farmers in Nigeria's Subhumid Zone. Cahiers d'Études Africaines 34(133/135):213-229.

Watson, D. J.

1999 The Faleme Valley Faunal Assemblages. In Archaeological Investigations of Long-Term Culture Change in the Lower Falemme (Upper Senegal Region) A.D. 500-1900. I. Thiaw, ed. Pp. 371-384: PhD Dissertation, Rice University.

2005 Under the Rocks: Reconsidering the Origin of the Kintampo Tradition and the Development of Food Production in the Savanna-Forest/Forest of West Africa. Journal of African Archaeology 3(1):3-55.

Webb, J. L. A., Jr.

1993 The Horse and Slave Trade between the Western Sahara and Senegambia. Journal of African History 34:221-246.

1995 Desert Frontier: Ecological and Economic Change Along the Western Sahel, 1600-1850. Madison: University of Wisconsin Press.

2005 Malaria and the Peopling of Early Tropical Africa. Journal of World History 16(3):269-291.

Weedman, K. J.

2006 An Ethnoarchaeological Study of Hafting and Stone Tool Diversity among the Gamo of Ethiopia. Journal of Archaeological Method and Theory 13(3):189-237.

Weiner, A. B.

1985 Inalienable Wealth. American Ethnologist 12(2):210-227.

Wells, T.

1998 Nail Chronology: The Use of Technologically Derived Features. Historical Archaeology 32(2):78-99.

Wendorf, F., and R. Schild

1998 Nabta Playa and Its Role in Northeastern African Prehistory. Journal of Anthropological Archaeology 17:97-123.

Whallon, R.

1972 A New Approach to Pottery Typology. American Antiquity 37(1):13-33.

1984 Unconstrained Clustering for the Analysis of Spatial Distributions in Archaeology. In Intrasite Spatial Analysis in Archaeology. H. Hietala, ed. Pp. 242-277. Cambridge: Cambridge University Press. 
Whallon, R., and J. A. Brown, eds.

1982 Essays in Archaeological Typology. Evanston, IL: Center for American Archaeology.

Whallon, R., and S. Kantmann

1969 Early Bronze Age Development in the Keban Reservoir, East-Central Turkey. Current Anthropology 10(1):128-133.

White, J. P., and D. H. Thomas

1972 What Mean These Stones? Ethno-Taxonomic Models and Archaeological Interpretations in the New Guinea Highlands. In Models in Archaeology. D. Clarke, ed. Pp. 275-308. New York: Methuen \& Co.

Wiessner, P.

1974 A Functional Estimator of Population from Floor Area. American Antiquity 39(2):343-350.

1982a Beyond Willow Smoke and Dogs' Tails: A Comment on Binford's Analysis of Hunter-Gatherer Settlement Systems. American Antiquity 47:171-178.

1982b Risk, Reciprocity and Social Influences On !Kung San Economics. In Politics and History in Band Societies. E. Leacock and R. B. Lee, eds. Pp. 61-84. Cambridge: Cambridge University Press.

1983 Style and Social Information in Kalahari San Projectile Points. American Antiquity 48(2):253-276.

2002 The Vines of Complexity: Egalitarian Structures and the Institutionalization of Inequality among the Enga. Current Anthropology 43(2):233-269.

Wilks, I.

1982a Wangara, Akan and Portuguese in the Fifteenth and Sixteenth Centuries. I. The Matter of Bitu. Journal of African History 23(3):333-349.

1982b Wangara, Akan and Portuguese in the Fifteenth and Sixteenth Centuries. Ii. The Struggle for Trade. Journal of African History 23(4):463-472.

Willey, G. R.

1951 Prehistoric Settlement Patterns in the Virú Valley, Perú. Volume 155. Washington, D.C.: Smithsonian Institution Press.

Wilmsen, E. N.

1989 Land Filled with Flies: A Political Economy of the Kalahari. Chicago: University of Chicago Press.

Wilmsen, E. N., D. Killick, D. D. Rosenstein, P. Thebe, C., and J. R. Denbow

2009 The Social Geography of Pottery in Botswana as Reconstructed by Optical Petrography. Journal of African Archaeology 7(1):3-40.

Wilson, D. C.

1994 Identification and Assessment of Secondary Refuse Aggregates. Journal of Archaeological Method and Theory 1(1):41-68.

Wobst, H. M.

1977 Stylistic Behavior and Information Exchange. In For the Director: Research Essays in Honor of James B. Griffin. C. Cleland, ed. Pp. 317-342. Anthropological Papers, Vol. 61. Ann Arbor: Museum of Anthropology, University of Michigan. 
Wobst, H. M.

1999 Style in Archaeology or Archaeologists in Style. In Material Meanings: Critical Approaches to the Interpretation of Material Culture. E. Chilton, ed. Pp. 118-132. Salt Lake City: University of Utah Press.

Wolf, E. R.

1982 Europe and the People without History. Berkeley: University of California Press. Woodhouse, J.

1998 Iron in Africa: Metal from Nowhere. In Transformations in Africa: Essays on Africa's Later Past. G. Connah, ed. Pp. 160-185. New York: Leicester University Press.

Wright, D. R.

1985 Beyond Migration and Conquest: Oral Traditions and Mandinka Ethnicity in Senegambia. History in Africa 12:335-348.

2010 The World and a Very Small Place in Africa: A History of Globalization in Niumi, the Gambia. Armonk, NY: M. E. Sharpe.

Wright, H. T.

1986 The Evolution of Civilizations. In American Archaeology Past and Future: A Celebration of the Society for American Archaeology 1935-1985. D. J. Meltzer, D. D. Fowler, and J. A. Sabloff, eds. Pp. 323-365. Washington, D. C.: Smithsonian Institution Press.

2000 Modeling Tributary Economies and Hierarchical Polities. G. M. Feinman and L. Manzanilla, eds. New York: Kluwer Academic / Plenum Publishers.

Wright, H. T., and G. A. Johnson

1975 Population, Exchange, and Early State Formation in Southwestern Iran. American Anthropologist 77(2):267-289.

Wright, J. B., D. A. Hastings, W. B. Jones, and H. R. Williams

1985 Geology and Mineral Resources of West Africa. London: George Allen \& Unwin. Wright, K. I.

1994 Ground-Stone Tools and Hunter-Gatherer Subsistence in Southwest Asia: Implications for the Transition to Farming. American Antiquity 59(2):238-263.

2008 Craft Production and the Organization of Ground Stone Technologies. In New Approaches to Old Stones: Recent Studies of Ground Stone Artifacts. Y. M. Rowan and J. R. Ebeling, eds. Pp. 130-143. London: Equinox.

World Wildlife Fund

2004 WWF Terrestrial Ecoregions. Redlands, CA: ESRI.

Wylie, A.

1982 An Analogy by Any Other Name Is Just as Analogical. Journal of Anthropological Archaeology 1:382-401.

2002 Thinking from Things. Berkeley: University of California Press.

Yaeger, J., and M. A. Canuto

2000 Introducing an Archaeology of Communities. In The Archaeology of

Communities: A New World Perspective. M. A. Canuto and J. Yaeger, eds. Pp. 115. New York: Routledge.

Yellen, J. E.

1977 Archaeological Approaches to the Present: Models for Reconstructing the Past. New York: Academic Press. 
Yerkes, R. W.

1983 Microwear, Microdrills, and Mississippian Craft Specialization. American Antiquity 48(3):499-518.

Yerkes, R. W., and P. N. Kardulias

1993 Recent Developments in the Analysis of Lithic Artifacts. Journal of Archaeological Research 1(2):89-119.

Yoffee, N.

2005 Myths of the Archaic State: Evolution of the Earliest Cities, States, and Civilizations. Cambridge: Cambridge University Press.

2007 Negotiating the Past in the Past: Identity, Memory, and Landscape in Archaeological Research. Tucson: University of Arizona Press.

York, R. N.

1978 Excavations at Dutsen Kongba, Plateau State, Nigeria. West African Journal of Archaeology 8:139-163.

Young, A.

1976 Tropical Soils and Soil Survey. Cambridge: Cambridge University Press.

Zangato, É., and A. F. C. Holl

2010 On the Iron Front: New Evidence from North-Central Africa. Journal of African Archaeology 8(1):7-23.

Zeltner, F.

1916 Quelques Gisements Préhistoriques De La Vallée Du Sénégal. Bulletins et Mémoires de la Société d'Anthropologie de Paris 7:238-244. 


\section{APPENDIX A \\ THE MADINA-SADATOU STUDY AREA: SURVEY METHODS AND SITE INVENTORY}

Diouboye, as with many archaeological sites across West Africa, endures as a series of low mounds whose dense artifacts and architectural remains signal a place of past, if not ancestral, occupation to nearby communities. As I discovered during a weeklong reconnaissance in the Madina-Sadatou study area in 2007, such well-known landmarks are readily identified with help from the local populace. In fact, strategies of guided or roadside survey have allowed archaeologists to locate several thousand sites in Senegambia (Martin and Becker 1984; Pélissier and Bâ 1980). Early research agendas influenced by the spirit of discovery, historical particularism, and nationalism generally worked to sample artifacts from different periods and/or find locales ripe for future excavation (see discussion in Richard 2009). The shift to processual and other empirical approaches over the past three decades, however, has led to more systematic surveys documenting habitation mounds and monumental cemeteries as well as ephemeral locales of past activity interspersed among them (e.g., A. Lawson 2003; S. K. McIntosh and R. J. McIntosh 1993b; Richard 2007).

Archaeology across the Upper Senegal region, and specifically along the Falémé River, nicely illustrates this century-long trend. During the colonial era a number of amateur scholars (Laforgue 1924; Zeltner 1916), and then professional researchers (Mauny 1963), made their way to eastern Senegal where they recorded several dozen sites and collected artifacts, dating mainly to the Middle to Late Stone Age, for curation at IFAN in Dakar (see Guitat 1970; Ravisé 1975). By the 1980s, an interest in the evolution of lithic technology led archaeologist Abdoulaye Camara and colleagues to more systematically document Stone Age sites along the lower Falémé River (Camara and Duboscq 1984, 1987, 1990), where they also encountered the site of Diouboye (Opper and Opper 1990). Finally, the 1990s saw the implementation of a truly systematic survey by Ibrahima Thiaw (1999) along the west bank of the Falémé River. Focusing on a fifty-kilometer stretch south from the Senegal-Falémé confluence, this pioneering project identified 154 sites dating anywhere from the Middle Stone Age to the early-20 ${ }^{\text {th }}$ century AD. By recording smaller sites associated with mobility strategies alongside the more formidable ruins of colonial outposts and fortified villages, this research has shed new light on local responses to interregional political trajectories over the longue-durée. More recently, Thiaw (2012) has further considered how local commoners, elites, and European interlopers negotiated political economic relations across the late precolonial landscape. The capacity to ask, let alone address, these sorts of anthropological and historical questions underscores the necessity of such survey methods in contemporary archaeological research. 
With this perspective in mind, the objectives of systematic archaeological survey in the Madina-Sadatou study area were as follows: (1) to document a representative sample of locales associated with the historical landscape centered on Diouboye; (2) to orient these locales within the cultural and chronological grid of the Upper Senegal region; and (3) to identify the material and spatial organization of economic (subsistence, craft, and long-distance exchange) activities performed at these locales. In addition, it was imperative to record survey data in a manner comparable to those from other surveys in Senegambia, particularly the one undertaken by Ibrahima Thiaw (see above).

Drawing upon the results of the 2007 guided survey, which revisited many of the sites previously documented by Marie-José and Howard Opper (1990), I led a systematic pedestrian survey during the subsequent field season in March-April 2008. While the initial research design called for a stratified random sampling of transects across the Madina-Sadatou study area, persistent vehicle problems limited survey to a contiguous area within walking or bicycling distance of Diouboye. Despite this constraint, it was possible to devise a survey methodology, described in the following section, capable of meeting the above research objectives. The second section of this appendix provides an inventory of the locales documented through both guided and systematic survey, including geomorphological and surface data relevant to analyses and interpretations of the historical landscape in Chapters 4-5.

\section{SURVEY METHODOLOGY}

\section{Sampling and Intensity}

Foremost in the survey design was the issue of documenting an adequate and representative sample of archaeological remains in the study area (Fish and Kowalewski 1990; Plog et al. 1978; Read 1975; Schiffer et al. 1978). As discussed in Chapter 3, the Madina-Sadatou study area is a survey universe lacking clearly defined physical boundaries. Geomorphological and ecological variation within the study area is strongly tied to distance from the Falémé River and its main tributaries. In general, fertile alluvium, archaic levees, and riparian bush give way to sparse savanna forest, exposed laterite, and sandstone ridges as one walks away from the river toward the Bundu Hills in the west or the Tomboura Escarpment in the east. The logistics of daily travel by foot and bicycle, however, made it difficult to access the latter landforms.

Because guided survey in 2007 indicated that sites, especially settlements, would be preferentially located near the Falémé River, I focused on complete coverage of a twokilometer buffer to the east and west of the main river channel. To this end, I defined kilometer-long survey parcels on the banks of the river running north and south of Diouboye. Over the course of six weeks, it was possible to survey 28 of these parcels to a distance of two kilometers from the river. In order to verify the fall-off in site density beyond this buffer, and explore the distribution of archaeological remains along smaller tributaries, I randomly selected 11 parcels for survey to a distance of four kilometers. The resulting pedestrian survey zone of 71.75 square kilometers provides a fairly complete window onto past occupations along the Falémé River near Diouboye (see Figure 4.1), but leaves open the question of how people related to the landscape of Madina-Sadatou as a whole. While contemporary villages $(n=14)$ and the few sites opportunistically recorded outside this core survey zone $(n=9)$ yield some insight in this regard, they also underline the need for further survey across the study area. 
Figure A.1: Survey through (a) dense savanna groundcover and (b) more typical sparse groundcover
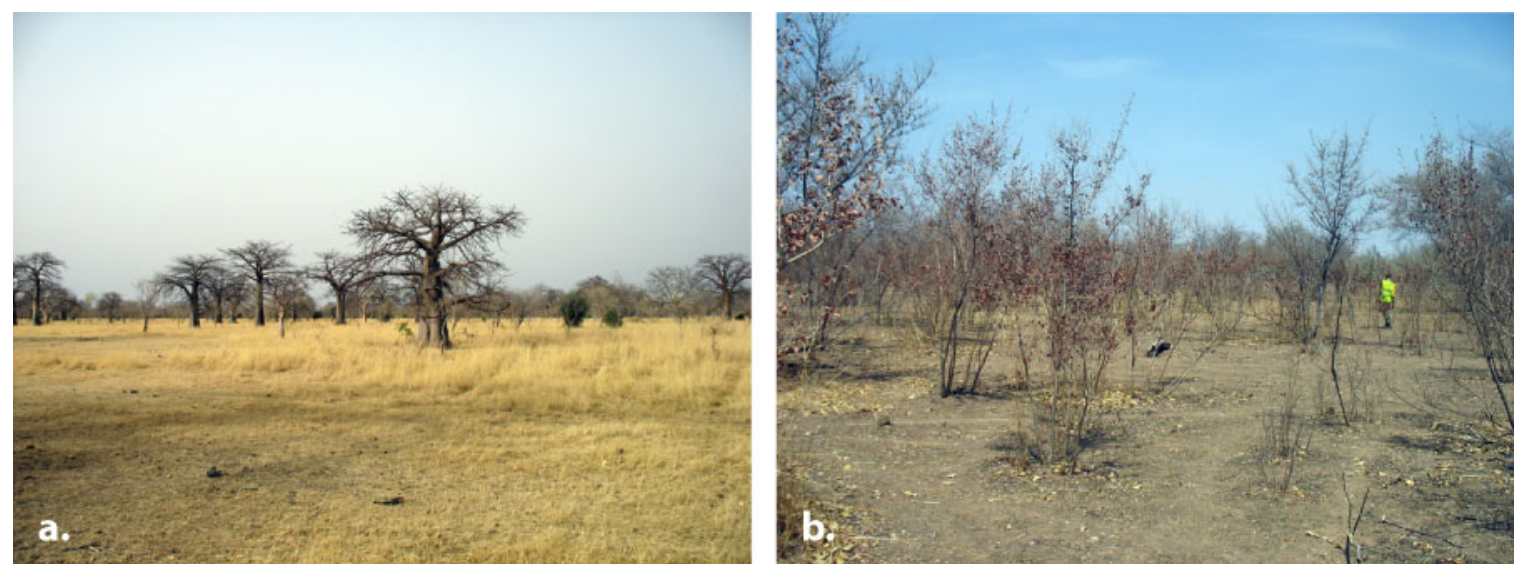

The representativeness of a survey further depends on overall visibility, the obtrusiveness of archaeological remains, and the intensity of survey coverage (Plog et al. 1978; Schiffer et al. 1978). Surface visibility within the survey zone was generally quite high. By the height of the dry season in March-April 2008, wild vegetation had been largely grazed by livestock or burned (Figure A.1b) and the agricultural fields next to villages had been cleared in preparation for the upcoming rains. Dense savanna grass did persist in some areas (Figure A.1a), largely at distances greater than two kilometers from the Falémé River or the nearest village. While this undoubtedly contributes to the perceived decline in site density away from the river, I would suggest that visibility across the survey zone was always sufficient to observe the more obtrusive hamlet and village sites of primary interest in this study.

The size and surface morphology of settlement sites in the West African savanna varies markedly according to past social strategies, environmental factors, and postdepositional processes (e.g., Connah 1984; Gallagher 2010; Holl 2002; Holl and Koté 2000; G. W. Lawson 1986; Richard 2007; Thiaw 1999; Togola 2008). Past settlements in the Madina-Sadatou study area were particularly obtrusive as low mounds of archaeological deposits invariably associated with stone features such as granary and hut foundations. With an average diameter of just over 53 meters, these sites were fairly easy to spot during pedestrian survey, especially given the high surface visibility in most transects. Stone Age sites lacking mounds were likewise fairly obtrusive due to the contrast of their white quartz and blue chert artifacts against the reddish soil.

Based on the above parameters and the desire to maximize coverage across a study area with a low density of archaeological remains, I opted for a survey of moderate intensity. Within each survey parcel, a team including myself, three young men from Sansanding, and one or two students from IFAN walked east-west transects usually spaced at 50 meters, although transects spaced at 30 meters were employed in several areas with exceptionally low surface visibility. Given the obtrusiveness of surface remains noted above, this coverage was sufficient to identify locales from all periods larger than 25 meters in diameter, in addition to a representative sample of smaller sites and isolated occurrences. 


\section{Locale Definition and Documentation}

Hundreds of thousands of years of human occupation coupled with low-energy erosion have made isolated pieces of lithic debitage and small sherds nearly ubiquitous throughout the study area, particularly along the margins of the Falémé River. While recognizing that all of these artifacts contain potential information about past landscapes and communities (Dunnell and Dancey 1983; Peterson and Drennan 2005), it was simply not feasible to record the majority of these solitary specimens (excepting the rare tobacco pipe fragment or metal tool).

Only when one of our team encountered unambiguous evidence for in situ activity did we halt to document the locale-defined as a relatively discrete place with one or more architectural features and/or an artifact scatter measuring more than ten meters in diameter or with a density of 20 artifacts or more per square meter. Although some locales were reclassified during analysis, I drew an in-field distinction between isolated occurrences and sites. Isolated occurrences $(n=65)$ are ephemeral assemblages appearing to represent a discrete event such as retouching a stone tool, breaking a pot, or dropping a groundstone axe. Sites (n=146), on the other hand, are locales with dense artifact densities and/or surface features resulting from repetitive or long-term human activity.

Each recorded locale, whether site or isolated occurrence, first received a unique Survey ID number. Using a Garmin handheld GPS receiver ( $\pm 5 \mathrm{~m}$ accuracy), I then plotted boundary coordinates for each site to encompass all visible surface features and scatters of artifacts greater than the above density threshold; isolated occurrences were recorded as a single point. While this method for estimating site area was less accurate than systematic artifact collection (Whallon and Kantmann 1969) or shovel-testing (Fleisher and LaViolette 1999; A. Lawson 2003), I personally recorded the boundaries of each site in order to exclude inter-observer bias. This method was sufficient to meet the above objectives by distinguishing discrete site size classes (i.e., homestead, hamlet, and village) defined in Chapters 4-5.

For each locale, I further made note of the surrounding environment and general state of preservation. As summarized in the inventory below, I broadly described the setting, including local relief and groundcover, as well as the soil matrix, with particular attention to gravel content. I also assessed the relative degree of erosion and other sources of disturbance working to either enhance or limit the visibility of archaeological features and artifacts. In the case of the former, I recorded the presence of stone alignments (circular, square, irregular), collapsed daub concentrations, pyrotechnic features, and groundstone implements. These observations, alongside collected surface artifacts (below), provide key insights into the activities undertaken at each site.

\section{Artifact Collection}

Despite the vagaries of post-depositional processes, surface artifacts often provide evidence for past technological practices and cultural traditions, as well as the relative or absolute timing of their deposition. For this reason, our field crew collected artifacts from the majority of sites $(n=125)$ and several isolated occurrences $(n=18)$ identified during survey. Adapting the two-part collection strategy developed by Thiaw (1999:245-246) for survey along the lowermost Falémé River, we took a systematic collection of all materials in a 25 square meter unit placed at the approximate center of each site; the location of such units were recorded using GPS. Although depositional and post-depositional 
processes usually make surface assemblages heterogeneous across any given site, systematic collection ensured that smaller lithic debris and sherds, such as those characteristic of cLSA pottery, would find their way into analysis and interpretation.

Appearing in surface assemblages at low frequencies, "diagnostic" artifacts such as stone tools, rare pottery forms, terracotta items, metallurgical debris, and imported materials such as glass and copper could yield a tremendous amount of information about past activities and social relations. For this reason, our field crew also undertook a purposive collection of these sorts of objects at most sites; this was the only form of surface collection employed for isolated occurrences. The intensity of this collection varied according to the area and artifactual composition of each locale, but usually continued until we had accumulated at least two or three dozen diagnostic artifacts. While these samples do not lend themselves to quantitative comparison or inferences based on absent types, the mere presence of diagnostic artifacts can help to situate them within the spatial, temporal, and cultural frames described in Chapters 4-5.

\section{SITE INVENTORY}

The following inventory provides a summary of surface and artifact data from each locale (including both sites and isolated occurrences) documented through both systematic and guided survey. Due to the reclassification of several locales and surface features during analysis in GIS (noted where appropriate), the survey data presented below differ somewhat from preliminary results previously published for the Central Falémé Archaeology Project (Gokee 2010). Where possible, I have made note of sites identified during previous surveys in the Madina-Sadatou study area (Camara 2000; Opper and Opper 1990; Ravisé 1975).

In addition to the geographic coordinates, area, and summaries of setting and soil, the FS numbers of systematic (s) and purposive (p) surface collection samples correspond to artifacts reported in this study and catalogued at IFAN. Descriptions of the associated artifact and features types and chronological designations appear in Appendices D-G and Chapter 4. 


\section{Systematic Survey: Sites}

Survey ID: FAL-1000 (Diouboye)

Coordinates: $13^{\circ} 54^{\prime} 28.86^{\prime \prime} \mathrm{N}, 12^{\circ} 11^{\prime} 08.05^{\prime \prime} \mathrm{W}$

Date recorded: 6/17/2007 Area $\left(\mathrm{m}^{2}\right)$ : 125,444

Setting: Low mounds in savanna, light groundcover

Soil: Consolidated clay loam with light to modest laterite gravel

FS (sample): 1739-1749, 1754-1769 (s)

Ceramic component(s): Iron Age

Lithic component(s): Unknown

Groundstone: Axe, Basin, Hachette, Handstone

Metallurgy: Iron tool/ornament, Slag

Other artifacts: Glass bead, Netweight

Surface feature(s): Stone circle, Stone alignment, Pyrotechnic feature

Primary function(s): Settlement

Notes: See Appendix B; initially recorded in 2007 as Pathé Djimba

Survey ID: FAL-1001

Coordinates: $13^{\circ} 58^{\prime} 59.61^{\prime \prime} \mathrm{N}, 12^{\circ} 11^{\prime} 44.87^{\prime \prime} \mathrm{W}$

Date recorded: $3 / 22 / 2008 \quad$ Area $\left(\mathrm{m}^{2}\right): 25,228$

Setting: Low mound in open savanna, light groundcover

Soil: Consolidated clay loam with dense laterite gravel

FS (sample): 2010-2011 (s); 2012 (p)

Ceramic component(s): Iron Age

Lithic component(s): Unknown

Groundstone: Handstone

Metallurgy: Slag

Surface feature(s): Stone circle

Primary function(s): Settlement

Notes: Features include six stone foundations (1-3 m in diameter)

\section{Survey ID: FAL-1002}

Coordinates: $13^{\circ} 59^{\prime} 00.73^{\prime \prime} \mathrm{N}, 12^{\circ} 12^{\prime} 16.13^{\prime \prime} \mathrm{W}$

Date recorded: 3/21/2008 Area $\left(\mathrm{m}^{2}\right)$ : 4692

Setting: Bluff in riparian savanna, light groundcover

Soil: Consolidated silt loam with laterite gravel

FS (sample): 2000-2001 (s); 2023 (p)

Ceramic component(s): None

Lithic component(s): MSA/LSA

Primary function(s): Unknown

Notes:

\section{Survey ID: FAL-1003}

Coordinates: $13^{\circ} 58^{\prime} 57.29 " \mathrm{~N}, 12^{\circ} 12^{\prime} 11.65^{\prime \prime} \mathrm{W}$

Date recorded: 3/21/2008 Area $\left(\mathrm{m}^{2}\right): 546$

Setting: Bluff in wooded savanna, light groundcover

Soil: Semi-consolidated sandy clay loam with dense laterite gravel

FS (sample): 2002 (s)

Ceramic component(s): None

Lithic component(s): MSA/LSA

Primary function(s): Unknown

Notes:

\section{Survey ID: FAL-1004}

Coordinates: $13^{\circ} 59^{\prime} 01.76^{\prime \prime} \mathrm{N}, 12^{\circ} 12^{\prime} 08.87^{\prime \prime} \mathrm{W}$

Date recorded: 3/21/2008 Area $\left(\mathrm{m}^{2}\right)$ : 26,125

Setting: Bank of seasonal drainage in open savanna, light groundcover

Soil: Consolidated sandy clay loam

FS (sample): 2003-2007, 2009 (s); 2024 (p)

Ceramic component(s): Atlantic Era

Lithic component(s): Unknown

Groundstone: Hachette, Handstone

Metallurgy: Nail (Hf), Iron tool/ornament, Slag

Other artifacts: Glass bead, Netweight, Pipe, Whorl

Surface feature(s): Stone circle

Primary function(s): Settlement

Notes: The Atlantic Era component appears to overlie an cLSA component eroding from the downward western slope of the site

\section{Survey ID: FAL-1005}

Coordinates: $13^{\circ} 58^{\prime} 54.96 " \mathrm{~N}, 12^{\circ} 11^{\prime} 43.96 " \mathrm{~W}$

Date recorded: 3/22/2008 Area $\left(\mathrm{m}^{2}\right): 13,160$

Setting: Low rise in open savanna, light groundcover Soil: Exposed laterite subsoil

FS (sample): 2013-2015 (s)

Ceramic component(s): None

Lithic component(s): MSA/LSA

Primary function(s): Unknown

Notes:

\section{Survey ID: FAL-1006-01}

Coordinates: $13^{\circ} 58^{\prime} 48.00^{\prime \prime} \mathrm{N}, 12^{\circ} 12^{\prime} 04.18^{\prime \prime} \mathrm{W}$

Date recorded: 3/22/2008 Area $\left(\mathrm{m}^{2}\right): 4144$

Setting: Bank of seasonal drainage in open savanna, modest groundcover

Soil: Consolidated clay loam with modest laterite FS (sample): 2016 (s); 2017 (p)

Ceramic component(s): Iron Age

Lithic component(s): Unknown

Groundstone: Handstone

Primary function(s): Settlement

Notes: The pottery assemblage appears transitional between cLSA (fine quartz/feldspar inclusions in the paste) and Iron Age (red slip and twisted cord roulette decoration)

\section{Survey ID: FAL-1006-02}

Coordinates: $13^{\circ} 58^{\prime} 43.46 " \mathrm{~N}, 12^{\circ} 12^{\prime} 06.69 " \mathrm{~W}$

Date recorded: 3/22/2008 Area $\left(\mathrm{m}^{2}\right): 3587$

Setting: Bank of seasonal drainage in open savanna, modest groundcover

Soil: Consolidated clay loam with light laterite gravel FS (sample): 2020 (s); 2019 (p)

Ceramic component(s): cLSA, Iron Age

Lithic component(s): MSA/LSA

Groundstone: Handstone, Bored stone

Primary function(s): Unknown

Notes: Initially recorded as FAL-1008 
Survey ID: FAL-1007

Coordinates: $13^{\circ} 58^{\prime} 45.56^{\prime \prime} \mathrm{N}, 12^{\circ} 12^{\prime} 20.92^{\prime \prime} \mathrm{W}$

Date recorded: 3/22/2008 Area $\left(\mathrm{m}^{2}\right): 743$

Setting: Gentle slope in riparian savanna, light groundcover

Soil: Semi-consolidated sandy clay loam

FS (sample): 2018 (s)

Ceramic component(s): None

Lithic component(s): MSA/LSA

Primary function(s): Unknown

Notes:

\section{Survey ID: FAL-1008}

Coordinates: $13^{\circ} 54^{\prime} 05.19^{\prime \prime} \mathrm{N}, 12^{\circ} 11^{\prime} 52.29^{\prime \prime} \mathrm{W}$

Date recorded: 4/17/2008 Area $\left(\mathrm{m}^{2}\right): 749$

Setting: Low ridge in wooded savanna, light groundcover

Soil: Consolidated sandy clay loam with laterite gravel

FS (sample): Not collected

Ceramic component(s): Iron Age

Lithic component(s): Unknown

Surface feature(s): Stone circle

Primary function(s): Settlement

Notes: A circular stone architectural foundation and associated scatter of Iron Age pottery sherds; initially recorded as FAL-IO-1067

\section{Survey ID: FAL-1009}

Coordinates: $13^{\circ} 58^{\prime} 40.07^{\prime \prime} \mathrm{N}, 12^{\circ} 12^{\prime} 20.68^{\prime \prime} \mathrm{W}$

Date recorded: 3/22/2008 Area $\left(\mathrm{m}^{2}\right): 782$

Setting: Gentle slope in savanna, light groundcover

Soil: Consolidated clay loam with laterite gravel

FS (sample): 2012 (s)

Ceramic component(s): None

Lithic component(s): MSA/LSA

Primary function(s): Unknown

Notes:

\section{Survey ID: FAL-1010}

Coordinates: $13^{\circ} 58 ' 35.24 " \mathrm{~N}, 12^{\circ} 12^{\prime} 16.96^{\prime \prime} \mathrm{W}$

Date recorded: 3/22/2008 Area $\left(\mathrm{m}^{2}\right): 1032$

Setting: Steep embankment in open savanna, no groundcover

Soil: Consolidated clay loam

FS (sample): 2022 (s)

Ceramic component(s): Atlantic Era

Lithic component(s): Unknown

Groundstone: Handstone

Surface feature(s): Collapsed daub

Primary function(s): Unknown

Notes:
Survey ID: FAL-1011

Coordinates: $13^{\circ} 55^{\prime} 57.52^{\prime \prime} \mathrm{N}, 12^{\circ} 11^{\prime} 55.69 " \mathrm{~W}$

Date recorded: 3/24/2008 Area $\left(\mathrm{m}^{2}\right)$ : 19,749

Setting: Gentle slope in open savanna, dense groundcover

Soil: Semi-consolidated sandy clay loam

FS (sample): 2025-2026 (s); 2027 (p)

Ceramic component(s): Atlantic Era

Lithic component(s): MSA/LSA

Surface feature(s): Collapsed daub

Primary function(s): Settlement

Notes:

\section{Survey ID: FAL-1012}

Coordinates: $13^{\circ} 56^{\prime} 07.36^{\prime \prime} \mathrm{N}, 12^{\circ} 11^{\prime} 56.47^{\prime \prime} \mathrm{W}$

Date recorded: 3/24/2008 Area $\left(\mathrm{m}^{2}\right): 8227$

Setting: Gentle slope in open savanna, no groundcover

Soil: Semi-consolidated sandy clay loam

FS (sample): 2028 (s); 2029 (p)

Ceramic component(s): Atlantic Era

Lithic component(s): MSA/LSA

Primary function(s): Unknown

Notes: A possible continuation of FAL-1011 from the south

Survey ID: FAL-1013

Coordinates: $13^{\circ} 56^{\prime} 10.41^{\prime \prime} \mathrm{N}, 12^{\circ} 11 ' 34.49^{\prime \prime} \mathrm{W}$

Date recorded: 3/24/2008 Area $\left(\mathrm{m}^{2}\right): 5108$

Setting: Level area in open savanna, light groundcover

Soil: Consolidated clay loam

FS (sample): 2030 (s)

Ceramic component(s): None

Lithic component(s): MSA/LSA

Primary function(s): Unknown

Notes:

\section{Survey ID: FAL-1014}

Coordinates: $13^{\circ} 56^{\prime} 17.60^{\prime \prime} \mathrm{N}, 12^{\circ} 11^{\prime} 23.21$ " W

Date recorded: 3/24/2008 Area $\left(\mathrm{m}^{2}\right)$ : 10,155

Setting: Low ridge in wooded savanna, light groundcover

Soil: Semi-consolidated clay loam with river gravel FS (sample): 2031 (s); 2032 (p)

Ceramic component(s): None

Lithic component(s): MSA/LSA

Primary function(s): Unknown

Notes: Possibly Sansandé Est (Camara 2000)

\section{Survey ID: FAL-1015}

Coordinates: $13^{\circ} 56^{\prime} 12.94 " \mathrm{~N}, 12^{\circ} 11^{\prime} 40.71^{\prime \prime} \mathrm{W}$

Date recorded: 3/24/2008 Area $\left(\mathrm{m}^{2}\right): 1183$

Setting: Gentle slope in wooded savanna, light groundcover

Soil: Consolidated clay loam with modest river gravel FS (sample): 2033 (s)

Ceramic component(s): cLSA

Lithic component(s): MSA/LSA

Groundstone: Handstone

Primary function(s): 
Survey ID: FAL-1016

Coordinates: $13^{\circ} 56^{\prime} 07.29^{\prime \prime} \mathrm{N}, 12^{\circ} 12^{\prime} 20.82^{\prime \prime} \mathrm{W}$

Date recorded: 3/24/2008 Area $\left(\mathrm{m}^{2}\right): 1200$

Setting: Bluff top in riparian savanna, modest groundcover

Soil: Consolidated clay loam

FS (sample): 2034 (s); 2035 (p)

Ceramic component(s): Iron Age

Lithic component(s): None

Surface feature(s): Collapsed daub

Primary function(s): Unknown

Notes: The pottery assemblage appears transitional between cLSA (fine quartz/feldspar inclusions in the paste) and Iron Age (twisted cord and cord-wrapped stick roulette decoration)

\section{Survey ID: FAL-1017}

Coordinates: $13^{\circ} 56^{\prime} 18.91 " \mathrm{~N}, 12^{\circ} 11^{\prime} 53.12^{\prime \prime} \mathrm{W}$

Date recorded: 3/24/2008 Area $\left(\mathrm{m}^{2}\right)$ : 53,936

Setting: Eroded embankment in wooded savanna, no groundcover

Soil: Consolidated clay loam with dense laterite gravel

FS (sample): 2037 (p)

Ceramic component(s): Atlantic Era, cLSA

Lithic component(s): MSA/LSA

Groundstone: Handstone

Surface feature(s): Stone square

Primary function(s): Settlement

Notes: Features include two to three square stone features and one complete pottery vessel eroding from a steep embankment

\section{Survey ID: FAL-1018}

Coordinates: $13^{\circ} 56^{\prime} 17.72^{\prime \prime} \mathrm{N}, 12^{\circ} 11$ '38.03" W

Date recorded: 3/24/2008 Area $\left(\mathrm{m}^{2}\right): 2143$

Setting: Level area in wooded savanna, light groundcover

Soil: Consolidated sandy clay loam

FS (sample): 2038 (s); 2039 (p)

Ceramic component(s): None

Lithic component(s): MSA/LSA

Primary function(s): Unknown

Notes:

\section{Survey ID: FAL-1019}

Coordinates: $13^{\circ} 56^{\prime} 36.59 " \mathrm{~N}, 12^{\circ} 12^{\prime} 21.95^{\prime \prime} \mathrm{W}$

Date recorded: 4/14/2008 Area $\left(\mathrm{m}^{2}\right): 2229$

Setting: Eroded embankment in open savanna, light groundcover

Soil: Semi-consolidated sandy clay loam with dense laterite gravel

FS (sample): 2148 (s)

Ceramic component(s): None

Lithic component(s): MSA/LSA

Groundstone: Handstone

Primary function(s): Unknown

Notes:
Survey ID: FAL-1020

Coordinates: $13^{\circ} 55^{\prime} 07.47^{\prime \prime} \mathrm{N}, 12^{\circ} 12^{\prime} 57.58^{\prime \prime} \mathrm{W}$

Date recorded: 3/25/2008 Area $\left(\mathrm{m}^{2}\right): 12,667$

Setting: Low ridge in open savanna, light

Soil: Consolidated sandy clay loam with modest laterite gravel

FS (sample): 2040 (s); 2041 (p)

Ceramic component(s): cLSA

Lithic component(s): Late Acheulean, MSA/LSA

Groundstone: Hachette

Primary function(s): Unknown

Notes: Located amid the alluvial terraces defined by Camara (2000) as Sansandé Sud

\section{Survey ID: FAL-1021}

Coordinates: $13^{\circ} 55^{\prime} 04.76^{\prime \prime} \mathrm{N}, 12^{\circ} 13^{\prime} 15.43^{\prime \prime} \mathrm{W}$

Date recorded: 3/25/2008 Area $\left(\mathrm{m}^{2}\right): 17,790$

Setting: Gentle slope in wooded savanna, no groundcover

Soil: Consolidated clay loam

FS (sample): 2042 (s); 2043-2044 (p)

Ceramic component(s): cLSA, Iron Age

Lithic component(s): MSA/LSA

Groundstone: Basin, Handstone

Metallurgy: Slag

Other artifacts: Glass bead

Surface feature(s): Stone circle

Primary function(s): Settlement

Notes: The cLSA component is concentrated in the eastern half of the site, discrete from several Iron Age stone features in the western half of the site

\section{Survey ID: FAL-1022}

Coordinates: $13^{\circ} 54 ' 59.78 " \mathrm{~N}, 12^{\circ} 13^{\prime} 33.44^{\prime \prime} \mathrm{W}$

Date recorded: 3/25/2008 Area $\left(\mathrm{m}^{2}\right): 4253$

Setting: Bank of seasonal drainage in wooded savanna, no groundcover

Soil: Consolidated clay loam

FS (sample): 2045 (s); 2046 (p)

Ceramic component(s): Atlantic Era

Lithic component(s): None

Groundstone: Handstone

Metallurgy: Iron tool/ornament

Other artifacts: Pipe

Surface feature(s): Stone alignment

Primary function(s): Settlement

Notes: Features include two possible rectilinear stone features 
Survey ID: FAL-1023

Coordinates: $13^{\circ} 54^{\prime} 50.24 " \mathrm{~N}, 12^{\circ} 13^{\prime} 51.11^{\prime \prime} \mathrm{W}$

Date recorded: $3 / 25 / 2008 \quad$ Area $\left(\mathrm{m}^{2}\right): 12,373$

Setting: Bank of seasonal drainage in wooded savanna, no groundcover

Soil: Consolidated sandy clay loam with modest river gravel and exposed bedrock

FS (sample): 2047 (s)

Ceramic component(s): None

Lithic component(s): MSA/LSA

Primary function(s): Unknown

Notes:

\section{Survey ID: FAL-1024}

Coordinates: $13^{\circ} 54^{\prime} 53.76^{\prime \prime} \mathrm{N}, 12^{\circ} 13^{\prime} 39.56^{\prime \prime} \mathrm{W}$

Date recorded: 3/25/2008 Area $\left(\mathrm{m}^{2}\right): 5809$

Setting: Low ridge in wooded savanna, light groundcover

Soil: Consolidated clay loam with laterite gravel

FS (sample): 2048 (s)

Ceramic component(s): cLSA

Lithic component(s): MSA/LSA

Groundstone: Hachette

Primary function(s): Unknown

Notes:

\section{Survey ID: FAL-1025}

Coordinates: $13^{\circ} 54 ' 55.86^{\prime \prime} \mathrm{N}, 12^{\circ} 13^{\prime 2} 28.28^{\prime \prime} \mathrm{W}$

Date recorded: 3/25/2008 Area $\left(\mathrm{m}^{2}\right): 2143$

Setting: Low ridge in wooded savanna, light groundcover

Soil: Consolidated clay loam with laterite gravel

FS (sample): 2049 (s)

Ceramic component(s): Atlantic Era, cLSA

Lithic component(s): MSA/LSA

Groundstone: Hachette, Handstone

Primary function(s): Unknown

Notes: The Atlantic Era component appears to be extremely sparse

\section{Survey ID: FAL-1026}

Coordinates: $13^{\circ} 544^{\prime} 42.49^{\prime \prime} \mathrm{N}, 12^{\circ} 13^{\prime} 46.14^{\prime \prime} \mathrm{W}$

Date recorded: $3 / 25 / 2008$ Area $\left(\mathrm{m}^{2}\right)$ : 23,040

Setting: Bank of seasonal drainage in riparian savanna, light groundcover

Soil: Consolidated sandy clay loam

FS (sample): 2050 (s); 2051 (p)

Ceramic component(s): Atlantic Era, cLSA

Lithic component(s): MSA/LSA

Groundstone: Basin, Handstone

Surface feature(s): Stone circle, Stone square

Primary function(s): Settlement

Notes: Features include ten circular and rectilinear stone architectural foundations
Survey ID: FAL-1027

Coordinates: $13^{\circ} 54^{\prime} 55.42^{\prime \prime} \mathrm{N}, 12^{\circ} 12^{\prime} 28.77^{\prime \prime} \mathrm{W}$

Date recorded: 3/25/2008 Area $\left(\mathrm{m}^{2}\right): 527$

Setting: Bluff in riparian savanna, light groundcover

Soil: Semi-consolidated sandy loam

FS (sample): 2052 (s); 2053 (p)

Ceramic component(s): cLSA

Lithic component(s): MSA/LSA

Primary function(s): Unknown

Notes:

Survey ID: FAL-1028

Coordinates: $13^{\circ} 56^{\prime} 27.06^{\prime \prime} \mathrm{N}, 12^{\circ} 14^{\prime} 14.71^{\prime \prime} \mathrm{W}$

Date recorded: 3/28/2008 Area $\left(\mathrm{m}^{2}\right): 1529$

Setting: Low ridge in wooded savanna, modest groundcover

Soil: Eroded laterite and bedrock

FS (sample): 2054 (s); 2055 (p)

Ceramic component(s): None

Lithic component(s): MSA/LSA

Primary function(s):

Notes: A possible isolated occurrence

\section{Survey ID: FAL-1029}

Coordinates: $13^{\circ} 56^{\prime} 10.63^{\prime \prime} \mathrm{N}, 12^{\circ} 13^{\prime} 09.71^{\prime \prime} \mathrm{W}$

Date recorded: 3/28/2008 Area $\left(\mathrm{m}^{2}\right): 2631$

Setting: Bank of seasonal drainage in cultivated field, no groundcover

Soil: Semi-consolidated sandy clay loam

FS (sample): 2056 (s)

Ceramic component(s): cLSA

Lithic component(s): MSA/LSA

Primary function(s): Unknown

Notes:

Survey ID: FAL-1030

Coordinates: $13^{\circ} 56^{\prime} 19.65^{\prime \prime} \mathrm{N}, 12^{\circ} 12^{\prime} 53.58^{\prime \prime} \mathrm{W}$

Date recorded: $3 / 28 / 2008 \quad$ Area $\left(\mathrm{m}^{2}\right): 7386$

Setting: Bank of seasonal drainage in open savanna, modest groundcover

Soil: Consolidated sandy loam with laterite gravel

FS (sample): 2057 (s)

Ceramic component(s): Atlantic Era, cLSA

Lithic component(s): MSA/LSA

Primary function(s): Unknown

Notes:

Survey ID: FAL-1031

Coordinates: $13^{\circ} 56^{\prime} 27.31 " \mathrm{~N}, 12^{\circ} 12^{\prime} 41.95^{\prime \prime} \mathrm{W}$

Date recorded: 3/28/2008 Area $\left(\mathrm{m}^{2}\right): 6119$

Setting: Gentle slope in wooded savanna, no groundcover

Soil: Consolidated clay loam with dense laterite gravel

FS (sample): 2058 (s); 2059 (p)

Ceramic component(s): Atlantic Era

Lithic component(s): MSA/LSA

Groundstone: Hachette

Primary function(s): Unknown

Notes: 
Survey ID: FAL-1032

Coordinates: $13^{\circ} 56^{\prime} 20.33^{\prime \prime} \mathrm{N}, 12^{\circ} 12^{\prime} 34.64^{\prime \prime} \mathrm{W}$

Date recorded: 3/28/2008 Area $\left(\mathrm{m}^{2}\right)$ : 6692

Setting: Low embankment in wooded savanna, no groundcover

Soil: Consolidated sandy clay loam with modest laterite gravel

FS (sample): 2060 (s); 2061 (p)

Ceramic component(s): cLSA

Lithic component(s): MSA/LSA

Groundstone: Handstone

Primary function(s): Unknown

Notes:

\section{Survey ID: FAL-1033}

Coordinates: $13^{\circ} 56^{\prime} 18.17^{\prime \prime} \mathrm{N}, 12^{\circ} 12^{\prime} 27.12^{\prime \prime} \mathrm{W}$

Date recorded: 3/28/2008 Area $\left(\mathrm{m}^{2}\right): 2211$

Setting: Bluff in riparian savanna, light groundcover

Soil: Semi-consolidated clay loam

FS (sample): 2062 (p)

Ceramic component(s): Iron Age

Lithic component(s): Unknown

Groundstone: Handstone

Primary function(s): Unknown

Notes: Small scatters of pottery sherds may originate from one or two reconstructable vessels

\section{Survey ID: FAL-1034}

Coordinates: $13^{\circ} 56^{\prime} 00.57 " \mathrm{~N}, 12^{\circ} 12^{\prime} 37.70^{\prime \prime} \mathrm{W}$

Date recorded: 4/2/2008 Area $\left(\mathrm{m}^{2}\right): 1118$

Setting: Low ridge in open savanna, light groundcover

Soil: Consolidated clay loam with dense laterite gravel

FS (sample): 2085 (s)

Ceramic component(s): None

Lithic component(s): MSA/LSA

Primary function(s): Unknown

Notes:

\section{Survey ID: FAL-1035}

Coordinates: $13^{\circ} 54$ '34.43" N, $12^{\circ} 11^{\prime} 26.41^{\prime \prime} \mathrm{W}$

Date recorded: 4/8/2008 Area $\left(\mathrm{m}^{2}\right): 1083$

Setting: Low rise in open savanna, no groundcover

Soil: Consolidated sandy clay loam

FS (sample): 1784 (s)

Ceramic component(s): Iron Age

Lithic component(s): Unknown

Primary function(s): Unknown

Notes: Initially recorded as FAL-1000, Area Q
Survey ID: FAL-1036

Coordinates: $13^{\circ} 52^{\prime} 42.93^{\prime \prime} \mathrm{N}, 12^{\circ} 10^{\prime} 02.56^{\prime \prime} \mathrm{W}$

Date recorded: 4/1/2008 Area $\left(\mathrm{m}^{2}\right)$ : 8755

Setting: Low embankment in riparian savanna, light groundcover

Soil: Consolidated sandy loam with dense laterite gravel

FS (sample): 2063 (s); 2064 (p)

Ceramic component(s): Atlantic Era, cLSA

Lithic component(s): MSA/LSA

Groundstone: Basin, Handstone

Metallurgy: Nail (Ct), Iron tool/ornament

Primary function(s): Unknown

Notes:

\section{Survey ID: FAL-1037}

Coordinates: $13^{\circ} 52^{\prime} 40.84^{\prime \prime} \mathrm{N}, 12^{\circ} 10^{\prime} 10.81^{\prime \prime} \mathrm{W}$

Date recorded: 6/22/2007 Area $\left(\mathrm{m}^{2}\right): 1969$

Setting: Gentle slope in open savanna, no groundcover

Soil: Consolidated clay loam with dense laterite gravel

FS (sample): 2065 (s); 2066 (p)

Ceramic component(s): Atlantic Era

Lithic component(s): MSA/LSA

Groundstone: Other

Primary function(s): Unknown

Notes: A few Atlantic Era pottery sherds appear intrusive from the adjacent site of Karé

\section{Survey ID: FAL-1038-01 (Karé)}

Coordinates: $13^{\circ} 52^{\prime} 53.70^{\prime \prime} \mathrm{N}, 1^{\circ} 10^{\prime} 10.37 " \mathrm{~W}$

Date recorded: $6 / 22 / 2007 \quad$ Area $\left(\mathrm{m}^{2}\right): 42,880$

Setting: Gentle slope in wooded savanna, light groundcover

Soil: Consolidated sandy clay loam

FS (sample): 2067 (s); 2068 (p)

Ceramic component(s): Atlantic Era, cLSA

Lithic component(s): MSA/LSA

Groundstone: Basin, Hachette, Handstone

Metallurgy: Nail (Hf)

Other artifacts: Netweight, Whorl

Surface feature(s): Collapsed daub, Stone circle, Stone square

Primary function(s): Settlement

Notes: The Atlantic Era component appears to overlie an cLSA component eroding from the downward eastern slope of the site; previously noted by Ravisé (1975) and Opper and Opper (1990:38) 
Survey ID: FAL-1038-02 (Karé)

Coordinates: $13^{\circ} 53^{\prime} 06.19^{\prime \prime} \mathrm{N}, 12^{\circ} 10^{\prime} 13.84^{\prime \prime} \mathrm{W}$

Date recorded: 6/22/2007 Area $\left(\mathrm{m}^{2}\right)$ : 61,485

Setting: Gentle slope in wooded savanna, light groundcover

Soil: Consolidated sandy clay loam

FS (sample): 2071 (s); 2072 (p)

Ceramic component(s): Atlantic Era

Lithic component(s): MSA/LSA

Groundstone: Basin, Handstone

Metallurgy: Nail (Ct, Hf), Iron tool/ornament,

Other artifacts: Coin, Glass bead

Surface feature(s): Collapsed daub, Stone circle, Stone square

Primary function(s): Settlement

Notes: Initially recorded as FAL-1040

\section{Survey ID: FAL-1039}

Coordinates: $13^{\circ} 52^{\prime} 47.79^{\prime \prime} \mathrm{N}, 12^{\circ} 09^{\prime} 55.52 " \mathrm{~W}$

Date recorded: 4/1/2008 Area $\left(\mathrm{m}^{2}\right): 6172$

Setting: Level area in riparian savanna, light groundcover

Soil: Semi-consolidated sandy loam with modest laterite gravel

FS (sample): 2069 (s); 2070 (p)

Ceramic component(s): cLSA

Lithic component(s): MSA/LSA

Groundstone: Handstone

Other artifacts: Netweight

Primary function(s):

Notes:

\section{Survey ID: FAL-1040}

Coordinates: $13^{\circ} 52^{\prime} 35.59 " \mathrm{~N}, 12^{\circ} 10^{\prime} 15.85^{\prime \prime} \mathrm{W}$

Date recorded: 4/1/2008 Area $\left(\mathrm{m}^{2}\right): 2087$

Setting: Low rise in wooded savanna, no groundcover

Soil: Consolidated sandy clay loam

FS (sample): Not sampled

Ceramic component(s): None

Lithic component(s): MSA/LSA

Primary function(s): Unknown

Notes: A relatively dense scatter of chipped stone debitage and microlithic/blade tools; initially recorded as FAL-IO-014

\section{Survey ID: FAL-1041}

Coordinates: $13^{\circ} 53^{\prime} 04.45^{\prime \prime} \mathrm{N}, 12^{\circ} 10^{\prime} 02.96 " \mathrm{~W}$

Date recorded: $4 / 1 / 2008 \quad$ Area $\left(\mathrm{m}^{2}\right): 11,055$

Setting: Bluff in riparian savanna, light groundcover

Soil: Consolidated sandy loam with laterite gravel

FS (sample): 2073 (s); 2074-2075 (p)

Ceramic component(s): cLSA

Lithic component(s): MSA/LSA

Groundstone: Hachette, Handstone

Primary function(s): Unknown

Notes:

\section{Survey ID: FAL-1042}

Coordinates: $13^{\circ} 57 ' 35.48^{\prime \prime} \mathrm{N}, 12^{\circ} 12^{\prime} 48.37^{\prime \prime} \mathrm{W}$

Date recorded: 4/2/2008 Area $\left(\mathrm{m}^{2}\right): 1445$

Setting: Blufftop in riparian savanna, no groundcover Soil: Consolidated clay loam with modest river gravel FS (sample): 2077 (s)

Ceramic component(s): None

Lithic component(s): MSA/LSA

Primary function(s): Unknown

Notes: A possible hunting stand

\section{Survey ID: FAL-1043-01}

Coordinates: $13^{\circ} 57^{\prime} 40.76^{\prime \prime} \mathrm{N}, 12^{\circ} 12^{\prime} 48.80 " \mathrm{~W}$

Date recorded: $4 / 2 / 2008 \quad$ Area $\left(\mathrm{m}^{2}\right): 4371$

Setting: Bluff top in riparian savanna, no groundcover Soil: Semi-consolidated clay loam

FS (sample): 2078 (p)

Ceramic component(s): Atlantic Era

Lithic component(s): MSA/LSA

Groundstone: Basin

Surface feature(s): Stone alignment

Primary function(s): Settlement

Notes: A perforated disc was found on the surface

Survey ID: FAL-1043-02

Coordinates: $13^{\circ} 57^{\prime} 46.36^{\prime \prime} \mathrm{N}, 12^{\circ} 12^{\prime} 47.25^{\prime \prime} \mathrm{W}$

Date recorded: 4/2/2008 Area $\left(\mathrm{m}^{2}\right): 2687$

Setting: Bluff top in riparian savanna, no groundcover Soil: Semi-consolidated clay loam

FS (sample): 2079 (p)

Ceramic component(s): Atlantic Era

Lithic component(s): MSA/LSA

Groundstone: Basin

Surface feature(s): Stone alignment

Primary function(s): Unknown

Notes:

Survey ID: FAL-1044-01

Coordinates: $13^{\circ} 58^{\prime} 07.26^{\prime \prime} \mathrm{N}, 12^{\circ} 12^{\prime} 44.75^{\prime \prime} \mathrm{W}$

Date recorded: 4/2/2008 Area $\left(\mathrm{m}^{2}\right): 6980$

Setting: Level area in wooded savanna, no groundcover

Soil: Semi-consolidated sandy clay loam with modest laterite gravel

FS (sample): 2081 (s); 2082 (p)

Ceramic component(s): cLSA, Iron Age

Lithic component(s): MSA/LSA

Groundstone: Handstone

Primary function(s): Unknown

Notes:

Survey ID: FAL-1044-02

Coordinates: $13^{\circ} 58^{\prime} 16.29 " \mathrm{~N}, 12^{\circ} 12^{\prime} 45.12^{\prime \prime} \mathrm{W}$

Date recorded: $4 / 20 / 2008 \quad$ Area $\left(\mathrm{m}^{2}\right)$ : 25,638

Setting: Level area in savanna, no groundcover

Soil: Semi-consolidated sandy clay loam with modest laterite gravel

FS (sample): 2206 (s); 2205 (p)

Ceramic component(s): cLSA

Lithic component(s): MSA/LSA

Primary function(s): Unknown

Notes: Initially recorded as FAL-1131 
Survey ID: FAL-1045 (Tomboura 1)

Coordinates: $13^{\circ} 57^{\prime} 33.13^{\prime \prime} \mathrm{N}, 12^{\circ} 12^{\prime} 53.76^{\prime \prime} \mathrm{W}$

Date recorded: 6/22/2007 Area $\left(\mathrm{m}^{2}\right): 3070$

Setting: Gentle slope in wooded savanna, no groundcover

Soil: Consolidated sandy clay loam with modest gravel from exposed bedrock

FS (sample): 2083 (s)

Ceramic component(s): None

Lithic component(s): MSA/LSA

Primary function(s): Unknown

Notes:

\section{Survey ID: FAL-1046}

Coordinates: $13^{\circ} 51^{\prime} 40.85^{\prime \prime} \mathrm{N}, 12^{\circ} 09^{\prime} 11.9^{\prime \prime} \mathrm{W}$

Date recorded: $4 / 6 / 2008 \quad$ Area $\left(\mathrm{m}^{2}\right)$ : 10,298

Setting: Gentle slope in wooded savanna, dense groundcover

Soil: Consolidated sandy clay loam

FS (sample): 2088 (p)

Ceramic component(s): Atlantic Era

Lithic component(s): None

Groundstone: Basin, Handstone

Other artifacts: Pipe

Surface feature(s): Collapsed daub

Primary function(s): Settlement

Notes: Features include several patches of collapsed daub or plaster from collapsed structures

\section{Survey ID: FAL-1047}

Coordinates: $13^{\circ} 51^{\prime} 40.57^{\prime \prime} \mathrm{N}, 12^{\circ} 08^{\prime} 42.34^{\prime \prime} \mathrm{W}$

Date recorded: 4/6/2008 Area $\left(\mathrm{m}^{2}\right): 2985$

Setting: Gentle slope in wooded savanna, dense groundcover

Soil: Semi-consolidated sandy clay loam

FS (sample): 2089 (p)

Ceramic component(s): Atlantic Era

Lithic component(s): None

Groundstone: Handstone

Surface feature(s): Collapsed daub

Primary function(s):

Notes:

\section{Survey ID: FAL-1048}

Coordinates: $13^{\circ} 51^{\prime} 44.15^{\prime \prime} \mathrm{N}, 12^{\circ} 08^{\prime} 54.08^{\prime \prime} \mathrm{W}$

Date recorded: 4/6/2008 Area $\left(\mathrm{m}^{2}\right): 617$

Setting: Level area in wooded savanna, modest groundcover

Soil: Consolidated sandy clay loam

FS (sample): 2091 (p)

Ceramic component(s): Atlantic Era

Lithic component(s): None

Groundstone: Handstone

Surface feature(s): Collapsed daub

Primary function(s): Settlement

Notes: Features include two patches of daub fragments from possible collapsed structures
Survey ID: FAL-1049

Coordinates: $13^{\circ} 51^{\prime} 47.57^{\prime \prime} \mathrm{N}, 12^{\circ} 09^{\prime} 20.06^{\prime \prime} \mathrm{W}$

Date recorded: $4 / 6 / 2008 \quad$ Area $\left(\mathrm{m}^{2}\right): 11,267$

Setting: Gentle slope in wooded savanna, light groundcover

Soil: Unconsolidated sandy clay loam

FS (sample): 2095 (s); 2094 (p)

Ceramic component(s): Atlantic Era

Lithic component(s): MSA/LSA

Groundstone: Basin, Handstone

Primary function(s): Unknown

Notes:

Survey ID: FAL-1050 (Tontèko)

Coordinates: $13^{\circ} 51^{\prime} 45.84^{\prime \prime} \mathrm{N}, 1^{\circ}{ }^{\circ} 09^{\prime} 29.32^{\prime \prime} \mathrm{W}$

Date recorded: $4 / 6 / 2008 \quad$ Area $\left(\mathrm{m}^{2}\right): 33448$

Setting: Bluff in riparian savanna, light groundcover Soil: Consolidated clay loam with laterite gravel FS (sample): 2096 (s); 2097 (p)

Ceramic component(s): Atlantic Era

Lithic component(s): MSA/LSA

Groundstone: Basin, Hachette, Handstone

Metallurgy: Iron tool/ornament, Slag

Other artifacts: Netweight, Pipe

Surface feature(s): Stone alignment

Primary function(s): Settlement

Notes:

\section{Survey ID: FAL-1051}

Coordinates: $13^{\circ} 51$ '35.06" N, 1209'35.03" W

Date recorded: 4/6/2008 Area $\left(\mathrm{m}^{2}\right): 4554$

Setting: Bluff in riparian savanna, light groundcover Soil: Consolidated sandy clay loam with laterite gravel

FS (sample): 2098 (s)

Ceramic component(s): Atlantic Era, Iron Age

Lithic component(s): None

Primary function(s): Settlement

Notes: A number of large cobbles may derive from eroded rectilinear stone architectural features

Survey ID: FAL-1052

Coordinates: $13^{\circ} 52^{\prime} 02.56^{\prime \prime} \mathrm{N}, 12^{\circ} 09^{\prime} 24.48^{\prime \prime} \mathrm{W}$

Date recorded: 4/7/2008 Area $\left(\mathrm{m}^{2}\right): 7403$

Setting: Bluff top in riparian savanna, modest groundcover

Soil: Consolidated sandy clay with laterite gravel FS (sample): 2099 (p)

Ceramic component(s): Atlantic Era, cLSA

Lithic component(s): MSA/LSA

Other artifacts: Netweight

Primary function(s): Unknown

Notes: The cLSA component is concentrated in northern half of site 
Survey ID: FAL-1053-01

Coordinates: $13^{\circ} 52^{\prime} 00.41^{\prime \prime} \mathrm{N}, 12^{\circ} 08^{\prime} 53.26^{\prime \prime} \mathrm{W}$

Date recorded: $4 / 7 / 2008 \quad$ Area $\left(\mathrm{m}^{2}\right): 7776$

Setting: Level area in wooded savanna, modest groundcover

Soil: Consolidated sandy clay loam with modest laterite gravel

FS (sample): 2102 (p)

Ceramic component(s): Atlantic Era

Lithic component(s): None

Groundstone: Handstone

Metallurgy: Nail (Hf)

Primary function(s): Unknown

Notes:

\section{Survey ID: FAL-1053-02}

Coordinates: $13^{\circ} 52^{\prime} 06.07^{\prime \prime} \mathrm{N}, 12^{\circ} 08^{\prime} 54.15^{\prime \prime} \mathrm{W}$

Date recorded: 4/7/2008 Area $\left(\mathrm{m}^{2}\right): 2100$

Setting: Low ridge in wooded savanna, modest groundcover

Soil: Consolidated sandy clay loam with modest laterite gravel

FS (sample): 2104 (p)

Ceramic component(s): Atlantic Era

Lithic component(s): None

Groundstone: Handstone

Surface feature(s): Collapsed daub

Primary function(s): Unknown

Notes: Initially recorded as FAL-1055

\section{Survey ID: FAL-1054}

Coordinates: $13^{\circ} 51^{\prime} 53.59 " \mathrm{~N}, 1^{\circ} 09^{\prime} 00.66^{\prime \prime} \mathrm{W}$

Date recorded: 4/7/2008 Area $\left(\mathrm{m}^{2}\right): 3185$

Setting: Level area in open savanna, light groundcover

Soil: Consolidated clay loam with dense laterite gravel

FS (sample): 2103 (p)

Ceramic component(s): Atlantic Era

Lithic component(s): None

Groundstone: Handstone

Primary function(s): Unknown

Notes:

\section{Survey ID: FAL-1055-01}

Coordinates: $13^{\circ} 55^{\prime} 16.79^{\prime \prime} \mathrm{N}, 12^{\circ} 13^{\prime} 33.78^{\prime \prime} \mathrm{W}$

Date recorded: 4/16/2008 Area $\left(\mathrm{m}^{2}\right): 2465$

Setting: Bank of seasonal drainage in open savanna, light groundcover

Soil: Consolidated clay loam with laterite gravel

FS (sample): Not sampled

Ceramic component(s): cLSA

Lithic component(s): MSA/LSA

Groundstone: Basin, Handstone

Primary function(s):

Notes: Initially recorded as FAL-IO-061
Survey ID: FAL-1055-02

Coordinates: $13^{\circ} 55^{\prime} 17.70^{\prime \prime} \mathrm{N}, 12^{\circ} 13^{\prime} 39.42^{\prime \prime} \mathrm{W}$

Date recorded: 4/16/2008 Area $\left(\mathrm{m}^{2}\right): 1668$

Setting: Bank of seasonal drainage in open savanna, light groundcover

Soil: Consolidated clay loam with modest laterite FS (sample): Not sampled

Ceramic component(s): $\quad$ cLSA

Lithic component(s): MSA/LSA

Primary function(s): Unknown

Notes: Initially recorded as FAL-IO-062

\section{Survey ID: FAL-1056 (Toranga)}

Coordinates: $13^{\circ} 52^{\prime} 43.23^{\prime \prime} \mathrm{N}, 12^{\circ} 09^{\prime} 09.25^{\prime \prime} \mathrm{W}$

Date recorded: 4/8/2008 Area $\left(\mathrm{m}^{2}\right): 104,027$

Setting: Low mounds in wooded savanna, modest groundcover

Soil: Consolidated sandy clay loam with laterite gravel

FS (sample): 2108 (p)

Ceramic component(s): $\quad$ Atlantic Era

Lithic component(s): Unknown

Groundstone: Basin, Handstone

Metallurgy: Nail (Ct), Iron tool/ornament, Slag

Other artifacts: Glass bead, Pipe

Surface feature(s): Collapsed daub, Stone circle,

Stone square, Pyrotechnic feature

Primary function(s): Settlement

Notes: Features include both rectilinear and circular stone architectural foundations

Survey ID: FAL-1057

Coordinates: $13^{\circ} 52^{\prime} 52.33^{\prime \prime} \mathrm{N}, 12^{\circ} 08^{\prime} 59.38^{\prime \prime} \mathrm{W}$

Date recorded: 4/8/2008 Area $\left(\mathrm{m}^{2}\right): 1257$

Setting: Low ridge in open savanna, light groundcover

Soil: Semi-consolidated sandy loam with dense laterite gravel

FS (sample): 2109 (p)

Ceramic component(s): None

Lithic component(s): MSA/LSA

Groundstone: Handstone

Primary function(s): Unknown

Notes: Possibly Karé Sud (Camara 2000)

\section{Survey ID: FAL-1058}

Coordinates: $13^{\circ} 52^{\prime} 48.83^{\prime \prime} \mathrm{N}, 12^{\circ} 09^{\prime} 19.62^{\prime \prime} \mathrm{W}$

Date recorded: 4/8/2008 Area $\left(\mathrm{m}^{2}\right): 384$

Setting: Level area in wooded savanna, no groundcover

Soil: Semi-consolidated sandy clay loam

FS (sample): 2110 (p)

Ceramic component(s): Atlantic Era

Lithic component(s): MSA/LSA

Groundstone: Hachette

Metallurgy: Slag

Primary function(s):

Notes: A few Atlantic Era pottery sherds and single piece of slag appear as intrusive elements in this site with an otherwise strong LSA 


\section{Survey ID: FAL-1059}

Coordinates: $13^{\circ} 52^{\prime} 46.36^{\prime \prime} \mathrm{N}, 1^{\circ} 09^{\prime} 35.86^{\prime \prime} \mathrm{W}$

Date recorded: 4/8/2008 Area $\left(\mathrm{m}^{2}\right): 19,003$

Setting: Bluff top in open savanna, light groundcover

Soil: Semi-consolidated clay loam with modest laterite gravel

FS (sample): 2111 (s); 2112 (p)

Ceramic component(s): Atlantic Era, cLSA

Lithic component(s): MSA/LSA

Groundstone: Basin, Handstone

Metallurgy: Nail (Ct), Iron tool/ornament, Slag

Other artifacts: None

Surface feature(s): Stone circle, Stone square

Primary function(s): Settlement

Notes: Features include both rectilinear and circular stone architectural foundations

\section{Survey ID: FAL-1060}

Coordinates: $13^{\circ} 52^{\prime} 59.42^{\prime \prime} \mathrm{N}, 1^{\circ} 09^{\prime} 29.28^{\prime \prime} \mathrm{W}$

Date recorded: 4/8/2008 Area $\left(\mathrm{m}^{2}\right): 5719$

Setting: Level area in wooded savanna, light groundcover

Soil: Consolidated sandy clay loam with modest laterite gravel

FS (sample): 2113 (s); 2114 (p)

Ceramic component(s): None

Lithic component(s): MSA/LSA

Primary function(s): Unknown

Notes:

\section{Survey ID: FAL-1061}

Coordinates: $13^{\circ} 53^{\prime} 06.03^{\prime \prime} \mathrm{N}, 12^{\circ} 09^{\prime} 20.63^{\prime \prime} \mathrm{W}$

Date recorded: 4/8/2008 Area $\left(\mathrm{m}^{2}\right): 805$

Setting: Gentle slope in wooded savanna, no groundcover

Soil: Consolidated sandy clay loam with modest laterite gravel

FS (sample): 2115 (s); 2116 (p)

Ceramic component(s): Atlantic Era

Lithic component(s): MSA/LSA

Metallurgy: Slag

Primary function(s): Settlement

Notes: A number of large cobbles may derive from eroded rectilinear stone features

\section{Survey ID: FAL-1062}

Coordinates: $13^{\circ} 54^{\prime} 50.08^{\prime \prime} \mathrm{N}, 12^{\circ} 12^{\prime} 00.76^{\prime \prime} \mathrm{W}$

Date recorded: 4/9/2008 Area $\left(\mathrm{m}^{2}\right): 649$

Setting: Eroded embankment in riparian savanna, dense groundcover

Soil: Semi-consolidated sandy clay loam

FS (sample): 2119 (s); 2120 (p)

Ceramic component(s): cLSA

Lithic component(s): MSA/LSA

Groundstone: Axe, Hachette, Handstone

Primary function(s): Unknown

Notes:
Survey ID: FAL-1063

Coordinates: $13^{\circ} 54^{\prime} 44.72 " \mathrm{~N}, 12^{\circ} 11^{\prime} 58.61^{\prime \prime} \mathrm{W}$

Date recorded: 4/9/2008 Area $\left(\mathrm{m}^{2}\right): 1875$

Setting: Bluff top in riparian savanna, no groundcover

Soil: Consolidated sandy clay loam

FS (sample): 2122 (s); 2123 (p)

Ceramic component(s): Atlantic Era, Iron Age

Lithic component(s): None

Groundstone: Basin, Handstone

Metallurgy: Slag

Surface feature(s): Collapsed daub, Stone alignment

Primary function(s): Unknown

Notes: Features include seven to eight small (1 m diameter) piles of river cobbles and two patches of collapsed daub

\section{Survey ID: FAL-1064}

Coordinates: $13^{\circ} 54 ' 48.41^{\prime \prime} \mathrm{N}, 12^{\circ} 11^{\prime} 53.42^{\prime \prime} \mathrm{W}$

Date recorded: 4/9/2008 Area $\left(\mathrm{m}^{2}\right): 12,199$

Setting: Gentle slope in wooded savanna, no groundcover

Soil: Consolidated sandy clay loam with laterite gravel

FS (sample): 2124 (s); 2125 (p)

Ceramic component(s): Atlantic Era, cLSA

Lithic component(s): MSA/LSA

Groundstone: Basin, Hachette, Handstone

Metallurgy: Slag

Other artifacts: Glass bottle, netweight

Surface feature(s): Stone circle

Primary function(s): Settlement

Notes:

Survey ID: FAL-1065-01

Coordinates: $13^{\circ} 54^{\prime} 41.46^{\prime \prime} \mathrm{N}, 12^{\circ} 11^{\prime} 36.64^{\prime \prime} \mathrm{W}$

Date recorded: 4/9/2008 Area $\left(\mathrm{m}^{2}\right): 10,201$

Setting: Level area in wooded savanna, no groundcover

Soil: Consolidated sandy loam with laterite gravel

FS (sample): 2128 (s); 2129 (p)

Ceramic component(s): cLSA, Iron Age

Lithic component(s): MSA/LSA

Groundstone: Hachette, Handstone

Surface feature(s): Collapsed daub

Primary function(s): Settlement

Notes:

Survey ID: FAL-1065-02

Coordinates: $13^{\circ} 54^{\prime} 45.29 " \mathrm{~N}, 12^{\circ} 11 ' 33.16^{\prime \prime} \mathrm{W}$

Date recorded: $4 / 9 / 2008 \quad$ Area $\left(\mathrm{m}^{2}\right): 10,891$

Setting: Level area in wooded savanna, no groundcover

Soil: Consolidated sandy loam with laterite gravel

FS (sample): 2130 (s)

Ceramic component(s): cLSA

Lithic component(s): MSA/LSA

Groundstone: Hachette

Primary function(s): Unknown

Notes: 


\section{Survey ID: FAL-1066}

Coordinates: $13^{\circ} 55^{\prime} 17.70^{\prime \prime} \mathrm{N}, 12^{\circ} 12^{\prime} 23.10^{\prime \prime} \mathrm{W}$

Date recorded: 4/9/2008 Area $\left(\mathrm{m}^{2}\right): 682$

Setting: Level area in wooded savanna, modest groundcover

Soil: Semi-consolidated sandy clay loam

FS (sample): 2131 (s)

Ceramic component(s): cLSA

Lithic component(s): MSA/LSA

Primary function(s): Unknown

Notes:

\section{Survey ID: FAL-1067}

Coordinates: $13^{\circ} 55^{\prime} 44.56^{\prime \prime} \mathrm{N}, 12^{\circ} 12^{\prime} 09.44^{\prime \prime} \mathrm{W}$

Date recorded: 4/9/2008 Area $\left(\mathrm{m}^{2}\right): 1455$

Setting: Level area in open savanna, modest groundcover

Soil: Semi-consolidated sandy clay loam

FS (sample): 2132 (p)

Ceramic component(s): Atlantic Era

Lithic component(s): None

Primary function(s): Settlement

Notes: A number of large cobbles may derive from eroded rectilinear stone features

\section{Survey ID: FAL-1068 (Sansanding 1)}

Coordinates: $13^{\circ} 54^{\prime} 48.52^{\prime \prime} \mathrm{N}, 12^{\circ} 11^{\prime} 16.69 " \mathrm{~W}$

Date recorded: 6/15/2007 Area $\left(\mathrm{m}^{2}\right)$ : 27,330

Setting: Gentle slope of exposed bedrock, light groundcover

Soil: Exposed bedrock with laterite gravel

FS (sample): Sansanding 1, IFAN 2007 (s, p)

Ceramic component(s): Iron Age

Lithic component(s): MSA/LSA

Groundstone: Handstone

Surface feature(s): Stone circle

Primary function(s): Settlement

Notes: Initially recorded as Sansanding 1

\section{Survey ID: FAL-1069}

Coordinates: $13^{\circ} 54^{\prime} 47.71 " \mathrm{~N}, 12^{\circ} 11^{\prime} 03.37^{\prime \prime} \mathrm{W}$

Date recorded: 4/10/2008 Area $\left(\mathrm{m}^{2}\right): 20,051$

Setting: Gentle slope of exposed bedrock, light groundcover

Soil: Exposed bedrock with laterite gravel

FS (sample): 2133 (p)

Ceramic component(s): Iron Age

Lithic component(s): None

Groundstone: Handstone

Surface feature(s): Stone circle

Primary function(s): Settlement

Notes: Features include 10-12 circular stone architectural foundations arranged in three discrete areas of the site
Survey ID: FAL-1070

Coordinates: $13^{\circ} 54^{\prime} 18.52^{\prime \prime} \mathrm{N}, 12^{\circ} 10^{\prime} 28.39 " \mathrm{~W}$

Date recorded: 4/7/2008 Area $\left(\mathrm{m}^{2}\right): 997$

Setting: Gentle slope in wooded savanna, light groundcover

Soil: Consolidated sandy clay loam with dense laterite gravel

FS (sample): 2134 (p)

Ceramic component(s): None

Lithic component(s): MSA/LSA

Surface feature(s): Stone alignment

Primary function(s): Unknown

Notes: An isolated stone installation associated with LSA component artifacts

\section{Survey ID: FAL-1071}

Coordinates: $13^{\circ} 54^{\prime} 17.99 " \mathrm{~N}, 12^{\circ} 10^{\prime} 38.85^{\prime \prime} \mathrm{W}$

Date recorded: 4/10/2008 Area $\left(\mathrm{m}^{2}\right): 1482$

Setting: Level area in wooded savanna, light groundcover

Soil: Consolidated clay loam with dense laterite gravel

FS (sample): 2135 (s)

Ceramic component(s): None

Lithic component(s): MSA/LSA

Primary function(s): Unknown

Notes:

Survey ID: FAL-1072

Coordinates: $13^{\circ} 54^{\prime} 18.35^{\prime \prime} \mathrm{N}, 12^{\circ} 10^{\prime} 44.87^{\prime \prime} \mathrm{W}$

Date recorded: $4 / 10 / 2008 \quad$ Area $\left(\mathrm{m}^{2}\right): 6327$

Setting: Level area in wooded savanna, no groundcover

Soil: Semi-consolidated sandy clay loam with light laterite gravel

FS (sample): 2136 (s)

Ceramic component(s): None

Lithic component(s): MSA/LSA

Surface feature(s): Stone alignment

Primary function(s): Unknown

Notes: A number of large cobbles form no clear feature

Survey ID: FAL-1073

Coordinates: $13^{\circ} 54 ' 31.20^{\prime \prime} \mathrm{N}, 12^{\circ} 11^{\prime} 28.91^{\prime \prime} \mathrm{W}$

Date recorded: 4/11/2008 Area $\left(\mathrm{m}^{2}\right): 1299$

Setting: Low embankment in open savanna, light groundcover

Soil: Semi-consolidated sandy loam with dense laterite gravel

FS (sample): 2137 (s)

Ceramic component(s): cLSA

Lithic component(s): MSA/LSA

Other artifacts: Netweight

Primary function(s): Unknown

Notes: 
Survey ID: FAL-1074

Coordinates: $13^{\circ} 54^{\prime} 22.69 " \mathrm{~N}, 12^{\circ} 11^{\prime} 19.09^{\prime \prime} \mathrm{W}$

Date recorded: 4/11/2008 Area $\left(\mathrm{m}^{2}\right): 8325$

Setting: Gentle slope in open savanna, light groundcover

Soil: Semi-consolidated sandy clay loam with light laterite gravel

FS (sample): 2138 (s)

Ceramic component(s): Atlantic Era, cLSA, Iron Age

Lithic component(s): MSA/LSA

Groundstone: Handstone

Surface feature(s): Collapsed daub, Stone circle

Primary function(s): Settlement

Notes: Features include one or two circular stone architectural foundations and four patches of collapsed daub

\section{Survey ID: FAL-1075-01}

Coordinates: $13^{\circ} 54^{\prime} 05.07^{\prime \prime} \mathrm{N}, 12^{\circ} 10^{\prime} 50.98^{\prime \prime} \mathrm{W}$

Date recorded: $4 / 13 / 2008 \quad$ Area $\left(\mathrm{m}^{2}\right): 19,145$

Setting: Bluff in riparian savanna, light groundcover

Soil: Semi-consolidated sandy clay loam with light laterite gravel

FS (sample): 2139 (s); 2141 (p)

Ceramic component(s): Atlantic Era, cLSA

Lithic component(s): MSA/LSA

Groundstone: Basin, Hachette, Handstone

Metallurgy: Slag

Other artifacts: Pipe

Primary function(s): Unknown

Notes: A deep ravine separates this northern half of the site from FAL-1075-02

Survey ID: FAL-1075-02

Coordinates: $13^{\circ} 54^{\prime} 01.41^{\prime \prime} \mathrm{N}, 12^{\circ} 10^{\prime} 44.64 " \mathrm{~W}$

Date recorded: 4/13/2008 Area $\left(\mathrm{m}^{2}\right): 19,534$

Setting: Bluff in riparian savanna, light groundcover

Soil: Semi-consolidated sandy clay loam with light laterite gravel

FS (sample): 2140 (s); 2141 (p)

Ceramic component(s): Atlantic Era, cLSA

Lithic component(s): MSA/LSA

Groundstone: Basin, Hachette, Handstone

Metallurgy: Iron tool/ornament, Slag

Other artifacts: Pipe

Surface feature(s): Stone circle, Other

Primary function(s): Settlement

Notes: A deep ravine separates this southern half of the site from FAL-1075-01

\section{Survey ID: FAL-1076}

Coordinates: $13^{\circ} 54^{\prime} 09.24 " \mathrm{~N}, 12^{\circ} 10^{\prime} 34.74$ " W

Date recorded: $4 / 13 / 2008$ Area $\left(\mathrm{m}^{2}\right): 481$

Setting: Level area in wooded savanna, light groundcover

Soil: Consolidated clay loam with light laterite gravel

FS (sample): 2142 (s)

Ceramic component(s): None

Lithic component(s): MSA/LSA

Primary function(s): Unknown

Notes:
Survey ID: FAL-1077

Coordinates: $13^{\circ} 54^{\prime} 01.83^{\prime \prime} \mathrm{N}, 12^{\circ} 10^{\prime} 29.53 " \mathrm{~W}$

Date recorded: 4/13/2008 Area $\left(\mathrm{m}^{2}\right): 8348$

Setting: Gentle slope in wooded savanna, light groundcover

Soil: Consolidated sandy loam with modest laterite gravel

FS (sample): 2143 (s); 2144 (p)

Ceramic component(s): cLSA, Iron Age

Lithic component(s): MSA/LSA

Groundstone: Hachette, Handstone

Metallurgy: Slag

Surface feature(s): Stone circle

Primary function(s): Settlement

Notes: Features include several circular stone architectural foundations interspersed by a number of displaced cobbles

\section{Survey ID: FAL-1078}

Coordinates: $13^{\circ} 53^{\prime} 54.48^{\prime \prime} \mathrm{N}, 12^{\circ} 10^{\prime} 22.98^{\prime \prime} \mathrm{W}$

Date recorded: 4/13/2008 Area $\left(\mathrm{m}^{2}\right): 3765$

Setting: Low ridge in open savanna, light groundcover

Soil: Consolidated sandy clay loam with dense laterite gravel

FS (sample): 2145 (s)

Ceramic component(s): None

Lithic component(s): MSA/LSA

Groundstone: Hachette, Handstone

Primary function(s): Unknown

Notes:

\section{Survey ID: FAL-1079}

Coordinates: $13^{\circ} 53^{\prime} 48.82^{\prime \prime} \mathrm{N}, 12^{\circ} 10^{\prime} 15.57 " \mathrm{~W}$

Date recorded: 4/13/2008 Area $\left(\mathrm{m}^{2}\right): 1341$

Setting: Low ridge in open savanna, no groundcover

Soil: Consolidated sandy clay loam with modest laterite gravel

FS (sample): Not collected

Ceramic component(s): None

Lithic component(s): MSA/LSA

Groundstone: Handstone

Primary function(s): Unknown

Notes:

\section{Survey ID: FAL-1080}

Coordinates: $13^{\circ} 53 ' 39.95^{\prime \prime} \mathrm{N}, 12^{\circ} 10^{\prime} 08.84^{\prime \prime} \mathrm{W}$

Date recorded: 4/13/2008 Area $\left(\mathrm{m}^{2}\right): 2943$

Setting: Bluff top in riparian savanna, no groundcover

Soil: Consolidated sandy loam with laterite gravel and angular cobbles

FS (sample): 2146 (s)

Ceramic component(s): cLSA

Lithic component(s): MSA/LSA

Surface feature(s): Stone square

Primary function(s): A possible hunting stand

Notes: 
Survey ID: FAL-1081

Coordinates: $13^{\circ} 53^{\prime} 16.53^{\prime \prime} \mathrm{N}, 1^{\circ} 09^{\prime} 51.96 " \mathrm{~W}$

Date recorded: 4/13/2008 Area $\left(\mathrm{m}^{2}\right): 2959$

Setting: Low ridge in cultivated field, no groundcover

Soil: Unconsolidated clay loam with laterite gravel

FS (sample): 2147 (s)

Ceramic component(s): None

Lithic component(s): MSA/LSA

Primary function(s): Unknown

Notes: A possible source of raw materials for chipped stone tool production

Survey ID: FAL-1082

Coordinates: $13^{\circ} 53 ' 23.59 " \mathrm{~N}, 12^{\circ} 09^{\prime} 44.65^{\prime \prime} \mathrm{W}$

Date recorded: 4/13/2008 Area $\left(\mathrm{m}^{2}\right): 7833$

Setting: Steep embankment in wooded savanna, no groundcover

Soil: Semi-consolidated sandy clay loam

FS (sample): Not collected

Ceramic component(s): None

Lithic component(s): MSA/LSA

Primary function(s): Unknown

Notes: Possibly identified by Ravisé (1975) as Karé, rive droite

\section{Survey ID: FAL-1083}

Coordinates: $13^{\circ} 56^{\prime} 40.21^{\prime \prime} \mathrm{N}, 12^{\circ} 12^{\prime} 16.60^{\prime \prime} \mathrm{W}$

Date recorded: 4/14/2008 Area $\left(\mathrm{m}^{2}\right)$ : 909

Setting: Eroded bluff top in riparian savanna, light groundcover

Soil: Semi-consolidated sandy loam

FS (sample): 2149 (p)

Ceramic component(s): Atlantic Era

Lithic component(s): None

Primary function(s): Unknown

Notes:

\section{Survey ID: FAL-1084}

Coordinates: $13^{\circ} 56^{\prime} 50.66^{\prime \prime} \mathrm{N}, 12^{\circ} 12^{\prime 22.88}$ " W

Date recorded: 4/14/2008 Area $\left(\mathrm{m}^{2}\right): 22,195$

Setting: Bluff top in riparian savanna, no groundcover

Soil: Consolidated sandy clay loam with moderate laterite gravel

FS (sample): 2151 (s); 2150, 2152 (p)

Ceramic component(s): Atlantic Era, CLSA, Iron Age

Lithic component(s): MSA/LSA

Groundstone: Basin, Handstone

Metallurgy: Iron tool/ornament

Other artifacts: Pipe

Surface feature(s): Stone circle, Pyrotechnic feature

Primary function(s): Settlement

Notes:

\section{Survey ID: FAL-1085}

Coordinates: $13^{\circ} 56^{\prime} 58.69^{\prime \prime} \mathrm{N}, 12^{\circ} 12^{\prime} 30.43^{\prime \prime} \mathrm{W}$

Date recorded: 4/14/2008 Area $\left(\mathrm{m}^{2}\right): 1777$

Setting: Low ridge in open savanna, no groundcover

Soil: Consolidated clay loam with dense laterite gravel

FS (sample): 2153 (s)

Ceramic component(s): Atlantic Era, cLSA

Lithic component(s): MSA/LSA

Groundstone: Hachette

Primary function(s): Unknown

Notes:

\section{Survey ID: FAL-1086}

Coordinates: $13^{\circ} 57^{\prime} 12.47^{\prime \prime} \mathrm{N}, 12^{\circ} 12^{\prime} 44.17^{\prime \prime} \mathrm{W}$

Date recorded: 4/14/2008 Area $\left(\mathrm{m}^{2}\right): 6013$

Setting: Steep embankment in open savanna, no groundcover

Soil: Consolidated sandy clay loam with dense laterite gravel

FS (sample): 2154 (s)

Ceramic component(s): None

Lithic component(s): MSA/LSA

Primary function(s): Unknown

Notes:

Survey ID: FAL-1087

Coordinates: $13^{\circ} 57^{\prime} 01.78^{\prime \prime} \mathrm{N}, 12^{\circ} 12^{\prime} 51.68^{\prime \prime} \mathrm{W}$

Date recorded: 4/14/2008 Area $\left(\mathrm{m}^{2}\right): 1063$

Setting: Gentle slope in open savanna, light groundcover

Soil: Consolidated clay loam with dense laterite gravel

FS (sample): 2155 (s)

Ceramic component(s): None

Lithic component(s): MSA/LSA

Groundstone: Hachette

Primary function(s): Unknown

Notes:

Survey ID: FAL-1088

Coordinates: $13^{\circ} 56^{\prime} 56.61^{\prime \prime} \mathrm{N}, 12^{\circ} 12^{\prime} 50.40^{\prime \prime} \mathrm{W}$

Date recorded: 4/14/2008 Area $\left(\mathrm{m}^{2}\right): 1559$

Setting: Gentle slope in open savanna, no groundcover

Soil: Consolidated clay loam with dense laterite gravel

FS (sample): Not collected

Ceramic component(s): cLSA

Lithic component(s): MSA/LSA

Groundstone: Handstone

Primary function(s): Unknown

Notes: Initially recorded as FAL-1079b 


\section{Survey ID: FAL-1089}

Coordinates: $13^{\circ} 56^{\prime} 51.61^{\prime \prime} \mathrm{N}, 12^{\circ} 12^{\prime} 57.99^{\prime \prime} \mathrm{W}$

Date recorded: 4/14/2008 Area $\left(\mathrm{m}^{2}\right): 271$

Setting: Level area in open savanna, no groundcover

Soil: Consolidated clay loam with modest laterite

FS (sample): 2156 (s)

Ceramic component(s): cLSA

Lithic component(s): MSA/LSA

Groundstone: Hachette

Primary function(s): Unknown

Notes:

\section{Survey ID: FAL-1090}

Coordinates: $13^{\circ} 56^{\prime} 51.09^{\prime \prime} \mathrm{N}, 1^{\circ} 13^{\prime} 05.14^{\prime \prime} \mathrm{W}$

Date recorded: 4/14/2008 Area $\left(\mathrm{m}^{2}\right): 1606$

Setting: Low ridge in open savanna, light groundcover

Soil: Consolidated clay loam with light laterite gravel FS (sample): Not collected

Ceramic component(s): cLSA

Lithic component(s): MSA/LSA

Primary function(s): Unknown

Notes:

\section{Survey ID: FAL-1091}

Coordinates: $13^{\circ} 57 ' 31.15^{\prime \prime} \mathrm{N}, 12^{\circ} 13^{\prime} 16.75^{\prime \prime} \mathrm{W}$

Date recorded: 4/14/2008 Area $\left(\mathrm{m}^{2}\right): 1670$

Setting: Gentle slope in open savanna, no groundcover

Soil: Consolidated clay silt with dense laterite gravel

FS (sample): 2157 (s)

Ceramic component(s): cLSA

Lithic component(s): MSA/LSA

Surface feature(s): Stone alignment

Primary function(s): Unknown

Notes:

\section{Survey ID: FAL-1092}

Coordinates: $13^{\circ} 57^{\prime} 00.25^{\prime \prime} \mathrm{N}, 12^{\circ} 13^{\prime} 15.17^{\prime \prime} \mathrm{W}$

Date recorded: $4 / 14 / 2008$ Area $\left(\mathrm{m}^{2}\right)$ : 39,824

Setting: Level area in cultivated field, no groundcover

Soil: Unconsolidated clay loam

FS (sample): 2158 (p)

Ceramic component(s): Atlantic Era

Lithic component(s): None

Surface feature(s): Stone circle

Primary function(s): Settlement

Notes: Features include several stone architectural foundations disturbed by plowing

Survey ID: FAL-1093-01

Coordinates: $13^{\circ} 56^{\prime} 51.96 " \mathrm{~N}, 12^{\circ} 13^{\prime} 13.74 " \mathrm{~W}$

Date recorded: 4/14/2008 Area $\left(\mathrm{m}^{2}\right): 1770$

Setting: Bank of seasonal drainage in open savanna, light groundcover

Soil: Consolidated sandy clay with dense laterite

FS (sample): 2159 (s)

Ceramic component(s): cLSA

Lithic component(s): MSA/LSA

Primary function(s): Unknown

Notes:
Survey ID: FAL-1093-02

Coordinates: $13^{\circ} 56^{\prime} 45.97^{\prime \prime} \mathrm{N}, 12^{\circ} 13^{\prime} 10.37^{\prime \prime} \mathrm{W}$

Date recorded: 4/14/2008 Area $\left(\mathrm{m}^{2}\right): 824$

Setting: Bank of seasonal drainage in open savanna, light groundcover

Soil: Consolidated sandy clay with dense laterite

FS (sample): Not collected

Ceramic component(s): cLSA

Lithic component(s): MSA/LSA

Primary function(s): Unknown

Notes:

\section{Survey ID: FAL-1094}

Coordinates: $13^{\circ} 56^{\prime} 30.66^{\prime \prime} \mathrm{N}, 12^{\circ} 12^{\prime} 08.09^{\prime \prime} \mathrm{W}$

Date recorded: 4/15/2008 Area $\left(\mathrm{m}^{2}\right): 1004$

Setting: Bluff top in riparian savanna, no groundcover

Soil: Consolidated sandy clay loam with dense laterite gravel

FS (sample): 2160 (s)

Ceramic component(s): cLSA

Lithic component(s): None

Primary function(s): Unknown

Notes:

\section{Survey ID: FAL-1095}

Coordinates: $13^{\circ} 56^{\prime} 35.60^{\prime \prime} \mathrm{N}, 12^{\circ} 12^{\prime} 03.96 " \mathrm{~W}$

Date recorded: 4/15/2008 Area $\left(\mathrm{m}^{2}\right)$ : 6494

Setting: Low rise in riparian savanna, no groundcover

Soil: Consolidated clay loam with dense laterite gravel

FS (sample): 2161 (s)

Ceramic component(s): None

Lithic component(s): MSA/LSA

Groundstone: Handstone

Primary function(s): Unknown

Notes:

Survey ID: FAL-1096-01

Coordinates: $13^{\circ} 56^{\prime} 53.83^{\prime \prime} \mathrm{N}, 12^{\circ} 12^{\prime} 10.43^{\prime \prime} \mathrm{W}$

Date recorded: 4/15/2008 Area $\left(\mathrm{m}^{2}\right): 1289$

Setting: Bluff in riparian savanna, light groundcover

Soil: Semi-consolidated sandy clay loam

FS (sample): 2162 (p)

Ceramic component(s): None

Lithic component(s): MSA/LSA

Primary function(s): Unknown

Notes:

Survey ID: FAL-1096-02

Coordinates: $13^{\circ} 56^{\prime} 56.27^{\prime \prime} \mathrm{N}, 12^{\circ} 12^{\prime} 14.86^{\prime \prime} \mathrm{W}$

Date recorded: 4/15/2008 Area $\left(\mathrm{m}^{2}\right): 3039$

Setting: Bluff in riparian savanna, light groundcover

Soil: Consolidated sandy clay loam

FS (sample): Not collected

Ceramic component(s): None

Lithic component(s): MSA/LSA

Primary function(s): Unknown

Notes: Initially recorded as FAL-IO-052 
Survey ID: FAL-1097

Coordinates: $13^{\circ} 57^{\prime} 01.75^{\prime \prime} \mathrm{N}, 12^{\circ} 12^{\prime} 19.08^{\prime \prime} \mathrm{W}$

Date recorded: 4/15/2008 Area $\left(\mathrm{m}^{2}\right): 856$

Setting: Bluff in wooded savanna, light groundcover

Soil: Consolidated sandy clay with laterite gravel

FS (sample): Not collected

Ceramic component(s): None

Lithic component(s): MSA/LSA

Primary function(s): Unknown

Notes:

\section{Survey ID: FAL-1098}

Coordinates: $13^{\circ} 57^{\prime} 12.74 " \mathrm{~N}, 12^{\circ} 12^{\prime} 19.56^{\prime \prime} \mathrm{W}$

Date recorded: $4 / 15 / 2008 \quad$ Area $\left(\mathrm{m}^{2}\right): 13,142$

Setting: Eroded basin in open savanna, light groundcover

Soil: Consolidated silty clay with extremely dense laterite gravel

FS (sample): 2164 (p)

Ceramic component(s): cLSA

Lithic component(s): MSA/LSA

Groundstone: Basin, Hachette, Handstone

Primary function(s): Unknown

Notes:

Survey ID: FAL-1099 (Tomboura Fulbe)

Coordinates: $13^{\circ} 57 ' 15.18^{\prime \prime} \mathrm{N}, 12^{\circ} 12^{\prime} 26.13^{\prime \prime} \mathrm{W}$

Date recorded: 4/15/2008 Area $\left(\mathrm{m}^{2}\right): 6287$

Setting: Gentle slope in open savanna, modest groundcover

Soil: Semi-consolidated sandy clay loam with light laterite gravel

FS (sample): 2165 (s); 2166 (p)

Ceramic component(s): Atlantic Era

Lithic component(s): Unknown

Groundstone: Basin, Handstone

Metallurgy: Slag

Surface feature(s): Collapsed daub, Stone alignment

Primary function(s): Settlement

Notes:

\section{Survey ID: FAL-1100}

Coordinates: $13^{\circ} 57 ' 24.07^{\prime \prime} \mathrm{N}, 12^{\circ} 12^{\prime} 33.29^{\prime \prime} \mathrm{W}$

Date recorded: 4/15/2008 Area $\left(\mathrm{m}^{2}\right): 1425$

Setting: Low embankment in wooded savanna, light groundcover

Soil: Consolidated sandy clay with modest laterite

FS (sample): 2167 (s)

Ceramic component(s): Atlantic Era, cLSA

Lithic component(s): MSA/LSA

Groundstone: Hachette

Primary function(s): Unknown

Notes:

\section{Survey ID: FAL-1101}

Coordinates: $13^{\circ} 57^{\prime} 32.61^{\prime \prime} \mathrm{N}, 12^{\circ} 12^{\prime} 35.26$ " W

Date recorded: 4/15/2008 Area $\left(\mathrm{m}^{2}\right): 8486$

Setting: Level area in open savanna, no groundcover

Soil: Semi-consolidated sandy clay loam with light sandstone gravel

FS (sample): 2168 (p)

Ceramic component(s): Atlantic Era, cLSA, Iron Age

Lithic component(s): MSA/LSA

Groundstone: Basin, Hachette

Other artifacts: Pipe

Primary function(s): Unknown

Notes:

\section{Survey ID: FAL-1102}

Coordinates: $13^{\circ} 57^{\prime} 00.87^{\prime \prime} \mathrm{N}, 12^{\circ} 12^{\prime} 08.54^{\prime \prime} \mathrm{W}$

Date recorded: 4/15/2008 Area $\left(\mathrm{m}^{2}\right): 5794$

Setting: Low ridge in open savanna, light groundcover

Soil: Consolidated silty loam with extremely dense laterite gravel

FS (sample): Not collected

Ceramic component(s): None

Lithic component(s): MSA/LSA

Primary function(s): Unknown

Notes:

\section{Survey ID: FAL-1103}

Coordinates: $13^{\circ} 56^{\prime} 39.02^{\prime \prime} \mathrm{N}, 12^{\circ} 11^{\prime} 59.06$ " W

Date recorded: 4/15/2008 Area $\left(\mathrm{m}^{2}\right): 2931$

Setting: Low ridge in open savanna, no groundcover Soil: Exposed bedrock and laterite duricrust

FS (sample): Not collected

Ceramic component(s): None

Lithic component(s): MSA/LSA

Primary function(s): Unknown

Notes:

\section{Survey ID: FAL-1104}

Coordinates: $13^{\circ} 56^{\prime} 32.30 " \mathrm{~N}, 12^{\circ} 11^{\prime} 53.79^{\prime \prime} \mathrm{W}$

Date recorded: 4/15/2008 Area $\left(\mathrm{m}^{2}\right): 4405$

Setting: Low ridge in open savanna, no groundcover

Soil: Consolidated sandy clay loam with dense laterite gravel

FS (sample): 2169 (s)

Ceramic component(s): None

Lithic component(s): MSA/LSA

Primary function(s): Unknown

Notes: Initially recorded as FAL-1103-02 
Survey ID: FAL-1105

Coordinates: $13^{\circ} 55^{\prime} 44.28^{\prime \prime} \mathrm{N}, 12^{\circ} 12^{\prime} 39.09^{\prime \prime} \mathrm{W}$

Date recorded: 4/15/2008 Area $\left(\mathrm{m}^{2}\right): 3203$

Setting: Bank of seasonal drainage in riparian savanna, light groundcover

Soil: Consolidated silty clay loam with laterite gravel FS (sample): 2171 (s); 2172 (p)

Ceramic component(s): Atlantic Era, cLSA, Iron Age

Lithic component(s): MSA/LSA

Groundstone: Handstone

Other artifacts: Netweight

Surface feature(s): Collapsed daub, Stone alignment

Primary function(s): Settlement

Notes:

\section{Survey ID: FAL-1106-01}

Coordinates: $13^{\circ} 55^{\prime} 29.15^{\prime \prime} \mathrm{N}, 12^{\circ} 12^{\prime} 41.50^{\prime \prime} \mathrm{W}$

Date recorded: 4/15/2008 Area $\left(\mathrm{m}^{2}\right)$ : 2149

Setting: Bluff in riparian savanna, light groundcover

Soil: Semi-consolidated sandy clay with laterite gravel

FS (sample): $2173(\mathrm{p})$

Ceramic component(s): cLSA, Iron Age

Lithic component(s): MSA/LSA

Groundstone: Hachette, Handstone

Primary function(s): Unknown

Notes:

\section{Survey ID: FAL-1106-02}

Coordinates: $13^{\circ} 55^{\prime} 24.92^{\prime \prime} \mathrm{N}, 12^{\circ} 12^{\prime} 44.60^{\prime \prime} \mathrm{W}$

Date recorded: $4 / 15 / 2008 \quad$ Area $\left(\mathrm{m}^{2}\right): 4428$

Setting: Bluff in riparian savanna, light groundcover

Soil: Semi-consolidated sandy clay with laterite gravel

FS (sample): Not collected

Ceramic component(s): cLSA

Lithic component(s): MSA/LSA

Groundstone: Handstone

Primary function(s): Unknown

Notes:

\section{Survey ID: FAL-1107}

Coordinates: $13^{\circ} 55^{\prime} 23.82^{\prime \prime} \mathrm{N}, 12^{\circ} 12^{\prime} 52.24^{\prime \prime} \mathrm{W}$

Date recorded: 4/15/2008 Area $\left(\mathrm{m}^{2}\right): 874$

Setting: Bank of seasonal drainage in wooded savanna, light groundcover

Soil: Consolidated sandy clay loam with modest laterite gravel

FS (sample): 2174 (p)

Ceramic component(s): Iron Age

Lithic component(s): None

Groundstone: Basin

Surface feature(s): Stone alignment

Primary function(s): Settlement

Notes:

\section{Survey ID: FAL-1108}

Coordinates: $13^{\circ} 55^{\prime} 21.17^{\prime \prime} \mathrm{N}, 12^{\circ} 13^{\prime} 06.40^{\prime \prime} \mathrm{W}$

Date recorded: 4/16/2008 Area $\left(\mathrm{m}^{2}\right)$ : 11,729

Setting: Level area in cultivated field, no groundcover Soil: Semi-consolidated sandy clay

FS (sample): 2175 (p)

Ceramic component(s): Atlantic Era, Iron Age

Lithic component(s): None

Metallurgy: Iron tool/ornament

Other artifacts: Glass bottle

Surface feature(s): Collapsed daub, Stone circle, Pyrotechnic feature

Primary function(s): Settlement

Notes:

\section{Survey ID: FAL-1109}

Coordinates: $13^{\circ} 55^{\prime} 14.83^{\prime \prime} \mathrm{N}, 12^{\circ} 13^{\prime} 17.39^{\prime \prime} \mathrm{W}$

Date recorded: 4/16/2008 Area $\left(\mathrm{m}^{2}\right): 7432$

Setting: Bank of seasonal drainage in open savanna, light groundcover

Soil: Consolidated silty clay loam with modest laterite gravel

FS (sample): 2178 (s); 2176 (p)

Ceramic component(s): cLSA

Lithic component(s): MSA/LSA

Groundstone: Axe

Primary function(s): Unknown

Notes:

Survey ID: FAL-1110

Coordinates: $13^{\circ} 55^{\prime} 11.66^{\prime \prime} \mathrm{N}, 12^{\circ} 13^{\prime} 25.78^{\prime \prime} \mathrm{W}$

Date recorded: 4/16/2008 Area $\left(\mathrm{m}^{2}\right): 2636$

Setting: Gentle slope in savanna, light groundcover

Soil: Consolidated clay loam with dense laterite gravel

FS (sample): 2177 (p)

Ceramic component(s): cLSA

Lithic component(s): MSA/LSA

Groundstone: Basin, Hachette, Handstone

Primary function(s): Unknown

Notes:

\section{Survey ID: FAL-1111}

Coordinates: $13^{\circ} 54 ' 38.09^{\prime \prime} \mathrm{N}, 12^{\circ} 12^{\prime} 19.23^{\prime \prime} \mathrm{W}$

Date recorded: 4/16/2008 Area $\left(\mathrm{m}^{2}\right): 2454$

Setting: Level area in open savanna, no groundcover

Soil: Consolidated sandy clay loam with laterite gravel

FS (sample): 2179 (s); 2180 (p)

Ceramic component(s): cLSA

Lithic component(s): MSA/LSA

Groundstone: Handstone

Primary function(s): Unknown

Notes: 
Survey ID: FAL-1112

Coordinates: $13^{\circ} 54^{\prime} 28.99 " \mathrm{~N}, 12^{\circ} 12^{\prime} 25.09^{\prime \prime} \mathrm{W}$

Date recorded: $4 / 16 / 2008 \quad$ Area $\left(\mathrm{m}^{2}\right): 713$

Setting: Low ridge in open savanna, no groundcover

Soil: Consolidated sandy clay loam with dense laterite gravel

FS (sample): Not collected

Ceramic component(s): None

Lithic component(s): MSA/LSA

Primary function(s): Unknown

Notes:

\section{Survey ID: FAL-1113}

Coordinates: $13^{\circ} 54^{\prime} 18.20^{\prime \prime} \mathrm{N}, 1^{\circ} 11^{\prime} 57.41^{\prime \prime} \mathrm{W}$

Date recorded: $4 / 16 / 2008 \quad$ Area $\left(\mathrm{m}^{2}\right): 1160$

Setting: Eroded basin in riparian savanna, light groundcover

Soil: Consolidated sandy clay with light laterite

FS (sample): 2181 (p)

Ceramic component(s): Iron Age

Lithic component(s): None

Metallurgy: Slag

Surface feature(s): Iron furnace (?)

Primary function(s): Unknown

Notes: Possible pit furnace

\section{Survey ID: FAL-1114}

Coordinates: $13^{\circ} 54^{\prime} 19.21^{\prime \prime} \mathrm{N}, 12^{\circ} 11^{\prime} 46.67^{\prime \prime} \mathrm{W}$

Date recorded: 4/16/2008 Area $\left(\mathrm{m}^{2}\right): 1826$

Setting: Eroded embankment in open savanna, modest groundcover

Soil: Consolidated silty clay loam with modest laterite gravel

FS (sample): 2182 (p)

Ceramic component(s): Iron Age

Lithic component(s): Unknown

Groundstone: Basin, Hachette, Handstone

Surface feature(s): Stone alignment

Primary function(s): Settlement

Notes:

\section{Survey ID: FAL-1115}

Coordinates: $13^{\circ} 54^{\prime} 18.08^{\prime \prime} \mathrm{N}, 12^{\circ} 11^{\prime} 38.19^{\prime \prime} \mathrm{W}$

Date recorded: 4/16/2008 Area $\left(\mathrm{m}^{2}\right): 460$

Setting: Bluff in riparian savanna, light groundcover

Soil: Consolidated silty clay loam with modest laterite gravel

FS (sample): 2184 (p)

Ceramic component(s): Iron Age

Lithic component(s): Unknown

Primary function(s): Unknown

Notes:
Survey ID: FAL-1116

Coordinates: $13^{\circ} 54^{\prime} 09.70^{\prime \prime} \mathrm{N}, 12^{\circ} 11^{\prime} 46.23^{\prime \prime} \mathrm{W}$

Date recorded: 4/16/2008 Area $\left(\mathrm{m}^{2}\right): 4558$

Setting: Low mound in wooded savanna, light groundcover

Soil: Consolidated sandy clay

FS (sample): 2185 (p)

Ceramic component(s): Iron Age

Lithic component(s): Unknown

Groundstone: Handstone

Surface feature(s): Stone alignment, Pyrotechnic

feature

Primary function(s): Settlement

Notes:

\section{Survey ID: FAL-1117}

Coordinates: $13^{\circ} 54^{\prime} 06.36^{\prime \prime} \mathrm{N}, 12^{\circ} 11^{\prime} 57.58^{\prime \prime} \mathrm{W}$

Date recorded: 4/16/2008 Area $\left(\mathrm{m}^{2}\right): 1385$

Setting: Level area in cultivated field, no groundcover

Soil: Semi-consolidated clay loam with laterite gravel

FS (sample): 2186 (p)

Ceramic component(s): Iron Age

Lithic component(s): None

Metallurgy: Slag

Primary function(s): Settlement

Notes:

\section{Survey ID: FAL-1118}

Coordinates: $13^{\circ} 53^{\prime} 59.15^{\prime \prime} \mathrm{N}, 12^{\circ} 11^{\prime} 48.52^{\prime \prime} \mathrm{W}$

Date recorded: 4/17/2008 Area $\left(\mathrm{m}^{2}\right): 2254$

Setting: Low rise in wooded savanna, no groundcover

Soil: Consolidated sandy clay loam with discrete concentrations of dense laterite gravel

FS (sample): 2187 (p)

Ceramic component(s): cLSA, Iron Age

Lithic component(s): Late Acheulean, MSA/LSA

Surface feature(s): Pyrotechnic feature

Primary function(s): Unknown

Notes:

\section{Survey ID: FAL-1119 (Goundafa)}

Coordinates: $13^{\circ} 54^{\prime} 04.18^{\prime \prime} \mathrm{N}, 12^{\circ} 11^{\prime} 33.26^{\prime \prime} \mathrm{W}$

Date recorded: 4/17/2008 Area $\left(\mathrm{m}^{2}\right)$ : 53,101

Setting: Low mounds in cultivated field, light groundcover

Soil: Consolidated sandy clay loam with modest laterite gravel

FS (sample): 2189 (s); 2188, 2190 (p)

Ceramic component(s): Iron Age

Lithic component(s): Unknown

Groundstone: Basin, Handstone

Metallurgy: Iron tool/ornament, Slag

Other artifacts: Netweight, Pipe

Surface feature(s): Stone circle, Pyrotechnic feature

Primary function(s): Settlement

Notes: Previously recorded by Opper and Opper (1990:23) 
Survey ID: FAL-1120

Coordinates: $13^{\circ} 53^{\prime} 48.30^{\prime \prime} \mathrm{N}, 12^{\circ} 10^{\prime} 50.97^{\prime \prime} \mathrm{W}$

Date recorded: 4/17/2008 Area $\left(\mathrm{m}^{2}\right)$ : 5855

Setting: Level area in riparian savanna, no groundcover

Soil: Semi-consolidated sandy loam

FS (sample): 2191 (p)

Ceramic component(s): Atlantic Era

Lithic component(s): None

Surface feature(s): Collapsed daub

Primary function(s): Unknown

Notes:

Survey ID: FAL-1121

Coordinates: $13^{\circ} 53 ' 28.87 " \mathrm{~N}, 12^{\circ} 10^{\prime} 16.26^{\prime \prime} \mathrm{W}$

Date recorded: 4/17/2008 Area $\left(\mathrm{m}^{2}\right): 6259$

Setting: Level area in riparian savanna, no groundcover

Soil: Consolidated sandy clay loam with laterite gravel

FS (sample): 2192 (s); 2193 (p)

Ceramic component(s): cLSA

Lithic component(s): MSA/LSA

Groundstone: Basin, Hachette

Other artifacts: Netweight

Primary function(s): Unknown

Notes:

\section{Survey ID: FAL-1122}

Coordinates: $13^{\circ} 53^{\prime} 21.27^{\prime \prime} \mathrm{N}, 12^{\circ} 10^{\prime} 10.91^{\prime \prime} \mathrm{W}$

Date recorded: 4/17/2008 Area $\left(\mathrm{m}^{2}\right): 724$

Setting: Bluff in riparian savanna, light groundcover

Soil: Consolidated clay loam

FS (sample): Not collected

Ceramic component(s): cLSA

Lithic component(s): MSA/LSA

Groundstone: Handstone

Primary function(s): Unknown

Notes:

\section{Survey ID: FAL-1126-01 (Tomboura E1)}

Coordinates: $13^{\circ} 58^{\prime} 29.68^{\prime \prime} \mathrm{N}, 12^{\circ} 12^{\prime} 17.28^{\prime \prime} \mathrm{W}$

Date recorded: $4 / 20 / 2008 \quad$ Area $\left(\mathrm{m}^{2}\right): 2639$

Setting: Gentle slope in open savanna, light groundcover

Soil: Semi-consolidated sandy clay with laterite gravel

FS (sample): 2196 (p)

Ceramic component(s): Atlantic Era, cLSA

Lithic component(s): MSA/LSA

Groundstone: Hachette, Handstone

Metallurgy: Iron tool/ornament

Other artifacts: Glass bead

Surface feature(s): Stone alignment

Primary function(s): Settlement

Notes:
Survey ID: FAL-1126-02 (Tomboura E1)

Coordinates: $13^{\circ} 58^{\prime} 11.92 " \mathrm{~N}, 12^{\circ} 12^{\prime} 22.90^{\prime \prime} \mathrm{W}$

Date recorded: $4 / 20 / 2008 \quad$ Area $\left(\mathrm{m}^{2}\right)$ : 95,082

Setting: Gentle slope in open savanna, light groundcover

Soil: Semi-consolidated sandy clay with laterite gravel

FS (sample): 2197-2198 (s)

Ceramic component(s): Atlantic Era, Iron Age

Lithic component(s): Unknown

Groundstone: Handstone

Metallurgy: Nail (Hf), Slag

Other artifacts: Netweight

Surface feature(s): Stone circle, Stone square, Pyrotechnic feature

Primary function(s): Settlement

Notes:

Survey ID: FAL-1127

Coordinates: $13^{\circ} 58 ' 21.40^{\prime \prime} \mathrm{N}, 12^{\circ} 12^{\prime} 28.43^{\prime \prime} \mathrm{W}$

Date recorded: 4/20/2008 Area $\left(\mathrm{m}^{2}\right)$ : 3309

Setting: Bluff in riparian savanna, light groundcover

Soil: Consolidated sandy clay with laterite gravel

FS (sample): 2199 (s); 2200 (p)

Ceramic component(s): cLSA

Lithic component(s): MSA/LSA

Groundstone: Basin, Handstone

Primary function(s): Unknown

Notes:

Survey ID: FAL-1128-01 (Tomboura E2)

Coordinates: $13^{\circ} 57^{\prime} 58.19^{\prime \prime} \mathrm{N}, 12^{\circ} 12^{\prime} 28.69^{\prime \prime} \mathrm{W}$

Date recorded: $4 / 20 / 2008 \quad$ Area $\left(\mathrm{m}^{2}\right): 329$

Setting: Gentle slope in open savanna, no groundcover

Soil: Consolidated sandy clay with laterite gravel FS (sample): Not collected

Ceramic component(s): cLSA

Lithic component(s): MSA/LSA

Primary function(s): Unknown

Notes:

Survey ID: FAL-1128-02 (Tomboura E2)

Coordinates: $13^{\circ} 57^{\prime} 52.70^{\prime \prime} \mathrm{N}, 12^{\circ} 12^{\prime} 29.00^{\prime \prime} \mathrm{W}$

Date recorded: 4/20/2008 Area $\left(\mathrm{m}^{2}\right): 4909$

Setting: Gentle slope in open savanna, light groundcover

Soil: Consolidated sandy clay loam with laterite gravel

FS (sample): 2201 (p)

Ceramic component(s): Atlantic Era, cLSA

Lithic component(s): MSA/LSA

Groundstone: Axe, Hachette, Handstone

Metallurgy: Nail (Hf), Iron tool/ornament

Other artifacts: Pipe

Surface feature(s): Stone square

Primary function(s): Settlement

Notes: Initially recorded as FAL-1129 
Survey ID: FAL-1129

Coordinates: $13^{\circ} 58 ' 28.97^{\prime \prime} \mathrm{N}, 12^{\circ} 12^{\prime} 24.66^{\prime \prime} \mathrm{W}$

Date recorded: 4/20/2008 Area $\left(\mathrm{m}^{2}\right): 2272$

Setting: Bluff in riparian savanna, modest groundcover

Soil: Consolidated sandy clay loam with laterite gravel FS (sample): Not collected

Ceramic component(s): Atlantic Era, cLSA

Lithic component(s): MSA/LSA

Primary function(s): Unknown

Notes: Initially recorded as FAL-IO-074

\section{Survey ID: FAL-1130}

Coordinates: $13^{\circ} 57^{\prime} 43.00^{\prime \prime} \mathrm{N}, 12^{\circ} 12^{\prime} 35.61^{\prime \prime} \mathrm{W}$

Date recorded: $4 / 20 / 2008 \quad$ Area $\left(\mathrm{m}^{2}\right)$ : 5589

Setting: Gentle slope in open savanna, light groundcover

Soil: Consolidated sandy clay loam with light laterite and river gravel

FS (sample): 2203 (p)

Ceramic component(s): Atlantic Era, cLSA

Lithic component(s): MSA/LSA

Groundstone: Hachette, Handstone

Other artifacts: Glass bead, Pipe, Whorl

Surface feature(s): Collapsed daub, Stone alignment

Primary function(s): Settlement

Notes:

\section{Survey ID: FAL-1131 (Tomboura North)}

Coordinates: $13^{\circ} 58^{\prime} 16.83^{\prime \prime} \mathrm{N}, 12^{\circ} 12^{\prime} 51.51^{\prime \prime} \mathrm{W}$

Date recorded: 6/22/2007 Area $\left(\mathrm{m}^{2}\right): 68,271$

Setting: Level area in cultivated field, no groundcover

Soil: Semi-consolidated sandy clay

FS (sample): 2204, IFAN 2007 (p)

Ceramic component(s): Atlantic Era

Lithic component(s): None

Groundstone: Hachette

Metallurgy: Nail (Ct, Hf), Iron tool/ornament

Other artifacts: Coin, Glass bead, Glass bottle

Surface feature(s): Collapsed daub, Stone circle, Stone square

Primary function(s): Settlement

Notes: Initially recorded as Tomboura 2

\section{Survey ID: FAL-1132-01}

Coordinates: $13^{\circ} 52 ' 28.94 " \mathrm{~N}, 12^{\circ} 10^{\prime} 08.79 " \mathrm{~W}$

Date recorded: 4/21/2008 Area $\left(\mathrm{m}^{2}\right): 5228$

Setting: Gentle slope in wooded savanna, modest groundcover

Soil: Semi-consolidated sandy clay with dense laterite and river gravel

FS (sample): Not collected

Ceramic component(s): None

Lithic component(s): MSA/LSA

Primary function(s): Unknown

Notes:
Survey ID: FAL-1132-02

Coordinates: $13^{\circ} 52^{\prime} 24.42^{\prime \prime} \mathrm{N}, 12^{\circ} 10^{\prime} 05.42^{\prime \prime} \mathrm{W}$

Date recorded: 4/21/2008 Area $\left(\mathrm{m}^{2}\right): 1114$

Setting: Gentle slope in wooded savanna, modest groundcover

Soil: Semi-consolidated sandy clay with dense laterite and river gravel

FS (sample): Not collected

Ceramic component(s): cLSA

Lithic component(s): MSA/LSA

Groundstone: Handstone

Primary function(s): Unknown

Notes:

Survey ID: FAL-1133

Coordinates: $13^{\circ} 51$ '52.05" N, 12¹0'04.04" W

Date recorded: 4/21/2008 Area $\left(\mathrm{m}^{2}\right): 3802$

Setting: Low rise of exposed bedrock, no groundcover

Soil: Sandstone and laterite gravel

FS (sample): 2207 (p)

Ceramic component(s): Iron Age

Lithic component(s): Unknown

Groundstone: Basin, Handstone

Surface feature(s): Stone circle

Primary function(s): Settlement

Notes:

\section{Survey ID: FAL-1134}

Coordinates: $13^{\circ} 51^{\prime} 37.89 " \mathrm{~N}, 12^{\circ} 10^{\prime} 03.65^{\prime \prime} \mathrm{W}$

Date recorded: 4/21/2008 Area $\left(\mathrm{m}^{2}\right): 3763$

Setting: Low rise of exposed bedrock, no groundcover

Soil: Sandstone and laterite gravel

FS (sample): 2208 (p)

Ceramic component(s): Iron Age

Lithic component(s): Unknown

Groundstone: Axe, Handstone

Metallurgy: Iron tool/ornament

Surface feature(s): Stone circle

Primary function(s): Settlement

Notes:

\section{Survey ID: FAL-1135}

Coordinates: $13^{\circ} 51^{\prime} 40.79 " \mathrm{~N}, 12^{\circ} 09^{\prime} 51.00^{\prime \prime} \mathrm{W}$

Date recorded: 4/21/2008 Area $\left(\mathrm{m}^{2}\right): 2700$

Setting: Level area in wooded savanna, modest groundcover

Soil: Semi-consolidated sandy clay loam with light laterite gravel

FS (sample): 2209 (p)

Ceramic component(s): Atlantic Era, cLSA

Lithic component(s): MSA/LSA

Groundstone: Handstone

Surface feature(s): Stone circle

Primary function(s): Settlement

Notes: 
Survey ID: FAL-1136-01

Coordinates: $13^{\circ} 51^{\prime} 56.22^{\prime \prime} \mathrm{N}, 1^{\circ} 09^{\prime} 53.65^{\prime \prime} \mathrm{W}$

Date recorded: $4 / 21 / 2008 \quad$ Area $\left(\mathrm{m}^{2}\right)$ : 963

Setting: Gentle slope in wooded savanna, modest groundcover

Soil: Semi-consolidated sandy loam

FS (sample): 2210 (p)

Ceramic component(s): cLSA

Lithic component(s): MSA/LSA

Groundstone: Basin, Hachette, Handstone

Primary function(s): Unknown

Notes:

Survey ID: FAL-1136-02

Coordinates: $13^{\circ} 51 ' 52.51^{\prime \prime} \mathrm{N}, 1^{\circ} 09^{\prime} 52.19^{\prime \prime} \mathrm{W}$

Date recorded: $4 / 21 / 2008$ Area $\left(\mathrm{m}^{2}\right): 421$

Setting: Low ridge in wooded savanna, modest groundcover

Soil: Semi-consolidated sandy clay loam

FS (sample): Not collected

Ceramic component(s): cLSA

Lithic component(s): MSA/LSA

Groundstone: Basin, Handstone

Primary function(s): Unknown

Notes:

Survey ID: FAL-1137

Coordinates: $13^{\circ} 52^{\prime 2} 29.85^{\prime \prime} \mathrm{N}, 1^{\circ} 09^{\prime} 49.84^{\prime \prime} \mathrm{W}$

Date recorded: 4/21/2008 Area $\left(\mathrm{m}^{2}\right): 2333$

Setting: Level area in wooded savanna, modest groundcover

Soil: Semi-consolidated sandy clay loam with light river gravel

FS (sample): 2211 (p)

Ceramic component(s): cLSA

Lithic component(s): MSA/LSA

Groundstone: Hachette, Handstone

Surface feature(s): Collapsed daub

Primary function(s): Unknown

Notes: Several concentrations of daub resemble collapsed pit furnaces, but associated artifacts are from cLSA component

\section{Survey ID: FAL-1138}

Coordinates: $13^{\circ} 52^{\prime} 31.32 " \mathrm{~N}, 12^{\circ} 09^{\prime} 44.31^{\prime \prime} \mathrm{W}$

Date recorded: 4/21/2008 Area $\left(\mathrm{m}^{2}\right): 11,803$

Setting: Bluff top in riparian savanna, no groundcover

Soil: Consolidated clay loam with moderate river

FS (sample): 2212 (s)

Ceramic component(s): Atlantic Era, cLSA

Lithic component(s): MSA/LSA

Groundstone: Axe, Hachette, Handstone

Metallurgy: Slag

Surface feature(s): Collapsed daub

Primary function(s): Iron smelting

Notes: Several concentrations of daub resemble collapsed pit furnaces,
Survey ID: FAL-1139-01

Coordinates: $13^{\circ} 52^{\prime} 19.24 " \mathrm{~N}, 12^{\circ} 09^{\prime} 35.84^{\prime \prime} \mathrm{W}$

Date recorded: 4/21/2008 Area $\left(\mathrm{m}^{2}\right): 9076$

Setting: Eroded basin in riparian savanna, light groundcover

Soil: Semi-consolidated sandy clay

FS (sample): Not collected

Ceramic component(s): cLSA

Lithic component(s): MSA/LSA

Groundstone: Axe, Handstone

Primary function(s): Unknown

Notes:

Survey ID: FAL-1139-02

Coordinates: $13^{\circ} 52^{\prime} 06.02^{\prime \prime} \mathrm{N}, 12^{\circ} 09^{\prime} 35.29 " \mathrm{~W}$

Date recorded: $4 / 21 / 2008$ Area $\left(\mathrm{m}^{2}\right): 10,861$

Setting: Bank of seasonal drainage in riparian savanna, moderate groundcover

Soil: Consolidated sandy clay loam with light laterite gravel

FS (sample): 2213 (p)

Ceramic component(s): Atlantic, cLSA

Lithic component(s): MSA/LSA

Groundstone: Handstone

Surface feature(s): Pyrotechnic feature

Primary function(s): Unknown

Notes:

\section{Survey ID: FAL-1140}

Coordinates: $13^{\circ} 51^{\prime} 55.88^{\prime \prime} \mathrm{N}, 12^{\circ} 09^{\prime} 38.86^{\prime \prime} \mathrm{W}$

Date recorded: 4/21/2008 Area $\left(\mathrm{m}^{2}\right): 3797$

Setting: Bank of seasonal drainage in riparian savanna, light groundcover

Soil: Consolidated sandy clay loam with light laterite gravel

FS (sample): Not collected

Ceramic component(s): cLSA

Lithic component(s): MSA/LSA

Groundstone: Handstone

Primary function(s): Unknown

Notes:

Survey ID: FAL-1141

Coordinates: $13^{\circ} 51^{\prime} 42.10^{\prime \prime} \mathrm{N}, 12^{\circ} 09^{\prime} 44.87^{\prime \prime} \mathrm{W}$

Date recorded: 4/21/2008 Area $\left(\mathrm{m}^{2}\right): 2114$

Setting: Bluff in riparian savanna, modest groundcover

Soil: Consolidated clay loam with modest laterite gravel

FS (sample): Not collected

Ceramic component(s): cLSA

Lithic component(s): MSA/LSA

Groundstone: Handstone

Surface feature(s): Collapsed daub

Primary function(s): Unknown

Notes: 
Survey ID: FAL-1142

Coordinates: $13^{\circ} 58^{\prime} 28.91^{\prime \prime} \mathrm{N}, 12^{\circ} 12^{\prime} 39.81^{\prime \prime} \mathrm{W}$

Date recorded: $4 / 21 / 2008 \quad$ Area $\left(\mathrm{m}^{2}\right): 1578$

Setting: Eroded basin in open savanna, light groundcover

Soil: Semi-consolidated sandy clay with patches of laterite gravel

FS (sample): 2220 (s); 2214 (p)

Ceramic component(s): cLSA, Iron Age

Lithic component(s): MSA/LSA

Groundstone: Basin, Hachette, Handstone

Primary function(s): Unknown

Notes:

\section{Survey ID: FAL-1143}

Coordinates: $13^{\circ} 58 ' 37.41 " \mathrm{~N}, 12^{\circ} 12^{\prime} 39.37^{\prime \prime} \mathrm{W}$

Date recorded: $4 / 21 / 2008 \quad$ Area $\left(\mathrm{m}^{2}\right): 23,469$

Setting: Eroded basin in open savanna, light groundcover

Soil: Consolidated sandy clay loam with modest laterite gravel

FS (sample): 2219 (s); 2216 (p)

Ceramic component(s): cLSA

Lithic component(s): Late Acheulean, MSA/LSA

Groundstone: Hachette

Other artifacts: Netweight

Primary function(s): Unknown

Notes:

\section{Survey ID: FAL-1144-01}

Coordinates: $13^{\circ} 58^{\prime} 50.25^{\prime \prime} \mathrm{N}, 12^{\circ} 12^{\prime} 31.96^{\prime \prime} \mathrm{W}$

Date recorded: 4/21/2008 Area $\left(\mathrm{m}^{2}\right): 1279$

Setting: Eroded basin in open savanna, light groundcover

Soil: Consolidated sandy clay loam with modest laterite gravel

FS (sample): Not collected

Ceramic component(s): None

Lithic component(s): MSA/LSA

Primary function(s): Unknown

Notes: Initially recorded as FAL-IO-084

\section{Survey ID: FAL-1144-02}

Coordinates: $13^{\circ} 58^{\prime} 46.73^{\prime \prime} \mathrm{N}, 12^{\circ} 12^{\prime} 39.08^{\prime \prime} \mathrm{W}$

Date recorded: 4/21/2008 Area $\left(\mathrm{m}^{2}\right): 2356$

Setting: Eroded basin in open savanna, light groundcover

Soil: Consolidated sandy clay loam with modest laterite gravel

FS (sample): Not collected

Ceramic component(s): cLSA

Lithic component(s): MSA/LSA

Groundstone: Handstone

Primary function(s): Unknown

Notes:
Survey ID: FAL-1144-03

Coordinates: $13^{\circ} 58^{\prime} 51.10^{\prime \prime} \mathrm{N}, 12^{\circ} 12^{\prime} 35.07^{\prime \prime} \mathrm{W}$

Date recorded: 4/21/2008 Area $\left(\mathrm{m}^{2}\right): 5394$

Setting: Eroded basin in open savanna, light groundcover

Soil: Consolidated sandy clay loam with modest laterite gravel

FS (sample): Not collected

Ceramic component(s): cLSA

Lithic component(s): MSA/LSA

Primary function(s): Unknown

Notes:

\section{Survey ID: FAL-1145}

Coordinates: $13^{\circ} 58^{\prime} 58.63^{\prime \prime} \mathrm{N}, 12^{\circ} 12^{\prime} 32.74^{\prime \prime} \mathrm{W}$

Date recorded: 4/21/2008 Area $\left(\mathrm{m}^{2}\right): 568$

Setting: Low ridge in open savanna, modest groundcover

Soil: Consolidated sandy clay loam with dense laterite gravel

FS (sample): Not collected

Ceramic component(s): cLSA

Lithic component(s): MSA/LSA

Primary function(s): Unknown

Notes:

\section{Survey ID: FAL-1146}

Coordinates: $13^{\circ} 59^{\prime} 04.44^{\prime \prime} \mathrm{N}, 12^{\circ} 12^{\prime} 29.30^{\prime \prime} \mathrm{W}$

Date recorded: 4/21/2008 Area $\left(\mathrm{m}^{2}\right): 1672$

Setting: Low ridge in open savanna, modest groundcover

Soil: Consolidated sandy clay loam with dense laterite gravel

FS (sample): Not collected

Ceramic component(s): cLSA

Lithic component(s): MSA/LSA

Groundstone: Handstone

Primary function(s): Unknown

Notes:

Survey ID: FAL-1147-01

Coordinates: $13^{\circ} 599^{\prime} 08.32^{\prime \prime} \mathrm{N}, 12^{\circ} 12^{\prime} 31.98^{\prime \prime} \mathrm{W}$

Date recorded: 4/21/2008 Area $\left(\mathrm{m}^{2}\right): 1226$

Setting: Steep embankment in wooded savanna, light groundcover

Soil: Consolidated sandy clay with patches of laterite gravel

FS (sample): 2217 (s)

Ceramic component(s): None

Lithic component(s): Late Acheulean, MSA/LSA

Groundstone: Handstone

Primary function(s): Unknown

Notes: 
Survey ID: FAL-1147-02

Coordinates: $13^{\circ} 59^{\prime} 08.15^{\prime \prime} \mathrm{N}, 12^{\circ} 12^{\prime} 35.88^{\prime \prime} \mathrm{W}$

Date recorded: 4/22/2008 Area $\left(\mathrm{m}^{2}\right): 579$

Setting: Steep embankment in wooded savanna, light groundcover

Soil: Consolidated sandy clay with patches of laterite gravel

FS (sample): 2218 (p)

Ceramic component(s): None

Lithic component(s): Late Acheulean

Primary function(s): Unknown

Notes: Initially recorded as FAL-IO-087

\section{Survey ID: FAL-1149}

Coordinates: $13^{\circ} 53^{\prime} 15.09^{\prime \prime} \mathrm{N}, 12^{\circ} 09^{\prime} 45.75^{\prime \prime} \mathrm{W}$

Date recorded: 4/19/2008 Area $\left(\mathrm{m}^{2}\right): 2332$

Setting: Level area in cultivated field, no groundcover

Soil: Semi-consolidated sandy clay loam

FS (sample): Not collected

Ceramic component(s): Atlantic Era

Lithic component(s): None

Groundstone: Handstone

Other artifacts: Pipe

Surface feature(s): Stone circle

Primary function(s): Settlement

Notes:

\section{Systematic Survey: Isolated Occurrences}

\section{Survey ID: FAL-IO-007}

Coordinates: $13^{\circ} 55^{\prime} 07.59 " \mathrm{~N}, 12^{\circ} 13^{\prime} 04.48^{\prime \prime} \mathrm{W}$

Date recorded: 3/25/2008 Area $\left(\mathrm{m}^{2}\right): 67$

FS (sample): Not sampled

Notes: A small scatter of chipped stone debitage

Survey ID: FAL-IO-008

Coordinates: $13^{\circ} 55^{\prime} 03.94 " \mathrm{~N}, 12^{\circ} 13^{\prime} 04.27^{\prime \prime} \mathrm{W}$

Date recorded: 3/25/2008 Area $\left(\mathrm{m}^{2}\right): 126$

FS (sample): Not sampled

Notes: A small scatter of chipped stone debitage

\section{Survey ID: FAL-IO-009}

Coordinates: $13^{\circ} 55^{\prime} 02.44^{\prime \prime} \mathrm{N}, 12^{\circ} 13^{\prime} 06.84^{\prime \prime} \mathrm{W}$

Date recorded: 3/25/2008

Area $\left(\mathrm{m}^{2}\right): 74$

FS (sample): Not sampled

Notes: A small scatter of chipped stone debitage

\section{Survey ID: FAL-IO-010}

Coordinates: $13^{\circ} 55^{\prime} 01.74 " \mathrm{~N}, 12^{\circ} 13^{\prime} 30.50^{\prime \prime} \mathrm{W}$

Date recorded: $3 / 25 / 2008$ Area $\left(\mathrm{m}^{2}\right): 80$

FS (sample): Not collected

Notes: A small scatter of chipped stone debitage

\section{Survey ID: FAL-IO-011}

Coordinates: $13^{\circ} 54^{\prime} 35.37^{\prime \prime} \mathrm{N}, 12^{\circ} 13^{\prime} 38.84^{\prime \prime} \mathrm{W}$

Date recorded: 3/25/2008 Area $\left(\mathrm{m}^{2}\right): 25$

FS (sample): Not collected

Notes: An isolated rectilinear stone feature
Survey ID: FAL-IO-012

Coordinates: $13^{\circ} 54^{\prime} 50.07 " \mathrm{~N}, 12^{\circ} 12^{\prime} 38.8^{\prime \prime} \mathrm{W}$

Date recorded: 3/25/2008 Area $\left(\mathrm{m}^{2}\right): 87$

FS (sample): Not collected

Notes: A small scatter of chipped stone debitage

\section{Survey ID: FAL-IO-015}

Coordinates: $13^{\circ} 52^{\prime} 44.36^{\prime \prime} \mathrm{N}, 12^{\circ} 11^{\prime} 23.23^{\prime \prime} \mathrm{W}$

Date recorded: 4/1/2008 Area $\left(\mathrm{m}^{2}\right): 90$

FS (sample): Not collected

Notes: A small scatter of chipped stone debitage

\section{Survey ID: FAL-IO-016}

Coordinates: $13^{\circ} 52^{\prime} 44.84^{\prime \prime} \mathrm{N}, 12^{\circ} 11^{\prime} 28.97 " \mathrm{~W}$

Date recorded: 4/1/2008

Area $\left(\mathrm{m}^{2}\right): 103$

FS (sample): 2076 (p)

Notes: A small scatter of chipped stone debitage and cLSA pottery sherds

\section{Survey ID: FAL-IO-017}

Coordinates: $13^{\circ} 57^{\prime} 52.74^{\prime \prime} \mathrm{N}, 12^{\circ} 12^{\prime} 47.75^{\prime \prime} \mathrm{W}$ Date recorded: 4/2/2008 Area $\left(\mathrm{m}^{2}\right): 77$

FS (sample): 2080 (p)

Notes: A small scatter of chipped stone debitage

Survey ID: FAL-IO-018

Coordinates: $13^{\circ} 58^{\prime} 02.12^{\prime \prime} \mathrm{N}, 12^{\circ} 14^{\prime} 09.22^{\prime \prime} \mathrm{W}$

Date recorded: 4/2/2008 Area $\left(\mathrm{m}^{2}\right): 83$

FS (sample): 2084 (p)

Notes: An isolated pyrotechnic feature associated with several Atlantic Era pottery sherds

\section{Survey ID: FAL-IO-019}

Coordinates: $13^{\circ} 56^{\prime} 02.14^{\prime \prime} \mathrm{N}, 12^{\circ} 12^{\prime} 31.61^{\prime \prime} \mathrm{W}$

Date recorded: 4/2/2008 Area $\left(\mathrm{m}^{2}\right): 20$

FS (sample): 2086 (p)

Notes: An isolated terracotta pipe fragment

\section{Survey ID: FAL-IO-020}

Coordinates: $13^{\circ} 51^{\prime} 41.48^{\prime \prime} \mathrm{N}, 12^{\circ} 07^{\prime} 56.73^{\prime \prime} \mathrm{W}$

Date recorded: $4 / 6 / 2008 \quad$ Area $\left(\mathrm{m}^{2}\right): 329$

FS (sample): 2090 (p)

Notes: A small scatter of Atlantic Era pottery sherds

\section{Survey ID: FAL-IO-021}

Coordinates: $13^{\circ} 51^{\prime} 45.53^{\prime \prime} \mathrm{N}, 1^{\circ} 08^{\prime} 51.17^{\prime \prime} \mathrm{W}$

Date recorded: 4/6/2008 Area $\left(\mathrm{m}^{2}\right): 87$

FS (sample): 2092 (p)

Notes: A small scatter of chipped stone debitage

\section{Survey ID: FAL-IO-022}

Coordinates: $13^{\circ} 52^{\prime} 18.89^{\prime \prime} \mathrm{N}, 12^{\circ} 09^{\prime} 14.02^{\prime \prime} \mathrm{W}$

Date recorded: 4/7/2008 Area $\left(\mathrm{m}^{2}\right): 5817$

FS (sample): 2106 (p)

Notes: An extremely diffuse scatter of non-diagnostic pottery sherds 
Survey ID: FAL-IO-023

Coordinates: $13^{\circ} 52^{\prime} 34.02^{\prime \prime} \mathrm{N}, 12^{\circ} 09^{\prime} 27.90^{\prime \prime} \mathrm{W}$

Date recorded: $4 / 7 / 2008 \quad$ Area $\left(\mathrm{m}^{2}\right): 94$

FS (sample): Not collected

Notes: A small scatter of chipped stone debitage

\section{Survey ID: FAL-IO-024}

Coordinates: $13^{\circ} 52^{\prime} 37.71^{\prime \prime} \mathrm{N}, 12^{\circ} 09^{\prime} 32.85^{\prime \prime} \mathrm{W}$

Date recorded: $4 / 7 / 2008 \quad$ Area $\left(\mathrm{m}^{2}\right): 65$

FS (sample): 2105 (p)

Notes: A small scatter of chipped stone debitage and cLSA pottery sherds

Survey ID: FAL-IO-025

Coordinates: $13^{\circ} 52^{\prime} 03.64 " \mathrm{~N}, 1^{\circ} 09^{\prime} 01.78^{\prime \prime} \mathrm{W}$

Date recorded: $4 / 7 / 2008 \quad$ Area $\left(\mathrm{m}^{2}\right): 6$

FS (sample): 2100 (p)

Notes: An isolated terracotta pipe fragment

Survey ID: FAL-IO-029

Coordinates: $13^{\circ} 52^{\prime} 44.12^{\prime \prime} \mathrm{N}, 1^{\circ} 08^{\prime} 47.99^{\prime \prime} \mathrm{W}$

Date recorded: $4 / 8 / 2008 \quad$ Area $\left(\mathrm{m}^{2}\right): 70$

FS (sample): Not collected

Notes: An extremely diffuse scatter of Atlantic Era pottery sherds

Survey ID: FAL-IO-030

Coordinates: $13^{\circ} 52^{\prime} 45.94^{\prime \prime} \mathrm{N}, 1^{\circ} 08^{\prime} 32.75^{\prime \prime} \mathrm{W}$

Date recorded: 4/8/2008

Area $\left(\mathrm{m}^{2}\right): 42$

FS (sample): Not collected

Notes: A small scatter of chipped stone debitage

\section{Survey ID: FAL-IO-032}

Coordinates: $13^{\circ} 53^{\prime} 09.71^{\prime \prime} \mathrm{N}, 12^{\circ} 09^{\prime} 37.23^{\prime \prime} \mathrm{W}$

Date recorded: 4/8/2008 Area $\left(\mathrm{m}^{2}\right): 101$

FS (sample): Not collected

Notes: A small scatter of chipped stone debitage

\section{Survey ID: FAL-IO-033}

Coordinates: $13^{\circ} 54^{\prime} 50.76^{\prime \prime} \mathrm{N}, 12^{\circ} 12^{\prime} 08.08^{\prime \prime} \mathrm{W}$

Date recorded: $4 / 9 / 2008 \quad$ Area $\left(\mathrm{m}^{2}\right): 81$

FS (sample): 2117 (p)

Notes: A small scatter of Iron Age pottery sherds

Survey ID: FAL-IO-034

Coordinates: $13^{\circ} 54^{\prime} 47.07^{\prime \prime} \mathrm{N}, 12^{\circ} 12^{\prime} 02.86^{\prime \prime} \mathrm{W}$

Date recorded: $4 / 9 / 2008 \quad$ Area $\left(\mathrm{m}^{2}\right): 60$

FS (sample): 2118 (p)

Notes: A small scatter of Iron Age pottery sherds

Survey ID: FAL-IO-035

Coordinates: $13^{\circ} 54^{\prime} 46.07^{\prime \prime} \mathrm{N}, 12^{\circ} 12^{\prime} 01.02^{\prime \prime} \mathrm{W}$

Date recorded: $4 / 9 / 2008 \quad$ Area $\left(\mathrm{m}^{2}\right): 76$

FS (sample): 2121 (p)

Notes: A small scatter of Atlantic Era pottery sherds

Survey ID: FAL-IO-036

Coordinates: $13^{\circ} 54^{\prime} 36.12^{\prime \prime} \mathrm{N}, 12^{\circ} 11^{\prime} 44.17^{\prime \prime} \mathrm{W}$

Date recorded: 4/9/2008 Area $\left(\mathrm{m}^{2}\right): 61$

FS (sample): 2126 (p)

Notes: A small scatter of Atlantic Era pottery sherds
Survey ID: FAL-IO-037

Coordinates: $13^{\circ} 54^{\prime} 35.54 " \mathrm{~N}, 12^{\circ} 11^{\prime} 42.49^{\prime \prime} \mathrm{W}$

Date recorded: $4 / 9 / 2008 \quad$ Area $\left(\mathrm{m}^{2}\right): 30$

FS (sample): 2127 (p)

Notes: A small scatter of Atlantic Era pottery sherds

Survey ID: FAL-IO-038

Coordinates: $13^{\circ} 54^{\prime} 23.83^{\prime \prime} \mathrm{N}, 12^{\circ} 11^{\prime} 24.86^{\prime \prime} \mathrm{W}$

Date recorded: 4/9/2008 Area $\left(\mathrm{m}^{2}\right): 193$

FS (sample): Not collected

Notes: A small scatter of non-diagnostic pottery

Survey ID: FAL-IO-039

Coordinates: $13^{\circ} 54^{\prime} 22.63^{\prime \prime} \mathrm{N}, 12^{\circ} 11^{\prime} 24.12^{\prime \prime} \mathrm{W}$

Date recorded: 4/9/2008

Area $\left(\mathrm{m}^{2}\right): 77$

FS (sample): Not collected

Notes: A small scatter of non-diagnostic pottery

Survey ID: FAL-IO-040

Coordinates: $13^{\circ} 54^{\prime} 14.87^{\prime \prime} \mathrm{N}, 12^{\circ} 11^{\prime} 19.86^{\prime \prime} \mathrm{W}$

Date recorded: 4/9/2008 Area $\left(\mathrm{m}^{2}\right): 85$

FS (sample): Not collected

Notes: A small scatter of non-diagnostic pottery

Survey ID: FAL-IO-041

Coordinates: $13^{\circ} 54 ' 16.02^{\prime \prime} \mathrm{N}, 12^{\circ} 11^{\prime} 12.43^{\prime \prime} \mathrm{W}$

Date recorded: 4/11/2008 Area $\left(\mathrm{m}^{2}\right): 2094$

FS (sample): Not collected

Notes: A diffuse scatter of chipped stone debitage

Survey ID: FAL-IO-042

Coordinates: $13^{\circ} 54^{\prime} 13.14 " \mathrm{~N}, 12^{\circ} 10^{\prime} 42.22^{\prime \prime} \mathrm{W}$

Date recorded: $4 / 13 / 2008 \quad$ Area $\left(\mathrm{m}^{2}\right): 76$

FS (sample): Not collected

Notes: A small scatter of chipped stone debitage and angular stone cobbles

Survey ID: FAL-IO-043

Coordinates: $13^{\circ} 53^{\prime} 04.29 " \mathrm{~N}, 12^{\circ} 09^{\prime} 47.97 " \mathrm{~W}$

Date recorded: $4 / 13 / 2008 \quad$ Area $\left(\mathrm{m}^{2}\right): 95$

FS (sample): Not collected

Notes: A small scatter of Atlantic Era pottery sherds

Survey ID: FAL-IO-044

Coordinates: $13^{\circ} 53^{\prime} 57.76^{\prime \prime} \mathrm{N}, 12^{\circ} 10^{\prime} 06.37^{\prime \prime} \mathrm{W}$

Date recorded: $4 / 13 / 2008 \quad$ Area $\left(\mathrm{m}^{2}\right): 590$

FS (sample): Not collected

Notes: An extremely diffuse scatter of chipped stone debitage

Survey ID: FAL-IO-046

Coordinates: $13^{\circ} 56^{\prime} 57.19^{\prime \prime} \mathrm{N}, 12^{\circ} 12^{\prime} 27.95^{\prime \prime} \mathrm{W}$

Date recorded: $4 / 14 / 2008 \quad$ Area $\left(\mathrm{m}^{2}\right): 74$

FS (sample): Not collected

Notes: An extremely diffuse scatter of non-diagnostic pottery sherds 
Survey ID: FAL-IO-047

Coordinates: $13^{\circ} 57^{\prime} 26.20^{\prime \prime} \mathrm{N}, 12^{\circ} 12^{\prime} 54.66^{\prime \prime} \mathrm{W}$

Date recorded: 4/14/2008 Area $\left(\mathrm{m}^{2}\right): 324$

FS (sample): Not collected

Notes: An extremely diffuse scatter of chipped stone debitage

Survey ID: FAL-IO-048

Coordinates: $13^{\circ} 56^{\prime} 43.63^{\prime \prime} \mathrm{N}, 12^{\circ} 12^{\prime} 37.66^{\prime \prime} \mathrm{W}$

Date recorded: $4 / 14 / 2008 \quad$ Area $\left(\mathrm{m}^{2}\right): 83$

FS (sample): Not collected

Notes: A small scatter of chipped stone debitage

Survey ID: FAL-IO-049

Coordinates: $13^{\circ} 57^{\prime} 20.59^{\prime \prime} \mathrm{N}, 12^{\circ} 13^{\prime} 14.43^{\prime \prime} \mathrm{W}$

Date recorded: 4/14/2008 Area $\left(\mathrm{m}^{2}\right): 508$

FS (sample): Not collected

Notes: An extremely diffuse scatter of Atlantic Era pottery sherds

\section{Survey ID: FAL-IO-050}

Coordinates: $13^{\circ} 56^{\prime} 24.92^{\prime \prime} \mathrm{N}, 12^{\circ} 12^{\prime} 13.30^{\prime \prime} \mathrm{W}$

Date recorded: 4/15/2008 Area $\left(\mathrm{m}^{2}\right): 4499$

FS (sample): Not collected

Notes: An extremely diffuse scatter of Atlantic Era pottery sherds

Survey ID: FAL-IO-052

Coordinates: $13^{\circ} 56^{\prime} 47.35^{\prime \prime} \mathrm{N}, 12^{\circ} 12^{\prime} 08.69^{\prime \prime} \mathrm{W}$

Date recorded: 4/15/2008 Area $\left(\mathrm{m}^{2}\right): 1683$

FS (sample): Not collected

Notes: A small scatter of chipped quartz debitage

\section{Survey ID: FAL-IO-053}

Coordinates: $13^{\circ} 57^{\prime} 10.93^{\prime \prime} \mathrm{N}, 12^{\circ} 12^{\prime} 25.59^{\prime \prime} \mathrm{W}$

Date recorded: 4/15/2008 Area $\left(\mathrm{m}^{2}\right): 297$

FS (sample): Not collected

Notes: A small scatter of chipped quartz debitage

Survey ID: FAL-IO-055

Coordinates: $13^{\circ} 577^{\prime} 17.19^{\prime \prime} \mathrm{N}, 12^{\circ} 12^{\prime} 06.87^{\prime \prime} \mathrm{W}$

Date recorded: 4/15/2008 Area $\left(\mathrm{m}^{2}\right): 127$

FS (sample): Not collected

Notes: A small scatter of non-diagnostic pottery

\section{Survey ID: FAL-IO-056}

Coordinates: $13^{\circ} 57^{\prime} 07.79^{\prime \prime} \mathrm{N}, 12^{\circ} 12^{\prime} 07.34^{\prime \prime} \mathrm{W}$

Date recorded: 4/15/2008 Area $\left(\mathrm{m}^{2}\right): 871$

FS (sample): Not collected

Notes: A diffuse scatter of chipped quartz debitage

Survey ID: FAL-IO-057

Coordinates: $13^{\circ} 56^{\prime} 26.24 " \mathrm{~N}, 12^{\circ} 11^{\prime} 58.85^{\prime \prime} \mathrm{W}$

Date recorded: 4/15/2008 Area $\left(\mathrm{m}^{2}\right): 1283$

FS (sample): Not collected

Notes: A diffuse scatter of chipped quartz debitage
Survey ID: FAL-IO-058

Coordinates: $13^{\circ} 55^{\prime} 36.81^{\prime \prime} \mathrm{N}, 12^{\circ} 12^{\prime} 42.79^{\prime \prime} \mathrm{W}$

Date recorded: 4/15/2008

FS (sample): Not collected

Area $\left(\mathrm{m}^{2}\right): 51$

Notes: A small scatter of chipped stone debitage

Survey ID: FAL-IO-059

Coordinates: $13^{\circ} 55^{\prime} 30.76^{\prime \prime} \mathrm{N}, 12^{\circ} 12^{\prime} 45.26^{\prime \prime} \mathrm{W}$

Date recorded: 4/15/2008 Area $\left(\mathrm{m}^{2}\right): 82$

FS (sample): Not collected

Notes: A deposit of chipped stone debitage, groundstone, and cLSA pottery sherds eroding from a seasonal drainage

\section{Survey ID: FAL-IO-060}

Coordinates: $13^{\circ} 54^{\prime} 44.75^{\prime \prime} \mathrm{N}, 12^{\circ} 14^{\prime} 15.42^{\prime \prime} \mathrm{W}$

Date recorded: 4/16/2008

Area $\left(\mathrm{m}^{2}\right): 46$

FS (sample): Not collected

Notes: An isolated circular stone installation

\section{Survey ID: FAL-IO-061}

Coordinates: $13^{\circ} 54^{\prime} 57.32^{\prime \prime} \mathrm{N}, 12^{\circ} 13^{\prime} 57.78^{\prime \prime} \mathrm{W}$

Date recorded: $4 / 16 / 2008 \quad$ Area $\left(\mathrm{m}^{2}\right): 79$

FS (sample): Not collected

Notes: A small scatter of chipped stone debitage and cLSA pottery sherds

Survey ID: FAL-IO-062

Coordinates: $13^{\circ} 54^{\prime} 37.47^{\prime \prime} \mathrm{N}, 12^{\circ} 12^{\prime} 05.95^{\prime \prime} \mathrm{W}$

Date recorded: $4 / 16 / 2008$ Area $\left(\mathrm{m}^{2}\right): 77$

FS (sample): Not collected

Notes: A small scatter of Atlantic Era pottery sherds

Survey ID: FAL-IO-063

Coordinates: $13^{\circ} 54^{\prime} 22.04^{\prime \prime} \mathrm{N}, 12^{\circ} 11^{\prime} 41.26^{\prime \prime} \mathrm{W}$

Date recorded: 4/16/2008 Area $\left(\mathrm{m}^{2}\right): 434$

FS (sample): 2183 (p)

Notes: An extremely diffuse scatter of chipped stone debitage

\section{Survey ID: FAL-IO-064}

Coordinates: $13^{\circ} 54^{\prime} 14.30^{\prime \prime} \mathrm{N}, 12^{\circ} 11^{\prime} 40.48^{\prime \prime} \mathrm{W}$

Date recorded: 4/16/2008

FS (sample): Not collected

Notes: A diffuse scatter of chipped stone debitage and cLSA pottery sherds

Survey ID: FAL-IO-065

Coordinates: $13^{\circ} 54^{\prime} 07.46^{\prime \prime} \mathrm{N}, 12^{\circ} 11^{\prime} 52.34^{\prime \prime} \mathrm{W}$

Date recorded: 4/16/2008

FS (sample): Not collected

Notes: A small scatter of Iron Age pottery sherds

\section{Survey ID: FAL-IO-066}

Coordinates: $13^{\circ} 54^{\prime} 05.85^{\prime \prime} \mathrm{N}, 12^{\circ} 12^{\prime} 08.74^{\prime \prime} \mathrm{W}$

Date recorded: $4 / 16 / 2008 \quad$ Area $\left(\mathrm{m}^{2}\right)$ : 90

FS (sample): Not collected

Notes: A small scatter of chipped quartz debitage 
Survey ID: FAL-IO-068

Coordinates: $13^{\circ} 53^{\prime} 50.30^{\prime \prime} \mathrm{N}, 12^{\circ} 11^{\prime} 00.87^{\prime \prime} \mathrm{W}$

Date recorded: 4/17/2008 Area $\left(\mathrm{m}^{2}\right): 1204$

FS (sample): Not collected

Notes: A small scatter of chipped quartz debitage

\section{Survey ID: FAL-IO-069}

Coordinates: $13^{\circ} 53^{\prime} 44.84^{\prime \prime} \mathrm{N}, 12^{\circ} 10^{\prime} 36.98^{\prime \prime} \mathrm{W}$

Date recorded: 4/17/2008 Area $\left(\mathrm{m}^{2}\right): 1472$

FS (sample): Not collected

Notes: An extremely diffuse scatter of Atlantic Era pottery sherds

\section{Survey ID: FAL-IO-070}

Coordinates: $13^{\circ} 53$ '33.72" N, 12¹0'58.45" W

Date recorded: 4/17/2008 Area $\left(\mathrm{m}^{2}\right): 75$

FS (sample): Not collected

Notes: A diffuse scatter of chipped stone debitage and Atlantic Era pottery sherds

\section{Survey ID: FAL-IO-071}

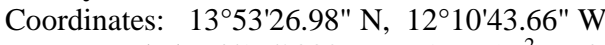

Date recorded: 4/17/2008 Area $\left(\mathrm{m}^{2}\right)$ : 94

FS (sample): Not collected

Notes: A small scatter of chipped stone debitage

\section{Survey ID: FAL-IO-073}

Coordinates: $13^{\circ} 58^{\prime} 52.92^{\prime \prime} \mathrm{N}, 12^{\circ} 11^{\prime} 09.85^{\prime \prime} \mathrm{W}$

Date recorded: 4/20/2008 Area $\left(\mathrm{m}^{2}\right): 4$

FS (sample): Not collected

Notes: An isolated quartz hammerstone

\section{Survey ID: FAL-IO-075}

Coordinates: $13^{\circ} 57^{\prime} 47.62^{\prime \prime} \mathrm{N}, 12^{\circ} 12^{\prime} 35.37^{\prime \prime} \mathrm{W}$

Date recorded: 4/20/2008 Area $\left(\mathrm{m}^{2}\right): 117$

FS (sample): Not collected

Notes: A diffuse scatter of chipped stone debitage and cLSA pottery sherds

\section{Survey ID: FAL-IO-081}

Coordinates: $13^{\circ} 58^{\prime} 28.92^{\prime \prime} \mathrm{N}, 12^{\circ} 12^{\prime} 36.55^{\prime \prime} \mathrm{W}$

Date recorded: $4 / 21 / 2008 \quad$ Area $\left(\mathrm{m}^{2}\right): 24$

FS (sample): 2215 (s)

Notes: An isolated reconstructable Atlantic Era pottery vessel

Survey ID: FAL-IO-082

Coordinates: $13^{\circ} 58^{\prime} 43.26^{\prime \prime} \mathrm{N}, 12^{\circ} 12^{\prime} 38.58^{\prime \prime} \mathrm{W}$

Date recorded: 4/21/2008 Area $\left(\mathrm{m}^{2}\right): 321$

FS (sample): Not collected

Notes: A diffuse scatter of chipped quartz debitage

\section{Survey ID: FAL-IO-085}

Coordinates: $13^{\circ} 58^{\prime} 51.45^{\prime \prime} \mathrm{N}, 12^{\circ} 12^{\prime} 43.14^{\prime \prime} \mathrm{W}$

Date recorded: 4/22/2008 Area $\left(\mathrm{m}^{2}\right)$ : 96

FS (sample): Not collected

Notes: A small scatter of chipped quartz debitage and cLSA pottery sherds
Survey ID: FAL-IO-090

Coordinates: $13^{\circ} 56^{\prime} 26.24 " \mathrm{~N}, 12^{\circ} 11^{\prime} 44.46^{\prime \prime} \mathrm{W}$

Date recorded: 3/24/2008 Area $\left(\mathrm{m}^{2}\right): 506$

FS (sample): Not collected

Notes: Initially recorded as FAL-1019

\section{Survey ID: FAL-IO-095}

Coordinates: $13^{\circ} 51^{\prime} 42.89^{\prime \prime} \mathrm{N}, 1^{\circ} 09^{\prime} 21.23^{\prime \prime} \mathrm{W}$

Date recorded: $4 / 6 / 2008 \quad$ Area $\left(\mathrm{m}^{2}\right): 7534$

FS (sample): 2093 (p)

Notes: An extremely diffuse scatter of Atlantic Era pottery; initially recorded as FAL-1049a

\section{Survey ID: FAL-IO-097}

Coordinates: $13^{\circ} 57^{\prime} 07.57^{\prime \prime} \mathrm{N}, 12^{\circ} 12^{\prime} 52.05^{\prime \prime} \mathrm{W}$

Date recorded: 4/14/2008

FS (sample): Not collected Area $\left(\mathrm{m}^{2}\right): 136$

Notes: A small scatter of chipped stone debitage and cLSA pottery sherds

\section{Survey ID: FAL-IO-099}

Coordinates: $13^{\circ} 56^{\prime} 38.21^{\prime \prime} \mathrm{N}, 12^{\circ} 12^{\prime} 36.93^{\prime \prime} \mathrm{W}$

Date recorded: 4/14/2008

FS (sample): Not collected Area $\left(\mathrm{m}^{2}\right): 553$

Notes: A small scatter of chipped quartz debitage; initially recorded as FAL-1088

\section{Survey ID: FAL-IO-100}

Coordinates: $13^{\circ} 55^{\prime} 54.83^{\prime \prime} \mathrm{N}, 12^{\circ} 12^{\prime} 34.45^{\prime \prime} \mathrm{W}$

Date recorded: 4/15/2008 Area $\left(\mathrm{m}^{2}\right): 1112$

FS (sample): 2170 (p)

Notes: A diffuse scatter of chipped stone debitage, cLSA pottery sherds, and a polished hematite; initially recorded as FAL-1104 


\section{Guided Survey: Sites}

\section{Survey ID: FAL-1123}

Coordinates: $13^{\circ} 48^{\prime} 45.21^{\prime \prime} \mathrm{N}, 1^{\circ}{ }^{\circ} 07^{\prime} 08.19^{\prime \prime} \mathrm{W}$

Date recorded: 4/19/2008 Area $\left(\mathrm{m}^{2}\right)$ : 44,623

Setting: Bank of seasonal drainage in wooded savanna, modest groundcover

Soil: Consolidated sandy clay loam

FS (sample): 2194 (p)

Ceramic component(s): Atlantic Era

Lithic component(s): None

Groundstone: Basin, Handstone

Metallurgy: Nail (Hf)

Other artifacts: Pipe

Surface feature(s): Collapsed daub, Stone circle, Stone square

Primary function(s): Settlement

Notes: Site comprises three low mounds surrounding a collapsed stone wall fortification (tata)

\section{Survey ID: FAL-1124}

Coordinates: $13^{\circ} 48^{\prime} 53.70^{\prime \prime} \mathrm{N}, 1^{\circ} 06^{\prime} 51.14^{\prime \prime} \mathrm{W}$

Date recorded: 4/19/2008 Area $\left(\mathrm{m}^{2}\right): 796$

Setting: Bank of seasonal drainage in open savanna, light groundcover

Soil: Consolidated sandy clay loam with light laterite gravel

FS (sample): Not collected

Ceramic component(s): Atlantic Era

Lithic component(s): None

Metallurgy: Iron tool/ornament

Surface feature(s): Stone circle

Primary function(s): Settlement

Notes:

Survey ID: FAL-1125-01

Coordinates: $13^{\circ} 49^{\prime} 33.55^{\prime \prime} \mathrm{N}, 12^{\circ} 06^{\prime} 18.73^{\prime \prime} \mathrm{W}$

Date recorded: $4 / 19 / 2008 \quad$ Area $\left(\mathrm{m}^{2}\right): 890$

Setting: Bank of seasonal drainage in open savanna, light groundcover

Soil: Consolidated sandy clay loam with laterite gravel

FS (sample): Not collected

Ceramic component(s): Atlantic Era

Lithic component(s): None

Other artifacts: Glass bottle

Surface feature(s): Stone circle

Primary function(s): Settlement

Notes:
Survey ID: FAL-1125-02

Coordinates: $13^{\circ} 49^{\prime} 33.04^{\prime \prime} \mathrm{N}, 1^{\circ} 06^{\prime} 20.14^{\prime \prime} \mathrm{W}$

Date recorded: 4/19/2008 Area $\left(\mathrm{m}^{2}\right): 1691$

Setting: Bank of seasonal drainage in open savanna, light groundcover

Soil: Consolidated sandy clay loam with laterite gravel

FS (sample): 2195 (s)

Ceramic component(s): cLSA

Lithic component(s): MSA/LSA

Groundstone: Hachette, Handstone

Primary function(s): Unknown

Notes:

Survey ID: FAL-1148 (Baganafara)

Coordinates: $13^{\circ} 51^{\prime} 42.04^{\prime \prime} \mathrm{N}, 12^{\circ} 03^{\prime} 51.63^{\prime \prime} \mathrm{W}$

Date recorded: 6/22/2007 Area $\left(\mathrm{m}^{2}\right): 88,162$

Setting: Low mounds in wooded savanna, light groundcover

Soil: Consolidated sandy clay loam with light laterite gravel

FS (sample): Madina Foulbé 1-3, IFAN 2007 (p)

Ceramic component(s): Iron Age

Lithic component(s): Unknown

Groundstone: Basin, Handstone

Metallurgy: Iron tool/ornament

Other artifacts: Whorl

Surface feature(s): Stone circle, Pyrotechnic feature

Primary function(s): Settlement

Notes: Features include several circular stone architectural foundations; initially recorded as Madina Foulbé 1-3

\section{Survey ID: FAL-1150 (Missira)}

Coordinates: $13^{\circ} 59 ' 35.16^{\prime \prime} \mathrm{N}, 12^{\circ} 12^{\prime} 33.84^{\prime \prime} \mathrm{W}$

Date recorded: 6/22/2007 Area $\left(\mathrm{m}^{2}\right): 4182$

Setting: Level area in cultivated field, no groundcover

Soil: A semi-consolidated clay loam with laterite gravel

FS (sample): Tomboura 3, IFAN 2007 (p)

Ceramic component(s): Atlantic Era

Metallurgy: Iron tool/ornament

Other artifacts: Glass bead

Surface feature(s): Stone square

Primary function(s): Settlement

Notes: Initially recorded as Tomboura 3

\section{Survey ID: FAL-1151 (Karé 2)}

Coordinates: $13^{\circ} 52 ' 37.24 " \mathrm{~N}, 12^{\circ} 08^{\prime 28.98 " ~ W}$

Date recorded: 6/20/2007 Area $\left(\mathrm{m}^{2}\right): 7415$

Setting: Low rise in wooded savanna, light groundcover

Soil: Consolidated clay loam with dense laterite gravel

FS (sample): Karé 2, IFAN 2007 (p)

Ceramic component(s): None

Lithic component(s): MSA/LSA

Primary function(s): Unknown

Notes: Initially recorded as Karé 2 
Survey ID: FAL-1152 (Diambaloye 1)

Coordinates: $13^{\circ} 56^{\prime} 04.75^{\prime \prime} \mathrm{N}, 1^{\circ}{ }^{\circ} 06^{\prime} 08.99^{\prime \prime} \mathrm{W}$

Date recorded: 6/19/2007 Area $\left(\mathrm{m}^{2}\right): 180$

Setting: Low ridge in wooded savanna, light groundcover

Soil: Consolidated sandy clay loam with dense laterite gravel

FS (sample): Diambaloye 1, IFAN 2007 (p)

Ceramic component(s): None

Lithic component(s): MSA/LSA

Primary function(s): Unknown

Notes: Initially recorded as Diambaloye 1

Survey ID: FAL-1153 (Diambaloye 2)

Coordinates: $13^{\circ} 55^{\prime} 31.19^{\prime \prime} \mathrm{N}, 1^{\circ} 06^{\prime} 01.47^{\prime \prime} \mathrm{W}$

Date recorded: 6/19/2007 Area $\left(\mathrm{m}^{2}\right): 734$

Setting: Low ridge in wooded savanna, light groundcover

Soil: Exposed bedrock and laterite

FS (sample): Diambaloye 2, IFAN 2007 (p)

Ceramic component(s): None

Lithic component(s): MSA/LSA

Primary function(s): Unknown

Notes: Initially recorded as Diambaloye 2

Survey ID: FAL-1154 (Diambaloye 3)

Coordinates: $13^{\circ} 54 ' 34.47^{\prime \prime} \mathrm{N}, 12^{\circ} 05^{\prime} 52.13^{\prime \prime} \mathrm{W}$

Date recorded: 6/19/2007 Area $\left(\mathrm{m}^{2}\right): 1384$

Setting: Low ridge in wooded savanna, light groundcover

Soil: Exposed bedrock and laterite

FS (sample): Diambaloye 3, IFAN 2007 (p)

Ceramic component(s): None

Lithic component(s): MSA/LSA

Primary function(s): Unknown

Notes: Initially recorded as Diambaloye 3 


\section{APPENDIX B DIOUBOYE: SURFACE ARCHAEOLOGY}

Beyond their role in defining site boundaries and guiding the placement of excavation trenches, surface archaeological data have not often found their way into studies of intra-site spatial dynamics in West Africa. In part, this may reflect the recognition that, while subsurface archaeological remains experience natural and cultural transformations, the cumulative processes affecting surface remains more strongly conflate their temporal dimensions and obscure their spatial ones (see discussions in Lewarch and O'Brien 1981; Redman 1987). Acknowledging these limitations, surface data nonetheless provide a broad, if necessarily coarse, window onto spatial relations among architectural features and other material practices within a site at a scale exceeding that achieved in the average horizontal exposure of subsurface deposits.

Surface distributions of artifacts have, for example, proven quite useful for investigating the spatial organization of food and craft production and refuse disposal activities in archaeological contexts ranging from villages in the American Southwest (Bayman and Sanchez 1998), to urban centers in Mesoamerica (Cowgill et al. 1984; Killion et al. 1989), to expansive tells in Anatolia (Redman and Watson 1970). In the Middle Niger region a surface-oriented study by Mary Clark (2003) successfully charted patterns of activity segregation and intensification as a means of evaluating models of social complexity, including institutions for economic specialization, at Jenné-jeno and surrounding sites. Paralleling research on standing ruins elsewhere around the world (e.g., Kent 1990; Marcus 2003), a growing number of studies in West Africa have looked at surface architecture to consider the economic practices, political strategies, and social identities materialized through the built environment (e.g., Agorsah 1986; DeCorse 2001a; Holl 1993, 2006; Monroe 2010; A. Smith and David 1995). All in all, these examples show how methodological tools that control for post-depositional biases can unlock the potential of surface data to address questions about the spatial distribution, intensity, and diversity of socioeconomic practices within a prehistoric village such as Diouboye.

Surface archaeology at Diouboye had three main objectives: (1) to evaluate the sources and intensity of post-depositional disturbance affecting spatial and temporal dimensions of surface remains; (2) to assess the potential stratigraphy and contents of subsurface deposits, thereby guiding the placement of excavation units (see Appendix C); and (3) to obtain data on the architecture and artifacts implicated in various subsistence, craft, and long-distance exchange activities across the site. In order to meet these objectives I directed a program of ground survey, feature mapping, and artifact collection over three successive phases of fieldwork undertaken by CFAP field crews.

During an initial phase of archaeological reconnaissance in 2007, our field crew identified and mapped the boundary of Diouboye using GPS. We also made a purposive 
collection of diagnostic surface artifacts (e.g., earthenware rim sherds, chipped stone tools, and iron artifacts) and noted possible residential areas marked by the presence of surface features resembling granary foundations. The second phase of surface investigation in 2008 comprised ground survey of the entire site and systematic artifact collection over three areas defined by discrete artifact concentrations (Area A, Area A-S, and Area $\mathrm{H}$ ). A comparison of surface and subsurface artifact assemblages in Area A and Area $\mathrm{H}$ then helped to configure strategies for excavation and further surface sampling in 2009. For this final phase of surface investigation, our field crew mapped all recognizable surface features and completed systematic artifact collection across the entire site.

In the following sections I recount the methods for ground survey, feature mapping, and systematic artifact collection at Diouboye. I then summarize the size, topography, post-depositional disturbances, and distributions of surface features and artifacts for each area - the primary spatial unit employed in the analysis of intra-site economic organization and social dynamics in Chapters 7-9.

\section{METHODOLOGY Ground Survey}

The ground survey of Diouboye had three sub-objectives: (1) to establish a network of fixed points for precise spatial control in surface sampling, mapping, and excavation; (2) to characterize the depth and areal extent of stratified archaeological deposits; and (3) to help model post-depositional biases introduced through natural and cultural processes.

Using a Nikon one-second optical theodolite, I first staked out a triangulated network of 16 major control points (Table B.1) with rebar posts at intervals of 50-100 meters. With an adjustment of less than four seconds for each angle in this network, it was possible to achieve a precision of \pm 16.64 centimeters for all major control points (following Pugh 1975:14-41). In the absence of a geological survey benchmark for anchoring this local network to geographic data at other scales, I used a Garmin handheld GPS to record the latitude and longitude of each major control point ( $\pm 5 \mathrm{~m}$ accuracy). I designated Datum 1 on Area A as the origin $(0,0)$ of a site-level grid system for surface artifact collection and feature mapping.

Stationing the theodolite at major control points, I recorded the elevation of 797 minor sample points at intervals chosen according to perceived ground surface variability -more points on heterogeneous terrain, fewer points on homogeneous terrain. Inputting these points into an ArcGIS geodatabase, I used natural-neighbor interpolation to generate an elevation model (1 $\mathrm{m}$ resolution) across the site.

As illustrated in Figure B.1, Diouboye occupies an archaic levee oriented parallel with the Falémé River. While some of the cultural deposits, ranging from 50.5 to 53.1 meters above sea level, conform to this natural topography, others coalesce into at least four discrete mounds. Broad spaces immediately to the east of these mounds may represent public spaces, corrals, and/or places for obtaining mud to build huts and other structures. Approximately 200 meters further to the east, the ground surface rises to form a terrace of weathered laterite and sandstone- the nearest source of rocks for constructing architectural foundations. A broad alluvial terrace stretches down gently west to the Falémé River some 500 meters away. 
Table B.1: Major control points for ground survey at Diouboye

\begin{tabular}{|l|rrc|cc|}
\hline \multirow{2}{*}{$\begin{array}{c}\text { Major Control } \\
\text { Point }\end{array}$} & \multicolumn{2}{|c|}{ Site-Level Grid Coordinates } & \multicolumn{2}{c|}{ UTM (Zone 28N) } \\
& East (m) & North (m) & Altitude (m asl) & Easting & Northing \\
\hline \hline Datum 1 & 0.000 & 0.000 & 53.00 & 803734 & 1539513 \\
\hline Datum 2 & 50.000 & 0.218 & 52.52 & 803794 & 1539518 \\
\hline Datum 3 & 122.783 & -92.662 & 51.99 & 803894 & 1539455 \\
\hline Datum 4 & 186.423 & -188.448 & 51.00 & 803968 & 1539368 \\
\hline Datum 5 & 240.368 & -155.906 & 51.45 & 804018 & 1539410 \\
\hline Datum 6 & 306.063 & -225.905 & 52.64 & 804091 & 1539350 \\
\hline Datum 7 & 391.768 & -240.863 & 52.40 & 804182 & 1539347 \\
\hline Datum 8 & 423.980 & -305.802 & 52.82 & 804213 & 1539288 \\
\hline Datum 9 & -19.919 & 111.846 & 49.39 & 803754 & 1539577 \\
\hline Datum 10 & 254.079 & 9.297 & 50.71 & 804052 & 1539502 \\
\hline Datum 11 & 333.317 & -50.052 & 51.19 & 804132 & 1539463 \\
\hline Datum 12 & 504.650 & -291.093 & 51.25 & 804304 & 1539321 \\
\hline Datum 13 & 578.647 & -290.404 & 51.13 & 804369 & 1539325 \\
\hline Datum 14 & 464.380 & -206.156 & 50.77 & 804241 & 1539388 \\
\hline Datum 15 & 647.356 & -327.323 & 51.04 & 804450 & 1539302 \\
\hline Datum 16 & 690.688 & -396.939 & 50.71 & 804503 & 1539241 \\
\hline
\end{tabular}

Figure B.1: Digital elevation model of Diouboye showing major control points

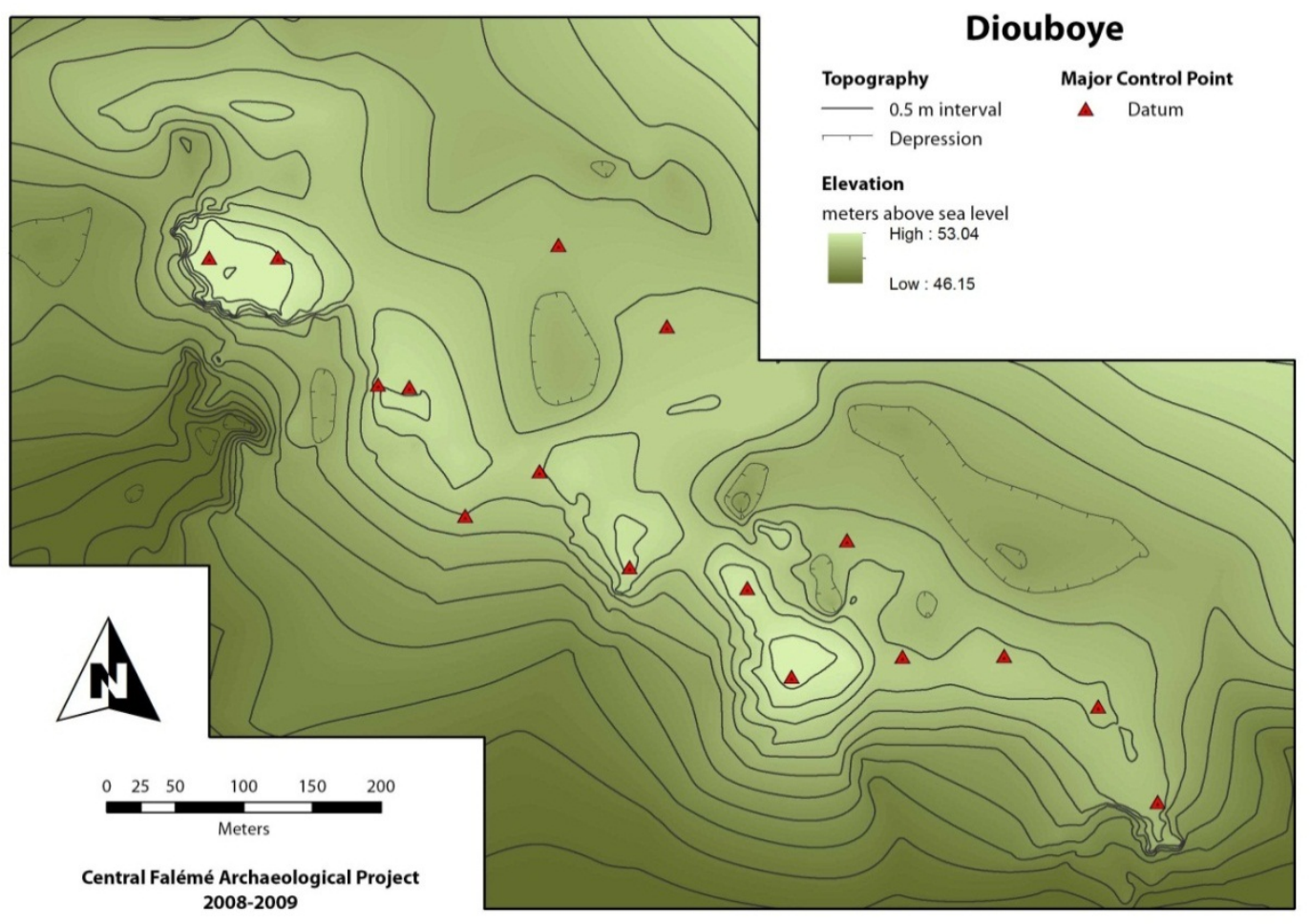




\section{Surface Erosion}

The above elevation model, alongside observations of modern land-use practices and artifact densities, can help to discern where and how erosional processes have contributed to the stratigraphic deflation and/or displacement of archaeological remains. Agro-pastoral practices, for example, have directly and indirectly impacted surface materials at Diouboye. A perennial water source in the nearby Falémé riverbed makes the site a regular encampment for Fulbe (Peul) pastoralists whose livestock tramples artifacts and consumes protective groundcover (Figure B.2a). Although farmers from Goundafa, a village located directly across the river, also incorporate land around the site into their cultivation cycle, they generally avoid the compact and rock-laden matrix of former residential areas (Figure B.2b). By removing natural ground cover, these agro-pastoral practices (with possible antecedents in the Iron Age) have increased levels of hydraulic erosion across the site.

Figure B.2: Modern agro-pastoral practices including (a) livestock pen north of Area M; and (b) land clearance for cultivation south of Areas $\mathbf{J} / \mathrm{K}$
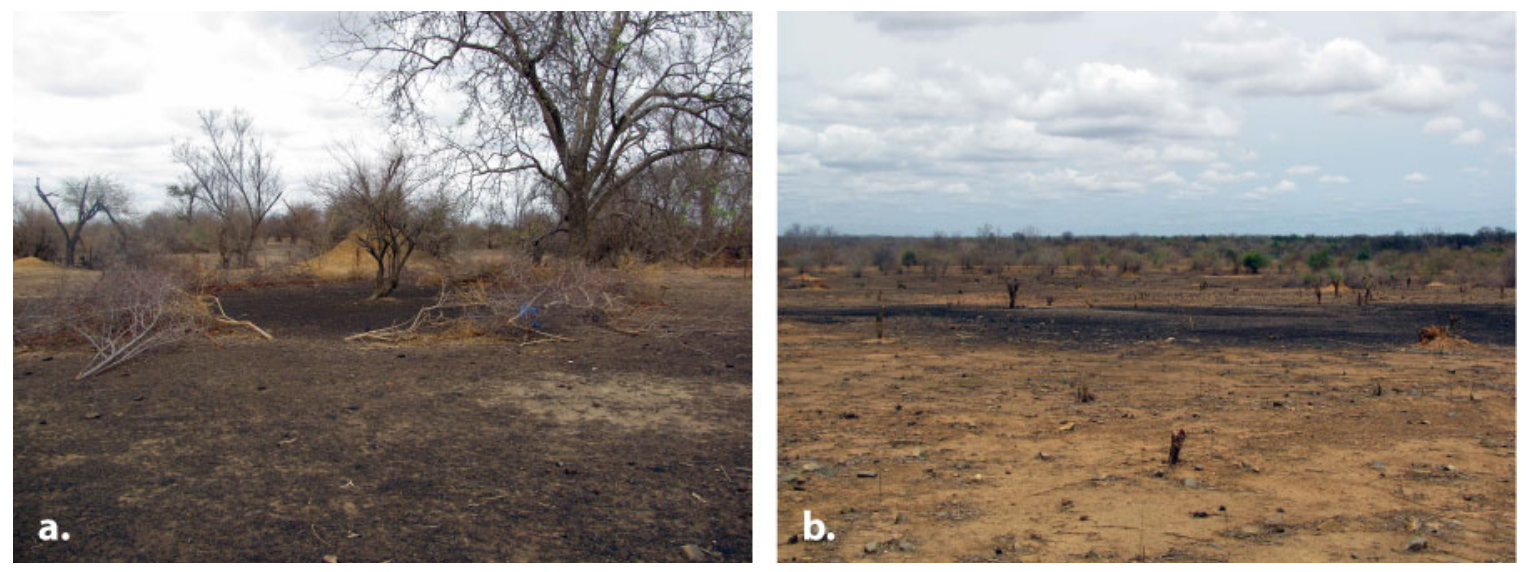

Surface topography and artifact densities indicate that hydraulic erosion is most pronounced along the western edge of the site where a steep slope contributes each rainy season to high energy run-off of precipitation and sedimentary matrix (Figure B.3). The resulting deflation and downslope migration of cultural deposits creates a high density "halo" of artifacts and angular sandstone rocks of unknown stratigraphic context, but which can nevertheless be traced upslope to their area of origin. Although the effect of sheet erosion away from these extant slopes is more difficult to determine, the integrity of surface features and low to modest artifact densities across residential areas points to more limited post-depositional deflation and displacement. 


\section{Figure B.3: View north across eroded southwestern slope of Area A}

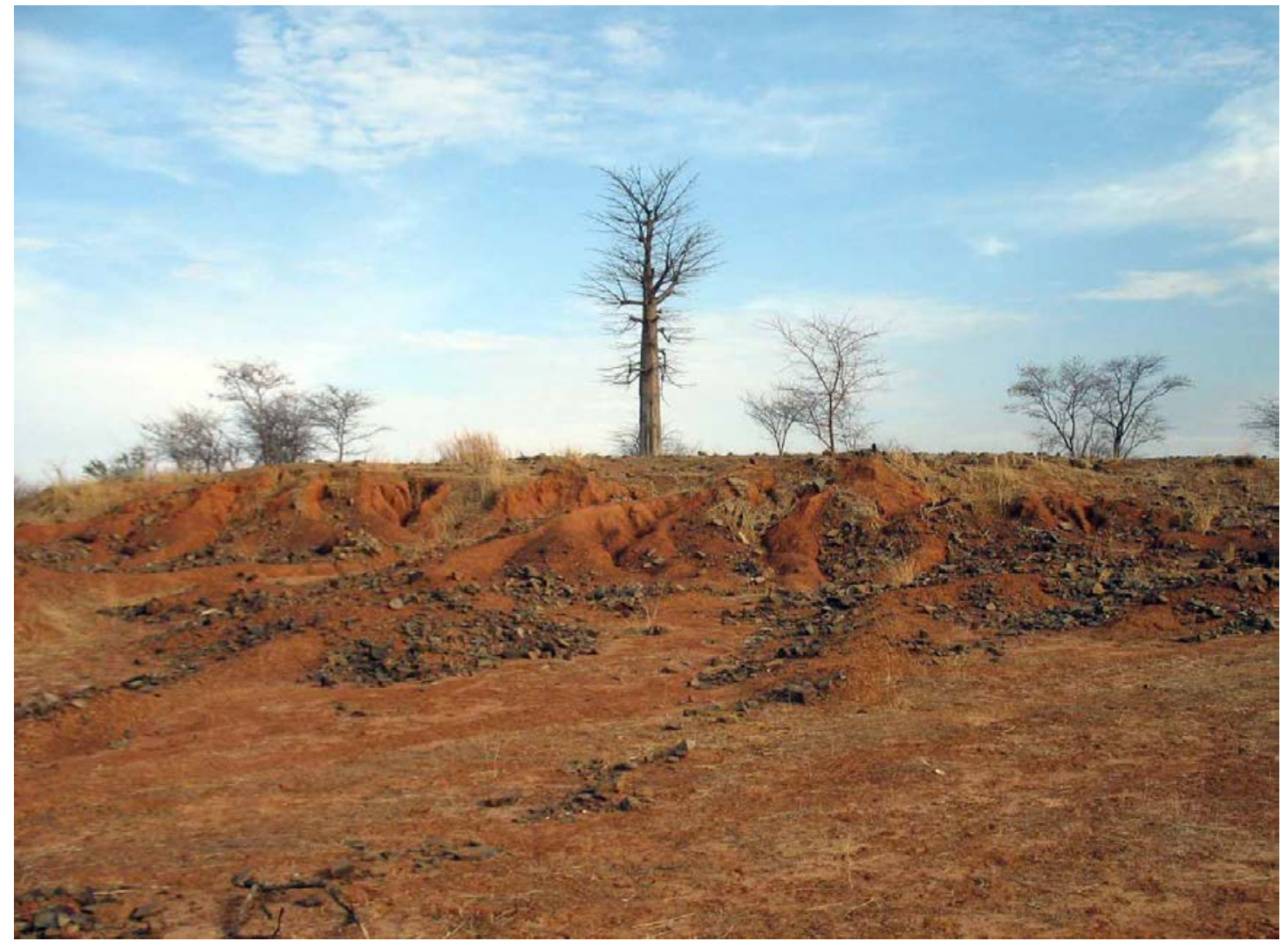

In order to assess the temporal dimensions of surface remains at Diouboye, I employed ArcGIS to develop models of underlying surface slope and erosion. Using the above elevation model, I calculated surface slope $(1 \mathrm{~m}$ resolution) to determine whether the proximity of any two features reflects contemporaneity, or simply the deflation of an intervening cultural deposit (Figure B.4). As discussed in Chapter 8, in-field observations, artifact densities, and a chronological index derived from the ceramic data all suggest that a $5 \%$ grade was the maximum slope between two features that could be considered contemporaneous for purposes of spatial analysis.

While a diachronic model for the transport and redeposition of artifacts lay beyond the scope of this study, I did produce a model of slope length and steepness ( $1 \mathrm{~m}$ resolution) to provide at least some handle on the effects of recent hydraulic erosion following the Revised Universal Soil Loss Equation (Erdogan et al. 2007). As shown in Figure B.5, length (L) incorporates the potential downslope accumulation of surface material from all points upslope. Steepness (S) represents a transformation of slope angle and more strongly determines the resulting LS values. Other factors in the soil loss equation, including soil type and coverage, precipitation, and management practices, were assumed to be relatively constant across the site.

With LS values ranging from 0.00 to 13.76 , it was necessary to determine the threshold beyond which erosion and artifact redeposition and/or deflation at Diouboye would have resulted in an analytically unacceptable level of "decomposition" in surface 
remains (Gregg et al. 1991). By comparing the LS model (see Figure 8.5) with field observations and the densities of surface ceramics and rocks from architectural features, I decided that LS values less than 2.0 represent an acceptable level of confidence in the integrity of the surface materials, while LS values greater than or equal to 2.0 define a surface too eroded for meaningful spatial analysis at a scale smaller than areas and wards.

As applied in Chapter 8, these models of slope and erosion together offer some, admittedly coarse, control over the post-depositional distortion of spatial and temporal dimensions among the surface features and artifacts at Diouboye.

\section{Figure B.4: Surface slope model of Diouboye}

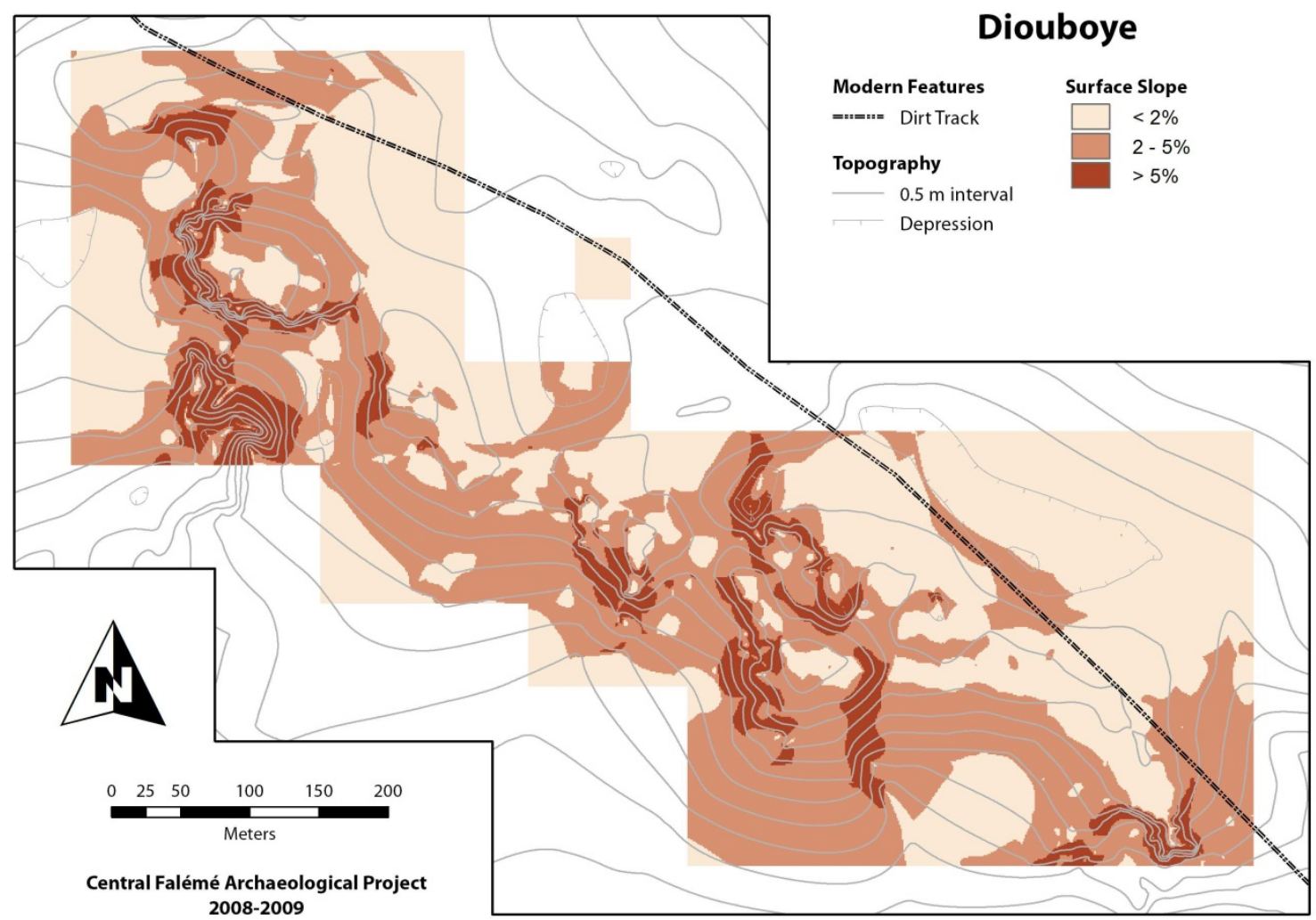

Figure B.5: LS erosion factor equation

$$
=\left(\frac{\text { Slope accumulation }}{22.13}\right)^{0.4} \quad \mathrm{x} \quad\left(\sin \left[\frac{\text { Slope angle }}{0.896}\right]\right)^{1.3}
$$




\section{Artifact Collection}

The collection of surface artifacts from Diouboye, particularly earthenware pottery, had several sub-objectives: (1) to evaluate post-depositional processes of trampling and other erosion; (2) to map the spatial organization of subsistence and/or craft production activities and refuse disposal; and (3) to establish the relative chronology of settlement growth and decline across the site. To these ends, I enacted a program of systematic sampling in the 2008 and 2009 field seasons informed by previous surface collection of diagnostic artifacts on the part of Marie-José and Howard Opper (1990), Hamady Bocoum and colleagues (IFAN collections SEN 1996.52), and an initial reconnaissance under the aegis of CFAP in June 2007 (IFAN collections SEN 2007.36).

Systematic surface collection in 2008 focused on Area A and Area $\mathrm{H}$ where the excavation of stratified deposits (Appendix C) provided an opportunity for comparison with subsurface materials. The methodology inaugurated during this field season, and adapted for the remainder of the site in 2009, deployed a simple sampling strategy. Since surface features revealed no periodic or stratified spatial structure, this avoided leaving gaps in the surface data that could adversely affect the subsequent interpolation of artifact densities. Using a grid tethered to major fixed control points, the CFAP field crew collected artifact samples at intervals of five meters (Figure B.6), stopping just past the discernible extent of surface artifacts in order to minimize an interpolated "edge effect."

\section{Figure B.6: Surface sampling points at Diouboye}

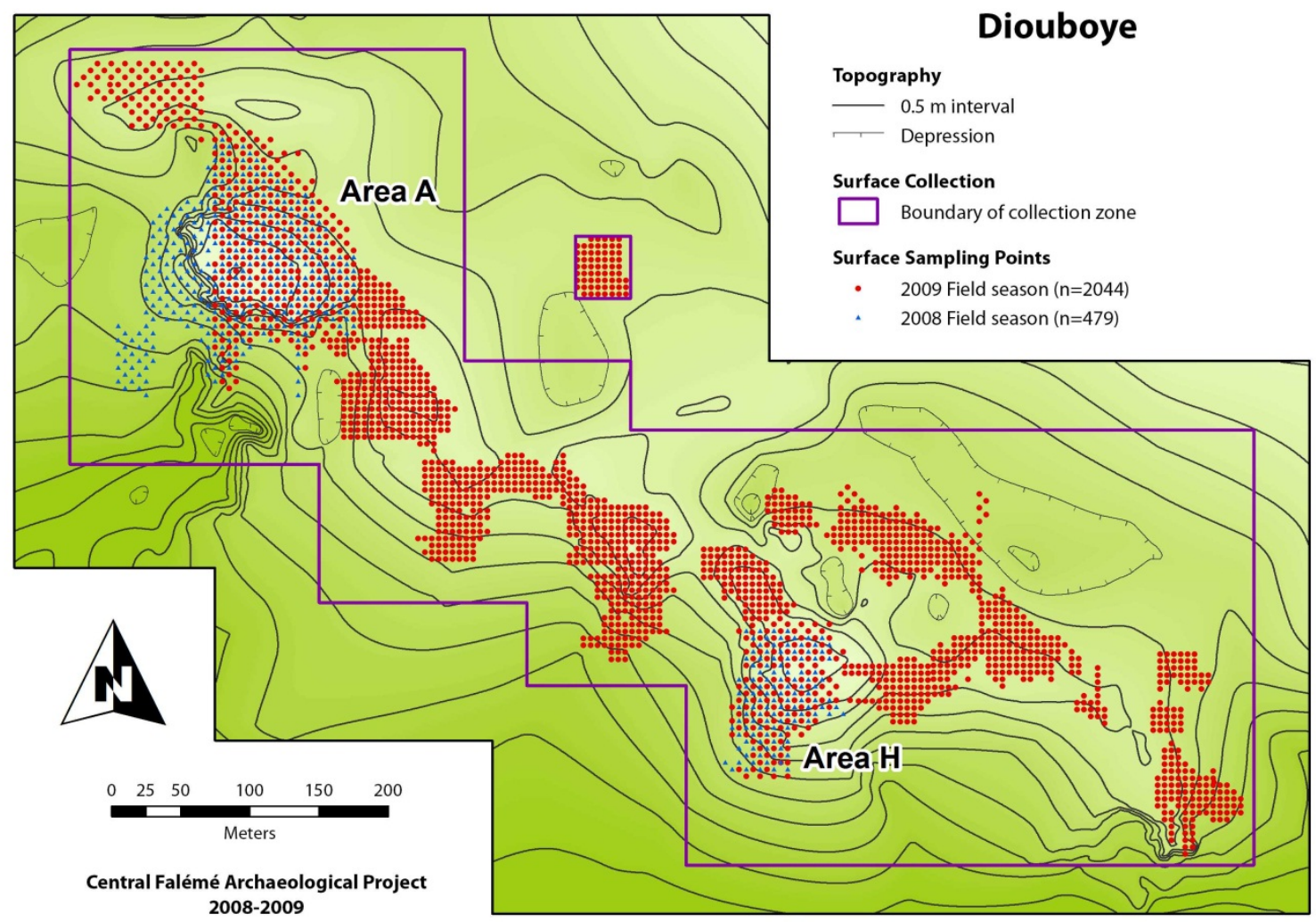


Although each sampling point received a separate FS number, the actual collection strategy was stratified by artifact category and revised slightly between the 2008 and 2009 field seasons. In 2008 we collected rim sherds and other diagnostic artifacts within circular units $\left(5 \mathrm{~m}^{2}\right)$ centered on sampling points set at ten-meter longitudinal and five-meter latitudinal intervals (FS\#1000-1728, 1770-1783), all while noting those units with excessive groundcover or other disturbances. We also counted the number of rock fragments ( $>10 \mathrm{~cm}$ diameter) in each circular unit to document the density of intact and disaggregated architectural features. Given their high frequencies, we only collected body sherds (all sizes) from the southeast quadrant of each circular unit $\left(1.25 \mathrm{~m}^{2}\right)$. Altogether, this strategy yielded an approximately $2.5 \%$ sample of body sherds and a $10 \%$ sample of rim sherds and other artifacts from Area A and Area $\mathrm{H}$.

Table B.2: $\quad$ Summary of sampling points by area

\begin{tabular}{|c|c|c|c|c|c|c|}
\hline \multirow{2}{*}{ Area } & \multirow{2}{*}{$\begin{array}{c}\text { Surface Collection } \\
\text { FS\# }\end{array}$} & \multicolumn{2}{|c|}{ Field Season } & \multicolumn{3}{|c|}{ Collected Artifacts } \\
\hline & & $2008\left(5 m^{2}\right)$ & $2009\left(4 \mathrm{~m}^{2}\right)$ & Present & Absent & Disturbed \\
\hline Area A & $\begin{array}{l}1001-1533,1537-1540, \\
\text { 1566-1601, 1770-1786, } \\
3150-3473\end{array}$ & 319 & 303 & 310 & 243 & 69 \\
\hline Area A-N & $1800-1887$ & & 90 & 25 & 46 & 19 \\
\hline Area A-S & $\begin{array}{l}\text { 1534-1536, 1541-1565, } \\
3735-3747\end{array}$ & 43 & 21 & 35 & 25 & 4 \\
\hline Area B & $3474-3734$ & & 236 & 96 & 140 & \\
\hline Area C & 964-979, 3036-3149 & & 135 & 66 & 68 & 1 \\
\hline Area D & 912-963, 980-996 & & 62 & 19 & 43 & \\
\hline Area F & 694-911 & & 252 & 124 & 125 & 3 \\
\hline Area G & $580-657$ & & 92 & 43 & 49 & \\
\hline Area $\mathrm{H}$ & $450-579,1610-1728$ & 117 & 132 & 117 & 124 & 8 \\
\hline Area J & $337-415$ & & 121 & 4 & 109 & 8 \\
\hline Area K & 1-152, 1995-1999 & & 153 & 20 & 123 & 10 \\
\hline Area L & $176-211$ & & 40 & 6 & 34 & \\
\hline Area $\mathrm{M}$ & $658-693$ & & 37 & 12 & 25 & \\
\hline Area N & $\begin{array}{l}416-449,997-999, \\
1888-1994,3000-3035\end{array}$ & & 168 & 48 & 118 & 2 \\
\hline Area P & $153-175,212-336$ & & 128 & 41 & 83 & 4 \\
\hline Area S & $3748-3811$ & & 64 & & 64 & \\
\hline
\end{tabular}

Estimation of surface artifact populations from the materials collected in 2008 allowed for a revision of the sampling strategy in 2009. Since one objective was to evaluate the spatial organization of past socioeconomic activities through their resulting artifact assemblages, the diversity of which depends to some extent on sample size 
(Sullivan and Tolonen 1998), it was necessary to obtain an adequate sample of infrequent, but potentially diagnostic, artifacts by sampling smaller circular units $\left(4 \mathrm{~m}^{2}\right)$ at intervals of only five meters (FS\#1-999, 1800-3811). To counteract the possible oversampling of body sherds, we only collected larger $\left(>4 \mathrm{~cm}^{2}\right)$ specimens from half of the southeast quadrant $\left(0.5 \mathrm{~m}^{2}\right)$ in each circular collection unit. At the same time, we estimated the density of all sherds within a quarter of each unit $\left(1 \mathrm{~m}^{2}\right)$ along an ordinal scale: (0) No sherds; (1) 1-5 sherds; (2) 6-10 sherds; (3) 11-20 sherds; (4) 21-50 sherds; (5) 51-100 sherds; (6) 101-200 sherds; (7) >200 sherds. Although surface coverage was slightly higher in Area A and Area $\mathrm{H}$ due to the sampling of larger units in 2008, overall this strategy yielded an approximately $2 \%$ sample of body sherds $(n=28,330)$ and $16 \%$ sample of rims and other sherds $(\mathrm{n}=2514)$ and diagnostic artifacts from the entire site surface (see Table B.2).

Figure B.7 illustrates the results of this systematic artifact collection as a map of interpolated (natural-neighbor) surface ceramic densities. Pottery sherds were far and away the predominant artifact class in the surface assemblage and zones of high density largely demarcate eroding margins and deflated stratigraphy across the site.

Figure B.7: Ceramic sherd density across Diouboye

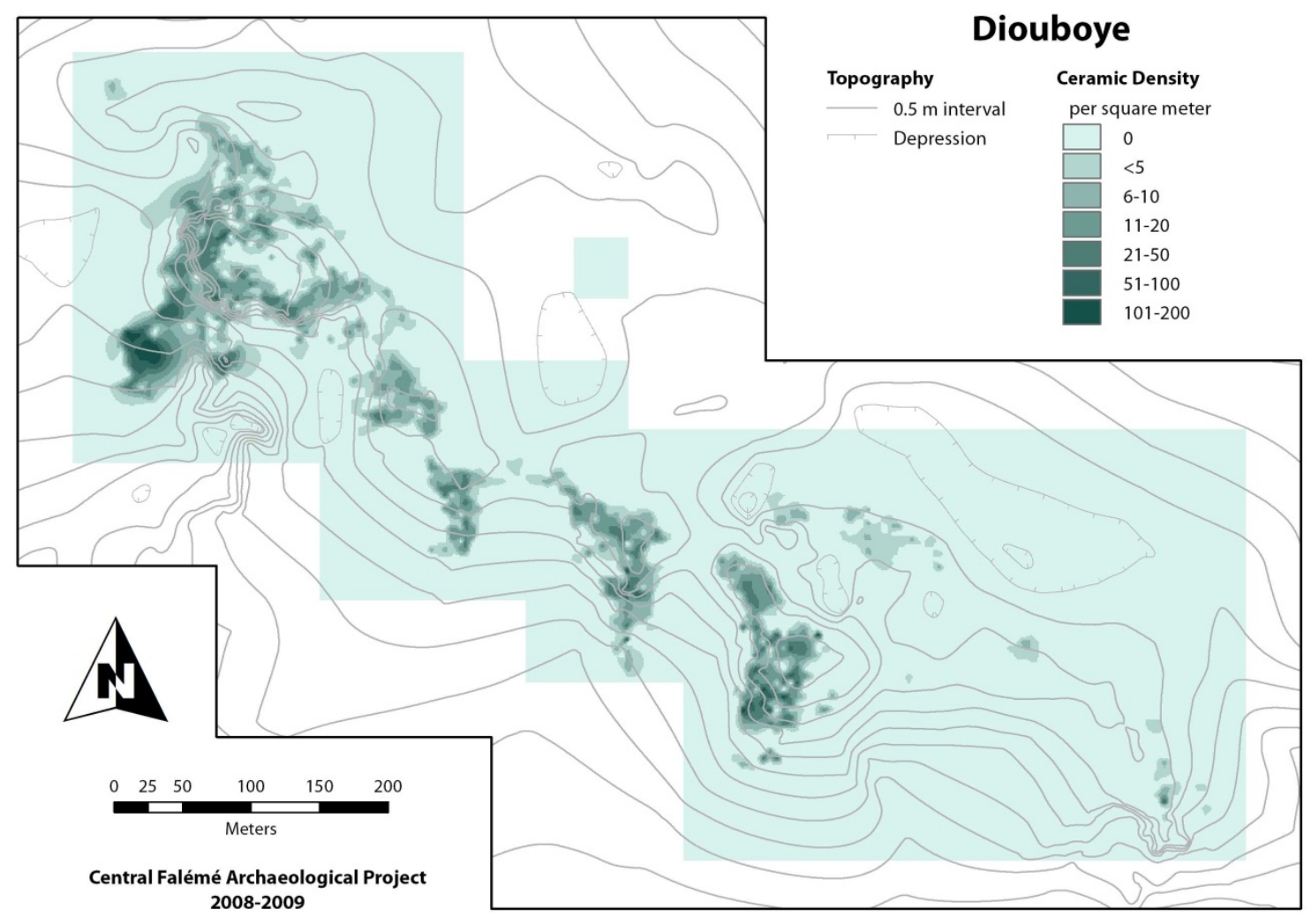




\section{Feature Mapping}

Concurrent with artifact collection in 2009, the CFAP field crew mapped 421 features across the surface of Diouboye. Many of these were circular or rectilinear arrangements of angular stones with clear spatial integrity, but others were either amorphous or partially dismantled. Erring on the side of caution, I recorded only those features that unmistakably resulted from deliberate placement, rather than natural deposition or erosion.

Using fiberglass tapes and two folding wooden rules, it was possible to obtain the coordinates of features relative to the site-level grid with an accuracy of \pm 10 centimeters. I also sketched each feature to capture relations among interior elements such as sandstone rock fragments, reconstructable pottery vessels, and lithic artifacts. Through comparison to similar features in subsurface contexts, these quantitative and qualitative variables formed the basis for a classification of features such as hut and granary foundations, pot rests, pavements, and miscellaneous circular and linear alignments, and their relation to less frequent grinding stones, lithic reduction scatters, collapsed daub, and even possible hearths (see Chapter 6). Figure B.8 portrays the surface features at Diouboye overlaying the density of angular sandstone rocks interpolated (naturalneighbor) from counts in each collection unit.

\section{Figure B.8: Intra-site areas and wards defined for Diouboye}

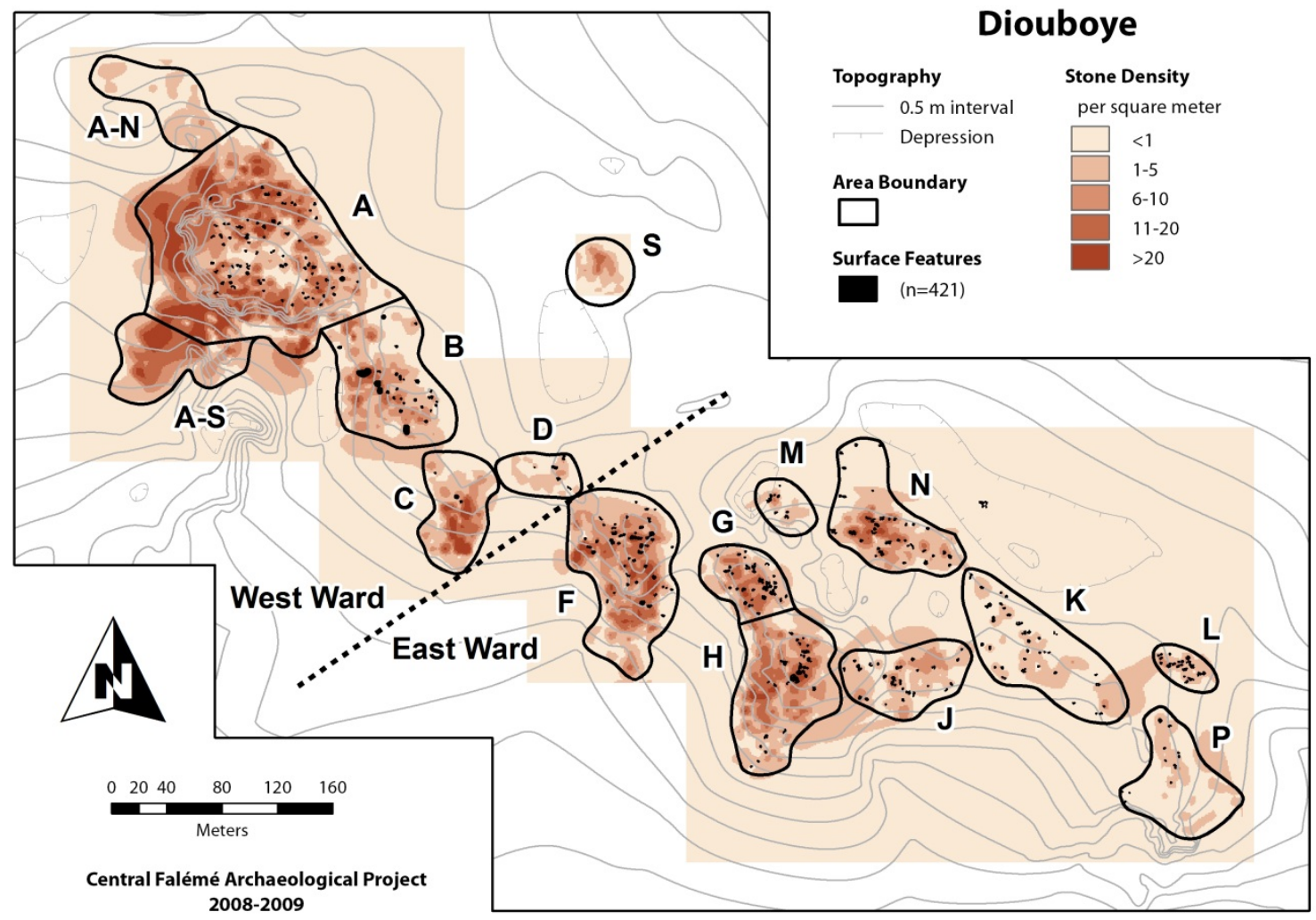




\section{INTRA-SITE SPATIAL UNITS: AREAS AND WARDS}

A visual inspection of ceramic densities (Figure B.7) and surface features (Figure B.8) reveals 16 more-or-less discrete concentrations of archaeological remains that I have deemed areas. Although these appear to comprise zones of repeated activity, postdepositional processes have clearly broadened the extent of some areas, and obscured the boundaries of others. In this latter regard, a continuous distribution of surface features made the recognition of areas more difficult in the eastern extent of Diouboye. For this reason, I parsed out coherent groups of surface features using a hierarchical cluster analysis (Average method) on their spatial coordinates. These results, largely coinciding with concentrations of surface artifacts, provided a means for inferring boundaries between those areas with low artifact frequencies such as Area $\mathrm{J}$ and Area K. The uneven distribution of architectural features underwrites a distinction between the relatively formalized space of residential areas, and more informal space of non-residential areas.

Cluster analysis further illustrated a higher-order spatial division of two intra-site wards. As shown in Figure B.7, the West Ward includes Area A, Area A-N, Area A-S, Area B, Area C, and Area D (and perhaps Area S), while the East Ward includes Area F, Area G, Area H, Area J, Area K, Area L, Area M, Area N, and Area P. Together with areas, these wards comprise the main units for spatial analyses in Chapters 7-9.

\section{Residential Areas}

Residential areas have unambiguous architectural features (excluding cairns and irregular stone alignments) along with artifacts such as earthenware pottery and chipped stone generally associated with quotidian domestic tasks. Since the average size of these residential areas $\left(5984 \mathrm{~m}^{2}\right)$ is nearly an order of magnitude larger than that of household compounds in contemporary West African villages (see Chapter 8), I assume that each represents the space occupied by several such compounds.

\section{Area A}

Area A $\left(19,222 \mathrm{~m}^{2}\right)$ is unequivocally the largest residential area at Diouboye, encompassing 96 stone architectural features and a diverse array of artifacts. Perched on the edge of an archaic levee, the steep western and southern slopes of Area A create the illusion of a mound much deeper than the 1.21 meters of stratified cultural deposits exposed during excavation (Units A1-A2). In fact, the fortified appearance of this embankment has led people from nearby communities to describe Diouboye as a tata (fortified village), thereby situating the site within the political tumult and violent regional conflicts of the mid- $19^{\text {th }}$ century (see Chapter 4 ). While neither excavation nor ground survey documented ruined walls similar to those found on unambiguous tata in the Madina-Sadatou study area, the slope of the embankment could have afforded the residents of Area A some degree of natural defense. With LS values exceeding 2.0, this prominent slope has, however, experienced substantial rill erosion (see Figure B.3), amplifying the estimated size of Area A with a halo of artifacts and sandstone fragments. Nearly all intact features were found above these heavily eroded margins on the lightly deflated crown of Area A and thus included in spatial analyses. Eight to ten small scatters of large pottery sherds document the abandonment of complete, or nearly complete, vessels. The excellent preservation of archaeological materials above the eroded margins of Area A suggests that they date to the terminal episode of site occupation. 


\section{Figure B.9: Surface features (granary foundations) in Area A}

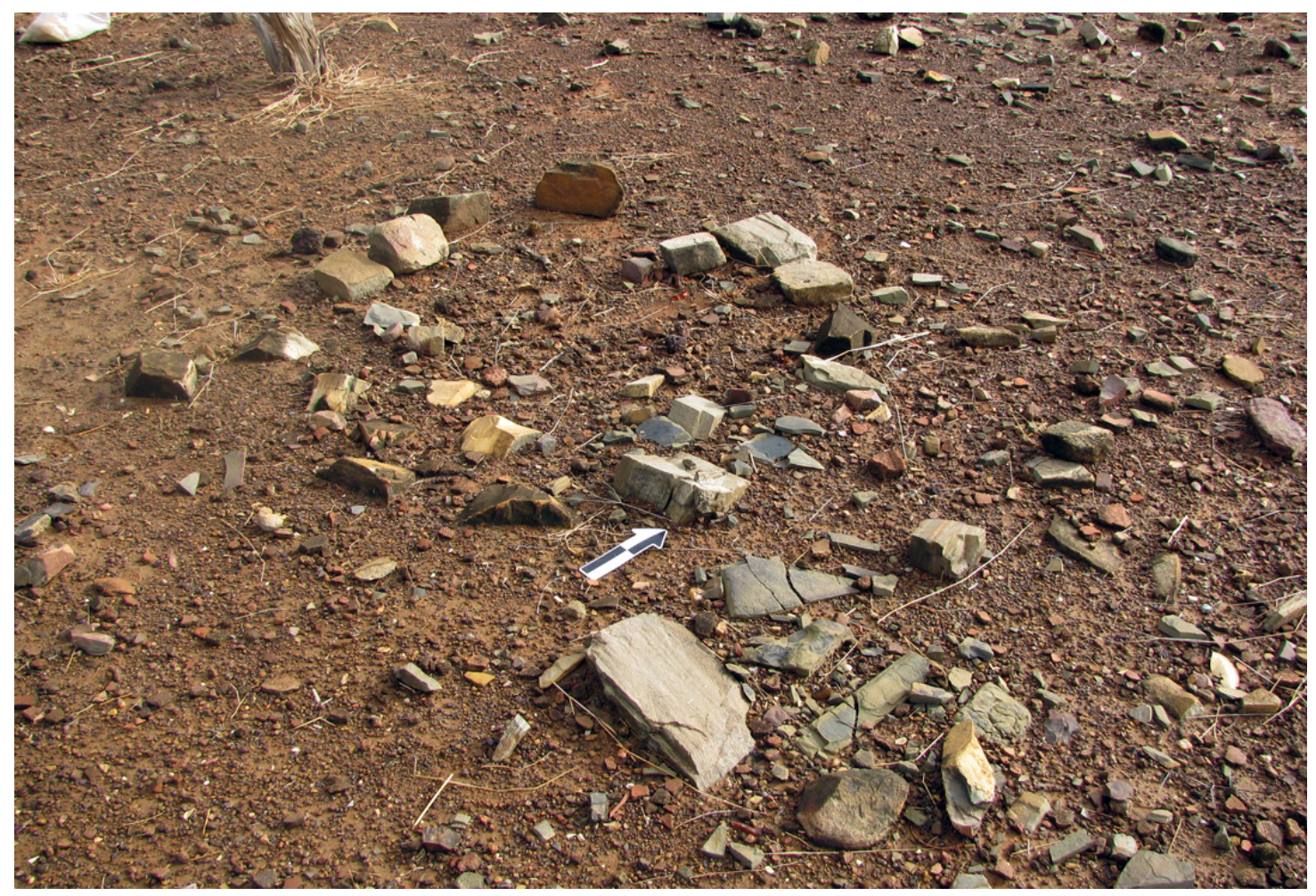

\section{Area B}

Area B comprises a discrete residential area $\left(6868 \mathrm{~m}^{2}\right)$ with 23 stone architectural features and scatters of pottery sherds, chipped stone debris, and groundstone fragments. Separated from Area A by a lightly depressed space devoid of artifacts, the archaeological deposits of this area form a low mound with an estimated depth of one meter based on stratigraphy visible in the eroded western margin. Alongside the preservation of architectural features, modest deflation across this area suggests that the surface data represent more or less contemporaneous activities. This includes the six macrolithic scatters of chipped mudstone debris situated downslope from the crown of Area B, but associated with Iron Age pottery sherds and groundstone.

\section{Area C}

Surface artifacts stretch continuously from Area B to Area D, but one can distinguish Area C (3606 $\left.\mathrm{m}^{2}\right)$ sloping gradually away to the south as a lightly mounded expanse of pottery sherds, chipped stone, groundstone, and displaced sandstone rocks attesting to a possible residential space that has undergone heavy stratigraphic deflation. In fact, low LS values (<2.0) coupled with high artifact densities point to long-term sheet erosion across this area. The only unambiguous surface features are a large granary, a concentration of collapsed daub, and a macrolithic scatter. The shallow profile $(<0.11 \mathrm{~m})$ exposed during excavation of this latter feature (see Chapter 7) suggests that these surface remains represent the near entirety of stratigraphy in Area C. 


\section{Figure B.10: View south across Area C}

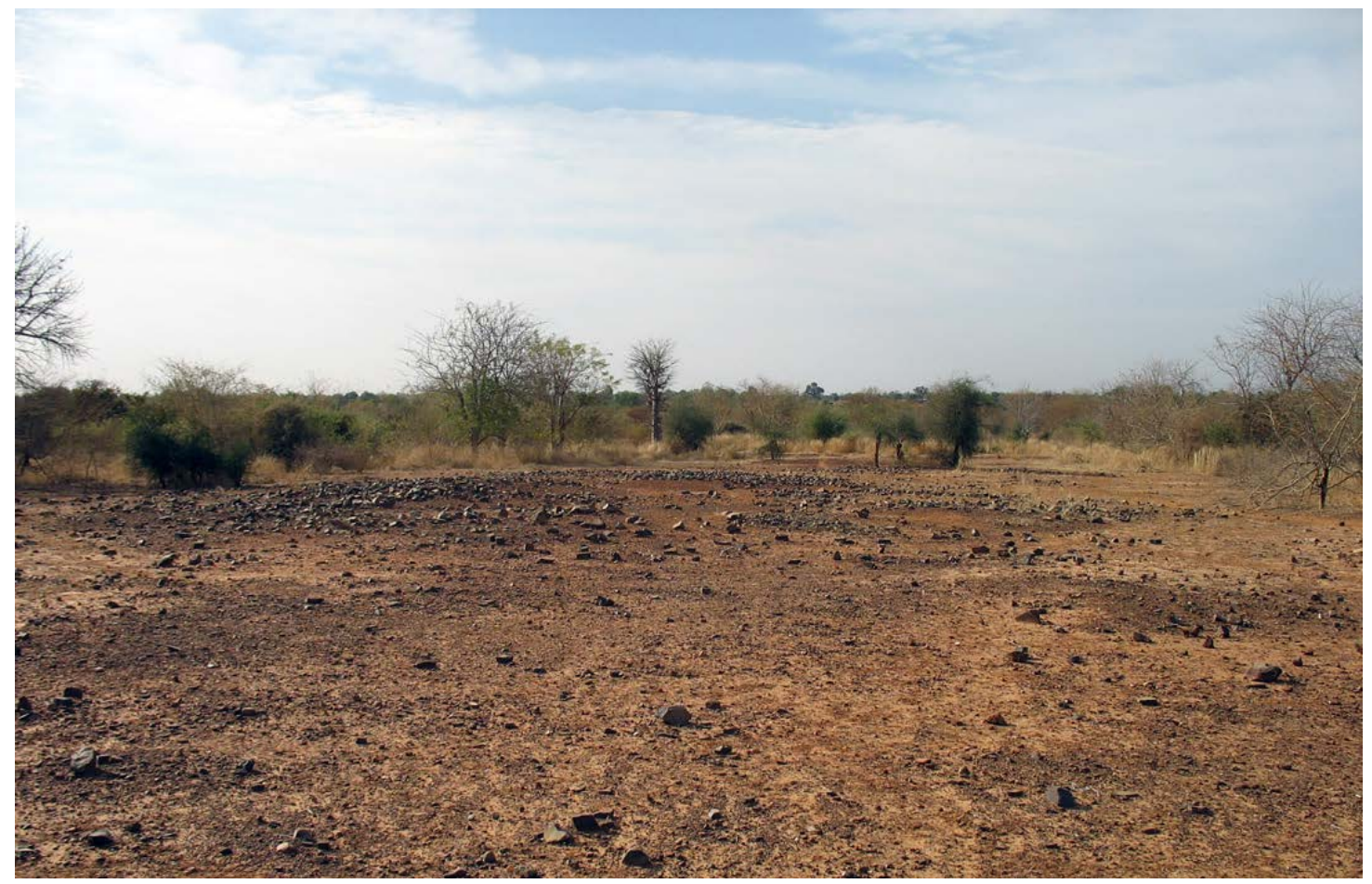

Area D

Occupying a lightly eroded stretch of the archaic levee underlying Diouboye, Area D $\left(1139 \mathrm{~m}^{2}\right)$ includes seven stone surface features and low frequencies of artifacts extending westward towards Area C. Beyond ubiquitous pottery sherds, these include chipped stone, groundstone, and metallurgical slag attesting to domestic activities as well as craft production, with the latter use of this space most likely preceding the former. Given the sparseness of these archaeological remains, I often subsume the artifacts and surface features from Area D together with Area C and Area F, respectively, for purposes of analysis and discussion throughout this study.

\section{Area $F$}

The northern half of Area F $\left(8493 \mathrm{~m}^{2}\right)$ is a low mound with high artifact densities and 53 surface features attesting to a modestly deflated zone of residential occupation. These materials presumably date to a terminal episode of occupation in this area where excavation (Unit F) revealed archaeological deposits extending to a depth of 0.80 meters below surface.

Beyond a steeply eroded embankment (LS value > 2.0), the southern half of Area F comprises a more heavily deflated zone with high densities of sandstone fragments, pottery sherds, and chipped stone debitage (Figure B.10). Given the questionable chronology of the nine surface features in this zone, I generally excluded them from spatial analyses in Chapter 8. 
Figure B.11: View north across Area F

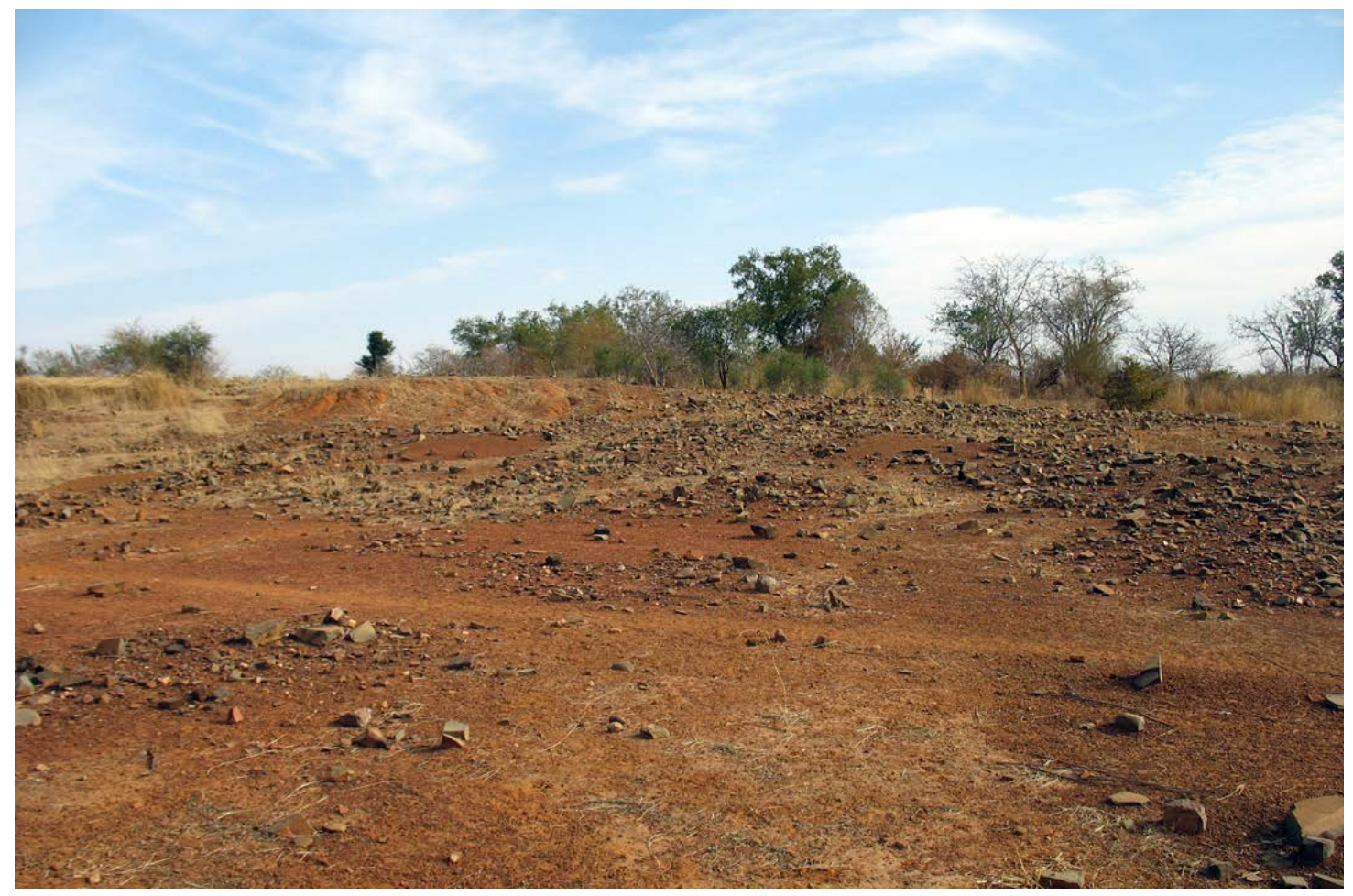

Figure B.12: View west across heavily deflated surface of Area G

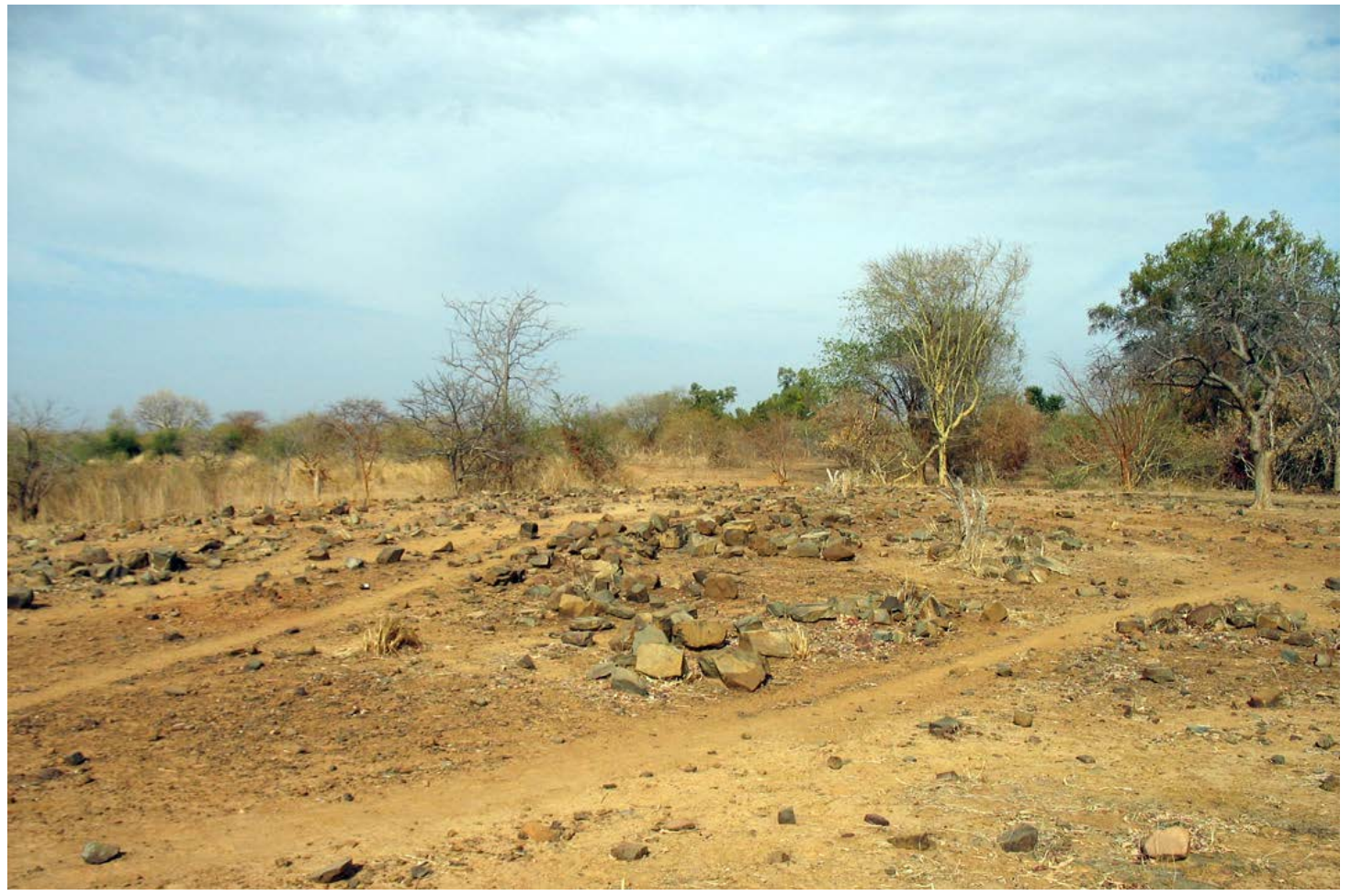




\section{Area $G$}

Area $\mathrm{G}\left(2595 \mathrm{~m}^{2}\right)$ is a compact mound capped by 42 surface features and an abundance of sandstone rocks, pottery sherds, and other artifacts attesting to residential occupation. Although it remains discrete from Area F in the west, this mound merges into that of Area H to the southeast. Despite low LS values $(<1.0)$ and nicely preserved architectural features, the high artifact density atop Area $G$ points to long-term sheet erosion. As a result, it is unlikely that all surface remains derive from the terminal phase of occupation in this area, where excavations (Unit $G$ ) revealed stratified deposits to a depth of 1.03 meters below surface. Although the western margin of Area G descends abruptly down the steep slope of the archaic levee underlying the site, the low density of artifacts below this point suggests that most occupation activity in this area was concentrated atop the mound.

\section{Area $\mathrm{H}$}

Thirty-two stone architectural features and modest densities of pottery sherds, chipped stone, groundstone, and slag littered across the crown of Area $\mathrm{H}\left(6690 \mathrm{~m}^{2}\right)$ document a final episode of residential occupation, possibly with limited craft production activities, lying atop more than two meters of archaeological deposits (Unit $\mathrm{H}$ ). The resulting elongated mound remains distinct from Area $G$ to the north and the low expanse of Area $\mathrm{J}$ to the east.

Descending to the southwest, higher artifact densities and the absence of unambiguous surface features highlight greater disturbance from both sheet erosion and contemporary agro-pastoral practices. For this reason, I generally excluded from spatial analysis those features found on and below the sloping margins of Area $\mathrm{H}$ where LS values exceed 2.0.

\section{Area J, Area K, and Area P}

Multivariate cluster analysis distinguished three groups of surface features attesting to residential occupation at Area J (3885 $\left.\mathrm{m}^{2}\right)$, Area K $\left(6596 \mathrm{~m}^{2}\right)$, and Area P $\left(4467 \mathrm{~m}^{2}\right)$. These share an archaic levee with a latitudinal orientation across the East Ward of the site and which slopes gently down to a wide basin in the north and a broad alluvial terrace in the south. Although LS values remain less than 2.0, the sparseness of artifacts across these areas suggests that surface vegetation has kept sheet erosion to a minimum. Meanwhile, the presence of unambiguous, yet relatively fragmentary, surface features in Area $J(n=28)$, Area $K(n=26)$, and Area $P(n=18)$ hints at past disturbance by cultivation practices. In fact, farmers from a nearby village were observed clearing the land immediately to the south for millet cultivation in 2009. Past farmers may have also occasionally brought the eastern extent of Diouboye under cultivation; this would account for the presence of cairns at Area L described below.

Since defining clusters of surface features is sensitive to post-depositional disturbances, I considered the architecture and artifacts from these areas together for many of the spatial analyses presented in Chapters 8-9. Stratified deposits with clearly seriated ceramic types and a depth of 0.46 meters visible in Unit K suggest that surface remains across these areas are more or less contemporaneous, but do not represent the terminal phase of occupation at the site. 
Figure B.13: View north across Area K

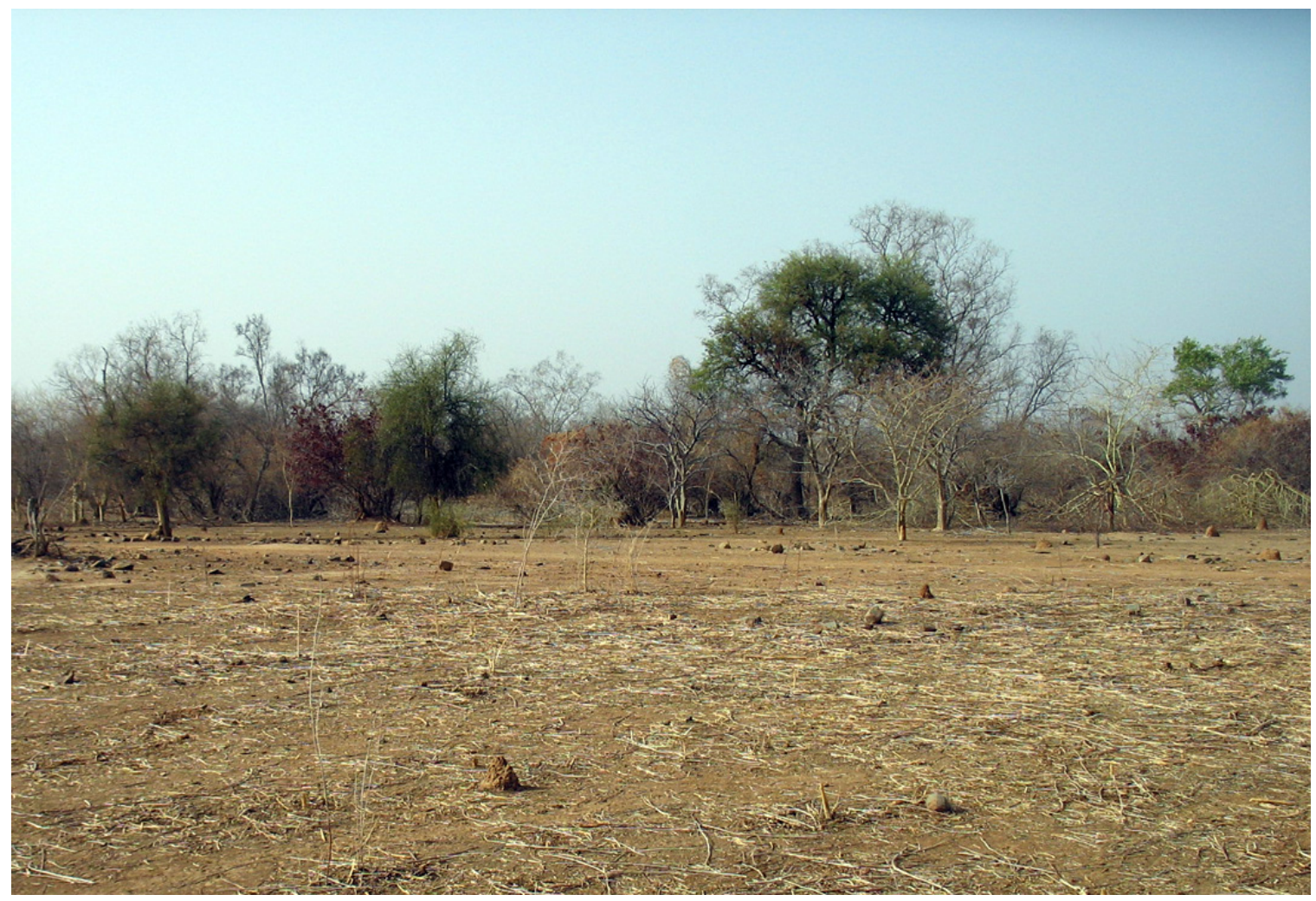

\section{Area $M$ and Area $N$}

Area M (1319 $\left.\mathrm{m}^{2}\right)$ comprises a cluster of nine architectural features situated on a low rise between broad depressions to the northwest and southeast. Along with modest frequencies of pottery sherds, the low LS values $(<1.0)$ across this area suggest that deflation has been minimal. Zones devoid of archaeological materials clearly separate Area $\mathrm{M}$ from Area $\mathrm{G}$ to the south and Area $\mathrm{N}$ to the east. Small sample sizes did, however, make it necessary to consider this area together with Area $\mathrm{N}$ together for many spatial analyses in Chapters 8-9.

Area N (5252 $\mathrm{m}^{2}$ ) appears as a relatively flat expanse of residential occupation debris, including modest frequencies of pottery sherds and 44 surface features-these latter distinguished from those of Area K only through a hierarchical cluster analysis. Gentle downward slopes to the north and south provide only a subtle indication of the stratified archaeological deposits (Unit N) extending to a depth of 0.84 meters below ground. As with Area M, low LS values $(<1.0)$ implicate light deflation of surface materials resulting mainly from sheet erosion.

\section{Non-Residential Areas}

Non-residential areas are scatters of pottery sherds and other artifacts associated with both subsistence and craft production activities. Despite the presence of dispersed sandstone fragments and cairns, the absence of unambiguous architectural features strongly suggests that these areas were not residential spaces. 


\section{Area A-N}

Area A-N $\left(3514 \mathrm{~m}^{2}\right)$ is a flat terrace stretching north of Area A where the absence of groundcover made it possible to sample a rather sparse population of surface artifacts. With no signs of permanent architecture, this area appears to represent ephemeral activities related to agro-pastoral and/or craft production.

\section{Area A-S}

Downslope to the south of Area A, a dense concentration of pottery sherds, chipped stone artifacts, and metallurgical slag forms Area A-S (4008 $\left.\mathrm{m}^{2}\right)$. Angular sandstone rocks scattered across this area do not, however, appear to represent redeposition since one can observe a clear break with the eroded slump from Area A. At the same time, their small size and lack of spatial coherence argues against the in situ decomposition of architectural features.

Erosion across this area is heterogeneous-a deep ravine (LS values $>2.0$ ) divides an otherwise flat expanse of archaeological materials (Figure B.14). That said, it is not possible to determine whether the high concentrations of artifacts derived from a rich midden deposit, or simply the deflation of a once-stratified deposit. In either case, the composition of the resulting assemblage with abundant slag and chipped stone appears consistent with (specialized?) activities related mainly to craft production.

\section{Figure B.14: View south across Area A-S}

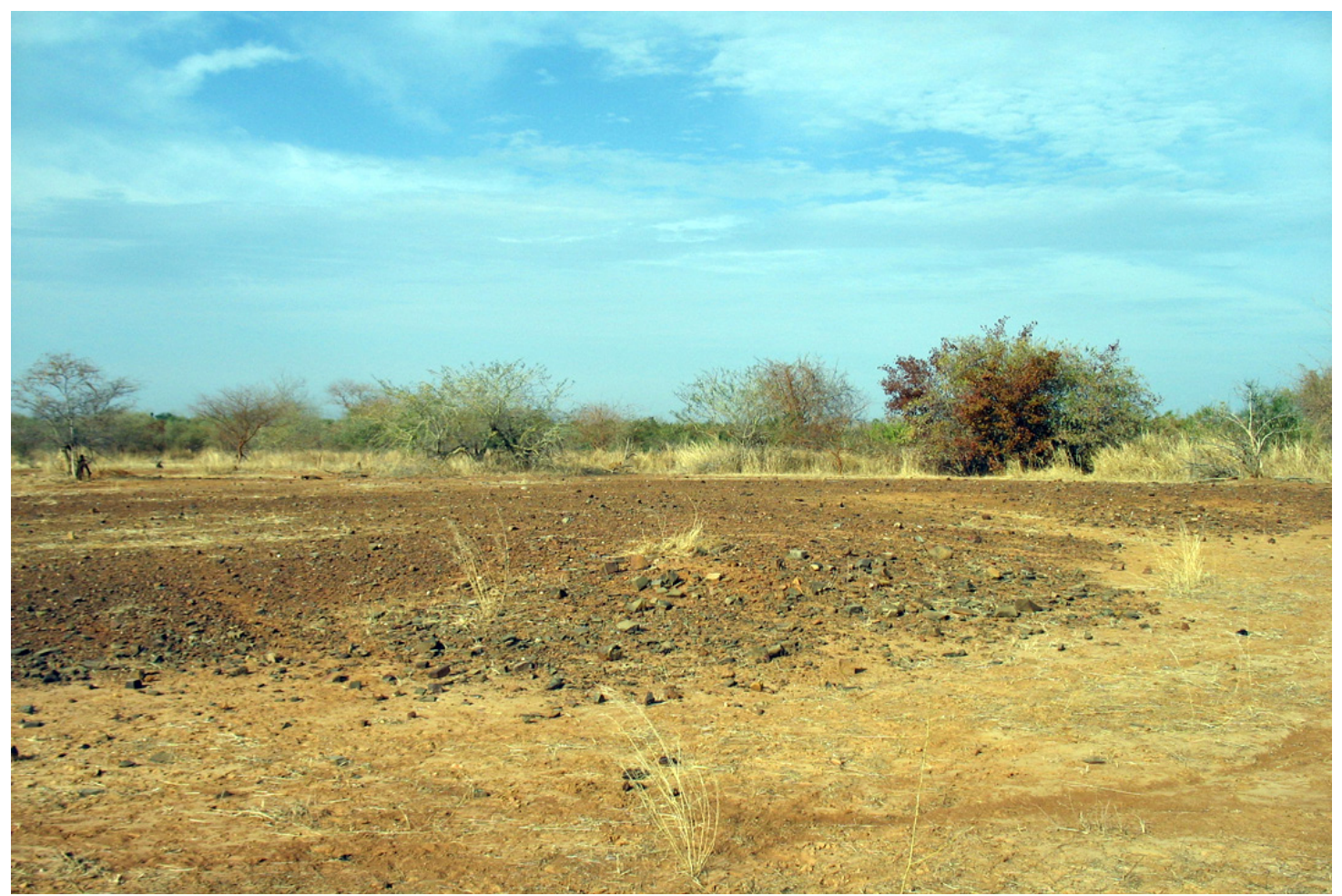




\section{Area L}

Area L (1066 $\left.\mathrm{m}^{2}\right)$ includes 24 discrete cairns of sandstone rocks laid out in various directions, but associated with almost no artifacts. While these surface features were initially thought to represent the remnants of a short-lived residential occupation or perhaps a cemetery, the absence of stratified deposits and human remains in a test unit over one cairn (Trench L) demonstrated that archaeological evidence in this area is completely superficial (Chapter 7). For this reason, I submit that these cairns derive from the dismantling and redeposition of surface features in order to better cultivate Area $\mathrm{K}$ and Area P to the southwest. Whatever the case may be, I subsumed the very small sample of artifacts from this area with those of Area P for the purposes of intra-site analyses.

\section{Area $S$}

Lying some 100 meters east of Area A, Area S $\left(1218 \mathrm{~m}^{2}\right)$ is a haphazard concentration of angular sandstone rocks interspersed with a few pottery sherds. Although some rocks may trace their origins to disaggregated architectural features, the excavation of one such feature (Unit S) revealed a near absence of subsurface deposits in this area. As with Area L above, I suggest that archaeological remains in this area represent ephemeral Iron Age activity and/or redeposition by local millet farmers looking to clear a stretch of land immediately to the south. 


\section{APPENDIX C DIOUBOYE: EXCAVATION METHODS AND STRATIGRAPHIC LOCI}

Regardless of shifting research paradigms, the excavation of settlement sites has been a mainstay of archaeological investigations in West Africa over the past century. The exploratory period of the mid- $20^{\text {th }}$ century, for example, witnessed large-scale excavation of towns mentioned in medieval Arabic geographies or local traditions as major political centers or êntrepots of trans-Saharan trade (e.g., Devisse 1983; EffahGyamfi 1985; Filipowiak 1979; Thilmans and Ravisé 1980; Thomassey and Mauny 1956). As archaeologists have drawn on this foundational research to pose novel questions about social processes and spatial organization, they have adopted excavation techniques oriented towards the recovery of fine-grained data in these urban centers, and they have turned to look increasingly at variation across different sorts of sites (e.g., Bocoum and McIntosh 2002; Connah 1981; Dueppen 2008; Haaland 1980; Holl 2002; S. K. McIntosh 1995; Nixon 2009; Stahl 2001; Thiaw 1999; Togola 2008). Most recently, targeted excavations have proven useful for tying regional survey data to cultural and chronological grids (Gallagher 2010; A. Lawson 2003; Richard 2007). While this is by no means a comprehensive review of excavation in West Africa, these studies attest to the incredible variability in depositional and post-depositional processes (including the preservation of metal and organic remains) that can characterize settlement sites across different culture areas, ecological zones, and time periods.

Given this variability, it was necessary to evaluate the temporal depth, stratigraphic integrity, and overall preservation of subsurface artifacts at Diouboye before committing to more intensive work at the site. During an initial foray to the MadinaSadatou study area in 2007, our CFAP field crew opened an exploratory unit (Trench AZ) along the southwestern margin of Area A. In order to maximize vertical exposure and minimize subsequent erosion, Trench AZ was cut into a steep embankment of archaeological sediments with visible pottery sherds and faunal remains. Excavation proceeded in arbitrary levels $(20 \mathrm{~cm})$. The deposits were not screened, but easily recognizable artifacts, such as sherds and faunal elements, were collected by hand.

Although the levee underlying Diouboye gave Area A the appearance of depth exceeding two meters, Trench AZ arrived at an extremely firm reddish yellow alluvium near the bottom of Level $5(0.70-0.90 \mathrm{~m})$. All told, the depositional sequence revealed four substrata and one heavily oxidized lens from a substantial hearth, all with excellent preservation of charcoal, ash, faunal remains, and pottery sherds. Despite the biased sherd sample, and stratigraphic mixing along the eroded surface of the unit, decorative attributes hinted at temporal trends across the arbitrary levels (Figure C.2). These data were instrumental in establishing an Iron Age chronology for Diouboye (and excluding a significant Late Stone Age component) as well as the potential for further excavations to document material and spatial practices constituting this village community. 
Figure C.1: Trench AZ stratigraphic profile

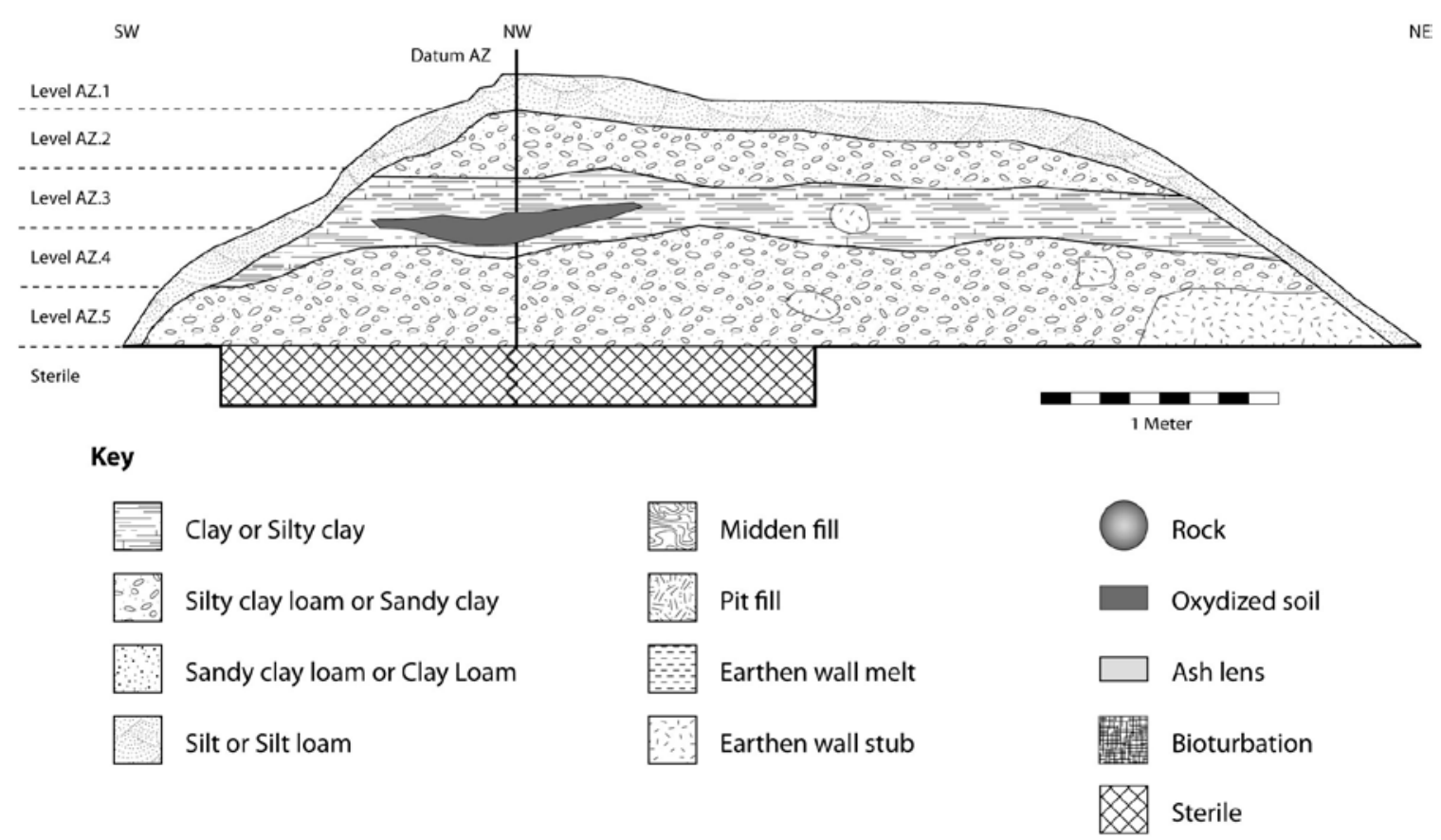

Figure C.2: Trench AZ body sherd seriation

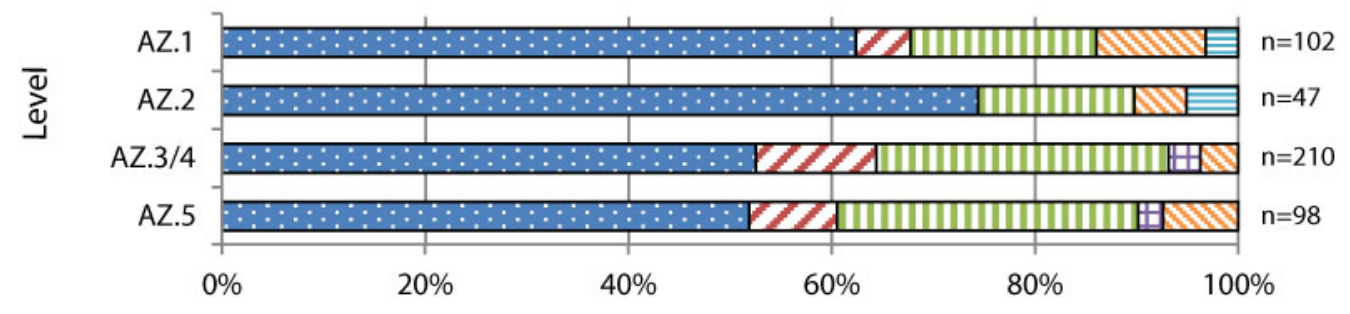

$\begin{array}{lll}\text { ⿴囗十 Twisted Cord Roulette } & \square \text { Folded Strip Roulette } & \text { B Braided Cord Roulette } \\ \square \text { Button Carved Roulette } & \boxplus \text { Cord-Wrapped Stick Roulette } & \text { 目 Chevron Carved Roulette }\end{array}$

Based on the results of Trench AZ, and the excellent preservation of stone architectural features across the site surface (see Appendix B), I undertook subsequent excavations at Diouboye with the following objectives in mind: (1) to document change and continuity in material practices across a sample of residential and non-residential areas; (2) to chart the spatial organization of these practices within and between these areas; (3) to establish a relative ceramic chronology for subsurface and surface contexts across areas (and on contemporaneous surveyed sites); and (4) to anchor these contexts to an absolute chronology for comparison with other sites across West Africa.

Achieving these objectives required a systematic methodology for excavation. In the following section, I describe the rationale for the size and placement of excavation 
units, as well as the strategies of excavation, documentation, and artifact recovery employed throughout the 2008-2009 field seasons. The second section of this appendix is an inventory of all loci, including substrata and features, excavated at Diouboye. As primary spatio-temporal units of analysis, these form the basis for addressing the above objectives in Chapters 7-9 and Appendix D.

\section{EXCAVATION METHODOLOGY Sampling}

In positioning units for subsurface investigation at Diouboye, I strove to balance the contiguous exposure of space in each area with a sample adequate for comparison between areas. Beyond the exploratory excavation of Trench AZ, it was possible, given the available resources and manpower, to excavate 79 square meters over nearly 18 weeks of fieldwork. As summarized in Chapter 7, I opened two units in residential Area A to assess spatial variability across this formidable mound; these included Unit A1 (2x2 $\mathrm{m})$ and Unit A2 (4x6 m). Elsewhere in the West Ward, I tested two non-residential spaces with Unit $\mathrm{C}(1 \mathrm{x} 1 \mathrm{~m})$ and Unit $\mathrm{S}(2 \mathrm{x} 2 \mathrm{~m})$. The greater number of residential areas in the East Ward mandated the excavation of Unit F (4x4 m), Unit G (4x4 m), Unit H (2x2 m), Unit K (2x2 m), Unit N (2x3 m); excavation of Trench L (1x2 m) focused on understanding the deposition of cairns in Area L. Altogether, this sample generated data from at least half the residential areas in each ward, as well as from each type of nonresidential area identified at the site.

By positioning units near the apex of sampled residential areas, it was possible to record the maximal stratified sequences of material practice needed for temporal and spatial comparison among past residential groups. This strategy was vital to reconstructing the processes of village settlement, expansion, and abandonment discussed in Chapters 8-9.

\section{Excavation and Documentation}

Consistent with protocol developed for other stratified sites in the West Africa (e.g., S. K. McIntosh 1995:25; Togola 2008:25-26), our field crew excavated each unit according to natural stratigraphy with thicker depositional layers subdivided at arbitrary $(10 \mathrm{~cm})$ intervals. Depending on soil texture and the presence of features, we employed light hand tools ranging from the pick and shovel to the hand hoe (daba), trowel, and broom or brush. With larger units partitioned into grid squares (2x2 m), I assigned each archaeological locus and architectural feature a unique field specimen (FS) number to maintain vertical and horizontal control over their contents. In this regard, the form filled out for each FS included a sketch of its horizontal position within the unit and its upper and lower depths below a local datum measured using a line level. In addition to photographs of each excavation level, I drew detailed plans of more substantial occupation surfaces and architectural features.

Although I noted soil characteristics for every FS during excavation, the archaeological sediments visible in the profiles of each completed excavation unit were further photographed, drafted, and described in detail. Using the standard Munsell Soil Color Charts (2000) and well-accepted field observations, such as the "ribbon test" (Reed et al. 2000), I recorded the color, texture, consistency, inclusion type and size, and possible sources of bioturbation for each stratigraphic deposit. 
For the purposes of analysis and interpretation, I eventually consolidated FS designations from each unit into loci representing more-or-less discrete depositional episodes (features) or processes (substrata). Those features, including ash lenses and some earthen wall elements, which were only identified ex post facto in excavated profiles each received an artificial FS number ending in ".1" to distinguish them in the database of excavation data. As described in Chapter 7, it was possible to organize these loci into substrata and strata representing continuity in material and spatial practices through time.

\section{Artifact Recovery}

With the notable exceptions of Trench AZ (above) and Trench L, our field crew sieved all excavated deposits through wire mesh screens $(2 \mathrm{~mm})$ to retrieve pottery sherds, faunal remains, and other artifacts. Daub was noted on the FS form where present, but not collected. Whenever possible, charcoal samples were collected from uncontaminated contexts for radiometric dating.

In order to recover macrobotanical remains and other small artifacts, we collected four to six liters of soil from each natural substratum, feature, and intact hearth or ash lens for bucket flotation. This process involved combining the sediment with ten liters of water (from the Falémé River) in a bucket, stirring to create a centrifuge, then pouring the water and suspended material through graduated brass sieves $(0.84 \mathrm{~mm}, 0.25 \mathrm{~mm})$. After repeating this process three times, the remaining sediment was passed through a mesh screen $(2 \mathrm{~mm})$ to recover a heavy fraction of artifacts sorted for later analysis.

These charcoal and soil samples, and other notable finds, such as objects abandoned on occupational surfaces, were given an in situ point provenience recording their precise vertical and horizontal coordinates from the local unit datum. 


\section{STRATIGRAPHIC INVENTORY}

\section{Unit A1}

\section{Stratum A1.1}

\section{Locus: A1 Surface}

FS \# 5001

Depth $(\mathrm{m}): 0.00-0.03 \quad$ Volume $\left(\mathrm{m}^{3}\right): \quad 0.040$

Description: Surface

The surface of Unit A1 was a loose, reddish-yellow (7.5 YR 6/6) sandy clay loam littered with laterite gravel (3-5\%), a few scattered sandstone rocks, and numerous ceramic sherds, including some from a partially reconstructable vessel directly to the east. Located on the apex of Area A, the unit surface was relatively flat, but sloped gently down to the north and east.

\section{Locus: A1.1a}

FS \# 5002, 5003

Depth (m): $0.00-0.13$

Description: Fill, genera

Volume $\left(\mathrm{m}^{3}\right): \quad 0.267$

A loose to friable, strong brown (7.5 YR 5/6) silty clay loam with fine charcoal mottles (1-3\%) and organic detritus (3-5\%). This substratum consisted of weathered topsoil easily distinguished from firmer deposits beneath. Surface A1.1a was a compact occupation floor with two small informal hearths (Feature 1).

Locus: Feature 1

FS \# 5002.1

Depth $(\mathrm{m}): 0.29-0.30 \quad$ Volume $\left(\mathrm{m}^{3}\right): \mathrm{n} / \mathrm{a}$

Description: Hearth

Two small ash lenses and three patches of heavily burnt silty clay loam indicative of an informal hearth area on Surface A1.1a.

\section{Locus: A1.1b.i}

FS \# 5004

Depth (m): $0.10-0.21 \quad$ Volume $\left(\mathrm{m}^{3}\right): 0.272$

Description: Fill, general

A moderately compact, reddish-yellow (7.5 YR 6/6) silt loam with fine to medium daub inclusions (3\%) and fine charcoal mottles (1-3\%). This substratum was a general fill deposit with pockets of increased artifact and charcoal densities. Surface A1.1b was an occupation surface defined, in part, by two in situ hearths (Features 2 and 3).

\section{Locus: A1.1b.ii}

FS \# 5005

Depth (m): $0.19-0.25 \quad$ Volume $\left(\mathrm{m}^{3}\right): 0.028$

Description: Fill, midden

A moderately loose, light yellowish-brown (10 YR 6/4) silt loam with ash lenses and fine to medium charcoal mottles (3\%), but no visible daub. High artifact and charcoal frequencies indicated that this deposit derives from deposition of household refuse.
Locus: Feature 2

FS \# 5004.1

Depth (m): $0.16-0.20 \quad$ Volume $\left(\mathrm{m}^{3}\right): \mathrm{n} / \mathrm{a}$

Description: Hearth

A small ash lens and associated patch of burnt sediment (diameter $0.63 \mathrm{~m}$ ) lying atop Surface A1.1b along the southern edge of the unit.

\section{Locus: Feature 3}

FS \# 5006

Depth (m): $0.15-0.21 \quad$ Volume $\left(\mathrm{m}^{3}\right): 0.054$

Description: Hearth

A shallow deposit of ash above a small patch of burnt silt loam lying atop Surface A1.1b in the northwestern corner of the unit.

\section{Locus: Feature 4}

FS \# 5008, 5012

Depth (m): $0.21-0.41 \quad$ Volume $\left(\mathrm{m}^{3}\right): 0.045$

Description: Fill, pit

A firm, reddish-brown (5 YR 4/3) silty clay loam with very coarse daub inclusions (7-10\%) and medium to coarse charcoal mottles (2-3\%). This shallow pit (20 cm in depth) extended down from Surface A1.1b and into Substratum A1.2b. The rich fill was consistent with the discard from a pyrotechnic feature.

Locus: A1.1c

FS \# 5007

Depth (m): $0.21-0.30 \quad$ Volume $\left(\mathrm{m}^{3}\right): 0.313$

Description: Fill, general

A firm, brownish-yellow (10 YR 6/6) clay loam with fine daub inclusions (1\%) and fine charcoal mottles (1-3\%). This substratum was general fill sharing a moderately diffuse boundary with Substratum A1.1b. An ash dump was present in the southwestern corner of the unit. In the northern half of the unit, Surface A1.1c appeared as a compact silty clay associated with two ash lenses, one overlying a shallow hearth (Feature 5).

\section{Locus: Feature 5}

FS \# 5007.1

Depth (m): $0.19-0.21 \quad$ Volume (m3): n/a

Description: Hearth

A small, informal hearth of burnt earth and overlying ash (diameter $0.40 \mathrm{~m}$ ) located on Surface A1.1c.

\section{Locus: Feature $\mathbf{N}$}

FS \# 5016.1

Depth (m): 0.34 - $0.66 \quad$ Volume (m3): n/a

Description: Fill, pit

A friable, reddish-yellow (7.5 YR 6/6) clay loam with fine to medium charcoal mottles (1-2\%). This feature cut down to a depth of at least 32 centimeters below Surface A1.2a. Based on the clear boundaries, shallow depth, and density of faunal remains, this pit may have been made specifically to dispose of refuse from a single episode of food preparation and consumption. 


\section{Stratum A1.2}

Locus: A1.2a.i/ii

FS \# 5009, 5010, 5011, 5014

Depth (m): 0.29 - $0.42 \quad$ Volume $\left(\mathrm{m}^{3}\right): 0.344$

Description: Fill, general

A firm, light yellowish-brown (10 YR 6/4) silty

clay with fine daub inclusions (1\%) and fine to medium charcoal mottles (3-7\%). Stratum A1.2a.i was a relatively thin $(4-7 \mathrm{~cm})$ horizon lying atop Surface A1.2a.i, defined in profile by horizontal sherd orientations and two shallow ash-filled hearths. Along with general occupation debris, this stratum also included earthen wall collapse from architectural features (L and 7) initially constructed on underlying surfaces. Stratum A1.2a.ii was a thin (5-7 cm) horizon of unknown origin, possibly deriving from either quotidian domestic activities or a brief construction episode. Surface A1.2a.ii supported two thick (3-4 cm) ash lenses or informal hearths, a rectilinear rock installation (Feature 6) overlying a third hearth, and collapsed fragments (Feature L) from an earthen wall.

\section{Locus: Feature 7}

\section{FS \# 5013}

Depth (m): $0.24-0.41 \quad$ Volume $\left(\mathrm{m}^{3}\right): 0.061$

Description: Earthen wall melt

An extremely firm, amorphous deposit of yellow (10 YR 8/6) clay loam with negligible inclusions. This sediment appeared to derive from episodic wall melt or collapse associated with an unidentified earthen wall structure contemporaneous with Substrata A1.2a.i and A1.2a.ii.

\section{Locus: Feature 6}

FS \# 5010.1

Depth (m): 0.23 - $0.32 \quad$ Volume $\left(\mathrm{m}^{3}\right): \mathrm{n} / \mathrm{a}$

Description: Stone alignment

A rectilinear alignment of sandstone rocks running east to southwest on Surface A1.2a.ii. Measuring 2.15 meters long and 0.22 meters wide, this feature was possibly a foundation or reinforcement to an earthen wall structure.

\section{Locus: Feature L}

\section{FS \# 5015.1}

Depth (m): $0.41 \quad-0.51 \quad$ Volume $\left(\mathrm{m}^{3}\right): \mathrm{n} / \mathrm{a}$

Description: Earthen wall stub

An earthen wall stub with very poor preservation on Surface A1.2a.ii. The matrix was an extremely firm, yellowish red (5 YR 5/6) sandy loam with light laterite gravel inclusions and very fine charcoal mottles (1-2\%).

\section{Locus: A1.2b}

FS \# 5015, 5016

Depth (m): $0.40-0.51 \quad$ Volume $\left(\mathrm{m}^{3}\right): 0.390$ Description: Fill, general A firm, strong brown (7.5 YR 5/6) silty clay with fine daub inclusions $(<1 \%)$, light ash, and very fine to fine charcoal mottles $(<1 \%)$. A few small sandstone rock fragments throughout the soil matrix suggested that this stratum derived partly from earthen wall demolition. Surface A1.2b included two ash-filled hearths (Features E and 9), a small ash lens, and a deposit of terre pisé collapse (Feature L). The surface was interrupted by a circular installation of sandstone rocks (Feature 8) extending upward from A1.2c.

\section{Locus: Feature 9}

FS \# 5017

Depth (m): $0.51 \quad-0.54 \quad$ Volume $\left(\mathrm{m}^{3}\right): 0.003$

Description: Hearth

An ash-filled hearth associated with Surface A1.2b.

\section{Locus: Feature E}

FS \# 5016.2

Depth (m): $0.49-0.51 \quad$ Volume $\left(\mathrm{m}^{3}\right): \mathrm{n} / \mathrm{a}$

Description: Hearth

A large ash lens and informal hearth on Surface A1.2b measuring 0.74 meters in diameter.

\section{Locus: Feature 10}

FS \# 5019, 5026, 5027

Depth (m): 0.53 - 0.81 Volume $\left(\mathrm{m}^{3}\right): 0.196$

Description: Fill, midden

A friable to firm, brown (7.5 YR 5/4) silty clay loam with medium to coarse charcoal mottles (10\%). Although this deposit had a poorly defined boundary, it filled a shallow depression extending down into Substratum A1.3a and was rich with artifacts, including one partially reconstructable vessel and a high density of large faunal elements. Whatever the origins of the depression, the fill was a general domestic refuse.

Locus: A1.2c

FS \# 5018, 5020, 5022

Depth $(\mathrm{m}): 0.49-0.65 \quad$ Volume $\left(\mathrm{m}^{3}\right): 0.393$

Description: Fill, general

A firm, strong brown (7.5 YR 5/6) silty clay with fine daub inclusions (1\%), light ash, and fine to fine charcoal mottles (1\%) nearly identical to Substratum A1.2b. The Feature 10 midden deposit encroached into the southern end of this substratum. The underlying Surface A1.2c supported a circular installation of sandstone rocks (Feature 8) and one informal hearth (Feature 18).

\section{Locus: Feature 8}

FS \# 5023

Depth (m): 0.58 - $0.63 \quad$ Volume $\left(\mathrm{m}^{3}\right): 0.022$

Description: Stone foundation, curvilinear

A nearly complete circular installation of seven sandstone rocks (diameter $0.80 \mathrm{~m}$ ). The stones all lay flush on Surface A1.2c. The soil matrix encasing the feature was a compact, light yellowish-brown (10 YR 6/4) silty clay. Directly beneath the rocks was a thin horizon $(4-5 \mathrm{~cm})$ of light brown silty clay combined with loose ash. 


\section{Locus: Feature 18}

FS \# 5018.1

Depth (m): 0.57 - $0.59 \quad$ Volume $\left(\mathrm{m}^{3}\right): \mathrm{n} / \mathrm{a}$

Description: Hearth

An informal hearth of burnt sediment (diameter 0.43

$\mathrm{m})$ overlaid with a thin lens of ash and associated

with a large fragment of daub (thickness $10 \mathrm{~cm}$ ).

Locus: A1.2d

FS \# 5021, 5025

Depth (m): $0.58-0.72 \quad$ Volume $\left(\mathrm{m}^{3}\right): 0.389$

Description: Fill, general

A firm, strong brown (7.5 YR 5/6) sandy clay with medium daub inclusions (3-7\%) and fine to medium charcoal mottles (1-5\%). Several angular sandstone rocks were present in the southeastern corner of the unit, but did not form a clear alignment. Based on the relatively high densities of charcoal, burnt daub, and large artifacts, this substratum most likely arose from a combination of domestic activities and earthen wall demolition. Beneath this deposit, a compact and plastered floor (Surface A1.2d) provided evidence for domestic occupation in the form of a hearth complex (Feature 11).

\section{Locus: Feature 11}

\section{FS \# 5024}

Depth (m): 0.63 - $0.66 \quad$ Volume $\left(\mathrm{m}^{3}\right): 0.017$

Description: Hearth complex

A thick horizon (3-4 cm) of three superimposed hearths, each defined by a reddish burnt sediment and separated by ash lenses.

\section{Stratum A1.3}

\section{Locus: A1.3a}

FS \# 5029, 5030, 5032

Depth $(\mathrm{m}): 0.71-0.81 \quad$ Volume $\left(\mathrm{m}^{3}\right)$ : 0.257

Description: Fill, general

A moderately firm, strong brown (7.5 YR 5/6) silty clay with medium daub inclusions (3-5\%) and fine charcoal mottles (1-5\%). Modest artifact frequencies, a solitary ash-filled hearth (Feature D), and a few sandstone fragments pointed to deposition through general occupation processes, including earthen wall maintenance and refuse disposal. A lens of sand and gravel in the eastern half of Unit A1 may have resulted from a brief episode of construction or surface leveling.

\section{Locus: Feature D}

FS \# 5031

Depth $(\mathrm{m}): 0.72-0.82 \quad$ Volume $\left(\mathrm{m}^{3}\right): 0.056$

Description: Hearth

A modest, informal hearth of burnt sediment $(3-5 \mathrm{~cm}$ in thickness) and overlying ash lens.
Locus: A1.3b.i

FS \# 5033

Depth (m): $0.81 \quad-0.92 \quad$ Volume $\left(\mathrm{m}^{3}\right): 0.119$

Description: Fill, midden

A friable to moderately firm, reddish-yellow (7.5 YR 6/6) sandy clay loam with dense inclusions of medium daub (3-5\%) and fine to medium charcoal mottles (5-7\%). The friable consistency of the soil matrix and high densities of daub, charcoal, and artifacts pointed to the presence of a sheet midden and/or episode of earthen wall collapse.

Locus: A1.3b.ii

FS \# 5034, 5035

Depth $(\mathrm{m}): 0.80-0.91 \quad$ Volume $\left(\mathrm{m}^{3}\right): 0.281$

Description: Fill, general

A moderately firm, brown (7.5 YR 5/4) silty clay with inclusions of medium daub (3-5\%) and fine to medium charcoal mottles (1-2\%). This matrix was associated with a linear alignment of small sandstone blocks (Feature 13) atop the poorly defined Surface A1.3b. This surface also articulated with Feature 14, a pyrotechnic feature originating in the underlying stratum.

\section{Locus: Feature 13}

FS \# 5035.1

Depth (m): 0.83 - $0.89 \quad$ Volume $\left(\mathrm{m}^{3}\right): \mathrm{n} / \mathrm{a}$

Description: Stone alignment

A rectilinear alignment of small angular sandstone rocks on Surface A1.3b just visible in the southwestern corner of the unit.

\section{Locus: Feature 15}

FS \# 5037

Depth (m): $0.91-0.96 \quad$ Volume $\left(\mathrm{m}^{3}\right): 0.006$

Description: Bioturbation

An amorphous pocket of loose fill in the southeastern corner of the unit attributable to an unknown source of biological disturbance.

Locus: A1.3c

FS \# 5038, 5039

Depth $(\mathrm{m}): 0.91-1.04 \quad$ Volume $\left(\mathrm{m}^{3}\right): 0.405$

Description: Fill, general

A moderately firm, reddish-yellow (7.5 YR 6/6) clay loam with fine to medium daub inclusions (3-5\%) and fine charcoal mottles (1\%). Given the high density of daub, this deposit likely formed, in part, from the collapse of an earthen wall structure. Below this stratum, Surface A1.3c was recognizable as a very compact occupational surface abutting Feature

\section{Locus: Feature 14}

FS \# 5036

Depth (m): $0.92 \quad-0.97 \quad$ Volume $\left(\mathrm{m}^{3}\right): 0.086$

Description: Pyrotechnic feature, other A relatively deep $(5-6 \mathrm{~cm})$ pyrotechnic feature marked by substantial burning of the underlying sediment (7.5YR 3/4) and a thick lens of ash and charcoal. Increased frequencies of metallurgical slag in the surrounding Substratum A1.3c matrix suggested that this feature resulted from smithing. 
Locus: A1.3d

FS \# 5040

Depth (m): 1.01 - $1.17 \quad$ Volume $\left(\mathrm{m}^{3}\right)$ : 0.532

Description: Fill, general

A moderately firm, light brown (7.5 YR 6/4) silty

clay with medium daub inclusions $(<1 \%)$ and fine charcoal mottles (1-2\%). The concentration of charcoal in the southern half of Unit A1 was suggestive of an nearby conflagration although no burnt soil was present. Surface A1.3d was not well defined, but did include an arrangement of possible post molds (Feature 12).

\section{Locus: Feature 12}

FS \# 5040.1

Depth (m): 1.14 - $1.19 \quad$ Volume $\left(\mathrm{m}^{3}\right)$ : n/a

Description: Post mold alignment

A possible arrangement of 4-5 post molds on Surface A1.3d with compact interior walls and nearly identical depths.

\section{Locus: Feature 16}

FS \# 5041.1

Depth (m): 1.14 - 1.17

Description: Ash dump

Volume $\left(\mathrm{m}^{3}\right): \mathrm{n} / \mathrm{a}$

A small pocket of ash along the southern edge of the unit just below Surface A1.3d.

Locus: A1.3e

FS \# 5041, 5042

Depth (m): $1.14-1.26 \quad$ Volume $\left(\mathrm{m}^{3}\right): 0.400$

Description: Fill, general

A firm, reddish yellow (7.5 YR 6/6) clay loam with inclusions of medium daub $(<1 \%)$ and charcoal mottles (2-3\%). This substratum derived from an initial episode of occupation in this area.

Locus: Feature 17

FS \# 5042.1

Depth (m): $1.21-1.23 \quad$ Volume $\left(\mathrm{m}^{3}\right): \mathrm{n} / \mathrm{a}$

Description: Ash dump

A small ash lens located at the transition to sterile soil in the northeastern corner of Unit A1.

\section{Stratum A1.4}

Locus: A1 Sterile

FS \# 5042.2

Depth (m): 1.26 - $1.46 \quad$ Volume $\left(\mathrm{m}^{3}\right): \quad 0.200$

Description: Sterile

An extremely firm, yellowish-red (5 YR 5/8) silty clay with trivial inclusions of laterite pea gravel $(<1 \%)$. No cultural remains or features were present in this deposit; sterile.

\section{Unit A2}

\section{Stratum A2.1}

\section{Locus: A2 Surface}

FS \# 5269, 5270, 5271, 5272, 5302

Depth (m): $0.00-0.07 \quad$ Volume $\left(\mathrm{m}^{3}\right): 0.220$

Description: Surface

A loose, brown (7.5 YR 4/4) silt loam littered with organic detritus, laterite pea gravel, and crushed sherds, including fragments of several partially reconstructable vessels. Located in the southcentral part of Area A, the surface of the unit sloped gently down from the north to the south. The dozen angular sandstone rocks dotting the surface formed no clear alignment, although circular foundations appeared directly to the north, east, and south.

\section{Locus: A2.1a}

FS \# 5275, 5276, 5301

Depth $(\mathrm{m}): 0.00-0.14 \quad$ Volume $\left(\mathrm{m}^{3}\right): 0.560$

Description: Fill, general

A loose, strong brown (7.5 YR 4/6) silty clay with light organic detritus. Although this substratum was weathered from the underlying general fill, it did not appear significantly deflated.

\section{Locus: Feature 114a}

FS \# 5273, 5274, 5279, 5282, 5286, 5289, 5293, 5295, 5298, 5303

Depth (m): $0.00-0.41 \quad$ Volume $\left(\mathrm{m}^{3}\right): 2.223$

Description: Fill, pit

A broad and amorphous pit filled with debris from domestic and craft production activities. While the form and dimensions suggested a borrow pit, the depression was subsequently filled with at least two different levels of refuse fill and capped with a more diffuse sheet midden. Feature 114a, the uppermost level, was a friable to moderately firm, strong brown (7.5 YR 4/6) sandy clay loam with medium charcoal mottles (1\%) and substantial frequencies of faunal remains and ceramic sherds, many from partially reconstructable vessels.

\section{Locus: Feature 114b}

FS \# 5317, 5331

Depth (m): $0.38-0.55 \quad$ Volume $\left(\mathrm{m}^{3}\right): 0.170$

Description: Fill, pit

Feature 114b, the intermediate level of within this borrow pit, was a moderately firm, strong brown (7.5 YR 4/6) silt loam with medium daub inclusions (1\%), fine to coarse charcoal mottles (3-5\%), ash lenses, and moderate artifact densities. 
Locus: Feature 114c

FS \# 5311, 5333, 5334, 5349, 5357

Depth (m): 0.34 - $0.87 \quad$ Volume $\left(\mathrm{m}^{3}\right): \quad 1.258$

Description: Fill, pit

Feature 114c, the lowest level of fill within the borrow pit, was a moderately firm, strong brown (7.5

YR 4/6) silt loam with medium daub inclusions (1\%) and fine to coarse charcoal mottles (3-5\%), but lacked the ash lenses of Feature 114b. Feature 114c contained substantial frequencies of ceramic sherds and large faunal remains. The boundaries of the pit were rather diffuse in all three levels. Although the bottom was not reached during excavation, the contours of the pit visible in the north profile and in the cross-section of Feature 122 (see below) both pointed to a maximum depth of 1.20 meters below the present-day ground surface.

\section{Locus: Feature 128}

FS \# 5345

Depth (m): 0.62 - $0.72 \quad$ Volume $\left(\mathrm{m}^{3}\right): 0.015$

Description: Stone foundation, irregular

A small dump of sandstone rocks and a laterite block on the bottom, eastern margin of the Feature 114 borrow pit.

\section{Locus: Feature 129}

FS \# 5346

Depth (m): 0.62 - $0.72 \quad$ Volume $\left(\mathrm{m}^{3}\right): 0.016$

Description: Stone foundation, irregular A small dump of sandstone rocks on the bottom, eastern margin of the Feature 114 borrow pit.

\section{Locus: A2.1b}

FS \# 5277, 5278, 5280, 5281, 5283, 5285, 5301, 5304, 5305, 5306

Depth (m): $0.03-0.27 \quad$ Volume $\left(\mathrm{m}^{3}\right): 2.425$

Description: Fill, general

A moderately firm, strong brown (7.5 YR 5/6) sandy clay with laterite pea gravel $(<1 \%)$ and light ash. This substratum was a weathered deposit produced from general occupation and construction activities on Surface A2.1b, which otherwise included two ash dumps (Features 119 and 120), a circular pit of unknown dimensions (Feature 116), and the collapse of an earthen wall structure (Feature 115).

\section{Locus: Feature 115 Collapse}

FS \# 5307

Depth (m): $0.16-0.18 \quad$ Volume $\left(\mathrm{m}^{3}\right):$ n/a

Description: Earthen wall collapse

This deposit included the entirety of Feature 118, a small ash dump falling within the boundaries of Feature 115 earthen wall structure. The entire contents were collected as a soil sample (PP\# 211).

\section{Locus: Feature 116}

FS \# 5284, 5324

Depth $(\mathrm{m}): 0.14-0.75 \quad$ Volume $\left(\mathrm{m}^{3}\right): 0.083$

Description: Fill, pit

A round pit, measuring at least 0.80 meters in diameter and 0.70 meters in depth below Surface
A2.1b. Whatever the initial function, the pit fill was a friable, brown (7.5 YR 5/4) sandy clay loam with fine to medium charcoal mottles (2-3\%), ash lenses, and moderate densities of ceramic sherds and faunal remains. The pit boundaries were clearly defined.

\section{Locus: Feature 119}

FS \# 5306.1

Depth (m): $0.19-0.23 \quad$ Volume $\left(\mathrm{m}^{3}\right): \mathrm{n} / \mathrm{a}$

Description: Ash dump

A small ash dump on Surface A2.1b.

Locus: Feature 120

FS \# 5305.1

Depth (m): $0.21-0.24 \quad$ Volume $\left(\mathrm{m}^{3}\right): \mathrm{n} / \mathrm{a}$

Description: Ash dump

A small ash dump on Surface A2.1b.

\section{Locus: Feature 115 Interior}

FS \# 5291, 5309

Depth (m): $0.18-0.29 \quad$ Volume $\left(\mathrm{m}^{3}\right): 0.165$

Description: Fill, room

An initial floor surface and horizon of fill within Feature 115. The interior surface and associated fill was a compact, strong brown (7.5 YR 5/6) clay loam with light daub inclusions (1\%).

\section{Locus: A2.1c}

FS \# 5288, 5290, 5292, 5294, 5308, 5310

Depth $(\mathrm{m}): 0.17-0.32 \quad$ Volume $\left(\mathrm{m}^{3}\right): 1.114$

Description: Fill, midden

A moderately firm, strong brown (7.5 YR 5/6) clay loam with coarse daub inclusions $(<1 \%)$ and fine charcoal mottles $(<1 \%)$. This substratum was a general fill from domestic activities contemporaneous with the use of a circular, earthen wall hut (Feature 115) on Surface A2.1c.

\section{Locus: Feature 115}

FS \# 5312

Depth $(\mathrm{m}): 0.17-0.37 \quad$ Volume $\left(\mathrm{m}^{3}\right): \quad 0.269$

Description: Earthen wall stub

A circular, earthen wall structure measuring some 2.20-2.30 meters in diameter on Surface A2.1c. The wall stub (20-26 cm thick) was a friable, yellowishbrown (10 YR 5/6) clay loam with very poor definition in the southwest quarter of the feature. A curvilinear arrangement of nine small sandstone rocks lay beneath the wall stub in the northeast corner, possibly demarcating an entryway.

\section{Locus: A2.1d}

FS \# 5296, 5297, 5299, 5300, 5313, 5314, 5315, 5316

Depth (m): 0.25 - $0.39 \quad$ Volume $\left(\mathrm{m}^{3}\right): 1.366$

Description: Fill, midden

A moderately firm, strong brown (7.5 YR 4/6) silt loam with coarse daub inclusions $(<1 \%)$ and medium charcoal mottles $(<1 \%)$. Despite the low density of charcoal, high artifact frequencies indicated that this substratum formed through refuse disposal. The underlying stratigraphic transition was not a surface per se although a cylindrical pit (Feature 122) did begin at this level. 
Locus: Feature 122

FS \# 5322, 5325, 5340, 5341, 5342

Depth (m): 0.35 - $1.25 \quad$ Volume $\left(\mathrm{m}^{3}\right): 1.340$

Description: Fill, pit

A slightly amorphous pit of unknown function, measuring at least 1.40 meters in diameter and 0.85 meters in depth below Surface A2.1d. The pit fill was a friable to moderately firm, dark yellowishbrown (10 YR 4/6) to strong brown (7.5 YR 5/6) sandy clay loam with medium to coarse daub inclusions (1-2\%) and fine to medium charcoal mottles (2-3\%). The density of artifacts, including ceramic sherds and large faunal remains, suggested that this deposit incorporated several episodes of household refuse.

\section{Stratum A2.2}

Locus: A2.2a

FS \# 5318, 5319, 5320, 5321, 5323

Depth $(\mathrm{m}): 0.35$ - $0.53 \quad$ Volume $\left(\mathrm{m}^{3}\right): \quad 1.928$

Description: Fill, midden

A moderately firm, strong brown (7.5 YR 4/6) sandy clay loam with medium to coarse daub inclusions (1-3\%) and fine to medium charcoal mottles (3-5\%). This substratum represented general occupation activity, including some earthen wall decay or collapse, although the source of elevated charcoal frequencies was not apparent.

Locus: A2.2b

FS \# 5326, 5329, 5330, 5332

Depth (m): $0.44-0.57 \quad$ Volume $\left(\mathrm{m}^{3}\right): 0.593$

Description: Fill, general

An extremely firm, strong brown (7.5 YR 5/6) silty clay with medium daub inclusions (2-3\%) and fine to medium charcoal (1\%). This substratum resulted from general residential activities, presumably associated with several angular rock installations (Features 123, 125, 126, 127) on the compact and well-defined Surface A2.2b.

\section{Locus: Feature 125}

FS \# 5329.1

Depth (m): $0.41-0.50 \quad$ Volume $\left(\mathrm{m}^{3}\right): \mathrm{n} / \mathrm{a}$

Description: Stone foundation, rectilinear

A rectilinear arrangement of four angular sandstone rocks, measuring 0.65 meters by 0.90 meters, on Surface A2.2b. The associated soil matrix did not differ from Substratum A2.2b. The stones lay directly above a horizon (3-5 cm thick) of crushed sandstone.

\section{Locus: Feature 127}

FS \# 5326.1

Depth (m): $0.52 \quad-0.56 \quad$ Volume $\left(\mathrm{m}^{3}\right): \mathrm{n} / \mathrm{a}$

Description: Stone foundation, irregular

An irregular installation of ten angular rocks, measuring up to 0.80 meters in diameter, on Surface A2.2b. The associated soil matrix did not differ from the surrounding Substratum A2.2b.
Locus: Feature 123

FS \# 5327, 5337

Depth (m): 0.46 - $0.66 \quad$ Volume $\left(\mathrm{m}^{3}\right): 0.288$

Description: Stone foundation, curvilinear

A circular installation of at least 25 rocks, with an estimated diameter of 1.70-1.80 meters, located on Surface A2.2b. The associated matrix was an extremely firm, strong brown (7.5 YR 4/6) clay with fine daub inclusions (2-3\%), fine to medium charcoal mottles (1\%), and high artifact densities. This deposit was either part of the rock foundation itself, or derived from the long-term melt of an earthen wall superstructure and associated activities in the immediate vicinity. This installation lay only 5-10 centimeters above, and within the same spatial footprint, as a nearly identical installation (Feature 130, see below).

\section{Locus: Feature 126}

FS \# 5328

Depth (m): $0.46-0.51 \quad$ Volume $\left(\mathrm{m}^{3}\right): 0.019$

Description: Stone foundation, irregular

An irregular installation of six small, angular sandstone rocks (diameter 0.55-0.70 m) on Surface A2.2b. The associated soil matrix did not differ from the surrounding Substratum A2.2b.

Locus: A2.2c.i

FS \# 5335, 5336, 5338

Depth $(\mathrm{m}): 0.50-0.72 \quad$ Volume $\left(\mathrm{m}^{3}\right): 1.347$

Description: Fill, general

A moderately firm to firm, strong brown (7.5 YR

4/6) silt loam with medium daub inclusions (1\%) and fine to medium charcoal mottles $(<1 \%)$. This substratum probably formed through general occupation activities and gradual earthen wall melt.

Locus: A2.2c.ii

FS \# 5339

Depth (m): 0.66 - $0.72 \quad$ Volume $\left(\mathrm{m}^{3}\right): 0.083$

Description: Stone foundation, curvilinear An extremely firm, strong brown (7.5 YR 5/6) silty clay with fine to medium daub inclusions (1-2\%) and fine to medium charcoal mottles $(<1 \%)$. This substratum occupied the space between two circular sandstone installations (Features 123 and 130); it likely constituted an intentional fill and repaving episode associated with their construction.

Locus: A2.2d

FS \# 5343, 5344, 5347, 5348

Depth (m): 0.65 - $0.77 \quad$ Volume $\left(\mathrm{m}^{3}\right): 0.583$ Description: Earthen wall collapse

An extremely firm, strong brown (7.5 YR 4/6) silty clay loam with fine to medium daub inclusions (5\%). This substratum presumably derived from earthen wall decay and collapse on the moderately welldefined Surface A2.2d. 


\section{Locus: Feature 130}

FS \# 5350

Depth (m): 0.68 - $0.76 \quad$ Volume $\left(\mathrm{m}^{3}\right): 0.065$

Description: Stone foundation, curvilinear

A circular installation of at least 29 sandstone rocks, with an estimated diameter of 1.80-1.90 meters, located on Surface A2.2d. The associated matrix was an extremely firm, strong brown (7.5 YR $5 / 6)$ silty clay with fine daub inclusions (1-2\%) and fine to medium charcoal mottles $(<1 \%)$ with an underlying lens of laterite gravel. This matrix was either part of the rock foundation itself, or derived from the long-term melt of an earthen wall superstructure and nearby activities.

\section{Locus: Feature 132}

FS \# 5335.1

Depth (m): $0.61 \quad-0.81 \quad$ Volume $\left(\mathrm{m}^{3}\right):$ n/a

Description: Earthen wall stub

A poorly preserved arc of earthen wall stub (10-15 $\mathrm{cm}$ thick) and associated ash lens on Surface A2.2d. The wall stub was a friable, yellowish-brown (10 YR 5/6) clay loam.

\section{Stratum A2.3}

Locus: A2.3a.i

FS \# 5351

Depth (m): $0.73-0.85 \quad$ Volume $\left(\mathrm{m}^{3}\right): \quad 0.320$

Description: Earthen wall collapse

An extremely firm, strong brown (7.5 YR 4/6) silt with fine to medium daub inclusions (7\%) and fine charcoal $(<1 \%)$. This substratum derived primarily from earthen wall melt and/or collapse.

Locus: A2.3b

FS \# 5352, 5354

Depth $(\mathrm{m}): 0.81-1.05 \quad$ Volume $\left(\mathrm{m}^{3}\right): 0.472$

Description: Fill, midden

A friable to moderately firm, light brown (7.5 YR

$6 / 4)$ sandy clay loam with fine to coarse daub (2\%), fine to medium charcoal (5-7\%), and elevated artifact densities.

Locus: A2.3a.ii

FS \# 5353

Depth (m): $0.81-0.94 \quad$ Volume $\left(\mathrm{m}^{3}\right): \quad 0.248$

Description: Earthen wall melt

An extremely firm, strong brown (7.5 YR 4/6) silt with fine to coarse daub inclusions (10-15\%) and fine charcoal $(<1 \%)$. This substratum derived primarily from earthen wall melt and/or collapse.

Locus: A2.3c

FS \# 5355, 5356

Depth (m): $0.93-1.21 \quad$ Volume $\left(\mathrm{m}^{3}\right)$ : 0.728

Description: Fill, general

An extremely firm, strong brown (7.5 YR 4/6) silty clay loam with very few inclusions. This substratum formed from general activity associated with initial occupation of the area.

\section{Stratum A2.4}

Locus: A2 Sterile

FS \# 5356.1

Depth (m): 1.21 - $1.26 \quad$ Volume $\left(\mathrm{m}^{3}\right): 0.200$

Description: Sterile

An extremely firm, reddish-yellow (7.5 YR 6/6) silty

clay loam with no mottles or inclusions. This

stratum contained no evidence of human activity;

culturally sterile.

\section{Unit C (Feature C-01)}

\section{Stratum C.1}

\section{Locus: Feature C-01}

FS \# 5400, 5401

Depth (m): $0.00-0.11 \quad$ Volume $\left(\mathrm{m}^{3}\right): 0.055$

Description: Macrolithic scatter

Feature C-01 was a macrolithic scatter of mudstone debitage. The associated matrix was a yellowish red silty clay deposited through aeolian erosion and long-term deflation.

\section{Locus: C Sterile}

FS \# 5402

Depth (m): $0.11-0.18 \quad$ Volume $\left(\mathrm{m}^{3}\right): 0.110$

Description: Sterile

A firm, reddish-yellow (7.5 YR 6/6) silty clay with no visible natural inclusions or cultural material.

\section{Unit F}

\section{Stratum F.1}

\section{Locus: F Surface}

FS \# 5085

Depth (m): $0.00-0.05 \quad$ Volume $\left(\mathrm{m}^{3}\right): 0.080$

Description: Surface

The surface of Unit F was a loose, light brown (7.5 YR 6/4) sandy clay littered with laterite pea gravel and angular sandstone fragments. Placed near several circular stone installations on the apex of Area F, the surface was fairly level with minimal erosion although high ceramic density attested to modest deflation.

\section{Locus: F.1a}

FS \# 5086, 5087, 5088

Depth $(\mathrm{m}): 0.00-0.10 \quad$ Volume $\left(\mathrm{m}^{3}\right): 1.120$

Description: Fill, general

A loose to friable, strong brown (7.5 YR 5/6) sandy loam with fine to coarse daub inclusions $(<1 \%)$, laterite pea gravel (1\%), and light organic detritus. This substratum was weathered from an underlying fill produced, in part, through the collapse and destruction of several earthen wall structures. 


\section{Locus: F.1b}

FS \# 5090, 5091, 5092, 5093, 5096, 5098

Depth (m): 0.04 - $0.19 \quad$ Volume $\left(\mathrm{m}^{3}\right)$ : $\quad 0.776$

Description: Earthen wall collapse

A firm to very firm, brownish yellow (10 YR 6/6)

silty clay loam with fine to medium daub inclusions

(2\%). This substratum appeared to result from

earthen wall collapse and subsequent weathering or leveling; it lay directly atop both Surface F.1b and

Surface F.1c where Substratum F.1c did not

intervene. Architectural structures (Features 54 and

57) from the lower surface extended into this one.

\section{Locus: Feature 51}

FS \# 5091.1

Depth $(\mathrm{m}): 0.10-0.12 \quad$ Volume $\left(\mathrm{m}^{3}\right): \mathrm{n} / \mathrm{a}$

Description: Ash dump

A small ash dump associated with the deposition of Stratum F.1b.

Locus: Feature 52/53

FS \# 5094, 5095

Depth (m): $0.09-0.18 \quad$ Volume $\left(\mathrm{m}^{3}\right): \quad 0.280$

Description: Hearth complex

A complex of two to three superimposed hearths comprised of burnt sediment horizons (each 2-4 cm thick) and intervening ash lenses comprising Surface F.1b. The sediment itself was a compact, reddishbrown (5 YR 5/4) clay loam with fine charcoal mottles (1\%) and ash lenses. Earthen wall melt visible in profile indicated that these features may have been circumscribed by, or abutted against, architectural walls.

\section{Locus: F.1c}

FS \# 5089, 5097

Depth (m): $0.10-0.18 \quad$ Volume $\left(\mathrm{m}^{3}\right): 0.346$

Description: Earthen wall melt

A compact, light brownish-yellow (10 YR 6/4) clay loam with fine to medium daub inclusions (1-2\%) and laterite pea gravel (2-5\%). This substratum occurred in amorphous deposits and its color and texture indicated episodes of earthen wall melt, possibly from several architectural features on the underlying occupational Surface F.1c, including an ill-defined wall stub (Feature 57), circular stone installation (Feature 54), and large hearth complex (Feature 52/53).

\section{Locus: Feature 54}

FS \# 5096.1

Depth (m): 0.11 - $0.18 \quad$ Volume $\left(\mathrm{m}^{3}\right): \mathrm{n} / \mathrm{a}$

Description: Stone foundation, curvilinear

A semi-circular installation of seven sandstone rocks on Surface F.1c measuring 1.23 meters in diameter. The feature appeared to have been partially dismantled. The associated sediment was a moderately firm, light brown (7.5 YR 6/4) clay loam with fine to medium daub inclusions (2-3\%), laterite pea gravel (1\%), and charcoal mottles $(<1 \%)$.

\section{Locus: Feature 58/59}

FS \# 5103

Depth (m): $0.18-0.28 \quad$ Volume $\left(\mathrm{m}^{3}\right): 0.199$

Description: Hearth complex

A complex of two to three superimposed hearths comprised of burnt sediment horizons (each 2-4 cm thickness) and intervening ash lenses on Surface F.1c. The sediment itself was a compact, reddishbrown (5 YR 5/4) clay loam with fine charcoal mottles (1\%) and ash lenses.

\section{Locus: Feature 57}

FS \# 5099

Depth (m): $0.07-0.24 \quad$ Volume $\left(\mathrm{m}^{3}\right): \quad 0.280$

Description: Earthen wall stub

A poorly-preserved circular earthen wall structure on Surface F.1c measuring at least 1.90 meters in diameter (wall width unknown). The sediment was an extremely firm, light brown (7.5 YR 6/4) silty clay loam with fine to medium daub inclusions $(<1 \%)$. An informal hearth of burnt sediment and ash was present on the interior floor. No replastering episodes were apparent within the structure.

\section{Stratum F.2}

Locus: F.2a.i

FS \# 5102, 5104, 5107

Depth $(\mathrm{m}): 0.13-0.28 \quad$ Volume $\left(\mathrm{m}^{3}\right): 0.502$

Description: Earthen wall melt

An extremely firm, light brown (7.5 YR 6/4) silty clay loam with fine to coarse daub inclusions (1\%) and fine to medium charcoal mottles (1\%). This substratum derived primarily from episodic earthen wall melt/collapse and subsequent leveling of the area for further domestic activities and architecture.

Locus: F.2a.ii

FS \# 5105, 5106

Depth (m): $0.17 \quad-0.31 \quad$ Volume $\left(\mathrm{m}^{3}\right): \quad 0.400$

Description: Earthen wall collapse

A firm, strong brown (7.5 YR 5/4) silty clay with

fine to coarse daub inclusions (5\%) and laterite pea gravel (5\%). Based upon the irregularity of deposition and overall consistency, this substratum likely derived from an episode of wall collapse and/or leveling.

Locus: F.2b

FS \# 5100

Depth $(\mathrm{m}): 0.24-0.32 \quad$ Volume $\left(\mathrm{m}^{3}\right): 0.281$

Description: Fill, general

A moderately to extremely firm, strong brown (7.5

YR 5/4) silty clay with fine charcoal mottles (1-2\%).

This substratum also contained large fragments of burnt daub oriented horizontally on a poorly defined surface. Based upon the heterogeneity and presence of charcoal in this deposit, particularly in the northern half of Unit F, it may have resulted from use of a possible roasting pit (Feature 65) on the underlying Surface F.05/06. 


\section{Locus: Feature 60 \\ FS \# 5105.1 \\ Depth (m): $0.18-0.28 \quad$ Volume $\left(\mathrm{m}^{3}\right)$ : n/a \\ Description: Stone foundation, irregular \\ An irregular scatter of five sandstone rocks on Surface F.2b/c, possibly from dismantling another installation (Feature 54?). The associated fill was an extremely firm, brown (7.5 YR 5/4) clay loam with laterite pea gravel (1-2\%) and fine charcoal flecks $(<1 \%)$, possibly indicating an eroded earthen wall superstructure.}

\section{Locus: Feature 62}

FS \# 5112

Depth (m): 0.25 - $0.46 \quad$ Volume $\left(\mathrm{m}^{3}\right): 0.089$ Description: Stone foundation, rectilinear

A square installation of twelve sandstone rocks stacked as two courses in a shallow pit excavated into Surface F.2b/c. The rectilinear stack measured 0.67 meters in diameter. Easily distinguishable from the surrounding sediment, the associated fill was a moderately firm, strong brown (7.5 YR 5/6) silty clay loam with fine to medium daub inclusions (1$2 \%)$ and fine charcoal mottles $(<1 \%)$.

\section{Locus: Feature 65}

FS \# 5115, 5117

Depth (m): $0.28-0.49 \quad$ Volume $\left(\mathrm{m}^{3}\right): \quad 0.300$

Description: Pyrotechnic feature, other

A shallow pit, measuring 1.30 meters in diameter, excavated through Surface F.2b/c and lined with a thin layer (2-3 cm thick) of friable, yellowish-white clay. The bottom of the pit was burned to produce a layer (4-5 cm thick) of oxidized, reddish sediment reminiscent of roasting activity. The fill was a moderately firm, strong brown (7.5 YR 5/6) sandy clay loam with daub inclusions (1\%) and dense fine to medium charcoal mottles (5\%), and containing a modest amount of faunal remains. Two small sandstone rocks capping the pit hinted at a functional association with Feature 62.

\section{Locus: Feature 61}

FS \# 5111.1

Depth (m): 0.23 - $0.52 \quad$ Volume $\left(\mathrm{m}^{3}\right): \mathrm{n} / \mathrm{a}$

Description: Earthen wall stub

A possible earthen wall stub of unknown dimensions protruding from the western profile of Unit F. The wall stub was an extremely firm, light Brownish-yellow (10 YR 6/4) clay loam with fine to medium daub inclusions (3\%). The structural fill, visible only in profile, was a moderately firm, strong brown (7.5 YR 4/6) silty clay loam with fine to coarse daub inclusions (5\%) and fine to medium charcoal mottles $(<1 \%)$.
Locus: F.2c.i

FS \# 5108, 5110, 5111, 5120, 5121

Depth (m): $0.23-0.48 \quad$ Volume $\left(\mathrm{m}^{3}\right): 1.234$

Description: Earthen wall collapse

A moderately to extremely firm, reddish-brown (5 YR 5/4) sandy clay loam with medium daub inclusions (3\%) and very light laterite pea gravel $(<1 \%)$. Based on its resemblance to the natural alluvium beneath Diouboye, this deposit most likely derived from terre pisé wall collapse. No clear occupation surface intervened between this substratum and the next.

\section{Locus: F.2c.ii}

FS \# 5109, 5119

Depth $(\mathrm{m}): 0.23-0.40 \quad$ Volume $\left(\mathrm{m}^{3}\right): 0.426$

Description: Earthen wall melt

An extremely firm, strong brown (7.5 YR 5/4) sandy to silty clay with fine to medium daub inclusions (3-5\%) and laterite pea gravel (1-5\%). This substratum probably resulted from the episodes of run-off from earthen wall structures initially constructed on Surface F.2d.

\section{Locus: Feature 63}

FS \# 5113

Depth $(\mathrm{m}): 0.38-0.41 \quad$ Volume $\left(\mathrm{m}^{3}\right): 0.002$

Description: Ash dump

A small ash and charcoal dump, possibly the result of bioturbation.

\section{Locus: Feature 66 Interior}

FS \# 5116

Depth $(\mathrm{m}): 0.40-0.46 \quad$ Volume $\left(\mathrm{m}^{3}\right): 0.089$

Description: Fill, room

An initial floor surface and first layer of fill within

Feature 66. The floor was a compact, light

yellowish-brown (10 YR 6/4) clay loam with some light ash and burning indicative of a small, informal earth in the center of the structure. The first layer of fill (3-6 cm thick) above this surface was a compact, Yellowish-brown (10 YR 5/4) clay loam with fine to coarse daub mottling (5-7\%) and fine ash lenses above and below, but contained very few cultural materials.

Locus: F.2d

FS \# 5122, 5123

Depth $(\mathrm{m}): 0.38-0.49 \quad$ Volume $\left(\mathrm{m}^{3}\right): 0.494$

Description: Fill, general

A firm, light brown (7.5 YR 6/4) brown silt with fine to medium daub inclusions $(<1 \%)$. This substratum contrasted with features constructed on Surface F.2d, including two earthen wall structures (Features 66 and 68), a stone installation (Feature 64), a hearth complex (Feature 70), and ash dump (Feature 67). While elevated concentrations of earthen wall melt/daub (5-7\%) in the northwestern corner of the unit attested to the demolition of Feature 71, the array of features and modest artifact densities otherwise attested to a general fill deposit. 


\begin{abstract}
Locus: Feature 66
FS \# 5124, 5127

Depth (m): $0.41 \quad 0.58 \quad$ Volume $\left(\mathrm{m}^{3}\right): \quad 0.251$

Description: Earthen wall stub

A poorly preserved, circular earthen wall structure built atop Surface F.2d with an estimated external diameter of 2.50 meters. The eroded earthen wall stub (26 cm thick) was an extremely firm, light brown (7.5 YR 6/4) clay loam with fine to medium daub inclusions (5-10\%) and light laterite gravel inclusions (1\%). Two small sandstone slabs lay beneath the wall stub as it entered the southern profile of Unit F, possibly marking an entryway.
\end{abstract}

\section{Locus: Feature 64 \\ FS \# 5114, 5126 \\ Depth (m): 0.33 - $0.56 \quad$ Volume $\left(\mathrm{m}^{3}\right): 0.216$ \\ Description: Stone foundation, curvilinear A circular installation of 13 sandstone rocks, measuring at least 1.15 meters in diameter and constructed on Surface F.2d. The associated fill was a moderately firm, strong brown (7.5 YR 5/6) silty clay with daub inclusions (2-3\%) and fine flecks of charcoal (1-2\%). Otherwise indistinguishable from the surrounding substratum, the associated matrix had an elevated frequency of faunal remains.}

\section{Locus: Feature 67}

FS \# 5126

Depth (m): $0.49-0.51 \quad$ Volume $\left(\mathrm{m}^{3}\right): 0.002$

Description: Ash dump

A small ash dump with charcoal on Surface F.2d.

\section{Locus: Feature 70}

FS \# 5133

Depth (m): 0.53 - $0.66 \quad$ Volume $\left(\mathrm{m}^{3}\right): 0.031$

Description: Hearth complex

A superimposed series of three or four hearths defined by ash lenses and heavily burnt sediment; associated with Surfaces F.2d and F.3a.

\section{Stratum F.3}

\section{Locus: F.3a}

FS \# 5125, 5128, 5129, 5131

Depth $(\mathrm{m}): 0.44-0.62 \quad$ Volume $\left(\mathrm{m}^{3}\right): 1.677$

Description: Fill, general

A firm, yellowish-red (5 YR 5/6) silty loam with fine to medium daub inclusions (1\%) and charcoal mottles (1\%). This substratum coincided with the continued use of several earthen wall structures (Features 69/71 and 72), construction of a compact surface or foundation (Feature 68), and the installation of a small stone foundation (Feature 73). An informal hearth (Feature 70), two ash dumps (Feature 75), modest artifact frequencies, and finely sorted sediment pointed to general domestic activities and natural sediment accumulation on the poorly defined Surface F.3a.
Locus: Feature 69

FS \# 5130, 5135

Depth (m): $0.53-0.62 \quad$ Volume $\left(\mathrm{m}^{3}\right): 0.049$

Description: Fill, room

A resurfacing and fill (demolition?) episode within the earthen walls of Feature 71. The sediment was a moderately loose, strong brown (7.5 YR 5/6) silty loam with fine to medium daub inclusions (2\%) and fine charcoal mottles (1\%). A thin lens of ash separated much of this fill from an underlying interior floor.

\section{Locus: Feature 73}

FS \# 5132

Depth (m): $0.48-0.58 \quad$ Volume $\left(\mathrm{m}^{3}\right): 0.060$

Description: Stone foundation, irregular

An installation of at least two sandstone rocks on

Surface F.3a. The associated sediment was a

moderately firm, yellowish red (5 YR 5/6) silty clay

loam with fine to medium daub inclusions (1\%) and

fine charcoal mottles (1\%) resembling earthen wall melt, but with elevated densities of sherds and faunal remains.

\section{Locus: Feature 68}

FS \# 5134

Depth (m): 0.49 - $0.62 \quad$ Volume $\left(\mathrm{m}^{3}\right): 0.135$

Description: Earthen wall melt

An extremely firm, strong brown (7.5 YR 5/6) silt with fine to coarse daub inclusions (3-5\%). Based on the texture and low density of artifacts and charcoal, this deposit was either an earthen wall melt, or a compact interior floor contemporaneous with Surface F.3a.

\section{Locus: Feature 72 Interior}

FS \# 5136

Depth (m): $0.58-0.63 \quad$ Volume $\left(\mathrm{m}^{3}\right): 0.091$

Description: Fill, room

The fill within Feature 72 was a firm, strong brown (7.5 YR 5/6) clay loam with light charcoal mottles (1\%).

\section{Locus: Feature 75}

FS \# 5138.1

Depth (m): $0.61-0.62 \quad$ Volume $\left(\mathrm{m}^{3}\right): \mathrm{n} / \mathrm{a}$

Description: Ash dump

A small ash dump with charcoal on Surface F.3a.

Locus: Feature 71 Interior

FS \# 5143

Depth (m): $0.62-0.70 \quad$ Volume $\left(\mathrm{m}^{3}\right): 0.258$

Description: Fill, room

An initial floor surface and primary layer of fill within Feature 71. The floor was compact clay loam with some light ash and oxidation indicative of a small, informal hearth. The layer of fill $(3-5 \mathrm{~cm}$ thick) above this surface was a firm, strong brown (7.5 YR 6/6) clay loam with fine to coarse daub inclusions (5-7\%), fine charcoal mottles (2-3\%), and light laterite gravel (1\%). 
Locus: F.3b

FS \# 5137, 5138, 5139, 5140

Depth (m): 0.58 - $0.67 \quad$ Volume $\left(\mathrm{m}^{3}\right)$ : 0.316

Description: Fill, general

An extremely firm, strong brown (7.5 YR 5/8) clay

loam with fine to coarse daub inclusions (5-7\%) and laterite pea gravel (1\%). A number of superimposed hearths (Feature 74), modest artifact frequencies, and the finely sorted sediment resulted from general domestic activities and/or natural sediment deposition over an extended period of time. This substratum overlaid Surface F.3b and was contemporaneous with the use of two earthen wall structures (Features 71 and 72).

\section{Locus: Feature 71}

FS \# 5143.1

Depth (m): $0.48-0.68 \quad$ Volume $\left(\mathrm{m}^{3}\right): \mathrm{n} / \mathrm{a}$

Description: Earthen wall stub

A well preserved, circular earthen wall structure built atop Surface F.3b with an estimated external diameter of 3.30 meters. The terre pisé wall stub, some 20-25 centimeters in thickness, was a firm, strong brown (7.5 YR 5/6) silty clay loam with fine to medium daub inclusions (10-15\%) and light laterite pea gravel (1\%).

\section{Locus: Feature 72}

FS \# 5146

Depth (m): $0.58-0.79 \quad$ Volume $\left(\mathrm{m}^{3}\right): 0.282$

Description: Earthen wall stub

A poorly preserved, circular earthen wall structure built atop Surface F.3b with a maximum diameter of 2.10 meters. The wattle-and-daub wall stub was a friable to moderately firm arc of yellowish-red (5 YR 5/6) clay loam (5-7 cm thick). A patch of burnt sediment in the southeast of the structure may have been an informal hearth.

\section{Locus: Feature 74}

FS \# 5145.1

Depth (m): $0.68-0.72 \quad$ Volume $\left(\mathrm{m}^{3}\right): \mathrm{n} / \mathrm{a}$

Description: Hearth

An extremely firm, yellowish-red (5 YR 5/6), silty clay loam with light charcoal mottles $(<1 \%)$. This modest horizon of burnt earth marked out an informal hearth area on Surface F.3b.

\section{Locus: F.3c}

FS \# 5141, 5142, 5144, 5145

Depth (m): 0.58 - $0.79 \quad$ Volume $\left(\mathrm{m}^{3}\right): 1.548$

Description: Fill, general

A moderately compact, yellowish-red (5 YR 5/6), sandy loam with medium daub inclusions $(<1 \%)$ and fine charcoal mottles $(<1 \%)$. As a transition to the sterile sediment beneath, this substratum yielded little evidence of human activity beyond a small ash dump along the eastern profile of the unit filling a depression in Surface F.3b.

\section{Stratum F.4}

Locus: F Sterile

FS \# 5147

Depth (m): $0.72-0.88 \quad$ Volume $\left(\mathrm{m}^{3}\right): 0.440$

Description: Sterile

An extremely firm, yellowish-red (5 YR 5/8) silt

loam with no visible inclusions; culturally sterile.

\section{Unit G}

\section{Stratum G.1}

\section{Locus: G Surface}

FS \# 5156, 5157, 5182, 5183

Depth (m): $0.00-0.06 \quad$ Volume $\left(\mathrm{m}^{3}\right): 0.080$

Description: Surface

A very loose, strong brown (7.5 YR 4/6) silt loam with organic detritus and laterite pea gravel.

Oriented near the apex of Area G, the unit surface was relatively flat and included several sandstone rocks, which formed no clear foundation, despite the high density of such features directly to the south, west, and north.

\section{Locus: G.1a.i}

FS \# 5159

Depth $(\mathrm{m}): 0.01-0.12 \quad$ Volume $\left(\mathrm{m}^{3}\right): 0.320$

Description: Fill, general

A loose, pale brown (10 YR 6/3) loamy sand with organic detritus and laterite pea gravel (2-3\%). This deposit was weathered from the underlying general fill and earthen wall collapse, but did not appear significantly deflated.

\section{Locus: G.1a.ii}

FS \# 5184, 5187

Depth (m): $0.00-0.15 \quad$ Volume $\left(\mathrm{m}^{3}\right): \quad 0.400$

Description: Fill, general

A friable, reddish-brown (5 YR 5/4) silty clay with laterite pea gravel (1\%), fine daub inclusions (15\%), and fine charcoal mottles (1\%). This deposit was weathered from the underlying deposit of general fill and a possible hearth complex, but did not appear significantly deflated.

\section{Locus: Feature 88}

FS \# 5187.1

Depth (m): $0.08-0.10 \quad$ Volume $\left(\mathrm{m}^{3}\right): \mathrm{n} / \mathrm{a}$

Description: Ash dump

A small ash dump presumably associated with the terminal phase of occupation. 
Locus: G.1a.iii

FS \# 5158, 5160, 5161, 5185, 5191

Depth (m): $0.00-0.18 \quad$ Volume $\left(\mathrm{m}^{3}\right): \quad 1.348$

Description: Fill, general

A loose to friable, strong brown (7.5 YR 4/6) silt

loam with organic detritus and fine charcoal mottles

$(<1 \%)$. This substratum was weathered from the

underlying deposit of general fill, but did not

appear significantly deflated.

\section{Locus: G.1b.i}

FS \# 5162, 5163, 5189

Depth (m): $0.10-0.24$

Description: Fill, general

Volume $\left(\mathrm{m}^{3}\right): \quad 0.568$

A firm, pale brown (10 YR 6/3) loamy sand with fine daub inclusions (1\%) and fine to medium charcoal mottles $(<1 \%)$. The absence of clay in the soil matrix suggested that this substratum was an intentional leveling episode, perhaps to create a more permeable surface and reduce mud during the rainy season.

\section{Locus: Feature 79}

FS \# 5164

Depth (m): $0.16-0.23 \quad$ Volume $\left(\mathrm{m}^{3}\right): 0.076$

Description: Fill, midden

A friable to moderately firm, strong brown (7.5 YR $5 / 6)$ sandy clay loam with coarse inclusions of burnt daub (5\%) and fine charcoal mottles $(<1 \%)$.

This substratum appeared as a small sheet midden or collapse of debris from an earthen wall structure on Surface G.1b.

\section{Locus: G.1b.ii}

FS \# 5190, 5192

Depth $(\mathrm{m}): 0.10-0.27 \quad$ Volume $\left(\mathrm{m}^{3}\right): 0.760$

Description: Fill, general

A moderately firm, yellowish-brown (10 YR 5/6) silty clay loam with fine charcoal mottles $(<1 \%)$.

This substratum was produced through general occupation activities on the underlying Surface G.1b.

\section{Locus: Feature 82}

FS \# 5170

Depth (m): 0.37 - $1.27 \quad$ Volume $\left(\mathrm{m}^{3}\right): \quad 0.032$

Description: Fill, pit

A cylindrical pit with an estimated diameter of 0.70 0.80 meters and extending at least 0.90 meters in depth below Surface G.1b. Whatever the original function, the pit fill was clearly a refuse deposit composed of heterogeneous and friable, strong brown (7.5 YR 5/6) clay loam with coarse daub inclusions (1\%), fine to coarse charcoal mottles (5$10 \%)$. Faunal remains, ceramic sherds, charcoal, and ash were all present in substantial frequencies.

\section{Locus: Feature 87}

FS \# 5193

Depth (m): $0.10-0.22 \quad$ Volume $\left(\mathrm{m}^{3}\right): 0.029$

Description: Stone foundation, curvilinear

A partially dismantled installation of five sandstone rocks on Surface G.1b with an estimated diameter of 0.50 meters. The associated soil matrix did not differ markedly from the surrounding G.1b stratigraphic deposit.

\section{Locus: Feature 90}

FS \# 5194, 5198, 5232, 5236, 5242, 5254, 5261, 5268

Depth $(\mathrm{m}): 0.21-1.03 \quad$ Volume $\left(\mathrm{m}^{3}\right): 0.744$

Description: Fill, pit

A cylindrical pit measuring $0.65-0.70$ meters in diameter and extending 0.80 meters in depth below Surface G.1b. Whatever the original function, the pit fill was clearly a refuse deposit comprised of heterogeneous and friable, strong brown (7.5 YR 4/6) clay loam with coarse daub inclusions (1\%) and fine to coarse charcoal mottles (5-10\%). Faunal remains, ceramic sherds, and angular and groundstone fragments were all present in substantial frequencies.

\section{Locus: G.1c.i}

FS \# 5199

Depth $(\mathrm{m}): 0.23-0.39 \quad$ Volume $\left(\mathrm{m}^{3}\right): 0.402$

Description: Earthen wall melt

A moderately firm, brownish-yellow (10 YR 6/6)

silty clay with laterite pea gravel $(<1 \%)$, fine daub inclusions (1\%), and fine charcoal (1\%). This substratum derived from the gradual decay and repair of earthen wall architecture (including Feature 89) on Surface G.1c.

\section{Locus: G.1c.ii}

FS \# 5165, 5166, 5195, 5196, 5197

Depth $(\mathrm{m}): 0.20-0.40 \quad$ Volume $\left(\mathrm{m}^{3}\right): 1.336$

Description: Fill, general

A firm to extremely firm, yellowish-brown (10 YR $5 / 6)$ silty clay with fine daub inclusions $(<1 \%)$ and fine to medium charcoal mottles (1-2\%). This substratum was a general fill produced through domestic activities undertaken in association with a possible small stone installation (Feature 91) and an informal hearth (Feature 92) on the well-defined Surface G.1c.

\section{Locus: Feature 91}

FS \# 5199.2

Depth (m): $0.33-0.38 \quad$ Volume $\left(\mathrm{m}^{3}\right): \mathrm{n} / \mathrm{a}$

Description: Stone foundation, irregular

A possible installation of at least two angular sandstone fragments resting on Surface G.1c.

\section{Locus: Feature 89}

FS \# 5230

Depth (m): $0.27-0.38 \quad$ Volume $\left(\mathrm{m}^{3}\right): 0.092$

Description: Earthen wall stub

A poorly preserved, curvilinear earthen wall foundation built atop Surface G.1c. The eroded wall stub (10-20 cm thick) was a very compact, light brown (7.5 YR 6/4) clay loam with fine to medium daub (5-10\%) and light laterite gravel inclusions (1\%). The diameter could not be estimated as a result of truncation by an intrusive pit (Feature 90). 
Locus: Feature 92

FS \# 5199.1

Depth $(\mathrm{m}): 0.35 \quad-0.38 \quad$ Volume $\left(\mathrm{m}^{3}\right): \mathrm{n} / \mathrm{a}$

Description: Hearth

An informal hearth comprised of an in situ horizon

(2-3 cm thick) of reddish, burnt sediment on Surface

G.1c, but lacking an associated ash deposit.

\section{Stratum G.2}

\section{Locus: G.2a}

FS \# 5167, 5168, 5231, 5233, 5234

Depth (m): $0.29-0.48 \quad$ Volume $\left(\mathrm{m}^{3}\right): 1.270$

Description: Earthen wall collapse

A firm, brownish-yellow (10 YR 6/6) silty clay with laterite pea gravel $(<1 \%)$, fine to coarse daub inclusions (1\%), and fine to medium charcoal mottles $(<1 \%)$. This substratum resulted from both earthen wall collapse and general domestic activities on the well-defined Surface G.2a. This surface included two informal hearths (Features 81/83 and 94) and the continued presence of a circular rock installation (Feature 93).

\section{Locus: Feature 94 \\ FS \# 5231.1 \\ Depth (m): $0.46-0.49 \quad$ Volume $\left(\mathrm{m}^{3}\right): \mathrm{n} / \mathrm{a}$ \\ Description: Hearth \\ An informal hearth comprised of a horizon $(2-3 \mathrm{~cm}$ thick) of burnt sediment on Surface G.2a, but lacking an associated ash deposit.}

\section{Locus: Feature 81/83}

FS \# 5171

Depth (m): $0.39-0.44 \quad$ Volume $\left(\mathrm{m}^{3}\right): \quad 0.014$

Description: Hearth complex

A large, informal hearth complex including a horizon (4-5 cm thick) of burnt earth and associated ash lenses on Surface G.2a. The oxidized sediment was a moderately firm, reddish-brown (5 YR 5/4) clay loam with fine to coarse charcoal (1-2\%).

\section{Locus: G.2b.i}

FS \# 5235, 5237, 5241

Depth (m): $0.42-0.56$

Description: Fill, general Volume $\left(\mathrm{m}^{3}\right): \quad 0.576$

An extremely firm, strong brown (7.5 YR 5/6) silty clay with laterite pea gravel (1\%), fine to medium daub inclusions (3-5\%), and fine to medium charcoal mottles (1\%). This substratum resulted from episodic earthen wall melt and general domestic activities, presumably those associated with circular stone installations (Features 93 and 96) on the underlying occupation Surface G.2b and continued use of a neighboring midden (G.2b.ii).

\section{Locus: G.2b.ii}

FS \# 5169, 5172, 5175

Depth $(\mathrm{m}): 0.37 \quad-0.51 \quad$ Volume $\left(\mathrm{m}^{3}\right): \quad 0.630$

Description: Fill, midden

A friable, light brown (7.5 YR 6/4) sandy loam with laterite pea gravel (1\%), medium daub inclusions
( $<1 \%$ ), fine to medium charcoal mottles (2-3\%), and ash lenses. This substratum was almost certainly a deflated sheet midden originating on Surface G.3a.

\section{Locus: Feature 93}

\section{FS \# 5247}

Depth $(\mathrm{m}): 0.38-0.54 \quad$ Volume $\left(\mathrm{m}^{3}\right): 0.145$

Description: Stone foundation, curvilinear

A large, probably circular, installation of at least 11 angular sandstone rocks on Surface G.2b with an estimated diameter of 1.70-1.90 meters. The structure was truncated in the southeast by an intrusive pit (Feature 90). The associated sediment was similar in texture to the surrounding G.2b.i matrix, but contained greater frequencies of charcoal, ash, and faunal remains.

\section{Locus: Feature 96}

FS \# 5238

Depth (m): $0.49-0.56 \quad$ Volume $\left(\mathrm{m}^{3}\right): 0.014$

Description: Stone foundation, curvilinear A circular installation comprising at least six angular sandstone rocks on Surface G.2b with an estimated diameter of $0.70-0.80$ meters. The associated sediment did not differ markedly from the surrounding G.2b.ii matrix.

\section{Stratum G.3}

\section{Locus: G.3a.i}

FS \# 5176, 5240, 5243

Depth (m): 0.48 - $0.68 \quad$ Volume $\left(\mathrm{m}^{3}\right): 0.502$ Description: Earthen wall collapse A firm, very pale brown (10 YR 7/4) clay with laterite pea gravel (5\%) and medium to coarse daub inclusions (5-10\%). This substratum probably resulted from the demolition and subsequent leveling of earthen wall architecture constructed on the poorly defined Surface G.3a.

\section{Locus: G.3a.ii}

FS \# 5173, 5178, 5239

Depth (m): $0.47-0.61$

Description: Fill, midden

Volume $\left(\mathrm{m}^{3}\right): \quad 0.235$

A friable, light brown (7.5 YR 6/4) sandy loam with laterite pea gravel (1\%), medium daub inclusions ( $<1 \%)$, fine to medium charcoal mottles (2-3\%), and ash lenses. This substratum was an episode of midden deposit that preceded G.2b.ii.

\section{Locus: G.3a.iii}

FS \# 5174, 5179, 5181

Depth (m): $0.47-0.64$

Description: Fill, general

Volume $\left(\mathrm{m}^{3}\right): \quad 0.621$

A moderately firm to firm, reddish-yellow (7.5 YR $6 / 6)$ silty clay with laterite pea gravel $(<1 \%)$, fine daub inclusions (1-2\%), and fine charcoal mottles $(<1 \%)$. This substratum was a general fill including intermittent episodes of wall melt. 


\section{Locus: Feature 84}

FS \# 5245, 5251

Depth (m): 0.48 - $0.59 \quad$ Volume $\left(\mathrm{m}^{3}\right): 0.067$

Description: Stone foundation, curvilinear

A circular installation of at least 16 angular sandstone rocks on Surface G.3a with an estimated diameter of 1.70-1.90 meters. The associated sediment, especially beneath the rocks, had a greater density of ceramic sherds and faunal remains than the surrounding G.3a.ii matrix.

\section{Locus: Feature 85}

FS \# 5177

Depth (m): 0.48 - $0.57 \quad$ Volume $\left(\mathrm{m}^{3}\right): 0.066$

Description: Stone foundation, curvilinear

A circular installation of seven sandstone rocks (diameter 0.55-0.60 m) located on Surface G.3a, and immediately post-dating Feature 86. The associated sediment did not differ from the surrounding G.3a.ii matrix.

\section{Locus: Feature 86}

FS \# 5180

Depth (m): 0.57 - $0.63 \quad$ Volume $\left(\mathrm{m}^{3}\right): 0.029$

Description: Earthen wall stub

A superimposed series of wattle-and-daub wall stubs or trenches and associated interior floors on Surface G.3a and, possibly, G.3b. Ranging from 510 centimeters in width, the wall stubs were curvilinear and composed of a friable, yellowishbrown (10 YR 5/6) clay. In the absence of significant inclusions, this matrix was probably a daub applied to the interior/exterior of a wooden wall frame. The interior floors exhibited light oxidation and ash from localized pyrotechnic activity.

\section{Locus: G.3b.i}

FS \# 5186, 5248, 5250, 5253

Depth (m): 0.60 - $0.74 \quad$ Volume $\left(\mathrm{m}^{3}\right)$ : 0.626

Description: Earthen wall collapse

An extremely firm, very pale brown (10 YR 7/4)

clay with laterite pea gravel (5-7\%) and medium to coarse daub inclusions (10-15\%). This substratum almost certainly resulted from the demolition and subsequent leveling of earthen wall architecture possibly built on the poorly defined Surface G.3b

Locus: G.3b.ii

FS \# 5244, 5246, 5252

Depth (m): 0.59 - 0.72

Description: Fill, general

Volume $\left(\mathrm{m}^{3}\right): \quad 0.442$

An extremely firm, strong brown (7.5 YR 5/6) clay with laterite pea gravel $(<1 \%)$, fine daub inclusions $(1-2 \%)$, and fine charcoal mottles $(<1 \%)$. This substratum appeared to derive from general occupation activities associated with Surface G.3b.

\section{Locus: Feature 98}

FS \# 5249, 5257, 5262

Depth (m): $0.60-0.73$

Description: Fill, midden

A sheet midden associated with initial occupation of Area G. The sediment was a moderately friable to firm, strong brown (7.5 YR 4/6) sandy clay loam with fine to medium charcoal mottles (2-3\%), ash, and elevated frequencies of ceramic sherds and faunal remains.

\section{Locus: Feature 99}

FS \# 5256, 5260

Depth (m): 0.66 - $0.74 \quad$ Volume $\left(\mathrm{m}^{3}\right): 0.037$ Description: Fill, midden

A sheet midden or ash dump associated with initial occupation of the area between strata G.3b and G.3c. The sediment was a moderately firm, reddish-yellow (7.5 YR 6/6) sandy clay loam with fine to medium charcoal mottles (3-5\%), ash, and modest frequencies of ceramic sherds and faunal remains.

\section{Locus: G.3c}

FS \# 5255, 5258, 5259, 5263, 5264, 5266

Depth (m): 0.69 - $0.83 \quad$ Volume $\left(\mathrm{m}^{3}\right): 1.395$

Description: Fill, general

A firm, reddish yellow (7.5 YR 6/6) sandy loam with medium daub inclusions $(<1 \%)$ and fine to medium charcoal mottles (2-3\%). This substratum appeared to derive from general domestic activities during the initial occupation of Area G.

\section{Stratum G.4}

Locus: G Sterile

FS \# 5267

Depth $(\mathrm{m}): 0.81-0.93 \quad$ Volume $\left(\mathrm{m}^{3}\right): 1.068$

Description: Sterile

An extremely firm, reddish-yellow (7.5 YR 6/6) silty clay with no mottles or inclusions. This stratum contained no evidence of human activity; culturally sterile.

\section{Unit H}

\section{Stratum H.1}

\section{Locus: H Surface}

FS \# 5043

Depth (m): $0.00-0.09 \quad$ Volume $\left(\mathrm{m}^{3}\right): \quad 0.020$

Description: Surface

The surface of Unit $\mathrm{H}$ was a loose, strong brown (7.5

YR 5/6) silty clay loam littered with laterite pea gravel (3-5\%), a few small sandstone rocks, and heavily crushed ceramic sherds. Located near the apex of Area $\mathrm{H}$, the unit was relatively flat, but sloped gently to the south. Three circular sandstone foundations appeared directly to the north and east; suggesting that the unit lay within the courtyard of a domestic compound. 


\begin{abstract}
Locus: H.1a
FS \# 5044

Depth (m): $0.00-0.17 \quad$ Volume $\left(\mathrm{m}^{3}\right): \quad 0.259$

Description: Fill, general

A loose to friable, strong brown (7.5 YR 5/6) silty clay loam with fine daub inclusions (1\%), fine charcoal mottles (1\%), and organic detritus. This substratum, a lightly deflated topsoil, differed in color and texture from the underlying deposit and may derive from long-term erosion.
\end{abstract}

\section{Locus: Feature A}

\section{FS \# 5044.1}

Depth (m): $0.13-0.20 \quad$ Volume $\left(\mathrm{m}^{3}\right): \mathrm{n} / \mathrm{a}$

Description: Ash dump

A dense ash lens (5-7 cm thick) overlying two angular sandstone rocks and possibly associated with two smaller patches of burnt sediment on the poorly defined Surface H.1a.

\section{Locus: H.1b}

FS \# 5045, 5046

Depth $(\mathrm{m}): 0.13-0.35 \quad$ Volume $\left(\mathrm{m}^{3}\right): \quad 0.816$

Description: Earthen wall collapse

A friable to moderately firm, light brown (7.5 YR

$6 / 4)$ silty clay loam with fine daub inclusions (2\%) and fine to coarse charcoal mottles (2\%); mediumsized gravels also present (3\%). Diffuse patches of burnt daub and ash and considerable quantities of earthen wall melt indicated that this substratum derived from general occupation activities, including the localized decay or collapse of one or more earthen wall structures. Features 21 and 22 were both visible in this stratum.

\section{Locus: H.1c}

FS \# 5047

Depth (m): $0.32-0.42 \quad$ Volume $\left(\mathrm{m}^{3}\right): 0.222$

Description: Earthen wall melt

An extremely firm, very pale brown (10 YR 7/4) sandy clay with coarse daub inclusions (2-5\%), medium charcoal mottles (1-3\%), and fine to medium sand and gravel (1\%). This substratum was clearly composed of earthen wall collapse. The underlying Surface H.1c supported two architectural features (Features 21 and 22).

\section{Locus: Feature 21}

FS \# 5047.1

Depth $(\mathrm{m}): 0.35$ - $0.45 \quad$ Volume $\left(\mathrm{m}^{3}\right): \mathrm{n} / \mathrm{a}$

Description: Stone alignment

A curvilinear alignment of three unevenly spaced sandstone rocks. The haphazard placement of the stones suggested an ad hoc technique for the preservation of an earthen wall structure on Surface H.1c, or simply the displacement following demolition of such a structure. One stone measured 60 centimeters in length and may have served a more specific architectural function.

\section{Locus: Feature 22 Interior}

FS \# 5048

Depth (m): $0.32-0.40 \quad$ Volume $\left(\mathrm{m}^{3}\right): 0.010$

Description: Fill, room

An initial floor surface and layer of fill within Feature 22. The sediment was a moderately firm, brown (7.5 YR 5/4) sandy clay and fine charcoal mottles (1\%). A thick lens $(2-4 \mathrm{~cm})$ of ash separated this fill from an underlying compacted surface.

\section{Locus: Feature 22}

FS \# 5048.1

Depth (m): $0.32 \quad-0.50 \quad$ Volume $\left(\mathrm{m}^{3}\right): \quad 0.044$

Description: Earthen wall stub

A curvilinear earthen wall stub composed of very compact, very pale brown (10 YR 7/4) sandy clay loam with almost no inclusions and estimated diameter of 1.80-2.00 meters. The foundation averaged 20-25 centimeters in thickness, although the build-up of wall melt made it slightly thicker at the interface with Surface H.1c.

\section{Locus: H.1d}

FS \# 5049

Depth (m): 0.42 - $0.56 \quad$ Volume $\left(\mathrm{m}^{3}\right): 0.445$

Description: Fill, general

A friable, light brown (7.5 YR 6/4) clay loam with medium daub inclusion (3-5\%) and fine to medium charcoal mottles (2-5\%). This substratum appeared to derive from general occupation activities and some earthen wall melt or collapse.

\section{Stratum H.2}

\section{Locus: H.2a}

FS \# 5050, 5051

Depth $(\mathrm{m}): 0.49-0.67 \quad$ Volume $\left(\mathrm{m}^{3}\right): 0.486$

Description: Fill, general

A moderately firm, brown (7.5 YR 5/4) sandy clay loam with medium to coarse daub inclusions (1-2\%) and fine to medium charcoal mottles (2-3\%); coarse gravels were rare $(<1 \%)$. This substratum was produced by general domestic activities taking place on the fire-hardened Surface H.2a. This surface was most discernible around the hearth complex (Feature F) in the eastern half of the unit.

\section{Locus: Feature F}

FS \# 5051.1

Depth (m): 0.65 - $0.67 \quad$ Volume $\left(\mathrm{m}^{3}\right): \mathrm{n} / \mathrm{a}$

Description: Hearth complex

A superimposed series of two or three hearths defined by ash lenses and horizons (1-2 cm thick) of heavily oxidized sediment; associated with Surface H.2a. 
Locus: H.2b

FS \# 5052

Depth (m): $0.63-0.76 \quad$ Volume $\left(\mathrm{m}^{3}\right): \quad 0.267$

Description: Fill, general

A moderately firm, strong brown (7.5 YR 5/6) silty clay loam with medium daub inclusions (1-2\%) and fine to medium charcoal mottles (1-2\%). Surface H.2b was easily defined around the hearth complex (Feature G) in the eastern half of the unit. The rapid succession of ash-filled hearths in this substratum indicated a relatively intensive use and maintenance of this space for domestic activities such as food preparation and consumption.

\section{Locus: Feature G \\ FS \# 5052.1 \\ Depth (m): $0.71-0.73 \quad$ Volume $\left(\mathrm{m}^{3}\right): \mathrm{n} / \mathrm{a}$ \\ Description: Hearth complex \\ A superimposed series of two or three hearths defined by ash lenses and horizons ( $2 \mathrm{~cm}$ thick) of heavily oxidized sediment; associated with Surface H.2b.}

\section{Locus: H.2c}

FS \# 5053

Depth (m): $0.70-0.79 \quad$ Volume $\left(\mathrm{m}^{3}\right): 0.176$

Description: Fill, general

A moderately firm, light brown (7.5 YR 6/4) silty clay with rare medium daub inclusions $(<1 \%)$ and fine to medium charcoal mottles (1-2\%). This substratum likely derived from general occupation activities and limited wall melt on Surface H.2d, defined by an informal hearth (Feature $\mathrm{H}$ ) in the eastern half of Unit $\mathrm{H}$.

\section{Locus: Feature $\mathbf{H}$}

FS \# 5054

Depth (m): $0.78-0.87 \quad$ Volume $\left(\mathrm{m}^{3}\right): 0.088$

Description: Hearth complex

An series of two or three informal hearths defined by ash lenses and horizons (4-5 cm thick) of heavily oxidized sediment; associated with Surface H.2c.

Locus: H.2d.i

FS \# 5055

Depth $(\mathrm{m}): 0.74-0.81 \quad$ Volume $\left(\mathrm{m}^{3}\right): \quad 0.230$

Description: Fill, general

An extremely firm, reddish-yellow (7.5 YR 6/6) silty clay loam with fine to medium daub inclusions (1\%) and fine charcoal mottles (1\%). This deposit was partially oxidized, probably the long-term result of cooking activity evidenced by numerous hearths.

\section{Locus: H.2d.ii}

FS \# 5056

Depth (m): $0.81-0.95 \quad$ Volume $\left(\mathrm{m}^{3}\right): \quad 0.276$

Description: Fill, general

A moderately firm, strong brown (7.5 YR 5/6) clay loam with medium daub inclusions (1\%), very fine charcoal mottles (1-2\%), and laterite pea gravel $(<1 \%)$. This substratum was a general fill produced through domestic activities and the decay of earthen wall structures. Although difficult to define in the western half of the unit, Surface H.2d was particularly compact around a hearth (Feature J).

\section{Locus: Feature J}

FS \# 5055.1

Depth (m): $0.90-0.95 \quad$ Volume $\left(\mathrm{m}^{3}\right): \mathrm{n} / \mathrm{a}$

Description: Hearth

An informal hearth defined by heavily oxidized sediment and a thin deposit of ash on Surface H.2d.

\section{Stratum H.3}

\section{Locus: H.3a}

FS \# 5057

Depth (m): $0.88-1.05 \quad$ Volume $\left(\mathrm{m}^{3}\right): 0.449$

Description: Fill, general

A moderately firm, strong brown (7.5 YR 5/6) silty clay loam with light gravel $(<1 \%)$, medium daub inclusions $(<1 \%)$, fine charcoal mottles $(1 \%)$. This substratum was a general occupational fill above the poorly defined Surface H.3a.

\section{Locus: H.3b}

FS \# 5058, 5059

Depth (m): 1.03 - $1.21 \quad$ Volume $\left(\mathrm{m}^{3}\right): 0.664$

Description: Fill, general

A firm, brownish-yellow (10 YR 6/6) silty clay with medium to coarse daub inclusions (2\%) and fine to medium gravel $(<1 \%)$. Based on sediment texture and color, this substratum may have included some wall melt, but otherwise appeared to represent gradual deposition from domestic activities.

\section{Locus: H.3c}

FS \# 5060, 5061

Depth (m): $1.20-1.40 \quad$ Volume $\left(\mathrm{m}^{3}\right): 0.691$

Description: Fill, midden

An extremely firm, strong brown (7.5 YR 5/6) silty clay loam with fine daub inclusions (1\%) and fine to medium charcoal mottles (1-2\%). This substratum had diffuse upper and lower boundaries, marking a general transition from the occupation horizons above to the midden fill below.

\section{Locus: Feature 27}

FS \# 5060.1

Depth (m): 1.40 - $1.45 \quad$ Volume $\left(\mathrm{m}^{3}\right): \mathrm{n} / \mathrm{a}$

Description: Ash dump

Two charcoal and ash lenses with no evidence of in situ burning. 


\section{Stratum H.4}

Locus: Feature 28a

FS \# 5061.1, 5062, 5063, 5063.1, 5064, 5065, 5070

Depth (m): 1.32 - $2.04 \quad$ Volume $\left(\mathrm{m}^{3}\right): 1.441$

Description: Fill, pit

A firm, strong brown (7.5 YR 5/6) silty clay loam mottled with medium daub inclusions $(<1 \%)$ and medium charcoal mottles $(<1 \%)$; laterite pea gravel also appeared in light bands $(<1 \%)$. This deposit included the uppermost layer of refuse fill within Feature 28, a broad borrow pit excavated to a maximum depth of 1.70 meters below Stratum H.3b/c. This deposit was clearly distinguished from the subsequent one which had an increased density of charcoal and daub.

\section{Locus: Feature 28b}

FS \# 5066, 5067, 5068, 5071

Depth (m): 1.72 - $2.00 \quad$ Volume $\left(\mathrm{m}^{3}\right): 0.563$

Description: Ceramic scatter

A dense cluster of sherds from partially

reconstructable vessel. This deposit corresponded to a single episode of disposal at the bottom of the Feature 28a. Based on the rim sherds, at least five vessels ranging from $29-87 \%$ complete were present.

\section{Locus: Feature 28c}

FS \# 5069, 5072, 5073, 5074, 5075, 5076

Depth (m): 1.93 - $2.95 \quad$ Volume $\left(\mathrm{m}^{3}\right): 1.029$

Description: Fill, pit

A friable, strong brown (7.5 YR 4/6) sandy clay loam with coarse daub inclusions $(<1 \%)$, laterite gravel $(<1 \%)$, and medium to coarse charcoal mottles (3-5\%). This deposit comprised the lower layer of fill within Feature 28, a large borrow pit. The diffuse boundaries of this deposit, however, point to some bioturbation by termites or burrowing animals.

\section{Stratum H.5}

\section{Locus: H Sterile}

FS \# 5076.1

Depth (m): 2.15 - $2.95 \quad$ Volume $\left(\mathrm{m}^{3}\right): 0.200$

Description: Sterile

An extremely firm, yellowish-red (5 YR 5/6) silty clay with trivial inclusions of laterite pea gravel $(<1 \%)$. No artifacts or features were present; culturally sterile.

\section{Unit K}

\section{Stratum K.1}

\section{Locus: K Surface}

FS \# 5148.1

Depth (m): $0.00-0.03 \quad$ Volume $\left(\mathrm{m}^{3}\right): 0.020$

Description: Surface

The surface of Unit K was a loose, strong brown (7.5 YR 5/6) loam with trivial inclusions of organic detritus and laterite pea gravel $(<1 \%)$. The unit surface was relatively level and located on the crest of a gentle rise near several stone installations attesting to past architecture in this area.

\section{Locus: K.1a}

FS \# 5148

Depth (m): $0.00-0.11 \quad$ Volume $\left(\mathrm{m}^{3}\right): \quad 0.280$

Description: Fill, general

A friable, strong brown (7.5 YR 5/6) loam with light organic detritus, but no daub inclusions or charcoal. This substratum comprised the weathered surface of an underlying general fill deposit.

\section{Locus: K.1b}

FS \# 5179

Depth (m): $0.04-0.17 \quad$ Volume $\left(\mathrm{m}^{3}\right): \quad 0.240$ Description: Fill, general

A firm, strong brown (7.5 YR 5/6) sandy clay loam with light root matter, medium daub inclusions (1\%), and fine charcoal mottles (1\%). This substratum was a general occupation fill with some evidence for earthen wall demolition or construction.

\section{Locus: Feature 76}

FS \# 5149.1

Depth (m): 0.10 - $0.17 \quad$ Volume $\left(\mathrm{m}^{3}\right): \mathrm{n} / \mathrm{a}$

Description: Stone foundation, irregular

An irregular deposition of six sandstone rocks on Surface K.1b with a maximum diameter of 1.10 meters. The associated sediment did not differ markedly from Stratum K.1b.

\section{Stratum K.2}

\section{Locus: K.2a}

FS \# 5150, 5151

Depth $(\mathrm{m}): 0.11 \quad-0.33 \quad$ Volume $\left(\mathrm{m}^{3}\right): 0.680$

Description: Earthen wall collapse A firm, brownish-yellow (10 YR 6/8) sandy clay loam with no daub inclusions or charcoal. This substratum formed a thick deposit of earthen wall collapse, possibly from the demolition of a structure (Feature 77) associated with Surface K.2a. 
Locus: Feature 78

FS \# 5151.2

Depth (m): $0.22 \quad-0.39 \quad$ Volume $\left(\mathrm{m}^{3}\right): \mathrm{n} / \mathrm{a}$

Description: Fill, pit

A shallow pit of unknown function excavated through Surface K.2a; filled with construction debris and other domestic refuse. The depositional fill was a moderately firm, strong brown (7.5 YR 5/6) sandy clay loam with medium to coarse burnt daub inclusions (5-10\%) and fine charcoal mottles (3-5\%).

\section{Locus: Feature 77}

FS \# 5151.1

Depth $(\mathrm{m}): 0.26-0.32 \quad$ Volume $\left(\mathrm{m}^{3}\right): \mathrm{n} / \mathrm{a}$

Description: Earthen wall melt

A thick deposit of earthen wall melt, or possible compacted foundation surface on Surface K.2a. The sediment was an extremely firm, light brownishyellow (10 YR 6/4) silty clay with fine to medium daub inclusions (1-2\%).

Locus: K.2b.i

FS \# 5152

Depth (m): $0.26-0.38 \quad$ Volume $\left(\mathrm{m}^{3}\right): 0.160$

Description: Fill, midden

A loose to friable, strong brown (7.5 YR 5/6) silty sand with fine to medium charcoal mottles (3-5\%). Root and termite action were visible. This deposit was a possible refuse dump from general occupation activities.

Locus: K.2b.ii

FS \# 5154

Depth $(\mathrm{m}): 0.30-0.43 \quad$ Volume $\left(\mathrm{m}^{3}\right): 0.426$

Description: Fill, general

A moderately firm, strong brown (7.5 YR 5/6) loam with medium daub inclusions $(<1 \%)$ and no appreciable charcoal. This deposit originated through general occupation activities on the poorly defined Surface K.2b.

Locus: K.2c

FS \# 5153

Depth (m): 0.35 - $0.55 \quad$ Volume $\left(\mathrm{m}^{3}\right): 0.299$

Description: Fill, general

A firm, yellowish-red (5 YR 5/6) sandy clay loam with fine to medium daub inclusions (1-2\%).

Transitioning to sterile, this substratum was produced during initial occupation of the area.

\section{Stratum K.3}

Locus: K Sterile

FS \# 5155

Depth (m): $0.46-0.67 \quad$ Volume $\left(\mathrm{m}^{3}\right): 0.600$

Description: Sterile

An extremely firm, yellowish-red (5 YR 5/6) silty

clay with no inclusions, but noticeable termite action or other bioturbation. This horizon contained no evidence of human activity; culturally sterile.

\section{Trench L (Feature L-31)}

\section{Stratum L.1}

\section{Locus: Feature L-31}

FS \# 5403

Depth (m): $0.00-0.12 \quad$ Volume $\left(\mathrm{m}^{3}\right): 0.200$

Description: Stone foundation, irregular

Feature L-31 was an irregular, elongated installation of sandstone rocks (length $1.76 \mathrm{~m}$ ) set on the modern ground surface. The uppermost stratum surrounding this feature was a loose, brown (7.5 YR 5/4) silty clay loam with no cultural materials.

\section{Locus: L.1}

FS \# 5404

Depth (m): $0.10-0.40 \quad$ Volume $\left(\mathrm{m}^{3}\right): 0.540$

Description: Sterile

A firm, brown (7.5 YR 5/4) silty clay loam with no artifacts and few natural inclusions of gravel and organic detritus; culturally sterile.

\section{Locus: L.2}

FS \# 5405

Depth (m): 0.36 - $1.15 \quad$ Volume $\left(\mathrm{m}^{3}\right): 1.400$

Description: Sterile

A leached soil horizon of extremely firm, yellowishred (5 YR 5/6) silty clay with no inclusions, but noticeable termite action or other bioturbation; culturally sterile.

\section{Unit N}

\section{Stratum N.1}

\section{Locus: N Surface}

FS \# 5200.1

Depth $(\mathrm{m}): 0.03-0.04 \quad$ Volume $\left(\mathrm{m}^{3}\right): 0.020$

Description: Surface

The surface of Unit $\mathrm{N}$ was a very loose, strong brown (7.5 YR 4/6) silt loam with light laterite pea gravel (1-2\%), a few sandstone rock fragments, and few artifacts. The unit was located at the southern margin of this off-mound residential area.

\section{Locus: N.1a}

FS \# 5200, 5201, 5212

Depth (m): $0.00-0.19$

Volume $\left(\mathrm{m}^{3}\right): 0.696$

Description: Fill, general

A loose to friable, strong brown (7.5 YR 4/6) silt with limited laterite pea gravel (1\%) and organic detritus. This substratum was weathered from the underlying sediment, but did not appear significantly deflated. This substratum overlay a poorly preserved occupation surface with a small circular installation of sandstone rocks (Feature 100 ) and a hearth (Feature 101). 


\section{Locus: Feature 100}

FS \# 5202

Depth (m): 0.05 - $0.17 \quad$ Volume $\left(\mathrm{m}^{3}\right): \quad 0.074$

Description: Stone foundation, curvilinear

A partially dismantled circular installation of 10-14 angular sandstone rocks, measuring some 0.92

meters in diameter. The stones lay nearly flush atop

Surface N.1a. The associated soil matrix did not

differ from the surrounding N.1a horizon.

\section{Locus: Feature 101}

FS \# 5201.1

Depth (m): $0.12-0.16 \quad$ Volume $\left(\mathrm{m}^{3}\right): \mathrm{n} / \mathrm{a}$

Description: Hearth

An informal hearth comprised of oxidized sediment and an ash lens, measuring 0.50-0.60 meters in diameter, on Surface N.1a.

\section{Locus: N.1b.i}

FS \# 5203, 5213

Depth $(\mathrm{m}): 0.11-0.22 \quad$ Volume $\left(\mathrm{m}^{3}\right): \quad 0.340$

Description: Fill, general

A friable to moderately firm, strong brown (7.5 YR $5 / 6)$ silty clay loam with laterite pea gravel (1-2\%), fine to medium daub inclusions (1\%), and fine charcoal mottles $(<1 \%)$. Root action was visible. This substratum appeared to result from a combination of domestic activities and earthen wall collapse (Feature 106/109).

\section{Locus: N.1b.ii}

FS \# 5204, 5214

Depth (m): $0.15-0.29 \quad$ Volume $\left(\mathrm{m}^{3}\right): \quad 0.360$

Description: Fill, general

A moderately firm, brown (7.5 YR 5/4) sandy clay with medium daub inclusions $(<1 \%)$ and fine charcoal mottles $(<1 \%)$. This substratum likely resulted from domestic activities and soil deposition on the poorly preserved Surface N.1b.

\section{Locus: Feature 109}

\section{FS \# 5215}

Depth $(\mathrm{m}): 0.24-0.30 \quad$ Volume $\left(\mathrm{m}^{3}\right): 0.024$

Description: Fill, room

A deposit of burnt earth (3-5 cm thick) and a thin lens of ash on Surface N.02 associated with a sediment of firm, strong brown (7.5 YR 5/6) silt loam with lateritic pea gravel $(<1 \%)$, fine daub inclusions (1\%), and fine to medium charcoal mottles $(<1 \%)$. Based on the circumscription of this feature within a curvilinear boundary, and its juxtaposition above Feature 106, this deposit most likely marked the interior collapse of this wattle-anddaub structure.

\section{Stratum N.2}

\section{Locus: N.2a}

FS \# 5205

Depth $(\mathrm{m}): 0.27-0.33 \quad$ Volume $\left(\mathrm{m}^{3}\right): 0.138$

Description: Fill, general

A firm, brownish-yellow (10 YR 6/6), sandy to silty clay with laterite pea gravel (1\%), fine to medium daub inclusions (1-2\%), and fine to medium charcoal mottles (1-2\%). This substratum included several episodes of earthen wall melt and/or collapse alongside debris associated with general domestic activities on Surface N.2a. These activities included the use of an informal hearth (Feature 103) and replastering of a circular earthen wall hut (Feature 106).

\section{Locus: Feature 103}

FS \# 5206

Depth (m): $0.28-0.33 \quad$ Volume $\left(\mathrm{m}^{3}\right): 0.022$

Description: Hearth

An informal hearth with a horizon of oxidized sediment (3-4 cm thick) and lens of ash on Surface N.2a.

Locus: Feature 106 Interior

FS \# 5217

Depth (m): $0.29-0.42 \quad$ Volume $\left(\mathrm{m}^{3}\right): 0.150$

Description: Fill, room

A firm, strong brown (7.5 YR 5/8) silt loam with lateritic pea gravel (1-2\%), medium daub inclusions (1-2\%), and charcoal mottles (<1\%). This deposit comprised an episode of fill and replastering above an ash lens and the original surface in Feature 106.

\section{Locus: N.2b}

FS \# 5207, 5216

Depth (m): $0.28-0.38 \quad$ Volume $\left(\mathrm{m}^{3}\right): 0.204$

Description: Earthen wall collapse

An extremely firm, very pale brown (10 YR 7/4) silty clay with lateritic pea gravel (2\%), fine to medium daub inclusions (5\%), and medium charcoal mottles $(<1 \%)$. This substratum most likely resulted from the leveling of the area after a major episode of earthen wall collapse over Surface N.2b and its associated features. These included a crushed ceramic vessel (Feature 104), small hearth (Feature 105) and two ash dumps (Features 110 and 111), along with a circular installation of tabular rocks (Feature 102), and an earthen wall structure (Feature 106).

\section{Locus: Feature 104}

FS \# 5210

Depth (m): $0.42-0.45 \quad$ Volume $\left(\mathrm{m}^{3}\right): 0.007$

Description: Ceramic scatter

A small scatter of sherds from one or two partially reconstructable ceramic vessels lying on Surface N.2b. 


\section{Locus: Feature 105}

FS \# 5207.1

Depth $(\mathrm{m}): 0.33 \quad-0.36 \quad$ Volume $\left(\mathrm{m}^{3}\right): \mathrm{n} / \mathrm{a}$

Description: Hearth

An informal hearth composed of a thin horizon of lightly oxidized sediment $(2-3 \mathrm{~cm})$ and an ash lens on Surface N.2b.

\section{Locus: Feature 102 Collapse \\ FS \# 5208 \\ Depth $(\mathrm{m}): 0.31 \quad-0.40 \quad$ Volume $\left(\mathrm{m}^{3}\right): 0.065$ \\ Description: Earthen wall collapse \\ An extremely firm, very pale brown (10 YR 7/4) \\ silty clay with fine to medium daub inclusions \\ ( $<1 \%$ ), fine charcoal mottles (1-2\%), and ash lenses \\ lying atop Feature 102. This deposit strongly \\ suggested the presence of an earthen wall structure above the stone foundation.}

\section{Locus: N.2c}

FS \# 5218, 5220

Depth (m): $0.32-0.47 \quad$ Volume $\left(\mathrm{m}^{3}\right): 0.061$

Description: Fill, general

An extremely firm, reddish-yellow (7.5 YR 6/6) silty clay with laterite pea gravel (3\%) and fine to coarse daub inclusions (5-7\%). This substratum arose from earthen wall collapse and subsequent leveling with deposition from other human activities on the underlying, and poorly defined, Surface N.2c. This surface did, however, correspond with the installation of sandstone rocks (Feature 102), the demolition of one earthen wall structure (Feature 112 Interior), and construction of another (Feature 106).

\section{Locus: Feature 106}

\section{FS \# 5219}

Depth $(\mathrm{m}): 0.26-0.47 \quad$ Volume $\left(\mathrm{m}^{3}\right): 0.038$

Description: Fill, room

A moderately well preserved, circular earthen wall stub (5-8 cm thick) and interior fill lying atop a floor of friable, yellowish-brown (10 YR 5/6) clay loam. With the absence of significant inclusions, this wall matrix was probably a daub applied to the interior/exterior of a wooden wall frame built on Surface N.2c and measuring an estimated 2.20-2.40 meters in diameter. The initial interior surface exhibited light oxidation from an small informal hearth.

\section{Locus: Feature 102}

FS \# 5211, 5222

Depth (m): 0.40 - $0.55 \quad$ Volume $\left(\mathrm{m}^{3}\right): 0.076$

Description: Stone foundation, curvilinear

An intact circular installation of at least 11 sandstone rocks on Surface N.2c measuring at least 1.20 meters in diameter. The sediment within this installation was a firm, reddish-yellow (7.5 YR 6/6) silty clay with fine to medium daub inclusions $(<1 \%)$, fine charcoal mottles (1-2\%), and ash lenses little different from the surrounding matrix.

\section{Locus: Feature 112 Interior}

FS \# 5209

Depth (m): $0.33 \quad-0.45 \quad$ Volume $\left(\mathrm{m}^{3}\right): \quad 0.180$ Description: Earthen wall collapse

A moderately firm, strong brown (7.5 YR 5/6) silty clay loam with laterite pea gravel $(<1 \%)$, fine to medium daub inclusions (3\%), and a lens of ash (2 $\mathrm{cm}$ thick) overlying the interior surface of Feature 112, a poorly preserved earthen wall structure. This deposit likely corresponded with wall collapse from the demolition of this structure coincident with the construction of other features on Surface N.2c.

\section{Locus: Feature 112}

FS \# 5224

Depth (m): $0.45-0.51 \quad$ Volume $\left(\mathrm{m}^{3}\right): 0.032$

Description: Earthen wall stub

A firm, brown (7.5 YR 5/4) clay loam with light oxidization and ash framed by large fragments of daub. This deposit included the interior surface and walls of a poorly preserved earthen wall structure of unknown dimensions.

\section{Locus: N.2d}

FS \# 5221, 5223, 5225, 5226

Depth $(\mathrm{m}): 0.43$ - $0.69 \quad$ Volume $\left(\mathrm{m}^{3}\right): 1.060$ Description: Fill, general

A moderately firm, reddish-yellow (7.5 YR 6/6) silty clay loam with laterite pea gravel (1-2\%) and fine to medium daub inclusions (1-3\%). The density of inclusions within this substratum was relatively heterogeneous and suggested a general episode of earthen wall construction, melt, and demolition. A number of horizontal sherds and an informal hearth (Feature 113) attested to the presence of the underlying Surface N.2d.

\section{Locus: Feature 113}

\section{FS \# 5227}

Depth (m): 0.59 - $0.66 \quad$ Volume $\left(\mathrm{m}^{3}\right): 0.016$

Description: Hearth complex

A large, informal hearth complex with a single horizon $(5 \mathrm{~cm})$ of oxidized sediment and ash lenses on Surface N.2d. The sediment was an extremely firm, strong brown (7.5 YR 5/6) sandy clay loam mottled with fine to coarse charcoal (1-2\%).

\section{Locus: N.2e}

FS \# 5228

Depth (m): $0.62-0.76 \quad$ Volume $\left(\mathrm{m}^{3}\right): \quad 0.420$

Description: Fill, general

A firm, reddish-yellow (7.5 YR 6/6), silty clay with laterite pea gravel (1-2\%) and fine to medium daub inclusions $(<1 \%)$. This substratum appeared to derive from general domestic activities associated with initial occupation of the area. 


\section{Stratum N.3}

\section{Locus: N Sterile}

FS \# 5229

Depth (m): $0.73-0.84 \quad$ Volume $\left(\mathrm{m}^{3}\right): 0.200$ Description: Sterile

An extremely firm, very pale brown (10 YR 7/4) clay with no inclusions, but noticeable termite action or other bioturbation. This horizon contained no evidence of human activity; culturally sterile.

\section{Unit S (Feature S-01)}

\section{Stratum S.1}

\section{Locus: Feature S-01}

FS \# 5082

Depth (m): $0.00-0.15 \quad$ Volume $\left(\mathrm{m}^{3}\right): \quad 0.196$

Description: Stone foundation, irregular An irregular installation of 18 angular sandstone rocks. Based on their haphazard arrangement, these stones may have been a relatively recent cairn by herders or farmers in the area. The associated sediment did not differ from the surrounding matrix, nor did it include any ash or charcoal.

\section{Locus: S Surface}

\section{FS \# 5080}

Depth (m): $0.03-0.05 \quad$ Volume $\left(\mathrm{m}^{3}\right): \quad 0.020$

Description: Surface

The surface of Unit S was a moderately loose, brown (7.5 YR 5/4) sandy clay loam littered with gravel (2\%). The unit was placed around a circular distribution of stones (Feature S-01), a possible architectural feature. Although very few ceramics were visible on the surface, further stones scattered to the north suggested possible features.

\section{Locus: S.1a}

FS \# 5081

Depth (m): $0.03-0.17 \quad$ Volume $\left(\mathrm{m}^{3}\right): \quad 0.273$

Description: Fill, general

A loose, brown (7.5 YR 5/4) sandy clay loam with few gravel inclusions. Fragments of possible daub were present in small quantities (2\%), but charcoal and artifact densities were minimal.

\section{Locus: S.1b}

FS \# 5083

Depth (m): $0.15-0.25 \quad$ Volume $\left(\mathrm{m}^{3}\right): 0.400$ Description: Fill, general

A friable, brown (7.5 YR 5/4) sandy clay loam with some organic detritus, but few other inclusions. The bottom of the substratum revealed some lightly oxidized sediment and a few small bone fragments just beneath Feature S-01.

\section{Stratum S.2}

\section{Locus: S.2}

FS \# 5084

Depth (m): $0.25-0.35 \quad$ Volume $\left(\mathrm{m}^{3}\right): 0.360$ Description: Sterile

A firm, brownish-yellow (10 YR 6/6) silty clay loam with light laterite pea gravel $(<1 \%)$, but no other material; culturally sterile. 


\section{APPENDIX D POTTERY ANALYSIS AND CLASSIFICATION}

Earthenware pottery sherds comprise the class of artifacts most frequently encountered and recovered during archaeological fieldwork in the Madina-Sadatou study area. By virtue of its ubiquity, this dataset offers an important baseline for building relative chronologies and identifying social activities at multiple scales-methodological steps in which pottery has long played a pivotal role in West Africa. Since the advent of archaeological reconnaissance and excavation early last century, pottery assemblages have helped to substantiate regional culture histories through the identification of fossiles directeurs sensitive to temporal change, and through stylistic boundaries supposedly consonant with past ethno-linguistic identities (see discussions in De Barros 1990; Richard 2009; Stahl 1999b). The growth of processual methodologies in West Africa has, however, led to a more critical use of data in both regards. Even as pottery remains fundamental to space-time systematics, individual sherd classification has been augmented by greater attention to assemblage variability (S. K. McIntosh 1995:130-132). Conceptual, methodological, and analytical advances have opened the way for research into the long-term dynamics of ethnic and other identities, as well as political institutions, economies, and ritual practices (e.g., Gijanto 2011a; Gosselain 2000; Haour 2011; Livingstone Smith 2007; Mayor 2010; Norman 2011; Ogundiran 2001; Stahl and Cruz 1998; Stahl et al. 2008). Interpretations of archaeological data have further benefitted from an ever-growing body of ethnoarchaeological literature on the economic and sociocultural dimensions of pottery manufacture and consumption in Africa and elsewhere (e.g., J. W. Arthur 2006; Gosselain 2010; Hegmon 2000; Stark 2003; Van der Leeuw 1993). All this is to say that, given the right methodological framework, the study of archaeological pottery from Madina-Sadatou can address a number of questions about the cultural economy of Diouboye and the surrounding historical landscape.

In order to relate artifacts to the past economic processes and social structures of interest, I have appealed to a middle-range interpretive framework loosely defined as a technological approach to material culture (Dobres and Hoffman 1994; Lechtman 1977; Lemmonier 1986; Leroi-Gourhan 1943). This approach has several merits, namely its widespread use in West Africa (Haour et al. 2010), its potential to integrate inferences from ethnoarchaeology (Roux 2007), and its capacity to directly relate material objects and assemblages to past socioeconomic practices (Dobres 2000). With this middle-range framework as guide, the analyses presented in this appendix had the following objectives: (1) to construct a regional ceramic chronology for the relative dating of surveyed sites; (2) to seriate stratified deposits and occupation phases at Diouboye; (3) to identify economic activities and possible sociocultural differences across Diouboye and the surveyed sites; and (4) to facilitate comparison between ceramic assemblages from the Madina-Sadatou study area and those found elsewhere in West Africa. 
In this appendix I first outline the tenets of a technological approach to ceramics in a discussion broadly applicable to artifact classes discussed in subsequent appendices. The second part presents a study of pottery production sequences in Madina-Sadatou based upon an analysis of the sherd assemblages recovered in the course of fieldwork. In this I draw upon ethnoarchaeological studies of pottery manufacture, particularly those in Senegambia, to tease out common logistical and social parameters for technical choices and to consider how these choices generate formal variation in single sherds and across assemblages. I then consider similar variability in the archaeological data to apprehend the range of techniques employed in past stages of production. The third part of this appendix employs the spatial and temporal distribution of these techniques, and their cooccurrences, to develop a set of typologies applicable to the objectives listed above.

\section{TECHNOLOGICAL SYSTEMS: AN INTERPRETIVE METHODOLOGY}

A technological approach to the study of past material culture views technologies as dynamic systems enjoining material objects and human actors together in networks of physical action, technical knowledge, social structures, and cultural schema (Lechtman 1977:6; Lemmonier 1986:154). In contrast to functional and behavioral perspectives that assume some measurable utility value drives the selection of technical choices (e.g., Dunnell 1978; Schiffer and Skibo 1997), this approach recognizes that technologies always implicate individual agency and sociocultural structures to a greater or lesser extent (Dobres 2000; Dobres and Hoffman 1994; Pfaffenberger 1992). This is not to deny the reality of functional and logistical constraints on (and motives for) technology, but only to assert that these choices have an important social content not easily reduced to "stylistic" variation among equivalent alternatives (see Conkey 2006; Hegmon 1998). This social content of technology can be at once the inertia of tradition (Sackett 1977, 1985), the assertion of social identity (Hodder 1982; Wiessner 1983; Wobst 1977), the reproduction of political economy (Bowser 2000; Brumfiel and Earle 1987; DeMarrais et al. 1996; Sinopoli 2003), and even the materialization of cultural schemas (M. J. Adams 1973; Lechtman 1996) —all of which are themselves reproduced and/or transformed in practice. It is for this reason that the remnants of past techniques and operational sequences (i.e., artifacts, features) provide analytical purchase for the study of economic processes and sociopolitical institutions from the archaeological record (see Chapter 2).

The operational sequence (chaîne opératoire) provides a convenient and wellknown analytic for parsing out the available and actual techniques employed for each stage in a given technological system of production (Dobres and Hoffman 1994; Lemmonier 1986; Leroi-Gourhan 1943). For each stage, inferences about past technical behaviors are drawn from formal variability in a given artifact or feature, usually with the aid of experimental or ethnoarchaeological studies (Roux 2007; Schiffer and Skibo 1997). With assumptions grounded in ethnographic, historic, or mechanical studies of the operational sequence under consideration, one can then make inferences about the logistical and social factors that recursively structured those choices (Carr 1995; Dobres 2000) and paved the way for individual agency and innovation (Hegmon and Kulow 2005; Wobst 1999). Over time and across space, the "genealogies” of techniques (sensu Gosden 2005) bundled together in material artifacts also provide a basis for interpreting the dynamic relations among social actors and institutions at multiple, interlocking scales. 
Technology, society, and culture of course interpenetrate well beyond systems of production. As material objects move through acts of use, exchange, repair, discard, and remembrance, they work to reproduce or even undermine the structural relations and cultural schemas in which they are enmeshed (Appadurai 1986; Keane 1997). Reading such object biographies requires careful attention to depositional contexts, patterns of use wear, and inter-object associations (see discussion in Chapter 6). While production does not exhaust the interpretive potential for material objects, the technical choices made in an operational sequence bundle together the material qualities that partly determine, or at least constrain, subsequent functions (Carr 1995; Schiffer and Skibo 1997) and semiotic interpretations (Keane 2005; Parmentier 1997). In sum, a technological approach, as I employ it here, provides a "middle-range" methodology (see Dobres and Robb 2005) for looking through archaeological objects onto past social worlds.

\section{A Note on Attributes versus Types}

A technological approach to objects and object classes requires identifying and recording a suite of attributes linked to the technical choices made at sequential stages of production. In contrast to a typological approach that assigns artifacts to intuited or paradigmatic classes, an attribute-based analysis allows one to consider formal variability within a dataset at the multiple scales needed to address a wide range of methodological, substantive, and theoretical issues (e.g., Adams and Adams 1991; Binford 1965; Carr 1995; Redman 1978; Sinopoli 1991; Whallon and Brown 1982). In West African archaeology, for example, the attribute-based analytical scheme advocated by Susan McIntosh (1995:130-132) has proven useful both for developing ceramic chronologies and for modeling social change in many different parts of the subcontinent (e.g., Dueppen 2008; Gallagher 2010; A. Lawson 2003; MacDonald 2011; McIntosh and Bocoum 2000; Richard 2007; Thiaw 1999; Togola 2008). Although a lack of consensus on attribute definitions and terminology has, until recently, handicapped interregional comparisons of pottery (see Haour et al. 2010:8-12), these studies have nonetheless highlighted a number of formal dimensions (e.g., paste type, vessel shape, decoration) broadly applicable to space-time systematics across West Africa and the study of pottery traditions as dynamic socio-technological systems.

This is by no means to reject typologies and taxonomic classification; these methodological tools are needed to relate the limitless amount of information latent in any artifact assemblage to a specific problem or interpretive purpose (Sinopoli 1991). An attribute-based analysis simply allows for the dialectical definition of useful types and for the construction of multiple practical typologies from one dataset (Adams and Adams 1991). Of course, all such typologies are constrained by the variables initially selected for measure. In line with a technological approach, my analyses of pottery (and other artifacts) focus on variables related to production and, to a lesser extent, those involved with use, exchange, and discard. As described in the final section of this appendix, these variables make possible the definition of typologies relevant to the analytical objectives outlined above.

\section{Sample Collection, Processing, and Analysis}

Fieldwork in 2008 and 2009, including systematic survey (Appendix A) and surface collection and excavation at Diouboye (Appendices B-C), generated a sample of 
77,109 pottery sherds. The sherds recovered each day were initially processed at a field laboratory in Sansanding by me and the CFAP field crew. For each FS unit (both surface and subsurface), we first counted and weighed all pottery as an aggregate. Next, we set aside non-body "diagnostic" sherds (i.e., rims, handles, bases, lids) for later analysis and continued to sort the bulky remainder of body sherds. With the exception of those from surveyed sites and from Unit $\mathrm{H}$ at Diouboye, we then discarded all "small" body sherds $\left(<4 \mathrm{~cm}^{2}\right)$ given the difficulty observing their paste and décor attributes; this is a common practice in West African archaeology (S. K. McIntosh 1995:138) and did not introduce bias into the ceramic assemblages (see below). The remaining body sherds were analyzed in the field lab mainly by me, but Massal Diagne and Aimé Kantoussan thankfully helped analyze the surface assemblage from Diouboye. We then selected a representative sample of body sherds (by decoration type) to catalogue at IFAN and disposed of the rest since resources for transportation and long-term storage were simply not available in this part of Senegal. Sherds collected during survey were dumped in the Falémé River, while those from Diouboye were interred with the backfill of excavation units. All diagnostic sherds, on the other hand, were washed in the field laboratory or in Dakar and transported to IFAN where I completed their analysis, drafting, and photography in 2008 and 2009.

The analysis of all pottery followed the same procedure, but the assemblages from regional survey and from Diouboye exhibited, not surprisingly, a different range of variability suited to different objectives. The assemblage from regional survey was fairly heterogeneous due to long-term changes in pottery technology, lending itself well to ceramic chronology and interregional comparison. The more homogeneous assemblages from surface collection and excavation at Diouboye also revealed sufficient diversity in technical attributes, albeit at finer scales, for the seriation of occupation phases and interpretation of vessel function(s). For these reasons, I generally distinguish between a Survey assemblage and Diouboye assemblage throughout this appendix.

The Survey assemblage was collected from 90 locales (84 sites and 6 isolated occurrences) and totaled 2297 rim and decorated body sherds from purposive sampling and 9638 sherds from systematic sampling; this included surface materials from Diouboye collected in three initial transects across Area C, Area N, and Area P (FS 1739$1749,1754-1769)$ where the area sampled $\left(25 \mathrm{~m}^{2}\right)$ was on par with that of systematic units on the surveyed sites. Broken down by form, this assemblage contains 10,716 body sherds, 1189 rim sherds, and 30 diagnostic base, handle, and lid sherds. As noted above, I did not exclude "small" $\left(<4 \mathrm{~cm}^{2}\right)$ sherds from the analysis since this size class includes a disproportionate number of the thin and heavily eroded sherds documenting Late Stone Age occupation in the study area.

The excavated component of the Diouboye assemblage totals 46,547 body sherds and 2777 rim and 62 other diagnostic sherds. After taking aggregate measures of all body sherds by FS unit, I analyzed the 19,337 "large" specimens $\left(>4 \mathrm{~cm}^{2}\right)$ from all excavation units. To account for possible biases introduced through a focus on large sherds, I also analyzed the 1790 small specimens from Unit $\mathrm{H}$. When compared to the large sherds from this same unit $(n=1196)$, the frequencies of 63 nominal attribute states (Tables D.4, D.10; Figure D.30) across the samples of large and small sherds are positively correlated $(r=0.997, \rho<0.001)$. Due to differences in preservation and visibility, decorative attribute frequencies are on average $15 \%$ greater for large body sherds than for small ones, but the 
strength of their correlation implies that this bias is consistent across all assemblages and should not affect classification or interpretation based on analysis of large sherds.

Table D.1: Sherds sampled for petrographic and compositional analyses; see below for attribute descriptions (Tables D.4, D.10; Figure D.22)

\begin{tabular}{|c|c|c|c|c|c|}
\hline Sherd ID & Site & Phase & Inclusions & Form & Decoration \\
\hline 5062.0001 & Diouboye & Early A/B & Grog & Body & Slip, Inc3 \\
\hline 5065.0029 & Diouboye & Early A/B & Grit & Body & CrB1 \\
\hline 5071.9001 & Diouboye & Early A/B & Grog & Rim S & Inc4, PnC \\
\hline 5019.0028 & Diouboye & Middle & Grit & Body & CrB1 \\
\hline 5019.9006 & Diouboye & Middle & Grog & Rim Ev1 & Slip, CrC, PnC \\
\hline 5027.0121 & \begin{tabular}{|l|} 
Diouboye \\
\end{tabular} & Middle & Grog & Body & CrB1 \\
\hline 5035.9014 & Diouboye & Middle & Grog & Rim ErR & Bevel, TwC \\
\hline 5042.0016 & Diouboye & Middle & Grog & Body & Slip \\
\hline 5057.9001 & Diouboye & Middle & Grog & Rim & TwC \\
\hline 5002.9006 & Diouboye & Late & Grog & Rim ErR & Slip, CrO, ImT \\
\hline 5010.9001 & Diouboye & Late & Grog & Rim ErA & Slip, Inc2 \\
\hline 5045.0003 & Diouboye & Late & Grit & Body & $\mathrm{CrC}$ \\
\hline 5045.0005 & Diouboye & Late & Sand/Organic & Body & Slip, CrC, PnC \\
\hline 5045.9012 & Diouboye & Late & Grit & Rim Ev1 & Bevel, CrD, Chnl \\
\hline 2064.0016 & FAL-1036 & LSA & Grit & Body & $\mathrm{Nr} 1$ \\
\hline 2064.9001 & FAL-1036 & LSA & Grit & Rim Ev1 & Bevel, ImC \\
\hline 2064.9009 & FAL-1036 & LSA & Grit & Rim EsT & Slip \\
\hline 2140.9020 & FAL-1075 & LSA & Grit & Rim E & Nr1 \\
\hline 2141.9008 & FAL-1075 & LSA & Grit & Rim EsT & - \\
\hline 2141.9009 & FAL-1075 & LSA & Grit & Rim S & Bevel, Polish, Nr1, ImC \\
\hline 6004.9001 & FAL-1075 & Tontèko & Grog/Organic & Rim Bd1 & $\mathrm{CmbR}$ \\
\hline 6004.0002 & FAL-1075 & Tontèko & Grit & Rim EsR & TwC, CmbR \\
\hline 6004.0003 & FAL-1075 & Tontèko & Grit & Body & CmbR, ImM \\
\hline 2097.0007 & Tontèko & Tontèko & Grog,/Organic & Body & $\mathrm{CmbR}$ \\
\hline 2097.9006 & Tontèko & Tontèko & Grit & Rim EfR & Polish, CmbS \\
\hline 2097.9008 & Tontèko & Tontèko & Grit & Rim EsR & CmbS, PnC, Add3 \\
\hline 2067.0155 & Karé & Karé & Grog & Body & TwC \\
\hline 2068.9010 & Karé & Karé & Grog,/Organic & Rim EfR & Organic Coat \\
\hline 2072.9012 & Karé & Karé & Grog,/Organic & Rim EfR & Organic Coat \\
\hline 2107.9001 & Toranga & Karé & Organic & Rim EwR & Organic Coat, Inc1 \\
\hline 2108.9004 & Toranga & Karé & Grit & Rim EsR & Polish \\
\hline 2108.9011 & Toranga & Karé & Grog/Organic & Rim S & Organic Coat, CmbR \\
\hline
\end{tabular}


The Diouboye assemblage also includes 28,330 body sherds and 2514 diagnostic (rim and non-body) sherds from surface contexts. Although these were not used to reconstruct the operational sequence of pottery production at Diouboye, I did assign them to seriated types in order to model occupational history and post-depositional processes across the site surface (see Chapter 8).

Finally, I chose a small subset of sherds from the Survey $(n=18)$ and Diouboye $(n=14)$ assemblages for exploratory petrographic and compositional analyses (Table D.1). This sample includes sherd types from each of the regional and Diouboye-specific ceramic phases defined in the final section of this appendix. Ostensibly, these analyses sought to determine whether or not compositional variability in the sherd population would be sufficient to address a wider set of questions on exchange and social boundaries. At the same time, this dataset helped to distinguish techniques for clay selection and processing in the pottery traditions of the Madina-Sadatou study area.

\section{POTTERY TECHNOLOGY IN THE MADINA-SADATOU STUDY AREA}

In the past, as today, hand-built pottery in West Africa was almost invariably earthenware-made of coarse porous clay and low-fired at temperatures around 800$1200^{\circ} \mathrm{C}$ (Rice 1987:6). Earthenware vessels can serve a wide variety of functions as water jars, cooking pots, feasting dishes, incense burners, and ritual offerings. In many West African societies, earthenware pottery is made by part- or full-time specialists, often, but not always, women belonging to a larger caste of craftspeople including blacksmiths, weavers, masons, and wood and leather workers (see Chapter 2). This is certainly the case for potters in Senegambia and surrounding regions whose traditions and techniques inform a substantial body of ethnoarchaeological literature and, by extension, much of the analysis below (B. Diop 1995, 2000; Frank 1998; Gallay 1970, 1992; Gelbert 1999, 2003; Guèye 1998; Huyescom 1994; Linares de Sapir 1969; Mayor 2010; Sall 2005; Thiam 2010). As with most craft activities, pottery manufacture requires a minimum of practical and discursive or ritual knowledge about how to transform raw materials into objects of functional and symbolic value. Potters acquire and reproduce this knowledge in a variety of social contexts ranging from prolonged apprenticeship with close family members to the adoption of techniques and aesthetics across broader communities of crafts producers and crafts consumers. Technologies of production are therefore integrated into the social fabric at several scales-from individual actors to intergenerational households and lineages to regional networks of artisans - each of which shapes technical choices in different ways across the operational sequence.

Earthenware pottery production consists of five general stages: (1) procuring clay and other resources; (2) preparing the paste; (3) vessel shaping; (4) decorating the vessel; and (5) firing. In the following analysis I offer a brief discussion of the logistical and social factors that tend to inform technical choices in ethnographic (and historic) contexts for each of these stages, and the potential repercussions of such choices for object biographies. I then describe the variables and their attribute states recorded to infer techniques of pottery manufacture and define the chronological and functional typologies employed throughout this study. Although my analysis focuses mainly on attributes pertaining to production, I also make note of those related to patterns of exchange, use, and deposition where possible. 


\section{Clay Procurement}

Earthenware pottery production in West Africa uses raw materials that are naturally abundant and/or low cost. Although I focus on clay, these materials include non-plastic tempering agents, tools for vessel shaping and decoration, pigments for coloring, and fuel for firing (Rice 1987:115). Along the Falémé River, as across much of West Africa, contemporary potters obtain clay from diverse sources, including river beds and embankments, rice paddies, and even termite mounds (David and Hennig 1972:15; Gelbert 1999; Gosselain and Livingstone Smith 2005; Guèye 1998:63; Linares de Sapir 1969:3; Livingstone Smith 2000; Sall 2005:36-40). These sources are often less than one kilometer from the potter's residence, and rarely more than three kilometers, since clay is heavy and must be transported back to household workshops. For this reason,

provenience studies of natural clay deposits can help to determine whether archaeological pottery was produced from one or more local sources or acquired through exchange (Stahl et al. 2008; Usman et al. 2005). In addition to distance, the color and stickiness of the natural clay (determined by its texture and mineral composition), ease of processing, social or political boundaries, and cultural taboos can influence potters within a community to exploit the same or different clay sources (B. Diop 2000:264-265; Gallay 1970:50-54; Gosselain 1999; Stark et al. 2000).

\section{Provenience Analysis}

I adopted two strategies for assessing variability in the clay sources exploited by past Madina-Sadatou potters. First, paste color provided a rudimentary measure of the iron and organic impurities in the initial clay source, at least to the extent that I could control for reduction in the firing atmosphere and post-production use (Rice 1987:333339). Using a Munsell (2000) soil color chart, I recorded the hue, value, and chroma of the most oxidized portion of paste visible in freshly broken cross-sections. Since this added considerable time to the analysis, I only did this for diagnostic sherds.

As illustrated in Figure D.1, the Diouboye assemblage has a greater range of paste color than the Survey assemblage where a greater proportion of sherds exhibit gray and reddish-gray paste (2.5 YR 5/2 to 7.5 YR 5/1). For the majority of sherds in both assemblages, paste color grades from light red to dark yellowish-brown with a mode of reddish-brown (5 YR 5/4). While this variability may derive largely from firing conditions, it does lend support to the typologies developed later in this appendix.

Second, I conducted an exploratory compositional analysis of the clay/silt matrix and non-plastic inclusions in a sample of 32 sherds (Table D.1) in the hopes of discriminating non-local clays introduced through exchange practices and/or migration. Although large-scale ceramic compositional studies are relatively new in West African archaeology (Stahl et al. 2008; Usman et al. 2005), they have long proven useful in other regions, particularly where natural clay sources can be included in the analysis (e.g., Bishop et al. 1982; Stark et al. 2000).

Since sample processing and instrument use can drive up the costs of large-scale studies, I set out with the modest goal of identifying the range of compositional variability in the Madina-Sadatou ceramic assemblages using laser ablation linked to inductively coupled plasma mass spectrometry (hereafter LA-ICP-MS). This method has several advantages over techniques such as neutron activation analysis - namely, low cost, rapid analytic time, and detection limits below ppm for 70 major, minor, and rare 
Figure D.1: Paste color distributions for the Diouboye and Survey assemblages showing confidence interval ellipsoids (90\%)

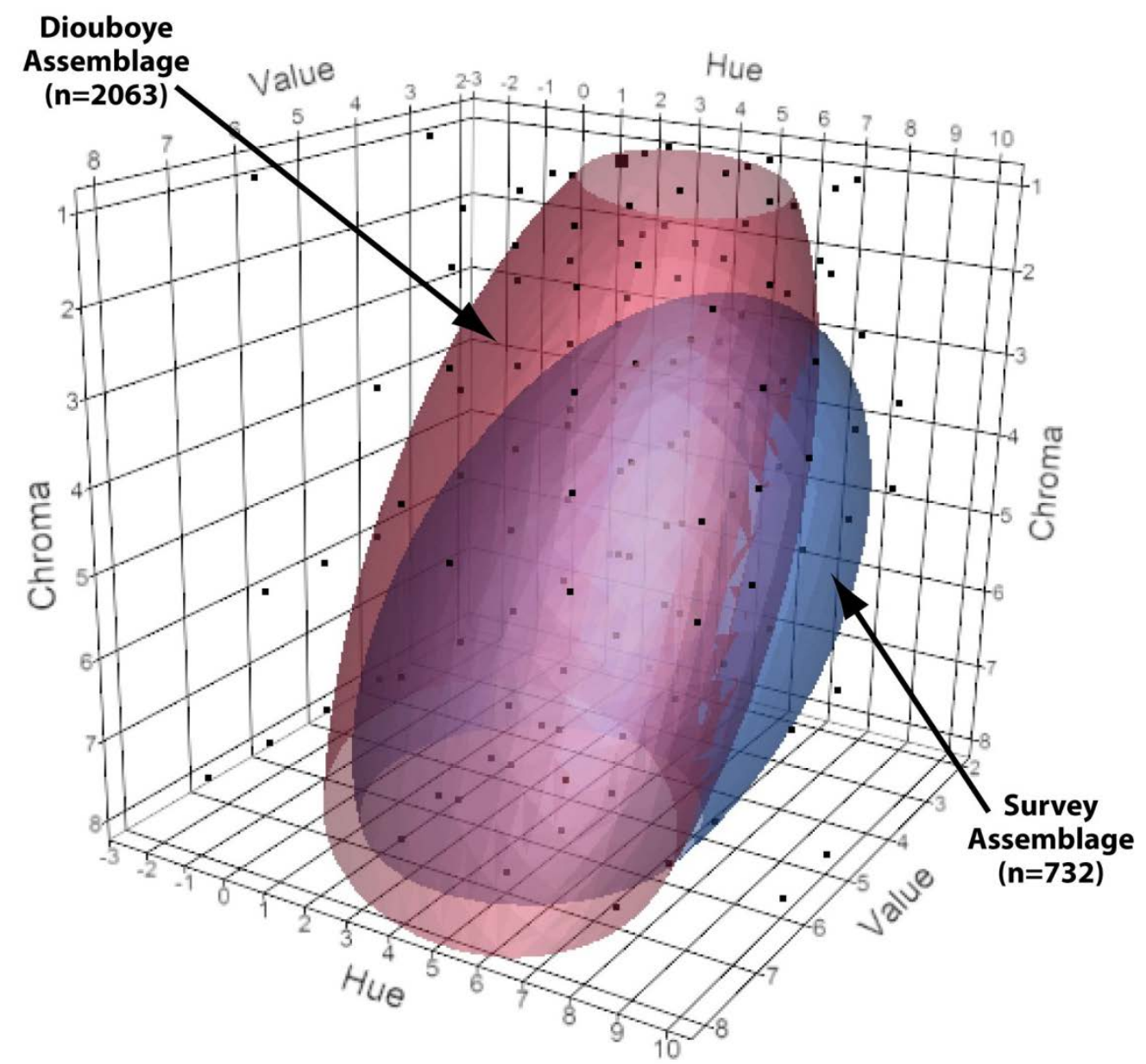

earth elements (Speakman and Neff 2005). Laser ablation also allows the analyst to pinpoint an area of less than $500 \times 500$ microns and thereby compare the composition of silt/clay matrix to that of non-plastic inclusions, slips, paints, or glazes. On the other hand, spectral interferences, elemental fractionation, and small discrepancies in the quantity of ablated material within and between samples all contribute to difficulty in measuring absolute elemental concentrations. In response, researchers have developed a number of data calibration methods to produce results equivalent to, or at least comparable with, those from other analytical techniques.

Using instruments available in the U-M Keck Elemental Geology Lab ${ }^{8}$, data acquisition generally followed a protocol established by Hector Neff (2003). To minimize the possibility of surface contamination, I directed ablation onto the freshly cut interior of each sherd and took three measurements of the paste matrix and two measurements from

\footnotetext{
${ }^{8}$ Analysis conducted under the supervision of Dr. Ted Huston.
} 
dominant grit or grog inclusions. After every two sherd samples, I took measurements on standardized NIST 612 glass tablets as well as "blank" readings in order to control for instrumental drift and calibrate data across the samples. In this way, the analysis generated counts of all scanned elements, with the exception of potassium $\left({ }^{39} \mathrm{~K}\right)$ and phosphorous $\left({ }^{31} \mathrm{P}\right)$, both of which posed a problem for the ICP-MS instrumentation. Because potassium oxide $\left(\mathrm{K}_{2} \mathrm{O}\right)$ can comprise $1-2 \%$ of potting clay in the greater Senegambia (Filipowiak 1979:90; Sall 2005:52), the results may slightly overestimate the concentrations of other scanned elements.

In order to convert the raw data obtained from LA-ICP-MS into actual element concentrations, I employed the Gratuze Method of calibration using aluminum $\left({ }^{27} \mathrm{Al}\right)$ as an internal standard (Gratuze 1999; Neff 2003). This method generates the concentrations of oxides in each sample, requiring me to back calculate the concentrations of all minor and trace elements for the following compositional analysis.

\section{Table D.2: $\quad$ Operating parameters for the laser ablation system and ICP-MS}

\begin{tabular}{ll}
\hline \hline Ablation Parameters & Instrument: Merchantek (New Wave) LUV266X \\
Laser power & $\sim 1.4 \mathrm{~mJ}$ \\
Laser frequency & $20 \mathrm{~Hz}$ \\
Laser speed & $10 \mathrm{microns} /$ second \\
Laser diameter & 100 microns \\
Ablation pattern & Line, $\sim 500$ microns in length \\
\hline \hline ICP-MS Settings & Instrument: Thermo Scientific Element ICP-HRMS \\
Coolant (Ar) & $\sim 16 \mathrm{~L} /$ min \\
Auxiliary (Ar) & $\sim 1 \mathrm{~L} /$ min \\
Carrier (Ar) & $\sim 1 \mathrm{~L} / \mathrm{min}$ \\
RF Power & $1400 \mathrm{~W}$ \\
Analytical time per run & 4 minutes \\
Segment time & $30 \mathrm{~ms}$ per run \\
Mass settling time & $\sim 300 \mathrm{~ms}$ \\
Analytical mode & Full peak scanning mode \\
\hline \hline
\end{tabular}

\section{Isotopes Measured}

Li-7, Na-23, Mg-24, Al-27, Si-30, P-31, K-39, Ca-43, Sc-45, Ti-47, V-51, Cr-52, Mn-55, Fe-57, Co-59, Ni-60, Cu-63, Zn-66, Rb-85, Sr-88, Y-89, Zr-90, Ag-107, Cd-111, In-115, Sn-118, Sb-121, Cs-133, Ba-137, La-139, Ce-140, Nd-146, Sm-147, Eu-151, Dy-163, Ho-165, Er-167, Lu-175, Ta-181, Pb-208, Th-232, U-238

\section{Compositional Classification}

Following Baxter (1989), classification proceeded at two analytical levels based on the concentrations of major constituents (normalized as oxides) and of minor and trace elements (Table D.3). For each of these levels, I used principal components analysis (PCA) to explore the data structure in both unstandardized and log-standardized form and to define three components accounting for most of the compositional variability among these constituents (Baxter 2003; Neff 1994:73-83). Unless otherwise specified, the following statistical analyses were performed on the log-normalized data. 


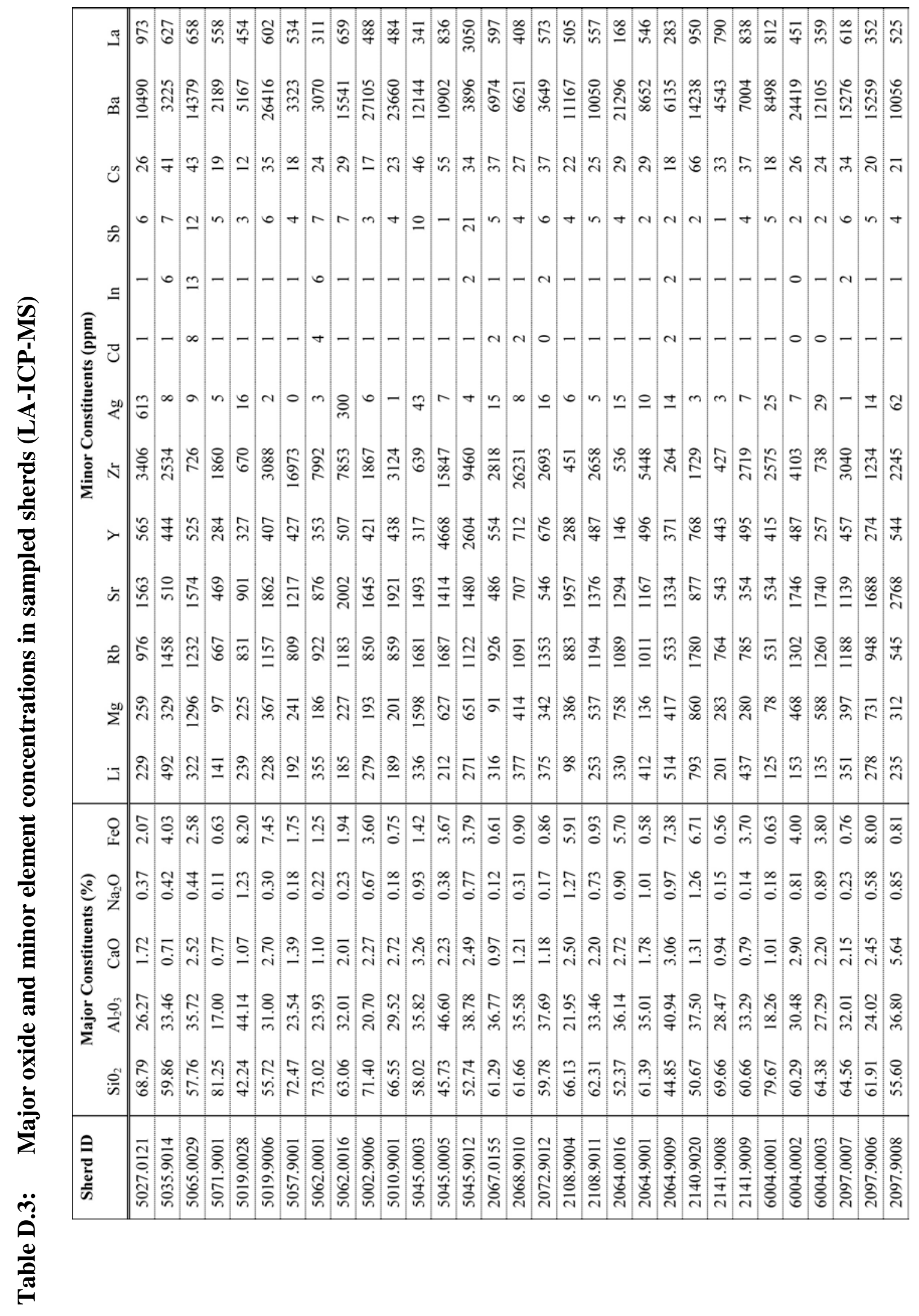




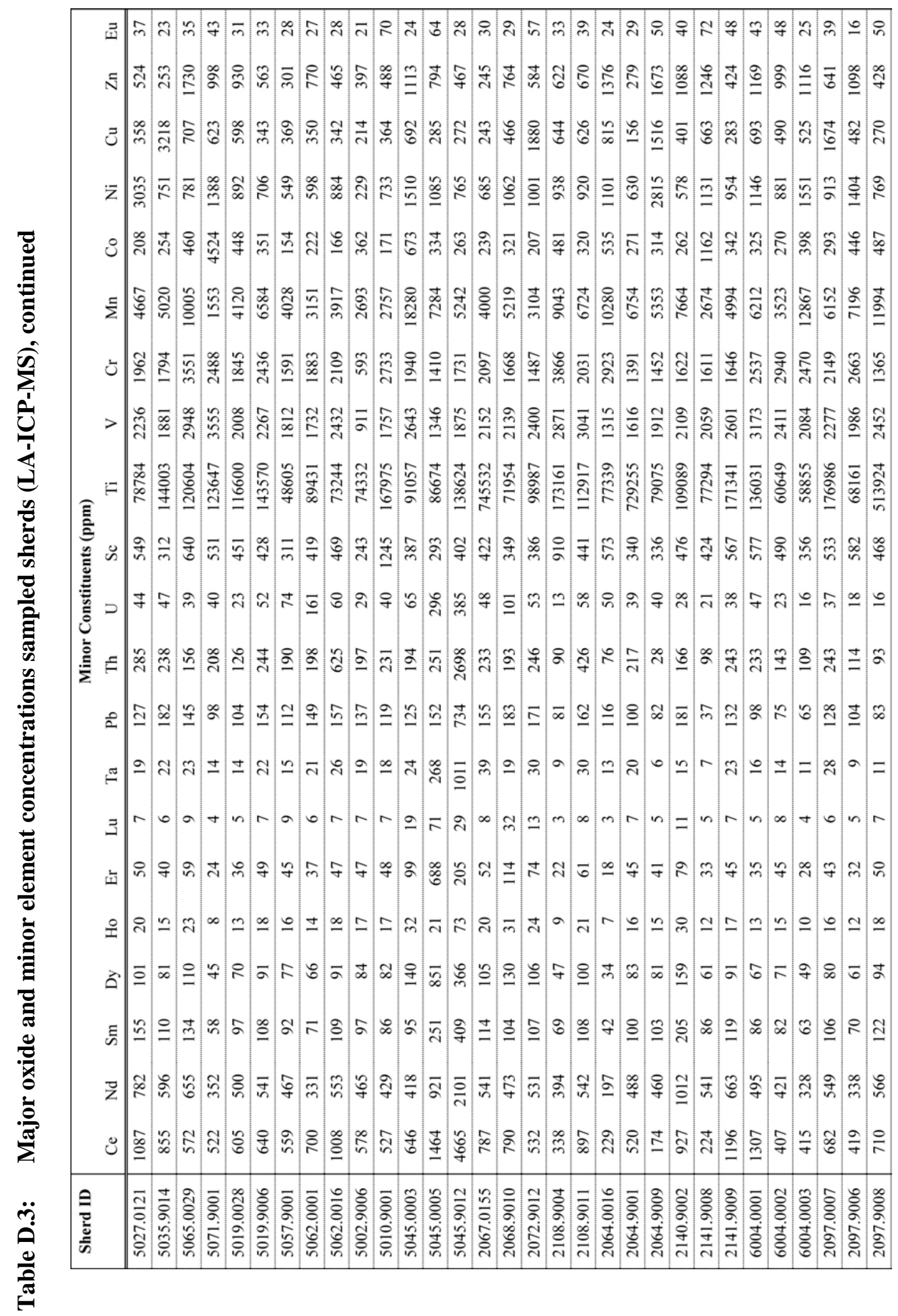


The oxides $\mathrm{SiO}_{2}, \mathrm{Al}_{2} \mathrm{O}_{3}, \mathrm{CaO}, \mathrm{Na}_{2} \mathrm{O}$, and $\mathrm{FeO}$ (or $\mathrm{Fe}_{2} \mathrm{O}_{3}$ ) are the dominant constituents in the Madina-Sadatou ceramic assemblages. PCA reveals heavy loading on the first principal component, with a strong negative correlation between $\mathrm{SiO}_{2}$ and $\mathrm{Al}_{2} \mathrm{O}_{3}$ $(\mathrm{r}=-0.9078, \rho<0.001)$ accounting for most of this variation. While modalities in the ratio of these two oxides could potentially discriminate among clay sources from a larger regional assemblage, the normal distribution within this sample is consistent with localized variation. The second and third principal components discriminate three compositional groups on the basis of variation in the other three oxides, but these are congruent with those groups identified from minor and trace elements.

PCA of the minor and trace constituents demonstrates a modest level of loading on the first three components, which account for $55 \%$ of the total variance. A cluster analysis (Ward's method) on these components reveals four compositional groups corresponding largely to differences in the relative concentrations of magnesium (Mg) and the rare earth elements (Figures D.2-D.3). As illustrated in Figure D.4, these groups match well with the variation of major constituents such as calcium oxide and iron oxide. Pending analysis of known clay sources, it is not yet possible to associate these groups with specific extraction locales on the landscape.

Figure D.2: Bivariate plots of four clay compositional groups plotted against the first two principal components showing confidence interval ellipses $(90 \%)$ (left) and the elemental vectors plotted against the first two principal components (right)
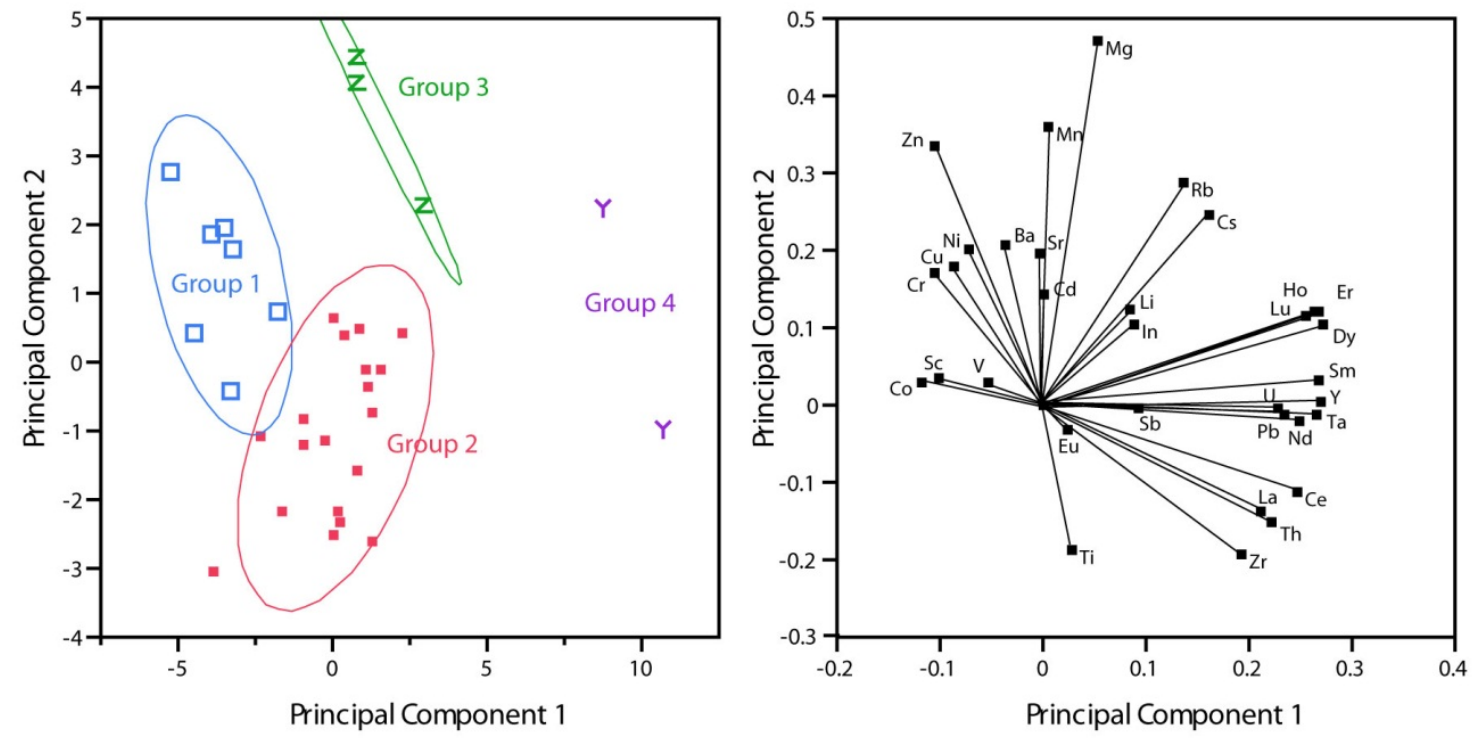
Figure D.3: Bivariate plot of magnesium and cerium concentrations in four clay compositional groups showing confidence interval ellipses $(90 \%)$

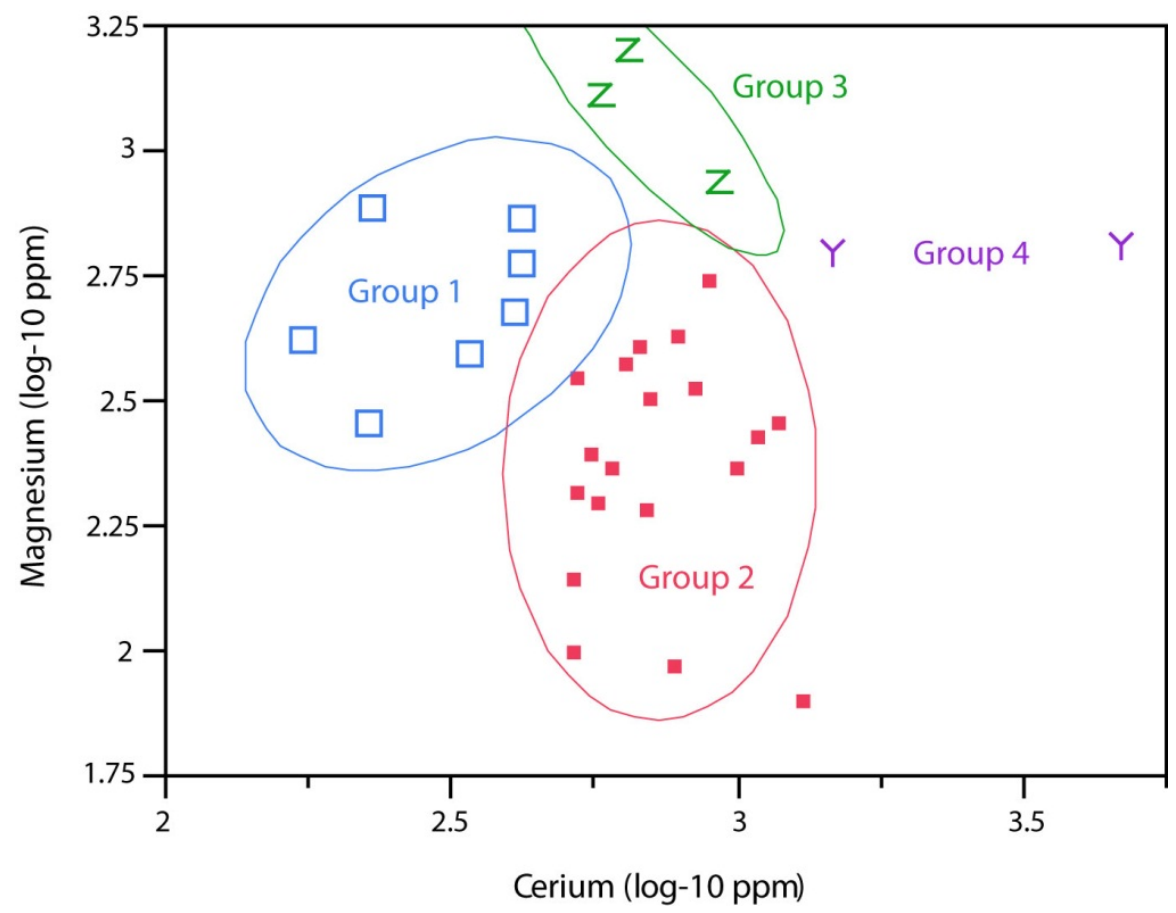

Figure D.4: Bivariate plot of calcium oxide and iron oxide concentrations in four compositional groups showing confidence interval ellipses $(90 \%)$

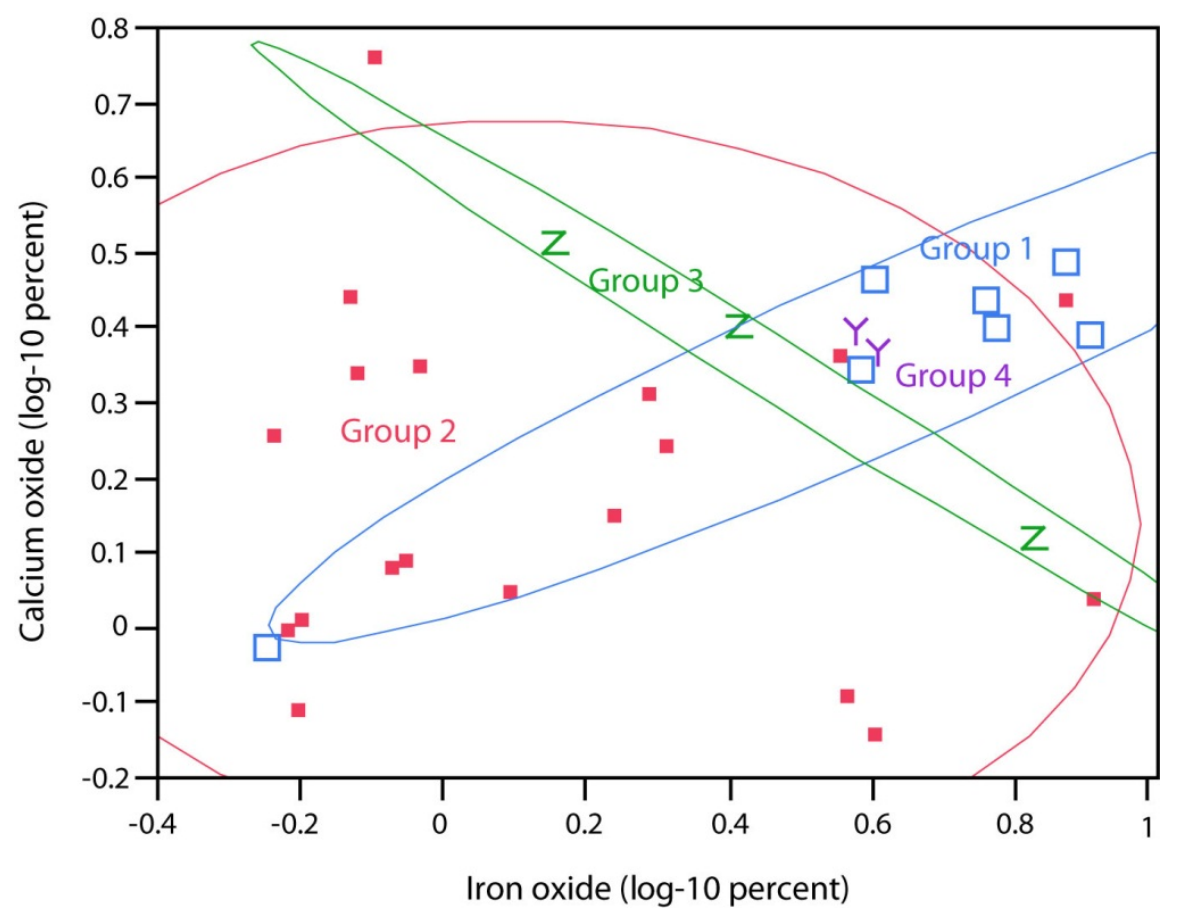


Although I ablated clay matrix and inclusions separately, it was important to verify that these compositional groups were not grossly affected by the so-called "tempering problem" (Neff et al. 1989). For this, I identified those elements that strongly and significantly discriminated between grit $(n=13)$ and grog $(n=11)$ inclusions in the sampled sherds. I then compared the concentrations of these elements with compositional Group 1 and Group 2, the former associated with grit temper (see Figure D.10 below).

Only two elements in these inclusions - cerium (Ce) and uranium (U) - mirror differences between the groups (Figure D.5). The concentrations of the other five elements show strong coherence between Group 1 and Group 2 and grog temper, in stark contrast to their concentrations in grit inclusions of crushed quartz-feldspathic rock. Scandium (Sc), for instance, has a greater mean concentration in Group 1 than in Group 2, despite a lower mean concentration in grit compared to grog (Figure D.5). Altogether these data indicate that grit inclusions do not significantly affect the results of compositional analysis on the surrounding clay matrix; the compositional groups represent different clay sources employed by potters in Madina-Sadatou.

Figure D.5: Boxplots of uranium (U) (left) and scandium (Sc) (right) concentrations for compositional groups and inclusion types
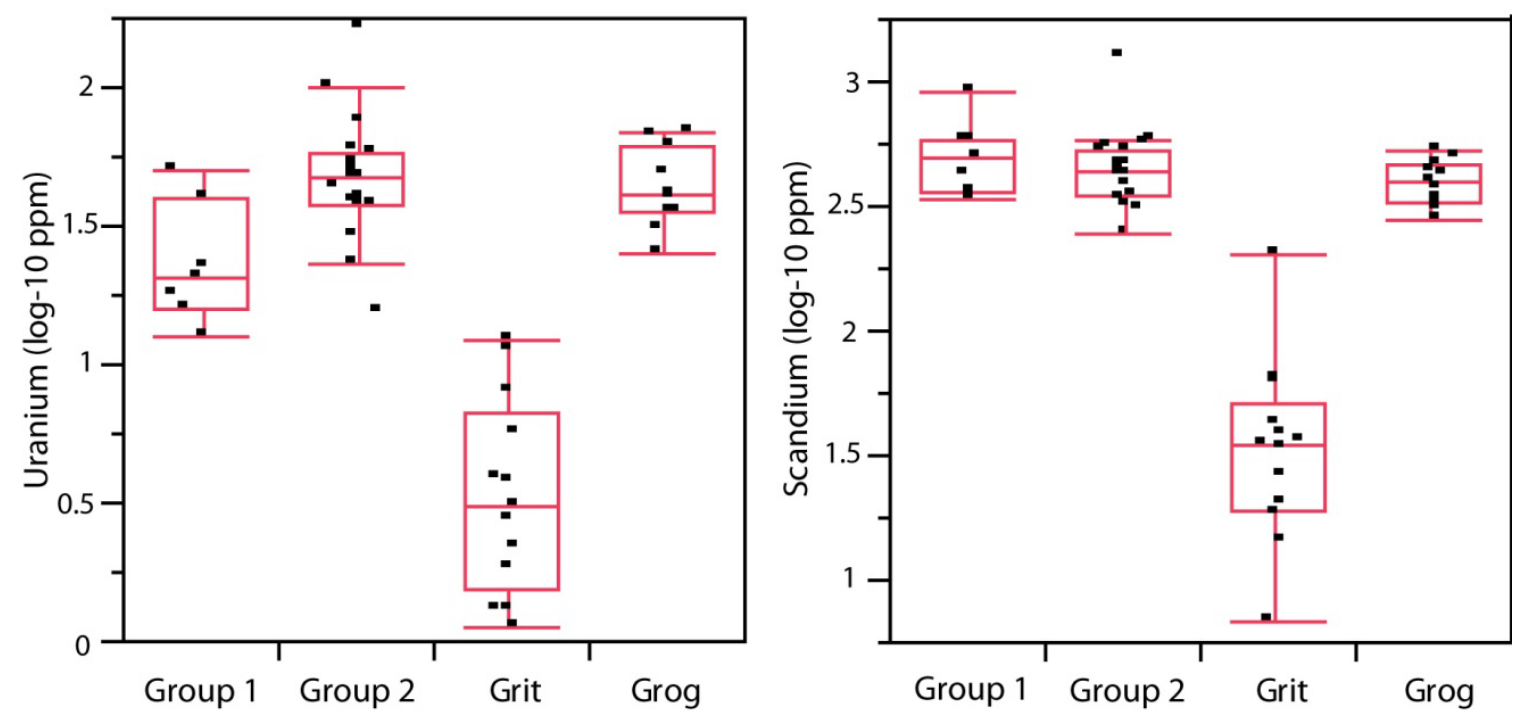

Despite the small samples, these compositional groups document continuity, and possible change, in clay procurement and/or exchange practices across the archaeological phases defined later in this appendix (Figure D.6). Most strikingly, Group 1 clay does not occur among the small sample of sherds from the Iron Age village of Diouboye, while the two outlier specimens comprising Group 4 clay both come from the final occupation phase of Area $\mathrm{H}$ at this site. Notably, one of these latter specimens (5045-0005) is thin (6 $\mathrm{mm}$ ) with a fine sand/organic paste resembling the "fine ware" observed in early contexts from Arondo and described by Thiaw (1999:172-173) as similar to pottery from the Middle Niger region. Group 3 clay does not show up in samples from Atlantic Era sites 
(Tontèko and Karé phases). Next to long-term continuity in the use of Group 2 clay, these patterns point to fairly homogeneous clay procurement strategies at Diouboye relative to those from earlier and later occupations, assuming for the moment that sherds with Group 4 clay represent vessels exchanged into the Madina-Sadatou study area.

Figure D.6: Distribution of compositional groups by horizon/phase

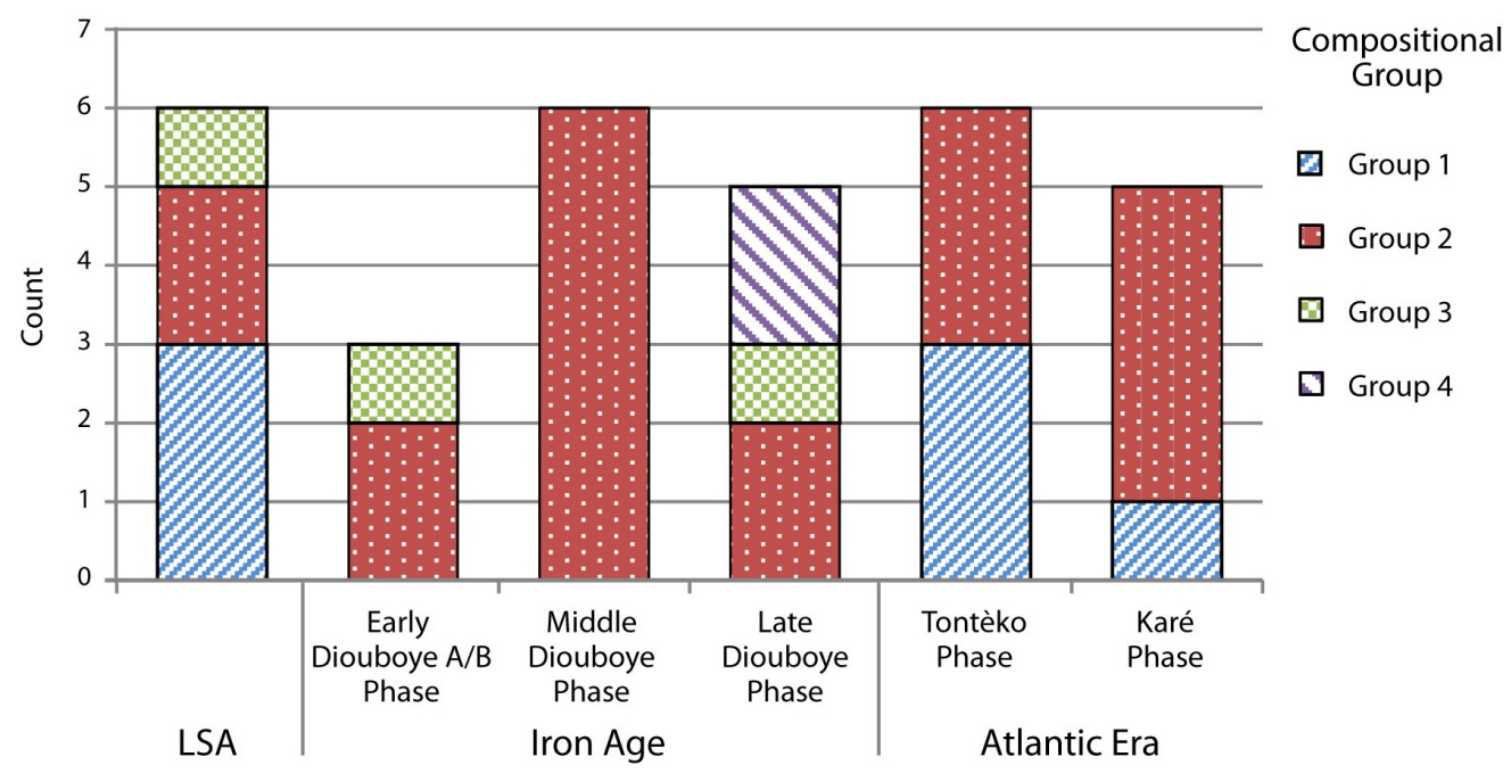

\section{Paste Preparation}

Wherever obtained, clay usually requires some manner of processing prior to vessel formation and firing. In some cases this involves treating the clay to alter its chemical composition - for example, adding calcareous materials or leaching out salt (Rice 1987:119; Sall 2005:41-42). In other cases potters adjust the strength, hardness, porosity, or thermodynamic properties of the clay by adding (or sifting out) non-plastic inclusions including, but not limited to, sand, grit, shell, crushed sherds (grog), grass, and even dung (Rice 1987:406-411); all of these have been documented in ethnographic and archaeological contexts across Senegambia (B. Diop 2000:265; Gelbert 1999, 2003; Guèye 1998:64-67; Sall 2005:42-45). Some inclusions, such as quartz and non-quartz sand, mica, and organic detritus need not signify a deliberate act of addition since these appear naturally in many clay deposits. Potters may, however, seek out clay sources with favorable quantities of such elements.

In contemporary West African villages, paste preparation most often takes place within relatively private workshops enclosed in family compounds. Potters (or, more often, their apprentices) mix and sift to adjust the paste "recipe" of dried clay and inclusions to suite cultural norms and tastes (Gosselain 1998; Gosselain and Livingstone Smith 2005; Livingstone Smith 2000). Given their consciousness of these techniques, potters can switch paste recipes quite rapidly, particularly in settings of cultural contact (Gelbert 1999, 2003), either by sharing technical knowledge with each other or by observing the appearances of other vessels. 
The paste recipes identified in archaeological ceramics thus emerge at the intersection of raw material availability, embodied habits, consumer tastes, and interaction within the often supra-local context of an artisanal network. Understanding the relative importance of these various factors begins with an adequate characterization of such paste recipes according to the techniques that generated them.

\section{Paste Analysis}

In order to identify techniques of paste preparation in the ceramic assemblages from Madina-Sadatou, I focused on the type, texture, shape, and density of non-plastic inclusions visible in the cross-section of each sherd (Table D.4). The type of inclusion allowed some initial distinction between intentional inclusions (grog) and potentially natural inclusions (i.e., organic chaff, dung, sand, and mineral). The shape of mineral grains permitted a tentative distinction between the heavily worn natural grains of sand and angular grains of grit temper (Stoltman 1989:149); this latter inclusion comprised further evidence for patterns of clay selection and procurement. The texture and density of inclusions together formed a proxy measure for the degree of sorting (or lack thereof) in paste preparation. Associations among these attributes then allowed me to define paste types (below) corresponding, at least in part, to the technical recipes used by past potters.

Analysis proceeded in two stages-macroscopic and microscopic. First, I conducted macroscopic analysis on all diagnostic and large body sherds from both the Survey and Diouboye assemblages. Breaking a corner from each sherd to observe a fresh cross-section, I recorded the type, texture, and density of inclusions and organic pores through visual comparison to an ordinal scale (after Figure 12.2 in Rice 1987:349). Second, I undertook a petrographic study of thin sections from the same subset of sherds chosen for compositional analysis (Table D.1). Using a point-count procedure (Stoltman 1989, 1991), I employed a polarizing microscope to observe (10X) and record the constituents of each thin section at fixed intervals of one millimeter. For better or worse, the petrographic slides were commissioned by a geological technician with no prior ceramic experience and the usable area (unscratched and $0.03 \mathrm{~mm}$ in thickness) of several thin sections was less than two square centimeters. In these cases, I rotated the slide $180^{\circ}$ and made another pass over its surface to obtain an acceptable sample size of at least 200 observations from each thin section (Stoltman 1991:107). To control for the possible bias of coarse inclusions, I noted multiple observations occurring on the same grain or void.

At each interval, I assigned the point directly beneath the microscope crosshairs to one of four general classes: void; clay matrix (grain size invisible); silt matrix (grain size visible up to $0.0625 \mathrm{~mm}$ ); and non-plastic inclusion (grain size $>0.0625 \mathrm{~mm}$ ). For the latter, I further recorded the material type as grog, quartz, mica, grit (a birefringent constituent variably comprised of feldspar, quartz, hornblende, and mica), or unidentified mineral. I measured grain size according to the scale used in macroscopic analysis, but with a further distinction between extremely fine and very fine grains (Table D.4). In order to determine the source of the inclusion, I made a somewhat subjective assessment of its sphericity and roundness, which together provide an index of natural wear. While these data helped to accurately quantify inclusions and refine the paste types defined through macroscopic analysis, a more detailed petrographic characterization of the mineral constituents in a larger sample of sherds could potentially discriminate among clay sources on a local or regional scale (e.g., Stark et al. 2000; Wilmsen et al. 2009). 


\section{Table D.4: Variables recorded for ceramic paste inclusions}

\begin{tabular}{|c|c|c|}
\hline Variable & Attribute (Code) & Description \\
\hline \multirow{6}{*}{ Type } & Grog & Crushed pottery or fragments of hardened clay \\
\hline & Grit & $\begin{array}{l}\text { Whitish, opaque rock fragments with quartz, feldspar, and flecks } \\
\text { of hornblende or mica }\end{array}$ \\
\hline & Mica & Dark, reflective mineral \\
\hline & Sand, quartz & Whitish, translucent mineral, usually rounded \\
\hline & Sand, unknown & Dark, opaque mineral, usually rounded \\
\hline & Organic & $\begin{array}{l}\text { (1) Unidentified } \\
\text { (2) Chaff (elongated voids, sometimes with carbonized edges) } \\
\text { (3) Shell/bone }\end{array}$ \\
\hline \multirow{7}{*}{ Texture } & Clay matrix & Grain in thin section is clay (invisible) or silt $(<0.0625 \mathrm{~mm})$ \\
\hline & $\begin{array}{l}\text { Extremely/ } \\
\text { Very fine (0) }\end{array}$ & $\begin{array}{l}<0.5 \mathrm{~mm} \text {; Grain in thin section is either extremely fine }(0.0625- \\
0.25 \mathrm{~mm}) \text { or very fine }(0.25-0.50 \mathrm{~mm})\end{array}$ \\
\hline & Fine (1) & $0.5-1 \mathrm{~mm}$ \\
\hline & Medium (2) & $1-2 \mathrm{~mm}$ \\
\hline & Coarse (3) & $2-3 \mathrm{~mm}$ \\
\hline & Very coarse (4) & 3-4 mm \\
\hline & $\begin{array}{l}\text { Extremely } \\
\text { coarse (5) }\end{array}$ & $>4 \mathrm{~mm}$ \\
\hline \multirow{6}{*}{ Density } & Very light (1) & $<1 \%$ \\
\hline & Light (3) & $1-5 \%$ \\
\hline & Medium (5) & $5-10 \%$ \\
\hline & Heavy (10) & $10-20 \%$ \\
\hline & Very heavy (20) & $20-30 \%$ \\
\hline & $\begin{array}{l}\text { Extremely } \\
\text { heavy (30) }\end{array}$ & $>30 \%$ \\
\hline \multirow{2}{*}{ Sphericity } & Spherical (1) & Grain in thin section has circular shape \\
\hline & Amorphous (0) & Grain in thin section has irregular or amorphous shape \\
\hline \multirow{2}{*}{ Roundness } & Round (1) & Grain in thin section has rounded corners and worn edges \\
\hline & Angular (0) & Grain in thin section has angular corners and unworn edges \\
\hline
\end{tabular}

\section{Paste Classification}

In order to classify techniques of paste preparation, it was first necessary to determine which inclusions might have been added by potters and which ones might have originated at the clay source. Grog was almost certainly an intended temper; many contemporary potters in West Africa crush broken sherds in a wooden mortar with the bodily gestures homologous to those that they, as women also responsible for food preparation, use to pound grain (Gosselain 2010:197). In contrast, the non-quartz sand 
grains visible in cross-section and in thin section were generally smaller and more heavily worn than other inclusions (Figures D.7-D.9), suggesting a natural presence in the raw clay. It was possible to infer the presence of organic inclusions from the voids visible, either macro- or microscopically, in the paste fabric. Although many voids derived from unknown, and possibly natural, inclusions, some elongated pores with carbonized edges were consistent with the chaff temper used today in the region (Gelbert 1999, 2003), and were classified as such. The origins of quartz sand remain somewhat ambiguous. Despite a modest proportion of unworn quartz grains visible in thin section, their size distribution resembles that of the unidentified, worn sand particles. Macroscopic inspection, on the other hand, revealed a trend towards many fewer, yet larger, quartz sand grains. The most parsimonious explanation for this discrepancy is that most quartz particles observed in the petrographic analysis were actually small grains of grit; only large quartz sand particles could be accurately identified without magnification. For this reason, quartz sand and mica have been subsumed into the grit category for analysis and classification.

The origins of grit inclusions are less certain. Although these particles are generally rounded and spherical, the proportion of unworn grains (20-30\%) and their size distribution are both on par with the intentionally crushed, albeit much softer, grog. This could signal an intended crushing of feldspathic-quartz (granitic) rock to dimensions prescribed by tradition for all tempering agents, an interpretation that finds indirect support from the discovery of several pieces of friable granitic rock in craft production contexts at Diouboye (see Chapter 6). Figure D.10 illustrates the association between clay compositional Group 1 and Group 3 and grit inclusions. In light of the above resolution of the analytical "tempering problem," this pattern probably represents some convergence in potters' choices about clay selection and processing techniques.

Figure D.7: Relative distribution of sphericity and roundness of grain types in the petrographic sherd (thin section) sample

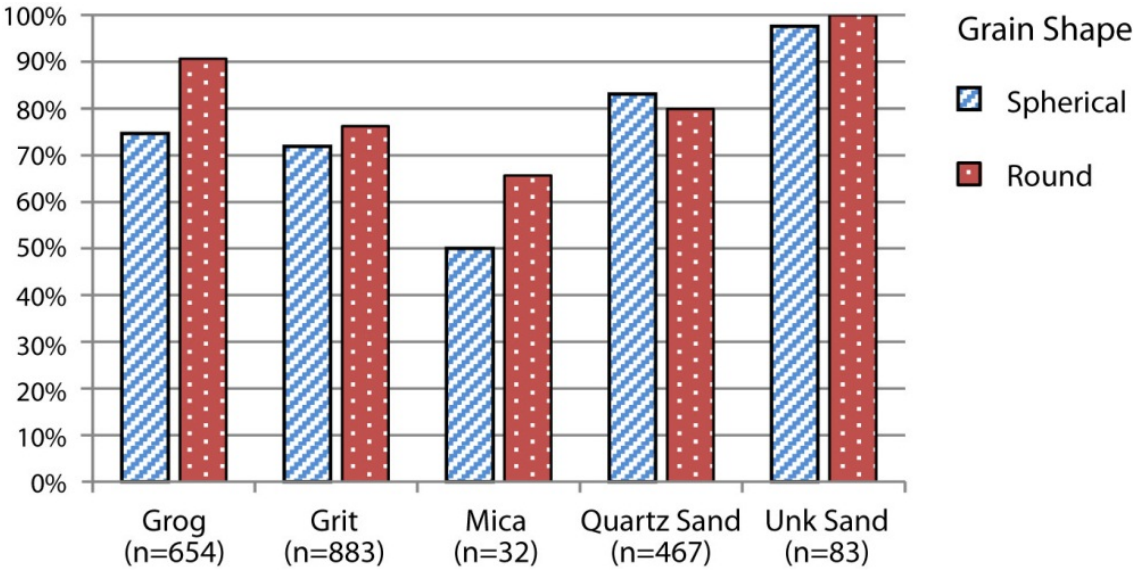


Figure D.8: Ordinal size distribution of grain types in the petrographic sherd sample (thin section)

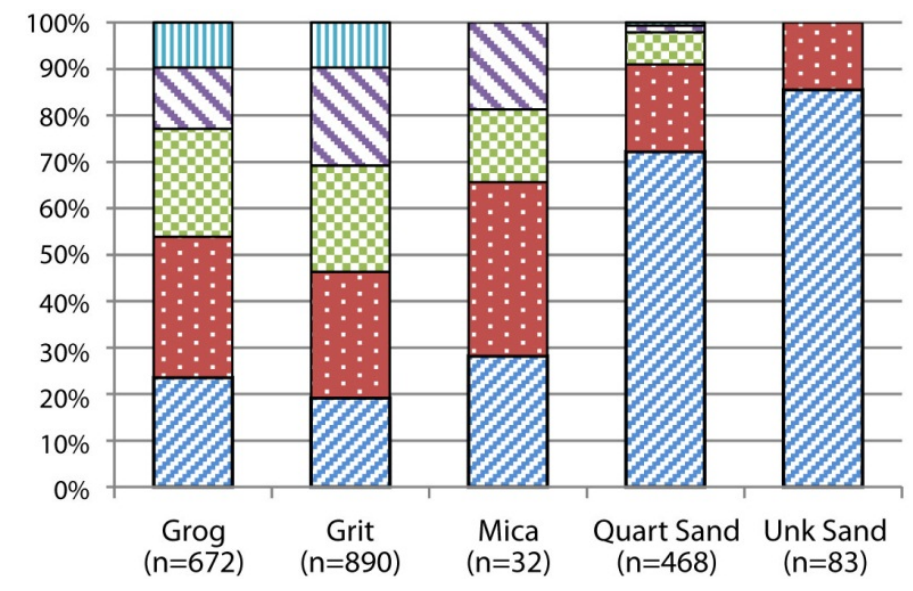

Grain Size

प Coarse

Q Medium

$\square$ Fine

$\square$ Very fine

Extremely fine

Figure D.9: Ordinal size distribution for grain types in the Survey assemblage (cross-section)

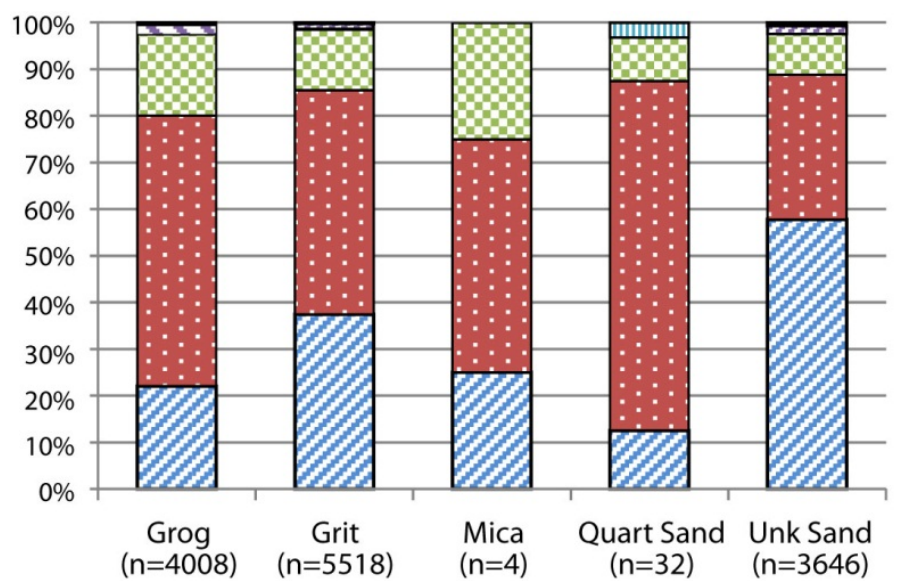

Grain Size

(1) Coarse

Medium

$\square$ Fine

Very fine

Extremely fine

Whether as natural element or added temper, potters in Madina-Sadatou clearly controlled for the type, quantity, and, to a lesser extent, size of the grog, grit, and organic inclusions in their paste recipes. Based upon associations among these inclusions (Table D.5), it was possible to identify three broad paradigmatic paste types in the Survey assemblage, only two of which appear in the Diouboye assemblage. First, grit inclusions are significantly dissociated from both grog $\left(\chi^{2}=4413, \rho<0.001\right)$ and from organic ones $\left(\chi^{2}=5292, \rho<0.001\right)$, suggesting that they comprise a distinct type of temper (grit).

Second, a positive association between grog and non-chaff organic inclusions $\left(\chi^{2}=1710\right.$, $\rho<0.001$ ) points to the salience of a grog paste recipe (grog). Third, a substantial number of sherds have voids resulting from the carbonization of relatively fine organic material, often associated with very light grog inclusions (organic). Since these three paste types- 
grit, grog, and organic - were identifiable during macroscopic analysis, they formed an important criterion in sampling sherds for microscopic analyses.

Figure D.10: Distribution of compositional groups by inclusion type

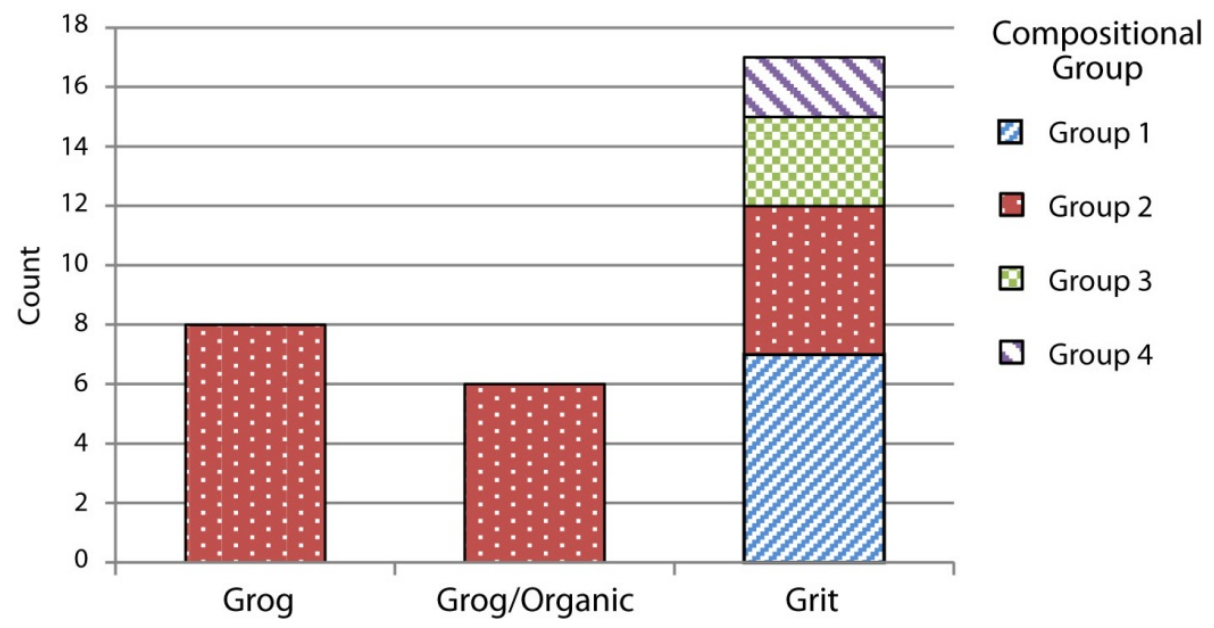

Table D.5: Cross-tabulation of inclusion types in the Survey assemblage

\begin{tabular}{|c|c|c|c|c|c|c|}
\hline & \multicolumn{6}{|c|}{ Grog } \\
\hline & \multirow[b]{2}{*}{ Grit } & \multicolumn{2}{|c|}{ Present } & \multicolumn{2}{|c|}{ Absent } & \multirow[b]{2}{*}{ Totals } \\
\hline & & Present & Absent & Present & Absent & \\
\hline \multirow{3}{*}{ Organic } & Chaff & 10 & 775 & 8 & 149 & 942 \\
\hline & Other & 47 & 2225 & 1137 & 2939 & 6348 \\
\hline & Absent & 107 & 844 & 4210 & 339 & 5500 \\
\hline Totals & & 164 & 3844 & 5355 & 3427 & 12,790 \\
\hline
\end{tabular}

The density of inclusions observed in sherd cross-sections varies within each paste type, with a slight trend for grit and grog temper to be denser than chaff (Figure D.11). Petrographic analysis shows the proportion of inclusions and voids to range from 9-43\%, including those small constituents invisible to the naked eye. Figure D.12 portrays the distinction between sherds tempered with grit and grog, although the latter often have small quantities ( $<10 \%$ ) of feldspathic-quartz grit, possibly from adding grittempered sherds. Bimodality in the relative abundance of grog inclusions corresponds to grog and chaff paste types, while bimodality in the relative abundance of grit inclusions corresponds to a potential subdivision of this type on the basis of density.

Figure D.13 illustrates the broad paste types and possible subdivisions identified through these macro- and microscopic analyses. While it is not yet possible to further classify paste types on the basis of inclusion size and density, some of this variability corresponds to differences in vessel formation and decoration techniques, lending credence to the chronological and functional typologies outlined later in this appendix. 
Figure D.11: Relative distribution of inclusion density in the Survey assemblage

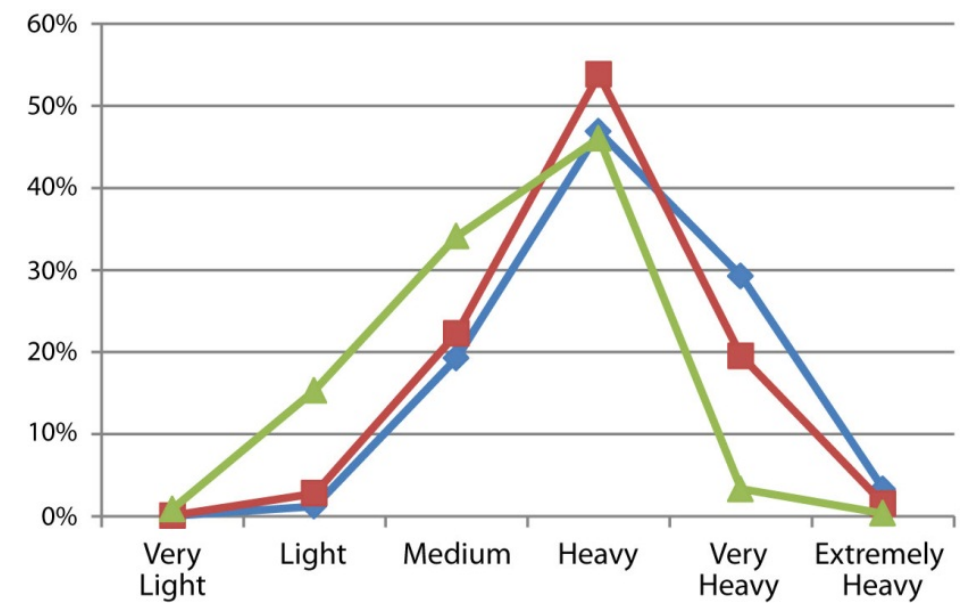

Inclusion Type

$\multimap$ Grit $(n=5499)$

$-5 \operatorname{trog}(n=4000)$

$-\Delta-$ Organic chaff $(n=3393)$

Figure D.12: Ternary diagram of clay/silt matrix, grog, and grit inclusions in the petrographic sherd sample (thin section)

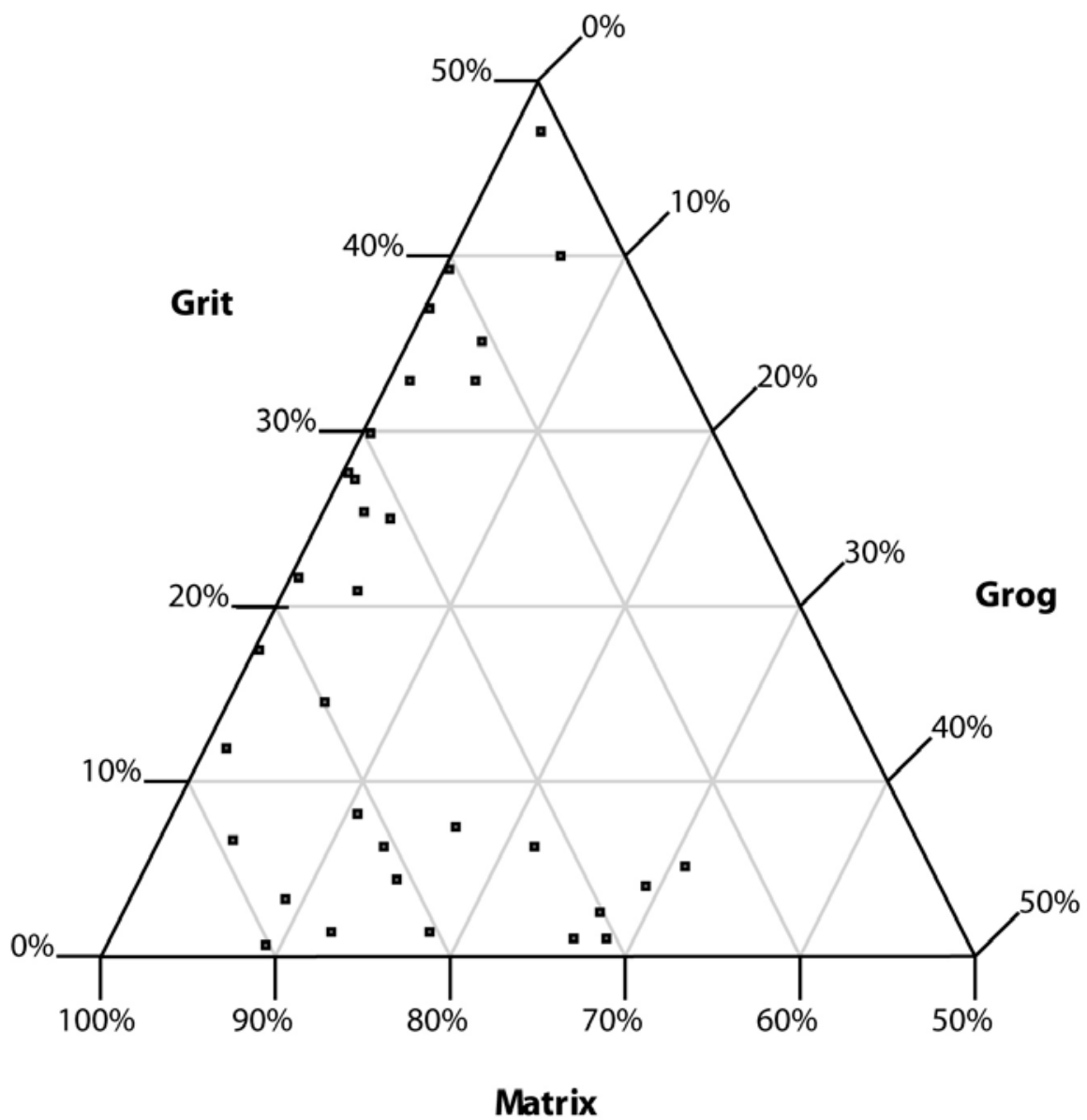


Figure D.13: Photomicrographs (5X) of paste types and possible sub-types in the petrographic sherd sample (plane-polarized light, scale is $500 \mu \mathrm{m}$ )

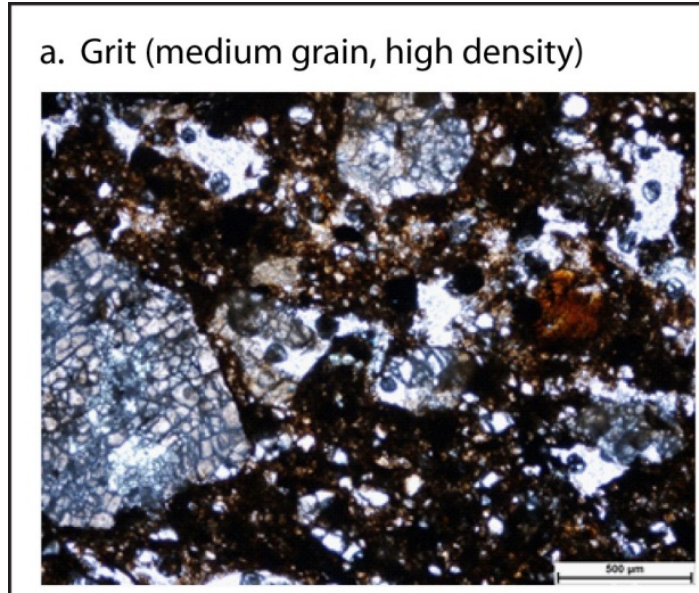

b. Grit (coarse grain, high density)

c. Grit (medium grain, moderate density)
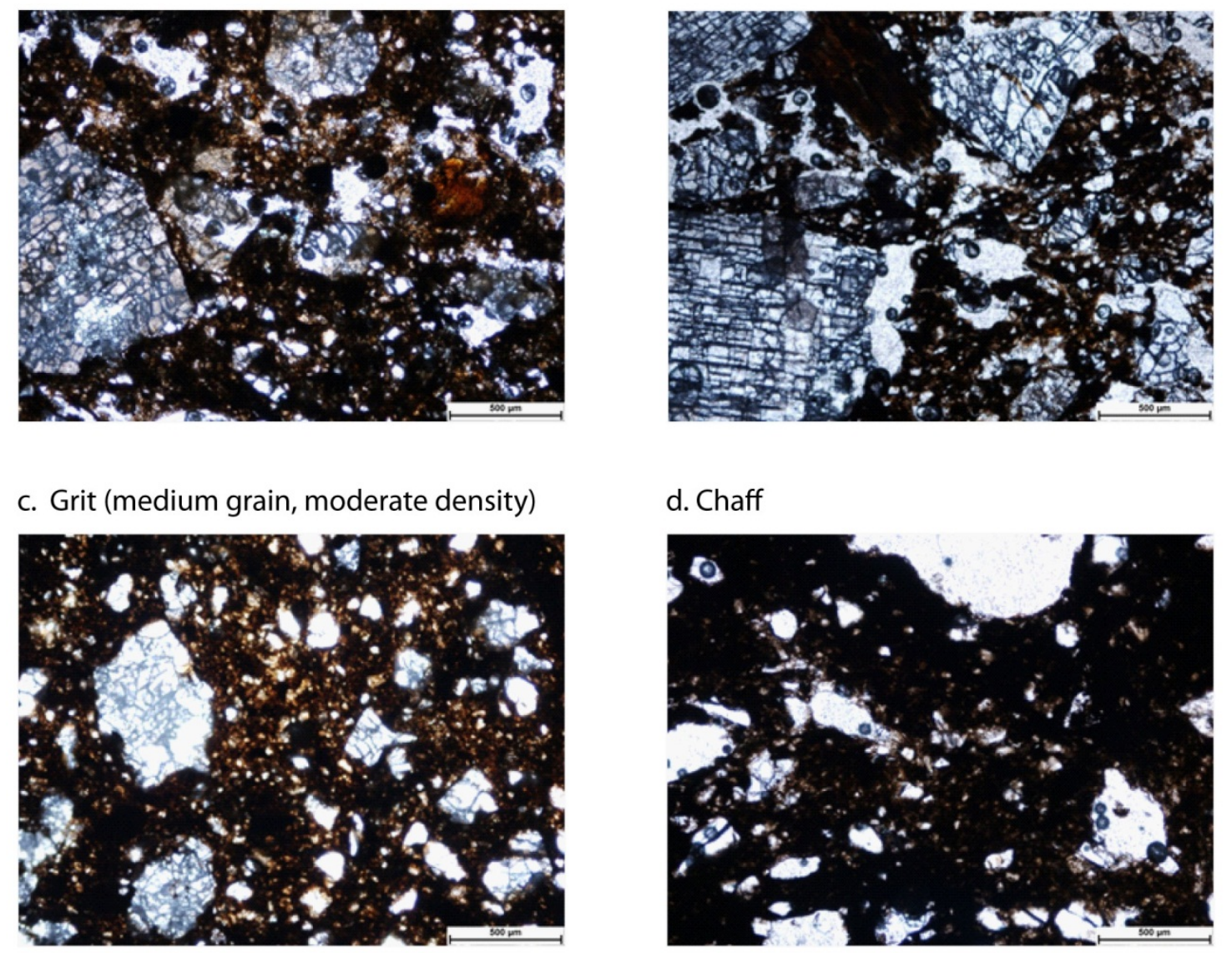

\section{d. Chaff}

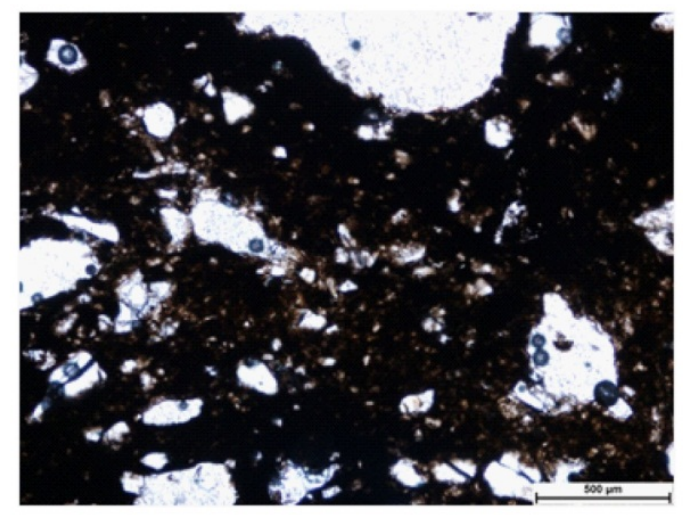

\section{e. Grog}

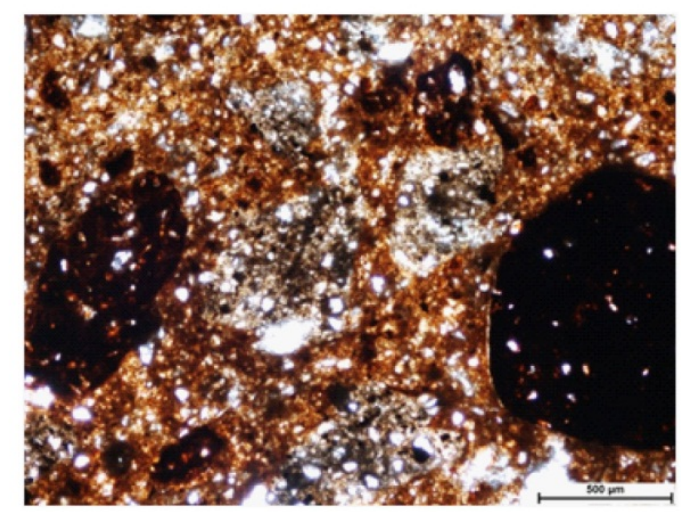

f. Grog and unidentified organic

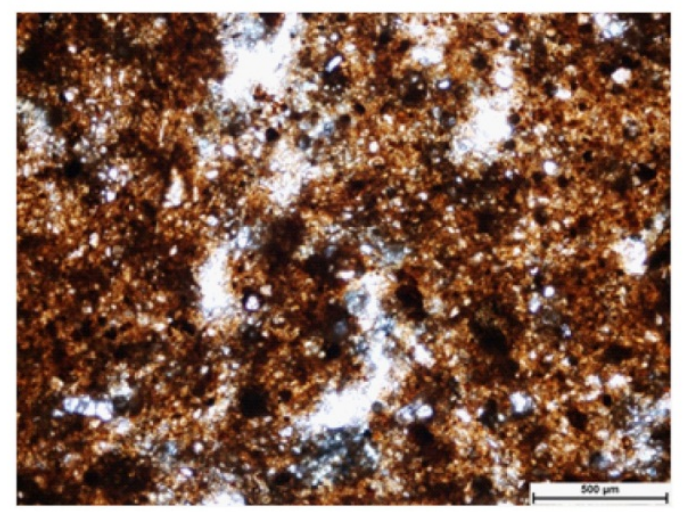




\section{Vessel Shaping}

The techniques employed to fashion a pot depend upon clay properties, desired vessel form, and of course, the repertoire of gestures known to the craftsperson. Ethnoarchaeological studies across Africa have shown that, because these gestures are embodied routines and relatively intangible in finished pots, they are often the most conservative elements within pottery traditions (Dietler and Herbich 1998; Gelbert 1999; Gosselain 2000). For this reason, techniques of vessel formation provide a latent index of social identities such as kinship, caste, and ethnicity in which networks of learning and apprenticeship are most often embedded (Mayor 2010; Roux 2007). This is not, however, to say that vessel shaping techniques never enter discursive consciousness, or that the transmission of technical knowledge is a discrete event. As Olivier Gosselain (2008) has recently emphasized in ethnographic research among Bella potters in Niger, the inculcation of bodily practices goes hand in hand, one might say literally, with what people think about those practices and their potential representations to self and others. Although the widespread interpretation of shaping techniques as cultural tradition has worked to reinforce their inertia among Bella potters, other historical contexts have documented their rapid appropriation-as in the case of base molding and hollowing in northeastern Senegal (Gelbert 1999). It is therefore important to keep in mind that techniques of vessel shaping, while often conservative, are not passively learned and reproduced, but may be an important medium through which craftspeople actively participate in the reproduction of social identities and political economies.

Shaping techniques for each element of a pottery vessel (i.e., base, body, neck, and rim) can, and often do, vary independently of one another within the normative operational sequences observed in ethnographic contexts, or inferred from archaeological ones (e.g., Gallay 1970; Gallay and Huysecom 1994; Gelbert 1999; Gosselain 2000; Guèye 1998; Mayor 2010; Sall 2005). Unfortunately, pottery assemblages from MadinaSadatou yielded few reconstructable vessels, so it was not possible to infer complete production sequences from macroscopic traces-a strategy that has proven fruitful with sufficient samples of complete vessels (e.g., Livingstone Smith and Viseyrias 2010; Mayor et al. 2005; Van Doosselaere 2005). Instead, I proceeded to classify sherds for each major section of a vessel on the basis of morphological differences. Although I attempt here to sort out formal variability relating to intended vessel use (function) and that deriving from divergent technical gestures and norms, both of these factors figure into chronological and functional typologies outlined in the final part of this appendix.

\section{Base Formation}

In West Africa today techniques for forming the base of a pot include drawing (direct pull), coiling, or molding clay over a pre-existing pot of the desired form (Gelbert 1999; Guèye 1998:67-70; Mayor 2010; Mayor et al. 2005; Sall 2005:55-57). Each of these can lead to variation in base thickness or morphology and leave macro-traces in complete vessels, but they are difficult to infer from incomplete ones, particularly where globular vessel shapes complicate any distinction between base and body sherds. In this regard, the very low numbers of unambiguous base sherds both in the Survey $(n=9)$ and Diouboye $(n=62)$ assemblages suggests that round-bottomed vessel forms predominated in the past, just as they do today. For each unambiguous base sherd, I recorded several basic attributes (Table D.6) including the gross morphological form (Figure D.14). 
Table D.6: Formal variables recorded for base sherds

\begin{tabular}{|l|l|l|}
\hline \multicolumn{1}{|c|}{ Variable } & \multicolumn{1}{|c|}{ Attribute (Code) } & \multicolumn{1}{c|}{ Description } \\
\hline \hline \multirow{3}{*}{ Form } & Round (1) & Base is rounded and slightly thickened \\
\cline { 2 - 3 } & Flattened (2) & Base is flat, but has no additional coil \\
\cline { 2 - 3 } & Pedestal, circular (3) & A coil has been added to form a circular pedestal \\
\cline { 2 - 3 } & Pedestal, conical (4) & A coil has been added to form a conical pedestal \\
\cline { 2 - 3 } & Footed (5) & Thick coils suspend the vessel body \\
\hline Diameter & Measured in cm & Maximum diameter of base that would rest on a surface \\
\hline Base Thickness & Measured in mm & Thickness of pedestal or foot, where applicable \\
\hline Base Height & Measured in mm & Height of base, measured on exterior of vessel \\
\hline Body Thickness & Measured in mm & Thickness of vessel wall, where applicable \\
\hline
\end{tabular}

\section{Figure D.14: Base forms}

\section{Round}

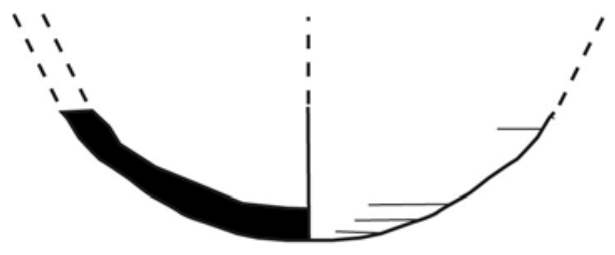

3. Pedestal, circular
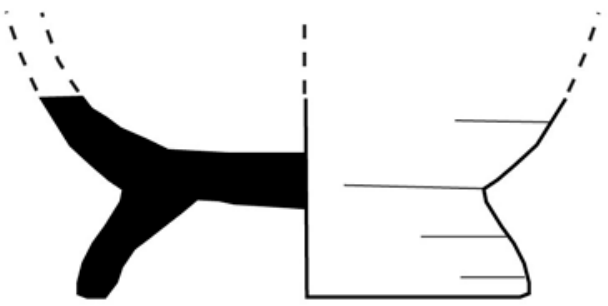

5. Footed

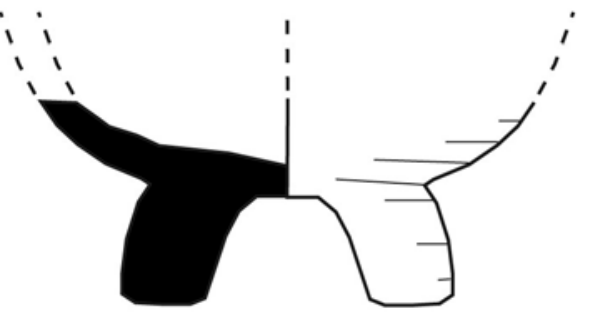

\section{Flattened}

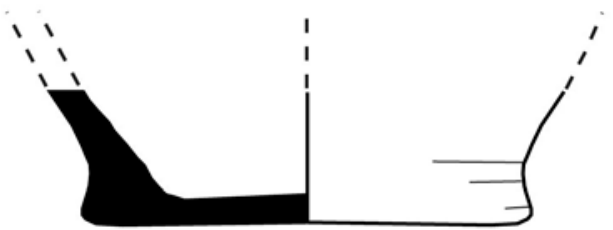

4. Pedestal, conical

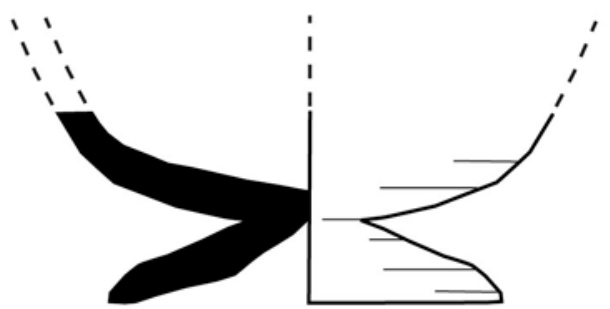




\section{Table D.7: Summary of base forms by assemblage}

\begin{tabular}{|l|c|c|c|c|c|}
\hline \multicolumn{1}{|c|}{ Assemblage } & \multicolumn{5}{c|}{ Base Form } \\
& Round & Flattened & Pestal, \\
circular & $\begin{array}{c}\text { Pedestal, } \\
\text { conical }\end{array}$ & Footed \\
\hline \hline Survey & 2 & 3 & 3 & 1 & \\
\hline Diouboye Excavation & 20 & 4 & 10 & & \\
\hline Diouboye Surface & 4 & 3 & 19 & & 2 \\
\hline \hline Total & $\mathbf{2 6}$ & $\mathbf{1 0}$ & $\mathbf{3 2}$ & $\mathbf{1}$ & $\mathbf{2}$ \\
\hline
\end{tabular}

The high number of round bases excavated at Diouboye (Table D.7) results from the recovery of reconstructable vessels in subsurface contexts. Round bases could have been fashioned by molding over a globular vessel or by drawing a lump of clay directly on a hand wheel (either a wooden or ceramic bowl); this latter technique may also have been responsible for the few flattened bases observed in the assemblages (Sall 2005:6682). The other three base forms required adding one or more clay coils to create a support; contemporary Mande potters undertake this step on rounded bases as they dry upside-down (Frank 1998:83). Such bases can also be manufactured by hollowing a lump, though this method is quite exceptional (Sall 2005:79) Notably, pedestal and footed base forms have a long history in the Upper to Middle Niger region where they occur assemblages dating to the Iron Age (Bedaux 1980; Bedaux and Raimbault 1993).

Modalities in the ratio of diameter to height for pedestal bases in the Diouboye assemblage (Figure D.15) define three general types: short narrow (Type 1); short wide (Type 2); and tall narrow (Type 3).

Figure D.15: Bivariate plot of base diameter by base height for circular pedestal base sherds in the Diouboye assemblage

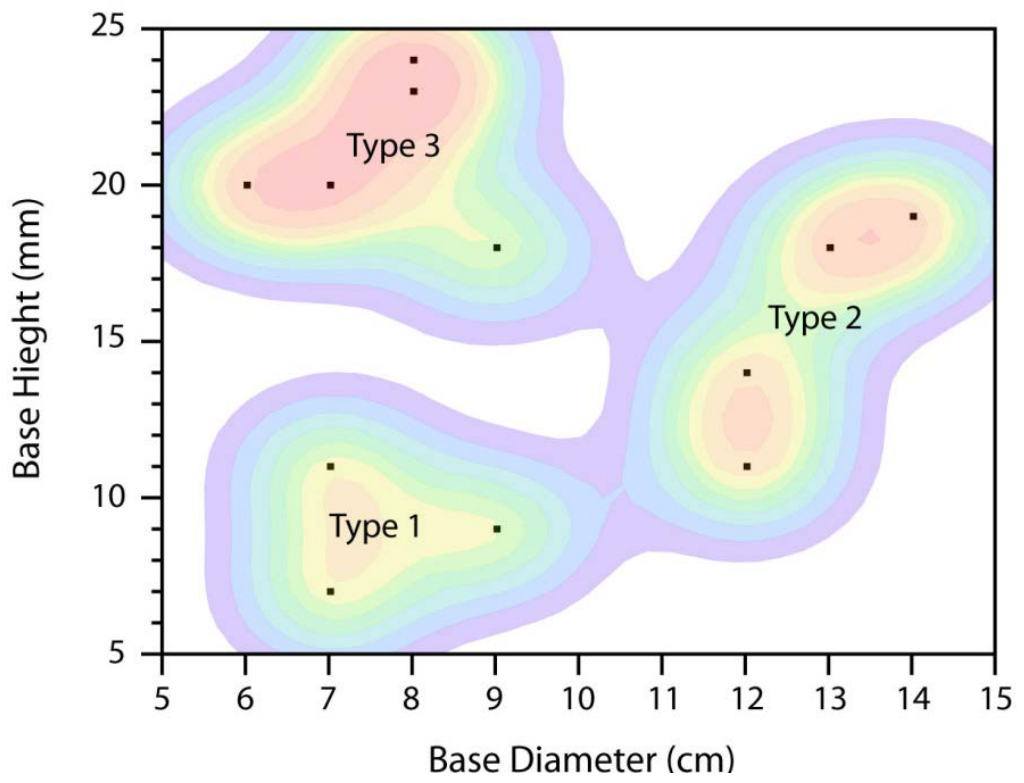




\section{Body Formation}

Ethnographic studies demonstrate a similar repertoire of techniques for vessel base and body formation in West Africa, although the latter need not be a continuation of the former. Broadly speaking, possible body formation techniques include molding, drawing, and several variants of coiling (Gelbert 1999; Guèye 1998:77-78; Sall 2005:60$61)$. One can decipher these techniques from complete vessels from macroscopic traces such as coil seams and breaks, paddle marks, or scrapes (Gelbert 2003, 2005; Huyescom 1994; Livingstone Smith and Viseyrias 2010), but only in rare cases did I note these attributes on the small and/or eroded sherds. As an extremely coarse proxy measure of body formation techniques and intended vessel function, I did, however, record the average body thickness for all possible sherds - though other technical constraints such

Figure D.16: Distribution of body sherd thickness for the Survey assemblage

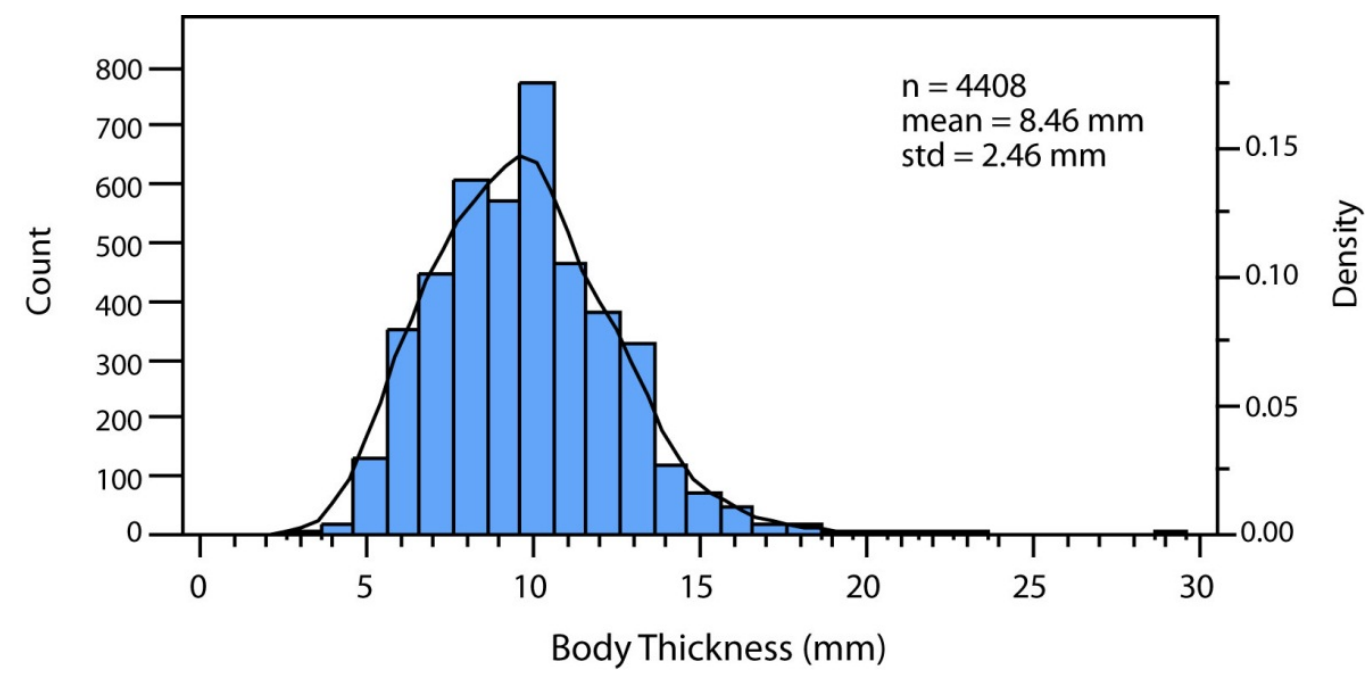

Figure D.17: Distribution of body sherd thickness for the Diouboye assemblage

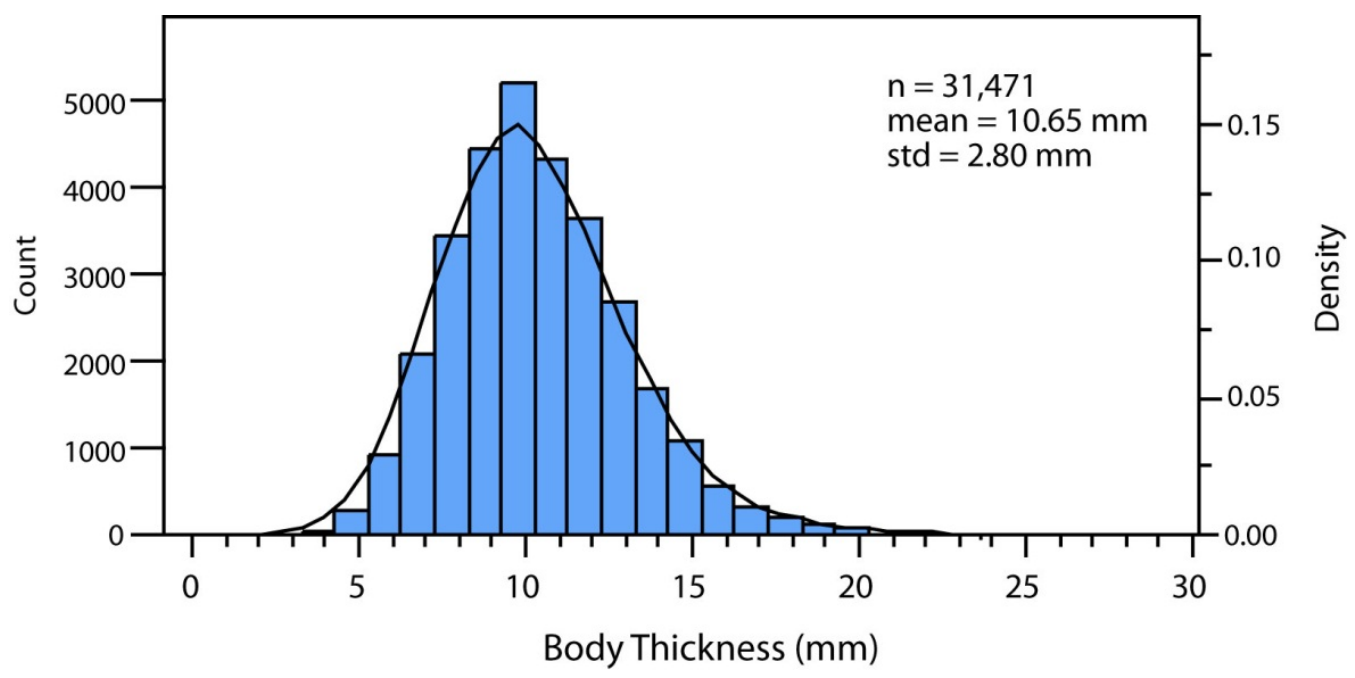


as wet clay strength must be kept in mind (Rice 1987:227-228). Of course, thickness varied within vessels, and even individual sherds, but I assume that significant differences across sherd populations do in fact correspond to some divergent techniques of vessel manufacture.

Although thickness is normally distributed for both the Survey and Diouboye assemblages (Figures D.16-D.17), vessel body thickness does differ significantly between archaeological horizons as discussed below.

\section{Handles}

Additive handles are extremely rare in all assemblages $(n=33)$. In general, it was only possible to identify three types of handle: small loops or straps; short knobs or cones; and long cylinders. Table D.8 provides the counts of these forms by assemblage, but handles do not figure greatly in the further analysis of pottery production sequences.

\section{Table D.8: $\quad$ Summary of handle forms by assemblage}

\begin{tabular}{|l|c|c|c|}
\hline \multicolumn{1}{|c|}{ Assemblage } & \multicolumn{3}{c|}{ Handle Form } \\
& Loop/Strap & Knob/Cone & Cylinder \\
\hline \hline Survey & 7 & 2 & - \\
\hline Diouboye Excavation & 9 & 2 & 2 \\
\hline Diouboye Surface & 7 & 2 & 2 \\
\hline \hline Total & $\mathbf{2 3}$ & $\mathbf{6}$ & $\mathbf{4}$ \\
\hline
\end{tabular}

\section{Pot Lids}

Pot lids identified on the basis of an acute rim angle (see below) are rare in the Survey assemblage $(n=8)$ and in excavation $(n=20)$ and surface $(n=18)$ contexts at Diouboye. Although the paucity of lid fragments may partly reflect the classification of ambiguous specimens as rims, it is plainly the case that ceramic lids were neither produced, nor used, in any great quantity in the Madina-Sadatou study area. The lid fragments from Diouboye display two or three modal diameters (Figure D.18), potentially related to different functional assemblages. 
Figure D.18: Distribution of pot lid diameters for the Diouboye assemblage

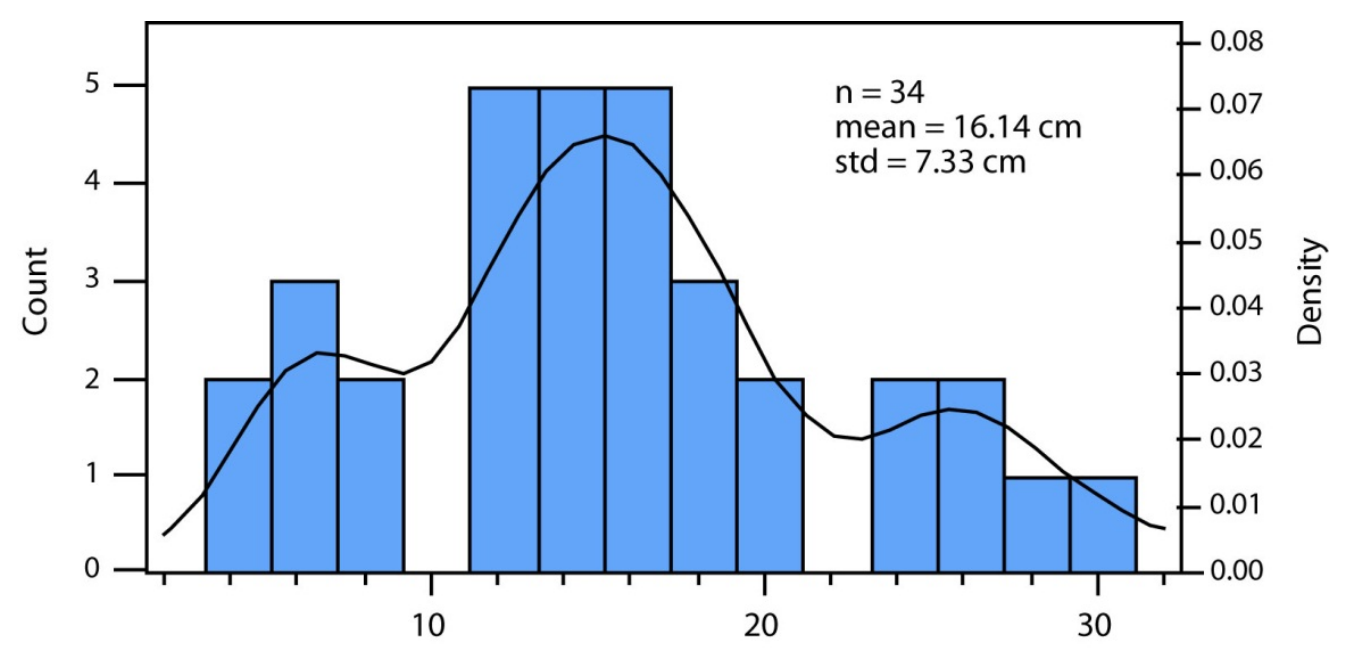

Lid Diameter $(\mathrm{cm})$

\section{Rim Formation}

Since rims comprise the bulk of the diagnostic sherds in the Survey and Diouboye assemblages, much of the analytical work of reconstructing past pottery production sequences and daily use necessarily falls to them. In ethnographic settings, gross formal characteristics such as rim shape and diameter often correspond to the intended function of the vessel, whereas micro-variation may arise from divergent technical choices and gestures - such as drawing the rim up from the vessel body, adding a coil, or folding the vessel body over into a thickened rim (Gelbert 2003; Guèye 1998:32-36; Sall 2005:6687). Starting with the rim types defined for assemblages from the lowermost Falémé (Thiaw 1999:157-161), I considered a suite of morphological variables (Table D.9; Figure D.19) to explore the interplay among logistical constraints (including use), technical knowledge, and aesthetic tastes in the shaping of vessel rims. Using these variables, as well as rim length (rim width divided by rim height), I propose a working classification of rim forms meant to capture these functional and social aspects.

\section{Rim Classification}

The vast majority of rims in the Madina-Sadatou ceramic assemblages fall into one of three general classes - simple, everted, and collared. These classes, employed in archaeological studies across West Africa, provide an important point of interregional comparison even as I define sub-classes and types from smaller scale variation within the data at hand (Figures D.20-D.22). Although classification provides one way to explore the technical choices within the operation stage of vessel shaping, it is important to bear in mind that these broad classes do not constitute static entities; variation within and between them help to interpret socioeconomic patterns and processes and technical traditions, as discussed below. 
Table D.9: Variables recorded for rim sherds

\begin{tabular}{|c|c|c|}
\hline Variable & Attribute (Code) & Description \\
\hline $\begin{array}{l}\text { Rim } \\
\text { Diameter }\end{array}$ & Measured in cm & External diameter of the vessel rim, measured on available arc \\
\hline $\begin{array}{l}\text { Orifice } \\
\text { Diameter }\end{array}$ & Measured in cm & Diameter of the vessel orifice, measured on available arc \\
\hline $\begin{array}{l}\text { Rim } \\
\text { Percentage }\end{array}$ & Percentage (\%) & $\begin{array}{l}\text { Percentage of the total vessel rim represented by the sherd, } \\
\text { estimated from available arc }\end{array}$ \\
\hline \multirow{3}{*}{ Restriction } & Restricted (1) & Orifice is restricted (jar), shoulder angle $>115^{\circ}$ \\
\hline & Semi-restricted (2) & Orifice is lightly restricted (pot), $90^{\circ} \leq$ shoulder angle $\leq 115^{\circ}$ \\
\hline & Unrestricted (3) & Orifice is unrestricted (bowl), shoulder angle $<90^{\circ}$ \\
\hline $\begin{array}{l}\text { Rim } \\
\text { Thickness }\end{array}$ & Measured in mm & $\begin{array}{l}\text { Average thickness of rim, measured midway between the } \\
\text { orifice and actual lip on everted rims }\end{array}$ \\
\hline Rim Angle & Measured in degrees & Angle formed between the planes of vessel lip and the rim \\
\hline $\begin{array}{c}\text { Shoulder } \\
\text { Angle }\end{array}$ & Measured in degrees & $\begin{array}{l}\text { Angle formed between the planes of the vessel orifice and the } \\
\text { shoulder } 3 \mathrm{~cm} \text { below the orifice }\end{array}$ \\
\hline Rim Width & Measured in mm & $\begin{array}{l}\text { Distance between vessel lip and the internal inflection point of } \\
\text { the orifice, measured on the plane of the lip }\end{array}$ \\
\hline Rim Height & Measured in mm & $\begin{array}{l}\text { Distance between vessel lip and the internal inflection point of } \\
\text { the orifice, measured perpendicular to the plane of the lip }\end{array}$ \\
\hline \multirow{3}{*}{$\begin{array}{l}\text { Inflected } \\
\text { Rim Shape }\end{array}$} & 1. Rounded & Interior inflection point forms a rounded "C" shape \\
\hline & 2. Angular & Interior inflection point forms an angular " $\mathrm{L}$ " shape \\
\hline & 3. Thick & $\begin{array}{l}\text { Interior inflection point forms a "swoosh" shape thickened in } \\
\text { relation to the rim and body }\end{array}$ \\
\hline $\begin{array}{l}\text { Body } \\
\text { Thickness }\end{array}$ & Measured in mm & Thickness of vessel wall $3 \mathrm{~cm}$ below orifice \\
\hline Bevel & Present/Absent & Presence or absence of beveling on lip \\
\hline
\end{tabular}

Figure D.19: Formal variables measured on rim sherds

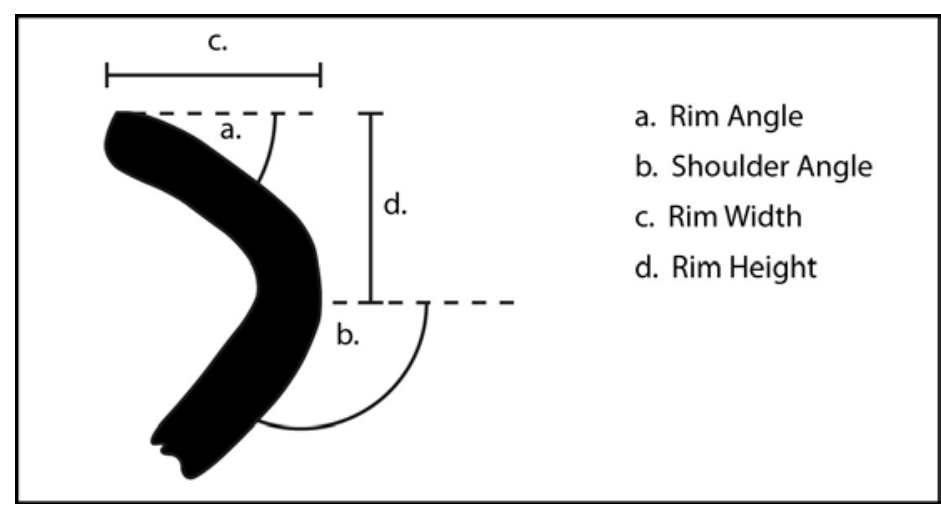


Figure D.20: Rim form classes and subclasses

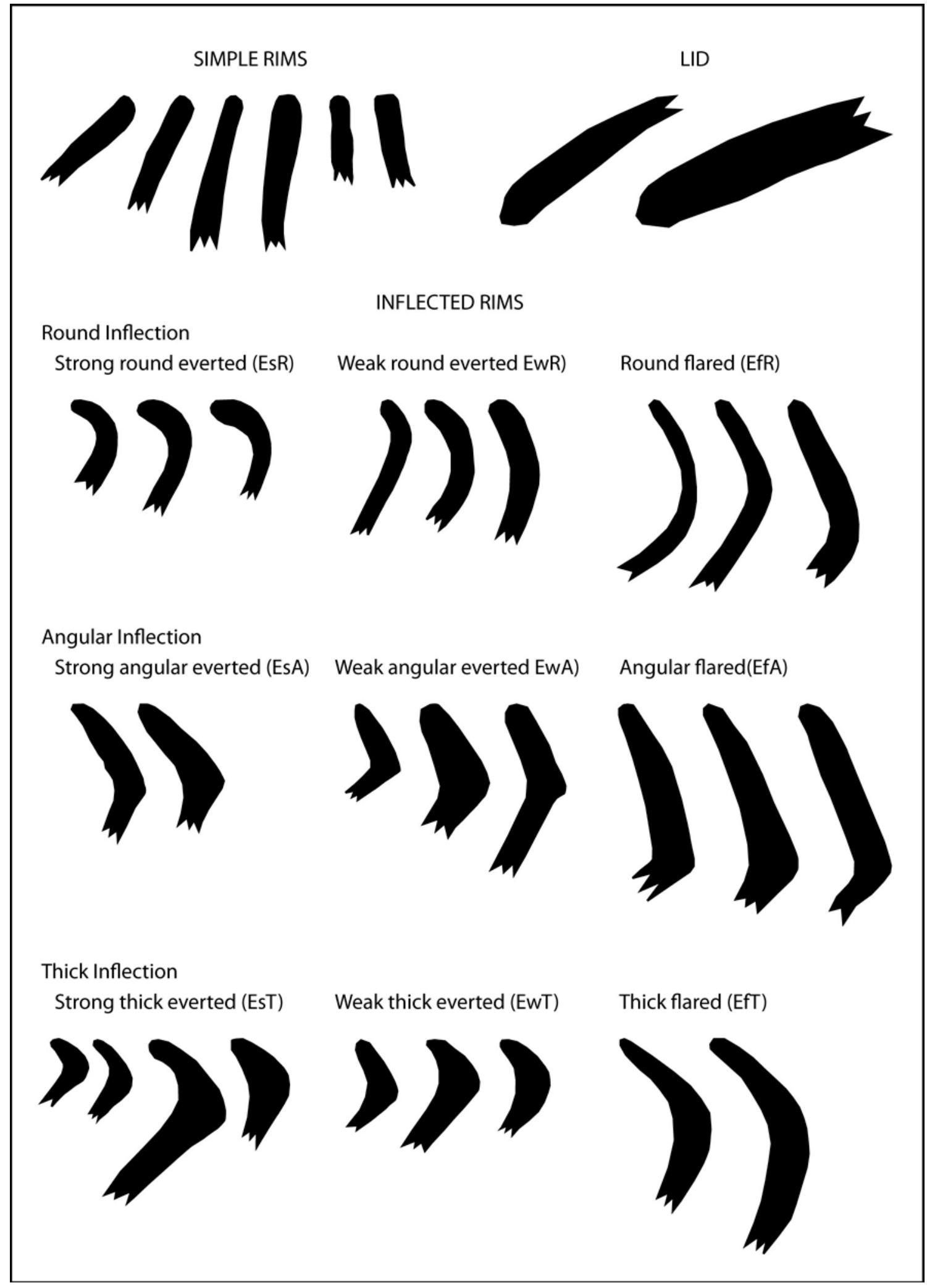


Figure D.20: Rim form classes and subclasses, continued

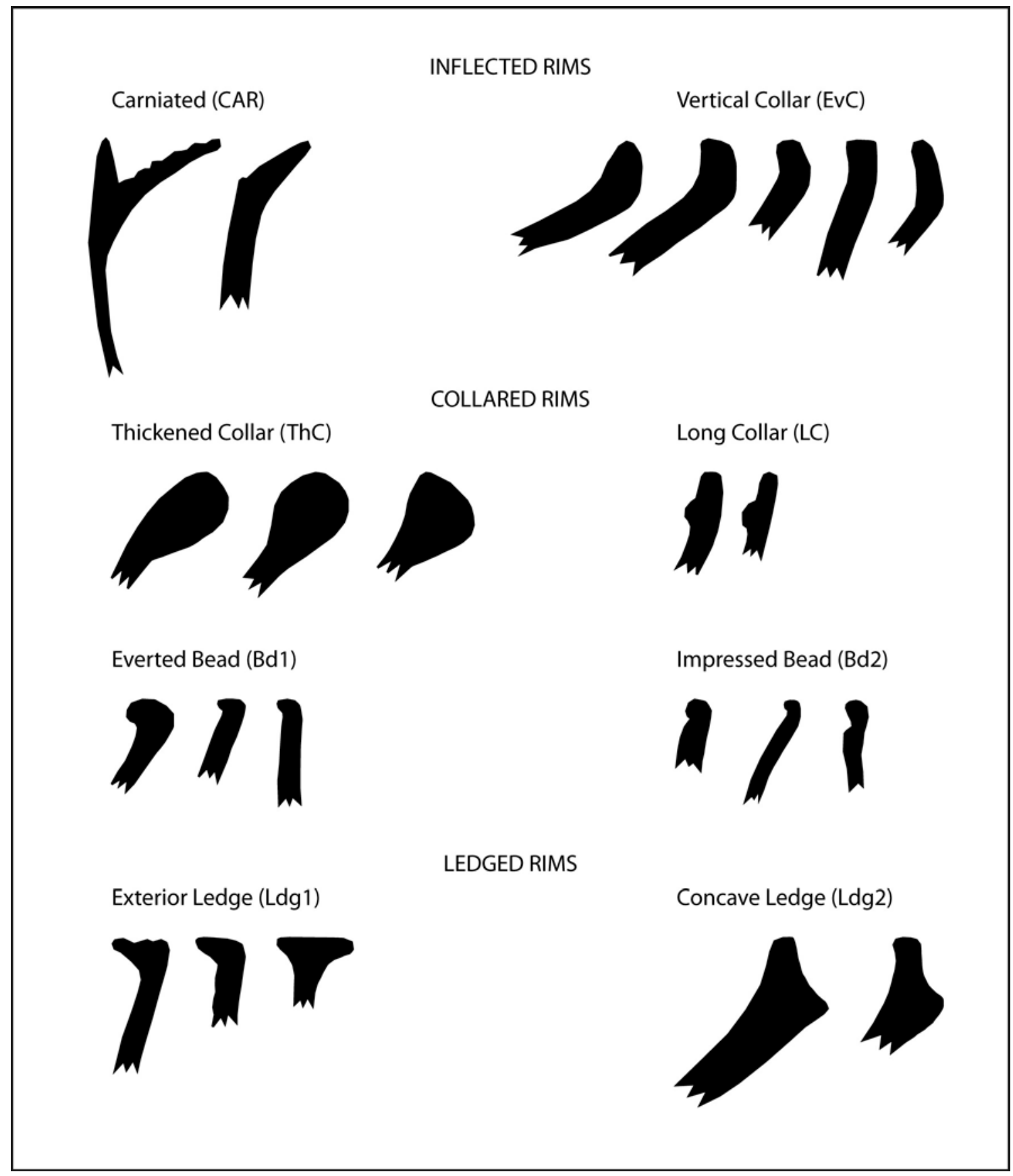

\section{$\underline{\text { Simple Rims }}$}

Simple rims mark the termination of the vessel body at the orifice with no discernible inflection or additional element. Rims of this class occur frequently in both the Survey $(n=135)$ and the excavated Diouboye assemblage $(n=351)$ where they are often beveled, channeled, tapered, or contoured slightly upwards (Figure D.20). To avoid misidentifying everted rims sherds broken above their inflection point, I only classified 
unambiguously simple rims as such. This may contribute to the low numbers of unrestricted bowl forms and pot lids in the final analysis.

\section{Inflected Rims}

Inflected rims have an outward or upward inflection of the vessel body created by either vertical scraping or adding an additional clay coil to the vessel shoulder (Figure D.20); they are the predominant rim class in both the Survey $(n=509)$ and the excavated Diouboye assemblage ( $\mathrm{n}=1037)$.

A visual sorting of drawn rim profiles and exploratory data analysis pointed to rim angle, relative rim length (length/thickness), and the shape of the internal inflection as the most salient dimensions of technical variability (see also Bocoum and McIntosh 2002:72-73; Thiaw 1999:158). In order to define subclasses, I first employed K-means cluster analyses to sort the everted rims in the Diouboye assemblage into flared, everted, and vertical collar rims, and those of the Survey assemblage into flared, weakly everted, sharply everted, and vertical collar rims. I then extrapolated the K-means clusters into paradigmatic subclasses (Figures D.21-D.22) useful for typing incomplete rims and those from surface contexts at Diouboye. Finally, I divided each of the everted and flared rim sherd subclasses within each assemblage according to the shape of the internal inflection (i.e., rounded, angular, thickened) to give the following classificatory scheme.

Vertical collar (EvC) rims have a rim angle of $80-100^{\circ}$ and a rim length/thickness ratio less than 3.5. This type appears as a short collar made by inflecting the vessel shoulder, rather than adding a coil or channel as for other collared rims (below).

Round everted (ErR) rims have a rim angle of $40-80^{\circ}$ and a rim length/thickness ratio in the vicinity of 1.5-4.0. In profile, the interior inflection point is rounded with rim thickness nearly the same as, or slightly less than, body thickness. Although the association of this subclass with a specific shaping technique must remain tentative, pending an analysis of macrotraces, contemporary potters in the Upper Senegal region make similar rim forms by squeezing clay coils onto the exterior of the vessel shoulder, then scraping horizontally with a wooden spatula or shell (Gelbert 2003; see also Sall 2005:66-82). Weak round everted (EwR) rims with a rim angle greater than or equal to $60^{\circ}$ and strong round everted (EsR) rims with a rim angle less than $60^{\circ}$ are two further sub-types distinguished in the Survey assemblage. Round flared (EfR) rims have a rim angle of $40-80^{\circ}$ and a rim length/thickness ratio greater than 3.5.

Angular everted (ErA) rims have a metric range similar to round everted rims. In profile, however, the interior inflection point displays a distinctive "L" shape with rim thickness nearly the same as, or slightly less than, body thickness. Contemporary potters in the Upper Senegal region make such rim forms by attaching clay coils to the vessel shoulder, then scraping upwards with a fragment of calabash (Gelbert 2003; see also Sall 2005:66-82). Again, I hesitate to associate angular everted rims with a specific shaping technique without a definitive study of macrotraces, but these do appear to represent a different suite of technical choices and gestures than other everted rim forms. For the Survey assemblage, weak angular everted (EwA) and strong angular everted (EsA) rims could be divided at a rim angle of $60^{\circ}$. Meanwhile, angular flared (EfA) rims have a rim angle of $40-80^{\circ}$ with a rim length/thickness ratio greater than 3.5. 
Figure D.21: Bivariate plot of rim length/thickness and rim angle for inflected rims in the Survey assemblage $(n=328)$

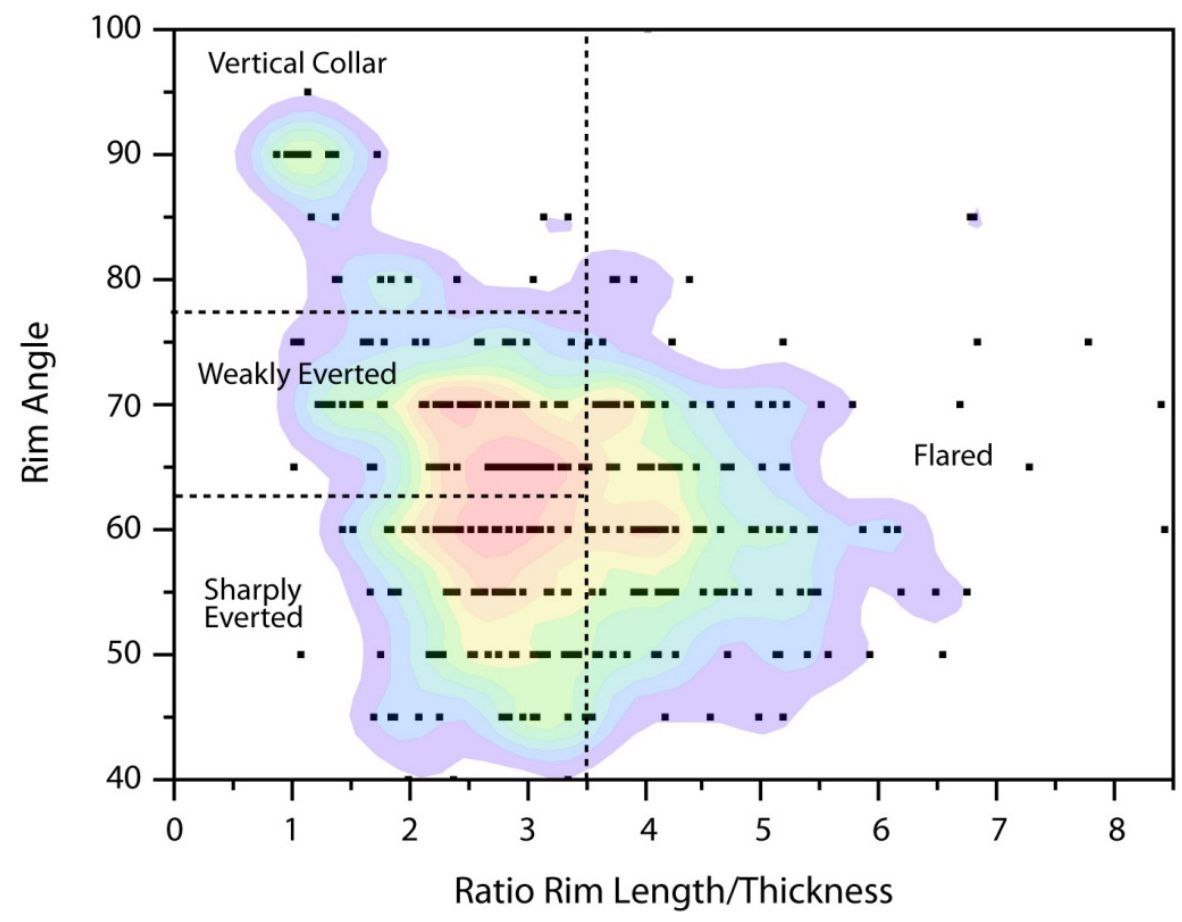

Figure D.22: Bivariate plot of rim length/thickness and rim angle for inflected rims in the excavated Diouboye assemblage $(n=632)$

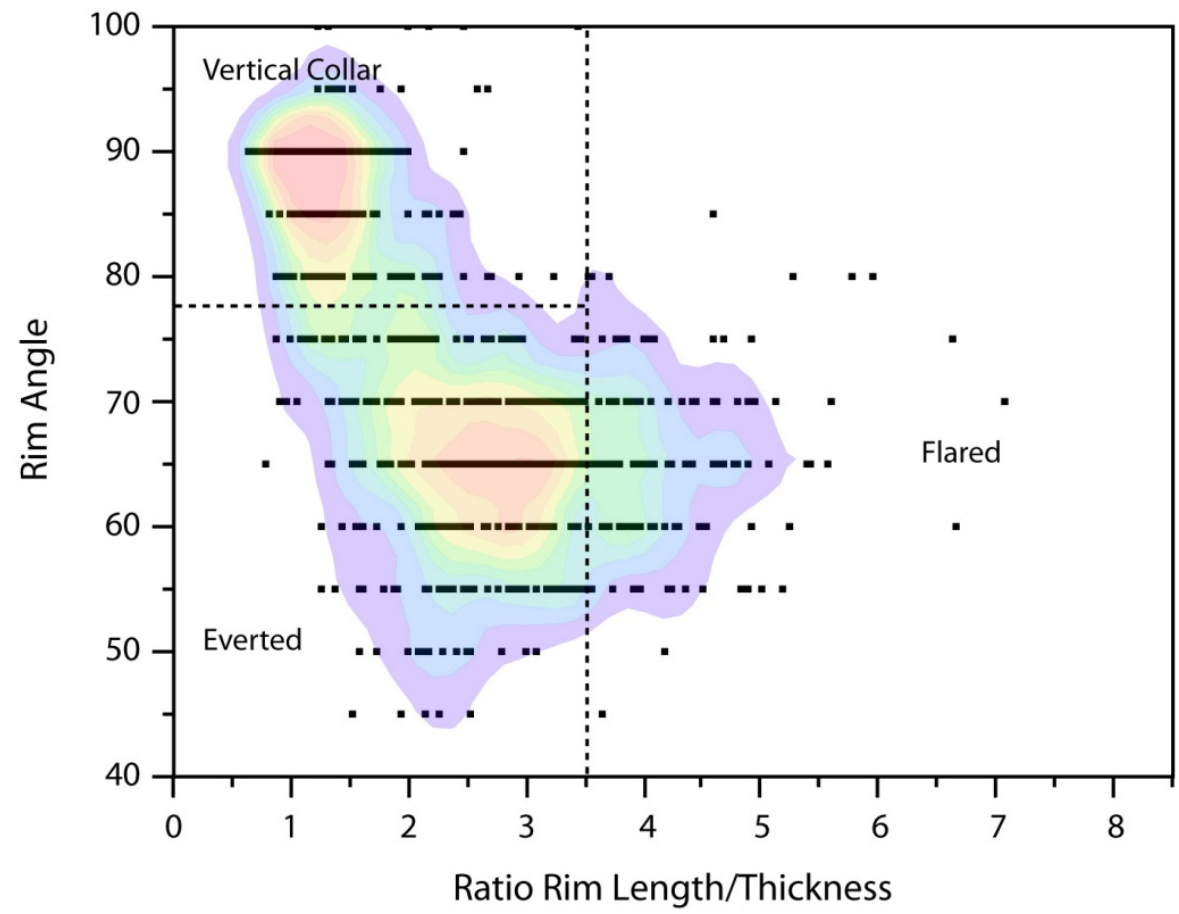


Thick everted (Et) rims have a metric range matching the other everted rim subclasses, but they exhibit a thickened inflection point tapering toward the rim and body. Although this rim form occurs in LSA contexts elsewhere across West Africa (e.g., Deme and McIntosh 2006:331; MacDonald 2011:61-62), it has no ethnographic parallels in Senegambia. However, I would hypothesize that potters added a coil of clay to the vessel orifice and gently drew outward to form a tapered eversion. As above, weak thick everted (EwT) and strong thick everted (EsT) rims could be divided at a rim angle of $60^{\circ}$. Thick flared (EfT) rims have a rim angle of $40-80^{\circ}$ with a rim length/thickness ratio greater than 3.5.

Carinated (CAR) rims, not shown in Figures D.20 and D.21, are inflected inwards with a rim angle in excess of $125^{\circ}$; these were not found at Diouboye, and only rarely on surveyed sites.

\section{Collared and Ledged Rims}

Collared and ledged rims encompass a greater range of variability than either simple or inflected rims, marking clear points of similarity and difference with pottery traditions elsewhere across the Middle and Upper Senegal regions (Bocoum and McIntosh 2002:72-73; Thiaw 1999:159-161), the Middle and Upper Niger regions (MacDonald 1997:184; S. K. McIntosh 1995:148-150; Togola 2008:57-58), and Gambia River basin (A. Lawson 2003:235-263). In contrast to inflected collar rims (see above), these rims resulted from additive or subtractive techniques of rim shaping. In total, collared and ledged rims accounted for a minor proportion of both the Survey $(n=57)$ and excavated Diouboye $(n=107)$ assemblages.

Thickened collar (ThC) rims have substantially thickened lip, but no eversion. The appearance of a long asymmetrical relief on the interior of suggests that potters made these rims by simply adding an additional clay coil around the vessel opening.

Long collar (LC) rims are otherwise simple rims with a thin coil added to form a cordon within two centimeters of the lip. Although these rims are found on sites in the Middle Senegal region and at Arondo (Thiaw 1999:160-161), they are extremely rare in assemblages from the Madina-Sadatou study area.

Everted bead (Bd1) rims have a slightly everted lip, possibly induced by flattening and smoothing the rim with either the fingers or a spatula. These differ from everted collar rims in that the lip bead is less than the thickness of the vessel body (rim length/thickness ratio $<1$ ).

Impressed bead (Bd2) rims have a beaded lip formed by an exterior groove or incision within two centimeters of the rim.

Exterior ledge (Ldg1) rims are similar to the everted rims described above, but they have a rim angle nearly flush with the plane of the orifice $\left(<30^{\circ}\right)$. This type also subsumes "T-shape" rims with a ledge on both the interior and exterior of the vessel orifice - a type whose scarcity in the Madina-Sadatou assemblages contrasts with their presence in the Upper and Middle Niger regions (MacDonald 1997:184; S. K. McIntosh 1995:150-151).

Concave Ledge (Ldg2) rims have a nearly vertical lip, presumably applied as a separate clay coil, offset by an interior concave ledge sufficient for supporting a lid. 


\section{Surface Decoration}

Decoration includes all those techniques that enhance or modify the appearance of the vessel subsequent to primary shaping. Since decorative techniques are usually consciously malleable, but leave a durable and visible imprint on the finished vessel that stands to frame social interactions at multiple scales, they often represent choices informed by both discursive and practical knowledge (Bowser 2000; David et al. 1988; Gosselain 2000, 2010, 2011; Hegmon and Kulow 2005; Hodder 1982). Ethnographic potters are usually aware of the decorative repertoire employed by their neighbors, but the conscious borrowing (or rejection) of these techniques occurs, by and large, in contexts of extensive contact such as the migration of a potter into a new community or prolonged inter-ethnic relations, often to suit the interests of consumers (Dietler and Herbich 1989; Gelbert 1999; Gosselain 2008; Guèye 2011). Here it is important to note that the complex carved and fiber roulettes prevalent in African pottery traditions may represent a mode of technical knowledge more akin to that exercised in vessel shaping. And seemingly decorative techniques may, of course, arise from mundane considerations of reducing the permeability of an earthenware vessel or enhancing surface texture to afford better gripping. Whatever the case, surface decoration techniques stand to document both long-term continuity or rupture in cultural traditions, as well as participation in nested spheres of economic and sociopolitical interaction.

I classified decorative techniques along three dimensions. Based on the overall form of alteration, I first distinguished between three general decoration types: surface treatment, additive elements, and impressed/subtractive elements. Second, I determined the tool used for subtractive decorative techniques (i.e., stylus, comb, carved or fiber roulette). For roulettes, identification was aided greatly by reference to the illustrated volume African Pottery Roulettes Past and Present (Haour et al. 2010; also Hurley 1979; Soper 1985), although some identifications remain tentative. Third, I described the specific gesture for applying the tool to the vessel surface (i.e., roulette, impression, incision). Since potters can employ techniques in combination, either on different parts of the same vessel or in compound motifs, I recorded attributes for the type, quantity, location (interior, exterior), and direction (including overlap) of decorative techniques on each sherd (Table D.10); I also observed whether or not a sherd was heavily eroded. For rim sherds, I noted techniques of lip decoration and the uppermost point of exterior decoration (Table D.11).

In order to aid comparison or possible reinterpretation, I describe each of the techniques identified in the Madina-Sadatou ceramic assemblages with reference to similar ethnographic and archaeological examples from surrounding regions. 
Table D.10: Variables recorded for surface decoration

\begin{tabular}{|c|c|c|}
\hline Variable & Attribute (Code) & Description \\
\hline \multirow{2}{*}{ Eroded } & Non-eroded $(0)$ & Sherd is not significantly eroded, decoration visible \\
\hline & Eroded (1) & Sherd is significantly eroded, decoration not visible \\
\hline \multirow{7}{*}{$\begin{array}{l}\text { Interior } \\
\text { Decoration }\end{array}$} & Undecorated $(0)$ & No decoration \\
\hline & Twine impression (1) & Impressions of a twine roulette \\
\hline & Single incision (2) & A single incision runs the circumference of the lip interior \\
\hline & Multi-incision/Comb (3) & Multiple incisions run the circumference of the lip interior \\
\hline & Button impression (4) & Impressions of a carved button roulette \\
\hline & Finger impression (5) & Impressions of fingertips \\
\hline & Other/Unknown (9) & Described on a sherd by sherd basis \\
\hline \multirow{6}{*}{$\begin{array}{l}\text { Surface } \\
\text { Treatment }\end{array}$} & Untreated (0) & No surface treatment \\
\hline & Slip (1) & A reddish slip on the vessel surface, usually polished \\
\hline & Polish/Burnish (2) & Vessel surface polished, but without slip \\
\hline & Organic coat (3) & Shiny black polish on the vessel surface \\
\hline & Soot (4) & Gritty black residue on the vessel surface \\
\hline & Glaze (5) & Glassy coating on the vessel surface \\
\hline \multirow{10}{*}{$\begin{array}{l}\text { Lip } \\
\text { Decoration } \\
\text { (Rim Only) }\end{array}$} & Undecorated (0) & No decoration present on the vessel lip \\
\hline & Button impression (1) & Impressions of a carved button roulette \\
\hline & Single incision (2) & A single incision runs the circumference of the vessel lip \\
\hline & Multi-incision/Comb (3) & Multiple incisions run the circumference of the vessel lip \\
\hline & $\begin{array}{l}\text { Vertical } \\
\text { incision/Comb (4) }\end{array}$ & $\begin{array}{l}\text { Multiple incisions run from the interior to the exterior of } \\
\text { the vessel lip }\end{array}$ \\
\hline & Circular punctate (5) & Circular impressions run the circumference of the vessel lip \\
\hline & Finger punctate (6) & Finger impressions run the circumference of the vessel lip \\
\hline & Bevel with punctate (7) & Rim flattened with circular impressions \\
\hline & Bevel (8) & Rim flattened with hard stick or spatula \\
\hline & Other/Unknown (9) & Described on a sherd by sherd basis \\
\hline \multirow{4}{*}{$\begin{array}{l}\text { Top Exterior } \\
\text { Decoration } \\
\text { (Rim Only) }\end{array}$} & Unknown $(0)$ & Uppermost point of exterior design cannot be determined \\
\hline & Lip (1) & Decorative elements begin at lip \\
\hline & Neck (2) & Decorative elements begin on neck \\
\hline & Shoulder (3) & Decorative elements begin on shoulder \\
\hline \multirow{7}{*}{$\begin{array}{l}\text { Exterior } \\
\text { Decoration } \\
\text { Direction }\end{array}$} & Horizontal (0) & Decorative elements applied horizontally \\
\hline & Vertical (1) & Decorative elements applied vertically \\
\hline & Oblique (2) & Decorative elements applied obliquely \\
\hline & Multi-directional (3) & Decorative elements applied in multiple directions \\
\hline & Curvilinear (4) & Decorative elements applied in curvilinear fashion \\
\hline & Crosshatch (5) & Decorative elements applied in overlapping fashion \\
\hline & Ziz-Zag (6) & Exterior decorative elements applied in sinuous fashion \\
\hline
\end{tabular}


Table D.10: Variables recorded for surface decoration, continued

\begin{tabular}{|c|c|c|}
\hline Variable & Attribute (Code) & Description \\
\hline \multirow{4}{*}{$\begin{array}{l}\text { Exterior } \\
\text { Decoration } \\
\text { Complexity }\end{array}$} & No decoration (0) & No decoration present (excluding surface treatment) \\
\hline & Single element (1) & One decorative element present (excl. surface treatment) \\
\hline & Double element (2) & Two decorative element present (excl. surface treatment) \\
\hline & Multiple elements (3) & $\begin{array}{l}\text { Three or four decorative elements present (excl. surface } \\
\text { treatment) }\end{array}$ \\
\hline \multirow{10}{*}{$\begin{array}{l}\text { Lip } \\
\text { Decoration } \\
\text { (Rim Only) }\end{array}$} & Undecorated (0) & No decoration present on the vessel lip \\
\hline & Button impression (1) & Impressions of a carved button roulette \\
\hline & Single incision (2) & A single incision runs the circumference of the vessel lip \\
\hline & Multi-incision/Comb (3) & Multiple incisions run the circumference of the vessel lip \\
\hline & $\begin{array}{l}\text { Vertical } \\
\text { incision/Comb (4) }\end{array}$ & $\begin{array}{l}\text { Multiple incisions run from the interior to the exterior of } \\
\text { the vessel lip }\end{array}$ \\
\hline & Circular punctate (5) & Circular impressions run the circumference of the vessel lip \\
\hline & Finger punctate (6) & Finger impressions run the circumference of the vessel lip \\
\hline & Bevel with punctate (7) & Rim flattened with circular impressions \\
\hline & Bevel (8) & Rim flattened with hard stick or spatula \\
\hline & Other/Unknown (9) & Described on a sherd by sherd basis \\
\hline \multirow{4}{*}{$\begin{array}{r}\text { Top Exterior } \\
\text { Decoration } \\
\text { (Rim Only) }\end{array}$} & Unknown (0) & Uppermost point of exterior design cannot be determined \\
\hline & Lip (1) & Decorative elements begin at lip \\
\hline & Neck (2) & Decorative elements begin on neck \\
\hline & Shoulder (3) & Decorative elements begin on shoulder \\
\hline \multirow{8}{*}{$\begin{array}{l}\text { Exterior } \\
\text { Decoration } \\
\text { Direction }\end{array}$} & Horizontal (0) & Decorative elements applied horizontally \\
\hline & Vertical (1) & Decorative elements applied vertically \\
\hline & Oblique (2) & Decorative elements applied obliquely \\
\hline & Multi-directional (3) & Decorative elements applied in multiple directions \\
\hline & Curvilinear (4) & Decorative elements applied in curvilinear fashion \\
\hline & Crosshatch (5) & Decorative elements applied in overlapping fashion \\
\hline & Ziz-Zag (6) & Exterior decorative elements applied in sinuous fashion \\
\hline & Other/Unknown (9) & Described on a sherd by sherd basis \\
\hline \multirow{4}{*}{$\begin{array}{l}\text { Exterior } \\
\text { Decoration } \\
\text { Complexity }\end{array}$} & No decoration (0) & No decoration present (excluding surface treatment) \\
\hline & Single element (1) & One decorative element present (excl. surface treatment) \\
\hline & Double element (2) & Two decorative element present (excl. surface treatment) \\
\hline & Multiple elements (3) & $\begin{array}{l}\text { Three or four decorative elements present (excl. surface } \\
\text { treatment) }\end{array}$ \\
\hline
\end{tabular}


Table D.10: Variables recorded for surface decoration, continued

\begin{tabular}{|c|c|}
\hline Decorative Elements & For descriptions, see below \\
\hline \multirow{3}{*}{ Additive (ADD) } & Plain cordon (Add1) \\
\hline & Baton-impressed cordon (Add2); Cordon with punctate (Add3) \\
\hline & Buttons or Knobs (Add4) \\
\hline \multirow{3}{*}{$\begin{array}{l}\text { Twisted Cord } \\
\text { Roulette (TCR) }\end{array}$} & Counter-twisted cord roulette (TwC) \\
\hline & Simple-twisted cord roulette (TwS) \\
\hline & Double-twisted cord roulette (TwD) \\
\hline \multirow{3}{*}{$\begin{array}{l}\text { Knotted Cord } \\
\text { Roulette (KCR) }\end{array}$} & Braided cord roulette: Single (BrS); Double (BrD) \\
\hline & Folded strip roulette (StF) \\
\hline & Knotted strip roulette (StK) \\
\hline \multirow{2}{*}{$\begin{array}{l}\text { Composite } \\
\text { Roulette (CWR) }\end{array}$} & Cord-wrapped stick roulette: Spaced (CwS1); Contiguous (CwS2) \\
\hline & Knot in cord roulette (CwK) \\
\hline $\begin{array}{l}\text { Natural } \\
\text { Roulette (NRT) }\end{array}$ & Shorn grass roulette: Fine (Nr1); Coarse (Nr2) \\
\hline \multirow{7}{*}{$\begin{array}{l}\text { Carved } \\
\text { Roulette (CRT) }\end{array}$} & Chevron carved roulette (CrC) \\
\hline & Dentate carved roulette (CrD) \\
\hline & Geometric carved roulette $(\mathrm{CrG})$ \\
\hline & Lattice carved roulette (CrT) \\
\hline & Oblique carved roulette (CrO) \\
\hline & Ladder carved roulette (CrL) \\
\hline & Button carved roulette (CrB1); Bead carved roulette (CrB2) \\
\hline \multirow{4}{*}{ Incision (INC) } & Round incision: Single (Inc1); Multiple (Inc2) \\
\hline & Sharp incision: Single (Inc3); Multiple (Inc4) \\
\hline & Comb: Round (CmbR); Sharp (CmbS); Channel (Chnl) \\
\hline & Finger drag (CmbF) \\
\hline \multirow{4}{*}{$\begin{array}{l}\text { Cord } \\
\text { Impression (IMP) }\end{array}$} & Knotted-cord impression (ImK) \\
\hline & Twisted-cord impression (ImT) \\
\hline & Cord-wrapped cord impression (ImC) \\
\hline & Cord-wrapped stick impression (ImS) \\
\hline \multirow{7}{*}{ Punctate (PNC) } & Ring punctate (PnR) \\
\hline & Circular punctate (PnC) \\
\hline & Cuneiform punctate (PnN) \\
\hline & Denticulate punctate $(\mathrm{PnD})$ \\
\hline & Baton impression (ImB) \\
\hline & Comb impression (ImM) \\
\hline & Finger impression (ImF) \\
\hline
\end{tabular}




\section{Surface Treatment}

Potters often finish their vessels by polishing the surface while leather-hard and/or applying a solution of clay slip or some organic compound prior to firing (Figure D.23).

Polish (P) is a lustrous surface made by rubbing a smooth, hard object across the vessel once it has become leather hard (Rice 1987:138). While some sherds display linear facets from burnish, these have been categorized as polish for analysis.

Slip (S) is a thin coating of clay suspended in water and applied to a vessel surface to alter its color, texture, or impermeability (Rice 1987:149-152). In the MadinaSadatou assemblages, slip fired to bright red to black adorned the upper shoulder and lips of many vessels; the corresponding presence of slip on rim interiors suggests that these were inverted and dipped into a solution. Slipped sherds invariably have a strong luster attesting to subsequent burnishing or polishing. It is important to note that both use wear and post-depositional factors worked to erode slip from vessel surfaces, contributing to an underrepresentation of this technique in the archaeological samples.

Organic coat (C) is a lustrous black carbon residue produced by rubbing some organic substance onto the vessel surface prior to firing. Senegambian potters today use baobab leaves to produce this veneer, which they describe in terms of aesthetics, rather than function (Gelbert 1999:215; also Guèye 1998:88; Sall 2005:116).

Glaze, a thin coating of melted glass, is extremely rare in the Survey assemblage where it appears on several imported sherds dating to the Atlantic Era.

In the Madina-Sadatou ceramic assemblages, some $25-30 \%$ of the non-eroded rim sherds, but only $14 \%$ of the non-eroded body sherds, exhibit surface treatment. These figures suggest that potters only treated a subset of vessels, with a strong emphasis on the shoulder, neck, and lip where such techniques would have had the highest visibility.

Figure D.23: Surface treatments (with keys to illustration)

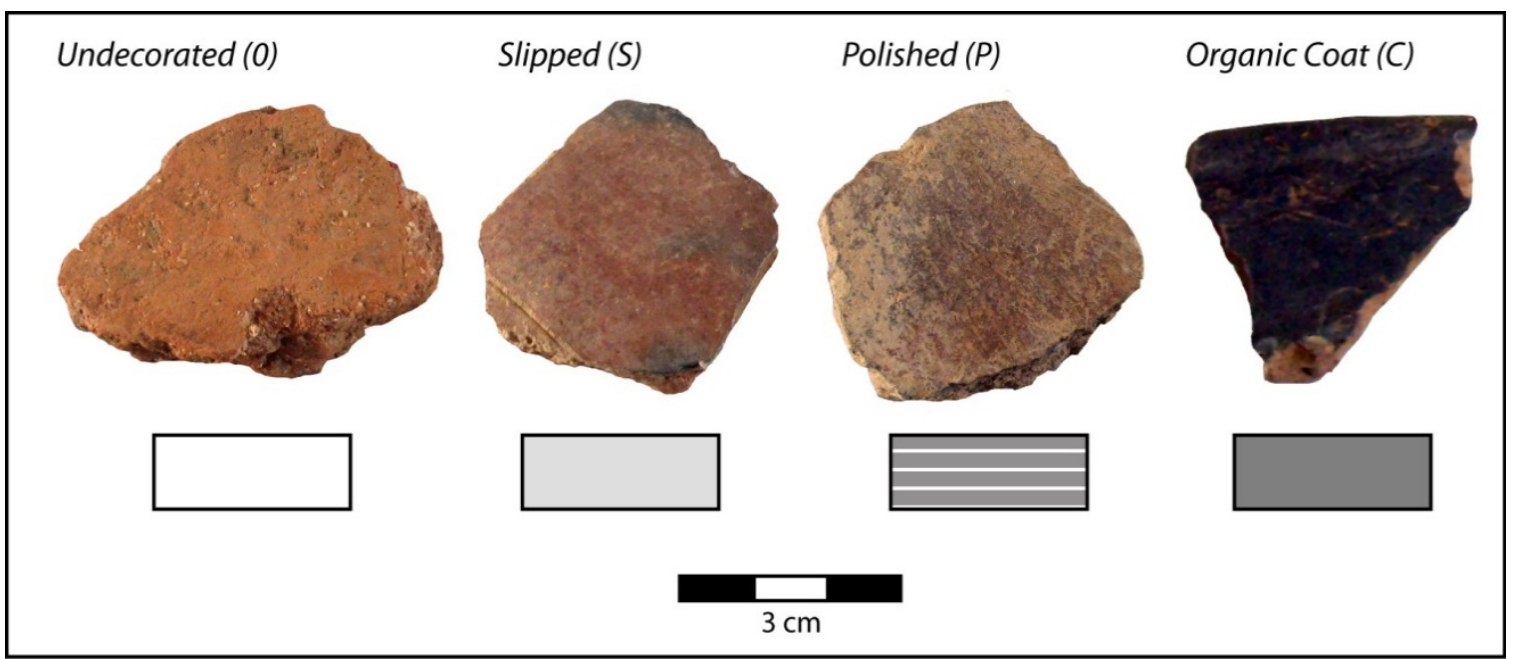


Figure D.24: Additive decorative elements

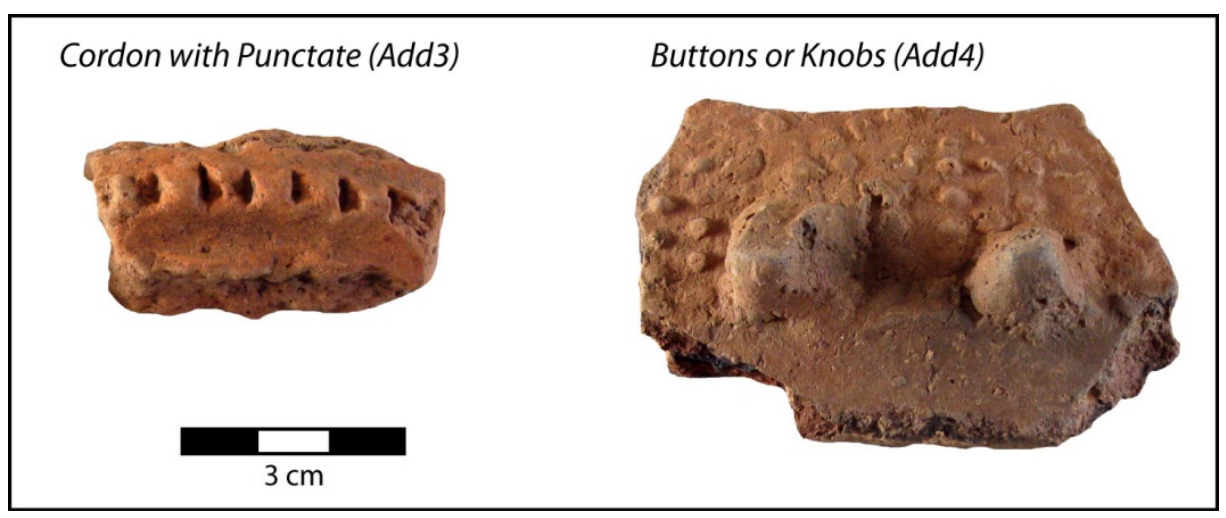

\section{Additive Decoration}

Additive decorative techniques involve attaching one or more plastic elements to the vessel surface (Guèye 1998:50-52; Sall 2005:113). Although rare in the MadinaSadatou assemblages, additive elements do include linear cordons and discontinuous buttons (Figure D.24) similar to those observed elsewhere in Senegambia (Bocoum and McIntosh 2002:70; Richard 2007:534; Thiaw 1999:156). Cordons are coils of clay applied as a raised band around the circumference of a vessel; these often bear the scalloped impression of a baton or other tool. Buttons subsume all non-linear appliqués ranging from round dots to tetons and other amorphous protuberances; these are significantly smaller than true weight-bearing handles (see above).

\section{Impressed/Subtractive Decoration}

Impressions or subtractive techniques are the most common (and diverse) decorative elements in the pottery assemblages from Madina-Sadatou. I categorized these techniques according to three broad gestures-rolling (roulette), dragging, and impression. Referring to ethnographic or experimental examples, I then identified the tools associated with one or more of these gestures. Here I describe all but the rarest or most idiosyncratic tool/gesture combinations.

\section{Twisted Cord Roulettes}

Winding together fiber strands or twines in different directions and quantities can yield a perplexing array of twisted cords, many of which form distinctive patterns of regular "bead" impressions when rolled across the moist surface of an unfired clay vessel (Haour et al. 2010:134-143; Hurley 1979; Soper 1985). Because the shape and size of these impressions may evolve during the use life of a twisted cord roulette as it gradually frays or loosens, the following classificatory scheme considers the number of constituent fiber cords and the complexity of twist (rather than metric variation). Figure D.25 illustrates the following types.

Simple-twisted cord (TwS) roulettes form a pattern of vertical fiber impressions with no clear beading, presumably the work of a cord with two groups of twines twisted together in the same direction (Hurley 1979 Types 1-2). In rare cases, these leave traces from a knot tied in the center of the twine. 
Counter-twisted cord (TwC) roulettes form an impression of oblique beads arranged in parallel rows of equal width, this made by a fiber roulette of two or more cords twisted in opposing directions from their initial twist (Hurley 1979 Types 2324). Although the nomenclature varies from region to region (Livingstone Smith 2007; Mayor et al. 2005:36; S. K. McIntosh 1995:137; Soper 1985:35-36), archaeological observations of this roulette décor indicate that it has been in widespread use across West Africa over the past several millennia.

Double-twisted cord (TwD) roulettes leave a distinctive imprint with rows of paired oblique or horizontal bead impressions that, based on comparison to experimental replicas by Hurley (1979 Types 87-92), may represent a cord made of three sequential combinations of two cords. Uncommon in the Madina-Sadatou study area, similar roulette décors have been noted in the Gambia River basin (A. Lawson 2003:243) and possibly in central Mali (Haour et al. 2010 Figure 3.5).

\section{Knotted Cord Roulettes}

Following Soper (1985), I draw a general distinction between twisted cords with circular cross-sections, and more complex roulettes made by braiding, folding, or otherwise knotting together simpler cords (see also Haour et al. 2010:58-62). I would argue that this distinction was salient for potters in the Madina-Sadatou study area since different types of twisted and knotted cord roulettes seldom appeared together on the same sherd (or presumably vessel). That is to say, co-occurring decorative elements could include one type of knotted cord roulette and one type of twisted cord roulette, but rarely two types from the same class-each presumably comprising a discrete, but complementary, suite of techniques within the available decorative repertoire. The following types are illustrated in Figure D.25.

Simple-braided cord $(\mathrm{BrS})$ roulettes have rows of alternating oblique impressions made by a tool composed of two or three fiber cords braided together with beaded segments on each side slanting in opposite directions (Hurley 1979 Types 210-211). A variant on this décor, the double-braided cord $(\mathrm{BrD})$ roulette, has two parallel beads within each oblique segment, indicating a roulette braided from at least four fiber cords (Hurley 1979 Type 212). Both of these fiber roulettes have been documented on archaeological pottery across West Africa (Mayor et al. 2005:36; Soper 1985:35-39), particularly in the Middle Niger and Middle Senegal regions where Susan McIntosh has identified several sub-types beyond those presented here (Bocoum and McIntosh 2002:67; S. K. McIntosh 1995:132-134).

Folded strip (StF) roulettes comprise columns of interlocking raised beads resulting from a tool with two or more fibrous elements-such as leaf strips or grass fronds-pleated together in an accordion-like fashion (Haour et al. 2010:60-62). While it was possible to identify some of the variants employed elsewhere in Africa (S. K. McIntosh 1995:136; Soper 1985:37-39), folded strip roulette decoration on pottery from Madina-Sadatou has squarish beads oriented perpendicular to the their respective rows with some variation related to the looseness or size of the fibers (see Thiaw 1999:154). Knotted strip roulette (StK) has parallel diagonal strips of undulating "staircase" impressions left by a complex woven tool; these are common in the Middle Niger region (Gallay 1992:38; Mayor et al. 2005), but virtually absent in Senegambian pottery assemblages, including those from Madina-Sadatou. 
Figure D.25: Twisted, knotted, and composite cord roulettes (with keys to illustration)

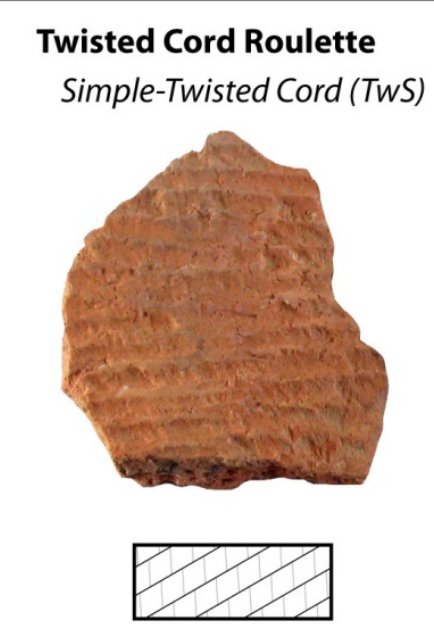

\section{Knotted Cord Roulette}

Simple-Braided Cord (BrS)
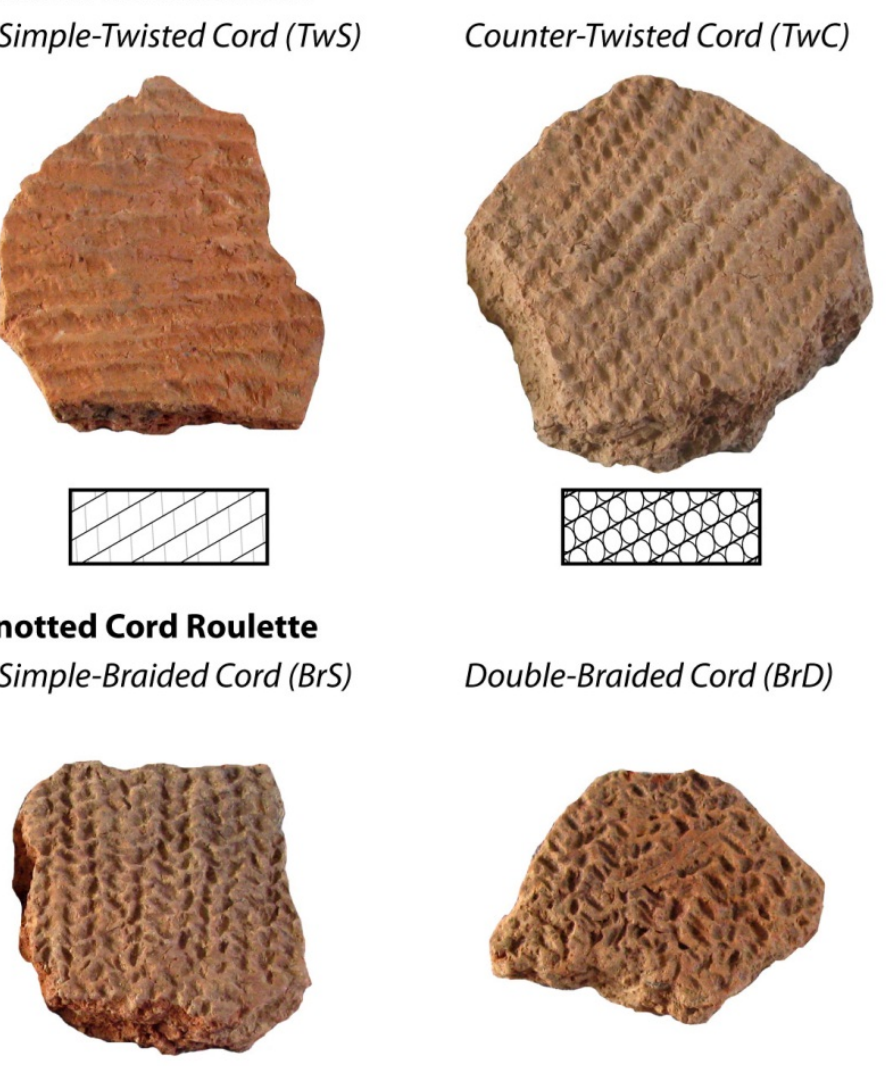

Double-Twisted Cord (TwD)

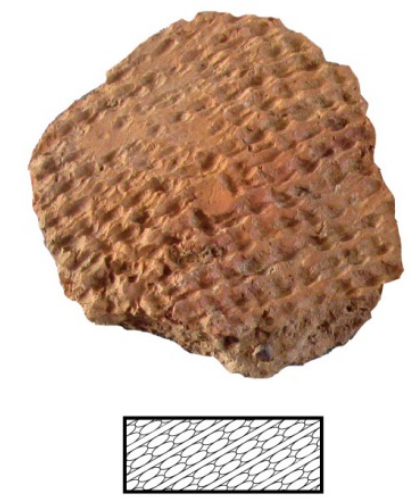

Double-Braided Cord (BrD)

Folded Strip (StF)
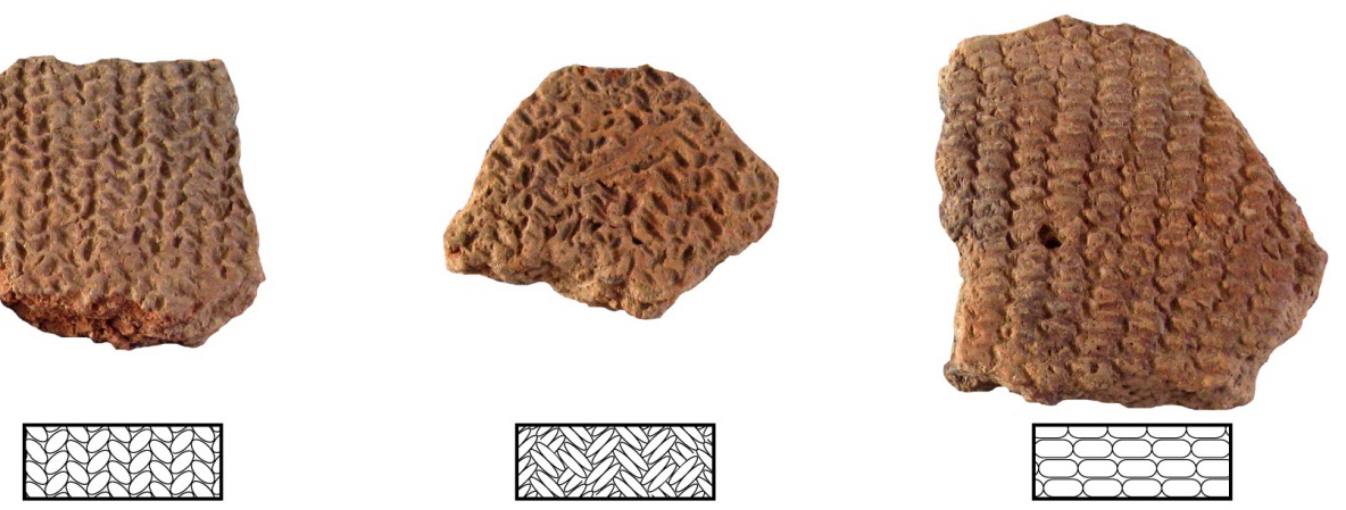

\section{Composite Roulette}

Cord-Wrapped Stick, Spaced (CwS1)

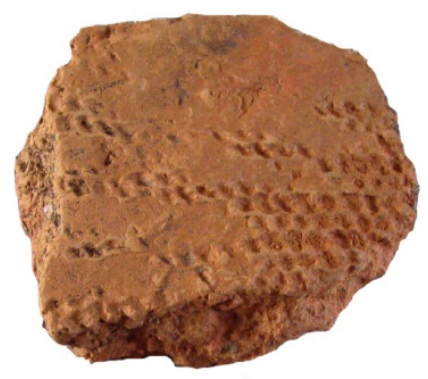

Cord-Wrapped Stick,

Contiguous (CwS2)
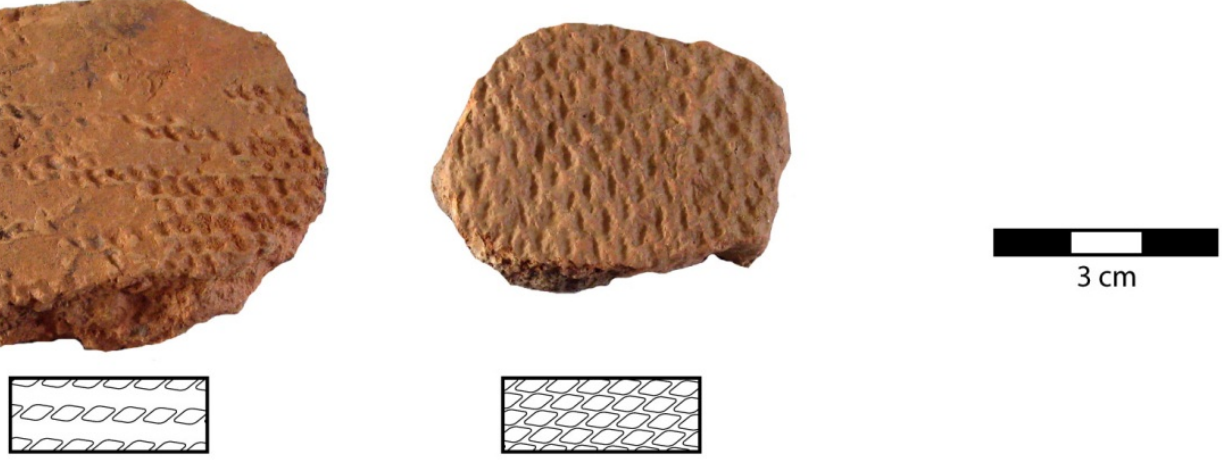


\section{Composite Roulettes}

Composite roulettes comprise a cord or other fibrous element wound around a more rigid vertical element-either another cord or a wooden stick (Haour et al. 2010:6877; Soper 1985:39). Composite roulettes have been identified in archaeological contexts across West Africa, particularly in Senegambia (Bocoum and McIntosh 2002:67; Richard 2007:529-531; Thiaw 1999:154), but only a few variants are present in the MadinaSadatou study area (Figures D.25).

Contiguous cord-wrapped stick (CwS2) roulettes have parallel rows of horizontal beaded segments generated by a cord wrapped in contiguous revolutions around an inflexible core element (Hurley 1979 Types 217-218, 221-222). This decorative element has been identified in archaeological contexts across West Africa, including Senegambia and the Upper to Middle Niger regions (Bocoum and McIntosh 2002:6768; Thiaw 1999:154; Togola 2008:47). Spaced cord-wrapped stick (CwS1) roulettes exhibit some spacing between the cord revolutions around the core element.

\section{Natural Roulettes}

Although marine shells and maize cobs have been, and continue to be, used as roulettes elsewhere in Senegambia (Richard 2007:532; Sall 2005:110), the two natural roulettes evidenced in the Madina-Sadatou ceramic assemblages both resemble shorn grasses (Figure D.26). Ethnographic comparisons indicate that a roulette of coarse shorn grass (Nr2) may represent the grass Blepharis sp. (Haour et al. 2010:84; Mayor et al. 2005:36). A fine shorn grass (Nr1) roulette coincides with the sabot pattern of deep imprints between triangular beads resembling hoof prints; this type has been observed in Late Stone Age and early Iron Age assemblages elsewhere in Senegambia (Bocoum and McIntosh 2002:67; Thiaw 1999:153). I tentatively suggest that the former type corresponds to a worn version of the latter, although it remains possible that some other organic or woven implement produced the impressions observed on pottery from Madina-Sadatou.

Figure D.26: Natural roulettes (with keys to illustration)

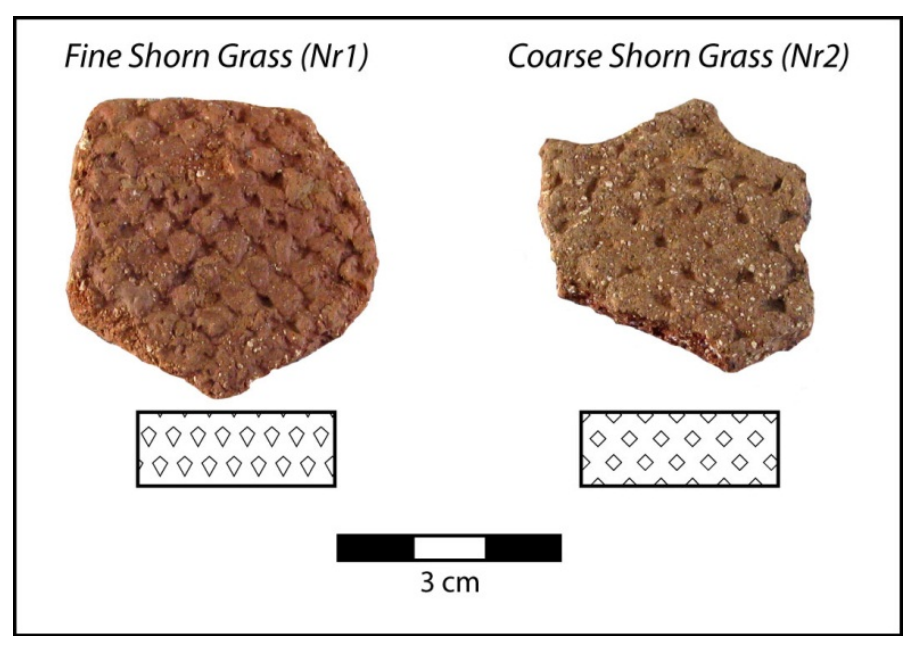




\section{Carved Roulettes}

Carved roulettes made from wood, bone, or terracotta can generate a diverse array of motifs (Figure D.27). Although they sometimes resemble knotted string roulettes, carved roulettes yield crisper edges and lack fibrous bead or segment outlines. In some cases these roulettes push up fine raised lines of clay along their edges. Archaeological and ethnographic data reveal a wide distribution of carved roulette decoration across West Africa, particularly in the southern savanna and equatorial forest (Livingstone Smith 2007). They rarely occur in pottery assemblages from northern Senegambia and the Sahel. In the Madina-Sadatou study area, carved roulettes usually appear on the shoulder of vessels otherwise decorated with twine roulette, but some patterns, such as button and beaded, do adorn the entire body of some larger pots. I recorded those few carved roulettes that did not fit the following typology as other/unknown.

Chevron ( $\mathrm{CrC}$ ) roulettes form distinctive undulating lines of alternating oblique raised segments separated by smooth depressions. Potters in the Middle Niger region employ similar roulettes today (Gallay 1992:38), but these have also been documented in pottery assemblages from the Upper Senegal (Thiaw 1999:155) and Upper Niger regions (Filipowiak 1968:225), in addition to those from megalithic sites in the Gambia River basin (Gallay 2010).

Dentate (CrD) roulettes yield "zig-zag” impressions similar to chevron roulette, but the raised rows resemble interwoven triangles rather than linear segments.

Button (CrB1) roulettes generate a regular and repetitive network of raised buttons generated by a cylindrical tool with circular divots (Soper 1985:34). Bead (CrB2) roulettes resemble a hybrid of carved chevron and button roulettes, with elongated segments aligned perpendicular to the axis of repetitive columns. These types have been observed in diverse archaeological contexts across West Africa, including the site of Niani in the Upper Niger region (Filipowiak 1979:186).

Lattice (CrT) roulettes comprise a network of deep squares or lozenges made by a tool etched with cross-hatching. Although uncommon, this motif has been noted in archaeological assemblages across Senegambia (Richard 2007:532; Thiaw 1999:155).

Oblique (CrO) roulettes form a pattern of oblique to near vertical ridges between flat-bottomed depressions; this points to a cylindrical tool engraved with several upward-spiraling grooves. When rolled across a vessel surface in alternating directions, this roulette can generate a cross-hatch pattern similar to, yet clearly distinguishable from, carved lattice roulette.

Ladder (CrL) roulettes yield rows of fine, raised "rungs" set between raised parallel ridges to form squarish depressions. The distribution of this roulette is not well known, but it has been identified in archaeological assemblages as far away as the Voltaic region (Dueppen 2008:88-90).

Geometric $(\mathrm{CrG})$ roulettes include motifs of triangles and, infrequently, squares produced by a finely carved tool. 
Figure D.27: Carved roulettes (with keys to illustration)

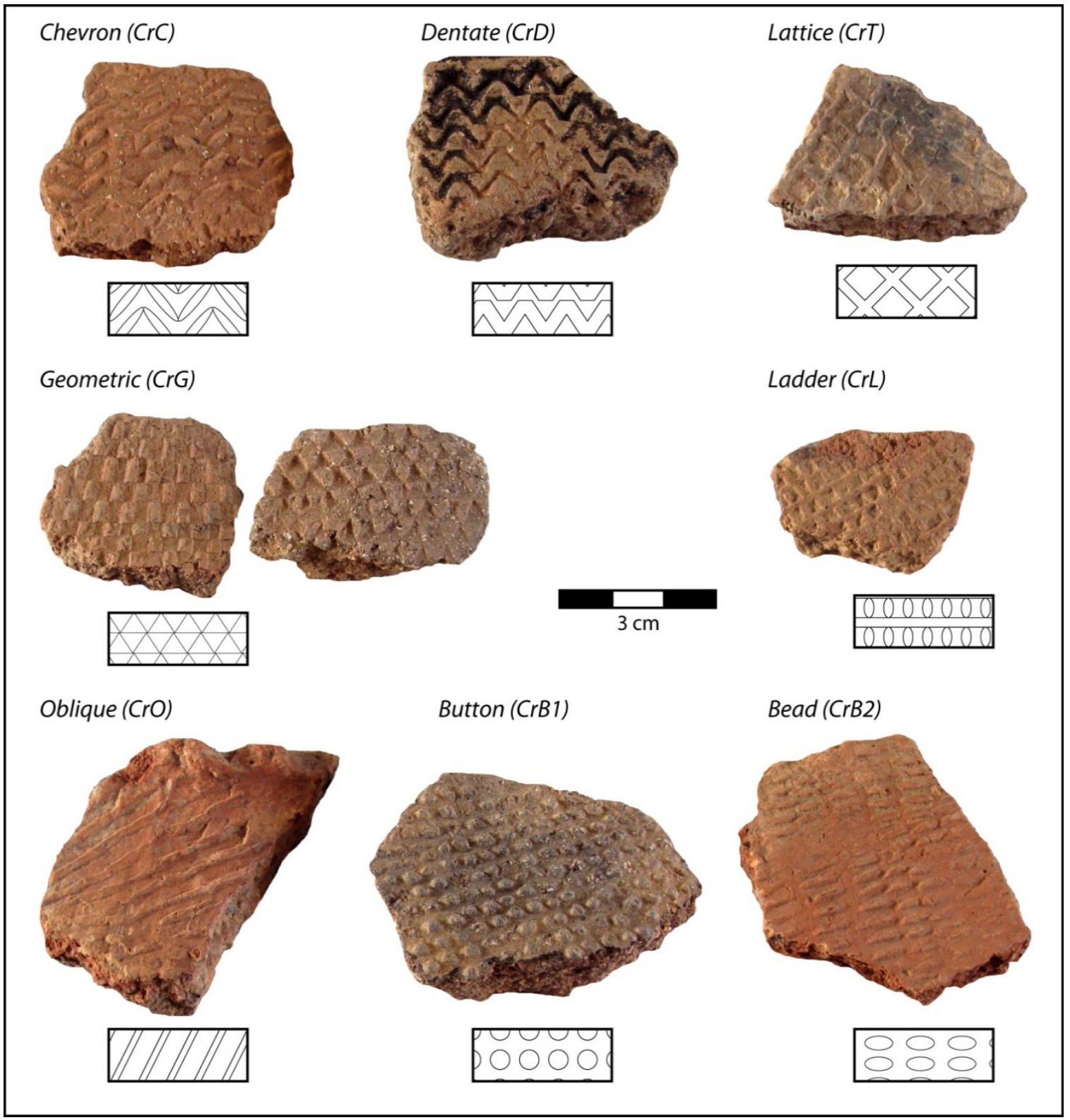

\section{Incisions}

Incisions arise from techniques for dragging or pushing a durable implement across a moist vessel surface. Incisions and combs have been a mainstay of pottery decoration in West Africa since the Late Stone Age and continue to find expression among contemporary potters (Gallay 1970:34, 1992:39-40; Gelbert 1999, 2003; Guèye 1998:50). With the exception of distinctive "rib-and-channel” wares from Iron Age sites in the Middle Senegal region (Bocoum and McIntosh 2002:68-69; Chavane 1985; Thilmans and Ravisé 1980), these decorative techniques are not strongly diagnostic.

For this analysis, I classified several types of incised decoration according to the bluntness/sharpness of the tool and its minimum number of applications (Figure D.28). 
Round incisions include single (Inc1) or multiple (Inc2) u-shaped grooves, usually around the circumference of the vessel, produced by a blunt stylus or other tool. Where multiple incisions could not be distinguished from comb, they were recorded as such.

Sharp incisions include single (Inc3) or multiple (Inc4) v-shaped grooves, usually around the circumference of the vessel, applied by a sharp stylus or other tool. As above, where multiple incisions could not be distinguished from comb, they were recorded as the latter.

Combs include round (CmbR) and sharp (CmbS) variants, as well as fingershaped grooves (CmbF) and broad shallow channels (Chnl). This latter did not, however, approach the heavily corrugated "rib-and-channel" decoration from the Middle Senegal region.

Figure D.28: Incised decorative elements (with keys to illustration)

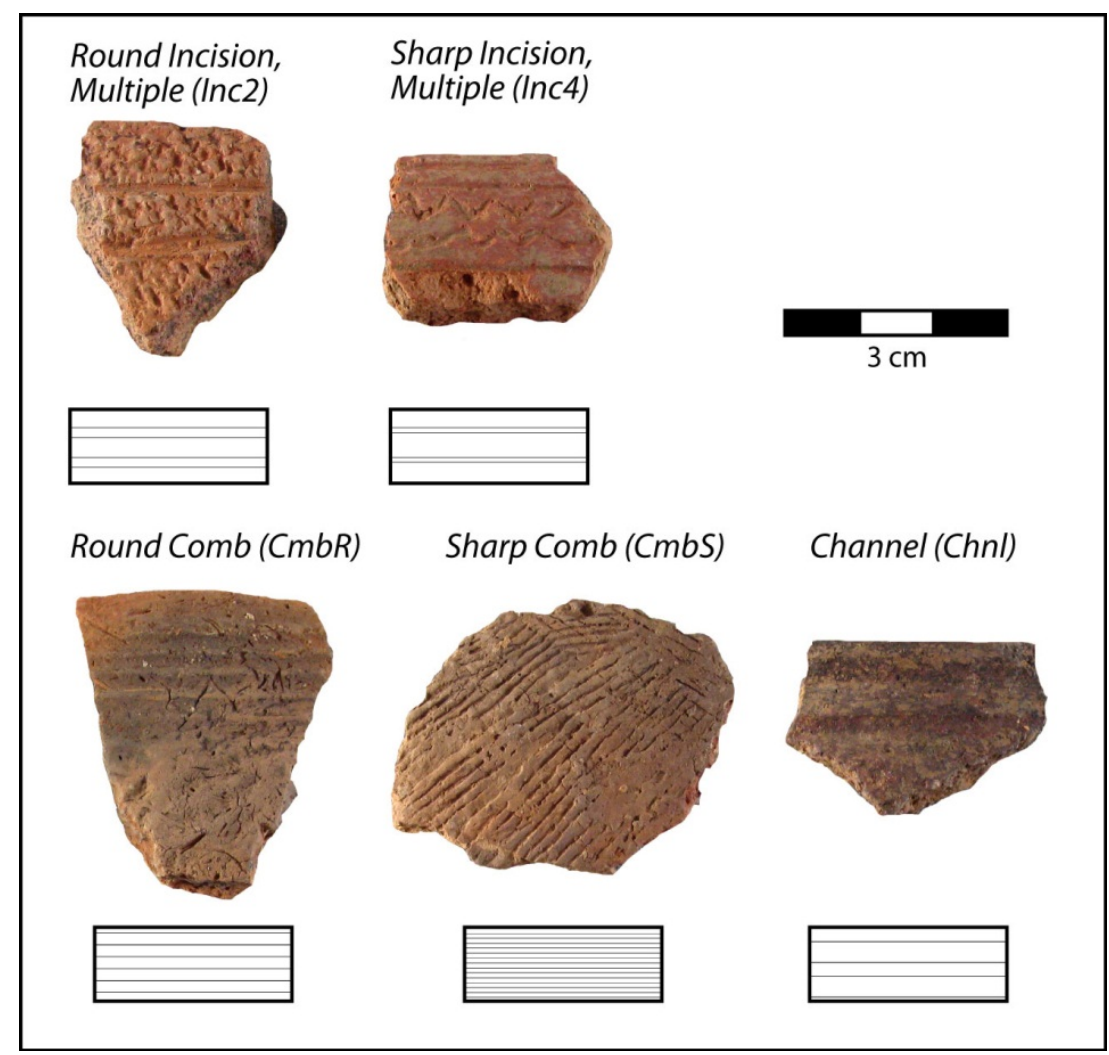

\section{Impressions}

Most tools used for roulettes and incisions can be impressed into the malleable clay of vessel surfaces, either once or repeatedly, to form decorative bands (Figure D.29). As a whole, impressions are widespread in West African pottery traditions, where their use extends back several millennia. Several varieties are, however, diagnostic of particular temporal horizons or regions. 
Knotted-cord impressions (ImK) make for bands of deep, evenly spaced, quasicircular divots, frequently located along the upper edge of twine roulette décor. Based on the appearance of fibers in some impressions, I have interpreted this motif as the result of a knotted cord, possibly lashed around the end of some twine roulettes.

Figure D.29: Impression decorative elements (with keys to illustration)

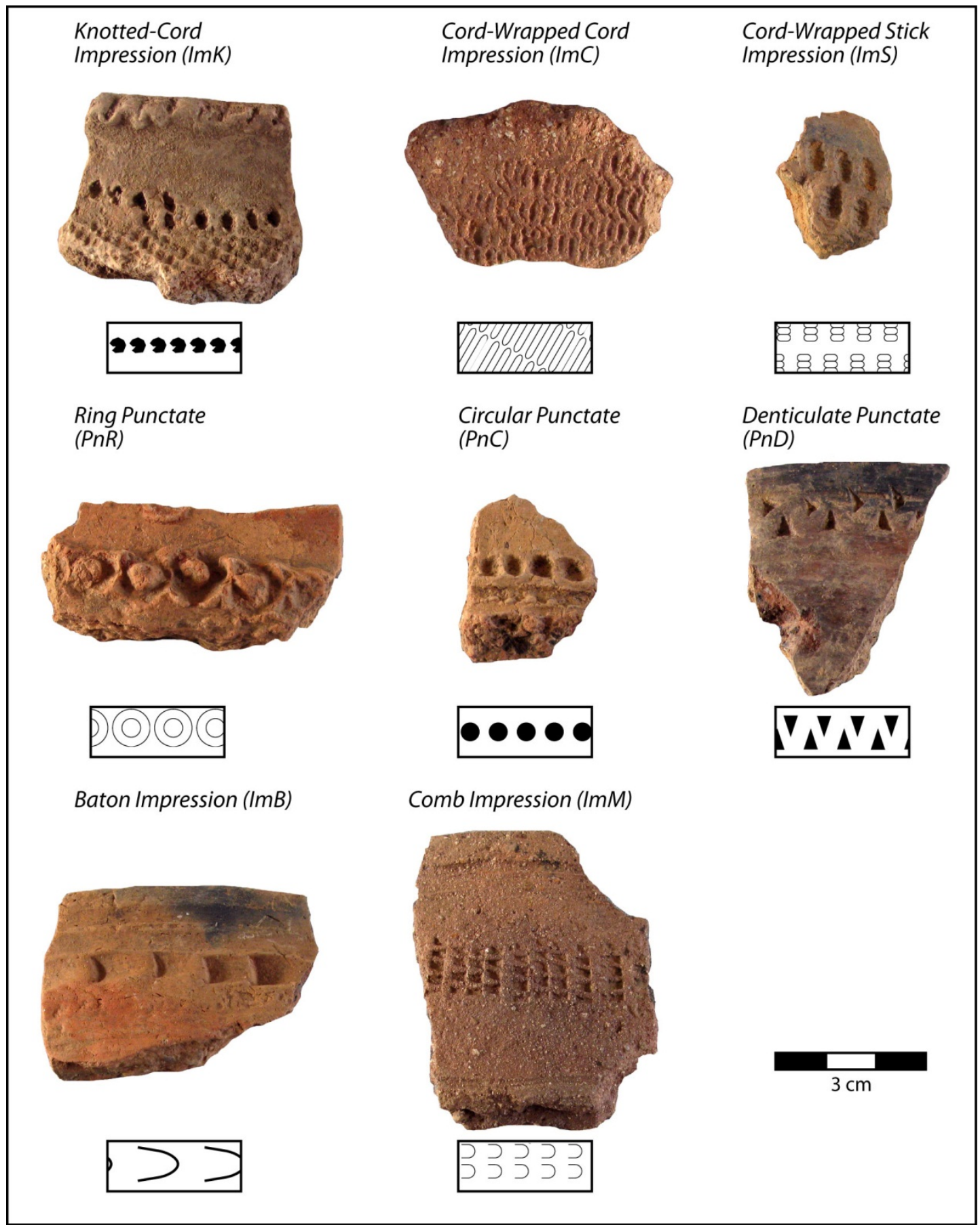


Twisted-cord impressions (ImT) bear the traces of a twisted or braided cord, usually in a solitary band around the upper body or shoulder of medium to large pots; this element is rare in the Madina-Sadatou ceramic assemblages.

Cord-wrapped cord impressions (CwM) are thin elongated marks set in columns, each representing a single impression, and applied repeatedly in an oblique and/or horizontal fashion to form dense bands around a vessel. The variant most commonly found in the Madina-Sadatou study area type resembles the "peigne fileté souple" associated with Late Stone Age occupations in the Sahara (Camps-Fabrer cited in Soper 1985:41), but increasingly documented at a number of sites across the Sahel and northern savanna (Bocoum and McIntosh 2002:67-68; Deme and McIntosh 2006:329-333; Huysecom 1990:47-48; MacDonald 1997:183, 2011:54; Thiaw 1999:154).

Cord-wrapped stick impressions (ImS) generate evenly spaced, vertical rows of beads aligned in columns. This decorative element probably derives from cordage wrapped in a non-contiguous fashion around a rigid central element.

Circular punctates $(\mathrm{PnC})$ and cuneiform punctates $(\mathrm{PnN})$ involve punching the rounded or rectilinear end of a baton, or more rarely, a sharp stylus, into the malleable clay of the vessel neck or shoulder. Using the same general technique, ring punctates (PnR) derive from an open cylinder-shaped tool such as a millet stalk. Denticulate punctates $(\mathrm{PnD})$ form a specific pattern of triangular punch marks, often associated with sharp incised lines, arranged in rows resembling teeth.

Baton impressions ( $\mathrm{ImB}$ ) form series of u-shaped depressions, deeper at one end than the other, made by pressing a blunt stylus laterally into the clay surface. Comb impressions (ImM) are columns of five or six rounded square imprints arranged in bands around the upper part of a vessel. Although a similar pattern has been linked to carved calabash roulettes among contemporary Soninke potters (Gallay 1970:34), the co-occurrence of this decorative element with dragged comb décor on many sherds points more convincingly to multiple gestures with the same tool. Finger impressions (ImF) appear occasionally on vessel surfaces.

\section{Firing}

Once pottery vessels have dried to leather hard, the final stage of production requires firing them to permanently transform the crystalline structure of the clay. Contemporary potters in Senegambia use an open-firing method in which they carefully pile vessels (sometimes in a borrow pit), cover them with fuel such as tinder or dung, and fire them at temperatures of at least $500-600^{\circ} \mathrm{C}$ until the fuel has been consumed (Gelbert 1999, 2003; Guèye 1998:78-81; Sall 2005:125-140). Since firing incurs high fuel costs and vessel attrition, this operational stage does much to account for the low economic return on traditional pottery manufacture (Rice 1987:172-176); this is particularly the case for open-firing where the critical variables of temperature, duration, and atmosphere are difficult to manage and can vary considerably within and between firing episodes, or even across single pots. Potters work to control these variables by adjusting the number of vessels fired together, the quantity and type of tinder, and the timing of removal from fire. Firings are more successful during the dry season when moisture fully evaporates from the leather hard vessels, reducing the chances of spalls and fractures. 
Figure D.30: Schematic oxidation patterns recorded for sherd cross-sections (after Teltser 1993: Figure 2)

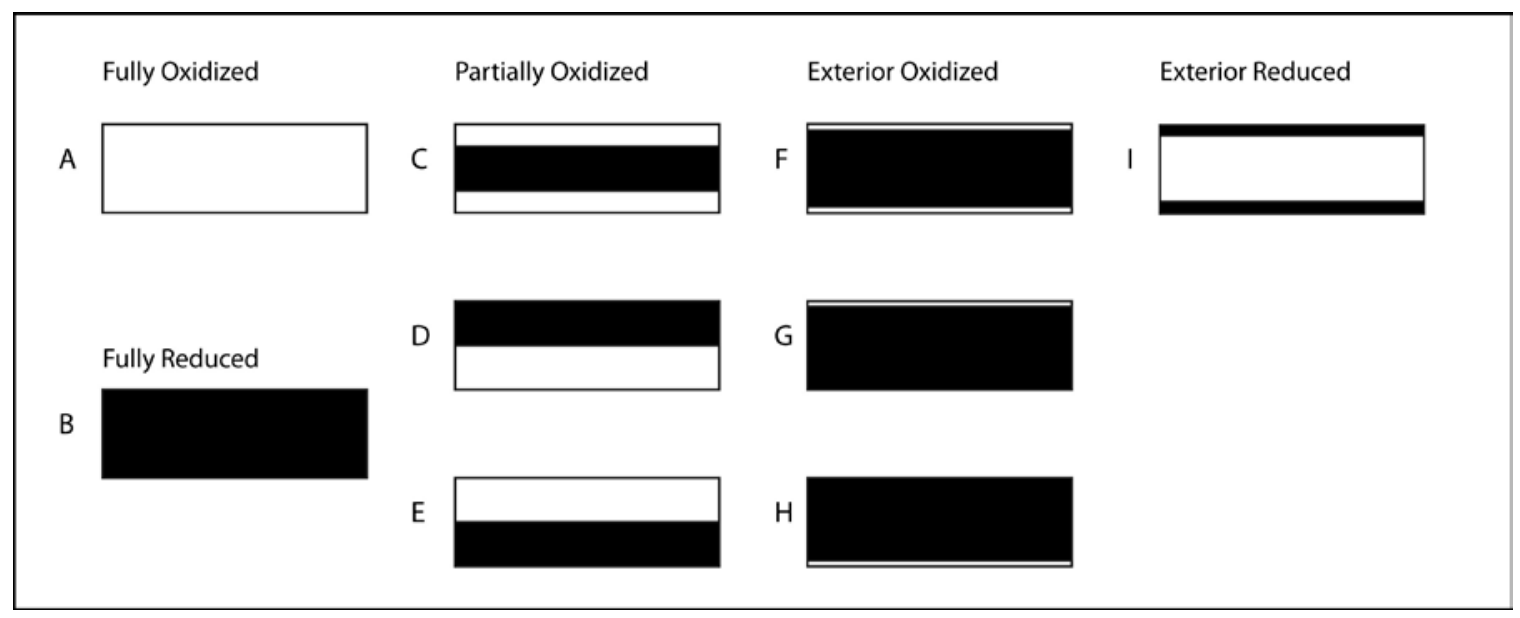

For pottery assemblages from the Madina-Sadatou study area, color variations visible in sherd cross-section attest to the gross differences in the atmospheric conditions of firing (see Rice 1987:334-335). When pots undergo firing with ample exposure to air, carbonaceous material in the clay matrix burns off as $\mathrm{CO}_{2}$ gas and iron oxidizes to produce a reddish cross-section, although the rate and extent of this process varies according to the type of clay, initial carbon content, and duration and intensity of heat exposure. Firing with limited air exposure (reducing conditions) retards iron oxidation and conserves carbonaceous material as bands of gray-colored paste in cross-section.

Using the chart shown in Figure D.30, I recorded the location of these unoxidized bands as a crude thermal history of each sherd. This attribute can vary from sherd to sherd, but I assume that significant differences between samples in the Madina-Sadatou ceramic assemblages correspond to broad differences in firing and/or cooking techniques. As I discuss in the following classification, oxidizing (A), partially oxidizing (C-E), and reducing $(\mathrm{B}, \mathrm{F}-\mathrm{H})$ conditions are never in and of themselves diagnostic of different potting traditions, but they do lend support to discrete differences between operational sequences otherwise inferred from techniques in other production stages.

\section{Use and Discard}

Despite an analytical focus on attributes most relevant to past production technologies, I noted several attributes related to practices of pottery use and discard. For example, the relative degree of erosion and size (calculated from bulk ceramic count and weight for each FS unit) can help to elucidate disposal behaviors and post-depositional processes acting on pottery sherds and the archaeological record (see Chapter 6).

\section{POTTERY CLASSIFICATION}

Two main objectives of this analysis were to devise chronological and functional typologies for ceramic assemblages from the Madina-Sadatou study area. In both cases I employed the above technical attributes to formulate sherd types whose frequencies or 
mere presence/absence could demonstrate change over time or assemblage-level differences in use.

For both the Survey and Diouboye assemblages, I first constructed a paradigmatic grid of provisional types using the variables of paste type, decorative element(s), and rim type. I next considered patterns of association among these provisional types and external temporal variables - either stratified contexts at Diouboye or other temporally-sensitive artifacts in surface contexts - in order to identify those types (and their attributes) most relevant for chronology. Although I do not address the issue of why ceramics change over time in this analysis, it is important to note that, beyond "stylistic drift," artifact variability can of course relate to a number of other socioeconomic processes, including functional needs for cooking and storage, associated clay preferences, or the need to signal cultural identities. After merging strongly co-occurring decorative and rim formation attributes, and discarding those less sensitive to chronology, I then proceeded to construct a hierarchical typology using those attributes with the strongest overall association (usually decorative element) as the criteria for each step of division (see Whallon 1972). Several iterations of this process resulted in types adequate for a ceramic chronology of Diouboye and the sites documented on survey.

Non-typed sherds either lacked visible decoration (or were too eroded), had extremely idiosyncratic attribute combinations, or were simply too abundant across multiple periods. Because all sherds convey information about past craft economies and other socioeconomic practices, I reserve the discussion of non-typed, but potentially quite informative, specimens for the actual contexts in which they were found (Chapters 4-9).

At the end of this section, I develop a preliminary categorization of rim sherds in terms of vessel function. Building upon the above classification of rim forms, I further consider shoulder angle and orifice diameter to compare the economic activities underlying ceramic assemblages at Diouboye and across the Madina-Sadatou study area.

\section{Survey Assemblage: Typology and Seriation Body Sherd Types}

Paste type and decorative techniques formed the two primary dimensions for chronological sherd types. Table D.11 summarizes the relationships between these two variables for all body sherds in the Survey assemblage.

Table D.12 lists the body sherd types formulated in order of their hierarchical division. That is, Type S2 only includes sherds remaining after the formulation of Type S1, Type S3 only includes sherds remaining after the formulation of Types S1 and S2, and so forth. While organic paste formed the criteria for the first division (Type S1), all remaining divisions (Types S2-S8) were based on impressed surface decoration-either single or commonly co-occurring sets of elements. The salience of these types as remnants of discrete operational sequences draws support from significant differences in technical variables that otherwise varied continuously or uniformly (i.e., body thickness, surface treatment, and relative oxidation or reduction). A total of 1927 sherds, accounting for $18 \%$ of the Survey assemblage, could be assigned to one of these types.

The remaining 8789 sherds are either undecorated, too idiosyncratic, or, as in the case of sherds decorated with twisted cord roulette, simply too common across strata from different periods. While these were not assigned to chronological types in the present study, they may prove useful in future analyses and interregional comparisons. 
Table D.11: Summary of decorative elements by paste type for body sherds in the Survey assemblage

\begin{tabular}{|c|c|c|c|c|c|c|c|}
\hline \multicolumn{2}{|c|}{ Decorative Attribute(s) } & \multicolumn{6}{|c|}{ Paste Type } \\
\hline & & \multicolumn{2}{|c|}{ Grit (n=4665) } & \multicolumn{2}{|c|}{ Grog (n=1946) } & \multicolumn{2}{|c|}{ Organic $(n=3287)$} \\
\hline & & $n$ & $\mathrm{Col} \%$ & $n$ & $\mathrm{Col} \%$ & $n$ & $\mathrm{Col} \%$ \\
\hline \multirow{2}{*}{$\begin{array}{c}\text { Interior } \\
\text { Treatment } \\
\end{array}$} & Slip/Polish & 191 & $4.09 \%$ & 98 & $5.04 \%$ & 35 & $1.06 \%$ \\
\hline & Organic Coat & 5 & $0.11 \%$ & 31 & $1.59 \%$ & 279 & $8.49 \%$ \\
\hline \multirow{2}{*}{$\begin{array}{l}\text { Surface } \\
\text { Treatment }\end{array}$} & Slip & 142 & $3.04 \%$ & 154 & $7.91 \%$ & 102 & $3.10 \%$ \\
\hline & Organic Coat & 0 & $0.00 \%$ & 0 & $0.00 \%$ & 190 & $5.78 \%$ \\
\hline Undecorated & & 3297 & $70.68 \%$ & 1219 & $62.64 \%$ & 2611 & $79.43 \%$ \\
\hline \multirow{2}{*}{$\begin{array}{l}\text { Additive } \\
\text { Décor }\end{array}$} & Add1 & 11 & $0.24 \%$ & 2 & $0.10 \%$ & 6 & $0.18 \%$ \\
\hline & Add2/Add3 & 6 & $0.13 \%$ & 4 & $0.21 \%$ & 2 & $0.06 \%$ \\
\hline $\begin{array}{l}\text { Twisted Cord } \\
\text { Roulette } \\
\end{array}$ & TwC/TwS & 280 & $6.00 \%$ & 274 & $14.08 \%$ & 293 & $8.91 \%$ \\
\hline \multirow{2}{*}{$\begin{array}{l}\text { Knotted Cord } \\
\text { Roulette }\end{array}$} & $\mathrm{BrS}$ & 2 & $0.04 \%$ & 4 & $0.21 \%$ & 1 & $0.03 \%$ \\
\hline & StF & 31 & $0.66 \%$ & 87 & $4.47 \%$ & 27 & $0.82 \%$ \\
\hline \multirow{2}{*}{$\begin{array}{c}\text { Composite } \\
\text { Roulette }\end{array}$} & CwS1 & 24 & $0.51 \%$ & 34 & $1.75 \%$ & 6 & $0.18 \%$ \\
\hline & CwS2 & 6 & $0.13 \%$ & 42 & $2.16 \%$ & 6 & $0.18 \%$ \\
\hline \multirow{2}{*}{$\begin{array}{l}\text { Natural } \\
\text { Roulette }\end{array}$} & $\mathrm{Nr} 1$ & 402 & $8.62 \%$ & 8 & $0.41 \%$ & 10 & $0.30 \%$ \\
\hline & Nr2 & 147 & $3.15 \%$ & 0 & $0.00 \%$ & 6 & $0.18 \%$ \\
\hline \multirow{5}{*}{$\begin{array}{l}\text { Carved } \\
\text { Roulette }\end{array}$} & $\mathrm{CrC}$ & 19 & $0.41 \%$ & 41 & $2.11 \%$ & 9 & $0.27 \%$ \\
\hline & $\mathrm{CrD}$ & 4 & $0.09 \%$ & 1 & $0.05 \%$ & 0 & $0.00 \%$ \\
\hline & $\mathrm{CrG}$ & 1 & $0.02 \%$ & 0 & $0.00 \%$ & 0 & $0.00 \%$ \\
\hline & CrT & 1 & $0.02 \%$ & 9 & $0.46 \%$ & 1 & $0.03 \%$ \\
\hline & CrB1/CrB2 & 15 & $0.32 \%$ & 40 & $2.06 \%$ & 7 & $0.21 \%$ \\
\hline \multirow{5}{*}{ Incision } & Inc1/Inc2 & 22 & $0.47 \%$ & 14 & $0.72 \%$ & 21 & $0.64 \%$ \\
\hline & Inc3/Inc4 & 5 & $0.11 \%$ & 0 & $0.00 \%$ & 6 & $0.18 \%$ \\
\hline & CmbR & 70 & $1.50 \%$ & 19 & $0.98 \%$ & 87 & $2.65 \%$ \\
\hline & $\mathrm{CmbS}$ & 14 & $0.30 \%$ & 14 & $0.72 \%$ & 12 & $0.37 \%$ \\
\hline & Chnl & 19 & $0.41 \%$ & 10 & $0.51 \%$ & 35 & $1.06 \%$ \\
\hline \multirow{2}{*}{$\begin{array}{l}\text { Cord } \\
\text { Impression }\end{array}$} & $\operatorname{ImK/ImT~}$ & 1 & $0.02 \%$ & 2 & $0.10 \%$ & 2 & $0.06 \%$ \\
\hline & $\operatorname{ImC}$ & 29 & $0.62 \%$ & 3 & $0.15 \%$ & 0 & $0.00 \%$ \\
\hline \multirow{5}{*}{ Punctate } & PnR & 2 & $0.04 \%$ & 3 & $0.15 \%$ & 1 & $0.03 \%$ \\
\hline & $\mathrm{PnC} / \mathrm{PnN}$ & 3 & $0.06 \%$ & 9 & $0.46 \%$ & 24 & $0.73 \%$ \\
\hline & PnB & 1 & $0.02 \%$ & 1 & $0.05 \%$ & 1 & $0.03 \%$ \\
\hline & PnM & 2 & $0.04 \%$ & 1 & $0.05 \%$ & 3 & $0.09 \%$ \\
\hline & $\mathrm{PnF}$ & 1 & $0.02 \%$ & 1 & $0.05 \%$ & 2 & $0.06 \%$ \\
\hline
\end{tabular}




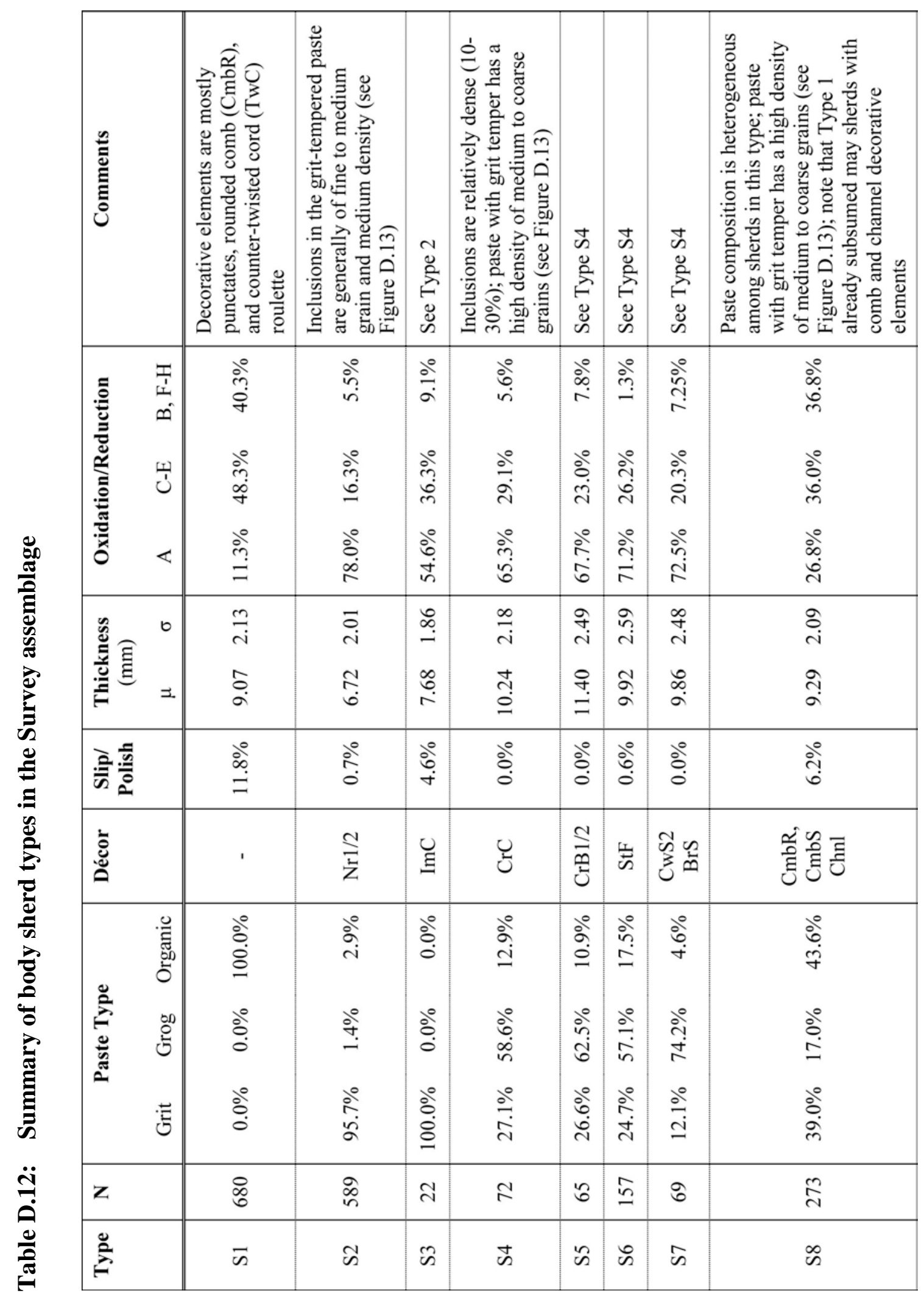




\section{Rim Types}

The classification of rim sherds began by looking at the relations between paste type and decorative elements (Table D.13). Although some rim subclasses were associated with paste types (Table D.14) and decorative elements, these relationships mostly proved useful (along with thickness, surface treatment and oxidation/reduction) for emphasizing types otherwise defined according to paste and decoration. Using the method of hierarchical division described above for body sherds, Table D.15 lists the rim types in order of their formulation. Again, organic paste type was the criteria for the first division (Type S10), with all remaining divisions (Types S11-S17) based on impressed surface decoration. In this way I assigned 542 out of 1189 total specimens to one of the rim types. On the basis of their paste type and visible décor, a further 75 simple rims were classified as one of the body sherd types described above. Illustrations of these types appear in Chapter 4.

Table D.13: Summary of decorative elements by paste type for rim sherds in the Survey assemblage

\begin{tabular}{|c|c|c|c|c|c|c|c|}
\hline \multicolumn{2}{|c|}{ Decorative Element(s) } & \multicolumn{6}{|c|}{ Paste Type } \\
\hline & & \multicolumn{2}{|c|}{ Grit $(n=478)$} & \multicolumn{2}{|c|}{ Grog $(n=390)$} & \multicolumn{2}{|c|}{ Organic $(n=255)$} \\
\hline & & $n$ & $\mathrm{Col} \%$ & $n$ & $\mathrm{Col} \%$ & $n$ & $\mathrm{Col} \%$ \\
\hline \multirow{2}{*}{$\begin{array}{l}\text { Interior/Lip } \\
\text { Treatment }\end{array}$} & Slip/Polish & 38 & $7.95 \%$ & 80 & $20.51 \%$ & 10 & $3.92 \%$ \\
\hline & Organic Coat & 7 & $1.46 \%$ & 18 & $4.62 \%$ & 102 & $40.00 \%$ \\
\hline \multirow{3}{*}{ Interior/Lip Décor } & Incision/Comb & 40 & $8.37 \%$ & 47 & $12.05 \%$ & 88 & $34.51 \%$ \\
\hline & Circular Punctate & 1 & $0.21 \%$ & 4 & $1.03 \%$ & 4 & $1.57 \%$ \\
\hline & Bevel & 23 & $4.81 \%$ & 44 & $11.28 \%$ & 43 & $16.86 \%$ \\
\hline \multirow{2}{*}{$\begin{array}{l}\text { Exterior Surface } \\
\text { Treatment }\end{array}$} & Slip/Polish & 49 & $10.25 \%$ & 86 & $22.05 \%$ & 7 & $2.75 \%$ \\
\hline & Organic Coat & 0 & $0.00 \%$ & 1 & $0.26 \%$ & 65 & $25.49 \%$ \\
\hline $\begin{array}{l}\text { Twisted Cord } \\
\text { Roulette }\end{array}$ & TwC/TwS & 27 & $5.65 \%$ & 40 & $10.26 \%$ & 18 & $7.06 \%$ \\
\hline Natural Roulette & Nr1 & 5 & $1.05 \%$ & 0 & $0.00 \%$ & 0 & $0.00 \%$ \\
\hline \multirow{3}{*}{ Carved Roulette } & $\mathrm{CrC}$ & 1 & $0.21 \%$ & 4 & $1.03 \%$ & 0 & $0.00 \%$ \\
\hline & $\mathrm{CrD}$ & 4 & $0.84 \%$ & 1 & $0.26 \%$ & 0 & $0.00 \%$ \\
\hline & CrB1/CrB2 & 0 & $0.00 \%$ & 10 & $2.56 \%$ & 0 & $0.00 \%$ \\
\hline \multirow{5}{*}{ Incision } & Inc1/Inc2 & 16 & $3.35 \%$ & 28 & $7.18 \%$ & 17 & $6.67 \%$ \\
\hline & Inc3/Inc4 & 3 & $0.63 \%$ & 4 & $1.03 \%$ & 1 & $0.39 \%$ \\
\hline & CmbR & 35 & $7.32 \%$ & 29 & $7.44 \%$ & 55 & $21.57 \%$ \\
\hline & $\mathrm{CmbS}$ & 3 & $0.63 \%$ & 10 & $2.56 \%$ & 19 & $7.45 \%$ \\
\hline & Chnl & 3 & $0.63 \%$ & 3 & $0.77 \%$ & 1 & $0.39 \%$ \\
\hline \multirow{2}{*}{ Impression } & $\operatorname{ImK} / \operatorname{ImT}$ & 3 & $0.63 \%$ & 6 & $1.54 \%$ & 4 & $1.57 \%$ \\
\hline & ImC & 65 & $13.60 \%$ & 6 & $1.54 \%$ & 0 & $0.00 \%$ \\
\hline \multirow{4}{*}{ Punctate } & PnR & 3 & $0.63 \%$ & 0 & $0.00 \%$ & 0 & $0.00 \%$ \\
\hline & $\mathrm{PnC} / \mathrm{PnN}$ & 9 & $1.88 \%$ & 13 & $3.33 \%$ & 17 & $6.67 \%$ \\
\hline & $\mathrm{PnB}$ & 1 & $0.21 \%$ & 10 & $2.56 \%$ & 1 & $0.39 \%$ \\
\hline & PnM & 4 & $0.84 \%$ & 1 & $0.26 \%$ & 0 & $0.00 \%$ \\
\hline
\end{tabular}


Table D.14: Summary of rim form subclass by paste type for rim sherds in the Survey assemblage

\begin{tabular}{|c|c|c|c|c|c|c|c|}
\hline \multicolumn{2}{|c|}{ Rim Class } & \multicolumn{6}{|c|}{ Paste Type } \\
\hline & Subclass & \multicolumn{2}{|c|}{ Grit $(\mathrm{n}=478)$} & \multicolumn{2}{|c|}{ Grog $(n=390)$} & \multicolumn{2}{|c|}{ Organic $(n=255)$} \\
\hline & & $n$ & $\mathrm{Col} \%$ & $n$ & $\mathrm{Col} \%$ & $n$ & $\mathrm{Col} \%$ \\
\hline Simple & & 38 & $7.95 \%$ & 57 & $14.62 \%$ & 32 & $12.55 \%$ \\
\hline \multirow{11}{*}{ Inflected } & EsR & 20 & $4.18 \%$ & 25 & $6.41 \%$ & 24 & $9.41 \%$ \\
\hline & EwR & 27 & $5.65 \%$ & 27 & $6.92 \%$ & 15 & $5.88 \%$ \\
\hline & EfR & 29 & $6.07 \%$ & 24 & $6.15 \%$ & 21 & $8.24 \%$ \\
\hline & EsA & 7 & $1.46 \%$ & 5 & $1.28 \%$ & 2 & $0.78 \%$ \\
\hline & EwA & 14 & $2.93 \%$ & 17 & $4.36 \%$ & 5 & $1.96 \%$ \\
\hline & EfA & 13 & $2.72 \%$ & 32 & $8.21 \%$ & 9 & $3.53 \%$ \\
\hline & EsT & 49 & $10.25 \%$ & 4 & $1.03 \%$ & 3 & $1.18 \%$ \\
\hline & EwT & 29 & $6.07 \%$ & 1 & $0.26 \%$ & 0 & $0.00 \%$ \\
\hline & EfT & 15 & $3.14 \%$ & 1 & $0.26 \%$ & 2 & $0.78 \%$ \\
\hline & EvC & 11 & $2.30 \%$ & 19 & $4.87 \%$ & 3 & $1.18 \%$ \\
\hline & CAR & 2 & $0.42 \%$ & 2 & $0.51 \%$ & 1 & $0.39 \%$ \\
\hline \multirow{4}{*}{ Collared } & LC & 1 & $0.21 \%$ & 1 & $0.26 \%$ & 1 & $0.39 \%$ \\
\hline & ThC & 6 & $1.26 \%$ & 4 & $1.03 \%$ & 0 & $0.00 \%$ \\
\hline & Bd1 & 2 & $0.42 \%$ & 1 & $0.26 \%$ & 5 & $1.96 \%$ \\
\hline & $\mathrm{Bd} 2$ & 4 & $0.84 \%$ & 14 & $3.59 \%$ & 2 & $0.78 \%$ \\
\hline \multirow{2}{*}{ Ledged } & Ldg1 & 2 & $0.42 \%$ & 0 & $0.00 \%$ & 6 & $2.35 \%$ \\
\hline & Ldg2 & 1 & $0.21 \%$ & 1 & $0.26 \%$ & 0 & $0.00 \%$ \\
\hline
\end{tabular}




\begin{tabular}{|c|c|c|c|c|c|c|c|c|}
\hline 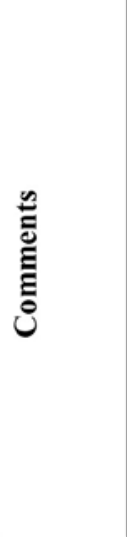 & 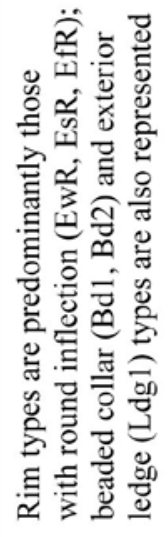 & 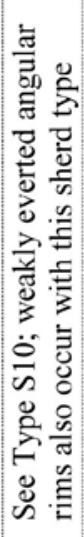 & 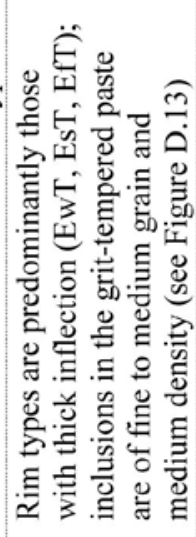 & 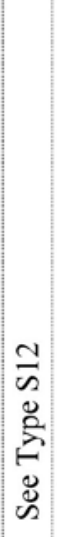 & 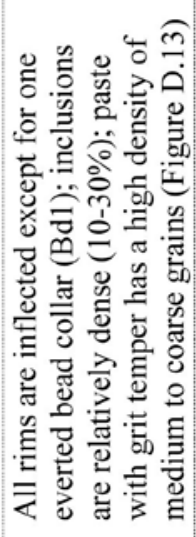 & 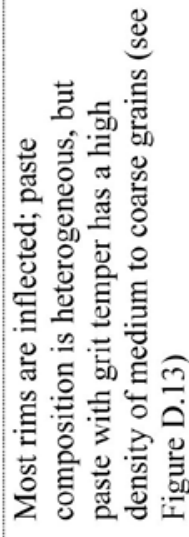 & 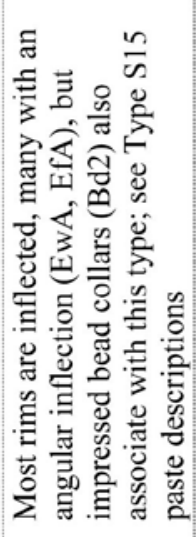 & 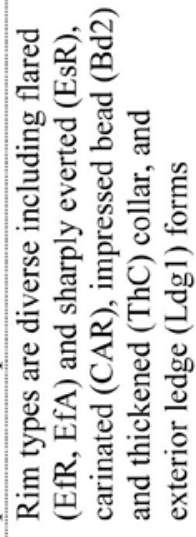 \\
\hline 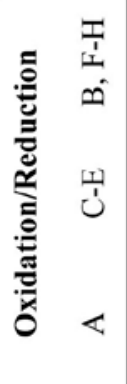 & 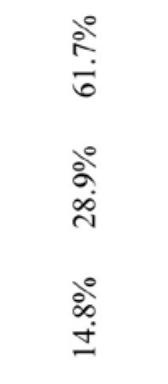 & 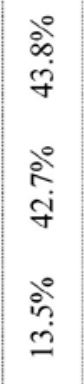 & 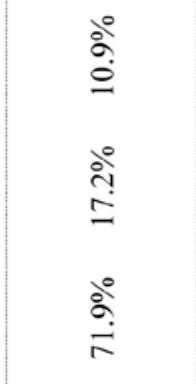 & 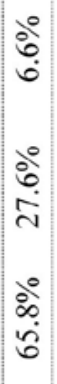 & 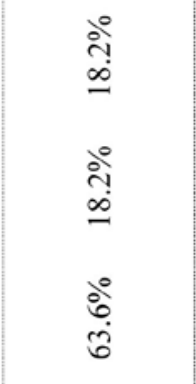 & $\begin{array}{l}\frac{0}{a} \\
\stackrel{0}{\circ} \\
\dot{+} \\
\dot{0} \\
\dot{\circ}\end{array}$ & 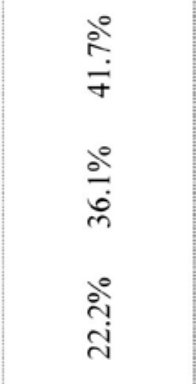 & $\begin{array}{l}\stackrel{0}{0} \\
\stackrel{+}{+} \\
\stackrel{0}{0} \\
\dot{+} \\
\stackrel{\circ}{\circ} \\
\stackrel{\infty}{\circ}\end{array}$ \\
\hline 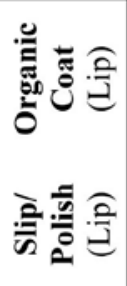 & $\begin{array}{l}\stackrel{\circ}{\mathrm{i}} \\
\stackrel{\mathrm{i}}{\mathrm{i}} \\
\stackrel{\circ}{0}\end{array}$ & $\begin{array}{l}\stackrel{\circ}{0} \\
\stackrel{0}{0} \\
\dot{0} \\
\dot{0}\end{array}$ & 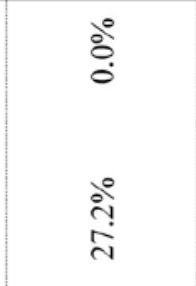 & ठें & ठ̊. & $\begin{array}{l}\stackrel{0}{\circ} \\
\stackrel{0}{0} \\
\stackrel{0}{\circ} \\
\stackrel{0}{0}\end{array}$ & $\begin{array}{l}\stackrel{0}{\circ} \\
\dot{0} \\
\stackrel{\circ}{0} \\
\stackrel{0}{0}\end{array}$ & $\begin{array}{l}\stackrel{\circ}{\circ} \\
\dot{\circ}\end{array}$ \\
\hline 它 & 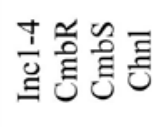 & ' & 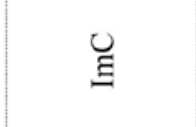 & $\frac{\mathrm{S}}{\mathrm{z}}$ & บِ & 导忌当氞 & 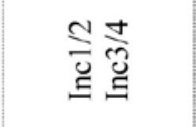 & 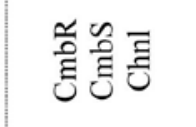 \\
\hline 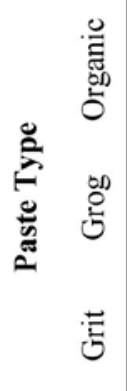 & $\begin{array}{l}\stackrel{0}{\circ} \\
\stackrel{0}{0} \\
\stackrel{0}{0} \\
\stackrel{0}{\circ}\end{array}$ & $\begin{array}{l}\stackrel{0}{0} \\
\stackrel{8}{0} \\
\stackrel{0}{0} \\
\stackrel{0}{\circ}\end{array}$ & $\begin{array}{l}\stackrel{\circ}{\circ} \\
\stackrel{\circ}{\circ} \\
\stackrel{\circ}{\circ}\end{array}$ & $\begin{array}{l}\stackrel{0}{2} \\
\stackrel{2}{2} \\
\stackrel{0}{0} \\
\infty \\
\infty\end{array}$ & $\begin{array}{l}\stackrel{\circ}{0} \\
\stackrel{\circ}{\infty} \\
\frac{\infty}{\infty} \\
\frac{\circ}{\circ}\end{array}$ & 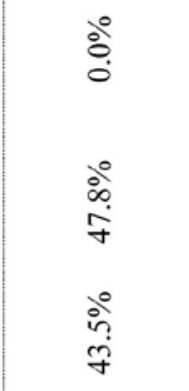 & 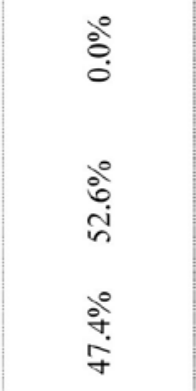 & 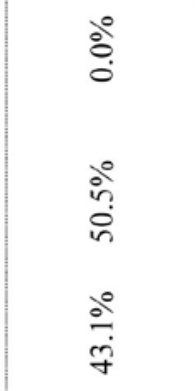 \\
\hline z & $\stackrel{ \pm}{=}$ & $\infty$ & $\overline{6}$ & $\approx$ & $=$ & $\stackrel{\sim}{\sim}$ & $\stackrel{\infty}{n}$ & $\stackrel{8}{ }$ \\
\hline 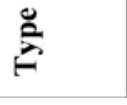 & $\frac{0}{n}$ & $\overline{\bar{n}}$ & $\frac{a}{n}$ & $\frac{m}{n}$ & $\frac{\Delta}{n}$ & $\frac{n}{n}$ & $\frac{0}{n}$ & $\frac{N}{n}$ \\
\hline
\end{tabular}




\section{Ceramic Seriation}

Seriation of body sherd and rim types in the Survey assemblage relied upon correspondence analysis (CA) to identify patterns of association among the samples from each site (see Baxter 2003:136-138; Duff 1996; Shennan 1997:308-327). Briefly, CA calculated a weighted chi-square distance for each assemblage away from the "average" assemblage; the sum of these distances then defined the overall inertia of the dataset. I then reduced the dimensionality of the dataset to those two axes accounting for most of the variance. By plotting types and assemblages in the same co-ordinate space of these two dimensions, I identified groups with similar chi-square inertia and could observe the relative contribution of one variable to inertia in the other. Figure D.31 displays the correspondence analysis plot for the composite systematic/purposive ceramic assemblages collected from sites on survey $(n=112)$, as well as the rim and body sherd types formulated above.

Notably, this plot does not conform to the "horseshoe-shaped" distribution of ceramic assemblages found on seriated sites where relative sherd frequencies shift gradually over time (see Figure D.33 below for Diouboye). This is to be expected for samples documenting punctuated shifts in technologies of pottery production over the course of several millennia combined with palimpsest surface assemblages. Sherd types and assemblages do, however, cluster into several groups, which help to define three archaeological components in the Madina-Sadatou study area when considered alongside other sources of archaeological and historical evidence,

Figure D.31: Correspondence analysis plot of rim and body sherd types in the Survey assemblage (blue dots represent individual site assemblages)

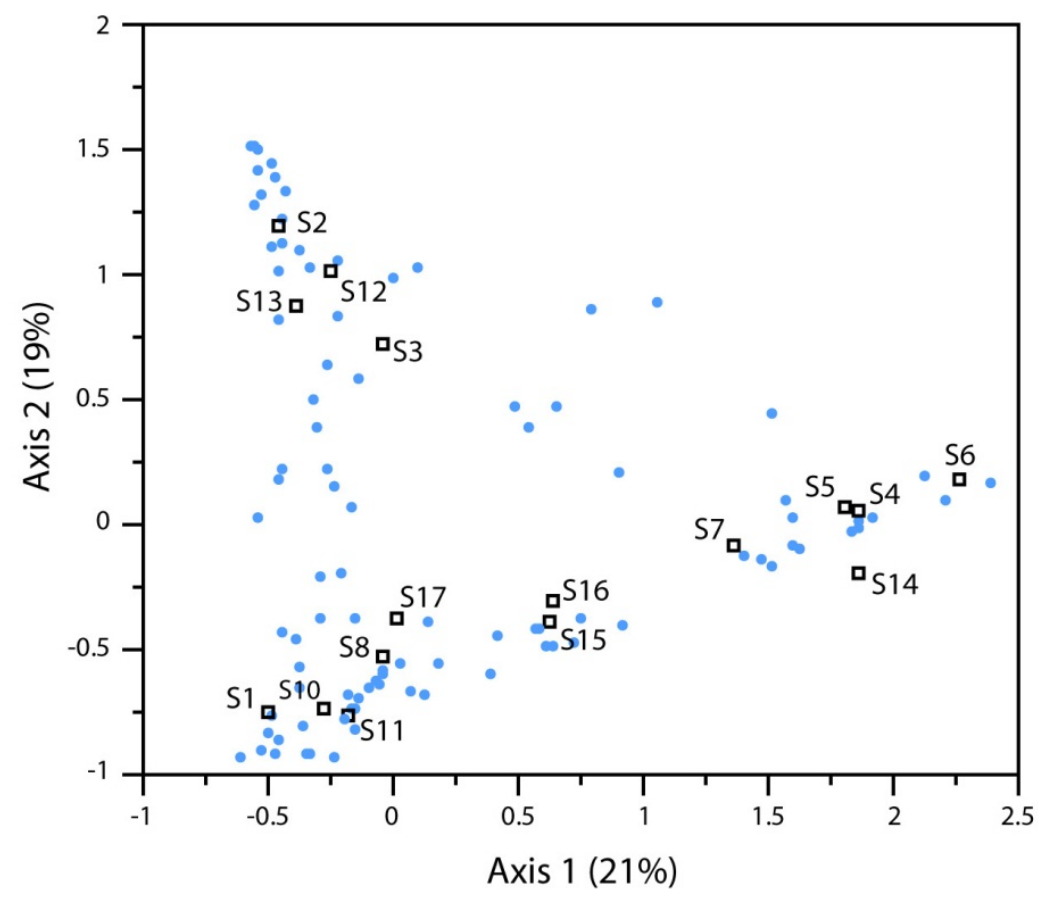


A Late Stone Age component includes sherd types S2, S3, S12, and S13 whose similar attributes highlight a relatively homogeneous operational sequence of pottery manufacture. Compositional and petrographic analyses of sherds $(n=6)$ from FAL-1036 and FAL-1075 show that potters accessed diverse clay sources (Groups 1, 2, and 3), even as they employed a consistent paste recipe with moderate to high densities of medium grit (quartz/feldspar) inclusions. Although shaping techniques are not known, the thinness of body sherds in this component (average thickness $<8 \mathrm{~mm}$ ) is more consistent with molding, than with coiling. Rim forms are generally simple or have a relatively short, thickened inflection. The limited repertoire of decorative elements includes natural roulette and/or cord-wrapped impression - the latter preferentially applied in bands around the upper body and vessel shoulder. The low frequencies of dark paste visible in sherd cross-sections point to firing within partially to fully oxidizing conditions. Sherd types from this component were not recovered from absolutely dated contexts in MadinaSadatou, but their strong association with chipped stone and groundstone artifacts, and similarity to pottery from firmly dated sites elsewhere in West Africa, clearly warrants an assignment to the Late Stone Age (see Chapter 4).

An Iron Age component includes the cluster of sherd types S4, S5, S6, S7, and S14. Compositional and petrographic analyses of excavated specimens from Diouboye $(n=14)$ revealed a more restricted use of clay from Groups 2, 3, and 4, while potters employed a diverse suite of paste recipes with grog, grit, and light organic inclusions. These types are significantly thicker (average thickness $>10 \mathrm{~mm}$ ) and more variable than those in the Late Stone Age component, hinting at a discrete shift in techniques of vessel shaping. Predominant rim forms include vertical collars and round and angular everted rims of varying length and angle. These illustrate a diverse decorative repertoire using twisted cord, knotted cord, and carved roulettes, incisions and punctates, and the application of red slip to the upper body, shoulder, and lip of selected vessels. The recovery of these sherd types in securely dated contexts at Diouboye, and similarity to Phase III ceramics from Arondo (Thiaw 1999:164-166), warrants their assignment to the Late Iron Age (ca. AD 500-1450). Indeed, the seriation of these types in stratified deposits allowed for the definition of Early/Middle Diouboye and Middle/Late Diouboye phase surface components (Chapters 4, 7-8).

An Atlantic Era component includes sherd types S1, S8, S10, S11, S17, as well as S15 and S16 whose relative inertias showed some affinity with the Iron Age component. Compositional analysis of specimens from this component $(n=11)$ revealed a restriction of clay sources to Groups 1 and 2 alongside a general shift towards the use of chaff temper. Body sherds had a moderate vessel thickness (averaging 9-10 mm). Rim forms illustrate considerable diversity - from angular and rounded everted rims to a variety of carinated, collared, and ledged forms. Decorative elements include some twisted cord roulette, but more numerous are incisions (including combing and channeling), punctates and comb impression, and the application of either slip or organic coating to the vessel surface. Possibly due to the increasing use of organic temper, dark bands visible in sherd cross-sections could also indicate firing in a relatively reducing atmosphere. Using external chronological evidence-including a radiometric date from FAL-1075, oral tradition, temporally-diagnostic smoking pipes, iron nails, and glass beads, and comparison to excavated materials at Senoudebou (Thiaw 1999:170-172) — it was possible to distinguish three ceramic phases within the Atlantic Era. As described in 
Chapter 4, these are an early Tontèko phase with types S15 and S16, a later Karé phase with types S1, S10, and S11, and, tentatively, an intermediary Tomboura phase associated with types S8 and S17.

Figure D.31 shows that the individual assemblages (sites) containing these sherd types form three or four clusters clearly associated with the aforementioned archaeological components. Although this pattern helped to identify the dominant component in each assemblage, the conflation of two, or in rare cases three, components made it necessary to evaluate ceramic and other artifact assemblages, associated features, and historical evidence on a site by site basis (see Appendix A).

\section{Diouboye Assemblage: Typology and Seriation Body Sherd Types}

The excavated Diouboye assemblage revealed only subtle temporal changes in many of the variables used to define types in the Survey assemblage (i.e., paste and inclusion type, oxidation, and body thickness). Although some decorative elements occurred more frequently with grit or grog paste (Table D.16), these relations were not significant enough to merit a hierarchical classification. The formulation of body sherd types thus depended exclusively on decorative attributes - a strategy that has proven useful for seriating ceramic assemblages from other stratified sites in West Africa (e.g., S. K. McIntosh 1995; McIntosh and Bocoum 2000; Thiaw 1999; Togola 2008).

I first examined the relative frequencies of decorative attributes across the stratified levels in each excavation unit to identify those with the clearest "battleship" distributions (see Chapter 7); these included braided cord roulette (BrS/BrD), button carved roulette ( $\mathrm{CrB} 1 / \mathrm{CrB} 2)$, chevron carved roulette (CrC), cord-wrapped stick roulette (CwS2), folded strip roulette (StF), and last, but not least, twisted cord roulette (TwC/TwS). Out of the total 19,337 large sherds recovered in excavation, I then assigned all possible specimens $(n=11,371)$ to one of these decorative types. I arbitrarily assigned those sherds with more than one decorative element to the type with the fewer total specimens.

\section{Rim Sherds}

Associations between rim form subclasses and decorative elements among the 2777 rim sherds excavated at Diouboye (Tables D.17, D.18, D.19, D.20) generated few types useful for seriation-either because they showed no directional change across stratified levels, or because they occurred in small frequencies. For this reason, I do not attempt a typology of rim sherds for Diouboye although patterns in these data do convey information about other socioeconomic dimensions of life in the village community when considered in their discrete spatio-temporal contexts (Chapters 6-9). 
Table D.16: Summary of surface treatment, thickness, and paste type by decorative elements for body sherds in the excavated Diouboye assemblage

\begin{tabular}{|c|c|c|c|c|c|c|c|c|c|c|}
\hline \multirow{3}{*}{\multicolumn{2}{|c|}{ Decorative Element }} & \multirow[t]{3}{*}{$\mathbf{N}$} & \multirow{2}{*}{\multicolumn{2}{|c|}{ Slip/Polish }} & \multirow{2}{*}{\multicolumn{2}{|c|}{$\begin{array}{l}\text { Thickness } \\
\text { (mm) }\end{array}$}} & \multirow{2}{*}{\multicolumn{4}{|c|}{ Paste Type }} \\
\hline & & & & & & & & & & \\
\hline & & & $n$ & $\%$ & $\mu$ & $\sigma$ & $n$ & $\%$ & $n$ & $\%$ \\
\hline \multicolumn{2}{|l|}{ Plain } & 4089 & 1609 & $39.3 \%$ & 9.73 & 2.76 & 1183 & $27.5 \%$ & 2849 & $66.2 \%$ \\
\hline \multicolumn{2}{|c|}{ Unknown Fiber Roulette } & 1809 & 81 & $4.5 \%$ & 10.07 & 0.06 & 159 & $8.8 \%$ & 1546 & $85.5 \%$ \\
\hline \multirow{2}{*}{$\begin{array}{l}\text { Twisted Cord } \\
\text { Roulette }\end{array}$} & $\begin{array}{l}\text { TwC, } \\
\text { TwS }\end{array}$ & 3429 & 114 & $3.3 \%$ & 10.55 & 2.69 & 84 & $2.4 \%$ & 3182 & $92.8 \%$ \\
\hline & TwD & 341 & 8 & $2.3 \%$ & 10.52 & 3.03 & 2 & $0.6 \%$ & 321 & $94.1 \%$ \\
\hline \multirow{2}{*}{$\begin{array}{l}\text { Knotted Cord } \\
\text { Roulette }\end{array}$} & $\begin{array}{l}\mathrm{BrS}, \\
\mathrm{BrD}\end{array}$ & 814 & 21 & $2.6 \%$ & 10.52 & 2.67 & 9 & $1.1 \%$ & 770 & $94.6 \%$ \\
\hline & $\mathrm{StF}$ & 2562 & 23 & $0.9 \%$ & 10.56 & 2.56 & 296 & $11.6 \%$ & 2196 & $85.7 \%$ \\
\hline \multirow{2}{*}{$\begin{array}{c}\text { Composite } \\
\text { Roulette }\end{array}$} & CwS1 & 86 & 5 & $5.8 \%$ & 10.24 & 2.68 & 9 & $10.5 \%$ & 73 & $84.9 \%$ \\
\hline & CwS2 & 2227 & 45 & $2.0 \%$ & 10.70 & 2.85 & 12 & $0.5 \%$ & 2060 & $92.5 \%$ \\
\hline \multirow{6}{*}{$\begin{array}{l}\text { Carved } \\
\text { Roulette }\end{array}$} & $\mathrm{CrC}$ & 1117 & 100 & $9.0 \%$ & 7.00 & 1.41 & 185 & $16.6 \%$ & 884 & $79.1 \%$ \\
\hline & CrG & 40 & 1 & $2.5 \%$ & 10.00 & 2.48 & 35 & $87.5 \%$ & 5 & $12.5 \%$ \\
\hline & CrT & 28 & 4 & $14.3 \%$ & 10.68 & 2.76 & 2 & $7.1 \%$ & 25 & $89.3 \%$ \\
\hline & $\mathrm{CrO}$ & 45 & 2 & $4.4 \%$ & 10.34 & 2.36 & 30 & $66.7 \%$ & 15 & $33.3 \%$ \\
\hline & CrL & 26 & 0 & $0.0 \%$ & 9.62 & 1.81 & 4 & $15.4 \%$ & 22 & $84.6 \%$ \\
\hline & $\begin{array}{l}\text { CrB1, } \\
\text { CrB2 }\end{array}$ & 1068 & 26 & $2.4 \%$ & 10.63 & 2.56 & 269 & $25.2 \%$ & 767 & $71.8 \%$ \\
\hline \multirow[b]{2}{*}{ Incision } & Inc1-4 & 170 & 60 & $35.3 \%$ & 9.09 & 2.27 & 39 & $22.9 \%$ & 113 & $66.5 \%$ \\
\hline & $\begin{array}{l}\text { CmbR, } \\
\text { CmbS, } \\
\text { Chnl }\end{array}$ & 144 & 42 & $29.2 \%$ & 9.89 & 2.28 & 35 & $24.3 \%$ & 102 & $70.8 \%$ \\
\hline \multirow[t]{2}{*}{ Impression } & $\begin{array}{l}\text { ImK, } \\
\operatorname{ImT}\end{array}$ & 24 & 6 & $25.0 \%$ & 10.50 & 2.64 & 0 & $0.0 \%$ & 24 & $100.0 \%$ \\
\hline & $\operatorname{ImS}$ & 13 & 6 & $46.2 \%$ & 8.77 & 1.48 & 2 & $15.4 \%$ & 10 & $76.9 \%$ \\
\hline Punctate & $\begin{array}{l}\text { PnC, } \\
\text { PnN }\end{array}$ & 99 & 9 & $9.1 \%$ & 9.32 & 2.41 & 2 & $2.0 \%$ & 92 & $92.9 \%$ \\
\hline \multirow{2}{*}{ Additive } & Add1-3 & 7 & 0 & $0.0 \%$ & 10.60 & 0.55 & 7 & $100.0 \%$ & 0 & $0.0 \%$ \\
\hline & Add4 & 3 & 2 & $66.7 \%$ & 8.67 & 3.79 & 1 & $33.3 \%$ & 2 & $66.7 \%$ \\
\hline
\end{tabular}


Table D.17: Summary of lip decoration and surface treatment by rim form subclass for rim sherds in the excavated Diouboye assemblage

\begin{tabular}{|c|c|c|c|c|c|c|c|c|c|c|c|c|}
\hline \multirow{3}{*}{\multicolumn{3}{|c|}{$\begin{array}{r}\text { Rim Class } \\
\text { Subclass } \\
n \\
\end{array}$}} & \multicolumn{6}{|c|}{ Lip Decoration } & \multicolumn{4}{|c|}{ Surface Slip/Polish } \\
\hline & & & \multicolumn{2}{|c|}{$\mathrm{PnC}$} & \multicolumn{2}{|c|}{$\mathrm{PnF}$} & \multicolumn{2}{|c|}{ Bevel } & \multicolumn{2}{|c|}{ Lip } & \multicolumn{2}{|c|}{ Body } \\
\hline & & & $n$ & $\%$ & $n$ & $\%$ & $n$ & $\%$ & $n$ & $\%$ & $n$ & $\%$ \\
\hline Simple & S & 426 & 8 & $1.9 \%$ & 1 & $0.2 \%$ & 104 & $24.4 \%$ & 126 & $29.6 \%$ & 114 & $26.8 \%$ \\
\hline \multirow{5}{*}{ Inflected } & ErR & 323 & 7 & $2.2 \%$ & 1 & $0.3 \%$ & 10 & $3.1 \%$ & 112 & $34.7 \%$ & 89 & $27.6 \%$ \\
\hline & EfR & 67 & 5 & $7.5 \%$ & 1 & $1.5 \%$ & 5 & $7.5 \%$ & 20 & $29.9 \%$ & 23 & $34.3 \%$ \\
\hline & ErA & 72 & 2 & $2.8 \%$ & 0 & $0.0 \%$ & 6 & $8.3 \%$ & 34 & $47.2 \%$ & 28 & $38.9 \%$ \\
\hline & EfA & 32 & 0 & $0.0 \%$ & 0 & $0.0 \%$ & 1 & $3.1 \%$ & 16 & $50.0 \%$ & 15 & $46.9 \%$ \\
\hline & $\mathrm{EvC}$ & 217 & 17 & $7.8 \%$ & 13 & $6.0 \%$ & 52 & $24.0 \%$ & 119 & $54.8 \%$ & 92 & $42.4 \%$ \\
\hline \multirow{4}{*}{ Collared } & ThC & 2 & 0 & $0.0 \%$ & 0 & $0.0 \%$ & 2 & $100.0 \%$ & 1 & $50.0 \%$ & 0 & $0.0 \%$ \\
\hline & LC & 3 & 0 & $0.0 \%$ & 0 & $0.0 \%$ & 0 & $0.0 \%$ & 0 & $0.0 \%$ & 0 & $0.0 \%$ \\
\hline & Bd1 & 37 & 1 & $2.7 \%$ & 0 & $0.0 \%$ & 5 & $13.5 \%$ & 24 & $64.9 \%$ & 15 & $40.5 \%$ \\
\hline & $\mathrm{Bd} 2$ & 36 & 1 & $2.8 \%$ & 0 & $0.0 \%$ & 1 & $2.8 \%$ & 22 & $61.1 \%$ & 22 & $61.1 \%$ \\
\hline \multirow{2}{*}{ Ledged } & Ldg1 & 8 & 1 & $12.5 \%$ & 0 & $0.0 \%$ & 2 & $25.0 \%$ & 2 & $25.0 \%$ & 3 & $37.5 \%$ \\
\hline & Ldg2 & 4 & 0 & $0.0 \%$ & 0 & $0.0 \%$ & 0 & $0.0 \%$ & 0 & $0.0 \%$ & 0 & $0.0 \%$ \\
\hline
\end{tabular}

Table D.18: Summary of fiber roulette decoration by rim form subclass for rim sherds in the excavated Diouboye assemblage

\begin{tabular}{|c|c|c|c|c|c|c|c|c|c|c|}
\hline \multicolumn{3}{|c|}{ Rim Class } & \multirow{2}{*}{\multicolumn{2}{|c|}{$\begin{array}{c}\text { Twisted Cord } \\
\text { Roulette } \\
\text { TwC/TwS }\end{array}$}} & \multicolumn{4}{|c|}{ Knotted Cord Roulette } & \multirow{2}{*}{\multicolumn{2}{|c|}{$\begin{array}{c}\text { Composite } \\
\text { Roulette } \\
\text { CwS1/CwS2 }\end{array}$}} \\
\hline \multirow{2}{*}{\multicolumn{3}{|c|}{$\begin{array}{c}\text { Subclass } \\
n\end{array}$}} & & & \multicolumn{2}{|c|}{$\mathrm{BrS} / \mathrm{Brd}$} & \multicolumn{2}{|c|}{ Stf } & & \\
\hline & & & $n$ & $\%$ & $n$ & $\%$ & $n$ & $\%$ & $n$ & $\%$ \\
\hline Simple & $\mathrm{S}$ & 426 & 75 & $17.6 \%$ & 18 & $4.2 \%$ & 21 & $4.9 \%$ & 18 & $4.2 \%$ \\
\hline \multirow{5}{*}{ Inflected } & ErR & 323 & 24 & $7.4 \%$ & 8 & $2.5 \%$ & 13 & $4.0 \%$ & 11 & $3.4 \%$ \\
\hline & EfR & 67 & 7 & $10.4 \%$ & 1 & $1.5 \%$ & 1 & $1.5 \%$ & 4 & $6.0 \%$ \\
\hline & ErA & 72 & 4 & $5.6 \%$ & 2 & $2.8 \%$ & 1 & $1.4 \%$ & 4 & $5.6 \%$ \\
\hline & EfA & 32 & 7 & $21.9 \%$ & 1 & $3.1 \%$ & 0 & $0.0 \%$ & 4 & $12.5 \%$ \\
\hline & Ev2 & 217 & 39 & $18.0 \%$ & 5 & $2.3 \%$ & 13 & $6.0 \%$ & 11 & $5.1 \%$ \\
\hline \multirow{4}{*}{ Collared } & ThC & 2 & 0 & $0.0 \%$ & 0 & $0.0 \%$ & 0 & $0.0 \%$ & 0 & $0.0 \%$ \\
\hline & LC & 3 & 0 & $0.0 \%$ & 0 & $0.0 \%$ & 0 & $0.0 \%$ & 0 & $0.0 \%$ \\
\hline & Bd1 & 37 & 5 & $13.5 \%$ & 0 & $0.0 \%$ & 0 & $0.0 \%$ & 1 & $2.7 \%$ \\
\hline & $\mathrm{Bd} 2$ & 36 & 2 & $5.6 \%$ & 0 & $0.0 \%$ & 1 & $2.8 \%$ & 0 & $0.0 \%$ \\
\hline \multirow{2}{*}{ Ledged } & Ldg1 & 8 & 1 & $12.5 \%$ & 0 & $0.0 \%$ & 0 & $0.0 \%$ & 0 & $0.0 \%$ \\
\hline & Ldg2 & 4 & 0 & $0.0 \%$ & 0 & $0.0 \%$ & 0 & $0.0 \%$ & 0 & $0.0 \%$ \\
\hline
\end{tabular}


Table D.19: Summary of carved roulette decoration by rim form subclass for rim sherds in the excavated Diouboye assemblage

\begin{tabular}{|c|c|c|c|c|c|c|c|c|c|c|}
\hline \multirow{2}{*}{\multicolumn{3}{|c|}{ Rim Class }} & \multicolumn{8}{|c|}{ Carved Roulette } \\
\hline & & & \multicolumn{2}{|c|}{$\mathrm{CrC}$} & \multicolumn{2}{|c|}{$\begin{array}{l}\text { CrD, CrG, } \\
\text { CrT, CrL }\end{array}$} & \multicolumn{2}{|c|}{$\mathrm{CrO}$} & \multicolumn{2}{|c|}{ CrB1, CrB2 } \\
\hline \multicolumn{3}{|c|}{$\begin{array}{c}\text { Subclass } \\
n\end{array}$} & $n$ & $\%$ & $n$ & $\%$ & $n$ & $\%$ & $n$ & $\%$ \\
\hline Simple & $\mathrm{S}$ & 426 & 30 & $7.0 \%$ & 6 & $1.4 \%$ & 18 & $4.2 \%$ & 33 & $7.7 \%$ \\
\hline \multirow{6}{*}{ Inflected } & ErR & 323 & 24 & $7.4 \%$ & 2 & $0.6 \%$ & 0 & $0.0 \%$ & 4 & $1.2 \%$ \\
\hline & EfR & 67 & 4 & $6.0 \%$ & 0 & $0.0 \%$ & 0 & $0.0 \%$ & 0 & $0.0 \%$ \\
\hline & ErA & 72 & 8 & $11.1 \%$ & 2 & $2.8 \%$ & 0 & $0.0 \%$ & 0 & $0.0 \%$ \\
\hline & EfA & 32 & 3 & $9.4 \%$ & 0 & $0.0 \%$ & 0 & $0.0 \%$ & 0 & $0.0 \%$ \\
\hline & Ev1 & 217 & 17 & $7.8 \%$ & 5 & $2.3 \%$ & 10 & $4.6 \%$ & 7 & $3.2 \%$ \\
\hline & Ev2 & 2 & 2 & $100.0 \%$ & 0 & $0.0 \%$ & 0 & $0.0 \%$ & 0 & $0.0 \%$ \\
\hline \multirow{4}{*}{ Collared } & ThC & 3 & 0 & $0.0 \%$ & 0 & $0.0 \%$ & 0 & $0.0 \%$ & 0 & $0.0 \%$ \\
\hline & LC & 37 & 6 & $16.2 \%$ & 1 & $2.7 \%$ & 0 & $0.0 \%$ & 2 & $5.4 \%$ \\
\hline & Bd1 & 36 & 3 & $8.3 \%$ & 1 & $2.8 \%$ & 1 & $2.8 \%$ & 1 & $2.8 \%$ \\
\hline & $\mathrm{Bd} 2$ & 8 & 0 & $0.0 \%$ & 0 & $0.0 \%$ & 1 & $12.5 \%$ & 1 & $12.5 \%$ \\
\hline \multirow{2}{*}{ Ledged } & Ldg1 & 4 & 0 & $0.0 \%$ & 0 & $0.0 \%$ & 0 & $0.0 \%$ & 0 & $0.0 \%$ \\
\hline & Ldg2 & 426 & 30 & $7.0 \%$ & 6 & $1.4 \%$ & 18 & $4.2 \%$ & 33 & $7.7 \%$ \\
\hline
\end{tabular}

Table D.20: Summary of impressed and incised decoration by rim form subclass for rim sherds in the excavated Diouboye assemblage

\begin{tabular}{|c|c|c|c|c|c|c|c|c|c|c|c|c|}
\hline \multirow{2}{*}{\multicolumn{3}{|c|}{ Rim Class }} & \multirow{2}{*}{\multicolumn{2}{|c|}{$\begin{array}{l}\text { Impression } \\
\text { ImK, ImT }\end{array}$}} & \multicolumn{4}{|c|}{ Punctate } & \multicolumn{4}{|c|}{ Incision } \\
\hline & & & & & \multicolumn{2}{|c|}{$\mathrm{PnR}$} & \multicolumn{2}{|c|}{ PnC, PnN } & \multicolumn{2}{|c|}{ Inc1-4 } & \multicolumn{2}{|c|}{$\begin{array}{c}\text { CmbS, } \\
\text { CmbR, Chnl }\end{array}$} \\
\hline & \multicolumn{2}{|c|}{$\begin{array}{c}\text { Subclass } \\
n\end{array}$} & $n$ & $\%$ & $n$ & $\%$ & $n$ & $\%$ & $n$ & $\%$ & $n$ & $\%$ \\
\hline Simple & $\mathrm{S}$ & 426 & 3 & $0.7 \%$ & 1 & $0.2 \%$ & 27 & $6.3 \%$ & 47 & $11.0 \%$ & 18 & $4.2 \%$ \\
\hline \multirow{6}{*}{ Inflected } & ErR & 323 & 21 & $6.5 \%$ & 2 & $0.6 \%$ & 27 & $8.4 \%$ & 8 & $2.5 \%$ & 6 & $1.9 \%$ \\
\hline & EfR & 67 & 4 & $6.0 \%$ & 0 & $0.0 \%$ & 9 & $13.4 \%$ & 3 & $4.5 \%$ & 3 & $4.5 \%$ \\
\hline & ErA & 72 & 8 & $11.1 \%$ & 0 & $0.0 \%$ & 5 & $6.9 \%$ & 10 & $13.9 \%$ & 2 & $2.8 \%$ \\
\hline & EfA & 32 & 4 & $12.5 \%$ & 0 & $0.0 \%$ & 4 & $12.5 \%$ & 8 & $25.0 \%$ & 0 & $0.0 \%$ \\
\hline & Ev1 & 217 & 13 & $6.0 \%$ & 1 & $0.5 \%$ & 19 & $8.8 \%$ & 26 & $12.0 \%$ & 11 & $5.1 \%$ \\
\hline & Ev2 & 2 & 0 & $0.0 \%$ & 0 & $0.0 \%$ & 0 & $0.0 \%$ & 0 & $0.0 \%$ & 0 & $0.0 \%$ \\
\hline \multirow{4}{*}{ Collared } & ThC & 3 & 0 & $0.0 \%$ & 0 & $0.0 \%$ & 0 & $0.0 \%$ & 0 & $0.0 \%$ & 0 & $0.0 \%$ \\
\hline & $\mathrm{LC}$ & 37 & 8 & $21.6 \%$ & 1 & $2.7 \%$ & 11 & $29.7 \%$ & 2 & $5.4 \%$ & 1 & $2.7 \%$ \\
\hline & Bd1 & 36 & 1 & $2.8 \%$ & 0 & $0.0 \%$ & 2 & $5.6 \%$ & 13 & $36.1 \%$ & 2 & $5.6 \%$ \\
\hline & $\mathrm{Bd} 2$ & 8 & 0 & $0.0 \%$ & 0 & $0.0 \%$ & 1 & $12.5 \%$ & 0 & $0.0 \%$ & 0 & $0.0 \%$ \\
\hline \multirow{2}{*}{ Ledged } & Ldg1 & 4 & 0 & $0.0 \%$ & 0 & $0.0 \%$ & 0 & $0.0 \%$ & 1 & $25.0 \%$ & 0 & $0.0 \%$ \\
\hline & Ldg2 & 426 & 3 & $0.7 \%$ & 1 & $0.2 \%$ & 27 & $6.3 \%$ & 47 & $11.0 \%$ & 18 & $4.2 \%$ \\
\hline
\end{tabular}




\section{Ceramic Seriation}

Following the procedure described above for the Survey assemblage, I employed correspondence analysis to identify those substrata having similar type frequencies within and between excavated sequences at Diouboye (Baxter 2003:204-206; Duff 1996). As shown in Figure D.32, the first two dimensions defined through CA accounted for a stepwise reduction in the chi-square inertia of the Diouboye body sherd assemblage by $61 \%$ and 24\%, respectively. The individual cases (substrata) illustrate a clear "horseshoe" pattern indicative of seriation according to the variables (sherd types) plotted in the same coordinate space. I then used K-means cluster analysis to parse the substrata into four fairly discrete groupings. With only four minor exceptions, the resulting groups were consistent with the actual stratigraphic relations between deposits in each excavation unit.

\section{Figure D.32: Correspondence analysis plot of body sherd types and substrata from Diouboye showing confidence interval ellipses (90\%) for four groups partitioned through $\mathrm{K}$-means cluster analysis}

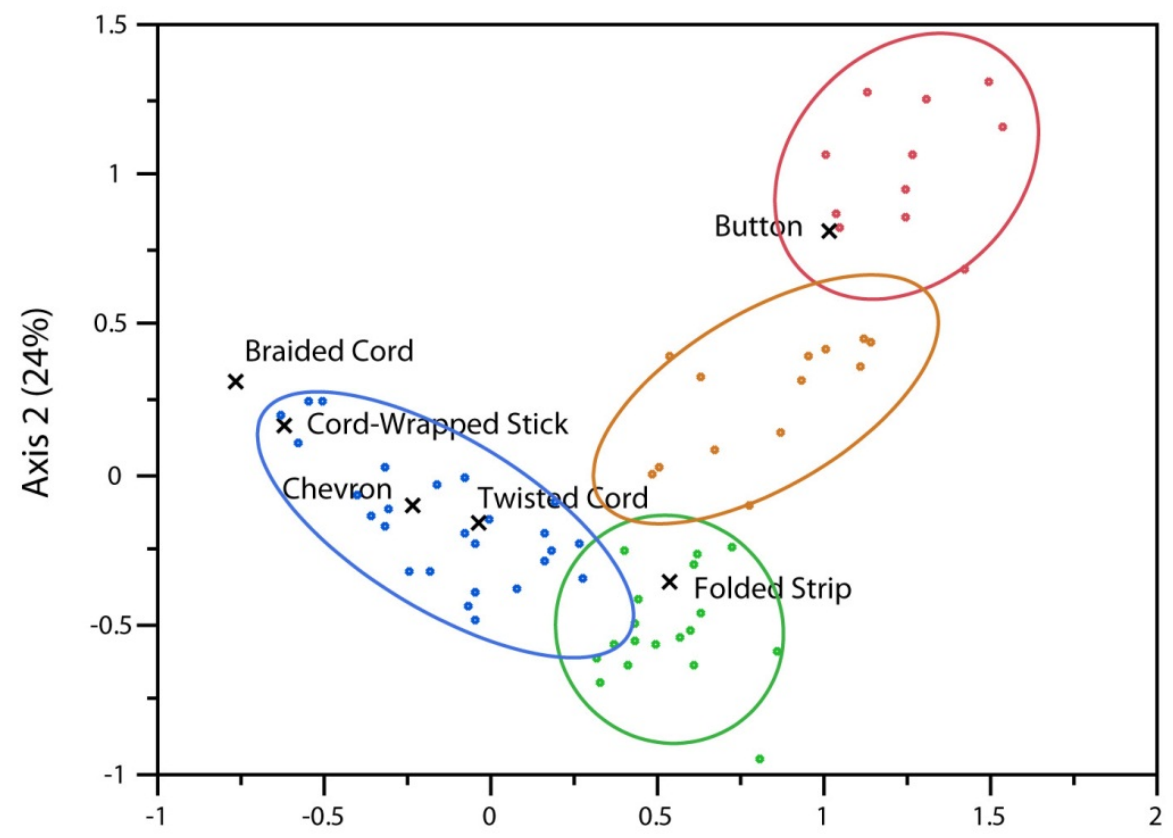

Axis $1(61 \%)$

This ceramic seriation yielded remarkably coherent results considering the marked variability in sherd sample sizes and the potential for bioturbation and other postdepositional processes such as pit digging and earthen wall construction, which can obscure temporal trends across substrata. In order to test the robustness of this initial seriation and mitigate any possible depositional biases, I also undertook a correspondence analysis for the larger sherd samples encompassed by entire strata in each excavation unit. The results, illustrated in Figure D.33, were nearly identical to those obtained for the substrata, further highlighting the appropriateness of this ceramic chronology for 
comparing these and other contexts across Diouboye. Based upon the contextual information and absolute radiocarbon dates presented in Chapters 7, I have labeled these the Early Diouboye A, Early Diouboye B, Middle Diouboye, and Late Diouboye phases.

Figure D.33: Correspondence analysis plot of body sherd types and strata from Diouboye showing confidence interval ellipses (90\%) for four phases partitioned through K-means cluster analysis

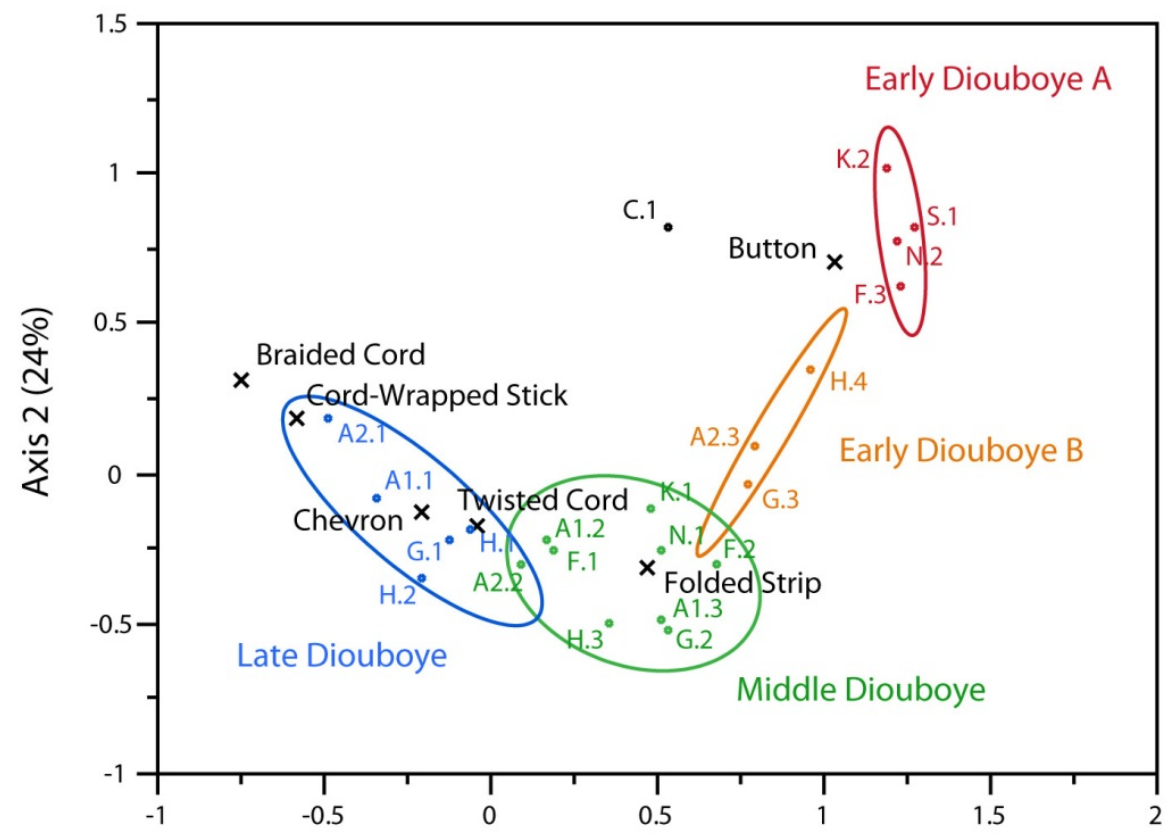

Axis 1 (67\%)

\section{Towards a Functional Typology}

Earthenware pottery vessels can play a variety of economic, political, and ritual roles as they move in and out of different social contexts, but I focus here on the more-orless practical functions shaped, literally, by their overall form. Ethnographic and experimental research have shown that the physical properties of a pot-including overall size, shape, and proportions between volume and orifice diameter, as well as rim form, decoration, and paste type-both constrain and contribute to its potential uses (Braun 1983; Henrickson and McDonald 1983; Rice 1987:211-226). In line with ethnographic studies of pottery across West Africa (e.g., David and Hennig 1972; B. Diop 2000; Frank 1998; Gallay 1970; Gelbert 1999, 2003; Guèye 1998; Sall 2005), archaeologist Susan McIntosh (1995:157-164) has suggested that variables of overall size and orifice restriction mainly distinguish among vessels used for storage, water transport, cooking, serving, washing and tanning, brewing beer, or specialized ritual performances. Given the paucity of complete vessels in the Diouboye and Survey assemblages, it becomes necessary to develop a working typology of vessel functions based from rim sherds. 


\section{Vessel Form and Size}

The low numbers of distinctive base sherds suggest that most pots and jars had a globular form, an interpretation that finds support from the few reconstructable vessels recovered during excavation. While relative orifice restriction (the ratio of orifice diameter to maximum body diameter) provides a good indication of functional vessel morphology, it was rarely possible to take this measure from single sherds. For this reason, I employed the shoulder angle on many rim sherds as a proxy for three main vessel forms identified as modal values in both the Diouboye and Survey assemblages (Figures D.34-D.35). Bowls have unrestricted, usually simple, rims with a shoulder angle of $85^{\circ}$ or less. Pots have lightly restricted orifices with a shoulder angle of $90-115^{\circ}$ and jars have severely restricted orifices with a shoulder angle of $120^{\circ}$ or greater. These modalities in shoulder angle hold more-or-less constant even as shoulder curvature and thickness vary across major rim classes and vessel size classes.

Vessel size also contributes to possible functions. Again, with few complete vessels providing measurements of height and maximal diameter, I found orifice diameter to provide an effective measure of vessel size when plotted against shoulder angle (Figures D.34-D.35). Although body and rim thickness also correlated weakly with shoulder angle, these size measures appeared to depend more on rim class and underlying formation techniques than on the overall vessel morphology.

Figure D.34: Bivariate plot of orifice diameter and shoulder angle for rim sherds in the Survey assemblage $(n=400)$

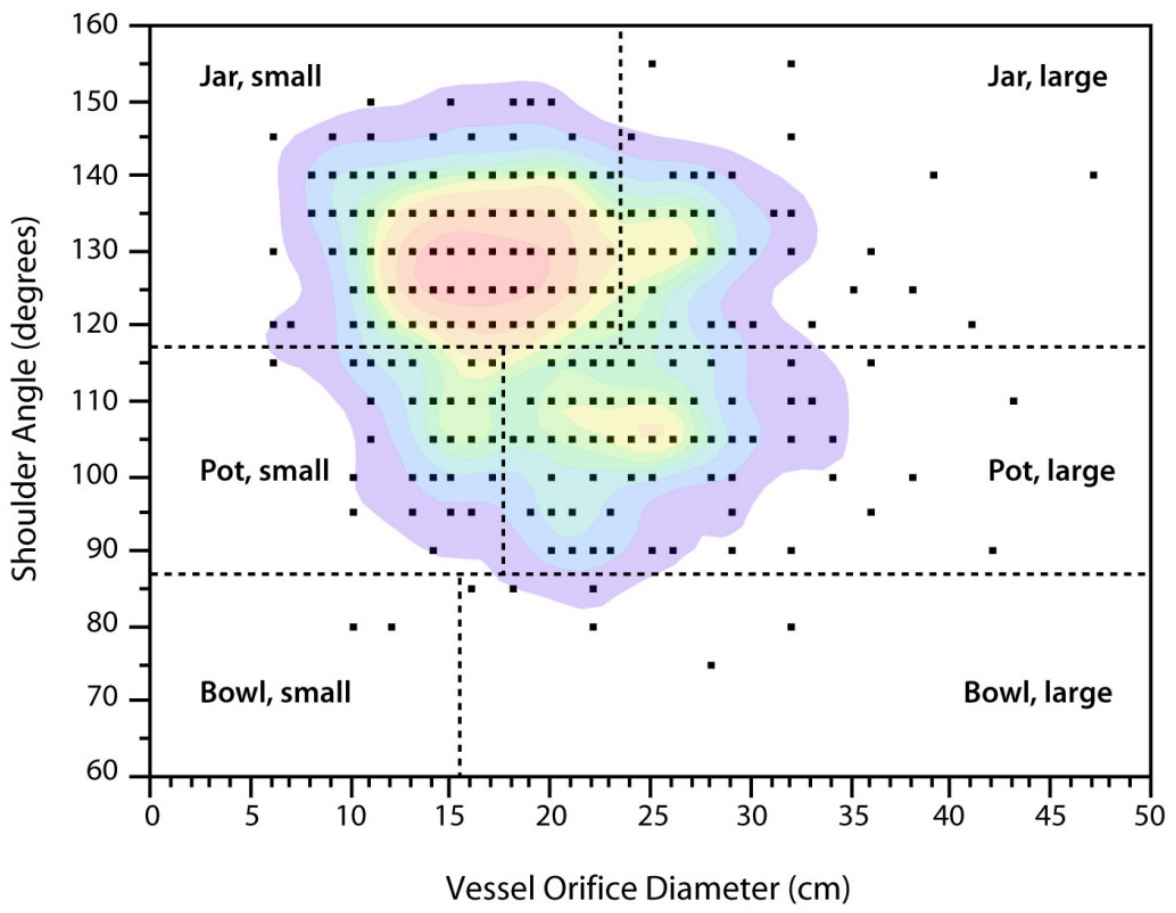


Figure D.35: Bivariate plot of orifice diameter and shoulder angle for rim sherds in the Diouboye assemblage $(n=728)$

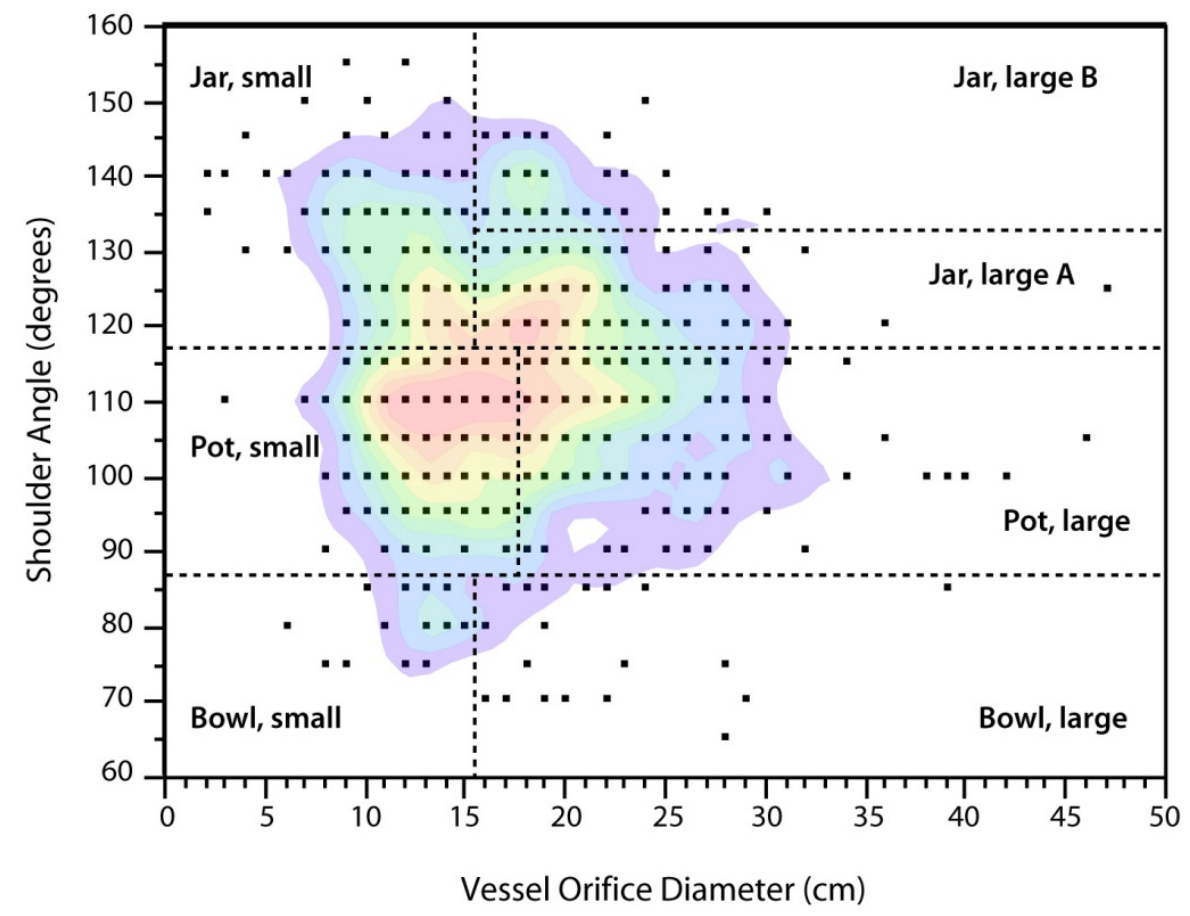

\section{Vessel Function}

A functional typology based on vessel form/size gains further support from associations with different rim subclasses (Figures D.36-D.37). In both the Survey and Diouboye assemblages, unrestricted bowl forms generally have unelaborated simple rims. In contrast, pots and jars boast collared and inflected rims able to provide additional grip, prevent spillage, or support a lid or another vessel (such as a couscous colander). Across the Survey assemblage, for example, lightly restricted pots with large and small openings accounted for most of the collared and ledged rims, while strongly restricted jars, again both large and small, mainly have inflected rims. The distinction between pots and jars in the Diouboye assemblage is more subtle, but beaded (Bd1/Bd2) and inflected rims are associated with small and large jars, while simple and vertical collar (EvC) rims are more frequent on small and large pots and, interestingly enough, large globular jars.

Following the above ethnographic studies, I propose the following vessel typology based on rim sherds in the Madina-Sadatou assemblages (and see Chapter 6).

Bowls

In both the Survey and Diouboye assemblages, I distinguished large and small bowls with estimated orifice diameters of greater or less than 15 centimeters, respectively (Figures D.34-D.35). With their unrestricted openings and unelaborated rims, small bowls could have been used for serving food, while large bowls could have been used for washing, tanning, or perhaps steaming grain (in the form of colanders). The low numbers 
of bowl sherds suggests that many tasks requiring a dish, but no application of heat, simply made use of organic calabashes as people living in the study area do today.

Pots

Similarly, I distinguished large and small pots within each assemblage according to estimated orifice diameters of greater or less than 18 centimeters, respectively (Figures D.34-D.35). The lightly restricted openings of pots work to keep their contents held in, even as they allow relatively unimpeded access to those contents. Although this makes pots useful for a variety of purposes, ethnographic observations in West Africa suggest that people in Madina-Sadatou would have used small ones for cooking sauces and/or cereals and large ones for cooking cereals or stews, brewing beer, washing, soaking, or even water storage.

Figure D.36: Relative abundance of rim subclass by vessel form for rim sherds in the Survey assemblage

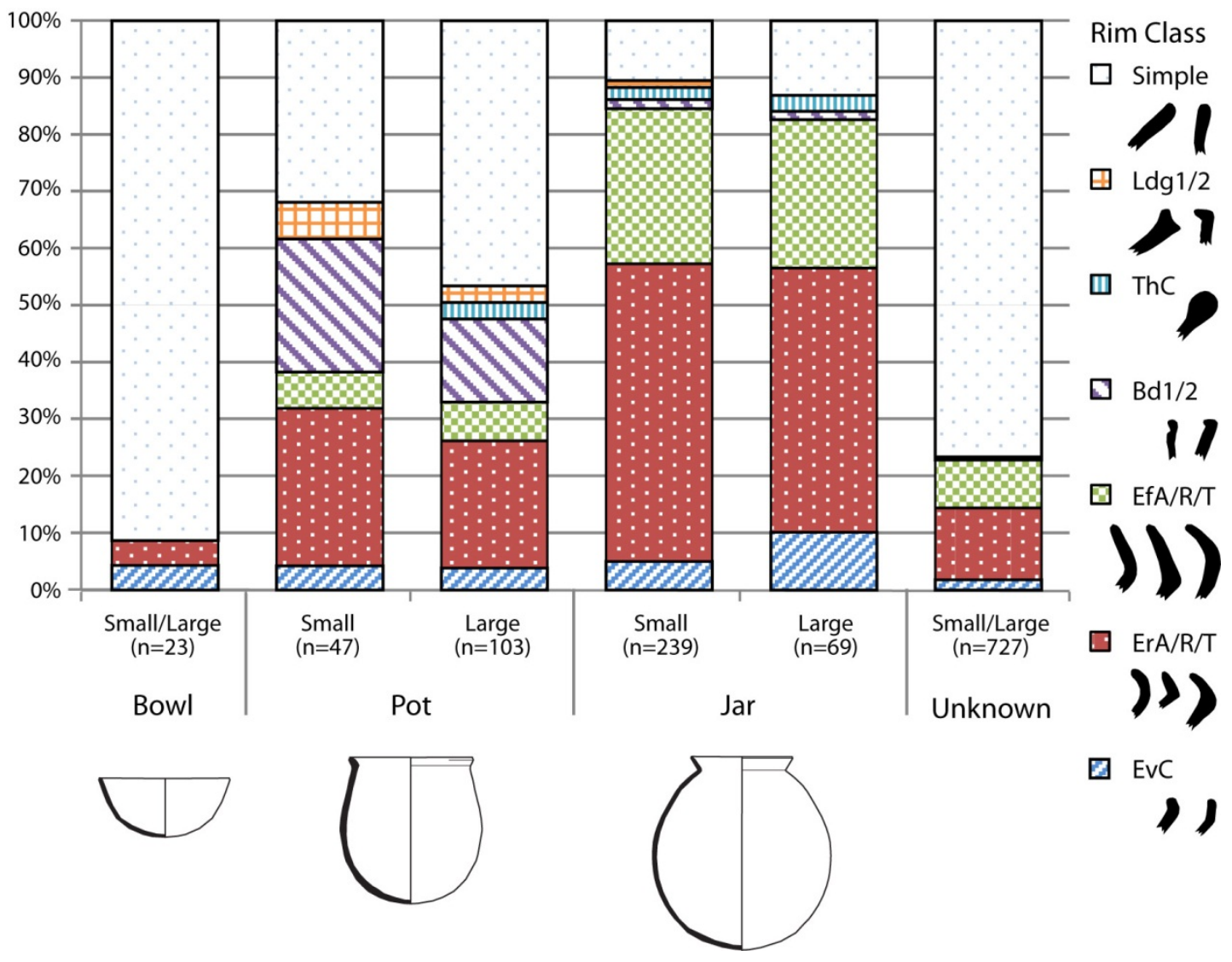


Figure D.37: Relative abundance of rim subclass by vessel form for rim sherds in the excavated Diouboye assemblage

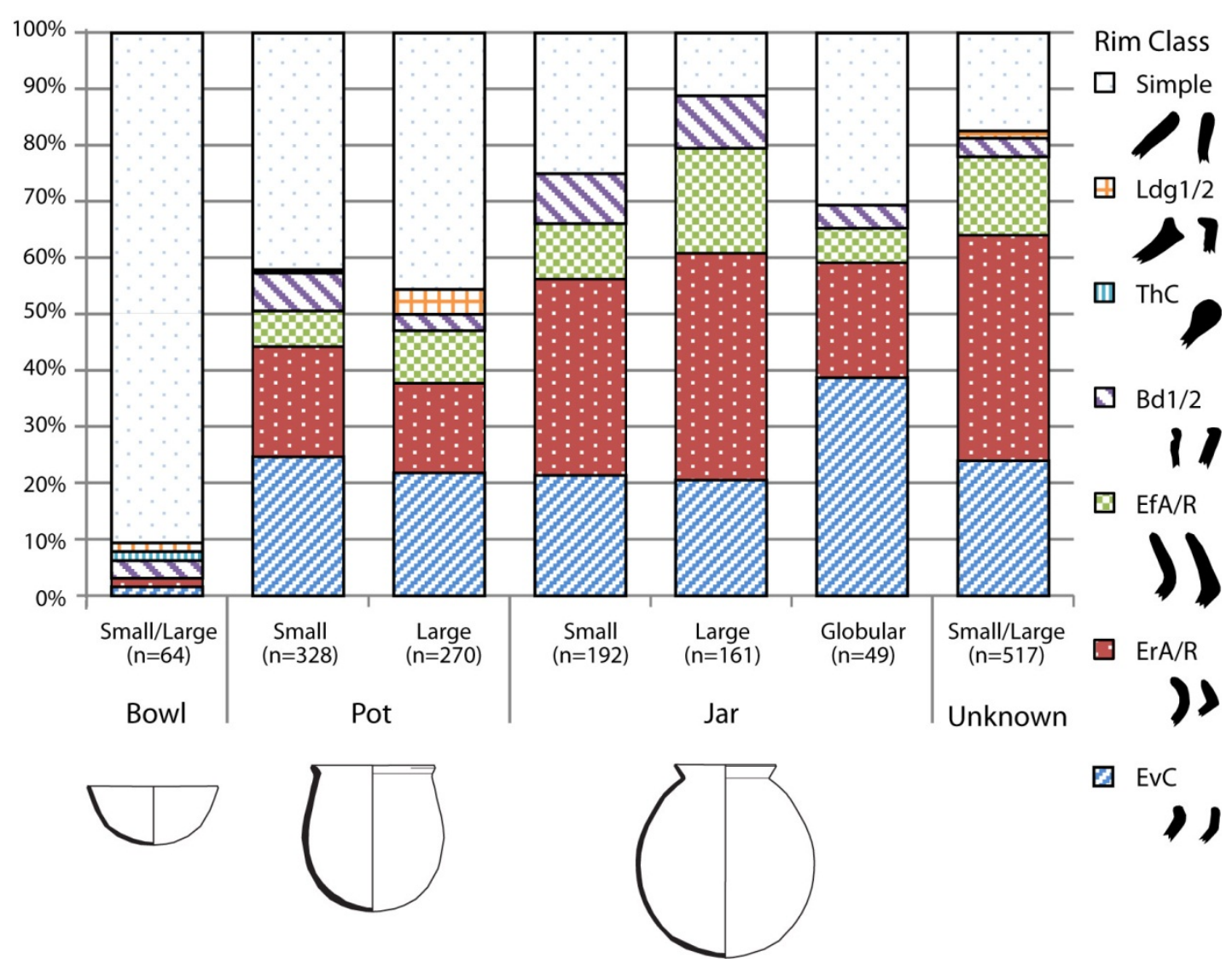

Jars

Jars exhibited greater variability across the Diouboye and Survey assemblages. In the latter assemblage, it was possible to classify small and large jars with orifice diameters less or greater than 23 centimeters, respectively (Figure D.34). In the Diouboye assemblage, however, I drew this distinction at an orifice diameter of only 15 centimeters and further identified a subset of the large globular jars whose shoulder angle of $135-160^{\circ}$ gave them a strongly restricted opening. With narrower openings than pots, jars further protect their contents from outside perturbations. These vessels are multifunctional, but ethnographic observations suggest that people probably used small jars for transporting water or possibly cooking and large ones for water storage or beer production. In this regard, the everted and flared rims found associated with jar forms would have been ideal for supporting a cover. 


\section{APPENDIX E LITHIC ARTIFACT ANALYSIS AND CLASSIFICATION}

Chipped stone and groundstone artifacts have historically received greater scholarly attention than other aspects of archaeology along the Falémé River. During the early $20^{\text {th }}$ century $\mathrm{AD}$, the Falémé region became known, at least among prehistorians, for its preponderance of polished hematite celts called hachettes (Laforgue 1924). Given their similarity to groundstone implements found elsewhere across West Africa, these artifacts quickly and deeply ensconced the region into literature on the Late Stone Age (LSA) or "Neolithic" tradition (Bocoum et al. 2002:49-50; Laforgue 1925; Lame 2000; Zeltner 1916). Archaeological interest in the Falémé River continued to focus on stone tool sites, with an archaeological mission by Raymond Corbeil and Raymond Mauny in 1948 and later expeditions by scholars from IFAN through the 1960s and 1970s (Guitat 1970, 1972; Ravisé 1975). This research program culminated in the 1980s with survey and excavations by Abdoulaye Camara (2000; Camara and Duboscq 1984, 1987, 1990), who defined a sequence of chipped stone industries dating back to the mid Pleistocene.

Beyond the recognition of hachettes, the Holocene lithic industries of the Falémé region nevertheless remain poorly defined, particularly in comparison to those found in the western Senegambia (Corbeil et al. 1948; Lame 2000; Ravisé 1970). Most summaries simply note the abundance of chipped quartz and polished stone tools, the dearth of microliths, and in many cases the presence of associated Iron Age pottery and metallurgical artifacts (Bocoum et al. 2002:49). The latter materials have been assumed to represent the contamination of earlier LSA assemblages, but it remains possible that people living along the Falémé River continued to make use of stone tools through the early-second millennium AD. While my express goal in this appendix is to outline the socioeconomic organization of lithic technologies at Diouboye, I offer a sketch of chipped stone and groundstone industries in the Madina-Sadatou study area as a baseline for future research.

Regarding lithic technology at Diouboye, my analysis had two main objectives: (1) to reconstruct the operational sequence(s) of materials and techniques involved in chipped stone and groundstone tool production, use, and curation; and (2) to identify the logistical and social parameters that most likely informed technical choices within these operational sequences.

In pursuing these objectives, I found it necessary to compare the lithic assemblage from Diouboye to those from three surveyed sites with LSA components (FAL-1022, FAL-1062, FAL-1075). First of all, terre pisé and wattle-and-daub architecture at Diouboye, which involved digging borrow pits and carrying sediment into the village, could have been responsible for the secondary deposition of lithic artifacts. In the absence of unambiguous lithic workshops, it was essential to demonstrate robust differences between LSA sites and Diouboye-differences most parsimoniously explained by 
primary deposition during the Iron Age occupation. Second, a comparison of lithic technology across two socioeconomic systems occupying the same physical landscape could shed light on the relative contribution of logistical and social parameters on the operational sequences evidenced at Diouboye.

As discussed in Appendix D, a technological approach to chipped stone and groundstone artifacts offers a middle-range methodology capable of accommodating potential diversity in the Diouboye and LSA assemblages and relating them to past technical choices. Below I briefly describe the methodology, including the sampling, sorting, and analytical procedures, applied to lithic artifacts recovered during survey and excavation in the Madina-Sadatou study area. In the following two sections, I work to reconstruct the operational sequences of chipped stone and groundstone production and use at Diouboye and the three LSA sites chosen for comparison. After addressing the objectives outlined above, I reserve discussion of the specific contexts and the spatiotemporal organization of these lithic technologies for Chapters 4-9.

\section{METHODOLOGY}

As a middle-range methodology, the technological approach is, not surprisingly, well-suited to the study of lithic artifact assemblages (Leroi-Gourhan 1943). This is due to the fact that lithic technologies are reductive; that is, the continual generation of debris - including cores, flakes, shatter, and so forth-potentially documents each technical act in the production and curation of a stone tool. It follows that the raw materials, size, and impact traces on these pieces, and their relative frequencies within an assemblage, provide a basis for inferring the materials, tools, and gestures involved in past operational sequences (J. L. Adams 2002; Andrefsky 2005; Inizan et al. 1995). In this regard, experimental and ethnoarchaeological studies can deliver crucial insights on the practical and social considerations underlying technical choices for raw material procurement, tool production, and even use/repair (Roux 2007; Yerkes and Kardulias 1993). The following technical study has drawn on a number of these analytical protocols and actualistic studies to identify and record macroscopic attributes resulting from sequences of tool production and use in Madina-Sadatou. In general, I have adapted analytical and classificatory schemes from comparable contexts in Africa (e.g., Casey 2000; Deacon 1984; Huysecom 1990; MacDonald 1997; Shaw and Daniels 1984) to facilitate interregional comparison.

\section{Artifact Sampling and Processing}

A total of 43,258 chipped stone and 338 groundstone artifacts were recovered from Diouboye and sites documented during survey (Appendices A-C). The sheer volume of these materials made it necessary to adopt the following strategy of sampling and aggregate analysis by archaeological context.

Guided and pedestrian survey identified chipped stone artifacts at 138 sites (118 surface-collected) and 37 isolated occurrences (9 surface-collected). Systematic surface collections accounted for 34,944 chipped stone artifacts (from 102 locales) and purposive collections yielded an additional 700 diagnostic pieces (from 69 locales). At the end of each day in the field, these samples were sorted on the basis of raw material and formal subclass (Table E.1), then counted and weighed as an aggregate (Table E.2). Following this aggregate analysis, lithic artifacts classified as unworked raw material or angular 
shatter were discarded in the Falémé River, while all remaining cores and detached pieces were transported to IFAN for further analysis and curation.

\section{Table E.1: $\quad$ Lithic artifact subclasses}

\begin{tabular}{|l|l|}
\hline \multicolumn{1}{|c|}{ Subclass (Code) } & \multicolumn{1}{c|}{ Description } \\
\hline Raw material (1) & A specimen of rock or mineral (Table E.3) with little or no modification \\
\hline Core (2) & $\begin{array}{l}\text { An objective specimen with negative impressions from detached pieces } \\
\text { and often with percussive wear along striking surfaces }\end{array}$ \\
\hline Detached piece (3) & $\begin{array}{l}\text { A positive specimen struck from a core; includes angular shatter, bipolar } \\
\text { flakes, and freehand flakes and blades }\end{array}$ \\
\hline Formal tool (4) & A specimen reduced and/or retouched into a specific form \\
\hline Hammerstone (5) & $\begin{array}{l}\text { A specimen with pitting, crushing, or other percussion wear along one or } \\
\text { more faces (subsumed with groundstone for purposes of analysis) }\end{array}$ \\
\hline Groundstone (6) & $\begin{array}{l}\text { A specimen with grinding or polishing on one or more faces (or a roughed } \\
\text { out blank probably intended for grinding/polishing) }\end{array}$ \\
\hline
\end{tabular}

Table E.2: $\quad$ Summary of chipped stone artifacts from systematic survey

\begin{tabular}{|l|l|ccccc|}
\hline Collection & Subclass/Type & \multicolumn{5}{|c|}{ Raw Material } \\
& & Quartz & Chert/Flint & Mudstone & Hematite & Other \\
\hline \hline \multirow{4}{*}{ Purposive } & Raw material & 1 & 9 & - & 3 & - \\
\cline { 2 - 7 } & Core & 2 & 16 & - & - & - \\
\cline { 2 - 7 } & Angular shatter & 92 & 45 & - & - & - \\
\cline { 2 - 7 } & Flake & 183 & 161 & 27 & 1 & - \\
\cline { 2 - 7 } & Formal tool & 60 & 85 & 12 & - & 2 \\
\hline Total Purposive Collection & $\mathbf{3 3 8}$ & $\mathbf{3 1 6}$ & $\mathbf{3 9}$ & $\mathbf{4}$ & $\mathbf{3}$ \\
\hline \multirow{5}{*}{ Systematic } & Raw material & 23 & 354 & - & 10 & - \\
\cline { 2 - 7 } & Core & 165 & 250 & 1 & - & - \\
\cline { 2 - 7 } & Angular shatter & 10,757 & 2992 & 189 & - & 8 \\
\cline { 2 - 7 } & Flake & 13,167 & 6513 & 366 & 119 & 5 \\
\cline { 2 - 7 } & Formal tool & - & 7 & 16 & - & 2 \\
\hline
\end{tabular}

During my stint at IFAN in 2010, it was only possible to analyze chipped stone artifacts collected from three sites for comparison with Diouboye. Excepting an Atlantic Era occupation confined to the southern extent of FAL-1075, these sites had dense and relatively homogeneous archaeological components classified as aceramic (FAL-1022) and ceramic Late Stone Age (FAL-1062, FAL-1075). The assemblages from these latter 
two sites were initially analyzed and interpreted by project archaeologist Massal Diagne (2010). While I have reanalyzed the chipped stone materials to ensure comparability with data from Diouboye, my results largely concur with those of Diagne whose study, which also examined groundstone and ceramic artifacts, provides a richer understanding of the regional LSA context (see summary in Chapter 4).

Surface collections across Diouboye in 2008 and 2009 generated 3749 chipped stone artifacts. After in-field processing and the discard of unworked raw material and angular shatter (see above), a total of 1930 specimens were returned to IFAN for cataloguing and further study. The excavation of all units other than Unit C turned up 193 specimens of chipped quartz, chert, mudstone, and hematite. Unit C, a 1x1 m unit excavated into a small pile of mudstone debitage (Feature C-01), yielded an additional 3797 pieces of angular shatter and flakes that were counted, weighed, and discarded in the field. Due to the nature of this sample, I present the data from Unit C in Chapter 7, rather than include them in this general analysis.

All groundstone artifacts recovered in the course of fieldwork were counted and weighed in the field, then transported to IFAN for analysis in 2008 and 2009. These materials derive from excavated $(n=22)$ and surface $(n=173)$ contexts at Diouboye and from systematic $(n=69)$ and purposive $(n=79)$ collection at surveyed sites. I present the analysis and interpretation of all these materials in the final section of this appendix.

\section{Analytical Variables}

In the course of processing lithic artifacts at the field laboratory in Sansanding, I quickly became familiar with the range of variability in this material class, both at Diouboye and among the surveyed sites. As outlined in Table E.1, I divided the materials from each sample into six subclasses based on their general position in operational sequences of tool production and use. In order to reconstruct the sorts of technical choices made during these sequences, I recorded a slightly different suite of formal and metric variables for each subclass (see below), keeping an eye out for traces of retouch and use wear on cores and detached pieces. Since no single attribute can be thought to correlate perfectly with a given reduction technique, variables were selected to tease out past technical choices from quantitative patterns at the assemblage level.

\section{CHIPPED STONE TECHNOLOGY IN THE MADINA-SADATOU STUDY AREA}

A comparison of the artifact assemblages from surveyed sites (Chapter 4) shows that chipped stone tools fell out of use in Madina-Sadatou by the advent of the Atlantic Era circa AD 1500 (precluding direct historical analogy to earlier lithic technologies). Fortunately, archaeological and ethnoarchaeological studies have over the past three decades have established a solid framework for linking lithic artifacts to the diverse choices comprising any given operational sequence (Andrefsky 2005; Inizan et al. 1995). Beyond the constraints of raw material availability (Andrefsky 1994), these technical choices articulate with broader socioeconomic strategies for food procurement and/or production (Bousman 2005; Diez-Martín et al. 2009a; I. Parsons 2011), mobility and sedentism (R. L. Kelly 1988; Shott 1986), craft manufacture (Shafer and Hester 1991; Yerkes 1983), and even exchange and social display (Ambrose 2002; Close 1978). Furthermore, a study of contemporary hide-workers in Ethiopia by Kathryn Weedman Arthur (2010; Weedman 2006) shows that chert and quartz scrapers require a modest 
level of technical knowledge to make and use- - knowledge held and maintained by women within an endogamous class of craftspeople including potters and smiths. It is with these perspectives in mind that the spatio-temporal organization of chipped stone technologies can inform the study of (political) economic practices at Diouboye and the LSA sites.

In this section I consider chipped stone technology as three interconnected stages of raw material procurement, reduction, and use; I discuss discard practices more specifically in Chapters 6-8. For each operational stage I outline the relevant variables, describe the recorded attributes, and summarize the resulting data through comparison of the Diouboye and LSA assemblages.

\section{Raw Material Procurement}

Primary materials strongly influence sequences of chipped stone reduction and potential uses of the resulting tools (Andrefsky 1994, 2009). Beyond the basic parameters of fine grain size and conchoidal fracturing, people select raw materials according to their hardness, purity, color, and of course, the abundance and spatial distribution of workable rock across the landscape. Two predominant constraints on access to material sources are patterns of mobility (Binford 1982; R. L. Kelly 1988; Shott 1986) and social boundaries

Table E.3: Raw materials in the chipped stone and groundstone assemblages

\begin{tabular}{|c|c|}
\hline Raw Material (Code) & Description \\
\hline Quartz (1) & $\begin{array}{l}\text { A translucent to milky white quartz mineral; derived from Birimian and/or } \\
\text { Mauritanide formations }\end{array}$ \\
\hline \multicolumn{2}{|c|}{ Sedimentary materials derived from Mauritanide formations } \\
\hline Chert, blue (2) & A blue to greenish-gray siliceous rock with waxy luster and veins of impurities \\
\hline Chert, brown (3) & A light brown to dark red siliceous rock with waxy luster and few impurities \\
\hline Flint, gray (4) & $\begin{array}{l}\text { A white to greenish-gray siliceous rock with chalky luster and few impurities; } \\
\text { unknown provenance (possible Mauritanide formations) }\end{array}$ \\
\hline Mudstone (5) & A light to dark brown sedimentary rock with very fine texture \\
\hline Sandstone, red (6) & A dark red (felsitic) sedimentary rock with medium texture \\
\hline Sandstone, gray (7) & A light to dark gray sedimentary rock with medium sand texture \\
\hline \multicolumn{2}{|c|}{ Igneous and metamorphic materials derived from Birimian formations } \\
\hline Hematite (8) & A dark red to black deposit of iron oxide (possible Birimian formations) \\
\hline Amphibolite (9) & A light pink to brown metamorphosed rock with nonfoliated texture \\
\hline Quartzite (10) & A pinkish-gray metamorphosed sandstone with nonfoliated texture \\
\hline Schist (11) & A greenish-gray metamorphosed igneous rock with schistose texture \\
\hline Rhyolite (12) & A light to dark gray igneous rock with porphyritic-aphanitic texture \\
\hline Granite (13) & A light pink to gray igneous rock with phaneritic texture \\
\hline Gabbro (14) & A dark igneous rock with strongly phaneritic texture \\
\hline
\end{tabular}


between communities (Weedman 2006). It follows that a comparison between the availability of raw materials and their actual exploitation can shed light on desired (or possible) tool forms as well as socioeconomic strategies within the historical landscape.

As described in Chapter 3, the Falémé River transects two major geological formations that contribute to the diversity of workable stones throughout its middle to lower basin (Table E.3). Birimian supracrustal folds some five to ten kilometers southeast of the pedestrian survey zone yield durable igneous and metamorphosed igneous rocks such as granite, gabbro, and amphibolite; this formation may also be the source of the hematite encountered as polished axes on many prehistoric sites. The Mauritanide belt of metamorphic and sedimentary rocks, which underlies the entire survey zone, contains the quartz, chert, and mudstone exploited for chipped stone tools; it also provides the sandstone used for architectural foundations at many settlement sites. Whereas sandstone can be found in exposed ridges of basement rock, fine-grained materials for chipped stone reduction can be readily obtained as cobbles in and along the Falémé River bed.

In fact, the size distributions of unworked raw materials (100\% cortex remaining) and lightly worked cores (with 50-99\% cortex remaining) from Diouboye and the LSA sites reveal a long-standing reliance on quartz and chert nodules smaller than five centimeters in diameter and weighing less than 50 grams (Figure E.1). Notably, the mean size of unworked and lightly worked quartz nodules does not differ significantly between the LSA (24.7 g) and Diouboye (27.1 g) assemblages ( $\mathrm{t}=-0.588, \rho=0.56)$, nor do quartz nodules differ significantly from chert (27.9 g) at Diouboye ( $\mathrm{t}=0.11, \rho=0.91)$. A significant difference in the weight of unworked (49.7 g) and lightly worked (14.6 g) chert nodules in the LSA assemblage $(t=-2.84, \rho=0.03)$ points convincingly to the

\section{Figure E.1: Boxplot of weights of unworked (raw) material and lightly worked} (core) nodules of quartz and chert in chipped stone assemblages

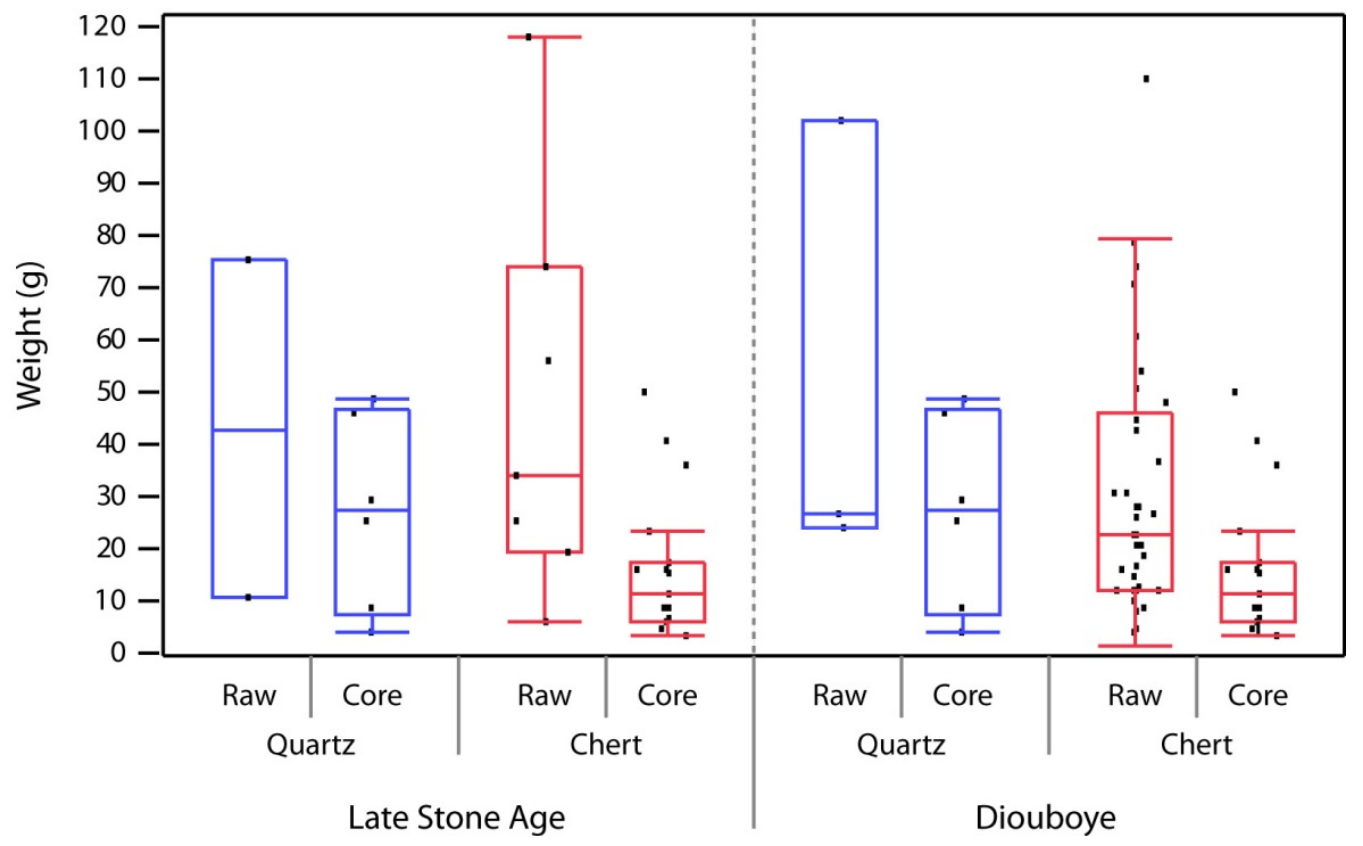


selective procurement of large chert nodules for reduction as freehand cores retaining much of their cortex (see below). Despite their greater selectivity, it is not clear that LSA populations obtained workable chert farther away than the Falémé River bed.

Figure E.2 shows that LSA populations also consumed relatively more chert than the occupants of Diouboye where contexts (excluding Unit C) reveal a more significant association with quartz $\left(\chi^{2}=9707.8, \rho<0.001\right)$. Since quartz appears to be more abundant than chert in the study area, this trend could reflect more expedient modes of tool production associated with sedentism at Diouboye. However, it is equally possible that the hardness and toughness of quartz made it the superior choice for the intensification of activities such as scraping (Greiser and Sheets 1979). Relatively limited mobility may also account for the lower frequencies of gray flint at Diouboye, but testing this hypothesis requires a more rigorous study of raw material provenance. I defer discussion of hematite debitage at Diouboye to the following section on groundstone technology.

Figure E.2: Relative abundance of raw materials in chipped stone assemblages

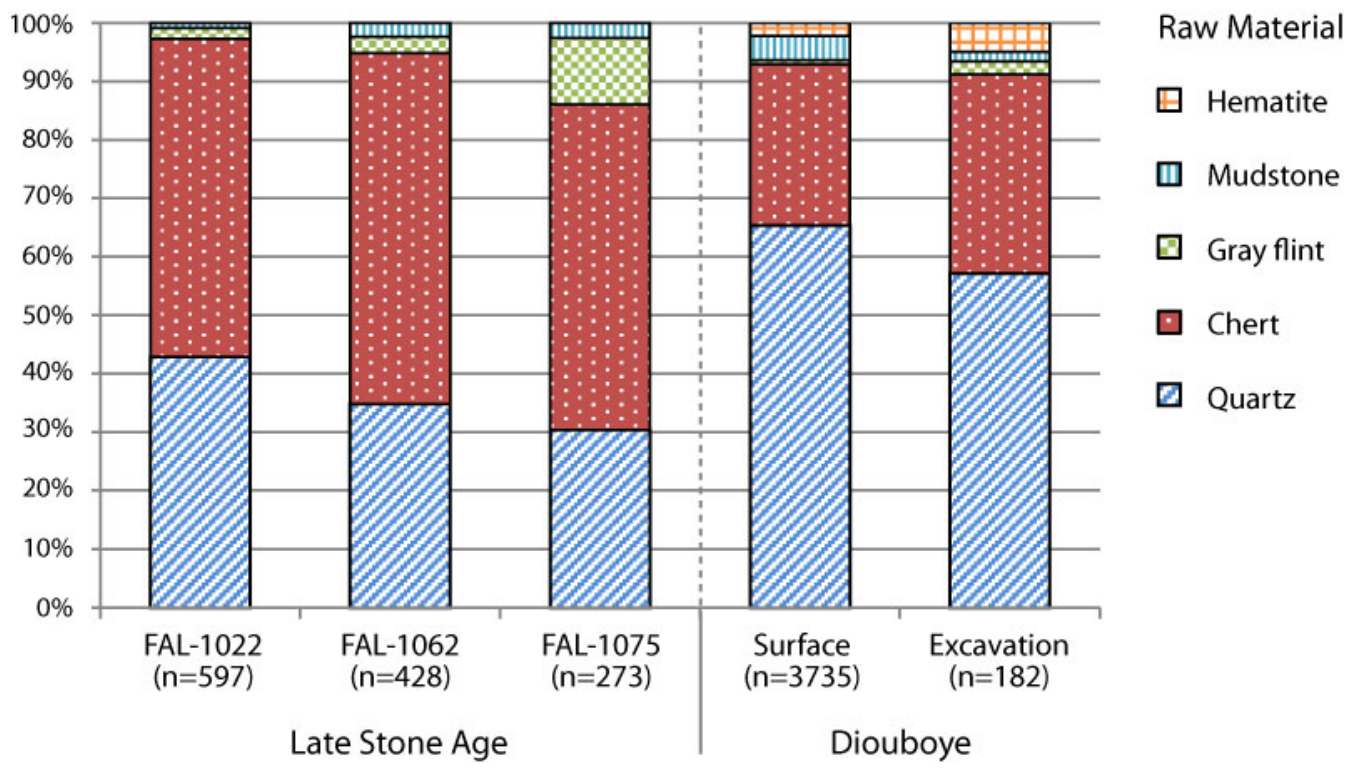

\section{Reduction}

Broadly speaking, reduction is the removal of detached pieces (flakes and shatter) from cores. The potential combinations of tools and gestures for reduction are diverse, but generally include soft- and hard-hammer techniques for freehand percussion, bipolar percussion, and pressure flaking (Cotterell and Kamminga 1987; Inizan et al. 1995:2932). In this analysis I have conceptualized reduction techniques as sub-stages of primary reduction and secondary reduction, acknowledging that any given artifact can shift back and forth between these sub-stages through practices of curation.

Embedded within broader socio-technological systems, choices about lithic reduction hinge upon several logistical and socioeconomic factors. First, as noted above, raw materials are more or less amenable to different reduction techniques depending on their physical properties (Andrefsky 1994; Barham 1987). In general, fine-grained 
siliceous stone such as chert can be worked with more precision than coarse-grained and less brittle materials such as rhyolite and quartzite. Fissures, inclusions, and other impurities can, however, decrease the homogeneity of an otherwise fine-grained material, thereby reducing its potential for controlled reduction. The size of a core-whether an unworked nodule of raw material or a nearly exhausted one-can also influence reduction techniques. That is to say, the small and compositionally heterogeneous nodules of quartz and chert exploited at Diouboye and nearby LSA sites would not have been amenable to the production of large formal tools.

Second, the distribution of workable stone resources can, in conjunction with mobility, strongly influence strategies for the manufacture and curation of chipped stone tools. All else being equal, mobile populations usually make more efficient use of raw materials than sedentary ones (Andrefsky 1994; Parry and Kelly 1987; Shott 1986). Freehand reduction techniques, for example, generate more effective working edge per mass raw material than bipolar techniques, while extensive secondary reduction (formalization) can yield multi-purpose cores/tools that can be curated to produce sharp flakes on the go (R. L. Kelly 1988).

Although curation has come to refer both to the transport of tools between activity areas and their maintenance over time (Andrefsky 2009:70-75), I use this concept to underline a useful, if necessarily broad, distinction between formal tools, which people maintain through extensive retouch, and informal tools made for short-term use, light regeneration, and discard. Curation may not have been a strict necessity in MadinaSadatou where workable stone sources are fairly abundant, but forays away from alluvial cobble deposits in and near the Falémé River may have benefitted from more judicious use of raw materials. People could have also curated higher quality materials, such as gray flint, possibly obtained through mobility and exchange.

Third, time-budgeting and the intended use of tools can likewise influence reduction strategies (Bousman 2005; Jeske 1992; Torrence 1989). Expedient or informal tools require limited production effort and skill although their natural shape and effective edges can make them suitable for specific tasks (Sillitoe and Hardy 2003; White and Thomas 1972). Formal tool production requires a greater investment in skill, time, and the search for appropriate raw materials, but the result may be a more versatile tool useful for a number of tasks (R. L. Kelly 1988), more specialized craft activities (Weedman 2006; Yerkes 1983), or at least the display of personal skill.

Finally, it is important to recognize that the transmission and reproduction of technical gestures for chipped stone reduction, as with all technological processes, are socially and cognitively mediated (Dobres 2000; Leroi-Gourhan 1943; Sackett 1982; Wiessner 1983). Even as they adapt to the aforementioned logistical parameters, stone tool makers also participate in cultural traditions that frame their known "ways of doing." In principle, shared reduction techniques can help to delineate social institutions and gender, class, or ethnic identities that perpetuate such technical styles in practice (K. W. Arthur 2010; Weedman 2006). For better or worse, it is not possible to pursue this perspective in Madina-Sadatou where variation in lithic assemblages from Diouboye and the sample of three LSA sites could not be most parsimoniously explained by raw material availability, mobility strategies, and (political) economic processes of subsistence and craft production. 
I now turn to the task of reconstructing the sequences for making and maintaining chipped stone tools in the Madina-Sadatou study area. In so doing, I define the variables and classificatory schemes employed to link cores, detached pieces, and formal tools to their respective reduction techniques. While individual lithic artifacts rarely yield definitive evidence for overall reduction strategies, robust assemblage-level patterns can attest to subtle differences in such strategies (Andrefsky 2009; Kuijt et al. 1995). I conclude this section with a reconstruction of the chipped stone reduction strategies at Diouboye and the three LSA sites chosen for comparison.

\section{Primary Reduction}

Primary reduction involves the application of force to a core in order to detach one or more pieces for either immediate use as informal tools or further reduction via retouch into formal tools. The shape and size of the resulting cores and detached pieces depend upon the choices of percussive implement(s), core support, direction(s) of force loading, and sequence of successive strikes (Cotterell and Kamminga 1987). Although these choices suppose an array of possible techniques, previous studies of latePleistocene and Holocene technology in Africa have outlined two main strategies of reduction—freehand and bipolar (e.g., Casey 2000; Deacon 1984; Diez-Martín et al. 2009a; Kusimba 2003; MacDonald 1997). These strategies, which are by no means mutually exclusive, represent frequently co-occurring sets of technical choices with known implications for patterning in lithic artifact assemblages.

Freehand reduction of a core involves sequential striking with a hard or soft percussive tool (knapping) and/or applying concentrated pressure with a pointed implement (pressure flaking) to detach pieces of two general types-freehand flakes and angular shatter. Since the former are usually preferable to the latter for use as tools, freehand reduction often employs techniques for core preparation to control the production of flakes of suitable size, shape, and effective cutting edge (Sillitoe and Hardy 2003; White and Thomas 1972). Exhausted freehand cores may be utilized informally or fashioned into formal tools such as scrapers and bifaces, or experience some combination thereof (R. L. Kelly 1988). As a freehand core dwindles from unworked nodule to expired nub, the size of detached pieces and their amount of dorsal cortex both decline, thereby permitting inferences about the socio-spatial organization of tool production and curation.

Bipolar reduction involves placing a core on an anvil and striking it from above with a hard percussive implement; the consequent application of force in two opposing directions leads to fracturing quite distinct from freehand reduction (Cotterell and Kamminga 1987). Wrapping the core in bark or fabric prior to reduction may result in a nice "package" of thinner flakes (White and Thomas 1972:278). Although cores generated through bipolar reduction have received some attention in the archaeological literature, particularly in their identification as pièces esquillées (see Goodyear 1993), it has long been difficult to document this technique in the archaeological record. Fortunately, a growing amount of experimental work (Barham 1987; Casey 2000; DiezMartín et al. 2011; Kuijt et al. 1995), combined with summaries of ethnographic observations (Parry and Kelly 1987; Shott 1989), have greatly expanded the inferential basis for recognizing bipolar reduction over the past three decades. These studies show 
that, in addition to pièces esquillées, bipolar detached pieces can include angular shatter and potentially distinctive flakes.

On average, this technique makes less efficient use of raw material (that is, less effective working edge per flake) than freehand reduction (Diez-Martín et al. 2011), but it can be used on lower quality and smaller materials, possibly even helping to maximize the exploitation of otherwise expired freehand cores (Barham 1987; Goodyear 1993). Many bipolar pieces have steep margins useful for cutting or scraping, but further bipolar crushing or knapping can transform them into formal tools if so desired (Jeske 1992).

With this background, the following artifact types and formal and metric variables provide a basis for interpreting the strategies of chipped stone reduction employed at Diouboye and selected LSA sites in the Madina-Sadatou study area.

Table E.4: Variables recorded for core specimens

\begin{tabular}{|c|c|c|}
\hline Variable & Attribute (Code) & Description \\
\hline \multirow{2}{*}{ Completeness } & Incomplete (0) & Specimen appears broken or incomplete \\
\hline & Complete (1) & Specimen appears complete \\
\hline \multirow{3}{*}{ Surface Cortex } & $0 \%(0)$ & No remaining cortex \\
\hline & $1-50 \%(1)$ & Cortex covers less than half of the specimen \\
\hline & $50-99 \%(2)$ & Cortex covers more than half of the specimen \\
\hline Weight & Grams (0.1 g) & Mass of the specimen \\
\hline Length & Millimeters (0.1 mm) & Maximum linear dimension of the specimen \\
\hline \multirow{3}{*}{ Core Type } & Freehand (1) & $\begin{array}{l}\text { Clear flake scars in one or more directions demonstrate a } \\
\text { sequential removal of detached pieces }\end{array}$ \\
\hline & Bipolar (2) & $\begin{array}{l}\text { Two or more points of opposite percussion with evidence of } \\
\text { crushing attest to bipolar force loading }\end{array}$ \\
\hline & Irregular (3) & Specimen cannot be assigned to either of the above types \\
\hline \multirow{4}{*}{$\begin{array}{l}\text { Freehand Core } \\
\text { Subtype }\end{array}$} & Discoid (1) & Radial flake scars occur on one or two faces \\
\hline & Polyhedral (2) & Irregular flake scars occur on three or more faces \\
\hline & Bladelet (3) & Parallel flake scars descend from a prepared striking surface \\
\hline & Keeled (4) & Light uni- or bifacial flaking produces a “chopping” edge \\
\hline \multirow{8}{*}{$\begin{array}{l}\text { Bipolar Core } \\
\text { Subtype }\end{array}$} & Area-Area (1) & Both striking surfaces have remaining area \\
\hline & Ridge-Ridge (2) & Both striking surfaces reduced to ridges \\
\hline & Point-Point (3) & Both striking surfaces reduced to points \\
\hline & Ridge-Area (4) & Opposing ridge and area striking surfaces \\
\hline & Point-Area (5) & Opposing point and ridge striking surfaces \\
\hline & Point-Ridge (6) & Opposing point and ridge striking surfaces \\
\hline & Simple crushed (7) & Lightly reduced nodule with two opposing striking surfaces \\
\hline & Rotated crushed (8) & Lightly reduced nodule with four or more striking surfaces \\
\hline \multirow{2}{*}{$\begin{array}{l}\text { Irregular Core } \\
\text { Subtype }\end{array}$} & Irregular (1) & Irregular or unknown core type \\
\hline & Fragment (2) & Broken core fragment \\
\hline
\end{tabular}




\section{Cores}

A core bears evidence for the removal of detached pieces, including negative impressions (scars) and crushing at the point(s) of impact called striking surfaces. Based on the number and orientation of these scars and striking surfaces, one can distinguish cores deriving from freehand and/or bipolar reduction, and, in some cases, subtler technical choices within these broader strategies. Although some analyses quantify these variables, I found that established core typologies captured the essential variability in assemblages from Madina-Sadatou (Table E.4). I did, however, measure the core size and record attributes for fragmentation and surface cortex in order to evaluate the edge-tomass efficiency and spatio-temporal organization of reduction.

\section{Freehand Cores}

In order to differentiate among techniques for freehand reduction, I adapted the classification of cores developed by Kevin MacDonald (1997) for the late prehistoric site of Korounkorokalé in Mali (see also Shaw and Daniels 1984). The following four subtypes (Figure E.3) describe modal patterns in the number of striking platforms and the directionality of detached piece removal—whether struck repeatedly in the same direction or rotated and struck from multiple directions.

\section{Figure E.3: Freehand core subtypes}

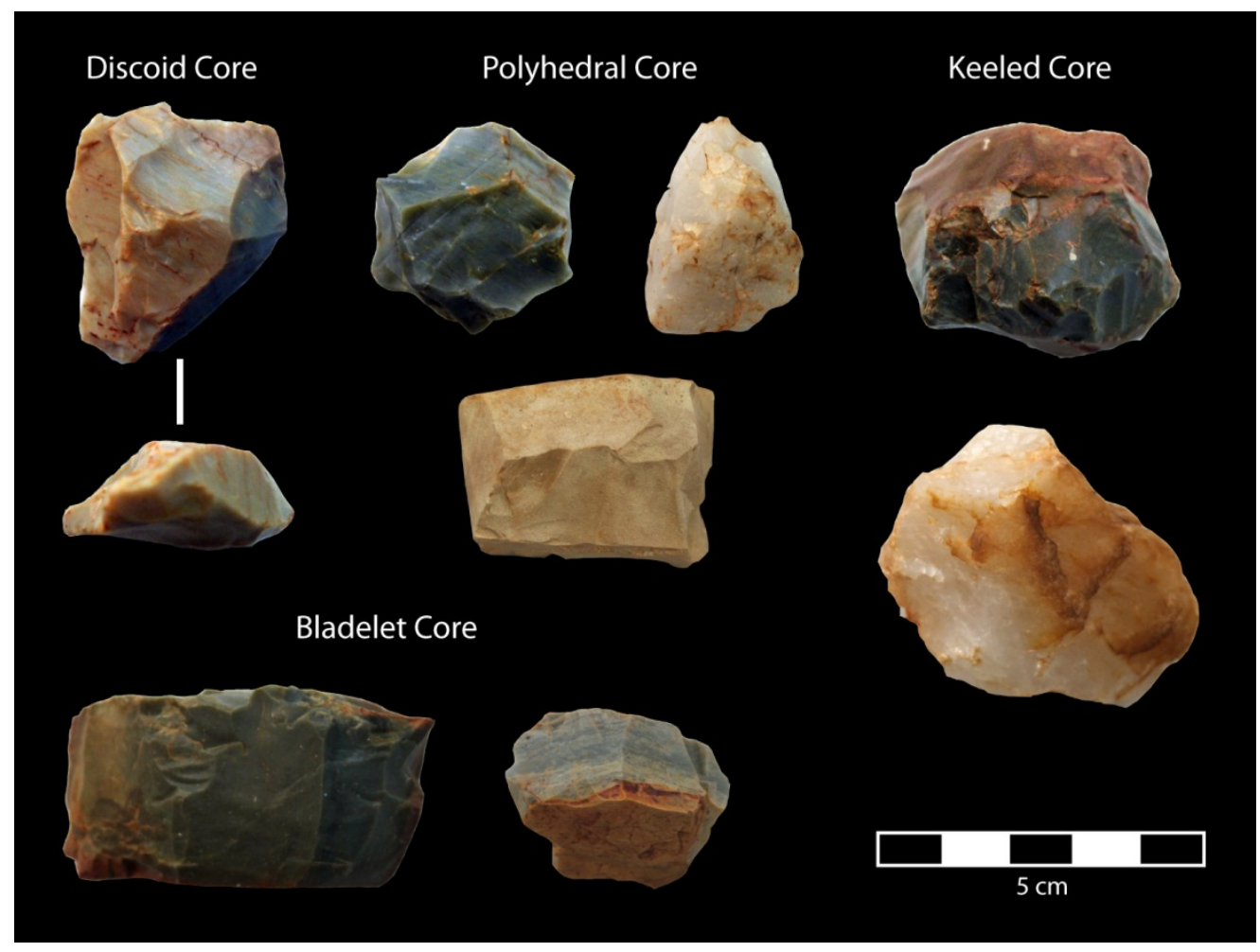


Discoid cores exhibit the sequential removal of flakes and other detached pieces in a clockwise or counterclockwise direction around one or two faces; these have been called nuclei en tortue in the francophone literature (Huysecom 1990:43). In addition to sharp freehand flakes, this process of unifacial or bifacial reduction (thinning) can generate core tools with a significant amount of effective working edge.

Polyhedral cores have three or more worked faces; they vary in shape from pyramidal to roughly cubical. Although the scars on these cores usually attest to the removal of blades, they lack a prepared striking surface. Rather, the continual rotation of the core generates a sequence of controlled flake removal from previous negatives.

Bladelet cores have parallel scars resulting from the removal of small, elongated flakes (bladelets) originating at a prepared striking surface. These cores vary in shape from cylindrical to pyramidal or prismatic.

Keeled cores have flakes removed along one or two faces to produce an effective working edge even as they retain at least half of their cortex. Also known as galets aménagés in the francophone literature (Camara and Duboscq 1990), these could represent lightly worked discoid cores, but they more likely served as small chopping tools unto themselves.

\section{Bipolar Cores}

A number of experimental studies have shown that force loading on a bipolar core produces striking surfaces in the shape of points, ridges, or flat areas, usually with crushing from repeated blows (Barham 1987; Casey 2000; Diez-Martín et al. 2011; Kuijt et al. 1995). Adapting the scheme outlined by Joanna Casey (2000; also Binford and Quimby 1963), I classified bipolar cores according to the shape of their two opposing striking surfaces: ridge-ridge; area-area; ridge-area; point-area; point-ridge; and pointpoint. It is not yet clear whether these subtypes represent discrete techniques of reduction and core rotation, or simply the specific properties of the raw material and percussive implements.

It was, however, possible to recognize traces of opposite force loading and crushing on partially worked quartz cobbles and other nodules of raw material (DiezMartín et al. 2009b). Simple crushed cobbles document the exploitation of two striking surfaces that remain stable throughout reduction. Rotated crushed cobbles appear to have been rotated during reduction to produce two or more exploited faces with evidence of opposite force loading. In all likelihood these cores represent the opening of raw material nodules for further freehand and/or bipolar reduction.

\section{Irregular Cores and Core Fragments}

Irregular cores bear ambiguous evidence for freehand and bipolar reduction or, in rare cases, unambiguous evidence of both.

Core fragments have scar impressions truncated by fracturing of the piece itself, often precluding inferences about prior reduction techniques. This subtype may represent core rejuvenation or bipolar reduction of nearly expired cores. 


\section{Figure E.4: Bipolar and irregular core subtypes}

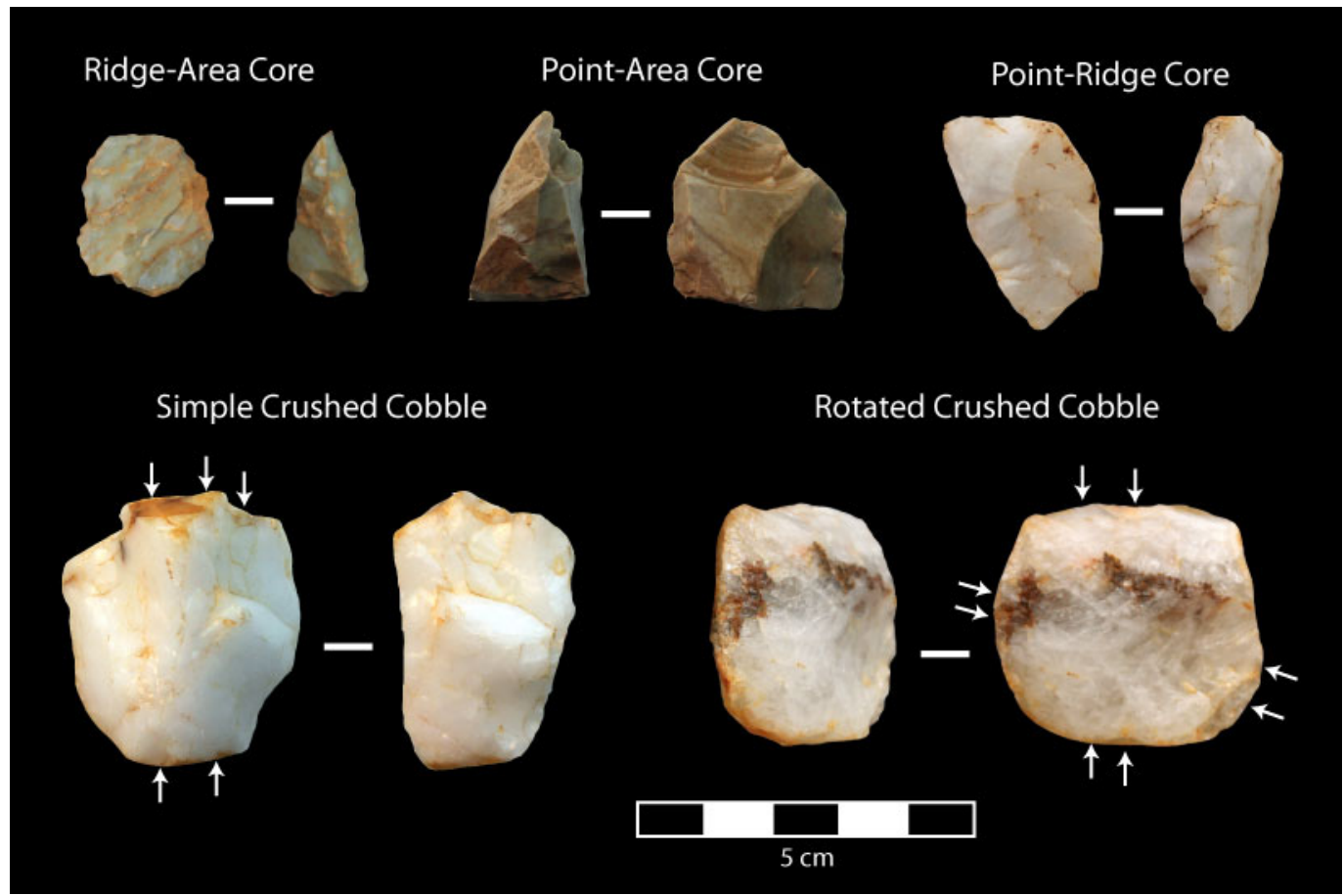

\section{Detached Pieces}

Detached pieces include the elements removed from a core during reduction. Traces of reduction techniques include the size and type of wear on striking platform(s), the shape of the distal termination, and bulbs of percussion and "ripples" on the ventral surface indicating the direction(s) of applied force (Cotterell and Kamminga 1987). Evidence for the progressive reduction of an unworked nodule into an exhausted core includes the relative size of detached pieces and the amount of cortex on their dorsal surfaces. While these variables allow one to attribute flakes to freehand or bipolar reduction, the wide range of pieces generated by these strategies makes it necessary to distinguish them at the assemblage level (Kuijt et al. 1995). Table E.5 outlines the variables recorded for detached pieces in the assemblages from Madina-Sadatou.

\section{Angular Shatter}

Both freehand and bipolar reduction generate substantial quantities of angular shatter-defined as those detached pieces that have neither a clear ventral surface nor exhibit a direct point of impact. Although I subdivided angular shatter into chips with no visible flake scars and chunks with one or two visible flake scars during analysis (Deacon 1984:371), these subtypes ultimately had no interpretive significance. Most angular shatter was counted, weighed, and discarded in the field, but those pieces with evidence for utilization or retouch underwent further analysis as described below. 
Table E.5: Variables recorded for detached pieces

\begin{tabular}{|c|c|c|}
\hline Variable & Attribute (Code) & Description \\
\hline \multirow{2}{*}{$\begin{array}{l}\text { Proximal End } \\
\text { Present }\end{array}$} & Absent (0) & Proximal end absent \\
\hline & Present (1) & Proximal end present \\
\hline \multirow{2}{*}{$\begin{array}{l}\text { Distal End } \\
\text { Present }\end{array}$} & Absent (0) & Distal end absent \\
\hline & Present (1) & Distal end present \\
\hline \multirow{4}{*}{ Dorsal Cortex } & $0 \%(0)$ & No cortex remaining on the dorsal surface \\
\hline & $1-50 \%(1)$ & Cortex covers less than half of the dorsal surface \\
\hline & $51-99 \%(2)$ & Cortex covers more than half of the dorsal surface \\
\hline & $100 \%(3)$ & Cortex completely covers the dorsal surface \\
\hline Weight & Grams $(0.1 \mathrm{~g})$ & Mass of the specimen \\
\hline Length & Millimeters $(0.1 \mathrm{~mm})$ & Maximum linear dimension of the specimen \\
\hline Width & Millimeters (0.1 mm) & Maximum width of the specimen \\
\hline \multirow{3}{*}{$\begin{array}{l}\text { Detached Piece } \\
\text { Type }\end{array}$} & Angular shatter (1) & No clear ventral surface or striking platform \\
\hline & Freehand flake (2) & Clear ventral surface and/or a single striking platform \\
\hline & Bipolar flake (3) & One or more crushed striking platforms \\
\hline \multirow{2}{*}{$\begin{array}{l}\text { Angular Shatter } \\
\text { Subtype }\end{array}$} & Chip (1) & No flake scars visible \\
\hline & Chunk (2) & One or two flake scars visible \\
\hline \multirow{2}{*}{$\begin{array}{l}\text { Freehand Flake } \\
\text { Subtype }\end{array}$} & Flake (1) & Clear ventral surface and/or single striking platform \\
\hline & Blade (2) & $\begin{array}{l}\text { Elongated flake with dorsal flake scars oriented in the } \\
\text { same direction of detachment }\end{array}$ \\
\hline \multirow{5}{*}{$\begin{array}{l}\text { Freehand Striking } \\
\text { Platform Type }\end{array}$} & Cortical (1) & Unmodified cortical surface of the core or objective piece. \\
\hline & Flat (2) & Smooth surface roughly perpendicular to the dorsal surface \\
\hline & Complex (3) & Angular surface from the removal of preparation flakes. \\
\hline & Abraded (4) & Surface abraded to remove flake scars and step fractures \\
\hline & Crushed (5) & $\begin{array}{l}\text { Shattered surface appearing as a ridge or point above a } \\
\text { clear bulb of percussion }\end{array}$ \\
\hline $\begin{array}{l}\text { Striking Platform } \\
\text { Width }\end{array}$ & Millimeters (0.1 mm) & Maximum width of the striking platform \\
\hline \multirow{4}{*}{$\begin{array}{l}\text { Freehand Distal } \\
\text { Termination } \\
\text { Type }\end{array}$} & Feathered (1) & Distal end gradually shears away from the objective piece \\
\hline & Hinged (2) & $\begin{array}{l}\text { Distal end rounded or sloped as a result of the impact } \\
\text { rolling away from the objective piece }\end{array}$ \\
\hline & Overshot (3) & $\begin{array}{l}\text { Distal end has adhering portion of the objective piece as a } \\
\text { result of the impact rolling inward }\end{array}$ \\
\hline & Stepped (4) & $\begin{array}{l}\text { Distal end snaps off to produce a roughly } 90^{\circ} \text { angle with } \\
\text { the ventral surface }\end{array}$ \\
\hline \multirow{3}{*}{$\begin{array}{l}\text { Bipolar Flake } \\
\text { Subtype }\end{array}$} & Areal wedge (1) & Specimen has one or two broad striking platforms \\
\hline & Pointed wedge (2) & Specimen has two narrow ridge/point platforms \\
\hline & Indeterminate (3) & Specimen has indeterminate striking platforms \\
\hline
\end{tabular}




\section{Freehand Flakes}

Freehand flakes have a smooth or lightly rippled ventral surface and, unless the proximal end is absent, a discrete striking platform. Depending on the applied distribution of compression, tensile, and shearing forces, flakes can have either a conchoidal form, with a discrete bulb of percussion, or bending form, which originate away from softer strikes near an acute edge. While these detached pieces are generally diagnostic of freehand reduction, they can also arise in small quantities during bipolar reduction.

As discussed below under informal tools, blades and bladelets are elongated flakes whose dorsal scars document the same orientation of percussion and detachment as preceding flakes. The striking platform, if present, usually shows signs of preparation.

\section{Figure E.5: Freehand and bipolar flake subtypes}

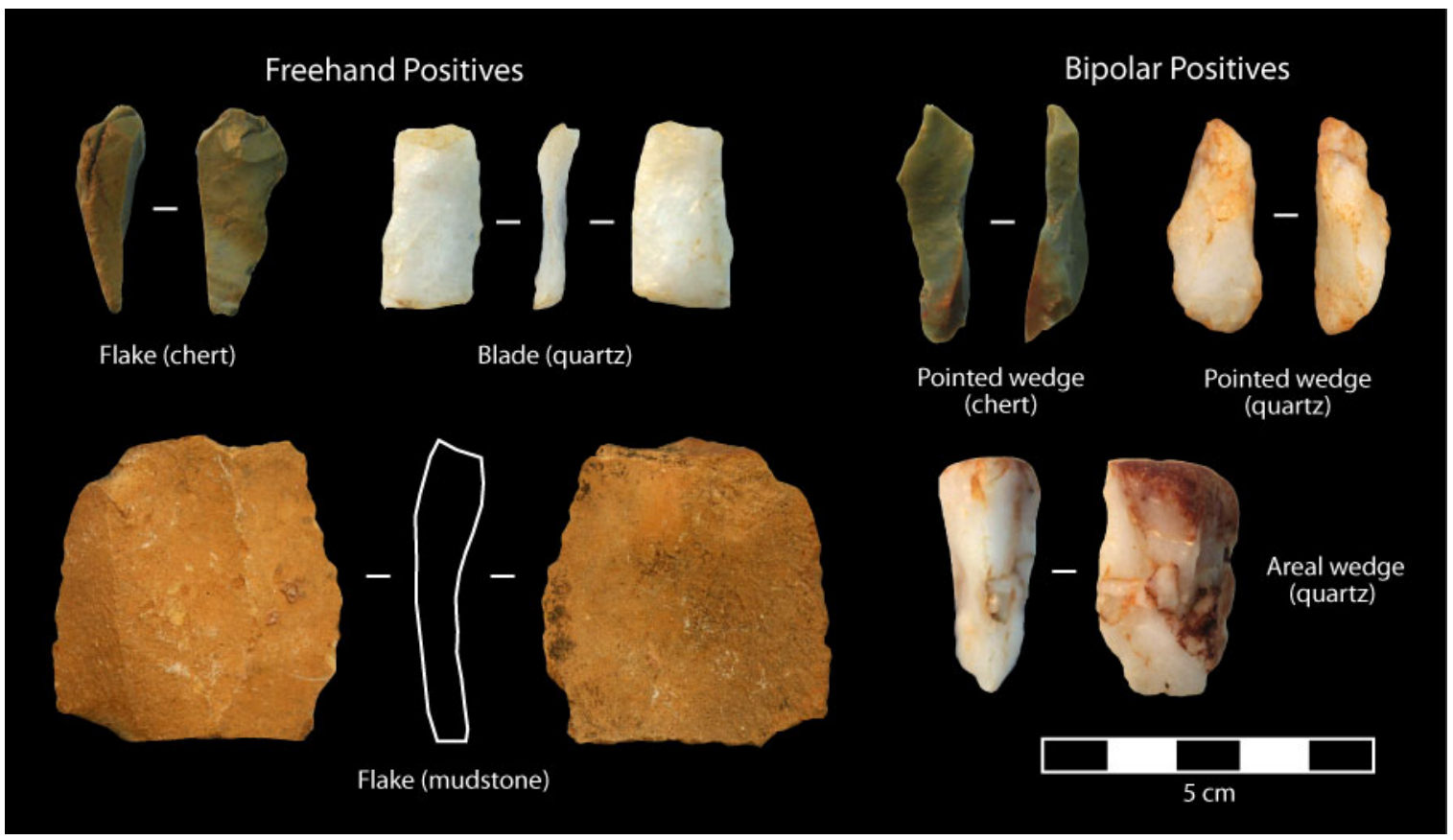

\section{Bipolar Flakes}

Building upon the association of so-called "compression” flakes with bipolar reduction (Cotterell and Kamminga 1987), experimental studies have developed a number of criteria to identify bipolar flakes: opposing pointed or crushed striking platforms; ventral scarring at these platforms; lack of a clear bulb of percussion; pronounced ripple marks; similar dorsal and ventral surfaces; steep lateral edge angles; and twisted ventral surfaces (Barham 1987:48; Diez-Martín et al. 2009b:282-284). Because the occurrence and visibility of these attributes depends upon raw material, bipolar flakes in quartz are more difficult to identify than those in chert. It is also important to note that, despite the nomenclature employed here, positives with these "bipolar" attributes can arise in small frequencies during freehand reduction.

Adapting a scheme developed by Fernando Diez-Martín and colleagues (2009b; 2011), I classified bipolar flakes by the size of their remaining striking platforms. Area 
wedges have one or two broad striking surfaces, often comprised of cortex. Pointed wedges have striking surfaces that have been reduced and crushed into ridges and points. Indeterminate wedges have extensive crushing or breaking that precludes an unambiguous identification of their striking platform.

\section{Secondary Reduction}

While primary reduction generates many flakes with effective working edges sufficient for a variety of tasks, past stone workers often undertook secondary reduction to maintain such pieces during use as informal tools and/or shape them into more formal tools. Such formal tools, when compared to assemblages elsewhere across West Africa, can also reveal how people participated in different cultural traditions across the prehistoric landscape. I defer discussion of tool form/function to the next section, so let it suffice here to briefly introduce the secondary reduction techniques observed in the lithic assemblages from Madina-Sadatou-backing and retouching.

Backing is the process of grinding, chipping, or otherwise dulling the margin(s) of a potential tool to allow for more efficient or safer force application onto an opposing edge. Although backed margins are distinctive of microlithic industries across Africa, they also occur on non-microlithic tool forms such as scrapers.

Retouch involves the removal of small flakes through soft hammer percussion or pressure flaking along the margins of a tool. I defined formal tools, somewhat subjectively, as those with "extensive” retouch-a continuous stretch or overlapping sequence of small flake scars marks an unambiguous tool edge. I did not attempt to distinguish retouch applied during initial production from that employed to regenerate a dulled edge over the lifespan of a tool.

\section{Comparison of Chipped Stone Reduction Strategies}

An interpretation of reduction strategies at Diouboye requires comparison with those evidenced by assemblages from neighboring LSA sites. Above all, this is necessary to establish the extent of primary chipped stone deposition at Diouboye versus possible redeposition from earlier LSA contexts. This can also expose shifts in socioeconomic organization across these two archaeological horizons within same physical landscape.

As illustrated in Figures E.6-E.7, the relative abundance of blue/brown chert and quartz artifacts indicate that raw material choices played a similar role in the chipped stone technologies of Diouboye and the LSA sites. First of all, bipolar flakes and angular shatter together demonstrate a significant and positive association with quartz $\quad\left(\chi^{2}=365.0\right.$, $\rho<0.001)$. The low frequencies of bipolar quartz cores — and quartz cores overall — could signify either the complete reduction of quartz nodules and/or the challenge of discriminating between bipolar cores and flakes for this material (Diez-Martín et al. 2011). Chert, on the other hand, was significantly associated with freehand flakes $\left(\chi^{2}=1293.1, \rho<0.001\right)$ and formal tools $\left(\chi^{2}=887.5, \rho<0.001\right)$. This is consistent with a quartz reduction strategy emphasizing the production of usable detached pieces, but limited secondary retouch for curation and tool formalization in comparison to chert. In sum, these data suggest that raw material availability along the Falémé River influenced reduction strategies by all prehistoric communities, even as these socially mediated strategies must have informed choices about procuring said raw materials. 
Figure E.6: Relative abundance of chipped quartz artifact types
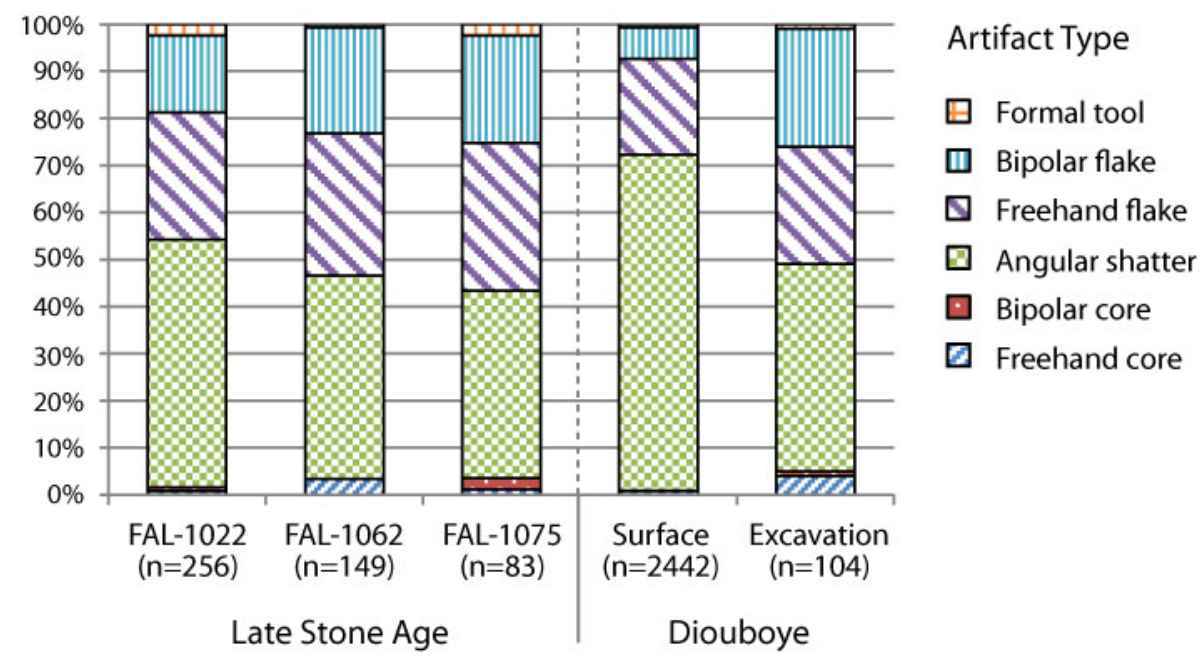

Figure E.7: Relative abundance of chipped chert artifact types

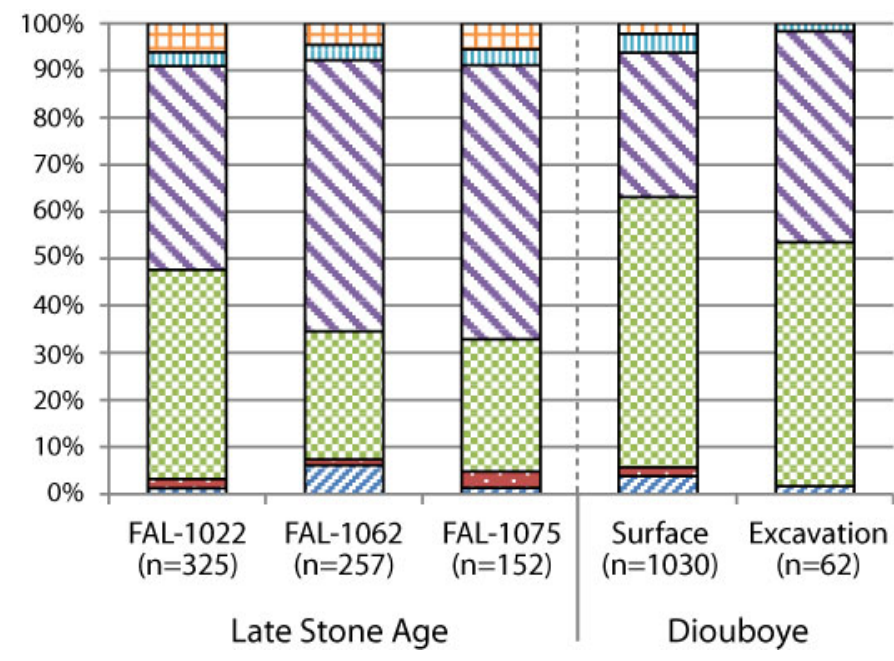

Artifact Type

日 Formal tool

口 Bipolar flake

Freehand flake

$\square$ Angular shatter

$\square$ Bipolar core

■ Freehand core

The available data also indicate that the occupants of Diouboye employed a different suite of reduction strategies than LSA populations. Not only did Diouboye yield relatively more quartz artifacts, but the site had significantly greater frequencies of angular shatter for all raw materials $\left(\chi^{2}=1717.4, \rho<0.001\right)$ and significantly fewer formal tools $\left(\chi^{2}=1634.3, \rho<0.001\right)$. While bipolar flakes occurred in similar frequencies across chert samples, they were significantly less frequent in the quartz assemblage from Diouboye $\left(\chi^{2}=1682.8, \rho<0.001\right)$. Relatively mobile LSA populations in Madina-Sadatou (see Chapter 4 ) could have reduced risk by carrying multifunctional, lightweight, and easily curated formal tools across the landscape (in addition to their expedient use of quartz). The more sedentary population of Diouboye, on the other hand, may have had 
less desire or incentive to make formal tools. One could hypothesize that the ability to convey social identities through iron, copper, and ceramic media within this Iron Age community further contributed to lower labor investment in chipped stone tools.

A comparison of detached piece size lends further support to the interpretation of greater bipolar reduction at Diouboye. Table E.6 shows that all detached piece types, including angular shatter, freehand flakes, and bipolar flakes, were significantly larger in the assemblage from Diouboye, where they also displayed greater variance. While this strategy would have produced fewer usable flakes per core (Diez-Martín et al. 2011), the larger detached pieces with moderate to steep margins could have immediately found use as informal tools.

Interestingly, the cumulative frequencies of relative dorsal cortex on detached pieces suggest that similar stages of reduction took place at Diouboye and the LSA sites (Figure E.8). The obvious exception to this generalization-bipolar reduced pieces from LSA sites - could reflect the biases of a small sample although this pattern dovetails with the size differential observed between unworked and lightly worked chert cores from LSA contexts (Figure E.1). If Late Stone Age knappers were selecting larger nodules to make more formalized tools, they could have opened these into cores with bipolar percussion prior to subsequent knapping with freehand techniques. This strategy would most parsimoniously account for the rapid diminution of cores noted above and the presence of dorsal cortex on $68 \%$ of the chert bipolar flakes arguably detached early in the reduction sequence.

Table E.6: Mean sizes of detached piece types

\begin{tabular}{|c|c|c|c|c|c|c|c|c|c|c|}
\hline \multirow[t]{3}{*}{ Assemblage } & \multicolumn{5}{|c|}{ Quartz } & \multicolumn{5}{|c|}{ Chert } \\
\hline & \multirow{2}{*}{$\begin{array}{c}\text { Total } \\
n\end{array}$} & \multicolumn{2}{|c|}{ Length (mm) } & \multicolumn{2}{|c|}{ Weight (g) } & \multirow{2}{*}{$\begin{array}{c}\text { Total } \\
n\end{array}$} & \multicolumn{2}{|c|}{ Length (mm) } & \multicolumn{2}{|c|}{ Weight (g) } \\
\hline & & $\mu$ & $\sigma$ & $\mu$ & $\sigma$ & & $\mu$ & $\sigma$ & $\mu$ & $\sigma$ \\
\hline \multicolumn{11}{|l|}{ Angular Shatter } \\
\hline LSA & 228 & - & - & 1.15 & 0.77 & 244 & - & - & 1.73 & 1.64 \\
\hline Diouboye & 1758 & - & - & 3.90 & 9.69 & 564 & - & - & 5.42 & 19.1 \\
\hline \multicolumn{11}{|l|}{ Freehand Flake } \\
\hline LSA & 138 & 16.24 & 5.59 & 1.29 & 1.68 & 359 & 16.42 & 6.18 & 1.26 & 1.62 \\
\hline Diouboye & 516 & 19.08 & 7.14 & 4.38 & 23.30 & 310 & 21.58 & 8.73 & 3.03 & 4.22 \\
\hline \multicolumn{11}{|l|}{ Bipolar Flake } \\
\hline LSA & 93 & 21.07 & 4.99 & 2.37 & 1.86 & 22 & 23.31 & 5.37 & 2.83 & 2.29 \\
\hline Diouboye & 185 & 23.30 & 6.56 & 3.23 & 3.10 & 38 & 25.31 & 7.65 & 3.28 & 2.81 \\
\hline
\end{tabular}


Figure E.8: Cumulative frequencies of distal cortex on detached pieces
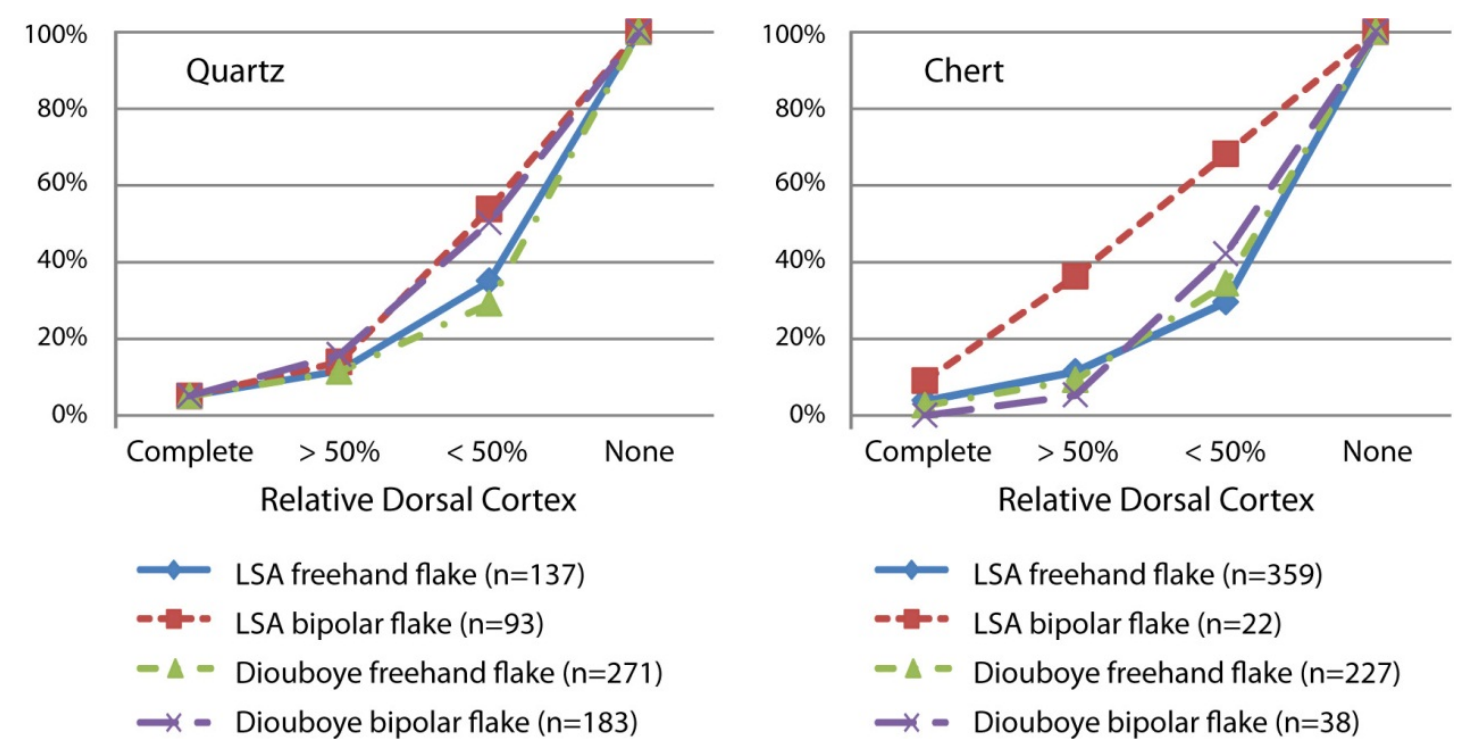

\section{Tool Use}

The durability and formal diversity of chipped stone tools make them well-suited for a variety of tasks: hunting and butchering animals; harvesting wild and domestic plants; and even working with leather, basketry, textiles, and other crafts (Odell 2001). Because these tasks implicate different organic and inorganic materials, as well as gestures such as scraping, cutting, and drilling, it is often possible to infer dominant modes of tool use and curation from size, shape, effective working edge shape and sharpness, and retouch and edge wear. These variables provide a basis for the classification of formal tools with extensive retouch, but they also allow for a consideration of pieces utilized and/or lightly retouched as informal tools.

For all detached pieces and cores, I recorded all visible edge and surface damage (Table E.7), keeping in mind the possible biases resulting from post-depositional processes. In the absence of more comprehensive microscopic and residue analyses (Odell 2001), this macroscopic assessment provides at least some check on the practical uses inferred from artifact size and form. Using these attributes, my classification of formal and informal tools in Madina-Sadatou (Table E.7) draws on a number of existing lithic artifact typologies from late Pleistocene to Holocene contexts in sub-Saharan Africa (Casey 2000; Corbeil et al. 1948; Deacon 1984; Huysecom 1990; MacDonald 1997; Shaw and Daniels 1984). 
Table E.7: Variables recorded for informal and formal chipped stone tools

\begin{tabular}{|c|c|c|}
\hline Variable & Attribute (Code) & Description \\
\hline \multirow{4}{*}{$\begin{array}{l}\text { Effective } \\
\text { Edge }\end{array}$} & Left (L) & \multirow{4}{*}{$\begin{array}{l}\text { Identifies the effective edge margin for each piece oriented } \\
\text { with ventral surface upward and distal end away from } \\
\text { observer. The following variables of Type, Shape, and } \\
\text { Angle were recorded for each relevant margin. }\end{array}$} \\
\hline & Right (R) & \\
\hline & Distal (D) & \\
\hline & Proximal (P) & \\
\hline \multirow{4}{*}{$\begin{array}{l}\text { Effective Edge } \\
\text { Wear/Retouch }\end{array}$} & Use wear (0) & Visible edge wear, but no retouch \\
\hline & Dorsal retouch (1) & Margin retouched on dorsal surface only \\
\hline & Ventral retouch (2) & Margin retouched on ventral surface only \\
\hline & Bifacial retouch (3) & Margin retouched on dorsal and ventral surfaces \\
\hline \multirow{5}{*}{$\begin{array}{l}\text { Effective } \\
\text { Edge Shape }\end{array}$} & Straight (0) & Utilized edge is straight \\
\hline & Convex (1) & Utilized edge curves outward \\
\hline & Concave (2) & Utilized edge curves inward \\
\hline & Pointed (3) & Utilized edge forms a point \\
\hline & Irregular (9) & Utilized edge is some combination of the above \\
\hline \multirow{3}{*}{$\begin{array}{l}\text { Effective } \\
\text { Edge Angle }\end{array}$} & Sharp (0) & Angle $<30^{\circ}$ \\
\hline & Moderate (1) & $30^{\circ} \leq$ Angle $<60^{\circ}$ \\
\hline & Steep (2) & Angle $\leq 60^{\circ}$ \\
\hline \multirow{4}{*}{ Backing Form } & Segment (1) & Single curved backed edge opposing the effective edge \\
\hline & Trapezoid (2) & $\begin{array}{l}\text { Two or three straight backed edges at least one of which is } \\
\text { parallel with effective edge }\end{array}$ \\
\hline & Triangle (3) & Two straight backed edges opening to third effective edge \\
\hline & Point (4) & Curved backed edge opening to pointed effective edge \\
\hline \multirow{6}{*}{$\begin{array}{l}\text { Formal Tool } \\
\text { Subtype }\end{array}$} & Geometric segment (1) & Detached piece with segment backing \\
\hline & Geometric, other (2) & Detached piece with trapezoid or triangle backing \\
\hline & Projectile point (3) & Core or detached piece thinned/retouched into a point \\
\hline & Burin (4) & Core or detached piece retouched into a long, thin point \\
\hline & End scraper (5) & Core or detached piece retouched along proximal/distal end \\
\hline & Side scraper (6) & Core or detached piece retouched along lateral margin(s) \\
\hline \multirow{5}{*}{$\begin{array}{l}\text { Informal Tool } \\
\text { Subtype }\end{array}$} & Blade (1) & Freehand blade with edge wear or light lateral retouch \\
\hline & Wedged blade (2) & $\begin{array}{l}\text { Wedge with cortex "backing" opposite edge with wear } \\
\text { and/or light lateral retouch }\end{array}$ \\
\hline & Utilized flake, sharp (3) & Detached piece with sharp to moderate effective edge angle \\
\hline & Utilized flake, steep (4) & Detached piece with steep effective edge angle \\
\hline & Utilized flake, point (5) & Detached piece with a pointed effective edge shape \\
\hline
\end{tabular}


Figure E.9: Formal chipped stone tool subtypes

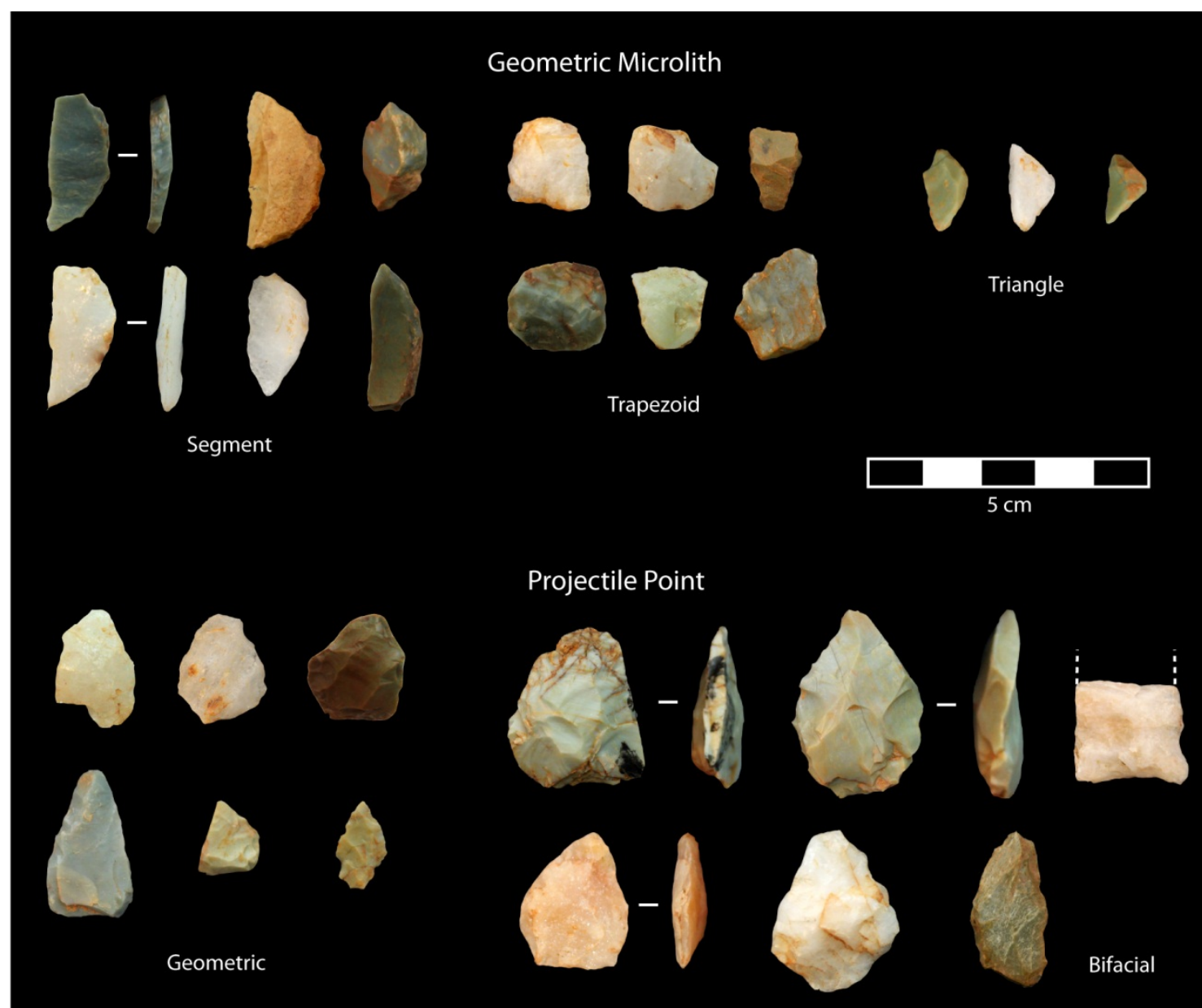

Burin

End Scraper

Side Scraper
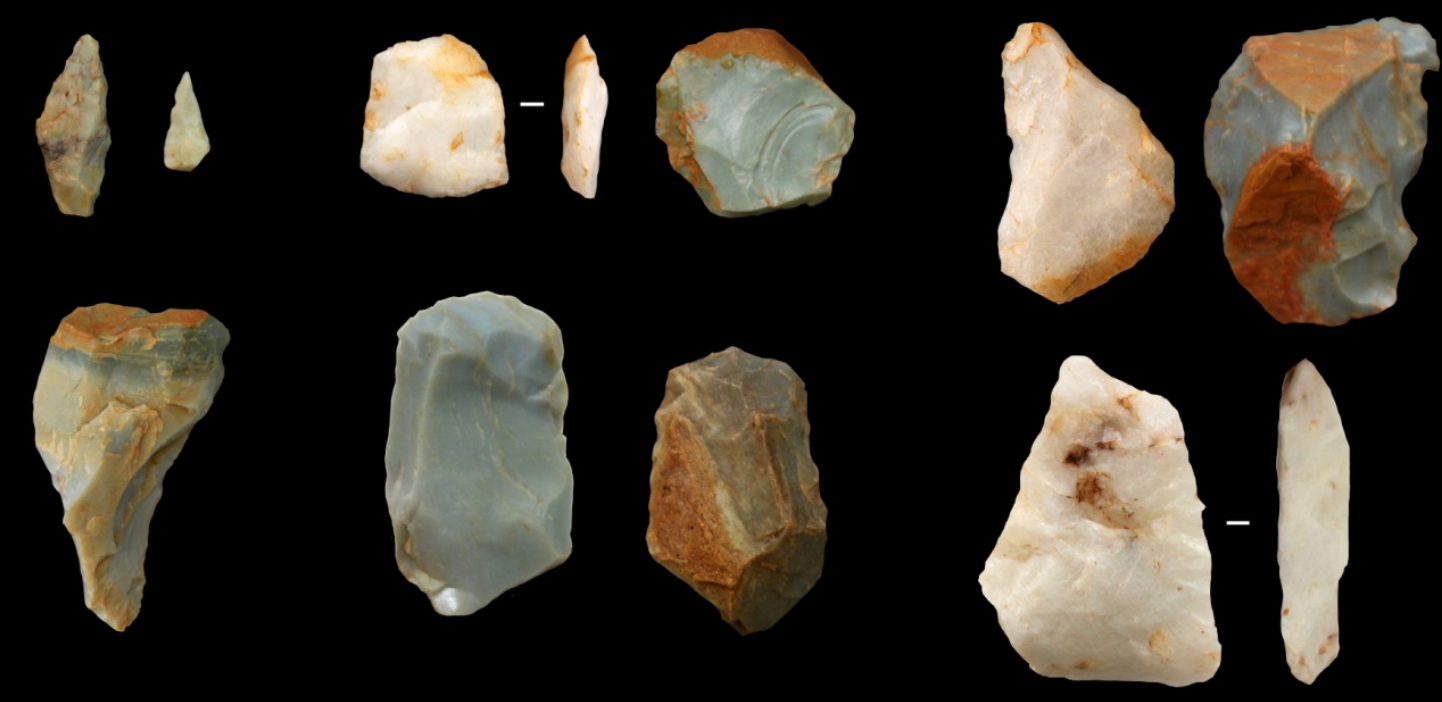


\section{Formal Tools}

Formal tools (Figure E.9) with backing and/or extensive retouch represent at least a modest investment in secondary reduction and curation.

Geometric microliths are freehand positives, usually blades, with strong backing along one or more margins and measuring less than 35 millimeters in length. On the basis of backing and effective margin shape, it was possible to define a number of subtypes for the Madina-Sadatou assemblages including segments, triangles, and trapezoids (Figure E.7). Due to their small size, it is often assumed that microliths were replaceable components in more complex tools such as sickles, harpoons, or other projectiles (Bousman 2005:212-213; Deacon 1984:274-276), although a growing number of microwear studies point to direct use by hand for cutting and scraping (see Kusimba 2003:180-183). Since they represent an investment of time and skill, geometric microliths may have also served as tokens of reciprocity in societies characterized by mobility and subsistence risk (Ambrose 2002). Whatever the case, geometric microliths were specialized components within the chipped stone tool kits of Madina-Sadatou.

Projectile points are freehand positives that have either one pointed margin or two effective working edges meeting to form an acute point. Two general subtypes exist within the Madina-Sadatou assemblages-geometric points and bifacial points. Geometric points are less than 30 millimeters long with light backing and unifacial, or in some cases bifacial, retouch. Bifacial points range up to 50 millimeters in length and have unifacial or bifacial retouch along all four margins. Presumably, projectile points were, depending on their size, mounted to spears, arrows, and harpoons to more effectively penetrate prey. That is to say, they denote diverse sorts of hunting activities.

Burins have a pointed distal margin and relatively straight lateral margins with retouch and moderate to steep edge angles. Derived from either freehand positives or heavily worked cores, most burins from Madina-Sadatou are "microliths" less than 30 millimeters in length, though some range up to 46 millimeters. The heavy investment in retouch suggests that these were specialized tools for piercing or perforating materials that could have included textiles, hides, wood, and even shell and stone.

Scrapers have one or two straight or convex working edges with moderate to steep angles and light to extensive retouch. More specifically, end scrapers have a dominant proximal/dorsal effective working edge, and side scrapers have a dominant lateral edge. Both scraper types usually derived from freehand positives with a flat ventral surface although core-based specimens also occur regularly. As broadly confirmed by ethnographic and microwear studies (Weedman 2006), the steep effective edges on scrapers make them useful for processing hides as either hand held (side scraper) or hafted (end scraper) implements.

\section{Informal Tools}

Informal tools (Figure E.10) lack significant technical investment beyond primary reduction, but have effective edges more or less amenable to certain types of tasks.

Blades are freehand positives with evidence of prepared core reduction and a length to width ratio generally greater than two. While they require greater technical investment and represent more efficient use of raw material than other informal tools, the effective working edges along the lateral margins of blades bear only traces of wear or very light retouch suggesting limited curation. The relative sharpness of the edges on 
blades makes them amenable for cutting and slicing tasks such as processing animal carcasses or harvesting plants.

Wedged blades are bipolar flakes with a lunate form, a wedge shape in cross section, and a "backing" of cortex opposite a moderate to steep effective working edge with traces of use wear or light retouch. Depending on the sharpness of the edge, these tools could have served in a variety of cutting and scraping tasks.

\section{Figure E.10: Informal chipped stone tool subtypes}

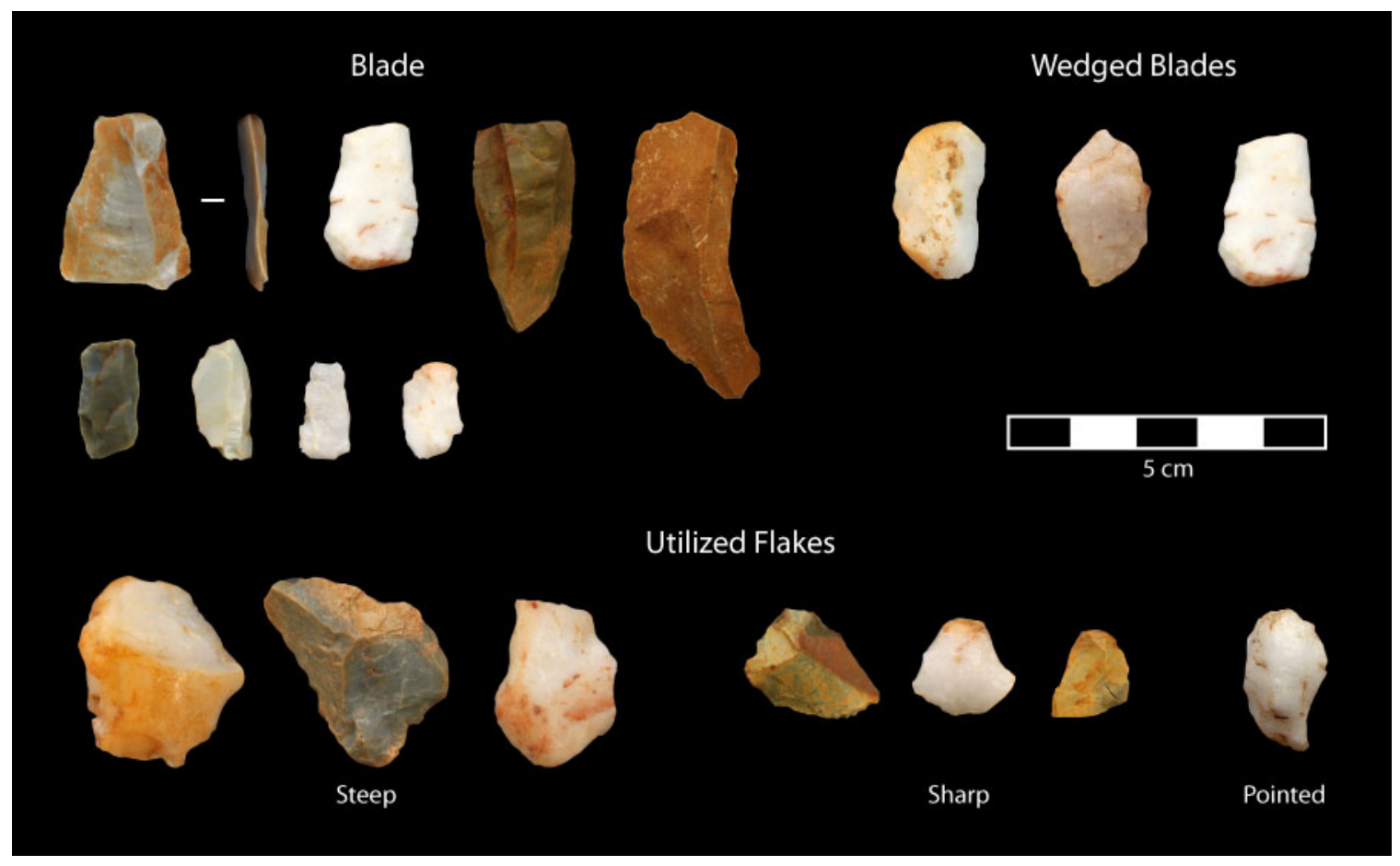

Utilized flakes include any detached piece-whether angular shatter, freehand flake, or bipolar flake-with wear or light retouch along one or two margins that does not correspond to any other type. In order to make inferences about possible use, utilized flakes in the Madina-Sadatou assemblages were further classified according to edge shape and angle: sharp utilized flakes have a sharp to moderate edge angle(s) for a diverse range of cutting and slicing tasks; steep utilized flakes have at least one steep edge angle ideal for scraping; and pointed utilized flakes have a pointed margin useful for perforation similar to more formalized burins.

Other possible functional types not subsumed within this scheme include fire starters and gunflints (see Appendix G regarding the latter). As summarized by Daphne Gallagher (2010:163), the contemporary use of quartz and flint with iron strikers may help to explain the presence of detached flint pieces at late prehistoric sites in Burkina Faso. Although it was not possible to identify chipped stone artifacts for fire starting, this was certainly one possible function of the pieces recovered on sites from all periods in the Madina-Sadatou study area. 
Table E.8: Summary of formal and informal quartz tool subtypes by site

\begin{tabular}{|c|c|c|c|c|c|c|c|c|c|c|c|}
\hline \multicolumn{2}{|l|}{ Site } & \multicolumn{10}{|c|}{ Formal Tools } \\
\hline \multirow{3}{*}{\multicolumn{2}{|c|}{$\begin{array}{c}\text { Total } \\
n \\
\end{array}$}} & \multirow{2}{*}{\multicolumn{2}{|c|}{$\begin{array}{l}\text { End/Side } \\
\text { Scraper }\end{array}$}} & \multirow{2}{*}{\multicolumn{2}{|c|}{ Point }} & \multirow{2}{*}{\multicolumn{2}{|c|}{ Burin }} & \multicolumn{4}{|c|}{ Geometric Microlith } \\
\hline & & & & & & & & \multicolumn{2}{|c|}{ Segment } & \multicolumn{2}{|c|}{ Other } \\
\hline & & $n$ & $\%$ & $n$ & $\%$ & $n$ & $\%$ & $n$ & $\%$ & $n$ & $\%$ \\
\hline FAL-1022 & 10 & 0 & $0.0 \%$ & 3 & $30.0 \%$ & 0 & 0.0\% & 4 & $40.0 \%$ & 3 & $30.0 \%$ \\
\hline FAL-1062 & 2 & 1 & $50.0 \%$ & 0 & $0.0 \%$ & 0 & $0.0 \%$ & 0 & $0.0 \%$ & 1 & $50.0 \%$ \\
\hline FAL-1075 & 3 & 0 & $0.0 \%$ & 1 & $33.3 \%$ & 0 & $0.0 \%$ & 0 & $0.0 \%$ & 2 & $66.7 \%$ \\
\hline Diouboye & 30 & 9 & $30.0 \%$ & 17 & $56.7 \%$ & 0 & $0.0 \%$ & 1 & $3.3 \%$ & 3 & $10.0 \%$ \\
\hline \multicolumn{2}{|l|}{ Site } & \multicolumn{10}{|c|}{ Informal Tools } \\
\hline \multirow{2}{*}{\multicolumn{2}{|c|}{ Total }} & \multirow{2}{*}{\multicolumn{2}{|c|}{ Blade }} & \multirow{2}{*}{\multicolumn{2}{|c|}{$\begin{array}{c}\text { Wedged } \\
\text { Blade }\end{array}$}} & \multicolumn{6}{|c|}{ Utilized Flake } \\
\hline & & & & & & \multicolumn{2}{|c|}{ Sharp } & \multicolumn{2}{|c|}{ Steep } & \multicolumn{2}{|c|}{ Point } \\
\hline & $n$ & $n$ & $\%$ & $n$ & $\%$ & $n$ & $\%$ & $n$ & $\%$ & $n$ & $\%$ \\
\hline FAL-1022 & 22 & 3 & $13.6 \%$ & 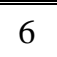 & $27.3 \%$ & 10 & $45.5 \%$ & 3 & $13.6 \%$ & 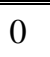 & $0.0 \%$ \\
\hline FAL-1062 & 21 & 2 & $9.5 \%$ & 7 & $33.3 \%$ & 6 & $28.6 \%$ & 6 & $28.6 \%$ & 0 & $0.0 \%$ \\
\hline FAL-1075 & 10 & 0 & $0.0 \%$ & 4 & $40.0 \%$ & 2 & $20.0 \%$ & 4 & $40.0 \%$ & 0 & $0.0 \%$ \\
\hline Diouboye & 75 & 6 & $8.0 \%$ & 22 & $29.3 \%$ & 15 & $20.0 \%$ & 31 & $41.3 \%$ & 1 & $1.3 \%$ \\
\hline
\end{tabular}

Table E.9: Summary of formal and informal chert tool subtypes by site

\begin{tabular}{|c|c|c|c|c|c|c|c|c|c|c|c|}
\hline \multicolumn{2}{|l|}{ Site } & \multicolumn{10}{|c|}{ Formal Tools } \\
\hline \multirow{3}{*}{\multicolumn{2}{|c|}{$\begin{array}{c}\text { Total } \\
n \\
\end{array}$}} & \multirow{2}{*}{\multicolumn{2}{|c|}{$\begin{array}{l}\text { End/Side } \\
\text { Scraper }\end{array}$}} & \multirow{2}{*}{\multicolumn{2}{|c|}{ Point }} & \multirow{2}{*}{\multicolumn{2}{|c|}{ Burin }} & \multicolumn{4}{|c|}{ Geometric Microlith } \\
\hline & & & & & & & & \multicolumn{2}{|c|}{ Segment } & \multicolumn{2}{|c|}{ Other } \\
\hline & & $n$ & $\%$ & $n$ & $\%$ & $n$ & $\%$ & $n$ & $\%$ & $n$ & $\%$ \\
\hline FAL-1022 & 27 & 6 & $22.2 \%$ & 2 & $7.4 \%$ & 2 & $7.4 \%$ & 15 & $55.6 \%$ & 2 & $7.4 \%$ \\
\hline FAL-1062 & 25 & 7 & $28.0 \%$ & 5 & $20.0 \%$ & 2 & $8.0 \%$ & 5 & $20.0 \%$ & 6 & $24.0 \%$ \\
\hline FAL-1075 & 12 & 4 & $33.3 \%$ & 5 & $41.7 \%$ & 1 & $8.3 \%$ & 1 & $8.3 \%$ & 1 & $8.3 \%$ \\
\hline Diouboye & 34 & 9 & $26.5 \%$ & 9 & $26.5 \%$ & 4 & $11.8 \%$ & 10 & $29.4 \%$ & 2 & $5.9 \%$ \\
\hline \multicolumn{2}{|l|}{ Site } & \multicolumn{10}{|c|}{ Informal Tools } \\
\hline \multirow{2}{*}{\multicolumn{2}{|c|}{ Total }} & \multirow{2}{*}{\multicolumn{2}{|c|}{ Blade }} & \multirow{2}{*}{\multicolumn{2}{|c|}{$\begin{array}{l}\text { Wedged } \\
\text { Blade }\end{array}$}} & \multicolumn{6}{|c|}{ Utilized Flake } \\
\hline & & & & & & \multicolumn{2}{|c|}{ Sharp } & \multicolumn{2}{|c|}{ Steep } & \multicolumn{2}{|c|}{ Point } \\
\hline & $n$ & $n$ & $\%$ & $n$ & $\%$ & $n$ & $\%$ & $n$ & $\%$ & $n$ & $\%$ \\
\hline FAL-1022 & 36 & 14 & $38.9 \%$ & 0 & $0.0 \%$ & 9 & $25.0 \%$ & 13 & $36.1 \%$ & 0 & $0.0 \%$ \\
\hline FAL-1062 & 37 & 1 & $2.7 \%$ & 0 & $0.0 \%$ & 25 & $67.6 \%$ & 11 & $29.7 \%$ & 0 & $0.0 \%$ \\
\hline FAL-1075 & 24 & 0 & $0.0 \%$ & 0 & $0.0 \%$ & 11 & $45.8 \%$ & 13 & $54.2 \%$ & 0 & $0.0 \%$ \\
\hline Diouboye & 74 & 8 & $10.8 \%$ & 4 & $5.4 \%$ & 32 & $43.2 \%$ & 26 & $35.1 \%$ & 4 & $5.4 \%$ \\
\hline
\end{tabular}


Figure E.11: Relative abundance of informal and formal quartz tool subtypes

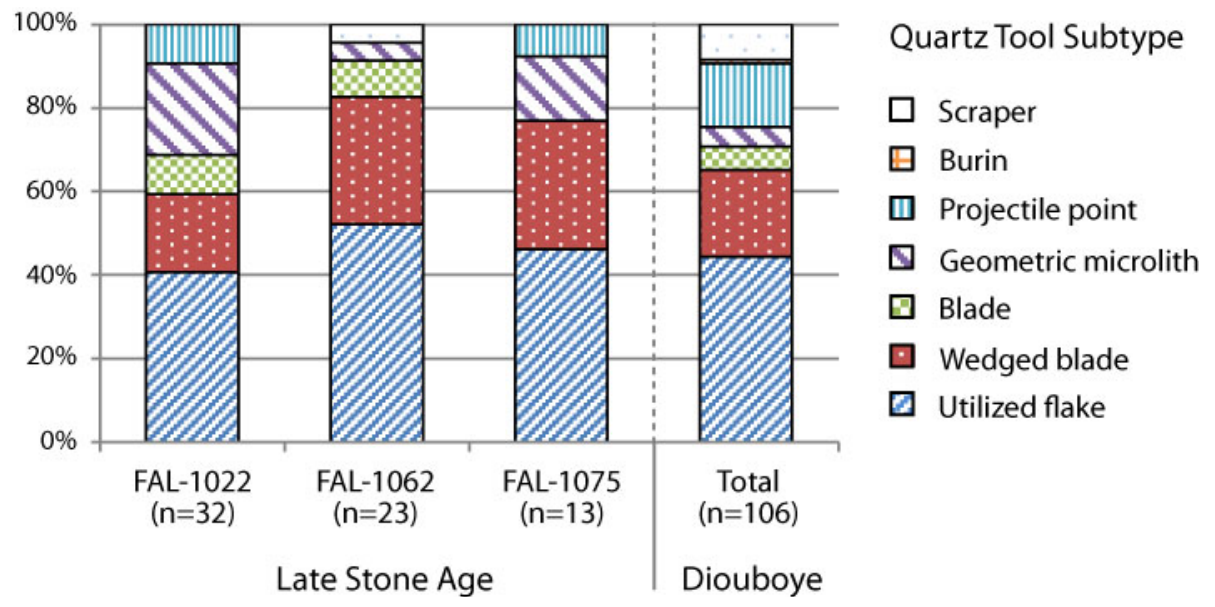

Figure E.12: Relative abundance of informal and formal chert tool subtypes

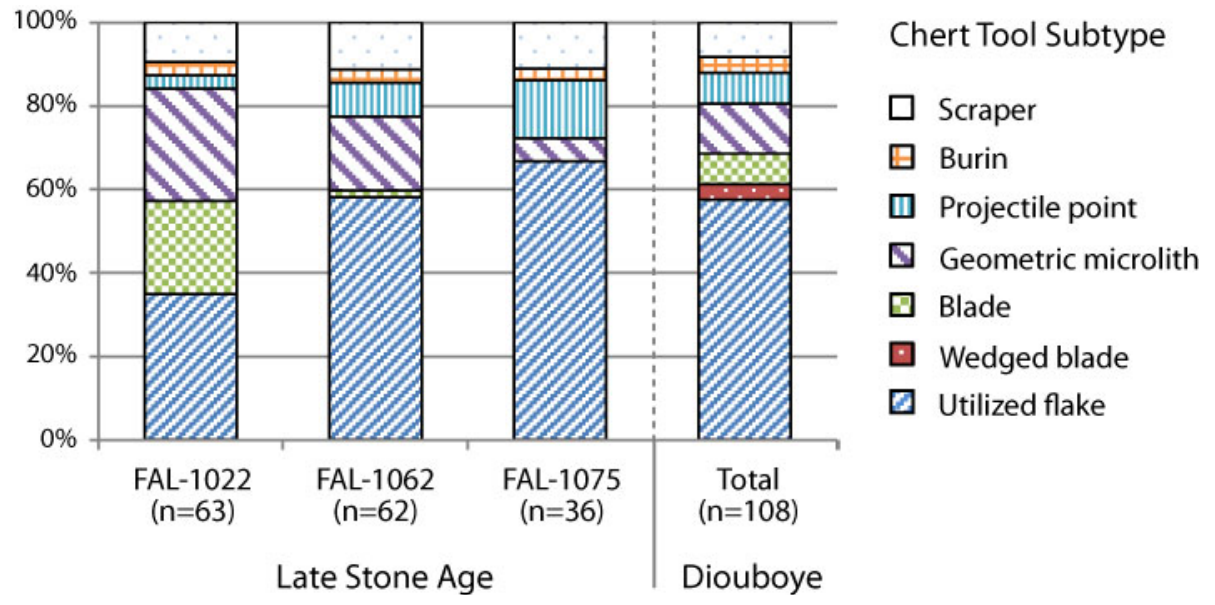

\section{Comparison of Chipped Stone Tool Use}

In the absence of direct evidence for tool use, a comparison of the LSA and Diouboye assemblages nonetheless points to continuity in formal and informal chipped stone tool kits and, presumably, some of their associated economic tasks. At the same time, subtle differences across these assemblages are consistent with a shift from relatively mobile to sedentary settlement strategies.

Although formal tools were relatively less abundant at Diouboye, their subtype distribution falls more or less within the range of variability observed at LSA sites in the study area (Tables E.8-E.9, Figures E.11-E.12). While the LSA assemblages boast an overall greater percentage of microliths, it is noteworthy that the relative frequencies of informal tools are both high and fairly consistent across the two archaeological components. Among the quartz artifacts, for example, the proportions of neither utilized flakes $\left(\chi^{2}=0.03, \rho=0.87\right)$, nor cortex blades $\left(\chi^{2}=0.43, \rho=0.51\right)$ differed significantly between the LSA component sites and Diouboye. This suggests that, despite predilections 
for different raw materials, both LSA and Iron Age economic practices relied upon a similar range of informal chipped stone tools.

Measures of assemblage diversity can reveal functional similarities or differences across the LSA and Diouboye toolkits, provided that one controls for the "sample-size effect” with bootstrap resampling to estimate confidence intervals (Baxter 2001). In Tables E.10-E.11 above, the Shannon H and Simpson 1-D indices each provide a measure of assemblage evenness that increases as a growing number of types become evenly dispersed within the sampled populations (Conkey 1989), while Margalef's richness SR index scales the number of types by the natural log of total specimens within the sampled populations. These measures show that quartz tool types are more evenly distributed at Diouboye although the significance of this pattern may be due to sample size bias. On the other hand, the chert tool assemblage at Diouboye appears to be significantly less rich than the LSA sites FAL-1062 and FAL-1075. The pooled assemblages from the three LSA sites, however, reveal no significant differences with those from Diouboye.

Table E.10: Diversity indices for quartz informal blades and formal tool subtypes with bootstrapped confidence intervals $(0.95)$

\begin{tabular}{|c|c|c|cc|cc|cc|}
\hline Site & n & S & \multicolumn{2}{|c|}{ Shannon H Entropy } & \multicolumn{2}{c|}{ Simpson Evenness } & \multicolumn{2}{c|}{ Margalef Richness } \\
& & & H & CI & 1-D & CI & SR & CI \\
\hline \hline FAL-1022 & 13 & 4 & 1.38 & $0.98-1.74$ & 0.75 & $0.56-0.82$ & 1.17 & $0.78-1.95$ \\
\hline FAL-1062 & 4 & 3 & 1.04 & $0.00-1.39$ & 0.63 & $0.00-0.75$ & 1.44 & $0.00-2.16$ \\
\hline FAL-1075 & 3 & 3 & 0.64 & $0.00-1.10$ & 0.44 & $0.00-0.67$ & 0.91 & $0.00-1.82$ \\
\hline LSA Total & 20 & 5 & 1.50 & $1.38-1.87$ & 0.77 & $0.70-0.84$ & 1.34 & $1.34-1.34$ \\
\hline Diouboye & 36 & 6 & 1.52 & $1.33-1.73$ & 0.75 & $0.67-0.81$ & 1.40 & $1.12-1.40$ \\
\hline
\end{tabular}

Table E.11: Diversity indices for chert informal blades and formal tool subtypes with bootstrapped confidence intervals (0.95)

\begin{tabular}{|c|c|c|cc|cc|cc|}
\hline Site & n & S & \multicolumn{2}{|c|}{ Shannon H Entropy } & \multicolumn{2}{c|}{ Simpson Evenness } & \multicolumn{2}{c|}{ Margalef Richness } \\
& & & H & CI & 1-D & CI & SR & CI \\
\hline \hline FAL-1022 & 41 & 6 & 1.55 & $1.58-1.90$ & 0.73 & $0.76-0.84$ & 1.62 & $1.35-1.62$ \\
\hline FAL-1062 & 26 & 6 & 1.83 & $1.49-1.89$ & 0.83 & $0.73-0.84$ & 1.84 & $1.23-1.84$ \\
\hline FAL-1075 & 12 & 6 & 1.58 & $1.20-1.86$ & 0.75 & $0.64-0.83$ & 0.83 & $0.76-0.84$ \\
\hline LSA Total & 79 & 7 & 1.85 & $1.68-1.88$ & 0.83 & $0.78-0.84$ & 1.37 & $1.37-1.37$ \\
\hline Diouboye & 42 & 7 & 1.85 & $1.58-1.90$ & 0.83 & $0.76-0.84$ & 1.61 & $1.34-1.61$ \\
\hline
\end{tabular}

Assuming that these indices provide some measure of the diversity or specialty of activities performed with chipped stone tools, they imply that the occupants of Diouboye employed quartz, but not chert, for a wider array of less specialized tasks than earlier LSA populations, whose toolkits varied subtly across the sampled sites. These patterns 
support the interpretation of more specialized activities occurring at shorter-term LSA sites in comparison to the sedentary community at Diouboye. At the same time, these data suggest that the occupants of Diouboye were less discriminant about what type of raw material to use for tasks involving both formal and informal tools.

Beyond diversity, a closer inspection of size variation reveals subtle differences in chipped stone tool use as well as socioeconomic strategies for mobility (Table E.12). With the notable exception of projectile points, both formal and informal chert tools are significantly smaller in the LSA than the Diouboye assemblage. Even the LSA chert microliths are more micro! Quartz tools also tend to be smaller in the LSA assemblages although not always significantly so. The greater variation in tool size at Diouboye, despite smaller sample sizes in many cases, further supports the case for lithic deposition resulting from economic activities at the site.

Possible interpretations for the larger tools at Diouboye include the production of smaller chert tools via freehand reduction by LSA stone knappers and/or more extensive retouch on such artifacts resulting from prolonged curation. As discussed above, a lighter and more formalized chert toolkit would have prepared mobile LSA communities for situations encountered away from raw material sources, even as they made somewhat expedient use of informal chert and quartz tools at the encampments of FAL-1022, FAL1062, and FAL-1075. Variability across these latter three sites gives a tantalizing glimpse of the dynamic social and spatial organization of lithic technologies during the mid- to late-Holocene-a topic that clearly merits investigation through further analysis of LSA materials from the Falémé River basin. The presence of larger microliths at Diouboye

\section{Table E.12: Summary of informal and formal tool subtype size (length)}

\begin{tabular}{|c|c|c|c|c|c|c|c|c|c|}
\hline \multirow{3}{*}{$\begin{array}{c}\text { Tool Type } \\
\text { Raw material }\end{array}$} & \multicolumn{6}{|c|}{ Length (mm) } & \multicolumn{3}{|c|}{ Two-Tailed T-Test } \\
\hline & \multicolumn{3}{|c|}{ LSA } & \multicolumn{3}{|c|}{ Diouboye } & & & \\
\hline & $n$ & $\mu$ & $\sigma$ & $n$ & $\mu$ & $\sigma$ & $t$ & $d f$ & $\rho$ \\
\hline \multicolumn{10}{|l|}{ Sharp util. flake } \\
\hline Quartz & 15 & 19.35 & 1.91 & 15 & 22.45 & 1.91 & 1.15 & 28 & 0.130 \\
\hline Chert & 34 & 18.12 & 0.92 & 25 & 24.86 & 1.07 & 4.77 & 57 & $<0.001$ \\
\hline \multicolumn{10}{|l|}{ Steep util. flake } \\
\hline Quartz & 12 & 23.08 & 2.09 & 23 & 28.23 & 1.51 & 1.99 & 33 & 0.027 \\
\hline Chert & 33 & 24.15 & 1.46 & 26 & 31.11 & 1.64 & 3.18 & 57 & 0.001 \\
\hline \multicolumn{10}{|l|}{ Side scraper } \\
\hline Quartz & 1 & 41.20 & - & 4 & 26.63 & 2.07 & - & - & - \\
\hline Chert & 9 & 27.47 & 2.21 & 6 & 37.28 & 2.70 & 2.81 & 13 & 0.007 \\
\hline \multicolumn{10}{|l|}{ Projectile point } \\
\hline Quartz & 3 & 17.30 & 2.86 & 15 & 20.47 & 1.28 & 1.01 & 16 & 0.328 \\
\hline Chert & 13 & 22.54 & 1.74 & 9 & 25.87 & 2.09 & 1.22 & 20 & 0.235 \\
\hline \multicolumn{10}{|l|}{ Microlith (all) } \\
\hline Quartz & 10 & 17.90 & 1.64 & 2 & 17.60 & 3.67 & 0.07 & 10 & 0.942 \\
\hline Chert & 27 & 15.57 & 0.87 & 10 & 22.16 & 1.43 & 3.93 & 35 & $<0.001$ \\
\hline
\end{tabular}


suggests either that people there did not need to minimize the weight of their toolkits or, more provocatively, that they intentionally scavenged larger microliths from LSA sites along the river. Whatever the case may have been, the available data indicate that the community of Diouboye relied strongly upon informal quartz tools for undertaking diverse economic activities within the village.

But what were these activities? Surface collection and excavation at Diouboye yielded a range of formal and informal tool types amenable for cutting, chopping, piercing, and scraping. Access to iron did not necessarily relegate chipped stone tools to one particular economic realm, but rather they probably continued to find use in hideworking and sewing, hunting and butchery, and perhaps the procurement and processing of wild and domesticated plants (see Chapter 6 for discussion). For this reason, the distributions of these tool types and their production debris also shed light on the spatial organization of chipped stone tool manufacture and use at Diouboye (Chapters 7-9).

\section{GROUNDSTONE TECHNOLOGY IN THE MADINA-SADATOU STUDY AREA}

Groundstone technology subsumes the production and/or use of stone tools through techniques of grinding, abrading, polishing, and pecking (J. L. Adams 2002). The resulting implements - including axes, handstones, pestles, grinding slabs, mortars, and hammerstones-are relatively bulky and require greater labor investment than chipped stone, but their durability and form are advantageous for redundant tasks such as chopping wood and processing grains or other organic and mineral resources. Since groundstone often appears in the technological repertoire of people engaged in intensive foraging or food production, technical choices must articulate with these broader subsistence strategies (David 1998; K. I. Wright 1994), patterns of mobility/sedentism (Graham 1994; Schlanger 1991), and even craft and political economies (Edmonds 1995; K. I. Wright 2008). This technology also entails embodied and practical knowledge mediated through inter-generational institutions and identities, most often ethnicity and gender (J. L. Adams 2002:54-57). Altogether the study of groundstone artifacts from Madina-Sadatou can help to chart shifting regimes of subsistence and mobility across the historical landscape and document the socio-spatial organization of food processing and craft activities within Diouboye.

Table E.13: Distribution of sites with groundstone by archaeological component

\begin{tabular}{|c|c|c|c|c|c|c|c|}
\hline \multirow{3}{*}{\multicolumn{2}{|c|}{$\begin{array}{r}\text { Archaeological Component(s) } \\
\text { Total Localities } \\
n \\
\end{array}$}} & \multicolumn{3}{|c|}{ Systematic Sampling } & \multicolumn{3}{|c|}{ Purposive Sampling } \\
\hline & & \multicolumn{2}{|c|}{ Localities } & \multirow{2}{*}{$\begin{array}{c}\text { Artifacts } \\
n \\
\end{array}$} & \multicolumn{2}{|c|}{ Localities } & \multirow{2}{*}{$\begin{array}{c}\text { Artifacts } \\
n \\
\end{array}$} \\
\hline & & $n$ & $\%$ & & $n$ & $\%$ & \\
\hline MSA/LSA & 10 & 2 & $20.0 \%$ & 4 & 3 & $30.0 \%$ & 3 \\
\hline cLSA & 35 & 9 & $25.7 \%$ & 14 & 13 & $37.1 \%$ & 19 \\
\hline cLSA/IA & 6 & 2 & $33.3 \%$ & 3 & 3 & $50.0 \%$ & 4 \\
\hline cLSA/IA/Atlantic & 4 & 1 & $25.0 \%$ & 1 & 2 & $50.0 \%$ & 2 \\
\hline cLSA/Atlantic & 18 & 9 & $50.0 \%$ & 21 & 8 & $44.4 \%$ & 32 \\
\hline IA & 13 & 6 & $46.2 \%$ & 12 & 2 & $15.4 \%$ & 8 \\
\hline IA/Atlantic & 2 & 1 & $50.0 \%$ & 1 & 0 & $0.0 \%$ & 0 \\
\hline Atlantic & 18 & 4 & $22.2 \%$ & 13 & 3 & $16.7 \%$ & 6 \\
\hline
\end{tabular}


The distinction between chipped stone and groundstone technologies is above all an analytical one since people frequently employ abrading techniques in the former, and knapping techniques in the latter (J. L. Adams 2002). Given this overlap, I have adapted the above operational sequence of raw material procurement, reduction, and useacknowledging that these latter two stages are deeply intertwined in groundstone technologies. Drawing upon ethnographic, experimental, and archaeological syntheses (J. L. Adams 2002; Hayden 1979; Rowan and Ebeling 2008), this section outlines the attributes recorded to identify past technical choices made within each stage, and thereby infer social and practical parameters on groundstone technologies in the Madina-Sadatou study area.

The ensuing analysis considers two assemblages of groundstone artifacts: (1) a Diouboye assemblage including 195 specimens from excavation and surface collection; and (2) a Survey assemblage including 148 specimens from systematic and purposive collections. In addition to the 55 sites and isolated occurrences sampled for the Survey assemblage (see Appendix A), groundstone artifacts (primarily handstone and grinding slab fragments) at an additional 51 localities, were observed, but not collected. As summarized in Table E.13, it is important to recall that, in contrast to the analysis of chipped stone tools, the Survey assemblage derives from sites and isolated occurrences whose archaeological components range from the aceramic LSA to the Atlantic Era (see Chapter 4). Although I attempt to draw distinctions among these components whenever possible, this is made difficult by the apparent longevity of groundstone technologies in Madina-Sadatou and the palimpsest nature of many surface assemblages.

\section{Raw Material Selection}

Raw material properties, particularly hardness, granularity, and size, all affect possible groundstone tool forms and techniques of manufacture (J. L. Adams 2002:1920). Since the relative abundance of desirable materials thus establishes parameters on groundstone technology, a comparison of available and exploited raw materials across the historical landscape of Madina-Sadatou can provide some indication of the economic strategies and cultural preferences shaped by stages of tool manufacture and use.

Despite some overlap with chipped stone materials such as quartz and schist, groundstone artifacts were generally composed of the more durable metamorphic and igneous rocks listed in Table E.3. While pieces large enough for grinding slabs may have required travel to outcrops more than ten kilometers distant, people from Diouboye could have feasibly obtained smaller cobbles sufficient for handstones and quartz

hammerstones from alluvial deposits around the Falémé River. Unfortunately, the source of hematite, which predominates in all the groundstone assemblages, has not been defined. While relatively unworked pieces appear smooth and water-worn, hinting at an origin in the Falémé River bed, the ubiquity of this material at a number of sites suggests either intensive exploitation of this source or perhaps a primary one farther to the south.

Figure E.13 illustrates the relative abundance of groundstone materials from Diouboye, systematic collections at all surveyed sites, and all collections from sites with a single cLSA, Iron Age, or Atlantic era component. The occupants of Diouboye and other Iron Age sites consumed a similar range of raw materials, including sandstone and rhyolite, and correspondingly less hematite than people associated with cLSA and 
Atlantic Era occupations. As discussed below, these raw material choices seem to correspond with decisions about tool use across archaeological horizons.

Figure E.13: Relative abundance of raw materials in groundstone assemblages

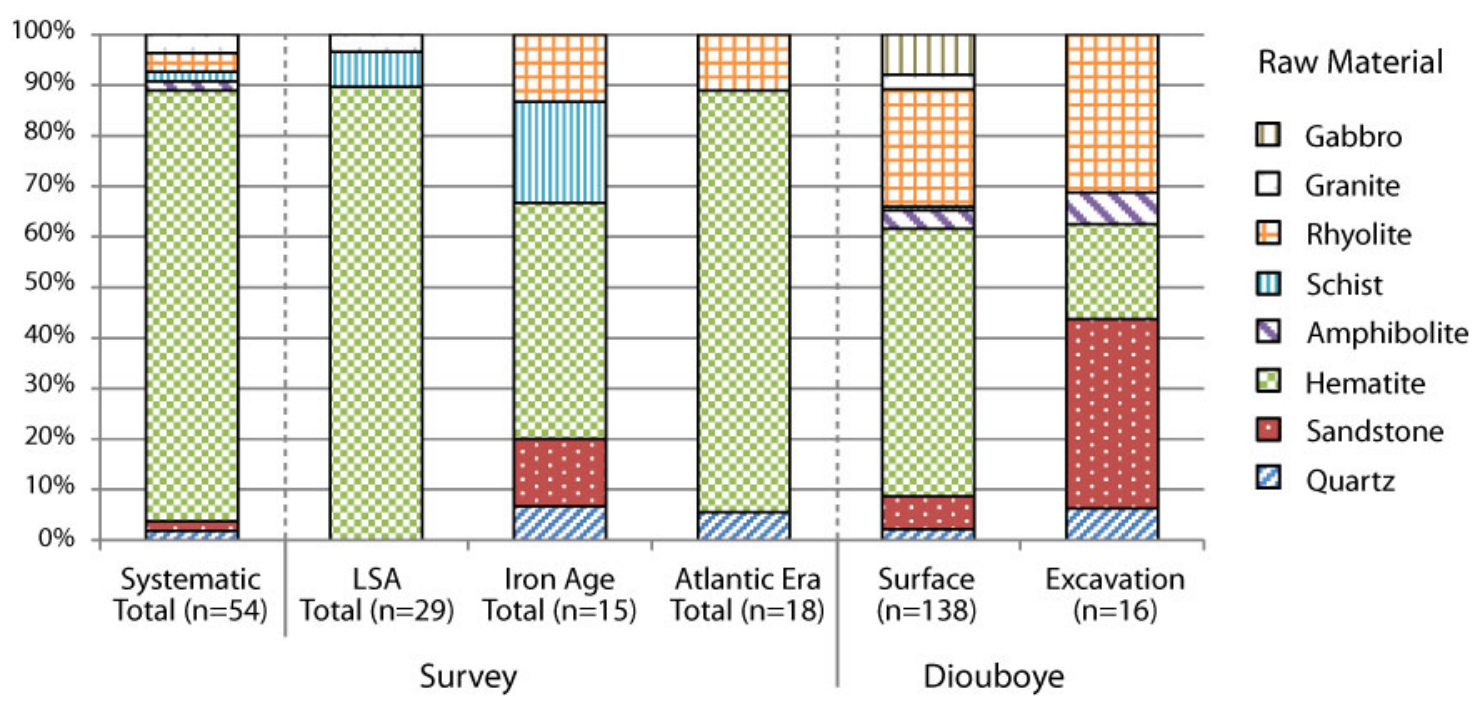

\section{Manufacture and Use}

Following archaeologist Jenny Adams (2002), it is useful to distinguish between expedient and strategic groundstone designs. Expedient tools are only altered through use, but strategic tools are modified to improve their use for specific tasks. Even as these strategies depend, in part, on the quality and abundance of raw materials, they each entail an idealized set of technical choices regarding reduction and function that can be traced through variables of form and wear.

Table E.14 outlines the groundstone artifact forms observed in the MadinaSadatou study area and the variables measured to record their size and fragmentation. Debitage from groundstone tool reduction and regeneration, including infrequent hematite and rare amphibolite specimens, was analyzed as part of the chipped stone assemblage described above. Along with finished tool forms, it was possible to study reduction sequences through associated blanks and preforms for hafted hematite tools.

\section{Percussion Tools}

Percussion tools have traces of extensive battering along one or more surfaces or edges, but no evidence of chipped stone reduction (thereby distinguishing them from bipolar cores). In addition to the variables listed in Table E.14, I recorded the number and position of worn surfaces and edges to identify two expedient types (Figure E.14).

Choppers display heavy chipping wear along one relatively sharp margin. These implements are rare in the assemblages from Madina-Sadatou, including only one rhyolite specimen from Diouboye and one hematite specimen from a cLSA component site. This latter, illustrated in Figure E.14, appears to be a broken hematite axe preform recycled as a small chopper. 
Table E.14: Variables recorded for groundstone tool form and size

\begin{tabular}{|c|c|c|}
\hline Variable & Attribute (Code) & Description \\
\hline \multirow{4}{*}{ Form } & Percussion tool (1) & $\begin{array}{l}\text { Surface(s) have pitting or fractures from percussion against } \\
\text { inorganic materials }\end{array}$ \\
\hline & Grinding tool (2) & $\begin{array}{l}\text { Surface(s) is smooth from repeated abrasion against organic } \\
\text { and/or inorganic materials }\end{array}$ \\
\hline & Polished tool (3) & $\begin{array}{l}\text { Surface(s) has sheen from polishing against inorganic } \\
\text { materials }\end{array}$ \\
\hline & Hafted tool (4) & $\begin{array}{l}\text { Surface(s) have pitting or sheen from percussion against } \\
\text { diverse materials; form is amenable to hafting }\end{array}$ \\
\hline \multirow{11}{*}{ Type } & Hammerstone (1) & See Figure E.14 \\
\hline & Chopper (2) & See Figure E.14 \\
\hline & Axe/Hachette (3) & See Figure E.15 \\
\hline & Bored stone (4) & See Figure E.15 \\
\hline & Handstone (5) & See Figure E.21 \\
\hline & Grinding Slab (6) & See Figure E.21 \\
\hline & Pestle (7) & See Figure E.21 \\
\hline & Polishing stone (8) & See Figure E.24 \\
\hline & Sharpening stone (9) & See Figure E.24 \\
\hline & Blade (10) & See Figure E.24 \\
\hline & Horn (11) & See Figure E.24 \\
\hline \multirow{4}{*}{ Fragmentation } & Complete (0) & Specimen appears complete or nearly complete \\
\hline & Proximal end only (1) & For hafted tools, proximal end, but not distal end, is present \\
\hline & Distal end only (2) & For hafted tools, distal end, but not proximal end, is present \\
\hline & Fragment (3) & Specimen is fragmentary or otherwise incomplete \\
\hline Weight & Grams (0.1 g) & Mass of the specimen \\
\hline Length & Millimeters $(0.1 \mathrm{~mm})$ & Length or maximum linear dimension of the specimen \\
\hline Proximal Width & Millimeters $(0.1 \mathrm{~mm})$ & Proximal or maximum width of the specimen \\
\hline Distal Width & Millimeters $(0.1 \mathrm{~mm})$ & Distal width of the specimen \\
\hline Thickness & Millimeters $(0.1 \mathrm{~mm})$ & Maximum thickness of specimen \\
\hline
\end{tabular}

Hammerstones have one or more heavily battered or worn surface. Comprising their own subclass of lithic artifact during initial sorting, hammerstones occur in modest frequencies in both the Diouboye $(n=40)$ and Survey $(n=5)$ assemblages. At Diouboye these come in a variety of materials although quartz (40.0\%) was by far predominant. Beginning as small cobbles with a diameter of $42-97$ millimeters $(\mu=69.2 \mathrm{~mm}, \sigma=10.5$ $\mathrm{mm})$, hammerstones were worked on one surface (22.2\%) or, more frequently, four to five surfaces (61.1\%), to yield a distinctively cubic stone with lightly concave depressions on several faces (Figure E.14). It was also possible to identify eight fragmentary hammerstones in the Diouboye assemblage, indicating that some chipped quartz likely derived from wear on these implements. The small size of the Survey assemblage precludes more detailed analysis, but hammerstones do appear on sites from all periods - one quartz specimen even turned up on a single component Atlantic era site. 
The functions of hammerstones and choppers from Madina-Sadatou cannot be independently ascertained without microscopic analyses of wear or residues, but the absence of two-handed percussive implements does steer interpretation away from activities that require heavy force such as mining. Although people could have used single-handed tools to process organic materials such as animal hides, grains, or other foodstuffs, experimental replications have demonstrated that quartz hammerstones with battered surfaces similar to those in the Madina-Sadatou assemblages most often result from chipped stone reduction and/or handstone and grinding slab rejuvenation (J. L. Adams 2002:151-153; David 1998:23, 35; Dodd 1979). Indeed, the limited numbers of hammerstones at LSA and Atlantic Era sites, and their spatial correlation with other groundstone implements at Diouboye, lends support to an interpretation of their use in semi-specialized socioeconomic activities.

\section{Figure E.14: Groundstone percussion tool types}

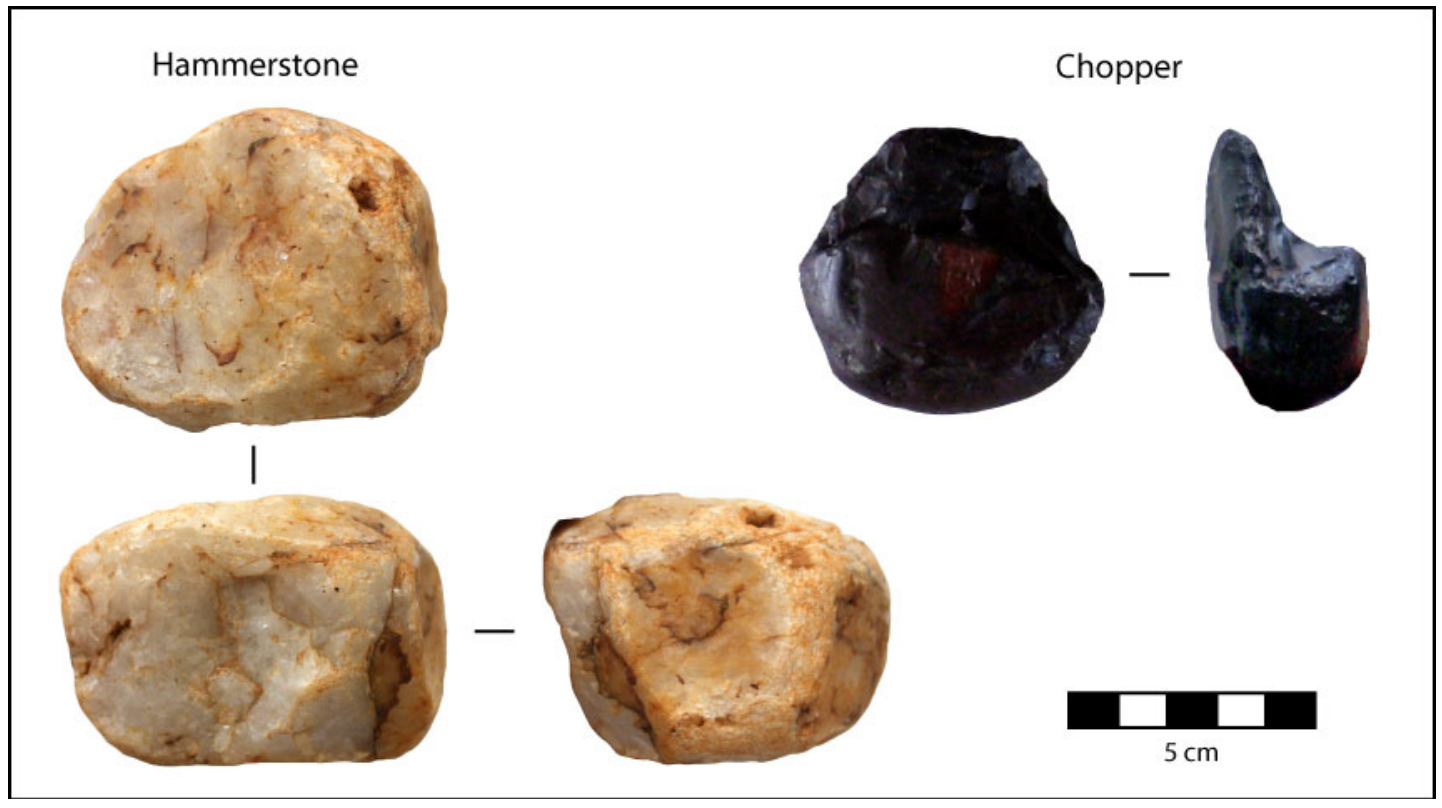

\section{Hafted Tools}

Hafted tools have strategic designs with elements or dimensions for attachment to a handle, thereby allowing more forceful hammering, chopping, and digging. Following a descriptive scheme established by André Leroi-Gourhan (1966:256-259; also Adams 2002), Table E.15 lists the variables recorded for hafted tools, and for grinding and polished tools where appropriate.

Bored stones include any ground or polished ring or sphere with an internal opening sufficient for mounting the object on a wooden haft, presumably as a weight. As discussed by Kevin MacDonald (1998), such objects are strongly associated with pastoral LSA components in the archaeology of West Africa. The two bored stone artifacts recovered during fieldwork in Madina-Sadatou (Figure E.15) include: one spherical 
weight (FAL-1006), possibly used as a mace head or digging stick weight; and one extremely fine polished ring (FAL-1125), possibly worn as a decorative arm ring.

Table E.15: Variables recorded for hafted groundstone tools

\begin{tabular}{|c|c|c|}
\hline Variable & Attribute (Code) & Description \\
\hline \multirow{4}{*}{$\begin{array}{l}\text { Reduction } \\
\text { Stage }\end{array}$} & Blank (0) & Raw material in rough shape and size of a hafted tool \\
\hline & Preform (1) & $\begin{array}{l}\text { Margins, but not blade, worked into shape of a hafted tool; } \\
\text { some cortex present and faces not ground }\end{array}$ \\
\hline & Semi-ground (2) & $\begin{array}{l}\text { Margins and blade worked into the shape of a hafted tool; } \\
\text { faces not fully ground }\end{array}$ \\
\hline & Fully-ground (3) & Hafted tool with fully ground margins, faces, and blade \\
\hline \multirow{4}{*}{ Tool Profile } & Rectilinear (1) & Rectangular in cross-section \\
\hline & Rounded rectilinear (2) & Rectangular with rounded corners in cross-section \\
\hline & Convex (3) & Elliptical in cross-section \\
\hline & Round (4) & Circular in cross-section \\
\hline \multirow{5}{*}{ Bit Shape } & Symmetrical convex (1) & Two symmetrical convex planes in cross-section \\
\hline & Asymmetrical convex (2) & Two asymmetrical convex planes in cross-section \\
\hline & Symmetrical bevel (3) & Two symmetrical flat planes in cross-section \\
\hline & Asymmetrical bevel (4) & Two asymmetrical flat planes in cross-section \\
\hline & Round (5) & Rounded in cross-section \\
\hline \multirow{5}{*}{ Blade Shape } & Rectilinear (1) & Rectangular proximal end \\
\hline & Oblique (2) & Oblique proximal end \\
\hline & Symmetrical convex (3) & Convex and symmetrical proximal end \\
\hline & Asymmetrical convex (4) & Convex but asymmetrical proximal end \\
\hline & Point (5) & Pointed proximal end \\
\hline \multirow{3}{*}{$\begin{array}{l}\text { Margin } \\
\text { Shape }\end{array}$} & Rectilinear (1) & Rectangular lateral margins \\
\hline & Convex (2) & Convex lateral margins \\
\hline & Concave (3) & Concave lateral margins \\
\hline \multirow{2}{*}{$\begin{array}{l}\text { Margin } \\
\text { Direction }\end{array}$} & Parallel (1) & Lateral margins run parallel \\
\hline & Convergent (2) & Lateral margins converge towards the distal end (base) \\
\hline \multirow{3}{*}{ Face Shape } & Flat (1) & Flat planes in cross-section \\
\hline & Convex (2) & Convex in cross-section \\
\hline & Concave (3) & Concave in cross-section \\
\hline \multirow{3}{*}{ Face Polish } & Unpolished (1) & No traces of polish \\
\hline & Semi-polished (2) & One face fully polished, or light polishing on both faces \\
\hline & Fully polished (3) & Both faces fully polished \\
\hline \multirow{3}{*}{ Base Shape } & Flat (1) & Truncated or flat distal end \\
\hline & Rounded (2) & Rounded distal end \\
\hline & Pointed (3) & Pointed distal end \\
\hline
\end{tabular}


Figure E.15: Hafted groundstone tool types and subtypes

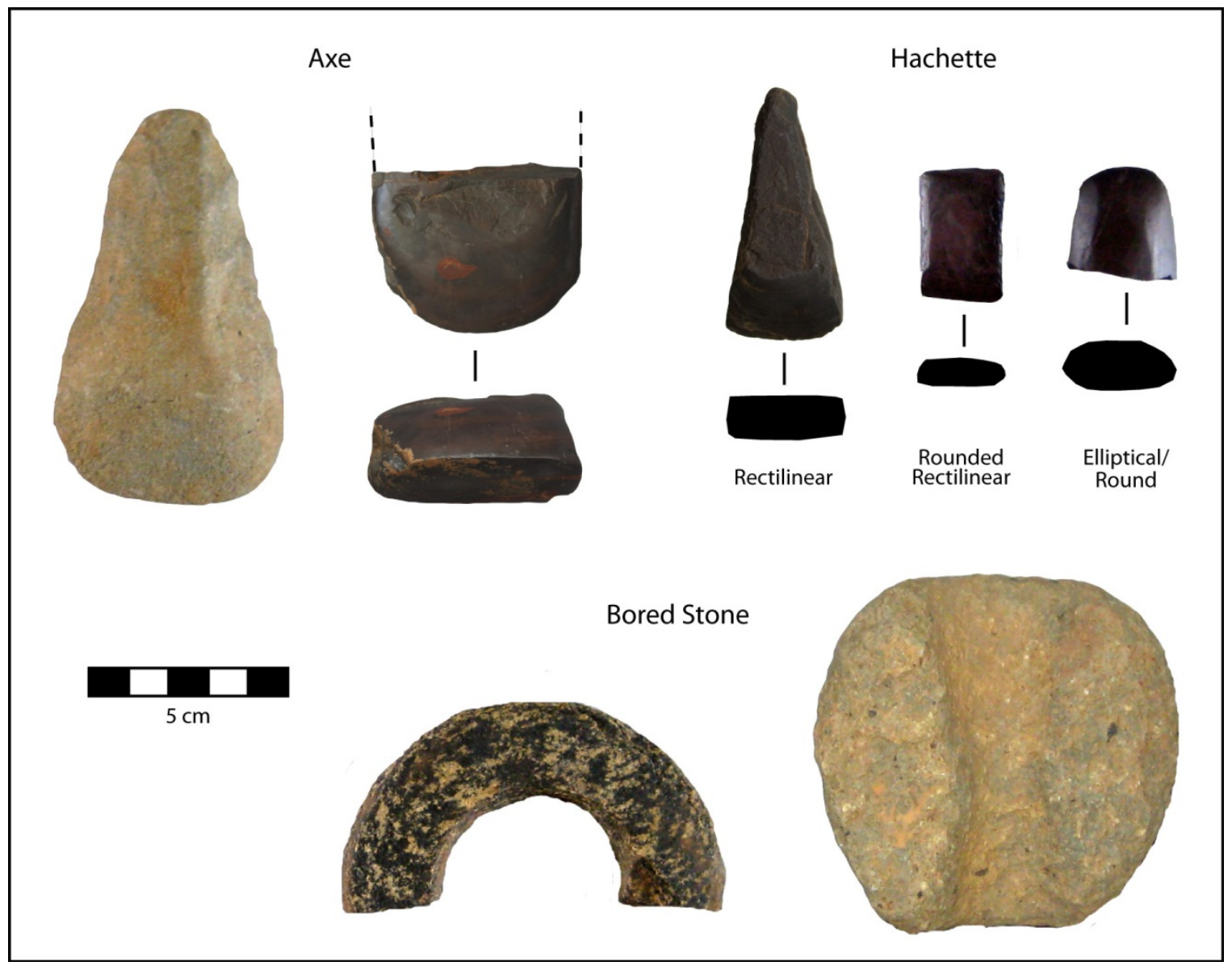

Axe heads are elongated implements with a bifacially polished proximal section (bit) opposite a tapered distal section (base) presumably designed for hafting (Figure E.13). Axe heads are far and away the most predominant ground stone tool type, accounting for nearly $44 \%$ of the combined Diouboye and Survey assemblages where modal configurations of size, shape, and ground finish on the margins, bit, blade, and faces document three different subtypes of axe head design and execution. And, since the vast majority (91.9\%) were made of hematite, it was generally possible to identify probable blanks and preforms from sequential stages of reduction, use, and recycling.

As illustrated for the sample of measurable specimens in Figure E.14, axe heads in the Madina-Sadatou study fall into at least two, and perhaps three, broad size categories. Across the entire assemblage, axes $(\mathrm{n}=24)$ have a length greater than 68 millimeters $(\mu=101.6 \mathrm{~mm}, \sigma=23.0 \mathrm{~mm}$ ), usually with a proximal blade width greater than 40 millimeters. Although the majority of these are hematite (62.5\%), large axes also come in a diverse array of other materials including amphibolite (8.3\%), schist (12.5\%), rhyolite (8.3\%), and granite (8.3\%). The majority of these have convex bits and blade edges with convergent lateral margins and convex faces amenable to hafting.

Following typological conventions in West Africa (MacDonald 1998:75), I refer to the smaller axe heads less than 68 millimeters in length $(\mu=41.5 \mathrm{~mm}, \sigma=8.3 \mathrm{~mm})$ as 
hachettes. These are almost always made of hematite (94.9\%), but rare specimens of quartz, schist, and rhyolite do occur. Independent of reduction stage, both fully- and semi-ground hachettes and their preforms come in a diversity of shapes with no clear modal attribute combinations, although it is useful to distinguish them on the basis of profile (Table E.16).

Figure E.16: Bivariate plot of axe head width and length (all complete specimens from Survey and Diouboye assemblages) with tenth-quantile kernel density estimate contours and confidence interval ellipses (90\%)

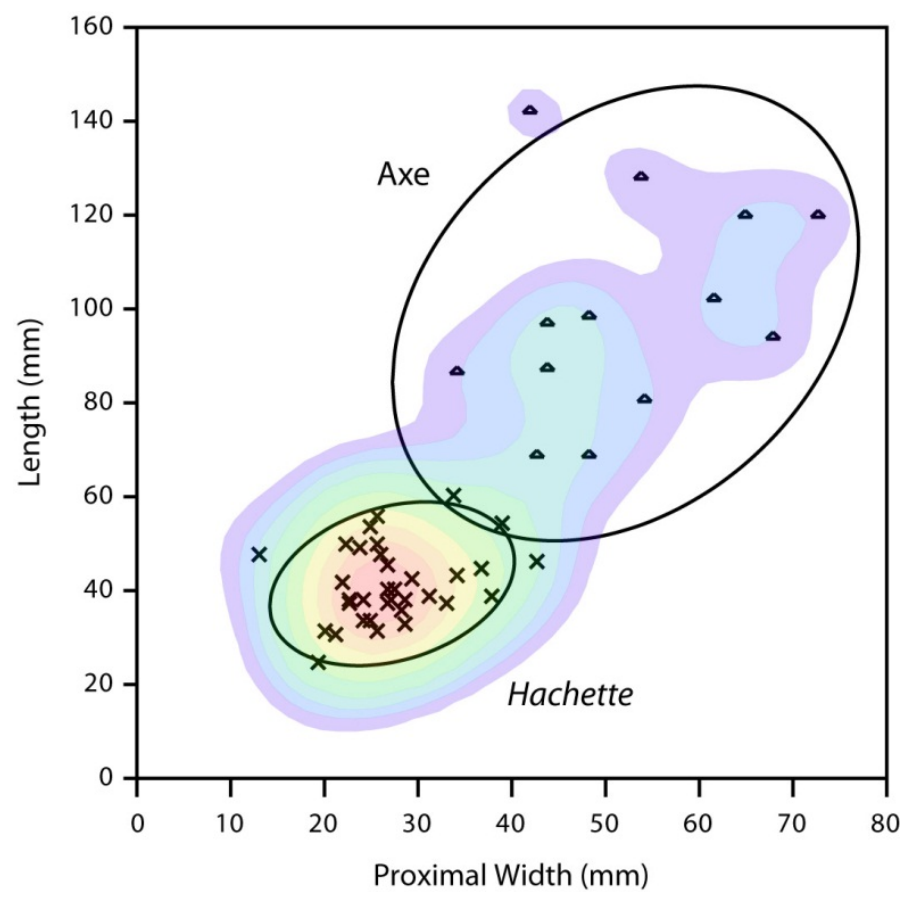

Table E.16: Summary of formal attributes for fully-ground, semi-ground, and preform hachettes from Survey and Diouboye assemblages

\begin{tabular}{|c|c|c|c|c|c|c|c|c|c|c|c|c|c|}
\hline \multicolumn{2}{|c|}{ Profile } & \multicolumn{4}{|c|}{ Bit Shape Present } & \multicolumn{4}{|c|}{ Margin Present } & \multicolumn{4}{|c|}{ Base Present } \\
\hline & \multirow{2}{*}{$\begin{array}{c}\text { Total } \\
\qquad n\end{array}$} & \multicolumn{2}{|c|}{ Convex } & \multicolumn{2}{|c|}{ Bevel } & \multicolumn{2}{|c|}{ Parallel } & \multicolumn{2}{|c|}{ Convergent } & \multicolumn{2}{|c|}{ Flat } & \multicolumn{2}{|c|}{$\begin{array}{l}\text { Rounded/ } \\
\text { Pointed }\end{array}$} \\
\hline & & $n$ & $\%$ & $n$ & $\%$ & $n$ & $\%$ & $n$ & $\%$ & $n$ & $\%$ & $n$ & $\%$ \\
\hline Rectilinear & 47 & 9 & $56.3 \%$ & 5 & $31.3 \%$ & 16 & $57.1 \%$ & 12 & $42.9 \%$ & 14 & $70.0 \%$ & 6 & $30.0 \%$ \\
\hline $\begin{array}{l}\text { Rounded } \\
\text { rectilinear }\end{array}$ & 16 & 0 & $0.0 \%$ & 4 & $100.0 \%$ & 8 & $57.1 \%$ & 6 & $42.9 \%$ & 4 & $33.3 \%$ & 8 & $66.7 \%$ \\
\hline $\begin{array}{l}\text { Elliptical/ } \\
\text { Round } \\
\end{array}$ & 12 & 2 & $100.0 \%$ & 0 & $0.0 \%$ & 3 & $37.5 \%$ & 5 & $62.5 \%$ & 3 & $33.3 \%$ & 6 & $66.7 \%$ \\
\hline Total & 75 & 11 & $14.7 \%$ & 9 & $12.0 \%$ & 27 & $36.0 \%$ & 23 & $30.7 \%$ & 21 & $28.0 \%$ & 20 & $26.7 \%$ \\
\hline
\end{tabular}


Interestingly, axe head subtypes show clear spatio-temporal differences across the Diouboye and Survey groundstone assemblages (Figure E.17). First of all, the low numbers of axe heads at single-component Atlantic Era sites points to limited use or secondary deposition. Second, the relative abundance of subtypes in LSA components, which account for most of the systematic and purposive samples from surveyed sites, differ markedly from the distributions observed at Diouboye and other single-component Iron Age sites. Indeed, a Fisher's exact test demonstrates that elliptical and rectilinear hachettes are significantly associated with LSA components, while rounded rectilinear hachettes and axes are significantly associated with the combined Diouboye and Iron Age samples $\left(\chi^{2}=17.49, \rho<0.001\right)$. While large axe counts may relate to divergent economic practices between LSA and Iron Age communities, as discussed below, the distribution of hachette subtypes hints at distinct designs and/or reduction strategies.

Figure E.17: Relative abundance of axe head subtypes

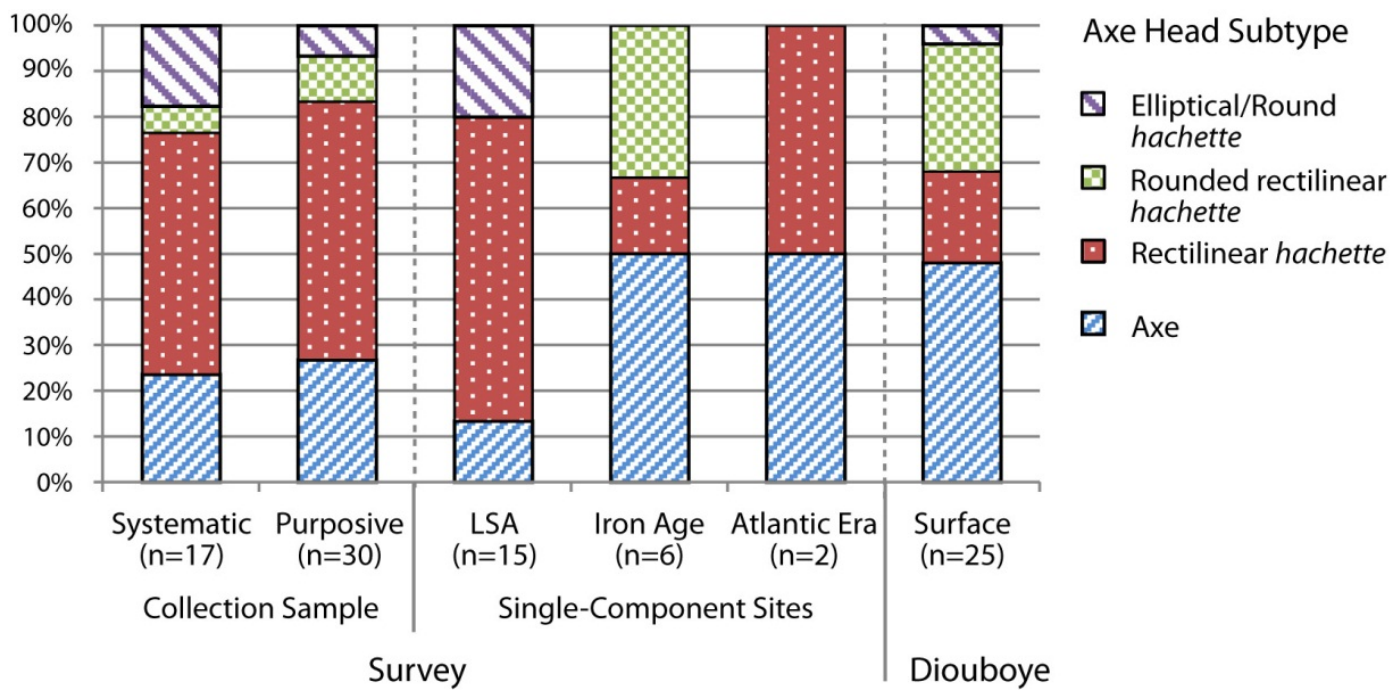

To consider these possibilities in more detail, I compared the relative frequencies of hachette blanks, preforms, and semi- and fully-polished tools, all excluding fragments, with hematite chipped stone debris from the Survey (predominantly LSA) and Diouboye assemblages. Figure E.18 shows that hematite debitage, while infrequent, does occur in similar proportions relative to hachettes at both Diouboye and systematic samples from surveyed sites. This suggests that chipping from initial manufacture and/or use took place on sites from both occupational horizons. Figure E.19 reveals that blanks and preforms comprise a substantial proportion of the hachettes at Diouboye, even surpassing their relative abundance in the Survey assemblage. This contrasts with an extremely tentative pattern from single-component Atlantic Era sites where the recovery of only two fullyground hachettes, despite the frequent conflation of LSA and Atlantic Era components (Table E.13), hints at the collection of LSA axes for votive objects. Although it remains possible, even likely, that Iron Age communities in Madina-Sadatou also collected hematite artifacts from earlier sites, the available data suggest that they continued to work 
and rework hachettes through a sequence of chipped and groundstone reduction techniques. This interpretation dovetails with subtle design differences between the Diouboye and the Survey assemblages.

Figure E.18: Distribution of hematite debitage and hachette by assemblage

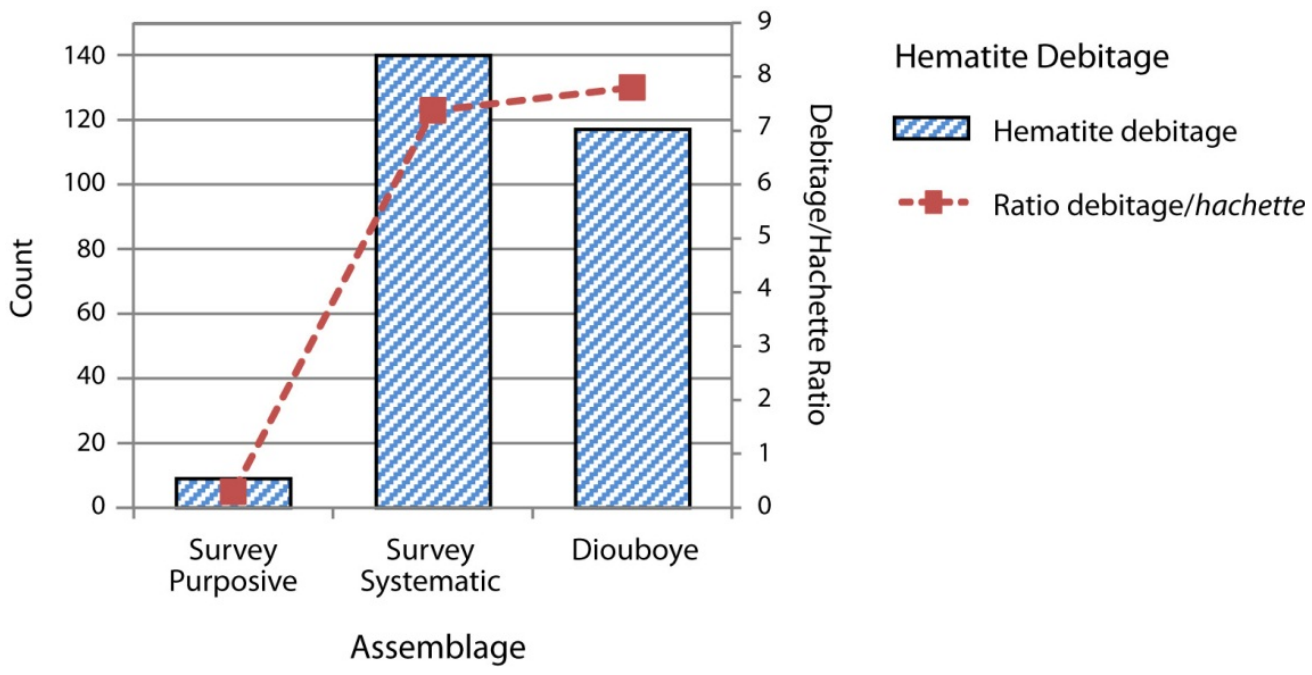

Figure E.19: Cumulative frequencies of hematite hachette reduction

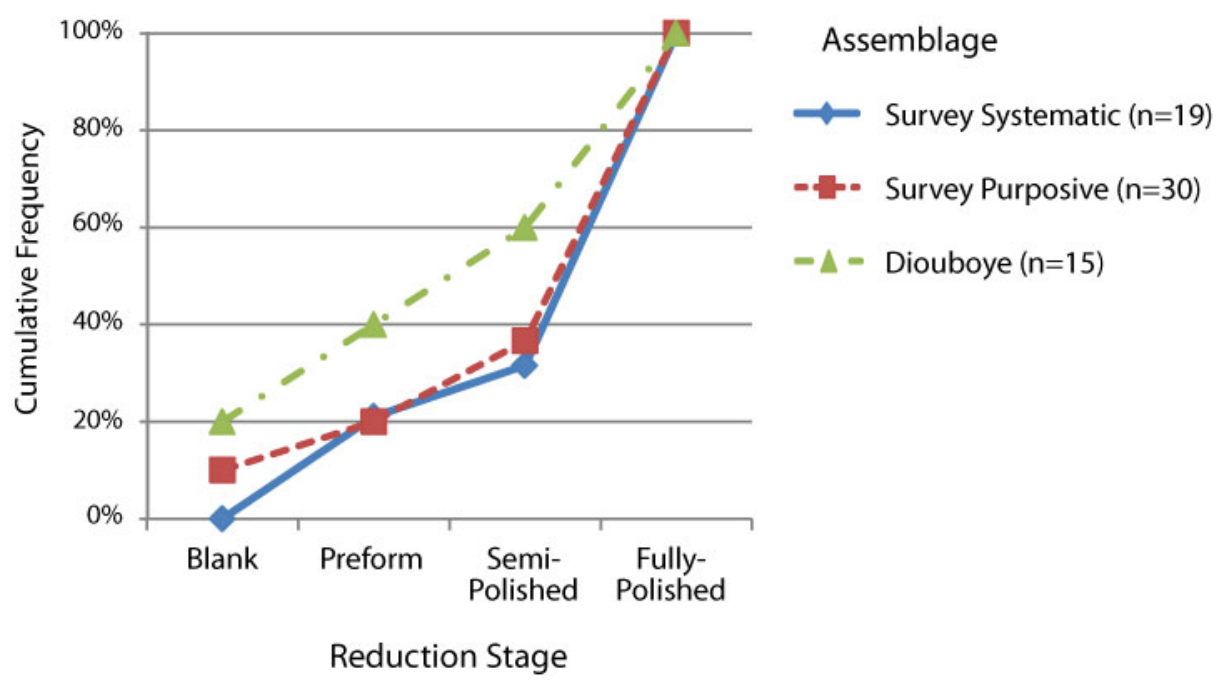

Despite their presence at mid- to late-Holocene archaeological sites across the Sahara and West Africa, the practical implications of groundstone axe heads remain poorly understood. Elsewhere their appearance coincides with an impetus to harvest hardwood trees and clear land as part of agricultural subsistence regimes (Edmonds 1995), but groundstone axes significantly predate agriculture across much of Africa (MacDonald 1998). Historically, people have attributed hachettes to supernatural forces, 
employing them as votive objects or amulets called gris-gris (Shaw 1944:28-32). This interpretation remains problematic for the archaeological record since past populations were actually making the hachettes to suite some economic purpose or cultural value. Alternative hypotheses summarized by Kevin MacDonald (1998) include woodworking and the ritualized shaping of cattle horns (Brown 1990), this latter proposing to explain their emergence alongside pastoral economies in Africa.

Regarding these functional possibilities, a number of experimental studies have evaluated the wear signatures generated by different materials and gestures (J. L. Adams 2002; Hayden 1979; MacDonald 1998:81). Percussive wood-working, for example, generally dulls the edges of polished stone axes, but rarely leads to the step fractures generated through contact with stone. Rubbing against materials such as wood, hides, and bone, which release organic compounds, produces tribochemical wear visible as a distinctive sheen, while abrasion against stone leads to pitting and striations. As outlined in Table E.17, I recorded attributes for macroscopic wear and use damage on groundstone hafted tools, as well as the grinding and polished tools discussed below.

Table E.17: Variables recorded for wear on groundstone tools

\begin{tabular}{|c|l|l|}
\hline Variable & \multicolumn{1}{|c|}{ Attribute (Code) } & \multicolumn{1}{c|}{ Description } \\
\hline \hline \multirow{4}{*}{ Bit Wear } & None/Light (0) & No visible wear \\
\cline { 2 - 3 } & Moderate (1) & Wear damage does not alter the tool form \\
\cline { 2 - 3 } & Heavy (2) & Wear damage that alters the tool form \\
\hline \multirow{4}{*}{ Blade Wear } & None (0) & No visible wear \\
\cline { 2 - 3 } & Chipped (1) & Step fractures visible \\
\cline { 2 - 3 } & Polished (2) & Polished sheen visible \\
\cline { 2 - 3 } & Chipped/Polished (3) & Step fractures and polished sheen visible \\
\hline \multirow{5}{*}{ Face Wear } & None (0) & No visible wear \\
\cline { 2 - 3 } & Chipped (1) & Step fractures visible \\
\cline { 2 - 3 } & Pitted (2) & Small pits or linear striations visible \\
\cline { 2 - 3 } & Unidentified (9) & Unidentified wear visible \\
\hline \multirow{5}{*}{ Base Wear } & None (0) & No visible wear \\
\cline { 2 - 3 } & Chipped (1) & Step fractures visible \\
\cline { 2 - 3 } & Pitted (2) & Small pits or linear striations visible \\
\cline { 2 - 3 } & Unidentified (9) & Unidentified wear visible \\
\hline
\end{tabular}

The distribution of wear patterns on groundstone axe heads reveals two interesting trends in the Madina-Sadatou assemblages (Figure E.20). First, patterns of wear are roughly equivalent for axes and hachettes, if not somewhat greater for the latter given the higher visibility of wear damage on hematite. Although pitting and chipping could represent post-depositional damage from trampling or erosion on materials derived in large part from surface collections, the presence of polish, most likely resulting from tribochemical processes, provides evidence for at least some primary usage. Second, wear 
damage in the form of lightly concave divots on hachette bases would be consistent with their use as small hammers or wedges (Casey 2000:72-80), rather than the trampling and erosion that would preferentially impact the broad faces, blade, and sharpened bit of these hematite tools.

Figure E.20: Relative distribution axe wear by subtype and assemblage
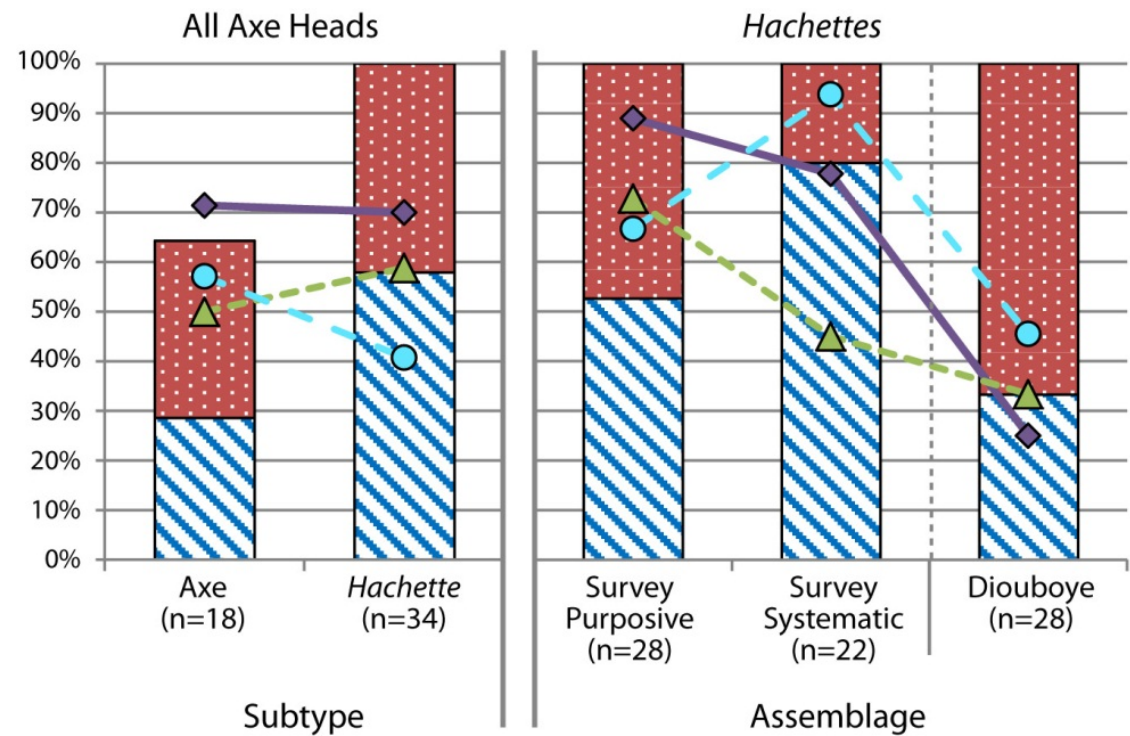

Wear Type

$\because \because$ Polished blade

01 Chipped blade

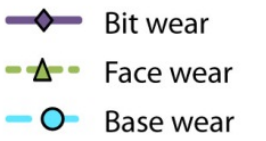

Returning to the hypotheses outlined above, the emphasis on partially or fully grinding these axe heads implies that their aesthetics had some cultural significance. Meanwhile, traces of chipping, pitting, abrasion, and organic polish document the practical application of hachettes in socioeconomic activities beyond ritual adornment. Although specific practices cannot be more clearly ascertained without microscopic analyses, the divergent patterns of wear suggest that techniques of groundstone axe use, as with production, took a different course at Diouboye in comparison to LSA societies. Whether reshaping the horns of livestock, working leather or wood, or practicing religious ritual, the available interpretations agree that groundstone axes, particularly hachettes, were employed in somewhat specialized socioeconomic activities whose cultural significance surpassed that of tasks completed with the expedient chipped stone tools found throughout the region.

\section{Grinding Tools}

Grinding tools have one or more surfaces made smooth through mechanisms of abrasion against organic and/or inorganic materials including other groundstone implements. In addition to the variables listed in Table E.14, I recorded the position and shape of these abraded surfaces in a manner similar to that employed for hafted tools (Table E.15). In this way it was possible to define three broad formal/functional typespestles, handstones, and netherstones. 
Pestles are long stones of expedient design with circular cross-sections and traces of wear and/or flattened ends from crushing and grinding other materials. Pestles, not illustrated in Figure E.21, are relatively rare in the Diouboye $(n=6)$ and Survey $(n=3)$ assemblages. Altogether these range in length from 60 to 133 millimeters $(\mu=78.8 \mathrm{~mm}$, $\sigma=36.2 \mathrm{~mm}$ ) and exhibit a diverse array of raw materials including sandstone (33.3\%), hematite (11.1\%), rhyolite (11.1\%), granite (22.2\%), and gabbro (22.2\%).

Handstones are round or elongated stones of either expedient or strategic design with one or more surfaces heavily abraded from repetitive grinding action against organic and inorganic materials. Both complete and fragmentary specimens were recovered from excavation $(n=10)$ and surface $(n=52)$ contexts at Diouboye. Only seven handstones were collected from the 79 localities in the study area where these artifacts were noted, so it is not possible to explore regional variability beyond the observation that amphibolite $(n=4)$ was the preferred material on surveyed sites in comparison to the rhyolite predominating at Diouboye (Figure E.23).

\section{Figure E.21: Grinding tool types and subtypes}

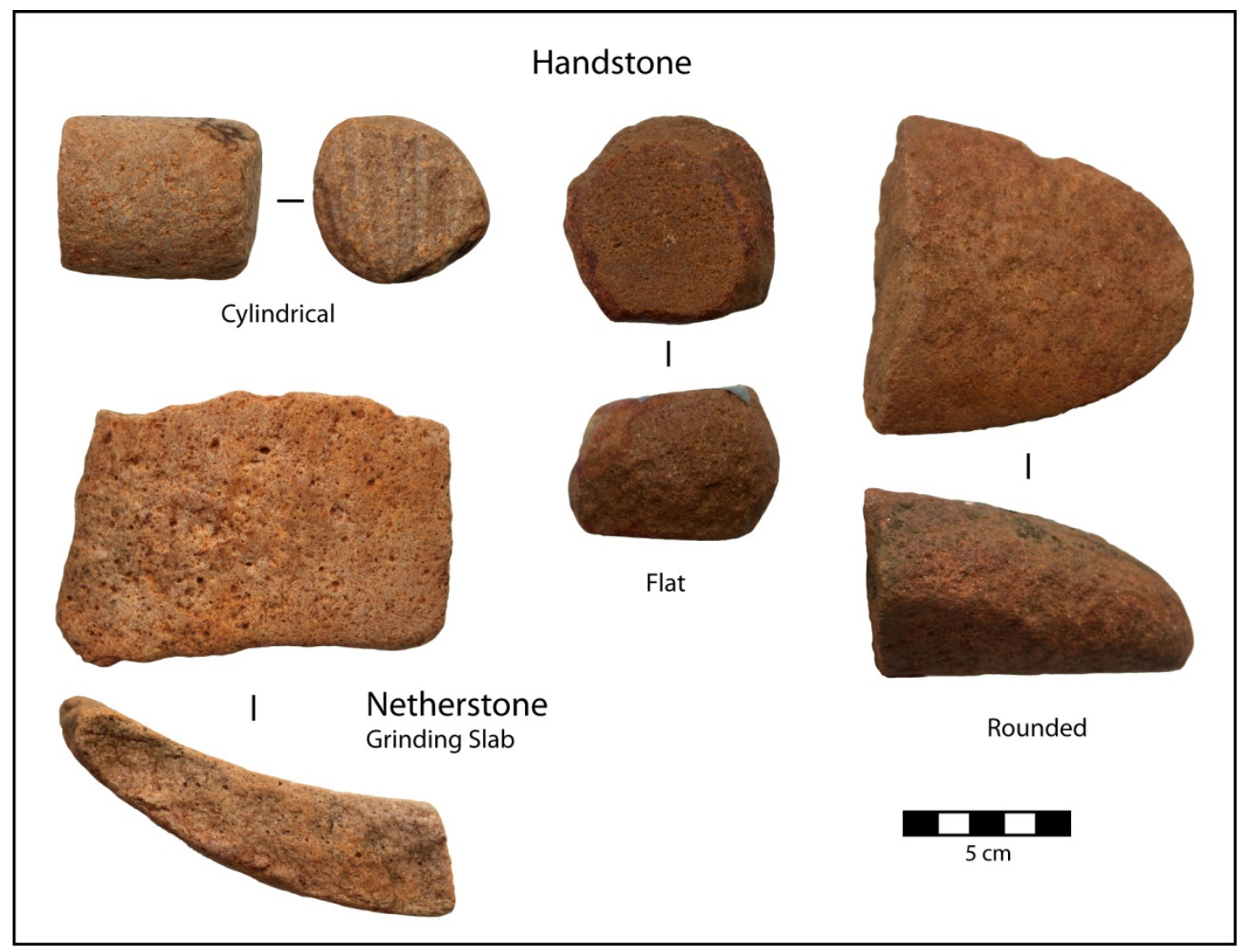

As illustrated in Figure E.22, handstones at Diouboye occur in two modal lengths of 48-100 millimeters and 110-148 millimeters, contrasting with their normal distribution by maximum width ( $\mathrm{n}=31, \mu=63.7 \mathrm{~mm}, \sigma=17.0 \mathrm{~mm}$ ) and thickness $(\mathrm{n}=31, \mu=45.1$, 
$\sigma=11.4 \mathrm{~mm}$ ). These modalities may represent one-handed and two-handed grinding implements, but this interpretation merits caution since people can work stones up to 150 millimeters with one hand. A more secure link to past handstone usage lies in the profile shape and number and position of abraded faces generated directly through grinding gestures (J. L. Adams 2002:98-114; David 1998:35-41). On the basis of these variables, I recognized three subtypes of handstone at Diouboye: cylindrical handstones with a round or elliptical profile; rounded handstones with at least one convex abraded face(s), and flat handstones with only flat abraded faces (Figure E.21). All of these subtypes occur in both the long and short modes.

\section{Figure E.22: Handstone length distribution}

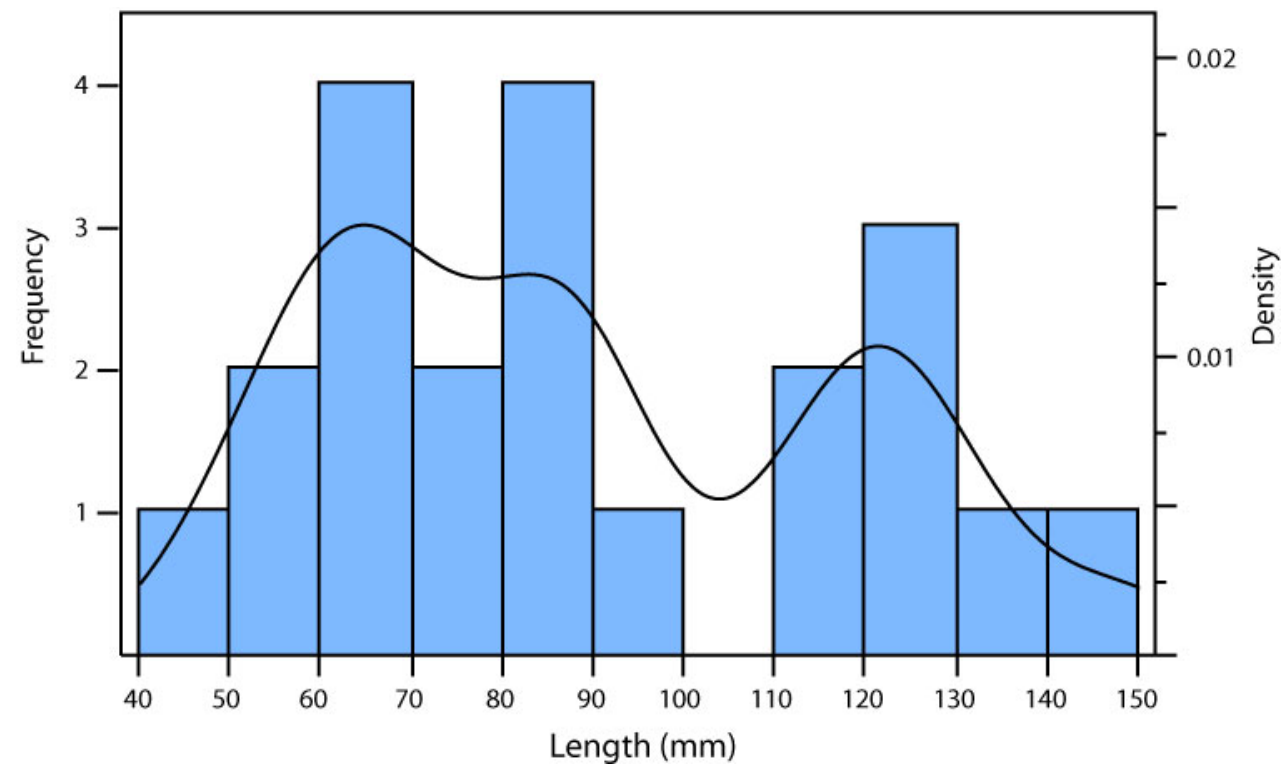

Netherstones are the functional counterpart to handstones and pestles (Figure E.21); they comprise the lower stationary stones against which softer, usually organic, products are ground with handheld stones. At Diouboye these artifacts were recovered from excavation $(\mathrm{n}=3)$ and surface contexts $(\mathrm{n}=16)$ where an additional three large basins were noted, but not collected, in the course of surface mapping. Although some 31 localities had netherstone fragments visible on the surface, only one specimen fell within systematic unit for collection.

On the basis of thickness and overall morphology, I distinguished two subtypes of netherstone at Diouboye: basins $(\mathrm{n}=2)$ with a deeply concave grinding surface and thickness greater than 50 millimeters for crushing activities; and grinding slabs with a lightly concave $(\mathrm{n}=10)$ or flat $(\mathrm{n}=3)$ grinding surface averaging 29.0 millimeters in maximum thickness $(\mu=8.7 \mathrm{~mm})$. With the exception of those large basins noted on the surface, netherstones are considerably more fragmented than the corresponding handstones and pestles from Diouboye. Since the strategic design of these netherstones entailed an investment in raw materials and labor, people probably continued to use and recycle them until completely expired (Schlanger 1991). 
The raw materials selected for netherstones and handstones reveal a preference at Diouboye for non-local stones, particularly rhyolite and granite (Figure E.23). Cobbles with the shape and size of handstones would have been available in the Falémé River bed less than a half kilometer distant, but obtaining wide pieces of rhyolite might have required exchange or travel beyond the catchment of the village. The relative proportions of raw materials in these admittedly small samples also show some correspondence across netherstone and handstone forms. Grinding tools with flat surfaces, for example, were made from sandstone, rhyolite, and granite. Concave grinding slabs and rounded handstones, on the other hand, made preferential use of rhyolite, perhaps indicating their use and wear together. Cylindrical handstones with a more diverse range of materials could have been used with either flat or concave grinding slabs. The fact that handstones outnumber netherstones at Diouboye and on surveyed sites could signal either more intensive wear on the former or, more provocatively, the presence of multiple handstones within any given household.

\section{Figure E.23: Relative abundance of grinding implements by material type}

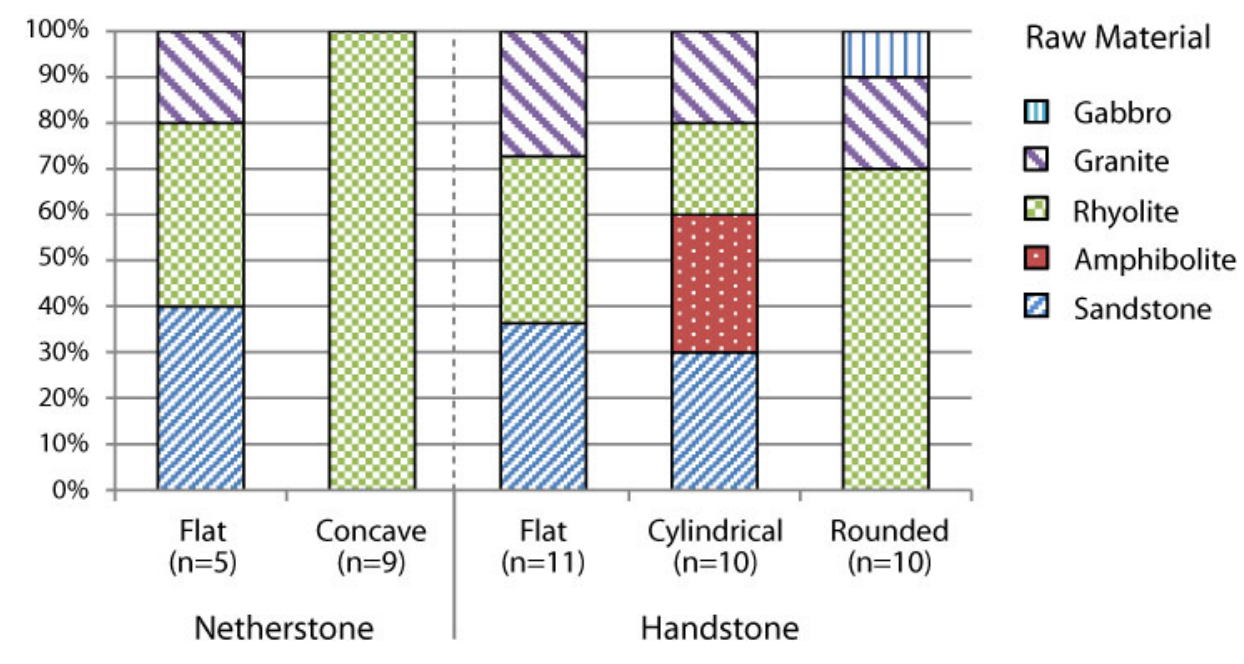

Ethnographic and archaeological studies have demonstrated that any single grinding tool can serve diverse functions, though many pertain to the processing of grains, legumes, nuts, and other vegetal foods (David 1998; K. I. Wright 1994). For this reason I associate these implements with food processing tasks throughout this study, recognizing that further microwear or residue analyses could eventually contribute to more nuanced interpretations of past activities. The appearance of grinding slabs and handstones on several single-component Atlantic Era sites suggests that lithic food processing technologies may have existed alongside the wooden mortars documented ethnographically and historically in the Upper Senegal region.

\section{Polished Tools}

Polished tools are miscellaneous ground stone artifacts with one or more surfaces made smooth through tribochemical processes and light abrasion against organic and/or 
inorganic materials. Following the procedure for hafted and grinding tools, I recorded the position and morphology of the relevant polished surfaces (Table E.15). This catch-all subclass included four quite discrete formal/functional types (Figure E.24).

Polishing stones are lightly flattened, usually circular, stones with sheen and/or light abrasion on one or more surfaces; the expedient design of these tools contrasts with the more strategic design of handstones. In the combined Diouboye $(n=14)$ and Survey $(\mathrm{n}=15)$ assemblages, these tools ranged from 38-90 millimeters in diameter $(\mu=58.3 \mathrm{~mm}$, $\sigma=16.4 \mathrm{~mm}$ ) and up to 57 millimeters in thickness $(\mu=19.8 \mathrm{~mm}, \sigma=14.2 \mathrm{~mm})$. Recovered from sites with LSA, Iron Age, and Atlantic Era components, polishing stones could have served any number of purposes within the associated communities: finishing hematite axes or other stone objects (J. L. Adams 2002:94); burnishing pottery (Gelbert 1999:215); and working hides, to name but a few possibilities. It is therefore likely that the polishing stones from Diouboye and surrounding sites were tools of expedient design within potentially specialized economic activities.

\section{Figure E.24: Polished tool types}

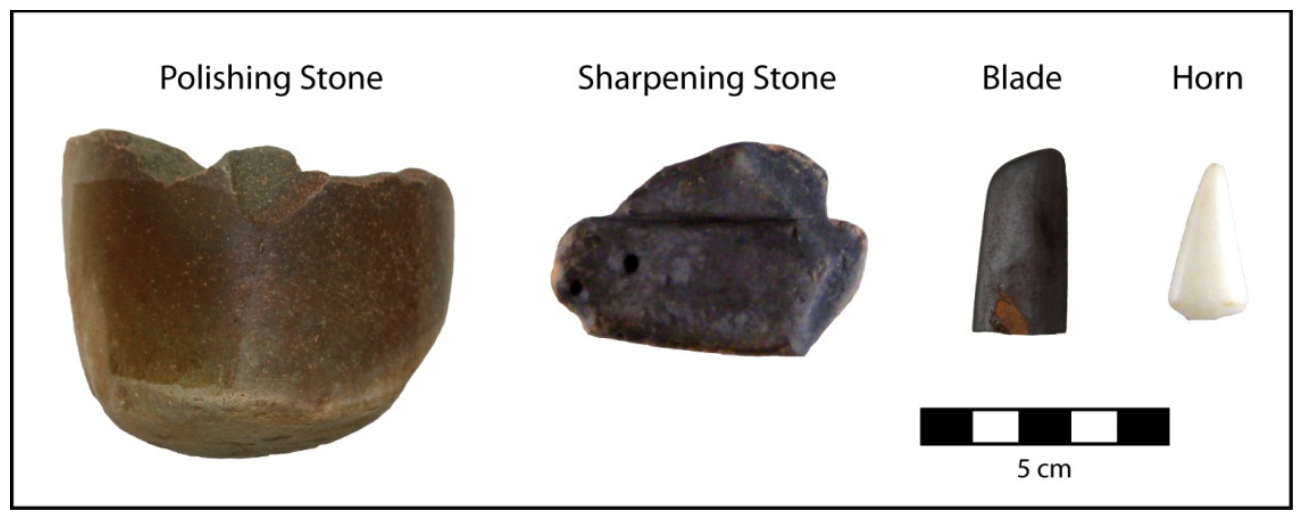

Sharpening stones include any rock with one or more grooves generated through the honing of inorganic tools or the straightening of organic ones. Rare in the MadinaSadatou archaeological record, only four such artifacts were collected as part of purposive sampling at four different LSA and Iron Age sites identified during survey.

Blades are relatively flat tools with at least one sharpened edge. These appear in low frequencies at both Diouboye $(\mathrm{n}=9)$ and LSA and Iron Age sites sampled for the Survey $(n=7)$ assemblage. With the exception of one rhyolite specimen, these were made of hematite with a high degree of polish. While the functions of these blades are not known, their strategic design clearly represents a greater investment in aesthetics than the chipped stone artifacts often found in association with them.

The horn is a unique object made of polished quartz found during systematic surface collection at FAL-1075. It measures 29.9 millimeters long by 15.4 millimeters in maximum width and weighs 4 grams. The cultural significance of this item is unknown. 


\section{Groundstone in the Madina-Sadatou Study Area: Implications for Interpretation}

The macroscopic analysis of groundstone artifact materials and morphology has revealed variability in past practices of resource procurement, tool design, and tool use by the Iron Age community at Diouboye. A limited consideration of wear patterns, in conjunction with ethnographic analogy, indicates that people employed diverse groundstone technologies both in daily household tasks such as food processing, as well as more specialized activities such as chipped stone reduction, tool-making, leatherworking, horn-shaping, and perhaps even religious ritual.

The spatial and temporal organization of these activities across the MadinaSadatou study area, and within Diouboye, are explored in Chapters 4 and 6-8. 


\section{APPENDIX F METALLURGICAL ARTIFACTS}

Metallurgy has long held a near-exalted place in the archaeology of West Africa where it remains subject to on-going field research and academic debate (e.g., Holl 2009; S. K. McIntosh and R. J. McIntosh 1988; Vogel 2000). Copper and gold have provided grounds for charting trans-Saharan trade networks (e.g., Deme and McIntosh 2006; Fenn 2006; Garenne-Marot 1995; Garrard 1982; Gondonneau and Guerra 2002; Nixon 2009) and precolonial regimes of value (Herbert 1984), but more ubiquitous iron has come to define an entire era in conventional techno-chronological frameworks (see S. K. McIntosh and R. J. McIntosh 1983). As discussed in Chapter 4, the so-called Iron Age (ca. 500 BC - AD 1500) witnessed the diffusion of iron metallurgy across West Africa alongside transitions to sedentary lifeways, food production, and the development of complex societies. Given the prominence of iron both in local histories and in evolutionary narratives eliding civilization and technological progress (Stahl 1999b), it should come as no surprise that debates surrounding the origins of metallurgyautochthonous invention versus external diffusion-figure prominently in the archaeology of the late-Holocene (e.g., Alpern 2005; Holl 2009; Killick 2004; D. E. Miller and Van Der Merwe 1994; Zangato and Holl 2010). While this topic lies well beyond the scope of the present study, it has motivated substantial research on the operational sequences of iron production that frame the analysis and interpretation of metallurgical evidence from Diouboye and other sites in the Madina-Sadatou study area.

The objectives of this analysis were threefold: (1) to identify technologies of local metal production and/or patterns of non-local acquisition, including their organization in space and time; (2) to develop a working typology for the function/use of metal artifacts, particularly those from Diouboye; and (3) to identify temporally sensitive artifact types capable of supporting the relative chronology developed for surveyed sites. As with pottery (see Appendix D), I examined variables related to logistical and social constraints on techniques for the production, exchange, and use of iron and copper-alloy objects.

In this appendix I trace the general operational sequences for metallurgical technologies at Diouboye and surveyed sites through production debris such as furnace and crucible fragments and slag, as well as rare metal artifacts. Through comparison to ethnographic, historic, and archaeological examples, I then propose functional and chronological typologies for dominant metal artifact classes. The contexts and interpretive significance of these archaeological data are discussed in Chapters 4-9.

\section{METHODOLOGY}

Diverse techniques for pre-industrial metallurgy in sub-Saharan Africa have been investigated by a number of scholars (e.g., Bocoum 2000; Childs and Killick 1993; D. E. Miller and Van Der Merwe 1994; Schmidt 1996; Vogel 2000) although artifacts of 
foreign (i.e., European) origin are now receiving greater coverage by archaeologists studying the Atlantic Era. For the metals of concern in this analysis, namely iron and copper, production began with the extraction of workable raw material by smelting (reducing) ores obtained through surface exploitation or mining. Using techniques of forging, smithing, casting, and cutting, people with specialized tools, skills, and knowledge then fashioned objects that continued to be repaired, refashioned, or even recycled. Although microscopic analyses of metal objects and production debris (and their interpretation by specialists) can distinguish among techniques for each of these operational stages, the present study depends on macroscopic analyses by a non-specialist (the author). For this reason, I considered only those variables necessary to reconstruct past metallurgy in broad terms, but with an eye towards meeting the above objectives.

\section{Artifact Sampling and Processing}

Metallurgical artifacts collected from surface and subsurface contexts in the Madina-Sadatou study area include iron and copper objects, slag, and furnace and crucible fragments. All such materials from each FS unit, with the exception of furnace fragments, were counted, weighed, and recorded in the field laboratory, and ultimately transported to IFAN for curation and analysis in 2008 and 2009. Because materials dating to the Atlantic Era predominated on sites collected during survey and contrasted with those Iron Age materials from Diouboye, I distinguish between the Survey and Diouboye assemblages in the following analysis and classification.

Metallurgical artifacts in the Survey assemblage included those from systematic and/or purposive surface collection at 32 sites documented during full-coverage survey and an additional four sites visited during guided prospection. Altogether, these materials include 67 iron (365.3 g) and two copper (2.1 g) artifacts, 77 pieces of slag (849.8 g), and two silver coins (see Appendix G). Only two sites hosted concentrations of oxidized daub possibly representing collapsed pit furnaces from smelting.

The Diouboye assemblage includes 47 iron (177.4 g) and five copper (3.8 g) artifacts from excavation, in addition to some 17 iron (89.1 g) and 3 copper (2.2 g) objects from surface collection units. Slag, on the other hand, occurred in higher frequencies on the site surface ( $\mathrm{n}=186,2921.8 \mathrm{~g})$, than in excavated contexts $(\mathrm{n}=29,91.2$ g), most likely because of its greater resistance to weathering and corrosion.

\section{Analytical Variables}

Following a cursory in-field examination of slag and metal artifacts from both the Survey and Diouboye assemblages, I relied upon established protocols to select variables with attribute states that I, as a non-specialist, could recognize by eye or with slight magnification under a hand lens (3X to 5X). In some cases, a steel file proved useful for removing patina.

\section{Slag}

In the absence of detailed microstructural and chemical studies, the visual classification of slag offers only a tentative distinction between smelting, smithing, and casting activities, and the type of metal involved therein (Bachmann 1993; Miller and Killick 2004). Contextual information from other artifacts and features can, however, narrow this range of technical possibilities (Greenfield and Miller 2004). Recognizing the 
limited value of these data, I nonetheless recorded the variables outlined in Table F.1. These have allowed me to differentiate metallurgical slag from other vitrified materials and at least hypothesize the sorts of underlying production techniques occurring at Diouboye and surrounding sites in the Madina-Sadatou study area.

Table F.1: $\quad$ Variables recorded for slag (after Bachmann 1993)

\begin{tabular}{|l|l|l|}
\hline \multicolumn{1}{|c|}{ Variable } & \multicolumn{1}{|c|}{ Attribute (Code) } & \multicolumn{1}{c|}{ Description } \\
\hline \hline Weight & Grams (g) & Mass of the specimen \\
\hline \multirow{5}{*}{ Color/Streak } & Black to very dark brown (1) & See Munsell Soil Color Chart \\
\cline { 2 - 3 } & Very dark gray (2) & See Munsell Soil Color Chart \\
\cline { 2 - 3 } & Dark gray (3) & See Munsell Soil Color Chart \\
\cline { 2 - 3 } & Dark reddish-gray (4) & See Munsell Soil Color Chart \\
\cline { 2 - 3 } & Dark brown (5) & See Munsell Soil Color Chart \\
\hline \multirow{5}{*}{ Texture } & Layered (1) & Slag has striated appearance \\
\cline { 2 - 3 } & Glassy Flow (2) & Slag appears glassy, often with conchoidal fractures \\
\cline { 2 - 3 } & Crystalline Flow (3) & Slag appears lustrous, often with crystalline structure \\
\hline \multirow{3}{*}{ Porosity } & Low (0) & Few gas holes present, high specific gravity \\
\cline { 2 - 3 } & High (1) & Many gas holes present, low specific gravity \\
\hline \multirow{2}{*}{ Inclusions } & Sand (1) & Sand grains visible in slag matrix \\
\cline { 2 - 3 } & Metal/Sulfides (2) & Metal prills or sulphides visible in slag matrix \\
\hline \multirow{2}{*}{ Rust } & Absent (0) & No rust visible on slag surface \\
\cline { 2 - 3 } & Present (1) & Light to dark brown rush visible on slag surface \\
\hline
\end{tabular}

\section{Iron and Copper-Alloy Artifacts}

Iron and copper objects are fairly diverse when considered across Iron Age and Atlantic Era occupations in the study area. While I recorded some metric variables for these artifacts (Table F.2), the functional typologies outlined in the next section generally represent a direct visual classification on the basis of form. For this, I referred to formal tool classes from archaeological and ethnographic contexts elsewhere in West Africa (e.g., Chavane 1985:152-163; Filipowiak 1979; Frank 1998; Thiaw 1999:218-222; Thilmans and Ravisé 1980; Togola 2008:218-222). For iron nails, the predominant artifact class in the Survey assemblage, I also recorded variables deemed useful for seriation in the Americas (Hume 1970:252-254; Wells 1998).

\section{METALLURGY IN THE MADINA-SADATOU STUDY AREA}

Although the technical skills and social identities of smiths continue to play a role in many present-day communities along the Falémé River and across West Africa (e.g., Appia 1965; De Barros 2000; Dilley 2000; Herbert 1993; McNaughton 1988), many, but not all, technologies of indigenous production rapidly declined with the growing availability of industrially-produced goods over the past few centuries (compare De Barros 2001; Goucher 1981). Even as these trends caution against projecting contemporary technologies too deeply into the past, ethnographic and historic accounts can highlight key parameters for production and exchange. In general, metallurgy requires mobilizing and coordinating labor via gender, age, lineage, or coresidential 
Table F.2: $\quad$ Variables recorded for metal artifacts

\begin{tabular}{|l|l|l|}
\hline \multicolumn{1}{|c|}{ Variable } & \multicolumn{1}{|c|}{ Attribute (Code) } & \multicolumn{1}{c|}{ Description } \\
\hline \hline Weight & Grams (g) & Mass of the specimen \\
\hline \multirow{4}{*}{ Material } & Iron (1) & Iron or steel \\
\cline { 2 - 3 } & Copper alloy (2) & Copper or copper-alloy \\
\hline \multirow{4}{*}{ Form } & Blade or projectile point (1) & See Figures F.2, F.4 \\
\cline { 2 - 3 } & Nail, rod, or other tool (2) & See Figures F.2, F.4 \\
\cline { 2 - 3 } & Wire (3) & See Figures F.2, F.4 \\
\cline { 2 - 3 } & Ornament (4) & See Figure F.3 \\
\cline { 2 - 3 } & Unidentified (9) & Unidentifiable metal fragment \\
\hline Tools and Ornaments & Millimeters (mm) & Length of specimen \\
\hline Length & Millimeters (mm) & Maximum width of specimen \\
\hline Width & Millimeters (mm) & Maximum thickness of specimen \\
\hline Thickness & Millimeters (mm) & Average diameter of shaft, if applicable \\
\hline Shaft Diameter & \multicolumn{2}{|l|}{} \\
\hline Nails & Millimeters (mm) & Length of specimen \\
\hline Length & Millimeters (mm) & Average diameter of specimen, if applicable \\
\hline Diameter & Round (1) & Point is rounded \\
\cline { 2 - 3 } Point Shape & Flat (2) & Point is truncated \\
\hline \multirow{3}{*}{ Shaft Taper } & None (1) & Shaft does not taper \\
\cline { 2 - 3 } & Two faces (2) & Shaft tapers along two faces \\
\cline { 2 - 3 } & Four faces (3) & Shaft tapers along four faces \\
\hline \multirow{2}{*}{$\begin{array}{l}\text { Shaft } \\
\text { Cross-Section }\end{array}$} & Square (1) & Shaft appears square in cross-section \\
\cline { 2 - 3 } & Rectangular (2) & Shaft appears rectangular in cross-section \\
\cline { 2 - 3 } & Round (3) & Shaft is round in cross-section \\
\hline \multirow{2}{*}{ Manufacture } & Hand-forged (1) & Nail has irregularities or evidence of hammering \\
\cline { 2 - 3 } & Machine-cut (2) & \multicolumn{2}{|c|}{} \\
\hline
\end{tabular}

community, depending on the cultural milieu and even the specific stage in the production sequence. The acquisition of technical skills and ritual knowledge through long-term apprenticeship and repetition may further work to reproduce the aforementioned social identities. In this section I summarize archaeological and historical evidence from precolonial West Africa to broadly sketch the array of possible techniques for obtaining metals through mining, smelting, and trade, and those for working them through forging, casting, and cutting.

\section{Mining}

The Falémé River and its tributaries transect a number of geological formations that yield gold and iron ores (see Chapter 3). Despite the absence of direct archaeological evidence for mining practices, historical accounts and the material remnants of smelting and smithing operations (below) point to local exploitation of these mineral resources.

Whether panning alluvial (placer) deposits or excavating shafts through lateritic soil, non-industrial gold mining strategies in sub-Saharan Africa are relatively laborintensive and require scheduling around the seasonal demands of subsistence agriculture 
(Curtin 1975a:204-205; Herbert 1998; Perinbam 1988). Furthermore, parallels in the tools and techniques of farming and mining - opening the earth, carrying heavy loads, grinding, sieving, and processing - mean that, historically, the division of labor and cultural proscriptions of the latter often mirrored those of the former.

Although historical sources from the $18^{\text {th }}$ century AD onwards document shaft mining in the heart of Bambuk along the Tomboura Escarpment (e.g., Boucard 1974 [1729]; Coste d'Arnobat 1789), people living along the lower Falémé River historically worked placer deposits of variable quality and depth (Curtin 1975a:198-206). With little or no oversight beyond a tax paid to local politico-ritual authorities, both men and women engaged in this activity during the dry season when other labor demands were at a minimum and low water levels provided access to profitable deposits. Even today women from Jakhanke and Fulbe villages in the Madina-Sadatou study area pan for gold in the Falémé River from March until June. The process involves digging a shallow pit into gold-bearing alluvium at the river margins, sifting out sandstone and quartz cobbles into small piles one to three meters in diameter (Figure F.1), and then panning out the gold dust from the remaining sand and silt with a calabash.

Although small cobble piles similar to those portrayed in Figure F.1b were observed at three riverside sites (FAL-1050; FAL-1063, FAL-1119), it is by no means clear that they resulted from panning. For the moment it is necessary to take at face value those historical accounts asserting the modest role of gold in the craft and long-distance exchange economies of communities in the Madina-Sadatou study area.

With regard to iron production, historical sources from the Upper Senegal region are decidedly less informative. High quality iron ores could have been obtained either directly from Birimian geological facies some 30 kilometers east of Madina-Sadatou, or perhaps as nodules of hematite and magnetite in the Falémé River bed. Presumably, people could have broken up and collected exposed lateritic crust containing 50-90\% iron oxides for smelting (Curtin 1975a:207; Sassoon 1963). While recognizing iron ores was undoubtedly an important source of practical knowledge (Appia 1965:329-334), acquiring this resource would have been less labor intensive than the exploitation of gold.

\section{Figure F.1: Gold extraction in the Falémé River: (a) digging pits into placer deposits; and (b) sifting and panning out cobbles (foreground) and sand (background) to obtain gold dust}
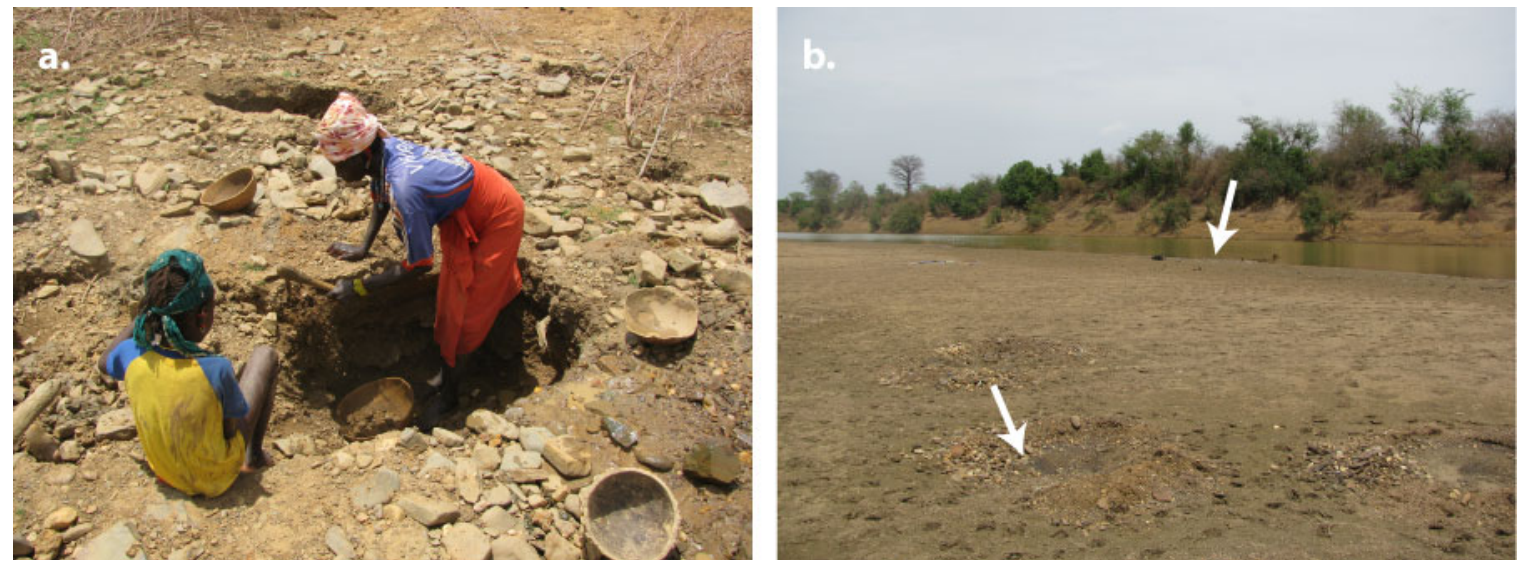


\section{Smelting}

Gold occurs naturally in an unoxidized state, but iron ores require smelting to obtain workable metals. This involves heating them to temperatures of at least $1150^{\circ} \mathrm{C}$ under the strongly reducing conditions of a furnace to produce an iron bloom of either wrought iron or low carbon-alloyed steel, in addition to the liquefied gangue called slag (Bocoum 2000:45-49; David et al. 1989). Archaeological evidence attests to a stunning diversity of techniques for manufacturing and operating smelting furnaces in sub-Saharan Africa-ranging from small pits aired by a bellows and tuyère pipe, to tall natural draft furnaces - with the size and scale of operations shaped by a complex array of ecological, economic, political, and cultural factors (Childs 1991; De Barros 2000; Kense 1983; Schmidt 1996). In many African societies the transformation of stone into metal was, or is, a danger-laden process that required mastery of technical skills as well as ritual knowledge often different from smithing proper (e.g., Appia 1965; Reid and MacLean 1995; Rowlands and Warnier 1993). On account of its clandestine and contaminating nature, smelting historically took place outside residential areas.

Figure F.2: Pit slag specimen from the site of Golmi on the lower Falémé River (IFAN Collections SEN 84.83e)

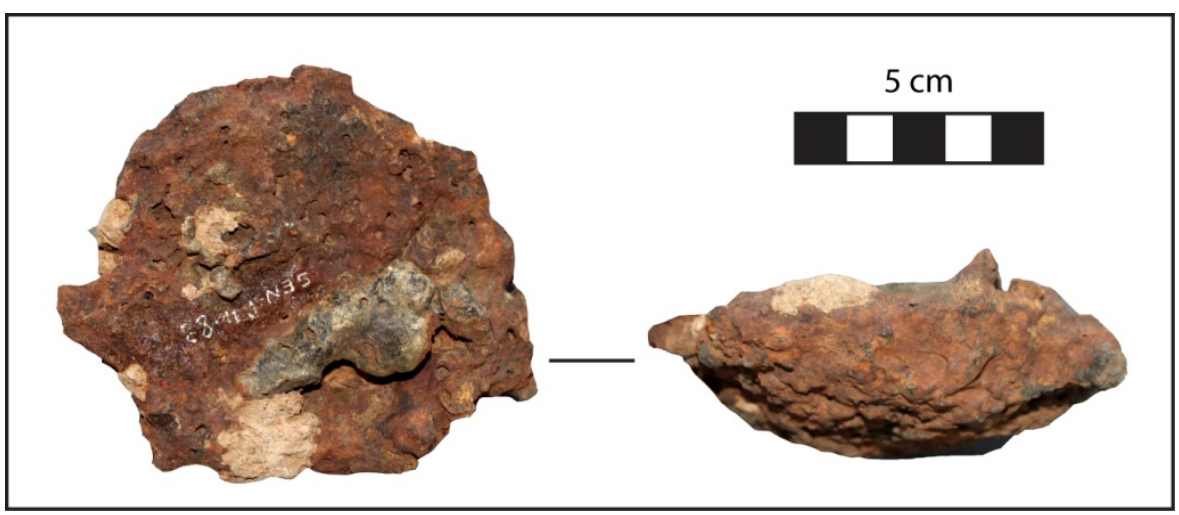

Table F.3: Summary of slag types in the Survey and Diouboye assemblages

\begin{tabular}{|l|c|c|c|c|c|l|}
\hline $\begin{array}{c}\text { Slag } \\
\text { Type }\end{array}$ & \multicolumn{2}{|c|}{$\begin{array}{c}\text { Assemblage } \\
\text { Survey }\end{array}$} & $\begin{array}{c}\text { Weight } \\
\text { Dean (g) }\end{array}$ & Texture & Porosity & \multicolumn{1}{c|}{ Notes } \\
\hline \hline Pit & 32 & 49 & 19.65 & Layered & Low & $\begin{array}{l}\text { Slag appears as thin cakes } \\
\text { some 2-5 cm thick with a } \\
\text { slightly convex surface, } \\
\text { often with sand inclusions }\end{array}$ \\
\hline Glassy & 2 & 44 & 10.76 & Glassy & Low/High & $\begin{array}{l}\text { Slag appears as small } \\
\text { nodules with glassy texture, } \\
\text { sometimes with metal or } \\
\text { sulfide inclusions }\end{array}$ \\
\hline General & 41 & 127 & 10.22 & - & - & $\begin{array}{l}\text { Includes all slag not } \\
\text { attributable to above types }\end{array}$ \\
\hline
\end{tabular}


Archaeological evidence for smelting in the Madina-Sadatou study area is remarkably slim, particularly when compared to the Middle Senegal region where batteries of smelting furnaces and slag heaps have revealed long-term and/or intensive production spanning the past two millennia (Bocoum 2000). Pedestrian survey documented only two locales with small heaps of possible furnace fragments associated with small round-bottomed pits and slag. While it remains possible that ore procurement and smelting took place away from the Falémé River, historical and archaeological evidence document more intense iron production with natural draft furnaces elsewhere across the Upper Senegal region—suggesting that communities in the Madina-Sadatou study area could have obtained this through regional exchange networks.

This overall interpretation finds tentative support from the range of slags present (and markedly absent) in the metallurgical assemblages (Bachmann 1993; Miller and Killick 2004). First, it was possible to distinguish pit slags whose layered texture, sand inclusions, and curvature point to consolidation within a small basin or pit; these resemble fragments of a well-preserved specimen from the site of Golmi on the lower Falémé River (Figure F.2). Although it is tempting to associate such slags with smelting activities, similar specimens from southern Africa appear to be forge bases related to smithing. Second, glassy slags have a glassy texture, low porosity, and limited inclusions; these may also derive from smithing. Finally, general slags with vesicular surface texture, low porosity, and varying amounts of rush and inclusions comprise the majority of slags in the Survey and Diouboye assemblages. Slags associated with large tapfurnaces, known elsewhere in Africa, are conspicuously absent, further pointing to the relatively small scale or low intensity of iron smelting and smithing in the study area.

\section{Smithing}

Smithing subsumes the techniques for heating, hammering, drawing, and welding metal into finished objects - techniques that smiths have necessarily adapted to the workable properties of imported metals over the past several centuries (Goucher 1981). The brief survey of blacksmithing in Chapter 2 reveals a strong trend towards specialized production of iron in precolonial West Africa, no doubt related to the difficulty in mastering the tools, techniques, and ritual knowledge of this craft (e.g., Herbert 1993; McNaughton 1988). Unlike smelting operations, smithing historically took place amid residential areas in workshops whose closure and scale depended on the broader sociopolitical and economic contexts for craft production.

Metal artifacts from Madina-Sadatou document at least three general operations for working or reworking metals into finished objects-forging, casting, and cutting. Although surveyed sites yielded no direct evidence for smithing activities, one probable iron forge did emerge from excavations at Diouboye where a conical crucible further demonstrates that smiths in the village possessed the tools and technical knowledge to cast metals such as copper and/or gold (Chapters 6-7).

Cutting of iron nail sheets by hand- or water-powered machines became prevalent in the Europe and the Americas during the $19^{\text {th }}$ century AD (Hume 1970:252-254; Wells 1998). The resultant tapering and burrs visible on some nails in the Survey assemblage thus document Atlantic-oriented trade, as opposed to local smithing activities. 


\section{Long-Distance Exchange}

While practices of gold mining and iron smelting in the precolonial economies of Madina-Sadatou remain far from clear, historical and archaeological evidence do document the import and export of metals over the past two millennia. According to European accounts from the $17^{\text {th }}$ century AD onwards (summarized in Curtin 1975a:207211; also Goucher 1981), people living along the Gambia River and the Atlantic littoral imported high quality iron from the Middle and Upper Senegal regions where people smelted ores in tall natural draft furnaces. In fact, the iron industry of the Upper Senegal was evidently productive enough to resist European imports until the late $19^{\text {th }}$ century $\mathrm{AD}$ - a reading of the historical evidence that dovetails with the relative dearth of machine-made objects from surface collections in Madina-Sadatou. In any event, if local iron consumption outweighed production, then people must have obtained the metal through regional exchange networks extending north to the Middle Senegal region, or south toward the Futa Jallon highlands, rather than through interregional trans-Saharan or Atlantic trade circuits.

In contrast to iron, which can be smelted locally, copper clearly represents an import to the Madina-Sadatou study area. The most likely sources are those of the Sahara Desert that supplied Arondo and other sites along the Senegal River (Bocoum and McIntosh 2002:96-104; Garenne-Marot 1995; Thiaw 1999:220-221), where archaeologists have found artifacts similar to the copper ornaments and wire recovered from Diouboye. Although this points cautiously to the import of finished objects, rather than metal ingots, the former could have been reworked by local metal smiths.

\section{CLASSIFICATION}

\section{Functional Typology}

One main objective of analysis was to classify metal artifacts according to their roles in day-to-day life. For the most part, a visual sort was sufficient to distinguish formal types in both the Diouboye (Figures F.3-F.4) and the Survey assemblages (Figure F.5), with some distinctions made on the basis of modal size variation. I have interpreted type functions through analogy to similar artifacts observed in ethnographic, historic, and archaeological contexts.

\section{Diouboye Assemblage}

Despite the high levels of corrosion on metal objects from Diouboye, only eight artifacts (iron metal nodules) could not be assigned to one of the following classes of blade/projectile, wire/rod, and ornament.

\section{Blades and Projectiles}

Blade tools from Diouboye include a number of relatively flat (usually 1-3 mm thickness) sheets of iron metal hammered into forms that I categorized as hoes, knives, projectile points, and harpoons (Figure F.3).

One fragmented iron blade resembling a rectangular spatula has been tentatively designated as small, hand-held hoe blade, similar to those used today by farmers in the Upper Senegal region (Chastanet 1984); this interpretation has been applied to similar blades from other Iron Age sites (Berthier 1997:87). Whatever the function of this blade, 
it is significantly broader and thicker $(6.8 \mathrm{~mm})$ than unequivocal projectile points from other archaeological contexts in Madina-Sadatou.

Knives are flat iron blades with a convex cutting edge welded to some form of rod (see below) for attachment to a handle. Small knife blades have a maximum width of 8-13 millimeters, while large knife blades have a maximum width of 20-31 millimeters. Knife blades of similar sizes are today employed for numerous subsistence and craft activities including butchery, meal preparation, and leather-working (Frank 1998:108-114), but they are also known from archaeological contexts dating to the Iron Age (Berthier 1997:77-78). The classification of large knives, which resemble both the hoe blade and large projectile points, must remain tentative.

Figure F.3: Blade and projectile tool types in the Diouboye assemblage

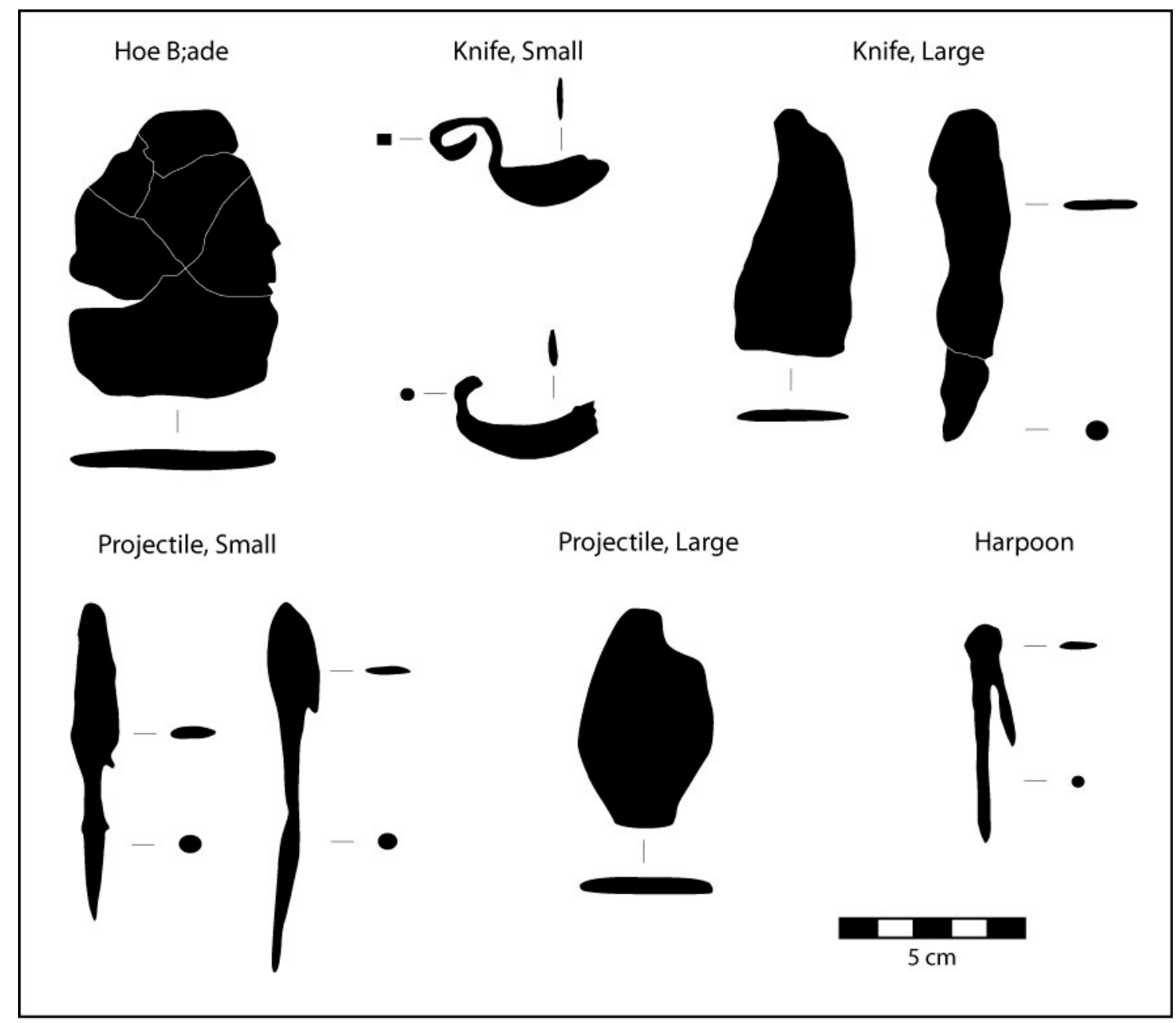

Projectile points have elongated, symmetrical iron blades tapering to a rod for attachment to a wooden shaft. Two small projectile points with basal tangs have a maximum width of 13-15 millimeters, while one possible large projectile point have a maximum width of 37 millimeters. Notably, the small points resemble those found at a 
number of Iron Age sites across the Sahel and savanna (Berthier 1997:77-78; Filipowiak 1979; Thiaw 1999:219); they also fall within the size range of, and may have had a similar function to, the arrowheads identified in the Survey assemblage (below).

A single harpoon consists of two rods hammered and welded to form an acute angle. Presumably, the longer rod attached to a wooden shaft so that the shorter, sharpened element could work as a piercing hook.

\section{Wires and Rods}

Rods and rod fragments predominate in the iron artifact assemblage from Diouboye (Figure F.4). Although some of these could have been tools such as awls and hooks, many probably represent broken shafts from blades and projectile points. These objects consistently measure 3-5 millimeters in diameter, so I classified them on the basis of shape-coil, bent, and s-shape. Only a single iron hook could be identified as a moreor-less complete artifact, but its intended use (e.g., fishing, latch, etc.) is unknown.

Two small pieces of drawn copper wire in the Diouboye assemblage measure 1.41.5 millimeters in diameter, similar to a "bronze ring" found previously at the site (Opper and Opper 1990:31). Presumably exported for ornamental purposes, wire of similar gauge has been documented at Iron Age sites elsewhere across the Sahel and Middle to Upper Senegal regions (Berthier 1997:74-75; Bocoum and McIntosh 2002:98-103; Thiaw 1999:219-222; Thilmans et al. 1980:34-35).

Figure F.4: Wire and rod types in the Diouboye Assemblage

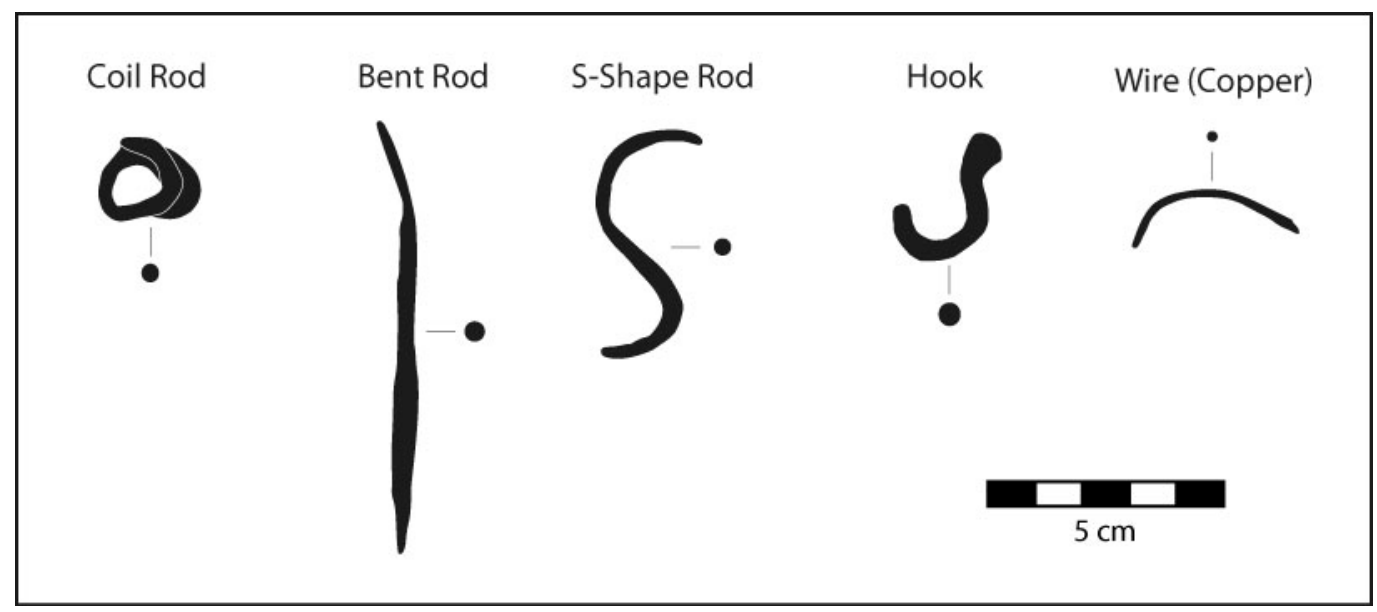

\section{Adornment}

Unequivocal objects of adornment are quite rare among the artifacts from

Diouboye (Figure F.5). I tentatively identified two iron bracelet fragments whose general curvature and weighted ends are reminiscent of those found elsewhere in Senegambia during the Iron Age. Admittedly, one or both of these artifacts could have been rod-like components of weapons or agricultural tools.

Four fragmentary copper ornaments of similar shape and size appear to derive from nearly identical spherical objects, possibly beads or small bells. As with the wire 
fragments, these bear a striking similarity to artifacts found at several other sites in the broader region (Chavane 1985:159-163).

Figure F.5: Adornment types in the Diouboye

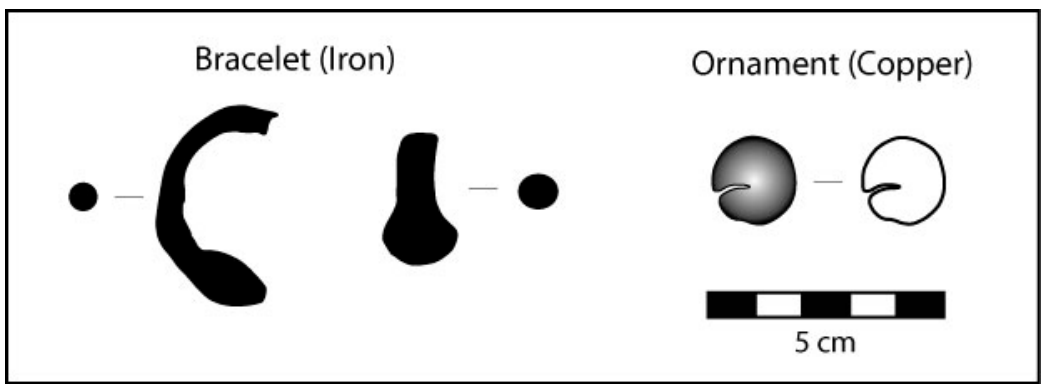

\section{Survey Assemblage}

The Survey assemblage includes artifacts classified as blades, projectiles, and rods, similar to those described above for the Diouboye assemblage, as well as iron nails deriving from Atlantic Era components.

\section{Blades and Projectiles}

Knife blades are elongated, thin (1-3 mm thick) sheets of iron with a convex cutting edge. Unlike those from Diouboye, however, they do not always have a welded rod for attachment to a handle. Knife blades measure 14-25 millimeters in width.

Hand sickles are oblong sheets of thin iron (1-2 mm thick) with a convex edge; these are considerably broader than knife blades. Farmers in the Upper Senegal today use similar tools to cut millet stalks during the harvest season (Ibrahima Thiaw, personal communication). Given their presence on Atlantic Era sites dating to the past three centuries, it seems likely that these blades served a purpose analogous to modern ones.

Arrowheads are relatively light iron projectile points (1-3 mm thick) with tangs and tapered shafts (2-3 mm diameter). The remarkable continuity in form among the five specimens recovered during survey hints at a shared mode of manufacture, if not origin.

\section{Nails}

Beyond a single fish hook, one eye-loop, and ten unidentified rod fragments, nails comprised the bulk $(n=24)$ of the wire/rod artifacts in the Survey assemblage. At a minimum, nails have a tapered iron shaft with a head or other evidence for hammering. Using variables of taper, shaft profile, and the presence of burrs, it was possible in most cases to distinguish between hand-forged nails and machine-cut nails. I did not observe any wire nails in the assemblage, although these are common at European outposts further north on the Falémé River (Thiaw 1999:219). By comparison to technological developments in Europe, these types convey information helpful for absolute dating. 
Figure F.6: Blade, projectile, and nail types in the Survey assemblage

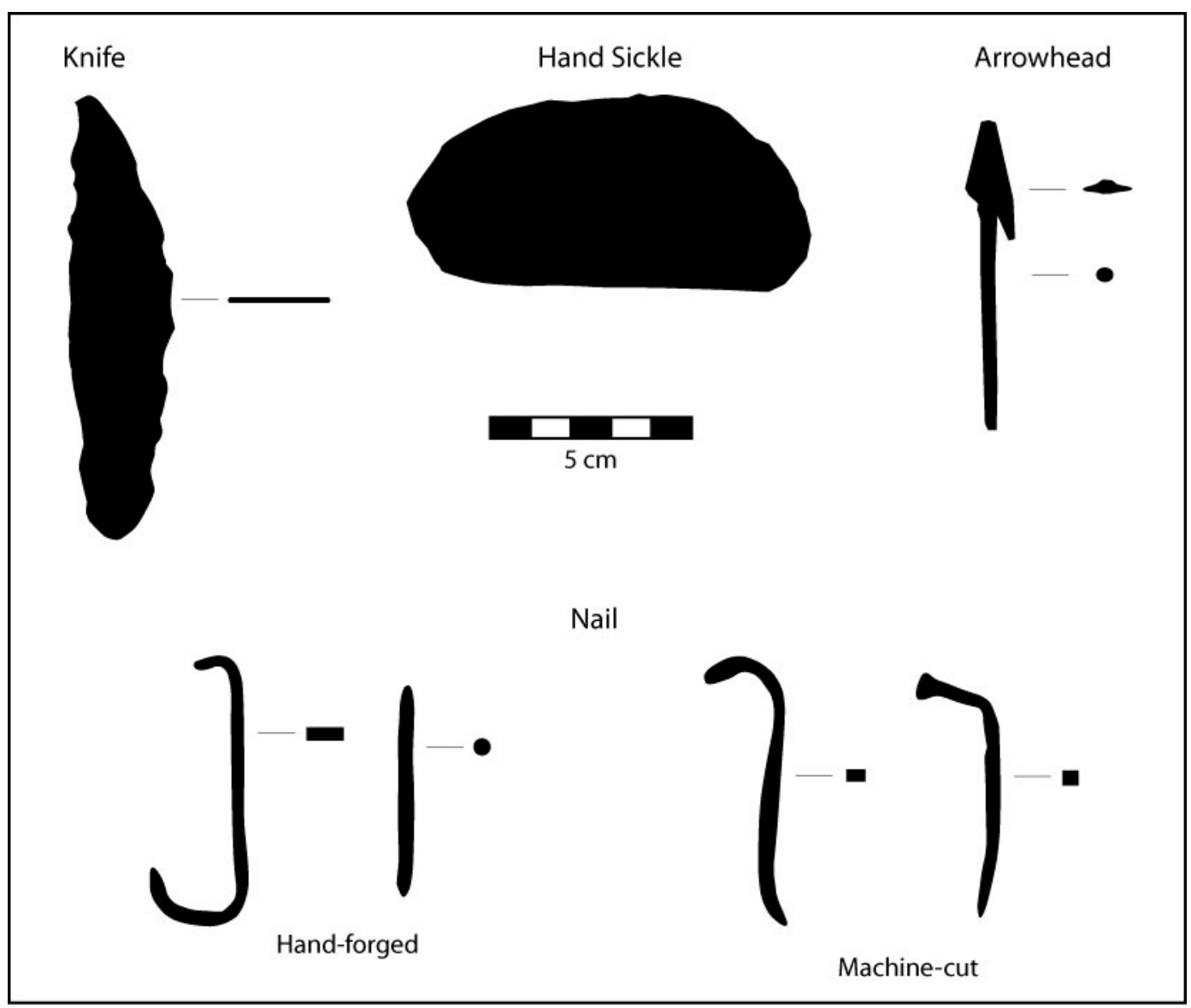

\section{Chronological Seriation}

Low frequencies of iron and copper objects from excavated contexts precluded their seriation in the Diouboye assemblage. With the exception of nondescript rod fragments, none of the artifact types from Diouboye occurred in the Survey assemblage, suggesting that the latter mainly included materials dating to Atlantic Era occupation of the study area.

Beyond a broad resemblance to ethnographic and historic materials documented elsewhere, the small numbers of projectile points and blades in the Survey assemblage were likewise insufficient for seriation. Nails, however, provided some indication of relative chronology based on the introduction of machine-cut nails across the region during the early-19 ${ }^{\text {th }}$ century AD (Thiaw 1999:219). Bearing in mind the purposive collection strategy and small sample size, the nail seriation in Table F.4 generally supports that of other artifact classes. 
Table F.4: Summary of metal artifact counts for surveyed sites

\begin{tabular}{|c|c|c|c|c|c|c|c|c|c|}
\hline \multirow[t]{2}{*}{ Site } & \multirow{2}{*}{$\begin{array}{l}\text { Ceramic } \\
\text { Component }\end{array}$} & \multirow{2}{*}{$\begin{array}{c}\text { Total } \\
\mathrm{n}\end{array}$} & \multicolumn{4}{|c|}{ Iron Tool } & \multicolumn{3}{|c|}{ Iron Nail } \\
\hline & & & Knife & Sickle & Arrowhead & Other & Hf & Cut & Unk \\
\hline Goundafa & Iron Age & 1 & & & & & & & \\
\hline FAL-1134 & Iron Age & 1 & 1 & & & & & & \\
\hline \multicolumn{2}{|l|}{ Iron Age Total } & 2 & 1 & - & - & - & - & - & - \\
\hline Tontèko & Tontèko & 2 & & & 1 & & & & \\
\hline FAL-1075 & Tontèko & 1 & & & & & 1 & & 1 \\
\hline \multicolumn{2}{|c|}{ Tontèko Phase Total } & 3 & - & - & 1 & - & 1 & - & 1 \\
\hline FAL-1053 & Tomboura & 1 & & & & & 1 & & \\
\hline Tomboura E1 & Tomboura & 2 & 1 & & & & 1 & & \\
\hline FAL-1004 & Tomboura & 16 & 1 & 1 & & 1 & 3 & & 2 \\
\hline FAL-1059 & Tomboura & 3 & & & & & & 2 & \\
\hline \multicolumn{2}{|c|}{ Tomboura Phase Total } & 22 & 2 & 1 & - & 1 & 5 & 2 & 2 \\
\hline Tomboura E2 & Karé & 2 & & & 1 & & 1 & & \\
\hline Tomboura N & Karé & 8 & & 1 & & & 1 & 4 & \\
\hline Karé & Karé & 16 & & & & 1 & 3 & 4 & \\
\hline Toranga & Karé & 6 & & 1 & & 1 & & 1 & \\
\hline FAL-1036 & Karé & 4 & & & 1 & & & 2 & \\
\hline FAL-1108 & Karé & 2 & 1 & & 1 & & & & \\
\hline \multicolumn{2}{|c|}{ Karé Phase Total } & 38 & 1 & 2 & 3 & 2 & 5 & 11 & - \\
\hline \multicolumn{2}{|l|}{ Total } & 65 & 4 & 3 & 4 & 3 & 11 & 13 & 3 \\
\hline
\end{tabular}




\section{APPENDIX G MISCELLANEOUS ARTIFACTS}

Glass, carved bone, shell, and terracotta (non-pottery) artifacts comprise a minor part of the archaeological record in West Africa, yet these "small finds" are often vital to space-time systematics. The original provenience and chronology of standardized goods produced in specialized, if not industrialized, workshops can help trace networks of exchange and establish the terminus post quem of surrounding archaeological contexts. In addition, these relatively scarce objects can shed light on past economic processes as they accrue or generate value through circulation-becoming tokens in local and regional political economies (e.g., DeCorse 2001a; Ogundiran 2002; Richard 2010; Stahl 2002). For these reasons, scholars working in West Africa have undertaken a number typological and historical studies that frame the present analysis of disparate glass, bone, shell, and terracotta artifacts recovered from Diouboye and other sites in the MadinaSadatou study area.

The study of miscellaneous artifacts had four objectives: (1) to shore up the relative chronology of surveyed sites using temporally sensitive objects; (2) to document local technologies for subsistence, craft production, and/or ritual; (3) to infer modes of long-distance exchange for objects of non-local origin; and (4) to consider practices of display and consumption underpinning political economies and social identities at multiple scales. Departing from the methodology deployed for major artifact classes, I make no attempt to reconstruct operational sequences for the assorted objects described in this appendix. Although I note technological implications whenever possible, I address the above objectives more directly through extant classification schemes, ethnographic and historic references to object use and exchange, and archaeological consideration of local versus non-local manufacture. Given the paucity of direct evidence for the use, reuse, and modification of these miscellaneous artifacts, however, it is necessary to adopt a more contextual approach to the study of actual consumption practices in Chapters 4 and 6-9.

\section{TERRACOTTA}

Given the quantity of earthenware pottery produced within the Madina-Sadatou study area, it should come as no surprise that people deployed their mastery of ceramic technology to make other sorts of objects-including smoking pipes, personal adornment, figurines, netweights, and spindle whorls. Based on the overall similarity of their operational sequences, the analytical procedures for these miscellaneous terracotta objects largely followed those outlined for pottery in Appendix D. Paste preparation and firing conditions, for example, were described using the same attribute states as for pottery sherds. Table G.1 lists the variables recorded for all terracotta objects before considering each subclass in greater detail. 
Table G.1: Variables recorded for terracotta artifacts

\begin{tabular}{|c|c|c|}
\hline Variable & Attribute (Code) & Description \\
\hline \multirow{7}{*}{ Subclass } & Smoking pipe (1) & A pipe, or pipe fragment, used for smoking \\
\hline & Bracelet (2) & A curved bracelet fragment \\
\hline & Figurine (3) & A discernible zoo- or anthropomorphic figurine fragment \\
\hline & Netweight (4) & $\begin{array}{l}\text { An undecorated object with a central bore, including the } \\
\text { following shapes: (1) small globular; (2) tubular; (3) } \\
\text { barrel; or (4) cigar }\end{array}$ \\
\hline & Bead (5) & A barrel-shaped or discoid object with a central bore \\
\hline & Spindle whorl (6) & $\begin{array}{l}\text { A decorated object with a central bore sufficient for } \\
\text { mounting on a spindle, including the following shapes: } \\
\text { (1) spherical; and (2) discoidal }\end{array}$ \\
\hline & Perforated disc (7) & A ceramic disc with multiple perforations \\
\hline \multirow{2}{*}{ Completeness } & Complete (0) & Specimen appears complete \\
\hline & Fragment (1) & Specimen is fragmentary \\
\hline Weight & Grams (0.1 g) & Mass of the specimen \\
\hline Length & Millimeters (mm) & Maximum length of the specimen \\
\hline Width/Diameter & Millimeters (mm) & Maximum width or diameter of the specimen \\
\hline Thickness & Millimeters (mm) & Maximum thickness of the specimen \\
\hline Bore Diameter & Millimeters (mm) & Diameter of the bore in pipe stems and perforated objects \\
\hline \multirow{4}{*}{ Inclusion Type } & None $(0)$ & No visible inclusions \\
\hline & Grog (1) & Crushed pottery or fragments of hardened clay \\
\hline & Grit/Sand (2) & Opaque rock fragments \\
\hline & Grog and Grit/Sand (3) & Crushed pottery and opaque rock fragments \\
\hline $\begin{array}{r}\text { Inclusion } \\
\text { Texture } \\
\end{array}$ & See Table D.4 & Relative size of the inclusion grains \\
\hline $\begin{array}{r}\text { Inclusion } \\
\text { Density } \\
\end{array}$ & See Table D.4 & Relative density of the inclusion grains \\
\hline \multirow{2}{*}{$\begin{array}{l}\text { Surface } \\
\text { Treatment }\end{array}$} & None $(0)$ & No surface treatment \\
\hline & Slip/Polish (3) & Slip and/or polished surface \\
\hline \multirow{3}{*}{ Decoration } & None $(0)$ & Undecorated \\
\hline & Twine (1) & Twine roulette or impression \\
\hline & Incisions (2) & One or more fine incisions \\
\hline Firing & See Figure D.30 & Schematic pattern of reduction/oxidation in cross-section \\
\hline
\end{tabular}

\section{Smoking Pipes}

Terracotta pipes of indigenous manufacture commonly turn up at archaeological sites in West Africa dating to the past five centuries. In contrast to contemporaneous European pipes, African-made pipes have socketed stems linking the bowl to a longer wooden reed for inhaling the smoke (S. K. McIntosh et al. 2003; Ozanne 1969; Philips 1983; Shaw 1960). Although these pipes may share techniques of surface treatment and decoration with earthenware vessels, they usually have a much finer and well-fired paste. 
Not surprisingly, smoking pipes figure prominently in debates around the timing and routes for the introduction of tobacco, an American domesticate, into and across West Africa. While it is conceivable that people smoked native fumigants prior to the emergence of trans-Atlantic commerce, no firm evidence for this exists at present (see discussions in S. K. McIntosh et al. 2003; Ozanne 1969). It follows that pipes are, at a minimum, diagnostic of archaeological assemblages dating to the $16^{\text {th }}$ century AD and later. A few studies have worked to further seriate pipe assemblages and to infer social interaction on the basis of the morphology and decorative elements of pipe stems, bases, and bowls (S. K. McIntosh et al. 2003; Shaw 1960); these form the basis for my analysis of terracotta pipes from Madina-Sadatou (Table G.2).

The sample of smoking pipes collected from 11 sites and isolated occurrences in the Madina-Sadatou study area includes only highly fragmented specimens-including 11 from purposive collection and 33 from systematic collection. An exploratory analysis of the metric and formal variables outlined in Table G.2 found few recurrent attribute associations sufficient for defining types. Pipe stems, for example, are invariably round, but decorative incisions, punctates, and slip vary markedly in their relationship to each other and to overall size and morphology.

\section{Table G.2: Variables recorded for terracotta smoking pipes}

\begin{tabular}{|c|c|c|}
\hline Variable & Attribute (Code) & Description \\
\hline \multirow{4}{*}{ Completeness } & Complete (0) & Specimen appears complete \\
\hline & Stem (1) & Complete stem or stem fragment \\
\hline & Bowl (2) & Complete bowl or bowl fragment \\
\hline & Base (3) & Complete base or base fragment \\
\hline Stem Diameter & Millimeters (mm) & Maximum diameter of the stem \\
\hline \multirow{4}{*}{$\begin{array}{l}\text { Stem/Bowl } \\
\text { Decoration }\end{array}$} & None $(0)$ & No decoration \\
\hline & Incisions (1) & Single or multiple incisions \\
\hline & Punctate (3) & Fine punctates arranged in bands or geometric pattern \\
\hline & Lipped (4) & Bowl has a fine everted lip around the orifice \\
\hline \multirow{2}{*}{$\begin{array}{l}\text { Stem Shape/ } \\
\text { Collar Shape }\end{array}$} & Circular (0) & Stem/collar appears circular cross-section \\
\hline & Rectilinear (1) & Stem/collar appear rectilinear in cross-section \\
\hline Base Diameter & Millimeters (mm) & Maximum diameter of the base \\
\hline \multirow{3}{*}{ Base Shape } & Single-angle footed (1) & Stem attaches to the side of a footed base \\
\hline & Double-angle footed (2) & Stem attached to the top of a footed base \\
\hline & Elbow (3) & Base is not footed \\
\hline Neck Height & Millimeters (mm) & Height of the neck between the foot and bowl \\
\hline Neck Diameter & Millimeters (mm) & Maximum diameter of the neck \\
\hline
\end{tabular}


Figure G.1: Smoking pipe stem decoration and base shapes

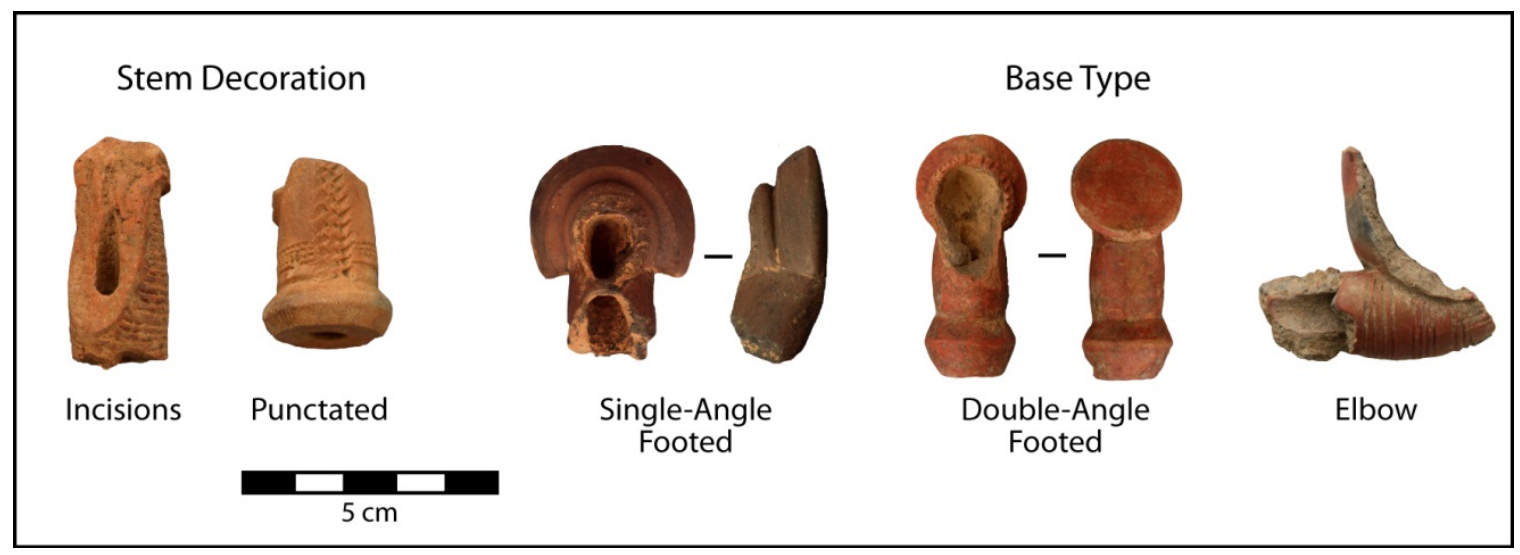

Nevertheless, base shape and collar shape, two temporally sensitive attributes on pipes from stratified deposits at Jenné in Mali (S. K. McIntosh et al. 2003) and Daboya in Ghana (Shinnie and Kense 1989), did seriate reasonably well alongside associated ceramic components defined for the Madina-Sadatou study area in Appendix D. Table G.3 shows a tentative trend from higher frequencies of pipes with double-angle footed bases associated with Tontèko phase ceramics to fewer pipes with single-angle footed and elbow bases associated with Tomboura and Karé phase ceramics. Despite the 800 kilometers that separates this study area from the Inland Niger Delta, it is notable that the Tontèko phase specimens most closely resemble those from $17^{\text {th }}$ to early-18 ${ }^{\text {th }}$ century AD contexts at Jenné where pipes with elbow bases, similar to the single specimen from Toranga, appear later in $19^{\text {th }}$ century AD contexts (Daget and Ligers 1962; S. K. McIntosh et al. 2003). Similar pipes have been recovered from roughly contemporaneous contexts along the lowermost Falémé River (Thiaw 1999:211-213) and at the site of Niani in Guinea (Filipowiak 1979:198). In contrast, regions further west along the Gambia River and the Atlantic littoral yield elbow base pipes, but few, if any, flared and double-angle footed base pipes (Gijanto 2007:16-17; A. Lawson 2003:266-269; Richard 2007:618). This suggests that a larger sample of terracotta pipes from contexts along the Falémé River would be amenable both to seriation and to the study of interregional exchange and smoking practices linking the communities of Madina-Sadatou to diverse spheres of cultural and economic interaction.

Further archaeological research might also shed light on who made and used these terracotta pipes. At least one historical account from the Senegambian coast attests to pipe production by potters (Richard 2007:615), while a compositional analysis by Ann Stahl and colleagues (2008) points to the more specialized production (or at least clay extraction strategies) and exchange of pipes in west-central Ghana. At present, however, it is not known which pipes from Madina-Sadatou were made locally and which were imported. Nor is it possible to ascertain whether or how smoking worked to reproduce shared identities and/or affirm social distinctions within Atlantic Era communities. 
Table G.3: Summary of smoking pipe collar and base shapes by Atlantic Era ceramic component

\begin{tabular}{|c|c|c|c|c|c|c|c|}
\hline \multirow[t]{2}{*}{ Locality } & \multirow[t]{2}{*}{ Phase } & \multirow{2}{*}{$\begin{array}{c}\text { Total } \\
\mathrm{n}\end{array}$} & \multicolumn{2}{|c|}{ Collar Shape } & \multicolumn{3}{|c|}{ Base Shape } \\
\hline & & & Round & Square & Double- Angle & Single-Angle & Elbow \\
\hline FAL-1075 & Tontèko & 5 & 1 & & 1 & & \\
\hline Tontèko & Tontèko & 16 & 4 & 2 & 4 & 1 & \\
\hline FAL-1004 & Tomboura & 11 & 2 & & 7 & 2 & \\
\hline FAL-1046 & Tomboura & 1 & & & & 1 & \\
\hline FAL-1101 & Tomboura & 2 & & & & & \\
\hline FAL-1130 & Tomboura & 2 & & & & 1 & \\
\hline FAL-1022 & Karé & 1 & & & & & \\
\hline FAL-1084 & Karé & 1 & 1 & & 1 & & \\
\hline Tomboura E2 & Karé & 1 & & & & & \\
\hline Toranga & Karé & 2 & & & & & 1 \\
\hline FAL-IO-019 & Unknown & 1 & & & & 1 & \\
\hline Total & & 44 & 8 & 2 & 13 & 6 & 1 \\
\hline
\end{tabular}

\section{Bracelets}

Surface collections at Diouboye by Marie-José and Howard Opper (1990) counted two nearly complete terracotta rings, tentatively identified as bracelets, with an exterior diameter of 80-90 millimeters and an interior one of 55-65 millimeters. These were wellfired with fine grog and organic inclusions, but they had no decoration or surface treatment of any kind. During the 2008 and 2009 field seasons, an additional four bracelet fragments were recovered from excavation contexts at Diouboye although one specimen boasts a fine red slip (Figure G.2).

With paste preparation and firing techniques identical to those for earthenware pottery, these bracelets could have been made by local potters. The use of these objects remains unknown, but their rarity in the archaeological record suggests a role in relatively infrequent activities such as ritual.

\section{Figurines}

Terracotta figurines occur in low frequencies at numerous late-Holocene archaeological sites in West Africa, where they range from the detailed ceramic statuary of the Middle Niger region (R. J. McIntosh 1989) to low-fired earthenware zoomorphic and anthropomorphic statuettes such as the three initially collected from the surface of Diouboye (Opper and Opper 1990). These specimens included one probable phallus and two nearly identical robust four-legged beasts, possibly cattle or hippopotami.

The present research program at Diouboye recovered unequivocal figurine fragments from both surface $(n=6)$ and excavation $(n=4)$ contexts, in addition to seven unidentifiable pieces of terracotta that most likely derive from figurines. With fine grog/organic inclusions and well-fired pasted, these strongly resemble the bracelets described above, suggesting a similar mode of manufacture by local craftspeople. The 
small sample precludes a rigorous analysis, but one large, well-worn specimen from the surface (Figure G.2) does resemble two zoomorphic statuettes found previously by the Oppers (1990:32). Quadrupedal figurines of similar appearance have been documented in the Middle Niger (S. K. McIntosh 1995:214-215) and Middle to Upper Senegal regions (Deme and McIntosh 2006:334; Thiaw 1999:208; Thilmans and Ravisé 1980:68). As with bracelets, terracotta figurines at Diouboye most likely played some symbolic role within community economic and ritual practices.

Figure G.2: Miscellaneous terracotta artifact subclasses

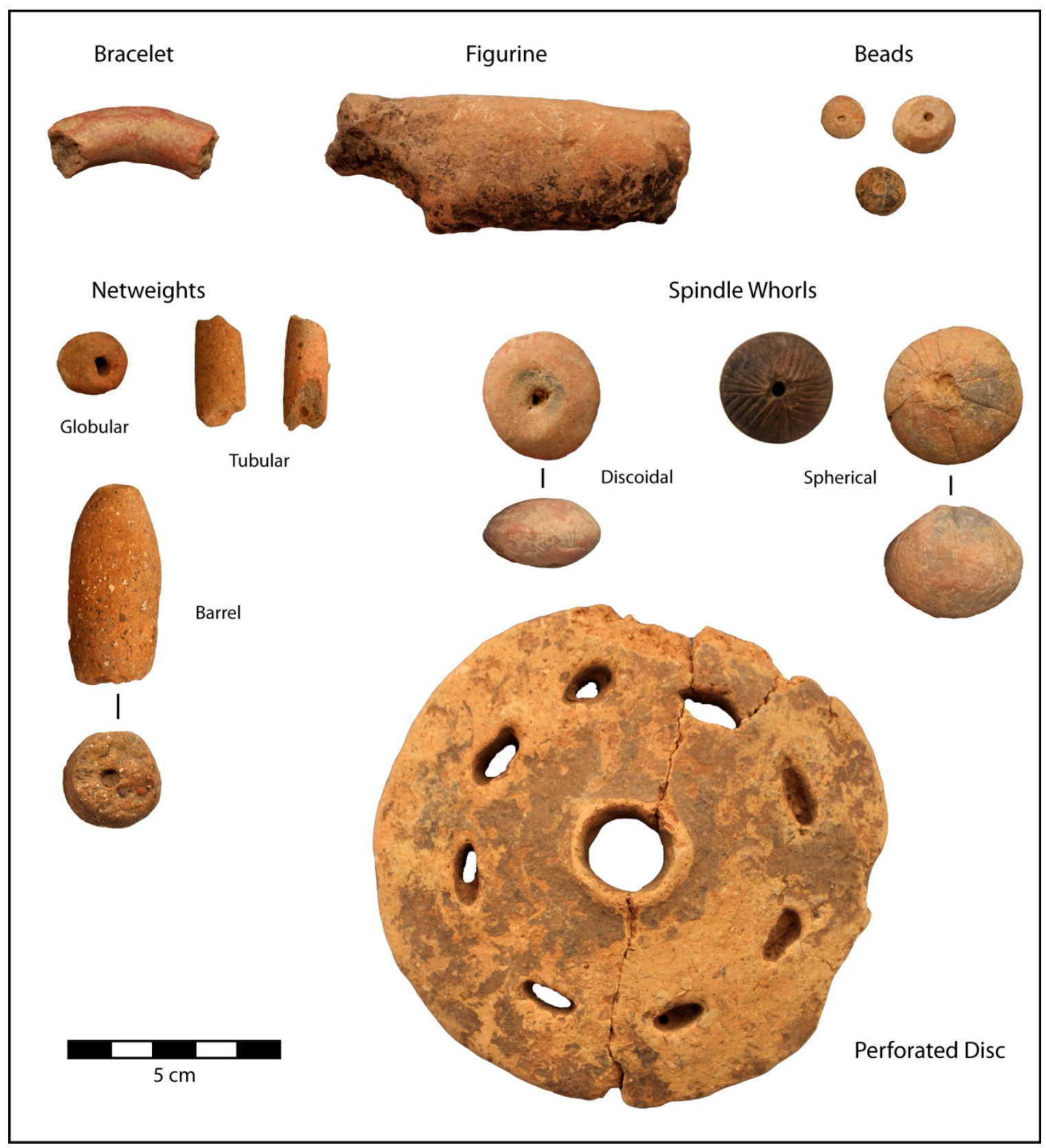


Figure G.3: Bivariate plot of length and diameter for perforated terracotta objects showing tenth-quantile kernel density estimate contours and confidence interval ellipses $(90 \%)$ for selected artifact subclasses

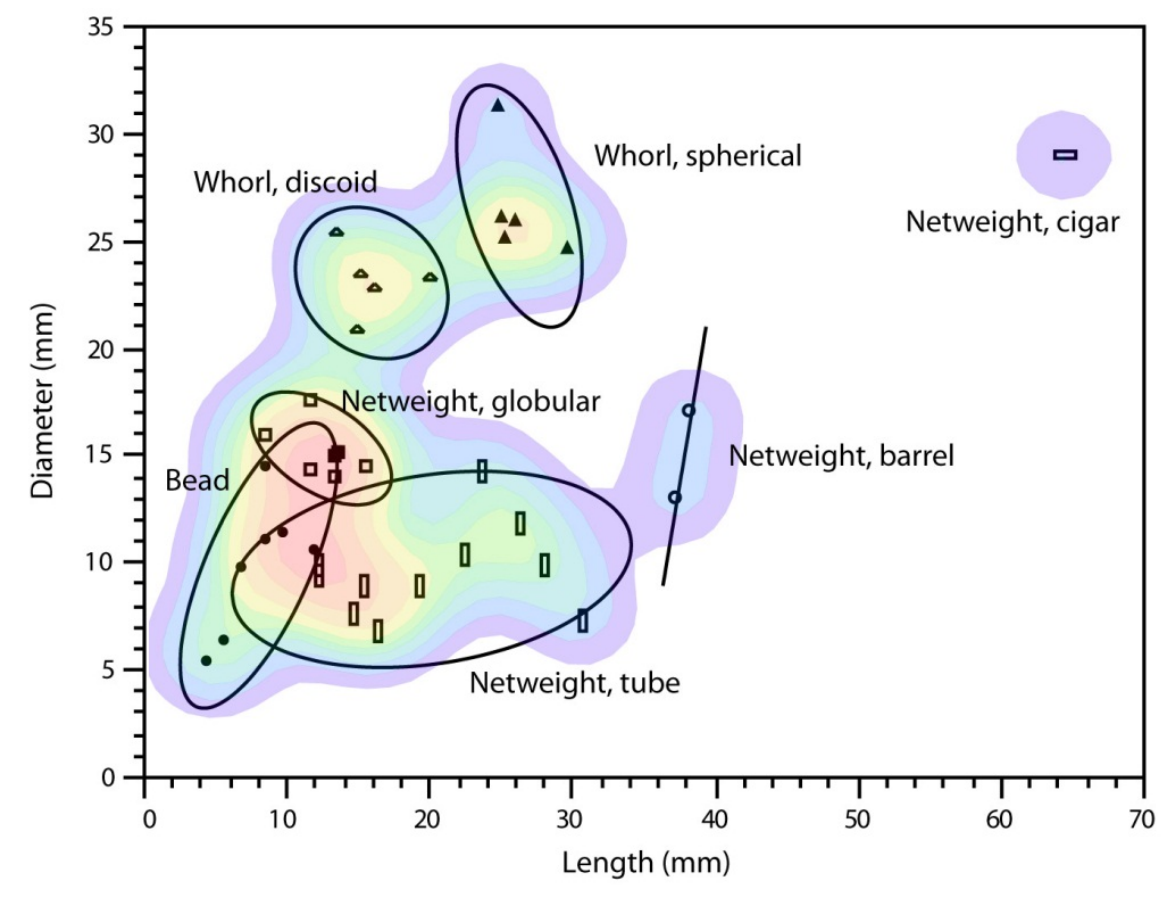

\section{Netweights}

Netweights are perforated objects with roughened or worn surfaces exposing course grog and/or grit temper and no hint of additional surface treatment or decoration. Although the 24 netweights recovered during fieldwork in Madina-Sadatou generally have a length exceeding their diameter (Figure G.3), they do overlap in size with beads, which were otherwise distinguished by greater symmetry and an emphasis on surface treatment (see below). Interestingly enough, the larger two barrel (length 37.0-38.0 mm, bore $3.0 \mathrm{~mm}$ ) and two cigar-shaped (length $>40 \mathrm{~mm}$, bore $5.0 \mathrm{~mm}$ ) specimens came from sites with an Iron Age or Tontèko phase ceramic component-in addition to a large cigarshaped weight previously recovered from Diouboye (Opper and Opper 1990:33). Seven globular netweights (length 7.9-15.1 mm, bore 2.1-3.7 mm) all derived from surface and excavated contexts within Diouboye. An assortment of 13 tubular netweights (length 11.8-30.4 mm, bore 1.8-3.5 mm) mainly came from surface contexts at 13 surveyed localities with cLSA or cLSA/Atlantic Era ceramic components.

As the name implies, I interpret these artifacts as the weights attached to fishing nets. Similar specimens have been documented along the Middle to Upper Senegal River (Bocoum and McIntosh 2002:178; Thiaw 1999:210, 222). Although the practical implications of a shift in modal netweight size and morphology between cLSA and Iron Age occupations in Madina-Sadatou are not yet known, this pattern could signal a shift in fishing techniques (Van Neer 2008:128-129). 


\section{Beads}

Terracotta beads exhibit spherical, biconical, or barrel shapes with a longitudinal perforation and a fine, well-fired paste (Figure G.3). These artifacts turned up in both excavation $(n=6)$ and surface $(n=1)$ contexts at Diouboye where prior fieldwork had yielded three similar specimens (Opper and Opper 1990:30-31). As shown in Figure G.2, these have a length of 4.1-11.7 millimeters $(\mu=7.8 \mathrm{~mm}, \sigma=2.6 \mathrm{~mm})$ and diameter of 5.414.5 millimeters $(\mu=9.9 \mathrm{~mm}, \sigma=3.1 \mathrm{~mm})$. Overlapping in size with the small tubular and globular netweights, the symmetrical shape and polished or slipped surface on beads clearly sets them apart from the former. In fact, terracotta beads were analyzed following the protocol for glass ones described below.

Terracotta beads of similar shape and/or size to those from Diouboye have been discovered in Iron Age contexts at Kumbi Saleh in southeast Mauritania (Berthier 1997:89), at sites in the Middle Niger region (S. K. McIntosh 1995:216; Togola 2008:4142), and along the Middle Senegal River (Bocoum and McIntosh 2002:178; Chavane 1985:163-167). The formal resemblance between these beads, presumably of local manufacture, and those made from exotic materials such as glass and carnelian may hint at the replication of imported objects.

\section{Spindle Whorls}

Spindle whorls generally occur in low frequencies on settlement sites in West Africa where they attest indirectly to the diffusion of cotton fiber processing and textile production from the late-first millennium AD onwards (Kriger 2005). Although they vary markedly across space and time, whorls are quite recognizable as perforated weights used to maintain the rotation of a wooden spindle while cotton or other fibers are drawn into thread from one end. It was possible to distinguish two types of whorls in the MadinaSadatou study area according to overall form-discoidal and spherical.

As shown in Figures G.2-G.3, spherical whorls $(n=10)$ are fairly symmetrical with a bore diameter of 3.0-4.7 millimeters and an exterior diameter of 23.1-31.2 millimeters ( $\mu=26.0 \mathrm{~mm}, \sigma=2.8 \mathrm{~mm}$ ). Beyond this shared morphology, most are decorated with a surface slip and/or incised geometric patterns - similar to whorls documented at Iron Age and Atlantic Era contexts in the Upper Niger (Filipowiak 1979:201) and Middle to Upper Senegal regions (Chavane 1985:149-151; Thiaw 1999:223-224). Notably, these differ stylistically from the conical flat-faced and intricately decorated whorls known from the Middle Niger region (S. K. McIntosh 1995:216, 227-231; Togola 2008:40-41). The Oppers (1990) had previously collected two such specimens from the surface of Diouboye and one from the site of Karé. My own fieldwork recovered these whorls from subsurface contexts at Diouboye $(n=4)$ and in surface collections $(n=6)$ at three sites representing substantial Atlantic Era settlements.

Discoidal spindle whorls, as illustrated in Figures G.2-G.3, have a biconical form with flattened ends and generally boast a surface treatment distinguishing them from globular netweights. These measure more than 20 millimeters in diameter $(\mu=22.9 \mathrm{~mm}$, $\sigma=1.8 \mathrm{~mm}$ ) with a central bore diameter of 1.1-2.4 millimeters. Most exhibit light wear, if not clear facets, around the perforation, leading to their tentative interpretation as spindle whorls (see Berthier 1997:89), although they could have feasibly served as objects of personal adornment and/or ritual, or perhaps even as netweights. Five of the six specimens from the Madina-Sadatou study area came from Diouboye. 
Spindle whorls were presumably made by potters, but it is not known whether those from Madina-Sadatou were of local or non-local manufacture. A compositional study by Ann Stahl and colleagues (2008) found six whorls from west-central Ghana to comprise a distinct clay group, possibly representing specialized production coincident with the growth of a household-based textile industry. This pattern may also hold for the Upper Senegal region where historical accounts describe ample cotton exports made possible by a household-level division of labor-women spinning and men weaving (Curtin 1975a:211-215). Testing this latter hypothesis will require more material evidence, but the present data support a scenario for the diffusion of cotton cultivation and loom technology across the region over the past millennium.

\section{Perforated Discs}

The perforated ceramic discs commonly found on Iron Age sites along the Middle to Upper Senegal River comprise something of an archaeological enigma. With diameters of 80-160 millimeters, they usually have one or two central holes surrounded by smaller holes of variable form. Based on finds from Sinçu Bara, Guy Thilmans and Anne Ravisé (1980) argued that these were disques à cordeler for spinning fibers into a thicker rope (see also Chavane 1985:143-150). Alternative hypotheses include censer lids or weighted components in fishing nets.

Given their ubiquity at sites in the Middle Senegal region and even at Arondo on the Senegal-Falémé confluence (Thiaw 1999:208-210), it is striking that only one perforated disc (Figure G.3) turned up in the Madina-Sadatou during purposive surface collection at the riverside site of FAL-1043. The absence of these artifacts at Diouboye suggests that economic or socio-technological practices within this community diverged from those of contemporaneous ones to the north.

\section{GLASS AND STONEWARE}

The production of glass and stoneware requires temperatures in excess of $1200^{\circ} \mathrm{C}$ using technologies that were unavailable in West Africa prior to the $20^{\text {th }}$ century AD. Artifacts made of these materials thus provide unequivocal evidence for long-distance exchange and contribute to relative chronologies, particularly where independent historical and archaeological evidence place their origins within firm spatial and temporal boundaries (DeCorse 1989).

\section{Imported Containers}

A single European stoneware jar fragment was found serendipitously as an isolated occurrence (FAL-IO-035) near the Atlantic Era site of Tontèko. Recorded following the protocol for earthenware pottery, the interior of this sherd had light channels from wheel-thrown manufacture and a greenish-gray glaze (Figure G.4); the buff-colored exterior was unglazed. The paucity of stoneware sherds in Madina-Sadatou contrasts with the situation further north along the lower Falémé River where the greater frequencies of imported European ceramics at Fort Senedebou and surrounding sites testifies to more direct interactions with Europeans during the mid- to late- $19^{\text {th }}$ century AD (Thiaw 1999:211-214, 224).

A total of eight glass container fragments turned up during purposive surface collections at five settlement sites, including FAL-1064 with Tomboura phase (AD 1700- 
1860) ceramics and Karé, Toranga, Tomboura North, and FAL-1108 with Karé phase (AD 1860-1925) ceramics. With the exception of one possible mold-blown green bottle shard and one lavender-tinted bottle stop, the remaining six specimens all derived from squarish bottles of aqua, lavender, or clear glass and mold-blown manufacture (Figure G.4). These artifacts, made from the $19^{\text {th }}$ to early- $20^{\text {th }}$ century $\mathrm{AD}$, lend support to the absolute chronology of their associated Atlantic Era ceramic components. As for stoneware, glass fragments document a heterogeneous participation in European trade circuits, numbering far more in the political core of Bundu further north (Thiaw 1999:215-216, 224) than in the relatively peripheral Madina-Sadatou study area.

\section{Figure G.4: Imported container types}

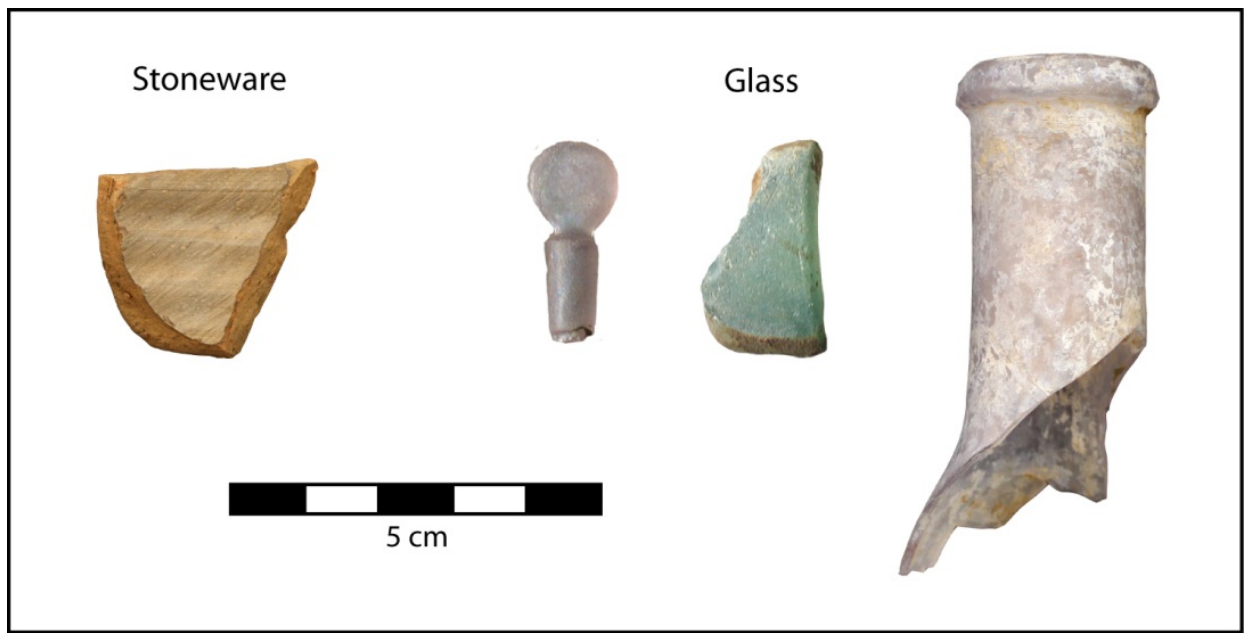

\section{Glass Beads}

Beads comprised the predominant imported artifacts both at surveyed sites and in surface and excavation contexts at Diouboye. Relying upon an analytical framework developed by Christopher DeCorse and colleagues (2003) for beads from the lower Falémé region, a mere 50-100 kilometers downriver from Madina-Sadatou, I recorded variables for size and overall morphology, structure, technique(s) of manufacture, luster, diaphaneity, color, and decoration. These attributes allowed me to fit the beads from Diouboye and surveyed Atlantic Era sites into extant typologies useful for interpreting chronology and provenience.

\section{Diouboye Assemblage}

In the course of their surface collection and test excavations, Marie-José and Howard Opper (1990) discovered eight glass beads at Diouboye. The CFAP fieldwork found additional glass beads during excavation $(n=6)$ and surface collection $(n=7)$. Two of these latter, however, were of European manufacture (see below Type 36, Type 103). The remaining beads, summarized in Table G.4, included the following types:

$G b-A$. Drawn green short cylindrical beads $(\mathrm{n}=3)$ measure 3.6-4.9 millimeters in length and 4.8-5.6 millimeters in diameter. Beyond their shared morphology and 
mode of manufacture, these beads vary in color from translucent dark green to an opaque light green. Their place of origin is not known.

$G b-B$. Drawn dichroic short cylindrical beads $(\mathrm{n}=4)$ measure 4.0-4.9 millimeters in length and 4.0-4.8 millimeters in diameter. Their translucent dichroic glass appears a cobalt blue when held in the hand, but changes to yellowish-green when held up to light (Davison et al. 1971). Although their ends have been ground, these beads could be broken or reworked versions of the dichroic tubular beads also found at Diouboye.

$G b-C$. Drawn dichroic tubular beads $(\mathrm{n}=4)$ measure 18.0-25.0 millimeters in length and 4.7-6.8 millimeters in diameter. A seam running the length of each bead marks a technical sequence of folding and then drawing the glass into shape, also resulting in a triangular cross-section and/or "corded” appearance. According to Marilee Wood (personal communication), compositional analyses of the two beads recovered during excavation link them to similar specimens produced at al-Basra in Morocco during the early-second millennium AD (Robertshaw et al. 2010).

$G b-D$. Compound red-on-transparent tubular beads $(\mathrm{n}=2)$ measure 9.7-12.0 millimeters in length and 3.2-5.0 millimeters in diameter. Beginning as tubes of transparent glass, these were rolled in translucent dark red glass and drawn into shape. According to Marilee Wood (personal communication), manufacturing techniques and chemical composition link these beads to the shorter, rounder specimens from the site of Igbo-Ukwu in Nigeria (Shaw 1970).

$G b-E$. A wound blue oblate bead fragment $(\mathrm{n}=1)$ measures 7.0 millimeters in length. The glass is a translucent, though not dichroic, blue of unknown origin.

$G b-F$. A molded gray short cylindrical bead $(n=1)$ measures 9.2 millimeters in length and 13.7 millimeters in diameter. This highly worn specimen has a speckled appearance similar to oblate molded lead-silica glass beads from al-Basra in Morocco (Robertshaw et al. 2010), although this relation has not been confirmed by compositional analysis.

Gb-G. Complex black pyramidal beads $(\mathrm{n}=3)$ measure 9.4-15.0 millimeters in length and 7.0-13.1 millimeters in maximum width. These artifacts are all dark gray to black opaque glass (?) pyramids with shallow depressions on each lateral face punctuated by a dot of opaque yellowish-white glass. Without destructive analyses, it has not been possible to more precisely define the materials and manufacturing techniques for these beads, nor to identify their place of origin.

Altogether quite consistent with an early-second millennium AD occupation, the glass bead assemblage from Diouboye resembles those from contemporaneous contexts at al-Basra in Morocco (Robertshaw et al. 2010), Kumbi Saleh (Berthier 1997:91-93; Thomassey and Mauny 1956:134-137) and Tegdaoust (Devisse 1983) in southeast Mauritania; and Gao (Insoll and Shaw 1997) and Igbo-Ukwu (Shaw 1970) along the middle to lower Niger River. Interestingly enough, some beads originated in the Maghreb, while others can be traced to Egypt or elsewhere in the Near East. This heterogeneity suggests that the people of Diouboye participated in a vast network including both the western and central/eastern trans-Saharan trade routes, most likely through intermediary relations with merchants and communities in the Upper Niger and Upper Senegal regions. 
Figure G.5: Glass, stone, bone, and shell ornament types from Diouboye

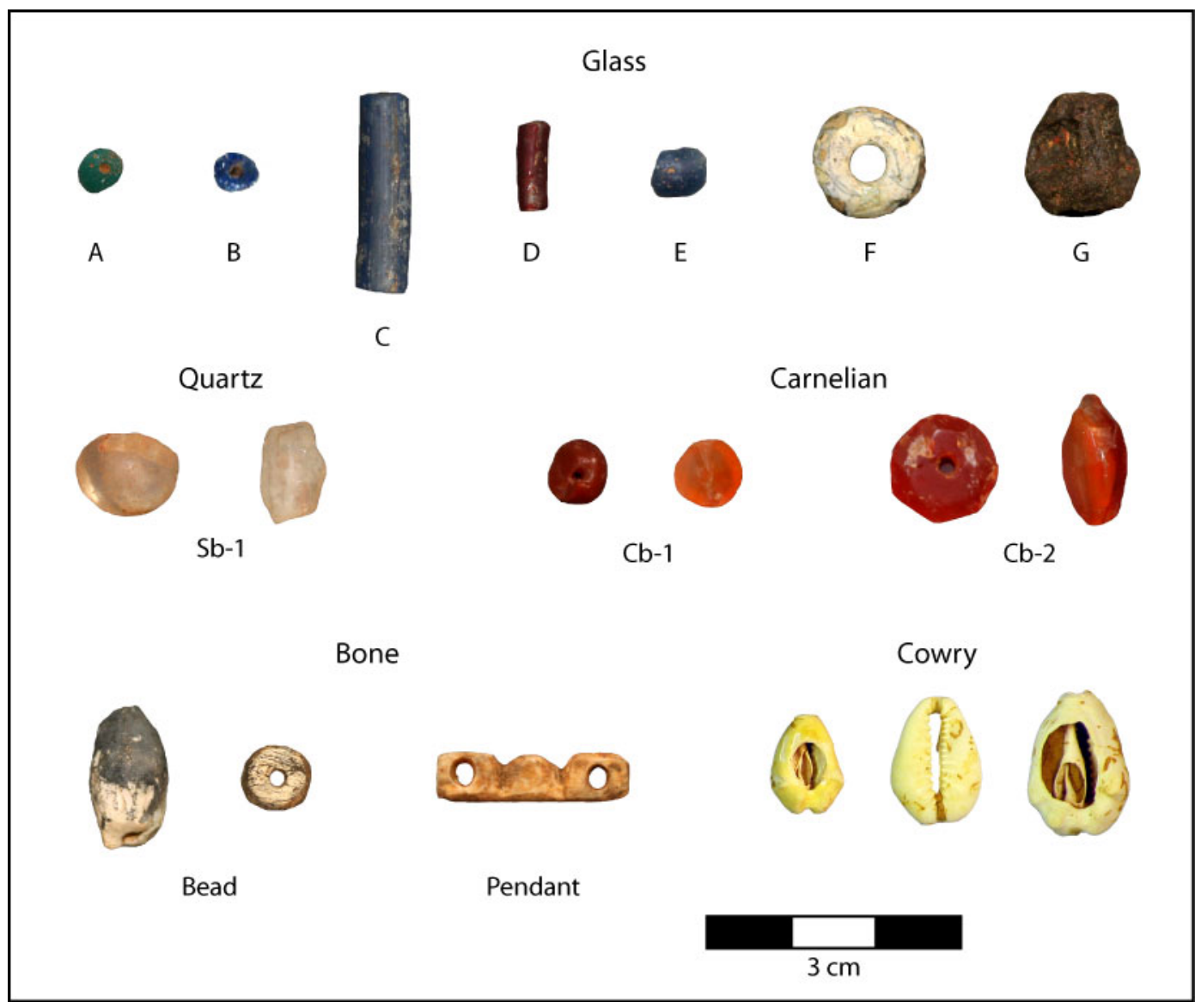

Table G.4: Summary of glass bead counts by sample from Diouboye

\begin{tabular}{|l|c|c|c|c|c|c|c|}
\hline \multicolumn{1}{|c|}{ Sample } & \multicolumn{7}{c|}{ Bead Type } \\
& Gb-A & Gb-B & Gb-C & Gb-D & Gb-E & Gb-F & Gb-G \\
\hline \hline Opper and Opper (1990) & 0 & 4 & 2 & 0 & 1 & 0 & 1 \\
\hline Surface (2008-2009) & 1 & 0 & 2 & 0 & 1 & 0 & 1 \\
\hline Excavation (2008-2009) & 2 & 0 & 0 & 2 & 0 & 1 & 1 \\
\hline \hline Total & $\mathbf{3}$ & $\mathbf{4}$ & $\mathbf{4}$ & $\mathbf{2}$ & $\mathbf{2}$ & $\mathbf{1}$ & $\mathbf{3}$ \\
\hline
\end{tabular}

\section{Atlantic Era Sites}

Nearly all of the glass beads collected during survey in the Madina-Sadatou study area could be assigned to the systematic typology developed for the lower Falémé region (DeCorse et al. 2003). Rather than detail each of these types (see Figure G.6), it is more useful for chronological purposes to summarize the observed beads by their period of manufacture. 
Eighteenth-century beads include only Type $38(\mathrm{n}=3)$. These complex wound beads of Venetian origin have a tubular to globular shape with opaque gold or white bands set into opaque black glass.

Late-eighteenth- to nineteenth-century beads include Type 37 (n=3), Type 39 $(n=3)$, Type $81(n=1)$, and Type 92a $(n=1)$. The first three types are complex wound beads of European origin with a spherical or ellipsoid form; these are opaque blue to black glass inlaid with white designs. A single variant of Type 92 consists of a biconical Bohemian bead molded from transparent glass.

Nineteenth-century beads include Type $2(n=1)$, Type $4(n=2)$, Type $7(n=1)$, Type $41(n=2)$, and Type $71(n=4)$. The first three types are simple wound beads of roughly oblate shape with transparent or translucent blue glass. The latter two types are compound drawn beads of globular or cylinder shape with red glass surrounding an opaque white core; these are often called "cornaline d'Aleppo."

Seventeenth- to early-nineteenth-century beads of European origin include Type $74(\mathrm{n}=4)$, Type $75(\mathrm{n}=13)$, Type $76(\mathrm{n}=12)$, Type $77(\mathrm{n}=72)$, and Type $84(\mathrm{n}=4)$. The first three types all comprise compound drawn beads of short cylindrical to globular shape with opaque red glass surrounding a translucent green core; these are often called "galet rouge." Beads assigned to Type 77 have a similar shape and mode of manufacture, but with colorless glass around an opaque white core; these are known as "galet blanc." Beads of Type 84 are compound drawn barrels of dark blue to black glass inlaid with vertical white stripes.

Mid- to late-nineteenth-century beads include Type $99(\mathrm{n}=1)$, Type $100(\mathrm{n}=3)$, Type $101(n=5)$, Type $102(n=1)$, Type $103(n=7)$, and Type 104a $(n=1)$. These are all short cylindrical prosser-molded ceramic beads of European origin ranging in color from white to light blue, green, and red.

Miscellaneous glass beads of apparent European manufacture dating to the past few centuries also include Type $16(n=5)$, Type $24(n=2)$, Type $25(n=4)$, Type 36 $(n=1)$, Type $47(n=1)$, Type $51(n=10)$, Type $52(n=7)$, Type $56(n=1)$, Type $58(n=1)$, Type $63(n=1)$, Type $65(n=1)$, and Type $69(n=1)$. These types subsume a diverse array of wound and drawn beads of simple to complex structure.

As shown in Table G.5, the abundance and chronological distribution of these beads aligns reasonably well with the Tomboura and Karé phase ceramic components defined in Appendix D. Sites with Tomboura phase (AD 1700-1860) ceramics yielded fewer and earlier beads than those with Karé phase (AD 1860-1925) ceramics, the dearth of beads from Toranga notwithstanding.

Beyond their support of the working ceramic chronology, imported glass beads show that people occupying the larger settlements in Madina-Sadatou began to participate in Atlantic-oriented exchange networks from the $18^{\text {th }}$ century AD onwards. While these small surface samples preclude a more rigorous discussion of exchange and display practices, it is curious that some Atlantic Era sites had more beads than others. Looking to case studies elsewhere across West Africa (e.g., DeCorse 2001a; Ogundiran 2002; Richard 2010; Stahl 2002), this pattern hints at the potential for future research to illuminate the cultural value and circulation of these objects through local and regional political economies. 
Figure G.6: Glass bead types from Atlantic Era sites (see DeCorse et al. 2003)

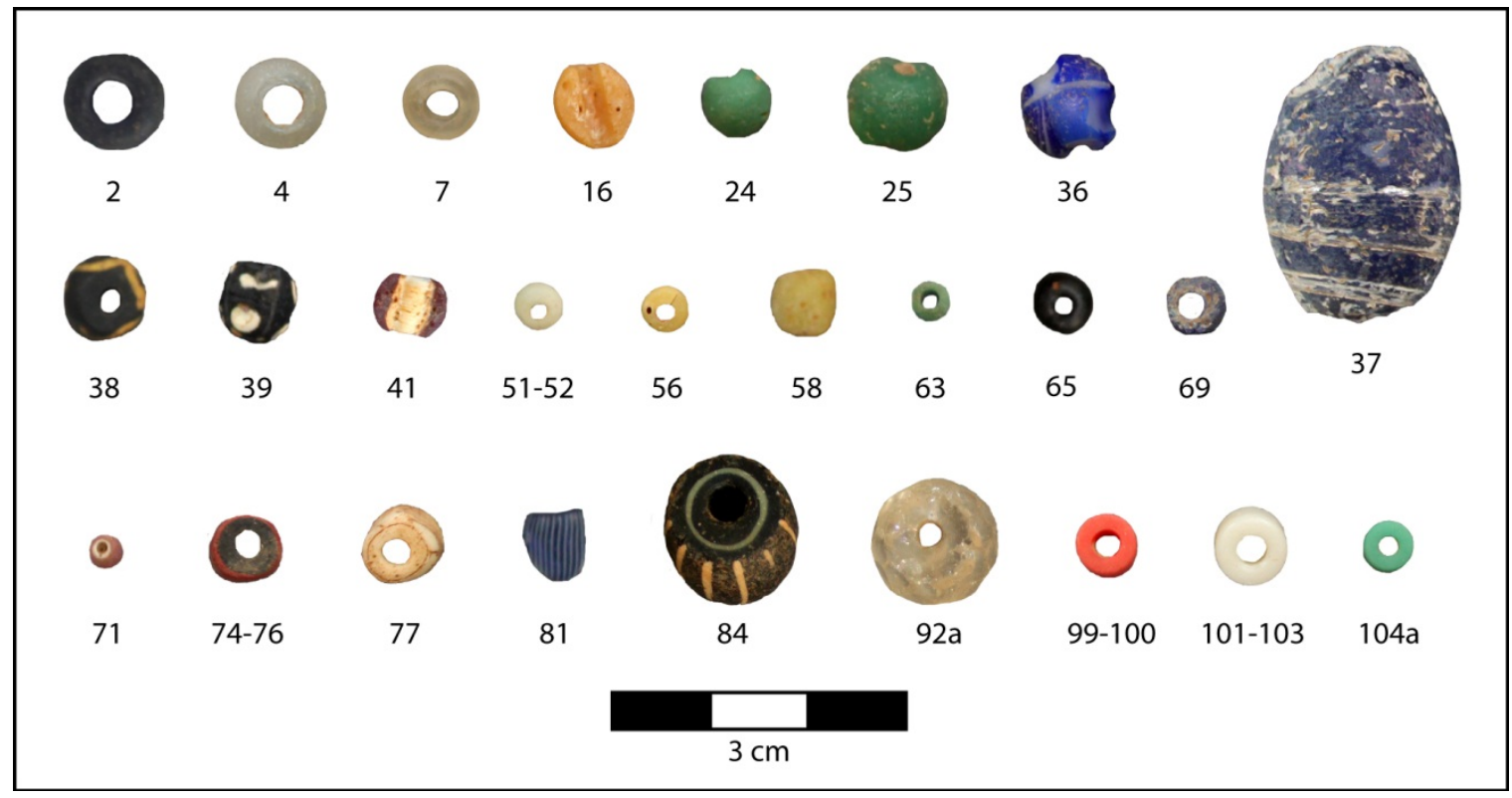

Table G.5: Summary of European glass bead counts by period of manufacture

\begin{tabular}{|c|c|c|c|c|c|c|c|}
\hline \multirow[t]{2}{*}{ Site } & \multirow[t]{2}{*}{ Phase } & \multirow{2}{*}{$\begin{array}{c}\text { Total } \\
\text { n }\end{array}$} & \multicolumn{5}{|c|}{ Period of Manufacture } \\
\hline & & & 18th c. & $\begin{array}{c}\text { Late 18th- } \\
\text { 19th c. }\end{array}$ & 19th c. & $\begin{array}{c}\text { 17th-early } \\
\text { 19th c. }\end{array}$ & $\begin{array}{l}\text { Mid- to } \\
\text { late- } 19^{\text {th }} \mathrm{C} \text {. }\end{array}$ \\
\hline FAL-1004 & Tomboura & 8 & 3 & & & 3 & \\
\hline Tomboura E1 & Tomboura & 1 & & & & 1 & \\
\hline FAL-1130 & Tomboura & 2 & & 1 & & 1 & \\
\hline Tomboura N & Karé & 54 & & 1 & 1 & 28 & \\
\hline Missira & Karé & 37 & & & 6 & 29 & \\
\hline Karé & Karé & 77 & & 6 & 3 & 42 & 18 \\
\hline Toranga & Karé & 1 & & & & & 1 \\
\hline FAL-1021 & Iron Age & 1 & & & & 1 & \\
\hline Total & & 181 & 3 & 8 & 10 & 105 & 19 \\
\hline
\end{tabular}

\section{STONE}

Several lithic artifacts, including beads and gunflints, merit more detailed consideration than was possible in the overview of chipped stone and groundstone technologies in Appendix E.

\section{Stone Beads}

Polished stone beads turned up infrequently during purposive surface collection at surveyed sites $(n=2)$ and during systematic surface collection $(n=2)$ and excavation $(n=7)$ 
at Diouboye where the Oppers (1990) previously found five specimens. On the basis of material and morphology, it was possible to distinguish the following types (Figure G.5) in the archaeological record of Madina-Sadatou.

$C b$-1. Spherical carnelian beads $(\mathrm{n}=5)$ have a globular to spherical shape with a length of 5.0-8.2 millimeters and maximum diameter of 5.5-8.1 millimeters. These are translucent with a rich orange to coral color.

$C b$-2. Facetted carnelian beads $(\mathrm{n}=3)$ are quite heterogeneous, ranging in overall shape from oblate to biconical; their size varies from 5.5-15.0 millimeters in length and 8.0-12.8 millimeters in maximum diameter. The ground facets on these beads point to similar techniques of manufacture distinct from those generating spherical ones. One hexagonal cylinder occurred with Tomboura phase ceramics at site FAL1004, but the rest of these facetted beads came from Diouboye.

$S b-1$. Translucent white quartz beads $(\mathrm{n}=2)$ include one spherical and one facetted specimen, both from Diouboye. These have a length of 11.8-12.3 millimeters.

$S b-2$. A single short cylindrical bead $(\mathrm{n}=1)$ fragment made from what appears to be local blue chert was found at site FAL-1036. This has a length of 4.8 millimeters and a diameter of 7.3 millimeters.

Similar types of stone beads, particularly those made of carnelian, have been observed at numerous sites dating from the late-first to early-second millennium AD elsewhere along the Senegal (Bocoum and McIntosh 2002:179; Chavane 1985:163-167; Thiaw 1999:210; Thilmans and Ravisé 1980) and Niger Rivers (Bedaux 1972:172; S. K. McIntosh 1995:247-248; Togola 2008:40-41), and at medieval towns in the Sahel such as Kumbi Saleh (Berthier 1997:91-93) and Tegdaoust (Devisse 1983). While the source of carnelian beads in West Africa has been the subject of some debate, a compositional analysis by Timothy Insoll and colleagues (2004) suggests that most specimens originated in the central Sahara or near the medieval town of Gao where archaeological evidence has even documented carnelian bead production. All this suggests that the stone beads from Diouboye were imported through exchange networks extending to the east along the Niger River.

\section{Gunflints}

Only two gunflints were discovered in the Madina-Sadatou study area-one during purposive survey in 2007 at the site of Missira (FAL-1150), and another one from the surface of Diouboye. Interestingly enough, the specimen from Diouboye, measuring 25 millimeters long by 20 millimeters wide, was a gray flint of English origin, while the specimen from Missira, measuring 28 millimeters long by 22 millimeters wide, was a honey-colored flint of French origin (Hume 1970:220). Although the precise dates of deposition are unknown, these two gunflints offer an apt material metaphor for local participation in both the British-dominated trade along the Gambia River and the Frenchdominated trade along the Senegal River during the Atlantic Era. They also hint at the presence of firearms on an increasingly violent political landscape.

\section{BONE AND SHELL}

Despite the abundance of bone and shell artifacts attesting to subsistence activities, the people occupying Diouboye did not frequently work such materials into 
formal tools and ornaments. Those worked objects that do exist document continuity in cultural practices with populations of adjacent regions.

\section{Ornaments}

The assemblage of carved shell and bone (or ivory) ornaments from MadinaSadatou is rather heterogeneous, so the following types remain provisionary.

Bone beads $(\mathrm{n}=7)$ comprise a group of roughly cylindrical specimens all from Diouboye, where the Oppers (1990:33) previously found one bead. One biconical bead resembling those of carnelian and quartz measures 17.3 millimeters in length, but the remaining seven all cluster into a coherent type ranging from 4.7-4.8 millimeters long and 7.5-11.8 millimeters in maximum diameter (Figure G.5).

A single bone pendant $(\mathrm{n}=1)$ serendipitously recovered during exploratory excavations at Diouboye in 2007 (Figure G.5) resembles a specimen found earlier by the Oppers (1990:33). These are elongated pieces of bone measuring 23.1-24.0 millimeters in length and 4.0-4.8 millimeters in width, with one perforation in each end and two symmetrical notches carved into the center. The congruence between these two objects is quite remarkable, suggesting that they shared some aesthetic or source of production.

Shell beads $(\mathrm{n}=2)$ were collected from two sites with Atlantic Era ceramics. A tabular piece of shell from FAL-1130 resembles those found at historic sites along the lowermost Falémé River (DeCorse et al. 2003 Type 108). A fine shell ring from Tomboura North (FAL-1131) measures at least 11.8 millimeters in diameter.

A possible ivory bracelet $(\mathrm{n}=1)$, or at least fragments thereof, was discovered during excavations at Diouboye. Measuring up to 5 centimeters in diameter, this specimen has similar dimensions to one recovered from Sinçu Bara on the Middle Senegal region (Thilmans and Ravisé 1980:72-73).

Although beads of bone and shell could have been made and consumed locally in Madina-Sadatou, they bear a resemblance to ornaments found at the sites elsewhere across Senegambia (Chavane 1985:163-167; Thilmans and Ravisé 1980:71) and those from Kumbi Saleh (Berthier 1997:90-91), hinting at shared craft traditions or interregional trade.

\section{Cowries}

Cowrie shells from the Indian Ocean, including the species Cypreae moneta and Cypraea annulus, have a long history as trade items in West Africa (M. Johnson 1970; Mauny 1961:373). Archaeological research shows that by the late-first millennium AD cowries had begun to make their way to towns and villages throughout the Senegal and Niger River basins (Berthier 1997:90-91; Nixon 2009:241; Thilmans and Ravisé 1980; Togola 2008:42). It was therefore not surprising to find nine of these artifacts in the course of excavation at Diouboye (Figure G.5), and one additional specimen on the surface of site FAL-1004. As with carnelian beads, cowries denote participation in vast networks for exchanging goods from east to west across the West African savanna. 


\section{COINS}

Silver coins were discovered at two sites with an Atlantic Era (Karé phase) ceramic component during purposive surface collection in 2007. The site of Karé (FAL1038) yielded a circular French one-franc coin minted in 1924. The site of Tomboura North (FAL-1131) yielded a Senegalese cinquante-centime coin from 1926. The dates on these coins are consistent with local histories of site abandonment and settlement leading to the consolidation of the present-day villagescape throughout the early- $20^{\text {th }}$ century AD. 


\title{
APPENDIX $\mathrm{H}$ \\ HARVESTING THE FALÉMÉ RIVER AND ITS SHORES: \\ PRELIMINARY RESULTS FROM ANALYSES OF FAUNA FROM DIOUBOYE
}

\author{
by \\ Stephen Dueppen \\ University of Oregon
}


Faunal remains are a critically important line of evidence for understanding multiple dimensions of past societies, from the subsistence economy to broader social processes and events such as rituals, changing social roles and statuses, and commerce. While significant advances have been made in recent decades, current understandings of Iron Age animal use in West Africa are limited by the small number of sites excavated and poor preservation of faunal remains in tropical contexts. In this report, I present the preliminary results of analyses of the animal remains from Diouboye, an ancient settlement occupied over several centuries between the late-first and early-second millennia AD. Faunal remains indicate that members of the community engaged in systematic and repeated exploitation of riverine animal resources, primarily of diverse animals large in size, in combination with the herding of domestic livestock. This strong riverine focus is found at multiple excavation units throughout the site, and appears to be a characteristic of all sampled households at the settlement.

\section{DIOUBOYE}

The site of Diouboye is located on the banks of the Falémé River, an important tributary of the Senegal River that rises in the Guinea highlands like many of West Africa's perennial streams. Today, this area of eastern Senegal receives circa 800-1000 millimeters of annual rainfall, and is characterized as a riparian zone surrounded by a lightly wooded savanna environment. However, during the site occupation it is likely that the area received greater rainfall, as attested by regional environmental data (see Chapter 3, summary in Dueppen 2012a), in addition to increasing evidence from faunal assemblages in comparable environments elsewhere in West Africa that vegetation zones may have been significantly north of modern distributions during the late-first millennium AD (e.g., the Mouhoun Bend of western Burkina Faso and the Gobnangou of southeastern Burkina Faso) (Dueppen 2008, 2012a; Gallagher 2010).

Diouboye itself is comprised of a linear series of mounds oriented northwest/southeast within one kilometer of the Falémé river. Given the site location relative to the river channel, it is likely that the inhabitants had direct access to the riverine system, as well as surrounding savanna environments to the east of the site. Riverine settings in West Africa can support large amounts of biomass in comparison to drier savanna environments, a favorable setting for productive hunting.

\section{METHODS AND BACKGROUND}

The Diouboye faunal assemblage is one of the largest ever recovered from an Iron Age West African site. Consequently, the results presented are derived from a sampling strategy developed in collaboration with Cameron Gokee to provide a preliminary glimpse of patterns and general socioeconomic strategies. The analyzed sub-sample (henceforth referred to simply as the "sample") has a focus on Mound A, but includes diverse contexts from seven excavation units in different parts of the site. Overall, the sample presented here constitutes circa $8-10 \%$ of the site faunal assemblage. This report focuses on a basic description of the general spectrum of mammalian, avian, reptilian, amphibian, fish and mollusk remains identified, which appear to be consistent across the site based on both the sample and informal spot-checking of bags not included in the sample. Future publications will explore the faunal assemblage in greater detail and with 
more quantitative precision including analyses of element metrics, body-part distributions, cooking techniques, et cetera.

Despite varying depositional contexts, the preservation of bone in the sample is similar from unit to unit. Bones were initially sorted, labeled, and analyzed at the University of Oregon's zooarchaeology laboratory, where preliminary identifications were made. These identifications were then confirmed during a research trip to the Smithsonian Institution, facilitated by the Archaeobiology program. In addition to the use of reference collections, distinctions between similar species were made by employing important published works (Peters 1986a, 1986b; van Neer 1989; MacDonald 1992; MacDonald and MacDonald 2000; Linseele 2007; Zeder and Lapham 2010, Zeder and Pilaar 2010; Dueppen 2011).

Deposits from Diouboye were screened using a $2 \mathrm{~mm}$ mesh and bagged separately according to FS number. The assemblage is highly fragmented, however a large number of bones from the sample could be designated to genus or species level, and many nonidentifiable bones could be assigned to lower level taxonomic levels or placed in a sizeclass using a system similar to those devised by Linseele (2007) and MacDonald (1995). As has been observed in other studies employing size class estimations, patterns in these data tended to mirror those from identified bones, supporting my reconstruction of the general structure of the assemblage. The analysis below is quantified according to NISP (Number of Identified Specimens) for each taxon in each FS number and by excavation unit. The calculation of MNI is currently not possible due to the nature of the sampling program, which in an effort to include a range of contexts often sampled only one FS number from a larger context composed of several FS numbers. Future analyses will include both the NISP and MNI quantifications. Overall, the sample included 442 elements attributed to taxa or size classes, from 54 FS numbers.

\section{THE DIOUBOYE FAUNA}

The faunal assemblage includes some taxa from most terrestrial and aquatic groupings commonly exploited by humans living in the West African savanna, in addition to domestic livestock and dogs (Tables 1 and 2). Ranging from river fish to megafauna, the inhabitants of Diouboye took advantage of the dense biomass found in the local riverine setting. Here I present preliminary description of the faunal spectrum, with information on their preferred habitats and relevant characteristics for reconstructing the Diouboye economy.

\section{Domestic Livestock}

The occupants of Diouboye kept a mixed herd evenly split between sheep/goats and cattle. Any discussion of livestock in a West African region with circa 800-1000 millimeters annual rainfall must consider that the area would have been home to the tsetse fly, and consequently trypanosomiasis (the disease is endemic in the region today) (see discussions in Blench and MacDonald 2000 and Dueppen 2012b). Unless local populations practiced long distance seasonal transhumance, it is likely that Diouboye's community kept livestock with some degree of trypanotolerance. Historically this area was home to the N'dama breed of cattle, a highly resistant longhorn taurine cattle variety that may have great antiquity in western West Africa, although most modern cattle in the region are mixed with more recently introduced indicine breeds (Epstein 1971). Similarly 
resistant small livestock have also been kept in the region, prior to deforestation in the $20^{\text {th }}$ century (Epstein 1971). Future in-depth analyses of livestock elements will provide insights into the breeds used by, and potentially the environmental context for human occupation in the area.

As will be explored below, it is difficult to assess the importance of domestic livestock at Diouboye. They comprise only a small percentage of the overall faunal sample, and are not even dominant among animals in their relative size classes. However, even small numbers of livestock can have a significant impact on lifestyles, and may play an important economic, symbolic and/or political role.

\section{Dogs}

Dogs are a minor component in the Diouboye faunal sample. Positive identification of dogs as distinct from jackals (Canis adustus) and African hunting dogs (Lycaon pictus) was made using MacDonald and MacDonalds' (2000) criteria. Specimens listed as probable dog remains were those of medium canids that couldn't be positively attributed to dog, but were slightly large for jackal and small for African hunting dog.

Dogs are kept in West African communities for a variety of purposes from herding and hunting companions to guarding households. In addition, in many areas dog meat is consumed regularly. All of these uses are possible given the nature of the site and its faunal assemblage, and future analyses will assess whether their remains indicate consumption.

\section{Very Small Duikers}

Several remains of very small duikers were identified at Diouboye. Based on size, these specimens all likely derive from the Red Flanked Duiker (Cephalophus rufilatus), as they would be unusually large for Maxwell's duiker (Cephalophus maxwelli). Red flanked duikers live in close proximity to water in West Africa's forests and wooded savannas, and their ideal habitat includes a mix of forest and moist grasslands with a permanent water source (Happold 1987; Estes 1991; Kingdon 1997). As a result, they tend today to be associated with savanna zones that receive over $1000 \mathrm{~mm}$ of annual rainfall, although they may be found in lower rainfall areas along riverine habitats such as the Falémé.

\section{Small Bovids}

The primary wild taxa in this size class are the bush duiker (Sylvicapra grimmia) and the oribi (Ourebia ourebi). While certain elements of these species can be distinguished (Van Neer 1989), none of the small bovid specimens in the sample are attributable at this level. The bush duiker occurs anywhere in the savanna or forest where there are enough thickets to provide cover (Happold 1987; Estes 1991; Kingdon 1997). Since they do not require regular drinking water, they are rarely found in riverine or gallery forest. Oribis also obtain moisture from vegetation and are rarely found in riverine contexts, but in comparison to the bush duiker they prefer open grasslands in a variety of savanna settings (Happold 1987; Estes 1991; Kingdon 1997). 
The small bovid size class likely also includes the remains of domestic sheep and goats (Ovis/Capra), which were found in relatively equal quantities to Sylvicapra/Ourebia.

\section{Medium Antelopes}

Medium antelopes, including Bushbuck (Tragelaphus scriptus), Reedbuck (Redunca redunca) and Kob (Kobus kob), comprise the largest single group in the Diouboye sample. Bushbucks are distributed widely in the forest, and wooded and grassland savannas in West Africa, but are restricted to habitats near permanent water with a preference for riverine forests (Happold 1987; Estes 1991; Kingdon 1997). Reedbucks are found in both wooded and grassland savannas, but also tend to be restricted to areas within $3 \mathrm{~km}$ of permanent water (Happold 1987), and prefer riverine settings with marshes and swamps (Happold 1987; Estes 1991; Kingdon 1997). Similarly, kobs inhabit wooded and grassland savannas within 3-6 km of permanent water (Happold 1997), and also prefer riverine forests with marshes and swamps (Happold 1987; Estes 1991; Kingdon 1997). This common emphasis on riverine environmental settings has significant implications for Diouboye hunting practices (see discussion below).

\section{Large Antelopes}

Two large antelope taxa were identified in the Diouboye assemblage, the Waterbuck (Kobus ellipsiprymnus) and the Roan Antelope (Hippotragus equinus). Similar to several of the medium antelopes, waterbuck inhabit wooded savannas within $10 \mathrm{~km}$ of permanent water (Happold 1987), and prefer riverine contexts with marshes and swamps (Happold 1987; Estes 1991; Kingdon 1997). The Roan Antelope is broader in its ecology, inhabiting wooded and grassland savannas (Happold 1987; Estes 1991; Kingdon 1997). Although generally found within $15 \mathrm{~km}$ of permanent water, they are less restricted in locale, as they browse, obtaining some water from leaves (Happold 1987). During the dry season they will enter marshes to feed (Happold 1987; Estes 1991).

The large antelope/bovid size class likely also includes the remains of domestic cattle (Bos), which were found in slightly higher quantities than the wild identified taxa.

\section{Very Large Bovids}

Several elements were identified as African Buffalo (Syncerus caffer), a very large bovid found in both forests and savannas in West Africa (Happold 1987; Estes 1991; Kingdon 1997). Buffalo require shade, green grass and daily water, and are consequently restricted to within five kilometers of permanent water in the dry season (Happold 1987), but are more widely distributed in the wet season (Happold 1987; Estes 1991). They have a strong preference for swamps and marsh environments.

\section{Suids}

Warthogs (Phacochoerus africanus) are the only suid identified in the Diouboye assemblage. They are hardy animals that can occur in a wide variety of environmental settings including fairly dry sahelian zones. However, they do prefer to be close to permanent water and tend to be found in floodplains and riverine forests, since they often graze grasses (Happold 1987; Estes 1991; Kingdon 1997). 


\section{Megafauna}

Two very large mammals were identified in the Diouboye assemblage, the Hippopotamus (Hippopotamus amphibius) and the Elephant (Loxodonta africana). The hippo is widespread in permanent rivers and lakes throughout Africa, as their main requirements are deep stretches of water to help them maintain their internal temperature, and adequate grass on the shoreline (Happold 1987; Estes 1991; Kingdon 1997). Elephants are less restricted to a particular environmental setting and while requiring water daily, they do range widely. Elephants may frequent riverine and swamp areas, when available, to feed and cool down (Happold 1987; Estes 1991; Kingdon 1997). These animals are unusual finds for a West African faunal assemblage, as they have rarely been identified at other sites.

\section{Felids}

Three different felids from different size classes were identified at Diouboye, the wildcat (Felis sylvestris), serval cat (Felis serval), and leopard (Panthera pardus). The wildcat is today found throughout sub-Saharan Africa, and their diet is extremely diverse (Happold 1987; Estes 1991; Kingdon 1997). Serval cats are common in wooded and grassland savanna, but are particularly abundant in riverine areas (Rosevear 1974; Happold 1987; Estes 1991; Kingdon 1997). They also consume a diverse diet, including duikers and oribis (Happold 1987), and may prey on domestic small stock. The Leopard is extremely widely distributed in sub-saharan Africa; all they require is broken terrain and heavy vegetation as stalking cover. They consume small to medium sized mammals (including possibly domestic animals), and often frequent riverine settings as the abundance of prey makes them excellent hunting grounds (Happold 1987; Estes 1991; Kingdon 1997). Felid remains are distributed throughout the site and would appear to have been regularly killed, possibly for protection of people and livestock, but also for their skins.

\section{Canids}

Two wild canids were found at Diouboye, the Side-Striped Jackal (Canis adustus) and the African hunting dog (Lycaon pictus). The jackal is an omnivorous canid that inhabits the wooded and grassland savannas, and is rarely found in the forest (Happold 1987; Estes 1991; Kingdon 1997). The larger African hunting dog is also found throughout the savannas of Africa. Pack hunters, they consume exclusively mammals, and tend to target medium-sized antelopes (Happold 1987; Estes 1991; Kingdon 1997). It is not known if either species commonly attacks livestock.

\section{Mongooses}

Several remains attributable to Mongooses (Herpestidae) have been identified. These will be studied in more detail in the future. More specific identification of the mongoose assemblage from Diouboye will be particularly valuable for environmental reconstruction, as mongooses vary greatly according to taxon in their habitats and diets.

\section{Small Mammals}

Two groups of small mammals have been identified in very low numbers at Diouboye, Gambian rats (Cricytomys gambianus) and Scrub hares (Lepus saxatilus). 
Gambian rats are omnivorous large rodents that live in all environmental zones in West Africa (Happold 1987; Estes 1991; Kingdon 1997). Scrub hares are widespread in savanna and farmland, as long as there is some dense vegetation in which to hide (Happold 1987; Estes 1991; Kingdon 1997). Both of these animals can cause damage to crops, and may be hunted in garden or field settings.

\section{Birds}

There are comparatively few avian remains in the Diouboye sample, both relative to the number of mammals overall and relative to small mammals. While differential preservation is a possibility, the identified avian remains are in excellent condition and a general pattern of poor preservation of birds appears unlikely. While one element from an unknown waterfowl was identified, the vast majority of bird remains are from galliforms.

As discussed elsewhere (MacDonald 1992; Linseele 2007; Dueppen 2011), there are three types of large galliform birds in West Africa, the smallest is the francolin (Francolinus sp.) and the largest is the Guinea Fowl (Numida meleagris), which occurs in both domestic and wild varieties. These two galliforms do not tend to overlap in size. However, the third galliform bird, the domestic chicken (Gallus gallus), is of medium size and can overlap with large francolins and small guinea fowl. The galliform remains from Diouboye tend to be of two discrete sizes comparable to francolins and guinea fowl, suggesting two populations rather than the continuum of sizes expected when chickens are present alone or in combination with either or both other galliform species.

Confirmation in this assessment is complicated by the fact that only certain elements can be used to positively distinguish these three birds when overlapping in size (the single conclusively identifiable element was of a guinea fowl in the large size class). The domestic status of guinea fowl at Diouboye is open to question, and some sites in the middle Senegal valley have produced large numbers of potentially domestic guinea fowl (MacDonald and MacDonald 2000), although no standard criteria differentiate the domestic and wild varieties. Avian eggshell is another potential line of evidence (see Dueppen 2011), but to date none has been recovered from Diouboye. Overall, data do not indicate the presence of either domestic fowl (chicken or guinea fowl) at Diouboye.

\section{Reptiles and Amphibians}

Three aquatic reptiles and amphibians were of some importance to the Diouboye economy- Crocodiles, Monitor Lizards (Varanus niloticus), and the African Softshell Turtle (Trionyx triunguis). Two crocodile genera appear in West Africa, Crocodylus and Osteolaemus (dwarf crocodiles). While dwarf crocodiles can reach 5 feet in length, some of the identified remains are from larger individuals exceeding this size, and are more likely derived from Crocodylus (either C. niloticus or C. cataphractus) (Villiers 1958). There are two species of monitor lizard in West Africa, of which Varanus niloticus is the most likely, since it prefers areas with rich vegetation and close to water (Mertens 1942). The African Softshell Turtle is confined to large permanent bodies of water, and can grow to fairly great size (Villiers 1958). All of these animals are expected residents of the Falémé River, particularly if it was flowing at sufficient volume to support hippos. 


\section{Mollusks}

Two types of freshwater bivalve have been identified at Diouboye. The first group is likely one of two genera of the family Mutelidae, Chambardia and Spathopsis, given their size and shell morphology (Van Damme 1984). Of these, Chambardia is more likely given Diouboye's location relative to the Falémé, as this genus prefers river settings, while Spathospsis is more commonly found in lakes. The second group can be attributed to the distinctive Nile Oyster (Etheria elliptica), which occurs in permanent rivers and lakes (Van Damme 1984). Neither bivalve is common in the assemblage, and it is unlikely freshwater mollusks were a significant component of the Diouboye economy. One landsnail of the family Achatinidae was identified as well. These snails prefer disturbed soils and moist swamp contexts, and are often consumed by people (Crowley and Pain 1970).

\section{Fish}

Fish remains were identified in only a few depositional contexts and were not particularly numerous for a settlement located so close to a major river. The fish that were caught were very large individuals, and these could have been captured with hunting tools, as part of hunting expeditions that included the large reptiles and amphibians. The most common, and largest, taxa were Lates niloticus, a main river channel fish (Daget 1954), and Synodontis sp., a catfish that also prefers the main river channel (Daget 1954; Welcomme and de Merona 1988). Several large Clarius sp. catfish were identified, that owing to their size would likely occupy the main river channel. The other, less common, remains also attest to opportunistic fishing in open water, including large individuals of Auchenoglanis sp., Bagris sp., Arias gigas, and Chrysichthys sp. (see Daget 1954).

\section{INTERPRETING THE FAUNAL ASSEMBLAGE}

The inhabitants of Diouboye kept domestic sheep/goats and cattle, and it is likely, unless engaged in extensive seasonal transhumance, that the breeds kept were to some degree trypanotolerant given the local environment. Interestingly, preliminary analyses of the livestock data indicate a focus on cattle, with a fairly even 1:1 ratio between large and small stock (in a balanced system, small stock generally occur in greater numbers within the herd and are slaughtered more frequently). The nature of the domestic assemblage will be better understood with continuing analyses to investigate which breeds were kept.

Despite the use of domestic livestock, the analyzed faunal assemblage is dominated by extensive and intensive hunting in the riverine zone around the Falémé. Medium-sized antelopes that are most common in riverine contexts were the largest component of the Diouboye economy, and account for three times the NISP as sheep/goats (and given their larger size, a much higher quantity of meat/skins/etc.). Although less common than medium antelopes, larger bovids typically found in marshes, such as waterbuck and African Buffalo were identified, as were Roan Antelope and African Elephant who seasonally graze and browse near rivers. Hippopotamus, a large aquatic mammal that cannot survive outside riverine environments was hunted and is found in multiple contexts. The focus on the riverine zone is also apparent in the relatively smaller numbers of small wild bovids that can survive without access to drinking water and are less commonly found in marsh/riverine contexts. 
Many of the identified carnivores, particularly the felids (Serval cats and Leopards), but also the African Hunting Dog (since it prefers medium Antelopes) also frequent marshes and other riverine contexts, and it is likely that some of these were killed during hunting expeditions, and possibly even for their skins. However, carnivores may have also been killed to prevent damage to domestic livestock herds. Although a systematic study has not yet been made, minimal carnivore damage is apparent in the Diouboye assemblage.

Mammalian riverine resources were supplemented by modest quantities of reptiles, amphibians and fish, some of great size. Ranging from crocodiles, large turtles, to enormous specimens of fish like Lates niloticus these animals represent a significant contribution of meat into the economy. In the case of crocodiles, meat may have been a secondary benefit as they may have been hunted to protect people and livestock. In comparison to large specimens and taxa found in riverine contexts, the Diouboye assemblage indicates a much less intensive exploitation of smaller-sized fish, mammals, and freshwater bivalves that would have been common in the area given the general faunal spectrum.

On the basis of the analyzed sample, Diouboye residents were targeting medium and large animals that represent significant carcass weight, rather than engaging in the intensive collection and hunting of smaller taxa that sometimes require larger infrastructural/technological input (e.g., basketry, fishing tackle, etc.). However, given the demonstrated richness of the riverine zone, these practices may not have been necessary.

At a broader level, domestic resources represent a much smaller contribution to subsistence than wild resources. This lack of emphasis on domesticates at the site might extend to farming, as the Diouboye faunal spectrum has very little evidence of garden hunting in comparison to contemporary sites in other parts of the West African savanna (see Dueppen 2008, 2012a; Gallagher 2010), although this hypothesis needs to be evaluated using other classes of evidence. Finally, any discussion of relative importance of resources at Diouboye requires the caveat that full analysis of the assemblage may result in significant revision of these patterns.

\section{ENVIRONMENTAL RECONSTRUCTION}

As described in detail above, the Diouboye faunal assemblage is primarily derived from a riverine zone and consequently has limited utility for reconstructing the surrounding environment from the species represented. Riverine contexts in West Africa are frequently home to a similar range of species regardless of whether they are located in wooded savanna, grassland savanna, or even sahelian zones. However, the late-first and at least the first century of the second millennium AD in West Africa is thought to have been significantly more humid, as has been indicated by the presence of forest margin species in contemporary assemblages from Burkina Faso (see Dueppen 2008, 2012a; Gallagher 2010).

At Diouboye, the presence of the Red Flanked Duiker, although infrequent, may indicate a more humid setting in eastern Senegal at the time. In addition, the large size of the fish in the Diouboye assemblage and the common presence of large aquatic animals may indicate that the Falémé was more substantial at the time of site occupation than it is 
today, likely also indicating wetter conditions. However, river flow is affected by rainfall over the entire catchment, and may not be indicative of local rainfall.

\section{CONCLUSION}

While results are preliminary and analyses on-going, faunal analyses from Diouboye have already revealed a dynamic riverine focus in addition to domestic animal use in this ancient community. Interestingly, the hunting and herding patterns identified appear to be generally consistent throughout the settlement, a remarkably niche-focused strategy for a West African site. As mentioned above, only 8-10\% of the assemblage has been analyzed to date, and the project when complete, will enable us to address myriad social, political, and economic questions in an area where few detailed archaeozoological data sets are available.

\section{Acknowledgements}

The research presented in this paper was primarily funded by an ACLS New Faculty fellowship to the author, with the support of the Andrew W. Mellon Foundation. Additional funds to visit comparative collections housed at the Smithsonian Institution were provided by an NSF Doctoral Dissertation Grant to Cameron Gokee (University of Michigan). Analyses were performed at the University of Oregon's Zooarchaeology Laboratory, and I thank Dr. Madonna Moss for providing space and access to comparative collections. Andrea Eller (University of Oregon) helped sort and label bones prior to transport to Washington D.C., and I appreciate her participation. At the Smithsonian I thank Dr. Melinda Zeder for providing space in the Archaeobiology laboratory and for facilitating access to the comparative collections. Lastly, I thank Cameron Gokee for initiating this fruitful collaboration on the archaeology of Diouboye and both Cameron Gokee and Dr. Ibrahima Thiaw (IFAN- Dakar) for coordinating the export of faunal remains for analysis. 


\section{BIBLIOGRAPHY}

Blench, R. M. and K. C. MacDonald, eds.

2000 The Origins and Development of African Livestock: Archaeology, Genetics, Linguistics, and Ethnography. UCL Press, London.

Crowley, T.E. and Pain, T.

1970 A monographic revision of the Arican landsnails of the genus Limicolaria, Schumacher. (Mollusca-Achatinidae), Annales du Musée Royal de l'Afrique Centrale, Sciences Zoologiques 177, Tervuren

Daget, J.

1954 Les Poissons du Niger Superieur. Memoires de l’Institut Français d'Afrique Noire 36, Dakar.

Dueppen, S.

2008 Reinventing Equality: The Archaeology of Kirikongo, Burkina Faso. Ph.D. dissertation, Department of Anthropology, University of Michigan.

2011 Early Evidence for Chickens at Iron Age Kirikongo (ca. AD 100-1450), Burkina Faso. Antiquity 85:142-157

2012a Egalitarian Revolution in the Savanna. Equinox Press; Sheffield

2012b Cattle in the West African Savanna: Evidence from $1^{\text {st }}$ millennium CE

Kirikongo, Burkina Faso. Journal of Archaeological Science 39:92-101

Epstein, $\mathrm{H}$.

1971 The Origin of the Domestic Animals of Africa. Africana Publishing, New York.

Estes, R.D.

1991 The Behavior Guide to African Mammals. University of California Press: Berkeley

Gallagher, D.

2010 Farming Beyond the Escarpment: Society, Environment, and Mobility in Precolonial Southeastern Burkina Faso. PH.D. dissertation, Department of Anthropology, University of Michigan

Happold, D.C.D.

1987 The Mammals of Nigeria. Clarendon Press, Oxford.

Kingdon, J.

1997 The Kingdon Field Guide to African Mammals. Academic Press Limited, London.

Linseele, V.

2007 Archaeofaunal Remains from the Past 4000 Years in Sahelian West Africa.

BAR International Series 1658, Oxford.

MacDonald, K.C.

1992 The Domestic Chicken (Gallus gallus) in Sub-Saharan Africa: A Background to its Introduction and its Osteological Differentiation from Indigenous Fowl (Numidinae and Francolinus sp.) Journal of Archaeological Science 19:303318. 
MacDonald, K.C.

1995 Analysis of the Mammalian, Avian, and Reptilian Remains. In Excavations at Jenné-Jeno, Hambarketolo, and Kaniana (Inland Niger Delta, Mali), The 1981 Season. edited by S. McIntosh, pp.291-318. University of California Press: Berkeley.

MacDonald, K.C. and R. H. MacDonald

2000 The Origins and Development of Domesticated Animals in Arid West- Africa. In The Origins and Development of African Livestock: Archaeology, Genetics, Linguistics, and Ethnography, edited by R. M. Blench and K. MacDonald, pp. 127-162. UCL Press, London.

Mertens, R.

1942 Die Familie der Warane (Varanidae). Abhandlungen der Senckenbergischen Naturforschenden Gesellschaft 462, 465, 466: 1-391

Peters, J.

1986a Osteomorphology and Osteometry of the Appendicular Skeleton of Grant's Gazelle, Gazella granti (Brooke, 1872), Bohor Reedbuck, Redunca redunca (Pallas, 1767) and Bushbuck, Tragelaphus scriptus (Pallas, 1766). Occasional papers, Laboratorium voor Paleontologie, Ghent University, Ghent 2.

1986b Osteomorphology and Osteometry of the Appendicular Skeleton of African Buffalo, Syncerus caffer (Sparrman 1779) and Cattle, Bos primigenius $\mathrm{f}$. taurus (Bojanus 1827). Occasional papers, Laboratorium voor Paleontologie, Ghent University, Ghent 1.

Rosevear, D.R.

1974 The Carnivores of West Africa, Trustees of the British Museum (Natural History), London

Van Damme, D.

1984 The Freshwater Mollusca of Northern Africa: Distribution, Biogeography and Palaeoecology. Dr. W. Junk, Dordrecht.

Van Neer, W.

1989 Contribution to the Archaeozoology of Central Africa. Annales Sciences

Villiers, A.

Zoologiques 259, Musée Royal de l’Afrique Centrale, Tervuren.

1958 Tortues et Crocodiles de l'Afrique Noire Francaise. Initiations africaines, Institut Francais d'Afrique Noire, Dakar

Welcomme R.L. and de Merona, B.

1988 Fish Communities of Rivers. In Biology and Ecology of African Freshwater Fishes, edited by C. Lévêque, M. N. Bruton and G. W. Ssentongo, pp. 251276. ORSTROM, Paris.

Zeder, M. and H. Lapham

2010 Assessing the reliability of criteria used to identify postcranial bones in sheep, Ovis, and Goats, Capra. Journal of Archaeological Science 37:2887-2905.

Zeder, M. and S. Pilaar

2010 Assessing the reliability of criteria used to identify mandibles and mandibular teeth in sheep, Ovis, and goats, Capra. Journal of Archaeological Science 37:225-242. 


\begin{tabular}{|c|c|c|c|c|c|c|c|c|c|c|c|c|c|c|c|c|}
\hline & है & ป & ণิ & $N$ & $\sim$ & - & $\approx$ & $\approx 0$ & $\theta \approx$ & ก & ల) & $\mathrm{No}$ & $m \infty$ & $\triangle$ & $m \mathrm{~m}$ & $=$ \\
\hline & $\stackrel{U}{:}$ & ナ & $m$ & $\sim$ & & & $\operatorname{in} 0$ & $a-$ & - & 0 & $\simeq$ & - & - & - & & - \\
\hline & 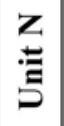 & - & $\nabla$ & & & & & & $N$ & $m$ & -1 & & & & & \\
\hline & 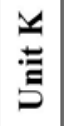 & & & & & & & - & - & & & & & & - & \\
\hline & 壱 & - & & - & - & & $m$ & & $m$ & - & $N$ & & & - & & \\
\hline & $\underset{5}{\mathbb{Z}}$ & m & $m$ & & & & -1 & $r ल$ & $m+$ & $N$ & $m$ & & -1 & -1 & - & - \\
\hline & 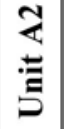 & $\cong$ & $r$ & $-N$ & - & - & $\cong=$ & $\simeq \cong$ & $=\infty$ & 0 & $\simeq$ & -7 & $-r$ & $\infty$ & $-N$ & $\infty$ \\
\hline 恶 & 导 & & $m$ & $\sim$ & & & -5 & $0 / 7$ & $N-$ & A & 0 & & & - & - & - \\
\hline 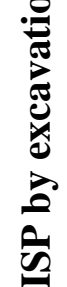 & 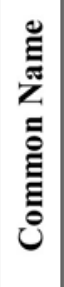 & 惫 & 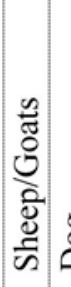 & 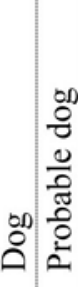 & 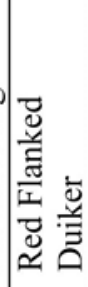 & 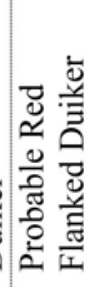 & 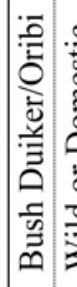 & 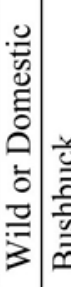 & 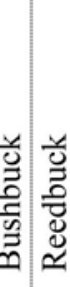 & $\begin{array}{l}0 \\
0 \\
2\end{array}$ & $\frac{7}{3}$ & : & 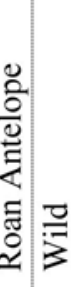 & 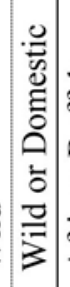 & 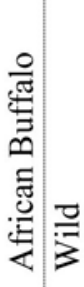 & 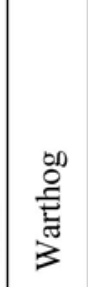 \\
\hline 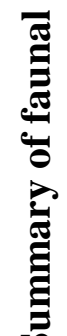 & 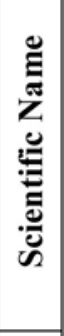 & $\mid$\begin{tabular}{c|}
$n$ \\
$\vdots$ \\
$\vdots$ \\
$\vdots$ \\
$\vdots$ \\
$\vdots$ \\
\hdashline \\
\end{tabular} & : & 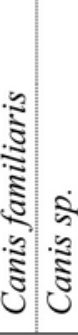 & 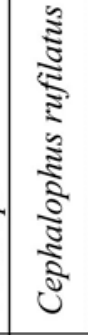 & 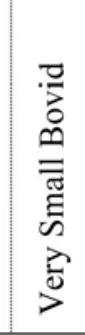 & 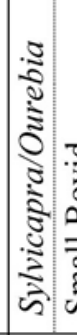 & च & 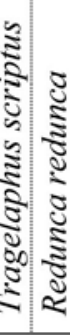 & $\mid \begin{array}{l}\mid \\
0 \\
0 \\
0 \\
0 \\
0 \\
0 \\
0\end{array}$ & 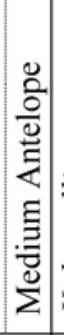 & 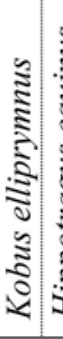 & 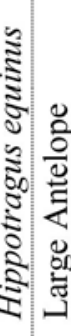 & 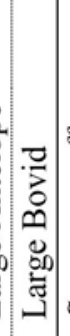 & 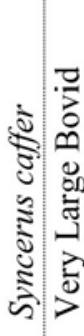 & 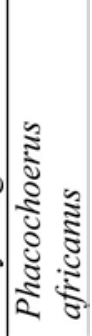 \\
\hline 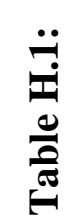 & 照 & \multicolumn{3}{|c|}{ 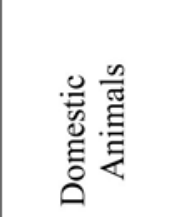 } & \multicolumn{2}{|c|}{ 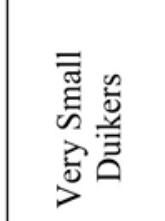 } & \multicolumn{2}{|c|}{ 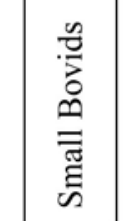 } & \multicolumn{3}{|c|}{ 害亭 } & \multicolumn{3}{|c|}{ 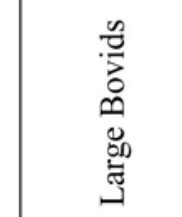 } & 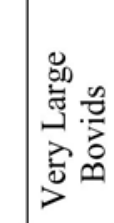 & $\stackrel{n}{\vec{y}}$ \\
\hline
\end{tabular}




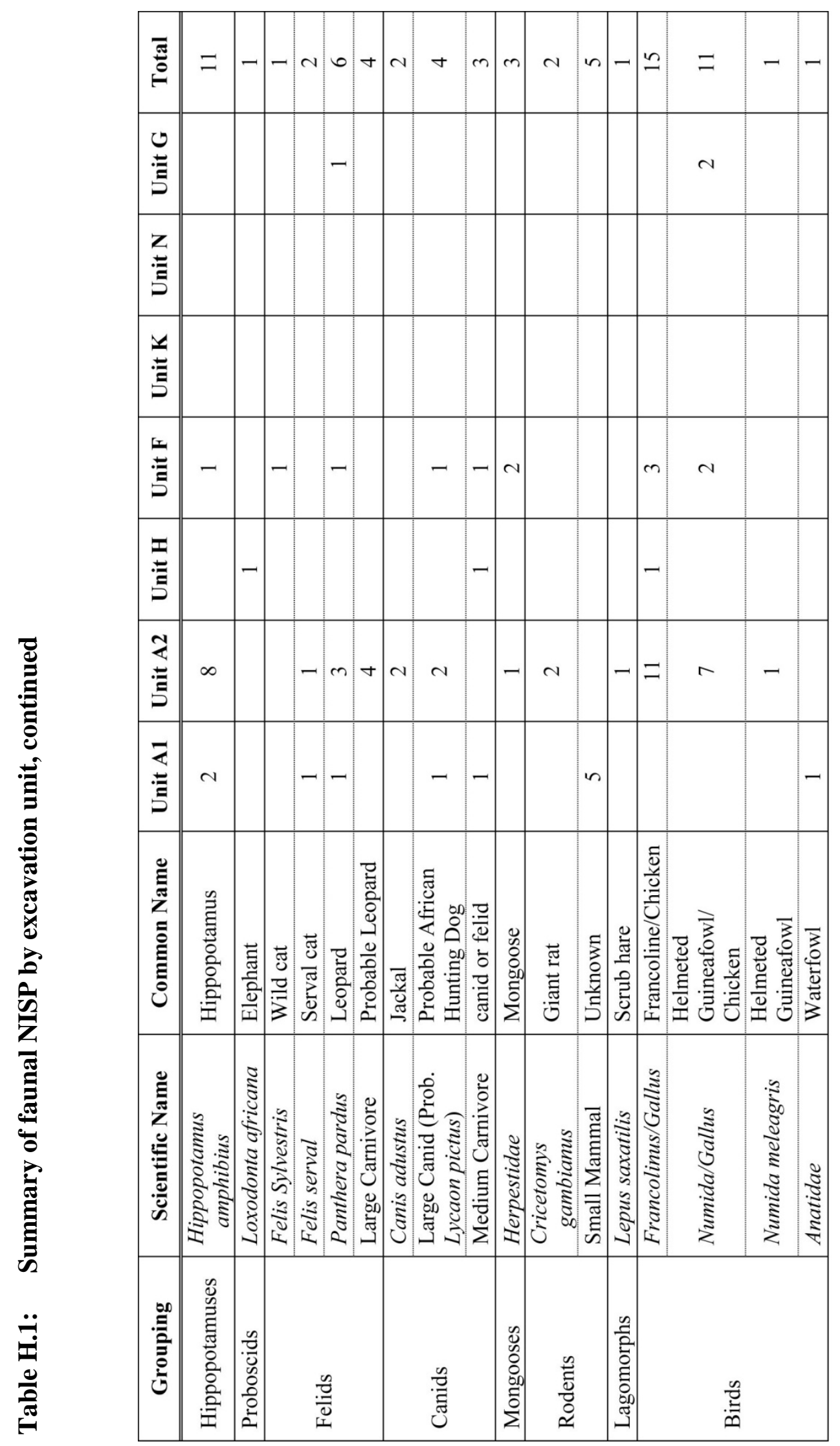




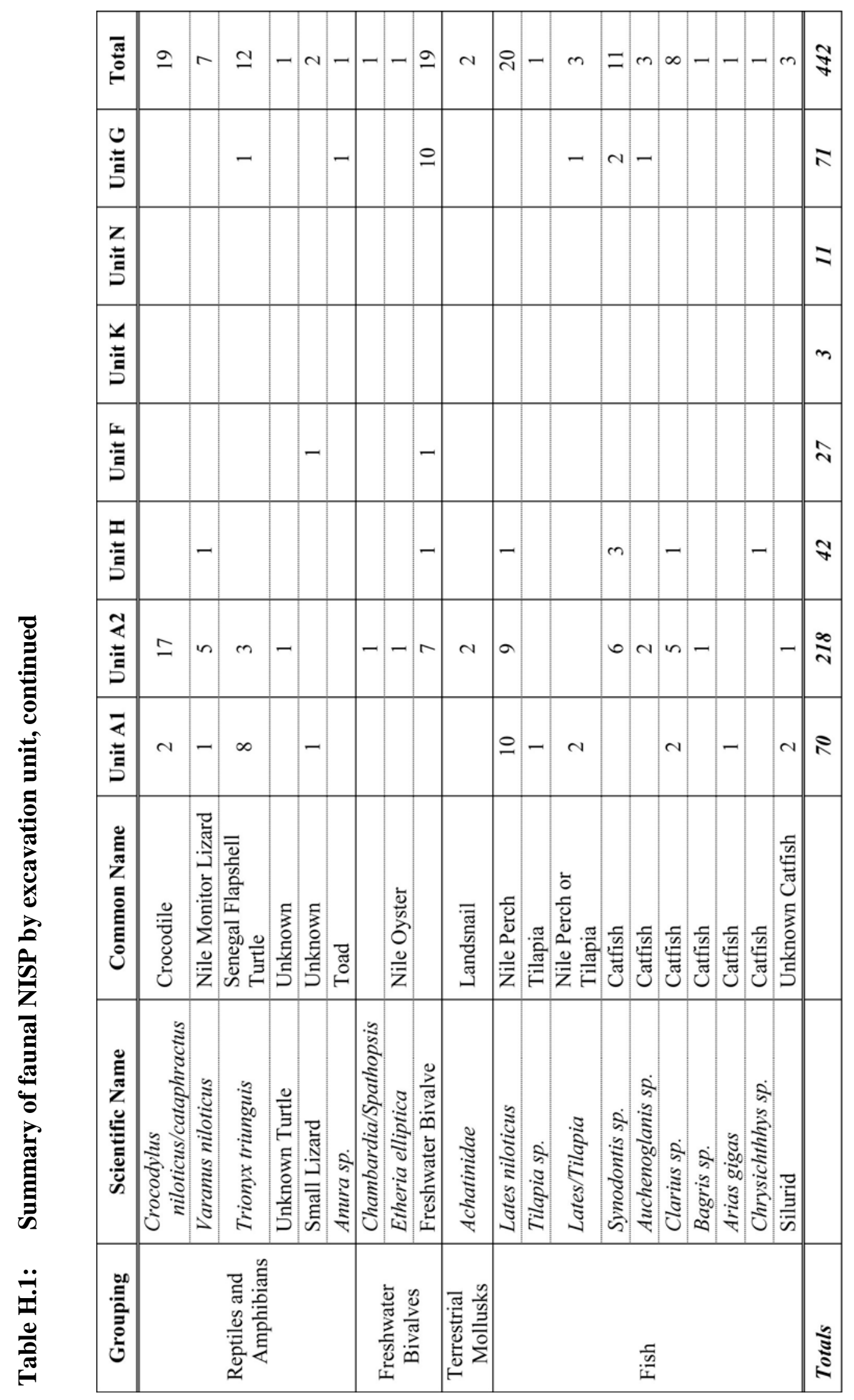




\title{
APPENDIX I \\ PRELIMINARY RESULTS OF MACROBOTANICAL ANALYSIS OF SEEDS AND FRUITS FROM DIOUBOYE
}

\author{
by \\ Daphne Gallagher \\ University of Oregon
}




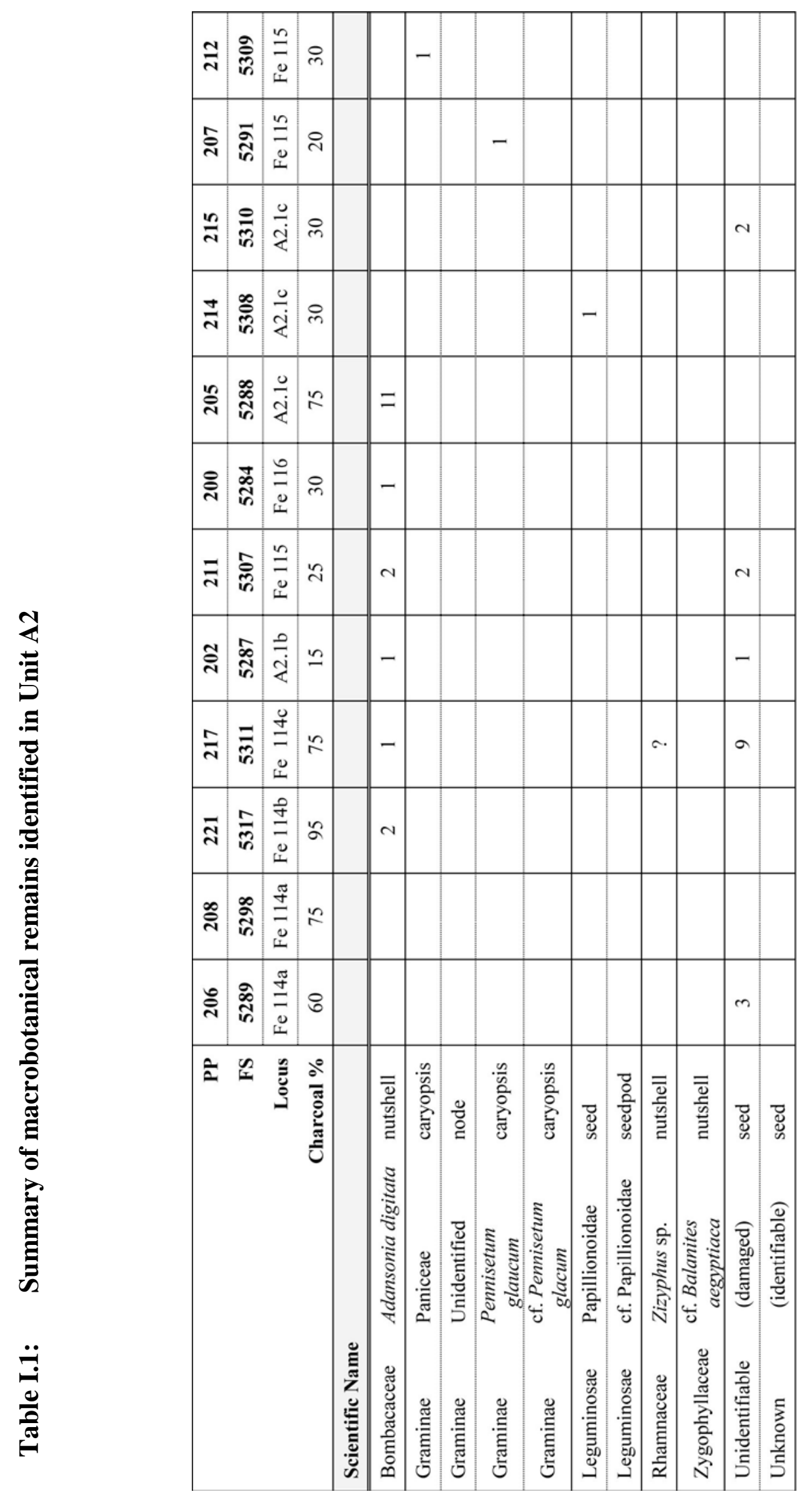




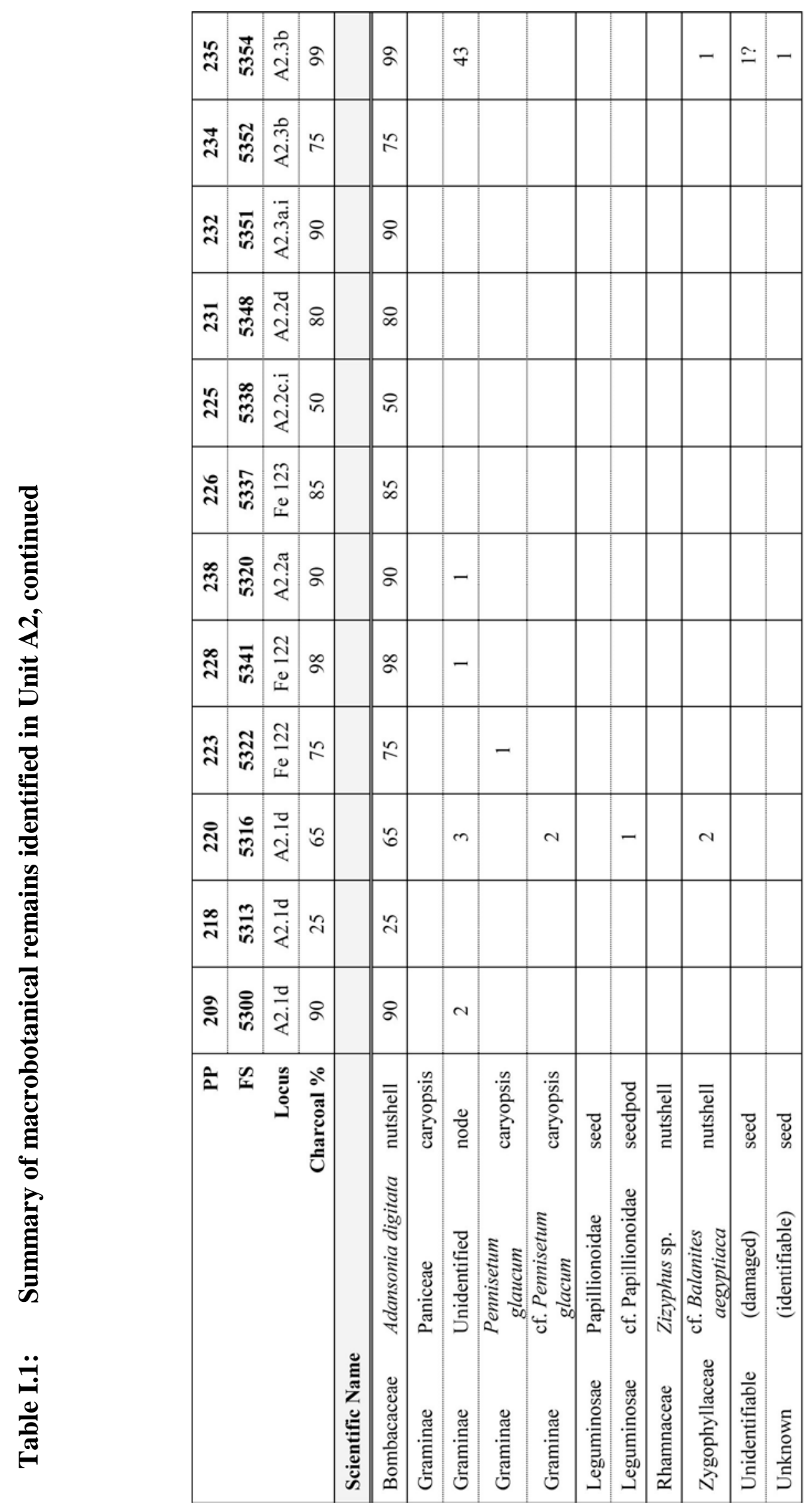




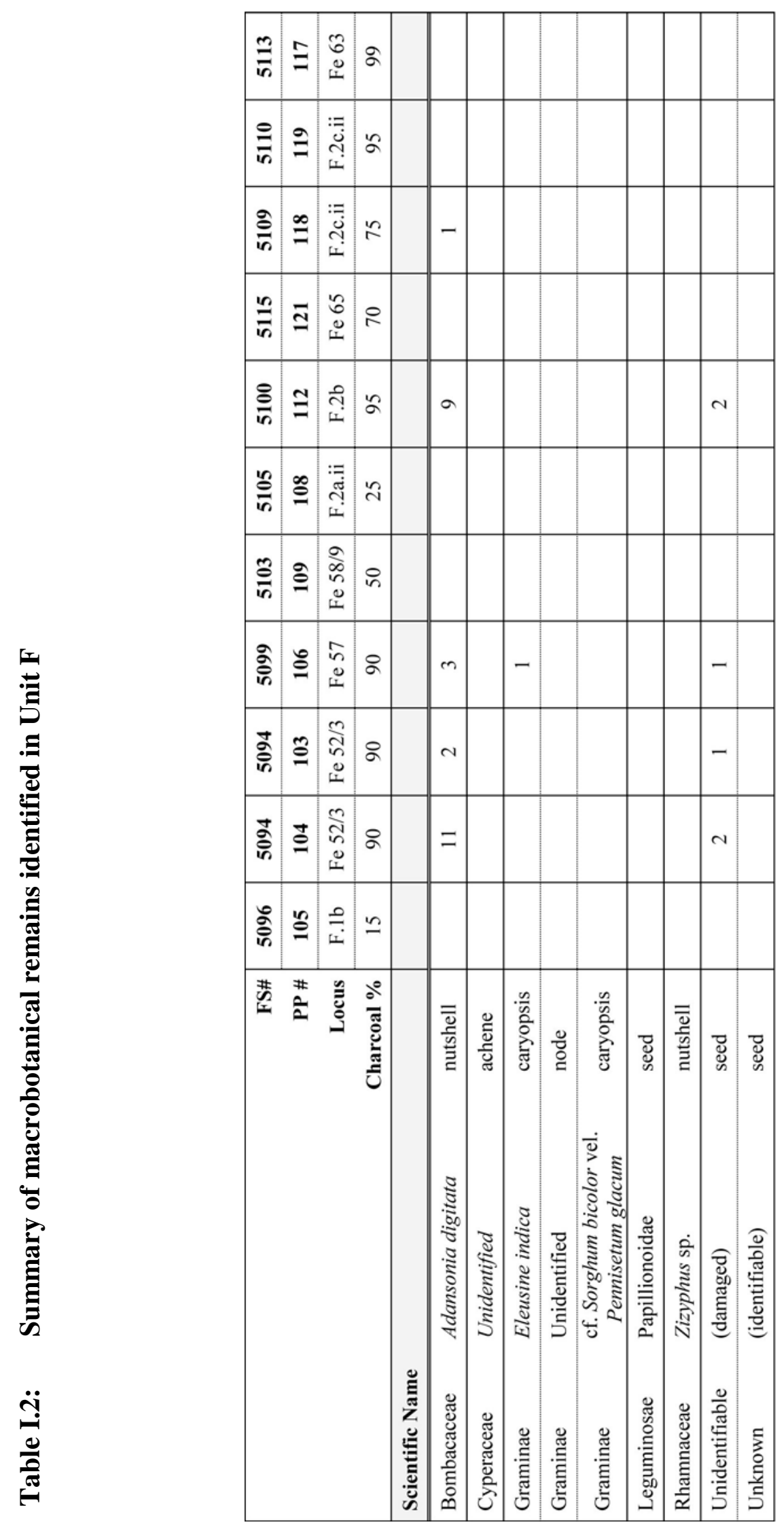




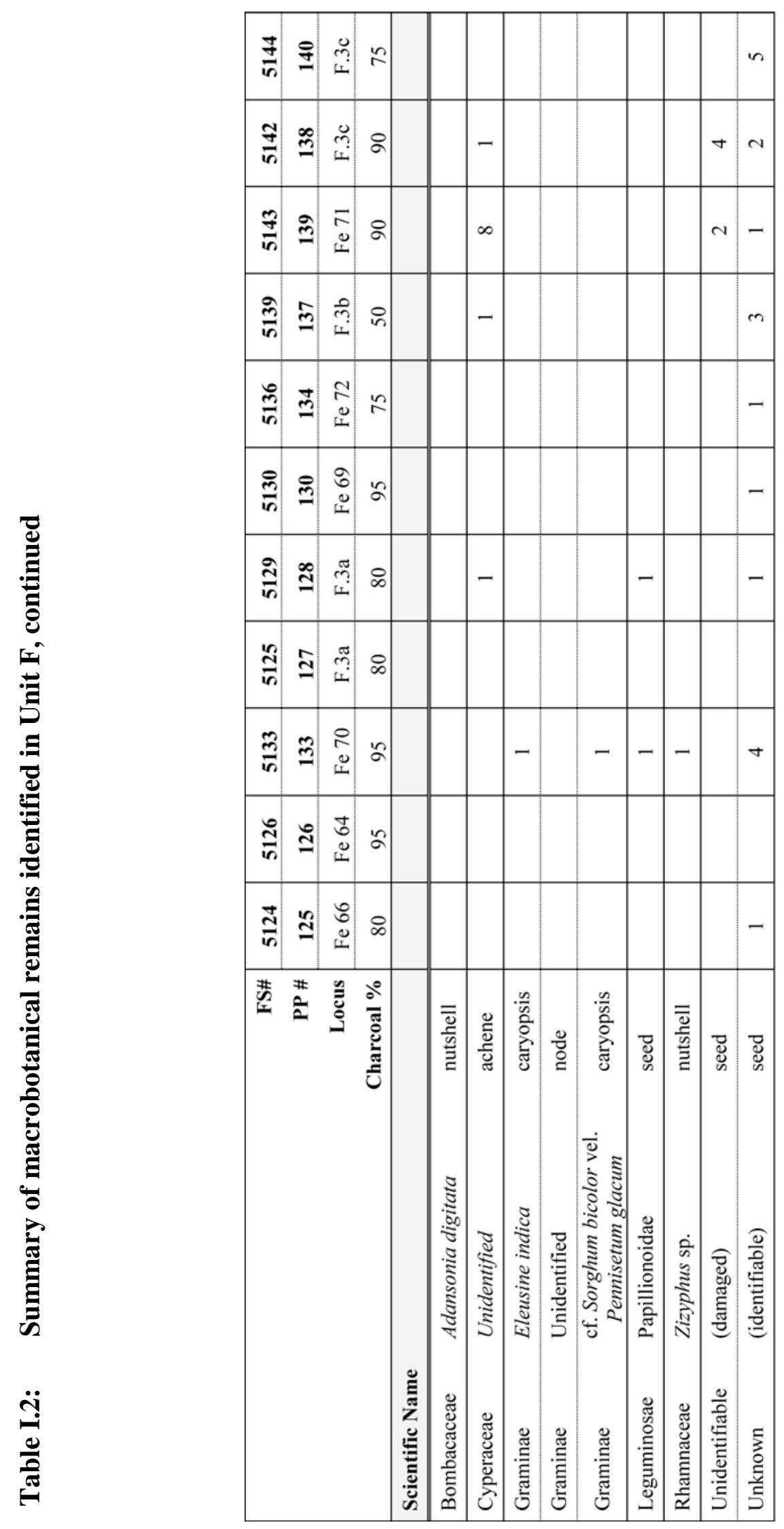




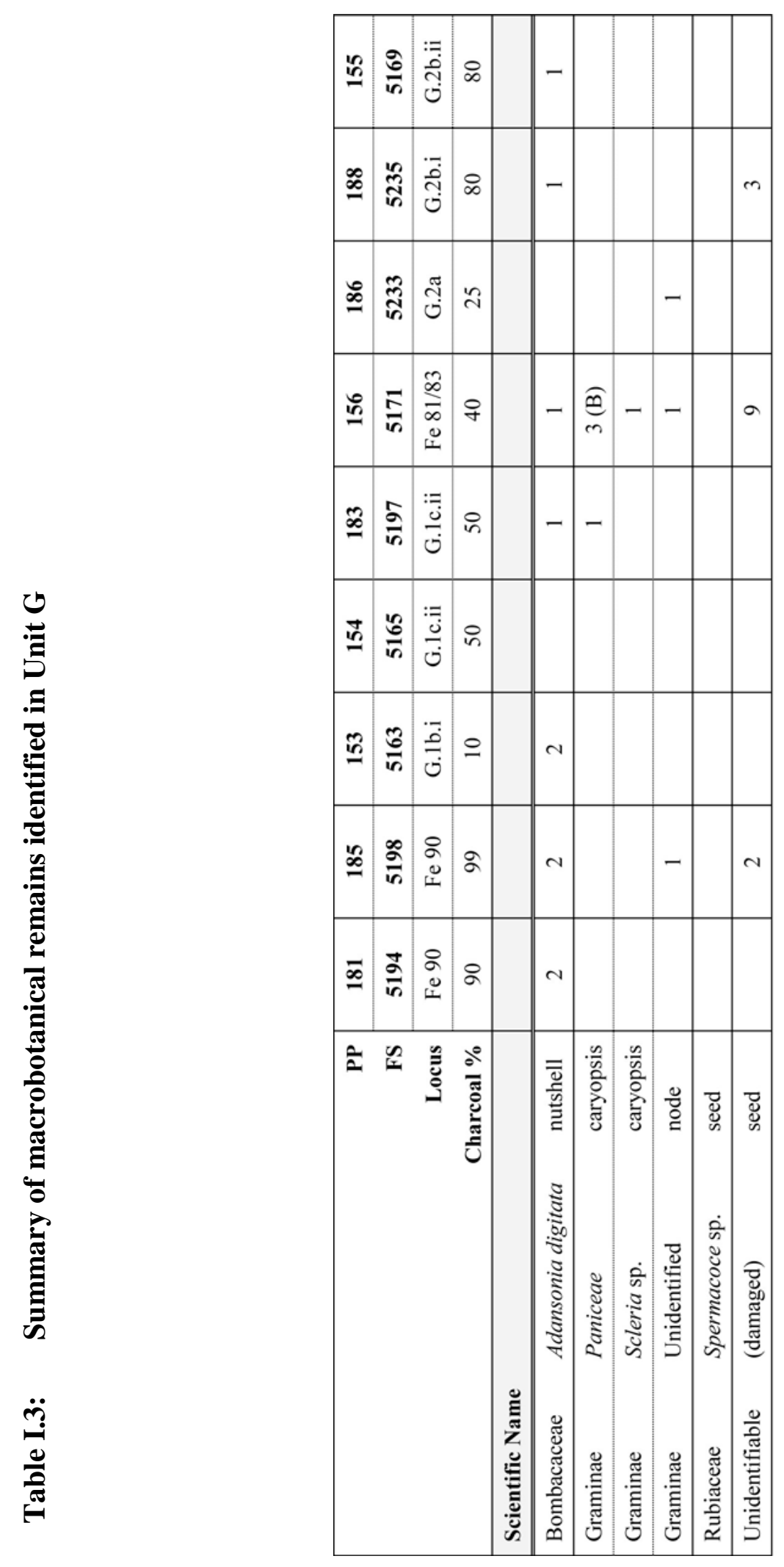




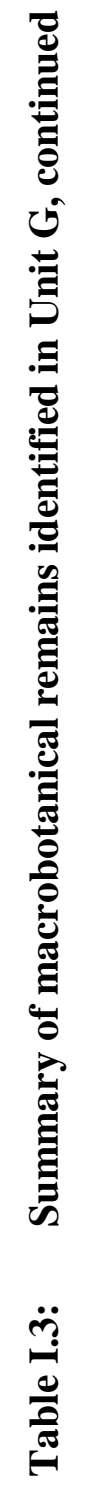

\begin{tabular}{|c|c|c|c|c|c|c|c|c|c|}
\hline$\stackrel{\infty}{\sigma}$ & 孛 & $\begin{array}{c}\mathscr{m} \\
\tilde{S}\end{array}$ & 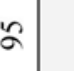 & $\infty$ & & & & & $r$ \\
\hline 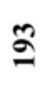 & 竞 & 仓ే & in & & & & & & \\
\hline$\hat{\jmath}$ & : & वे & 2 & $\sim$ & & & & & \\
\hline$\stackrel{2}{\cong}$ & : & $\begin{array}{ll}8 \\
0\end{array}$ & 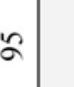 & - & & & & - & \\
\hline$\stackrel{\infty}{10}$ & $\frac{m}{i n}$ & 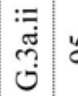 & 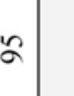 & - & & & & & $\mathrm{N}$ \\
\hline$\Xi$ & 亲 & 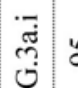 & $\approx$ & & & & & & r \\
\hline ळ & $\frac{\infty}{n}$ & 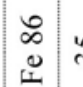 & $m$ & & & & & & \\
\hline$\Xi$ & : & $\begin{array}{ll} \\
0 \\
0 \\
0\end{array}$ & in & & & & & & \\
\hline & 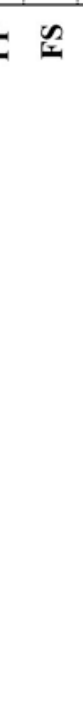 & 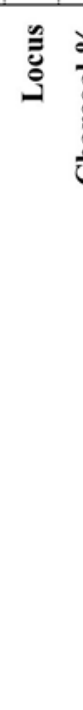 & 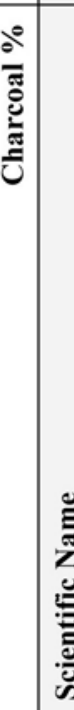 & 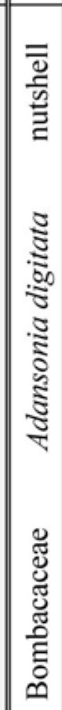 & 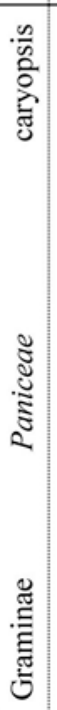 & 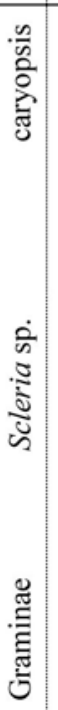 & 总 & 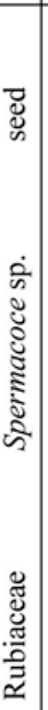 & 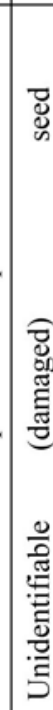 \\
\hline
\end{tabular}

